\title{
ORAL SESSIONS
}

Wednesday, 17 May 2017

\section{OS1 - Psychology \& Behaviour}

\author{
OS1:OC19 \\ Is food addiction a singular and distinct entity from binge \\ eating? \\ Long, C. ; Blundell, J.' ; Finlayson, G. ${ }^{1}$ \\ 'University of Leeds
}

Introduction: The concept of 'food addiction' (FA) has stimulated a surge in research and debate in the scientific literature recently. Much research depends on the use of the Yale Food Addiction Scale (YFAS) to categorise individuals as 'food addicts'. A systematic review found that YFAS 'diagnoses' are consistently associated with binge eating, warranting investigation into whether the YFAS is able to identify any unique 'addiction-like' qualities beyond those already accounted for by the Binge Eating Scale (BES). Methods: A large cross-sectional study of male and female UK adults (N: 667, minimum age: 18, mean: 26.27, SD: 11.05) was conducted between April 2015 and March 2016. The questionnaires measured a range of eating-related behaviours, consumption of alcohol and drugs, addictive behavioural traits and psychological wellbeing, including the Control of Eating Questionnaire, Power of Food Scale, Three Factor Eating Questionnaire (disinhibition subscale), Eating Disorder Examination (restraint subscale), Alcohol Use Disorders Identification Test, Drug Abuse Screening Test, Addiction Prone Personality Scale, Beck Depression Inventory, World Health Organisation Quality of Life, State-Trait Anxiety Inventory and the Perceived Stress Scale.

Results: $7.6 \%$ of the sample met the YFAS criteria for 'food addiction' $(\mathrm{N}=51)$ whilst the mean 'symptom count' was 1.88 (SD: 1.45). YFAS 'symptom count' correlated most strongly with BES $(\mathrm{R}=0.66)$. The BES correlated as strongly as YFAS with all other measures of eating pathology, addictive personality and psychological wellbeing.

Conclusions: Based on the remarkably similar overlap between the YFAS and BES with other eating, wellbeing or addiction-related behaviours, these results suggest that any unique behaviours supposedly identified by the YFAS do not appear to be distinct from binge eating. A clear definition of FA as a distinct condition is needed before there can any scientific basis for its validation.

Conflict of Interest: None Disclosed

Funding: Sugar Nutrition UK funded research relating to this abstract

\section{OS1:OS20}

\section{How important is fatness to young children's friendship} choices, preferences, and self-identification?

Charsley, J. '; Collins, S. '

${ }^{1}$ University of Leeds

Introduction: Evidence that young children may display anti-fat attitudes is testimony to the pervasiveness of stigma and prejudice directed at people with obesity. This also presents a practical challenge to those working in early intervention or prevention; how not to introduce or escalate this negativity. In fact, there's a lot we don't know about young children's views on fatness, especially relative to other physical differences. Accordingly, this study used a Personal Construct methodology to investigate whether fatness dominated young children's judgements of physical appearance. Methods: $\mathrm{N}=85$ Primary school children $(\mathrm{m}=5.7 \mathrm{yr})$ were presented with detailed colour drawings of visibly different child characters: fat, opposite sex, in a wheelchair, or same sex and health weight ('standard'). Using a simple repertory grid, children identified the main differences between pairs of characters, which of each pair they would choose as a friend, and which they were most similar/different to and preferred. The choices and reasons given were recorded and transcribed.

Results: Being fat, the opposite sex, or in a wheelchair were equally likely to be identified as most different from the standard character. A difference in body weight or shape was mentioned in only $8 \%$ of the reasons given. More common were differences in sex $(12 \%)$, hair (20\%), being in a wheelchair (27\%), and the clothes worn (29\%). There were no differences in friendship choices between characters, with children significantly more likely to choose the character who was the same sex, not in a wheelchair, and not fat. Fatness was referred to in only $11 \%$ of reasons for rejection as a friend. More physical than social reasons were apparent in children's preferences for, and rejection of, character features for themselves. Again, there was little distinctive about the fat character in comparison with that of the opposite sex or in a wheelchair. There was variation in children's views and occasional extreme views. One of the 2 most overweight children identified fatness as the most important difference and as the character they would least like to be. They also expressed strong anti-fat views. Conclusion: This combined Personal Construct and listening approach allowed young children to give 'first person' views on fatness relative to other visible differences. That a character's fatness was of low importance in the great majority of these social and identity choices does not contradict the wider literature on obesity stigma. Rather, it is a reminder to those working with young children that how we see the world may be different to their world view, for a time at least.

Conflict of Interest: None

Funding: None

\section{OS1:OC21}

\section{Associations between health-related quality of life and body mass index in Portuguese adolescents: LabMed Physical Activity Study}

Evaristo, S. ; Moreira, C. ' ; Santos, R. ${ }^{2}$; Lopes, L. ' ; Abreu, S. ' ; Mota, J. ${ }^{1}$ ${ }^{1}$ CIAFEL - FADEUP, ${ }^{2}$ Ciafel - Fadeup; 2 Early Start Research Institute, Faculty Of Social Sciences. University Of Wollongong, Australia

Introduction: In adolescence there are several biological and phycological changes associated with overweight and obesity. The purpose of this study was to analyze the associations between Health-Related Quality of Life (HRQoL) and body mass index (BMI) in adolescents.

Methods: This is a cross-sectional analysis of 957 Portuguese adolescents (446 girls and 511 boys) aged 12-18 years. A series of variables were collected: a) HRQoL was assessed using the Kidscreen-10 questionnaire; b) Socioeconomic status (SES) was measured using the Family Affluence Scale; c) body composition (weigh, height) were measured according to standard protocols and, d) pubertal stage was assessed with Tanner stages. Results: HRQoL was significantly higher in boys than girls $(\mathrm{p}<0.05)$. The prevalence of overweight and obesity was $23.5 \%$ and $6.7 \%$ in girls and $21.3 \%$ and $5 \%$ in boys, respectively. The association between HRQoL with 
BMI controlling for age, pubertal stage and SES, showed that HRQoLwas inversely and significantly associated with $\mathrm{BMI}$ in boys $(\beta=-0.130$; $p<0.05)$ and girls $(\beta=-0.148 ; p<0.05)$.

Conclusions: Our results suggest that HRQoL was significantly and inversely associated with BMI in both sexes. Public health policies targeting a healthy weight could be a strategy of particular interest for improving the HRQoL of adolescents.

Conflict of Interest: None Disclosed.

Funding: The Research Centre on Physical Activity Health and Leisure (CIAFEL) is supported by FCT grant: UID/DTP/00617/2013.

\section{OS1:OC22}

\section{Patterns of public opinions about obesity and people with obesity in Sweden, UK, Germany, and Italy}

Kyle, T. ; Pietrobelli, A. ${ }^{2}$; Nadglowski, J. ${ }^{3}$; Pauli, D. ${ }^{4}$; Hilbert, A. ${ }^{5}$; Salas, X. ${ }^{6}$; Thomas, D.; Puhl, R. ${ }^{8}$

${ }^{1}$ ConscienHealth, ${ }^{2}$ Pediatric Unit, Verona University Medical School, Verona, Italy, ${ }^{3}$ Obesity Action Coalition, Tampa, FL, USA, ${ }^{4}$ G.B. Rossi Hospital, Verona, Italy, ${ }^{5}$ Integrated Research And Treatment Center Adiposity Diseases, University Of Leipzig Medical Center, Leipzig, Germany, ${ }^{6} \mathrm{Canadian}$ Obesity Network, Edmonton, Alberta, Canada, ${ }^{7}$ Center For Quantitative Obesity Research, Montclair State University, Montclair, NJ, USA, ${ }^{8}$ Rudd Center For Food Policy And Obesity, University Of Connecticut, Hartford, CT, USA

Introduction: Weight bias (WB) is an impediment to progress toward adoption of evidence-based strategies to address obesity. Substantial social, economic, medical, and economic harm to people with obesity $(\mathrm{PwO})$ results from WB. Key elements of WB include blame directed at $\mathrm{PwO}$, assumptions of laziness or poor discipline, and social rejection. Recent evidence found a consistent presence of WB in 4 countries ${ }^{*}$, but limited international comparisons exist to understand the nature and patterns of these attitudes across countries. The present research measured beliefs about the causes of obesity, and also beliefs and attitudes about PwO in Sweden (SE), UK, Germany (DE), and Italy (IT).

Methods: Random samples totaling 34,220 adults completed anonymous, voluntary online surveys between Oct 2016 and Jan 2017. Microsurveys required only one response per participant to reduce fatigue with multiple questions. Likert scales (5-points) measured agreement with 4 narratives regarding causes of obesity, as well as perceptions of blame, social acceptance, laziness, and self-discipline of PwO. Descriptive statistics were analyzed to identify significant differences between and within the four countries.

Results: Agreement that obesity results from addictive junk food was high in all 4 countries, highest in SE (mean agreement $3.8 \pm 0.1$ ) and IT $(3.9 \pm 0.1)$. Belief in irresponsibility as a cause of obesity was higher in UK respondents $(3.3 \pm 0.1)$ than in $\mathrm{DE}(2.8 \pm 0.1)$. Respondents agreed with environmental causes of obesity in all 4 countries. Agreement that obesity is a medical concern was higher in $\mathrm{DE}(3.2 \pm 0.1)$ than in $\mathrm{UK}(2.9 \pm 0.1)$. Belief that $\mathrm{PwO}$ should be blamed was highest in UK $(3.4 \pm 0.1)$, lowest in $\mathrm{DE}(2.9 \pm 0.1)$. Measures of social acceptance varied less among the different countries than attribution of blame. Respondents in IT were more likely to attribute laziness to $\mathrm{PwO}(3.5 \pm 0.1$ vs 2.8 to 3.2$)$.

Conclusion: Public beliefs associated with WB vary among these four countries. Belief that addictive junk food plays a causative role is widespread and especially strong in Italy and Sweden. Further research is needed to assess changes in these beliefs over time and to assess reasons for the patterns observed here. Understanding these patterns and reasons for them may be helpful for strategies to reduce WB.

Conflict of Interest: None disclosed

Funding: Research relating to this abstract was funded by a grant from Novo Nordisk

References:

1 Puhl et al, 2015, IJO, 39(7).
OS1:OC23

Bidirectional associations between psychosocial well-being and adherence to healthy dietary guidelines in European children: Prospective findings from the IDEFICS study

Arvidsson, L. ${ }^{1}$; Eiben, G. ${ }^{\prime}$; Hunsberger, M. '; Bourdeaudhuij, I.'; Molnar, D. ${ }^{3}$; Jilani, H. ${ }^{4}$; Thumann, B. ${ }^{4}$; Veidebaum, T. ${ }^{5}$; Russo, P. ${ }^{6}$; Tornatitis, M. ${ }^{7}$; Santaliestra-Pasías, A. '; Pala, V.'; Lissner, L.'

${ }^{1}$ Section for Epidemiology and Social Medicine (EPSO), The Sahlgrenska Academy, University of Gothenburg, ${ }^{2}$ Department Of Movement And Sport Sciences, Ghent University, ${ }^{3}$ Department Of Paediatrics, Clinical Center, University Of Pécs, ${ }^{4}$ Leibniz Institute For Prevention Research And Epidemiology - Bips, ${ }^{5}$ National Institute For Health Development, ${ }^{6}$ Institute Of Food Sciences, Cnr, ${ }^{7}$ Research And Education Institute Of Child Health Ref, ${ }^{8} \mathrm{Genud}$ (Growth, Exercise, Nutrition, And Development) Research Group, University Of Zaragoza, ${ }^{9}$ Nutritional Epidemiology Unit, Department Of Preventive Medicine, Fondazione Ircss Istituto Nazionale Dei Tumori

Introduction: In children the relationship between a healthy diet and psychosocial well-being has not been fully explored and the existing evidence is inconsistent. This study investigates the chronology of the association between children's adherence to healthy dietary guidelines and well-being, with special attention to the influence of weight status on the association. Methods: 7,675 children 2 to 9 years old from the eight-country cohort study IDEFICS were investigated. They were first examined between September 2007 and June 2008 and re-examined again two years later. Psychosocial well-being was measured using self-esteem and parent relations questions from the KINDL ${ }^{\circ}$ and emotional and peer problems from the Strengths and Difficulties Questionnaire. A Healthy Dietary Adherence Score (HDAS) was calculated from a 43 -item food frequency questionnaire as a measure of the degree to which children's dietary intake follows nutrition guidelines. The analysis employed multilevel logistic regression with bidirectional modelling of dichotomous dietary and well-being variables as both exposures and outcomes.

Results: A higher HDAS at baseline predicted better self-esteem (OR 1.2, 95\% CI 1.0;1.4) and fewer emotional and peer problems (OR 1.2, 95\% CI 1.1;1.3 and OR 1.3, 95\% CI 1.2;1.4) two years later. For the reversed direction, better self-esteem predicted higher HDAS two years later (OR $1.195 \%$ CI 1.0;1.29). The stratified analysis by weight status revealed that the associations between higher HDAS at baseline and better well-being at follow-up were similar in both normal weight and overweight children. Conclusion: Present findings suggest a bidirectional relation between diet quality and self-esteem. Additionally, higher adherence to healthy dietary guidelines at baseline was associated with fewer emotional and peer problems at follow-up, independent of children's weight status.

Conflict of Interest: None Disclosed

Funding: Research relating to this abstract was funded by the European Commission within the Sixth RTD Framework Programme Contract No. 016181 (FOOD) with additional financial support from Epilife TEENS

\section{OS1:OC24}

\section{Tracking diet variety in childhood and its association with appetitive traits}

\section{Vilela, S. ${ }^{1}$; Hetherington, M. ${ }^{2}$; Oliveira, A. ' ${ }^{1}$ Lopes, C. $^{1}$}

${ }^{1}$ EPIUnit Institute of Public Healt of Porto University, ${ }^{2}$ School Of Psychology, University Of Leeds

Introduction: Research on influence of early eating habits on appetite-related eating behaviors using a prospective approach is scarce, especially in children. The aim of this study was to explore the relationship between changes in diet variety from 4 to 7 years of age and child appetitive traits measured at 7 years of age.

Methods: Participants are from the population-based birth cohort Generation XXI, assembled in Porto, Portugal during 2005/2006. The present analysis included 4371 evaluated at both $4 y$ and $7 y$ follow-ups, with complete data on food frequency questionnaire (FFQ) at $4 y$, and complete information on the Children's Eating Behaviour Questionnaire (CEBQ), 
at $7 y$. Weight and height were measured at both evaluations. A healthy diet variety index (HDVI) was calculated at both ages using FFQ, considering variety within and among the five main food groups (starchy foods (including potatoes), fruit, vegetables, meat, fish and alternatives and dairy foods). The weighted numbers of servings consumed of each food group were summed; the maximum score was 5 . To assess tracking of dietary variety, tertiles of HDVI scores were calculated at both ages then re-categorized as 'maintain' for those children who remained within their allocated tertile from 4 to 7 years, 'increase' for those children whose dietary variety score moved to a higher tertile and 'decrease' for those children whose dietary variety fell to a lower tertile. Associations between tracked tertile (maintain, increase and decrease) and eating traits measured by the CEBQ were evaluated through linear regression models.

Results: The HDVI score decreased from 4 to 7 years (mean (SD): 4.01 $(0.52)$ and $3.60(0.35), \mathrm{p}<0.001$, respectively), with a large effect size $(0.8)$. In both girls and boys HDVI scores showed a high stability $(>0.33)$ and a positive predictive value (>1.0). Compared to girls, boys presented a higher tracking of diet variety. Increasing dietary variety was inversely associated with food fussiness at age $7 \mathrm{y}$ in both girls and boys $(\beta:-0.12 ; 95 \% \mathrm{CI}$ : $-0.19,-0.04$ and $\beta:-0.13 ; 95 \%$ CI: $-0.20,-0.05$, respectively) and satiety responsiveness', only in girls ( $\beta$ : $-0.09 ; 95 \% \mathrm{CI}:-0.15,-0.02)$. Decreased dietary variety was linked to higher scores in food fussiness in both girls and boys ( $\beta$ : 0.15 ; 95\% CI: 0.07, 0.23 and $\beta$ : 0.19; 95\% CI: 0.10,0.27, respectively).

Conclusion: Overall dietary variety decreased from 4 to 7 years of age with a moderate tracking in both girls and boys. A low dietary variety was predicted by fussy eating in both boys and girls, and with satiety responsiveness in girls.

Conflict of Interest: None Disclosed

Funding: FCT (FCT-PTDC/SAU-EPI/121532/2010) - Operational Programme Factors of Competitivenes (COMPETE).

\section{OS2 - Metabolic Outcomes}

\section{OS2:OC50}

MRI-FAT quantification of the liver, subcutaneous and visceral fatty tissue and correlation with blood lipids in patients before and after bariatric surgery

Wölnerhanssen, B. '; Nabers, D. ${ }^{2}$; Moor, M. ${ }^{3}$; Borgwardt, S. ${ }^{3}$; Peterli, R. ${ }^{3}$; Beglinger, C. ${ }^{3}$; Meyer-Gerspach, A. ${ }^{3} ;$ Bieri, O. $^{4}$

${ }^{1}$ Department of Research, St. Claraspital Basel, Basel, Switzerland, ${ }^{2}$ Division Of Medical And Biological Informatics, German Cancer Research Center (Dkfz), Heidelberg, Germany, ${ }^{3} \mathrm{~S} / \mathrm{S}$, ${ }^{4}$ Department Of Radiology, University Hospital Basel, Basel, Switzerland

Introduction: Non-alcoholic fatty liver disease (NAFLD) is characterized by atherogenic dyslipidemia. NAFLD patients are at an increased risk for both cardiac and non-cardiac vascular diseases. The effect of bariatric surgery on fat distribution in the liver has so far been studied with liver biopsies and single voxel MR techniques. In this study, liver fat fraction (LFF), subcutaneous (SAT) and visceral adipose tissue (VAT) was assessed using MRI in combination with blood lipids to monitor the efficiency of bariatric surgery.

Methods: In 11 morbidly obese patients an iterative decomposition of water and fat with echo asymmetry and least-squares estimation (IDEAL) approach was used for whole liver fat quantification in combination with a 2-point Dixon technique for volumetric fat imaging of adipose tissue pre- and 3,6, and 12 months after surgery. SAT and VAT adipose tissue volumes were assessed from fat images and separated by statistical shape models. Serum: total cholesterol, HDL, LDL and TG were measured.

Results: LFF, SAT and VAT volumes decreased postoperatively with a good phenomenological description using an exponential model of form $t$ is the time (in days) after intervention, $\mathrm{A}=\left[A_{1}, A_{2}, \ldots, A_{11}\right]$ refers to the individual patient specific LFF, SAT or VAT volumes, in combination with a global time constant $T_{L F F V A T S A T}$ for each measure. The LFF reduced the fastest, (time constant: $T_{L F F}^{\text {LFF }}=96 \pm 28 \mathrm{~d}$ ), followed by SAT $\left(T_{S A T}=140 \pm 18\right.$ $\mathrm{d})$, and $\operatorname{VAT}\left(T_{V A T}=170 \pm 21 \mathrm{~d}\right)$. LFF measures showed a clear correlation with total cholesterol, cholesterol/HDL ratio and LDL (each: $\mathrm{p}<0.001$ ) and a trend for HDL $(p=0.006)$. Decrease in VAT and SAT did not correlate with blood lipids.

Conclusions: 1) Decrease of body fat after bariatric surgery follows a distinct time pattern: liver fat decreases fastest followed by subcutaneous and last visceral fat, 2) decrease in liver fat correlates with changes in blood lipids.

Conflict of Interest: No conflict of interest

The trial was funded by the Swiss National Science Foundation (SNSF: Marie HeimVoegtlin subsidy: PMPDP3-145486/1)

\section{OS2:OC51 \\ Effect of a lifestyle intervention in obese infertile women on cardiometabolic health and quality of life}

Dammen, L. ${ }^{1}$; Wekker, V. ${ }^{2}$; Oers, A. ${ }^{3}$; Mutsaerts, M. ${ }^{4}$; Painter, R. ${ }^{2}$; Zwinderman, A. ${ }^{5}$; Groen, H. ; Beek, C. ${ }^{2}$; Kobold, A. ${ }^{6}$; Land, J.'; Kuchenbecker, W. ${ }^{7}$; Golde, R. ${ }^{8}$; Oosterhuis, G. ${ }^{9}$ Vogel, N. ${ }^{10}$; Mol, B. ${ }^{11}$; Roseboom, $T^{2}$; Group, A. $^{3}$

${ }^{1}$ Department of Epidemiology, University of Groningen , University Medical Center Groningen, P.O. Box 30001, 9700 RB Groningen, the Netherlands, 'Department Of Obstetrics And Gynecology, Academic Medical Center, University Of Amsterdam, Meibergdreef 5, 1105 Az Amsterdam, The Netherlands, ${ }^{3}$ Department Of Obstetrics And Gynecology, University Of Groningen , University Medical Center Groningen, P.O. Box 30001, $9700 \mathrm{Rb}$ Groningen, The Netherlands, ${ }^{4}$ Department Of General Practice, University Medical Center Utrecht, University Of Utrecht, 3508 Ga Utrecht, The Netherlands, ${ }^{5}$ Department Of Clinical Epidemiology, Biostatistics And Bioinformatics, Amsterdam Public Health Research Institute, Academic Medical Center, University Of Amsterdam, Meibergdreef 5, 1105 Az Amsterdam, The Netherlands, ${ }^{6}$ Department Of Laboratory Medicine, University Of Groningen, University Medical Center Groningen, P.O. Box 30001, 9700 Rb Groningen, The Netherlands, ' Department Of Gynecology And Obstetrics, Isala Clinics, Dokter Van Heesweg 2, 8025 Ab Zwolle, The Netherlands, ${ }^{8}$ Department Of Gynecology And Obstetrics, Maastricht University Medical Center, Maastricht University, Maastricht P. Debyelaan 25, 6229 Hx Maastricht, The Netherlands, ${ }^{9}$ St. Antonius Hospital, Koekoekslaan 1, $3435 \mathrm{Cm}$ Nieuwegein, The Netherlands, ${ }^{10}$ Martini Hospital, Van Swietenplein 1, 9728 Nt Groningen, The Netherlands, ${ }^{11}$ Robinson Research Institute, School Of Medicine, University Of Adelaide, Sa 5006, Australia

Introduction: The prevalence of obesity, an important cardiometabolic disease risk factor, is rising in women. Current international guidelines state that lifestyle adjustments are the cornerstone of the primary prevention and treatment of obesity and cardiometabolic diseases. The success of lifestyle interventions depend on intrinsic motivation, but also timing. The start of infertility treatment might be a perfect window of opportunity to improve lifestyle. We hypothesize that a lifestyle intervention program prior to infertility treatment improves cardiometabolic health and quality of life in obese infertile women.

Methods: Between 2009-2012, 577 obese infertile women were randomized to a 6 month lifestyle intervention preceding infertility treatment (intervention group), or to prompt infertility treatment (control group). Women were between 18 and 39 years old and had a body mass index $(\mathrm{BMI}) \geq 29$. The intervention aimed at $5-10 \%$ weight loss or a $\mathrm{BMI}<29$. Outcome measures were anthropometrics, blood pressure, high sensitive C-reactive protein (hs-CRP), lipids, glucose, insulin, the homeostasis model assessment of insulin resistance (HOMA-IR), and prevalence of metabolic syndrome (MetS) at 3 and 6 months. Quality of life (QoL) was measured at 3, 6, and 12 months. Mixed effects regression models analyses were performed. Results are displayed as estimated mean differences between the groups.

Results: Weight ( $-3.1 \mathrm{~kg} 95 \% \mathrm{CI}:-4.0--2.2 \mathrm{~kg}$; P $<0.001)$, waist circumference $(-2.4 \mathrm{~cm} \mathrm{95 \%} \mathrm{CI:}-3.6--1.1 \mathrm{~cm} ; \mathrm{P}<0.001)$, hip circumference $(-3.095 \%$ CI: $-4.2--1.9 \mathrm{~cm}$; P $<0.001)$, BMI $\left(-1.2 \mathrm{~kg} / \mathrm{m}^{2} 95 \%\right.$ CI: -1.5 $\left.--0.8 \mathrm{~kg} / \mathrm{m}^{2} ; \mathrm{P}<0.001\right)$, systolic blood pressure $(-2.8 \mathrm{mmHg} 95 \% \mathrm{CI}$ : 
$-5.0--0.7 \mathrm{mmHg} ; \mathrm{P}=0.01)$ and HOMA-IR $(-0.5$ 95\% CI: $-0.8--0.1 ; \mathrm{P}$ $=0.01)$ were lower in the intervention group as compared to controls. HsCRP and lipids did not differ between groups. The odds ratio for MetS in the intervention group was 0.53 (95\% CI: $0.33-0.85$; P < 0.01) compared to controls. Physical QoL scores were higher in the lifestyle intervention group (2.2 95\% CI: 0.9 - 3.5; P = 0.001) while mental QoL scores did not differ.

Conclusion: In obese infertile women, a lifestyle intervention leads to better cardiometabolic health and physical quality of life compared to prompt infertility treatment.

Trial registration: NTR1530.

Conflict of Interest:The department of obstetrics and gynecology of the UMCG received an unrestricted educational grant from Ferring pharmaceuticals BV, The Netherlands. B. W. Mol is a consultant for ObsEva.

Funding: Research relating to this abstract was funded by ZonMw grant: 50-5011096-518 and supported by Dutch Heart Foundation grant: 2013T085.

\section{OS2:OC52}

\section{Changes in physical fitness, hepatic markers, and insulin resistance after an 8-months multicomponent intervention program with Down syndrome adolescents}

\section{Silva, E. '; Silva, T. ${ }^{2}$; Lemos, L. ${ }^{3}$; Mota, J. ${ }^{4}$; Martins, C. ${ }^{1}$}

${ }^{1}$ Universidade Federal da Paraíba, ${ }^{2}$ Faculdade Maurício De Nassau, ${ }^{3}$ Universidade Federal Da Paraíba; Centro Universitário Unipê; Faculdade Maurício De Nassau, ${ }^{4}$ Universidade Do Porto

Introduction: The Down Syndrome population has low levels of physical fitness (PF) (EID., 2015) and higher risk for diabetes (PIKORA et al., 2015) and hepatic dysfunction (MAY and KAWANISH., 1996) when compared to the population without the syndrome.

Objective: To analyze pre and post values of insulin resistance (HOMA-IR), alanine aminotransferase (ALT), aspartate aminotransferase (AST), and PF after an 8-months multicomponent intervention with Down Syndrome adolescents.

Method: This is a quasi-experimental, time series study, with before and after exposure measurements. The sample was comprised by 17 adolescents with Down Syndrome (15.3 \pm 2.4 year-olds; 10 boys and 7 girls). An 8-months intervention based on physical exercise classes, nutritional education, and parental support was offered. Physical exercises classes took 60 minutes, twice a week, and prioritized physical fitness and motor coordination exercises. Nutritional education happened once a month, and was proposed for parents and participants. Parental support component consisted of physical exercise classes for parent and the same time as their sons. The cardiorespiratory fitness was evaluated by Shuttle-Run Test, and the upper limbs strength by dynamometry (Jamar). Blood was collected through venipuncture and Homa-IR values were calculated. ALT and AST were determined by blood collection through venipuncture. The paired t-test was used to identify the differences between pre and post-intervention mean values, with SPSS software (21.0).

Results: Volunteers participated in $85 \%$ of the physical exercise sessions. Statistically significant decreases were observer for HOMA-IR (pre $=2.7$ \pm 1.8 versus post $=1.5 \pm 0.5, \Delta=-1.2, \mathrm{p}=0.01$, ) ALT $($ pre $=22.2 \pm 1.4$ Versus post $=17.8 \pm 0.9 \Delta=-4.4, \mathrm{p}=0.01)$ and AST $($ pre $=21.4 \pm 1.1$ versus post $=19.5 \pm 0.7 \Delta=-1,9, \mathrm{p}=0.02)$. It was also observed a better profile for cardiorespiratory fitness (pre $=4.6 \pm 0.9$ versus post $=6.2 \pm 0.8$, $\Delta=1.6, \mathrm{p}=0.01$ ) and upper limbs strength (pre $=8.2 \pm 1.1$ versus post $=$ $9.7 \pm 1.2 ; \Delta=1.5 \mathrm{p}=0.01)$.

Conclusion: Down Syndrome adolescents involved in an 8-months multicomponent intervention program presented a better insulin resistance and hepatic marker profiles, as well as increased values for physical fitness after the proposed intervention.

Conflict of Interest: We declare that you have no conflict of interest

Funding: National Council for Scientific and Technological Development

\section{OS2:OC53}

A randomised controlled trial assessing the impact of intermittent vs continuous energy restriction on blood pressure and anthropometry in healthy subjects with central obesity. "The Met-IER study"

Pinto, A. '; Bordoli, C. ; Buckner, L. ' ; Kaplan, P. ; Arenal, I. ' Jeffcock, E. '; Kim, C. ' 'Johnston, . $^{2}$; Hall, W. ${ }^{1}$

${ }^{1}$ Faculty of Life Sciences and Medicine, Kings College London, ${ }^{2}$ Lighterlife Uk Ltd

Intermittent energy restriction (IER) as a method of weight management is gaining interest as an alternative to continuous energy restriction (CER). Here we present data assessing changes in anthropometry and blood pressure (BP) from a study which compared the short-term effects of IER versus CER. A parallel-design, RCT in 45 healthy men and women (aged 35-75 y) with central obesity was undertaken. IER was 2 consecutive days of very low energy diet (VLED; LighterLife UK Ltd) plus a 5-d healthy eating pattern for 4 weeks. CER comprised $-500 \mathrm{kcal} /$ day healthy eating pattern. The primary outcome was insulin sensitivity (Pinto et al., abstract). Secondary outcomes included ambulatory BP, body weight, BMI, waist circumference and \% body fat. Treatment effects were assessed at endpoint (at least 2 days after the last VLED day for IER) by ANCOVA (adjusted for baseline, sex and compliance). Baseline weights were $($ mean \pm SD, $\mathrm{n}=43) 102 \pm 17 \mathrm{~kg}$ in men and $83 \pm 17 \mathrm{~kg}$ in women. Percentage weight loss did not significantly differ between groups: IER, $-3.1 \%$ (95\%CI -3.9, -2.3); CER, -2.7\% (95\%CI -3.5, -1.9). There were no significant differences in percentage reductions in waist circumference (IER, $-3.4 \%$, 95\%CI -4.8, -2.0; CER, -3.0\%, 95\%CI -4.4, -1.6) nor percentage body fat reduction between groups. Twenty-four hour systolic ambulatory $\mathrm{BP}(\mathrm{ABP})$ was $6.7 \mathrm{~mm} \mathrm{Hg}$ lower (95\% CI 1.1, 12.3, P = 0.020) following IER compared to CER, and $24 \mathrm{~h}$ diastolic ABP was $4.3 \mathrm{~mm} \mathrm{Hg}$ lower (95\% CI 1.2, 7.3, $P=0.009)$. Similar differences were found for awake systolic ABP (CER-IER mean difference $7.9 \mathrm{~mm} \mathrm{Hg}$; 95\% CI 1.4, 14.4, $P=0.019$ ) and awake diastolic ABP (CER-IER mean difference $4.5 \mathrm{~mm} \mathrm{Hg}$; 95\% CI $0.9,8.1, P=0.017)$. There were no treatment differences in night-time ABP. Selecting either a standard CER diet or " $5: 2$ " IER diet, in combination with adherence to healthy eating guidelines, results in equivalent weight loss and changes in body composition, but IER resulted in greater reductions in $24 \mathrm{~h}$ and day-time systolic and diastolic ABP.

Conflicts of Interest: KLJ is employed by LighterLife UK Ltd

Funding: Research relating to this abstract was funded by LighterLife UK Ltd

\section{OS2:OC54}

\section{Metabolically healthy obese and incident cardiovascular} disease events among $\mathbf{3 . 5}$ million men and women in the UK

Caleyachetty, R. '; Thomas, G. ; Toulis, K. ; Mohammed, N. ; Gokhale, K. ' Nirantharakumar, $K{ }^{\text {' }}$

${ }^{1}$ The Institute of Applied Health Research, University of Birmingham

Introduction: Whether metabolically healthy obese (MHO) is associated with excess risk of cardiovascular disease(CVD) events is a subject of debate. Important limitations to the evidence base include inconsistent definitions of metabolic health, inconsistent control for confounders, and small sample sizes. We sought to address these limitations in a large contemporary cohort, based on linked electronic health records. Our objective was to examine the association of $\mathrm{MHO}$ with 4 different presentations of incident CVD.

Methods: We used linked electronic health records from 1995 to 2015 in The Health Improvement Network (THIN) to assemble a cohort of 3.5 million individuals aged 18 years or older and initially free from CVD. We categorised our population in groups according to BMI and the presence or absence of 3 metabolic abnormalities (diabetes, hypertension, hyperlipidemia) which were summed to create a metabolic abnormalities score $(0,1,2$ and 3$)$. We classified MHO individuals as having 0 metabolic abnormalities. The primary endpoints were the first record of one of 4 
cardiovascular presentations [coronary heart disease (CHD), cerebrovascular disease, heart failure, and peripheral vascular disease (PVD)]. We used Cox proportional hazard models to calculate hazard ratios (HRs) and $95 \%$ confidence intervals (CIs) for associations between $\mathrm{MHO}$ and CVD events. Patient's age, sex, self-reported smoking status, and social deprivation were included in models.

Results: Compared to normal weight individuals with 0 metabolic abnormalities, individuals who were obese with 0 metabolic abnormalities had an increased risk of CHD (adjusted HR 1.49, 95\% CI 1.45,1.54), cerebrovascular disease (adjusted HR 1.07, 95\% CI 1.04,1.11) and heart failure (adjusted HR 1.96, 95\% CI 1.86, 2.06). MHO individuals had a lower risk of PVD (adjusted HR 0.91, 95\% CI 0.86,0.96). In sensitivity analyses that excluded cigarette smokers, obese individuals with 0 metabolic abnormalities had a significantly increased risk of developing PVD (adjusted HR 1.11, 95\% CI 1.00,1.24). Risk of CVD events in obese individuals increased with increased number of metabolic abnormalities.

Conclusion: This is the largest prospective study of the association between the MHO and a range of incident CVD events. MHO individuals are at higher risk of $\mathrm{CHD}$, cerebrovascular disease and heart failure than normal weight metabolically healthy individuals. The priority of health professionals should be to promote and facilitate weight loss among obese persons, regardless of the presence or absence of metabolic abnormalities.

Conflict of Interest: None Disclosed

Funding: No Funding

\section{OS3 - Adipose Tissue Biology}

\section{OS3:OC1}

\section{The actions of elevated BNP on adipose tissue adaptations during chronic pressure overload}

Gonçalves, N. ${ }^{1}$; Moura, C. ${ }^{1}$; Silva, A. ${ }^{1}$; Rodrigues, $P .{ }^{1}$; Conceição, G. ${ }^{1}$; Santos, M. ${ }^{1}$; Jr, R. ${ }^{1}$; Leite-Moreira, A. ${ }^{7}$; Falcão-Pires, I. ${ }^{1}$

${ }^{1}$ Departamento de Cirurgia e Fisiologia - Faculdade de Medicina da Universidade do Porto

Introduction: The progression of chronic pressure overload (CPO) is associated to cardiac cachexia as a consequence of insufficient energy supply Additionally, some studies demonstrate that the heart secretes cardiokines able to modulate the adipose tissue (AT) structure and function promoting adiposopathy. In this study we investigate the effects of CPO in AT.

Methods: Wistar rats were submitted to aortic banding (Ba group; $0.6 \mathrm{~mm}$ diameter) or sham procedure (Sham group). After 8 weeks, left ventricular (LV) function and structure (echocardiography and invasive hemodynamics) was evaluated and samples (LV and AT) collected for histological and molecular evaluations. Plasma was obtained for quantification of circulating B-type natriuretic peptide (BNP). Finally, visceral AT from normal rats was incubated with the BNP plasma concentrations detected in the Sham and Ba group ( 0.27 and $0.47 \mathrm{ng} / \mathrm{ml}$ respectively) for $24 \mathrm{~h}$ and then collected for molecular studies. Data presented as mean \pm SEM. * $\mathrm{p}<0.05 \mathrm{vs}=$ ="' sham $=$ "' br $=$ "'” $>$

Results: Eight-weeks of banding increased LV systolic pressure (Ba: $153 \pm 10.5^{\star}$ vs Sham $110 \pm 3.6$ ) and triggered cardiac remodeling (Heart weight $\mathrm{Ba}: 3.3 \pm 0.30^{*}$ vs Sham $\left.2.3 \pm 0.05\right)$ with fibrosis $\left(\mathrm{Ba}: 6 \pm 0.9^{*}\right.$ vs Sham $4 \pm 0.5$ ) and cardiomyocytes' hypertrophy (Ba: $484 \pm 33.6^{*}$ vs Sham $382 \pm 23.6)$ when compared to Sham animals. The same group was at a compensated stage of the disease with higher ejection fraction (Ba: $89 \pm 1.9^{\star}$ vs Sham $\left.78 \pm 0.9\right)$, however a stiffer myocardium was observed with increased end diastolic pressure-volume relation $\left(\mathrm{Ba}: 0.11 \pm 0.031^{*}\right.$ vs Sham $0.04 \pm 0.006)$ and passive force of isolated cardiomyocytes ( $\mathrm{Ba}$ $4.4 \pm 0.57^{\star}$ vs Sham $\left.3.3 \pm 0.29\right)$. Despite similar adiposity between the 2 groups (Ba: $7.5 \pm 0.25$ vs Sham $7.9 \pm 0.88$ ), aortic constriction triggered adipocyte atrophy (Ba: $1287 \pm 85.1^{\star}$ vs Sham $\left.1659 \pm 103.8\right)$ as well as AT increased fibrosis (Ba: $8.7 \pm 0.61^{\star}$ vs Sham $7.2 \pm 0.31$ ) and dysfunction, as observed by overexpression of pro-inflammatory adipokines (TNFa Ba: $0.06 \pm 0.018^{\star}$ vs Sham $0.03 \pm 0.013$; IL1 $\beta$ Ba: $0.28 \pm 0.15^{*}$ vs Sham $0.04 \pm 0.01)$. The incubation of AT from normal rats with BNP confirmed that the elevated circulatory levels of this cardiokine were able to induced increased expression of pro-inflammatory adipokines by the AT (TNFa Ba: $31125 \pm 1455^{\star}$ vs Sham $26380 \pm 1428$; IL1 $\beta$ Ba: $12221 \pm 1086^{\star}$ vs Sham $9038 \pm 678)$.

Conclusion: We demonstrated that higher circulatory levels of BNP promoted by LV CPO are able to induce adiposopathy characterized by remodeling of the AT and overexpression of pro-inflammatory adipokines. Research relating to this abstract was funded by Projeto DOCnet - Diabetes \& obsity at the crossroads between Oncological and Cardiovascular diseases - a system analysis NETwork towards precision medicine, Ref. ${ }^{a}$ NORTE-01-0145-Feder-000003,financiado pelo N2020.

\section{OS3:OC2 \\ Insulin resistance is associated with a specific methylation profile of visceral adipose tissue in obese patients}

González-Izquierdo A. '; Carreira M.C. '; Moreno-Navarrete J.M. ${ }^{3}$; Amil M. ${ }^{2}$; Díaz-Lagares A. ${ }^{4}$; Casanueva F.F. ; Fernandez-Real J.M. ${ }^{3}$; Crujeiras A.B. ${ }^{2}$

${ }^{1}$ Molecular Endocrinology Department, Complejo Hospitalario Universitario de Santiago (CHUS-IDIS) and Santiago de Compostela University (USC); CIBER Fisiopatología de la Obesidad y Nutrición, Santiago de Compostela, Spain., ${ }^{2}$ Molecular Endocrinology Department, Complejo Hospitalario Universitario De Santiago (Chus-Idis) And Santiago De Compostela University (Usc); Ciber Fisiopatología De La Obesidad Y Nutrición, Santiago De Compostela, Spain., ${ }^{3}$ Department Of Diabetes, Endocrinology And Nutrition, Institut D'Investigació Biomédica De Girona (Idibgi); Ciber Fisiopatología De La Obesidad Y La Nutrición (Ciberobn), Girona, Spain., ${ }^{4}$ Cancer Epigenetics And Biology Program (Pebc), Bellvitge Biomedical Research Institute (Idibell), L'Hospitalet, Catalonia, Spain.

Introduction: Elucidating the potential mechanisms involved in controlling insulin resistance is an important priority in counteracting obesity-associated diseases. We propose that an epigenetic regulation can mediate the susceptibility to insulin resistance in obesity and aimed this study to disentangle the epigenetic basis of insulin resistance by performing a genome-wide epigenetic analysis in visceral adipose tissue (VAT) from morbidly obese patients.

Methods: The insulin sensitivity was determined by the clamp technique (M-clamp value $<4.7$ ) in 12 morbidly obese patients. DNA isolated from VAT of 7 insulin-resistant (IR) and 5 insulin-sensitive (IS) patients was hybridized in the HumanMethylation450 Infinium BeadChip array.

Results: Comparing DNA methylation levels between IR and IS, 982 CpG sites were identified with significant differences between both groups. These differentially methylated CpGs (DMCpGs) were associated with 538 genes, which were able to completely distinguish patients IR of IS. The majority were located in the CpG islands, mainly in the open-sea region. With respect to the chromosomic distribution, those DMCpGs with increased methylation levels in IR were distributed in chromosomes 1, $5,6,10,11$, and 13 , whereas those DMCpGs with decreased methylation levels in IR were mainly found in chromosomes 12, 16, and 19. The Gene Ontology (GO) analysis determined a high number of genes were linked to signal transduction, transcription regulation and cell adhesion. Most of the genes associated with the DMCpGs were significantly $(P<0.001)$ related with insulin signalling pathways and $10 \%$ of them were associated with Diabetes according to the ' Human Diabetes Proteome Project"'. The current work identified novel IR-related genes epigenetically regulated in VAT, such as COL9A1, COL11A2, CD44, MUC4, ADAM2, IGF2BP1, GATA4, TET1, ZNF714, ADCY9, TBX5, and HDACM

Conclusion: This study demonstrates the existence of a methylome profile associated with insulin resistance in VAT. The results identify potential epigenetic biomarkers and novel therapeutic targets in the prevention and treatment of disturbances in insulin sensitivity associated with obesity. The observations of the current work are of foremost relevance as they provide tools for additional personalized treatment for prevention of type 2 diabetes based on epigenetic biomarkers. 


\section{Conflict of Interest: None Disclosed}

Funding: Research relating to this abstract was funded by grants from the Fondo de Investigacion Sanitaria, PE13/00024 and PI14/01012 research projects and CIBERobn (CB06/003) from the Instituto de Salud Carlos III (ISCIII)-Subdireccion General de Evaluacion y Fomento de la Investigación; Fondo Europeo de Desarrollo Regional (FEDER)

\section{OS3:OC3}

Ethnic differences in body fat distribution and the relationship with adipose tissue oestrogen receptor expression.

\section{Keswell, D. ${ }^{1}$; Tootla, M. ${ }^{1}$; Goedecke, J. ${ }^{2}$}

${ }^{1}$ University of Cape Town, ${ }^{2}$ South African Medical Research Council

Background: Sex hormones play an important role in body fat distribution. We have previously shown that fat deposition differs between black and white South African (SA) women. Thus the aim of this study was to examine the associations between subcutaneous adipose tissue (SAT) expression of oestrogen receptors (ER $\alpha$ and ER $\beta$ ) and aromatase (CYP19A), and body fat distribution among black and white women.

Methods: Body fat distribution (DXA) and gene expression of ER $\alpha$, ER $\beta$ and CYP19A in abdominal deep SAT (DSAT) and superficial SAT (SSAT), and gluteal SAT were measured in 13 normal-weight and 13 obese black and 12 normal-weight and 10 obese white women.

Results: Gluteal ER $\alpha$ expression was significantly lower $(p=0.002)$ and CYP19A expression higher $(\mathrm{p}<0.001)$ in obese versus normal-weight black and white women, while ERa expression was higher $(1.078 \pm 0.054$ vs. $0989 \pm 0.052,(\mathrm{P}<0.001))$ and $\mathrm{ER} \beta$ expression lower $(0.992 \pm 0.058$ vs. $1.095 \pm 0.065,(\mathrm{P}<0.001))$, in black compared to the white women irrespective of BMI. Furthermore within the gluteal depot CYP19A expression was higher in black compared to white women $(1.089 \pm 0.109$ vs. $1.033 \pm 0.105, \mathrm{P}=0.030)$. Gluteal ERa expression was associated with reduced trunk fat mass (FM) in both black and white women $(\mathrm{r}=-0.41$, $\mathrm{p}=0.04 ; \mathrm{r}=-0.49, \mathrm{p}=0.02$; respectively) and with reduced leg FM in white women only $(\mathrm{r}=-0.564, \mathrm{p}=0.006)$.

Conclusions: Ethnic differences in body fat distribution were associated with regional differences in expression of ERs and support the fundamental differences in the effects of sex hormones on metabolism and body composition in black and white women.

Conflict of Interest: None Disclosed

Funding: Research relating to this abstract was funded by the National Research Foundation of South Africa and the Astrazeneca Research Trust.

\section{OS3:OC4}

\section{Unbalanced proteostasis in preadipocytes in obesity-related type 2 diabetes}

JSánchez-Ceinos, J. '; Ovelleiro, D. 2; del Río-Moreno, M. ';

Pedraza-Arévalo, S. ' Luque, R. '; Castaño, J.P. ' ; Navarro-Ruiz, M.C. ';

Membrives, A. ${ }^{3}$; López-Miranda, J. ${ }^{4}$; Vázquez-Martínez, $R .{ }^{1}{ }^{~}$.

Guzmán-Ruiz, R. ${ }^{1}$;Malagon, M.M. ${ }^{1}$

${ }^{1}$ IMIBIC/University of Córdoba/Reina Sofia University Hospital, Córdoba; CIBER Fisiopatología de la Obesidad y Nutrición (CIBERobn), ISCIII, Spain., ${ }^{2}$ Área De Innovación Tecnológica Y Bioinformática, Imibic/University Of Córdoba/Reina Sofia University Hospital, Córdoba, Spain, ${ }^{3}$ Unidad De Gestión Clínica De Cirugía General Y Digestivo, Reina Sofia University Hospital, Córdoba, Spain., ${ }^{4}$ Lipids And Atherosclerosis Unit, Imibic/Reina Sofia University Hospital/University Of Córdoba; Ciberobn, Spain.

Introduction: In obesity, adipocytes present a constellation of stress processes that compromise their function, including oxidative- and endoplasmic reticulum (ER)-stress which, together with impaired protein homeostasis (i.e., proteostasis), contribute to the development of insulin resistance (IR). Adipogenesis is also altered in obese individuals with IR/ type 2 diabetes (T2D). However, the molecular challenges undergone by preadipocytes in obesity remain not fully elucidated.
Methods: Primary preadipocytes were isolated from the stromal-vascular fraction of subcutaneous (SC) and omental (OM) adipose tissue of normoglycaemic (NG) and T2D obese patients. Comparative proteomic analysis of SC vs. OM preadipocytes from the two groups of patients was carried out using iTRAQ-coupled LC-MS/MS. Significant pathways identified by quantitative proteomics were further investigated by gene expression and immunoblot analyses.

Results: The human preadipocyte proteome is enriched in proteins involved in signal transduction and cell cycle, metabolism of proteins and nucleic acids, chromatin organization and intracellular traffic. Comparative proteomics of human preadipocytes revealed significant depot-specific differences in several key pathways between NG and T2D obese patients, namely mRNA splicing in SC preadipocytes and protein folding in OM preadipocytes. Further analyses confirmed the dysregulation of spliceosome components and splicing factors in T2D SC preadipocytes, while changes in members of the unfolded protein response were observed in T2D OM preadipocytes.

Conclusion: Our data suggest that T2D in obesity is associated with the dysregulation of the cellular machinery involved in protein biogenesis, folding and degradation in the cells responsible for the renewal and maintenance of the adipose tissue, the preadipocytes.

Conflict of Interest:None disclosed.

Funding: MINECO/FEDER (BFU2013-44229-R; BFU2015-70454-REDT); JJAA/ FEDER (PI-0200/2013); FIS (PIE14 00005), ProteoRed $\left(\mathrm{PRB}^{2}\right)$, CIBERobn (ISCIII).

\section{OS3:OC5}

\section{Alpha-MSH induces "browning" of subcutaneous white adipose tissue in mice}

Rodrigues, A. '; Salazar, M. '; Rocha-Rodrigues, S. ${ }^{2}$; Gonçalves, I. '; Cruz, C. '; Almeida, H. ${ }^{1}$; Magalhães, J. ${ }^{2}$; Gouveia, $A{ }^{3}$

${ }^{1}$ Departamento de Biomedicina- Unidade de Biologia Experimental, Faculdade de Medicina do Porto; IBMC - Instituto de Biologia Molecular e Celular and I3S - Instituto de Investigação e Inovação em Saúde, Universidade do Porto, Portugal, ${ }^{2}$ Ciafel - Centro De Investigação Em Actividade Física, Saúde E Lazer, Fadeup - Faculdade De Desporto Da Universidade Do Porto, Portugal, ${ }^{3}$ Departamento De Biomedicina- Unidade De Biologia Experimental, Faculdade De Medicina Do Porto; Ibmc - Instituto De Biologia Molecular E Celular And I3S - Instituto De Investigação E Inovação Em Saúde; Fcnaup, Universidade Do Porto, Portugal

Introduction: White adipose tissue (WAT) has the ability to expand by storing fat but reaches a limited accretion during later stages of obesity, setting off a systemic metabolic dysfunction. The presence of inducible brown-like adipocytes was recently described in WAT able to convert fat into heat, for that reason WAT "browning" become a key strategy for managing obesity over the last years. Melanocortins, such as $\alpha-\mathrm{MSH}$ are able to increase lipolytic activity of white adipocytes and also thermogenesis of brown adipocytes. Whether they can induce the transdifferentiation of white to beige adipocytes is unknown and constitutes the basis of this work

Methods: C57BL/6J mice were fed a high fat diet (DIO: $45 \%$ energy from fat) or standard diet (control) for 10 weeks and treated with $\alpha-\mathrm{MSH}$ $(150 \mu \mathrm{g} / \mathrm{kg} /$ day) or vehicle (saline) during 14 days. Paraffin-embedded subcutaneous WAT (SAT) sections were used for determination of adipocyte cross-sectional area. Immunohistochemistry was carried out for detection of the "browning" marker protein uncoupling protein 1 (UCP1). Expression analysis of UCP-1 was also performed by real-time PCR in SAT and in 3T3-L1 adipocytes stimulated with the $\alpha$-MSH $(1 \mu \mathrm{M})$ for $4 \mathrm{~h}$ or $24 \mathrm{~h}$. Mitochondria content and oxygen consumption of 3T3-L1 adipocytes was measured through NAO incorporation and a Clark-type electrode, respectively.

Results: $\alpha$-MSH induces an increased expression of UCP- 1 mRNA in SAT depot from DIO mice of about 8 times relative to saline animals. In lean mice (control), $\alpha$-MSH has a less pronounced effect, increasing UCP1 levels 1.7 above saline. In agreement, the appearance of beige adipocytes, 
UCP-1 positive, dispersed around SAT is clearly visible by immunohistochemistry in SAT from a-MSH-treated mice. A significant reduction of adipocyte area was also observed in animals treated with $\alpha-\mathrm{MSH}$ compared to saline animals. In 3T3-L1 adipocytes, $\alpha-\mathrm{MSH}$ was able to induce a 20-fold upregulation of UCP-1 gene. The a-MSH treatment also increases the number of mitochondria (1.5-fold) and oxygen consumption rates (2-fold) in 3T3-L1 adipocytes, suggesting the occurrence of uncoupled respiration.

Conclusion: The melanocortin a-MSH promotes structural and transcriptional alterations on SAT depot from lean and DIO mice inducing beige/brown-like features. These effects seem to be accomplished by a functional capacity for thermogenesis in 3T3-L1 adipocytes. These findings confer on the $a-\mathrm{MSH}$ potential as future obesity therapy.

Conflict of Interest: None Disclosed

Funding: FCT/MEC (PIDDAC) and FEDER-Fundo Europeu de Desenvolvimento Regional, COMPETE 2020-Programa Operacional Competitividade e Internacionalização (PTDC/BIM-MET/2123/2014); Tanita Healthy Weight Community Trust; Adriana Rodrigues is supported by FCT (SFRH/BPD/92868/2013).

\section{OS3:OC6}

\section{Involvement of the dermal white adipose tissue in cutaneous wound healing delay during obesity}

\section{Begey A.L. '; Nguyen-Tu M.S. '; Sigaudo-Roussel D. ${ }^{1}$ \\ ${ }^{1}$ UMR 5305 CNRS, 69367 Lyon cedex 07, France; University of Lyon 1, 69367 Lyon cedex 07, France}

Introduction: Dermal white adipose tissue was shown to increase during obesity at the expense of surrounding tissues and could participate to cutaneous fragility. Extremely obese patients are subjected to higher risk for pressure ulcers than normal weight or overweight patients. The aim of the present study was 1) to study the cutaneous healing process depending on the adiposity in a diet-induced model of obesity in mice and 2) to characterize the dermal and epididymal white adipose tissues (dWAT and eWAT) responses to lipolytic stimulation as well as their ability to respond to insulin.

Methods: C57Bl6 male mice were randomly assigned to a high calorie diet for 4 or 12 weeks (HCD4 or HCD12). First, we induced a skin pressure injury and we studied the time for skin healing using digital photographs taken at different times. The surface measurement of the injured skin was evaluated using ImageJ software. Skin functionality was evaluated before injury and after the skin was fully healed using laser Doppler flowmetry in response to a local pressure application or vasoactive drugs. Intraperitoneal glucose and insulin tolerance tests were performed to evaluate the systemic sensitivity. The lipolytic response was investigated measuring the glycerol secretion by either dWAT or eWAT after an incubation with noradrenalin. The response to insulin was tested in the same way after an incubation with insulin plus noradrenalin.

Results: We reported that the skin healing process is delayed by 5 to 10 days in HCD12 mice compared to HCD4 mice. The injured surface area showed that the necrotic surface of the skin was 75\% bigger in HCD12 mice than HCD4 mice. Skin functionality appears to be altered after skin closure in both HCD groups compared to their respective control groups. The skin blood flow was decreased in HCD4 and HCD12 mice in response to a local pressure application after skin closure compared to the non-wounded skin. In response to vasoactive drugs, the skin blood flow was also decreased in HCD12 mice after skin closure compared to the to the non-wounded skin, but not in HCD4 mice. The lipolytic response and the insulin response were decreased in the eWAT in HCD12 mice compared to HCD4 in contrast to the dWAT where the responses were not different between HCD4 and HCD12 mice.

Conclusion: This study suggests that the increase of dermal white adipose tissue during obesity leads to a cutaneous fragility. The severity of the skin lesion and the time to heal were dependent to the increase in adiposity but not dependent to dermal adipose tissue insulin resistance. In addition, it appears that at the time of skin closure the skin responses to stimuli were not restored.

Conflict of Interest: No conflict of interest.

Funding: Research was funded by University of Lyon1/CNRS.

\section{Thursday, 18 May 2017}

\section{OS4 - Energy Balance}

\section{OS4:OC7}

\section{Sustainability of changes in appetite at 1-year follow-up after initial weight loss with a very-low energy diet}

$\underline{\text { Nymo S }}{ }^{1}$; Coutinho SR ; Torgersen HC-L'; Truby $H^{2}$; Kulseng $B^{3}$; Martins C.

${ }^{1}$ Centre for Obesity Research, Department of Cancer Research and Molecular Medicine, Faculty of Medicine, Norwegian University of Science and

Technology, Trondheim, Norway, ${ }^{2}$ Department Of Nutrition, Dietetics \& Food, Monash University, Melbourne, Australia., ${ }^{3}$ Centre For Obesity, Department Of Surgery, St. Olav Hospital-Trondheim University Hospital, Trondheim, Norway

Introduction: Weight loss (WL) induced by non-ketogenic diets has been acutely associated with a compensatory increase in appetite, which may lead to overeating and potential weight regain. Few studies have assessed the sustainability of this compensatory increase in appetite in the longterm. The aim of this study was to determine if changes in subjective and objective appetite markers observed with WL are sustained at 1-year (1Y) follow-up.

Method: 36 adults (10 females) with obesity (BMI: $36.6 \pm 0.7 \mathrm{~kg} / \mathrm{m}^{2}$, age: $41 \pm 1.6 y r$ ) followed 8 weeks (wks) of a very-low energy diet (VLED), followed by 4 wks refeeding and $1 Y$ maintenance program. Fasting/postprandial subjective feelings of hunger, fullness, desire to eat and prospective food consumption (PFC) were assessed and plasma concentrations of active ghrelin (AG), total peptide YY (PYY) and active glucagon-like peptide 1 and insulin were measured every 30 minutes for 2.5 hours. Measurements were taken at baseline, week 13 (W13) and 1Y. Statistical analysis was performed using linear mixed model, with Bonferroni adjustments. Data are presented as mean \pm SEM.

Results: A WL of $17.1 \pm 1.1 \%(\mathrm{P}<0.001)$ at $\mathrm{W} 13$ was associated with a significant increase in feelings of hunger in fasting $(\mathrm{P}<0.05)$ and significant reduction in feelings of PFC in fasting $(\mathrm{P}<0.001)$ and postprandially $(\mathrm{P}<0.05)$. There was also a significant increase in fasting/postprandial concentrations of AG $(\mathrm{P}<0.001$ for both $)$ and a reduction in fasting/ postprandial concentrations of insulin ( $\mathrm{P}<0.001$ for both). At $1 \mathrm{Y}$ follow-up and with sustained WL $(15.2 \pm 1.1 \%)$, a significant reduction in postprandial PFC feelings $(\mathrm{P}<0.05)$ and a significant increase in fasting/ postprandial AG plasma concentrations $(\mathrm{P}<0.001$ for both) and a significant reduced in fasting/postprandial insulin plasma concentration were still observed.

Conclusion: With a $15 \%$ sustained WL at $1 Y$ follow-up, plasma concentrations of AG do not revert to baseline and are still increased, despite no changes in hunger and a reduction in PFC feelings. Future studies should evaluate how this impacts on actual food intake and how it relates to longterm WL maintenance.

Conflict of Interest: The authors declare no conflicts of interest.

Funding: Liaison Committee between the Central Norway Regional Health Authority (RHA) and the Norwegian University of Science and Technology (NTNU). Clinic of Surgery, St. Olav University Hospital, Trondheim. Norway. Allevo, Karo Pharma AS. Sweden, for providing the VLED products (no commercial interest). 
OS4:OC8

\section{Habitual physical activity and satiety: Confirmatory evidence for enhanced homeostatic appetite control in physically active individuals}

\section{Beaulieu, K. ' ${ }^{\prime}$ Long, C. ' Hopkins, M. ${ }^{2}$; Blundell, J. ' ; Finlayson, G.}

${ }^{1}$ University of Leeds, ${ }^{2}$ Leeds Beckett University; University Of Leeds

Introduction: Evidence suggests that higher levels of physical activity (PA) improve the ability to compensate for previous energy intake (EI) shown by decreased ad libitum EI following high-energy preloads. However, prior studies lacked objective assessment of habitual PA and 24-h EI. The purpose of this study was to assess the effect of PA level on EI and appetite sensations following high-energy-density (HED) and low-energy-density (LED) preloads.

Methods: High (HiPA; $\mathrm{n}=12,8 \mathrm{~F}$ ), moderate (ModPA; $\mathrm{n}=11,8 \mathrm{~F}$ ) and low (LoPA; $\mathrm{n}=11,8 \mathrm{~F}$ ) physically active individuals, matched for age and $\mathrm{BMI}$, were grouped based on tertiles of moderate-to-vigorous habitual PA obtained from objective and quantified PA. Individuals attended a preliminary assessment where resting metabolic rate, body composition, cardiorespiratory fitness and eating behaviour traits were measured. This was followed by 2 intensive laboratory probe days where ad libitum EI was determined at lunch, dinner and evening snack box after consumption of a fixed breakfast. Prior to the ad libitum lunch meal, porridge preloads varying in energy density (HED: $690 \mathrm{kcal}$ and LED: $250 \mathrm{kcal}$ ) but matched for volume and macronutrient composition were consumed. Appetite sensations were assessed periodically throughout the day.

Results: Following the consumption of the preloads, ad libitum EI at the lunch meals was significantly different between activity groups, such that HiPA ( $p=0.013)$ and ModPA ( $p=0.004)$ consumed less after HED compared to LED. No differences existed for LoPA ( $p=0.593)$. No differences were seen in EI between activity groups for dinner or snack box consumption. Total EI was greater in HED compared to LED $(p<0.001)$, whereas daily hunger was greater in LED compared to HED ( $p=0.01)$.

Conclusion: These results demonstrate that ModPA and HiPA reduced ad libitum EI following HED compared to LED preload, while LoPA did not. This suggests that LoPA were insensitive to the nutritional manipulation, indicating blunted satiety signaling. Therefore, habitual PA enhances satiety signaling and homeostatic appetite control.

\section{OS4:OC9}

\section{Promoting satiation and satiety within a weight management} programme improves appetite control and weight loss.

Buckland, N. '; Camidge, D. '; Croden, F. ; Hetherington, M. '; Blundell, J.' ; Finlayson, $G$.

'University of Leeds

Introduction: Weight management programmes (WMPs) that promote satiation and satiety by encouraging behaviour changes towards unre $\neg$ stricted intake of low energy density (LED) food choices might be more effective than self-led traditional calorie reduction WMPs. The SWIPSS trial examined the effect of a commercial weight loss programme pro $\neg$ moting unrestricted intake of LED foods [Slimming World, UK (SW)] on body weight and weight loss experience compared to a self-led calorie-re $\neg$ duction standard care WMP [Live Well, NHS standard care (SC)]. The tri $\neg$ al also examined the effects of meals varying in energy density (consistent with SW approach) on appetite and energy intake.

Methods: Ninety-six women who were overweight or obese (age: $41.8 \pm$ 1.4y; BMI: $33.3 \pm 0.4 \mathrm{~kg} / \mathrm{m}^{2}$ ) were recruited from SW and SC weight management arms of a 14-week clinical trial. Weight loss experi-ence was assessed with weekly questionnaires. In weeks 3 and 12 as part of laboratory intensive probe days, participants' diurnal appetite ratings and ad libitum meal intake were assessed in response to iso-caloric LED and high energy dense breakfast and lunch meals.

Results: LED meals increased participants' subjective sensations of fullness and reduced sensations of hunger throughout the day and, re $\neg$ duced total day energy intake compared to high energy dense meals (all ps<.001). The SW group lost more weight and were more likely to achieve clinical weight loss ( $>5 \%)$ compared to the SC group. The SW group ex $\neg$ perienced greater control over eating, found the WMP easier to adhere to, experienced more enjoyment losing weight, were more satisfied with the WMP and were more motivated to continue the programme compared to the SC group.

Conclusion: A commercial WMP supporting behaviour changes towards LED food choices is an effective strategy for weight loss with health bene $\neg$ fits and improved experience above self-led calorie reduction programmes [CT\#NCT02012426]

Conflict of Interest: None

Funded by Slimming World, UK

\section{OS4:OC10}

Associations between genetic susceptibility, eating behaviour and growth in children up to 5 years, results from the EDEN mother-child cohort

de Lauzon-Guillain, $B^{1}$; Charles, $M A^{1}$; Akoli Koudou, $Y^{1}$; Forhan, $A^{1}$; Ong, $K^{2}$; Heude, $B^{1}$; on behalf of the EDEN mother-child cohort study group ${ }^{3}$ ${ }^{1}$ INSERM, UMR1153 Epidemiology and Biostatistics Sorbonne Paris Cité Center, Early ORigin of the Child's Health and Development Team (ORCHAD), Paris, France; Paris Descartes University, France, ${ }^{2}$ Medical Research Council Epidemiology Unit, University Of Cambridge, Addenbrooke'S Hospital, Cambridge, England, ${ }^{3}$ INSERM

Introduction: Many genetic polymorphisms identified by genome-wide-association studies on obesity appeared involved in the regulation of food intake. Our objective was to examine the links between a genetic obesity risk score, eating behavior and growth of children up to age 5 years.

Methods: In 1,142 children from the EDEN mother-child cohort, a combined obesity risk-allele score (BMI-GRS) was calculated from genotypes at 16 variants identified by genome-wide association studies of childhood body mass index (BMI) or early-onset obesity. Children's appetite and food neophobia were reported by parents at 1,2, 3 and 5 years and energy intake (restricted to infants non breastfed during 3-d food records) was assessed at 4, 8 and 12 months. Associations between BMI-GRS and eating behaviour were tested using logistic regressions and associations between eating behaviour at a given age and predicted BMI at subsequent ages were tested by linear regressions.

Results: A high BMI-GRS was associated with a higher energy intake at 1 year, higher appetite at 2 and 5 years and a lower food neophobia at 1 year. Higher energy intake in infancy and higher appetite from 1 year onwards were related to higher subsequent BMI. Associations observed between BMI-GRS and BMI from 3 years were weaker and even no longer significant at 3 and 4 years after adjustment for appetite at 2 years.

Conclusion: The associations observed between BMI-GRS and eating behaviour precede those found between this score and child's BMI. Genes involved in childhood obesity could therefore promote higher appetitive traits in the first years of life, followed by a subsequent increase in BMI.

Conflict of Interest: None Disclosed

OS4:OC11

Evaluation of the accuracy of predictive equations in estimating resting energy expenditure in obese patients

Sammarco, R. ${ }^{1}$; Cioffi, I. ${ }^{1}$; Marra, M. ${ }^{1}$; Rosa, E. ${ }^{1}$; Onufrio, M. ${ }^{1}$; Caldara, A. ${ }^{\prime}$; Contaldo, F.' ; Pasanisi, F. ${ }^{1}$

${ }^{1}$ Department of Clinical Medicine and Surgery, University Federico II

Introduction: Several predictive equation are available for the evaluation of resting energy expenditure (REE) in obese patients. This study compares resting energy expenditure (REE) measured (MREE) by indirect 
calorimetry (IC) with REE predicted (PREE) using different equations in Caucasian obese adults.

Methods: We studied 1851 (1181 females, 670 males) obese patients (BMI $>30 \mathrm{~kg} / \mathrm{m}^{2} ; 18-65$ years). REE was measured by indirect calorimetry ( $V$ max29-Sensormedics).Data were obtained comparing MREE with PREE derived from several published equations for normal weight or obese subjects. Mean differences between PREE and MREE as well as the accuracy prediction within $\pm 10 \%$ level were investigated in all sample as well as in BMI sub-groups (Group $1=30-40 \mathrm{~kg} / \mathrm{m}^{2}$; Group $2=40-50 \mathrm{~kg} / \mathrm{m}^{2}$; Group $\left.3>50 \mathrm{~kg} / \mathrm{m}^{2}\right)$.

Results: We observed that FAO, Henry and Muller (BC) equations provide a satisfactory mean differences P-M (bias - 0.7, - 0.3 and $0.9 \%$; RMSE 273, 263 and $269 \mathrm{kcal} / \mathrm{d}$, respectively); HB and Henry equation resulted accurate individually (57 and 56.9\%). Only Muller (BC) gave the lowest REE difference P-M (-1.7\%; RMSE $228 \mathrm{kcal} / \mathrm{d})$ in females, while Johnstone and De Lorenzo equations were more accurate (55.1 and 54.8\%) When sample was split up in three BMI subgroups, no difference was found for males, whereas the majority of equations included in this study failed to estimate REE in severely obese females (BMI $\left.>40 \mathrm{~kg} / \mathrm{m}^{2}\right)$. Overall, the accurate prediction was considered low $(\sim 55 \%)$ for all predictive equations, unelated to BMI.

Conclusion: REE predictive equations developed in obese patients and for specific age groups are more suitable than those for the general population. Inaccuracy of predicted REE could affect dietary prescription and consequently dietary compliance. Measurements of REE with indirect calorimetry is highly recommended in severely obese patients.

Conflict of Interest: None Disclosed

Funding: No Funding

\section{OS4:OC12}

\section{Acute exercise modifies levels of adiponectin and other} (adipo) cytokines in cerebrospinal fluid of young individuals

\section{Janakova, Z. ${ }^{1}$; Schon, M. ${ }^{1}$; Kosutzka, Z. ${ }^{2}$; Ukropec, J. ${ }^{3}$; Valkovic, P. ${ }^{2}$;}

\section{Ukropcova, $B$. $^{3}$}

${ }^{1}$ Institute of Pathological Physiology, Faculty of Medicine, Comenius University, Bratislava, Slovakia; Institute of Experimental Endocrinology, Biomedical Research Center, Slovak Academy of Sciences, Bratislava, Slovakia, ${ }^{2} 2 \mathrm{Nd}$ Department Of Neurology, Faculty Of Medicine, Comenius University \& University Hospital, Bratislava, Slovakia, ${ }^{3}$ Institute Of Experimental Endocrinology, Biomedical Research Center, Slovak Academy Of Sciences, Bratislava, Slovakia

Introduction: Obesity and physical inactivity increase the risk of cognitive decline and physical activity can improve body composition and metabolism as well as cognitive functions and psychological well-being. Interestingly, adipose tissue-derived adipokine adiponectin has been shown to mediate antidepressive effects of exercise in mice. However, mechanisms and mediators behind the exercise action on the human brain remain to be elucidated. We measured the effect of acute aerobic exercise on spectrum of (adipo) cytokines in cerebrospinal fluid (CSF) of young volunteers.

Methods: A sample of CSF was obtained by atraumatic lumbar puncture in a basal state (non-exercise day1) and after 90 minutes of running $\left(<5.30\right.$ min. $\mathrm{km}^{-1},>75 \%$ maximal heart rate; exercise day 2$)$ from 6 healthy individuals $\left(3 \mathrm{M} / 3 \mathrm{~F}\right.$; age $\left.=24.8 \pm 6.0 \mathrm{yrs} ; \mathrm{BMI}=23.1 \pm 2.5 \mathrm{~kg} / \mathrm{m}^{2}\right)$. Levels of 174 cytokines were measured in CSF before and after exercise using antibody array (RayBiotech). Physical fitness (maximal aerobic capacity, $\left.\mathrm{VO}_{2} \max \right)$ was assessed by bicycle spiroergometry. Cognitive functions were measured by computerized CogState tests.

Results: Running induced a $21.3 \%$ decrease in adiponectin $(\mathrm{p}<0.01)$, more than $10 \%$ decrease in IL-18R beta and $\operatorname{PDGF}(\mathrm{p}<0.05)$ and more than 5\% decrease in IL5R alpha, TGF beta1 and 2, CXCL9, MMP13, Tie-1, Activin A, IL-18 binding protein alpha $(\mathrm{p}<0.05)$ and IGF-2 $(\mathrm{p}<0.01)$ levels in CSF. CXCL16, IGF-1 and SDF-1 decreased in men only $(\mathrm{p}<0.05)$, while an increase in IGFBP-4, CCL4, IGF-1 and IL-16 was observed in women only $(\mathrm{p}<0.05)$. Adiponectin in CSF positively correlated with $\mathrm{VO}_{2} \max (\mathrm{n}=6, \mathrm{R}=0.82, \mathrm{p}<0.05)$ albumin $\mathrm{CSF} /$ serum ratio $(\mathrm{n}=12, \mathrm{R}=0.65, \mathrm{p}<0.05)$ and the $\%$ change of adiponectin after run with cognitive test score $(n=6, R=0.89, p<0.05)$.

Conclusion: Acute aerobic exercise significantly modified levels of adiponectin and several other cytokines in cerebrospinal fluid of young individuals, suggesting their potential role as mediators of exercise-induced effects on human brain. Our results also suggest sex-related differences in response to exercise. Importance of these changes for both obesity-related cognitive decline and exercise-induced benefits requires further studies.

Conflict of Interest: None Disclosed

Funding: SAS-NSC Joint Research Cooperation grant 2013/17,VEGA 2/0191/15, APVV-15-0253.

\section{OS5 - Environment and Policy}

\section{OS5:OC31}

\section{Exploring the relation of exposure to fast food outlets with obesity}

Mackenbach, J. ${ }^{1}$; Charreire, H. $^{2}$; Glonti, K. ${ }^{3}$; Bardos, H. ${ }^{4}$; Rutter, H. ${ }^{3}$; Compernolle, S. ${ }^{5}$; Bourdeaudhuij, I. ${ }^{5}$; Nijpels, G. 6 ; Brug, J.7; Oppert, J.8; Lakerveld, J. ${ }^{1}$

${ }^{1}$ Department of Epidemiology \& Biostatistics, VU University Medical Center, ${ }^{2}$ Equipe De Recherche En Epidémiologie Nutritionnelle (Eren), Centre De Recherche En Epidémiologie Et Statistiques, Inserm (U1153), Inra (U1125), Cnam, Comue Sorbonne Paris Cité, Université Paris 13 \& Paris Est University, Lab-Urba, Upec, Urban School Of Pa, ${ }^{3}$ Ecohost - The Centre For Health And Social Change, London School Of Hygiene And Tropical Medicine, ${ }^{4}$ Department Of Preventive Medicine, Faculty Of Public Health, University Of Debrecen, ${ }^{5}$ Department Of Movement And Sport Sciences, Ghent University, Ghent, ${ }^{6}$ Department Of General Practice And Elderly Care, Emgo Institute For Health And Care Research, Vu University Medical Center, ${ }^{7}$ Department Of Epidemiology \& Biostatistics, Vu University Medical Center \& Faculty Of Social And Behavioral Sciences, University Of Amsterdam, ${ }^{8}$ Equipe De Recherche En Epidémiologie Nutritionnelle (Eren), Centre De Recherche En Epidémiologie Et Statistiques, Inserm (U1153), Inra (U1125), Cnam, Comue Sorbonne Paris Cité, Université Paris 13 \& Sorbonne Universités, Université Pierre Et Marie Curie,

Introduction: It is unknown via which pathways exposure to fast food outlets could influence levels of obesity. In this study we explored whether perceptions about availability of fast food outlets in the neighbourhood, and fast food consumption, could explain any observed associations between exposure to fast food outlets and obesity in adults.

Methods: We analysed data from 5084 SPOTLIGHT survey participants residing in five European urban regions. Participants reported on socio-demographics, fast food consumption, weight and height and neighbourhood perceptions. A virtual neighbourhood audit was conducted to geolocalize and categorize fast food outlets. Direct associations and mediating pathways of exposure to fast food outlets, perceived availability and usage of fast food outlets, fast food consumption, and self-reported weight status were explored using multilevel logistic and multinomial regression analyses.

Results: After full covariate adjustment, living in a neighbourhood with higher spatial access to fast food outlets was associated with increased perceived availability and usage of fast food outlets $(\mathrm{RRR}=3.30$, $95 \% \mathrm{CI}=1.71 ; 6.34)$, but not with fast food consumption or obesity. Perceived availability and usage of fast food outlets was associated with greater reported consumption $(\mathrm{OR}=1.55,95 \% \mathrm{CI}=1.05 ; 2.28)$.

Conclusion: Exposure to fast food outlets was not directly associated with obesity, but we did find an association between objective and perceived availability. The perceptions of availability were, in turn, related to levels of fast food consumption. These result emphasize the complexity of individual and environmental influences on lifestyle behaviours and weight status and highlight the importance of taking into account both objective and subjective measures of the food environment. 
Funding: Research relating to this abstract was supported by the Seventh Framework Programme (CORDIS FP7) of the European Commission, HEALTH (FP7 HEALTH-2011-two-stage) [278186]. The content of this article reflects only the authors' views and the European Commission is not liable for any use that may be made of the information contained therein.

\section{OS5:OC32}

\section{Associations between the food environment and obesity: The impact of methodological choices on outcomes}

Hobbs, M. ${ }^{1}$; Griffiths, C. ${ }^{2}$; Green, M. ${ }^{3}$; Jordan, H. ${ }^{4}$; Saunders, J. ${ }^{5}$; Mckenna, J. ${ }^{4}$

${ }^{1}$ Leeds Beckett University \& Leeds Trinity University, ${ }^{2}$ Leeds Beckett University, ${ }^{3}$ The University Of Liverpool, ${ }^{4}$ The University Of Sheffield, ${ }^{5}$ Public Health Team, Rotherham Borough Council

Introduction: The food environment is increasingly considered an im portant factor in determining elevated obesity prevalence. However, evidence outside of the US linking the food environment to obesity continues to be equivocal, perhaps due to methodological heterogeneity.

Objective: To compare three methodological approaches used to investigate associations between obesity and food environment.

Methods: Representative data $(n=22,889)$ from the Yorkshire Health Study were used. Body mass index (BMI) was calculated using self-reported height and weight; obesity $=\mathrm{BMI} \geq 30$. Food outlets were mapped using two databases for comparison: Ordnance Survey Points of Interest (PoI) and Local Authority (LA). Food outlets within each neighbourhood (2km radial buffer around home postcode) were summed to indicate availability and were categorised as 'fast-food', 'large supermarkets', 'convenience and other food retail outlets' and 'all food outlets'. Logistic regression (odds ratios, 95\% confidence intervals) was used for all analyses. Environmental data was modelled in quartiles (Q1 least exposed). Age, gender, ethnicity, deprivation and rural/urban classification were included as covariates Three different methodological approaches were explored:

- Approach 1 estimated associations between food outlets and obesity using PoI data.

- Approach 2 investigated the impact of adjusting for supermarket exposure when modelling the association between fast-food outlets and obesity.

- Approach 3 used a sub-sample of participants $(n=4,723)$ to compare associations when using PoI and Local Authority (LA) data.

Results: Approach 1 showed statistically significant increases in the odds of obesity in Q2 and Q3 of all types of food outlets compared to Q1. However, there was no difference in the likelihood of obesity for Q4. Approach 2 showed that the association between fast-food outlet exposure and obesity did not change when adjusting for supermarkets. Approach 3 demonstrated different associations when comparing LA and PoI data Unlike PoI, LA data suggested that increases in exposure to fast-food was associated with increased likelihood of obesity across all quartiles of exposure (Q2-Q4).

Conclusions: Associations between the food environment and obesity changed dependent upon methodological approach. Caution should be exercised in collating study findings that have used different methodological approaches.

Conflict of Interest: None Disclosed.

Funding: No Funding.
OS5:OC33

How does parents' perception of food insecurity impacts on adolescents' nutritional status, diet, and psychological wellbeing?

\section{Prioste, A. ' ; Fonseca, $\mathrm{H}^{2}$; Gregório, M. ${ }^{3}$; Canhão, $\mathrm{H}^{3}$}

${ }^{1}$ Faculdade de Psicologia, Universidade de Lisboa, Portugal; Escola de Psicologia e Ciências da Vida, COPELABS, Universidade Lusófona, Portugal., ${ }^{2}$ Department Of Paediatrics, Hospital De Santa Maria, Faculdade De Medicina, Universidade De Lisboa, Portugal. Rheumatology Research Unit, Instituto De Medicina Molecular, Faculdade De Medicina, Universidade De Lisboa, Lisboa, Portugal., ${ }^{3}$ Faculdade De Ciências Médicas, Universidade Nova De Lisboa

Introduction: Food insecurity concept includes uncertainty or worry about having enough food, inadequate quality of food, lack of consistent access to adequate food and food acquired through socially unacceptable means. Rates of food insecurity have been rising worldwide and Portugal is no exception. The literature on the impact of food insecurity on adolescents is scarce, although adolescents may be particularly vulnerable to insecure households. The current study aims at investigating the potential differences between adolescents who are living in food secure and those living in food insecure households as regards to anthropometric, nutritional and psychological variables. Moreover, this study sought to analyze whether food insecurity households can be predicted based on adolescents' anthropometric, nutritional and psychological variables.

Methods: The sample was comprised by 149 family dyads, i.e., mother or father and the adolescent $(\mathrm{n}=298)$. Adolescents were evaluated using anthropometric measurements and self-reported questionnaires. Parents were asked to complete a measure to assess food insecurity. Adolescents living in food secure households were compared with those living in food insecure ones, according to a set of variables: nutritional status, diet, psychological variables, and health and body perception.

Results: $25.5 \%$ of the parents perceived their household as food insecure. On average, adolescents living in food insecure households had a higher body mass indexthan the adolescents living in food secure households. Adolescents living in food secure households had breakfast on a regular basis more often and had a lower consumption of soft drinks than the adolescents living in food insecure households.

Conclusion: Food insecurity, as perceived by parents, had a significant impact on the adolescents' nutritional status, body perception and dieting habits. Our results support previous studies that showed a paradoxical association between household food insecurity and overweight status in children. Future studies should assess potential discrepancies between parents' and adolescents' perception and focus on the long-term impact of food insecurity.

Conflict of Interest: None Disclosed

Funding: Research relating to this abstract was funded by the Public Health Initiatives Programme (PT06), financed by EEA Grants Financial Mechanism 2009-2014.

\section{OS5:OC34}

A systematic review of the effectiveness of individual, community and societal-level interventions at reducing socioeconomic inequalities in obesity among adults and children

\author{
Hillier-Brown, F. ${ }^{1}$; Bambra, C. ${ }^{1}$; Cairns, J. ${ }^{1}$; Kasim, A. ${ }^{2}$; Moore, H. ${ }^{3}$; \\ Summerbell, $C$ \\ ${ }^{1}$ Institute of Health and Society, Newcastle University, ${ }^{2}$ Wolfson Research \\ Institute For Health And Wellbeing, Durham University, ${ }^{3}$ Obesity Related \\ Behaviours Research Group, Durham University
}

Introduction: Socioeconomic inequalities in obesity are well established in high-income countries. There is a lack of evidence of the types of intervention that are effective in reducing these inequalities among adults and children.

Methods: A systematic review of the effectiveness of individual, community and societal interventions in reducing socio-economic inequalities 
in obesity among adults and children. Nine electronic databases were searched from start date to October 2012 along with website and grey literature searches. The review examined the best available international evidence (both experimental and observational) in any setting and country. Studies were included if they reported both a body fatness-related outcome and a measure of socioeconomic status. Data extraction and quality appraisal were conducted using established mechanisms and narrative synthesis was conducted.

Results: The 'best available' evidence was provided by 20 adult studies and 23 child studies. At the individual level, there was evidence of the effectiveness of primary care delivered tailored weight loss programmes among deprived groups amongst adults, and there was indicative evidence that screen time reduction and mentoring health promotion interventions could be effective in reducing inequalities in obesity amongst children Community based behavioural weight loss interventions and commu nity diet clubs (including workplace ones) had some evidence of effectiveness, at least in the short term, amongst adults. For the community level interventions aimed at children, evidence was inconclusive - with some studies suggesting that school-based health promotion activities and community-based group-based programmes were effective in reducing obesity - others not. Societal level evaluations were few, low quality and inconclusive. Further, there was little evidence of long term effectiveness, and few studies of pre-school children, adolescents and men or outside the USA. However, there was no evidence to suggest that interventions increase inequalities.

Conclusion: The best available international evidence suggests that some individual and community-based interventions may be effective in reducing socio-economic inequalities in obesity among adults and children in the short term. Further research is required particularly of more complex, multi-faceted and societal-level interventions.

Conflict of Interest: None Disclosed

Funding: Research relating to this abstract was funded by the National Institute for Health Research Public Health Research Programme (project number 09/3010/14)

\section{OS5:OC35}

Impact of a soft drink-free policy on sales of unhealthy and all drinks in YMCA Victoria aquatic and recreation centres

Boelsen-Robinson, $T^{1}$; Kurzeme, A. ${ }^{2}$; Jerebine, A. ${ }^{2}$; Gilham, B. ${ }^{1}$; Backholer, K. '; Chung, A. '; Peeters, A.

${ }^{1}$ Deakin University, ${ }^{2}$ YMCA Victoria

Background: Food retail within sports, aquatic and recreation centres is often nutritionally poor. As health-promoting settings they represent an opportunity to implement healthy food retail strategies. YMCA Victoria, the Australian state of Victoria's largest aquatic and recreation provider recently committed to removing soft drink from all its centres.

Aim: This study aimed to determine the impact of the removal of soft drinks from a sample of recreation and aquatic centres in Melbourne on sales of unhealthy drinks and of all drinks.

Methods: Monthly sales data from January 2013 to May 2016 was collected from nine YMCA centres with a kiosk or cafe. All centres removed soft drinks by December 2015. Drinks were classified using state Government nutritional guidelines as 'green' (best choice), 'amber' (choose carefully) or 'red' (limit). Interrupted time series analysis was conducted to determine the effect of the policy, adjusted for autocorrelation and seasonal effects. Analysis was conducted on changes to the volume of ready-to-drink beverages, as well as dollar sales value of all drinks.

Results: Sales volume of the 'red' ready-to-drink beverages significantly decreased by $54.8 \%(-37.8 \%,-71.8 \%)$ and 'green' drink volume increased by $23.8 \%(10.9 \%, 36.8 \%)$, with no change in 'amber' drinks. The dollar value of all beverages sold did not change after the intervention compared to the pre-intervention period.

Conclusion: This innovative policy had its intended effect of reducing purchase of unhealthy drinks, without negatively impacting on overall drinks sales. The development of stakeholder-relevant outcome measures is important to support the spread of such policies at scale.

Disclosure: AK and AJ are employed by YMCA Victoria.

\section{OS5:OC36}

Maternal generation and overweight and obesity in 2-11 year old children: Evidence from birth cohort of the longitudinal study of Australian children

\section{Zulfiqar, T. '; Strazdins, L. ' D $^{\prime}$ Este, C. ${ }^{1}$; Dinh, H. ${ }^{\text {; }}$ Banwell, C. \\ ${ }^{1}$ The Australian National University}

Introduction: First and second generation children of immigrants from low-income countries are at higher risk of overweight and obesity compared to the native children from the developed countries. However, such evidence is scarce in Australia. The current study determines the association of maternal generation and economic development of origin country with the overweight and obesity of Australian children aged 2-11 years.

Methods: Secondary data analysis of the longitudinal study of Australian children waves 2-6 of the Birth cohort was conducted. Exposure variable was mother's generation according to the economic development of her origin country. The main outcome of interest was overweight and obesity in children according to the International Obesity Taskforce age- and sex-specific BMI cut points.

Results: Nationally representative sample of 4,606, 2-3-year-old children is followed for 10 years (2,349 boys and 2,257 girls]. The final sample in wave 6 comprises of 3764, 10-11-year-old children (1929 boys and 1835 girls). At baseline, $24 \%$ of children (aged 2-3 years) of the first generation and $22 \%$ of children of second-generation mothers from developing countries were overweight and obese compared to $23 \%$ children of native mothers. The proportion of overweight and obese children was $19 \%$ in the first generation and $23 \%$ in second-generation mothers from other developed countries at the baseline. Cross-sectional multivariable logistic regression models of wave 2 to wave 6 , show that after controlling for the child gender and socioeconomic position, children of first generation mothers from developing countries had 37\% (95\% CI 1.05 1.79) higher odds of overweight and obesity relative to the children of native mothers at age of 4-5 years (wave 3) and 52\% (95\% CI 1.12 2.04) higher odds at age 10-11 years (wave 6). Children of first generation mothers from developed countries had 25\% (95\% CI .56 .98) lower odds of overweight and obesity at age $2-3$ years (wave 2) and $30 \%$ (95\% CI .50 .95) lower odds at age of 8-9 years (wave 5) relative to children of native mothers.

Conclusion: Maternal generation and economic development of origin country provide important insight to the risk of overweight and obesity in children of immigrants.

Conflict of Interest: None

Funding: No Funding

\section{OS6 - Childhood Obesity Management: from the Lab to the Community}

\section{OS6:0C37}

\section{Targeting parental recognition and understanding of childhood overweight to improve child weight outcomes:} The impact of the MapMe intervention at 12 months follow-up

Jones, A. ${ }^{1}$; Mann, K. ${ }^{1}$; Cutler, L. ${ }^{1}$; Parkinson, K. ${ }^{1}$; Arnott, B. ${ }^{1}$; Araújo-Soares, V. '; Pearce, M. ' ; Tovee, M. ${ }^{2}$; Ells, L. ${ }^{3}$; Harris, J. ${ }^{4}$; Adamson, A. ${ }^{1}$ ${ }^{1}$ Newcastle University, ${ }^{2}$ University Of Lincoln, ${ }^{3}$ Teesside University, ${ }^{4}$ University Of St Andrews

Introduction: Evidence indicates parents typically do not classify their child as overweight (OW) even when they are, according to commonly 
used clinical criteria. This is postulated to be a barrier to parents taking appropriate action for their child's weight gain. We developed the MapMe intervention to improve parental recognition of childhood OW in paperand web-based formats and targeted families of 4-5 and 10-11 year olds. MapMe includes body image scales of known weight status and information on childhood OW and modifiable health related behaviours. The impact of MapMe on recognition of OW has been reported ${ }^{1}$. The aim of this study was to assess its impact on child weight outcomes 12 months post intervention.

Methods: A 3 arm randomised controlled trial tested the impact of MapMe on child weight outcomes 12 months post intervention ${ }^{2}$. Parents of 4-5 and 10-11 year old children were recruited via schools which were randomised to either the paper-based, web-based or control (no intervention) group. Child height and weight measurements were taken at baseline and 12 months follow up and body mass index (BMI) calculated. Weight status was determined using UK90 criteria $^{3}$.

Results: 2933 families were recruited (53\% boys; 48\% 4-5 years). 2210 children had valid BMI data at both timepoints of which 334 were OW/ very OW at baseline. Of these OW/very OW children 137 improved their weight status between baseline and follow up, $68 \%$ of which were in an intervention group. There was a significant reduction in BMI centile in OW/very OW children in all study groups (web-based: $-2.4, \mathrm{p}<0.001$ paper-based: $-2.1, \mathrm{p}<0.001$; control: $-1.0, \mathrm{p}=0.009)$. When the difference in change between groups was assessed there was a significantly greater reduction in those children in an intervention group compared to control $(-1.19, \mathrm{p}=0.033)$. When the intervention groups were examined separately the difference in change between the web-based and control groups approached significance $(-1.31, \mathrm{p}=0.059)$

Conclusion: Since most OW/very OW children who improved their weight status were part of a family allocated to an intervention group and differences in BMI centile change were more marked in those in an intervention group, these data indicate that the MapMe intervention, particularly in its web-based format, can help OW/very OW children's weight outcomes. Further work is required to replicate these findings and investigate mechanisms of action.

Conflict of Interest: None disclosed

Funding: National Prevention Research Initiative; Gateshead Council; Newcastle City Council; North Tyneside Council; Redcar \& Cleveland Borough Council; Sunderland City Council

\section{References:}

1 Jones et al.: (2016) Obes Rev.

2 Parkinson et al.: (2015) BMC Public Health.

3 Cole et al.: (1995) Arch Dis Child.

\section{OS6:OC38}

\section{Gaining consensus on obesity-related co-morbidities to include in a screening tool for children and adolescents attending community weight management services}

\section{Sharma, V. ${ }^{1}$; Coleman, D. ${ }^{1}$; Sharples, $P^{2}{ }^{2}$; Nixon, $P^{1}{ }^{1}$; Hamilton-Shield, $P^{3}{ }^{3}$; Bryant, D. ${ }^{\prime}$ \\ ${ }^{1}$ The University of Leeds, ${ }^{2}$ London School Of Hygiene And Tropical Medicine,} ${ }^{3}$ The University Of Bristol

Aim: To gain consensus on which obesity-related co-morbidities to include in a child/adolescent $(<18)$ screening tool.

Introduction: NICE guidance (PH47) recommends that children/adolescents attending community weight management services are screened for obesity-related co-morbidities to identify those at risk, and provide support and treatment at an earlier stage to minimise future complications. A systematic review and meta-analyses conducted as part of the research identified 24 co-morbidities and their prevalence amongst healthy weight, overweight and obese children/adolescents.

Methods: A modified version of the Research and Development/University of California at Los Angeles (RAND/UCLA) Appropriateness Method was utilised over two cycles to gain consensus on which co-morbidities (cycle 1) and screening methods (cycle 2) to include in the screening tool. Each cycle consisted of: 1) a pre-meeting questionnaire for a group of health professionals and researchers to review the systematic review and meta-analyses results; 2) a focus group with service users (parents and children) to obtain their views; 3 ) a face-to-face consensus meeting with the health professionals and researchers; 4) a post-meeting questionnaire for the health professionals and researchers.

Results: The cycle 1 results indicated seven co-morbidities were considered appropriate for inclusion: diabetes, hypertension, dyslipidaemia, obstructive sleep apnoea, depression, anxiety, and low self-esteem. However cycle 2 results showed uncertainty over the suitability of the screening methods for use within a weight management setting. Additionally during cycle 2 the appropriateness of conditions, such as dyslipidaemia and low self-esteem, was questioned.

Conclusion: Despite agreement over the co-morbidities in cycle 1 , the group of researchers and health professionals were unable to achieve consensus on screening methods which they deemed suitable for administration by community weight management services. Concerns over the length of questionnaires, staff training requirements - including inter-rater reliability, interpretation of test results, and conflicting evidence on the current prevalence of the co-morbidities were some of the reasons provided. Future plans are to re-visit the NICE guidance the research was based on, as well as literature relating to population screening to identify appropriate steps forward and highlight avenues for future research.

Conflict of Interest: Professor Shield chaired the NICE group which developed the NICE guidance

Funding: Research relating to this abstract was part of a University of Leeds funded $\mathrm{PhD}$

\section{OS6:OC39 \\ New reference values for cardiorespiratory fitness in children and adolescents with obesity}

Johansson, L. ${ }^{1}$; Westerståhl, M. ${ }^{2}$; Brissman, M. ${ }^{3}$; Janson, A. ${ }^{4}$; Morinder, G. ${ }^{5}$; Marcus, $C^{3}$

${ }^{1}$ National Childhood Obesity Centre, Karolinska University Hospital, ${ }^{2}$ Department Of Laboratory Medicine, Karolinska Institutet, ${ }^{3}$ Department Of Clinical Science, Intervention And Technology, Karolinska Institutet, ${ }^{4}$ National Childhood Obesity Centre, Karolinska University Hospital And Department Of Women'S And Childrens Health, Karolinska Institutet, ${ }^{5}$ National Childhood Obesity Centre, Karolinska University Hospital And Department Of Clinical Science, Intervention And Technology, Karolinska Institutet

Introduction: Low cardiorespiratory fitness (CRF) in children and adolescents is associated with present and future morbidity. However, previously published reference values for CRF in children stem from a general population and they are of limited value when cardiometabolic risk among children with obesity is evaluated. Therefore, the purpose was to present age- and gender specific reference values for CRF among Swedish children and adolescents with obesity, and to describe to what extent VO2 max was explained by anthropometric values.

Methods: CRF was assessed in 705 children (356 girls, 8-20 years) with an Åstrand submaximal bicycle test at the National Childhood Obesity Centre, Karolinska University Hospital, Stockholm, Sweden. Data was collected from 1999-2013. All participants were classified as obese according to IOTF,

Results: Absolute $\mathrm{VO} 2 \mathrm{max}(1 / \mathrm{min})$ increased with age, while relative VO$2 \mathrm{max}(\mathrm{ml} / \mathrm{kg} / \mathrm{min})$ decreased with age. Age, length and weight explained $19.5 \%$ of absolute $\mathrm{VO}_{2} \max (1 / \mathrm{min})$ in girls and $26.6 \%$ in boys. No association was found between degree of obesity and absolute $\mathrm{VO}_{2} \max (1 / \mathrm{min})$. Absolute $\mathrm{VO}_{2} \max (1 / \mathrm{min})$, for both boys and girls, was similar to previously published reference values of CRF in a general population. However, relative $\mathrm{VO}_{2} \max (\mathrm{ml} / \mathrm{kg} / \mathrm{min})$ was lower and none of the boys, and only $5 \%$ of the girls, with obesity exceeded the $25^{\text {th }}$ percentile when comparing CRF to subjects of normal weight.

Conclusion: These are, to our knowledge, the first reference values of CRF in children with obesity. They enable comparison of CRF in children 
and adolescents with obesity which can help clinicians assessing risks and provide guidelines for treatment as well as serve as an important clinical outcome.

Conflict of Interest: None Disclosed

Funding: No Funding

\section{OS6:OC40}

The parent-led PEACH ${ }^{\mathrm{TM}}$ program for child weight management improved parenting and child diet, and was associated with reduced child adiposity when delivered at scale

\section{Moores, C. ' ;iller, J. '; Chan, L. ${ }^{1}$; Daniels, L. ${ }^{2}$; Croyden, D. ${ }^{2}$; Vidgen, H. ${ }^{2}$;} Magarey, $A .^{\prime}$

${ }^{1}$ Nutrition and Dietetics, School of Health Sciences, Faculty of Medicine, Nursing and Health Sciences, Flinders University, Adelaide, South Australia, Australia, ${ }^{2} S c h o o l$ Of Exercise And Nutrition Sciences, Faculty Of Health, Queensland University Of Technology, Brisbane, Queensland, Australia

Introduction: Parenting, Eating and Activity for Child Health ( $\left.\mathrm{PEACH}^{\text {тм }}\right)$ is a 6-month, parent-led, parenting and healthy lifestyle program for families with overweight/obese children of primary school age. While our previous RCT demonstrated efficacy of the $\mathrm{PEACH}{ }^{\mathrm{TM}}$ program (1), this presentation aims to report pre-post outcomes of $\mathrm{PEACH}^{\mathrm{TM}}$ when upscaled and delivered in community settings.

Methods: 926 overweight/obese children from 817 families were enrolled in $105 \mathrm{PEACH}^{\mathrm{TM}}$ groups across Queensland, Australia from October 2013 to September 2016. Parents completed questionnaires (pre- and post-program) for outcome measures including parenting self-efficacy and children's dietary questionnaire (CDQ; 2). Children were measured (height, weight and waist) pre- and post-program. Complete paired pre-post data for these outcomes were available for up to $49 \%$ (between $n=299-356$ ) of children who attended $\geq 1$ session $(n=720)$. Bootstrapped paired-samples t-tests were performed, data are presented as mean difference, 95\% CI [LL, UL].

Results: A comparison of children with paired pre- and post-program data to children with baseline data only indicates that those withou paired data were proportionately less female ( $52 \%$ to $57 \% ; \mathrm{P}=0.07$ ), older $(0.29 \mathrm{y}[0.03,0.54])$, and had a higher BMI z-score $(0.039[-0.026,0.100])$ At baseline, children with paired data were $57 \%$ female with mean age 8.8 $\pm 1.8 \mathrm{y} ; 22 \%$ were overweight; $33 \%$ obese; and $45 \%$ morbidly obese (IOTF extended cut-points).

Post-program, parents had higher parenting self-efficacy scores $(0.17$ $[0.10,0.25])$. Children had improved intakes (CDQ scores), eating more fruits and vegetables $(1.14[0.65,1.59])$, fewer discretionary (high fat/high sugar) foods $(-0.65[-0.82,-0.49])$, and fewer non-milk sugar-sweetened beverages $(-0.40[-0.60,-0.24])$. BMI z-score (US CDC2000) was lower $(-0.112[-0.138,-0.089])$ and waist $\mathrm{Z}$-score (UK1990) had also reduced $(-0.049[-0.086,-0.013])$. An overall BMI $z$-score decrease of $5.4 \%$ $[-6.8 \%,-4.2 \%])$ corresponded with an $8 \%$ decrease in the proportion of morbidly obese children.

Conclusion: There were significant improvements in parenting self-efficacy and child diet when PEACH ${ }^{\mathrm{TM}}$ was upscaled and delivered in community settings. Ultimately, these parenting and diet improvements were associated with statistically and clinically significant reductions in the level of child adiposity. When delivered at scale, $\mathrm{PEACH}^{\mathrm{TM}}$ is an effective family-based, multicomponent, lifestyle weight management service for obese children, in alignment with a key recommendation from the Report of the Commission on Ending Childhood Obesity (3).

References:

1 Magarey AM et al.: 2011 Pediatrics 127;2:214-222.

2 Magarey AM et al.: 2009 Int J Pediatr Obes 4:4:257-265

3 World Health Organization, 2016 ISBN 9789241510066.
OS6:OC41

User and provider perspectives on the recruitment process of a family-focused, multicomponent childhood weight management programme delivered in the community setting

Kelleher, M. ${ }^{1}$; Harrington, D. ${ }^{1}$; Shiely, D. ${ }^{1}$; Perry, P. '; Mchugh, D. ${ }^{1}$

${ }^{1}$ University College Cork

Introduction: The recruitment of participants for childhood obesity treatment programmes is a particularly challenging issue. Few children who are referred to weight management treatment end up enrolling. This qualitative study explored (1) the process, barriers and successes experienced by public health nurses (PHNs) whilst recruiting families of obese children to a government-funded, multidisciplinary weight management programme, and (2) parents' experience of being referred as well as their reasons for enrolling and completing the programme.

Methods: In-depth, semi-structured interviews were conducted with 10 PHNs and 10 parents listed on the programme database. Topic guides were developed based on literature and were used as a tool rather than a rule, to allow participants to construct a narrative about their experience. Interviews continued until data saturation was reached, were transcribed verbatim and transcripts were analysed using Interpretive Phenomenological Analysis.

Results: PHNs identified a lack of practical training and fear of approaching the issue of weight with parents as key barriers to referral. Furthermore, they felt that the development of a trust-worthy app to calculate Body Mass Index could help alleviate anxiety over misclassification while also reducing the time required to assess eligibility. Motivating factors for parents included an underlying concern their child was carrying excess weight, the desire to acquire new skills around healthy eating and to learn how to become more active as a family. Once enrolled, the group support they received and seeing their child having fun motivated them to complete the programme.

Conclusion: We consider it vital that all professionals involved in referring families to weight management programmes receive more practical training to increase their self-efficacy and skills in motivating parents to participate in childhood obesity treatment programmes. Furthermore, programme material should highlight the opportunities to acquire new skills and knowledge around attaining and maintaining healthy lifestyles.

\section{Conflict of Interest: No competing interests exist}

Funding: This research was funded by the Health Research Board SPHeRE/2013/1. The Health Research Board (HRB) supports excellent research that improves people's health, patient care and health service delivery. We aim to ensure that new knowledge is created and then used in policy and practice. In doing so, we support health system innovation and create new enterprise opportunities

\section{OS6:OC42 \\ Raising healthy kids - a national health target to help tackle childhood obesity}

\section{Mcrobbie. H. ${ }^{1}$; Evison, K. ${ }^{1}$; Mccormack, L. ${ }^{1}$ \\ ${ }^{1}$ Ministry of Health, New Zealand}

Introduction: A healthy body weight is important for good health and mental wellbeing, but approximately $7 \%$ of children under the age of five in New Zealand are obese, with higher rates among Maori and Pacific children. The New Zealand Ministry of Health has a comprehensive package of 22 initiatives to combat childhood obesity that include targeted interventions for children who are obese, increased support for those at risk of becoming obese, and broad approaches to make healthier choices easier for all New Zealanders. One of the targeted initiatves is a Health Target that was implemented in July 2016 and stipulates 'By December 2017, 95\% of obese children identified in the Before School Check (B4SC) programme will be offered a referral to a health professional for clinical assessment and family based nutrition, activity and lifestyle interventions.' The B4SC occurs at age four, has $>90 \%$ coverage and focuses on early intervention to ensure positive, sustained effects on health. 
Methods: Data collected during the B4SC are captured in a national database. Each quarter the health target result is calculated from the number of children identified as obese (BMI>98th percentile on the NZ-WHO growth charts) from all completed B4SC processed in the previous six month period and the number of children where their referral was acknowledged within 30 days or who are already under care or the referral was declined by the parent/caregiver. Results are publically reported and monthly meetings are held with District Health Board (DHB) leads to identify barriers and facilitators to achieving the target.

Results: The national achievement in the first quarter was $49 \%$, but ranged from $17 \%$ to $83 \%$ across the DHB areas. Barriers identified include lack of skill/confidence in raising the issue of obesity with parents, inadequate referral pathways, limited clinical pathways and treatment options.

Conclusion: This health target is helping to put obese children and their families in touch with healthcare professionals who can check for any clinical risk associated with obesity, encourage families to take action and importantly monitor the child's growth. On its own it is not expected that this health target will decrease the rates of childhood obesity but through the interventions developed in the Childhood Obesity Plan we have health professionals, government, the private sector, communities, schools and families on board helping to address the issue.

Conflict of Interest: None Disclosed

Funding: No Funding
Friday, 19 May 2017

\section{OS7 - Treatment Interventions and Outcomes}

\section{OS7:0C61 \\ Impact of menstrual cycle phase and oral contraceptive use on the greater thermic effect of a high vs normal protein meal}

Duhita, M. ' ; Schutz, Y. ; Montani, J. '; Dulloo, A. ; Miles-Chan, J.

${ }^{1}$ Department of Medicine / Physiology, University of Fribourg, Fribourg, Switzerland

Introduction: Successful approaches to weight control should elicit effects on both sides of the energy balance equation, i.e., energy intake and expenditure. For this reason there has been much interest in the role of dietary protein, which has been demonstrated to favourably affect satiety, thermogenesis, body weight and body composition. However, there are a number of discrepancies in study findings. As such there is a need to characterise individual determinants of the thermogenic effects of dietary protein. In this context, the aim of the present study was to determine the influence of menstrual cycle phase and oral contraceptive use on the thermogenic response to a standardised high protein (HP) meal in healthy, young women.

Methods: Resting energy expenditure (EE; by indirect calorimetry) and heart rate $(\mathrm{HR})$ were measured in 16 healthy young women $(20-30 \mathrm{y}$; BMI $18.5-30 \mathrm{~kg} / \mathrm{m}^{2}$ ), split evenly between those taking and not taking the combined, monophasic oral contraceptive pill. Following an overnight fast, EE and HR monitoring was conducted for $30 \mathrm{~min}$ at rest, and continued for 3-5 h following the ingestion of either a normal protein (NP; $11 \%$ energy from protein) or HP (24\%), isocaloric meal (590 kcal). The two meal tests were conducted in a randomised order and repeated in each phase of the menstrual cycle (follicular and luteal).

Results: There was no effect of menstrual phase or contraceptive pill use on baseline EEor HR, nor in the response of either variable to the NP meal. However, whereas the HP meal increased EE significantly more than the NP meal in women without contraceptive pill use (+34\% versus $+20 \%$; $\mathrm{p}<0.001)$, this greater HP-induced thermogenesis was not observed in women taking the pill $(+26 \%$ versus $+25 \%$; $p=14)$.

Conclusion: This study shows an abolishment of the greater thermic effect of a HP versus NP meal in women taking the oral contraceptive pill. Although the mechanism remains to be determined, this finding has important implications for the effective use of HP for body weight regulation in women taking oral contraception. With current obesity treatment and prevention strategies largely ineffective in women (with women generally losing less weight during weight management programmes than men), understanding the relationship between sex hormone status and our metabolic response to dietary factors, such as protein intake, is key to enabling the successful re-calibration of existing diet/lifestyle recommendations.

Conflict of Interest: The authors declare that there is no conflict of interest.

Funding Disclosure: Research relating to this abstract was funded by intramural Funding from the Department of Medicine, University of Fribourg, and a Swiss Government Excellence Scholarship for Foreign Scholars.

OS7:0C62

Galacto-oligosaccharides supplementation increased bifidobacteria, but not insulin sensitivity, in obese prediabetic humans

Canfora, E. '; Beek, C. '; Hermes, G. ${ }^{2}$; Goossens, G. '; Jocken, J. '; Holst, J.3.

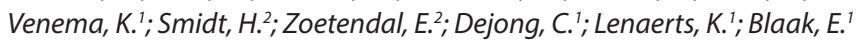
${ }^{1}$ Maastricht University Medical Centre+ ${ }^{2}$ Wageningen University, ${ }^{3}$ University Copenhagen 
Introduction: The gut microbiota may influence host lipid and glucose metabolism, satiety and chronic low-grade inflammation, thereby contributing to the aetiology of obesity and type 2 diabetes mellitus. Fermentation end products, in particular the short-chain fatty acid (SCFA) acetate, are believed to be involved in these processes. Here, we investigated the long-term effects of supplementation with galacto-oligosaccharides (GOS), an acetogenic fibre, on gut microbiota composition and host metabolism in humans.

Methods: Forty-four overweight/obese (BMI $28-40 \mathrm{~kg} / \mathrm{m}^{2}$ ) prediabetic men and postmenopausal women completed this double-blind, placebo-controlled, parallel intervention study. The participants were randomly assigned to daily $15 \mathrm{~g}$ GOS or isocaloric placebo intake for 12 weeks. Before and after the intervention, peripheral and adipose tissue insulin sensitivity, faecal microbiota composition, plasma and faecal SCFA, energy expenditure and substrate oxidation, body composition, as well as hormonal and inflammatory responses, were determined.

Results: Supplementation of GOS, but not placebo, to the daily dietled to a marked 5-fold increase in faecal Bifidobacterium spp. $(P=0.009$, $\mathrm{q}=0.144)$. Microbial richness or diversity were not affected.No alterations of faecal and fasting plasma SCFA concentrations, changes in systemic concentrations of gut-derived hormones, incretins, lipopolysaccharide-binding protein or other systemic inflammatory markers were observed. In addition, no significant alterations in peripheral and adipose tissue insulin sensitivity, body composition, and energy and substrate metabolism, were found.

Conclusions: Twelve-week supplementation of GOS selectively increased faecal Bifidobacterium spp. abundance, but this did not translate into significant changes in insulin sensitivity and related host substrate and energy metabolism in overweight/obese prediabetic men and women

\section{OS7:0C63}

\section{Modelling the cost-effectiveness of referrals to open-group behavioural programmes: A microsimulation over $\mathbf{2 5}$ years using data from the WRAP trial}

Pimpin, L. ${ }^{\prime}$; Jaccard, A. '; Webber, L. '; Retat, L. '; Aveyard, P. ${ }^{2}$; Boyland, E. ${ }^{3}$; Halford, J. ${ }^{3}$; Suhrcke, M. ; Turner, D. ${ }^{4}$; Irvine, L. ; Jebb, S. ${ }^{2}$; Ahern, A. ${ }^{5}$

${ }^{1}$ UK Health Forum, ${ }^{2}$ University Of Oxford, ${ }^{3}$ University Of Liverpool, ${ }^{4}$ University Of East Anglia, ${ }^{5}$ University Of Cambridge

Introduction: The Weight loss Referral for Adults in Primary care (WRAP) trial showed that referring individuals with $\mathrm{BMI} \geq 28 \mathrm{~kg} / \mathrm{m}^{2}$ to an open-group behavioural weight loss programme for 12 weeks resulted in greater weight loss than a brief intervention (BI; self-help booklet), and a 52 -week programme was more effective than a 12 -week programme ${ }^{1}$. The longer programme costs more in the short-term, but it is unclear whether it is cost-effective in the longer-term.

Methods: We conducted a microsimulation ${ }^{2}$ based on outcomes in the three arms of the WRAP trial (BI, 12-week referral, 52-week referral) over a 25-year horizon. Our starting population was a cohort of 20 million, representative of the age, sex, and initial BMI distribution in the whole trial cohort. For each scenario, the mean 2-year BMI change for that intervention group was applied to the whole population and we assumed all weight lost was regained by 5 years after baseline. Thereafter individuals followed population-based weight trajectories. The incidence of 13 chronic diseases, associated direct healthcare costs, and quality-adjusted life years (QALY) were summarised annually for each scenario, between 2014 and 2039. We compared the cumulative incremental cost per QALY of each scenario.

Results: Over a 25-year period, the 52-week programme was cost-effective compared to the 12-week and BI scenarios. Compared to the 12-week programme, the 52-week programme resulted in 1786 fewer incident cases and 1282 more QALYs per 100000 individuals, at a cost of $£ 4.9$ million, per 100000 individuals ( $£ 3804 / \mathrm{QALY}$ ). Compared to the BI, the 52-week programme avoided 2409 cases of incident disease per 100000 individuals, resulting in 1925 additional QALYs at a cost of $£ 4.8$ million per 100000 individual (£2498/QALY).The incremental cost per QALY for the 12-week programme was dominant compared to BI, with 643 additional QALYs per 100000 individuals, at a cost-saving of $£ 68,000$ per 100000 individuals. Conclusion: Microsimulations predicted that over a 25 -year period, offering a behavioural programme to people seeking to lose weight reduces the incidence of obesity-related disease and is cost-effective compared to offering a self-help booklet. A 52 -week programme offers greater potential health gains than the 12-week programme and the brief intervention and is cost-effective by UK usual standards of $£ 20000 / \mathrm{QALY}^{3}$.

\section{References:}

1 Amy $L$ Ahern et al.: Extended and standard duration weight loss referrals for adults in primary care (WRAP): a pragmatic randomised controlled trial. Lancet (Under Review) 2017.

2 McPherson K, et al.: Tackling obesities: future choices: Modelling future trends in obesity and the impact on health: Citeseer; 2007.

3 NICE. Guide to the methods of technology appraisal. London; 2013.

\section{OS7:0C64 \\ Controlled comparison of stepped-care multi-modal treatment versus behavioral weight loss for binge eating disorder in patients with obesity}

\section{Grilo, C.';}

${ }^{1}$ Yale University School of Medicine

Introduction: This randomized controlled trial tested the effectiveness of a stepped-care multi-modal approach versus behavioral weight loss (BWL) for patients with obesity and binge eating disorder (BED).

Methods: 191 patients (mean age $48,71 \%$ female, $79 \%$ white) with BED and co-morbid obesity (mean BMI 39) were randomly assigned to 6 months of BWL $(\mathrm{N}=39)$ or stepped-care $(\mathrm{N}=152)$. Within stepped-care, patients started with BWL for one month; treatment-responders continued with BWL while non-responders switched to specialist treatment (CBT) and all stepped-care patients were additionally randomized to either anti-obesity medication or placebo (double-blind) for the remaining five months. Independent assessments were performed at baseline, during treatment, post-treatment (6 months), and 6- and 12-month follow-ups after completing treatments (through 18 months) with reliably-administered structured interviews and measures.

Results: ITT analyses of abstinence rates (zero binges/month) revealed BWL and stepped-care did not differ significantly overall at post-treatment $(74 \%$ vs $64 \%$ ) or 12 -month follow-up ( $45 \%$ vs $41 \%)$. Mixed-models regression analyses of binge-eating frequency through post-treatment revealed significant time effects but that BWL and stepped-care did not differ overall; within stepped-care, however, medication was significantly superior to placebo overall and among initial non-responders switched to CBT. Mixed-models of binge-eating frequency during the 12-months after treatment revealed good maintenance that did not differ across treatments. Mixed models revealed significant \% weight loss through post-treatment but BWL and stepped-care did not differ overall; within stepped-care, however, medication was significantly superior to placebo overall and among both initial responders continued on BWL and non-responders switched to CBT. Mixed-models during follow-up revealed significant time effects with \% weight change larger at 6 than 12 months; \% weight loss at 12-month follow-up was 4\% (BWL), 6\% (BWL+placebo), and $7.5 \%$ (BWL+medication).

Conclusion: Overall, BWL and stepped-care treatments produced significant improvements in binge-eating and weight loss that were maintained through 18 months in obese patients with BED. Anti-obesity medication enhanced outcomes with behavioral treatments within stepped-care.

Conflict of Interest: None disclosed.

Funding: This research was funded by a grant from the National Institutes of Health (2R01 DK098492). 


\section{OS7:OC65}

\section{Distribution matters - body composition profiling associated} with prior health care burden

\section{West, J. ${ }^{1}$; Linge, J. ${ }^{2}$; Romu, T. ${ }^{1}$; Borga, M. ${ }^{1}$; Bell, J. ${ }^{3}$; Leinhard, O. ${ }^{1}$ \\ ${ }^{1}$ Advanced MR Analytics AB; Linköping University, ${ }^{2}$ Advanced Mr Analytics Ab, ${ }^{3}$ University Of Westminster}

Introduction: There are significant economic costs associated with obesity. Yet the consensus definition of obesity, based on body mass index (BMI), lacks detail on precise body composition and distribution of fat-compartments within the body. Magnetic resonance imaging, currently the gold standard for body composition profiling, allows for accurate quantification of body fat content and distribution. The aim of this study was to determine the association between body composition measures and prior health care burden (HCB), measured as number of nights hospitalization, and to characterize subjects with the highest prior HCB.

Methods: 2,864 males and 3,157 females, age range at imaging 46 to 78 years, were included from the UK Biobank imaging cohort. Visceral adipose tissue index $\left(\mathrm{VATi}=\mathrm{VAT} /\right.$ height $\left.^{2}, 1 / \mathrm{m}^{2}\right)$, abdominal subcutaneous adipose tissue index (ASATi=ASAT/height ${ }^{2}, 1 / \mathrm{m}^{2}$ ) and intra-muscular adipose tissue in anterior thighs (IMAT, \%) were measured using a $1.5 \mathrm{~T}$ MR scanner. The MR-images were analyzed using AMRA Profiler research (AMRA, Sweden). The HCB was derived from UK Biobank hospital in-patient data gathered prior to, and during the imaging study. Three univariate linear models were constructed investigating the associations of each of the body composition measurements to HCB. Subsequently, multivariable linear regression was used to assess the relationship between $\mathrm{HCB}$ and all the body composition measurements jointly. All models were adjusted for age, sex, smoking, alcohol intake, and physical activity. Finally, body composition was determined for subjects in the $90^{\text {th }}$ percentile of prior HCB and compared to a group with no hospital nights matched on sex, age, and BMI, using Mann-Whitney U test without correction for multiple comparisons.

Results: Univariate tests showed significant relationships $(\mathrm{p}<0.001$, $\left.\mathrm{b}_{\mathrm{VATi}}=0.85 \pm 0.11, \mathrm{~b}_{\mathrm{ASATi}}=0.39 \pm 0.07, \mathrm{~b}_{\mathrm{IMAT}}=0.34 \pm 0.04\right)$ with $\mathrm{HCB}$ GLM showed that HCB was associated with increased VATi $(\mathrm{p}<0.001$ $\mathrm{b}=0.60 \pm 0.17)$ and increased IMAT $(\mathrm{p}<0.001, \mathrm{~b}=0.23 \pm 0.05)$. ASATi was not significant $(\mathrm{p}=0.85)$ in the GLM analysis. The group with highest prior HCB consisted of 382 females and 292 males, age at imaging 64 (5769) (median, $25^{\text {th }}-75^{\text {th }}$ percentile), and were hospitalized for at least nine nights. Body composition characteristics of this group were as follows: BMI 27.2 (24.4-30.7), VATi 1.35 (0.81-1.95), ASATi 2.56 (1.77-3.54) and IMAT 5.96 (4.72-7.47). Comparing to subjects with no hospital nights, VATi and IMAT were significantly higher $(\mathrm{p}<0.001)$.

Conclusion: This study demonstrated that VATi and IMAT had a significant positive relationship with prior hospitalization. ASATi did not show significant relationship using multivariable analysis.

Conflict of Interest ODL, JW, TR, JL, MB (AMRA)

Funding: Financial support by Pfizer

\section{OS7:0C66}

Impact of bariatric surgery on cancer incidence in morbidly obese subjects: Results from a French nationwide populationbased cohort

Vandendorpe, S. ; Ficheur, G. ${ }^{2}$; Czernichow, S. ${ }^{3}$; Verkindt, H. ${ }^{4}$; Nesslany, P. ; Pattou, F. ${ }^{4}$; Romon, M. ' ${ }^{\prime}$ Caiazzo, R. ${ }^{4}$; Pigeyre, M. ${ }^{1}$

${ }^{1}$ Nutrition Department, University-Hospital of Lille, France, ${ }^{2}$ Public Health Department, University-Hospital Of Lille, France, ${ }^{3}$ Nutrition Department, European Hospital Of Georges-Pompidou, Paris, France, ${ }^{4}$ Endocrine Surgery Department, University-Hospital Of Lille, France

Introduction: With a prevalence estimate of $1.2 \%$ in French adults, morbid obesity (body mass index (BMI) $\geq 40 \mathrm{~kg} / \mathrm{m}^{2}$ ) is an established risk factor for chronic diseases, such as cancer. The effects of bariatric surgery on cancer risk remain uncertain, and only few studies, conducted in Canada,
United States and Sweden, have reported inconstant effect of bariatric surgery on cancer incidence. Our objective consists in evaluating impacts of bariatric surgery on cancer occurrence in morbidly obese subjects, using the nationwide French Healthcare Insurance System database.

Methods: A retrospective cohort of morbidly obese adults (18-60 years old) has been constituted from hospital national administrative database (Diagnosis Related Group Program, "Programme De Médicalisation des Systèmes d'Information" (PMSI)), which is an exhaustive source of all medical and surgical care covered by the Healthcare Insurance System in hospitals. Subjects having undergone bariatric surgery in 2010 were included and matched with non-operated obese control subjects, on age, gender, class of obesity and Charlson Comorbidity Index (weighted sum of comorbidities of subjects, based on the International Classification of Diseases (ICD) diagnosis codes). Bariatric procedures included gastric banding $(n=3663)$, gastric bypass $(n=3425)$, vertical banded gastroplasty $(n=3255)$, sleeve gastrectomy $(n=651)$, biliopancreatic diversion $(n=237)$. Incidence of cancer was defined on a period of 3.5 years, by the occurrence of principal or secondary diagnosis codes of "cancer" in the PMSI. A Cox regression model has been used to assess association between risk of cancer and bariatric surgery, stratified on matching variables. A specific analysis on obesity-related cancer was also performed. HR were computed and are presented along with 95\% confidence intervals $(95 \% \mathrm{CI})$

Results: 11321 subjects (mean age $37.5 \pm 10.8$ years, $82.5 \%$ women) have been included in the bariatric surgery group and 11321 in the control group. 152 cancers were diagnosed in bariatric surgery group (1.35\%) and 165 in control group (1.47\%). Incidence of cancer was 4.7 per 1000 person-years in the bariatric group versus 5.1 per 1000 person-years in the control group (hazard-ratio $(\mathrm{HR})=0.90,95 \% \mathrm{CI}[0.67-1.12], p=0.332$ ). Obesity-related cancer was diagnosed on 84 subjects in bariatric group and 82 subjects in control group ( $\mathrm{HR}=1.05,95 \% \mathrm{CI}[0.74-1.36], p=0.75)$. Conclusion: Our study did not show any significant association between bariatric surgery and cancer incidence decrease after 3.5 years of follow-up. A long-term study seems necessary to demonstrate the beneficial effects of bariatric surgery on cancer occurrence in morbidly obese subjects.

Conflict of interest: None Disclosed

Funding: No Funding

\section{OS8 - Lifecourse Epidemiology}

\section{OS8:OC25}

Age modified the association between BMI and Parkinson's disease - a cohort study

\section{Sääksjärvi, K. ' ; Knekt, P. ' Lyytinen, J.2. Lundqvist, A. '; Jääskeläinen, T. ';} Männistö, S.'

${ }^{1}$ National Institute for Health and Welfare, ${ }^{2}$ Helsinki University Central Hospital

Introduction: Obesity has been associated with a greater PD risk, but previous studies have been inconsistent. Thus, investigation of potential effect modifying factors is needed.

Methods: This cohort study included subjects from the Finnish Mobile Clinic Health Examination Survey conducted in 1966-1972, aged 15 years and over and free from PD at the baseline $(n=57,178)$. Height and weight were measured at the baseline, and body mass index (BMI) was calculated. A questionnaire provided information on several background factors, such as smoking and leisure-time physical activity. During 29 years of follow-up, 359 incident PD cases occurred. The multivariate Cox's model estimated the strength of association between BMI and PD risk, adjusting for age, sex, marital status, community density, geographical area, smoking, and leisure-time physical activity.

Results: A non-significant positive association between BMI and PD risk was observed in total population ( $\mathrm{p}$ for trend 0.11 ). However, age at base- 
line modified the association as higher BMI was statistically significantly associated with increased PD risk among those who were $<50$ years of age at baseline: compared to individuals with $\mathrm{BMI}<23$, the relative risk of PD was 2.49 (95\% confidence interval (CI) 1.52-4.06), 3.05 (95\% CI $1.88-4.97), 2.95$ (95\%CI 1.64-5.29), and 2.67 (95\%CI 1.32-5.42) for the BMI categories of 23 to $24.99,25$ to $27.49,27.5$ to 29.99 , and $30+$, respectively. There was no association between BMI and PD risk among those who were 50 years of age and older at baseline.

Conclusion: Being overweight or obese before the age of 50 predicted higher PD risk. There might be a critical time window during the life course where obesity increases the risk of PD.

Conflict of Interest: None disclosed

Funding: Research related to this abstract was funded by The Finnish Parkinson Foundation

\section{OS8:OC26 \\ Vitamin D status and obesity - a prospective study in the Finnish adult population}

\section{Jääskeläinen, ${ }^{1}{ }^{1}$; Männistö, S. ${ }^{1}$; Härkänen, $T^{1}$; Lundqvist, $A .{ }^{1}$}

${ }^{1}$ National Institute for Health and Welfare, Helsinki, Finland

Introduction:Vitamin D status and obesity has shown to be inversely associated in many cross-sectional studies. However, the evidence from the prospective studies is limited and the causal relationship is unclear. The aim of the present study was to examine whether vitamin D status predicts the incidence of overweight or obesity during the 11-year follow-up.

Methods: The study population comprised normal-weight individuals aged 30-79 years at the baseline from the Health 2000 Survey and its follow-up, the Health 2011 Survey $(\mathrm{n}=1288)$. Both surveys included questionnaires, interviews and the health examination. Weight and height were measured in light clothing without shoes as a part of health examination. Overweight or obesity was defined as body mass index (BMI) $\geq 25 \mathrm{~kg} / \mathrm{m}^{2}$. Serum 25-hydroxyvitamin D concentration (S-25(OH) D), the biomarker of vitamin D status, was determined from frozen samples $\left(-70{ }^{\circ} \mathrm{C}\right)$ by radioimmunoassay. Statistical analyses were based on linear and logistic regression models adjusted for age and the month of blood sampling. Multivariate model was additionally adjusted for marital status, education, leisure-time physical activity and smoking.

Results: During the follow-up 28\% $(\mathrm{n}=135)$ of normal-weight men and $29 \%(\mathrm{n}=232)$ of women became overweight or obese $\left(\mathrm{BMI} \geq 25 \mathrm{~kg} / \mathrm{m}^{2}\right)$. In women, S-25(OH) D concentration at the baseline was higher (47.9 $\mathrm{nmol} / \mathrm{l}$ ) in those who stayed normal weight compared to those whose $\mathrm{BMI}$ reached $25 \mathrm{~kg} / \mathrm{m}^{2}(44.7 \mathrm{nmol} / \mathrm{l})(\mathrm{p}=0.01)$. In normal-weight women odds ratio (OR) for becoming overweight or obese during the follow-up was $0.56(95 \%$ CI $0.33,0.94$; p for trend $=0.03)$ for the highest quintile of $\mathrm{S}-25(\mathrm{OH}) \mathrm{D}$ versus the lowest. After adjustment for marital status, education, leisure-time physical activity and smoking, the association weakened (OR for the highest vs. lowest quintile $0.65,95 \%$ CI 0.38, 1.10; p for trend $=0.14)$. Among men, vitamin $\mathrm{D}$ status did not predict the incidence of overweight or obesity (OR for the highest vs. lowest quintile 1.13, 95\% CI $0.57,2.25$; $\mathrm{p}$ for trend $=0.34$ ).

Conclusion: The results of the present prospective study based on nationally representative data suggest that low vitamin D status may predict the incidence of overweight or obesity in women, but not in men. However the association weakened after further adjustment for sociodemographic and lifestyle factors. Thus, it may be possible that vitamin D is a biomarker of healthy lifestyle rather than an independent risk factor for obesity.

Conflict of Interest: None disclosed

Funding: Research relating to this abstract was funded by the Juho Vainio Foundation.
OS8:OC27

Prevalence of hypertension and its association with obesity and other risk factors in Federal District, Brazil: The study of cardiovascular risk in adolescents (ERICA)

Gonçalves, V.'; Galvão, T.'; Silva, M.'; Pardo, F.'; Dutra, E. ; Carvalho, K. ${ }^{1}$ University of Brasilia, Brazil, ${ }^{2}$ State University Of Campinas, Brazil, ${ }^{3}$ Federal University Of Amazonas, Brazil

Introduction: Despite being a more frequent condition in adults and the elderly, adolescents have presented changes in blood pressure (BP) at increasingly early ages. In this sense, the aim of this study was to estimate the prevalence of hypertension and associated risk factors in participants of the study of cardiovascular risk in adolescents.

Methods: Cross-sectional study, school-based with a sample divided into conglomerates of public and private schools and classes from rural and urban areas in the Federal District, Brazil. Anthropometry, BP measurements and biochemical tests were performed. The adolescents completed a questionnaire using an electronic data collector on sociodemographic aspects. The mean systolic or diastolic BP $\geq 95$ th percentile of the reference curve was considered to be hypertension, obesity was classified according to the BMI for age greater than $\mathrm{z}$-score $+2 \mathrm{SD}$ and the altered fasting insulin corresponded to levels $\geq 15 \mathrm{mU} / \mathrm{L}$. Bi and multivariate analyzes were performed by Poisson regression. We adopted $\mathrm{p}<0.20$ in the selection of variables for the multivariate model. The data were analyzed in Stata 14, using the Survey function to calibrate the weights of the complex sampling used. The study was approved by the Research Ethics Committee of the Medical School of the University of Brasília.

Results: The sample consisted of 2.587 adolescents aged 12 to 17 years. The female gender represented $50.4 \%$ of the sample and the prevalence of hypertension was $7.9 \%$ (95\% CI 6.3-9.1). Of these, 1.200 performed the biochemical tests. The parameters associated with hypertension, after the adjusted analysis, were: rural school (PR 4.1, 95\% CI 3.2-5.2), male (PR 2.7, 95\% CI 2.0-3.7), age between 15 and 17 years (PR 2.3, 95\% CI 1.7-3.2), obesity (PR 4.0, 95\% CI 2.5-6.4), and hyper insulinemia (PR 2.0, 95\% CI 1.2-3.5).

Conclusion: The prevalence of hypertension in school adolescents was $7.9 \%$, associated with demographic variables and modifiable risk factors, especially obesity and insulin resistance marker. This reinforces the importance of improving lifestyle of adolescents in order to preserve a better health condition.

\section{OS8:OC28 \\ Association of obesity with chronic conditions during transition to retirement in the GAZEL cohort: A 15-year prospective study}

Matta, J. ${ }^{1}$; Carette, C. ${ }^{2}$; Zins, M. ${ }^{\text {; }}$ Lemogne, C. ${ }^{3}$; Godlberg, M. ${ }^{\text {' }}$; Czernichow, $S^{\prime}$

${ }^{1}$ INSERM UMS 011, ${ }^{2}$ Hopital Europeen Georges Pompidou, ${ }^{3}$ Paris Descartes University

Introduction: The burden of disease in terms of perceived health problems is generally relieved by retirement. Obese participants might not benefit from the same improvement compared to normal weight ones. To date, no data has compared the association of obesity with perceived health problems around the timing of retirement. The objective is to assess the odds of bad health perception, mental, physical fatigue and depression in obese versus normal weight participants over a 15-year follow-up period ( 7 years prior to retirement, period of retirement and 7 years following retirement).

Methods: $10300-10316$ men and women with a mean age of 55 from the GAZEL cohort with data on anthropometrics, self-rated health, mental and physical fatigue and depression were included. The interaction of body mass index (BMI) categories with time for each health component were calculated using generalized estimating equations controlling for sex and OR 95\% CI were determined. 
Results: There was no interaction between time and BMI categories regarding bad health perception and mental fatigue. Physical capacity was decreased in obese participants around the timing of retirement versus normal weight (OR 0.89, 95\% CI 0.82-0.97) and was higher following retirement (OR 1.05 95\% CI 1.02-1.09) in obese versus normal weight. Obese participants had higher odds of depression after retirement versus normal weight participants (OR 1.06 95\% CI 1.01-1.12).

Conclusion: Obesity around the timing of retirement affected health in different ways and has increased the odds of physical fatigue and depression 7 years following retirement compared to normal weight participants.

Funding: The GAZEL cohort was funded by EDF-GDF and INSERM

\section{OS8:OC29}

The association between duration of breastfeeding and type 2 diabetes in adulthood: Influence from potential confounders

\author{
Bjerregaard, L. '; Mortensen, E. '; SøRensen, T.'; Baker, J. ${ }^{1}$
}

${ }^{1}$ Department of Clinical Epidemiology (formerly Institute of Preventive Medicine), Bispebjerg and Frederiksberg Hospitals, The Capital Region, Copenhagen, Denmark, ${ }^{2}$ Institute Of Public Health And Center For Healthy Aging, University Of Copenhagen, Copenhagen, Denmark

Introduction: Although a number of observational studies suggest that breastfeeding protects against the development of type 2 diabetes later in life, there are inconsistencies across studies, potentially due to differences in study size and adjustment for potential confounders. Moreover, most studies are limited to comparison of breast-fed versus bottle-fed infants. Therefore, the aim of this study was to examine the association between duration of breastfeeding and type 2 diabetes in adulthood in a cohort with a wide range of potentially confounding variables.

Methods: We included 4,786 individuals from the Copenhagen Perinatal Cohort born 1959-61 in Denmark. The duration of any breastfeeding as assessed by a physician interviewing the mothers at the infant's 1 -year examination was divided into quartiles $(\leq 1,>1-2,>2-4,>4$ months). Type 2 diabetes status (age $\geq 30$ years) was obtained from the National Patient Register $(n=171)$. Hazard ratios (HR) and 95\% confidence intervals for the association between duration of breastfeeding and type 2 diabetes were estimated by Cox proportional hazards regressions without and with adjustment for sex, birth weight, maternal body mass index, maternal diabetes during pregnancy, maternal smoking during the third trimester and parental social status and education.

Results: In the unadjusted analysis, there was a significant and inverse trend between quartiles of duration of breastfeeding and risk of type 2 diabetes in adulthood $(P=0.01)$. In unadjusted analyses, compared with infants breastfed for 1 month or less, among infants breastfed for $>2-4$ months there was a non-statistically significant, albeit inverse, association with type 2 diabetes $(\mathrm{HR}=0.84$ [0.56-1.26]). In unadjusted analyses, compared with infants breastfed for 1 month or less, infants breastfed for $>4$ months had a $40 \%$ reduced risk of type 2 diabetes $(\mathrm{HR}=0.60[0.38-$ 0.96]). However, after adjustment for potential confounders this was attenuated to a $29 \%$ reduced risk $(\mathrm{HR}=0.76[0.47-1.23])$ and was no longer statistically significant.

Conclusion: When a range of type 2 diabetes risk factors are considered, breastfeeding is not associated with adult type 2 diabetes. Although breastfeeding has a modest but non-significant protective effect against this disease, the results suggest that it is likely an indirect indicator of diabetes risk factors, highlighting the potential effects of unknown or poorly measured confounders. In addition, mediation by later life risk factors should be considered.

Conflict of Interest: None Disclosed

Funding: Research relating to this abstract was funded by the European Union's Horizon 2020 research and innovation programme under grant agreement $\mathrm{n}^{\circ} 633595$, DynaHEALTH.
OS8:OC30

The "paradox of overweight, obesity and fat mass" in older adults population: The 10-year follow-up of the health, wellbeing and aging (SABE) study

\section{Roediger, M. ${ }^{1}$; Marucci, M. ${ }^{1}$; Dourado, D. ${ }^{1}$; Duarte, $Y^{2}{ }^{2}$; Lebrão, M. ${ }^{3}$}

${ }^{1}$ Department of Nutrition, School of Public Health, University of São Paulo/ Brazil, ${ }^{2}$ Department Of Nursing Medical-Surgical School Of Nursing, University Of São Paulo/ Brazil, ${ }^{3}$ Department Of Epidemiology, School Of Public Health, University Of São Paulo/ Brazil

Introduction: Epidemiological studies have been demonstrated that overweight, obesity and high fat mass increase the risk of mortality in middle-aged and younger people, however,in older adults, this relation is still is not clear. This study verified the association of overweight, obesity and high fat mass with 10-years mortality, according by age groups and gender, in older adults community-dwelling Brazilian cohort.

Methods: A longitudinal, epidemiological, and home-based study was carried out using data from the SABE Study (Health, Well-Being and Aging) conducted in the city of São Paulo - SP/Brazil, in 2000 and 2010. In 2000 , the population was composed of 2143 elderly people ( $\geq 60$ years). In 2010, deaths were verified by the Mortality Information System of the city of São Paulo. The variables of this study were overweight (determined through by of body mass index - BMI, when BMI $\geq 28$ and $<30 \mathrm{~kg} / \mathrm{m}^{2}$ ), obesity (class I when BMI $\geq 30 \mathrm{~kg} / \mathrm{m}^{2}$ to $<35 \mathrm{~kg} / \mathrm{m}^{2}$, classes II and III when $\mathrm{BMI} \geq 35 \mathrm{~kg} / \mathrm{m}^{2}$ ) and fat mass (determined through by the triceps skinfold thickness - TSF, when low fat mass $<25^{\text {th }}$ percentile and high fat mass $\geq 25^{\text {th }}$ percentile, using the percentiles of the same population). The Cox regression-based test was used to calculate the mortality rate. The association between variables of this study with mortality using Poisson regression estimate (hazard ratios - HR), according by age groups (60-79 and $\geq 80$ years) and gender. Statistical analyses were conducted using version 13.0 of STATA statistical software.

Results: Over the 10 years-follow-up period, there were 769 deaths $(40.2 \%)$, being $58 \%$ in men and $73 \%$ in older adults $\geq 80$ years. The total mortality rate was higher in individuals with low fat mass (66.6), in relation the high fat mass (43.5). It was observed that men with low fat mass had a higher mortality rate (89.7), than women (52.8), in relation high fat mass. For men was found associated between obesity I (HR: 1.66) and obesity II (HR: 1.91) with mortality, whereas for women, low fat (HR: 1.34) mass was associated with mortality, however obesity I (HR: 0.66) demonstrated protection for mortalitly. In relation to age groups differences, it was observed a higher mortality rate was observed for those in from the $\geq 80$ y group with low fat mass (HR: 140.7).

Conclusion: The "obesity paradox" showed be the low fat mass that most contributed to elucidate the mortality risk in Brazilian older people. It seems that the conditional nutritional performs a different role under with regard to the risk of mortality in this group, considering gender and age.

Conflict of Interest: None Disclosed

Funding: Fundação de Amparo à Pesquisa do Estado de São Paulo (FAPESP) - Brazil 
Saturday, 20 May 2017

\section{OS9 - Inter-organ Crosstalk}

\section{OS9:OC13 \\ Impairment of intestinal barrier integrity in human obesity: Involvement of dietary lipids and links with bioclinical phenotypes}

Genser, L. ; Dong, L. ; Barbot-Trystram, L. ${ }^{3}$; Torcivia, A. ${ }^{4}$; Vaillant, J. ${ }^{4}$; Wind, P.5; Salem, J.6; Michalski, M. ; Laugerette, F. ; Drai, J.9.; Clément, K. ${ }^{10}$; Oppert, J. ${ }^{11} ;$ Rousset, M. ${ }^{12}$; Brot-Laroche, E. ${ }^{13}$; Leturque, A. ${ }^{12}$; Thenet, S. ${ }^{14}$; Poitou-Bernert, C. $^{10}$

${ }^{1}$ Institute of Cardiometabolism and Nutrition, ICAN, Pitié-Salpêtrière Hospital, Paris, F-7513, France. UMR 1166 INSERM UPMC UPD, Pitié-Salpêtrière Hospital, Paris, F-75013 France. Assistance Publique Hôpitaux de Paris, Department of digestive surgery, live, ${ }^{2}$ Umr 1138 Inserm Upmc Upd, Cordeliers Research Center, Paris, F-75006 France, ${ }^{3}$ Assistance Publique Hôpitaux De Paris, Department Of Functional Coprology, Pitié-Salpêtrière Hospital. Paris, F-75013 France, ${ }^{4}$ Assistance Publique Hôpitaux De Paris, Department Of Digestive Surgery, Liver Transplantation, Pitié-Salpêtrière Hospital.Paris, F-75013 France, ${ }^{5}$ Assistance Publique Hôpitaux De Paris, Department Of Digestive Surgery, Avicenne Hospital, 93000, Bobigny, F-93000, France., ${ }^{6}$ Institute Of Cardiometabolism And Nutrition, Ican, Pitié-Salpêtrière Hospital, Paris, F-7513, France; Assistance Publique Hôpitaux De Paris, Cic-1421, Pitié-Salpêtrière Hospital.Paris, F-75013 France, ${ }^{7}$ Lyon University, Carmen Laboratory, Inra U1397, Univ Lyon-1, Inserm U1060, Insa Lyon, Villeurbanne, France, ${ }^{8}$ Lyon University, Carmen Laboratory, Inra U1397, Univ Lyon-1, Inserm U1060, Insa Lyon, Villeurbanne, France ${ }_{\text {, }}^{9}$ Hospices Civiles De Lyon, Centre Hospitalier Lyon Sud, Department Of Biochimie, Pierre-Bénite, France, ${ }^{10}$ Institute Of Cardiometabolism And Nutrition, Ican, Pitié-Salpêtrière Hospital, Paris, F-7513, France; Umr 1166 Inserm Upmc Upd, Pitié-Salpêtrière Hospital, Paris, F-75013 France; Assistance Publique Hôpitaux De Paris, Department Of Nutrition, Pitié-Salpêt, ${ }^{11}$ Institute Of Cardiometabolism And Nutrition, Ican, PitiéSalpêtrière Hospital, Paris, F-7513, France; Assistance Publique Hôpitaux De Paris, Department Of Nutrition, Pitié-Salpêtrière Hospital.Paris, F-75013 France, ${ }^{12}$ Institute Of Cardiometabolism And Nutrition, Ican, Pitié-Salpêtrière Hospital, Paris, F-7513, France; Umr 1138 Inserm Upmc Upd, Cordeliers Research Center, Paris, F-75006 France, ${ }^{13}$ Institute Of Cardiometabolism And Nutrition, Ican, Pitié Salpêtrière Hospital, Paris, F-7513, France; Institute Of Cardiometabolism And Nutrition, Ican, Pitié-Salpêtrière Hospital, Paris, F-7513, France., ${ }^{14}$ Institute Of Cardiometabolism And Nutrition, Ican, Pitié-Salpêtrière Hospital, Paris, F-7513, France; Umr 1138 Inserm Upmc Upd, Cordeliers Research Center, Paris, F-75006 France; Ephe, Psl Research University, Paris F-75006 France

Background \& aims: Obesity is associated with a low-grade inflammation, which could be promoted by intestinal barrier impairment as suggested by studies in fat-fed rodents. Intestinal permeability (IP) is poorly described in human obesity. We characterized IP in obese subjects and evaluated the impact of acute lipid supplies.

Methods: IP was evaluated ex vivo in Ussing chambers using FITC-labeled tracers at baseline and after exposure to lipid micelles, on jejunum samples of obese and non-obese subjects obtained from surgical waste of gastric bypass and surgeries performed in non-obese subjects. In vivo, IP was assessed by lactitol/mannitol urinary excretion ratio (L/M). Tight junction proteins were studied by immunofluorescence. LPS activity and zonulin levels were measured in serum. Effect of lipid micelles on epithelial permeability was studied in Caco-2/TC7 cells.

Results: In fasting condition, ex vivo IP to 0.4 and $4 \mathrm{kDa}$ tracers was slightly but not significantly increased in obese as compared to non-obese subjects $(\mathrm{p}=0.18)$. Occludin and tricellulin labelling was reduced at tight junction. L/M ratio and seric LPS levels did not differ between groups but increased circulating zonulin levels were observed in obese subjects $(+13 \%, \mathrm{p}=0.02)$. In Caco-2/TC7 cells, lipid micelles increased paracellular permeability through a Src kinase-dependent mechanism. After a lipid micelle supply, higher values of ex-vivo IP were recorded for the jejunum of obese compared to non- obese subjects $(+92 \%$; $<<0.05)$, with a decreased tricellulin intensity. Ex vivo IP to $0.4 \mathrm{kDa}$ tracer was positively correlated with fat mass and systemic inflammation; IP to $4 \mathrm{kDa}$ tracer was correlated with insulin-resistance surrogates (insulinemia, adiponectin; $\mathrm{p}<0.05)$. After lipid challenge, correlations between IP and altered metabolic status observed at fasting state were statistically reinforced.

Conclusions: Increase of jejunum permeability in obese subjects is exacerbated after an acute lipid supply and associated with tight junction impairments. Jejunum permeability is linked to metabolic status and systemic but not local inflammation.

Conflict of Interest: None disclosed

Funding: Research relating to this abstract was funded by ICAN, Institut Benjamin Delessert, Groupe Lipides et Nutrition, UPPIA, SFCD, fondation obelisque, Fondation Nestlé

\section{OS9:OC14}

\section{Maternal lipids and delivery mode predict neonatal gut colonization}

Guzzardi, M. ${ }^{\text {; }}$ Sanguinetti, E. ${ }^{1}$; D'Aurizio, R. ${ }^{2}$; Rizzo, F. ${ }^{3}$; Saggese, $P^{3}{ }^{3}$; Weisz, A. ${ }^{3}$; Kemeny, A. ${ }^{4}$; Pellegrini, M. ${ }^{2}$; lozzo, P. ${ }^{1}$

${ }^{1}$ Institute of Clinical Physiology, National Research Council (IFC-CNR), Pisa, Italy, ${ }^{2}$ Laboratory Of Integrative System Medicine, Institute Of Informatics And Telematics, National Research Council (lit-Cnr), Pisa, Italy, ${ }^{3}$ Laboratory Of Molecular Medicine And Genomics, Department Of Medicine, Surgery And Dentistry 'Schola Medica Salernitana', University Of Salerno, Salerno, Italy, ${ }^{4}$ Department Of Obstetric And Gynecology, Asl 1 Massa E Carrara, Italy

Introduction: Neonatal gut colonization influences microbiota stabilization and health in later life. We explored the effects of maternal metabolic profile and delivery mode on meconium microbiota composition.

Methods: Microbiota was assessed in 74 meconium samples by deep $16 \mathrm{~S}$ rRNA sequencing. Anthropometrics, metabolic and inflammatory markers (by multiplex immunoassay), and leukocyte telomere length (by Real-Time PCR) were measured in newborns and their mothers.

Results: Firmicutes (50.7\%), Proteobacteria (26.6\%), Actinobacteria (15.6\%) and Bacteroidetes (4.9\%) were the most represented phyla in the meconium. Maternal pregnancy BMI and newborn's weight were positively related to Cyanobacteria $(\mathrm{p} \leq 0.05)$, while maternal pregnancy triglycerides (TG) and caesarean section (C-section) were associated with greater Simpson diversity, higher Bacteroidetes, and lower Proteobacteria $(\mathrm{p} \leq 0.03)$ frequencies. Maternal TG tended to increase with Firmicutes, but was negatively related to Enterocuccus frequency $(p=0.006)$. C-section was negatively associated with Bacilli $(\mathrm{p}=0.007)$, particularly Lactobacillus ( $\mathrm{p}=0.031)$, and positively with Clostridia $(\mathrm{p}=0.004)$ frequencies. A progressive increment in Coriobacteria ( $\mathrm{p}=0.04)$, Clostridia and Deltaproteobacteria $(\mathrm{p} \leq 0.01)$, and a decrement in Bacilli and Gammaproteobacteria $(\mathrm{p}<0.01)$ were observed moving from vaginal delivery with low or high maternal TG ( $<$ or $>$ median) to C-section with low or high maternal TG.

The above microbial classes were related to c-peptide and TNF-alpha levels in cord blood; no relationship with telomere length was detected.

Conclusion: We showed that maternal obesity, dyslipidemia and C-section independently and synergistically modulate neonatal gut bacterial colonization, which is related to newborn inflammatory profile. Gut microbiota is a potential mechanism linking maternal obesity to offspring health.

Conflict of interest: The authors have no conflict of interest to declare. Research related to this abstract was funded by the EU-FP7-HEALTH DORIAN project (Grant Agreement \#278603) 


\section{OS9:OC15}

\section{Inflammatory markers are elevated with advanced age,} obesity and type 2 DM - a study of young and old MZ BMIand DM-discordant twins

Heinonen, S. ${ }^{1}$; Jokinen, R. ${ }^{1}$; Bakker, L. ${ }^{1}$; Kaprio, J. ${ }^{2}$; Rissanen, A. ${ }^{\text {; }}$; Pietiläinen, . $^{\text {' }}$

${ }^{1}$ Obesity Research Unit, Research Programs Unit, Diabetes and Obesity, University of Helsinki, Helsinki, Finland, ${ }^{2}$ Finnish Twin Cohort Study, Department Of Public Health, University Of Helsinki, Helsinki

Introduction: Low-grade inflammation increases in obesity and type 2 diabetes, but the roles of ageing or genetic influences on inflammation are unclear.

Methods: We used a genetically matched monozygotic (MZ) BMI-discordant co-twin comparison design to study how acquired obesity in young (22-36 y) and old (59-79 y) twin pairs, with or without type 2 diabetes (T2DM) affects plasma inflammatory markers (Olink Proseek" Multiplex Inflammation $196 \times 96$ panel), of which 74 could be analysed. The BMI-discordant pairs $\left(\triangle \mathrm{BMI} \geq 3 \mathrm{~kg} / \mathrm{m}^{2}\right)$ were divided into four groups: 1) young and healthy twin pairs, $n=26 ; 2$ ) old and healthy twin pairs, $n=9 ; 3$ ) old and T2DM discordant pairs, $\mathrm{n}=5$; and 4) old and T2DM concordant pairs, $\mathrm{n}=6$. Body composition was measured by DEXA and insulin sensitivity by oral glucose tolerance test.

Results: Compared to their leaner co-twins, the heavy co-twins had more body fat in all 4 groups and lower insulin sensitivity in all but the T2DM concordant pairs $(\mathrm{p}<0.05)$. In young and healthy pairs, 12 out of 74 inflammatory markers (IL18R1, CDCP1, FGF21, IL6, DNER, HGF, TRANCE, SCF, TRAIL, CXCL1, OSM, SLAMF1, p < 0.05) were increased in heavy compared with lean co-twins. In old and healthy pairs, the corresponding numbers were 8/74 (FGF21, CCL4, HGF, IL18, SCF, ADA, IL8, BDNF), in T2DM discordant pairs 6/74 (CST5, TRAIL, GDNF, IL17c, IL10rb, CCL25), and no differences in T2M concordant pairs.

Comparisons of the heavy co-twins between the four groups (all with BMIs $31.0-35.8 \mathrm{~kg} / \mathrm{m}^{2}$ ) revealed that $30 / 74$ inflammatory markers were elevated in the healthy but old twins, 19/74 in the T2DM discordant pairs, and $7 / 74$ in the heavier twins of the T2DM concordant pairs compared with heavy twins who were young and healthy $(\mathrm{p}<0.001)$. In the lean co-twins (BMIs $25.3-29.9 \mathrm{~kg} / \mathrm{m}^{2}$ ) the respective values across groups were $20 / 74,24 / 74$ and 24/74, $\mathrm{p}<0.001$. No differences were detected across the three older groups

Conclusion: Inflammatory markers rise with age both in lean and heavy co-twin. The relative role of body weight differences on inflammatory markers is modified by age and T2DM. T2DM is a strong driving factor for low-grade inflammation as in BMI-discordant but T2DM-concordant pairs, both twin pair members had high inflammation levels.

\section{OS9:OC16}

\section{Low muscle mass is associated with non-alcoholic fatty liver disease independently with obesity and insulin resistance}

\section{Yang, . $^{1} ;$ i Lu, C. ${ }^{2}$; Huang, . $^{2}$}

${ }^{1}$ Department of Family Medicine, National Taiwan University Hospital, Bei-hu Branch, ${ }^{2}$ Department Of Family Medicine, National Taiwan University Hospital

Introduction: Non-alcoholic fatty liver disease (NAFLD) is an emerging chronic liver disease. We aimed to determine the association between the muscle mass and NAFLD severity using semi-quantitative ultrasonography.

Methods: This is a cross-sectional study. A total of 614 participants were recruited from the community. NAFLD was evaluated according to the Ultrasonographic Fatty Liver Indicator (US-FLI), which is a semi-quantitative liver ultrasound score (score: $0-8)$. NAFLD severity was defined as normal (US-FLI score: 0-1), mild (US-FLI score: 2-4) and moderate-to-severe (US-FLI score $\geq 5$ ). Appendicular Skeletal muscle mass (ASM) was measured and skeletal muscle index (SMI) was defined as ASM/body weight (ASM\%) value. SMI was further divided into tertiles.
The associations between the severity of NAFLD and SMI were assesd by ordinal logsitic regression model.

Results: NAFLD was found in $53.7 \%$ of the participants. SMI was inversely correlated significantly with the increment of NAFLD severity $(31.7 \%, 30.8 \%$ and $30.3 \%$ in normal, mild and moderate to severe NAFLD, respectively; P for trend $<0.0001)$. Participant with lower SMI also had worse metabolic characteristics, such as higher fasting sugar, higher triglycerides, higher low density lipoprotein, lower high density lipoprotein, higher body mass index (BMI) and arger waist circumference ( $\mathrm{P}$ for trend $<0.0001$ from low to high tertiles of SMI). Similarly, the US-FLI score was inversely correlated with SMI $(3.4,1.8$ and 0.9 in the tertile1 [T1], tertile2 [T2] and tertile3 [T3] of SMI, P for trend $<0.0001)$. Ordinal logistic regression showed the less SMI was associated with NAFLD severity (T1 odds ratio[OR]: 14.8 [95\% CI: 9.2-23.2]; T2 OR: 3.2 [95\% CI: 2.1-4.9] compared with T3) after adjustment for age and gender. After further adjustment for BMI, homeostasis model assessment index for insulin resistance (HOMA-IR), smoking, exercise and betel nuts, the association was still significant (T1 OR: 3.1; 95\% CI: 1.73-5.6; T2 OR: 1.7, 95\% CI:1.05-2.77). Serum vitamin D (Vit-D) was further adjusted and showed protective for getting NAFLD (OR: 0.9, 95\% CI: 0.84-0.96).

Conclusion: In conclusion, low muscle mass was significantly associated with more severe NAFLD independently with obesity and insulin resistance. Furthermore, Vit-D seems to have protective effects against NAFLD.

Conflict of Interest: No conflict of interest

Funding: No Funding

\section{OS9:OC17}

\section{Search for potential glucose/insulin-sensitive myokines in} humans

\section{Galgani, J. ${ }^{1}$; Mizgier, M. '; Ravussin, E. ${ }^{2}$}

${ }^{1}$ Pontificia Universidad Catolica de Chile, ${ }^{2}$ Pennington Biomedical Research Center

Introduction: Glycemia is determined by a tight control of insulin sensitivity and its secretion. Indeed, an inverse relation between both processes is found. Recently, skeletal muscle was proposed to interact with pancreas through myokines. It is hypothesized that insulin-mediated glucose disposal triggers myokine release, which can then target insulin secretion. Abnormal insulin secretion in genetic mice models having altered muscle glucose metabolism supports this hypothesis. In addition, muscle cell-derived media also influences in vitro glucose-stimulated insulin secretion. The physiological relevance of such findings for humans is unknown. We aimed to identify glucose/insulin-sensitive myokines through a euglycemic clamp at a high insulin dose to maximize muscle glucose uptake. Proteins known to be released from muscle cells and having their receptors expressed in pancreatic islets are candidate myokines mediating a muscle-pancreas crosstalk.

Methods: In 15/15 non-diabetic males/females (35 \pm 12 [SD] y old; $27 \pm 4$ $\left.\mathrm{kg} / \mathrm{m}^{2}\right)$ a 2 -h euglycemic clamp $\left(120 \mathrm{mU} / \mathrm{m}^{2} / \mathrm{min}\right)$ was conducted. Blood was drawn under fasting and steady-state conditions. By a PCR-based technology, 92 proteins were quantified (Proseek Multiplex Inflammation I96'96; OLINK Proteomics). Proteins having a change upon glucose/insulin infusion $(\mathrm{p}<0.05$; paired $\mathrm{t}$-test and corrected by the false discovery rate) and being related with insulin-mediated glucose disposal (by Spearman test) were searched for their human muscle origin and presence of receptors in human/murine pancreatic islets from published studies. Glucose disposal rate was divided by estimated metabolic body size (EMBS=fat-free mass $[\mathrm{kg}]+17.7)$. Body composition was measured by DXA. Results: Insulin-mediated glucose disposal was $7 \pm 3 \mathrm{mg} / \mathrm{kg}$ EMBS/min (range: 4 to $13 \mathrm{mg} / \mathrm{kg}$ EMBS/min). Glucose/insulin infusion modified plasma concentration of 24 out of 73 detected proteins. The change in plasma concentration upon glucose/insulin infusion for 16 of these proteins related with insulin-mediated glucose disposal $(r=-0.39$ to -0.60 ; $\mathrm{p}<0.05$ ). Four proteins (SCF, CCL11, IL10 and IL12B) were detected in 
conditioned media of human skeletal muscle cells from at least two studies. IL10 and IL12B do express their receptors in human or murine pancreatic islets or beta cells.

Discussion: IL10 and IL12B represent candidate myokines found in circulation, which are affected by glucose and insulin in humans. The fact that these proteins express their receptors in pancreatic islets encourages determining the effect of their recombinant forms on in vitro insulin secretion. Eventually, IL10 and IL12B play a role mediating this hypothetical skeletal muscle-pancreas crosstalk.

Conflict of Interest: None

Funding: FONDECYT-Chile 1130217 and NORC-USA P30 DK072476

\section{OS9:OC18}

\section{Protein kinase CK2 is involved in adipose tissue insulin} signaling and remodeling during obesity and weight loss

Sanna, M. ${ }^{\text {; }}$ Borgo, C. ${ }^{2}$; Favaretto, F. ${ }^{3}$; Fabris, R. ${ }^{1}$; Salizzato, V. ${ }^{2}$; Cesaro, L. ${ }^{4}$; Belligoli, A. ' ; Foletto, M. '; Prevedello, L. '; Vindigni, V.5 ; Bardini, R. ${ }^{6}$; Busetto, L. ' ${ }^{1}$ Donella-Deana, A. ${ }^{4}$; Milan, G. ${ }^{3}$; Vettor, ${ }^{1}{ }^{1}$

${ }^{1}$ Center for the Study and the Integrated Management of Obesity, University of Padova, Italy, ${ }^{2}$ Department Of Biomedical Sciences, University Of Padua, 35131 Padua, Italy, ${ }^{3}$ Department Of Medicine, University Of Padua, Internal Medicine 3, 35128 Padua, Italy, ${ }^{4}$ Department Of Biomedical Sciences, University Of Padua, 35131 Padua, Italy., ${ }^{5}$ Department Of Neurosciences, University Of Padua, 35128 Padua, Italy., ${ }^{6}$ Department Of Surgical, Oncological And Gastroenterological Sciences, University Of Padua, Division Of General Surgery, 35128 Padua, Italy.

Introduction: CK2 is a protein kinase implicated in several essential cellular processes, over-expressed in cancer and described to regulate insulin signaling cascade. Recently CK2 has been described to negatively regulate thermogenesis (Shinoda K et al, 2015, Cell Metabolism) and to inhibit insulin release (Rossi $\mathrm{M}$ et al, 2015, PNAS). Nevertheless, the role of CK2 in adipose tissue (AT) and its involvement in human obesity development and therapy has been poorly investigated.

Methods: Our multi-disciplinary team performed biochemical analysis of signaling pathways by WB and in vitro kinase activity assays, and glucose handling studies using glucose uptake assay and IF in adipocyte cultures and glucose and insulin tolerance test in mice. Moreover we quantify CK2 expression/activity in human AT specimens of 27 obese patients, clinically characterized, in 12 obese patients underwent relevant weight loss and 11 normal-weight controls.

Results: We proved that CK2 amount and activity were not influenced by insulin stimulation and that CK2 activity was efficiently inhibited by specific inhibitors, structurally unrelated. We worked with CX-4945, a CK2 inhibitor currently used in cancer clinical trials, using the minimal concentration $(2.5 f \hat{Y} \mathrm{M})$ and pre-treatment time (1hr) able to efficiently inhibit CK2 activity, avoiding any cytotoxic effect. Pharmacological inhibition of CK2 did not significantly affect in vitro adipogenic differentiation or expression profiling of mature adipocytes. Conversely, we showed that in human and murine adipocytes CK2-inhibition decreases the insulin-induced glucose uptake by counteracting Akt-signaling and GLUT4-translocation to the plasma membrane. We compared CK2 expression and activity in different mouse tissues highlighted that white skeletal muscle fibres and liver contained the highest quantity of this kinase. CK2 was expressed more in brown AT than in white AT depots. We show that CK2 promotes insulin-signaling in mouse AT, liver and skeletal muscle and that in vivo acute treatment with CX-4945 impairs glucose-tolerance in mice. Studies in tissues of ob/ob and $\mathrm{db} / \mathrm{db}$ mice highlights an up-regulation of CK2 expression and activity only in WAT. CK2 hyper-activation is strongly evident also in SAT and VAT of obese patients and weight loss obtained by bariatric surgery or hypocaloric diet reverts CK2 up-regulation to normal level.

Conclusion: We show that CK2 is involved in insulin sensitivity, glucose handling and remodeling of WAT. Moreover we identify CK2 hyper-activation as a hallmark of human obesity, suggesting a new potential therapeutic target for metabolic diseases.
Conflict of Interest: None Disclosed

Funding: This research was funded by University of Padova, Italian Ministries of Health and MIUR.

\section{OS10 Child Health and Obesity Prevention}

\section{OS10:OC43}

\section{Macronutrient composition of early childhood diet in relation to growth and body composition}

\section{Voortman, $T^{1}$; Jaddoe, . $^{1}$; Franco, $0 .{ }^{1}$ \\ ${ }^{1}$ Erasmus MC, University Medical Center}

Background: Previous studies suggest that high protein intake in early childhood leads to a higher body mass index. However, it is not clear whether this reflects a higher fat mass and hence more adiposity, or a difference in lean mass, as observed in adults. Furthermore, it is not clear what the role is of other macronutrients in the diet and its replacement effects. We examined associations of macronutrient composition of the diet in early childhood with growth and detailed measures of body composition up to the age of 9 years.

Methods: This study was performed among 3,564 children participating in a population-based cohort study in the Netherlands. Dietary intake was assessed with food-frequency questionnaires at age 1 year. We calculated intakes of total protein and protein from different sources; of total carbohydrates, fiber, polysaccharides, monosaccharides and disaccharides; and of total, saturated, monounsaturated and polyunsaturated fat. Height and weight were repeatedly measured between the ages of 1 and 9 years. Fat and fat-free mass were measured at 6 and 9 years using dual X-ray absorptiometry. We calculated age- and sex-specific SD scores for height, weight, body mass index (BMI), fat-mass index (FMI), and fat-free mass index (FFMI). Macronutrient intakes were expressed in energy percentages and entered in multivariable linear mixed models in which we examined different macronutrient replacement effects. Models were adjusted for maternal age, education, BMI, and smoking and for child sex, age, ethnicity, birth weight, breastfeeding, diet quality, television watching, and sports. Results: Results from multivariable models showed that higher intake of total and animal protein (both dairy and non-dairy) was associated with a higher height, weight and BMI up to the age of 9 years, irrespective of whether it was replacing carbohydrates or fats in the diet. Further analyses showed that the positive association with BMI was completely explained by a higher FMI and not FFMI. No significant associations were observed for intakes of fat or carbohydrates specifically.

Conclusions: Our results suggest that high protein intake, particularly from animal food sources, in early childhood is associated with higher body fat mass, but not fat-free mass. Future studies are needed to examine the optimal range of protein intake and macronutrient composition of the diet for infants and young children and translate these findings into dietary guidelines targeted at this specific age group.

\section{OS10:OC44}

Prenatal risk factors influencing childhood BMI and overweight independent of birth weight and infancy BMI a path analysis within the Danish national birth cohort

Morgen, C. ${ }^{1}$; Ängquist, L. '; Baker, J. ' ; Andersen, A. ${ }^{2}$; Michaelsen, . $^{3}$; SøRensen, $T^{3}$

${ }^{1}$ Department of Clinical Epidemiology, Bispebjerg and Frederiksberg Hospital, The capitol Region of Denmark., ${ }^{2}$ Section Of Social Medicine, Department Of Public Health, University Of Copenhagen., ${ }^{3}$ Department Of Nutrition, Exercise And Growth, Faculty Of Sciences, University Of Copenhagen, Denmark.

Introduction: Prenatal risk factors for childhood overweight may operate indirectly through the development in body size in early life and/or directly independent hereof. We quantified the effects of maternal and 
paternal body mass index (BMI), maternal age, socioeconomic position (SEP), parity, gestational weight gain, maternal smoking during pregnancy, caesarean section, birth weight, and infancy BMI (5 and 12 months) on BMI at 7 and 11 years.

Methods: Family triads with information on maternal, paternal and child BMI at ages $7(n=29374)$ and 11 years $(n=18044)$ were selected from the Danish National Birth Cohort. Information originated from maternal interviews and medical health examinations. Path analysis was used to estimate the direct and indirect effects of prenatal risk factors on childhood BMI z-scores (BMIz per unit score of the risk factor), and logistic regression analyses were used to estimate the associations with childhood overweight.

Results: The strongest direct effects on BMIz at age 7 years were found for maternal and paternal BMI (0.19 BMIz per maternal BMIz and 0.14 BMIz per paternal BMIz), low SEP (0.08 BMIz), maternal smoking (0.12 BMIz) and higher BMIz at 5 and 12 months (up to 0.19 BMIz per higher infant BMIz). For BMIz at age 11 years, with BMIz at age 7 years included in the model, similar effects were found, but the direct effects of BMIz at age 5 and 12 months on BMIz at age 11 years were mediated through BMI at age 7 (0.62 BMIz at age 11 years per higher BMIz at age 7 years). Same patterns were seen from analyses with overweight as the outcome. For a child with a mean height and BMI, the effects can be translated onto absolute measures. The sum of the direct effects can be translated to a 2.3 and a 5.7 $\mathrm{kg}$ higher weight at age 7 and 11 years, respectively.

Conclusions: Parental BMI, low SEP and smoking during pregnancy have persisting, strong and direct effects on child BMI and overweight independent of birth weight and infancy BMI.

\section{OS10:OC45}

\section{Maternal and offspring intelligence in relation to BMI across childhood and adolescence}

Wraw, C. '; Gale, C. ${ }^{2}$; Der, G. ${ }^{3}$; Deary, I. ${ }^{1}$

${ }^{1}$ University of Edinburgh, ${ }^{2}$ University Of Edinburgh \& University Of Southampton, ${ }^{3}$ University Of Glasgow

Objective: To examine the associations between both maternal and offspring's intelligence and offspring's body mass index (BMI) in childhood and adolescence.

Method: Participants were members of the National Longitudinal Survey of Youth 1979 (NLSY-79) Children and Young Adults cohort ( $\mathrm{n}=11,512$ ) and their biological mothers who were also members of the NLSY-79 ( $\mathrm{n}=4,932)$. Offspring's IQ was measured with the Peabody Individual Achievement Test. Mother's IQ was measured with the Armed Forces Qualification Test when they were aged between 15-23 years. A series of regression analyses tested the association between IQ and offspring's BMI in middle childhood (age 5-7 years), late childhood (age 8-10 years), early adolescence (age 11-13 years), and middle adolescence (age 14-18 years) for boys and girls separately, while adjusting for potential confounding and mediating factors (i.e. maternal ethnicity, education and pre-pregnancy BMI).

Results: After adjustment for potential mediating and confounding factors, mother's IQ was inversely associated with son's BMI in middle childhood (beta $=-0.081,95 \%$ C.I. -0.160 to $-0.002, p=0.045$ ) and daughter's BMI in early (beta $=-0.102,95 \%$ C.I. -0.172 to $-0.004, p=0.004$ ) and middle adolescence (beta $=-0.100,95 \%$ C.I. -0.170 to $-0.032, \mathrm{p}=0.004$ ). After adjustment for potential confounding factors, girl's IQ was negatively associated with BMI in middle adolescence (beta $=-0.095$, 95\% C.I -0.146 to $-0.044, \mathrm{p}<0.001)$ and boy's IQ was positively associated with BMI in late childhood (beta $=0.085,95 \%$ C.I. 0.028 to $0.142, \mathrm{p}=0.004$ ) and early adolescence (beta $=0.094,95 \%$ C.I. 0.037 to $0.150, \mathrm{p}=0.001$ ). Conclusion: Higher maternal IQ was associated with lower offspring BMI. Higher childhood IQ was associated with a lower BMI in girls but a higher BMI in boys. Future studies could further investigate the roll played by mother's education and ethnicity in the IQ-BMI association.
Acknowledgements: This work was undertaken in The University of Edinburgh Centre for Cognitive Ageing and Cognitive Epidemiology, part of the cross council Lifelong Health and Wellbeing Initiative (MR/K026992/1).

Funding: Funding from the BBSRC and the Medical Research Council (MRC) is gratefully acknowledged.

Conflict of Interest: None.

\section{OS10:0C46}

\section{Associations between body size from childhood to young adulthood and adult colon cancer}

Jensen, B. ${ }^{1}$; Bjerregaard, L. ${ }^{1}$; Ängquist, L. '; Gögenur, I. ${ }^{2}$; Renehan, A. ${ }^{3}$; Osler, M. ${ }^{4}$; SøRensen, T.'; Baker, J.'

${ }^{1}$ Department of Clinical Epidemiology, (former Institute of Preventive Medicine), Bispebjerg and Frederiksberg Hospital, The Capital Region, Copenhagen, Denmark, ${ }^{2}$ Department Of Surgery, Zealand University Hospital, Copenhagen University, KøGe, Denmark, ${ }^{3}$ Division Of Cancer Sciences, School Of Medical Sciences, Faculty Of Biology, Medicine And Health, University Of Manchester, Manchester, United Kingdom, ${ }^{4}$ Research Center For Prevention And Health, Glostrup Hospital, Glostrup, Denmark

Introduction: Elevated body mass index (BMI) in adulthood is associated with increased colon cancer incidence. We have shown that excess $\mathrm{BMI}$ in childhood is also associated with increased colon cancer risk in adulthood, but it is unclear whether changes in body size from childhood to young adulthood influences risk. Here, we evaluated patterns of weight status in childhood and young adulthood with colon cancer risk in later adulthood.

Methods: A total of 61,446 boys from the Copenhagen School Health Record Register were identified in the Danish Conscription Database (including virtually all Danish men born 1939-1959). Weight and height were measured at age 7 years and in young adulthood (17-26 years). These young men were linked to the Danish National Cancer Register and followed from age 40 years to identify cases of colon cancer $(n=706$, median age $=62.7$ years). Based on recommended cut-offs from the International Obesity Task Force ( $<18$ years) and the World Health Organisation ( $\geq 18$ years) the boys and men were categorised as normal-weight or overweight. Weight status patterns were categorized as: normal-weight/ normal-weight (reference), overweight/ normal-weight, normal-weight/ overweight and overweight/ overweight. Cox proportional hazard regression models were used to estimate hazard ratios (HR) and 95\% confidence intervals.

Results: The men were followed for a median of 24.5 years. Boys that were overweight at age 7 years $(3.0 \%)$ or in young adulthood $(8.4 \%)$ had a significantly higher risk of adult colon cancer compared with normal-weight peers (HR: 1.54 [1.06-2.25] and HR: 1.45 [1.14-1.86], respectively). Compared with normal-weight boys who remained normal-weight as young men, overweight boys who became normal-weight as young men had a similar risk of adult colon cancer (HR: 0.62, [0.28-1.38]) but boys who were overweight and remained overweight as young men had an increased risk of adult colon cancer (HR: 2.69 [1.76-4.12]).

Conclusions: Overweight boys that lose weight and achieve a normal-weight status by young adulthood do not carry an increased risk of adult colon cancer compared with boys who remain normal-weight as young men. However, overweight boys who remain overweight as young men have an increased risk of adult colon cancer. These results highlight the importance of weight management in childhood.

\section{Conflicts of Interest: None disclosed}

Funding: The European Research Council, Grant Agreement no. 281419 and the Danish Council for Independent Research (DFF), Grant Agreement no. 133100218 . 


\section{OS10:OC47}

\section{The prevention of obesity in infancy by targeting sleep or} food and activity: RCT outcomes at 5 years

Taylor, B. '; Taylor, R. '; Gray, A. '; Galland, B. ' Heath, A. '; Lawrence, J. '; Hanna, M. ${ }^{1}$; Hatch, B. ${ }^{1}$

${ }^{1}$ University of Otago

Background: Obesity prevention initiatives targeting sleep in infants are rare despite emerging evidence of potential benefit. Our Prevention of Overweight in Infancy (POI) study determined that a conventional approach targeting food, activity, and breastfeeding was less effective than an approach targeting sleep when outcomes were measured at 2 years. We now report outcomes at 3.5 and 5 years of age.

Methods: The POI RCT studied 802 parent/infant dyads randomized antenatally to one of four groups: Control, FAB (food, activity, and breastfeeding), Sleep, or Combination (FAB and Sleep). All received standard well-child care. FAB participants received additional guidance/support promoting breastfeeding, healthy eating, and physical activity (to 18 months). Sleep participants attended an antenatal education session covering normal development of sleep and self-settling techniques, received a home visit at 3 weeks addressing prevention of sleep problems, and a sleep treatment programme upon request (from 6 months). Body mass index (BMI) was measured at 24 months, and secondary outcomes including diet, physical activity and sleep, were assessed by questionnaire, diary or accelerometry, at multiple time points including 3.5 and 5 years.

Results: Although there was no significant intervention effect for BMI at 24 months, there was a group difference in the prevalence of obesity (overall $\mathrm{P}=0.027$ ) driven by lower rates of obesity in the Sleep (OR $0.46,95 \%$ CI $0.25-0.83, \mathrm{P}=0.011$ ) and Combination (OR 0.51, 95\% CI 0.28-0.90, $\mathrm{P}=0.022$ ) groups compared with $\mathrm{FAB}$, but not compared with Control. At five years, there continued to be significant differences between groups, this time for both BMI, BMI z-score as well as \% obese. The FAB group were now significantly worse than the control group while the sleep and combination groups were better than FAB, but not better than the control group.

Conclusions: An early life sleep intervention halved the odds of obesity in children at two years of age. By 5 years the sleep intervention effects were no longer significant but the conventional intervention targeting diet and activity appear to have made obesity measures worse.

\section{Conflict of Interest: None}

Funding: Research relating to this abstract was funded by the Health Research Council of NZ, the Southern District Health Board of NZ and the University of Otago.

\section{OS10:OC48}

\section{Metabolically healthy obesity in young adulthood: is it explained by duration of obesity?}

\section{Araújo, J. ${ }^{1}$; Cabral, M. ${ }^{1}$; Ramos, E. ${ }^{2}$}

${ }^{1}$ EPIUnit - Institute of Public Health, University of Porto, ${ }^{2}$ Epiunit - Institute Of Public Health, University Of Porto. University Of Porto Medical School.

Introduction: Metabolically healthy obesity (MHO) is a phenotype identified in subgroups of obese individuals who do not present metabolic abnormalities. However, its mechanisms are not well understood yet, and some authors claim that $\mathrm{MHO}$ may represent those with a lower duration of exposure to obesity. Therefore, we studied the association between duration of obesity from adolescence to early adulthood and MHO.

Methods: Participants of the EPITeen cohort (Porto, Portugal) evaluated at 13, 17, 21 and 24 years of age were included $(n=1042)$. Duration and degree of body mass index (BMI) in the 11-year period was summarized through the area under the curve of body mass index $\left(\mathrm{BMI}_{\mathrm{AUC}}\right)$. Metabolically healthy participants at 24 years were considered those presenting $<2$ of the following criteria defined according to ATP III: blood pressure, fasting glucose, high-density lipoprotein-cholesterol and triglycerides.
The association between $\mathrm{BMI}_{\mathrm{AUC}}$ and the different categories of $\mathrm{MHO}$ was estimated through multinomial logistic regression models.

Results: Combining obesity and pre-obesity categories, there was $22.7 \%$ of metabolically healthy overweight (MHOW) at 24 years of age. When specifically considering the obesity category, there was $4.7 \%$ of $\mathrm{MHO}$. The median $\mathrm{BMI}_{\mathrm{AUC}}$ was $242.1 \mathrm{Kg} / \mathrm{m}^{2}$ (25th-75th percentiles: 223.1-265.2) in 11 years, corresponding to $22.0 \mathrm{Kg} / \mathrm{m}^{2}$ on average per year. After adjusting for sex and education, 1 unit increase in the $\mathrm{BMI}_{\mathrm{AUC}}$ on average per year in the period studied was associated with an $18 \%$ lower odds of MHOW (OR $=0.88,95 \%$ CI: $0.79-0.99)$, in comparison to metabolically unhealthy overweight.

Conclusion: Participants with higher duration and degree of obesity were less likely to be metabolically healthy. These results highlight the relevance of the time of exposure to obesity in the definition of the metabolic profile.

Conflict of Interest: None disclosed.

Funding: This study was supported through FEDER from the Operational Programme Factors of Competitiveness - COMPETE and through national Funding from the Portuguese Foundation for Science and Technology - FCT (Portuguese Ministry of Education and Science) within the project PTDC/DTP-EPI/6506/2014, and by the Epidemiology Research Unit - Institute of Public Health, University of Porto (UID/DTP/047507/2013). The individual doctoral grant to MC (PD/ BD/105824/2014) by the Portuguese Foundation for Science and Technology - FCT is gratefully acknowledged.

\section{OS11 Bariatric and Metabolic Surgery}

\section{OS11:OC55}

\section{The advanced DiaRem score improves prediction of diabetes} remission one-year post gastric bypass

Aron-Wisnewsky, J. '; Sokolovska, N. '; Liu1, Y. ${ }^{1}$; Comaneshter, D. ${ }^{2}$; Vinker, S. ${ }^{2}$; Pecht, T. ${ }^{3}$; Poitou, C. '; Oppert, J. ${ }^{4}$; Bouillot, J. ${ }^{5}$; Genser, L. ${ }^{6}$; Dicker, D. '; Zucker, J. ${ }^{8}$; Rudich, A. ${ }^{3}$; Clément, K. $^{1}$

${ }^{1}$ ICAN, APHP, Nutrition department, Pitié-Salpêtrière Hospital, F-75013, Paris, France, Sorbonne Universités, UPMC Université Paris 06 and INSERM, UMR_S 1166, Team 6 Nutriomics, ${ }^{2}$ Sackler School Of Medicine, Tel Aviv University, Israel, ${ }^{3}$ Clinical Biochemistry And Pharmacology Department, Negev (Nibn), BenGurion University, Beer-Sheva, 84103, Israel, ${ }^{4}$ Ican, Aphp, Nutrition Department, Pitié-Salpêtrière Hospital, F-75013, Paris, France, Sorbonne Universités, Upmc Université Paris 06, ${ }^{5} \mathrm{Aphp}$, Ambroise Paré Hospital, Surgery Department, 92100 Boulogne-Billancourt, France, ${ }^{6} \mathrm{Aphp}$, Pitié-Salpétrière Hospital, Hepato-Biliary And Digestive Surgery Department, F-75013, Paris, France, ${ }^{7}$ Internal Medicine D And Obesity Clinic, Hasharon Hospital-Rabin Medical Center, Petach-Tikva, Israel, ${ }^{8}$ Ican, Aphp, Nutrition Department, Pitié-Salpêtrière Hospital, F-75013, Paris, France;Ummisco Umi 209 Ird/Upmc, Paris, France

Objective: T2D patients, but not all, experience diabetes remission (DR) after bariatric surgery (BS). It is thus critical to develop predictive scores applicable in clinical routine. The DiaRem score is a relevant score in DR prediction post-Roux-en-Y gastric bypass (RYGB), but, might be not accurate for all patients and in some score categories. We aimed to develop an optimized DR predictive score (the advanced-DiaRem).

Methods: We used a retrospective French cohort $(\mathrm{N}=1866)$ with $352 \mathrm{~T} 2 \mathrm{D}$ patients followed one year post-RYGB. We developed the Advanced-Diarem in a test cohort $(\mathrm{N}=213)$ and examine its accuracy in independent cohorts from France $(\mathrm{N}=134)$ and Israel $(\mathrm{N}=99)$.

Results: Adding to the original DiaRem two clinical parameters (diabetes duration and anti-diabetic drugs numbers), and modifying the score weighting penalization, led in improved predictive performance for the Ad-DiaRem. Ad-DiaRem score displayed an improved Area under the ROC and predictive accuracy as compared to the DiaRem score (respectively 0.911 vs. 0.856 and $A c c=0.841$ vs.0.789; $p=0.03$ ), thus leading to the correct reclassification of $8 \%$ more patients with the Ad-Diarem, initially misclassified with the Diarem. Furthermore, using the Ad-Diarem, there was less misclassification in the middle scoring zone. This improved prediction was confirmed in the confirmation cohorts. 
Conclusion We propose the Ad-DiaRem score, which includes two additional clinical parameters, as an optimized scoring system with improved faccuracy to predict DR one-year post-RYGB. Consequently, this scoring system might be helpful for personalized management of diabetic subjects when considering BS in routine care, ultimately contributing to precision medicine.

\section{Conflict of Interest: None Disclosed}

Funding: Research relating to this abstract was funded by Funds obtained by the Campus program (Maimonide "Franco-Israeli project" in cooperation with Ben Gurion University. Clinical study was supported by the Assistance Publique-Hôpitaux de Paris, and the Direction of Clinical Research (CRC) for clinical investigation (PHRC 02076 to KC, CRC P050318 to CP and CRC-FIBROTA to JAW and $\mathrm{KC}$ ), and the National Agency of Research (ANR Adipofib, and the national program "Investissements d'Avenir" with the reference ANR-10-IAHU-05)

\section{OS11:OC56}

\section{Surgical revision in 1000 consecutives patients who had sleeve gastrectomy for morbid obesity in a single center}

Antonio, D. ${ }^{1}$; Elias, C. ${ }^{1}$; Marco, N. ${ }^{1}$; Elie, C. $^{1}$

${ }^{1} \mathrm{CHIPS}$ - Centre Hopitalier Poissy Saint-Germain-en Laye, Poissy, France

Introduction: Bariatric surgery is the best available, long-term treatment for morbid obesity. Currently, laparoscopic sleeve gastrectomy (LSG) is the most commonly performed bariatric procedure in France. Despite its safety and efficacy, long-term complications of LSG are not rare including weight regain, insufficient weight loss, gastroesophageal reflux disease (GERD), and twisting or stenosis. The goal of this study was to analyze the pattern and short-term results of surgical revision in patients with LSG.

Methods: Revisional surgery, regardless of its motivation, was always a multidisciplinary decision after thorough clinical, biological, endoscopic, and radiological assessment. Patients who had revisional surgery were retrospectively identified and subsequently divided in 4 subgroups according to preoperative body mass index $(\mathrm{BMI})\left(<\right.$ or $\left.>50 \mathrm{~kg} / \mathrm{m}^{2}\right)$ and the presence or not of GERD. The minimal follow up period was 12 months.

Results: Between December 2004 and September 2015, 1000 patients had LSG. 114 patients were lost to follow-up (11.4\%). The rate of excess weight loss $(\mathrm{EWL})>50 \%$ was $68 \%$. Out of 886 patients, 47 had a revisional surgery $(5.3 \%)$ for inadequate weight loss, GERD, or stenosis, respectively. The performed procedures included Roux en Y Gastric Bypass (60\%), reSleeve (18\%), Duodenal Switch (DS) (12\%), Single Anatomosis Duodeno-Ileal Shunt (6\%), and Sero-myotomy (4\%). Median interval from the initial surgery to conversion was 27 months (18-41). Median operating room time was $170 \mathrm{~min}$ (range, 100-290). Median length of stay was 72 hours (range, $48-120$ ). Mortality was nil. Overall complication rate was $8 \%(4 / 47)$. The follow-up rate at 12 months was at $96 \%$. Satisfaction index at 12 month was $88 \%$.

Conclusions: Revisional options after LSG are safe and lead rarely to complications. Nowadays, LSG could be considered as the first step of a potentially multi-step bariatric pathway. Longer follow-up will be needed to establish the correct algorithm of choice of the surgical option for post LSG revision.

Conflict of Interest: None

Funding: No Funding

\section{OS11:OC57}

\section{Effect of resistance exercise training on body composition and physical fitness after gastric bypass (PROMISE study) - a randomized controlled trial}

\author{
Oppert, J. ${ }^{1}$; Bellicha, A. ${ }^{2}$; Roda, C. ${ }^{3}$; Bouillot, J. ${ }^{4}$; Torcivia, A. ${ }^{5}$; Tanguy, M. ${ }^{6}$;
} Clément, K.7; Poitou, C. '; Ciangura, $C^{1}$

${ }^{1}$ Department of Nutrition, Pitié-Salpêtrière Hospital, Assistance PubliqueHôpitaux de Paris; Sorbonne Universités, UPMC, Univ Paris 06, Institute of Cardiometabolism and Nutrition (ICAN); Paris, France, ${ }^{2}$ Department Of Nutrition, Pitié-Salpêtrière Hospital, Assistance Publique-Hôpitaux De Paris; Sorbonne Universités, Upmc, Univ Paris 06, Institute Of Cardiometabolism And Nutrition (Ican); Paris, France, Laboratory Bioingenierie, Tissus And Neuroplasticity ${ }^{3}{ }^{3}$ Barcelona Institute For Global Health; Barcelona, Spain, ${ }^{4}$ Department Of Visceral Surgery, Ambroise Paré Hospital, Assistance Publique-Hôpitaux De Paris; University Versailles-Saint-Quentin; Boulogne, France, ${ }^{5}$ Department Of Digestive And Hepato-Pancreato-Biliary Surgery, Pitié-Salpêtrière Hospital, Assistance Publique-Hôpitaux De Paris; Sorbonne Universités, Upmc, Univ Paris 06; Paris, France, ${ }^{6}$ Unité De Recherche Clinique, Pitié-Salpêtrière Hospital, Assistance Publique-Hôpitaux De Paris; Paris, France, ${ }^{7}$ Department Of Nutrition, Pitié-Salpêtrière Hospital, Assistance PubliqueHôpitaux De Paris; Sorbonne Universités, Upmc, Univ Paris 06, Institute Of Cardiometabolism And Nutrition (Ican); Paris, France, Nutriomics Team, Inserm, Umr_S U1166; Paris, France

Introduction: Bariatric surgery leads to marked weight loss, which is associated with lean body mass loss and decreased muscle strength. We aimed to determine whether resistance training with protein supplementation is effective in maintaining lean body mass and muscle strength 6 months after Roux-en-Y gastric bypass (RYGB).

Methods: The PROMISE study is an open RCT conducted at the obesity clinic of Pitie-Salpetriere University Hospital in Paris, France, between May 2010 and December 2014. Out of 290 eligible patients, 76 women with severe obesity undergoing RYGB participated in the study. Participants were randomly assigned at the time of surgery either to usual care with regular medical and nutritional follow-up (CON group), or to usual care and additional oral protein intake (48g/d of whey protein, PRO group), or to usual care, additional protein intake and supervised strength training (3 sessions/wk for $18 \mathrm{wk}, \mathrm{PRO}+\mathrm{EX}$ group). Patients were followed for 6 months after surgery. Primary outcomes were presurgery to 6-month postsurgery changes in lean body mass (assessed by DXA) and muscle strength (assessed by accelerometry). Secondary outcomes included changes in cardiorespiratory fitness (peak $\mathrm{VO}_{2}$ measured during a maximal exercise test), habitual physical activity (measured by the Actigraph GT3X accelerometer during a usual week), dietary protein intake (as assessed by a dietician), nutritional status (serum albumin, iron, vitamins), obesity comorbidities and health-related quality of life (SF-36 questionnaire).

Results: Baseline characteristics of participants (mean (SD) BMI: 44.0 (5.8) $\mathrm{kg} / \mathrm{m}^{2}$, age: $42.4(9.9) \mathrm{y}$ ) did not differ between the 3 groups. Mean weight loss $(-28 \mathrm{~kg})$ and lean body mass loss $(-8.8,-8.2$ and $-7.7 \mathrm{~kg}$ in the CON, PRO and PRO+EX groups, respectively) did not differ between groups. Lower limb muscle strength increased significantly in the $\mathrm{PRO}+\mathrm{EX}$ group compared to the CON and $\mathrm{PRO}$ groups $(+8.4 \mathrm{~kg}$ vs -21.6 and $-21.3 \mathrm{~kg}$, respectively, $\mathrm{p}<0.05)$. Changes in peakVO $\mathrm{O}_{2}$ and daily steps (mean increases: $+2.8 \mathrm{~mL} / \mathrm{kg} / \mathrm{min}$ and $+1178 \mathrm{steps} / \mathrm{d}$, respectively) did not differ between groups. Quality of life, obesity comorbidities and nutritional status improved 6 months after RYGB, without differences between groups.

Conclusion: This is the first randomized trial testing a combination of supervised strength training and additional protein intake after bariatric surgery on body composition and muscle functional changes. Significantly improved muscle strength was achieved 6 months after RYGB, although no differences were found regarding weight loss and body composition changes. Future studies should investigate the added value of combining resistance and endurance training and explore the appropriate time point to perform such exercise post-surgery. 


\section{OS11:OC58}

Long-term incidence of nephropathy in patients with obesity treated by bariatric surgery or usual care: Results from the Swedish obese subjects (SOS) study

Svensson, P. '; Shulman, A. '; Andersson-Assarsson, J. ${ }^{1}$; Peltonen, M. ${ }^{2}$; Carlsson, L. ${ }^{1}$

${ }^{1}$ Institute of Medicin. Sahlgrenska Academy at the University of Gothenburg, Sweden, ${ }^{2}$ Chronic Disease Prevention Unit, National Institute For Health And Welfare, Helsinki, Finland

Introduction: Obesity, as well as diabetes and hypertension, are risk factors for kidney disease and nephropathy. We have previously shown that bariatric surgery results in long-term sustained weight-loss but also in amelioration of obesity-comorbidities, such as diabetes. However, the long-term effect of bariatric surgery on prevention of kidney disease is not well established.

Methods: The SOS study consists of 4037 patients with obesity that has been treated by bariatric surgery or usual care (controls). The current investigation includes 1894 bariatric surgery patients and 1954 controls, al without kidney disease at baseline.

Nephropathy was defined using a composite endpoint consisting of macroalbuminuria (300 $\mathrm{mg} / 24$ hours) or $\mathrm{eGFR}<45 \mathrm{ml} / \mathrm{min}$ combined with a doubling of s-creatinine or diagnosed severe kidney disease (CKD 4 or 5, end stage renal disease, end-stage of chronic renal insufficiency, treatment with chronic hemodialysis or peritoneal dialysis).

Data on nephropathy events were obtained both from scheduled follow-up visits in the SOS study and from searching the Swedish National Patient Register.

Results: During the follow-up time (median 18.2 years), nephropathy was observed in 114 patients in the control group and 52 in the bariatric surgery group (corresponding to incidence rates of 3.5 and 1.6 evets per 1000 person years, respectively). The cumulative incidence of nephropathy was significantly lower in the bariatric surgery group compared with the control group (Hazard ratio $=0.43 ; 95 \%$ CI: 0.31-0.60, $\mathrm{p}<0.001$ ). Similar results were obtained after adjustments for baseline risk factors or when only register-based outcomes were used in the analyses.

Conclusion: Bariatric surgery reduces the incidence of nephropathy in patients with obesity.

Conflict of Interest: Payment received in form of lecture fees from AstraZeneca, Johnson\&Johnson, and MSD.

Funding: Research relating to this abstract was funded by US National Institutes of Health, Swedish Research Council, Sahlgrenska University Hospital ALF research grant, Swedish Diabetes Foundation.

\section{OS11:OC59}

\section{How should post-bariatric hypoglycaemia be evaluated?}

\section{Monteiro, M. ${ }^{1}$; Kirk, A. ${ }^{2}$; Batterham, . $^{1}$}

${ }^{1}$ University College London Hospitals, Bariatric Centre for Weight Management and Metabolic Surgery; Centre for Obesity Research, University College London London, UK, ${ }^{2}$ University College London Hospitals, Bariatric Centre For Weight Management And Metabolic Surgery, London, Uk

Introduction: Post bariatric hypoglycaemia is a rare yet challenging condition. Post-bariatric patients can present with a large spectrum of signs and symptoms consistent with hypoglycaemia. Although most often these are vasogenic symptoms attributed to dumping syndrome, real hypoglycaemia may also occur as a direct consequence of the surgical procedure or to other concomitant endocrine and systemic disorders that warrant differential diagnosis workup and specific clinical management.

Our aim is to present a protocol proposal for post bariatric hypoglycaemia investigation supported by the outcomes of its implementation at our bariatric centre.

Investigation protocol and Results: We report a case series of post-bariatric patients $(n=20)$ with clinical suspicion of hypoglycaemia that underwent the comprehensive diagnostic work-up protocol herein described.

A detailed food daily record coupled with ambulatory continuous glucose monitoring for 14 days using the commercially available FreeStyle Libre (Abbott, UK) device were used to document the occurrence and pattern of hypoglycaemic episodes. This approach allowed to confirm $(n=18)$ or exclude $(n=2)$ hypoglycaemia as the cause of the patients symptoms, thus preventing the need for further investigations. Fasting glucose, insulin, C-peptide, morning cortisol, IGF-1 and growth hormone levels were measured as a screening for endocrine causes of hypoglycaemia when documented by continuous glucose monitoring. These were found to be within normal limits in every patient with documented hypoglycaemia. A mix meal test was then performed and gut hormone levels, including GLP1 levels were measured to assess post-prandial hormone response; while a $72 \mathrm{~h}$ supervised fast was performed whenever a fasting hypoglycaemia pattern was found on the glucose monitoring.

Individualized dietician conselling for diet modification was provided to every patient presenting postprandial reactive hypoglycaemia with the aim of improving symptoms and managing hypoglycaemia. Four patients required additional pharmacological treatment that included an initial trial of acarbose followed by the use of somatostin analogue treatment for effective hypoglycaemia control.

Conclusion: With the increasing demand for obesity surgery, the number of patients presenting with post-bariatric hypoglycaemia is becoming more common than initially appreciated. Early recognition, evaluation and management of post-bariatric hypoglycaemic episodes are crucial, which can be eased by implementing a stepwise investigation protocol including a detailed food record coupled with ambulatory continuous glucose monitoring and a mix meal test.

\section{OS11:OC60 \\ Effects of RYGB on beta-cell function and glucose metabolism in response to a mixed meal test in diabetic and non-diabetic patients: A 7 years follow-up study}

\section{Astiarraga, B. ${ }^{1}$; Palumbo, M. ${ }^{1}$; Gaggini, M. ${ }^{2}$; Ciociaro, D. ${ }^{2}$; Mari, A. ${ }^{3}$;}

Anselmino, M. ${ }^{4}$; Ferrannini, E. ${ }^{1}$; Gastaldelli, A. ${ }^{2}$; Camastra, S. ${ }^{1}$

${ }^{1}$ Department of Clinical and Experimental Medicine, University of Pisa, Italy, ${ }^{2}$ Institute Of Clinical Physiology, Cnr, Pisa, ${ }^{3}$ Institute Of Neuroscience, Cnr, Padua, Italy, ${ }^{4}$ Bariatric Unit, Santa Chiara Hospital, Pisa, Italy

Introduction: Gastric bypass surgery determines changes in glucose metabolism (1) that lead to improvement or remission of Type 2 Diabetes (T2D). Long term studies indicate that T2D remission is greater at $2 \mathrm{y}$ than $10 \mathrm{y}(2)$, however, if the effect of surgery on beta cell function and glucose fluxes in response to a meal are maintained unaltered long term after surgery, has not been determined.

Methods: We studied, seven years after Roux-en Y Gastric Bypass (RYGB), 15 patients (8 T2D and 7 nondiabetic (ND) before surgery). In each subject was performed a double tracer mixed meal test (MTT) to evaluate post meal glucose flux and b-cell function (b-cell GS) with a mathematical model. The patients had been previously studied before surgery and 1 year later.

Results: ND and T2D lost $24 \%$ and $34 \%$ of their initial weight $1 \mathrm{y}$ after surgery and their weight was maintained at $7 y(-21 \%$ and $-28 \%$ respectively). T2D subjects resolved diabetes after RYGB and this was maintained after 7 years. The glucose curves were similar in ND and T2DM after surgery and similar at $1 y$ and $7 y$ after RYGB. In both groups postsurgery, the tracer-derived appearance of oral glucose showed a rapid increase followed by a sharp drop, a pattern that was mirrored by postprandial glucose levels and insulin secretion. The endogenous glucose production was less suppressed during the MMT. In T2D the improvement in insulin secretion rate and in the $b$-cell GS observed 1 year $(\mathrm{p}=0.001)$ after surgery was maintained 7 years after surgery $(34 \pm 6$ vs $67 \pm 13$ vs $85 \pm 21$ pmol . min- 1 . m-2 $\mathrm{mM}-1$, respectively before $v s$ y $v s 7 \mathrm{y})$ ) without differences compared to $\mathrm{ND}$, in the latter the slight worsening in the b-cell GS observed at 1 year 
$(\mathrm{p}=0.02)$ was maintained at 7 years $(140 \pm 19$ vs $89 \pm 9$ vs $80 \pm 11 \mathrm{pmol}$ min-1. m-2.mM-1; respectively before $v s$ y $v s 7 \mathrm{y}$ ).

Conclusion: We conclude that in both ND and T2D metabolic changes are maintained 7 years after RYGB surgery with similar postprandial dumping-like pattern and glucose tolerance.

\section{References:}

1 Camastra S. et al.: Diabetes. 2013;62:3709-3717.

2 Carlsson LM, et al.: N Engl J Med 2012;67:695-704.

Funding: Italian Ministry of Health, Grant RF-2011-02348446
EASO SYMPOSIA

\section{Thursday, 18 May 2017}

\section{AS2 - The MooDFOOD EU Project}

\section{AS2:1 \\ The Mindful Eating Behaviour Scale: Development psychometric properties and correlations with BMI}

L.H.H. Winkens ${ }^{1}$; T. van Strien ${ }^{1,2}$; J.R. Barrada ${ }^{3}$; I.A. Brouwer'; B.W.J.H. Penninx ${ }^{4}$; M. Visser ${ }^{1,5}$

${ }^{1}$ Department of Health Sciences, Faculty of Earth \& Life Sciences, Vrije Universiteit Amsterdam, Amsterdam Public Health research institute, The Netherlands, ${ }^{2}$ Radboud University Nijmegen, Behavioural Science Institute, Nijmegen, the Netherlands, ${ }^{3}$ Facultad de Ciencias Sociales y Humanas, Universidad de Zaragoza, Teruel, Spain, ${ }^{4}$ Department of Psychiatry, VU University Medical Center / GGZ ingeest, Amsterdam Public Health research institute, the Netherlands, ${ }^{5}$ Department of Internal Medicine, Nutrition and Dietetics, VU University Medical Center, Amsterdam, Amsterdam Public Health research institute, the Netherlands

Introduction: The increasing prevalence of overweight and obesity calls for effective strategies regarding weight loss and weight loss maintenance. Mindful eating is eating with awareness and attention and might be an effective approach for healthy weight regulation. To be able to measure mindful eating we developed and evaluated a new scale: the Mindful Eating Behaviour Scale (MEBS).

Methods: A selection of 20 items from existing instruments was used to design the MEBS. In a sample of 1279 adults aged 55 years and older from the Longitudinal Aging Study Amsterdam, the internal structure of this scale was evaluated using an exploratory structural equation modelling approach on half of the sample. Confirmatory factor analysis (CFA) was conducted on the whole sample to develop the final version of the scale. Cronbach's alpha and correlations with BMI were calculated.

Results: Two items were deleted because of low item loadings and one item because of high correlated uniqueness. The final CFA model with 17 items and four domains (Focused Eating, Hunger and Satiety Cues, Eating with Awareness, Eating without Distraction) showed good fit $(\mathrm{CFI}=0.97$, TLI $=0.96$, RMSEA $=0.04)$. Cronbach's alpha's were medium to high $(0.70$ to 0.89$)$. Higher BMI was correlated with lower Focused eating $(r=-0.10)$, lower Hunger and Satiety Cues $(r=-0.12)$ and lower Eating with Awareness $(r=-0.17)$, but not with Eating without Distraction $(r=0.03)$.

Conclusion: In this study, the Mindful Eating Behaviour Scale was successfully developed consisting of 17 items and four domains. This scale showed good internal consistency reliability and convergent validity in a sample of Dutch people of 55 years and older. Correlations of Focused eating, Hunger and Satiety Cues and Eating with Awareness with BMI indicate that these domains potentially may play a role in weight gain. Further research into associations between mindful eating and obesity is needed.

Conflict of Interest: None Disclosed.

Funding: Research relating to this abstract was funded by the European Union FP7 MooDFOOD Project 'Multi-country cOllaborative project on the rOle of Diet, FOod-related behaviour, and Obesity in the prevention of Depression' (grant agreement no. 613598) The Longitudinal Aging Study Amsterdam is supported by a grant from the Netherlands Ministry of Health Welfare and Sports, Directorate of Long-Term Care. The data collection in 2012-2013 was financially supported by the Netherlands Organization for Scientific Research (NWO) in the framework of the project "New Cohorts of young old in the 21 st century" (file number 480-10-014). 
AS2:2

\section{Which biopsychosocial variables contribute to more weight} gain in depressed persons?

Paans, ${ }^{1}{ }^{1}$

'VU University Medical Center, Amsterdam, Netherlands

Introduction: Depression appears to be associated with weight gain. Little is known about whether this association is independent of, or partly due to, several biopsychosocial variables. This study aims to investigate which biopsychosocial variables contribute to weight gain over a 4-year period in persons with major depressive disorder or high depressive symptoms. Methods: Data from 1658 adults who participated in the Netherlands Study of Depression and Anxiety were used. Baseline depression was measured with a DSM-IV based psychiatric interview and with a depressive symptom measure. Four year weight gain was classified as stable weight (within $5 \%$ gain or loss) versus weight gain ( $>5 \%$ gain), excluding those with $>5 \%$ weight loss. Twenty-one baseline psychological, lifestyle and biological variables and antidepressant use were considered as potential contributing variables.

Results: The mean BMI of the weight stable group $(\mathrm{N}=1075)$ was 25.5 $(\mathrm{SD}=4.7)$, and they gained on average $0.3 \mathrm{~kg}(\mathrm{SD}=2.1)$ over 4 -years The weight gain group $(\mathrm{N}=583)$ had a mean $\mathrm{BMI}$ of $24.8(\mathrm{SD}=5.0)$, and gained on average $7.9 \mathrm{~kg}(\mathrm{SD}=4.3)$. In sociodemographic adjusted models, both major depressive disorder and depressive symptoms were associated with subsequent weight gain significant (odds ratio (OR) 1.92, 95\% confidence interval (CI) $1.48-2.50$ and OR 1.26, 95\% CI 1.14-1.41 respectively). However, none of the biopsychosocial variables or use of antidepressants was associated with weight gain, thus did not contribute to the observed increased weight gain risk in depression, except for alcohol intake $(\mathrm{ab}=0.03$, (95\% CI 0.01-0.08).

Conclusion: Our results confirmed larger 4-year weight gain in depression, this was found to be independent of many different biopsychosocial variables and use of antidepressants. Future research should explore other potential factors that may be responsible for the increased risk for subsequent weight gain in depression, e.g. unhealthy dietary patterns or eating styles, or underlying intrinsic factors such as genetics.

Conflict of Interest: None Disclosed

Funding: Funding for this abstract was provided by the European Union FP7 MooDFOOD Project 'Multi-country cOllaborative project on the rOle of Diet, FOod-related behaviour, and Obesity in the prevention of Depression' (grant agreement no. 613598).

\section{AS2:3}

\section{Obesity and depressive symptoms in a multi-ethnic population}

\section{Gibson-Smith, D. ${ }^{1}$}

'VU University Medical Center, Amsterdam, Netherlands

Introduction: It is generally known that obesity and depression are positively associated. E Contributions for this could be due to psychosocial factors such as body satisfaction, weight discrimination, coping mechanisms, lifestyle and health status, all of which are dependent on social norms which differ between cultures. Hence this study aims to examine whether obesity and depressive symptoms are linked among six ethnic groups, and explores to what extent this association is independent of differences in lifestyle, health status.

Methods: Data on 21,030 men and women (18-70 years) was sourced from the HELIUS (Healthy life in an urban setting) study. The cross-sectional relationship between the level of obesity (by body mass index, BMI) and waist circumference and presence of depressive symptoms (by Patient Health Questionnaire-9) was analysed by logistic regression analyses, adjusted for socio-demographic variables, lifestyle factors, the number of chronic diseases. Consistency across ethnic groups (Dutch, South-Asian
Surinamese, African Surinamese, Ghanaian, Turkish and Moroccan) was examined by testing interaction terms.

Results: BMI and waist circumference were significantly associated with depressive symptoms (odds ratio $[\mathrm{OR}]=1.09,95 \%$ confidence interval [CI]:1.05-1.10, OR = 1.07, CI:1.02-1.11 per SD increase in BMI and waist circumference respectively after adjustment for socio-demographic variables, lifestyle and the number of chronic diseases. Interaction analyses revealed significant variation in this association between ethnic groups. Among Dutch and African Surinamese obesity (BMI>30) and the highest quartile of waist circumference were significantly associated with depressive symptoms in all models, whilst for South-Asian Surinamese, Ghanaian, Turkish and Moroccan obesity was not associated with depressive symptoms.

Conclusion: Obesity was associated with a higher risk of depressive symptoms, although this link was stronger in the Dutch than in other ethnic groups ( South-Asian Surinamese, Ghanaian, Moroccan,Turkish) confirming that cultural differences impacts on the link between obesity and depression.

\section{Conflict of Interest: None Disclosed}

Funding: The infrastructure for the Netherlands Study of Depression and Anxiety study (www.nesda.nl) is funded through the Geestkracht program of the Netherlands Organisation for Health Research and Development (Zon-Mw, grant number 10-000-1002) and is supported by participating universities and mental health care organizations: VU University Medical Center, GGZ inGeest, Arkin, Leiden University Medical Center, GGZ Rivierduinen, University Medical Center Groningen, Lentis, GGZ Friesland, GGZ Drenthe, Scientific Institute for Quality of Healthcare (IQ Healthcare), Netherlands Institute for Health Services Research (NIVEL), and Netherlands Institute of Mental Health and Addiction (Trimbos). DGS, MB, NP, MV, IB and BP were supported by the EU-MoodFood grant. Funding for this research is provided by EU FP7 MooDFOOD Project 'Multi-country cOllaborative project on the rOle of Diet, FOod-related behaviour, and Obesity in the prevention of Depression', Grant agreement no. 613598.

\section{AS2:4}

Bidirectional association between weight and waist circumference change with common mental disorder: Prospective findings from the Whitehall II study

Knüppel, A. ${ }^{\prime}$

${ }^{1}$ University College London, United Kingdom

Introduction: Few studies have investigated the bidirectional association of weight and waist circumference (wc) changes with common-mental disorder (CMD), while taking into account the potential U-shape of the association. Aim of this study was to examine both the association of common mental disorder with subsequent weight and wc changes and the association of weight and wc changes with incident CMD.

Methods: We analysed repeat measures from adults (18,422 person observations) enrolled in the Whitehall II cohort study, recruited at age 35-55 years and examined every 5 years over 25 years. CMD was measured using the 30-item General Health questionnaire (GHQ). Measured weight and wc data were used to calculate 5 year weight and wc changes, categorised into four groups (loss $<-3 \%$, stable $-3 \%$ to $3 \%$, moderate gain $>3 \%$ to $\leq 5 \%$ and high gain $>5 \%)$. We used random effects regression to model the association of weight and wc change with CMD over 5 years and with a 5 year lag-period.

Results: CMD was associated with increased odds of weight and wc gain (weight: OR 1.25, 95\%-CI 1.14, 1.37; wc: OR 1.22, 95\%-CI 1.09, 1.36) and reduced odds for weight loss (OR $0.89,95 \%$-CI $0.80,1.00$ ) compared to stable weight and wc in models adjusting for socio-demographic factors, health behaviours, baseline body weight measures and disease status (CVD, diabetes, cancer). However, CMD was not associated with weight or wc changes after a 5 year lag period.

Both weight and wc gain and loss were associated with increased odds for incident CMD adjusted for the same factors as listed above (weight loss: OR 1.19, 95\%-CI 1.01, 1.39; high gain: OR 1.20 95\%-CI 1.05, 1.39; wc loss: OR 1.28, 95\%-CI 1.01, 1.61; high gain: 1.29, 95\%-CI 1.08, 1.53). Weight 
gain and loss were also associated with incident CMD after a 5-year time lag (loss: OR 1.19, 95\%-CI 1.00, 1.42; high gain: OR 1.23, 95\%-CI 1.05 1.44).

In sensitivity analyses excluding participants with baseline anti-depressant intake, the association of weight loss and subsequent incident CMD attenuated marginally (OR 1.18, 95\%-CI 0.98, 1.41).

Conclusion: The findings suggest that 5 -year weight gain and weight loss might increase the odds of CMD 5 years later compared to stable weight change, while the association of CMD with subsequent weight and wc changes might not be causal.

Conflict of Interest: None Disclosed

Funding: Research relating to this abstract was funded by the Seventh Framework Programme of the European Commission (FP7-KKBE-2013-2-1-01) as part of the Multi-country collaborative project on the role of Diet, Food-related behaviour, and Obesity in the prevention of Depression (MooDFOOD)

\section{AS3 - Developing and Designing Evidence- Based Digital Tools for Weightloss Maintenance: The H2020 NoHoW Project}

\section{AS3:1 \\ Losing weight and keeping it off in Europe: Representative population weight control surveys in 3 countries}

E. Evans ${ }^{1}$, K. Sainsbury' ${ }^{1}$, M.M. Marques' ${ }^{1,2}$ S. Pederson ${ }^{3}$, L. Lähteenmäki ${ }^{3}$, P.J. Teixeira ${ }^{2}$, J. Stubbs ${ }^{4}$, B.L. Heitmann ${ }^{5}$, F.F. Sniehotta ${ }^{1}$

${ }^{1}$ Institute of Health \& Society, Faculty of Medical Sciences, Newcastle University, UK, ${ }^{2}$ CIPER-Self-Regulation, Faculty of Human Kinetics, University of Lisbon, Portugal; ${ }^{3}$ Aarhus University, Denmark, ${ }^{4}$ School of Psychology, Faculty of Medicine and Health University of Leeds, UK, ${ }^{5}$ Research Unit for Dietary Studies at The Parker Institute, Frederiksberg, Denmark;

Introduction: Most weight loss (WL) attempts take place in the 'real world', outside of controlled clinical or research contexts. Consequently, little is known about factors influencing the success of these efforts. We undertook the largest European survey to date of WL, WL maintenance and their correlates in overweight and obese adults.

Methods: Nationally-representative population samples from the UK, Portugal and Denmark ( $\mathrm{N}=2000 ; 1021 \mathrm{men})$ completed an online survey. Inclusion criteria were $\mathrm{BMI} \geq 25 \mathrm{~kg} / \mathrm{m}^{2}$ and $\geq 1$ completed WL at tempt. Respondents reported details of their most recent completed WL attempt, any subsequent regain, WL strategies used, psychological and demographic data. Using regression analyses, correlates of WL and maintenance were identified.

Results: Mean reported WL was 9\%. 1310 respondents (67\%) achieved clinically significant WL ( $\geq 5 \%)$. Respondents with greater WL used professional support, more self-regulatory strategies and higher initial BMI. Most respondents did not use evidence-based weight management strategies, and explicitly discontinued them once active WL efforts ended.

Overall, respondents regained weight to $2 \%$ below start weight. However 516 (26\%) respondents maintained $\geq 5 \%$ WL. Overall WL maintenance was predicted by larger \% weight loss, use of more WL maintenance strategies and higher initial BMI.

Conclusion: These findings highlight novel, ecologically-valid correlates of self-reported 'real world' WL and WL maintenance success in large, nationally-representative samples. The strategies and baseline predictors identified can potentially inform the development of interventions to support WL and WL maintenance in the overweight/obese European general population.

Conflict of Interest: None Disclosed

Funding: This project has received Funding from the European Union's Horizon 2020 research and innovation programme under grant agreement No 643309

\section{AS3:2}

A qualitative investigation of the role of emotion regulation in weight loss maintenance and its interaction with motivation and behavioural self-regulation

\section{K.Sainsbury', E. Evans 1, L. Lähteenmäki², P.J. Teixeira ${ }^{3}$, F. Sniehotta}

Institute of Health \& Society, Faculty of Medical Sciences, Newcastle University, UK ${ }^{2}$ Aarhus University, Denmark; ${ }^{3}$ CIPER-Self-Regulation, Faculty of Human Kinetics, University of Lisbon, Portugal;

Introduction: The roles of motivation and self-regulation in weight loss and maintenance (WLM) are reasonably well understood. In contrast, little is known about the role that emotions and emotion regulation may play in WLM. The aim of this study was to summarise the ways in which emotions and emotion regulation influence WLM efforts, and to characterise both the between- and within-participant variability in how emotional experiences interact with, and potentially undermine, otherwise good motivation and self-regulatory capacity.

Methods: Qualitative interviews were conducted with 44 previously obese individuals (UK: $\mathrm{N}=25$; Denmark: $\mathrm{N}=19$ ) who had successfully lost $>5 \%$ of their body weight following a deliberate weight loss attempt, and were currently attempting maintenance of that loss. Topic guides were developed to elicit information about emotion regulation and its links with self-regulation and motivation. Data was analysed in NVivo.

Results: Responses could be categorised as indicating a predominantly emotional or reasonable style for managing the impact of emotions on eating and WLM. There was also evidence of considerable within-participant variation in coping responses over time and across contexts. Emotional and reasonable participants differed in the intensity of emotional experiences and the likelihood that their emotions and regulation would lead to regain via lapses in motivation and active self-regulation.

Conclusion: Emotion regulation is an important consideration when understanding the process of WLM for many individuals. Motivation and self-regulation can be undermined by strong emotions and an emotional coping style, and this should therefore be considered when designing interventions and support to prevent weight regain.

Conflict of Interest: None Disclosed

Funding: This project has received Funding from the European Union's Horizon 2020 research and innovation programme under grant agreement No 643309

\section{AS3:3}

Evidence-based behaviour change in a weight loss maintenance e-Health program: The H2020 NoHoW intervention toolkit

A.L. Palmeira', M.M. Marques ${ }^{1,2}$, J. Encantado', S. Carvalho3, C. Duarte ${ }^{3}$, M. Ermes $^{4}$, E. Evans ${ }^{2}$, M. Harjumaa ${ }^{4}$, B.L. Heitmann ${ }^{5}$, V. Huotari ${ }^{4}$, T. Kinnunen ${ }^{4}$, M. Matos $^{3}$, L. Palmeira ${ }^{3}$, S. Scott ${ }^{6}$, F.F. Sniehotta ${ }^{2},{ }^{4}$ R.J. Stubbs ${ }^{6}$, P.J. Teixeira ${ }^{1}$

${ }^{1}$ CIPER-Self-Regulation, Faculty of Human Kinetics, University of Lisbon, Portugal; 2Institute of Health \& Society, Faculty of Medical Sciences, Newcastle University, UK: ${ }^{3}$ Cognitive and Behavioural Centre for Research and Intervention, University of Coimbra, Portugal; ${ }^{4} \mathrm{VTT}$ Technical Research Centre of Finland Ltd, Tampere, Finland ${ }^{5}$ Research Unit for Dietary Studies at The Parker Institute, Frederiksberg, Denmark; ${ }^{6}$ School of Psychology, Faculty of Medicine and Health University of Leeds, UK;

Introduction: Weight loss maintenance (WLM) encompasses the development of the individual „weight self-regulation know-how" and sustained behaviour change (BC). Evidence shows that some BC theories (e.g. self-determination theory) and techniques (e.g., mindfulness) can be effective in WLM, albeit resource demanding. E-Health has the potential to lessen the demand for resources, but they seldom consider $\mathrm{BC}$ theories and techniques. This presentation will focus on the process of development of the NoHoW Toolkit (TK) focusing on its content and results from the pilot study.

Methods/Results: The NoHoW project (www.nohow.eu) is a European Union $\mathrm{H} 2020$-funded project that is carrying out a large-scale international randomised controlled trial to test whether a Toolkit (TK) for (1) motivation and self-regulation, (2) emotion regulation or (3) both factors in combination, will improve WLM, compared to (4) only regular self-monitoring. The TK is 
a web-based interface of up-to-date data collected with the participants' activity tracker and smart scale. The BC content is presented in a map with 19 to 24 short sessions (5-8 min). Informed by the guidelines for the development of complex interventions, we used a systematic approach to the translation of theory and evidence to e-Health intervention components, by (1) developing theory-driven logic models and identifying key theoretical constructs targeted, (2) selecting intervention techniques expected to impact on target constructs, and (3) translating these techniques into the TK principles and technical specifications.

Conclusion: A systematic approach to state-of-the art evidence while designing and developing e-Health tools may provide sustainable solutions to WLM across the Europe.

\section{Conflict of Interest: None}

Funding: The project has received Funding from the European Union's Horizon 2020 research and innovation programme under grant agreement No 643309

AS3:4

The development of the NoHoW trial for weight loss maintenance: Design, analyses, challenges and solutions.

S. Scott' ${ }^{1}$ J. Encantado ${ }^{2}$, M. Ermes ${ }^{3}$, M. Harjumaa ${ }^{3}$, B.L. Heitmann ${ }^{4}$, G.W. Horgan $^{5}$, V. Huotari ${ }^{3}$, T. Kinnunen ${ }^{3}$, J. Leppänen ${ }^{3}$, S. C. Larsen ${ }^{4}$, M.M. Marques $^{2,6}$, M. Matos ${ }^{7}$, M. L. Mikkelsen ${ }^{4}$, D. Nutter ${ }^{8}$, A. L. Palmeira ${ }^{2}$, I. Santos $^{2}$, C. Stalker ${ }^{9}$, F.F. Sniehotta ${ }^{6}$, J. Stubbs ${ }^{1}$, P.J. Teixeira².

1 School of Psychology, Faculty of Medicine and Health University of Leeds, UK; ${ }^{2}$ CIPER-Self-Regulation, Faculty of Human Kinetics, University of Lisbon, Portugal; ${ }^{3}$ VTT Technical Research Centre of Finland Ltd, Tampere, Finland; ${ }^{4}$ Research Unit for Dietary Studies at The Parker Institute, Frederiksberg Denmark; ${ }^{5}$ Biomathematics and Statistics Scotland, Rowett Institute of Nutrition and Health, University of Aberdeen, UK; ${ }^{6}$ Institute of Health \& Society, Faculty of Medical Sciences, Newcastle University, UK; 7 Cognitive and Behavioural Centre for Research and Intervention, University of Coimbra, Portugal, 8 Biomathematics and Statistics Scotland, JCMB, UK; 9 University of Derby, UK;

Introduction: The NoHoW trial is a 3 -centre $2 \times 2$ randomised controlled trial testing behaviour change approaches for self-regulation and motivation versus emotion regulation/stress management for Weight Loss Maintenance (WLM). These approaches are delivered through a digital architecture including real-time tracking technologies.

Methods: This presentation describes: (1) the trial design, its relationship to the toolkit structure and hypotheses; (2) digital architecture development for intervention delivery and data collection; (3) planned primary and secondary outcome analyses; (4) challenges and solutions encountered during trial development.

Results: An overview is provided of:

(1) Trial design, logic models, primary outcomes (weight \& health biomarkers), secondary outcomes (e.g., psychological mediators of WLM, dietary and physical activity behaviours), and process evaluation;

(2) The ICT architecture for recruitment, data collection and analysis including the toolkit, trial management software, online questionnaires (Qualtrics), dietary recalls (INTAKE24), wireless tracking technologies (Fitbit Charge2 \& Aria scales);

(3) Planned analyses for primary (weight, body composition, cortisol, full lipids \& HbAlc) and secondary outcomes: diet, physical activity, sleep, moderators and mediators of behaviour change, predictors of relapse/maintenance, process evaluation and cost-effectiveness;

(4) Challenges and solutions generated in development of a large-scale digital behaviour change trial including data source synchronisation, cross-cultural data collection, harmonisation, and recruitment of a hard-to-reach population.

Conclusion: Recommendations and practical implications for large scale trials will be shared. The next steps for the NoHoW trial will be discussed.

Conflict of Interest: None Disclosed

Funding: This project has received Funding from the European Union's Horizon 2020 research and innovation programme under grant agreement No 643309

\section{AS4 - Young Adult Obesity: The Transition from Adolescence to Emerging Adulthood}

\section{AS4:1 \\ Young adult obesity: The transition from adolescence to emerging adulthood}

\author{
K. Steinbeck; A. Poobalan; L. Aucott; S. Thomee; C. Nikolaou; A. Hill; \\ L. Baur; T. Gill
}

Introduction: Young people in transition from adolescence to adulthood are vulnerable for developing unhealthy lifestyle behaviours and consequently obesity. Many undergo significant life course changes such as leaving home, going to university/college, starting work etc, which all impact on behaviours relevant to energy balance. This group risks the same obesity-related physical complications as older adults and is affected by psychological distress. None-the-less, this age group is neglected in terms of research and policy, and are hard to engage. Although this crucial period of transition presents several challenges for traditional approaches to interventions, it should be viewed as an opportunity to explore more insightful solutions.

Methods: This symposium will focus on current issues of obesity research in young adults, based on the critical mass of emerging data. The format will be interactive with short presentations from experts followed by a facilitated panel discussion:

Prof Kate Steinbeck, University of Sydney, Australia: Why should we focus on weight issues in young adults?

Dr Amudha Poobalan/Dr Lorna Aucott, University of Aberdeen, UK: Young adults' lifestyle factors and risky behaviours and their interactions with obesity

Dr Sara Thomee, University of Gothenburg, Sweden: Modern day technology \& weight gain in young adults

Dr Charoula Nikolaou, Universite Catholique de Louvain, Belgium: Obesity, emerging adulthood \& e-learning

Prof Andrew Hill, University of Leeds, UK: Psychosocial issues in young adults with obesity

Prof Louise Baur, University of Sydney, Australia: Clinical management of weight issues in young adults

Facilitated by Prof Tim Gill, University of Sydney, Australia PANEL DISCUSSION: 'Engaging young adults on weight and health'

Conflict of Interest: None Disclosed

Funding: No Funding 


\section{AS5 - Epicardial and Perivascular Adipose Tissue Dysfunction in Obesity}

\author{
AS5:1 \\ Transcriptional alterations in epicardial adipose tissue during \\ early life
}

\section{Michael E Symonds}

The Early Life Research Unit, Academic Division of Child Health, School of Medicine, University of Nottingham, Nottingham, NG7 2UH

Introduction: The anatomical and final functional architecture of each adipose tissue within the body is mainly determined by the pronounced changes in transcriptional processes during early postnatal life. To date this has not been examined using a bioinformatics approach that would be predicted to provide novel insights on fat development. Presented here are the transcriptional alterations in the epicardial adipose tissue that occur during early life.

Methods: To gain insight into the developmental origins of the molecular changes within fat in early life we conducted a multi-region transcriptome analysis from five major (epicardial, subcutaneous, perirenal, sternal and omental) adipose depots sampled at 7 and 28 days of age from young sheep. Furthermore, we analysed the epicardial adipose transcriptome of neonates, infants and children undergoing surgery for congenital heart disease.

Results: By weighted gene co-expression network analysis, we found that each developmental stage and fat depot can be segregated into a concise group with a small number of unique functional modules of co-expressed genes. Each module exhibited temporal and spatial specificity and were associated with distinct biological processes. This indicates a consistent and a sequential order of transcriptional changes in each depot with age. These are indicative of different cellular origins, metabolism, environmental influence and functionality. In particular, we uncovered specific anatomical regional clusters of gene markers which were associated with distinctive cellular progenitor of brown adipocytes that were validated by cross-age modular preservation. In humans our results also indicated that the pattern of gene expression represents a distinct developmental stage, even after the rebound in abundance of thermogenic genes in later childhood. Using weighted gene coexpression network analyses, we found precise anthropometric-specific correlations with changes in gene expression and the decline of thermogenic capacity within EAT.

Conclusion: Our results demonstrate that each adipose tissue depot follows an independent path of postnatal development, which could be influenced by cell progenitor type as well as the immediate endocrine environment. Finally, we demonstrate that weighted gene co-expression network analysis provides a valuable resource to dissect gene regulatory mechanisms. Together, these results provide a resource to elucidate gene regulatory mechanisms underlying the progressive development of BAT during early life.

Conflict of Interest: None Disclosed

Funding:

\section{Transcriptomic analysis reveals a strong down regulation of immune response pathways in ferret aortic perivascular adipose tissue by cold exposure: Potential implications for cardiovascular health}

Bàrbara Reynés'; Evert M. van Schothorst ${ }^{2}$; Estefanía García-Ruiz'; Jaap Keijer'; Paula Oliver'; Andreu Palou'; Andreu Palou Oliver

${ }^{1}$ Laboratory of Molecular Biology, Nutrition and Biotechnology, Universitat de les Illes Balears and CIBER de Fisiopatología de la Obesidad y Nutrición (CIBERobn), Palma de Mallorca, Spain; ${ }^{2}$ Human and Animal Physiology, Wageningen University, Wageningen, the Netherlands

Introduction: The role of perivascular adipose tissue (PVAT) in cardiovascular disease (CVD) is under active investigation, given its ability to regulate vascular inflammation, positively and negatively, which, in turn, modulates vascular health. A pro-inflammatory state of PVAT has been related with atherosclerosis development, while an anti-inflammatory state has been shown to be protective. Cold exposure is known to affect immune response and, thus, could affect the pathogenesis of cardiovascular diseases. Here we used a promising animal model (ferrets, Mustela putorius furo) aiming to explore the effect of cold exposure on PVAT transcriptomic profile to gain insight into potential CVD treatment strategies. Methods: We designed a ferret-specific Agilent microarray and performed a global gene expression analysis in aortic PVAT (aPVAT) of cold-exposed ferrets (one week at $4^{\circ} \mathrm{C}$ ) in comparison to controls. Ferrets were selected due to their similarity to humans in terms of immune response and adipose tissue organization and metabolism making them a well-suitable translational animal model.

Results: Our transcriptome analysis using a ferret-specific whole genome microarray revealed a clear and strong down-regulation of immune response-related genes induced by cold exposure in aPVAT. Affected genes, including the top down-regulated, were related to processes such as antigen recognition and presentation, cytokine signalling and immune system maturation and activation. This immunosuppressive effect was depot-specific, as it was not observed in the inguinal subcutaneous depot. Interestingly, the immunosuppressive gene expression pattern observed in aPVAT was also evident in peripheral blood mononuclear cells (PBMC).

Conclusion: These results reveal that cold exposure produces an inhibition of immune response-related pathways in aPVAT, reflected in PBMC, indicative of an anti-inflammatory response, which could contribute to a protective cardiovascular profile. Moreover, PBMC could be used to examine the potential of cold exposure as cardioprotective anti-inflammatory strategy in translational human studies.

None Disclosed

CIBER de Fisiopatología de la Obesidad y Nutrición is an initiative of the ISCIII. This work was supported by the Spanish Government (Ministerio de Economía y Competitividad, INTERBIOBES -AGL2015-67019-P), by the EU FP7 project DIABAT (HEALTH-F2-2011-278373) and by the University of the Balearic Islands (BIOTHERM, FA42/2016). E. García-Ruiz was recipient of a fellowship from the University of the Balearic Islands.

\section{AS5:3}

\section{The molecular phenotype of epicardial adipose tissue}

Eugenia Carvalho

Center for Neuroscience and Cell Biology, University of Coimbra, E-3000 Coimbra, Portugal

Introduction: The age-adjusted mortality from cardiovascular disease (CVD) has decreased an astonishing $75 \%$ over the past 40 years [1]. However, due to the dramatic increase in obesity and diabetes, the prevalence of related complications is rising, including heart failure [2]. Epicardial adipose tissue (EAT) has recently been identified as an important fat depot around the heart and is implicated in cardiac function and its morphology [3]. EAT has also been considered a potential risk factor for CVD development [4]. Not much is known regarding the metabolic phenotype 
of this cardiac fat depot. Therefore, we sought to evaluate glucose and lipid metabolism in EAT explants from heart failure patients, with and without diabetes.

Methods: Insulin-stimulated 14C-glucose uptake and isoproterenol-stimulated lipolysis were carried out on freshly isolated adipocytes from heart failure patients with and without diabetes. In addition, gene and protein expression were evaluated by real-time PCR and western blot techniques respectively.

Results: Not only was insulin-stimulated 14C-glucose uptake impaired in freshly isolated EAT cells but lipolysis was greatly decreased in EAT compared to SAT of the same patients. Moreover, excessive or insufficient levels of autophagy may contribute to heart disease and CVDs can alter endoplasmic reticulum (ER) function. However, no studies that have evaluated autophagy and ER stress in EAT from these patients. Furthermore, EAT has been described as brown-like fat, with increased expression of UCP1. However, no studies that have evaluated mitochondrial respiration in EAT compared to SAT biopsies in this population. Our hypothesis is that dysregulated autophagy and ER stress in EAT play an important role in heart failure and that mitochondrial respiration is significantly different in EAT compared to SAT biopsies due to its phenotypic metabolic differences. Our preliminary results indicate an increase in UPR markers, such as GRP78 and GRP94, as well as in autophagy markers, such as Beclin1 and LC3II/LC3I ratio. Furthermore, by high resolution respirometry (Oroboros), we observed that EAT is bioenergetically more active and more sensitive to mitochondrial substrate supply and has higher relative oxidative capacity than SAT in heart failure patients.

Conclusion: Our recent studies identified significant metabolic differences between EAT and subcutaneous adipose tissue (SAT) from the same patients and highlights EAT as a possible therapeutic target in situ in the cardiac microenvironment [5]. These findings highlight important stress responses and thermoregulation diversity between SAT and EAT in heart failure patients, bringing to light that EAT has a unique signature, important in heart physiology.

Conflict of Interest: None Disclosed

Funding: Research relating to this abstract was funded by the Portuguese Diabetes Foundation, PTDC/SAU-OSM/104124/2008 and the EXCL/DTP-PIC/0069/2012,

\section{References:}

1 Nabel and Braunwald, 2012 (PMID: 22216842)

2 Wang and Hill, 2015 (PMID: 25651176)

3 lacobellis et al., 2014 (PMID: 24557503)

4 Salazar et al., 2016 (PMID: 27213076)

5 Burgeiro et al., 2016 (PMID: 26814014)

\section{AS5:4}

\section{Impact of regional differences in PVAT on vascular health and disease}

\section{Marta Gil-Ortega}

Departamento de Ciencias Farmacéuticas y de la Salud, Facultad de Farmacia, Universidad CEU-San Pablo, 28668 Madrid, Spain

Perivascular adipose tissue (PVAT) is a local adipose tissue surrounding blood vessels, which is phenotypically different from other fat depots. It releases a number of important vasoactive factors with physiological and pathophysiological paracrine effects. Under physiological situations, PVAT releases a number of vasoactive substances that elicit a net beneficial anti-contractile effect on vascular function and are essential for the maintenance of vascular homeostasis. The amount and phenotype of PVAT can vary under pathophysiological conditions. Pathophysiological situations, such as obesity, diabetes or hypertension induce a dysfunction of PVAT due to changes in its amount and in the expression pattern of vasoactive factors. As a consequence, in established obesity PVAT loses its anti-contractile properties due to an increase of contractile, pro-oxidant and pro-inflammatory factors thus leading to endothelial dysfunction and vascular disease. However, a moderate enlargement of PVAT is associated with an increase of vasodilatory adipokines, thus beneficially impacting vascular homeostasis. Moreover, a large body of evidence suggests regional phenotypic and functional differences among PVAT depots, depending on the specific vascular bed. These non-uniform and separate PVATs exert different paracrine effects on vascular structure and function that largely impact disease states, such as endothelial dysfunction, atherosclerosis or insulin resistance associated to obesity. This emerging view of PVAT function requires considering heterogeneous PVATs as a specialized organ that can differentially regulate vascular function depending on its anatomical location. In this context, the adipose-vascular axis may represent a novel target for pharmacological intervention of vasculopathy in cardiometabolic disorder.

Conflict of Interest: None Disclosed

Funding: Research relating to this abstract was funded by Fundación Eugenio Rodríguez-Pascual and GESCAMET.

\section{AS5:5 \\ Modulation of cardiac visceral adipose tissue by obesity: Effects in cardiac structure}

\section{G. Conceição' J. Matos'2 N. Gonçalves'; D. Fontoura'; AP. Lourenço'; R. Vitorino ${ }^{2}$; AF. Leite-Moreira'; I. Falcão-Pires' ${ }^{1}$ G. Conceição}

${ }^{1}$ Departamento de Cirurgia e Fisiologia, Faculdade de Medicina, Universidade do Porto, Portugal; '² Department of Medical Sciences, iBiMED, University of Aveiro, Portugal

Introduction: Heart failure with preserved ejection fraction (HFpEF) is recognized as an important cause of cardiovascular mortality and morbidity reaching approximately $50 \%$ of heart failure cases. Several risk factors, such as obesity, are associated with its development. Obesity represents a condition where excessive energy intake results in the expansion of adipose tissue, contributing to a systemic pro-inflammatory state. The heart itself is surrounded by cardiac visceral adipose tissue (CVAT), which represents an additional risk factor to pathological alterations in the cardiovascular system. The adipokines produced by CVAT can act in a paracrine manner directly on the myocardium and can influence their structure and function. So, we aim to characterize the profile of CVAT in an obese animal model of HFpEF and its cardiac impact.

Methods: CVAT from 20-weeks-old lean (control group) and obese ZSF1 rats (HFpEF - obese group) was collected for: 1) proteins identification using mass spectrometry; 2) characterization of adipokines' expression; 3 ) assessment of adipocytes cross-sectional area and 4) collection of 24h-DMEM CVAT-derived conditioned medium for: incubation of myocardial organo-cultures prepared from 7-days-old Wistar Kyoto cardiac explants and characterization of the profile of secreted adipokines. After incubation with conditioned media from lean or obese rats, cross-section area of cardiomyocytes and fibrosis were evaluated.

Results: CVAT from obese ZSF1 rats presented a decrease in mitochondrial metabolism and an increasein processes associated with extracellular matrix expansion and triglyceride metabolism. The histological studies of CVAT revealed hypertrophy of adipocytes in obese ZSF1 rats, as well as a unilocular appearance compared with a multilocular appearance of CVAT in lean ZSF1 rats. Molecular studies of CVAT from obese ZSF1 rats presented a significantly increase in expression of several adipokines involved in fibrotic and hypertrophic pathways, namely in leptin, apelin, chemerin or visfatin. Moreover, CVAT from obese ZSF1 rats showed an increase expression of PPAR $\gamma$ supporting adipogenesis of adipose tissue. Conditioned media from CVAT obese rats exhibited an inflammatory profile and, in organo-cultures, induced cardiomyocytes hypertrophy and fibrosis compared to the conditioned media from lean rats.

Conclusion: Obesity promotes alterations in CVAT structure, composition, metabolism and secretion profile, which significantly affects myocardial structure, inducing collagen deposition and cardiomyocyte hypertrophy. 
AS5:6

\section{An Adrenergic System in Perivascular Adipose Tissue}

\section{Nadia Ayala-Lopez, Gregory D Fink and Stephanie W. Watts}

Dept of Pharmacology and Toxicology, Michigan State University, East Lansing MI USA 48824-1317

Introduction: Perivascular adipose tissue (PVAT, the fat immediately adjacent to blood vessels) is a fat depot beginning to be appreciated for the contributions it makes to vascular function and human health. PVAT is normally viewed as being anticontractile and vasculoprotective. PVAT in the splanchnic vessels is the visceral fat that is particularly important because of its association with the greatest risk of cardiovascular disease. We have made the novel discovery that PVAT contains a significant concentration of norepinephrine (NE) and test the hypothesis that mesenteric PVAT/visceral fat contains a functional, active adrenergic system, defined as the ability to synthesize, release, take up and metabolize catecholamines. Because vascular function is largely governed by adrenergic receptor stimulation, this work is significant in elevating a system that could directly regulate vessel function.

Methods: HPLC, isometric contraction assays, Westerns, immunohistochemistry and other techniques were used to test this hypothesis using multiple PVATs but primarily that in the mesenteric bed.

Results: NE was present in isolated PVATs (aortic, superior mesenteric, mesenteric resistance vessel). The indirect sympathomimetic tyramine caused a PVAT-dependent, a adrenergic receptor-dependent contraction in multiple isolated vessels; contraction and PVAT NE content was minimally dependent on sympathetic innervation. These findings suggest NE can be released. Multiple transporters for NE were tested for their ability to uptake NE. Using a combination of Westerns, RT-PCR and transporter dye uptake, the organic cation transporter-3 (OCT-3) rose to the top as the primary PVAT transporter for NE uptake. Last, the enzyme semicarbazide sensitive amine oxidase (SSAO) possessed the greatest amine oxidase activity in PVATs; the question remains whether NE is metabolized by SSAO directly.

Conclusion: An adrenergic system that at least encompasses release, uptake and metabolism of NE exists in PVAT. This system is important because of the recognized contributions NE makes in determining vessel tone but also adipocyte lipolysis.

Conflict of Interest: none

Funding: NIH P01 HL70687

\section{AS6 - Functional Body Composition, Obesity \& Cardiometabolic Health: Beyond the Adipocentric View}

\section{AS6:1 \\ Functional body composition: Insights into energy balance regulation.}

Müller M.

Department of Human Nutrition, Christian-Albrechts-Universität zu Kiel, Germany

Functional body composition integrates body components into regulatory systems, that is, it relates body composition to its corresponding in vivo functions and metabolic processes. The concept relates to interpretation of body functions (e.g. energy expenditure, EE) and their disturbances in the context of body components and vice versa (ii) interpretation of the meaning of individual body components in the context of their functional consequences (e.g. EE). Functional body composition extends the traditional view of static compartment models (e.g. a 2 compartment model on fat mass and fat free mass, FFM), crossing as well as combining established body composition levels (i.e. the anatomic, the cellular and the molecular level). Current research on EE relates to metabolic adaptation to negative energy balance, mitochondrial metabolism associated with aging, obesity and type 2 diabetes mellitus, the role of EE in hunger and appetite control, non-shivering thermogenesis and brown adipose tissue activity, cellular bioenergetics as a target of obesity treatment and the evolutionary and ecological determinants of EE in humans and other primates. As far as regulation of energy balance is concerned there is recent evidence that $\mathrm{EE}$ (rather than body weight or energy intake, EI) is under tight control. Biologically, EE is maintained within a narrow physiological range. An EE-set point has been proposed as the width between the upper and lower boundaries of the individual EE range. Regulation of EE may fail in very obese patients with an EI above their upper boundary and after drastic weight loss when obese patients go far below their lower EE boundary and thus are loosing control. Adaptive thermogenesis (AT) refers to changes in resting and non-resting energy expenditure (REE and nREE) with weight changes which are independent from changes in FFM and FFM composition. With negative energy balance, AT is directed towards energy sparing. It relates to a reset of biological defense of body weight and mainly refers to REE. After weight loss, AT of nREE adds to weight maintenance. During overfeeding, energy dissipation is explained by AT of the nREE component only. As to body weight regulation during weight loss, AT relates to two different set points with a settling between them. During early weight loss, the first set is related to depleted glycogen stores associated with the fall in insulin secretion where AT adds to meet brain's energy needs. During maintenance of reduced weight, the second set is related to low leptin levels keeping EE low to prevent triglyceride stores getting too low which is a risk for some basic biological functions (e.g., reproduction). Innovative topics of AT in humans are on its definition and assessment, its dynamics related to weight loss and its constitutional and neuro-endocrine determinants.

Conflict of Interest: The author is a consultant of Seca company, Hamburg, Germany.

Funding: Research relating to this abstract was funded by a grant of the German Ministry of Education and Research (BMBF 0315681), BMBF Competence Network Obesity (CNO) and the German Research Foundation (DFG Bo 3296/1-1 and DFG Mü 714/ 8-3).

AS6:2

\section{Collateral fattening: When a deficit in lean body mass drives overeating.}

Dulloo AG.

Department of Medicine, Division of Physiology, University of Fribourg

In his last review entitled 'Some adventures in body composition', Gilbert Forbes reminded us that 'lean body mass and body fat are in a sense companions. To what extent the lean body mass or fat-free mass (FFM) component in this companionship impacts on energy intake is rarely a topic for discussion, amid a dominant adipocentric view of the long-term control of food intake. Yet, an analysis of the few human studies that have investigated the relationships between objectively-measured food intake and body composition reveals a potentially important role for both an increase and a decrease in FFM in the drive to eat. However, it is important to distinguish between (i) a relatively 'passive' role of FFM on energy intake which is mediated by 'energy-sensing' mechanisms that translate FFM-induced energy needs to energy intake, and (ii) a more 'active' role of FFM in the drive to eat through feedback signals between FFM deficit and energy intake. Thus, a loss or deficit of FFM should be viewed as a risk factor for increased fatness not only because of the impact of the FFM deficit in lowering the maintenance energy requirement, but also because of the body's attempt to restore FFM by overeating - a phenomenon referred to as 'collateral fattening. These findings have implications for research directed as much towards the elucidation of peripheral signals and energy-sensing mechanisms that drives hunger and appetite, as towards understanding the mechanisms by which dieting and sedentariness predispose to fatness. 
Conflict of Interest: There is no conflict of interest to disclose.

Funding: This research work was supported by the Swiss National Science Foundation (grant no. 310030-152870).

\section{AS6:3 \\ Body composition and cardiometabolic health: The need for novel concepts}

\section{Bosy-Westphal A.}

Institute of Nutritional Medicine, University of Hohenheim, Stuttgart, Germany

Due to experts consensus overweight and obesity should be defined based on body composition rather than BMI. The use of conventional parameters like visceral fat or percentage fat mass is however of similar and limited value for disease risk prediction at the population level and does not extend much beyond the use of simple BMI. This conundrum is partly explained by an inappropriate definition of a normal body composition as well as the limited understanding of dynamic changes between body compartments with weight gain.

Innovative body composition concepts integrate lean and fat compartments in order to derive indices that are both predictive and causative for cardiometabolic risk. Novel concepts that reveal the positive association of metabolic load and the inverse association of metabolic capacity with cardiometabolic risk include (i) a proper normalization of body composition parameters for body height as well as (ii) a combination of body composition parameters using (a) nonlinear relationships, (b) mathematical modeling and (c) categorical classifications based on a decision tree. For example, the relationship between fat and lean mass is nonlinear and the definition of a normal lean mass depends on the corresponding fat mass, sex, age and body region. In addition, adipocyte-myocyte crosstalk via adipokines adds to explain the relationship between body composition and cardiometabolic risk.

Application of these novel concepts will not only improve the diagnosis of overweight and obesity but will also lead to a better understanding of the pathophysiology of obesity associated cardiometabolic risk.

Conflict of Interest: None Disclosed/Payment received from ......

Funding: No Funding/Research relating to this abstract was funded by ......

\section{AS6:4}

When and to what extent do fat mass and fat-free mass drive energy intake?

\section{Stubbs RJ.}

School of Psychology, Faculty of Medicine and Health, University of Leeds

For the last two decades models of appetite and energy balance control have focused heavily on acute gastrointestinal signals modulated by tonic signaling from adipose tissue. More recent models have attempted to integrate the role of body fat mass (FM) and fat free mass (FFM) in appetite control and the relationships of these functional body composition compartments to appetite control (1).

There is cross-sectional evidence that at or close to energy balance, FFM is positively related to energy intake (EI) and FM either shows a weak negative or no association with EI in subjects feeding ad libitum. Further analysis suggests that the effects of FFM and FM on EI are fully mediated by resting metabolic rate (RMR) (2). Analyses of free-living subjects where activity patterns are more variable suggests activity energy expenditure also correlates with EI. These cross-sectional studies suggest energy turnover drives EI and the largest determinant of energy requirements in most humans is FFM. During chronic positive energy balances both FM and FFM expand, increasing energy demands. There is little evidence that an expanding FM exerts strong negative feedback on longer term EI.

However, during chronic negative energy balances FM, FFM and RMR all decrease and energy turnover decreases but appetite increases. Some studies suggest that proportionate loss of FFM during weight loss predicts subsequent weight regain.

Taken together these lines of evidence suggest that changes in the size and functional integrity of FFM may influence appetite and energy intake. Increases in FFM associated with either weight gain or high levels of exercise may "pull" EI upwards but energy deficits that decrease FFM may exert a stronger pull on appetite.

The ratio of FFM:FM lost during energy deficits can be influenced by (i) rate and extent of weight loss, (ii) initial body composition prior to weight loss, (iii) protein:energy ratio of the diet consumed at a given level of energy balance and (iv) degree of activity/inactivity (3).

Thus, changes in body structure that influence physiological and psychological function may affect energy balance behaviours. These relationships should be tested in prospective studies that examine the relationship between physiology and behaviour during chronic periods of altered energy balance. Such studies may have relevance for weight loss and maintenance strategies and the nutritional management of malnutrition.

\section{References:}

1 Blundell, J., et al. Obes. Rev: 16 S1, 67-76

2 Hopkins et al. IJO 2016; 40, 312-318

3 Heymsfield at al. Obes Rev. 2014; 15: 310-321

Conflict of Interest: R James Stubbs is a consultant to Slimming World through University of Leeds Consulting (Trading as Consulting Leeds).

Funding: No Funding 


\section{REVIEW / WORKSHOP SESSIONS}

Wednesday, 17 May 2017

\section{RS1 - Size matters: How can Big Data Help Obesity}

RS1:1

How to make the most out of big data: Best practice examples

Ilja C.W. Arts

Maastricht Centre for Systems Biology (MaCSBio) and Dept. of Epidemiology, CARIM School for Cardiovascular Diseases, Maastricht University, Maastricht, The Netherlands

In the past 15 years, technological advances have led to an enormous increase in the availability of data. Starting with the initial publication of the human genome in 2001 and followed by similar advances in transcriptomics, proteomics, metabolomics and metagenomics, we now have access to near-complete biological datasets for large groups of people. In parallel we are witnessing a 'quantified-self' revolution, which allows individuals to monitor their health on a daily basis using wearables.

These developments have great promise to radically increase our understanding of obesity, to improve and personalize disease risk prediction in individuals with obesity, and to provide novel targets for the prevention and treatment of obesity and its comorbidities. It is becoming increasingly clear that there is substantial heterogeneity in the multitude of short and long term effects that obesity has on well-being and health. In this talk I will present a number of examples of the successful use of big data to achieve these goals.

Before we can make the most of big data, however, a number of challenges have to be addressed. While omics data are being generated in an unprecedented manner, it remains extremely difficult and time-consuming to carefully (clinically) phenotype individuals with sufficient detail Once generated, data should become available for re-use, and with the adoption by the European Commission of the FAIR Guiding Principles for scientific data management and stewardship, steps are being made to make data Findable, Accessible, Interoperable, and Reusable. In order to increase our understanding of obesity, we have to go beyond 'black-box' correlations and develop data-analysis and modelling approaches that enable us to 'connect everything to everything' while at the same time incorporating biological knowledge that has been generated in decades of careful hypothesis driven research. Examples will be given from the field of systems biology where methods are being developed, that allow us to integrate such data and knowledge at different spatial (intracellular, organ, whole body) and time scales (from glucose dynamics to development of type 2 diabetes).

\section{RS1:2}

\section{Personalized nutrition by prediction of glycemic responses}

\section{David Zeevi}

Weizmann Institute of Science, Rehovot, Israel

The past decades have witnessed a surge in the prevalence of obesity, diabetes and the metabolic syndrome. Many of these disorders are associated with high post-meal blood glucose responses, but common dietary methods for controlling these responses have limited efficacy, mainly due to high interpersonal variability in the response to even the same meal. One of the factors underlying this variability is the gut microbiome: a huge ecosystem of bacteria, archaea, viruses and eukaryotes with vast potential metabolic capacity. In our work we developed new tools for the analysis of the gut microbiome and used these tools, along with blood parameters, dietary habits, anthropometrics and physical activity to accurately predict post-meal blood glucose responses to real-life meals. These predictions were then used to design personalized diets which successfully reduced hyperglycemia. Our results suggest that personalized diets can successfully lower post-meal blood glucose and its grave metabolic consequences.

$\mathrm{RS} 1: 3$

The body composition profile - enhancing the understanding of obesity using UK biobank imaging data

Linge, J. ${ }^{1}$; West, J. ${ }^{2}$; Romu, T. ${ }^{2}$; Borga, M. ${ }^{2}$; Bell, J. ${ }^{3}$; Leinhard, O. ${ }^{2}$

${ }^{1}$ Advanced MR Analytics AB, ${ }^{2}$ Advanced Mr Analytics Ab; Linköping University, ${ }^{3}$ University Of Westminster

Introduction: The definition of obesity, based on body mass index (BMI), lacks detail on fat distribution. The body composition profile (BCP) quantifying fat compartments and relations between fat and muscle volume, may enable the identification of obese subgroups. The aim of this work was to illustrate how the BCP can be used on a well-characterized, large imaging database to differentiate the obese population based on different metabolic disease groups: subjects with self-reported history of previous cardiovascular events (HCE) and subjects with self-reported diabetes mellitus (DM), as compared to subjects without HCE or DM.

Methods: 1,169 obese (BMI > =30) subjects ( 595 females and 574 males, 45 to 77 years) from the UK Biobank imaging cohort were included. Visceral adipose tissue index (VATi=VAT/height ${ }^{2}, 1 / \mathrm{m}^{2}$ ), abdominal subcutaneous adipose tissue index (ASATi=ASAT/height $\left.{ }^{2}, 1 / \mathrm{m}^{2}\right)$, lean thigh muscle volume divided by total body weight (muscle ratio, $1 / \mathrm{kg}$ ), intra-muscular fat in the anterior thigh muscles (IMAT, \%) and liver proton density fat fraction (PDFF, \%) were measured with a $1.5 \mathrm{~T}$ magnetic resonance (MR) scanner. The MR-images were analyzed using AMRA"Profiler research (AMRA, Sweden). 74 subjects with HCE (heart attack and angina) were sex and age matched to subjects with DM and to controls (no HCE or $\mathrm{DM}$ ). Kruskal-Wallis test (KW) and Mann-Whitney U test (where KW was significant) were used to test the differences for each measurement and group without correction for multiple comparisons.

Results: VATi in HCE was 2.45 (2.05-2.94) $1 / \mathrm{m}^{2}$ (median, 25th-75th percentile) compared to $2.66(2.19-3.21)$ in DM and $1.98(1.71-2.74)$ in controls. The differences were significant comparing HCE $(\mathrm{p}=0.005)$ and DM $(\mathrm{p}<0.001)$ to controls. ASATi in HCE was $3.24(2.56-4.39)$ compared to $3.6(2.85-4.63) \mathrm{l} / \mathrm{m}^{2}$ in DM and $3.28(2.54-4.35)$ in controls, with no significant differences. Muscle ratio in HCE was $0.12(0.11-0.13) 1 / \mathrm{kg}$ compared to $0.11(0.1-0.13)$ in DM and $0.13(0.11-0.14)$ in controls, and IMAT in HCE was 7.11 (5.73-8.89) \% compared to 7.79 (6.43-9.62) in $\mathrm{DM}$ and 7.04 (5.31-8.01) in controls. Significant differences for muscle ratio and IMAT were found comparing DM to controls $(\mathrm{p}=0.007, \mathrm{p}=0.01$ respectively). Liver PDFF in HCE was 6.14 (3.57-12.89) \% compared to 9.02 (5.38-16.23) in DM and 4.76 (3.11-7.62) in controls. The PDFF differences were significant comparing DM to HCE $(\mathrm{p}=0.01)$ and controls $(\mathrm{p}<0.001)$

Conclusion: Different BCP parameters were found to be associated with specific diseases identified in an obese subcohort from the UK Biobank. Additional research is needed to determine whether these parameters are associated with prospective development of disease.

Conflict of Interest: ODL, JW, TR, JL, MB (AMRA)

Funding: Financial support by Pfizer. 
RS1:4

\section{Metabolic profiling of tissue-specific insulin resistance in} human obesity

Vogelzangs, N. ${ }^{1}$; Kolk, B. ${ }^{2}$; Jocken, J. ${ }^{2}$; Goossens, G. ${ }^{2}$; Stehouwer, C. ${ }^{3}$; Greevenbroek, M. ${ }^{3}$; Kallen, C. ${ }^{3}$; Evelo, C. ; Valsesia, A. ; Hankemeier, T.6; Astrup, A. ${ }^{7}$; Saris, W. ${ }^{2}$; Arts, I. ${ }^{1}$; Blaak, E. ${ }^{2}$; Consortium, T. ${ }^{8}$

${ }^{1}$ Department of Epidemiology, Cardiovascular Research Institute Maastrich (CARIM) \& Maastricht Centre for Systems Biology (MaCSBio), Maastricht University, Maastricht, The Netherlands, ${ }^{2}$ Department Of Human Biology And Movement Sciences, Nutrim School Of Nutrition And Translational Research In Metabolism, Maastricht University, Maastricht, The Netherlands, ${ }^{3}$ Department Of Internal Medicine, Cardiovascular Research Institute Maastricht (Carim), Maastricht University, Maastricht, The Netherlands, ${ }^{4}$ Department Of Bioinformatics - Bigcat, Nutrim School Of Nutrition And Translational Research In Metabolism \& Maastricht Centre For Systems Biology (Macsbio), Maastricht University, Maastricht, The Netherlands, ${ }^{5}$ Nestlé Institute Of Health Sciences, Lausanne, Switzerland, ${ }^{6}$ Netherlands Metabolomics Centre, Leiden, The Netherlands, ${ }^{7}$ Department Of Nutrition, Exercise And Sports, Faculty Of Science University Of Copenhagen, Copenhagen, Denmark, ${ }^{8}$ The Diogenes Consortium

Introduction: Recent evidence indicates that insulin resistance (IR) in obesity may develop independently in different organs, representing different etiologies towards cardiometabolic diseases. The aim of this study is to characterize differences in the metabolic profile of persons with IR in skeletal muscle or in the liver.

Methods: Overweight/obese participants $(\mathrm{n}=755)$ of the European $\mathrm{DiO}$ Genes project, a randomized, controlled weight maintenance trial (after weight loss), were classified at baseline into 4 groups: (1) no IR $(\mathrm{n}=381)$, (2) IR in muscle only $(n=124)$, (3) IR in liver only $(n=123)$, (4) IR in both muscle and liver $(\mathrm{n}=127)$. The IR phenotype was based on the lowest tertile of the muscle insulin sensitivity index (glucose (slope_peak-nadir) / insulin (meanOGTT)) and the top tertile of the hepatic insulin resistance index (glucose (AUC_0-30)* insulin (AUC_0-30)), derived from a 5-point oral glucose tolerance test. At baseline 18 plasma metabolites were identified and quantified by NMR and compared across groups using multinomial logistic regression analyses.

Results: Mean age was $41(\mathrm{SD}=6)$ years, $66 \%$ were women, $81.5 \%$ were obese and mean BMI was $34.5(\mathrm{SD}=5)$. Participants with liver-IR were more often men $(\mathrm{p}<.001)$ and had a higher waist-to-hip-ratio $(\mathrm{p}<.001)$ compared to participants with muscle-IR. Before and after adjustment for sex, BMI and waist-to-hip-ratio, participants with liver-IR showed higher concentrations of (precursors) of branched-chain (valine, leucine, isoleucine, oxo-isovaleric acid) and other (alanine, proline, tyrosine) amino acids, as well as higher levels of glucose, lactate, creatine, and triglycerides (compared to no IR: all adjusted OR $=1.3-1.7$ per SD increase in metabolite; all $\mathrm{p}<0.05)$ and lower concentrations of the ketone body acetoacetate (adjusted OR per $\mathrm{SD}=0.62, \mathrm{p}=0.02$ ). Muscle-IR was associated with lower concentrations of glycine (adjusted OR per $\mathrm{SD}=0.73, \mathrm{p}=0.01$ ). No consistent differences in hydroxyisobutyrate, 3-OH-butyrate, creatinine, $\mathrm{n}$-acetyl, and acetate levels were found between groups.

Conclusion: Liver and skeletal muscle IR are characterized by clearly distinct metabolic profiles. Persons with liver-IR have elevated concentrations of (branched-chain) amino acids, glycolysis-related metabolites and triglycerides, whilst persons with primarily muscle-IR present with reduced glycine concentrations. The role of these metabolites as markers of tissue-specific insulin resistance warrants further investigation.
RS2 - A Year in Adipose Tissue: Novel Players and Future Treatments in WAT and BAT Biology

\section{RS2:1 \\ FGF21 and energy balance}

\section{Francesc Villarroya}

Department of Biochemistry and Molecular Biomedicine, and Institute of Biomedicine, University of Barcelona. CIBERobn, Spain

Fibroblast growth factor (FGF)-21 is an endocrine member of the FGF family with healthy effects on glucose and lipid metabolism. FGF21 improves glycemia and lipidemia in rodent models of obesity and insulin resistance. In addition to its effects improving insulin sensitivity, FGF21 causes weight loss by increasing energy expenditure. Activation of the thermogenic activity of brown adipose tissue and promotion "browning" (i.e., the appearance of the beige/brite type of brown adipocytes in white adipose tissue) are considered relevant mechanisms involved in the action of FGF21 promoting energy expenditure. Both, direct actions of FGF21 on adipose tissue and indirect effects mediated by central actions of FGF21, have been found to contribute to these physiological effects of FGF21. However, obesity in rodents and humans is paradoxically associated with abnormal high levels of blood FGF21. It has been proposed that obesity is a state of resistance to the actions of FGF21. In adipose tissue from obese mice and in obese patients, there are low levels of the FGF co-receptor $\beta$-Klotho which is known to be required for the cellular actions of FGF21. There are indications that the pro-inflammatory environment and the endoplasmic reticulum-stress occurring in adipose tissues in obese individuals causes the $\beta$-Klotho down-regulation. Despite this scenario, native FGF21 and FGF21 derivatives retain their capacity to improve the metabolic profile and promote weight-loss when used as pharmacological tools both in rodent models of obesity and in pilot studies in obese patients. FGF21 derivatives or molecules mimicking FGF21 action are currently under active research as attractive candidates for the development of novel anti-obesity drugs designed to increase energy expenditure. Moreover, recent findings identified a novel pathway of regulation of FGF 21 production by brown and beige adipose tissues based on the activation of the GPR120 receptor for omega-3 polyunsaturated fatty acids. Nutritional and pharmacological strategies to increase the endogenous FGF21 levels and to promote by this way its healthy metabolic effects are also under way.

Research relating to this abstract was funded by MINECO (SAF2004- 55725-TR) Instituto Danone and Generalitat de Catalunya (SGR-2014SGR141). Spain.

\section{$\mathrm{RS} 2 \cdot 2$}

\section{Plasticity of WAT}

Karpe, F.; Pinnick, K.; Todorcevic, M.; Hilton, C.; Denton, N.; Kuo, F.-C.; Neville, M. J.; Christodoulides, C.

Oxford Centre for Diabetes, Endocrinology and Metabolism, University of Oxford, Oxford, UK

Introduction: The study of genetics of obesity and human fat distribution have provided us with overwhelming evidence that overall fatness is driven by central mechanisms involving satiety whereas fat distribution is a consequence of variation in peripherally (largely adipocyte) expressed genes. The genetic component of fat distribution therefore provides a paradigm for exploring the plasticity of regional adipose tissue, expansion, contraction and remodelling.

Methods: Gene loci identified from genome wide association studies of human fat distribution helped to select candidates for functional studies of regional adipose tissue plasticity. Two independent targets from $\mathrm{HOXC13}$ locus displayed genetic and epigenetic interactions and these effect were evaluated in immortalised human abdominal and gluteofemoral cell lines. A further target was selected from a type 2 diabetes (T2D) 
risk locus with insulin resistant phenotypes (KLF14). This locus has in later studies been associated with fat distribution as well. In vitro studies were evaluated against in vivo phenotypes such as adipose tissue cellularity quantified by microscopy.

Results: The HOXC13 locus consists of three independent sites which maps to the HOTAIR and mir196b genes which showed site-specific expression under epigenetic regulation. Beyond this expression QTLs were identified in both genes. Stable knockdown of the genes demonstrated clear effects on either proliferation or adipogenesis. The KLF14 T2D risk variant was associated with profound enlargement of adipocyte cell size and in vitro effect on adipogenesis.

Conclusion: Selection of GWAS loci for fat distribution appears to generate informative nodes explaining adipose tissue plasticity using in vitro and in vivo studies.

Conflict of Interest: None Disclosed

Funding: The British Heart Foundation, the Medical Research Council

\section{RS2:3}

\section{Calorie restriction decreases thermogenic potential of subcutaneous WAT in humans.}

Barquissau, V. ' Léger, B. '; Beuzelin, D. '; Martins, F. ' Maoret, J. '; lacovoni, J. '; Stich, V. ${ }^{2}$; Déjean, S. ${ }^{3}$; Viguerie, ${ }^{1}{ }^{1}$; Langin, $D .{ }^{1}$

${ }^{1}$ INSERM, ${ }^{2}$ Prague Charles University, ${ }^{3}$ Toulouse University

Introduction: The presence of brown-like adipocytes in rodent white adipose tissue (WAT), so-called "brite" or "beige" adipocytes, has been shown to protect from obesity and insulin resistance. Recently, brite adipocyte development was suggested to mediate, at least in part, metabolic improvements during calorie restriction in mice (S. Fabbiano et al., Cell metabolism 2016). In humans, occurrence of those brite adipocytes and their potential benefits on metabolic health are poorly documented.

Methods: Herein, we studied human subcutaneous WAT biopsies harvested from 289 overweight or obese subjects, before and after an 8-week very low calorie diet (VLCD). Expression of more than 90 genes related to adipocyte subtype (i.e. white, brite or brown) and to adipose metabolism was quantified by qPCR. Clinical parameters including fat percentage and insulin resistance (HOMA-IR) were also assessed.

Results: At baseline, UCP1 mRNA levels were highly heterogeneous. Hierarchical clustering led to distinguish three subgroups of patients displaying elevated ("high-UCP1"), intermediate ("medium-UCP1") or weak ("low-UCP1") UCP1 expression. Sexual dimorphism was evidenced by much higher proportion of females in high- and medium-UCP1 groups. UCP1 expression was tightly correlated with genes involved in brite/brown adipocyte metabolism such as ELOVL3, PLIN5 and CKMTla/CKMT2. High- and medium-UCP1 groups displayed lower insulin resistance and higher plasma adiponectin and HDL cholesterol compared to low-UCP1 group. After VLCD, fat mass was decreased and insulin sensitivity was strongly improved. Simultaneously, UCP1 expression was sharply reduced as well as genes involved in thermogenesis, such as $C K$ $M T 1 a / C K M T 2$, and in mitochondrial electron transport chain, such as $\mathrm{COX} 4$.

Conclusion: In conclusion, UCP1 expression in subcutaneous white adipose tissue of overweight/obese patients was associated with healthy metabolic status. However, calorie restriction consistently impaired thermogenic potential of WAT. Hence, WAT browning cannot be considered as a mediator of metabolic improvements following VLCD.

Conflict of Interest: None disclosed.

Funding: EU FP7 project DIABAT (HEALTH-F2-2011-278373).

\section{RS2:4}

\section{Adipocyte hypertrophy and associated inflammatory phenotype as major determinants of insulin resistance in male obese humans}

Verboven, K. '; Wouters, K. ${ }^{2}$; Gaens, K. ${ }^{2}$; Hansen, D. ${ }^{3}$; Bijnen, M. ${ }^{2}$; Wetzels, S. ${ }^{2}$; Stehouwer, C. ${ }^{2}$; Goossens, G. '; Schalkwijk, C. ${ }^{2}$; Blaak, E. ; Jocken, J. ${ }^{1}$

${ }^{1}$ NUTRIM School of Nutrition and Translational Research in Metabolism, Maastricht University Medical Centre,$+{ }^{2}$ Carim Cardiovascular Research Institute Maastricht, Maastricht University Medical Centre,$+{ }^{3}$ Reval Rehabilitation Research Center, Biomed Biomedical Research Institute, Hasselt University

Introduction: Obesity is associated with adipose tissue (AT) dysfunction characterized by adipocyte hypertrophy, impaired lipolysis and elevated AT inflammation. It is suggested that AT dysfunction in obesity, rather than an elevated fat mass per se, is associated with insulin resistance (IR). Therefore, we investigated whether AT morphology, lipolysis and inflammation are associated with IR in different AT depots of obese subjects with and without type 2 diabetes mellitus (T2DM).

Methods: Subcutaneous (SC) and visceral (V) AT biopsies from agematched (39-65 year) lean (BMI $23 \pm 0.3 \mathrm{~kg} / \mathrm{m}^{2}$, HOMA-IR $2.1 \pm 0.2$ ), obese (BMI $38 \pm 0.9 \mathrm{~kg} / \mathrm{m}^{2}$, HOMA-IR $\left.5.6 \pm 0.7\right)$ and obese T2DM men (BMI $38 \pm 1.2 \mathrm{~kg} / \mathrm{m}^{2}$, HOMA-IR $7.9 \pm 1.7$ ) were included. AT phenotype was characterized by ex vivo measurement of basal and maximal stimulated lipolysis (mature adipocytes), adipocyte size distribution (AT tissue sections) and AT immune cells (flow cytometry).

Results: Adipocyte hypertrophy was present in obese SCAT and VAT compared to lean, accompanied by a shift in adipocyte size distribution. In VAT, CD45 ${ }^{+}$leukocytes and M1 macrophages were significantly increased in both obese groups compared to lean. In SCAT no group differences were observed in immune cell populations. In VAT, adipocyte size $(r=0.603)$, CD $45^{+}$leukocytes $(r=0.515)$ and maximal isoprenalin responsiveness $(r=-0.364)$ were significantly correlated with HOMA-IR, together accounting for 56\% of the variance in HOMA-IR (multiple linear regression adjusted $\mathrm{R}^{2}, 0.564$ ), adjusted for age and fat mass. In SCAT, adipocyte size $(r=0.460)$, basal lipolysis $(r=0.526), \mathrm{CD}^{+} \mathrm{T}$ cells $(r=0.389)$, $\mathrm{CD}^{+} \mathrm{T}$ cells $(\mathrm{r}=0.506)$ and NK cells $(r=0.452)$ were significantly correlated with HOMA-IR, together accounting for $40 \%$ of the variance in HOMA-IR (multiple linear regression adjusted $\mathrm{R}^{2}, 0.406$ ), adjusted for age and fat mass.

Conclusion: Adipocyte hypertrophy in both SCAT and VAT is a major determinant of IR. In hypertrophic visceral adipocytes, M1 macrophage-mediated inflammation may contribute to IR, while in hypertrophic subcutaneous adipocytes infiltration of cytotoxic T cells and an attenuated lipolysis may be more important. 


\section{RS3 - Loss versus Maintenance: The False Dichotomy}

\section{RS3:1 \\ Predictors of weight regain after moderate diet-induced weight loss}

\section{M. van Baak}

NUTRIM School for Nutrition and Translational Research in Metabolism, Department of Human Biology and Movement Sciences, Maastricht University Medical Centre+, Maastricht, The Netherlands

Introduction: One of the most difficult problems in obesity management is the prevention of weight regain after successful weight loss. Several factors have already been identified that are associated weight regain. For this presentation, the focus will be on the potential role of adipose tissue-related factors as predictors of weight regain.

Methods: 57 Individuals who were overweight or obese lost approximately $10 \%$ of their initial weight by means of a very low calorie diet (VLCD) during 5 weeks or a low calorie diet (LCD) for 12 weeks. Subsequently they were put on a strict weight maintenance diet for 4 weeks. Thereafter they were advised to maintain weight, but did not receive further dietary advice for 9 months to mimic normal life conditions. Subcutaneous fat biopsies and blood samples were obtained at different time points and postprandial exogenous fatty acid uptake into adipose tissue was measured with stable isotope tracer methodology.

Results: Weight loss-induced changes in meal-derived fatty acid uptake, adipocyte size, expression of genes and proteins, with a focus on stress and the extracellular matrix (ECM), and circulating factors and their association with weight regain will be presented. We found evidence for a role of weight loss-induced cellular stress and ECM remodeling in determining weight regain. We also found evidence for a role for adipose tissue leukocytes. Meal-derived fatty acid uptake did not change with weight loss and was not associated with weight regain. Changes in the levels of a number of circulating factors, such as RBP4, FFA, FGF21 and ACE, were associated with weight regain.

Conclusion: Inter-individual differences in weight loss-induced cellular stress and the ECM remodeling response in adipose tissue are likely to play a role in weight regain after moderate diet-induced weight loss. The role of inflammation in these inter-individual differences in adaptations to weight loss requires further study.

Conflict of Interest: none

Funding: Research relating to this abstract was funded by Netherlands Organisation for Scientific Research TOP, grant number: 200500001

RS3:2

\section{Long-term weight loss maintenance for obesity: A multidisciplinary approach.}

\section{Paolo Sbraccia}

Department of Systems Medicine, University of Rome Tor Vergata, and Obesity Center, University Hospital Policlinico Tor Vergata, Rome, Italy.

Obesity arise from a chronic positive energy balance and has huge consequences in terms of morbidity, mortality and health care costs. Its rising prevalence, in the developed/developing world, is an indirect indicator of the lack of an efficient unidirectional long-term strategy to induce and maintain weight loss. After an initial weight loss, when a negative energy balance is induced by diet, increasing physical activity, or both, most patients regain weight. This is due to a progressive loss of adherence to diet and activity prescriptions for complex endogenous compensatory mechanisms. This is particularly unfortunate, since a moderate and stable weight loss of 5-10\% would translate in significant clinical improvement of obesity-related cardio-metabolic risk factors. However, so far, long-term weight maintenance is defined in a time frame that range from one to two years, whilst the obvious expectation would be a lifelong weight reduction. Many studies have engaged in multidisciplinary approaches aiming at successfully reduce body weight; however, quite few of them had the features of large RCT with long-term follow-up. Among them, deserve to be mentioned the Look AHEAD (Action for Health in Diabetes) study, the Diabetes Prevention Program (DPP) and, with the addition of pharmacotherapy, the XENDOS (XENical in the prevention of Diabetes in Obese Subjects) and the SCALE (Satiety and Clinical Adiposity - Liraglutide Evidence) Obesity and Prediabetes trials. Powerful data came also from two large prospective studies: the National Weight Control Registry (NWCR) and the 1999-2006 national Health and Nutrition Examination Survey (NHANES). Overall, these studies demonstrated that the adoption of the latest generation weight reduction lifestyle modification program with the possible addition of cognitive behavioral procedures and/or drugs, leads to a long-term weight loss maintenance in a significant proportion of the study groups. In this regard, the search for the determinants that induce weight regain vs those that enable weight maintenance is highly warranted while certainly representing a challenging task. Finally, in the daily practice, the severity of obesity together with the presence and severity of complications and age need to be taken into consideration in order to grade multidisciplinary interventions (lifestyle modification, pharmacotherapy, bariatric surgery and/or rehabilitation). In this regard, we took advantage of the now well-established Edmonton Obesity Staging System (EOSS) to develop a therapeutic algorithmic chart that includes BMI, age and EOSS stages (Management algorithm for patients with overweight or obesity: consensus statement of the Italian Society for Obesity - SIO, Eat Weight Disord. 2016).

RS3:3

Early responders to liraglutide $\mathbf{3 . 0} \mathbf{m g}$ as adjunct to diet and exercise from the scale maintenance trial

\section{Wharton, S. ${ }^{1}$; Jacobsen, P. ${ }^{2}$; Aronne, L. ${ }^{3}$}

${ }^{1}$ Wharton Medical Clinic, Weight and Diabetes Management, Hamiliton, ON, Canada, ${ }^{2}$ Novo Nordisk A/S, SøBorg, Denmark, ${ }^{3}$ Weill Cornell Medical College, New York, Ny, Usa

Introduction: The SCALE Maintenance trial randomised adults with obesity $\left(\mathrm{BMI} \geq 30 \mathrm{~kg} / \mathrm{m}^{2}\right)$ or overweight $\left(\mathrm{BMI} \geq 27 \mathrm{~kg} / \mathrm{m}^{2}\right)$ with comorbidities who lost $\geq 5 \%$ of initial body weight during a 4 -12-week low-calorie diet (1200-1400 kcal/day) run-in period prior to randomisation to liraglutide $3.0 \mathrm{mg}$ or placebo as adjunct to diet and exercise. Mean weight loss during the run-in period was $6.0 \%$.

Methods: This post-hoc analysis of the SCALE Maintenance trial compared outcomes in liraglutide $3.0 \mathrm{mg}$ early responders vs. early non-responders (ERs vs. ENRs; $\geq 5 \%$ vs. $<5 \%$ weight loss at week 16 after randomisation) who completed 56 weeks of treatment.Efficacy outcomes for liraglutide $3.0 \mathrm{mg}$ ERs vs. ENRs are observed means or proportions for individuals completing 56 weeks' treatment. The same analysis set was also used for adverse events.

Results: Mean characteristics at randomisation $(\mathrm{n}=212)$ for patients on liraglutide $3.0 \mathrm{mg}$ were: 46 years of age, $84 \%$ female, BMI $36 \mathrm{~kg} / \mathrm{m}^{2}$. At week 16, of those who completed 56 weeks' treatment with a measurement at week $16(\mathrm{n}=159) ; 108(68 \%)$ were ERs to liraglutide $3.0 \mathrm{mg}$ and $51(32 \%)$ ENRs. At 56 weeks, the mean relative change in body weight was $-9.9 \%$ in ERs vs. $0.0 \%$ in ENRs. Percentage of individuals who maintained their run-in weight loss during the 56 weeks of treatment or lost additional weight was $91.7 \%$ vs. $47.1 \%$, and the percentage of individuals who at week 56 had regained at least the run-in weight loss was $0.0 \%$ vs. $3.9 \%$ for ERs vs. ENRs, respectively. The percentage of individuals achieving $\geq 5 \%,>10 \%$ or $>15 \%$ weight loss from randomisation at 56 weeks was $73.1 \%, 47.2 \%$ and $20.4 \%$ for ERs vs. $11.8 \%, 0.0 \%$, and $0.0 \%$ for ENRs. ERs achieved greater reduction in mean waist circumference: $-7.9 \mathrm{~cm}$ vs. 0.0 $\mathrm{cm}$ in ENRs. Changes from randomisation in systolic/diastolic blood pressure was $-0.1 /+2.0 \mathrm{mmHg}$ in ERs vs. $-0.5 /+1.3 \mathrm{mmHg}$ in ENRs. Changes in glycaemic outcomes in ERs and ENRs were $\mathrm{HbA}_{1 c}:-0.2$ and $-0.1 \%$ and fasting plasma glucose: -0.6 and $-0.5 \mathrm{mmol} / \mathrm{L}$. ERs experienc- 
ing any adverse events (AEs) was $92.5 \%$ vs. $89.7 \%$ for ENRs. For serious AEs it was $2.7 \%$ vs. $0.0 \%$ and for gastrointestinal AEs $77.8 \%$ vs. $41.4 \%$ for ERs vs. ENRs respectively.

Conclusion: Among those who completed 56 weeks' treatment, liraglutide $3.0 \mathrm{mg}$ ERs achieved greater additional weight loss than liraglutide $3.0 \mathrm{mg}$ ENRs.

RS3:4

Long-term follow-up (4 years) of patients receiving cognitive behavioural therapy (CBT) or usual care before bariatric surgery: A randomized controlled trial

\section{Gade, H. ${ }^{1}$; Friborg, O. ${ }^{2}$; Sandbu, R. ${ }^{1}$; Rosenvinge, J. ${ }^{2}$; Hjelmesaeth, J. ${ }^{1}$}

${ }^{1}$ Morbid Obesity Centre, Vestfold Hospital Trust, Tonsberg, Norway,

${ }^{2}$ Department Of Psychology, Uit - The Arctic University Of Norway

Introduction: We examined whether an adapted cognitive behavioural therapy (CBT) preoperative intervention exceeded usual care in the improvement of dysfunctional eating behaviours, symptoms of anxiety and depression and body weight 4 years after bariatric surgery.

Methods: A total of 102 patients participated in a randomized clinical trial (RCT) of two arms, i.e. a 10-week CBT-programme $(n=28,16$ women $)$ or usual pre-surgical care $(\mathrm{n}=33,27$ women) (http://clinicaltrials.gov/ ct2/show/NCT01403558). In the present 4-year follow-up study 61 patients participated. Outcome variables: Dysfunctional eating behaviours (emotional eating, uncontrolled eating, cognitive restraint) and mood symptoms (anxiety and depression) were assessed web-based with standard self-report questionnaires (TFEQ R- $21^{4}$ and $\mathrm{HADS}^{5}$, respectively). Paired t-tests were used to examine total and within-group changes, and analysis of covariance (ANCOVA) to examine between-group differences at 4 years with the baseline outcome score as a covariate.

Results: The average scores of dysfunctional eating and depression, but not anxiety, improved significantly from commencing the study to 4 -years after bariatric surgery. The degree of improvements in uncontrolled eating, cognitive restraint, emotional eating and depression were $41 \%, 17 \%$, $27 \%$ and $48 \%$, respectively (all $p$ 's $<.005$ ). BMI and body weight were reduced by $28 \%$ (35 kg, 12 BMI-points, both $p$ 's $<.001$ ). The CBT-group did not surpass the control group in any of the outcome variables. On the contrary, patients in the control displayed a significantly higher mean improvement in scores of depression (62\% vs 33\%, respectively, $p=.023$ ) Conclusion: This 4-year follow-up study confirmed beneficial long-term effects of bariatric surgery on dysfunctional eating behaviours and depression, and that a pre-surgical CBT intervention did not improve these outcomes. Limitations include selection bias and low power due to relatively high attrition rate $(40 \%)$, as well as gender imbalance at follow-up.

${ }^{4}$ Three-Factor eating questionnaire R-21

${ }^{5}$ Hospital anxiety and depression scale

Conflict of Interest: None Disclosed

Funding: Hege Gade has received grants from South-Eastern Norway Regional Health Authority

\section{RS4 - Population Based Approaches}

\section{RS4:1}

\section{Regulations around the food environment}

Tim Lobstein

Director of Policy, World Obesity Federation, London, UK

'Regulation' covers a range of meanings, from school rules (e.g. no candy in the lunch box) to global trade arrangements and Codex standards-setting. In between lie the national policies and local community regulations that most directly shape the markets and choices we all make. Particularly important are the power-relationships inherent in making the rules and regulations: Who designs the rules, who administers and monitors them, who decides the sanctions for breaking the rules? For example, rules on the marketing of tobacco products are set at national level under an international framework, enshrined in legal statutes, and penalised through civil and criminal law. Rules on the marketing of soft drinks are - with some exceptions - largely voluntary in Europe, administered by the industry, with no sanctions imposed on rule-breakers. The word 'voluntary', like the word 'responsibility' makes the industry look good - and a better phrase might be 'non-binding' to indicate that the rules can be broken at any time. The development of research to support regulations is increasing rapidly in the area of food policies. This can strengthen the arguments for public health, against the opposing policies it faces from other interests. Recent advance have been made in several areas: (i) surveillance of policies - e.g. INFORMAS, especially FoodEPI and EPI-BIA, and ATNI; (ii) policy implementation - e.g. nutrition labelling (using crowd sourcing); (iii) policy acceptability - e.g. PorGrow and PolMark projects in the EU; (iv) policy databases - e.g. NOURISHING and WHO; (v) inequities in policy impact - e.g. HEPP in the EU. Lastly, rules and regulations are not 'burdens' for most of us: they are benefits. They ensure protection and high standards, they hold the powerful to account, and they can actually help the private sector do what the best of its members want to do.

Conflict of Interest: None disclosed

Funding: No Funding

\section{RS4:2}

\section{Global Active City}

Gabriel Messmer, Evaleo, and Nathalie Farpour-Lambert

Obesity Prevention and Care Program Contrepoids, University Hospitals of Geneva, Switzerland

Obesity and other non-communicable diseases (NCDs) have a worldwide impact on societies and economies. In particular, declining levels of physical activity, unhealthy diet, social inequalities and poor mental health contribute to this burden and necessitate a particular attention. As the urban population is continuously growing, cities have a unique position to identify social needs, engage individuals on health issues, and empower communities to build an environment facilitating lifestyle changes and promoting sustainable health. Opportunities for behavioural changes are centrally important to the development and maintenance of individual and collective health and well-being, and the treatment and recovery of ill-being.

News standards scheme to support the promotion of physical activity, sport and sustainable health for all within an urban environment or an organisation have been developed. The first step is to ensure that a city, or an organisation, implements a system for the management and control of "Physical Activity and Sport for All" principles.

Individual sustainable health management is an approach with allows the incorporation of sustainable health actions and good practices into one's daily life. The project includes seven sustainable health action areas: 1) personal development; 2) physical activity; 3) food culture, nutrition and hydration; 4) functional health; 5) relaxation, sleep and self-care; 6) art and cultural engagement; and 7) supportive contextual environment.

The standards specifies the requirements applicable to cities, or organisations wishing to gain accreditation. A set of accompanying user-friendly guidelines will be published and regularly updated by the standard scheme owner.

Conflict of interest: None disclosed.

Funding: International Olympic Committee 
RS4:3

\section{Upstream levers for changing the obesity prevalence}

\section{Jeroen Lakerveld}

VU University Medical Center, Amsterdam, Netherlands

Overconsumption of high-energy foods and lack of physical activity are the main behavioural risk factors for adult obesity. These behaviours are influenced by a range of downstream, individual-level factors, as well as upstream, environmental-level factors. In this presentation, these upstream determinants will be defined and a historical background will be provided. In addition, the current evidence base regarding these determinants across various types of environments will be summarised, and put in perspective.

Conflict of Interest: None Disclosed

Funding: No Funding

\section{Thursday, 18 May 2017}

\section{RS5 - EASO COMs Session: Post-Bariatric Surgery Challenges}

\section{RS5:2}

\section{Metabolic bone disease after bariatric surgery}

\section{Dr. Nuria Vilarrasa}

Bellvitge University Hospital-IDIBELL

Bariatric surgery is an effective treatment for morbidly obese patients that leads to improvement or even resolution of associated comorbidities, but it is not exent of nutritional and metabolic complications. In this sense, concerns have been raised over its adverse effects on the skeletal health as increased bone loss and fragility has been described after bariatric surgery that may lead to increased bone fractures. Bone changes following bariatric surgery have been analysed in multiple prospective studies in the short-term and suggest that weight loss preferentially affects hip BMD and is greater during the first year after surgery when the largest weight reduction occurs. This decrease according to the different series based mainly on patients undergoing gastric bypass, is $9-10 \%$ in the femoral neck and $3-4 \%$ at lumbar spine. It has been speculated that this initial bone mass loss may be a normal adaptation to the decrease in mechanical load on bone that stabilizes along with weight. Few reviews and meta-analysis have compared BMD after bariatric surgery with nonsurgical controls with controversial results being obtained. In some, BMD at femoral neck but not at lumbar spine was lower, in others the decrease was observed at both sites only after mixed surgical procedures. On the contrary, other authors showed that BMD at the spine and radius was greater in postsurgical patients compared to an obese population. Moreover, contradictory results have also been described regarding fracture risk after bariatric surgery. Three retrospective studies based on community medical records, showed greater fracture risk after bariatric surgeries with a malabsortive component compared to general population. However, the only retrospective study using weight-matched controls performed by Lahmohamed including 2079 patients with a mean follow-up of 2 years, did not observe a change in fracture risk following surgery. A limitation of the previous studies is their retrospective design, difficulties in selecting appropriate controls, and missing data regarding vitamin $\mathrm{D}$ and nutritional factors. The most important risk factors for bone mass decline are age, menopause, and increased loss of lean mass. Also, vitamin D deficiency, secondary hyperparathyroidism, and changes in adipokines may affect bone strength.

To date, in the search of a new non-invasive tool for assessing skeletal microarchitecture, trabecular bone score has been developed. The applica- bility of this method in the assessment of bone microarchitecture changes and fracture risk after bariatric surgery seems promising but has been scarcely analyzed.

\section{RS6 - Biological Rhythms and Behavioral Patterns}

\section{RS6:1}

\section{Biological rhythms, lifestyle choices and insulin resistance}

Scott, E.M.

Senior Lecturer in Medicine, University of Leeds, and Consultant Diabetologist, Leeds Teaching Hospitals NHS Trust, Leeds UK

Life on earth is governed by the continuous 24-hour cycle of light and dark. Organisms have adapted to this environment with clear circadian rhythms in their physiology and metabolism enabling them to anticipate predictable environmental fluctuations over the day and to optimise the timing of relevant biological processes to this cycle. Central to the control of these rhythms is the circadian clock system. Current evidence suggests that interactions between central and peripheral molecular clocks are important in metabolic function. Modern man often works during the night, is exposed to artificial light exposure after daylight ends and chooses to sleep fewer hours than his forbears. When considered in the light of increasing epidemiological evidence linking shift work and sleep disturbance to the metabolic syndrome, and in view of their central role in organising the circadian rhythmicity of cell and tissue metabolism, clock genes are potential candidate genes for the metabolic syndrome and their dysregulation provides a potential molecular mechanism through which environmental disturbances can be translated into disease. Disrupting this process through mutations in the core clock genes or by interfering with sleep and the environmental zeitgebers that entrain the central clock appear to modulate the function of cells and tissues leading to the development of obesity/type 2 diabetes. Building on our basic science research examining the effect of clock disruption in the development of insulin resistance and the metabolic syndrome we have developed novel techniques of analysing complex data obtained by $24 \mathrm{hr}$ continuous glucose monitoring and applied it to understand the temporal glucose profiles that are associated with poorer outcomes in diabetes and more recently the relationship of sleep to temporal glucose control. This has opened up new possibilities for understanding how both genetic and environmental circadian factors, including sleep, may influence $24 \mathrm{hr}$ glucose control and diabetes in man. This talk will outline the importance of the circadian clock and sleep to the development and progression of obesity/type 2 diabetes, focusing on its clinical relevance.

\section{RS6:3 \\ Habitual sleep duration and quality in individuals with obesity and appetite markers}

Kleppe, M. ${ }^{1}$; Nymo, S. ${ }^{2}$; Coutinho, S. ${ }^{1}$; Salater, S. ${ }^{3}$; Kulseng, B. ${ }^{4}$; Martins, C. ${ }^{2}$ ${ }^{1}$ Norwegian University of Science and Technology, ${ }^{2}$ Department Of Cancer Research And Molecular Medicine, Faculty Of Medicine, Norwegian University Of Science And Technology (Ntnu), Trondheim, Norway, ${ }^{3}$ Centre For Obesity And Innovation (Obece), Clinic Of Surgery, St. Olav University Hospital, Trondheim, Norway, ${ }^{4}$ St. Olavs University Hospital, Trondheim, Norway

Introduction: Laboratorial studies have shown that acute sleep deprivation is associated with increased appetite. However, no population studies have been done regarding the potential association between habitual sleep duration/quality and both subjective and objective appetite markers. The aim of this study was to look for differences in both subjective and objective appetite measures between short and normal sleepers, and good and bad sleepers in individuals with obesity. 
Method: 95 adults (55 females) with obesity (BMI: $36.6 \pm 3.9 \mathrm{~kg} / \mathrm{m}^{2}$, age $42.7 \pm 9.8$ years) took part in the study. Sleep duration was measured both subjectively with Pittsbourgh Sleep Quality Index (PSQI) and objectively using SenseWear armbands for 1 week and sleep quality was also assessed with PSQI. Fasting/postprandial subjective feelings of hunger, fullness, desire to eat (DTE) and prospective food consumption (PFC) were assessed and plasma concentrations of active ghrelin (AG), total peptide YY (PYY), active glucagon-like peptide 1 (GLP-1) and insulin measured every 30 minutes for 2.5 hours. Short sleep was defined as $<7$ and normal sleep $\geq 7$ hours/night. A PSQI global score $<5$ was defined as good sleep and $>5$ as bad sleep quality.

Results: Sleep duration was $6.7 \pm 1.0$ and $6.2 \pm 1.2$ hours/night from PSQI and armbands, respectively. $46.3 \%$ and $58.8 \%$ participants were classified as short and $53.7 \%$ and $41.2 \%$ as normal sleepers according to PSQI and armband data, respectively. $61.1 \%$ presented with bad sleep quality according to the PSQI. Neither the PSQI or the armbands showed any significant difference in any appetite variables between either short and normal duration sleepers, or good and bad sleepers. There was also no significant difference found between the groups in gender, age and BMI.

Conclusion: In our study neither habitual sleep duration, nor quality, seem to be associated with either subjective or objective appetite markers in individuals with obesity.

\section{Conflict of Interest: None}

Funding: Liaison Committee between the Central Norway Regional Health $\mathrm{Au}$ thority (RHA) and the Norwegian

University of Science and Technology (NTNU). Clinic of Surgery, St. Olav University Hospital, Trondheim, Norway

\section{RS6:4}

Impact of season on measured body mass index: Artefact of age or effect of the environment? - The childhood obesity surveillance initiative in the Republic of Ireland - 2008, 2010, $2012 \& 2015$

\section{Bel-Serrat, S. ${ }^{1}$; Heinen, M. ${ }^{1}$; Mehegan, J. ${ }^{1}$; Murrin, C. ${ }^{1}$; Kelleher, $C^{1}$ ${ }^{1}$ UCD School of Public Health, Physiotherapy and Sports Science}

Introduction: Routine measurement of weight and height is crucial to monitor the progress of childhood obesity. Even when age in years is assessed there may be growth spurts or seasonal influences on weight and height affecting body mass index. This analysis aims to evaluate the potential implications of taking anthropometric measurements at different time points of the year on the prevalence of overweight and obesity in children. Methods: A nationally representative sample of 7,650 First class children (52.5\% girls), mean age $7.2( \pm 0.4 \mathrm{SD})$ years, participating in the Irish Childhood Obesity Surveillance Initiative (COSI) in four rounds were included. Height and weight were objectively measured by trained nutritionists. The study followed the WHO COSI protocol. BMI was categorised according to International Obesity Task Force cut-offs (1). Measurements took place between April and June in 2008 (summer term), October and November in 2010 (autumn term), November and January in 2012 and November and February in 2015 (winter-spring term). Children's age was calculated in months and split into four age sub-categories. Three logistic regression models were undertaken with gender, chronological age, round or season, separately and together included.

Results: Mean age was $7.5( \pm 0.3 \mathrm{SD})$ years, $7.0( \pm 0.4 \mathrm{SD}), 7.1( \pm 0.4 \mathrm{SD})$ and $7.1( \pm 0.4 \mathrm{SD})$ in each round respectively. The prevalence of overweight and obesity among children declined over time from $23.2 \%$ in 2008 to $18.3 \%$ in 2015 ( $\mathrm{p}_{\text {trend }}<0.001$ ). This persisted when stratified by age sub-category in those aged $84-89$ months, prevalence was $22.7 \%$ in $2008,21.2 \%$ in $2010,18.6 \%$ in 2012 and $15.7 \%$ in 2015 ( $\mathrm{p}_{\text {trend }}<0.001$ ). All regression models showed gender and chronological age were significant but collinearity between season and round was seen. The final model included girls, OR 1.67(1.49-1.87, p<0.001), chronological age, OR 0.93(0.87-0.89, $\mathrm{p}=0.043)$ and round, OR 0.88(0.83-0.93, $\mathrm{p}<0.001)$.
Conclusion: Although examinations in 2008 took place in summer term, the observed higher overweight and obesity rates in that round seem to be an effect of chronological age rather than season. Moreover, a real time trend towards stabilisation in obesity rates appears to be occurring in Irish First class children. Nevertheless, date of measurement in relation to birthdate needs to be considered when investigating obesity in 7-year-old children, a 6-month age difference could represent a large gap in growth. Our findings support the use of age range (in months) to provide more reliable estimates, rather than a single age point (in years).

Reference:

1 Cole TJ et al. BMJ 2000;320(7244):1240-1243.

Conflict of Interest: None Disclosed

Funding: Research relating to this abstract was funded by the Health Service Executive (HSE) of Ireland.

\section{RS7 - EASO PPHTF Session: Integrating Insights}

\section{RS7:1}

\section{The Systems Approach: Theory}

\section{Kremers, S.P.J.}

School of Nutrition and Translational Research in Metabolism (NUTRIM), Maastricht University, the Netherlands

Introduction: Obesity prevention efforts have often resulted in recommendations regarding the application of a systems approach. Often, such recommendations are translated into approaches that are actually lacking true Systems Theory assumptions.

Methods: Salient basic assumptions of Systems Theory are presented and applied to research and practice of obesity prevention. These include the existence of non-linear determinant - behaviour relationships and interactions between types and levels of environmental influences. Potential consequences of the theoretical assumptions for research in terms of feedback loops, synergy and higher-order moderation will be outlined.

Results: Examples are provided of recent empirical applications of systems theory to the study of obesity-related behaviours among various target groups.

Conclusion: It will be argued that research is strengthened when obesity prevention interventions are viewed as changes to the system and that a mechanistic view on implementation should be replaced by a contextual view. Implications of applying a systems approach in obesity prevention research will be discussed, e.g. for study design, analytical approaches, intervention design and implementation.

Conflict of Interest: None Disclosed

Funding: No Funding

\section{RS7:2}

\section{Complex systems thinking: From theory to practice to address obesity}

\section{H. Rutter}

London School of Hygiene and Tropical Medicine, London, England

The UK Government Foresight report in 2007 placed the concept of complexity at the core of our understanding of obesity. This has been followed by extensive discussion of this approach, and its relevance for obesity, since then, with frequent reference to complexity in the academic literature.

This rhetoric has not, however, been matched by reality. There are very few published strategies or policies that explicitly and genuinely take account of complexity, and equally few evaluations of interventions. We have studied the distribution of research funding by a major UK funder and found that the overwhelming majority of funded projects address small scale, 
individual level interventions, with little if any consideration of complexity. There are strong indications that an equivalent situation obtains with other funders in other countries.

At the same time progress to tackle obesity has been limited across Europe and the rest of the world. Some countries have seen indications of a levelling off in the increase in prevalence of obesity. But there have been few if any reports of sustained declines in prevalence, and there are also strong indications of widening inequalities in the distribution.

In this talk I will argue that one of the reasons for the persistence of obesity is a failure adequately to grapple with it as an emergent property of a complex adaptive system by research funders, researchers, practitioners, and policymakers. If we are successfully to address the problem we will need both to reconceptualise the problem and pursue approaches that move us from discussion of a theoretical approach to widespread, practical action. This will require changes to the ways in which research is funded and conducted; the wider use of existing approaches and tools for evaluation of complex system actions, alongside the development of new methodological approaches; and the adoption of novel conceptual approaches to the policy challenges by policymakers and practitioners.

These changes will take some time, and many of them may initially fee uncomfortable, but until we make them the likelihood of identifying ef fective combinations of responses to the challenge of obesity is likely to remain elusive. The time is long overdue to move our discussions of the role of complexity in obesity from theory to practice.

Conflict of interest: None

Funding: This work is funded by The Health Foundation

\section{RS7:3}

\section{Applying Systems Modeling to Obesity Prevention and Control}

Bruce Y. Lee, MD, MBA; Marie C. Ferguson, MSPH; Daniel Hertenstein BS; Atif Adam, MD; Sindiso Nyathi, BS; Molly Domino, BS; Mario Solano Gonzalez, BS; Michelle S. Wong, PhD; Peggy I. Wang, PhD; Shawn T. Brown, PhD

Global Obesity Prevention Center at Johns Hopkins University, Baltimore, MD, USA

Preventing and controlling obesity requires understanding and addressing multiple complex systems (biological, behavioral, social, environmental). Unaided this can be difficult without the help of computational methods. Computational simulation modeling has helped transform decision making in numerous other field such as meteorology, transportation and manufacturing. In this session, we will describe computational simulation modeling and how it can help address obesity prevention and control, it's strengths and weaknesses and give examples of how simulation models can help with decision-making.

\section{RS8 - Beyond the Minister of Health: Lessons from countries where obesity is classified as a} disease

RS8:1

\section{Access to Obesity Treatment: Lessons from Canada where obesity is classified as a disease}

Ximena Ramos Salas, Mayank Rehani, Brad Hussey, Arya M. Sharma, on behalf of the Canadian Obesity Network.

'Canadian Obesity Network, Canada

Introduction: In 2006, the Canadian Clinical Practice Guidelines on the Management and Prevention of Obesity in Adults and Children were released, calling for the treatment of obesity as a chronic disease 1. In Octo- ber 2015, the Canadian Medical Association declared obesity as a disease requiring enhanced research, treatment and prevention efforts 2 .

Objective: The Canadian Obesity Network (CON-RCO) sought to quantify and qualify access to publicly provided medical care for obesity, as well as interventions covered by private health plans. Specifically, CON-RCO aimed to capture the level of:

a) Access to specialists and interdisciplinary teams for behavioural intervention;

b) Access to weight management programs that include meal replacement;

c) Access to anti-obesity medications through public and private means; and

d) Access to bariatric surgery and wait times.

Methods: Survey instruments were used to acquire information on provincial and territorial policies and services, private drug benefit plans offered by the health insurance industry, and bariatric surgical services and wait times. Interviews were conducted with representatives of health insurance companies, healthcare staff in bariatric surgical centres, and representatives of provincial and territorial governments or health service authorities.

Results: None of the provincial or territorial governments have officially recognized obesity as a chronic disease. Health Canada has also not officially recognized obesity as a chronic disease and has continued to consider obesity as a lifestyle risk factor. There is a profound lack of interdisciplinary teams for obesity management in Canada. Canadians who may benefit from a medically supervised weight management programs with meal replacements are expected to pay out-of-pocket for the meal-replacement. Less than $20 \%$ of the Canadian population with private drug insurance plans has access to anti-obesity medications. These medications are not covered under any provincial drug benefits programs either. Bariatric surgery is available to less than $0.54 \%$ or 1 in 183 of adults (with severe obesity) per year. Wait times for bariatric surgery range from 2-5 years and are the longest for any surgically treatable disease.

Conclusion: Obesity has not received official recognition as a chronic disease by the federal government, any of the provincial/territorial governments, despite the Canadian Medical Association's recognition. The lack of recognition of obesity as a chronic disease by public and private payers, health systems, the public, and media has a trickle-down effect on access to treatment. Obesity continues to be treated as a self-inflicted risk factor, which affects the type of interventions and approaches that are implemented by governments and health benefit plans.

References:

1 Lau DCW, et al. 2006 Canadian clinical practice guidelines on the management and prevention of obesity in adults and children. CMAJ: 2007;176(8):S1-S13.

2 Canadian Medical Association (2015). CMA recognizes obesity as a disease. https://www.cma.ca/En/Pages/cma-recognizes-obesity-as-a-disease.aspx.

Conflict of Interest: none disclosed

Funding: Research related to this abstract was funded by a grant from Novo Nordisk Canada.

\section{RS8:3}

\section{Tackling obesity: Turkey Experience}

Yardim, N.

Turkish Ministry of Health, Public Health Institute; Head of Obesity, Diabetes \& Metabolic Diseases Department

Introduction: Chronic diseases are also the most important cause of death and burden of disease in Turkey. The rate of obesity frequency was seen $20 \%$ for adults in 2014 health survey by TURKSTAT. In the COSI 2013 study obesity is found $22.5 \%$ among children aged $7-8$ (\%14.2 overweight); In school study obesity was found $30.2 \%$ (19.5\% overweight) in 11-15 years old. In the 18th Development Plan of Turkish Government the 21 st priority program is the wellness program which coordinator is the Ministry of Health. 
Since 2010, healthy nutrition and active living programs has been implemented. Diabetes control and salt reduction programs are implemented also.

Methods: Within the scope of obesity prevention in Turkey; studies, successes and challenges were shared.

Results: Healthy nutrition and active living program is implemented as the Prime Ministry circular and all sectors are concerned with this program. Studies are carried out on awareness studies; public and in-service training; regulations; developing guidelines, reorganization health services and monitoring.

Within awareness and advocacy studies, special days and weeks are celebrated nationwide; walking organisations are held every month in each province by health directorate. In 2015, 40,000; in 2016, 300,000 bicycle was provided for schools; municipalities and universities. Food and beverages standards was developed for school canteens, the sale of cola, fizzy drinks, chocolate, candy in school canteens was prohibited. Since 2012 in the second term of primary schools, school milk and raisin distribution program is applied. Fitnessgram card was improved in secondary schoo and high school. The Nutrition Guide for Turkey; National Physical Activity Guidelines and Physical Activity Guidelines in Chronic Diseases were prepared. In order to reduce the advertising and marketing pressure for children, the nutrition profile and application guide was prepared because of Law Radio and Television Supreme Council. Labelling regulation was updated in accordance with the EU. In primary health care services, obesity counselling units and wellness centres were established. Diets therapy is given by dieticians. Obesity counselling guideline was developed for family practitioner and nurses. One million were published and distributed. Trainings of trainer (ToT) were held. Obesity in children is monitored within COSI with WHO Euro. National Nutrition and Health Survey was conducted in 2010 and it will be conduct in autumn 2017.

The program was evaluated by WHO and the report was printed. Currently, www.saglik.gov.tr; and www.beslenme.gov.tr addresses, social media addresses of the ministry are actively used in public information and awareness.

Conclusion: Follow-up of school's regulations should be realized . A school lunch program should be developed. Trans fats should be eliminated. In order to increase physical activity in the community, the use of bicycles as a means of transportation should be increased and wide pavements with trees should be constructed by municipalities. Infrastructures of schools should be increased in terms of physical activity applications. Pay for prevention must be provided in health services.

\section{RS9 - Information Technology: A cause and a tool to treat obesity}

\section{RS9:2 \\ Developing tools for weight loss maintenance: The NoHoW toolkit}

Stubbs RJ.

School of Psychology, Faculty of Medicine and Health, University of Leeds

While evidence based weight management programmes (WMPs) are effective for initial weight loss the majority of those who engage with such programmes drop out and/or relapse in the longer-term. Current evidence suggests that behavioural support for weight loss maintenance (WLM) could be improved through theoretically informed, evidence-based approaches: (i) self-regulation of behaviour, self-efficacy and motivation may be helpful for longer-term maintenance, (ii) emotion regulation and stress management may help decrease drop out and improve coping with weight relapse, (iii) personalising solutions to meet individual needs in WMPs may improve programme adherence and (iv) scale could be maximized and cost minimized through us of digital technologies for WML. There are many digital offering for weight management but the most popular WLM apps and digital approaches appear to be less evidence based; those that are more evidence-based tend to be less popular. This is the concept of a broken market.

The NoHoW project aims to understand consumer practices in relation to WL and WLM and combine this knowledge with the most promising evidence for behaviour change to develop a new digital toolkit for WLM. The NoHoW toolkit combines aspects of behavioural self-regulation and motivation with emotion regulation and stress management and tracking technologies, delivered in 4 versions. The four versions of the toolkit will be evaluated in a $2 \times 2$ randomised controlled trial that will allow (i) formal evaluation of efficacy through primary and secondary outcome analysis and (ii) examination of moderators and mediators of WLM and weight relapse in a sample of 1002 European adults (iii) health economics analysis.

The results of this evaluation will help us better understand which evidence based components work and how they can be personalised to meet the needs of different people in WMPs. By working with commercial partners we aim to exploit the synergies between academia and industry (i) in developing a more refined and effective toolkit for consumer use and (ii) to enhance commercial offerings for WLM. These pathways to impact will accelerate development of digital solutions to maximize the reach of WLM solutions in the general population.

Conflict of Interest: R James Stubbs is a consultant to Slimming World through University of Leeds Consulting (Trading as Consulting Leeds).

Funding: This project has received funding from the European Union's Horizon 2020 research and innovation programme under grant agreement No 643309. 


\section{RS11 - Discussion Session: Obesity and the Food Industry - collaboration or conflict}

\author{
RS11:1
}

\section{Environmental drivers and food policy}

Peeters, $A$.

Deakin University, Melbourne, Australia

Today, an unhealthy diet is the largest modifiable contributor to ill health and death globally, with substantial impact on health, wellbeing and productivity. One of the key drivers of an unhealthy diet is the pervasiveness of unhealthy food and drink in our daily food environments. Our global lack of progress to date in improving population nutrition underscores the futility of building nutrition skills and education across the community in a context of unchanging food environments.

In this context there is a need for a comprehensive, multi-level and multi-setting approach to improving population nutrition. It is important that the policy approaches proposed to improve population nutrition align with key environmental drivers of unhealthy population diets. It is also critical that we think broadly about the various policy contexts for improving population nutrition, from policy set by national governments to that introduced by local community settings.

The evidence base for the feasibility, sustainability and effectiveness of such policy is growing but major gaps remain if we are to see widespread implementation of the comprehensive suite of policies required at scale. To effectively fill these gaps there is a need to include those involved in policy implementation in the development of the evidence base.

Conflict of Interest: None Disclosed

Funding Received: Research relating to this abstract was funded by the National Health and Medical Research Council in Australia

\section{RS11:2}

\section{How can we encourage change in the food industry to improve public health nutrition?}

Jebb, S.A.

Nuffield Department of Primary Care Health Sciences, University of Oxford

There is now widespread agreement that (i) the food environment is an important determinant of eating habits, (ii) improvements in public health nutrition cannot be achieved solely through public education messages and (iii) there is a need to engage the food industry to make it easier for individuals to achieve a healthier diet. The outstanding question is how best to encourage the changes required in business practices.

To date a range of approaches have been deployed including legislation aimed primarily at specific product reformulation goals, such as the Danish ban on trans fats or mandatory salt targets in South Africa. There are also examples of more diverse voluntary agreements, negotiated with industry by policymakers, as used in the Public Health Responsibility Deal in England or led by subgroups of the food industry, such as the US Healthy Weight Commitment. Other changes have resulted from concerted campaigning by consumer groups usually on very specific issues. There is also growing interest from investors in driving change; recognising the long term financial risk in some parts of the food sector from increased regulation, scrutiny by the public health community and changing consumer behaviour.

This presentation will look across these initiatives to consider what has been achieved and the obstacles to progress. It will use reductions in portion size of foods high in fat or sugar as a specific example to illustrate the complexity of aligning public health goals, commercial interests and public acceptability to achieve sustained change

Conflict of Interest: Chair of the Public Health Responsibility Deal Food Network in England (2010-2015)

Funding: University of Oxford

\section{RS11:3}

\section{Obesity and the Food Industry - collaboration or conflict: An industry perspective}

Black, $R$.

PhD, Principal at Quadrant D Consulting, Adjunct Professor Tufts University Friedman School of Nutrition Science and Policy

For the developed world, the packaged food \& beverage industry produces a majority of the food consumed. Within the broader food context, many argue that the most pressing nutrition driven public health issues are overweight \& obesity (with increased resin for a variety of disease endpoints), and excessive sodium consumption (with possible increased risk of stroke and cardio-vascular disease). Taken together, this has lead some to demand (and achieve) government intervention via regulations to change the diet with a goal to improve public health (for example, Mexico's tax on sugar sweetened beverages, various restrictions on advertising). This approach presumes: i) that our knowledge of what is consumed and why, is sufficiently deep, broad and robust to allow for informed policy decisions on what change is necessary; and ii) that partnering with food companies to address the issues is not possible and only regulations can change our food supply. Both of these assumptions can be challenged to varying degrees. If we accept that all who are involved in the food supply system (whether from a public health and/or industry position, that is public or private) have knowledge that is unique to their business / profession / experience, then we must also accept that an optimal solution can only be achieved when all of this knowledge is leveraged. We must learn to recognize those opportunities where public-private partnerships are unavoidable, where neither the private sector nor the public health sector can achieve success in the absence of the other. Failure to do so will surely lead to wasted efforts, ineffective or suboptimal solutions, and delay in reversing current dietary trends that contribute to these chronic diseases.

\section{RS12 - The Definition of Treatment Success: Lifecourse Perspective}

\section{RS12:1 \\ What defines success in the treatment of children with obesity?}

Baker, J.L.

Department of Clinical Epidemiology, Frederiksberg and Bispebjerg Hospital, Denmark

With more than $25 \%$ of children in Europe classified as overweight or obese, there is an incredible need for treatment. First and foremost, the goals of treatment are to reduce weight and to maintain this weight loss. Although there are numerous approaches to treatment, they almost always have these goals in common. However, there are several challenges in defining what constitutes a successful treatment. These challenges arise since the treatment of children with obesity occurs in many settings, is provided by a broad range of health personnel, varies greatly in its programme components, eligibility criteria, intensity, and duration, and is delivered to patients across a wide range of developmental ages (infants to adolescents). Additionally, as childhood obesity is a chronic disease, it is challenging to define an end to follow-up. In this talk, in addition to these challenges and their impact on defining treatment success, other key issues of which metrics are used to assess weight loss and the potential 
consideration of other health outcomes in defining treatment success will be explored.

\section{RS12:2}

\section{Defining treatment success: An adult life course perspective}

\section{Dicker, $D$.}

Israeli association for the study of Obesity, Hasharon Hospital-Rabin Medical Center

Obesity is a chronic and multifactorial disease that has a pivotal role in several health problems; hence, lifelong treatment is necessary and challenges are ongoing. Weight loss can be achieved by lifestyle changes, pharmacotherapy (1) and bariatric / metabolic surgery (2). Weight loss of $5-15 \%$ by means of lifestyle changes and / or pharmacotherapy has been shown to alleviate disease burden(3). Bariatric surgery has demonstrated that decreased morbidity and mortality correlates with the long term maintenance of $10-30 \%$ weight loss(4). In the SOS study, maintaining $18 \%$ weight loss after 20 years translated into reduced overall mortality and decreased cardiovascular, diabetes and cancer morbidity(4). Severa definitions for successful long term weight loss maintenance have been proposed. Wing and Hill defined successful weight loss as the maintenance of $10 \%$ or more of intentional weight reduction over one year According to this definition, in more than $20 \%$ of overweight / obese persons, successful weight loss can be maintained by means of lifestyle changes (5). Difficulties in long term weight loss maintenance can be attributed to non adherence to lifestyle and pharmacotherapy guidance, heterogeneity of treatment effect, the microbiome effect and mainly to physiological responses, such as metabolic adaptation, which counteract weight loss and promote weight regain. The changing perspective of obesity to an adipose-based chronic disease and the focus on complications may promote better understanding and a more successful approach to long term weight loss maintenance (6). This may be reached by incorporating lifestyle, cognitive support, new and effective medications, endoscopic solutions and bariatric / metabolic surgery into a comprehensive and early armamentarium to prevent weight regain.

Conflict of Interest: None

Funding: No Funding

References:

1 Johansson K, Neovius M, Hemmingsson E. Effects of anti-obesity drugs, diet, and exercise on weight-loss maintenance after a very-low-calorie diet or low-calorie diet: a systematic review and meta-analysis of randomized controlled trials. Am J Clin Nutr 2014;99:14-23.

2 Sjostrom L, Lindroos AK, Peltonen $\mathrm{M}$ et al. Lifestyle, diabetes, and cardiovascular risk factors 10 years after bariatric surgery. N Engl J Med 2004; 351: 2683-2693.

3 Garvey WT, Mechanick Jl, Brett EM et al. American association of clinical endocrinologists and american college of endocrinology comprehensive clinical practice guidelines for medical care of patients with obesity. Endocr Pract. 2016 Jul;22 Suppl 3:1-203.

4 Sjostrom L. Review of the key results from the Swedish Obese Subjects (SOS) trial - a prospective controlled Intervention study of bariatric surgery. J Intern Med 2013; 273: 219-234.

5 Wing RR, Hill JO. Successful weight loss maintenance. Annu. Rev. Nutr. 2001. 21:323-41.

6 Mechanick Jl, Hurley DL, Garvey WT . Adiposity-based chronic disease as a new diagnostic term: american association of clinical endocrinologists and the american college of endocrinology position statement. Endocr Pract. 2016 Dec 14. [Epub ahead of print].

\section{RS12:3}

\section{Ageing population - sarcopenic obesity}

Verdijk, L.B.

Maastricht University, Netherlands

Sarcopenia is defined as the age-related progressive loss of muscle function in the presence of loss of muscle mass, and has recently been recognized as an independent clinical condition in the International Classification of Diseases system. Sarcopenia is associated with physical/functional as well as metabolic deterioration, resulting in the loss of independence, reduced quality of life, and increased mortality. As such, sarcopenia culminates in ever increasing health care costs, and represents one of the major health care challenges in our aging society. Similar to sarcopenia, obesity is widespread, its prevalence increases with age, and its manifestation has a major impact on physical function, health, and the health care system. The combined presence of sarcopenia and obesity has been termed "sarcopenic obesity" and appears to act synergistically, thereby maximizing the functional and metabolic deterioration.

Muscle mass maintenance is determined by the intricate balance between muscle protein synthesis and muscle protein breakdown rates. It has been well established that physical activity and food intake (primarily protein intake) represent the two main anabolic stimuli affecting this balance and, as such, play an essential role in muscle maintenance throughout life. Though the etiology of sarcopenia is likely multifactorial, recent work suggests that a diminished response to protein intake and physical activity in elderly versus young individuals may be a key factor driving the loss of skeletal muscle tissue throughout the aging process. This phenomenon has been termed 'anabolic resistance'. Importantly, the presence of excess body fat (along with e.g. associated insulin resistance and inflammation) has multiple negative effects on muscle tissue, and may further enhance the anabolic resistance already present in senescent muscle.

In this lecture, an overview will be given on the process of sarcopenia, the role of muscle protein turnover in the development and treatment of sarcopenia, and the interaction with (sarcopenic) obesity. With a focus on the role of exercise and (protein) nutrition, treatment options will be discussed that aim to offset the imbalance between muscle protein synthesis and breakdown, on the long-term reaching a healthy body-weight, while maintaining or, if possible, improving muscle mass and function in order to support overall health in the aging population.

Conflict of Interest: None Disclosed

Funding: No Funding

\section{RS13 - Environmental Pollutants}

\section{RS13:1}

\section{Adipose tissue pollutants and obesity}

\section{Monteiro, R.; Teixeira, D.; Pestana, D.; Calhau, C.}

Departamento de Biomedicina, Unidade de Bioquímica, Faculdade de Medicina, Universidade do Porto, 4200-319 Porto, Portugal; Instituto de Investigação e Inovação em Saúde (I3S), Universidade do Porto, 4200-135 Porto, Portugal; Unidade de Saúde Familiar Pedras Rubras, Agrupamento de Centros de Saúde Maia-Valongo, 4470-105 Maia, Portugal; Nutrição e Metabolismo, NOVA Medical School, Faculdade de Ciências Médicas, Universidade Nova de Lisboa, Lisboa, Portugal; ProNutri - Clinical Nutrition \& Disease Programming, CINTESIS - Center for Health; Technology and Services Research, Porto, Portugal

The social and economical burden of obesity, due to its increasing prevalence and associated comorbidities has led to its consideration as a priority in health management. Apart from being of utmost importance in the genesis of obesity, the imbalance between calories ingested and energy expenditure explains only part of obesity etiology. Alongside with this, the mere increase in adipose tissue accumulation is not responsible for obesity-associated pathologies, which seem more dependent on location of adipose tissue accumulation, adipose tissue cellularity, and adipocyte dysfunction. Recently, the accumulation of lipophilic environmental pollutants on virtually all living organisms has been highlighted. With the acknowledgement that adipose tissue serves as the main reservoir for these compounds, came the recognition that their presence in that tissue goes beyond serving as main accumulation. In fact, adipose tissue constitutes one of the main targets of environmental toxicant actions. Evidence is being gathered, from different research groups, including our own, on the 
effects of adipose tissue pollutants and obesity and its comorbidities. In this regard, the growing production and release into the environment of chemical compounds from different chemical classes, along with human exposure from environmental and food sources bring an additional player in to the discussion of obesity origins. Furthermore, either acting locally or being released from their adipose tissue reservoir, these chemicals compounds, some of which have been demonstrated to possess endocrine-disrupting ability, impose pressure onto energy homeostasis regulation, predisposing to obesity and adipocyte dysfunction.

Conflict of Interest: None disclosed.

Funding: Research relating to this abstract was funded by FCT (Fundo Social Europeu, Programa Operacional Potencial Humano da EU (POPH); PEst-OE/ SAU/UI0038/2011, SFRH/BPD/40110/2007, SFRH/BD/46640/2008, SFRH/ $\mathrm{BD} / 64691 / 2009)$.

\section{RS14 - Pharmacotherapy}

\section{RS14:1 \\ Current drugs for obesity: From actual availability to future perspectives}

Van Gaal, L.F.

Department of Endocrinology, Diabetology and Metabolism, Faculty of Medicine, Antwerp University / Belgium

Obesity is becoming increasingly common and is recognized as a major public health problem worldwide. Overweight (BMI > 25) prevalence ranges from $30-50 \%$ and clear obesity $(\mathrm{BMI}>30)$ is also becoming increasingly prevalent.

It has been shown that an intentional weight reduction of 5-10 \% may lead to a marked improvement in cardiovascular risk factors and a substantial reduction up to $20-25 \%$ in co-morbidity. The $10 \%$ weight loss option has been reported to be associated with a reduction of $25-30 \%$ of visceral fat associated with an improvement of glucose tolerance (30\%), triglyceride levels (30\%), blood pressure (10\%) and factors of hemostasis and fibrinolysis (10-30\%). Although dietary approach and lifestyle adaptation remains the cornerstones of obesity therapy, long-term success is extremely disappointing - even after intensified therapeutic approaches, such as VLCD, dietary therapy, physical exercise and behavioral treatment.

Over the last years, obesity research has been focused on the exploration of new biochemical pathways and on new pharmacological intervention potentials. Large-scale, long term studies have demonstrated that the use of pharmacological agents are able to induce significant weight loss (5$10 \%)$ in addition to dietary/physical activity approaches and important reduction of co-morbidities as well, fulfilling the targets of 20-30\% risk reduction. Characteristics of an ideal anti-obesity agent include safe molecules without major side effects, promoting a weight reduction in a dose dependent manner, having long lasting effects not leading to addictive properties and/or toxicity.

Among anti-obesity agents in Europe, we have orlistat, the naltrexone/ bupropion combination and high dose liraglutide.

In the US we have additionally lorcaserin as a 5-HT2c serotonin agonist and the low dose phentermine/topiramate as approved drugs.

Orlistat as a lipase inhibitor has been used for many years in the $120 \mathrm{mg}$ formulation, as well as the $60 \mathrm{mg}$ OTC format. Orlistat shows modest weight reduction, has a beyond weight loss effect on LDL cholesterol and delays the onset of type 2 diabetes in obese individuals with prediabetes ( $37 \%$ risk reduction beyond lifestyle).

The tolerability is very good, except for the expected gastro intestinal side effects of steatorrhea in case of too high fat intake.

Bupropion is an anti-depressant, also used for smoking cessation; when used in combination with naltrexone, a $\beta$-opioid receptor antagonist, weight loss effects reach an absolute weight loss of 7-8 kg, depending on the patients studied, roughly $5.5 \%$ placebo substracted. It has an acceptable safety profile (a cardiovascular outcome trial is ongoing) but is un- fortunately, although approved, not yet available in the majority of the EU countries. In the injectable drug categories, liraglitude $3 \mathrm{mg}$ as a GLP-1 receptor agonist, recently became available: liraglutide offers an absolute weight loss, close to $10 \mathrm{~kg}$ (6-7 kg difference approx. when compared to placebo). From the extensive Scale trial program, it was shown liraglutide has a very good efficacy and safety; besides the well-known gastro intestinal adverse events (nausea, vomiting) it shows additional benefit on glucose tolerance (reduction of pre diabetes), on blood pressure and cardio vascular biomarkers. In a maintenance study after a LCD significant weight maintenance could be obtained.

Recently, the consistent findings of 3 years' observation have been published, focusing on both efficacy, tolerability and safety.

Future development perspectives of anti-obesity drug will very likely focus on combination therapy, mainly in the peptide field.

RS14:3

\section{Triple hyoglycaemic therapy as a novel therapeutic strategy in medical management of obesity}

Lipworth, S. '; Iftikhar, M. 2. Amir, Z. ; Balaratnam, J. ${ }^{3}$; Boparai, A. ${ }^{3}$;

Coppack, S. ${ }^{3}$; Emmanuel, J. ${ }^{3}$

${ }^{1}$ University of Oxford, ${ }^{2}$ Barts And The London School Of Medicine, ${ }^{3}$ Barts Health Nhs Trust

Intruduction: Obesity affects just over a quarter of adults in England (Public Health England, 2016). There is an urgent need for novel evidence based therapeutic regimes to underpin the emerging field of medical management of obesity. Recent randomised placebo controlled trials (RCTs) have shown the glucagon-like peptide-1 agonist (GLP1) liraglutide to be efficacious in a dose dependent manner for weight loss in both diabetics and non-diabetics. In diabetic patients Sodium-glucose co-transporter 2 inhibitors (SGLT2i) are associated with weight loss which is significantly increased when they are used in conjunction with a GLP1 agonist. Use of hypoglycaemic agents in combination with the primary aim of weight loss is currently not widespread practice.

Methods: 504 patient records were examined between January 2014 and September 2016. Of these 202 met the inclusion criteria of being in one of the treatment groups and having at least two BMI readings available. Data was treated non-parametrically and corrections for multiple comparisons performed using the Bonferonni method. Mean and median time adjusted ratio of BMI change was calculated for each group. Groups were as follows: Metformin alone, metformin + GLP, metformin + SGLT2i, or metformin + GLP + SGLT2i (triple).

Results: There was no statistically significant difference between baseline characteristics of the cohort between the treatment groups as determined by one-way ANNOVA. Our cohort contains a disproportionately large number of females $(66.3 \%)$ and all groups had a mean and median first BMI recording $>40 \mathrm{Kg} / \mathrm{m}^{2}$ (Class III obesity). There was a significant difference between distributions of \% change in BMI per year between the treatment groups $(\mathrm{p}=0.030)$. An inspection of pairwise tests revealed a significant comparison between triple therapy and metformin (median $\%$ BMI change per year 1.74 vs $-0.495, \mathrm{p}=0.004$ (0.04 after adjustment for multiple comparisons)). None of the other pairwise comparisons were significant (median \% BMI change 1.91, 1.37 for Metformin + GLP/Metformin + SGLT respectively). Patients on metformin as single therapy gained weight (median increase $0.5 \%$ in BMI per year).

Conclusion:Our results are important as they highlight the potential use of SGLT2i/GLP combinations in obese patients for whom surgery is not viable and other interventions such as lifestyle changes alone or medication such as Orlistat are inadequate. They are also notable because our cohort suffers from more severe obesity than those from recent RCTs. Further expansion and analysis of this dataset is likely to strengthen the case for an RCT looking at combination hypoglycaemic therapy in obese patients.

We have no conflicts of interest to declare. 
RS14:4

Naltrexone/bupropion is well tolerated and had no effect on serious adverse events in participants receiving antidepressant medication, including SSRIs, in a large randomized double-blind study

\section{Halseth, A. ${ }^{1}$, Gilder K. ${ }^{1}$, Shan K. ${ }^{1}$, Acevedo L. ${ }^{1}$, Smith $S .{ }^{2}$}

${ }^{1}$ Orexigen Therapeutics, Inc., La Jolla, CA, USA, ${ }^{2}$ Florida Hospital Translational Research Institute For Metabolism And Diabetes, Orlando, Fl, Usa

Introduction: Extended-release naltrexone $32 \mathrm{mg} /$ bupropion $360 \mathrm{mg}$ (NB) is approved for chronic weight management as an adjunct to diet and physical activity. This study assessed the effect of NB on cardiovascular (CV) events in overweight/obese participants at elevated CV risk. Randomized participants (NB or placebo [PBO]) received an internet-based weight management program. Participants were required to lose $\geq 2 \%$ body weight at 16 weeks, without a sustained increase in blood pressure, to continue study drug.

Methods: This study was terminated early after the second interim analysis, which corresponded to $50 \%$ of the primary endpoint data being collected. Data on CV endpoints were previously published. The current analyses focused on adverse events in participants on antidepressants at baseline, as they were excluded from Phase 3 trials.

Results: The intent-to-treat (ITT) population ( $\mathrm{PBO} \mathrm{N}=4450$, NB $\mathrm{N}=4455$ ) was $54.5 \%$ female, $83.5 \%$ white, mean age of $61 \mathrm{yrs}$, mean BMI $37.3 \mathrm{~kg} / \mathrm{m}^{2}, 22.8 \%$ with a history of depression, $23.1 \%$ on antidepressant medication, including $15.4 \%$ on SSRI. The incidence SAEs in participants receiving antidepressants was similar between NB (10.7\%) and PBO $(9.9 \%)$ and was also similar to that observed in the overall population $(9.5 \% \mathrm{NB}, 8.1 \% \mathrm{PBO})$. SAEs in those on SSRIs were similar between NB (10.1\%) and PBO (9.4\%). For those on antidepressants or just SSRIs, AELDSDs were similar to those observed in the overall population and were primarily GI disorders.

Conclusion: Obesity increases the risk of developing depression. Data from these analyses demonstrate that for participants taking NB and antidepressants, including SSRIs, there is a similar adverse event profile as the overall population and revealed no evidence of an additional health risk with combined use.

Conflict of Interest: Some authors are employees of Orexigen Therapeutics, Inc.

Funding: Research relating to this abstract was funded by Orexigen Therapeutics, Inc.

\section{RS14:5}

Effect on body weight of naltrexone/bupropion in overweight and obese participants with cardiovascular risk factors in a large randomized double-blind study

Halseth, A. ${ }^{1}$ Gilder K. ${ }^{1}$, Shan K. ${ }^{1}$, Acevedo L. ${ }^{1}$, Buse J. ${ }^{2}$

${ }^{1}$ Orexigen Therapeutics, Inc., La Jolla, CA, USA, ${ }^{2}$ University Of North Carolina School Of Medicine; Chapel Hill, Nc, Usa

Introduction: Extended-release naltrexone $32 \mathrm{mg} /$ bupropion $360 \mathrm{mg}$ (NB) is approved for chronic weight management as an adjunct to diet and physical activity. This study assessed the effect of NB on cardiovascular (CV) events in overweight/obese participants at elevated CV risk. Randomized participants ( $\mathrm{NB}$ or placebo [PBO]) received an internet-based weight management program. Participants were required to lose $\geq 2 \%$ body weight at 16 wks, without a sustained increase in blood pressure, to continue study drug.

Methods: This study was terminated early after the second interim analysis, which corresponded to $50 \%$ of the primary endpoint data being collected. Data on CV endpoints were previously published. The current analyses focus on change in body weight.

Results: The intent-to-treat (ITT) population ( $\mathrm{PBO} \mathrm{N}=4450$, NB $\mathrm{N}=4455$ ) was $54.5 \%$ female, $83.5 \%$ white, mean age 61 yrs, mean BMI $37.3 \mathrm{~kg} / \mathrm{m}^{2} ; 85.2 \%$ had T2DM, 32.1\% had CV disease. At $52 \mathrm{wks}$, the ITT-LOCF analysis showed greater least squares mean percent change in body weight $(\mathrm{LSM} \% \Delta \mathrm{BW})$ with $\mathrm{NB}(-3.1 \%$; $95 \% \mathrm{CI}-4.8,-1.4)$ vs $\mathrm{PBO}$ $(-0.3 \% ; 95 \%$ CI $-1.9,1.4)$. Both groups lost more weight in an ontreatment analysis (NB [-7.3\%] and PBO [-3.9\%]). The odds ratios of $5 \%$ and $10 \%$ weight loss were 3.3 and 4.1, respectively, in NB over PBO. At 104 wks, the on-treatment $\mathrm{LSM} \% \Delta \mathrm{BW}$ was $-6.3 \%$ with $\mathrm{NB}(\mathrm{n}=1137)$ vs $-3.5 \%$ with PBO $(\mathrm{n}=741)$. Major reasons for NB withdrawal were adverse events (AE, 29\%) and patient decision (21\%), with GI disorders being the most common AEs leading to NB withdrawal.

Conclusion: Weight loss with NB in this study, in an older population predominantly with diabetes and elevated CV risk, was somewhat lower than that observed in overweight/obese participants without T2DM and similar to participants with T2DM in Phase 3 studies.

Conflict of Interest: Some authors are employees of Orexigen Therapeutics, Inc.

Funding: Research relating to this abstract was funded by Orexigen Therapeutics, Inc.

\section{RS15 - EASO Knowledge Gap Session. Household Food Insecurity: Where are we now - lessons learnt?}

\section{RS15:2}

\section{Food insecurity and aging during economic crisis}

Graça, P.1,2; Gregório, M.J. ${ }^{1,2,3}$

${ }^{1}$ Directorate-General of Health, Portugal; ${ }^{2}$ Faculty of Nutrition and Food Sciences of University of Porto, Portugal; 3 Escola Superior de Leiria do Instituto Politécnico de Leiria; 4 EpiDoC Unit, Centro de Estudos de Doenças Crónicas (CEDOC) da NOVA Medical School, Universidade Nova de Lisboa (NMS/UNL), Lisboa, Portugal

Introduction: Food insecurity (FI) has received much attention in recent years in high-income countries due to the increasing of poverty and social inequalities indicators, as a result of the global economic crisis. In Europe, since 2009, it was observed an increasing trend in food insecurity. Data from EuroStat database showed that the prevalence of food insecurity in Europe increased 2.2 percent points between 2009 and 2012, despite the decreasing trends until 2010

Methods: Data derived from the national FI survey in Portugal - INFOFAMÍLIA Survey - conducted by the Directorate-General of Health. INFOFAMÍLIA survey was a cross-sectional study in a sample of users of primary health care. A systematic random sampling was performed, in which one in five users aged more than 18 years old that resorted to the national health system in (Health Centres) was recruited, during the collection periods. Data were collected using face-to-face interviews by nurses in primary health care and household. Data analysed includes data from four surveys, conducted between 2011-2014. FI was evaluated using a psychometric scale adapted from the Brazilian Food Insecurity Scale and data were collected by face-to-face interviews.

Results and discussion: This national survey on food insecurity, implemented in 2011 in order to evaluate food insecurity trends during the period that Portugal was under the International Monetary Fund financial assistance program (2011-2014), found a high prevalence of food insecurity in the Portuguese population. In 2014, 45.8\% of the Portuguese households included in this study were food insecure.

A higher risk of food insecurity was observed in the ages groups of 30-64 years, when comparing to adults aged 65 and over. Data from Infofamília survey in Portugal are consistent with results obtained in other countries. Food insecurity have been less well studied in elderly, however studies about food insecurity in USA and Canada suggested that food insecurity is less prevalent among this age group. The social support mechanisms available for elderly might explain this result. Moreover, food insecurity in elderly could be underestimated because of this age group self-perception. 
Conclusion: Despite these results, food insecurity among older adults should be considered as an important public health problem and a priority action for nutrition policies.

Food insecurity in elderly might be associated with poor health conditions, such as functional impairments, obesity and other chronic diseases that are more prevalent in this group.

Conflict of Interest: None

Funding: No Funding

\section{RS15:4}

\section{Ireland's 'Let's take on childhood obesity 2013-2016'}

\section{Nolan, C. F. 1}

\section{'Safefood, Ireland}

Introduction: Ireland is on track to become the most obese nation in Europe1. Childhood obesity has complex origins and progress in tackling the issues has to date, been slow2. One in four Irish children is either overweight or obese with most recent data indicating that rates may have stabilised3. Safefood (the all-island Food Safety Promotion Board), coordinated a three year integrated multimedia campaign based on six practical messages (3 diet-related)

Methods: A nationally representative fact to face survey was undertaken pre-campaign $(n=909)$ and post-campaign $(n=935)$ of adults with children under 12 years of age. The digital and print media impacts of the campaign were also recorded.

Results: Most parents (77\%) felt that they were primarily responsible, the majority $(70 \%)$ saw a health professional as the point of contact and less than half $(45 \%)$ were aware of the health consequences of overweight in childhood.

The diet related messages achieved some traction. The majority (68\%), had attempted to reduce sugary drink intake in the past year and there were a reported increase of $10 \%$ to $36 \%$ of drinking water only with meals. Two thirds (67\%) of parents reported giving their child(ren) a half adult portion but $30 \%$ stated that they didn't mind how much 'healthy food' they ate.

The daily intake of treat foods actually increased to $40 \%$ during the 3 year period and $53 \%$ of parents gave these as a reward for good behaviour. Only $27 \%$ of parents tried to increase hours of sleep but $57 \%$ of parents reported reducing screen time to less than 2 hours per day. There was also a $5 \%$ improvement in children achieving 1 hours of physical activity daily - up to $54 \%$ respondents.

There was strong engagement on digital media channels with over 750,000 Safefood website visits and almost 3 million organic Facebook views.

Conclusion: The comprehension and uptake of the diet-related messages were better than that for the sleep, screen and play ones. There was a social class gradient campaign response which mirrors that of the childhood obesity pattern globally. Further collaboration with primary care health professionals is indicated.

\section{References}

1 The Lancet journal, Volume 387, No. 10026, p1377-1396, 2 April 2016: Trends in adult body-mass index in 200 countries from 1975 to 2014: a pooled analysis of 1698 population-based measurement studies with 19.2 million participants. NCD Risk Factor Collaboration (NCD-RisC) $\dagger$

+NCD Risk Factor Collaboration members are listed at the end of the paper

2 World Health Organization (2015a) Draft Final Report of the Commission on Ending Childhood Obesity, available at: http://www.who.int/end-childhood-obesity/commission-ending-childhood-obesity-draft-final-report-en. pdf

3 The Childhood Obesity Surveillance Initiative (COSli) in The Republic of Ireland: Findings from 2008, 2010 and 2012, Dr Mirjam Heinen, Dr Celine Murrin, Professor Leslie Daly, Dr John O'Brien, Dr Patricia Heavey, Ms Jean Kilroe, Ms Michelle O'Brien, Ms Helena Scully, Ms Lisa-Marie Mulhern, Ms Adrienne Lynam, Dr Catherine Hayes, Ms Ursula O'Dwyer, Dr Nazih Eldin, and Professor Cecily Kelleher http://www.ucd.ie/t4cms/COSI\%20report\%20 (2014).pdf

\section{RS16 - EASO COTF Session: Interventions in Childhood Obesity}

\section{RS16:2}

\section{Addressing comorbidities with activity and exercise}

\section{Jorge Mota \\ Ciafel-Fadeup}

The high prevalence of sedentary behavior and physical inactivity is a major health risk and a leading cause of death worldwide. Several population studies worldwide showed that around two-thirds of youngsters are considered to be overweight or obese, generating a global obesity epidemic. Obesity comorbidities are also an extension of this problem and it has become critical to highlight the necessity of self-care and healthy living habits. Exercise is a viable way to help prevent and combat many chronic non-communicable diseases such as obesity. However, both the public health and education sectors have carried out several initiatives aimed at preventing childhood obesity. Nonetheless, the results of this action are controversial and note of all of those initiatives have succeeded in producing reductions in the prevalence of overweight and obesity in targeted groups. Thus, on this presentation we are looking forward to highlight the best available evidence regarding the effects of exercise, namely the type and intensity, on obesity and related comorbidities considering different domains (eg: transportation) and settings (eg. Schools) trying to emphasizes the important role of preventive measures in changing the obesogenic environment taking into consideration the role of different actors and policies.

Conflict of Interest: None

Funding: No Funding

\section{RS16:3}

\section{Altered glucose metabolism in childhood obesity: Treating a diverse phenotype}

Santoro, $N$.

Yale University, Department of Pediatrics

Prediabetes and type 2 diabetes usually manifest during mid-puberty together with a peak of transient insulin resistance. In healthy adolescents, transient insulin resistance is balanced by an increase in insulin secretion. In obese adolescents pancreatic beta cells are not always able to cope with the rising insulin resistance, this would result in a progressive failure of the beta cell to balance the insulin resistance. Notably, beta-cell failure in youth occurs faster than in adults; whereas in adults the transition towards type 2 diabetes takes about 10 years with roughly $7 \%$ yearly reductions in beta-cell function in obese adolescents beta cells deteriorate at a rate of roughly $15 \%$ per year, with a mean transition time from prediabetes to overt diabetes of about 2,5 years.

Recent studies in obese children and adolescents have also elucidated that the onset and the progression of the disease is influenced not only by the progressive impairment of beta cells, but also by the presence of non alcoholic fatty liver disease (NAFLD). In fact, several studies clearly indicate that there is a deleterious effect of intra-hepatic fat accumulation on insulin sensitivity at a multi-organ level. In general, obese children and adolescents with NAFLD tend to be more insulin resistant than age and sex matched pairs and tend to show an adverse metabolic pattern characterized by dyslipidemia and adverse changes in glucose metabolism.

Overall, recent clinical studies clearly indicate that impaired glucose metabolism in pediatrics is a more severe and complex disease than in adults and therefore needs a more thorough investigation in order to tailor novel therapies more appropriate to the severity of the disease at this young age. References:

1 Tuomi T, Santoro N, Caprio S, Cai M, Weng J, Groop L. The many faces of diabetes: a disease with increasing heterogeneity. Lancet. 2014; 383: 1084-94. 
2 D'Adamo E, Cali AM, Weiss R, Santoro N, Pierpont B, Northrup V, Caprio S. Central role of fatty liver in the pathogenesis of insulin resistance in obese adolescents. Diabetes Care. 2010; 33: 1817-22.

O’Malley G, Santoro N, Northrup V, D’Adamo E, Shaw M, Eldrich S, Caprio S. High normal fasting glucose level in obese youth: a marker for insulin resistance and beta cell dysregulation. Diabetologia. 2010; 53:1199-209.

Nowicka P, Santoro N, Liu H, Lartaud D, Shaw MM, Goldberg R, Guandalini C, Sa voye $\mathrm{M}$, Rose $\mathrm{P}$, Caprio S. Utility of hemoglobin A(1c) for diagnosing prediabetes and diabetes in obese children and adolescents. Diabetes Care. 2011; 34: 1306-11

\section{Saturday, 20 May 2017}

\section{RS17 - Physical Activity Strategies}

\section{RS17:1}

\section{Physiology of sedentary behaviour - uncovering the muscle proteomics}

Duarte, J.A

Ciafel, Faculty of Sport, University of Porto, Portugal

The word "sedentary" derives from the Latin word "sedentariu", which in tends to qualify someone who spends a lot of time sitting. Sedentary is invariably linked to a reduction of functional demands imposed to skeletal muscles and to organic systems responsible for the reestablishment of systemic homeostasis, such as cardiovascular and respiratory systems. This situation, when acute, transient, and sporadic, is physiologically favourable, because it facilitates the reestablishment of homeostasis (at muscular and systemic level) lost during previous physical efforts. However, when regular, the long-lasting low functional requirement favours, at the cellular level in each involved organ, a progressive and irreversible loss of molecular redundancy, which compromises the efficiency of cell signalling pathways and the organ maximal functionality. Regarding skeletal muscle, this chronic low functional exigency imposed to muscle fibbers favours the changes in the contractile apparatus, with less abundant myofibrillar and cytoskeletal proteins, in parallel with a reduced amount of enzymes involved in oxidative energetic metabolism and in the cellular antioxidant defence. Moreover, the molecular mechanisms responsible for the maintenance of the quality of intracellular proteins and organelles are also neg atively affected, with a weakened expression of some chaperones involved in cell stress response as well as proteins involved in detoxification and cellular protective functions, conditions that promote the accumulation of aggregated (dysfunctional) proteins and the enhancement of mitochondrial heteroplasmy, with loss of mitochondrial maximal function. It must be highlighted that this low chronic functional demands of skeletal muscle also compromises its hormonal production, with negative repercussions in the target organs which influence the occurrence of metabolic dysfunction/diseases. In summary, the sedentary behaviour limits the ability of local and systemic homeostatic control mechanisms that restrict the adaptive capacity to tolerate episodes of increased demands during daily life, such as food intake, dehydration, physical exercise, and infections, among others, and predisposes the subjects to dysfunction conditions, with increased risk of disease and premature death. In other words, sedentary life-style seems to accelerate the biological aging process.
RS17:2

Promoting Physical Activity and Reduced Sedentary Time in Football Clubs: Lessons From EuroFIT

Teixeira, P. J. ' ; Silva, M. S. '; Gray, C. M. ${ }^{2}$; Hunt K. ${ }^{3}$; Ploeg, H. ${ }^{4}$; Roberts, G. ${ }^{5}$; Bunn, C. ${ }^{6}$; Nassau, F. ; Andersen, E. ${ }^{8}$; Wyke, S. ${ }^{2}$

${ }^{1}$ CIPER Self-Regulation, Faculty of Human Kinetics, University of Lisbon, Portugal; ${ }^{2}$ Institute of Health and Wellbeing, College of Social Sciences, University of Glasgow, UK; ${ }^{3} \mathrm{MRC} / \mathrm{CSO}$ Social and Public Health Sciences Unit, Institute of Health and Wellbeing, University of Glasgow, UK; ${ }^{4}$ Department of Public and Occupational Health, and EMGO Institute for Health and Care Research, VU University Medical Center, Netherlands; ${ }^{5}$ Norwegian School of Sport Sciences, Department of Coaching and Psychology, Norway; ${ }^{6}$ Institute of Health and Wellbeing, College of Social Sciences, University of Glasgow, UK; ${ }^{7}$ Department of Public and Occupational Health, and EMGO Institute for Health and Care Research, VU University Medical Center, Netherlands

Purpose: The European Fans in Training (EuroFIT) program was delivered in elite professionals football clubs in four European countries and aimed to engage overweight and obese men in initiating and maintaining improvements in physical activity, sedentary, and dietary behaviours. It seeks also to overcome the 'know-do' gap in the implementation of public health programs, by developing, in partnership with stakeholders, policy-makers and end-users, a set of validated guidelines to maximize replication beyond the funded project.

Methods: Pragmatic randomised controlled trial comprising a gender-sensitized 12-week lifestyle program in 1200 middle-aged (30-65y) men in Portugal, Norway, the Netherlands, and the UK. The program was led by club coaches, and affiliation to clubs leveraged to extend reach to 'hard-toreach' men, and increase group relatedness, interest in the program, and adaptive motivation for behaviour change. Real-time, self-relevant feedback on step count and sedentary time was provided via an accelerometer (SitFit) and challenge and connection to other participants promoted with a proprietary app (MatchFit). Analyses of effectiveness seeking how well it works and by which mechanisms it works and cost-effectiveness will be conducted. An extensive process evaluation is also embedded in the RCT assessing implementation, mechanisms, and context, seeking to build an evidence base that informs policy and practice.

Results: The EuroFIT RCT involved 15 participating clubs. Perspectives from sociology and gender studies, Self-Determination Theory and Achievement Goal Theory were integral components of the intervention and measurement protocol. A key strength of the EuroFIT project is the existing and developing relationships with all partners, through participative, inclusive, approaches to designing, delivering and scaling up the program. It follows an iterative, action research style approach conductive to guidelines and a licensing system firmly embedded in experience, allowing replication with fidelity in delivery

Conclusion: The European Fans in Training project development was framed to address two of the major problems in health promotion research and translation: Long-term sustainability of health-related behavioural changes and the scaling-up of research-based interventions, reaching a population impact.

\section{RS17:3}

\section{Has sport a role to play in preventing obesity? The Healthy Stadia initiative}

Ireland, $R$

'Director of the European Healthy Stadia Network

Introduction: Whilst professional athletes are often portrayed as being at the pinnacle of physical health, the spectator side of professional sport has long been associated with the promotion of highly processed food and drink and alcohol both at mega events (1) and in community sport (2). The coverage of sport both for those in attendance and those observing through the media may be considered as contributing to the obesogenic environment across Europe therefore. But can sport play a more produc- 
tive role? This paper will provide some more positive examples of the role sport can play using the example of the Healthy Stadia movement.

Healthy Stadia: Public health has long been concerned about sport's commercial links. In contrast, Healthy Stadia (3) was developed as a settings approach to "promote the health of visitors, fans, players, employees and the surrounding community" in North West England in 2005. Since then, Healthy Stadia has developed tools and programmes working with both individual stadia and governing bodies across Europe and has been able to promote health benefitting both sport itself and its many beneficiaries. The presentation will include examples of good practice such as working with UEFA and its European football tournaments on the promotion of active travel to and from host venues, and European Fans in Training (EuroFIT [4]), a physical activity and sedentary time reduction programme for overweight football fans being piloted at 15 European football clubs. Much of Healthy Stadia's work in promoting healthier eating and physical activity can be seen to impact on obesity.

\section{References:}

1 Garde A, Rigby N. Going for gold - Should responsible governments raise the bar on sponsorship of the Olympic Games and other sporting events by food and beverage companies? Communications Law. 2012;Vol.17(2):42-49.

2 Kelly B, Baur LA, Bauman AE, King L, Chapman K, Smith BJ. Food and drink sponsorship of children's sport in Australia: who pays? Health Promotion International. 2010:Vol.26(2):188-195.

3 European Healthy Stadia Network: http://www.healthystadia.eu/. Last accessed: 16.02.17.

4 European Fans in Training: http://wwweurofitfp7.eu Last accessed: 16.02.17.

Conflict of Interest: Robin Ireland is a Non-Executive Director of the European Healthy Stadia Network.

Funding: No Funding was received for this abstract.

\section{RS18 - Debate: Challenges of Managing Obesity-Related Disorders}

\section{RS18:1}

\section{Arterial hypertension}

\section{Jordan, J.}

Institute of Aerospace Medicine, German Aerospace Center and Chair of Aerospace Medicine, University of Cologne, Cologne, Germany

Arterial hypertension is one of the most common comorbidities in obese individuals. Moreover, obesity and arterial hypertension are additive in terms or cardiovascular risk. Obese patients require more medications to have their blood pressure controlled and are prone to treatment resistant arterial hypertension. Data from good quality clinical trials on the utility of weight loss in the management of hypertension is surprisingly scarce and the available data is rarely translated into clinical practice. Information on how to combine weight loss interventions with antihypertensive medications, which are ultimately required in many patients, is virtually non-existent. Antihypertensive treatment recommendations for lean hypertensives may not be simply extrapolated to obese hypertensives and have to take into account the metabolic comorbidities, particularly insulin resistance and dyslipidemia. Given their positive metabolic profile, renin angiotensin system inhibitors are a good choice for the treatment of obese hypertensive patients. Yet, most patients will require antihypertensive combination therapy. Dihydropyridine calcium channel blockers are a good choice as combination partner. From an antihypertensive point of view, low dose thiazide diuretics are an excellent combination partner for renin angiotensin system inhibitors while potential metabolic side effects may occur. Beta-blockers while efficiently lowering blood pressure can lead to significant weight gain. In obese patients not achieving target blood pressure with first line drugs, mineralocorticoid receptor antagonists may be particularly useful. However, there is a substantial risk for hyperkalemia, particularly in patients with type 2 diabetes mellitus
RS18:3

\section{The role of stress (hormones) in obesity}

\section{van Rossum, E.F.C.}

Department of Internal Medicine, division of Endocrinology, Erasmus University Medical Center, and the Obesity Center CGG, Rotterdam, the Netherlands

An imbalance between energy (food) intake and energy expenditure is currently regarded as the major cause of obesity with multiple contributing environmental and genetic factors. However, evidence is now mounting that stress, and in particular the stress hormone cortisol is a key player in this pandemic.

In pathological conditions such as Cushing's disease or use of high doses of exogenous corticosteroids, increased corticosteroid exposure causes all components of the metabolic syndrome (MetS) and cardiovascular disease (CVD), as well as depression.

It is known that corticosteroids can 1. increase appetite with a preference for energy-dense food ("comfort food"), 2. cause a redistribution of white adipose tissue to the abdominal region, and 3. suppress the activity of brown adipose tissue, resulting in abdominal obesity and its adverse metabolic sequelae. Interestingly, coinciding with the rise in obesity, MetS and CVD, the intake of food with a high glycemic index and levels of stress have increased, while the average hours of sleep has decreased. These are all factors known to induce an increase in daily cortisol production. From this perspective, it could be postulated that a continuous loop may exist between obesity, unhealthy lifestyle and increased cortisol, which maintain or worsen the obese condition, and may counteract weight loss. Scalp hair cortisol analyses provide a relatively novel way to study the relationships between cortisol and obesity and its cardiometabolic sequelae, now clearly showing that on average systemic cortisol levels in adults and children with obesity are elevated. Importantly, mechanisms of underlying increased cortisol exposure in obesity will be discussed, as well as potential new stress(hormone) reducing treatment targets.

\section{RS19 - Nutrition and Foetal Programming}

\section{RS19:1}

\section{Epigenetics and the environment: Implications for obesity and metabolic disease}

\section{Constância, $M$.}

University of Cambridge, Metabolic Research Laboratories, MRC Metabolic Diseases Unit and Department of Obstetrics \& Gynaecology, Cambridge CB2 OSW, UK

The 'term' epigenetics (which literally means 'on top' of genetics) is commonly used to describe the study of stable alterations in gene expression that cannot be explained by changes in DNA sequence. Epigenetic processes are essential for development and differentiation but may also arise in later life, either by random change of under the influence of the environment. 'Environmental' epigenetics is a theme that evokes a great deal of excitement because epigenetic alterations are involved in environmentally triggered phenotypes that have long-lasting effects on metabolism and health (sometimes even in subsequent generations). Thus, epigenetic mechanisms such as DNA methylation, histone modifications and non-coding RNAs, including small RNAs, seem to allow an organism to respond to the environment through changes in gene expression. In this talk I will review the evidence, from work on model systems, which shows that environmental effects can provoke epigenetic responses. I will consider critically our current knowledge of the epigenetic responses that are brought about by nutrition, but also chemical pollutants and external stresses across the life-course. The following key questions will be discussed: Do epigenetic changes actually contribute to environmentally induced phenotypes? What do we know about the mechanistic links between the environmental triggers and the epigenetic alterations? Which are the developmental and metabolic phenotypes that are associated with 
environmentally induced epigenetic responses? How should data from experimental models be extrapolated to humans? What are the implications of environmentally induced epigenetic alterations for human health, in particular obesity and type 2 diabetes, and what could be the impact for intergenerational risk of metabolic disease?

\section{RS19:2}

\section{Learnings from the DALI project}

\section{Desoye, G.; on behalf of the DALI consortium}

Medical University of Graz

Introduction: Obesity in pregnancy is a risk factor for gestational diabetes mellitus (GDM). GDM is associated with adverse outcomes and increased long term risk for features of the metabolic syndrome in mother and offspring including obesity. Therefore, GDM prevention is a major public health goal, but successful interventions have not been found. In a quest to tackle the current obesity burden by reducing GDM, the DALI project was set up. At its core is a pan-European multicenter RCT testing different approaches for the reduction of GDM risk. This was accompanied by a health economic evaluation of costs vs benefits of the interventions.

Methods: Multicenter RCT with factorial design conducted in 9 European countries in 2012-2015. Women $(n=1035)$ with a BMI $\geq 29$ were enrolled in week 15 of their pregnancy. After an oGTT to exclude pre-existing women with insulin resistance, the women $(n=436)$ were randomized to either a control group, healthy eating (HE), physical activity (PA) or HE + PA groups. They underwent an intensive coaching program based on motivational interviewing principles (BMC Pregn Childb 2013:13, 142) including 5 face-to-face and up to 4 phone coaching sessions. At weeks $24-28$ and 35-37 oGTTs were performed and women filled PA and dietary questionnaires to assess intervention effectiveness. Primary outcomes were gestational weight gain at 35-37 weeks (GWG) and fasting glucose and insulin resistance at 24-28 weeks. Secondary outcomes included, among others, fasting insulin concentrations, GDM, birth weight and small (SGA) or large-for-gestational age (LGA) babies. In addition quality adjusted life years (QALYs), and societal costs were assessed.

Results: $23 \%$ of the women were excluded because of pre-existing insulin resistance. These women were older, with higher pre-pregnancy BMI, blood pressure, triglycerides and free fatty acids, higher insulin resistance and secretion, but lower disposition index. In the HE+PA group $(\mathrm{n}=108)$ but not in the HE $(\mathrm{n}=113)$ or PA $(\mathrm{n}=110)$ group alone, women achieved substantially less GWG than controls $(\mathrm{n}=105)$ by $35-37$ weeks $(-20.2$ $(95 \%$ CI $-3.58 ;-0.46 \mathrm{~kg})$ ). Despite this reduction there were no improvements in fasting or post-glucose load glucose or insulin concentrations or IR. Birthweight as well as SGA and LGA rates were similar. The HE\&PA intervention was cost-effective for quality-of-life adjusted life-years (QALYs) at delivery, predominantly caused by a reduction in delivery-related costs. Neither the HE\&PA intervention, nor any of the other interventions, was cost-effective for fasting glucose or insulin resistance.

Conclusion: The DALI Lifestyle Study clearly shows for the first time that a combined physical activity and healthy eating intervention is the intervention of choice among overweight/obese pregnant women over either intervention alone for both effectiveness and cost effectiveness. Although confirming an inability to prevent GDM, the intervention was still associated with health care savings largely by reducing delivery costs. This lends support for a broad implementation of this intervention.

Conflict of Interest: None disclosed

Funding: Research related to this abstract was funded by grant 242187 of the European Commission within FP7-Health

\section{PLENARY SESSION}

\section{Wednesday, 17 May 2017}

\section{PL 1 - Obesity as a disease}

PL1:2

\section{The challenges in defining obesity}

Schindler, K. ', Ludvik, B.',

${ }^{1}$ Medical University Vienna, Div. Endocrinology and Metabolism, Vienna, Austria 'Dept. of Medicine 1 and Karl Landsteiner Institute for Obesity and Metabolic Disorders, Rudolfstiftung Hospital, Vienna, Austria

The Body Mass Index was established in the 1950s by Ancel Keys. It is simple to measure and to calculate. The easy application is an advantage for the use in epidemiological studies. BMI has a good correlation with body fat and is used to classify a person as under-, normal-, overweight or obese. Several studies have shown the problematic aspects of using solely BMI to define obesity and the associated health risk. BMI does not distinguish between fat mass and lean body mass. Consequently, BMI does not capture differences in body composition related to ethnicity, sex, age and physical performance. There is evidence that waist circumference is superior in assessing the cardiovascular risk associated with central body fat. Thus, there is a controversy regarding the validity of measurement of BMI as a tool for medical decisions such as the advice to loose weight. This also related to the use of the BMI as a measure to assess the benefits of weight loss interventions. It has also been recognized, that BMI does not provide information about loss of muscle mass and muscle function - both playing a key role in the development of the frailty syndrome.

BMI seems to be a good screening tool for identifying obesity. However, for making the decision on who should change his/her body status and to assess the success of any intervention other measures than BMI are needed.

\section{PL1:3}

\section{Beyond cardiometabolic disease: The myth of healthy obesity}

\section{Matthias Blüher}

University of Leipzig, Germany

Expansion of adipose tissue upon weight influences whole body metabolism and obesity is an important risk factor for type 2 diabetes, dyslipidemia, fatty liver disease, hypertension, cardiovascular disease and certain types of cancer. However, the individual obesity-related risk for these diseases is not determined by increased fat mass alone. Therefore, there must be factors, which protect a subgroup of obese individuals - so called metabolically healthy obese - against obesity-related diseases. Heterogeneity of body composition, fat distribution and adipose tissue function may underly the variable risk to develop metabolic and cardiovascular diseases associated with increased body fat mass. Central body fat distribution has been shown to better predict obesity-related cardiometabolic diseases than whole body fat mass or body weight. Dysfunction of adipose tissue maybe initiated by an inability of adipose tissue to increase body fat mass by recruiting new (healthy) adipocytes, which activates a sequence of pathological mechanisms including cellular insulin resistance and increased lipolytic capacity, intracellular accumulation of toxic molecules, activation of stress pathways, visceral (ectopic) fat accumulation, changes in the cellular and intracellular matrix composition, increased number of immune cells within adipose tissue, increased autophagy and apoptosis, fibrosis, alterations in gene and protein expression patterns. Most likely, impaired adipocyte function is caused by genetic, behavioural and environmental factors which are not entirely understood. As a result of 
impaired subcutaneous adipose tissue expandability, adipocytes become larger and release signals (e.g. hormones, cells, metabolites) resulting in a proinflammatory, diabetogenic and atherogenic serum profile. These adverse signals may contribute to inflammation of adipose tissue and secondary organ damage in target tissues such as liver, brain, endothelium, vasculature, endocrine organs and skeletal muscle.

However, it is at least debatable whether "healthy obesity really exists. Beyond the cardiometabolic perspective, there are several disease entities, including osteoarthritis, pulmonary diseases, certain types of cancer, psychologic conditions and psychiatric diseases, which do not justify the term "healthy obesity". The myth of "healthy obesity" will be discussed and current analyses of obesity without any accompanied disease or adverse health conditions will be critically reviewed.

Conflict of Interest: Speaker and/or AdBoard honoraria from AstraZeneca, Bayer Boehringer-Ingelheim, Lilly, Novartis, Novo Nordisk, Sanofi

Funding: No Funding relating to this abstract.

\section{Thursday, 18 May 2017}

\section{PL2 - Childhood Obesity Plenary}

\section{PL2:1}

\section{Educating HCPs into a new paradigm: A Danish success story}

\section{Holm, J. C.}

Consultant in Paediatrics, Associate Clinical and Research Professor, PhD, Head of Research, The Children's Obesity Clinic, and The Danish Childhood Obesity Biobank at the Department of Pediatrics Holbæk University Hospital, Denmark.

The Children's Obesity Clinic's Treatment (TCOCT) protocol reduce approximately $75-85 \%$ of the degree of obesity in patients (children and youth with obesity) and some of those increasing BMI actually attain an improved body composition. The cumulative drop out rate of $9 \%$ after 1 year and $22 \%$ after 2 years of treatment and those stopping treatment tend to maintain a favorable weight status over time. TCOCT protocol has been transferred to another Pediatric Department and to 8 primary care clinics across Denmark where 75 and $74 \%$ of patients reduced their degree of obesity. Further, cholesterols, degree of hypertension, parental degree of obesity, visceral and hepatic fat, and bullying are all reduced, and appetite, body self-esteem, and quality of life are improved during treatment with the use of 5 health professional hours per patient per year.

The Children's Obesity Clinic convey a paradigm shift where 1. obesity is regarded as a disease, where 2. the fat mass is neuroendocrinely regulated in order to preserve fat mass, and with the development of a 3 new pedagogy, which provides authenticity and self-identification so that the patient develop a perspective where the patient understands that the treatment is lifelong which is internalized providing empowerment. Altogether, this strategy has proven successful with translation of science into daily clinical practice where course including programmes and materials are well developed and received by approximately 450 course participants. Further, all clinical contacts are integrated to various scientific programs including consortia studies like "TARGET", "BIOCHILD", "EGG", and "MicrobLiver".

Altogether, these initiatives have enabled the establishment of The Danish Childhood Obesity Biobank, which has included more than 6,500 overweight/obese and normal weight children.

\section{Friday, 19 May 2017}

\section{PL4 - Plenary Lecture}

\section{PL4:1 \\ Sympathetic control of adiposity}

\section{Ana Domingos}

Instituto Gulbenkian de Ciência, Lisboa, Portugal

Leptin is a hormone produced by the adipose tissue that acts in the brain, stimulating white fat breakdown. We find that the lipolytic effect of leptin is mediated through the action of sympathetic nerve fibers that innervate the adipose tissue. Using intravital two-photon microscopy, we observe that sympathetic nerve fibers establish neuro-adipose junctions, directly "enveloping" adipocytes. Local optogenetic stimulation of sympathetic inputs induces a local lipolytic response and depletion of white adipose mass. Conversely, genetic ablation of sympathetic inputs onto fat pads blocks leptin-stimulated phosphorylation of hormone-sensitive lipase and consequent lipolysis. Thus, neuro-adipose junctions are necessary and sufficient for the induction of lipolysis in white adipose tissue and are an efferent effector of leptin action. Direct activation of sympathetic inputs to adipose tissues may represent an alternative approach to induce fat loss, circumventing central leptin resistance or difficult drug delivery to the brain.

\section{References:}

1 http://www.cell.com/cell/abstract/S0092-8674(15)01107-1

2 http://www.cell.com/cell/abstract/S0092-8674(15)01177-0

3 http://www.nature.com/nrn/journal/v16/n11/full/nrn4050.html

4 http://www.nature.com/nature/journal/v527/n7576/full/527043a.htm

\section{PL5 - Plenary Lecture}

\section{PL5:1}

\section{Man-microbe symbiosis, a key feature in metabolic health}

Joel Doré

INRA, Jouy-en-Josas, France

The human intestinal tract harbours a complex microbial ecosystem which plays a key role in nutrition and health. Interactions between food constituents, microbes and the host organism derive from a long co-evolution that resulted in a mutualistic association.

Current investigations into the human faecal metagenome are delivering an extensive gene repertoire representative of functional potentials of the human intestinal microbiota. The most redundant genomic traits of the human intestinal microbiota are identified and thereby its functional contribution. These observations show a unique segregation of the human population into individuals with low versus high gene-counts. It significantly expands our ability to look for specificities of the microbiota associated with human diseases and to ultimately validate microbial signatures of prognostic and diagnostic value in immune mediated diseases. In obesity, the microbiota is a key player in the development of obesity. the overall phenotypic characteristics are worse in individuals with low gene counts (LGC) microbiota, which represent $25 \%$ of overweight to moderately obese subjects and up to $75 \%$ of extreme obese (candidates for bypass surgery). LGC patients present a low grade inflammatory context also associated with insulin-resistance, and the worst response to dietary constraints in terms of weight loss or improvement of biological and inflammatory characteristics. Conversely, a calorie restricted diet, with low fat, high protein and especially high-diverse fiber content, was able to correct the low gene count microbiota, raising by more than $25 \%$ the gene count during a 6 weeks intervention. Gastric bypass surgery rapidly cor- 
rects average gene richness and restores higher proportions of symbionts that appear deprived in extreme obesity. The effects seem durable over a 12 months follow up.

The human intestinal microbiota should hence be regarded as a true organ, amenable to rationally designed modulation for human health. Dysbiosis appears in essence as an alteration of man-microbe symbiosis which triggers interest in the application of functional metagenomics to better understand the crosstalk between intestinal symbionts and human cells and tissues.

\section{PL7 - European and Portuguese Obesity Day Session}

PL7:2

\section{Prevention of childhood obesity: Lessons learned from a school-based intervention program}

Rosário, R.; Araújo, A.; Padrão, P.; Lopes, O. Moreira, A.; Pereira B., Moreira, $P$.

Obesity is a serious public health condition and is increasing all over the world (1). Previous studies have been developed aiming at preventing childhood obesity (2), nonetheless, best practices are far from complete. The present study aimed to analyze the impact of an intervention program, based on healthy lifestyles, on childhood overweight and dietary intake.

We developed a trial with 464 children (239 female, 6 to 12 years old) from seven elementary Portuguese schools, three of them were allocated to intervention and four to control group. The intervention program was implemented over 24 weeks based on two terms: teachers' education and training, and their intervention in the classroom with children. Sociodemographic, anthropometric, physical activity, and dietary assessments were performed before and at the end of the intervention.

Results: Children from intervention group reported a significantly increase in fruit and vegetable intake and a reduction in low nutrition, energy dense foods, when compared to their counterparts from the control group. Additionally the incidence of overweight was significantly lower in intervened children when compared to the controllers.

Conclusion: Our study provides further support for the success of intervention programs aimed at preventing childhood overweight and in improving a dietary intake able to control obesity.

\section{References:}

1 NCD Risk Factor Collaboration (NCD-RisC): Trends in adult body-mass index in 200 countries from 1975 to 2014: a pooled analysis of 1698 population-based measurement studies with 19.2 million participants. Lancet 2016;387:1377-1396.

2 Waters E, de Silva-Sanigorski A, Hall BJ, Brown T, Campbell KJ, Gao Y, Armstrong R, Prosser L, Summerbell CD: Interventions for preventing obesity in children. The Cochrane Database Of Systematic Reviews 2011:CD001871.

PL7:4

\section{New hypothalamic targets and strategies to fight obesity}

Cavadas, $C$.

Center for Neuroscience and Cell Biology \& Faculty of Pharmacy; University of Coimbra, Portugal

During the last 8 years, by using different cellular and rodent models, we have been investigating pathophysiological changes that occur in the hypothalamus and in the adipose tissue upon high fat diet-induced obesity and insulin resistance, and how can we interfere to reduce the deleterious impact of those changes. We have been focusing our research on the impact of obesity and high fat diet on hypothalamic neurogenesis, microRNA pathways and sirtuins. By using dietary, pharmacological or gene therapy approaches, combined with relevant animal models, our results suggest that those

\section{PL7:5}

Numbers of childhood obesity: The Generation XXI study.

\section{Ana Cristina Santos}

ISPUP, Faculty of Medicine University of Porto

Obesity is at epidemic levels among European children, and precursors of type 2 diabetes and cardiovascular disease are now being described in children. Portugal is characterized by a high prevalence of childhood overweight and obesity, placing Portugal among the highest in Europe. A major public health challenge in the 21 st century is to device an effective policy and practice to combat the epidemic of obesity, particularly regarding young ages. Thus, it is essential to identify the critical periods of postnatal life implicated in the development of childhood obesity and metabolic health. Birth cohorts are the best way to approach this problem, as children are followed from birth to adulthood, providing an unique opportunity to longitudinally collect information on dynamic variables such as growth and adiposity accrual throughout the life span. Embed in Generation XXI, a previously assembled cohort of 8647 babies born in Porto, Portugal, between 2005 and 2006, we aimed to study to estimate the prevalence and incidence of obesity and different body fat distribution phenotypes in the Portuguese at different stages of child development.

Generation XXI data was collected using structured questionnaires to obtain information on mothers' and children demographic and lifestyle variables and on past and current medical history. Clinical registries at birth were also used to collect information on delivery, post-partum complications, and anthropometrics at birth. The whole cohort has been invited for several revaluations (at $4(n=5824), 7(n=5722)$ and 10 (now finishing) years of age), and the same standard procedures were used. Data were collected by trained interviewers, using structured questionnaires, measurements of anthropometrics (weight, height, waist circumference and body composition assessed through tetrapolar bioimpedance and in a subsample $(n=2,408)$ by dual-energy X-ray absorptiometry) and blood pressure were performed and blood samples collected.

Results from the 4 and 7 years of age evaluations showed that, at 4 years of age $21.7 \%$ (22.6\% in females and $20.9 \%$ in males) and $10.7 \%(11.5 \%$ in females and $9.8 \%$ in males, $\mathrm{p}=0.033$ ) of the evaluated children were overweight and obese, respectively. As expected, the prevalence of obesity increased when evaluated at 7 years of age to $14.5 \%(14.2 \%$ in females and $14.8 \%$ in males, $\mathrm{p}=0.031$ ), but the prevalence of overweight remained stable. We observed 329 new cases of obesity ( $7.3 \%$ in 3 years) from the 4 to the 7 years of age evaluation, and this incidence was higher in males that $\mathrm{n}$ females $(8.2 \%$ vs. $6.4 \%$, respectively, $\mathrm{p}=0.001)$. We are now finalizing the data set for the 10 years of age evaluation and these data as well as for the other indicators of obesity will soon be available.

These data show as expect, that the prevalence of childhood obesity is high in Portugal, but also is the incidence of this condition. Data produced by this research provide value input for national public health purposes, but also for designing evidence based strategies as to curb the obesity epidemic and related health outcomes in the Portuguese society

\section{PL7:6}

\section{Healthy Eating Promotion: A Portuguese experience}

\section{Rodrigo Quaresma}

Nutri Ventures

Nutri Ventures is the first children's entertainment brand in the world to promote Healthy Eating. At a time when child obesity is considered by the World Health Organization to be the pandemic of the 21st-century, Nutri Ventures is a fun and effective response to the issue of healthy eating for children between the ages of 4 and 10. Our mission is to develop entertaining content that encourages children to adopt healthy lifestyles, 
particularly with regard to nutrition and physical activity and create an exciting and positive overall environment about them, so that children can associate good feelings to healthy behaviors. As the Project is also a movement and a cause, in every country Nutri Ventures set up partnerships with organizations whose mission is aligned with its. This is the case with government departments for Education and Health, NGO's that promote the cause of Healthy Eating, food distributors and producers. The company is helping to strengthen the message of each one of these partnerships through its story and characters. The Nutri Ventures' movement hopes to be a cause that will unite the whole of society in the quest to encourage children to develop healthier eating habits. That's why the company:

- Supports the cause of healthy eating, creating and using the contents of Nutri Ventures and its institutional partnerships for this end;

- Promotes not just healthy eating but healthy life styles;

- Promotes confidence, self-esteem, and a positive body image;

- Encourages children to be physically active;

- Transmits its messages always in a positive way and reinforcing healthy behaviors;

- Associates its image and contents only with partners who share its mission: to encourage healthy eating and life styles in children. For this reason, in every region Nutri Ventures has a recognized and independent partnership to evaluate the nutritional quality of food products licensed by Nutri Ventures;

- Endeavors to promote food production which respects the principles of ecological balance and sustainable farming;

- $\quad$ Requires that all products developed by partners licensed by Nutri Ventures will be subject to a thorough evaluation of methods and production techniques both in terms of environmental issues, human rights and child safety." 


\section{Thursday, 18 May 2017}

\section{P01 - Basic and Experimental Science}

\section{T1P1}

\section{A novel adipokine progranulin augments NO-mediated vasorelaxation in hydrogen sulfide-dependent manner}

\section{Beltowski, J.';}

${ }^{1}$ Department of Pathophysiology, Medical University, Lublin, Poland

Introduction: Progranulin (PGRN) is a growth factor and regulatory protein involved in embryonic development, wound healing, carcinogenesis, neuroprotection and host defense. PGRN serves as the agonist or antagonist of several receptors of the tumor necrosis factor (TNF) protein superfamily. Recently, progranulin has been identified as the new adipokine involved in the regulation of adipose tissue inflammation and insulin sensitivity. In addition, progranulin augments nitric-oxide (NO)-mediated vasorelaxation by increasing NO-stimulated cGMP production (Kazama K et al. Acta Physiol 2016, doi: 10.1111/apha.12739). We examined the mechanism of PGRN-induced vasorelaxation with special emphasis on the role of endogenous hydrogen sulfide (H2S) which cooperates with $\mathrm{NO}$ in the regulation of vascular tone.

Methods: The effect of PGRN on vascular tone of the first- and second-order rat mesenteric arteries was examined by wire myography. Cyclic GMP was measured by enzyme immunoassay. $\mathrm{H} 2 \mathrm{~S}$ production by vascular preparations was assayed by the polarographic sensor

Results: PGRN (5-100 ng/ml) augmented acetylcholine and spermine NONOate (the NO donor)-induced relaxation of endothelium-intact and endothelium-denuded arterial rings, respectively. PGRN augmented spermine NONOate-induced cGMP production. In contrast, PGRN had no effect on vasorelaxation induced by protein kinase $\mathrm{G}$ activator, 8-bromo-cGMP. Progranulin increased H2S production by endothelium-denuded arterial rings and its effect on NO-induced vasorelaxation was mimicked by $\mathrm{H} 2 \mathrm{~S}$ donor, $\mathrm{Na} 2 \mathrm{~S}$, and abolished by cystathionine $\gamma$-lyase (the H2S)-synthesizing enzyme) inhibitor, propargylglycine. Na2S, but not PGRN, augmented the NO-induced stimulation of recombinant soluble guanylyl cyclase. PGRN had no effect on $\mathrm{H} 2 \mathrm{~S}$ production by endothelial cells. Augmenting effect of PGRN on NO-mediated vasorelaxation was abolished by antibodies specific for TNF-related apoptosis-inducing ligand (TRAIL) receptor but not by antibodies specific for TNF receptor-1 or -2. NO-induced vasorelaxation was impaired in rats made insulin-resistant by high fructose feeding. Serum PGRN was increased in fructose-fed rats, however, augmenting effect of PGRN on NO-mediated vasorelaxation tended to be impaired in insulin-resistant animals which was accompanied by reduced expression of TRAIL receptor in the arterial wall.

Conclusion: Progranulin stimulates $\mathrm{H}_{2} \mathrm{~S}$ production by vascular smooth muscle but not endothelial cells, and augments NO-induced vasorelaxation through $\mathrm{H}_{2} \mathrm{~S}$-dependent stimulation of NO-activated soluble guanylyl cyclase. Vascular effect of progranulin is mediated by TRAIL receptors. TRAIL signalling in the vascular wall and vasodilating effect of PGRN are impaired in the metabolic syndrome.

\section{T1P2}

\section{Abdominal obesity in Algerian students}

Corinne, D. ${ }^{1}$; Rabiaa, K. ${ }^{1}$; Djamel-Eddine, M. ${ }^{1}$; Lahcène, N. $^{1}$ ${ }^{1}$ ALNUTS - Université Constantine Algérie
Introduction: Central obesity indices predict cardiometabolic risk factors more accurately than the general obesity defined by BMI in both men and women. This study aims to estimate abdominal obesity in Algerian students with indicators of central fat distribution.

Methods: The study was carried out in 2012-2014 on voluntary and healthy students of the University of Constantine in Algeria. The results concern 398 girls and 201 boys aged between 20 to 25 years. Four obesity indices with their optimal cut-off values were investigated: BMI ( $\geq 30 \mathrm{~kg} /$ $\mathrm{m}^{2}$ ), Waist Circumference (WC; male: $>102 \mathrm{~cm}$; female: $>88 \mathrm{~cm}$ ), Waistto-Hip Ratio (WHR; male: $>1.0$; female: $>0.85$ ), Waist to Height Ratio (WHtR: >0.50).

Results: For boys the average BMI and WC is respectively $22.8 \pm 3.0$ $(16.9-32.8) \mathrm{kg} / \mathrm{m}^{2}$ and $80.7 \pm 7.5(65.2-103.9) \mathrm{cm}$. In girls WC mean is $75.9 \mathrm{~cm} \pm 8.5(58.0-105.2) \mathrm{cm}(\mathrm{p}<0.001)$ and BMI mean is $22.8 \pm 3.0$ $(15.8-36.4) \mathrm{kg} / \mathrm{m}^{2}(\mathrm{p}<0.05)$. According to BMI, 3.3\% of girls and $2.0 \%$ of boys classifies as obese and according to WC, $10.3 \%$ girls and $1.0 \%$ boys are obese. The WHR average is $0.76 \pm 0.06(0.63-0.98)$ for girls and $0.82 \pm 0.05(0.72-0.99)$ for boys $(\mathrm{p}<0.001)$. This indicator classifies $6.0 \%$ of girls with android obesity. $7.8 \%$ of girls $(0.47 \pm 0.06)$ and $4.0 \%$ of boys $(0.46 \pm 0.04)$ have central fat distribution $(\mathrm{p}<0.05)$, defined by WHtR $>$ 0.5 .

Conclusion: In Algeria, obesity is often assessed by body mass index (BMI). There is not published information of other obesity indices. Further research is needed for this group of population to determine an optimal cut-off for various obesity indices to predict CVD risk factors and MetS.

T1P3

\section{Accuracy of predictive equations for estimating resting energy expenditure in extreme obese females}

\section{Sammarco, R. ${ }^{1}$; Marra, M. ${ }^{\prime}$; Caprio, C. '; Silvestri, E. '; Vincenzo, O. ${ }^{1}$;} Contaldo, F.'; Pasanisi, F.'

${ }^{1}$ Department of Clinical Medicine and Surgery, Federico II University

Introduction: During the last three decades the prevalence of overweight and obesity has increased in European people. Because of the ongoing obesity, in particular severely obese, the assessment of resting energy expenditure (REE) provides useful information for weight management. The study aimed to compare resting energy expenditure (REE) measured (MREE) by indirect calorimetry (IC) in extreme obese females patients with REE predicted (PREE) using different equations.

Methods: We studied 224 extreme obese female patients (age $34.7 \pm 9.0$ y, $149 \pm 14 \mathrm{~kg}$, height $161 \pm 6 \mathrm{~cm}$, BMI $55.7 \pm 4.7 \mathrm{~kg} / \mathrm{m}^{2}$ ). Data were obtained comparing MREE with PREE derived from published equations for normal and overweight (Harris-Benedict, Schofield and FAO, Mifflin, De Lorenzo, Owen), and obese (Lazzer, Muller and Bernstein). The average differences between PREE and MREE, as well as the accuracy at 10\% level, were evaluated.

Results: Mean REE was evaluated in 224 females $(2434 \pm 366 \mathrm{kcal} / \mathrm{d})$, the smallest difference in percentage between PREE and MREE was observed with De Lorenzo $(-3.8 \pm 14.9 \%)$ and Muller equation $(7.3 \pm 12.4 \%)$. Accuracy at $10 \%$ level resulted very low $(<50 \%)$ in all equations considered (Bernestein 7.1\%; Muller 48.2\%)

Conclusions: The study shows that De Lorenzo and Muller equations can be used for population studies whereas in clinical practice (individual patient) no equation seems to be accurate. Inaccuracy of predicted REE could affect both dietary prescription and compliance.Measurement with Indirect Calorimetry shoud be widely available.

\section{References:}

1 Marra et al. Accuracy of predictive equations for estimating resting energy expenditure in obese adolescents. J Pediatr. 2015 Jun;166(6):1390-6. 
2 Marra et al. BMR variability in women of different weight. Clin Nutr. 2007Oct;26(5):567-72.

Conflict of Interest: None Disclosed

Funding: No Funding

\section{T1P4}

\section{Adiponectin and glucose metabolism in obese women}

Veiga, L. ${ }^{1}$; Brito, M. ${ }^{1}$; Oliveira, A. ${ }^{1}$; Nunes, J. ${ }^{2}$

${ }^{1}$ Escola Superior de Tecnologia da Saúde de Lisboa, Portugal, ${ }^{2}$ Centro Hospitalar De Lisboa Central, Epe

Introduction: Obesity represents a major risk factor for type 2 diabetes. Adiponectin is a hormone associated with an antidiabetic action and obese individuals tend to present low circulating levels of that hormone. The aim of our study was to evaluate the association between adiponectin plasma levels and several parameters related to carbohydrate metabolism in obese women, independently from the effect of anthropometrics.

Methods: We studied 112 obese otherwise healthy women, characterized for anthropometrics. They were submitted to an oral glucose tolerance test (OGTT) with plasma glucose, insulin, and C-peptide levels assessed at fasting and 30', 60', 90' and 120' after $75 \mathrm{~g}$ of oral glucose. Insulin resistance (IR) was assessed by the homeostatic model assessment (HOMA-IR) and an assessment of $\mathrm{HbAlc}$ was performed. Women were classified as normoglycemic, prediabetic and diabetic according to HbAlc and glucose values (fasting and 120'). We looked for direct associations of adiponectin levels with anthropometrics. We also study eventual direct and anthropometric-independent associations between adiponectin levels and carbohydrate metabolism parameters.

Results: Obese women were characterized by mean age $=34.6 \pm 8$.3years $\mathrm{BMI}=43.6 \pm 7.9 \mathrm{Kg} / \mathrm{m}^{2}, \quad$ body $\quad$ fat $\quad$ mass $=53.9 \pm 14.8 \mathrm{Kg}$ waist $=117.5 \pm 15.1 \mathrm{~cm}$, and waist:hip ratio $(\mathrm{WHR})=0.88 \pm 0.07$. HO$\mathrm{MA}-\mathrm{IR}=4.28 \pm 3.5, \mathrm{HbAlc}=5.6 \pm 0.8 \%$, glucose in OGTT $=93,4 \pm 28.6 /$ $144.4 \pm 38.3 / 145.6 \pm 55.6 / 131.0 \pm 55.5 / 117.8 \pm 53.0 \mathrm{mg} / \mathrm{dl}$, insulin in $\mathrm{OGTT}=17.6 \pm 11.2 / \quad 76.4 \pm 51.3 / \quad 91.0 \pm 52.1 / \quad 86.6 \pm 53.0 /$ $72.9 \pm 52.6 \mathrm{iU} / \mathrm{l}$, and C-peptide in OGTT $=3.5 \pm 1.2 / 9.0 \pm 8.9 / 10.2 \pm 3.0 /$ $10.4 \pm 3.2 / 10.0 \pm 6.5 \mathrm{ng} / \mathrm{ml}$. Normoglycemia was considered in 80 women, prediabetes in 22 and diabetes in 10 and adiponectin was significantly different among these groups $(\mathrm{p}=0.032)$. Adiponectin was inversely and significantly correlated with waist $(p=0.029 ; r=-0.207)$, WHR $(\mathrm{p}<0.001 ; \mathrm{r}=-0.335)$, HbA1c $(\mathrm{p}=0.035 ; \mathrm{r}=-0.201)$, HOMA-IR $(p=0.001 ; r=-0.312)$, glucose $60^{\prime}(p=0.002 ; r=-0.295)$, glucose 90' $(p=0.01 ; r=-0.25)$, glucose $120^{\prime}(p=0.003 ; r=-0.284)$, fasting insulin $(p<0.001 ; r=-0.362)$, insulin 60' $(p=0.004 ; r=-0.278)$, insulin 90 $(\mathrm{p}=0.009 ; \mathrm{r}=-0.249)$, insulin $120^{\prime}(\mathrm{p}=0.004 ; \mathrm{r}=-0.271)$, fasting C-peptide $(\mathrm{p}<0.001 ; \mathrm{r}=-0.429)$, and C-peptide 90' $(\mathrm{p}=0.018 ; \mathrm{r}=-0.229)$. After adjustment for WHR a significant inverse association with adiponectin levels was still present for HOMA-IR, insulin at fasting, 60', 90' and 120', and C-peptide at fasting

Conclusion: Dysglycemic obese women tend to present lower levels of circulating adiponectin which is dependent on abdominal fat content. Adiponectin has its own insulin-sensitizing action that is independent of anthropometrics.

Conflict of Interest: None Disclosed

Funding: Research relating to this abstract was partially funded by Merck SA

\section{T1P5}

\section{Adiponectin and vitamin D in obese patients}

Corina, M. ${ }^{1}$; Valeriu, A. '; Irina, S. ' Laura, G. '; Madalina, P. '; Bogdan, C. ' ; Laura, $S{ }^{1}$

${ }^{1}$ Carol Davila University of Medicine and Pharmacy

Introduction: Obesity represent a major health problem worldwide and it is correlated with the expansion of white adipose tissue especially in ab- dominal circumference. Our pourpose was to assess impact of body mass index (BMI) to level of adiponectin and 25 hydroxy-vitamin D.

Methods: We performed a study including -15 obese subjects (all men), with $\mathrm{BMI}>30$ and waist circumference $>102 \mathrm{~cm}$, and 15 normal subjects (all men). On plasma samples we determined the levels of triglycerides, total cholesterol, LDL cholesterol, HDL cholesterol, 25 hydroxy-vitamin $\mathrm{D}$ and adiponectin.

Results: Obese subjects had significantly lower concentration of 25 hydroxy-vitamin D and adiponectin than normal subjects $(\mathrm{p}<0.05)$. Body mass index (BMI) was inversely correlated with adiponectin and 25 hydroxy-vitamin D concentrations

Conclusion: Adiponectin and 25 hydroxy-vitamin D are decreased in obese patiens and inversly corelated with BMI.

Conflict of Interest: None Disclosed

Funding: No Funding

T1P6

\section{Adipose tissue gene expression level in mitochondrial pathways associates with weight loss success}

Jokinen, R. ${ }^{1}$; Rinnankoski-Tuikka, R. ${ }^{2}$; Kaye, S. '; Saarinen, L. ${ }^{3}$;

Myöhänen, M. '; Rappou, E. '; Rissanen, A. '; Velagapudi, V. ; Virtanen, K. ; Pirinen, E. ${ }^{2}$; Pietiläinen, K. $^{1}$

${ }^{1}$ Obesity Research Unit, Research Programs Unit, Diabetes and Obesity, University of Helsinki, Finland, ${ }^{2}$ Research Programs Unit, Molecular Neurology, University Of Helsinki, Helsinki, Finland, ${ }^{3}$ Research Programs Unit, GenomeScale Biology, Faculty Of Medicine, University Of Helsinki, Helsinki, Finland, ${ }^{4}$ Metabolomics Unit, Institute For Molecular Medicine Finland Fimm, University Of Helsinki, Finland, ${ }^{5}$ Turku Pet Centre, Turku University Hospital And University Of Turku, Finland

Introduction: Mitochondrial biogenesis and metabolic pathways in white adipose tissue are downregulated in obesity, and this mitochondrial decline associates with metabolic complications. Yet it has not been thoroughly investigated if weight loss as the first line treatment for obesity remedies the adipose tissue mitochondrial decline, or if differences in white adipose tissue mitochondria between individuals influence weight loss success.

Methods: We analyzed the subcutaneous adipose tissue gene expression profile from clinically well-characterized obese patients on a diet based weight loss intervention at baseline and after 5 and 12 months. The patients were divided into good and poor responders based on the degree of weight loss. To extend our transcriptomics findings we analyzed the quantity of mitochondrial DNA (mtDNA) and mtDNA derived transcripts.

Results: Genes in mitochondrial pathways were expressed at a higher lev$\mathrm{el}$ in the good responders at baseline and total expression level in these pathways correlated with weight loss success and adipose tissue glucose uptake rate. Higher $M T$-CO1 mRNA level and a trend for higher mtDNA quantity were observed in the good responders by comparison to the poor. Unexpectedly weight loss induced a downregulation of nuclear encoded mitochondrial pathways. MtDNA quantity initially increased followed by return to baseline level in the poor responders. In the good responders a tendency for initial increase and a significant decrease between five to 12 months was observed.

Conclusions: Higher baseline gene expression level in mitochondrial pathways associates with better long-term weight loss success, potentially due to higher adipose tissue metabolic activity. In contrast to previous findings, nuclear encoded mitochondrial pathways were further downregulated by weight loss in our study material.

Conflict of Interest: None disclosed.

Funding: Research relating to this abstract was funded by Helsinki University Hospital Research Funds, the Novo Nordisk Foundation, Diabetes Research Foundation, Jalmari and Rauha Ahokas Foundation, Finnish Foundation for Cardivascular Research, Academy of Finland (266286, 272376 and 285963), Emil Aaltonen Foundation, Orion-Farmos Research Foundation, Maud Kuistila Foundation, Finnish Medical Foundation, Biocentrum Helsinki and Sigrid Jusélius Foundation. 
T1P7

\section{Adrenergic induction of beige adipocytes in white adipose tissue is attenuated with aging in mice}

Shin, W. ${ }^{1}$; Okamatsu-Ogura, Y. ${ }^{1}$; Machida, K. ${ }^{1}$; Tsubota, A. ${ }^{\text {; }}$; Nio-Kobayashi, J. ${ }^{2}$; Kimura, $K .^{1}$

${ }^{1}$ Department of Biomedical Sciences, Graduate School of Veterinary Medicine, Hokkaido University, Sapporo, Japan, '2Laboratory Of Histology And Cytology, Graduate School Of Medicine, Hokkaido University, Sapporo, Japan

Objective: There are two types of thermogenic adipocytes that regulate energy expenditure and express uncoupling protein (UCP)-1 in a mitochondria: the brown adipocyte within brown adipose tissue (BAT) that is activated by adrenergic stimulation and beige adipocyte that appears within the white adipose tissue (WAT) in response to chronic adrenergic stimulation. This study examined age-related changes in responses of both type of adipocytes to adrenergic stimulation in mice.

Methods: Aged (age 20 months) and young (age 4 months) mice were injected daily with either saline or beta-3 adrenergic receptor agonist CL316,243 (CL; $0.1 \mathrm{mg} / \mathrm{kg}$, once a day) for 1 week.

Results: The body and WAT weight tended to be higher in aged mice. CL treatment increased UCP-1 protein amounts in both BAT and inguinal WAT, suggesting activation of brown and beige adipocytes. However, induction of beige adipocytes was impaired in aged mice, whereas brown adipocyte activation was comparable to young mice. There were no differences in the beta- 3 adrenergic receptor mRNA expression; however, the number of platelet-derived growth factor receptor alpha-expressing progenitor cells, which were reported to differentiate into beige adipocytes, significantly decreased in inguinal WAT of aged mice compared with that of young mice.

Conclusions: Inductive ability of beige adipocyte in WAT declines with aging in mice. It may be partly because of a decreased number of progenitor cells associated with aging and contribute to development of obesity.

\section{T1P8}

\section{Age dynamics of adipose tissue accumulation and obesity types formation in men and women}

Pinkhasov B.B.; Deev D.A.; Mohova I.G.; Lutov Yu.V.; Kuzminova O.I.; Selyatitskaya V.G.

Research Institute Experimental and Clinical Medicine, Novosibirsk, Russia

Introduction: The purpose of the study was to investigate features of adipose tissue accumulation and its topography in men and women with overweight and obesity in ontogenesis.

Methods: The study included 251 men and 352 women. All patients were divided into 3 age groups: in group 1 were carried patients of the first mature age period (from 22 to 35 years), in group 2 - the second mature age period (from 36 to 60 years), in group 3 - elderly age period (from 61 to 75 years). Measurements of body weight $(\mathrm{kg})$, body height $(\mathrm{m})$, waist circumference $(\mathrm{WC}, \mathrm{cm})$ and hips circumference $(\mathrm{HC}, \mathrm{cm})$ were taken. The existence of overweight and obesity was determined by calculating the body mass index (BMI). Obesity type was determined by the $\mathrm{WC} /$ $\mathrm{HC}$ relation: in men if $\mathrm{WC} / \mathrm{HC}<0.95$ assigned gynoid type, and if $\mathrm{WC} /$ $\mathrm{HC} \geq 0.95$ - android type of obesity; at women if $\mathrm{WC} / \mathrm{HC}<0.85$ assigned gynoid type, and if $\mathrm{WC} / \mathrm{HC} \geq 0.85$ - android type of obesity.

Results: It is shown that increase of relative adipose tissue amount occurs throughout all studied periods of ontogenesis in men and women. At the same time, the most intense increase was appeared at the first mature age period in men while in women it was appeared during at the second mature age period. The comparative analysis of gender differences has confirmed the fundamental distinctions consisting in authentically higher relative adipose tissue accumulation presented more by subcutaneous fat in organism of women and by mainly accumulation of abdominal fat in men. The irregularity of fatty tissue accumulation in gluteofemoral and abdominal areas was reflected in a ratio of obesity types. It is shown that at the beginning of the first mature age period the gynoid type of obesity dominates also in man. However, active accumulation of abdominal adi- pose tissue leads to the fact that by the end of the first mature age period the occurrence of gynoid and android type equalized, and then the android type of obesity begins to dominate in men. In women gynoid type of obesity dominates until the end of the second mature age period, and then the occurrence of android type begin to increase, and continues to increase in the elderly.

Conclusion: Women versus men have two decades "metabolic wellbeing", which largely determines the later development of chronic non-communicable diseases.

\section{T1P9 \\ An artificial neural network to predict resting energy expenditure in obesity}

Cécile, B. ${ }^{1}$; Emmanuel, D. ${ }^{2}$; Séverine, $L^{3}$; Cyrielle, C. ${ }^{2}$; Christine, $M .{ }^{4}$; Muriel, C. '; Martine, L. ${ }^{2}$; Chantal, S. ${ }^{2}$

${ }^{1}$ Hospices Civils De Lyon, ${ }^{2}$ Hospices Civils De Lyon, Centre Intégré De L’Obésité Rhone Alpes, Crnh-Ra, Laboratoire Carmen, ${ }^{3} \mathrm{Cinfo}, \mathrm{Ap}-\mathrm{Hp},{ }^{4} \mathrm{Crnh}-\mathrm{Ra}$

Introduction: The resting energy expenditure (REE) determination is important in nutrition for adequate dietary prescription. The gold standard i.e. indirect calorimetry is not available in clinical settings. Thus, several predictive equations have been developed, but they lack of accuracy in subjects with extreme weight including obese populations. Artificial neural networks (ANN) are useful predictive tools in the area of artificial intelligence, used in numerous clinical fields. The aim of this study was to determine the relevance of ANN in predicting REE in obesity.

Methods: A Multi-Layer Perceptron (MLP) feed-forward neural network with a back propagation algorithm was created and cross-validated in a cohort of 565 obese subjects (BMI within 30-50 kg.m-2) with weight, height, sex and age as clinical inputs and REE measured by indirect calorimetry as output. The predictive performances of ANN were compared to those of 23 predictive REE equations in the training set and in two independent sets of 100 and 237 obese subjects for external validation.

Results: Among the 23 established prediction equations for REE evaluated, the Harris \& Benedict equations recalculated by Roza were the most accurate for the obese population, followed by the USA DRI, Müller and the original Harris \& Benedict equations. The final 5-fold cross-validated three-layer 4-3-1 feed-forward back propagation ANN model developed in that study improved precision and accuracy of REE prediction over linear equations (precision $=68.1 \%, \mathrm{MAPE}=8.6 \%$ and $\mathrm{RMSPE}=210$ $\mathrm{Kcal} / \mathrm{d}$ ), independently from BMI subgroups within 30-50 kg.m-2. External validation confirmed the better predictive performances of ANN model (precision $=73 \%$ and $65 \%, \mathrm{MAPE}=7.7 \%$ and $8.6 \%, \mathrm{RMSPE}=187$ $\mathrm{Kcal} / \mathrm{d}$ and $200 \mathrm{Kcal} / \mathrm{d}$ in the 2 independent datasets) for the prediction of REE in obese subjects.

Conclusion: We developed and validated an ANN model for the prediction of REE in obese subjects that is more precise and accurate than established REE predictive equations independent from BMI subgroups. For convenient use in clinical settings, we provide a simple ANN-REE calculator.

Conflict of Interest: None Disclosed

Funding: No Funding 
T1P10

\section{Anti-obesigenic effects of OBEX in mice fed with high fat diet and 3T3-F442A cell line.}

\author{
Izquierdo, A. ${ }^{1}$; Andrade, S. ${ }^{1}$; Folgueira, C. ${ }^{2}$; Sanz, E. ${ }^{3}$; Crujeiras, A. ${ }^{4}$; \\ Casanueva, F. ${ }^{5}$ ' Carreira, M. ${ }^{1}$ \\ ${ }^{1} \mathrm{CIBER}$ obn, ${ }^{2} \mathrm{IDIS},{ }^{3}$ Catalysis, ${ }^{4} \mathrm{C}$ hus, Fundación Ramón Dominguez, Ciberobn, \\ ${ }^{5}$ Usc, Ciberobn
}

Introduction: The recent explosion in prevalence of obesity has grown over the last decades achieving a worldwide epidemic dimension which demands novel and true effective therapeutic approaches. OBEX is an oral nutritional supplement specifically made with natural antioxidants with recognized anti-obesity effects. OBEX contains slimaluma, phaseolamina, L-carnitine, inulin and açaí berry, products whose anti-obesity action and control on metabolic parameters such as fasting glucose, cholesterol and triglycerides levels has been demonstrated in several studies. In this work, we evaluated the anti-obesity effect of OBEX using both in vivo and in vitro models.

Methods: 57BL6/J normal weight mice were randomized into five weightmatched groups ( $\mathrm{n}=10 /$ group). One group had ad libitum access to standar diet. The other four groups were fed with a high fat diet. From the HFD groups, one group had access to water and the others had OBEX in three different concentrations $(0.10,0.25$ and $0.50 \mathrm{~g} / \mathrm{day} / \mathrm{mice})$ diluted in the drinking water. Body weight, food and water intake were measured during the experimental period.

3T3-F442A cells. Once they reach confluence, 3T3-F442A pre-adipocytes can be differentiated into mature adipocyte with $10 \%$ foetal bovine serum, $1 \%$ penicillin-sreptomycin, $0.5 \mathrm{mM}$ IBMX, $25 \mu \mathrm{M}$ dexamethasone and 5 $\mu \mathrm{g} / \mathrm{ml}$ of insulin for 3 days. In the next 7 days cells were maintained in DMEM media with $10 \%$ of foetal bovine serum, $1 \%$ of penicillin-sreptomycin and supplemented with $1 \mu \mathrm{g} / \mathrm{ml}$ of insulin.

Results: We demonstrate that OBEX administration in high fat diet fed mice reduces weight gain by decreasing adiposity gain, increasing energy expenditure and thermogenesis independently of feeding behavior. In addition, in 3T3-F442A cell line, OBEX is able to decrease the proliferation of precursors, the differentiation process and lipid load in mature adipocytes suggesting a final effect of decreasing fat mass.

Conclusions: OBEX is a possible new treatment for obesity reducing weight gain by decreasing adiposity gain, increasing energy expenditure and thermogenesis without changes in food intake.

Conflict of Interes: Eduardo Sanz is an employee of Catalyis S.L. The investigation costs associated to this work were partially supported by Catalysis S.L.

Funding: This work has been partially supported by Catalysis S.L. and CIBERobn, an initiative of the Instituto de Salud Carlos III of Spain which is supported by the regional development Funding program (FEDER)

\section{T1P11}

\section{Appetite reduction in overweight adults after using Redusure $^{\mathrm{TM}}$ (IQP-AK-102), a proprietary composition of soluble dietary fibres}

Bongartz, U. ${ }^{1}$; Uebelhack, R. '; Alt, F. ${ }^{1}$; Grube, B. ${ }^{2}$; Chong, P. ${ }^{3}$; Khoo, J. ${ }^{3}$; Teng, E. $^{3}$

${ }^{1}$ Analyze \& Realize $\mathrm{GmbH},{ }^{2}$ Practice For General Medicine, ${ }^{3}$ Inqpharm Europe Ltd.

Introduction: IQP-AK-102, a novel and proprietary composition of three soluble fibres, has the ability to enhance satiety sensation in the upper gut by increasing gastric distension and delaying gastric emptying; ultimately reducing food intake. ${ }^{1}$ In a previous study, it was shown that IQP-AK-102 can provide significant benefits for weight reduction already within 4 weeks. ${ }^{2}$ The aim of the present study was to evaluate efficacy and safety of IQP-AK-102 on appetite reduction in overweight adults.

Methods: 36 healthy, adult overweight subjects took 2 capsules of IQPAK-102 three times daily for 4 weeks after a 2-week run-in phase. Visual analogue scales (VAS) and Haber (Hunger/Satiety) scale were used to as- sess appetite sensations for 3 days a week during the run-in phase as well as in the first and last week of the treatment period. The scales were completed before breakfast (at -30 and $0 \mathrm{~min}$ ) and after breakfast (at 15, 30, $45,60,90,120$ and $180 \mathrm{~min}$ ). Additionally, food intake was also recorded in a diary.

Results: 35 subjects were included in the final analysis. Analysis of the pre/ post 4-week supplementation showed a statistically significant increase in satiety feelings and fullness (both $\mathrm{p}<0.001$ ); and statistically significant reduced hunger and prospective food consumption (PFC) scores (both $\mathrm{p}<0.001)$. Subjects experienced a statistically significant $(\mathrm{p}=0.002)$ and clinically relevant decrease in energy intake. No serious product-related adverse events were reported.

Conclusion: The results indicate that IQP-AK-102 is an effective intervention for appetite reduction with a good safety profile.

\section{Reference:}

1 Halford, J.C.G., \& Harrold, J.A. (2012). Satiety-enhancing products for appetite control: science and regulation of functional foods for weight management. The Proceedings of the Nutrition Society, 71(2), 350-62.

2 Bongartz, U., Uebelhack, R., Grube, B., Pee-Win, C., \& Alt, F. (2016). Double-blind, randomized, placebo-controlled, bicentric clinical investigation to evaluate the benefit and tolerability of Redusure IQP-AK-102 in reducing body weight in overweight and obese subjects. Advancement in Medicinal Plant Research, 4(3): 73-82.

Conflict of Interest: JooLian Khoo, Pee-Win Chong and Emily Teng are employees of InQpharm Europe Ltd.

Funding: Research relating to this abstract was funded by InQpharm Europe Ltd.

\section{T1P12}

Exercise Training associated to Low-Level Laser Therapy (LLLT) improves neuropeptide-Y, visceral fat and inflammatory state in Obese Women

Dâmaso, A. '; Campos, R. ${ }^{2}$; Masquio, D. ${ }^{3}$; Sene-Fiorese, M. ${ }^{2}$; Duarte, F. ; Junior, A. ${ }^{4}$; Tock, L. ${ }^{5}$; Bagnato, V. ${ }^{4}$; Parizotto, N. ${ }^{2}$

${ }^{1}$ Universidade federal de Sao Paulo, ${ }^{2}$ Federal University Of Sao Carlos, ${ }^{3}$ Federal University Of Saopaulo, ${ }^{4}$ University Of Sao Paulo, ${ }^{5}$ Weight Science

Introduction: The obesity was strongly associated to both deregulation in energy balance and inflammatory state leading to metabolic syndrome and cardiometabolic risk, and showing the importance of new approach. In this way, the purpose of this study was to investigate if aerobic plus resistance training associated to phototherapy is more effective than this type of exercise training alone on energy balance and inflammatory markers in obese women.

Methods: it was involved 49 obese women with age of 20-40 years, BMI $\geq 30 \mathrm{~kg} / \mathrm{m}^{2}$ and $\leq 40 \mathrm{~kg} / \mathrm{m}^{2}$ during 4 month of weight loss therapy (clinical and nutritional approach; and exercise 3 session x 60 minutes week). The voluntaries were assigned to physical training plus phototherapy ( $\mathrm{n}=$ $24)$; and physical training plus SHAM group $(\mathrm{n}=25)$. The interventions consisted on phototherapy application $(830 \mathrm{~nm})$, immediately after the physical training. Body composition, neuroendocrine, metabolic and inflammatory parameters were evaluated.

Results: In both groups was finding a reduction in body mass, BMI, body fat mass, LDL-cholesterol, hip and waist circumferences; and increase in lean body mass. However, only in physical training plus phototherapy group it were showed a reduction in neuropeptide $\mathrm{Y}$ and interleukin-6, associated to accentuated reduction in visceral fat.

Conclusion: In the present investigation, exercise training plus phototherapy is more effective than exercise training alone to improve metabolic and neuroendocrine regulation of energy balance in obese women during weight loss therapy.

\section{Disclosure: None Disclosed.}

Funding: Research relating to this abstract was funded by Support Foundation of São Paulo Research - FAPESP (2013/041364; 2013/19046-0; 2013/08522-6; 2015/14309-9), National Council for Scientific and Technological Development 
- CNPq (573587/2008-6; 300654/2013-8; 150177/2014-3) and Coordination of Higher Education Personnel Training - CAPES

\section{T1P13 \\ Association between body fat and bone mineral density in normal-weight middle-aged Koreans}

\section{Choi, M. ${ }^{1} ; K^{\prime}$ im, D. ${ }^{1}$}

${ }^{1}$ Department of Family Medicine Hallym Sacred Heart Hospital

Background: Osteoporosis and osteopenia are characterized by reduced bone mineral density (BMD) and increased fracture risk. Although the risk of fractures is higher in underweight people than that in overweight people, the accumulation of body fat (especially abdominal fat) can increase the risk of bone loss. This study aimed to evaluate the association between body fat and BMD in normal-weight Koreans.

Methods: This study included 5,515 adults (the mean age: 50.3 years old, $56.2 \%$ women). BMD and body fat were measured using dual-energy X-ray absorptiometry (DXA). Correlation and regression analyses were used to assess the association between BMD and body fat. The cut-off values of normal-weight obesity (NWO) were more than $>20.6 \%$ body fat for men with a body mass index (BMI) of $18.5 \sim 22.9 \mathrm{~kg} / \mathrm{m}^{2}$ and $>33.4 \%$ body fat for women.

Results: Body fat \% tended to be negatively associated with BMD, while BMI tended to be positively associated with BMD. However, there was no association between body fat and BMD in normal-weight men.

Conclusion: Increased body fat was associated with reduced BMD in normal-weight adults, but the effects of body fat on BMD in normal-weight men could not be confirmed.

\section{T1P14}

\section{Association of FTO and apelin gene expression with dietary glycemic index and glycemic load among morbid obese and non-obese subjects}

Safarian, M. ${ }^{1}$; Yuzbashian, E. ${ }^{2}$; Zarkesh, M. ${ }^{3}$; Asghari, G. ${ }^{2}$; Mirmiran, $P^{2}{ }^{2}$. Mahmoodi, B. ${ }^{2}$; Hedayati, M. ${ }^{3}$; Daneshafrooz, A. $^{3}$; Khalaj, $A .{ }^{4}$

${ }^{1}$ Biochemistry of Nutrition Research Center, School of Medicine, Mashhad University of Medical Sciences, Mashhad, Iran, ${ }^{2}$ Nutrition And Endocrine Research Center, Research Institute For Endocrine Sciences, Shahid Behesht University Of Medical Sciences, Tehran, Iran, ${ }^{3}$ Cellular And Molecular Endocrine Research Center, Research Institute For Endocrine Sciences, Shahid Beheshti University Of Medical Sciences, Tehran, Iran, ${ }^{4}$ Tehran Obesity Treatment Center, Department Of Surgery, Shahed University, Tehran, Iran

Objective: To investigate the association between the apelin and FTO gene expression in adipose tissue and dietary glycemic index (GI) and glycemic load (GL) among non-diabetic morbid obese and non-obese subjects.

Methods: Visceral and subcutaneous adipose tissues were obtained from 32 morbidly obese and 32 age- and sex-matched non-obese subjects, who underwent open abdominal surgery. All of the subjects are free of diabetes and cancers and without using anti-lipid medication. Usual dietary intake during the previous year was measured using a validated semi-quantitative food frequency questionnaire. Dietary GI and GL were calculated using International Tables of Glycemic Index and Glycemic Load Values (2008). The gene expressions of apelin and FTO in visceral and subcutaneous adipose tissue were assessed by Real-Time PCR.

Results: The mean age was 39.6 years for both groups and body mass index for morbidly obese and non-obese subjects were 45.3 and 25.6 $\mathrm{kg} / \mathrm{m}^{2}$, respectively. Apelin gene expression in visceral adipose tissue was correlated with dietary GI $(r=0.455, P=0.009)$ and GL $(r=0.445$, $P=0.011)$ among non-obese subjects and, with GL $(\mathrm{r}=0.351, P=0.049)$ among morbid obese subjects. Apelin gene expression in subcutaneous adipose tissue was correlated with GI among morbid obese $(r=0.395$, $P=0.025)$ and non-obese $(\mathrm{r}=0.571, P=0.001)$ subjects. Moreover, we found significant correlations between apelin concentration and dietary GI among morbid obese $(\mathrm{r}=0.388, P=0.028)$ and non-obese $(\mathrm{r}=0.566$,
$P=0.001)$ subjects. In addition, the positive correlation was observed between dietary GL and apelin concentration among non-obese subjects $(\mathrm{r}=0.420, \mathrm{P}=0.026)$. There were no significant findings between FTO gene expression and dietary GI and GL.

Conclusion: It seems that the apelin concentration and mRNA expression have the positive correlation with dietary GL and GI among morbid obese and non-obese subjects; suggesting an important role for insulin pathways in the regulation of apelin expression.

\section{T1P15}

Associations between serum osteocalcin level and insulin resistance

\section{Kang, J. ${ }^{1}$}

${ }^{1}$ Dept. of family physician, Konyang Univ. Hospital

Introduction: To determine the associations between serum osteocalcin level and insulin resistance measured by using homeostasis model assessment of insulin resistance index (HOMA-IR)

Methods: A total of 98 subjects ( 24 men and 74 women) were selected for this retrospective cross-sectional study who voluntarily visited a health examination center for routine health check-up including the blood test for serum osteocalcin level. Multiple regression analysis was used to determine which independent variables were independently related to osteocalcin levels

Results: Stepwise multiple regression analysis adjusted for age, sex, menopausal status, body mass index, serum alkaline phosphatase, serum calcium and phosphate showed that osteocalcin negatively correlated with serum glucose $(\hat{\mathrm{a}}=-0.145, \mathrm{P}=0.001)$ and homeostasis model assessment of insulin resistance index (HOMA-IR) ( $\hat{\mathrm{a}}=-1.794 \mathrm{P}=0.027)$ independently. ConclusionStepwise multiple regression analysis adjusted for age, sex, menopausal status, body mass index, serum alkaline phosphatase, serum calcium and phosphate showed that osteocalcin negatively correlated with serum glucose $(\hat{\mathrm{a}}=-0.145, \mathrm{P}=0.001)$ and homeostasis model assessment of insulin resistance index $(\mathrm{HOMA}-\mathrm{IR})(\hat{\mathrm{a}}=-1.794 \mathrm{P}=0.027)$ independently.

Disclosure: None Disclosed

Funding: No Funding

T1P16

\section{Associations between the saliva microbiota and body size among 9-12 year old Finnish girls}

\section{Raju, S. ${ }^{1}$; Weiderpass, E. ${ }^{2}$; Rounge, T. $^{3}$}

${ }^{1}$ Folkhälsan Research Center, Helsinki, Finland, ${ }^{2}$ Folkhälsan Research Center, Helsinki, Finland. Cancer Registry Of Norway, Oslo, Norway. Karolinska Institutet, Stockholm, Sweden. University Of Tromsø, The Arctic University Of Norway, Tromsø, Norway., ${ }^{3}$ Folkhälsan Research Center, Helsinki, Finland. Cancer Registry Of Norway, Oslo, Norway.

The epidemic of obesity escalating around the world and the number of overweight and obese people worldwide has increased to 2.1 billion in 2013. Data from the Finnish Health in Teens Study (Fin-HIT,www.finhit. fi, $\mathrm{N} \sim 11000$ ), show that about $13 \%$ of 9-12-year-old Finnish girls are overweight, $2.2 \%$ are obese and $12 \%$ are underweight. The human intestinal microbiota may play a role in body size development. However, associations between saliva microbiota and body mass index (BMI) have been sparsely studied. The aim of our study is to investigate the association between the saliva microbiota and body size of girls.

16S rDNA (V3-V4 region) sequencing of 517 randomly selected saliva samples from the Fin-HIT cohort have produced about $\sim 5$ million $16 \mathrm{~S}$ sequences. Identification of species (operational taxonomic units) and the diversity index have been calculated by the software package Mothur and comparison to the ribosomal RNA sequence database SILVA. Alpha-diversity using Shannon index and Inverse Simpson index, and beta-diversity using Bray Curtis- and Thetayc dissimilarity index were calculated. 
Differentially abundant bacteria were identified at different taxonomic levels using the Zero inflated Gaussian model - fitZig (metagenomeSeq). Alpha and beta diversity were statistically significantly different in the normal vs. overweight and obese combined, and obese vs. all other lower body sizes, respectively. Compared to normal weight children, bacteria belonging to the genus Kingella and Bradyrhizobium were significantly more abundant in underweight girls, bacteria Shuttleworthia, Anaerovorax, Moraxella, Mycoplasma, Bordetella and Alysiella were more abundant in overweight girls, bacteria Enhydrobacter was more abundant in obese girls. Selenomonas spp. known for associated with obesity in women was also a statistically significant in our study too.

Our results show that the saliva microbiota differs significantly between girls of different body size and may potentially be used for early identification of girls at risk for developing underweight and obesity.

Conflict of Interest: None Disclosed

Funding: The study was funded by Samfundet Folkhälsan, the Academy of Finland Medicinska Understödsföreningen Liv och Hälsa rf, the Swedish Cultural Foundation, Signe and Ane Gyllenberg Foundation and the Yrjö Johansson Foundation.

\section{T1P18}

\section{Beneficial effects of Tucum-do-Pantanal (Bactris setosa Mart) extract on diet induced obesity, liver steatosis and insulin resistance in C57BL/6J mice}

\section{Ballard, C. '; Santos, E. '; Dubois, M. ${ }^{3}$; Pilon, G. ; Cazarin, C. ${ }^{3}$; Marette, A. ${ }^{3}$;} Jr., M. ${ }^{1}$

${ }^{1}$ University of Campinas, ${ }^{2}$ Federal University Of Mato Grosso Do Sul, ${ }^{3}$ Université Laval

Introduction: Polyphenols are bioactive compounds found in plants with potential beneficial effects in preventing chronic diseases. The aim of present study was to analyze the consumption of Tucum-do-Pantanal extract (TE), rich in polyphenols, on body weight gain, energy intake, adiposity, lipid and glucose homeostasis.

Methods: Eight-week-old C57BL/6J male mice ( $n=12$ /group) were fed either a Standard diet (10\% fat, $L F$ group) or a High Fat High Sucrose diet (45\% fat, HFHS and Tucum groups). LF and HFHS groups received daily doses $(100 \mathrm{mg} / \mathrm{kg}$ ) of vehicle (water) by gavage, and Tucum group received $100 \mathrm{mg} / \mathrm{kg}$ of TE. Body weight gain was assessed twice week and energy intake, three times a week. Adiposity was evaluated by TD-NMR (Bruker"). Glucose (GTT) and insulin (ITT) tolerance tests were performed in 7 and 8 weeks. Blood samples were collected during GTT for insulinaemia. After 8 weeks, mice were sacrificed and liver tissue was collected for triglycerides, cholesterol measures and Akt/PKB signaling pathway. Data was expressed as media \pm SEM. Statistical analysis was performed using ANOVA (Sigmaplot, USA, $\mathrm{p} \leq 0.05$ ). All procedures were approved by the Laval University Animal Ethics Committee.

Results: TE induced a lower total weight gain (32\%), adiposity (29\%), triglycerides (25\%), cholesterol (8\%) compared to HFHS vehicle. TE treatment also decreased glucose-induced hyperinsulinaemia during the GTT and promoted a superexpression of PAKT (SER473) in the liver. Energy intake was similar between HFHS and Tucum groups. HFHS group obtained higher body weight gain and body fat mass than LF in supporting the establishment of obesity model.

Conclusion: TE, an extract rich in polyphenols, protected against obesity, liver steatosis and insulin resistance induced by the HFHS diet without change the energy intake. Further studies are necessary to establish the mechanism of the polyphenols in energy expenditure, glicemic control and lipid metabolism.

\section{Conflict of Interest: None}

Funding: Research relating to this abstract was funded by National Council for Scientific and Technological Development (CNPq/Brazil)
T1P19

\section{Biotransformed citrus residue act as an anti-inflammatory in} co-culture of 3T3-L1 and RAW 264.7

\author{
Nakajima, V. '; Ávila, A. ; Macedo, G. ; Macedo, J.' \\ ${ }^{1}$ University of Campinas
}

Introduction: In obesity, more macrophage infiltrate on adipose tissue, causing increased secretion of pro-inflammatory cytokines. This pro-inflammatory state contributes to the development of other non-communicable diseases. Thus, reduction in cytokines secretion would be beneficial. This study evaluated the effect of citrus residue extracts (Biotransformed, In Natura and Autoclaved) on TNF- $\alpha$, IL- 6 and adiponectin secretion in co-culture of 3T3-L1 adipocytes and RAW 264.7 macrophages.

Methods: 3T3-L1 were cultivated for 12 days for differentiation, after that a transwell was added in the well and RAW 264.7 were grown in upper layer. After 24 hours, cells were incubated with citrus residue extracts for another 24 hours. Cell supernatant was collected and stored until analysis. Cytokines concentration was measured using Milliplex" MAP Mouse Adipocyte Luminex assay.

Results: Addition of $0.2 \mathrm{mg} / \mathrm{ml}$ of extract did not have any positive effect on pro-inflammatory cytokines. Treatment with $1.0 \mathrm{mg} / \mathrm{ml}$ of Biotrasformed and In Natura extracts were able to reduce the secretion of TNF- $\alpha$ (30.7\% and $14.9 \%$ ) and IL-6 (43.4\% and $42.7 \%$ ) compared to Control with any treatment. Biotransformed extract at a concentration of $1.0 \mathrm{mg} / \mathrm{ml}$ promoted greater increase in adiponectin in relation to In Natura $(66.0 \%$ and $35.3 \%$ above Control, respectively). In view of the flavonoids profile, In Natura extract contains mainly hesperidin and naringin, and because of the biotranformation process by fermentation, the Biotransformed extract contains hesperitin and naringenin.

Conclusion: The different flavanone profile of the Biotransformed extract caused a better anti-inflammatory activity in vitro, being a product with promising results to combat inflammation associated with obesity.

Conflict of Interest: None disclosed.

Funding: Research relating to this abstract was funded by São Paulo Research Foundation (FAPESP)

\section{T1P20 \\ Body mass index is associated with region-dependent metabolic reprogramming of adipose tissue beyond insulin influence}

Alves, M. ${ }^{1}$; Moreira, A. ${ }^{1}$; Guimaraes M. ${ }^{2}$; Nora, M. ${ }^{2}$; Sousa, M. ${ }^{1}$; Oliveira, P. Monteiro, $M^{4}$

${ }^{1}$ UMIB-ICBAS, UP, ${ }^{2}$ Department Of General Surgery Of Hospital São Sebastião, Centro Hospitalar De Entre O Douro E Vouga, Portugal, ${ }^{3}$ Umib-Icbas, Up \& I3S \& Fmup, ${ }^{4}$ University College Of London Uk \& Umib-Icbas, Up

Introduction: Adipose tissue (AT) is involved in dysmetabolism pathogenesis. Regional fat distribution and functioning may contribute to obesity-related metabolic disorders and adverse health outcomes. Specific fat depots are suggested to possess unique biological properties, but specific metabolic profiles of subcutaneous (SAT) and visceral adipose tissue (VAT) remain unknown. We aimed to characterize VAT and SAT glucose metabolism, and their correlation with body mass index (BMI).

Methods: AT samples from patients $(\mathrm{n}=12 ; \mathrm{F}: \mathrm{M}, 9: 3)$ with a mean age of 46 years (26-83 years) and an average BMI of $29.6 \mathrm{~kg} / \mathrm{m}^{2}\left(18-37 \mathrm{~kg} / \mathrm{m}^{2}\right)$ were used. VAT and SAT explants were obtained during elective laparoscopy, either cholecystectomy for uncomplicated cholelithiasis or gastric bypass for severe obesity. Explants were placed in insulin-free cell culture media and their metabolic profile was established by proton nuclear magnetic resonance.

Results: AT explants display a glucose and pyruvate consumption and acetate production that is region-dependent according with the patients BMI. In VAT, glucose consumption was positively correlated with BMI, while alanine and lactate production were negatively correlated with BMI, whereas in SAT the patients BMI did not affect AT secretome suggesting 
that increased BMI promotes a metabolic reprogramming of VAT towards de novo lipogenesis. This region-dependent metabolic reprogramming of AT associated with BMI was autonomous of insulin.

Conclusion: BMI-related metabolic remodeling in VAT occurs beyond the effects known to be mediated by insulin. Targeting this BMI-induced metabolic remodeling may represent a novel therapeutic target to counteract the increased health risks derived from visceral adiposity.

Conflict of Interest: none disclosed

Funding: This work was supported by the "Fundação para a Ciência e a Tecnologia"-FCT co-funded by Fundo Europeu de Desenvolvimento Regional - FEDER via Programa Operacional Factores de Competitividade - COMPETE/QREN to UMIB (PEst-OE/SAU/UI0215/2014); CICS-UBI (Pest-C/SAU/UI0709/2014); MG Alves (IFCT 2015) and PF Oliveira (IFCT 2015)

\section{T1P21}

Body proportion (relative leg length) has a moderator effect on fat-free mass and resting energy expenditure relationship

Silva, C. ; Teles, J.' ; Fragoso, I. ${ }^{1}$

${ }^{1}$ Faculty of Human Kinetics, University of Lisbon

Introduction: Low relative leg length is a sensitive indicator of negative growth conditions (low availability of nutrients and energy). It has been suggested that early energy restriction can lead to compensatory adaptations translated in lower metabolism and energy expenditure. The aim of this study was to investigate the role of relative leg length on the resting energy expenditure-to-fat-free mass ratio.

Methods: A sample of 611 young individuals (11-16 years) was recruited. Leg length was determined subtracting sitting height from stature and relative leg length was calculated (leg length/stature $\times 100$ ). Resting energy expenditure was estimated using Muller equation. Slaughter equations for children and youth of both sexes were used to estimate percentage of fat mass. Percentage of fat mass and body mass were used to determine fatfree mass in kilograms. Physical activity level was estimated with Questionnaire RAPIL II and maturation status with Tanner-Whitehouse III method. Preliminary analysis of covariance and Sidak's post hoc test were conducted. To identify an eventual moderator effect of relative leg length moderation analysis based on multiple linear regression was conducted.

Results: The resting energy expenditure-to-fat-free mass ratio was higher in the fourth quarter of relative leg length, compared with the first quarter $(p=0.003)$. Moderation analysis confirmed the moderator effect of relative leg length on resting energy expenditure-to-fat-free mass ratio $(p=0.003)$. Differences can reach about $60 \mathrm{kcal}$ by kilogram of fat-free mass a day, which represents a loss of $2.9 \mathrm{~kg}$ of body mass a year.

Conclusion: It can be concluded that relative leg length influences the relationship between fat-free mass and resting energy expenditure. More investigation is needed in this area.

Conflict of Interest: None Disclosed

Funding: Research relating to this abstract was funded by the Science and Technology Foundation (PTDC/DES/113156/2009).

\section{T1P22}

Capsinoids increase energy expenditure without overt 18 F-FDG uptake in humans proven bat-positive by cold stimulation using PET scanning

Sun, L. ${ }^{1}$; Camps, S. '; Goh, H. ${ }^{2}$; Govindharajulu, P. ${ }^{3}$; Schaefferkoetter, J. ${ }^{4}$; Verma, S. ; Sadananthan, S. ; Totman, J. '; Sendhil, V.5 ; Townsend, D.7; Leow, M. ${ }^{8}$

${ }^{1}$ Singapore Institute for Clinical Sciences, $A^{*}$ STAR, Singapore, ${ }^{2}$ Singapore Institute For Clinical Sciences, A*Star,Singapore, ${ }^{3}$ Singapore Institute For Clinical Sciences, $A^{*}$ Star, Singapore, ${ }^{4}$ Clinical Imaging Research Center,

A*Star,Singapore, ${ }^{5}$ Singapore Bioimaging Consortium, $A *$ Star,Singapore, ${ }^{6}$ Clinical Imaging Research Center, $A^{*}$ Star,, Singapore, ${ }^{7}$ Clinical Imaging Research Center, A*Star, Singapore, ${ }^{8}$ Tan Tock Seng Hospital, Singapore Institute For Clinical Sciences, $A^{*}$ Star,Singapore

Background: Brown adipose tissue (BAT) contributes to whole-body energy expenditure (EE), during cold-induced thermogenesis (CIT) in humans. Food ingredients such as capsaicin and its non-pungent analogue (capsinoids) have also been shown to affect energy expenditure in healthy human subjects with metabolically active BAT, but not in those with undetectable BAT subjects. However, there is no direct evidence to support the role of capsinoids in BAT activation and modulation of energy expenditure.

Aim: The aim of the present work is to compare capsinoids and cold exposure on BAT activation and whole-body EE.

Methods: Eleven healthy subjects ( 8 males, 3 females) having a mean age of 28 years (range: 23-38), a mean BMI of 22.7 (range: 20-26) underwent ${ }^{18} \mathrm{~F}-\mathrm{FDG}$ PET/MR scans after oral ingestion of $12 \mathrm{mg}$ capsinoids or $1-2 \mathrm{~h}$ of cold exposure $\left(17^{\circ} \mathrm{C}\right)$, each performed on separate days. FDG was intravenously administered for about 20 minutes prior to the PET/MR scan (3 $\mathrm{mCi}$ ). Whole-body EE after oral ingestion of capsinoids $(12 \mathrm{mg})$ and cold exposure were measured for $2 \mathrm{~h}$ in a randomised, crossover design. SUVmean of the region of interest were evaluated in all scans.

Results: All subjects showed no marked FDG uptake after capsinoid ingestion. Under normal conditions $\left(24^{\circ} \mathrm{C}\right)$, there was a $9.4 \%$ increase in $\mathrm{EE}(1.2 \pm 0.06$ vs $1.1 \pm 0.06 \mathrm{kcal} / \mathrm{min})$ after ingestion of capsinoids compared to baseline in the BAT-positive group and no significant change in $\mathrm{EE}$ in BAT negative subjects ( $1.18 \pm 0.16 \mathrm{vs} 1.14 \pm 0.15 \mathrm{kcal} / \mathrm{min})$. Upon cold exposure, 7 subjects showed marked FDG uptake into adipose tissue of the supraclavicular and neck regions (BAT-positive group), whereas the remaining 4 subjects (BAT-negative group) showed no detectable uptake. There was an increase of $18 \%$ in EE after cold exposure in BAT positive subjects $(1.38 \pm 0.07$ vs $1.16 \pm 0.05 \mathrm{kcal} / \mathrm{min})$. Cold stimulus induced a smaller increase in $\mathrm{EE}(6.5 \% ; 1.26 \pm 0.17 \mathrm{vs} 1.19 \pm 0.18 \mathrm{kcal} / \mathrm{min})$ in BAT-negative subjects. In comparison, cold exposure induced a twofold increase in whole-body EE compared with capsinoids ingestion in BAT-positive subjects $(\mathrm{P}=0.003)$.

Conclusion: The preliminary findings suggest that capsinoids only evoked increases EE in BAT-positive subjects, suggesting that BAT play a significant role in the increases of EE stimulated by capsinoids without BAT activation. It is possible that capsinoids stimulate BAT to much lesser degree than cold when using FDG uptake as a measure. Therefore it's premature to state that capsinoids increase EE via a BAT-independent mechanism. 


\section{T1P23}

\section{Carbohydrate intake is associated with higher apelin gene expression in visceral and subcutaneous adipose tissues}

Yuzbashian, E. ; Zarkesh1, M. ${ }^{2}$; Asghari, G. ${ }^{3}$; Mahmoodi, B. ${ }^{3}$; Mirmiran, P. $^{3}$; Hedayati, M. ${ }^{2}$; Safarian, M. ${ }^{4}$, Daneshafrooz, A. ${ }^{2}$; Khalaj, $A .{ }^{5}$

${ }^{1}$ Nutrition and Endocrine Research Center, Research Institute for Endocrine Sciences, Shahid Beheshti University of Medical Sciences, and Student Research Committee, Biochemistry of Nutrition Research Center, School of Medicine, Mashhad University of Medica, ${ }^{2}$ Cellular And Molecular Endocrine Research Center, Nutrition And Endocrine Research Center, Research Institute For Endocrine Sciences, Shahid Beheshti University Of Medical Sciences, Tehran, Iran, ${ }^{3}$ Nutrition And Endocrine Research Center, Research Institute For Endocrine Sciences, Shahid Beheshti University Of Medical Sciences, Tehran, Iran, ${ }^{4}$ Biochemistry Of Nutrition Research Center, School Of Medicine, Mashhad University Of Medical Sciences, Mashhad, Iran, ${ }^{5}$ Obesity Treatment Center, Department Of Surgery, Shahed University, Tehran, Iran

Introduction: The evidence showed that the insulin level concentrations have an important role in apelin gene regulation and circulation. Diet may affect the level of insulin. The aim of the current study was to investigate the association of the apelin gene expression and concentration with habitual carbohydrate intake.

Methods: Visceral and subcutaneous adipose tissues were obtained from 32 morbidly obese and 32 age- and sex-matched non-obese subjects, who underwent open abdominal surgery. Dietary intake was collected using a valid and reliable food frequency questionnaire, and daily intake of carbohydrates, fiber, glucose, fructose, and total sugar were calculated. The mRNA expressions of apelin gene in visceral and subcutaneous adipose tissues were analyzed by Real-Time PCR.

Results: The mean age was 39.6 years for both groups and body mass index for morbidly obese and non-obese subjects were 45.3 and $25.6 \mathrm{~kg} / \mathrm{m}^{2}$, respectively. Apelin gene expression was more increased in morbid obese than non-obese subjects in both subcutaneous ( 3.42 vs $0.49, P<0.05)$ and visceral adipose $(3.65$ vs $0.19, P<0.05)$ tissues. Apelin expression in visceral adipose tissue among non-obese subjects was marginally correlated with fructose $(\mathrm{r}=0.314, P=0.061)$; however, among morbid obese subjects, carbohydrate $(\mathrm{r}=0.429, P=0.014)$, fiber $(\mathrm{r}=-0.360, P=0.034)$, glucose $(r=0.341, P=0.048)$, and fructose $(r=0.310, P=0.057)$ was correlated with apelin gene expression in visceral adipose tissue. Moreover, among obese subjects we found significant correlations between apelin expression in subcutaneous adipose tissue and carbohydrate $(r=0.447$, $\mathrm{P}=0.010)$, fiber $(\mathrm{r}=-0.443, \mathrm{P}=0.013)$, total sugar $(\mathrm{r}=0.369, P=0.038)$, and among non-obese subjects we found significant correlations with glucose $(r=0.481, P=0.006)$, fructose $(r=0.467, P=0.008)$, and total sugar ( $\mathrm{r}=0.435, P=0.014)$. Apelin concentration was correlated with percentage of energy from carbohydrate $(r=0.379, P=0.046)$, fiber $(r=-0.501$, $\mathrm{P}=0.001)$ only in non-obese subjects.

Conclusions: The results of the current study showed that apelin among morbid obese had a positive correlation between carbohydrate and simple sugar intakes and a negative correlation with fiber intake.

Conflict of Interest: None of the authors has any personal or financial conflict of interest.

Funding: Not applicable.

Acknowledgment: The authors would like to thank Dr Mohammad-Reza Ebrahimi for surgical procedures.

\section{T1P24}

\section{Cariprazine influence on PPAR- $\gamma$ expression during in vitro adipogenesis}

Melinda, K. ${ }^{1}$; Zsolt, G. ${ }^{1}$; József-Attila, P.2 ; Tamás, T. ${ }^{3}$; Zoltán, $K_{.}{ }^{4}$

${ }^{1}$ Department of Pharmacology and Clinical Pharmacy, University of Medicine and Pharmacy of Tîrgu Mures, Romania, ${ }^{2}$ Student Of The Faculty Of Pharmacy, University Of Medicine And Pharmacy Of Tîrgu. Mures, Romania, ${ }^{3}$ Department Of Pharmacodynamics, Faculty Of Pharmacy, Semmelweis University Budapest, Hungary, ${ }^{4}$ Doctoral School, University Of Medicine And Pharmacy Of Tîrgu Mures, Romania
Background: Second generation antipsychotics (SGAs) generally increase the risk of the metabolic syndrome. Cariprazine is a novel SGA with partial agonist activity at dopamine $\mathrm{D} 2 / 3$ receptors. The aim of our study was to investigate the influence of cariprazine (compared with aripiprazole) on PPAR- $\gamma$ expression during in vitro adipogenesis.

Materials and Method: To induce adipocyte differentiation from mouse embryonic fibroblasts, these were grown to confluence and then cultured in adipogenic medium for 21 days. Aripiprazole and cariprazine was used at different concentrations, similar to and higher than their therapeutic plasma concentrations $(0.5 \mu \mathrm{M}$ and $20 \mu \mathrm{M}$, respectively $0.1 \mu \mathrm{M}$ and 2 $\mu \mathrm{M})$. On 7 th, 14 th, and 21 th days after induction, PPAR- $\gamma$ expression was assessed by Western blot technique. Because GAPDH is often stably and constitutively present at high levels in most cells, it was used as loading control. The obtained film was evaluated with the ImageJ software. The amount of protein in a sample was proportional with the black spots area of the film. Data analysis was performed using one way ANOVA test and the statistical significance level was established at $\mathrm{p}$ value $<0.05$.

Results: The mean $( \pm \mathrm{SD})$ values of PPAR- $\gamma / \mathrm{GAPDH}$ area ratio in the control/aripiprazole 0.5 / aripiprazole 20/cariprazine 0.1/cariprazine 2 groups were the followings: $2.22 \pm 0.97 / 1.45 \pm 0.96 / 1.39 \pm 0.36 / 2.04 \pm 0.79$ / $1.93 \pm 1.55$ on the 7 th day after induction; $1.69 \pm 1.08 / 1.68 \pm 0.50$ / $1.96 \pm 0.31 / 1.36 \pm 0.26 / 1.69 \pm 1.48$ on the 14 th day of adipocyte differentiation and $1.28 \pm 0.80 / 1.56 \pm 0.25 / 1.89 \pm 0.17 / 0.97 \pm 0.36 /$ $1.05 \pm 0.70$ on the 21 th day of adipogenesis. No differences were found between the groups compared, so either drug did not influence PPAR- $\gamma$ expression.

Discussion: Olanzapine, risperidone are SGAs with important transcriptional and metabolic effects. These drugs increase the expression of the most important pro-adipogenic factor, PPAR- $\gamma$, especially during early phase of adipocyte differentiation. Conversely, aripiprazole and perhaps cariprazine, have only slight metabolic effects, although about cariprazine there are only a few data concerning its molecular mechanisms in cell culture. In our study, this novel SGA, seems to be without influence on PPAR- $\gamma$ expression in the early, intermediate and late phases of adipogenesis.

Conclusion: Our in vitro results agree with other short time reports concerning safety and tolerability of cariprazine, sustaining its lack of influence on pro-adipogenic factor PPAR- $\gamma$. Even so, further long time clinical and in vitro studies on adipogenesis are necessary to complete the metabolic profile of this new drug.

Funding: This work was funded by the Studium Prospero Foundation and Hungarian Academy of Sciences, grant Nr. 0347/26.02.2016.

\section{T1P25}

\section{Cell type specific inhibition of co-stimulatory CD40 interactions in obesity}

\section{Aarts, S. ${ }^{1}$; Berg, S. ${ }^{1}$, Toom, M. ${ }^{1}$; Shami, A. ${ }^{\text {; }}$ Lutgens, E. ${ }^{1}$}

${ }^{1}$ Department of Medical Biochemistry, Academic Medical Center, University of Amsterdam

Introduction: The co-stimulatory molecule pair CD40-CD40L plays a central role in fine-tuning immunological reactions, including obesity-induced inflammation. Genetic ablation of CD40L results in amelioration of adipose tissue inflammation, but in contrast the CD40-/- mice displayed worsened insulin resistance and excessive inflammation of adipose tissue compared with wild-type mice.

Methods: To investigate which cells are responsible for the phenotype observed in the CD40-/- mice, we have generated cell type specific CD40 or CD40L knock out mice for macrophages (LysMcreCD40 $0^{\mathrm{fff}}$ ), dendritic cells/adipose tissue macrophages $\left(\mathrm{CD} 11 \mathrm{C}^{\mathrm{cre}} \mathrm{CD} 40^{\mathrm{ffl}}\right.$ ), adipocytes (Adipo$\left.\mathrm{Q}^{\text {cre }} \mathrm{CD} 40^{\mathrm{fff}}\right)$, and $\mathrm{T}$ cells $\left(\mathrm{CD} 4^{\text {cre }} \mathrm{CD} 40^{\mathrm{ffl}}, \mathrm{CD} 4^{\mathrm{cre}} \mathrm{CD} 40 \mathrm{~L}^{\mathrm{fffl}}\right)$ which are fed a high fat diet for 18 weeks.

Results: LysM ${ }^{\text {cre }} \mathrm{CD} 40^{\mathrm{ffl}}$ mice show that loss of macrophage CD40 increases macrophage accumulation in the visceral adipose tissue. However, deficiency of macrophage CD40 has no effect on adipose tissue mass, insulin 
tolerance, or plasma triglyceride concentrations. The $\mathrm{CD} 11 \mathrm{C}^{\mathrm{cre}} \mathrm{CD} 40^{\mathrm{fl}}$ ${ }^{\mathrm{fl}}$ mice show that CD40 deficiency on DCs and adipose tissue macrophages decreases visceral adipose tissue weight, increases lipid content in the liver and slightly decreases leukocyte numbers and pro-inflammatory gene expression in the adipose tissue.

Conclusion: To this point, the first experiments show that macrophage CD40 does have an effect in obesity-induced inflammation, but is probably not the key player. Future experiments with the AdipoQ ${ }^{\text {cre }} \mathrm{CD} 40^{\mathrm{ffl}}$ and the $\mathrm{CD} 4^{\text {cre }} \mathrm{CD} 40(\mathrm{~L})^{\mathrm{ffll}}$ mice will provide clarity on the exact role of CD40CD40L interactions in diet-induced obesity.

Conflict of Interest: None Disclosed

Funding: Research relating to this abstract was funded by the Dutch heart foundation (CVON2011-19).

\section{T1P26}

\section{Chronic mild-cold exposure promotes weight gain in obese rats raised at thermoneutrality}

Aldiss, P. ' ; Lewis, J. ' 'Ebling, F. ; Budge, H. '; Symonds, M. ${ }^{1}$

${ }^{1}$ The Early Life Research Unit, Academic Division of Child Health, School of Medicine, University of Nottingham, Nottingham, NG7 2UH, ${ }^{2} \mathrm{School}$ Of Life Sciences, University Of Nottingham Medical School, Queen'S Medical Centre, Nottingham Ng7 2Uh

Introduction: Therapeutic activation of brown adipose tissue (BAT) in rodent models of obesity rapidly induces weight loss and ameliorates obesity. However, these studies are typically conducted in animals raised at sub-thermoneutrality and potentially confounded by abnormally large and active BAT depots which are not representative of those found in adult humans. The purpose of this study therefore was to determine whether BAT activation induced weight loss in obese animals raised at thermoneutrality.

Methods: Three week old Sprague-Dawley male rats were immediately transferred to thermoneutrality $\left(28^{\circ} \mathrm{C}\right)$ on arrival and fed either a chow $(n=6, C)$ or high fat diet (HFD) $(n=16, O B)$ for the duration of the study. At 12 weeks of age sub-groups of HFD animals were exposed to mild-cold $\left(20-22^{\circ} \mathrm{C}, \mathrm{n}=6, \mathrm{COB}\right)$ or administered with the clinically approved $\beta 3$-AR agonist Mirabegron $(0.75 \mathrm{mg} / \mathrm{kg}, \mathrm{n}=6, \beta 3 \mathrm{OB})$ for 4 weeks At the end of the intervention period all animals were placed in CLAMS metabolic cages for up to $48 \mathrm{~h}$ to assess energy expenditure/intake and activity followed by tissue collection.

Results: Final body weight $(\mathrm{p}<0.01)$ and fat mass $(\mathrm{p}<0.05)$ were raised with obesity and neither intervention induced weight loss. Instead, $\mathrm{COB}$ were heavier than both $\mathrm{OB}(650.7$ vs $585.6 \mathrm{~g}, \mathrm{p}<0.05)$ and $\beta 3 \mathrm{OB}(592.0$, $\mathrm{p}<0.05)$ groups, with weight gain raised with cold exposure. Only the inguinal fat pad was heavier in COB animals (i.e. 24.4 vs. $12.0 \mathrm{mg} / \mathrm{g} \mathrm{BW}$, $\mathrm{p}<0.05)$. Food intake per cage increased in COB animals yet when assessed individually in metabolic cages there was no difference in energy intake relative to body mass. COB animals also demonstrated a reduced 24h VO2 (9376 vs $8469 \mathrm{ml} / \mathrm{kg} / 24 \mathrm{~h}, \mathrm{p}<0.01$ ) and dark cycle ambulatory activity $(\mathrm{p}<0.01)$. Acute dosing with Mirabegron in CLAMS had no effect on energy expenditure.

Conclusion: When raised in conditions more comparable to adult humans (i.e. at thermoneutrality and free access to an obesogenic diet) interventions designed to stimulate BAT do not promote weight loss, with chronic mild-cold exposure promoting weight gain. We hypothesise obesity may compromise the ability to respond to cold by adaptive thermogenesis such that the response is not just to stimulate BAT but to increase energy storage primarily through subcutaneous adipose tissue deposition in order to improve insulation. The molecular changes in both brown and subcutaneous adipose tissues are currently under investigation.

Funding: None

Disclosure: PA is in receipt of a BHF studentship ref no. FS/15/4/31184

\section{T1P27}

\section{Circulating MOTS-c (a mitochondrial-released peptide) concentration is similar in lean/insulin-sensitive and obese/ insulin-resistant humans}

\section{Cataldo, R. ${ }^{\text {; }}$ Santos, J. ' ; Galgani, J.'}

${ }^{1}$ Pontificia Universidad Catolica de Chile

Introduction: MOTS-c (mitochondria open reading frame [sORF] of the $12 \mathrm{~S}$ rRNA type c) is a recently described mitochondrial-encoded and -released peptide. In mice, MOTS-c has systemic action being proposed as a mitochondrial-derived hormone. MOTS-c influences fuel metabolism in distant organs including skeletal muscle. Indeed, mice treated with MOTS-c improve muscle insulin sensitivity and prevent diet-induced obesity and insulin resistance. In addition, MOTS-c overexpression in HEK293 cells enhances AMP-kinase-dependent fatty acid oxidation, glucose utilization and lactate production. Whether MOTS-c plays a role in humans is elusive. We compared plasma MOTS-c concentration in lean/ insulin-sensitive and obese/insulin-resistant humans. Additionally, we compared MOTS-c determination by a non-commercial and commercial ELISA.

Methods: After an overnight fasting, plasma MOTS-c concentration was determined in sex- and age-matched lean/insulin-sensitive (5/5 males/ females; $33 \pm 7$ [SD] y old; $22.1 \pm 1.1 \mathrm{~kg} / \mathrm{m}^{2} ;$ HOMA $\left.1.10 \pm 0.49\right)$ and obese/insulin-resistant (5/5 males/females; $36 \pm 8$ y old; $32.4 \pm 1.5 \mathrm{~kg} / \mathrm{m}^{2}$; HOMA $2.77 \pm 1.25$ ) healthy volunteers. In addition, circulating glucose, lactate and insulin concentrations were measured before and for 3 hours after a 75-g glucose load. From postprandial response, Matsuda index was also calculated as an insulin sensitivity surrogate. MOTS-c was determined by a non-commercial (Reference Service, U. of Southern California. USA) and commercial (Cloud Clone; CEX132Hu. USA) ELISA. Means were compared by $\mathrm{t}$ test and associations by Pearson correlation. Results: Lean vs.obese volunteers had lower fasting plasma lactate $(1.00 \pm 0.33$ [SD] vs. $1.38 \pm 0.34 \mathrm{mmol} / \mathrm{l} ; \mathrm{p}=0.02)$ and borderline lower fasting plasma glucose $(89 \pm 4$ vs. $93 \pm 3 \mathrm{mg} / \mathrm{dl} ; \mathrm{p}=0.06)$, while postprandial plasma lactate $(\mathrm{p}=0.61)$ and glucose $(\mathrm{p}=0.14)$ responses were similar between groups. As expected, a higher Matsuda index was detected in lean vs. obese individuals $(6.9 \pm 3.7$ vs. $2.6 \pm 0.8$, respectively; $\mathrm{p}<0.01)$. Plasma MOTS-c concentration (by the Reference Service) was similar in lean and obese volunteers $(0.48 \pm 0.16$ vs. $0.52 \pm 0.15 \mathrm{ng} / \mathrm{mL}$, respectively; $\mathrm{p}=0.59$ ), with a low size effect (mean difference/SD $=0.24$ ) found. Plasma MOTS-c concentration was much higher when assessed by the commercial vs. non-commercial ELISA ( $117 \pm 62$ fold). Furthermore, null association between assays was detected $(\mathrm{r}=0.12 ; \mathrm{p}=0.63)$.

Conclusion: Circulating MOTS-c concentration does not appear to be influenced by obesity and/or insulin resistance in humans. Human plasma MOTS-c determination requires validation for future studies.

Conflict of interest: None

Funding: FONDECYT 1130217

\section{T1P28 \\ Cognitive function in bariatric surgery versus non-surgical patients for obesity}

Matsumoto, J.'; Hirano, Y.'; Kitahara, A. ${ }^{3}$; Tokuyama, H. ; Yamaga, M. ${ }^{3}$; Kitamoto, T. ${ }^{3}$ Y Yokote, K. $^{3}$

${ }^{1}$ Department of Regional Disaster Medicine, Graduate School of Medicine, Chiba University, ${ }^{2}$ Research Center For Child Mental Development, Chiba University, ${ }^{3}$ Department Of Clinical Cell Biology And Medicine, Graduate School Of Medicine, Chiba University, ${ }^{4}$ Department Of Advanced Aging Medicine, Graduate School Of Medicine, Chiba University

Introduction: Recent research suggests that bariatric surgery might help stop the decline in cognitive function associated with obesity, and bariatric surgery has been shown to improve many aspects of cognitive function. Factors underlying improved cognition following surgery are not yet fully established. The aim of this study was to investigate whether the cog- 
nitive function such as memory, attention, inhibition or decision-making abilities were altered in patients with obesity who undergoing bariatric surgery (BS) and non-surgical patients (NS) at baseline and after 6 months or point in postoperative time.

Methods: A total of 9 severe obese patients (age, 25-65; BMI, 39.6-51.2) including four participants who were scheduled to undergo the bariatric surgery and five patients who were non-surgical were enrolled in this study. All participants had their intelligence quotient, cognitive function were assessed using the Wechsler Adult Intelligence Scale, Stroop test, Trail Making Test and the Iowa Gambling Task (IGT), and their eating-related pathophysiology and depressive, anxiety symptoms, self-esteem were also evaluated at baseline and after 6 months.

Results: Compared with NS group, BMI in BS individuals who underwent bariatric surgery was significantly lower than in the NS at the 6 months later from a baseline ( $\mathrm{p}=0.002)$. Regard with decision-making abilities, in particular in the last trial on IGT, a significant interaction arose when considering the relationship between both groups. However, no significant differences among intelligence quotient, cognitive function such as memory, attention, inhibition, and depressive, anxiety symptoms, self-esteem in BS and NS at the time of baseline and after 6 months.

Conclusion: The findings indicated that in some obese patients undergoing bariatric surgery may not be associated with improved cognitive function. Future studies should investigate to prove the certainty of these results by a larger sample size, and also prospective studies with extended follow-ups (e.g, 3 years) should examine whether bariatric surgery can attenuat cognitive decline in severely obese patients.

Conflict of Interest: None Disclosed.

Funding: Research relating to this abstract was funded by JSPS KAKENHI Grant Number JP15K01703.

\section{T1P29 \\ Comparable effects of breakfast meals varying in protein source on appetite and subsequent energy intake in healthy males}

\section{Dougkas, A. ${ }^{1}$;Ostman, . $^{2}$}

${ }^{1}$ Institut Paul Bocuse, ${ }^{2}$ Food For Health Science Centre, Lund University

Introduction: Increased protein intake has the greatest putative role in appetite control followed by carbohydrates and fat (1). However, the satiating effect of animal vs plant proteins remains unknown (2). The present study examined the effects of breakfasts containing animal proteins [milk (AP)], a blend of plant proteins [oat, pea and potato (VP)] or 50:50 mixture of the two (MP) compared with a carbohydrate-rich meal $(\mathrm{CHO})$ on appetite, energy intake (EI) and metabolic measures.

Methods: A total of 28 males [mean age 27.4 ( \pm SD 4.2$)$ years, BMI 23.4 ( \pm $2.1) \mathrm{kg} / \mathrm{m}^{2}$ ] consumed three isoenergetic $(1674 \mathrm{~kJ})$ rice puddings matched for energy density and macronutrient content as breakfast (25\% E from protein) in a single-blind, randomised, cross over design. Appetite ratings and blood samples were collected and assessed at baseline and every 30 and $60 \mathrm{~min}$, respectively, until an ad libitum test meal was served $3.5 \mathrm{~h}$ later. Free-living appetite was recorded hourly by visual analogue scales and EI in weighed food records for the remainder of the day.

Results: A repeated measures ANOVA showed that fullness was 15\% after consumption of the CHO test breakfast compared with the protein breakfasts $(P=0.019)$. No differences in subjective appetite ratings were observed after consumption of the AP, VP and MP. Furthermore, there were no differences between the AP, VP, MP and CHO breakfasts in ad libitum EI and self-reported EI during the remainder of the day. Although insulin metabolism was not affected, $\mathrm{CHO}$ induced a higher glucose response $(\mathrm{P}=0.001)$ and total amino acids concentration was in the order of $\mathrm{AP}=\mathrm{MP}>\mathrm{VP}>\mathrm{CHO}$ breakfast $(\mathrm{P}=0.001)$.

Conclusion: Manipulating the protein source of foods consumed as breakfast, elicited comparable effects on appetite and EI at both laboratory and free-living environment in healthy men. Thus, whole or partial replacement of animal by plant proteins may offer an effective and sus- tainable alternative when targeting the satiating capacity of high protein foods.

\section{References:}

1 Paddon-Jones D. et al. (2008) Protein, weight management and satiety. Am J Clin Nutr 87 (5): 1558S-1561S

2 Gilbert JA et al. (2011) Effects of proteins from different sources on body composition. Nutr Metab Cardiovasc Dis 21 Suppl 2: B16-31.

Conflict of Interest: None disclosed

Funding: Lund University Antidiabetic Food Center, a VINNOVA (Sweden's Innovation Agency) Excellence Center

\section{T1P30 \\ Contribute of cognitive reserve for weight loss in severely obese treatment}

Ribeiro, O. ${ }^{1}$; Carmo, I. ${ }^{2}$; Fagundes, M. ${ }^{2}$; Horácio, J. ${ }^{1}$; Figueira, M. ${ }^{3}$; Paiva, T. ${ }^{4}$

${ }^{1}$ Neuropsychology Unit of Hospital de Egas Moniz, Lisbon, ${ }^{2}$ Endocrinology, Diabetes And Metabolism Service, Hospital De Santa Maria, Lisbon, ${ }^{3}$ Psychiatric Department, Hospital De Santa Maria, Lisbon, ${ }^{4}$ Cenc - Sleep Medicine Center, Lisbon

Introduction: The prevalence of obesity has been steadily increasing and is a major public health problem worldwide. It is associated with multiple medical and psychological conditions with a probable link to deficits in several cognitive domains. Cognitive Reserve (CR) is one of the possible moderators of the association between Body Mass Index (BMI) and cognitive function contributing to more efficient results in cognitive performance (CP). The aim of this study is to examine changes in anthropometric measures, cognitive performance $(\mathrm{CP})$ and psychopathological symptoms (PS) and to evaluate the contribution of CR for weight loss in severely obese patients after one year of structured Psychological and $\mathrm{Nu}$ tritional intervention.

Method: We examined CP, PS and CR values of 56 severely obese patients from a convenience sample of a Portuguese Referral Center for Severely Obese Treatment. Sociodemographic data, clinical data, and neuropsychological assessment were collected in a session with a mean duration of 45 minutes. Subjects were reassessed one year later after a Psychological and Nutritional intervention. Results were statistically analyzed through the SPSS program.

Results: The population predominantly female, aged between 31 and 40 years had mostly 9 years of education and low income. At baseline, CR was not significantly related to sex, age, and with hypertension, diabetes, cholesterol, alcoholic and smoking habits $(p<.05)$. The highest group (with low CR), presented greater anthropometric measures and a higher Body Mass Index (BMI) than the group with high CR. After intervention, BMI significantly decreased $(p<.05)$ and weight loss was within the expected average $(39.4 \% ; M=6.7 \mathrm{~kg} ; 1 / 2 S D=2.8 \mathrm{~kg})$; there were more patients with low CR that lost weight; the mean difference in weight loss between groups of CR was positive and significant for BMI $(\mathrm{p}<0.05)$; CP showed a significant increase in measures like Immediate Recall, Digit Symbol, Trail Making Test and Stroop Interference Test $(p<0.05)$ and a significantly decreased on Learning and Digit Span (Backward) $(p<0.05)$; PS showed a significant decrease $(\mathrm{p}<0.05)$. Age, monthly income, and PS explained $44.9 \%$ of the results CR didn't show to be a significant predictor of weight loss.

Conclusion: Psychological and Nutritional intervention had a positive and significantly influence on anthropometric measures, PS and CP contributing for their decrease in severe obese. CR didn't significantly contribute to weight loss.

Disclosure: None Disclosed/Payment received from Hospital Egas Moniz, Hospital de Santa Maria or Sleep Medicine Center

Funding: Funding/Research relating to this abstract was funded by Hospital Egas Moniz, Hospital de Santa Maria or Sleep Medicine Center 
T1P31

\section{Depletion of the small GTPase RAB4B in T cells leads to adipose tissue dysfunction and insulin resistance}

Jérôme, G. '; Gwennaelle, B. ${ }^{1}$; Stoyan, I. ${ }^{2}$; Bastien, V. ${ }^{1}$; Laurent, Y.' ; Nicolas, V. ${ }^{3}$; Jean-François, T. $^{1}$; Mireille, $\mathrm{C}^{1}{ }^{1}$

${ }^{1}$ Université Côte d'Azur, INSERM U1065, C3M, Team «Cellular and Molecular Physiopathology of Obesity and Diabetes », Nice, France, ${ }^{2}$ Université Côte D’Azur, Inserm U1065, C3M, Team «Metabolism And Cancer», Nice, France, ${ }^{3}$ Université Pierre Et Marie-Curie, Inserm U1138, Cordeliers Research Center, Paris, France

Introduction:Changes in adipose immune cells in obesity is involved in the inflammation of adipose tissue (AT) and in its impaired expendability leading to the development of insulin resistance (IR) and metabolic disease. However, the underlying mechanisms involved in this change remain not completely understood. A fine-tune control of intracellular trafficking is crucial for the function and fate of the immune cells. The Rab GTPases govern intracellular trafficking and we recently found a down-regulation of Rab4b, a key protein of the early/recycling endosomes, in the T lymphocytes of AT, both in obese mice and humans. We thus aimed at determining the consequences of the down-modulation of Rab4b in T cells on AT function and glucose and lipid homeostasis.

Methods: We generated mice with a specific invalidation of Rab4b in T cells (Rab4b $\mathrm{b}^{\text {Tcell KO})}$ ) by crossing Rab4b flox/fox mice with Lck-Cre mice. We analysed the metabolic phenotype of these mice fed a normal chow diet (NCD) or a high-fat diet (HFD) and characterized the cellular events underlying the phenotypic changes.

Results: Compared with wild-type mice, Rab $4 b^{\text {Tcell KO }}$ mice on NCD have the same body and AT weight but exhibited a defect in AT expandability with hypertrophic and insulin resistant adipocytes and with an increase in the number of adipose mesenchymal stem cells suggesting a defect in adipocyte differentiation. Accordingly, the secretome of this AT inhibited adipocyte differentiation. We found that the lack of Rab4b in T cells skewed the balance between adipose Treg/Th17 towards Th17 leading to an inflammation of AT that could explain the defect in its expandability. As a consequence, Rab4b $\mathrm{b}^{\text {Tcell KO }}$ mice exhibited ectopic lipid deposition in liver and muscles and IR with age. Finally, those mice when fed a HFD, became as obese as wild-type mice but exhibited an exacerbation of IR.

Conclusion: This study uncovers a control of $\mathrm{T}$ cell fate by Rab4b and reveals that the loss of Rab4b expression in T cell during obesity could contribute to IR by promoting disturbance in AT T cell homeostasis leading to inflammation and impaired AT expandability AT.

Funding: INSERM and the Société Francophone du Diabète

\section{T1P32}

Determination of brown adipose tissue activity in mice using a new hyperpolarized $\left[1-{ }^{13} \mathrm{C}\right]$ pyruvate MRI method

Riis-Vestergaard, M. ${ }^{1}$; Breining, P. ${ }^{1}$; Pedersen, S. ${ }^{1}$; Laustsen, C. ${ }^{2}$; Borghammer, $P^{3}$; Richelsen, $B{ }^{1}$

${ }^{1}$ Department of Endocrinology and Internal Medicine, Aarhus University Hospital, ${ }^{2} \mathrm{Mr}$ Research Center, Institute Of Clinical Medicine, Aarhus University, ${ }^{3}$ Department Of Clinical Physiology And Nuclear Medicine, Aarhus University Hospital

Introduction: Brown adipose tissue (BAT) is a highly metabolically active organ which during activation leads to respirational uncoupling, heat loss and increased energy expenditure. BAT is present in humans and can be a potential target for treatment of obesity and metabolic syndrome. ${ }^{13} \mathrm{C}$-labelled hyperpolarized pyruvate MRI is a new diagnostic tool without radiation that can be used for real time assessment of metabolic processes. The aim of the study is to identify the BAT organ and activity in mice using this new method compared to the more conventional method FDG (fluorodeoxyglucose) PET/MRI, in order to determine BAT.

Methods: Chronic cold (gradually cooling from $22 \mathrm{C}$ to $6 \mathrm{C}$ for $2-3$ weeks, $\mathrm{n}=12$ ) and thermoneutral (30C for $2-3$ weeks, $\mathrm{n}=12$ ) acclimated mice were scanned with hyperpolarized $\left[1-{ }^{13} \mathrm{C}\right]$ pyruvate MRI for assessment of the interscapular BAT. Corresponding FDG PET/MRI was performed. Finally, the mice were sacrificed; interscapular BAT was removed and assessed for thermogenic markers by qPCR.

Results: BAT activity could be visualized by hyperpolarized $\left[1-{ }^{13} \mathrm{C}\right] \mathrm{py}$ ruvate MRI. After cold exposure the $\left[1-{ }^{13} \mathrm{C}\right]$ bicarbonate $/\left[1-{ }^{13} \mathrm{C}\right]$ pyruvate signal was significantly increased compared to thermoneutral conditions. Similar results were seen for the metabolites $\left[1-{ }^{13} \mathrm{C}\right]$ lactate and $\left[1-{ }^{13} \mathrm{C}\right]$ alanine. FDG PET/MRI showing increased FDG uptake in the cold acclimated mice confirmed the results. Finally, qPCR on interscapular BAT revealed increased levels of the thermogenic genes including UCP1 in cold acclimated mice compared to thermoneutral mice.

Conclusion: Chronic cold exposure is a potent activator of interscapular BAT in rodents and can be visualized and determined by $\left[1-{ }^{13} \mathrm{C}\right]$ hyperpolarized pyruvate MRI. This radiation-free method could pave the way for future human BAT studies.

Conflict of interest: none

\section{T1P33}

\section{Dietary n-3 fatty acids as phospholipids improve insulin sensitivity of the liver and skeletal muscle in dietary obese} mice

Rossmeisl, M. ${ }^{1}$; Pavlisova, J. ${ }^{1}$; Kucharikova, P. ${ }^{1}$; Kalendova, V. ${ }^{1}$; Kopecky, J. ${ }^{\prime}$ ${ }^{1}$ Institute of Physiology, Czech Academy of Sciences

Introduction: Nutrition could aid in the prevention of obesity and associated metabolic comorbidities. Previous studies in rodent models of obesity indicated that dietary n-3 fatty acids (Omega-3), namely EPA and DHA, could exert beneficial effects on metabolism, while their phospholipid (PL) form might be more effective than fish oil, i.e. triacylglycerols (TG). Here, we compared the TG and PL form regarding their effects on glucose homeostasis in obese mice.

Methods: Male C57BL/6N mice $(n=7-10)$ were fed for 8 weeks a corn oil-based high-fat diet (lipids $~ 32 \mathrm{wt} \%$; cHF) supplemented with the Omega-3 concentrate either as TG (Epax 1050 TG from Epax AS; Omega-3 TG) or PL (Krill oil from Olympic Seafood AS; Omega-3 PL) at a dose $\sim 30 \mathrm{~g}$ EPA+DHA per kg diet. Glucose homeostasis was assessed by means of glucose tolerance tests and hyperinsulinemic-euglycemic clamps using $\mathrm{D}-[3-3 \mathrm{H}]$ glucose as a tracer. Comparisons were judged to be significant at $\mathrm{p} \leq 0.05$ (t-test).

Results: Compared to cHF, both Omega-3 TG and Omega-3 PL reduced body weight by $8 \%$ and $26 \%$, and hepatic steatosis by $30 \%$ and $64 \%$, respectively. However, only Omega-3 PL reduced fasting blood glucose and plasma insulin by $18 \%$ and $59 \%$, while improving glucose tolerance, i.e. reducing AUC by $38 \%$. Clamp studies showed elevations of glucose infusion rate, glucose turnover, as well as whole-body glycolysis and glycogen synthesis by $303 \%, 198 \%, 177 \%$, and $299 \%$, respectively, and reductions in hepatic glucose production by $54 \%$, in the Omega-3 PL group. In contrast, Omega-3 TG only increased whole-body glycogen synthesis by $172 \%$. Moreover, Omega-3 PL increased the rate of glycogen synthesis in quadriceps muscle by $286 \%$.

Conclusion: Our data confirm the superior efficacy of the PL form of Omega-3 regarding the effects on hepatic steatosis and glucose homeostasis, and provide a rationale for the preferential use of Omega-3 PL in clinical practice.

Conflict of Interest: None Disclosed.

Funding: Research relating to this abstract was funded by the Czech Science Foundation (project no. 17-11027S). 
T1P34

Different combinations of maternal and postnatal diet are reflected in changes of hepatic parenchyma and hepatic TNFalpha expression in male rat offspring

KačArević, Ž. ${ }^{1}$; Grgić, A. ${ }^{1}$; Šnajder, D. ${ }^{2}$; Bakula, M. ${ }^{3}$; Bijelić, N. ${ }^{4}$; Lekšan, I. ${ }^{1}$

${ }^{1}$ Faculty of Medicine University of Osijek, Department of Anatomy and Neuroscience, Osijek, Croatia, ${ }^{2}$ University Hospital Center Osijek, Clinical Department For Nuclear Medicine And Radiaton Protection, Osijek, Croatia,

${ }^{3}$ University Hospital Center Osijek, Institute Of Pathology, Osijek, Croatia, ${ }^{4}$ Faculty Of Medicine University Of Osijek, Department Of Histology And Embryology, Osijek, Croatia

Introduction: Obesity is related to increased TNF-alpha production in different tissues and TNF-alpha is connected to mitochondrial dysfunction in the liver and development of fatty infiltration of the liver. Also, postnatal change from normal to high-fat diet causes a significant increase in TNF-alpha serum levels. The aim of this research was to determine how maternal diet during pregnancy and lactation and switching male offspring to a different dietary regime influences rat liver.

Methods: Ten female Sprague Dawley rats at nine weeks of age were randomly divided in two groups and fed either standard laboratory chow or high-fat diet during five weeks, and then mated with the same male subject. After birth and lactation male offspring from both groups were further divided into four subgroups depending on their subsequent diet. At 22 weeks of age, the animals were weighted, sacrificed and major organs were collected and weighted. Immunohistochemistry for TNF-alpha was performed on liver, and liver samples were analysed for pathohistological changes.

Results: The group in which mothers were fed standard chow and offspring high-fat diet had the most pronounced changes being the heaviest liver, poorest histopathological findings and strongest TNF-alpha immunohistochemical staining of liver parenchyma.

Conclusion: High-fat diet during pregnancy and lactation and switching to high-fat diet postnatally affects liver weight, histological structure and TNF-alpha expression.

Conflict of Interest: None Disclosed.

Funding: Research relating to this abstract was supported in part by a grant of the Ministry of Science, Education and Sports of the Republic of Croatia (ID: 219-2192376-2092) and also by the grant of the University of Rijeka, Croatia (ID: 13.06.2.2.63).

\section{T1P35}

Differential expression of miRNAs related to angiogenesis and adipogenesis in subcutaneous fat of obese and non-obese women

Gasparotto, A. '; Borges, D. ${ }^{2}$; Sassi, M. ; Milani, A. ${ }^{3}$; Rech, D. ${ }^{3}$; Terres, M. ${ }^{3}$; Ely, P. ${ }^{3}$; Ramos, M. ${ }^{4}$; Meinhardt, N. ${ }^{4}$; Mattevi, . $^{1}$

${ }^{1}$ Universidade Federal de Ciências da Saúde de Porto Alegre, ${ }^{2}$ Chronic Diseases Research Center - Nova Medical School, ${ }^{3} \mathrm{Irmandade}$ Santa Casa De Misericórdia De Porto Alegre, ${ }^{4}$ Hospital Nossa Senhora Da Conceição

Introduction: Recent evidences suggests that obesity is associated with a state of adipose tissue remodeling, characterized by adipocyte hypertrophy, increased angiogenesis and production of proinflammatory adipokines. In order to better understand these alterations, we compared expression of microRNAs (miRNAs) that target genes involved in angiogenesis, inflammation and adipogenesis in adipose tissue of obese and non-obese women.

Methods: Subcutaneous adipose tissue (SAT) samples were collected during bariatric and cosmetic surgery for obese and non-obese women respectively. Mature miRNAs were quantified by real-time polymerase chain reaction. Clinical and anthropometrics measurements were obtained for further comparisons.

Results: miR-16 was overexpressed in SAT of non-obese women (n-expression ratio $=-151.1 ; \mathrm{P}<0.001)$. Additionally, the expression of miR-27b and miR-424-5p was significantly correlated with waist circumference in non-obese women $(\mathrm{r}=0.453 ; \mathrm{P}=0.027$ and $\mathrm{r}=0.502, \mathrm{P}=0.014$, respectively). Total and central subcutaneous adipose tissue thicknesses were correlated with miR-424-5p levels $(r=0.475, \mathrm{P}=0.046$ and $\mathrm{r}=0.506$, $\mathrm{P}=0.034$, respectively). miR-424-5p expression was also correlated with body mass index in the obese group $(\mathrm{r}=0.582, \mathrm{P}=0.018)$.

Conclusion: Both miR-16 and miR-424 have shown associations with different body fat mass-related parameters and have vascular endothelial growth factor (VEGF) and its receptors as target genes. These data suggest they may be involved in the alterations of angiogenesis observed in obesity.

Conflict of insterest: The authors delcare that they have no conflict of interest

Funding: Research relating to this abstract was funded by Conselho Nacional de Desenvolvimento Científico e Tecnológico (CNPq, Brazil) and Fundação de Amparo à Pesquisa do Estado do Rio Grande do Sul (FAPERGS, Brazil).

\section{T1P36}

\section{Disturbed NK cell function in obesity is associated with an increased colon tumor risk}

\section{Bähr, l. ${ }^{1}$; Goritz, V. ${ }^{1}$; Doberstein, $H_{.}{ }^{1}$; Kielstein, $H^{1}{ }^{1}$}

${ }^{1}$ Institute of Anatomy and Cell Biology, Martin Luther University HalleWittenberg

Introduction: Natural killer (NK) immune cells control tumor progression and metastases. Previous studies showed altered NK cell functions in obese individuals. Obesity is associated with a higher colon cancer incidence, but underlying mechanisms remained unclear. We investigated the impact of an altered NK cell functionality on the increased colon cancer risk in obesity.

Methods: The cytotoxicity of leptin-incubated NK cells against colon tumor cells was determined using an Europium release assay. Cytokine secretion of NK cells was measured by luminex immunoassay. Moreover, in vivo experiments in normal weight and obese rats were performed. Colon cancer growth was induced in half of the animals by injection of azoxymethane (AOM). Body weight and visceral fat mass were determined, colon tumors were characterized and tumor, splenic and hepatic NK cells were analyzed by real-time RT-PCR and immunohistochemnistry.

Results: The cytotoxicity of NK cells against colon tumor cells was significantly reduced after leptin incubation. Additionally, the reduced NK cell cytotoxicity by leptin was associated with a decreased IFN $\gamma$ secretion. In obese rats, body weight and visceral fat mass were significantly increased compared to the normal weight animals. AOM-treated obese rats showed an increased quantity, size and weight of colon tumors compared to the normal weight AOM group. Molecular and immunohistological analyses indicate tissue specific changes in NK cell numbers as well as expression levels of activating NK cell receptors in obese animals.

Conclusion: The results showed that the impaired NK cell function may be one cause for the elevated colon cancer risk in obesity.

Conflict of Interest: The authors declare no conflict of interest.

Funding: This project was supported by a grant from the Dr. Werner Jackstädt Foundation.

\section{T1P37 \\ Do different protein intakes influence body composition and muscle function in obese rats?}

Rossignon, F.'; Martin, V. ${ }^{2}$; Salles, J.'; Patrac, V. ; Bacquer, O. ; Giraudet, C. ; Ratel, S. ${ }^{2}$; Faria, C. ' Boirie, Y.' ; Guillet, C. ${ }^{1}$

${ }^{1}$ University Clermont Auvergne, UMR1019, ${ }^{2}$ University Clermont Auvergne, Ea3533, Inra, Umr1019

Introduction: During obesity, impairments in oxidative capacities, lipid infiltration and insulin resistance within skeletal muscle may act in concert to lead to loss of muscle mass and function. Considering the role of 
dietary proteins in preserving mass and improving muscle function, the aim of the study was to determine the potential preventive effects of increased protein intake on changes in metabolism and function muscle induced by obesity.

Methods: Male Wistar rats, aged 9 months, were randomized in 4 groups $(\mathrm{n}=15)$ and were fed during 10 weeks either with a standard diet (STD) with normal lipid intake (13.8\% of total energy intake (TEI)), or with a high fat diet (HFD) (45\% of TEI). For each diet, two levels of protein intakes were tested: $12 \%$ of TEI (STD12 and HFD12) and 25\% of TEI (STD25 and HFD25). For each animal, body weights and food consumption were quantified weekly. Measurements of body composition and muscle functionality were performed. The data are presented as mean \pm standard error and analyzed by two-way ANOVA (diet and protein intake) and a Duncan's Multiple-Comparison Post-hoc Test.

Results: Total calories intakes were not different between the groups. The increases in weight and fat mass were significantly greater in the HFD12 group (respectively: $+176.2 \pm 13.9 \mathrm{~g}$ and $+121.5 \pm 7.8 \mathrm{~g}$ ) than in STD groups (STD12: $+95.3 \pm 20.2 \mathrm{~g}$ and $+62.5 \pm 4.3 \mathrm{~g}$; STD25: $+65.6 \pm 15.3 \mathrm{~g}$ and $+53.4 \pm 3.6 \mathrm{~g}, \mathrm{P}<0.05$ vs HFD12. These increases were less important in the HFD25 group $(+133.9 \pm 17.2 \mathrm{~g}$ and $+98.2 \pm 6.0 \mathrm{~g}, \mathrm{P}=\mathrm{NS}$ vs HFD12). The weights of skeletal muscles taken during sacrifices were not different between groups. At the end of the 10 weeks diet, muscle strength and power values were decreased in all groups. However, the decrease of muscle strength and power tended to be higher in HFD12 group $(-0.97 \pm 0.33 \mathrm{~N}$ and $-0.23 \pm 0.07 \mathrm{~W}$ ) than in STD groups (STD12:-0.39 $\pm 0.32 \mathrm{~N}$ and $-0.07 \pm 0.10 \mathrm{~W}$; STD25: $-0.35 \pm 0.31 \mathrm{~N}$ and $-0.07 \pm 0.01 \mathrm{~W})$. These variations observed in HFD12 group were more attenuated in HFD25 group $(-0.51 \pm 0.18 \mathrm{~N}$ and $-0.17 \pm 0.06 \mathrm{~W})$.

Conclusion: Differences in the macronutrient composition of diets influence the evolution of body weight and composition more than calorie intake. Moreover, protein enrichment in the diet during lipid overnutrition could attenuate the deleterious consequences of obesity on muscle function.

Conflict of Interest: None Disclosed

\section{T1P38 \\ Does fatness exacerbate frailty? The effects of age and dietary-induced obesity on the contractile function of isolated locomotory and respiratory skeletal muscles}

\section{Hill, C. ${ }^{1}$; James, R. ${ }^{1}$; Cox, V. ${ }^{1}$; Tallis, J. ${ }^{1}$}

${ }^{1}$ Centre for Applied Biological and Exercise Sciences, Faculty of Health and Life Sciences, Coventry University, Coventry, CV1 5FB, U.K

The effect of increasing age on the decline in skeletal muscle contractility has been well established. Given that the normal ageing process is further associated with increased adiposity, and recent evidence demonstrating that obesity and the related increase in muscular lipid content may evoke a significant reduction in skeletal muscle contractility, the present study is the first to examine whether obesity exacerbates the age related decline in skeletal muscle function.

Methods: 70 week old female mice (CD-1) were split into two experimental groups, control and obese. Obese animals were fed a calorie rich high fat diet and controls standard lab chow for 9 weeks. Following this, either the soleus (SOL), predominantly slow twitch, extensor digitorum longus (EDL), predominantly fast twitch, or a section of the costal diagram (DIA) was isolated from each individual and isometric tetanus stress (force/cross-sectional area), work loop (WL) power $(\mathrm{W} / \mathrm{kg}$ ) and fatigue resistance were measured.

Results: Obese animals had a significantly greater body mass, Lee Index of Obesity \& fat pad mass compared to the lean counterparts. Obese SOL and EDL muscle mass was significantly greater, but maximal isometric stress and work loop power $(\mathrm{W} / \mathrm{Kg})$ were not significantly different to the controls. By contrast, obese DIA maximal isometric stress had a statistical tendency to be lower than controls whilst WL power was significantly lower, with a $25 \%$ reduction in obese animals. There was no difference in the fatigue resistance between obese and control for both the SOL and the EDL, although the results indicate that the DIA from obese animals would fatigue faster than the lean controls due to producing less power.

Conclusion: The present findings demonstrate that an increased adiposity will significantly reduce the contractile performance of the diaphragm but not locomotory muscles. It should be considered that the locomotory muscles of the obese animals would have to overcome greater bodily inertia due to the elevated body weight of these individuals, thus having important whole body mechanical implications. The given reduction in the contractility of the diaphragm may result in an impaired respiratory function and in turn increase the demand on the cardiovascular system. A reduction in diaphragm function may therefore contribute to a decline in functional capacity and the negative cycle of obesity due to impaired oxygen uptake.

\section{T1P39 \\ Effect of consumption chronic of high fat diet on the role of central glucose in production control hepatic glucose.}

Andrade I.S. '; Freitas N.T. '; Sousa I.F.'; Nascimento C.M.O. '; Oyama L.M. '; Ribeiro E.B.

${ }^{1}$ Federal University of São Paulo

Introduction: A high fat diet is a great risk factor for obesity and it is associated comorbities, such insulin resistance and diabetes. Diet nutrients act on hypothalamic neurons, which control food intake, glycemia and energetic homeostasis. Glucose administration induces feeding suppression. We found an increase in extracellular glucose in the ventromedial hypothalamus, normoglycemia and failure in response to glucose injection on food intake (Andrade et al 2015). The hepatic production of glucose (EPG) is crucial for the maintenance of normoglycemia. The objective of the present study was to verify the effect of high-fat diet on phosphoenolpyruvate carboxykinase (PEPCK).

Methods: Two-months old male Swiss mice were randomly assigned to standard chow $(3.76 \mathrm{kcal} / \mathrm{g})$ or high-fat diets $(5.1 \mathrm{kcal} / \mathrm{g})$ enriched with lard (saturated fat source). Body weight and food intake were recorded weekly for 12 weeks. Mice were then intracebroventricular injected with either saline or Glucose $(100 \mu \mathrm{g} / 5 \mu \mathrm{L})$ and had food available for 2 and 4 hours. Mice were euthanizied $4 \mathrm{~h}$ after glucose injection and liver were dissected for PEPECK analysis by Western blot. The adipose tissues were dissected and weighed. Blood glucose levels, triglyceride and cholesterol were determined by enzymatic Methods: Eight groups were studied, according to diet and injection received: saline control (Csal), control Glucose (CGli), High-fat saline (high-fat sal), High-fat glucose (high-fat Gli). Data were analyzed by ANOVA followed by either Turkey or Student's " $t$ " test.

Results: During diet treatment, daily food intake was lower $(\mathrm{p}=0.0001)$ in high-fat group in comparison control group with similar energy intake. The high-fat diet increased body weight gain $(p=0.0001)$, adipose tissue Retroperitoneal $(p=0.05)$ and Mesenteric $(p=0.001)$. After 4 hours at glucose injection the food intake was lower in the control group $(\mathrm{p}=0.05)$, but did not cause effect in the high- fat group, and a decreased serum triglyceride in both groups $(C, p=0.001$ and High-fat, $p=0.0001)$ in comparison to groups that received saline injections, but did not alter glucose and cholesterol. The saline high-fat groups had higher blood glucose levels $(\mathrm{p}=0.01)$ and cholesterol $(\mathrm{p}=0.001)$ and lower triglyceride $(\mathrm{p}=0.01)$ in comparison to the saline control group. The PEPCK was similar between the groups and was not altered by glucose injection.

Conclusion: The glucose inhibited food intake in control group but failed in the group high-fat diet. The PEPCK was similar in both groups and were not unchanged by glucose injection. It is necessary to investigate the effect of glucose on another hepatic gluconeogenic enzyme, glucose 6-phosphatase (G6Pase).

Disclosure: None Disclosed

Funding: Research was funded by State of Sao Paulo Research Foundation (FAPESP). 
T1P40

Effect of diet-induced weight loss on angiopoietin-like protein 4 and adipose tissue lipid metabolism in overweight and obese humans

Kolk, B. ${ }^{1}$; Vink, R. ${ }^{1}$; Jocken, J. '; Goossens, G. ${ }^{1}$; Roumans, N. ${ }^{1}$; Mariman, E. ${ }^{1}$; Baak, M.'; Blaak, E. ${ }^{1}$

${ }^{1}$ Department of Human Biology and Movement Sciences, NUTRIM School of Nutrition and Translational Research in Metabolism, Maastricht University, Maastricht, The Netherlands

Introduction: Angiopoietin-like protein 4 (ANGPTL4) plays a role in lipid partitioning by inhibiting lipoprotein lipase (LPL)-dependent plasma clearance of triacylglycerol (TAG) in adipose tissue (AT). In this study, we investigated the effects of diet-induced weight loss on plasma ANGPTL4 concentrations in relation to in vivo AT LPL activity and lipolysis and AT ANGPTL4 release in overweight/obese individuals.

Methods: Sixteen participants (BMI: $28-35 \mathrm{~kg} / \mathrm{m}^{2} ; 10$ women) were randomized to a dietary intervention composed of either a low-calorie diet $(1250 \mathrm{kcal} / \mathrm{d})$ for 12 weeks or a very-low-calorie diet $(500 \mathrm{kcal} / \mathrm{d})$ for 5 weeks, followed by a weight stable period of 4 weeks. Before and after intervention, AT arteriovenous concentration differences were measured in combination with AT blood flow before and after intake of a high-fat mixed-meal enriched with $\left[\mathrm{U}-{ }^{13} \mathrm{C}\right]$-palmitate to determine in vivo AT LPL activity and lipolysis and AT ANGPTL4 release.

Results: The intervention significantly reduced body weight $(-8.6 \pm 0.6$ $\mathrm{kg}, \mathrm{P}<0.001)$ and plasma ANGPTL4 concentrations were unaffacted. Significant postprandial AT ANGPTL4 release into the circulation was observed $(\mathrm{P}<0.01)$ and this release tended to be higher after intervention $(\mathrm{P}=0.072)$. No significant association was observed between plasma ANGPTL4 and AT chylomicron-TAG extraction, which reflects in vivo LPL activity. After intervention fasting and postprandial plasma ANGPTL4 concentrations were positively associated with net AT free FA and glycerol release, both reflecting in vivo AT lipolysis (fasting glycerol: $\mathrm{r}=0.503 ; \mathrm{P}=0.047$ and postprandial glycerol: $\mathrm{r}=0.597 ; \mathrm{P}=0.015$ ).

Conclusion: Plasma ANGPTL4 is unaffected by weight loss. ANGPTL4 is released from human AT after a high-fat meal in overweight/obese individuals. Plasma ANGPTL4 was not related to in vivo AT LPL activity, but was positively associated with AT lipolysis after weight loss. The role of ANGPTL4 in human AT lipolysis requires further investigation.

\section{T1P41}

\section{Effect of high intensity interval training and diet on} concentrations of vaspin in rats fed with hyperlipidic diet

Costa, L. ${ }^{1}$; Castro, C. ${ }^{1}$; Silva, F. ' Sene-Fiorese, M. ' Fabrizzi, F. ' ; Anibal, F. ; Duarte, A. ${ }^{1}$; Furino, $V .{ }^{1}$

${ }^{1}$ Federal University of São Carlos

Introduction: Vaspin is an adipokine derived from visceral adipose tissue and a member of the serine protease inhibitor family, which is highly expressed by visceral adipose tissue in obesity conditions. Literature suggests that physical exercise, performed aerobically or anaerobically, is able to provide metabolic adaptations favorable to health parameters. In this context, high intensity interval training (HIIT) has been considered a promising time-efficient strategy, since the its adaptations are similar to those promoted by long-term aerobic exercises. Thus, this study aimed to examine the effects of HIIT on systemic vaspin and visceral adipose tissue of rats with experimental obesity.

Methods: 40 male Wistar rats were randomly assigned to four experimental groups: sedentary + standard diet (S); Trained + Standard Diet (STR); Sedentary + Hyperlipidic Diet (H); Trained + Hyperlipid Diet (HTR). The obesity induction protocol lasted 4 weeks and it was followed by training involving 3 workouts per week for 10 weeks. The training treatment consisted of 5 minutes of warm-up and 4 to 6 intervals of 4 minutes of vigorous activity plus 3 minutes of active recovery.
Results: Group " $H$ " showed higher serum vaspin values than the groups "STR" and "HTR" (S, $112.79 \pm 35.07$, STR, $103.74 \pm 22.88, \mathrm{H}$, $220.20 \pm 97.00$, HTR, $144.41 \pm 32.71)$. In the visceral adipose tissue (S, $0.48 \pm 0.30$, STR, $0.42 \pm 0.24, \mathrm{H}, 0.79 \pm 0.31$, HTR, $0.76 \pm 0.54)$ the values did not suffer changes as a result of Diet or exercise.

Conclusion: It was concluded that the hyperlipidc diet was able to increase the concentration of circulating Vaspine and the exercise HIIT was not able to reverse this process.

Disclosure: None Disclosed

Funding: FAPESP (2013/12797-0) and CAPES.

\section{T1P42}

\section{Effect of intermittent fasting on the expression of SREBP 1c and 2 and acetyl CoA carboxylase in the hypothalamus of female rat}

\section{Fernandez, M. ${ }^{\text {; }}$, Vilca, C. ${ }^{1}$; Pedroso, A. ${ }^{2}$; Cinelli, L. ' ; Batista, L. ${ }^{\text {; }}$;}

Albuquerque, $K{ }^{1}$

${ }^{1}$ Universidade Federal do Rio de Janeiro, ${ }^{2}$ Universidade Federal De São Paulo

Introduction: Intermittent fasting has been used as a strategy for loss or maintenance of body weight, however hypothalamic lipogenic events may affect the regulation of food intake and although some studies show fast weight loss, the hypothalamic effect of intermittent fasting on the central lipogenic mechanism is not elucidated. In this way, was evaluated the effect of intermittent fasting on the expression of the sterol regulatory element-binding protein (SREBP) and the acetyl-CoA carboxylase (ACC) enzyme.

Methods: Wistar female of 67 days of life, composed three experimental groups treated for 6 weeks: Control group (C), mantained in free feed consumption throughout the treatment; Restricted 3: 2 (R3), mantained with food restriction at $50 \%$ of $C$ group consumption for two days, followed by three days of free consumption; and Restricted 5:2 (R5) mantained with food restriction at $50 \%$ of $C$ group consumption for two days, followed by five days of free consumption. All treated groups had free access to water. After treatment the female rats were euthanized and the hypothalamus was dissected to evaluate the mRNA levels of the SREBP-1c and 2 transcription factors and the ACC lipogenic enzyme by qPCR.

Results: The hypothalamic expression of SREBP 1c and 2, as well as the ACC enzyme, were not statistically different vs $\mathrm{C}$ group, however elevation of SREBP-1c (R5: 93\%) and SREBP2 (R3: 70\%: R5: 75\%) was observed, with greater repercussion on SREBP2 and R5 group.

Conclusion: Intermittent fasting for 6 weeks in females rats did not alter the expression of early proteins of the lipogenic cascade, but suggests that hypothalamic damage in the expression of SREPB 1c and 2 may be time-dependent.

Conflict of Interest: No conflict of interest

Acknowledgement: This study was supported by Fundação Carlos Chagas Filho de Amparo à Pesquisa do Estado do Rio de Janeiro - FAPERJ and the Coordenação de Aperfeiçoamento de Pessoal de Nível Superior - CAPES.

\section{T1P43 \\ Effect of phosphorus supplementation on diet induced thermogenesis of a normal protein-low phosphorus meal}

\section{Abdouni L. '; Olabi A. '; Obeid O.'}

${ }^{1}$ American University of Beirut

Introduction: Diet induced thermogenesis (DIT), the energy needed to digest and metabolize food, is primarily related to ATP production, which is known to be affected by phosphorus availability. On the other hand, the high inconsistency in findings related to DIT (1) triggered our interest to determine the role of phosphorus in this process. Thus, a study was designed to explore the impact of phosphorus on DIT of a normal pro- 
tein-low phosphorus meal using a low phosphorus containing protein, egg white.

Method: A crossover design study was conducted where 12 overnight fasted healthy lean male subjects were asked to consume a $554 \mathrm{Kcal}$ normal protein-low phosphorus meal (15\% of total E from protein) with phosphorus $(500 \mathrm{mg}$ ) or placebo tablets in a random order. Energy expenditure was measured at baseline and for the next four hours in a period of 15 minutes interval with 15 minutes break, using a ventilated hood and canopy system (COSMED QUARK CPET UNIT) for indirect calorimetry measurement. Changes in energy expenditure from baseline were calculated and data were analyzed using two-way analysis of variance (ANOVA).

Results: Postprandial energy expenditure increased following the ingestion of the different meals (Time: $P<0.000$ ). While, postprandial energy expenditure of the phosphorus supplemented group was found to be higher than that of the control (Phosphorus: $P<0.001$ ) especially from 60 min and onward.

Conclusion: Phosphorus was able to significantly increase and prolong postprandial energy expenditure and therefore, it is involved in diet induced thermogenesis.

Conflict of Interest: The authors declare that they have no conflict of interest.

Funding: The work was supported by a grant from the University Research Board at the American University of Beirut and the Lebanese National Council for Scientific Research.

\section{Reference:}

1 Methodological considerations for meal-induced thermogenesis: measurement duration and reproducibility. Br J Nutr. 2013 Dec 14;110(11):19781986.

\section{T1P44}

\section{Effect of vitamin D on muscle mitochondria size and function in high fat diet-induced obese rats and palmitic acid-treated C2C12 cells}

Chang, E. ${ }^{1}$; Shin, Y. ${ }^{1}$; Lee, S. ${ }^{1}$; Jung, S. ${ }^{1}$; Kim, Y. ${ }^{1}$

${ }^{1}$ Department of Nutritional Science and Food Management, Ewha Womans University

Introduction: Decreased mitochondrial number and dysfunction in skeletal muscle are associated with obesity and insulin resistance. In previous studies, vitamin D deficiency develops muscle atrophy, muscle weakness, and falls in accompanied with changes in gene expression related to muscle growth and differentiation in human and animals. However, the relationship between vitamin $\mathrm{D}$ and muscle mitochondria biogenesis and function in obese rats has never been investigated. The purpose of this current study is to investigate effect of vitamin D on muscle mitochondria size and function.

Method: Male Sprague-Dawley rats were randomized and fed a normal diet (NOR), a $45 \%$ high-fat diet (HF), or a $45 \%$ high-fat diet containing $25 \mathrm{IU}$ vitamin $\mathrm{D} / \mathrm{kg}$ diet (HF+LVD) for 12 weeks. Body weight and food intake were monitored twice a week. Mitochondrial morphology was determined by electron microscopic analysis. 24 hour palmitic acid (PA)-pretreated $\mathrm{C} 2 \mathrm{C} 12$ differentiated myobutes were incubated with 1,25-dihydroxyvitamin D (1,25(OH)2D; 100 nM, 24 hour). Reverse transcription polymerase chain reaction was performed to determine the effects of $1,25(\mathrm{OH}) 2$ on gene expression involved in mitochondria biogenesis and function.

Results: Vitamin D insufficiency (HF+LVD) significantly exacerbates HFC-increased body weight and adipose tissue mass $(\mathrm{P}<0.05)$. In addition, muscle mitochondria number and size were remarkably reduced in vitamin $\mathrm{D}$ deficient obese rats compared to HFC-induced obese rats. In PA-treated C2C12 cells, 1,25(OH)2D significantly upregulates mRNA levels involved in mitochondria biogenesis and function such as peroxisome respiratory factor 1 (NRF1), proliferator-activated receptor coactivator-1a (PGC-1a), nicotinamide adenine dinucleotide (NAD)-dependent deacetylase, sirtulin1 (SIRT1), and nuclear and mitochondrial transcription factor A (Tfam) $(\mathrm{P}<0.05)$.

Conclusion: These results suggested at least partial involvement of vitamin $\mathrm{D}$ in muscle mitochondria suggest the beneficial role of vitamin $\mathrm{D}$ in obesity.

\section{Conflict of Interest: None Disclosed}

Funding: Research relating to this abstract was funded by the Basic Science Research Program through the National Research Foundation of Korea (NRF) funded by the Ministry of Science, ICT, and Future Planning (2014R1A1A3050953)

\section{T1P45 \\ Effects Of High-Fat Feeding On Liver Proteome Of An Ovariectomized Rat Model}

\section{Pedroso, A. ${ }^{1}$; Dornellas, A. ${ }^{2}$; Boldarine, V. ; Oyama, L. ' ; Nascimento, C. ${ }^{1}$; Tashima, A. ' ; Ribeiro, E. ${ }^{1}$ \\ ${ }^{1}$ Universidade Federal de São Paulo, ${ }^{2}$ Universidade Federal Do Paraná}

Introduction: Obesity prevalence is increased in menopausal women. The incidence of non-alcoholic fatty liver disease (NAFLD) increases with obesity and ovarian hormone deprivation. Dietary factors, such as the intake of saturated or polyunsaturated fatty acids, may impact this process. This study investigated the effects of high-fat feeding on the liver proteome of ovariectomized rats.

Methods: 2-months-old Wistar rats were either sham-operated (Sham) or ovariectomized (Ovx). For 2 months from surgery Sham group was fed with control diet and Ovx rats were fed with either control diet (OvxC) or high-fat diets, enriched with lard (OvxL) or fish oil (OvxF). Hepatic proteins were analyzed by mass spectrometry. String database was used to perform the analysis of affected pathways.

Results: Despite the similar food intake, OvxC had higher body weight in relation to Sham, and OvxL showed significantly higher body weight than the other groups. OvxL also had increased fat pads weight when compared to the other groups, while OvxF showed lower fat pads weight than OvxC and heavier livers than the other groups. Ovx rats showed high levels of serum glucose, independently of the diet consumed. OvxL presented hyperinsulinemia and hyperleptinemia. Cholesterol levels were higher in OvxL than in Sham, and lower in OvxF than in OvxL. OvxF presented reduced triglyceride levels. Only OvxL group showed hepatic fat accumulation. Shotgun proteomics enabled the quantification of 373 proteins. Comparison between Sham and OvxC groups showed that ovariectomy altered the expression of 40 hepatic proteins. The main effects of such changes are related to impairment of peroxisome function. The analysis among Ovx groups showed altered expression of 81 and 104 hepatic proteins in OvxL and OvxF in relation to OvxC, respectively. Pathway analysis indicated that these alterations could lead to imbalances in degradation of fatty acids and branched chain amino acids regardless the type of fat consumed. However, OvxL and OvxF showed distinct effects concerning glutathione metabolism. Increased activity in glycine, serine and threonine metabolism and oxidative phosphorylation was observed only in OvxF.

Conclusion: This study showed that either lard or fish oil intake induced specific changes in the liver of ovariectomized rats, with signs of hepatic fat accumulation only in OvxL. These results provide important insight for future studies ascertaining the molecular mechanisms by which saturated or polyunsaturated fatty acids could influence the development of NAFLD in menopausal women. The data suggests that modulation of redox homeostasis and energy metabolism have a pivotal role in this process.

Conflict of Interest: none disclosed

Funding: Research relating to this abstract was funded by FAPESP 


\section{T1P46}

\section{Effects of peripherally acting neuropeptide $Y$ receptor antagonists BIBO3304 and BIIE0246 on obesity}

\section{Ailanen, L. ' ; Vähätalo, L. ' ; Salomäki, H. ${ }^{1}$; Ruohonen, S. '; Savontaus, E. ${ }^{2}$}

${ }^{1}$ Department of Pharmacology, Drug Development and Therapeutics and Turku Center for Disease Modeling, University of Turku, Turku, Finland, ${ }^{2}$ Department Of Pharmacology, Drug Development And Therapeutics And Turku Center For Disease Modeling, University Of Turku, Turku, Finland; Unit Of Clinical Pharmacology, Turku University Hospital, Turku, Finland

Introduction: Neuropeptide Y (NPY) plays an important role in the regulation of energy homeostasis in central and sympathetic nervous system, and mice overexpressing NPY in noradrenergic neurons (OE-NPY ${ }^{\mathrm{DBH}}$ ) display obesity with related metabolic disorders. Peripheral NPY Y1- and Y2-receptors (YRs) are known to mediate obesogenic effects via inhibition of lipolysis or via stimulation of adipogenesis, respectively, but their importance in regulating energy homeostasis is not known. The aim of this study was to elucidate peripheral blockade of YRs on the metabolic phenotype of OE-NPY ${ }^{\mathrm{DBH}}$ and WT mice with healthy and high caloric diet.

Methods: Specific Y1R-antagonist BIBO3304, Y2R-antagonist BIIE0246, or vehicle were administered (i.p.) for 2 or $4 \frac{1}{2}$ weeks on chow- or western-diet-fed OE-NPY ${ }^{\text {DBH }}$ vs. WT mice ( $n=6-11$ /group). Weight gain, food intake and body composition were monitored throughout the experiment.

Results: Y1R-antagonism had no long-lasting effects on body composition on either diet. However, Y1R-antagonism decreased serum and liver triglyceride in chow-fed WT mice, but increased serum cholesterol levels in both genotypes on western diet. Y2R-antagonist on chow-diet increased body weight gain and predisposed WT mice to metabolic disturbances i.e. increased serum insulin and cholesterol levels and hepatic triglycerides. Similarly on western-diet Y2R-antagonist had obesogenic effect in WT mice, which is suggested to result from blockage of presynaptic Y2Rs increasing the NPY levels. Oppositely, Y2R-antagonist treated OE-NPY ${ }^{\mathrm{DBH}}$ mice on western diet had reduced body fat mass gain and hepatic triglycerides, and decreased serum cholesterol levels relative to fat mass.

Conclusion: On a healthy diet or with normal NPY levels, peripheral Y1R- or Y2R-antagonism seems not to have advantages in the control of body weight and adiposity. However, during energy surplus combined with elevated NPY levels, e.g. stress combined with an unhealthy diet, Y2R-antagonism has beneficial effect on fat deposition.

Acknowledgement: The authors would like to thank Satu Mäkelä, Wendy Orpana and Sanna Bastman for technical assistance.

Conflict of Interest: None Disclosed

Funding: Research relating to this abstract was funded by Academy of Finland, the Finnish Funding Agency for Technology and Innovation, and Turku University Hospital Research Fund.

\section{T1P47}

\section{Effects of two long bouts of aerobic exercise on energy} expenditure and appetite during an oral glucose tolerance test

Boule, N. ${ }^{1}$; Eshghi, S. ${ }^{1}$; Myette-Côté, É. ${ }^{2}$

${ }^{1}$ University of Alberta, ${ }^{2}$ University Of British Columbia

Introduction: The impact of exercise on energy balance can extend beyond the energetic cost of the exercise itself. Changes in appetite or energy metabolism can persist for hours and can accentuate or attenuate the energy deficit caused by exercise. Two long bouts of moderate intensity aerobic exercise have been shown to reduce some of the typical responses to hypoglycemia, such as neuroglycopenic symptoms, increased counter-regulatory hormones and the suppression of glucose metabolism. However, the impact of this form of exercise on appetite and glucose metabolism during hyperglycemia has not been studied.
Methods: Twelve men, six with and six without type 2 diabetes (T2D) came to the laboratory for two separate conditions according to a randomized crossover design. On day 1 of each condition, participants either rested or performed two bouts of 90 minutes exercise at $80 \%$ of their ventilatory threshold. On day 2 of each condition, data from indirect calorimetry and appetite ratings (150 mm visual analogue scales) were collected during fasting and every hour of a 4-hour oral glucose tolerance test (OGTT). For all variables below, there was a significant effect of time (i.e., of the OGTT). Therefore, for simplicity, analyses were presented separately for the fasting and postprandial states according to $2 \times 2$ factorial ANOVA which examined the effects of exercise (Ex vs Rest) and diabetes (T2D vs Ctrl), as well as their interactions.

Results: Respiratory exchange ratio (RER) was higher in T2D in both the fasting and postprandial states (both $\mathrm{p}<0.01$ ). In both healthy and T2D, exercise lowered postprandial RER $(\mathrm{p}<0.01)$, but the decrease in fasting RER did not reach statistical significance $(\mathrm{p}=0.08)$. Energy expenditure was unaffected by diabetes or exercise. Overall, ratings for prospective food consumption were higher during the OGTT from the exercise condition compared to the rest condition $(\mathrm{p}=0.03)$. However, only in T2D did postprandial fullness decreased following exercise (Diabetes by Exercise interaction $\mathrm{p}=0.05)$. Participants with T2D had a lower desire to eat something sweet in the fasting $(\mathrm{p}=0.01)$ and postprandial state $(\mathrm{p}=0.03)$, but a Diabetes by Exercise interaction $(\mathrm{p}=0.045)$ indicated that exercise tended to increase the desire to eat something sweet in T2D while decreasing this rating in healthy participants during the OGTT.

Conclusion: Two long bouts of exercises reduced following-day glucose oxidation and increased prospective food consumption in both healthy and T2D participants during an OGTT. However, the desire to eat something sweet was affected differently in healthy versus T2D.

Conflict of Interest: None Disclosed

Funding: This study was supported by a pilot grant from the Alberta Diabetes Institute.

T1P48

Elevated M30 and L-FABP concentrations in morbid obesity decrease after dramatic weight loss due to bariatric surgery

Meyer-Gerspach, A.1; Wölnerhanssen, B.1; Senn, A.2; Moor,

M.3; Borgwardt, S.4; Peterli, R.5; Beglinger, C.1;Bieri, 0.3

${ }^{1}$ Department of Research, St. Claraspital Basel, Basel, Switzerland, ${ }^{2}$ Department Of Biomedicine, University Hospital Basel, Basel, Switzerland, ${ }^{3}$ Department Of Radiology, University Hospital Basel, Basel, Switzerland, ${ }^{4}$ Department Of Psychiatry, University Hospital Basel Basel, Switzerland, ${ }^{5}$ Department Of Surgery, St. Claraspital Basel, Basel, Switzerland

Introduction: Morbid obesity is associated with a number of co-morbidities, including lipotoxic effects in the liver. Accumulation of lipid intermediates in the liver results in lipotoxicity leading to cellular dysfunction and death. Liver biopsy is the gold standard for the diagnosis of fatty liver disease; however, liver biopsy is invasive emphasizing the need for noninvasive diagnostic biomarkers. Liver fatty acid-binding protein (L-FABP, plays a significant role in preventing lipotoxicity) and M30 (a marker for severe liver disease) are two circulating biomarkers of fatty liver disease and might serve as diagnostic tools. The marked changes of bariatric surgery on body weight and fatty liver disease assessed by circulating levels of L-FABPs and M30 is not known.

Methods: In total, 51 morbidly obese patients $\left(\mathrm{BMI}>35 \mathrm{~kg} / \mathrm{m}^{2}\right)$ scheduled for bariatric surgery and 43 lean controls were included in the study. Fasting plasma samples were taken from lean controls and morbidly obese patients before and 6 months after bariatric surgery. L-FABPs and M30 were analysed by a commercially available ELISA kit. Student's unpaired t-test was used to test for significant differences between lean and obese; paired t-test was used to compare pre- and postoperative values.

Results: Morbidly obese patients exhibit increased levels of L-FABPs and M30 compared to the lean controls (L-FABPs: $50 \pm 11$ vs $9 \pm 2 \mathrm{ng} / \mathrm{mL}$, $\mathrm{p}=0.001 ; \mathrm{M} 30: 205 \pm 54$ vs $60 \pm 4 \mathrm{U} / \mathrm{L}, \mathrm{p}=0.01)$. A significant decrease in 
of both biomarkers was observed 6 months post-surgery (L-FABPs: $9 \pm 1$ vs $50 \pm 11 \mathrm{ng} / \mathrm{mL}, \mathrm{p}<0.001 ; \mathrm{M} 30: 81 \pm 5$ vs $205 \pm 54 \mathrm{U} / \mathrm{L}, \mathrm{p}=0.018)$. Conclusion: L-FABP and M30 are markedly increased in morbid obesity; the reduction of both markers after weight loss could play an important role in the beneficial effects of bariatric surgery.

Conflict of Interest: None Disclosed

Funding: The trial was funded by the Swiss National Science Foundation.

\section{T1P49}

Emerging inflammation and dysbiosis caused by a high fat diet administration in zebrafish

Jayo, N. ${ }^{1}$;

${ }^{1}$ Azti Tecnalia

Introduction: The obesity epidemic is characterised by an excess accumulation of body fat and a low-grade systemic inflammatory tone. Energy-dense foods and overnutrition represent major starting points altering lipid metabolism, systemic inflammation and the gut microbiota ${ }^{1}$ The role that microbes have in energy homeostasis has driven research to study the association of intestinal microbiota and obesity ${ }^{2}$. Zebrafish has become a popular model organism in biomedical research because its immune and inflammatory pathways are conserved to humans, as well as other metabolic processes such as lipid metabolism ${ }^{3}$.

Methods: 5 days post-fertilization (dpf) zebrafish larvae were maintained during 30 days with a control diet and a control diet supplemented with $20 \%$ fat (HFD). Intestinal inflammation was studied by gene expression profile, histology of larvae intestinal epithelium, alcian blue staining of goblet cells and neutrophils count by flow citometry; furthermore, dysbiosis was tested by metabarcoding and qPCR.

Results: A differential expresion of a number of genes, related with the antiinflammatory and proinflamatory cascade, signaling receptors, and antimicrobial metabolism was detected; Neutrophils and goblet cells count showed the development of an incipient and mild inflammation, which was confirmed with the cellular apoptosis and mucosal disruption observed in histology. Furthermore, there is a proven dysbiosis in the total number and taxons of intestinal microbiota.

Conclusion: A HFD causes a change in the intestinal microbiota closely related to an emerging inflammation in $30 \mathrm{dpf}$ zebrafish larvae.

\section{Conflict of Interest: None Disclosed}

Funding: Research relating to this abstract was funded by LIFE+ program (project LIFE13 ENV/ES/001048, ENVIPHAGE), MAROMEGA Project (Basque Country Government, Economic Development and Structure; Technology, Innovation and Competitivness Department; Technology Direction) and Basque Country DAPA ( Agriculture, Fisheries and Food Department)

\section{References:}

1 Patterson, E., Ryan, P., Cryan, J., Dinan, T., Ross, R., Fitzgerald, G. and Stanton, C. (2016). Gut microbiota, obesity and diabetes. Postgraduate Medical Journal, 92(1087), pp.286-300.

2 Lopez-Cepero A. and Palacios C. (2015). Association of the intestinal Microbiota and Obesity. Puerto Rico Health Science Journal 34(2), pp.60-4.

3 Holtta-vuori m., Salo v., Nyberg I., Brackmann c., Enejder a., Panula p. and Ikonen e. (2010). Zebrafish: gaining popularity in lipid research. Biochemical Journal. 429, 235-242.

\section{T1P50}

\section{Evaluation of indirect calorimetry in subjects with extreme obesity}

Fidilio, E. '; Guerrero, M. ${ }^{1}$; Velásquez, M. '; Ortiz, A. ' ; Giribes, M. '; Cardenas, G. ${ }^{\text {; }}$ Balibrea, J. ${ }^{2}$; Burgos, R. ${ }^{1}$; Mesa, J. ${ }^{1}$; Fort, J. ${ }^{2}$; Ciudin, A. ${ }^{1}$

${ }^{1}$ Endocrinology and Nutrition Department, Vall d'Hebron University Hospital, Barcelona, Spain, ${ }^{2}$ Endocrine And Metabolic Surgery Department, Vall D'Hebron University Hospital, Barcelona, Spain
Introduction: Obesity is a major public health problem, now out of control, with a very significant economic cost, both for its high prevalence and the associated comorbidities. Bariatric surgery (BS) is at present the most effective treatment for morbid obesity. However, the percentage of excess weight lost (\%EWL) between the individuals is variable and has been associated with age, sex, comorbidities and energetic balance. Indirect calorimetry (IC) is the gold standard method for measuring the resting energy expenditure (REE) in the clinical practice. In the case of the extreme obesity, defined as BMI $>50 \mathrm{~kg} / \mathrm{m}^{2}$, there is little data in the literature regarding the basal energy expenditure, and the factors that might influence the \%EWL after BS.

Objectives: On these bases, we aimed to describe the baseline characteristics of the IC of subjects with extreme obesity in our centre and to assess the relationship between the IC and \%EWL after BS.

Methods: We designed an observational study of patients with extreme obesity who attended our center between January 2014 and December 2015. Baseline REE was determined by IC in 39 subjects with a Vmax metabolic monitor. In this period of time 9 patients underwent BS and had 12 month of follow-up. The REE was also determined at 1 and 12 months after BS. An adequate response to BS was considered when 12 month \%EWL was $>50 \%$.

Results: The mean age was $46.5 \pm 11.77$ years and $64.10 \%$ were women. Baseline BMI and REE were $56.23 \pm 5.58 \mathrm{~kg} / \mathrm{m}^{2}$ and $2320.38 \pm 750.81$ $\mathrm{kcal} /$ day, respectively. In the subgroup of patients that underwent BS, the REE decreased in the first month from baseline to $1537.6 \pm 117.46 \mathrm{Kcal} /$ day $(\mathrm{p}=0.023)$ and remained similar at 12 months $(1526.00 \pm 123.35$ $\mathrm{Kcal} /$ day, $\mathrm{p}=0.682$ ). The REE after BS is similar with the REE of the normal weight Spanish population. The BMI after BS was $47.7 \pm 7.54 \mathrm{~kg} / \mathrm{m}^{2}$ at 1 month and $36.17 \pm 5.17 \mathrm{~kg} / \mathrm{m}^{2}$ at 12 month. The $\% E W L$ at 12 month after BS was $60.38 \pm 17.93 \%$. The REE at 1 and 12 months was negatively correlated with the initial excess of weight $(\mathrm{r}=-0.714, \mathrm{p}=0.047$ and $\mathrm{r}=-0.681, \mathrm{p}=0.014$, respectively). The patient's age and \%EWL in the first month were independent predictors of \%EWL at 12 months.

Conclusion: In the patients with extreme obesity evaluated in our study, the REE at 1 month after BS reduced significantly until levels comparable with normal weight Spanish population while the BMI was still in the range of morbid obesity. The REE remained unchanged at the 12 months after BS. However, baseline REE was not a predictor of weight lost one year after CB. Further studies are needed in order to deepen the factors that influence the \%EWL after BS and the evolution of the REE.

Conflict of Interest: None Disclosed

Funding: No Funding

\section{T1P51}

Exercise training modulates inflammation in rats with metabolic syndrome and heart failure with preserved ejection fraction

Schmidt, C. ${ }^{1}$; Gonçalves, N. ${ }^{2}$; Silva, A. ${ }^{2}$; Coelho, J. ${ }^{2}$; Fontoura, D. ${ }^{2}$; Leite, S. ${ }^{2}$; Miranda-Silva, D. ${ }^{2}$; Falção-Pires, I. ${ }^{2}$; Lourenço, A. ${ }^{2}$; Oliveira, J. ${ }^{1}$; LeiteMoreiro, A. ${ }^{2}$; Moreira-Gonçalves, D. ${ }^{1}$

${ }^{1} \mathrm{CIAFEL}$, Faculty of Sport, University of Porto, ${ }^{2}$ Surgery And Physiology Department, Faculty Of Medicine, University Of Porto

Introduction: Patients with heart failure with preserved ejection fraction (HFpEF) have a high prevalence of comorbidities such as obesity, diabetes mellitus and hypertension, which are associated with a chronic low-grade inflammatory state. There is currently evidence that this inflammatory condition is an important mediator in the development of HFpEF. It is known that exercise training has pleiotropic effects in several pathologies, especially for its anti-inflammatory properties. In the present study, we evaluated if exercise training would be able to improve exercise capacity, diastolic function and modulate inflammation, endothelial dysfunction, oxidative stress, neurohumoral activation and extracellular matrix remodeling in rats with metabolic syndrome and HFpEF. 
Methods: The study was performed with nine-week old ZSF1 obese rats $(\mathrm{Ob}, \mathrm{n}=20)$. At 16 th week, animals were randomly divided into two groups: sedentary (ObSed; $\mathrm{n}=10)$ and exercised $(\mathrm{ObEx} ; \mathrm{n}=10$; aerobic exercise on treadmill during 4 weeks, 5 days/week, $60 \mathrm{~min} /$ day, at a speed of $20 \mathrm{~m} / \mathrm{min})$. At the 18 th week, animals were submitted to an echocardiographic evaluation. At the end of the protocol (20th week), all animals performed maximal oxygen uptake test (VO2max), were sacrificed and blood samples were collected for plasma protein analysis by ELISA [interleukin-6 (IL-6), C-reactive protein (CRP), tumor necrosis factor alpha (TNF- $\alpha$ ), intercellular adhesion molecule-1 (ICAM-1), vascular cell adhesion molecule-1 (VCAM-1), malondialdehyde (MDA), protein carbonyl content (PCC), N-terminal pro-brain natriuretic peptide (NT-proBNP)] and the ratio of matrix metalloproteinase- 9 and its inhibitor, tissue inhibitor of metalloproteinase-1 (MMP9/TIMP1)].

Results: Exercise training improved VO2max $(21,1 \pm 2.5$ vs $17,2 \pm 1.8$ $\mathrm{mL} . \mathrm{Kg}-1 . \mathrm{min}-1)$ and attenuated diastolic dysfunction in ObEX group $\left(\mathrm{E} / \mathrm{E}^{\prime}\right.$ ratio $13,8 \pm 1,8$ vs $\left.16,5 \pm 1,9\right)(\mathrm{p}<0.05)$. Exercised animals showed reduced levels of IL-6, CRP, TNF- $\alpha$, ICAM-1, PCC, NT-proBNP and MMP9/TIMP1 $(\mathrm{p}<0.05)$. No differences were found for VCAM-1 and MDA. In addition, the ratio E/E' was positively correlated with IL-6 $(\mathrm{r}=0,63 ; \mathrm{p}=0,02)$, NT-proBNP $(\mathrm{r}=0,64 ; \mathrm{p}=0,02)$ and MMP9/TIMP1 $(\mathrm{r}=0,69 ; \mathrm{p}=0,01)$.

Conclusion: Regular exercise training improved exercise capacity and diastolic function in rats with metabolic syndrome and HFpEF. This was associated with reduced circulating levels of inflammatory cytokines and markers of endothelial dysfunction, oxidative stress, neurohumoral activation and extracellular matrix remodeling.

Funding: Grant from the European Commission FP7-Health-2010; MEDIA-261,409. CIAFEL is funded by ERDF through the Operational Competitiveness Programme, and by FCT (UID/DTP/00617/2013).

\section{Reference:}

1 Schmidt, C.: CAPES (BEX 0554/14-6).

2 Moreira-Gonçalves, D.: FCT (SFRH/BPD/90010/2012).

\section{T1P52}

\section{Fat free mass predicts resting energy expenditure in extreme} obese females patients

Marra, M. ${ }^{1}$; Sammarco, $R .{ }^{1}$; Amato, V. $^{1}$; Naccarato, M. ${ }^{1}$; Onufrio, M. ${ }^{1}$; Contaldo, F. ' Pasanisi, F. $^{1}$

${ }^{1}$ Department of Clinical Medicine and Surgery, University Federico II

Introduction:The relationship between Resting Energy Expenditure (REE) and body composition has not extensively been studied in extreme obese patients. Aim of this study is to develop predictive equations for REE in extremely obese females using both general characteristics and body composition measures.

Methods: Two-hundred twenty four extreme obese females (range 18-50 y) (weight $149 \pm 14 \mathrm{~kg}$, BMI $55.7 \pm 4.7 \mathrm{~kg} / \mathrm{m}^{2}$, FFM $66.3 \pm 8.3 \mathrm{Kg}$, FAT $78.5 \pm 12.0 \mathrm{Kg}$, FAT $54.1 \pm 4.8 \%)$ participated in the study.

REE was measured by indirect calorimetry ( $V$ max29-Sensormedics) and body composition was estimated by bioimpedance analysis, performed at $50 \mathrm{kHz}$ (DS Medica) in all patients. The following variables were considered as possible predictors of REE:

1) general characteristics: age, height, weight, BMI;

2) Body composition: FFM, FAT, FAT\%.

Statistical analysis was performed using linear correlation and multiple regression analysis (SPSS vers. 15.0).

Results:Unexpectedly, REE was not closely correlated (linear correlation) with body weight $(\mathrm{r}=.260, \mathrm{p}<0.005)$; the most strictly correlated parameter was FFM $(r=.284)$ whereas age, Fat and FAT\% were not correlated with REE.

Therefore the following predictive equations were obtained by multiple regression analysis:

Forindividual general characteristics (age, weight, height, BMI):

REE $(\mathrm{kcal} / \mathrm{d})=6.572^{\prime}$ weight $+1482(r=.260$ SEE $354 \mathrm{kcal} / \mathrm{d})$

For Body composition (FFM, FAT, FAT\%):
REE $(\mathrm{kcal} / \mathrm{d})=59.8^{\prime} \mathrm{FFM}+128.4 \times$ FAT \% $-35.5 \times$ FAT $-5688 ;(r=.356$ SEE $345 \mathrm{kcal} / \mathrm{d})$

For individual general characteristics + Body composition:

$R E E(\mathrm{kcal} / \mathrm{d})=95.4^{\prime} \mathrm{FFM}+128.4 \times \mathrm{FAT} \%-35.6 x$ body weight -5688 ; $(r=.356$ SEE $345 \mathrm{kcal} / \mathrm{d})$

Conclusion:This study suggests that in extreme obese adult females, REE can be estimated from FFM; the inclusion of FAT\% and body weight into the predictive equation improve the evaluation of REE. A larger multicenter study is required to confirm our preliminary observation.

\section{References:}

1 Marra et al. Accuracy of predictive equations for estimating resting energy expenditure in obese adolescents. J Pediatr. 2015 Jun;166(6):1390-6.

2 Marra et al. The prediction of basal metabolic rate in young adult, severely obese patients using single-frequency bioimpedance analysis. Acta Diabetol. 2003 Oct;40 Suppl 1:S139-41.

Conflict of interest: None Disclosed

Funding: No Funding

\section{T1P53 \\ Fat overshooting during weight regain in humans: Its relationship with adiposity before weight loss}

Jacquet, P. '; Jacquet, J.' ; Dulloo, A. ${ }^{\text {' }}$

${ }^{1}$ Department of Medicine, Division of Physiology, University of Fribourg, Switzerland

Introduction: The notion that dieting and weight cycling makes people fatter has been a subject of considerable controversy in past decades. More recent prospective studies, however, suggest that it is dieting to lose weight in people who are in the normal range of body mass index (BMI), rather than in those who are overweight or obese, that most strongly and consistently predict future weight gain. In addressing the plausibility that dieting-induced weight loss predisposes the lean (rather than the overweight or obese) to regaining more body fat than what had been lost (i.e. fat overshooting), we have assessed the nature of the relationship between initial adiposity and the extent of fat overshooting from data on body composition in men participating in the classic Minnesota Experiment - a longitudinal study of experimental energy restriction and refeeding. Methods: Individual data on body composition - fat mass (FM) and fatfree mass (FFM) - were analyzed for the 12 men who completed the entire Minnesota Experiment consisting of a control (baseline) period, 24 weeks of semistarvation during which they lost $\sim 25 \%$ of body weight, followed by 20 weeks of refeeding. Fat overshooting was calculated as the difference between FM regained and FM lost, and expressed in absolute terms (as excess $\mathrm{kg}$ ) or as a percentage of FM at baseline before weight loss.

Results: In these men of normal BMI and initial body fat $\%$ in the range of 6 and 25\%, there was a large inter-individual variability in excess FM regained, with the amount of fat overshot (after complete FFM recovery) being in the range of 1 and $12 \mathrm{~kg}$. The data for both the initial body fat $\%$ and the fat overshot (in absolute or relative terms) were normally distributed. The application of various regression models shows a strong inverse exponential relation between the absolute or relative body fat overshoot and the initial body fat $\%$, the correlation coefficient ( $r$ value) being in the range of 0.65 to 0.90 .

Conclusion: The demonstration here of a robust inverse exponential relationship between fat overshooting during weight regain and initial adiposity prior to weight loss reinforce the notion that the risks for excess weight gain in response to dieting and weight cycling is greater in those of normal body weight than in those who are overweight or obese.

\section{Conflict of Interest: None Disclosed}

Funding: Research relating to this abstract was supported by intramural Funding of the University of Fribourg, Switzerland 
T1P54

\section{Gastrointestinal sensing of xylitol, erythritol and glucose affects brain networks associated with appetite regulation}

Wölnerhanssen, B. '; Zanchi, D. 2. Rehfeld, J. ${ }^{3}$; Roux, C. ${ }^{4}$; Borgwardt, S. ${ }^{5}$; Beglinger, C. '; Meyer-Gerspach, A. ${ }^{1}$

${ }^{1}$ Department of Research, St. Claraspital Basel, Basel, Switzerland, ${ }^{2}$ Department Of Psychiatry, University Hospital Basel, ${ }^{3}$ Department Of Clinical Biochemistry, Rigshospitalet, University Of Copenhagen, ${ }^{4}$ Diabetes Complications Research Centre, Conway Institute, University College Dublin, ${ }^{5}$ Department Of Psychiatry, University Hospital Basel Basel, Switzerland

Introduction: Xylitol (X) and erythritol (E), two polyols, are naturally occurring non-nutritive sweeteners with distinct effects on satiation hormone release. Functional magnetic resonance imaging (fMRI) can be used to elucidate brain-gut interactions. The effects of polyol ingestion on brain resting state functional networks has not been investigated before. Methods: Eighteen healthy volunteers received $75 \mathrm{~g}$ glucose, $50 \mathrm{~g}$ xylitol or $75 \mathrm{~g}$ erythritol or tap water (placebo) in a randomized fashion. Glucose, insulin, GLP-1 and CCK were measured before and after brain fMRI examination in a resting state paradigm. Independent component analysis (ICA) using a dual regression technique was performed focusing on 3 resting-state networks involved in appetite regulation: Default Mode Network (DMN), Sensorimotor Network (SMN) and Saliency Network (SN). Results: fMRI analyses showed higher SMN activity after glucose administration compared to placebo $(\mathrm{p}<.001), \mathrm{E}(\mathrm{p}<.001)$ and $\mathrm{X}(\mathrm{p}<.05)$. Higher SMN activity was shown after $\mathrm{X}$ and $\mathrm{E}$ administration compared to placebo $(\mathrm{p}<.001$ resp. $\mathrm{p}<.05)$. X had a small effect on plasma insulin and glucose, whereas $\mathrm{E}$ had no effect. All 3 carbohydrates stimulated CCK and GLP-1 release. Positive correlations $(\mathrm{p}<0.05)$ were found between insulin and the SN activity after all 3 carbohydrates. Positive correlations $(\mathrm{p}<0.05)$ were found between glucose levels and the SN activity after glucose administration.

Conclusion: Glucose,xylitol and erythritol affect SMN activity, which is associated with appetite regulation. SN activity correlated to glucose resp. insulin levels.

Conflict of Interest: None Disclosed

Funding: The trial was funded by the SNSF (Marie Heim-Voegtlin subsidy: PMPDP3-145486/1)

\section{T1P55 \\ Genetic dissection of brown adipose tissue function in spontaneously hypertensive rats}

Pravenec, M. ' ; Zidek, V. '; Landa, V. '; Mlejnek, P. '; Silhavy, J. '; Simakova, M. '; Strnad, H. ${ }^{2}$; Kazdova, L. ${ }^{3}$; Skop, V. ${ }^{3}$; Mracek, T. ${ }^{1}$; Houstek, J. ${ }^{1}$

${ }^{1}$ Institute of Physiology, Czech Academy of Sciences, Prague, Czech Republic, ${ }^{2}$ Institute Of Molecular Genetics, Czech Academy Of Sciences, Prague, Czech Republic, ${ }^{3}$ Institute For Clinical And Experimental Medicine, Prague, Czech Republic

Introduction: Recently, brown adipose tissue (BAT) was suggested to play an important role in lipid and glucose metabolism in rodents and possibly also in humans.

Methods: In the current study, we used genetic and correlation analyses in the BXH/HXB recombinant inbred (RI) strains, derived from Brown Norway (BN) and spontaneously hypertensive rats (SHR), to identify genetic determinants of BAT function and its role in the pathogenesis of metabolic disturbances.

Results: Linkage analyses revealed significant quantitative trait locus (QTL) associated with interscapular BAT mass on chromosome 4 and two closely linked QTL associated with glucose oxidation and glucose incorporation into BAT lipids on chromosome 2. Using weighted gene co-expression network analysis (WGCNA) we identified gene co-expression module eigengenes QTL in the BXH/HXB RI panel. The Coral4.1 co-expression module eigengene QTL includes $\mathrm{Cd} 36$ hub gene, a prominent candidate which codes for fatty acid translocase and plays an important role of fatty acid transport into BAT and triglyceride utilization in BAT. The Darkseagreen co-expression module eigengene QTL includes Sort1 (sortilin 1) candidate gene and is closely linked to Wars2 (tryptophanyl tRNA synthetase 2 (mitochondrial)) positional candidate gene. Since $\mathrm{Cd} 36$ and Wars2 genes are dysfunctional in the SHR, we prioritized these genes for in vivo functional analyses. SHR-Cd36 transgenic rats with wild type Cd36 when compared to the SHR had reduced BAT mass. SHR-chr.2 congenic and SHR-Wars2 transgenic rats, with wild type Wars2 gene, showed increased glucose oxidation and incorporation into BAT lipids when compared to SHR with mutant Wars2. Bat mass, glucose oxidation and incorporation into BAT lipids significantly correlated with metabolic and hemodynamic parameters in RI strains.

Conclusion: These results demonstrate important role of Cd36 and Wars2 genes in regulating BAT mass and function and consequentially lipid and glucose metabolism.

\section{Conflict of Interest: None Disclosed}

Funding: Research relating to this abstract was funded by grant 13-04420S from the Czech Science Foundation

\section{T1P56}

\section{Ghrelin, leptin and GLP-1 as modulators of the nutritional support of spermatogenesis}

Martins, A. ${ }^{1}$; Moreira, B. ${ }^{2}$; Barros, A. ${ }^{3}$; Sousa, M. ${ }^{1}$; Monteiro, M. ${ }^{4}$; Oliveira, P. $^{5}$

${ }^{1}$ UMIB-ICBAS, UP, ${ }^{2}$ Cics-Ubi \& Umib-Icbas-Up, ${ }^{3}$ Reproductive Genetics Clinic Prof. Alberto Barros, ${ }^{4}$ University College Of London, Uk \& Umib-Icbas, Up, ${ }^{5}$ UmibIcbas, Up \& I3S \& Fmup

Introduction: Male infertility has arisen as one important comorbidity associated with the energy imbalance promoted by obesity. Spermatogenesis is under nutritional support by the somatic Sertoli cells that sustain the energetic needs of the developing germ cells. Overweight and obese men present severe alterations in their hormonal profile, particularly in ghrelin, leptin and glucagon-like peptide-1 (GLP-1) levels. We hypothesized that those hormones could modulate the nutritional support of spermatogenesis thus, we studied the effect of ghrelin, leptin and GLP-1 in human Sertoli cells (hSCs) metabolic phenotype.

Methods: hSCs were exposed to increasing concentrations of those hormones mimicking levels reported in men with different BMI. The concentrations used were 20, 100 and $500 \mathrm{pM}$ of ghrelin; 5, 25 and $50 \mathrm{ng} /$ $\mathrm{mL}$ of leptin and 10, 1000 and 100000 pM of GLP-1. The intermediary metabolism of the cells was analyzed using an NMR-based metabolomics approach. The protein levels of key glycolysis-related transporters and enzymes were assessed. Mitochondrial activity was also studied. Oxidative damages were evaluated by studying lipid peroxidation, protein carbonilation and nitration.

Results: Ghrelin, leptin and GLP-1 receptors were identified in hSCs. Exposure to ghrelin showed an inverse association with the production of lactate and also modulated mitochondrial bioenergetics. All leptin concentrations decreased hSCs acetate production, which may impact developing germ cells proliferation. No alterations were noted in the oxidative profile after exposure to leptin. Interestingly, exposure to GLP-1 dose-dependently increased lactate production, which has been highlighted as crucial to improve the male reproductive potential. The highest concentration of GLP-1 decreased mitochondrial membrane potential.

Conclusion: Our results suggest that ghrelin, leptin and GLP-1 may serve as energy status sensors with an important impact on the nutritional support of the spermatogenesis. Further studies will be needed to unveil the possible physiological role of these hormones in male reproductive function, particularly in the etiology of subfertility/infertility in overweight/ obese individuals.

\section{Conflict of Interest: None Disclosed}

Funding: Funded by "Fundação para a Ciencia e a Tecnologia" - FCT: MG Alves (IFCT2015 and PTDC/BIM-MET/4712/2014)); PF Oliveira (PTDC/BBBBQB/1368/2014 and IFCT2015); AD Martins (SFRH/BD/108726/2015); CICS-UBI (Pest-C/SAU/UI0709/2014) and UMIB (Pest-OE/SAU/UI0215/2014) co-funded 
by Fundo Europeu de Desenvolvimento Regional d FEDER via Programa Operacional Factores de Competitividade COMPETE/QREN, FSE and POPH funds.

\section{T1P57 \\ Habitual physical activity and apelin gene expression in visceral and subcutaneous adipose tissues among morbid obese and non-obese subjects}

Mahmoodi, B. ${ }^{\text {'; }}$ Yuzbashian, E. '; Zarkesh, M. ${ }^{2}$; Asghari, G. '; Safarian, M. ${ }^{3}$; Hedayati, M. ${ }^{2}$; Mirmiran, $P$.

${ }^{1}$ Nutrition and Endocrine Research Center, Research Institute for Endocrine Sciences, Shahid Beheshti University of Medical Sciences, Tehran, Iran

${ }^{2}$ Cellular And Molecular Endocrine Research Center, Research Institute For Endocrine Sciences, Shahid Beheshti University Of Medical Sciences, Tehran, Iran, ${ }^{3}$ Biochemistry Of Nutrition Research Center, Department Of Biochemistry And Nutrition Sciences, School Of Medicine, Mashhad University Of Medical Sciences, Mashhad, Iran

Introduction: adipose tissue is an expanded endocrine organ affects body hemostasis by a secreting protein named adipokine. Plasma level of apelin (a new adipokine) decreased after exercise; however, the evidence on gene regulation in adipocyte is rare. The objective of the present study was to investigate the association between the apelin gene expression and habitual physical activity among morbid obese and non-obese subjects.

Methods: Visceral and subcutaneous adipose tissues were obtained from 32 morbidly obese and 32 non-obese age- and sex-matched subjects, who underwent open abdominal surgery. Physical activity was collected using a valid and reliable International Physical Activity Questionnaire (IPAQ)-long form, and the metabolic equivalent of task (MET) was calculated. The mRNA expressions of apelin gene in visceral and subcutaneous adipose tissues were analyzed by Real-Time PCR.

Results: The mean age was 39.6 years for both groups and body mass index for morbidly obese and non-obese subjects were 45.3 and $25.6 \mathrm{~kg} / \mathrm{m}^{2}$, respectively. Apelin gene expression was more increased in morbid obese than non-obese subjects in both subcutaneous ( 3.42 vs $0.49, P<0.05)$ and visceral adipose $(3.65$ vs $0.19, P<0.05)$ tissues. Apelin expression in visceral adipose tissue among morbidly obese subjects was correlated with total MET ( $\mathrm{r}=-0.345, P=0.045)$; however, apelin gene expression in subcutaneous adipose tissue was not significantly correlated with total MET. Among morbid obese subjects, total home MET $(r=-0.412, P=0.019)$ was correlated with visceral adipose tissue.

Conclusions: Decreased apelin gene expression in visceral adipose tissue is linked to total MET in morbidly obese subjects, suggesting an important role of physical activity pathways in the causal relationship between consequences of higher physical activity and the development of obesity.

\section{T1P58}

Hepatic lipogenesis, beta-oxidation and autophagy are modulated by acylated and desacyl ghrelin - role in NAFLD resolution after sleeve gastrectomy in diet-induced obese rats

Ezquerro, S. '; Méndez-Giménez, L. '; Becerril, S. ${ }^{2}$; Moncada, R. ${ }^{3}$; Valentí, V. ${ }^{4}$; Catalán, V. ' ;ómez-Ambrosi, J. ${ }^{1}$; Frühbeck, G. ${ }^{5}$; Rodríguez, A. ${ }^{1}$

${ }^{1}$ Metabolic Research Laboratory, Clínica Universidad de Navarra, IdiSNA, Pamplona, Spain, ${ }^{2}$ Ciber Fisiopatología De La Obesidad Y Nutrición (Ciberobn), Instituto De Salud Carlos lii, Madrid, Spain, ${ }^{3}$ Department Of Anesthesia, Clínica Universidad De Navarra, Idisna, Pamplona, Spain, ${ }^{4}$ Department Of Surgery, Clínica Universidad De Navarra, Idisna, Pamplona, Spain, ${ }^{5}$ Department Of Endocrinology \& Nutrition, Clínica Universidad De Navarra, Idisna, Pamplona, Spain

Introduction: Our goal was to evaluate the potential role of ghrelin isoforms in the amelioration of non-alcoholic fatty liver disease (NAFLD) after sleeve gastrectomy in diet-induced obese rats.

Methods: Male Wistar rats $(\mathrm{n}=161)$ underwent surgical (sham operation and sleeve gastrectomy) or dietary interventions [fed ad libitum a normal or a high-fat diet or pair-fed].
Results: Reduced $(P<0.05)$ plasma desacyl ghrelin levels, without changes in acylated ghrelin, were observed in obese rats. Circulating desacyl ghrelin levels were dramatically decreased $(P<0.05)$, whereas the acylated/desacyl ghrelin ratio was increased $(P<0.05)$, after sleeve gastrectomy. Morever, a significant $(P<0.05)$ reduction in hepatic triacylglycerol content and lipogenic enzymes Mogat2 and Dgat1, increased mitochondrial DNA and induced AMPK-activated mitochondrial FFA $\beta$-oxidation and autophagy to a higher extent than pair-feeding was shown. Primary rat hepatocytes stimulated with both ghrelin isoforms significantly $(P<0.05)$ increased triacylglycerol content, triggered AMPK-activated mitochondrial FFA $\beta$-oxidation and autophagy.

Conclusion: The reduction in desacyl ghrelin after sleeve gastrectomy appears to contribute to the decrease in lipogenesis, whereas the increased relative acylated ghrelin levels activate factors involved in mitochondrial FFA $\beta$-oxidation and autophagy in obese rats, thereby ameliorating NAFLD.

Conflict of Interest: None Disclosed

Funding: Supported by FIS-FEDER (FIS PI12/00515, PI13/01430 and PI16/00221) from ISCIII and the Department of Health of the Gobierno de Navarra (61/2014).

\section{T1P59}

\section{Hepatic steatosis is associated with lower prior health care burden in visceral obesity}

\section{Romu, T. ; Linge, J. ${ }^{2}$; Borga, M. ${ }^{1}$; West, J. ${ }^{1}$; Bell, J. ${ }^{3}$; Leinhard, O. ${ }^{1}$}

${ }^{1}$ Advanced MR Analytics AB; Linköping University, ${ }^{2}$ Advanced Mr Analytics Ab, ${ }^{3}$ University Of Westminster

Introduction: Non-alcoholic fatty liver disease (NAFLD) is associated with increased mortality and morbidity. As NAFLD is associated with insulin resistance, hepatic steatosis may develop during the progression of the metabolic syndrome. As NAFLD has a fairly high prevalence in the overweight population it is important to understand if an elevated liver fat is related to increased morbidity in the overweight and obese. The aim of this study was to determine if hepatic steatosis is associated with previous health care burden (HCB), defined as accumulated hospital nights, in the overweight population when controlled for body composition.

Methods: A total of 3,686 overweight (BMI $>=25)$ subjects (1,699 females and 1,987 males, age 45 to 78 years), were included from the UK Biobank imaging cohort. Visceral adipose tissue index $\left(\mathrm{VATi}=\mathrm{VAT} / \mathrm{height}^{2}, 1 / \mathrm{m}^{2}\right)$, abdominal subcutaneous adipose tissue index (ASATi=ASAT/height ${ }^{2}, 1 /$ $\mathrm{m}^{2}$ ) and liver proton density fat fraction (PDFF, \%) were measured from 1.5T MR images using AMRA profiler research (AMRA, Sweden). Based on the in-patient data available in UK Biobank, the accumulated hospitalization nights was extracted as an indicator of previous health care burden. One univariate linear model was constructed investigating the association between liver PDFF and HCB. Subsequently, two multivariable regression models were constructed; one model with liver PDFF and $\mathrm{BMI}$ as representatives of body composition; and another with liver PDFF, VATi and ASATi. All models were adjusted for age, sex, smoking, alcohol intake and physical activity.

Results: The univariate test showed a significant positive relationship ( $\mathrm{p}=0.03,=0.510 .24)$ between liver PDFF and HCB. The BMI-adjusted model of the overweight group showed that liver PDFF was non-significant $(\mathrm{p}=0.72)$, and BMI was positively associated with accumulated hospital nights $(\mathrm{p}<0.001,=0.170 .03)$. The VATi- and ASATi-adjusted GLM analysis showed that liver PDFF was negatively associated with HCB ( $p=0.02,=-0.650 .29)$, VATi had a positive association $(p<0.001$, $=1.270 .22)$ and ASATi had no significant association $(\mathrm{p}=0.43)$.

Conclusion: High liver PDFF was associated with high previous hospitalization in general. However, when controlling for overall obesity (BMI), no such association was found. Further, when controlling for abdominal fat distribution (VATi and ASATi), the association was negative. This observation suggests that hepatic steatosis may have a linkage to visceral obesity and that there may exist a body composition with a skewed ectopic 
fat accumulation, i.e. high visceral fat but low liver fat, that is associated with previous health care burden.

Conflict of Interest: ODL, JW, TR, JL, MB (AMRA)

Funding: Financial support by Pfizer.

\section{T1P60}

\section{Hesperidin treatment reduces excess body weight gain and metabolic alterations associated with the intake of an obesogenic diet in rats}

\section{Andrea, M. ' ; Juana, S. '; Andreu, P. ${ }^{2}$; Portillo, M. ' ; Catalina, P. ${ }^{2}$}

${ }^{1}$ Nutrition and Obesity Group. Dpt. Nutrition and Food Science. Faculty of Pharmacy. University of the Basque Country (UPV/EHU), ${ }^{2}$ Laboratory Of Molecular Biology, Nutrition And Biotechnology (Lbnb), University Of The Balearic Islands (Uib)

Introduction:Hesperidin, a flavanone present in fruit citrus and derivatives, has been described to possess interesting biological properties related to the lipid metabolism. The aim of the present study was to assess whether hesperidin treatment may prevent the effects of a western diet on obesity development and related metabolic alterations in rats.

Methods: 23 male Wistar rats aged 3 months were randomly divided into 3 groups: C: Control group fed with standard diet, WD: Western Diet group fed with western diet (rich in fat and sugar), and HESP: Hesperidin group fed WD+100mg/kg/day of Hesperidin by gavage. Food intake and body weight were followed and blood pressure was measured after 1-month treatment. After 2 months of treatment, animals were sacrificed and blood parameters and gene expression in liver were analysed. Morphological and immunohistochemistry analysis were also performed in retroperitoneal white adipose tissue (WAT).

Results:Animals of the WD group displayed higher body weight gain than C animals, whereas HESP animals showed intermediate values and not different than controls. In addition, animals of the HESP group showed lower systolic and diastolic blood pressure than animals of the WD group. Plasma leptin levels, and hepatic expression of lipogenesis-related genes (Srebf1, Fasn and Scd1) and fatty acid oxidation-related genes (Cptla and Ppara) were increased in WD animals versus $C$ animals, but these parameters were generally normalised in HESP animals. Analysis of WAT revealed the presence of multilocular adipocytes with positive immunostaining for UCP1 and CIDE-A in the HESP group, but not in the $\mathrm{C}$ and WD groups.

Conclusion:Hesperidin treatment in rats reduces excess body weight gain and other alterations associated to the intake of an obesogenic diet. These effects may be related, at least in part, to the normalisation of the expression of lipid metabolism-related genes in liver, as well as the induction of browning in WAT.

Conflict of Interest: None Disclosed

Funding: Research funded by Institute of Health Carlos III (CIBERObn), Spanish government (AGL2015-67019-P), and Government of the Basque Country. A. Mosqueda is a recipient of a fellowship from the National Council of Science and Technology (CONACYT), Mexico.

\section{T1P61}

\section{Histomorphological and molecular characterization of the infrapatellar adipose body}

Hadi, H. ${ }^{1}$; Granzotto, M. ; Favero, M. ${ }^{2}$; Belluzzi, E. ${ }^{2}$; Porzionato, A. ${ }^{3}$; Sarasin, G. ${ }^{3}$; lacobellis, C. ; Fontanella, C. ${ }^{5}$; Caro, . $^{3}$; Vettor, R. ${ }^{1}$; Macchi, V. ${ }^{3}$; Rossato, M. ${ }^{1}$

${ }^{1}$ Department of Medicine - DIMED, Clinica Medica 3, University of Padova, School of Medicine, Padova, Italy, ${ }^{2}$ Rheumatology Unit, Department Of Medicine - Dimed, University Hospital Of Padova, Italy, ${ }^{3}$ Institute Of Human Anatomy, Department Of Molecular Medicine, University Of Padova, Italy, ${ }^{4}$ Orthopaedic Clinic, Department Of Surgery, University-Hospital Of Padova, Italy, ${ }^{5}$ Centre For Mechanics Of Biological Materials, University Of Padova, Italy
Introduction: Obesity is considered the greatest modifiable risk factor for knee osteoarthritis (KOA). The mechanisms that link the two conditions are still not fully clarified but recent data suggest an important role mediated by the infrapatellar fat pad (IFP). Recently, IFP has been shown to be a local producer of adipocytokines suggesting a potential role in osteoarthritis (OA) pathogenesis. We aimed to evaluate the histopathological and molecular characteristics of OA IFP compared to controls.

Methods: We enrolled 28 overweight/obese subjects undergoing total knee replacement for OA. The controls were represented by IFP and adjacent synovial membrane specimens sampled from bodies or bodies part of 8 healthy subjects without history of osteoarthritis involved in the Body Donation Program 'Donation to Science' held by the University of Padova. The microscopic anatomy of IFP was analyzed through histological and morphometrical Methods: The histology of the synovial membrane adjacent to the IFP was also analyzed. We determined mRNA expression using Quantitative real time PCR for adipokines (leptin, adiponectin) and cytokines IL-6, TNF- $\alpha$, MCP-1, VEGF in the IFP specimens of KOA subjects. Results: All the evaluated IFPs showed microscopical characteristics similar to white adipose tissue. IFPs were organized in adipose lobuli separated by fibrous septa. No differences were detected in the mean diameter of the adipose lobuli of the IFP in KOA subjects and controls. Mononuclear infiltration was present in $22 \mathrm{KOA}$ patients while it was not observed in any of the IFP used as control $(p=0.001)$. The average number of vessels and the thickness of interlobular septa were significantly higher in KOA subjects compared to controls ( $\mathrm{p}<0.0001$ and $\mathrm{p}=0.004$ respectively). BMI correlated positively with the thickness of the interlobular septa in IFP. Concerning synovial membrane, the presence of lymphocytic infiltration, hyperplasia and fibrosis was significantly higher in KOA compared to controls. In IFP samples it was noticed an expression of genes typical of inflamed adipose tissue such as IL-6, TNF- $\alpha$, MCP-1 and VEGF. Moreover, we observed a significant positive correlation between the number of blood vessels of KOA synovial membrane and mRNA expression of VEGF in IFP.

Conclusion: This study describes for the first time the histopathological characteristics of IFP in a large cohort of overweight/obese subjects affected by KOA. IFP showed pathologic structural changes in the lobule dimension, interlobular septa, vascularization and inflammatory infiltrate. These changes were associated with synovial inflammation. Moreover, we added evidence about the existence of a probable crosstalk between cytokines produced by IFP and the adjacent synovial membrane.

\section{T1P62 \\ How does weight loss affect appetite? A systematic review and meta-analysis}

\section{Roekenes, J.' ; Lyngstad, A. '; Torekov, S. '; Sainsbury, A. '; Svendsen, M.4; Martins, C. ${ }^{1}$}

${ }^{1}$ Department of Cancer Research and Molecular Medicine, Faculty of Medicine and Health Sciences, Norwegian University of Science and Technology, Trondheim, Norway, ${ }^{2}$ Department Of Biomedical Science, University Of Copenhagen, Copenhagen, Denmark, ${ }^{3}$ The Boden Institute Of Obesity, Nutrition, Exercise \& Eating Disorders, The University Of Sydney, Charles Perkins Centre, Sydney, New South Wales, Australia, ${ }^{4}$ Department Of Endocrinology, Obesity And Preventive Medicine, Oslo University Hospital, Oslo, Norway

Background: Several studies have reported increased appetite after diet-induced weight loss (WL) in people with overweight/obesity, however the evidence is contradictory.

Aim: This systematic review and meta-analysis aims to conduct a critical appraisal of the effect of diet-induced WL on subjective and objective markers of appetite.

Methods: A systematic literature search was performed on 22.09.2016 in databases (PubMed, Cochrane, SCOPUS, OVID, CINAHL/Medline, EMBASE, PsychINFO, HaPI, AMED, OVID Nursing Database, Maternity and Infant Care, Global Health) using appropriate MeSH terms for original articles written in English. Randomized and non-randomized controlled trials with a dietary intervention performed on adults (aged $\geq$ 
18 years) with overweight/obesity $\left(\mathrm{BMI} \geq 25 \mathrm{~kg} / \mathrm{m}^{2}\right)$ were included, provided that the study employed a within-subject, repeated measure design for appetite markers. To be included, the duration of the intervention had to be $\geq 7$ days and measures not be taken while study participants were in ketosis, report a minimum weight loss of $\geq 2 \mathrm{~kg}$, and provide results on subjective appetite feelings assessed using visual analogue scales and/ or circulating concentration of appetite-regulating hormones. The risk of bias will be evaluated using the Cochrane risk of bias tool. Effect sizes will be calculated using Hedges' g score, using both fixed and random effect models.

Results: The search yielded a total of 6,109 potentially relevant articles. After removing duplicates, a total of 3,341 abstracts were reviewed. Full texts of 124 articles have been acquired and are currently being screened independently by two reviewers and assessed against the inclusion / exclusion criteria and for quality and data extraction.

Conclusion: The results of this review will provide up-to-date evidence on the impact of diet-induced WL on appetite markers in the overweight/ obese population, which will be a useful guide for clinical practice.

Acknowledgments: None disclosed

Conflict of interest: None disclosed

Funding: Norwegian University of Science and Technology - Department of Cancer Research and Molecular Medicine, Faculty of Medicine and Health Science, Trondheim, Norway

\section{T1P63}

\section{Human subcutaneous adipose tissue does not secrete FGF21}

Baak, M. ${ }^{1}$; Vink, R. ${ }^{1}$; Roumans, N. ${ }^{1}$;Mariman, E. ${ }^{1}$

${ }^{1}$ Maastricht University Medical Centre+

Subcutaneous adipose tissue does not secrete FGF21 in overweight and obese individuals

Introduction: FGF21 has been shown to be an important regulator of energy metabolism with adipose tissue as its main target. Although some studies suggest that FGF21 is produced in human adipose tissue, it is not known whether adipose tissue is also a source of circulating FGF21 in humans.

Methods: Total and active FGF21 concentrations were determined invenous (ven) blood draining the subcutaneous adipose tissue (scAT) and in arterialised (art) blood in 16 overweight and obese individuals (BMI 27$35 \mathrm{~kg} / \mathrm{m}^{2}$ ) before and after diet-induced weight loss. Measurements were performed in the fasting state and for 5 hours after consumption of a highfat mixed meal. ScAT biopsies were collected in the fasting state before and after weight loss and gene expression was determined by microarray. The measurements after weight loss were performed after a 4-week weight stable period in a situation of energy balance.

Results: No difference in art and scAT ven total FGF21 concentrations was found (fasting total FGF21 art $121.3 \pm 190.6 \mathrm{pg} / \mathrm{ml}$, scAT ven $120.5 \pm 188.9 \mathrm{pg} / \mathrm{ml}$ (mean \pm interquartile range), $\mathrm{p}=0.91)$. Similarly, no arteriovenous differences were found postprandially or after weight loss. The weight loss of around 10\% of initial body weight did not affect art or scAT ven total FGF21 concentrations (before vs after: art 121.3 \pm 190.6 vs $111.2 \pm 73.0 \mathrm{pg} / \mathrm{ml}(\mathrm{p}=0.191) ; \mathrm{scAT}$ ven $120.5 \pm 188.9$ vs $111.6 \pm 73.4$ $\mathrm{pg} / \mathrm{ml}(\mathrm{p}=0.211))$. Meal consumption increased total FGF21 concentrations to a similar extent in art and scAT ven blood $(\mathrm{p} \leq 0.002)$. Results for active FGF21 were similar to those of total FGF21 under all conditions. No FGF21 gene expression was detectable in scAT biopsies before or after weight loss.

Conclusion: No secretion of FGF21 from human scAT was detectable in overweight and obese individuals before nor after weight loss. In addition no FGF21 gene expression could be detected in scAT biopsies. This suggests that scAT is not a source of circulating FGF21 in humans.

Conflict of Interest: None disclosed

Funding: Research relating to this abstract was funded by Netherlands Organisation for Scientific Research TOP, grant number: 200500001

\section{T1P65 \\ Hypothalamic inflammatory makers and POMC are differentially regulated in response to dietary fats in obese prone as compared to obese resistant mice}

\section{Razolli, D. '; Araújo, T.'; Velloso, L.'}

${ }^{1}$ State University of Campinas

Hypothalamic dysfunction has emerged as an important determinant of diet-induced obesity (DIO). However, it is unknown what mechanism is the trigger of this process. Here, we employed obese prone (OP) and obese resistant (OR) mice to evaluate three hypotheses. The first hypothesis was that consumption of dietary fats (HF) would promote early changes in gut microbiota leading to increased gut permeability to LPS and/or fatty acid (FA) that could trigger hypothalamic dysfunction. Sequencing of the $16 \mathrm{~S}$ rRNA revealed no differences in gut bacterial communities between OP and OR prior or one day after HF. The second hypothesis was that HF or systemic infusion of FAs would lead to an early differential increase of blood free FAs. Non-esterified FA determination revealed that after oneday treatment, systemic FAs were similar in OP and OR submitted to either treatment. Finally, the third hypothesis was that FAs would promote different regulations of either inflammation or neuropeptide expression in hypothalamus. Upon continuous infusion of palmitate, OP mice presented an early (24h) increase in the expression of POMC, TNF $\alpha, F 4 / 80$, $\mathrm{CD} 11 \mathrm{~b}$ and CX3CL1 as compared to OR. Increased expression of POMC was accompanied by a lower increment in the hypothalamic $\alpha$-MSH levels following FA infusion and different proconvartase 1/3 expression between OP and OR mice. Thus, inflammatory markers and POMC are differently regulated in $\mathrm{OP}$ as compared to OR mice following the systemic treatment with FA. This data places early defective hypothalamic responsiveness to dietary fats as a mechanism potentially involved in the predisposition to DIO.

\section{T1P66}

\section{Hypothyroidism leads to leptin resistance in male wistar rats}

\section{Mironova, D. '; Handjieva-Darlenska, T.}

${ }^{1}$ Medical University Sofia

Introduction:Thyroid disorders play a crucial role in regulation of metabolism. The animal model of hypothyroidism with metabolic complications shows the important interplay between thyroid function and adipose tissue. There are not enough data in the literature on the effect of thyroid disorders on leptin secretion. Thus, the aim of our study was to develop experimentally induced models of hypo- and hyperthyroidism, and to examine their effects on leptin.

Methods:The study was performed in 18 male male Wistar rats. Animals were divided into three groups: first group with hyperthyroidism, second group with hypothyroidism, and third group was a control. In order to induce hypo- and hyperthyroidism the experiment lasted for 30 days. During this period all rats received chow food and water ad libitum. At the end of the study rats were anaesthetized. Blood samples were taken from rat's tails for measurement of TSH, fT4, fT3 and leptin levels by ELISA method (BioVendor).

Results: Our results showed the development of experimentally induced models of hypo- and hyperthyroidism with changes in blood levels of TSH, fT4, and fT3, respectively. Moreover, leptin levels were significantly increased in the hypothyroidism compared to the hyperthyroidism (2.28 $\mathrm{pg} / \mathrm{ml}$ vs.1.81 $\mathrm{pg} / \mathrm{ml}, \mathrm{p}<0.05$ ) which suggested a leptin resistance.

Conclusion: The experimentally induced models of hypo- and hyperthyroidism seem to be easy models to examine the metabolic disturbances rats. Our data suggest that despite the high leptin signaling to the brain to suppress appetite, rats with hypothyroidism were obese. 
T1P67

IL-32 levels are increased in obesity promoting adipose tissue inflammation and extracellular matrix remodelling

Catalán, V. ' Gómez-Ambrosi, J. '; Rodríguez, A. '; Ramírez, B. '; Valentí, V. '; Moncada, R. ${ }^{3}$; Landecho, M. ${ }^{4}$; Silva, C. ${ }^{5}$; Salvador, J. ${ }^{5}$; Frühbeck, G. ${ }^{6}$

${ }^{1}$ Metabolic Research Laboratory, Clínica Universidad de Navarra, IdiSNA, CIBEROBN, Pamplona, Spain, ${ }^{2}$ Department Of Surgery, Clínica Universidad De Navarra, Idisna, Ciberobn, Pamplona, Spain, ${ }^{3}$ Department Of Anesthesia, Clínica Universidad De Navarra, Idisna, Ciberobn, Pamplona, Spain, ${ }^{4}$ Department Of Internal Medicine, Clínica Universidad De Navarra, Idisna, Pamplona, Spain, ${ }^{5}$ Department Of Endocrinology \& Nutrition, Clínica Universidad De Navarra, Ciberobn, Pamplona, Spain, ${ }^{6}$ Department Of Endocrinology \& Nutrition, Clínica Universidad De Navarra, Idisna, Ciberobn, Pamplona, Spain

Introduction: Interleukin (IL)-32 is a recently described cytokine involved in the regulation of inflammation. Our aim was to explore the circulating IL-32 concentrations in normal weight, obesity and obesity-associated type 2 diabetes (T2D) volunteers as well as to analyse the impact of weight loss induced by bariatric surgery. Furthermore, we investigated the potential regulatory roles of IL-32 in inflammation and extracellular matrix (ECM) remodelling in human adipocytes.

Methods: Visceral (VAT) and subcutaneous (SAT) adipose tissue gene expression levels as well as circulating concentrations of IL-32 were analysed in 90 individuals. Serum IL-32 concentrations were also measured before and after weight loss achieved by Roux-en-Y gastric bypass (RYGB, $\mathrm{n}=20$ ). The effect of the stimulation and silencing of mRNA of IL-32 on the expression levels of inflammation and ECM remodelling related-genes in cultures of human differentiated adipocytes was also explored.

Results: Increased $(\mathrm{P}=0.015)$ circulating levels of IL-32 in obese patients decreased $(\mathrm{P}=0.031)$ after weight loss achieved by Roux-en-Y gastric. Obese patients exhibited higher $(\mathrm{P}<0.01)$ expression levels of IL-32 in VAT as well as in SAT. Inflammatory stimuli (LPS and TNF-á) and hypoxia significantly enhanced $(\mathrm{P}<0.001)$ the expression of IL32 expression whereas a significant downregulation $(\mathrm{P}<0.05)$ after anti-inflammatory cytokines (IL-4 and IL-13) treatment was observed. The addition of exogenous IL-32 induced $(\mathrm{P}<0.05)$ the expression of inflammation markers (IL1B, IL6, CCL2, COX2 and TNF) and ECM-related genes (HIF1A, CHI3L1, VEGFA, MMP9, SPP1 and TNC) in human adipocyte cultures and IL32-silenced adipocytes showed a downregulation $(\mathrm{P}<0.05)$ of inflammatory genes (CCL2, TNF, SPP1 and IL1B).

Conclusion:These findings provide evidence that the upregulated levels of IL-32 in human obesity might be implicated in its characteristic chronic pro-inflammatory state. IL-32 emerges as a nexus in adipose tissue biology at which the pathways of inflammation, ECM remodeling and the development of obesity-associated comorbidities converge.

Conflict of Interest: The authors declare that they have no conflict of interest.

Funding: Supported by FIS-FEDER, Instituto de Salud Carlos III (ISCIII)

(PI13/00460 and PI14/00950 and PI16/01217) and CIBEROBN, Spain.

\section{T1P68}

\section{Impact of breakfast skipping vs. dinner skipping on regulation of energy balance and metabolic risk}

Nas, A. ${ }^{1}$; Hägele, F. ${ }^{1}$ Kahlhöfer, J. ${ }^{1}$; Keller, J. ${ }^{1}$; Rising, R. ${ }^{2}$; Bosy-Westphal, A. ${ }^{1}$ 'University of Hohenheim, ${ }^{2} \mathrm{D}$ \& S Consulting Services Inc., New York

Background: Meal skipping has become an increasing trend of the modern life style that may lead to obesity and type 2 diabetes.

Objective: To investigate if timing of meal skipping impacts these risks by affecting circadian regulation of energy balance and glucose metabolism. Design: In a randomized controlled crossover trial, 17 participants (BMI $23.7 \pm 4.6 \mathrm{~kg} / \mathrm{m}^{2}$ ) underwent three isocaloric 24-hour interventions ( 55 30 and $15 \%$ carbohydrates, fat, and protein, respectively): a breakfast skipping day (BS) and a dinner skipping day (DS) separated by a conventional three-meal-structure day (control). Energy and macronutrient balance were measured in a respiration chamber. Postprandial glucose, insulin and daylong glycaemia as well as insulin secretion were analyzed.

Results: When compared with three-meal control, 24-hour energy expenditure was higher at both skipping days (BS: $+43 \mathrm{kcal} / \mathrm{d}, \mathrm{DS}:+92 \mathrm{kcal} / \mathrm{d}$; both $\mathrm{p}<0.01)$ whereas fat oxidation increased with BS only $(+16 \mathrm{~g} / \mathrm{d}$, $\mathrm{p}<0.001)$. Spontaneous physical activity, daylong glycaemia and 24-hour insulin secretion did not differ between intervention days. Postprandial HOMA index (+54\%) and glucose levels after lunch $(+46 \%)$ were however higher with BS compared to DS (both $\mathrm{p}<0.05$ ).

Conclusion: Compared to three meals a day, meal skipping increased energy expenditure. By contrast, higher postprandial insulin levels and increased fat oxidation with breakfast skipping suggest the development of metabolic inflexibility in response to prolonged fasting that may in the long-term lead to impaired glucose homeostasis.

Conflicts of interest statement: The authors declare no conflict of interest

Funding: This study was funded by budgetary resources of the University Hohenheim.

T1P69

\section{In vitro evaluation of anti-lipogenic potential by biotransformed soy extracts}

\section{Ávila, A. ' ; Queirós, L. '; Ueta, T. ' Lopes, D. '; Macedo, G. ; Macedo, J.'} ${ }^{1}$ University of Campinas

Introduction: Obesity is characterized by adipose tissue excess and, in many cases, with increased macrophage infiltration and pro-inflammatory cytokines secretion, leading to a subclinical chronic inflammatory state. Many compounds have been evaluated in an attempt to prevent and treat obesity and its complications. Among the compounds studied, phenolics are of great interest, including soy isoflavones. However, to be efficiently absorbed, the isoflavones must be transformed from glycosylated to aglycone form. For this purpose, the enzymatic biotransformation has shown great potential. Thus, this study aimed to evaluate, in in vitro assays, the anti-lipogenic potential of the enzymatically biotransformed soymilk.

Methods: The soymilk was biotransformed using tannase and $\beta$-glycosidase enzymes separately, both in immobilized forms. The optimization process was conducted, followed by HPLC-DAD, to select the best reaction parameters in order to obtain enriched extracts in aglycone isoflavones. In vitro evaluation of anti-lipogenic potential was performed using 3T3-L1 cell line and the cell cytotoxicity was conducted by MTT assay. After that, the anti-lipogenic assay was carried out adding samples in differentiation medium, followed by Oil Red $\mathrm{O}$ staining and absorbance reading at $492 \mathrm{~nm}$.

Results: In both extracts, the enzymatic biotransformation process was able to increase significantly the content of aglycones, forms often found in low quantity in soymilk. The concentration increased about 35-36 times to daidzein and 38 times to genistein after soymilk reaction with tannase and $\beta$-glycosidase. The extracts showed low cytotoxicity in 3T3$\mathrm{L} 1$ cell line in concentrations ranging from 2.5 to $25 \mu \mathrm{L} / \mathrm{mL}$. However, in the anti-lipogenic assay, there was a discreet effect in the adipocytes differentiation process from fibroblasts, decreasing from 1.7 to $6.6 \%$ on the total lipid accumulation for biotransformed extract with tannase, while the use of $\beta$-glycosidase led to a decrease of 2.1 to $5.5 \%$.

Conclusion: The biotransformation process with tannase and $\beta$-glycosidase enzymes was able to promote significant changes in isoflavones profiles present in soymilk, bringing on the bioconversion of the glycosidic forms (daidzin and genistin) in their respective aglycone forms (daidzein and genistein). Although the samples showed a discreet effect in adipocyte cell inhibiting differentiation, for further tests the modulation by the samples in lipolysis processes will be investigated, in order to contribute to the development of a product or ingredient with potential use in obesity prevention or treatment.

Conflict of Interest: None disclosed 
Funding: Research relating to this abstract was funded by São Paulo Research Foundation (FAPESP)

T1P70

Increased iFGF23 plasma levels in older obese patients with vitamin D deficiency

Holecki, M. ${ }^{1}$; Maria Bożentowicz-Wikarek ${ }^{2}$, Magdalena OlszaneckaGlinianowicz ${ }^{3}$, Jan Duława ${ }^{1}$, Andrzej Wiecek', Małgorzata Mossakowska ${ }^{5}$, Jerzy Chudek ${ }^{2}$

${ }^{1}$ Department of Internal Medicine and Metabolic Diseases, Medical University of Silesia, Katowice, Poland, ${ }^{2}$ Pathophysiology Unit, Department of Pathophysiology, Medical Faculty in Katowice, ${ }^{3}$ Health Promotion and Obesity Management Unit, Department of Pathophysiology, Medical Faculty in Katowice, ${ }^{4} \mathrm{Nephrology}$, Transplantation and Internal Medicine, Medical Faculty in Katowice, Medical University of Silesia, ${ }^{5}$ International Institute of Molecular and Cell Biology, Warsaw

Background: Obesity is generally considered as frequently accompanied by vitamin $\mathrm{D}$ deficiency with secondary hyperparathyroidism.

A factor potential responsible for escalating active vitamin $\mathrm{D}$ deficiency is fibroblast growth factor 23 (FGF-23) which down-regulates renal 1,25 $(\mathrm{OH}) 2 \mathrm{D} 3$ synthesis.

Aim: The aim of the study was to analyze the plasma level of iFG-23 in relation to vitamin D status in older, obese participants of nationwide study PolSenior.

Material and methods: The study involved 1050 obese patients aged 65 yrs and above (443men and 607 women).

4 patients with 25(OH)D3 excess were excluded. The plasma levels of iFGF-23, PTH, 25(OH)D3, total Ca and P were determined.

Results: Sufficient vitamin D levels were found in $12.0 \%$, insufficiency was observed in $32.5 \%$, severe insufficiency in $46.1 \%$ and deficiency in $9.4 \%$ of obese participants. There was a progressive increase in plasma iFGF-23 concentration along with stages of vitamin D deficiency from $9.9 \pm 14.3$ to $14.4 \pm 14.9 \mathrm{ng} / \mathrm{ml}(\mathrm{p}=0.07)$. Additionally, a parallel progressive increase in PTH serum levels from $40.6 \pm 20.5$ to $64.9 \pm 112 \mathrm{pg} / \mathrm{ml}(\mathrm{p}<0.001)$ with stable calcium and phosphate serum levels across vitamin $\mathrm{D}$ deficiency classes were found. The prevalence of secondary hyperparathyroidism was $16.8 \%$ in subjects with optimal vitamin D status and in $23.3 \%, 32.2 \%$ and $52.0 \%$ subjects with insufficiency, severe insufficiency and vitamin D deficiency, respectively.

Results: Increased plasma iFGF-23 levels in obese patients with already observed low vitamin D status may enhance consequences of vitamin D deficiency by inhibiting renal hydroxylation of $25(\mathrm{OH}) \mathrm{D}_{3}$

\section{T1P71}

\section{Influence of FTO RS9939609 on appetite, hunger and} postprandial ghrelin on morbid obese women

\section{Magno, F. ${ }^{1}$; Guaraná, H. ' Carneiro, J. ${ }^{2}$; Fonseca, A. ${ }^{3}$; Rosado, E.}

${ }^{1}$ Universidade Federal do Rio de Janeiro, ${ }^{2}$ Hospital Universitário Clementino Fraga Filho, ${ }^{3}$ Instituto Oswaldo Cruz - Fiocruz

Introduction: The polymorphism on Fat Mass and Obesity Associated (FTO) gene has been indicated as an obesity predictor, as well as being related to food intake regulation. The aim of this study was to evaluate the influence of FTO single nucleotide polymorphism (SNP) rs9939609 on appetite, hunger and postprandial ghrelin.

Methods: 38 morbid obese women were divided into 3 groups according to their FTO genotype (restriction fragment length polymorphism (PCRRFLP) method). Anthropometric, laboratory and dietary variables were evaluated at basal period. All subjects received a standard meal. Visua Analogy Scales were applied for assessment of hunger and satiety sensations, with 30 minutes break between each one. Ghrelin was evaluated in basal and postprandial period.

Results: The frequency of genotypes was $34.2 \%$ (TT), $42.1 \%$ (TA) and $23.7 \%$ (AA). It was observed an increase in postprandial ghrelin in TT and decrease in TA and AA genotypes. TT presented greater hunger than TA and AA after 60 minutes. TA presented greater satisfaction at 30 and 90 minutes, compared with TT. TA showed greater fullness than TT after 30 minutes and TT showed lower fullness than TA and AA after 90 minutes. Desire to eat was higher in TT at 60 and 90 minutes when compared to TA and AA.

Conclusion: FTO rs9939609 influenced on appetite. The presence of TT genotype in obese woman showed increased hunger and craving to eat, in addition to post prandial increased ghrelin.

Conflict of Interest: None Disclosed.

Funding: FAPERJ (Fundação de Amparo à Pesquisa do Estado do Rio de Janeiro).

\section{T1P72}

Influence of the SNP RS9939609 in the fat mass and obesityassociated (FTO) gene on satiety, food intake and leptinaemia of morbid obese women

\section{Guarana, H. ${ }^{\text {; }}$ Magno, F. ; Carneiro, J. ${ }^{2}$; Fonseca, A. ${ }^{3}$; Rosado, E. ${ }^{1}$}

${ }^{1}$ Universidade Federal do Rio de Janeiro, ${ }^{2}$ Hospital Universitário Clementino Fraga Filho, ${ }^{3}$ Instituto Oswaldo Cruz - Fiocruz

Introduction: Obesity represents one of the most serious public health problems in the developed world, with exponential growth observed in the recent decades. Some studies indicate that FTO gene polymorphism may influence the control of food intake, but knowledge is still few for this Conclusion. The aim of this study was evaluate the influence of SNP rs9939609 of FTO gene on postprandial satiety, food intake and leptinaemia.

Methods: This is a cross-sectional analytical study, which included adult women with morbid obesity without other chronic diseases. The individuals were divided in 3 groups according to their FTO genotype. At basal period anthropometric, laboratory (glucose, insulin, lipid profile and leptin) and dietary variables were evaluated. Also blood samples were collected for genotyping of the FTO gene (restriction fragment length polymorphism (PCR-RFLP) method) and Visual Analogue Scale was used to evaluated hunger and satiety. Then all volunteers received a standard meal balanced in lipids, carbohydrates and proteins to assess postprandial leptin. Data analyzes were performed using SPSS software version 21.0, considering $\mathrm{p} \leq 0.05$

Results: Thirty-eight morbid women aged $35.5 \pm 7.4$ years and body mass index of $45.4 \pm 7.03 \mathrm{~kg} / \mathrm{m}^{2}$ were evaluated. The frequency of genotypes AA, AT and TT was 23.7\% $(n=9), 42.1 \%(n=16)$ and $34.2 \%(n=13)$, respectively. No differences were observed among genotypes for glucose, insulin, total cholesterol, HDL-C, LDL-C, VLDL -C, triglycerides and caloric and macronutrient intake. Women with TT genotype showed higher hunger and desire to eat 180 minutes after having the standard meal. Satiety and leptin concentration did not differ between genotypes at times 0 and 180, but women with TT genotype had decreased serum leptin in the postprandial period.

Conclusion: Food intake and satiety did not differ between genotypes but TT showed higher desire to eat when compared to AA and AT, what agrees to a reduced leptinaemia seen in TT genotype in the post prandial period.

Conflict of Interest: None disclosed.

Funding: FAPERJ (Fundação de Amparo à Pesquisa do Estado do Rio de Janeiro). 


\section{T1P73}

Insulin resistance is associated with increased $\mathrm{mRNA}$ expression of inflammation-related cytokines in both subcutaneous abdominal and visceral fat

Stich, V.1; Rossmeislova, L.1; Siklova, M.1; Kracmerova, J.1; Sramkova, V.1; Koc, M.1; Klimcakova, E.1; Roussel, B.2; Viguerie, N.2; Langin, D.2

${ }^{1}$ Third Faculty of Medicine, Charles University, Prague, Czech Republic, ${ }^{2}$ Institut Des Maladies Métaboliques Et Cardiovasculaires, Inserm Université Paul Sabatier, Umr1048, Toulouse, France

Background and aim: Excessive accummulation of visceral adipose tissue (VAT) is considered to be a risk factor of insulin resistance and metabolic diseases. The aim of this cross sectional study in lean and obese subjects was to investigate the association of expression of inflammation-related cytokines in VAT and subcutaneous abdominal adipose tissue (SAAT) with whole-body insulin resistance.

Subjects and Methods: mRNA expresssion of twelve cytokines was measured, using qRT-PCR, in paired samples of VAT and SAAT obtained during elective abdominal operations in 53 women with a wide range of BMI $\left(20.2\right.$ to $\left.48.9 \mathrm{~kg} / \mathrm{m}^{2}\right)$. Prior to the operation, anthropometric indices and glucose disposal rate (GDR) using hyperinsulinemic euglycemic clamp were measured.

Results: mRNA expression of TNFalpha, hepatic growth factor, IL 10, IL $1 \mathrm{RN}$ and leptin in both, VAT and SAAT, were in positive correlation with adiposity indices (BMI, fat percentage, waist circumference) and in negative correlation with GDR adjusted to fat free mass. The impact of fat depot was differential for other genes: GDR was in negative correlation with serum amyloid and IL-1B expression in VAT and IL-18 and serpin -1 expression in SAAT. In addtion, TNFalpha, IL 10, IL 1RN expression in both fat depots were in positive correlation with plasma triacylglycerol levels. No correlations with GDR were found in either depot for adiponectin, CCL2, IL6 and IL8.

Conclusion: Whole body insulin resistance is associated with increased expression of a number of pro-inflammatory as well as anti-inflammatory cytokines in both, VAT and SAAT. This suggests that metabolic disturbances in obesity should not be ascribed just to pro-inflammatory features of VAT but rather are due to complex changes of inflammatory state in both VAT and SAAT.

Acknowledgement: This work was supported by grant LIMEX from the Grant Agency of Czech Republic, and by projects PRVOUK P31 and UNCE 20431 from Charles University in Prague.

\section{T1P74 \\ Insulin sensitivity differentially alters body fat and its distribution in black and white women}

Victor, $H_{.}{ }^{1}$; Goedecke, J. ${ }^{1}$

${ }^{1}$ University of Cape Town

Background: Studies in South Africa (SA) and the USA have shown that despite less central fat mass, black women are more insulin resistant than their white counterparts, but hypersecrete insulin to maintain normoglycaemia. It is not known whether these differences in insulin sensitivity and secretion determine the ethnic-specific body fat distribution of these women.

Objective: To determine whether the differences in insulin sensitivity and secretion in black and white South African (SA) women predict body fat and its distribution after a 5-year follow-up period.

Methods: In a sample of black $(n=13)$ and white $(n=9)$ premenopausal SA women, body fatness and distribution were measured using dual energy $\mathrm{x}$-ray absorptiometry) and insulin sensitivity (SI ) and secretion (acute insulin response to glucose, AIRg) were measured using a frequently sampled intravenous glucose tolerance test at baseline and after a 5-year free-living follow-up period. The association between baseline insulin sensitivity and secretion and body fat and its distribution at 5-year follow up were determined using multivariate regression, adjusting for baseline fat mass in black and white women separately.

Results: Baseline SI correlated negatively with total fat mass (kg and \%) at follow up in black $(\beta=-0.402, P=0.036$ and $\beta=-0.392, P=0.002)$, but not white $(\beta=-0.006, P=0.954$ and $\beta=-0.032, P=0.686)$ women, and with body fat distribution at follow-up in the white (android/gynoid ratio: $\beta=-0.663, P=0.022)$, but not the black women $(\beta=-0.086, P=0.801)$. In contrast, AIRg did not correlate with total fat mass or body fat distribution in black or white women at follow-up.

Conclusion: Greater insulin sensitivity predicts less fat mass in black women and reduced centralization of body fat in white women over a 5 year free-living follow-up period

\section{Conflict of Interest: None Disclosed}

Funding: Research relating to this abstract was funded by South African National Research Foundation Competitive Programme for Rated Researchers (Grant No: 78824).

\section{T1P75 \\ Intermittent hunger inhibits the central anorexigenic response of insulin, serotonin and leptin in Wistar females}

\author{
Vilca, C. ' Fernandez, M. ' ${ }^{1}$ Pedroso, A. ${ }^{2}$; Batista, L. ' ; Carmo, M. ${ }^{\text {; }}$ \\ Albuquerque, $K^{\prime}$ \\ ${ }^{1}$ Universidade Federal do Rio de Janeiro, ${ }^{2}$ Universidade Federal De São Paulo, \\ São Paulo
}

Introduction: The nutritional transition ongoing in Latin America, demonstrates the prevalence of obesity. From this perspective, considering the diffusion about the complications that may result from obesity or about of self-image, the search for the rapid loss of body mass led individuals to the practice of the induction of intermittent cycles of hunger, which consists of food restriction followed by periodic feeding ad libitum. However, the results on the health benefits of this strategy are still controversial. In the hypothalamus, insulin, serotonin and leptin have anorexigenic action, but it is not clear whether intermittent sense of hunger may alter this inhibitory response and compromise the regulation of food intake.

Methods: Wistar female of 67 days of life, composed three experimental groups treated for 6 weeks: Control group (C), Kept in free feed consumption throughout the treatment; Restricted 3: 2 (R3), with food restriction at $50 \%$ of $\mathrm{C}$ consumption for two days, followed by three days of free consumption; and the Restricted 5:2 (R5) with food restriction at 50\% of $\mathrm{C}$ consumption for two days, followed by five days of free consumption. All treated groups had free access to water. To the intracerebroventricular infusion, the rats were subjected to stereotactic surgery and the implantation of a guide cannula in the left lateral hypothalamic ventricle. On the 7 th postoperative day the rats received infusion of $5 \mu \mathrm{L}$ vehicle (saline $0.9 \%)$ or insulin $(10 \mathrm{mU})$ or serotonin $(20 \mu \mathrm{g})$ or leptin $(10 \mu \mathrm{g})$ and the feed intake was evaluated in two periods of 12-hours post-infusion. Data were analyzed using SPSS 20.0 software and treated with Student's t test and significance for $\mathrm{p}<0.05$.

Results: After the respective infusions, it was verified that the $\mathrm{C}$ group presented anorexigenic response, with inhibition of the consumption in the first 12 hours and in the total $24 \mathrm{~h}$, but no difference in the food consumption of the R3 and R5 groups was observed in the periods of time evaluated after the respective infusions.

Conclusion: Repeated hunger sensations in Wistar rats have led to central alterations in three important anorexigenic regulations systems, and more human studies need to be identified before recommending this strategy.

Conflict of Interest: None Disclosed

Acknowledgement: This study was supported by Fundação Carlos Chagas Filho de Amparo à Pesquisa do Estado do Rio de Janeiro - FAPERJ and the Coordenação de Aperfeiçoamento de Pessoal de Nível Superior - CAPES. 


\section{T1P76}

\section{Interrelation between urine VEGF and plasma adipokines} with kidney function in patients with metabolic syndrome

\section{Shulkina, S. ; Smirnova, E.}

${ }^{1}$ Perm State Medical University, Perm, Russian Federation

Introduction: In most studies hyperfiltartion, caused by obesity, and decreasing of glomerular filtration rate (GFR) coming with arterial hypertension is presented. Metabolic syndrome (MS) combines these two factors. We have studied the interrelation of leptin, resistin, insulin of plasma and Vascular endothelial growth factor (VEGF) in urine and microalbuminuria (MAU) coming with MS and uncomplicated obesity (O).

Methods: 80 MS patients (age 42,3 $\pm 8,3$ years, BMI $38.0 \pm 7,8 \mathrm{~kg} / \mathrm{m}^{2}$ ), were compared to the control group of 30 patients with $\mathrm{O}$ (age 39,4 $\pm 5,3$ years, BMI $36,7 \pm 5,5 \mathrm{~kg} / \mathrm{m}^{2}$ ). The concentration of insulin, leptin, resistin in blood, VEGF in urine, MAU were studied. HOMA IR and eGFR (CKDEpi formula) were calculated.

Results:In group MS compared to group O the levels of HOMA IR (5,2 \pm 2 vs. $2,1 \pm 1 ; \mathrm{p}<0.01$ ), leptin ( $46 \pm 12,7$ vs. $36 \pm 11 ; \mathrm{p}<0,01)$, resistin $(4,6 \pm 1,2$ vs. $3,4 \pm 1,4 ; \mathrm{p}=0,02)$, MAU $(25,8 \pm 7,8$ vs. $8,2 \pm 1,5 ; \mathrm{p}<0,01)$ VEGF $(79 \pm 22,3$ vs. $28 \pm 13,5$; $p=0,01)$ were higher. Ingroup MS hyperfiltration (eGFR $\left.>125 \mathrm{ml} / \mathrm{min} / 1,73 \mathrm{~m}^{2}\right)$ was present in $8 \%(\mathrm{n}=6)$, optimal filtration (eGFR $\left.>90 \mathrm{ml} / \mathrm{min} / 1,73 \mathrm{~m}^{2}\right)$ in $50 \%(\mathrm{n}=40)$, insignificantly lower filtration (eGFR $\left.<90>60 \mathrm{ml} / \mathrm{min} / 1,73 \mathrm{~m}^{2}\right)$ in $44 \%(\mathrm{n}=34)$. In group O hyperfiltration was not present, optimal filtration was present in $87 \%$ $(\mathrm{n}=26)$. In group MS MAU $(>30 \mathrm{mg} / \mathrm{ml})$ was present in $21,4 \%(\mathrm{n}=15)$ patients, $42,8 \%$ had a higher level of MAU $(10-29 \mathrm{mg} / \mathrm{ml})(\mathrm{n}=30)$. In group O MAU $(>30 \mathrm{mg} / \mathrm{ml})$ was not present.In the group of patients with GFR $<90 \mathrm{ml} / \mathrm{min} / 1,73 \mathrm{~m}^{2}$ with MAU $>30 \mathrm{mg} / \mathrm{ml}(\mathrm{n}=12)$, the rise of concentration of resistin compared to the group of patients with GFR $<90$ $\mathrm{ml} / \mathrm{min} / 1,73 \mathrm{~m}^{2}$ withMAU $<30 \mathrm{mg} / \mathrm{ml}(5 \pm 1,2 \mathrm{ng} / \mathrm{mlvs} .3,5 \pm 1,3 \mathrm{ng} / \mathrm{ml}$ $\mathrm{p}=0,01)$ was identified. In group MSthe relation of the MAU level and leptin $(R=0,53 ; p=0,02)$, VEGF $(R=0,36 ; p=0,03)$, eGFR $(R=-0,5$; $p=0,03)$ was observed.VEGFwas related to the level of uric acid ( $R=0,49$ $\mathrm{p}=0,04)$, leptin $(\mathrm{R}=0,57 ; \mathrm{p}=0,02)$, resistin $(\mathrm{R}=0,33 ; \mathrm{p}=0,04)$, eGFR $(\mathrm{R}$ $=-0,46 ; \mathrm{p}=0,03)$, DBP level $(\mathrm{r}=0,7 ; \mathrm{p}=0,02)$ andSBP $(\mathrm{r}=0,49 ; \mathrm{p}=0,03)$ Conclusion:With optimal GFR in group MS compared to $\mathrm{O}$ the signs of glomerural dysfunction were identified, which manifested in the increasing of MAUandVEGF. The decreasing of GFR is related to increased adipose tissue activity.

\section{T1P77}

\section{Is 24-hour continuous core body temperature monitoring in humans using an ingestible capsule telemetric sensor reliable?}

\section{Monnard, C. ' ; Fares, E. '; Calonne, J. '; Miles-Chan, J. ' ; Montani, J.';}

Durrer, D. ${ }^{2}$; Schutz, Y. ${ }^{1}$; Dulloo, A. ${ }^{1}$

${ }^{1}$ Department of Medicine, Division of Physiology, University of Fribourg, Switzerland, ${ }^{2}$ Cabinet Médical Com'S, Eurobesitas, Vevey, Switzerland

Introduction: There is increasing interest in the use of pill-sized ingestible capsule telemetric sensors for measuring core body temperature (Tc) following renewed interest in Tc as a potential indicator of metabolic efficiency and thrifty metabolic traits. The aim of this study was to investigate the feasibility of measuring Tc using the CorTemp system in vivo and to validate the accuracy of these capsule sensors ex vivo.

Methods: Tc was measured over an average of 20 hours in 27 subjects with simultaneous energy expenditure measurement in the overnight fasted state at rest and during standardized low-intensity physical activity involving either dynamic low-power cycling esercise (10-50W) or intermittent leg press isometric exercise (against 5-25 kg load), as well as after a $600 \mathrm{kcal}$ mixed meal ingestion at rest. Validation of the accuracy of the capsule sensors was made ex vivo against mercury thermometers across the physiological range $\left(35-40^{\circ} \mathrm{C}\right)$ in the morning and afternoon of $2-3$ consecutive days. Comparisons among capsule sensors and thermometers were made using Bland-Altman analysis. Systematic bias, error and temperature drift over time were assessed.

Results: The circadian temperature profile classically reported in free-living humans was confirmed. Significant increases in Tc were found in response to low-level dynamic cycling $>40 \mathrm{~W}$ (i.e. $>2.5$ METs) but no significant changes were detectable during intermittent low-level isometric exercise ( $<2$ METs) nor during the peak postprandial thermogenesis of the meal. Issues of particular interest for investigators included: $i$ ) fast 'turbo' gut transit time $(<15 \mathrm{~h})$ in one out of every 5 subjects; $i$ ) sudden erratic readings in tele-transmission of Tc; iii) non-consistent bias in capsule temperature measurement vs mercury thermometers: the mean systematic bias averaged $-0.25^{\circ} \mathrm{C}$ (limits of agreement -0.91 to $+0.41{ }^{\circ} \mathrm{C}$ ); substantial drift of capsule temperature over time was observed in one of every 4 capsules.

Conclusion:Since Tc is tightly regulated within a very narrow range in the healthy homoeotherm's body, physiological investigations of Tc require great accuracy and precision (better than $0.1^{\circ} \mathrm{C}$ ). Although the ingestible capsule sensor methodology appears of great interest for non-invasively monitoring the transit gut temperature, new technology requires a reduction in the inherent error of measurement, and warrants more inter-laboratory investigation on the above factors.

\section{Conflict of Interest: None Disclosed}

Research relating to this abstract was supported by the Swiss National Science Foundation (no. 310030_152870).

\section{T1P78 \\ Is there any correlation between anthropometric measurements with miR-143 and -34a expression in two different adipose tissues?}

Yuzbashian, E. '; Tabaei, K. '; Zadeh-Vakili, A. ; Daneshafrooz, A. ; Asghari, G. ${ }^{1}$; Mirmiran, P. $^{1}$; Hedayati, M. ${ }^{3}$; Khalaj, . $^{4}$

${ }^{1}$ Nutrition and Endocrine Research Center, Research Institute for Endocrine Sciences, Shahid Beheshti University of Medical Sciences, Tehran, Iran, ${ }^{2}$ School Of Advanced Sciences In Medicine, Tehran Medical Sciences Branch, Islamic Azad University, Tehran, Iran, ${ }^{3}$ Cellular And Molecular Endocrine Research Center, Research Institute For Endocrine Sciences, Shahid Beheshti University Of Medical Sciences, Tehran, Iran, ${ }^{4}$ Tehran Obesity Treatment Center, Department Of Surgery, Shahed University, Tehran, Iran

Introduction: Obesity changes the metabolic functions of adipose tissues, which may contribute to the development of obesity-associated non-communicable chronic disease. MicroRNAs are a short non-coding RNA may regulate the post-transcriptionally gene expression. Recent in vivo studies showed that miR-143 and miR-34a play an important role in the metabolic regulation of adipocytes. We aimed to investigate the association of miR-143 and miR-34a expression in omental and subcutaneous adipose tissues with anthropometric indices and blood pressure (BP) among healthy adults.

Methods: We selected 42 subjects (21 non-obese, 10 obese, and 11 morbid obese) who were eligible according to the study criteria. Omental and subcutaneous adipose tissues were obtained during open abdominal surgery. The miR-143 and miR-34a expression in omental and subcutaneous adipose tissues were assessed using Real-Time PCR. Height, weight, body mass index (BMI), waist, hip, and neck circumferences, and BP were determined according to standard protocols.

Results: MiR-143 expression were significantly correlated with BMI $(\mathrm{r}=0.746, \mathrm{p}=0.006)$, weight $(\mathrm{r}=0.750, \mathrm{P}=0.008)$, waist $(\mathrm{r}=0.687$, $\mathrm{P}=0.028)$, and neck $(\mathrm{r}=0.862, \mathrm{P}=0.003)$ in omental adipose tissue among morbid obese subjects. Moreover, miR-34a expression in omental adipose tissue were correlated with height $(\mathrm{r}=0.631, \mathrm{P}=0.035)$ and neck $(r=0.611, P=0.045)$. There was no significant correlation between miR-143 and -34a with anthropometric measurements in subcutaneous adipose tissues.

Conclusion: Our study suggested that several obesity-related indices have a positive correlation with miR-143 in omental adipose tissues. 
Conflict of Interest: None Disclosed

Funding: No Funding

Acknowledgement: The authors would like to thank Dr Mohammad-Reza Ebrahimi for surgical procedures

\section{T1P79 \\ Leptin and LPS induced ECM remodelling of human adipose tissue}

Kos, K. ${ }^{1}$; Pastel, E. ${ }^{1}$; Rittig, N. ${ }^{2}$; Mcculloch, L. ${ }^{1}$; Moeller, N. ${ }^{2}$

${ }^{1}$ University of Exeter, ${ }^{2}$ Aarhus University Hospital

Introduction: Extracellular matrix (ECM) fibrosis and subsequent stiffening hinder adipose tissue proliferation and impair its fatty acid storage function. Whilst ECM remodelling is part of the physiological response of wound healing, obesity is associated with increased inflammation and raised leptin levels which may contribute to excessive collagen production and development of adipose tissue fibrosis. We examined the influence of acute inflammation and hyperleptinaemia on ECM remodelling and fibrosis regulators.

Methods: Abdominal adipose tissue biopsies from six lean non-diabetic men treated in a randomised crossover trial with 1) saline bolus injection and 2) a bolus of $1 \mathrm{ng} / \mathrm{kg}$ lipopolysaccharide endotoxin (LPS) were obtained $135 \mathrm{~min}$ after each intervention. In addition, omental and subcutaneous abdominal adipose tissue (SCAT) from overweight women $(\mathrm{n}=10)$ was treated with recombinant leptin $(0.1,10$ and $100 \mathrm{nM})$ over 24 hours to evaluate its effect on profibrotic factors. Transcript accumulation was analysed by Taqman technology.

Results: In comparison to placebo, LPS infusion led to a dramatic response with rise of FFA, circulating TNFalpha and Interleukin 6 as well as increased expression of the inflammatory cytokines CCL2/MCP-1 (58\%) and TNFA (16\%) in adipose tissue but without a notable change in macrophage marker expression at the time of biopsy. The pro-fibrotic factor TGF betal and leptin increased with LPS infusion $(1.04 \pm 0.1$ (mean \pm SEM) $\mathrm{AU}$ versus $1.9 \pm 0.3 \mathrm{AU}, \mathrm{p}=0.03$ and $1.03 \pm 0.1$ versus $1.5 \pm 0.4 \mathrm{AU}, \mathrm{n}=6$ $\mathrm{p}=0.03$ respectively) with only an insignificant increase in COL1A1 and COL3A1 expression. When studying whether leptin could contribute to ECM remodelling we found that leptin resulted in a dose-dependent increase in TGFbetal in omental tissue only (Control, 0.1,10 and 100nM: $1.14 \pm 0.14,1.25 \pm 0.22,1.23 \pm 0.13$ and $1.38 \pm 0.22 \mathrm{AU}, \mathrm{n}=10, \mathrm{p}<0.05)$ and reduction in SCAT expression of COL1A1 $0.68 \pm 0.07,0.69 \pm 0.08$ $0.71 \pm 0.1,0.47 \pm 0.08 \mathrm{AU}, \mathrm{n}=9, \mathrm{p}<0.05)$ as well as COL6A3 in both fat depots.

Conclusion: LPS induces an inflammatory ECM response in human adipose tissue independent of macrophage infiltration. Independently, the adipokine leptin however appears to counter-regulate collagen production in subcutaneous tissue by which it may limit the development of adipose tissue fibrosis.

Conflict of Interest: None Disclosed

\section{T1P80}

\section{Leptin intake at physiological doses throughout lactation in rats restores the altered stomach sympathetic drive caused by mild gestational calorie restriction}

\section{Szostaczuk, N. ${ }^{1}$; Sánchez, J. ${ }^{1}$; Konieczna, J. ${ }^{1}$ :Palou, A. ' Pico, C. ${ }^{1}$}

${ }^{1}$ Laboratory of Molecular Biology, Nutrition and Biotechnology (Nutrigenomics), University of the Balearic Islands and CIBER de Fisiopatología de la Obesidad y Nutrición (CIBEROBN), Palma de Mallorca, Balearic Islands, Spain.

Introduction: Gestational undernutrition in rats has been linked with lower sympathetic innervation in offspring, affecting different peripheral tissues, such as the stomach. In this tissue, the lower sympathetic drive has been proposed to account for the lower gastric ghrelin secretion and for the resulting lower circulating levels. In the light of previous findings showing the essential role of leptin during the suckling period to prevent obesity and reverse developmental malprogramming effects, we aimed to assess whether the supplementation with physiological doses of leptin throughout the suckling period may reverse the alterations in sympathetic innervation in the stomach caused by mild maternal calorie restriction during pregnancy.

Methods: Three groups of male rats were studied: the offspring of ad libitum fed dams (controls), the offspring of $20 \%$ calorie restricted dams during the first part of pregnancy (CR), and CR rats supplemented with physiological doses of oral leptin throughout lactation (CR-Leptin). The study was performed in juvenile ( 25 days) and adult ( 6 months) animals. Tyrosine hydroxylase (TyrOH) levels and the density of TyrOH-immunoreactive $\left(\mathrm{TyrOH}^{+}\right)$fibers, used as indicators of sympathetic innervation, were determined in the stomach of these animals. Serum ghrelin levels were also determined at three ages: 25 days, and 3 and 6 months.

Results: At 25 days old, CR rats displayed significantly lower TyrOH levels and lower density of $\mathrm{TyrOH}^{+}$fibers in the stomach than controls. Notably, these alterations were not found in CR-Leptin animals, suggesting that the altered sympathetic innervation was reverted by leptin treatment. The aforementioned alteration found in young CR animals was mitigated at the age of 6 months, and differences between control and CR animals did not reach significance level. Nevertheless, the trend to a lower density of TyrOH+fibers in $\mathrm{CR}$ animals compared to controls was also reverted in CR-Leptin animals. According to the decreased innervation, CR animals, but not CR-Leptin animals, also showed lower serum ghrelin levels than controls, particularly at the age of 3 months, while differences between groups were also mitigated in 6-month-old animals.

Conclusion: These findings show the ability of leptin intake during lactation to reverse the malprogrammed alterations in the sympathetic innervation of the stomach caused by mild maternal calorie restriction during gestation in rats and support the relevance of leptin as an essential factor during lactation.

Conflict of Interest: None Disclosed

Funding: Research relating to this abstract was funded by AGL2012-33692 and AGL2015-67019-P.

\section{T1P81 \\ Leptin raises defended body temperature without activating thermogenesis}

Fischer, A. ${ }^{1}$; Hoefig, C. ${ }^{2}$; Abreu-Vieira, G. ${ }^{3}$; Jong, J. ${ }^{3}$; Petrovic, N. ${ }^{3}$; Mittag, J. ${ }^{2}$; Cannon, B. ${ }^{3}$; Nedergaard, J. ${ }^{3}$

${ }^{1}$ The Wenner-Gren Institute \& University of Hamburg/Eppendorf, ${ }^{2}$ Karolinska Institute ${ }^{3}$ The Wenner-Gren Institute

Leptin has been believed to exert its weight-reducing action not only by inducing hypophagia but also by increasing energy expenditure/thermogenesis. Leptin-deficient ob/ob mice have correspondingly been thought to be thermogenically limited and to show hypothermia, mainly due to atrophied brown adipose tissue (BAT). In contrast to these established views, we found that BAT is fully functional and that leptin treatment did not increase thermogenesis in wildtype or in ob/ob mice. Rather, ob/ob mice showed a decreased but defended body temperature (i. e., were anapyrexic, not hypothermic) that was normalized to wild-type levels after leptin treatment. This was not accompanied by increased energy expenditure or BAT recruitment but, instead, was mediated by decreased tail heat loss. The weight-reducing hypophagic effects of leptin are, therefore, not augmented through a thermogenic effect of leptin; leptin is, however, pyrexic, i. e., it alters centrally regulated thresholds of thermoregulatory mechanisms, in parallel to effects of other cytokines. 
T1P82

Leptin regulates adipocyte autophagy: A potential autocrine regulatory contributing to the up-regulation of adipose tissue autophagy in obesity

Goldstein, N. ${ }^{1}$; Haim, Y. ${ }^{1}$; Beck-Sickinger, A. ${ }^{2}$; Blüher, M. $^{2}$; Rudich, A. ${ }^{1}$

${ }^{1}$ Ben-Gurion University of the Negev, ${ }^{2}$ University Of Leipzig

Background/Objectives: Adipose tissue (AT) dysfunction in obesity is characterized by altered autophagic activity and adipocytokines secretion profile. Here we hypothesized that adipocytokines could act in an auto-paracrine manner to dysregulate AT autophagy.

Subjects/Methods: Human adipocytes (chub-s7) were treated with con dition media (CM) from visceral adipose tissue explants and autophagy genes and flux were assessed. Complementarily, we studied the effect of selected adipocytokines on autophagy genes and protein expression. Finally, human adipose tissue samples $(\mathrm{n}=186)$ were assessed for clinical associations between human visceral AT autophagy gene expression and circulating leptin by multivariate models.

Results: In differentiated human pre-adipocytes CM increased both autophagy gene expression and flux. Among several selected adipocytokines, progranulin, and more robustly - leptin, but not chemerin, increased autophagy gene expression. Leptin treatment also showed increased autophagic activity as measured by flux. In the human cohort, circulating leptin levels positively associated with human omental-AT expression of ATG5 $m R N A$, associations that remained significant (beta $=0.344, \mathrm{p}<0.001$ ) in a multivariate model adjusted for age, sex and BMI.

Conclusions: Obesity-associated increased leptin secretion may act in an autocrine manner to increase autophagic activity in adipocytes.

Funding: Israel Science Foundation; Deutsche Forschungsgemeinschaft (DFG)

T1P83

\section{Lipolytic effect of Uroguanylin and Guanylin in human omental adipocytes}

Rodríguez, A. '; Gómez-Ambrosi, J.'; Catalán, V. '; Ezquerro, S. '; Becerril, S. '; Moncada, R. ${ }^{3}$; Valentí, V. ; Silva, C. ${ }^{5}$; Salvador, J.5.; Frühbeck, G. ${ }^{5}$

${ }^{1}$ Metabolic Research Laboratory, Clínica Universidad de Navarra, IdiSNA, Pamplona, Spain, ${ }^{2}$ Ciberobn, Instituto De Salud Carlos lii, Madrid, Spain, ${ }^{3}$ Department Of Anesthesia, Clínica Universidad De Navarra, Pamplona, Spain ${ }^{4}$ Department Of Surgery, Clínica Universidad De Navarra, Pamplona, Spain, ${ }^{5}$ Department Of Endocrinology \& Nutrition, Clínica Universidad De Navarra, Pamplona, Spain

Introduction: Intestinal uroguanylin and guanylin can regulate appetite and food preference. We evaluated the impact of obesity and type 2 diabetes (T2D) on proguanylin and prouroguanylin expression/secretion as well as their potential role in lipolysis in human adipocytes.

Methods: Plasma concentrations and gastrointestinal expression of proguanylin (GUCA2A) and prouroguanylin (GUCA2B) were measured in 134 subjects.

Results: Reduced $(P<0.05)$ circulating prouroguanylin, but not proguanylin, were found in obesity in relation to adiposity. Obese T2D individuals exhibited higher $(P<0.05)$ intestinal GUCA2A expression as well as of the receptors of the guanylin system, GUCY2C and GUCY2D, in omental adipocytes. Guanylin and uroguanylin treatment significantly $(P<0.05)$ induced lipolysis in differentiated omental adipocytes, as evidenced by HSL phosphorylation at Ser563, an increase in fatty acids and glycerol release together with an up-regulation of AQP3, AQP7, FATP1 or CD36.

Conclusion: Uroguanylin and guanylin stimulate lipolysis in human omental adipocytes. Given the lipolytic action of the guanylin system on omental adipocytes, the herein observed reduction in plasma prouroguanylin levels in obese patients points to a role in excessive fat accumulation in obesity.

Conflict of Interest: None Disclosed
Funding: Supported by FIS-FEDER (FIS PI12/00515, PI13/01430 and PI16/00221) from ISCIII and the Department of Health of the Gobierno de Navarra $(61 / 2014)$.

As requested, in both groups the caloric intake reduction was similar $(-10 \%)$ and the analysis of the 22 type and number of servings showed almost identical modifications in both groups (Anova $\mathrm{p}>0.05$ ).

Table 1 summarizes the results of the study. In the placebo group the BW and $\mathrm{AC}$ reductions were respectively $7.8 \mathrm{~kg}$ and $10 \mathrm{~cm}$, whereas the $\mathrm{PG}$ group the BW lost was $11.9 \mathrm{~kg}$ (Anova Vs placebo $\mathrm{P}<0.001$ ) and the $\mathrm{AC}$ reduction was of $13.6 \mathrm{Cm}$ ( Anova Vs placebo $\mathrm{P}<0.001$ ).

Furthermore, despite at baseline most of the labs variables were within the normal range, in both group there was also found a significant reduction of plasma lipids, glucose and blood pressure, that was more evident in the group treated with PG (Anova $\mathrm{p}<0.05)$.

Particularly the decline of HsCRP was evident in both groups, and in this case also PG was shown to be more effective than placebo (Anova $\mathrm{p}<$ $0.01)$. From the correlation analysis it seems that the lipids intake was the most responsible for the increase of HsCRP, and it was also shown that the extra virgin olive oil in the quantity that was used was not correlated with HsCRP increase.

Conclusions: In moderately obese subject following a 10\% caloric restriction together with a moderate increase of physical activity, PG was shown to be more effective than placebo (Anova: $p<0.01$ ) in reducing body weight, abdominal circumference and inflammation determined through HsCRP levels. The control of the diet using the 22 weekly servings system was found to be an interesting and effective tool to support the diet compliance.

\section{T1P85 \\ Long-term effects of FGF21 in maternal milk on metabolism and adiposity in adult mice}

\section{Gavaldà-Navarro, A. ${ }^{1}$; Cereijo, R. ${ }^{1}$; Morón, S. ${ }^{1}$; Giralt, M. ' ; Villarroya, F. ${ }^{1}$}

${ }^{1}$ Department of Biochemistry and Molecular Biomedicine, Institute of Biomedicine (IBUB), University of Barcelona, Av. Diagonal, 643, 08028 Barcelona, Spain, and Centro de Investigación Biomédica en Red de Fisiopatología de la Obesidad y Nutrición (CIBEROBN)

Introduction: The presence of hormonal factors in maternal milk is considered to influence metabolic health in adulthood, including propensity to obesity. Fibroblast growth factor 21 (FGF21) is a hormonal factor that plays important roles in controlling glucose homeostasis and energy metabolism. Previous studies found that FGF21 is present in human and rodent milk and FGF21 in milk is necessary to ensure optimal digestive and endocrine functions in developing intestine in neonatal mice. However, the long-term effects of the presence of FGF21 in milk on adult metabolism and adiposity are unknown.

Methods: In order to investigate the consequences of being fed milk containing FGF21 or not during lactation, wild-type (WT) and FGF21-KO mice were cross-fed during lactation obtaining four experimental groups: WT mice fed by WT dams, WT mice fed by FGF21-KO dams, FGF21-KO mice fed by WT dams and FGF21-KO mice fed by FGF21-KO dams. Male mice were maintained under standard conditions until 12-month-old, when glucose and insulin tolerance (ITT) tests were performed. After sacrifice, interscapular brown (iBAT), epididymal (eWAT), inguinal (iWAT) adipose tissues, liver and ileum were dissected and analysed. Gene expression was analysed using qRT-PCR.

Results: FGF21-KO adult mice showed a slight decrease in body weight and adiposity (especially iWAT and iBAT), which was enhanced when FGF21-KO mice had been fed by WT dams during lactation No overt changes in glycaemia were found. However, there were signs of lowered insulin sensitivity (impaired ITT) in FGF21-KO mice fed by FGF21-KO dams. Otherwise, ITT was improved in FGF21-KO mice which had been nursed by WT dams. No major changes in gene expression were found in iBAT, eWAT and liver. In iWAT, marker genes of "browning" (Bmp8b, Prdm16, Pgc-1 $\alpha$ and, especially, Ucp1) were expressed more intensely in FGF21-KO mice fed by WT dams than in FGF21-KO mice fed by FGF21- 
KO dams WT mice fed by FGF21-KO dams. WT mice fed by FGF21-KO dams during lactation showed increased FGF21 expression in iBAT and iWAT, but not in the liver. In ileum, gastric inhibitory peptide and genes involved in fatty acid oxidation (carnitine octanoyltransferase, carnitine palmitoyltransferase-1- $\alpha$ ) were down-regulated in FGF21-KO mice fed by FGF21-KO dams but not in FGF21-KO mice fed by WT dams.

Conclusion: Exposure to FGF21 present in maternal milk has mild longterm consequences in adulthood. However, signs of altered glucose/insulin homeostasis and disturbed function in subcutaneous WAT and small intestine occur in adult mice that were fed milk devoid of FGF21, especially when these adults were also on a FGF21 null background.

Conflict of Interest: No competing financial interests.

Funding: Recercaixa and Instituto Danone. Spain.

\section{T1P87}

\section{Maternal high-fat feeding and/or overweight and the early programming of metabolic and cognitive risk in the offspring: A study in mice and human infants}

Sanguinetti, E. ; Guzzardi, M. ${ }^{1}$; Panetta, D. ${ }^{1}$; Tripodi, M. ${ }^{1}$; Zega, A. ${ }^{1}$; Telleschi, M. ; Ditaranto, F.'; Muratori, F. ${ }^{2}$; Burchielli, S. ; Kemeny, A. ${ }^{3}$; Salvadori, P. ${ }^{1} ;$ lozzo, P. ${ }^{1}$

${ }^{1}$ Institute of Clinical Physiology, National Research Council (CNR), Pisa, Italy ${ }^{2}$ Irccs, Stella Maris Foundation, Pisa, Italy, ${ }^{3}$ Gabriele Monasterio Foundation (Ftgm), Pisa, Italy, ${ }^{4}$ Department Of Obstetric And Gynecology, Asl 1 Massa E Carrara, Italy

Introduction: Exposure to an adverse nutritional-metabolic environment during intra-uterine development increases the predisposition to metabolic-cognitive disorders. We examined the effects of maternal high-fat dieting (HFD)/overweight on offspring's growth, eating behaviour, cerebral metabolism and cognitive development.

Methods: Mice born to normal diet (ND) and HFD-fed dams ( $\mathrm{n}=45$ ) were studied at 1 or 6 months of age to assess growth, food intake, cognitive-exploratory behaviour (Y-maze test), and cerebral glucose metabolism (fasting and intranasally insulin-stimulated, by positron emission tomography). Serum adipokine profile was measured by multiplex immunoassay. Ninety-one human lean and overweight mothers were recruited. Growth and eating behaviour during the first 2 years of life, and neurodevelopment at $6(\mathrm{n}=24), 12(\mathrm{n}=26), 18(\mathrm{n}=26)$ and 24 months of age $(\mathrm{n}=22)$, were monitored by diaries, and by feeding and mental developmental scales in their offspring.

Results: At weaning, mice born to HFD mothers had higher body weight, glycemia, brain glucose metabolism and insulin sensitivity compared to controls. In adulthood, they showed overweight and hyperglycemia, resulting from hyperphagic and hypolocomotor behavior, hyperresistenemia, brain hypermetabolism and cerebral insulin resistance. In human infants, maternal overweight predicted higher birth weight and growth, greater dietary lipid intake and a positive feeling towards food. In the offspring of HFD/overweight mothers (mice and humans) compared to offspring of ND/lean mothers, cognitive function was superior at the time of weaning, but declined at a faster rate thereafter.

Conclusion: Maternal overweight results in alterations in eating behaviour, body weight, cerebral metabolism and cognition in the offspring.

The authors have no conflict of interest to declare.

Research related to this abstract was funded by the EU-FP7-HEALTH DORIAN project (Grant Agreement \#278603).

\section{T1P88}

Melatonin treatment increases oxygen comsuption in isolated adipocytes preventing body weight gain in mice induced to obesity by high fat diet $\frac{\text { Farias, } T^{1}}{\text { Vale, } M .{ }^{1}}$ 'Cruz, M. ${ }^{1}$; Sá, R. ${ }^{1}$; Paixão, R. ${ }^{1}$; Silva, N. ${ }^{1}$; Batini, F. ; Andrade, P. ${ }^{2}$;

${ }^{1}$ Federal University of São Paulo, ${ }^{2}$ Cruzeiro Do Sul University

Introduction: Obesity is becoming a global epidemic and is linked directly or indirectly to other diseases. Recent data suggests that adipocyte mitochondria might play an important role in the development of obesity through defects in the regulation of b-oxidation, mitochondrial respiratory system and production of oxygen radicals, all these effects are under control of a fine genic adjustment, for example, by Pgc-1 $\alpha$, the largest transcription regulator of thermogenic program and a mitochrondrial biogenesis activator. Melatonin, pineal gland's hormone, has been described as a regulator of mitochondrial bioenergetic function. In this context, melatonin has emerged as a potential therapeutic tool for treating obesity. Thus, the aim is to investigate how melatonin supplementation acts on physiology of white adipose tissue in obese mice

Methods: C57BL/6J mice were submitted to obesity by a high fat diet for 10 weeks. Simutaneously, they received (or not) melatonin $(1 \mathrm{mg} / \mathrm{kg})$ in drinking water.The weight of the animals were weekly evaluated. At the end of the protocol, inguinal adipose tissue were isolated and evaluated for basal mitochondrial respiration (Oroboros oxygraph-2k equipment) and total RNA was extracted for Pgc-1 $\alpha$, Ppar $\gamma$ and Prdm-16 mRNA expression and evaluation by real-time quantitative PCR analyses.

Results: Melatonin treatment was effective in preventing body weight gain in C57BL/6J mice [final weight (grams): control 26,33 $\pm 0,37$; obese $39,98 \pm 2,40 ;$ obese+melatonin $33,03 \pm 0,77 ; n=7-10]$. Furthermore the oxygraph analyses show that melatonin increased significantly basal oxygen consupmtion (OCR: control $100 \pm 5,95$; obese 48,03 $\pm 9,53$; obese+melatonin $102,55 \pm 20,94 ; \mathrm{n}=5-6)$. The analysis of gene expression showed that melatonin supplementation increased (2,7X) Pgc-1a mRNA expression as weell as increased Ppar $\gamma$ and Prdm-16RNA expression (2,8X and $1,5 \mathrm{X}$ respectivey) in animals induced to obesity by a higt fat diet. Conclusions: Ours data suggest that melatonin can acts in the prevention of obesity through improves mitochondrial function in adipocytes.

Conflict of interest: No Disclosure

Financial Support: Research relating to this abstract was funded by FAPESP (2015/03554-2--2016/07638-9)

\section{T1P89 \\ Metabolic dysfunction in translocator protein knockout mice: Role in inflammation}

Naik, R. ${ }^{1}$; Chatterjee, P. ${ }^{2}$; Ji, B. ${ }^{2}$; Suhara, T. ${ }^{2}$; Higuchi, M. ${ }^{2}$; Barron, A. ${ }^{1}$

${ }^{1}$ Neurobiology of Aging and Disease Laboratory, Lee Kong Chian School of Medicine, Nanyang Technological University, 59 Nanyang Drive, Singapore 636921, 2Department Of Functional Brain Imaging Research, National Institutes For Quantum And Radiological Science And Technology, 4-9-1 Anagawa, InageKu, Chiba-Shi, Chiba 263-8555, Japan

Introduction: The translocator protein (TSPO) is a multi-functional, mitochondrial transmembrane protein, which has been implicated in the regulation of mitochondrial bioenergetics, glucose metabolism and inflammation. Pharmacological studies have indicated that TSPO-targeted drugs may promote metabolic health in animal models of diet-induced obesity. However, TSPO function in metabolic homeostasis remains to be corroborated in vivo via genetic studies.

Methods: Global TSPO knockout (TSPO-KO) mice were developed to investigate TSPO function in the regulation of metabolic homeostasis and obesity-associated inflammation. Three month old male wild type (WT) and TSPO-KO mice were either fed standard or high fat (HF) diet for four months and body weight, fasting blood glucose, leptin and insulin levels were monitored. Adiposity was examined in living mice by computerized 
tomography (CT) and abdominal and subcutaneous fat depots were isolated to corroborate in vivo findings. Inflammation was assessed in adipose and brain by immunohistochemistry and quantitative PCR analyses. Results: Metabolic dysfunction was observed in TSPO-KO mice including increased body weights and adiposity, and elevated levels of leptin fasting glucose and insulin. While HF feeding resulted in comparable end-point levels of obesity and metabolic dysfunction between WT and TSPO-KO mice, the development of hyperglycemia was accelerated in $\mathrm{HF}$ fed TSPO-KO mice. Interestingly, although TSPO-KO was associated with detrimental metabolic outcomes, it was protective against markers of neuroinflammation in HF fed TSPO-KO mice.

Conclusion: TSPO plays an important role in the regulation of metabolic homeostasis and may represent a therapeutic target for the management of metabolic dysfunction and associated inflammation.

Conflict of Interest: None Disclosed

Funding: No Funding

\section{T1P90}

\section{Metabolic syndrome contributes to the natural history of heart failure with preserved ejection fraction}

Conceição, G. '; Matos, J. ${ }^{2}$; Sampaio-Pinto, V. ${ }^{3}$; Miranda-Silva, D. ' ; Fontoura, D. '; Sena, C. ; Lourenço, A. '; Nascimento, D. ${ }^{3}$; Vitorino, R. ${ }^{2}$; LeiteMoreira, A. '; Falcão-Pires, I. ${ }^{1}$

${ }^{1}$ Departamento de Cirurgia e Fisiologia, Faculdade de Medicina, Universidade do Porto, Portugal, ${ }^{2}$ Department Of Medical Sciences, Ibimed, University Of Aveiro, Portugal, ${ }^{3} \mid 3 S$ - Instituto De Investigação E Inovação Em Saúde, Universidade Do Porto, Portugal, ${ }^{4}$ Laboratory Of Physiology, Institute Of Biomedical Imaging And Life Sciences (Ibili), Faculty Of Medicine, University Of Coimbra, Portugal

Introduction: Heart failure with preserved ejection fraction (HFpEF) represents $50 \%$ of heart failure cases. These patients exhibit metabolic syndrome (MetS) linked to a large number of comorbidities, including abdominal obesity. The new paradigm proposes that comorbidities drive structural and functional remodelling in HFpEF through systemic endothelial inflammation. The mechanisms underlying cardiovascular disease in MetS are complex and can culminate with increased oxidative stress. Thus, we intend to clarify the contribution of metabolic syndrome in development of endothelial dysfunction potentially driven by oxidative stress.

Methods: Lean ZSF1 (ZSF1Ln, $\mathrm{n}=7$ ) and obese ZSF1 (ZSF1Ob, $\mathrm{n}=7$ ) rats were echocardiographically evaluated at 20-25 weeks. Visceral adipose tissue (VAT) was collected for histological and mass spectrometry analysis. Urine and plasma were evaluated for detection of 8-hydroxydeoxyguanosine (8-OHdG) and malondialdehyde (MDA), respectively. Left ventricular (LV) samples were used for flow cytometry, protein expression by immunoblotting and in situ detection of myocardial oxidative stress by immunofluorescence.

Results: Echocardiographically, the ZSF1Ob group showed increased LV mass, preserved ejection fraction and LV diastolic dysfunction. Histological studies of VAT presented adipocytes hypertrophy in obese ZSF1Ob. Proteome analysis of VAT revealed the prevalence of processes related to oxidative metabolism in ZSF1Ob rats, which was evidenced by a decrease of the aldehyde dehydrogenase mitochondrial and catalase proteins. Urinary levels of 8-OHdG and plasma levels of MDA were significantly higher in ZSF1Ob rats, which evidence systemic oxidative stress. Flow cytometry analysis exhibited cardiac inflammation and endothelial cell activation by increased expression of ICAM-1 and VCAM-1. Protein expression studies revealed a significant increase in the expression of NADPH oxidase 2 and a significant decrease in NADPH oxidase 4 in ZSF1Ob rats, enzymes responsible for regulating the formation of reactive oxygen species.In situ detection of superoxide anion was significantly higher in ZSF1Ob rats. Conclusion: Obesity prompts an increase of oxidative stress by alterations in adipose tissue. The main cause of development/progression diastolic dysfunction complications can be oxidative stress associated to endothelial cell activation.

\section{T1P91 \\ Modified SJH alleviates FFAs-induced hepatic steatosis through leptin signaling pathways}

Lim, D. ' ; Kim, H. $^{1}$

Introduction: Samjunghwan $(\mathrm{SJH})$ is an herbal formula used in traditional Korean medicine. This prescription has long been used in treatment of aging and lifestyle diseases. The current study showed the effect and mechanisms of anti-hepatic steatosis action of modified $\mathrm{SJH}(\mathrm{mSJH})$ in vitro and in vivo.

Methods: HepG2 cell line was used as a model in evaluation of the anti-hepatic steatosis effect. Steatosis was induced by treatment with a mixture of free fatty acids (FFAs). Effect of $\mathrm{mSJH}$ on the intracellular lipid levels of FFAs-induced cells was assessed using Oil Red O staining and biochemical assays. Moreover, the expression of genes and proteins as well as signaling pathways related to lipid metabolism were also examined using PCR array, quantitative real-time PCR, and western blotting. As in vivo study, dyslipidemic mice model was made with $\mathrm{C} 57 \mathrm{BL} / 6$ mice fed high-fat diet. Each group consist of four groups; normal diet (ND), high fat diet (HFD), HFD + low dose mSJH (LD), HFD + high dose mSJH (HD). After 10 weeks, serous, hepatic and fecal lipid profiles were investigated. Genes and proteins were analyzed by western blot and real-time PCR.

Results: Treatment with $\mathrm{mSJH}$ resulted in significantly decreased intracellular lipid accumulation in steatosis-induced cells. Furthermore, mSJH triggered the phosphorylation of AMP-activated protein kinase and acetyl-CoA carboxylase as well as increased the expression of leptin at both protein and gene levels. In addition, C57BL6 mice fed high-fat diet (HFD) showed significant improvements in body, liver weights and fat weights; and serum, hepatic and fecal lipid parameters in response to the treatment with $\mathrm{mSJH}$. Furthermore, $\mathrm{mSJH}$ showed favorable effects on the hepatic expression of several genes related to lipid metabolism. Betaine, one of constituents of $\mathrm{mSJH}$ exerted fundamental beneficial impact on FFAs-induced cells. However, the beneficial effects of $\mathrm{mSJH}$ were diminished upon blocking of leptin signaling by dexamethasone, suggesting the leptin signaling as a key component in $\mathrm{mSJH}$-mediated modulation of lipid homeostasis.

Conclusion: Our results suggest that $\mathrm{mSJH}$ exerts an anti-hepatic steatosis effect via activation of leptin and associated signaling cascades related to lipid metabolism.

\section{Conflict of Interest: None Disclosed}

Funding: This study was supported by the Traditional Korean Medicine R\&D program funded by the Ministry of Health \& Welfare through the Korea Health Industry Development Institute (KHIDI). (HI14C0556)

\section{T1P92 Effects in cardiac structure}

\section{Conceição, G. ${ }^{\text {; }}$ Matos, J. ${ }^{2}$; Gonçalves, N. '; Fontoura, D. '; Lourenço, A. ';} Vitorino, R. ${ }^{2}$; Leite-Moreira, A. '; Falcão-Pires, I. ${ }^{1}$

${ }^{1}$ Departamento de Cirurgia e Fisiologia, Faculdade de Medicina, Universidade do Porto, Portugal, ${ }^{2}$ Department Of Medical Sciences, Ibimed, University Of

Introduction: Heart failure with preserved ejection fraction (HFpEF) is recognized as an important cause of cardiovascular mortality and morbidity reaching approximately $50 \%$ of heart failure cases. Several risk factors, such as obesity, are associated with its development. Obesity represents a condition where excessive energy intake results in the expansion of adipose tissue, contributing to a systemic pro-inflammatory state. Aveiro, Portugal 
The heart itself is surrounded by cardiac visceral adipose tissue (CVAT), which represents an additional risk factor to pathological alterations in the cardiovascular system. The adipokines produced by CVAT can act in a paracrine manner directly on the myocardium and can influence their structure and function. So, we aim to characterize the profile of CVAT in an obese animal model of HFpEF and its cardiac impact.

Methods: CVAT from 20-weeks-old lean (control group) and obese ZSF1 rats (HFpEF - obese group) was collected for: 1) proteins identification using mass spectrometry; 2) characterization of adipokines' expression; 3 ) assessment of adipocytes cross-sectional area and 4) collection of 24h-DMEM CVAT-derived conditioned medium for: incubation of myocardial organo-cultures prepared from 7-days-old Wistar Kyoto cardiac explants and characterization of the profile of secreted adipokines. After incubation with conditioned media from lean or obese rats, cross-section area of cardiomyocytes and fibrosis were evaluated.

Results: CVAT from obese ZSF1 rats presented a decrease in mitochondrial metabolism and an increasein processes associated with extracellular matrix expansion and triglyceride metabolism. The histological studies of CVAT revealed hypertrophy of adipocytes in obese ZSF1 rats, as well as a unilocular appearance compared with a multilocular appearance of CVAT in lean ZSF1 rats. Molecular studies of CVAT from obese ZSF1 rats presented a significantly increase in expression of several adipokines involved in fibrotic and hypertrophic pathways, namely in leptin, apelin, chemerin or visfatin. Moreover, CVAT from obese ZSF1 rats showed an increase expression of PPAR $\gamma$ supporting adipogenesis of adipose tissue. Conditioned media from CVAT obese rats exhibited an inflammatory profile and, in organo-cultures, induced cardiomyocytes hypertrophy and fibrosis compared to the conditioned media from lean rats.

Conclusion: Obesity promotes alterations in CVAT structure, composition, metabolism and secretion profile, which significantly affects myocardial structure, inducing collagen deposition and cardiomyocyte hypertrophy.

\section{T1P93}

\section{Modulation of glut4 during adipogenesis in 3T3L1 correlates with expression of LXR alpha- a cofactor study}

\section{Krishna, M. ${ }^{1}$; Jaleel, $A .{ }^{1} ;$; A, A. ${ }^{1}$; Surendran, A. ${ }^{1}$; Chandran, $M .{ }^{1}$}

\section{${ }^{1}$ Rajiv Gandhi Centre for Biotechnology}

Introduction: Adipogenesis is a complex biological process involving synchronised interplay of different nuclear receptors. Aberration in the process leads to obesity and associated disorders. Insulin signaling and glucose is supposed to be the first cue for the initiation of protein-protein interaction leading to adipogenesis [1]. Based on the gene expression pattern and protein level showed during initial hours of adipogenesis, expression of LXR alpha precedes Glut4. This led us to check the role of LXR alpha, a known glucose sensor in cells, in the initiation stages of adipogenesis.

Methods: Gene expression and protein levels of PPAR gamma, LXR alpha, eight co-factors and eight target genes were assessed with real-time PCR and western blots. Change in expression of Glut4 and LXR alpha in presence of FMOC-L-leucine, a PPAR gamma selective agonist, were assessed with immuno-cytochemical studies. Immuno-precipitation of PPAR gamma and interactome analysis using LC-MS were done.

Results: Gene expression and protein levels of PPAR gamma and LXR alpha showed a gradual increase from $6^{\text {th }}$ hour after adipogenic cocktail administration. FMOC-L-leucine enhanced Glut4 expression much earlier in the adipogenic process and was associated with enhanced LXR alpha expression. The translocation of Glut4 from nuclear region to cytoplasm and inner membrane side was associated with high degree of co-localization with LXR alpha protein. IP experiments and mass spectral data revealed the interacting co-factors of PPAR gamma.

Conclusion: Even though the uptake of glucose through insulin action starts within short time period, the Glut4 expression is followed by LXR alpha activation. In pancreatic $\beta$-cells, increase in glucose concentration leads to nuclear translocation of LXR alpha [2]. But here, we report the cytoplasmic translocation of LXR alpha upon glucose uptake. LXR alpha is known be a glucose sensor and induces specific co-factor recruitment upon glucose binding [3]. LC-MS data obtained in the present study also denote altered co-factor recruitment of PPAR gamma in presence of FMOC-L-Leucine.

Reference

1 Rosen ED, MacDougald OA. Adipocyte differentiation from the inside out. Nat Rev Mol Cell Biol 2006; 7:885-96

2 Chapman AH, Helleboid S, Jakel H, et al. Cell Res 2006; 16:661-670.

3 Mitro N, Mak PA, Vargas $L$ et al. The nuclear receptor LXR is a glucose sensor. Nature 2007; 445:219-223.

Conflict of Interest: None Disclosed

Funding: The present research is supported by Department of Science and Technology, Ministry of Science and Technology, Govt. of India

\section{T1P94}

\section{Modulation of gut microbiota during probiotic-mediated lead to reduction of body weight in Iraqi obese female.}

\section{Alajeeli, $F^{1}$}

${ }^{1}$ Tikrit University

Gut flora play important role in regulation of body weight and all study share that is may be used to safety treatment of obesity.

Methods: 25 healthy obese female volunteers was participated in this study and divided in two group, G1 used probiotic with 4 strain one capsule per day and G2 was used placebo capsule both group used same diet program for 8 weeks, stool sample was collected before and after study finished and extracted DNA form stood and used q PCR technology by sued specific primers.

Results: Microbial abundance measured by quantitative polymerase chain reaction (qPCR), the Bacteroids spp. showed significant increased in probiotic group G1 in initial of study it was percentage $36 \%$ and after consumed probiotic it was increased to $42 \%$ compared with placebo groups G2 it showed decreased 33\%. In Ruminocococus spp, the probiotic group decreased to $18 \%$ compared with baseline $23 \%$, but in the placebo group the percentage was still $23 \%$ without any change In the Bafidobacterium spp. Bifidobacterium was consist of $0.05 \%$ and in the probiotic group G1 it was increased to $24 \%$ and in the placebo group G2 its decreased to $0.01 \%$.the Bifidobaiterium spp. Show highly significant $\mathrm{p} \leq 0.0001$. The lactobacillus spp. showed significant deference with $\mathrm{p} \leq 0.026$ it showed increased to $11 \%$ in the probiotic group compared with baseline $(0.005) \%$ and compared with placebo group it was decreasd to $0.004 \%$. Bacteroidetes to Firmicutes $(\mathrm{B} / \mathrm{F})$ ratio was increased in $\mathrm{G} 1$ group (2.33) compared with placebo group (1.4) and in baseline the ratio was (1.56). In the body weight The group $(\mathrm{G} 1)$ who consumed probiotic showed decrease $(5.4 \%)$ $5.4 \mathrm{~kg}$ from (92.92) to (87.88) and the placebo group showed decreased $4.14 \%(3.4) \mathrm{kg}$ from $(91.28)$ to $(87.5)$ but there was no significant difference between two groups.

T1P95

Molecular biomarkers for weight control in obese individuals subjected to a multi-phase dietary intervention

Bolton, J. '; Montastier, E. ; Carayol, J. ${ }^{2}$; Bonnel, S. ; Mir, L. ' ; Marques, M. ' ; lacovoni, J. ${ }^{1}$; Villa-Vialaneix, $N .^{3}$; Valsesia, A. ${ }^{2}$; Langin, $D .{ }^{1}$; Viguerie, $N .^{1}$ ${ }^{1}$ INSERM, ${ }^{2} \mathrm{NIHS},{ }^{3}$ INRA

Introduction: While calorie restriction has proven beneficial for weight loss, long-term weight control is variable between individuals. We aimed to identify biomarkers of weight control during a dietary intervention, which included 8-weeks of calorie-restriction and 6-months of follow-up. Methods: Subcutaneous abdominal adipose tissue transcriptomes were compared between 21 obese individuals that had either good (maintained weight loss) or poor (regained weight) weight control during the dietary intervention. Selected genes were validated on 310 individuals from the 
same study using RT-qPCR, and protein levels of potential circulating biomarkers were measured by ELISA.

Results: We evaluated 4 genes that had altered expression during the dietary intervention, encode secreted proteins, and have not previously been implicated in weight control (EGFL6, FSTL3, CRYAB, IGFBP3); as well as 2 genes for which baseline expression was different between those with good and poor weight control (ASPN, USP53). Changes in plasma concentration of EGFL6, FSTL3, and CRYAB mirrored adipose tissue mRNA expression, all decreased during dietary intervention in individuals with good weight control. ASPN and USP53 had higher baseline expression in individuals that went on to have good weight control, and eQTL analysis found polymorphisms associated with expression levels of USP53 in adipose tissue. A regulatory network was identified in which TGF $\beta 1$ was responsible for down-regulation of a series of genes during dietary intervention in good-controllers. Interestingly, ASPN is a TGF $\beta 1$ inhibitor. Conclusion: This study found circulating biomarkers released by adipose tissue and associated with weight control, which could serve to adjust weight management strategies and genes that may be prognostic for successful weight control.

Conflict of interest: DL is a member of InstitutUniversitaire de France, JC and AV are employed by Nestlé, the other authors have nothing to disclose.

Funding: Research relating to this abstract was funded by the Innovative Medicines Initiative Joint Undertaking (grant agreement $n^{\circ} 115372$ ), Inserm, Paul Sabatier University and the Commission of the European Communities (FP6-513946 DiOGenes).

\section{T1P96 \\ Natural killer cell functionality is impaired by diet-induced obesity in a postmenopausal breast cancer mouse model}

Mattheis, L. '; Jung, J. '; Kielstein, H. '; Spielmann, J.'

${ }^{1}$ Martin Luther University Halle-Wittenberg, Institute of Anatomy and Cell Biology

Introduction: Obesity is a widespread disease and was identified as a major risk factor for malignant diseases, including postmenopausal breast cancer. Although the underlying mechanisms are poorly understood, it is known from in-vitro studies that essential functions of natural killer (NK) cells such as targeting tumor cells are disturbed in obese individuals. Thus, the aim of the present study is the investigation of NK cell functionality of obese mice in a postmenopausal breast cancer model.

Methods: To induce obesity, female mice (BALB/c) received either a standard chow ( $4 \%$ fat) or a high fat diet (34\% fat) for up to 13 weeks. Thereafter, mice were ovarectomized and after 3 weeks of recovery, syngeneic 4T1-Luc2 mouse mammary tumor cells were injected orthotopically into the fat pad of the mammary gland. Twenty hours or 3 weeks after tumor cell challenging blood, tissues and tumors were collected and analyzed. Different techniques such as flow cytometry, luminex and real-time PCR aimed to analyze numbers, activity and physiological properties of NK cells.

Results: Body weight, visceral fat amount, plasma leptin and IL-6 levels were significantly increased in diet-induced obese BALB/c mice. Tumor burden was increased in the obese animals as compared to their lean littermates. Interestingly, the functionality of NK cells was altered in the obese mice with breast cancer.

Conclusion: The present study on a postmenopausal breast cancer model helps to understand basic molecular mechanisms regulating NK cell functionality in obese individuals and the association of the elevated breast cancer risk in obesity.

Conflict of Interest: Not disclosed.

Funding: Research relating to this abstract was funded by Institute Danone and the Wilhelm Roux Program of the Martin Luther University Halle-Wittenberg.

\section{T1P97}

\section{No effect of 12 weeks of weight loss on plasma INSL5 in humans}

\section{Edwards, K. ' ; Prendergast, L. ${ }^{2}$; Kalfas, S. ; Sumithran, P. ' ; Proietto, J.}

${ }^{1}$ University of Melbourne, Department of Medicine (AH), Heidelberg, Victoria, Australia, ${ }^{2}$ La Trobe University, Department Of Mathematics And Statistics,

Bundoora, Victoria, Australia

Introduction: Ghrelin is widely considered to be the only known human gut hormone that stimulates appetite and food intake. However, insulin-like peptide 5 (INSL5) was recently identified as an orexigenic hormone in mice (1). Currently little data exists on the appetite modulating effects of INSL5 in humans.

Methods: Subjects were placed on a VLED program to achieve a $15 \%$ weight loss. Of 97 subjects who began the trial, 50 successfully lost $15 \% \pm 2.5 \%$ of their starting body weight. Plasma INSL5 levels were measured in these subjects during a mixed meal test (at 0, 30, 60 and 240 minutes) at baseline and $15 \% \pm 2.5 \%$ weight loss. INSL5 levels were determined using a USCN Life Science ELISA kit.

Results: Prior to weight loss (baseline), postprandial INSL5 levels (ng/ $\mathrm{mL}$ ) were significantly higher at 30,60 and 240 minutes when compared to fasting levels (30 mins: $4.02 \mathrm{ng} / \mathrm{mL}$ higher, $95 \%$ CI $[0.72,7.31], \mathrm{p}=$ 0.018; 60 mins: 3.84ng/mL higher, [0.98, 6.70], $\mathrm{p}=0.009 ; 240$ mins: 4.46ng/ mL higher, $[1.99,6.92], \mathrm{p}<0.001)$.

After $15 \% \pm 2.5 \%$ weight loss, there was no significant change in fasting INSL5 compared to baseline ( $\mathrm{p}=0.772)$.

Conclusion: It is concluded that INSL5 levels change in the opposite direction to reported changes in ghrelin following a meal, and after weight loss. These results indicate that INSL5 may not act as an orexigenic hormone in humans. However, the study is limited by a small sample size, and further research is necessary.

Conflict of Interest: None disclosed

Funding: KE is supported by the Australian Government Research Training Scheme References

1 Grosse J, Heffron H, Burling K, Akhter Hossain M, Habib AM, Rogers GJ, et al. Insulin-like peptide 5 is an orexigenic gastrointestinal hormone. Proceedings of the National Academy of Sciences. 2014;111(30):11133-8.

\section{T1P98 \\ Normolipidic fish oil diet reduces visceral fat and preserves glucose hypophagia in mice}

\section{Sousa, I. ' ; Andrade, I. '; Freitas, N. '; Nascimento, C. '; Oyama, L. '; Ribeiro, E. ${ }^{1}$}

'Universidade Federal de São Paulo

Introduction: The type of dietary fat may affect the mechanisms controlling energy homeostasis. Glucose acts at the hypoyhalamus to inhibit food intake. We have previously shown that a hyperlipidic fish oil diet impaired glucose-induced hypophagia (de Sousa et al. 2013). The present work evaluated the effect of a normolipidic fish oil diet on the feeding response induced by glucose.

Methods: Two-months-old Swiss mice $(\mathrm{n}=15)$ were fed for 12 weeks with a balanced diet ( $9.57 \%$ energy from fat) containing either soy oil or fish oil as the fat source. Body weight and food intake were measured once weekly throughout the treatment. Mice were then i.p. injected either vehicle or glucose in the doses of 400,800 or $2000 \mathrm{mg} / \mathrm{kg}$ b.w. and the amount of food ingested was measured after $1 \mathrm{~h}, 6 \mathrm{~h}, 12 \mathrm{~h}$ and $24 \mathrm{~h}$. After euthanasia, the retroperitoneal, mesenteric and epididymal fat were weighed and serum glucose and lipids were measured. Data were analyzed by Student's $t$ test or two-way Anova followed by Bonferroni post-test, with significance set at $\mathrm{p}<0.05$.

Results: Body weight gain, food intake, glycaemia, and blood triglycerides levels were similar between the soy and the fish group. The fish group showed a significant $24 \%$ reduction of the sum of fat pads weight $(p=0.004)$ and a $43 \%$ reduction of total cholesterol levels $(p=0.014)$. The glucose dose of $400 \mathrm{mg} / \mathrm{kg}$ b.w failed to significantly inhibit feeding. The 
$800 \mathrm{mg}$ glucose dose inhibited feeding in both groups after 1 hour (soy: $67 \%, \mathrm{p}<0.05$; fish: $74 \%, \mathrm{p}<0.01)$ and did so only in the fish group after 6 hours $(41 \%, \mathrm{p}<0.01)$. The $2000 \mathrm{mg}$ glucose dose inhibited feeding similarly in both groups after 1 and 6 hours. Both the 800 and the $2000 \mathrm{mg}$ glucose doses failed to significantly inhibit feeding after 12 and 24 hours. Conclusion: In relation to normolipidic soy oil intake, normolipidic fish oil intake improves body composition and serum lipid profile and preserves the hypophagic response to glucose.

Conflict of Interest: None Disclosed.

Funding: Research relating to this abstract was funded by National Counsel of Technological and Scientific Development (CNPq).

Reference:

1 de Sousa IF, de Souza AP, Andrade IS, Boldarine VT, Nascimento CM, Oyama LM, Telles MM, Ribeiro EB. Effect of fish oil intake on glucose levels in rat prefrontal cortex, as measured by microdialysis. Lipids Health Dis 2013; 12:188.

\section{T1P99}

\section{Nutritional status of vitamin D and its relationship with insulin resistance in individuals with obesity classified as metabolically healthy and unhealthy phenotype}

\section{Cordeiro, A. ${ }^{1}$; Campos, B. ' ; Pereira, S. ${ }^{1}$; Saboya, C. ${ }^{1}$; Ramalho, A. ${ }^{1}$}

${ }^{1}$ Research Center of Micronutrients - Federal University of Rio de Janeiro

Introduction: High prevalence of vitamin D deficiency (VDD) has been observed associated with obesity worldwide. Insulin resistance (IR) is a frequent metabolic condition present in some of individuals with obesity, which besides of the disease per se, present other metabolic changes and/ or risks. The aim was relate the nutritional status of vitamin D with IR in individuals with healthy (MHO) and unhealthy metabolically (MUHO) phenotype classified by HOMA-IR.

Methods: A descriptive cross-sectional study was conducted with individuals of both sexes, aged 21-63 years. Anthropometric data [weight, waist circumference (WC), BMI, besides body roundness index] and metabolic parameters [blood glucose, insulin, hemoglobin A1c, HOMA-IR, HOMA- $\beta$ and $25(\mathrm{OH}) \mathrm{D}$ were obtained. The cut-off points for deficiency and insufficiency were $\leq 20$ and $21-29 \mathrm{ng} / \mathrm{mL}^{1}$, respectively. Individuals were classified as MUHO according to HOMA-IR $\geq 2.5^{2}$.

Results:232 individuals were evaluated, 76,7\% were female. For all, 23,3\% were classified as MHO (G1) and $76,7 \%$ as MUHO (G2). Mean of age, weight, BMI, WC and body roundness index were $42.5 \pm 9.9 / 41.8 \pm 10$. $9[p=.662] ; 116.5 \pm 18.1 / 118.0 \pm 19.1[p=.593] ; 41.8 \pm 3.8 / 42.8 \pm 5.0[p=$ $.204] ; 117.0 \pm 13.1 / 120.6 \pm 13.8[p=.041] ; 7.9 \pm 1.8 / 8.5 \pm 2.0[p=.021]$ in G1 and G2, respectively. Nutritional status of vitamin D showed $44.4 \%$ insufficiency (mean of $25.7 \pm 2.6$ ) versus $43.8 \%$ (mean of $24.5 \pm 2.9$ ) [ $p=.077]$, and $35.2 \%$ deficiency (mean of $17.1 \pm 2.7$ ) versus $41.6 \%$ (mean of $14.5 \pm 3.6$ ) $[p=.004]$ in G1 and G2, respectively. The highest value of HOMA-IR was found in individuals with VDD in G2 $(5.9 \pm 4.7) \mathrm{com}-$ pared with same nutritional status profile in $\mathrm{G} 1(3.7 \pm 2.5)[p=0.000]$ Serum insulin levels and WC were negatively correlated with $25(\mathrm{OH}) \mathrm{D}$ values, $\mathrm{r}=-0.121 / p=0.065$ and $\mathrm{r}=-0.131 / p=0.046$, respectively. The odds ratio of $25(\mathrm{OH}) \mathrm{D}$ levels relates with risk of MUHO was observed [OR $=1.051 ; p=0.012 ; 95 \% \mathrm{CI}=1.011-1.093$ ].

Conclusion: The nutritional status of vitamin D was significant difference between MHO and MUHO classified by HOMA-IR, presenting high prevalence of VDD and high incidence of IR in MUHO.

\section{Reference:}

1 Holick MF, J Clin Endocrinol Metab 2011.

2 Durward CM, J Obes 2012.

Conflict of Interest: None Disclosed

Funding: No Funding

\section{T1P102}

\section{Obesity-induced decreases in muscle performance are not} reversible by short-term weight loss

Tallis, J. '; Seebacher, F. ${ }^{\text {; }}$ Mcshea, K. $^{2}$; James, . $^{\text {' }}$

${ }^{1}$ Centre for Applied Biological and Exercise Sciences, Faculty of Health and Life Sciences, Coventry University, Coventry, CV1 5FB, U.K, ${ }^{2}$ School Of Life And Environmental Sciences A08 University Of Sydney, Science Road, Sydney, Nsw, 2006, Australia

Introduction: Recent evidence has demonstrated a link between muscular lipid accumulation and a decline in the contractility of isolated skeletal muscle performance, which may therefore lead to altered locomotor performance. Weight loss is a common and intuitive intervention for obesity, and some but not all obesity-induced physiological changes are reversible by weight loss. Here we tested whether obesity-induced changes in metabolic and muscle phenotypes are reversible by weight loss.

Methods: Zebrafish (Danio rerio) were split into three groups: 1) control fish fed once a day for eight-nine weeks; 2) obese fish fed three times per day for eight-nine weeks; 3) obese-lean fish fed three times per day for four weeks, then once per day for four-five weeks. Metabolic scope, swimming performance, myosin heavy chain concentration, citrate synthase activity and muscle contractility of a section of rostral (anterior dorsal) muscle fibres were compared between experimental groups.

Results: We show that obesity-induced decreases in metabolic scope, which resulted from concomitant increases in resting metabolic rate and decreases in maximal metabolic rate, are reversible with weight loss in a zebrafish (Danio rerio) model. Changes in metabolic rate were not associated with altered mitochondrial abundance or capacities (citrate synthase activity). Obesity caused a decrease in locomotor capacities, which were accompanied by decreases in isolated muscle work-loop power output and isometric force, when these variables were normalized to muscle size, but not by changes in fatigue resistance. Myosin heavy chain concentrations decreased, and there was a shift towards fast myosin heavy chains in obese individuals.

Conclusion: Surprisingly, and contrary to our hypotheses, these obesity-induced changes in locomotor and muscle phenotypes were not reversible by short-term weight loss. These results show that obesity has persistent effects that are not solely associated with changes in body mass, and that weight loss alone may not be a sufficient intervention.

\section{T1P104 \\ Pharmacological targeting of glucose-6-phosphate dehydrogenase in human erythrocytes by Bay 11-7082, parthenolide and dimethyl fumarate}

Ghashghaeinia, M. '; Giustarini, D. ${ }^{2}$; Koralkova, P. ${ }^{3}$; Köberle, M. ${ }^{4}$; Alzoubi, K. ${ }^{7}$; Bissinger, R. ${ }^{1}$; Hosseinzadeh, Z. ${ }^{5}$; Dreischer, P. ${ }^{6}$; Bernhardt, I. ; Lang, F. ${ }^{1}$; Toulany, M. ${ }^{8}$; Wieder, T. ${ }^{9}$; Mojzikova, . $^{3}$; Rossi, R. $^{2}$

'Department of Cardiology, Vascular Medicine and Physiology, University of Tübingen, Germany, ${ }^{2}$ Department Of Life Sciences, University Of Siena, Siena, Italy, ${ }^{3}$ Department Of Biology, Palacky University, Olomouc, Czech Republic, ${ }^{4}$ Department Of Dermatology And Allergy, Technische Universität München, Germany, ${ }^{5}$ Centre For Ophthalmology, University Of Tübingen, Germany, ${ }^{6}$ University Of Tübingen, Department Of Physiology l, Germany, ${ }^{7}$ Saarland University, Campus A2.4, Saarbrücken, Germany, ${ }^{8}$ Division Of Radiobiology And Molecular Environmental Research, Department Of Radiation Oncology, University Tübingen, Germany, ${ }^{9}$ Department Of Dermatology, University Tübingen, Germany

Introduction and Methods. In mature erythrocytes, glucose-6-phosphate dehydrogenase $(\mathrm{G} 6 \mathrm{PDH})$ and 6-phosphogluconate dehydrogenase (6PGDH) yield NADPH, a crucial cofactor of the enzyme glutathione reductase (GR) converting glutathione disulfide (GSSG) into its reduced state (GSH). GSH is essential for detoxification processes and survival of erythrocytes. We explored whether the anti-inflammatory compounds Bay 11-7082, parthenolide and dimethyl fumarate (DMF) were able to deplete a common target (GSH), and to impair the function of upstream enzymes of GSH recycling and replenishment. Reduced and oxidised glu- 
tathione was measured by HPLC and erythrocyte enzyme activities by spectrophotometric assays.

Results: Long-term treatment $(24 \mathrm{~h})$ of erythrocytes $(0.6 \%$ hematocrit) with Bay 11-7082, parthenolide or DMF lead to concentration-dependent programmed erythrocyte death (eryptosis), decrease of MCV values along with a parallel increase of MCHC values, negligible hemolysis and complete depletion of both GSH and GSSG. At pharmacologically relevant concentrations, Bay 11-7082 had the highest depletory effect on the intracellular GSH and GSSG concentrations. The complete depletion was achieved at $20 \mu \mathrm{M}$ Bay 11-7082; a 2.5-fold resp. 7-times lower concentration as compared with parthenolide or DMF.

GSH depletion was due to strong inhibition of G6PDH activity. Both, 20 $\mu \mathrm{M}$ Bay 11-7082 or $50 \mu \mathrm{M}$ parthenolide led to complete G6PDH inhibition whereas $140 \mu \mathrm{M}$ DMF caused only 50\% enzyme inhibition. Bay 11-7082 and DMF, but not parthenolide, were also able to inhibit GR activity. GR activity was entirely inhibited by $20 \mu \mathrm{M}$ Bay 11-7082 and only partially by $140 \mu \mathrm{M}$ DMF.

Conclusions: the specific G6PDH inhibitory effect of these anti-inflammatory compounds offer up new opportunities in the treatment of human diseases prone to high NADPH and GSH consumption rates, among them malaria, trypanosomiasis, cardiovascular diseases, psoriasis, multiple sclerosis, cancer or obesity.

\section{T1P105 \\ Association of human adipose tissue autophagy-related genes with gluco-metabolic status}

Xu, Q. '; Mariman, E. '; Roumans, N. ${ }^{1}$; Vink, R. ${ }^{1}$; Goossens, G. '; Blaak, E. '; Jocken, J.'

Maastricht University

Background: Adipose tissue (AT) autophagy is closely linked to human obesity and increased metabolic risk. In addition, recent findings establish a role for autophagyin the regulation of lipid metabolism. Here, we examined the expression of autophagy- and lipolysis-related genes in human abdominal subcutaneous AT of overweight/obese in comparison to lean healthy individuals.

Subjects/Methods: Human subcutaneous adipose tissue biopsies derived from 26 well-phenotyped lean $(n=9)$ and overweight/obese $(n=17)$ participants were used to perform RT-PCR of the major autophagy-related genes (ATGs) and lipases (ATGL and HSL). Associations between ATG expression and clinical characteristics were studied. Differentiated human adipocytes were used for mechanistic deepening.

Results: The expression of ATG5, 12 and 7 were higher in subcutaneous AT of overweight/obese compared to lean men $(p=0.043, p=0.004$ $\mathrm{p}=0.015$, respectively), while expression of the classical lipolysis markers HSL and ATGL tended to be lower in overweight/obese AT ( $p=0.092$, $p=0.084)$. Positive correlations were found between ATG12 and BMI $(\mathrm{r}=0.407, \mathrm{p}=0.039)$. ATG7 correlated positively with BMI $(\mathrm{r}=0.380$ $p=0.055)$, WHR $(r=0.420, p=0.041)$, 2h glucose $(r=0.488, p=0.011)$ insulin $(\mathrm{r}=0.419, \mathrm{p}=0.033)$ and HOMA-IR $(\mathrm{r}=0.407, \mathrm{p}=0.039)$. Multivariate linear regression revealed that ATG7 gene expression correlated positively with $2 \mathrm{~h}$ glucose independent of BMI and HOMA-IR. Gene expression interaction analysis using correlation clustering showed that ATG7 negatively correlated with HSL $(\mathrm{p}<0.01)$ and ATGL $(\mathrm{p}<0.01)$ In line, treatment of human adipocytes with a HSL inhibitor resulted in increased starvation-induced LC3 accumulation, a marker of increased autophagic flux.

Conclusion: A decreased subcutaneous AT expression of lipases is accompanied by an increased expression of ATGs in overweight/obese subjects. An increased ATG7 expression seems to be mainly dependent on glucose tolerance per se rather than adiposity or insulin resistance.

\section{T1P106}

\section{Physical exercise and body mass index in adolescents}

Oliveira, A. ${ }^{1}$; Vázquez, J. ${ }^{2}$; Albuquerque, C. ${ }^{1}$; Oliveira, M. ${ }^{3}$; Cunha, M. ${ }^{1}$; Andrade, A. ${ }^{1}$; Bica, ${ }^{1}{ }^{1}$

${ }^{1} \mathrm{Cl} \& D E T S$-Instituto Politécnico de Viseu - Portugal, ${ }^{2}$ Upsa - Salamanca - Spain, ${ }^{3}$ Aces Dão-Lafões - Portugal

Background: The World Health Organization (WHO) put obesity among the ten major risk factors to health these days, considering the prevention of the obesity epidemic as a priority public health challenge in the twenty-first century.

Objectives: Analyze the existence of causality of the physical activity variables (frequency of adequate physical exercise achievement for wheezing and sweating out of school hours; hours of enough exercise to stay out of breath and sweating out of school hours; desire to increase the sport), as determining factors in the body mass index of adolescents.

Methods: This study was observational and transversal, quantitative, non-experimental, descriptive and correlational, was conducted with 1467 adolescents with a mean age of $14.01( \pm 1,43 \mathrm{Dp})$, mostly sex female $(52.3 \%)$, living in rural areas $(63.32 \%)$ in various districts of mainland Portugal, attending between the 5 th and 12 th grade public education, it is carried out anthropometric assessment and stratification index obesity based on the framework of the National Center for Health and Statitics (NCHS) of CDC (2000).

Results: It was possible to see that $7.2 \%$ of adolescents are obese, $14.5 \%$ pre-obesity, $74.3 \%$ normal weight and $4 \%$ underweight. In boys: $10.0 \%$ are obese and $15.1 \%$ show pre-obesity, while girls have $4.6 \%-13.8 \%$ and obesity - pre-obesity. The inferential analysis confirms significant effect of some of the determinants studied in body mass index (BMI) showing the teenagers, who are described below.

The daily practice of daily physical exercise is associated with body mass index ( $\left.\chi^{2}=26.077, \mathrm{p}=048\right)$, and is in the obese group where daily practice is more evident (res $=2.0$.); those who do not have normal weight.

The total absence of physical exercise is more evident in the group of adolescents who have a low body mass index ( $\chi 2=18.487, \mathrm{p}=, 038)$.

The desire to change habits associated with physical exercise has an effect on body mass index ( $\chi 2=10.120, p=0.018)$; It is the group of obese adolescents expressing, with statistical significance, the desire to increase physical exercise (res = 2.5.); since those with normal weight do not intend to increase this practice (res. $=2.3$ ).

Conclusion: The factors studied in this research can assume importance in the identification of groups at risk, with implications for planning health promotion activities in the group of adolescents.

Keywords: BMI, OBESITY, ADOLESCENTS, PHYSICAL EXERCISE.

\section{T1P108 \\ Plasma lipid profiling of tissue-specific insulin resistance in human obesity}

Kolk, B. ${ }^{1}$; Vogelzangs, N. ${ }^{2}$; Jocken, J. '; Valsesia, A. ${ }^{3}$; Hankemeier, T. ${ }^{4}$; Astrup, A. ${ }^{5}$; Saris, W. ${ }^{1}$; Arts, I. ${ }^{2}$; Blaak, E. ${ }^{1}$; Consortium, D. ${ }^{6}$

${ }^{1}$ Department of Human Biology and Movement Sciences, NUTRIM School of Nutrition and Translational Research in Metabolism, Maastricht University, Maastricht, The Netherlands, ${ }^{2}$ Department Of Epidemiology, Cardiovascular Research Institute Maastricht (Carim) \& Maastricht Centre For Systems Biology (Macsbio), Maastricht University, Maastricht, The Netherlands, ${ }^{3}$ Nestlé Institute Of Health Sciences, Lausanne, Switzerland, ${ }^{4}$ Netherlands Metabolomics Centre, Leiden, The Netherlands, ${ }^{5}$ Department Of Nutrition, Exercise And Sports, Faculty Of Science, University Of Copenhagen, Copenhagen, Denmark, ${ }^{6}$ Diogenes Consortium

Introduction:Insulin resistance (IR) in obesity may develop independently in different organs (i.e. liver and skeletal muscle), representing different etiologies towards type 2 diabetes mellitus. The aim of this study is to identify plasma lipid profiles of persons with IR in either skeletal muscle or in the liver. 
Methods: Overweight/obese participants $(n=640)$ of the European DiOGenes project, a randomized, controlled weight maintenance trial (after weight loss), were classified at baseline into 4 groups: (1) no IR ( $\mathrm{n}=325)$, (2) IR in muscle only ( $\mathrm{n}=108)$, (3) IR in liver only $(\mathrm{n}=99)$, (4) IR in both muscle and liver $(\mathrm{n}=108)$. The IR phenotype was derived from a 5-point oral glucose tolerance test and based on the lowest tertile of the muscle insulin sensitivity index (glucose (slope_peak-nadir) / insulin (meanOGTT)) and the top tertile of the hepatic IR index (glucose (AUC_0-30)* insulin (AUC_0-30)). 140 plasma lipids were identified and quantified by liquid chromatography-mass spectrometry and compared between these groups using multinomial logistic regression analyses and Benjamini \& Hochberg FDR-adjusted p-values. In additional analyses, sex and waistto-hip ratio adjustments were taken into account.

Results: Mean age was $41(\mathrm{SD}=6)$ years, $66 \%$ were women, $81.5 \%$ was obese and mean BMI was $34.5(\mathrm{SD}=5)$. Participants with liver-IR were more often men $(\mathrm{p}<0.001)$ and had a higher waist-to-hip ratio $(\mathrm{p}<0.001)$ when compared to participants with muscle-IR. Participants with liver-IR showed higher concentrations of plasma triacylglycerol (TAG) (FDR: $\mathrm{p}=0.02$ ) and alkylphosphatidylethanolamine ( $\mathrm{PE}(\mathrm{O}-)$ species (FDR: $\mathrm{p}=0.002$ ), but lower relative concentrations of odd-chain TAG species (FDR: $\mathrm{p}=0.04$ ), when expressed as percentage of total TAG. Additional analyses showed that differences in (odd-chain) TAG disappeared after sex and waist-to-hip ratio adjustment. Muscle-IR was associated with lower concentrations of lysophosphatidylcholine (LPC) (FDR: p = 0.02). Conclusion: Liver-IR and skeletal muscle-IR are characterized by distinct plasma lipid profiles. Liver-IR may be associated with elevated TAG and $\mathrm{PE}(\mathrm{O}-)$ concentrations, whilst muscle-IR is associated with reduced LPC concentrations. The mechanisms relating these lipids to tissue-specific IR require further study.

\section{T1P109}

\section{Polydextrose changes the gut microbiome and attenuates fasting triglyceride and cholesterol levels in mice fed with Western diet}

\section{Putaala, H. ${ }^{1}$; Raza, G. ${ }^{2}$; Hibberd, A. ${ }^{3}$; Alhoniemi, E. ${ }^{4}$; Tiihonen, K. ${ }^{\text {; }}$; Mäkelä, K. ${ }^{2}$; Herzig, K. $^{2}$}

${ }^{1}$ DuPont Nutrition and Health, Global Health and Nutrition Science, Kantvik, Finland, ${ }^{2}$ Institute Of Biomedicine, Department Of Physiology And Biocenter Of Oulu, University Of Oulu, Finland, ${ }^{3}$ Dupont Nutrition And Health, Genomics \& Microbiome Science, St. Louis, Mo, Usa, ${ }^{4}$ Avoltus Oy, Turku, Finland

Introduction: Polydextrose is a non-viscous soluble fiber that is sustainably and partially fermented throughout the colon with concomitant short chain fatty acid production (1). Polydextrose has been reported to confer lipid-modulating effects in humans as well as in animal studies. It has been shown to reduce both total and LDL cholesterol in hypercholesterolemic subjects (2), and to reduce LDL cholesterol and increase HDL cholesterol in subjects with impaired glucose metabolism (3). It also reduced the plasma total and LDL cholesterol and liver cholesterol in gerbils fed a cholesterol-rich diet (2). As gut microbiota have been recognized as a key environmental factor influencing whole-body metabolism by affecting energy balance (4), we investigated the effect of polydextrose during Western diet feeding in mice on gut microbiota.

Methods: C57BL/6 male mice $(n=20)$ were fed Western diet ( $5 \%$ fiber, $41 \% \mathrm{kcal}$ fat) with $150 \mathrm{mg} /$ day polydextrose (Litesse Ultra, DuPont Nutrition and Health) in water (PDX) or with water alone (control) by oral gavage for 14 days. Body weight and food intake were monitored daily. After the feeding period, 12-hour fasting blood samples were collected for total cholesterol and triglyceride measurement, in addition to which caecal digesta were collected for barcoded $16 \mathrm{~S}$ rRNA amplicon sequencing. Results: Over feeding period, significant reduction $(\mathrm{p}<0.001)$ in the cumulative food intake in the PDX mice was observed but no significant change in the body weight $(\mathrm{p}=0.07)$ was seen. When blood parameters were measured, the fasting plasma triglyceride $(\mathrm{p}<0.001)$ and total cholesterol $(p<0.05)$ were significantly reduced in PDX mice. When the microbiota between the treatments were compared at phylum level, Bac- teroidetes, Proteobacteria and Deferribacteres were enriched in control treatment while Actinobacteria were enriched in PDX treatment.

Conclusion: PDX supplementation to mice fed with Western diet significantly reduced cumulative food intake and induced a positive hypolipidemic effect, supporting the previous preclinical and clinical findings. Furthermore, microbiome analysis revealed that PDX has a clear modulating effect on composition of gut microbiota during Western diet feeding and might in part explain the systemic changes in blood lipids.

\section{References:}

1 Fava et al. (2007) Br J Nutr 98, 123-133.

2 Pronczuk and Hayes(2006) Nutr Res 26, 27-31.

3 Schwab et al. (2006) Eur J Clin Nutr 60, 1073-1080.

4 Cani and Delzenne (2009) Curr Pharm Des 15, 1546-1558.

Conflict of Interest: DuPont Nutrition \& Health manufactures and sells polydextrose as a trade name Litesse.

Funding: Research relating to this abstract was funded partially by a grant from DuPont Nutrition \& Health Kantvik Finland and partially by University of Oulu.

\section{T1P110 \\ Predictive value of serum concentrations of a panel of eleven different adipokines for components of the metabolic syndrome}

\section{Ebert, T.'; Gebhardt, C. '; Scholz, M. ${ }^{2}$; Wohland, T. ${ }^{3}$; Schleinitz, D. ';} Fasshauer, M. ${ }^{1}$; Blüher, M. ${ }^{1}$; Stumvoll, M. ${ }^{1}$; Kovacs, $P^{3}{ }^{3}$; Tönjes, A. ${ }^{1}$

${ }^{1}$ University of Leipzig, Department of Endocrinology and Nephrology, Leipzig, Germany, ${ }^{2}$ Institute Of Medical Informatics, Statistics And Epidemiology, University Of Leipzig, Leipzig, Germany, ${ }^{3}$ Leipzig University Medical Center, Ifb Adipositydiseases, Leipzig, Germany

Background/Objectives: Adipocytokines potentially link obesity with other metabolic disease states. However, human studies on adipocytokine regulation are often limited to smaller trials in specific disease states and only consider a few adipose tissue-secreted factors. Thus, it still remains unclear which adipocytokines are the best predictors for a certain facet of the metabolic syndrome, i.e. obesity, hypertension, dyslipidemia, and insulin resistance.

Subjects/Methods: Eleven adipocyte-derived factors (adiponectin, adipocyte fatty acid binding protein [AFABP], chemerin, progranulin, fibroblast growth factor [FGF] 19, FGF21, FGF23, irisin, vaspin, angiopoietin-related growth factor, interleukin-10, and insulin-like growth factor-1) were measured in a large and extensively phenotyped, cross-sectional cohort of $>1000$ subjects. A standardized, step-wise statistical approach was performed using hypothesis-free cluster analysis and multivariate regression analyses to assess correlations among the adipokines and between adipokines and markers of the metabolic syndrome, such as insulin resistance, serum lipid profile and fat distribution.

Results: A close association between adipocytokines and metabolic markers was proposed if both, hypothesis-free cluster analysis and a highly standardized multivariate model approach, give concordant Results Based on this approach, adiponectin is strongly associated with high density lipoprotein cholesterol. Furthermore, the adipocytokine AFABP predicts fat mass, whereas FGF21 can serve as an independent predictor of circulating triglycerides in our cohort. Various other adipocytokines are associated with several clinical parameters but are not independently clustered to anthropometric and/or metabolic markers.

Conclusions: Adiponectin, AFABP, and FGF21 could serve as markers for distinct metabolic disease states in a general population. Other adipocytokines are not associated with metabolic disease states in both hypothesis-free and multivariate model approach and might, therefore, only have limited potential for metabolic risk prediction in general cohorts. Further studies need to confirm these results in different cohorts that include more patients with metabolic disease states. 
T1P111

\section{Prefronto-cerebellar transcranial direct current stimulation (tDCS) modulates the desire to eat in overweight subjects: A pilot study}

\section{Redolar-Ripoll, D. '; Viejo-Sobera, R. '; Marrón, E. '; Cuatrecasas, G. $;$} Lorda, P. ${ }^{3}$; Magerowski, G. ; Alonso-Alonso, M. ${ }^{4}$

${ }^{1}$ Cognitive NeuroLab. Faculty of Health sciences. Universitat Oberta de Catalunya. Barcelona, Spain., ${ }^{2}$ Dpt. Endocrinology. Clinica Sagrada Família. Barcelona. Spain., ${ }^{3}$ Faculty Of Health Sciences. Universitat Oberta De Catalunya. Barcelona, Spain, ${ }^{4}$ Laboratory Of Bariatric And Nutritional Neuroscience, Beth Israel Deaconess Medical Center, Harvard Medical School. Boston, Usa.

Introduction: Overweight and obesity are associated with impairments in neurocognitive function that may contribute to the development and maintenance of maladaptive eating behaviors. Recent neuroimaging studies have identified dysregulation in brain pathways that connect the cerebellum with the cerebral cortex in obesity. In this investigation, we examined, for the first time, the effects of noninvasive neuromodulation of prefronto-cerebellar pathways in human subjects with excess body weight using transcranial direct current stimulation (tDCS).

Methods: This was a randomized, sham-controlled, crossover pilot study in 8 subjects with overweight or class I obesity ( $5 \mathrm{f}, 3 \mathrm{~m}$; mean age: $42.6 \mathrm{y}$; mean BMI $28.9 \mathrm{~kg} / \mathrm{m}^{2}$ ). Participants received two tDCS sessions, real and sham, on two consecutive days (same time of the day, 2-4-hour fasting). Stimulation was delivered for 20 minutes at $2 \mathrm{~mA}$ using a tDCS device with the anode placed over F3 (left dorsolateral prefrontal cortex), and cathode over the right cerebellum. This tDCS montage was selected to simultaneously enhance the excitability of the prefrontal cortex and decrease the excitability of the cerebellum. Before and after tDCS, patients filled in visual analogue scales (VAS) for appetite and mood. We also evaluated performance in a food-modified N-back working memory task with 3 levels of cognitive load (1-back, 2-back, and 3-back).

Results: Active tDCS caused an increase in the desire to eat following food cue exposure $(p=0.005)$. This was also observed when subjects were asked about their desire to eat in general, i.e. independent of food cues, but it was present only at trend level $(\mathrm{p}=0.074)$. The increase in food-cuetriggered desire to eat after $\mathrm{tDCS}$ was positively correlated with individual scores in a measure of emotional eating $(p=0.051)$. In line with these data, subjects tended to worsen their performance in the food-modified $\mathrm{N}$-back task after active tDCS, specifically, making more errors during the 2-back condition compared with 1-back $(\mathrm{p}=0.079)$. There were no changes in basic motor performance or any additional effects on appetite, mood or working memory (digit span).

Conclusion: This study represents the first demonstration that modulation of prefronto-cerebellar pathways can affect brain mechanisms related to appetite and food craving. The observed effects go against our study hypothesis, suggesting that the specific tDCS montage that we used may have enhanced motivation to eat, and possibly reduced cognitive control over food. Future studies should explore whether reversing the polarity or changing the targets of this tDCS montage could lead to different effects with potential application in patients with overeating and obesity.

\section{T1P112}

\section{Prospective memory in severely obese patients}

Costa, P.'; Ribeiro, O. ${ }^{2}$; Horácio, J.'; Silva, D. ${ }^{1}$

${ }^{1}$ Human and Social Sciences Faculty of Algarve University, ${ }^{2}$ Neuropsychology Unit Of Egas Moniz Hospital

Introduction: Obesity is a clinical condition of multifactorial etiology, resulting from a combination of factors that influence individual behavior. Severe obesity is considered a risk factor that affects people's lives and is associated with cognitive difficulties, particularly in memory and executive functioning.Since prospective memory (PM) depends heavily on executive functioning, it is expected that PM will be compromised. Thus, this work aims to study the PM in severe obese candidates for bariatric surgery and in patients with a normal Body Mass Index (BMI) through neuropsychological assessment (NA), to study the influence of adiposity in this cognitive domain.

Methods: Participants were selected from a convenience sample, at the Neuropsychology Unit of the Egas Moniz Hospital. The control group was selected in the community. The sample consisted of 48 female participants: 24 morbidly obese candidates for bariatric surgery and 24 participants with a normal BMI. NA used the following tools: the Zoo Map, the Stroop Interference Test, Digit Span, the Complex Rey Figure (CRF), the Trail Making Test (TMT), the Rivermead Behavioral Memory Test-III (RBMT-III), and the Hospital Anxiety and Depression Scale (HADS). A sociodemographic and clinical data were collected, before the NA in a single session. After the NA, the results were statistically analyzed through the SPSS program.

Results: The results showed that PM is affected by severe obesity, as assessed by the RBMT-III; Patients with severe obesity had lower results on tests of spatial and visual functions, including planning, attention, and processing speed; Depressive symptoms in obese people is higher than the normal population. There was a relationship between PM and executive functioning; No evidence was found indicating that there is a relationship between depression or anxiety and prospective memory.

Conclusion: PM and executive functions were decreased in patients with severe obesity. Deficits in PM can lead to relapse in obese patients since they must remember that they should not eat certain foods or that they should not consume them, following the dietary plan. More important is the adherence to therapy and the learning and remembering of techniques necessary for its effectiveness. This study evidenced that prospective memory is compromised in patients with severe obesity, with no similar data on this subject in the literature.

None Disclosed/Payment received from Egas Moniz Hospital.

No Funding/Research relating to this abstract was funded by Egas Moniz Hospital.

\section{T1P113 \\ Prospective studies exploring the possible impact of a functional ID3 polymorphism on changes in obesity measures}

Svendstrup, M. ${ }^{1}$; Appel, E. ; Sandholt, C. ; Ahluwalia, T. '; Ängquist, L. ${ }^{2}$; Thuesen, B. ${ }^{3}$; JøRgensen, M. ${ }^{4}$; Pedersen, O. '; Grarup, N. ${ }^{1}$; Hansen, T. ${ }^{1}$; SøRensen, T. $^{2}$; Vestergaard, $H{ }^{1}$

${ }^{1}$ Novo Nordisk Foundation Center for Basic Metabolic Research, Section of Metabolic Genetics, University of Copenhagen, ${ }^{2}$ Department Of Clinical Epidemiology, (Former Institute Of Preventive Medicine), Bispebjerg And Frederiksberg Hospital, ${ }^{3}$ Research Centre For Prevention And Health, Glostrup Hospital, ${ }^{4}$ Steno Diabetes Center

Introduction: Genetic factors determining changes in adiposity over time have been sparsely examined. The Inhibitor of differentiation-3 (ID3) protein is regulating adipocyte differentiation and angiogenesis, and Id3 knock-out mice showed attenuated increase in body mass index (BMI) and visceral adipose tissue mass over time. We hypothesized that a functional missense variant (rs11574) of ID3 reducing the function of the ID3 protein would lead to an attenuated increase over time in fat mass, BMI, waist circumference and waist-hip-ratio in humans.

Methods: The genotyped Danish study populations included ORGGEN, a cohort of obese men $(\mathrm{N}=716)$ and a randomly selected group (the random cohort) $(\mathrm{N}=826)$ from the Danish draft boards who were examined at mean age 20 and 46 years, and Inter99 $(\mathrm{N}=6116)$ and Health2006 $(\mathrm{N}=2761)$, two population-based samples of non-diabetic middle-aged people, who were both followed up after five years.

Results: In meta-analyses of all data, we found no significant association between rs11574 A-allele and BMI change over an average follow-up of 26 years $\left(\mathrm{N}=7094, \beta-0.13 \mathrm{~kg} / \mathrm{m}^{2},[-0.28 ; 0.02], \mathrm{p}=0.083\right)$ or $3-5$ years $\left(\mathrm{N}=5664, \beta:-0.06 \mathrm{~kg} / \mathrm{m}^{2},[-0.130 ; 0.003] \mathrm{p}=0.060\right)$. We did not observe associations between rs 11574 minor A-allele and changes in waist circumference, waist-hip-ratio or fat mass. We found a statistically significant association between rs11574 A-allele and cross-sectional BMI ( $\mathrm{N}=10359, \beta$ 
$\left.-0.16 \mathrm{~kg} / \mathrm{m}^{2},[-0.30 ;-0.01], \mathrm{p}=0.033\right)$ and fat mass $(\mathrm{N}=4188, \beta-0.52 \mathrm{~kg} /$ $\left.\mathrm{m}^{2},[-1.03 ;-0.01], \mathrm{p}=0.046\right)$ in a meta-analysis of all three cohorts. Conclusion: Despite experimental evidence of dysfunction of the rs 11574 ID3 polymorphism, we found no consistent impact of the genetic variant on changes in fat mass, BMI or fat distribution over time in three Danish cohorts. We did find an association with cross-sectional BMI, but this finding could not be confirmed by large consortium data look-up.

Conflict of Interest: None Disclosed

Research relating to this abstract was funded by Danish Diabetes Academy who funded $1 / 3$ of the PhD salary of the first author

\section{T1P114}

\section{Rab34, a novel regulator of lipid droplet metabolism in adipocytes and hepatocytes}

Travez-Garcia A. '; López-Alcalá J. '; Guzmán-Ruiz R. ; Rodríguez A. ${ }^{2}$; Rabanal Y.; Fernández-Vega A. ; López-Miranda J. ; Nogueiras R. ; Frühbeck G. ${ }^{2}$; Vázquez-Martínez R. ; Malagon M.M. ${ }^{5}$

${ }^{1}$ IMIBIC/University of Córdoba/Reina Sofia University Hospital, Córdoba; CIBER Fisiopatología de la Obesidad y Nutrición (CIBERobn), ISCIII, Spain, ${ }^{2}$ Metabolic Research Laboratory, Department Of Endocrinology \& Nutrition, Clínica Universidad De Navarra, Idisna, Pamplona; Ciberobn, Spain, ${ }^{3}$ Lipids And Atherosclerosis Unit, Imibic/Reina Sofia University Hospital/University Of Córdoba; Ciberobn, Spain, ${ }^{4}$ University Of Santiago De Compostela-Instituto De Investigación Sanitaria, Santiago De Compostela; Ciberobn, Spain, 5 mibic/ University Of Córdoba/Reina Sofia University Hospital, Córdoba; Ciber Fisiopatología De La Obesidad Y Nutrición (Ciberobn), Isciii, Spain.

Introduction: Adipocytes store excess energy as triglycerides (TAGs) in lipid droplets (LDs), which are surrounded by a phospholipid monolayer that serves as an anchoring surface for diverse proteins. These proteins participate in the regulation of lipid storage and mobilization and play crucial roles in LD biogenesis, movement and interaction with other cellular organelles. Recently, we identified by subcellular proteomics a novel member of the Rab family of small GTPases, Rab34, as a component of the LD cover in adipocytes. In this work, we aimed at characterizing the molecular and functional features of this GTPase with regards to lipid homeostasis in cells accumulating lipids under either normal or pathological conditions, adipocytes and hepatocytes.

Methods: The intracellular localization of Rab34 was explored by immunocytochemistry and confocal microscopy in undifferentiated and differentiated murine 3T3-L1 adipocytes and human adipocytes, as well as in human HepG2 hepatocytes exposed to oleate to induce LD formation. The effects of Rab34 silencing and/or overexpression on cellular morphometric parameters and lipid accumulation were also studied. A BioID proximity-dependent biotinylation method was employed to identify Rab34 molecular partners in adipocytes and hepatocytes. Rab34 levels in samples of human adipose tissue and liver were also analysed.

Results: Microscopy studies revealed that the appearance of LDs in adipocytes during adipogenesis or in HepG2 cells upon oleate treatment evoked Rab34 translocation from the Golgi apparatus to the LD surface, likely through a retrograde pathway via the endoplasmic reticulum-Golgi intermediate compartment (ERGIC). A close spatial relationship between Rab34 labeling and ER membranes was observed. Colocalization and BioID studies showed that Rab34 interacts with the intracellular traffic mediators, Arf1 and COPI components. Furthermore, overexpression and silencing studies demonstrated that this GTPase regulates the number of LDs and the lipogenic and lipolytic rates in adipocytes. Finally, Rab34 protein content was dysregulated in both adipose tissue and liver of obese patients as well as in in vitro models of adipocyte hypertrophy or insulin resistance.

Conclusion: Our studies indicate that Rab34 may regulate LD biogenesis and/or growth by controlling the intracellular traffic of lipids and LD-associated proteins, likely through its interaction with the Arf1/COPI traffic system. Rab34 represents a novel marker of LDs under both normal and pathological conditions.
Conflict of Interest: None disclosed.

Funding: MINECO/FEDER (BFU2013-44229-R; BFU2015-70454-REDT); JJAA/ FEDER (PI-0200/2013); FIS/FEDER (PIE14_00005; PI13/01430; PI16/00221), ProteoRed $\left(\mathrm{PRB}^{2}\right)$, CIBERobn (ISCIII).

\section{T1P115 \\ Reduced skeletal muscle protein turnover and deiodinase-3 upregulation in the adaptive thermogenesis that facilitates body fat recovery during weight regain}

\section{Calonne, J. '; Isacco, L. '; Miles-Chan, J. '; Arsenijevic, D. '; Montani, J.' ;} Guillet, C.'; Boirie, Y.'; Dulloo, A. ${ }^{1}$

${ }^{1}$ Department of Medicine, Division of Physiology, University of Fribourg, Switzerland, ${ }^{2}$ Ea3920 And Epsi Platform, Bourgogne Franche-Comté University, F-25000 Besançon, France, ${ }^{3}$ Clermont Auvergne Université, Inra, Unité De Nutrition Humaine, Clermont-Ferrand, France

Introduction The recovery of body weight after weight loss is characterized by an accelerated rate of fat recovery (preferential catch-up fat) resulting partly from an adaptive suppression of thermogenesis. Although the skeletal muscle has been implicated as an effector site for such thrifty metabolism driving catch-up fat, the underlying mechanisms remain to be elucidated. We test here the hypothesis that this thrifty metabolism driving catch-up fat could reside in a reduced rate of protein turnover (an energetically costly 'futile' cycle) and in altered local thyroid hormone metabolism in skeletal muscle.

Methods Using a validated rat model of semi-starvation-refeeding in which catch-up fat is driven solely by suppressed thermogenesis, we assessed after 1 week of refeeding in refed and control animals the following: (i) in-vivo rates of protein synthesis in hindlimb skeletal muscles using the stable isotope flooding dose technique of incorporation of ${ }^{13} \mathrm{C}$-labeled valine in muscle protein, (ii) ex-vivo muscle assay of net formation of thyroid hormone tri-iodothyronine (T3) from precursor hormone, thyroxine (T4), and (iii) protein expression of muscle deiodinases (type 1,2 and 3) using validated antibodies.

Results We show that after 1 week of calorie-controlled refeeding, the fractional protein synthesis rate was lower in skeletal muscles of refed animals than in controls (by $30-35 \%, \mathrm{p}<0.01$ ) despite no between-group differences in the rate of skeletal muscle growth or whole-body protein deposition - thereby underscoring concomitant reductions in both protein synthesis and protein degradation rates in skeletal muscles of refed animals compared to controls. These differences in skeletal muscle protein turnover during catch-up fat were found to be independent of muscle type and fiber composition, and were associated with a slower net formation of muscle T3 from precursor hormone T4, together with a significant increase $(\sim 50 \%)$ in muscle expression of deiodinase type 3 (which inactivates $\mathrm{T} 3$ and $\mathrm{T} 4$ ), and a decreased or unaltered expression of the T3-activating enzyme, deiodinase type 2 .

Conclusions These results suggest that diminished skeletal muscle protein turnover, together with altered local muscle metabolism of thyroid hormones leading to diminished intracellular T3 availability, are features of the thrifty metabolism that drives the rapid restoration of the fat reserves during weight regain after caloric restriction.

The authors declare no conflict of interest

Research was supported by Swiss National Science Foundation (no. 310030_152870). 


\section{T1P116}

Relationship between REE and serum reactive $C$ protein indexes in extreme obese females patients

Sammarco, R. ${ }^{1}$; Marra, M. ${ }^{\text {; }}$ Pagano, M. ${ }^{1}$; Vaccaro, A. ' Speranza, E. ' Santarpia, L. ${ }^{1}$; Contaldo, F. ; Pasanisi, F. ${ }^{1}$

${ }^{1}$ Department of Clinical Medicine and Surgery, University Federico II

Introduction:In the literature, different studies showed that chronic inflammatory diseases (Crohn disease, dialysis patients) could increase Resting Energy Expenditure (REE). Also, obese patients may have a chronic inflammatory status, so the aim of this study was to evaluate the possible relationship between inflammatory parameters such as serum reactive $\mathrm{C}$ protein (PCR) and REE in extreme obese female patients.

Methods: Two-hundred fifty six extreme obese females (range 18-60 y) participated in the study (weight $123 \pm 22 \mathrm{~kg}$, BMI $46.7 \pm 7.9 \mathrm{~kg} / \mathrm{m}^{2}$ ) REE was measured by indirect calorimetry (V max29-Sensormedics) and body composition was estimated by bioimpedance analysis, performed at 50 $\mathrm{kHz}$ (DS Medica).

Data were analized according to PCR tertiles: (group1 n. 84: $<0.56 \mathrm{mg}$ / $\mathrm{dl}, 0.37 \pm 0.07$; group 2 n. 87 : 0.56-1.21 mg/dl, $0.83 \pm 0.19$; group $3 \mathrm{n} .85$ $>1.21 \mathrm{mg} / \mathrm{dl}, 2.00 \pm 0.90$ ).

Statistical analysis was performed using ANOVA for comparing the groups (SPSS vers. 15.0)

Results: Weight (group 1:112 $\pm 15 \mathrm{~kg}$, group 2: $123 \pm 24 \mathrm{~kg}$,group 3 $132 \pm 22 \mathrm{~kg}$ ) and BMI ( group 1: $43.0 \pm 5.6 \mathrm{~kg} / \mathrm{m}^{2}$, group 2: $46.3 \pm 7.1 \mathrm{~kg} /$ $\mathrm{m}^{2}$, group 3: $\left.50.1 \pm 8.7 \mathrm{~kg} / \mathrm{m}^{2}\right)$ were significantly different between groups whereas age and height were similar. FAT e FAT\% increased progressively in the subgroups. FFM was significantly different between group 1 $(57.2 \pm 7.6 \mathrm{~kg})$ and group $3(61.8 \pm 10.3 \mathrm{~kg})$.

REE was highest $(\mathrm{p}=0.001)$ in the group $3(2463 \pm 454 \mathrm{kcal})$ whereas group 1 and group 3 was not significantly different $(2117 \pm 325 \mathrm{kcal} / \mathrm{d}$ vs $2249 \pm 355 \mathrm{kcal} / \mathrm{d}$ ).

REE, corrected for FFM $(40.1 \pm 5.4 \mathrm{kcal} / \mathrm{kg})$, was higher $(\mathrm{p}=0.001)$ in the subgroup 3 than group 1 and $2(37.3 \pm 4.8 \mathrm{kcal} / \mathrm{kg}, 37.5 \pm 5 \mathrm{kcal} / \mathrm{kg})$.

Conclusion:REE, both in absolute values and corrected for FFM, was higher in the group with higher serum PCR, suggesting chronic inflammation could affect REE in obesity. These preliminary data warrant further investigation.

Conflict of interest: None Disclosed

Funding: No Funding

T1P117

Resistance training improves metabolic profile and inflammation in adipose tissue of obese high-fat diet-induced rats which is maintained after one month of cessation

Seraphim, $P^{1}{ }^{\text {; }}$ Freitas, M. ${ }^{1}$; Veras, A. ${ }^{1}$; Festuccia, W. ${ }^{2}$; Teixeira, M. ${ }^{\text {; }}$; Teixeira, G. ${ }^{1}$; Nunes, $M{ }^{2}$

${ }^{1}$ Faculdade de Ciências e Tecnologia - Universidade Estadual Paulista (UNESP) - Campus Presidente Prudente - Brazil, ${ }^{2}$ Instituto De Ciências Biomédicas Universidade De São Paulo - Usp - Brazil

Introduction: The development of the adipose tissue is a condition that leads to metabolic damage in adipocytes and in other tissues due to subclinical inflammation state ${ }^{(1)}$. Aerobic training has been an important too to improve these alterations, mainly by promoting reduction of adiposity (2). But it is unclear in the literature the effect of resistance training on these alterations. Another important point is that the cessation of training is typically adopted by most part of population, and the consequences of this life style have not already been well-stablished.This study aimed to investigate the effects of resistance training and detraining on PPAR $\gamma$ and TNF-alpha expression in adipose tissue of obese high-fat diet-induced rats.

Methods: Thirty Wistar rats were divided into: high-fat diet (HF), highfat diet trained (HFT) and high-fat diet detrained (HFD) groups. Resistance training (jump squat) was performed for 12 weeks, $3 \mathrm{x} /$ week, 3 sets of 12 repetitions. HFD interrupted the training at eighth week, characterizing detraining during 4 weeks. Insulin tolerance test (ITT), histological analysis of adipocyte size, and RT-PCR and Western blotting assays were performed in periepidydimal adipose tissue. All procedures involving live animals were conducted in accordance with Ethics Committee on Animal Use (CEUA FCT / Unesp - Campus Presidente Prudente, Protocol $\left.\mathrm{n}^{\circ} 02 / 2015\right)$.

Results: HFT group presented the lowest body weight, weight gain, adipocyte size and fat deposits compared to the other groups $(P<0.05)$. Detraining reversed all these alterations. HFT showed a 30\%-increase of kITT compared to HF, which was similar in HFD $(\mathrm{P}<0.05)$. HFT presented lower TNFa protein/mRNA levels $(P<0.05)$ compared to HF, and also showed an increase of PPAR $\gamma$ protein/mRNA levels compared to HF $(P<0.05)$, which were similar in HFD.

Conclusion: Resistance training attenuated the effects of high-fat diet provokes, reducing weight and fat mass, and improving insulin resistance. One month of cessation of training promoted rapid gain of weight and fat mass. Resistance training was effective on improvement of the inflammatory profile in adipose tissue, with prevention of reduction of PPAR $\gamma$ even under ingestion of a high-fat diet. In addition, even with body fat gain after cessation of training, TNFa levels remained low and PPAR $\gamma$ levels remained elevated, suggesting that the detraining can accelerate the gain of body fat, but without eliciting an inflammatory response.

Confilict of interest: The authors declare no conflict of interest.

Funding: CAPES - Coordenação de Aperfeiçoamento de Pessoal de Nível Superior. Reference:

1 Gao et al.: PlosOne. 2015;10: e0119784; 2- O'Leary VB, et al. J Appl Physiol. 2006;100:1584-1589.

\section{T1P118}

Resting energy expenditure is associated with insulin resistance and inflammatory status before and after weight loss in patients with severe obesity

Bettini, S. ${ }^{1}$; Bordigato, E. ' Fabris, $R .{ }^{1}$; Serra, $R .{ }^{1}$; Prà, C. ${ }^{1}$; Belligoli, A. ' ; Sanna, M. ${ }^{1}$; Foletto, M. ${ }^{1}$; Prevedello, L. ${ }^{1}$; Vettor, R. ${ }^{1}$; Busetto, L. ${ }^{1}$

${ }^{1}$ Center for the Study and the Integrated Management of Obesity, University of Padova, Italy

Introduction: Resting energy expenditure (REE) is mainly driven by body composition but tends to decline after caloric restriction more than what is expected according to body composition changes (metabolic adaptation). Determinants of REE beyond body composition values are less known. Our aim was to investigate the role of metabolic and inflammatory markers in determining REE and metabolic adaptation after weight loss. Methods: REE (indirect calorimetry), body composition (fat-free mass or FFM, fat mass or FM by bioelectrical impedance analysis), metabolic markers (fasting plasma glucose, fasting insulin, HOMA index, lipids levels) and adipocytokines (leptin, TNF-alfa, IL-6, hsCRP) were determined before and after weight loss in 175 patients with severe obesity (mean BMI: $44.6 \pm 7.4 \mathrm{~kg} / \mathrm{m}^{2}$ ) treated with laparoscopic sleeve gastrectomy (LSG) or a lifestyle modification program.

Results: At baseline, REE values were significantly related to sex (male $=1$; female $=2 ; \mathrm{r}=-0.593 ; \mathrm{p}<0.001)$, BMI $(\mathrm{r}=0.600 ; \mathrm{p}<0.001)$, FFM $(r=0.758 ; \mathrm{p}<0.001)$, FM $(\mathrm{r}=0.388 ; \mathrm{p}<0.001)$ waist $(\mathrm{r}=0.664$; $\mathrm{p}<0.001)$, HDL-cholesterol $(\mathrm{r}=-0.225 ; \mathrm{p}<0.01)$, fasting glucose $(\mathrm{r}=0.166 ; \mathrm{p}<0.05)$, fasting insulin $(\mathrm{r}=0.301 ; \mathrm{p}<0.001)$, HOMA index $(\mathrm{r}=0.305 ; \mathrm{p}<0.001)$, hsCRP $(\mathrm{r}=0.214 ; \mathrm{p}<0.01)$. Only FFM, FM and HOMA index were independently related to baseline REE in a multiple regression model. Weight loss was $28.1 \pm 11.2 \%$ of the baseline body weight. In order to account for body composition changes, a predictive equation for REE was derived by using the baseline FFM and FM values. A predicted post-weight loss REE was then calculated by using this equation and by entering the individual body composition values measured after weight loss. Metabolic adaptation was defined as the difference between observed and predicted REE after weight loss. Metabolic adaptation was 
$168 \pm 230 \mathrm{kcal} /$ day. Metabolic adaptation was significantly related to the reduction of hsCRP observed after weight loss $(\mathrm{r}=0.172 ; \mathrm{p}<0.05)$.

Conclusion: REE was independently associated with body composition values and insulin-resistance state in patients with severe obesity. Metabolic adaptation during weight loss, defined as any reduction of REE beyond what is can be expected by FFM and FM loss, was related to the improvement of inflammatory status after body weight reduction.

Conflict of Interest: None Disclosed

Funding: No Funding

\section{T1P119}

\section{Reward processing alteration after bariatric surgery in obesity}

hirano, Y.'; Matsumoto, J.2.; Kitahara, A. ; Tokuyama, $H^{4}{ }^{4}$; Yamaga, M. ; Kitamoto, T.; Matsumoto, K. ; Masuda, Y.5; Yokote, K. ${ }^{3}$

${ }^{1}$ Research Center for Child Mental Development, Chiba University, ${ }^{2}$ Department Of Regional Disaster Medicine, Graduate School Of Medicine, Chiba University, ${ }^{3}$ Department Of Clinical Cell Biology And Medicine, Graduate School Of Medicine, Chiba University, ${ }^{4}$ Department Of Advanced Aging Medicine, Graduate School Of Medicine, Chiba University, ${ }^{5}$ Department Of Radiology, Chiba University Hospital

Introduction: Similarities in cognitive domain between obesity and addiction are recently focused. In a recent meta-analysis, the enhancement of activation in amygdala and striatum in obesity and addiction was reported and they proposed these similarities may be associated with an enhanced focus on reward [1]. Weight regain after bariatric surgery is a well-known problem. We hypothesized that the reward processing was not changed after bariatric surgery and cognitive education is needed to keep reduced weight. The aim of the study is to investigate reward processing during the monetary incentive delayed (MID) task in obese patients before and after bariatric surgery (sleeve gastrectomy) using neuropsychological tests and functional magnetic resonance imaging (fMRI).

Method: Twelve obese participants $(\mathrm{BMI}>35)$ were recruited for this study. Five of them were scheduled to undergo the bariatric surgery. So far, three participants (age, 38-65; BMI, 45.9-51.2) completed fMRI sessions before and after six months bariatric surgery in a 3T MRI scanner A MID task was modified from a design added fixation on a crosshair after a press button to a target [2] from original [3]. During two 9-minutes fMRI sessions, participants saw one of 5 cues expecting win/lose $\$ 1$ or $\$ 5$ or neutral, then pressed a button when a target was shown after a short fixation in a trial. After the second fixation after a response, they saw their feedback of win or lose and total winnings. Functional images were analyzed using SPM12 and group analysis in each prospect, anticipation, and feedback of reward phase was conducted.

Results: Response time during MID task was not changed after six months surgery. However, bilateral thalamus and left putamen and amygdala activation were increased in the prospect reward phase $(\mathrm{p}<0.05$, uncorrected for multiple comparisons) although the sample size was still limited.

Conclusion: The result suggested that the reward processing supposed to be enhanced in obesity was not decreased even after bariatric surgery. Further study is needed to prove the certainty of these results by increasing sample size.

Conflict of Interest: None Disclosed.

Funding: Research relating to this abstract was funded by JSPS KAKENHI Grant Number JP15K01703.

References:

1 García-García et al., Obes Rev 2014;15:853-69

2 Andrews et al., Biol Psychiatry 2011;69:675-83

3 Knutson et al., Neuroimage 2000;12:20-7
T1P121

Role of nicotinamide nucleotide transhydrogenase (NNT) in obesity: Studies in independent and congenic mutant mice sub-strains

\section{Rovani, J. ${ }^{\text {; }}$ Raposo, $\mathrm{H}^{1}{ }^{\text {; }}$, Vercesi, A. ${ }^{1}$; Oliveira, $\mathrm{H} .{ }^{1}$}

${ }^{1}$ State University of Campinas

Introduction: The nicotinamide nucleotide transhydrogenase (NNT) is a mitochondrial enzyme that catalyzes the formation of NADPH from $\mathrm{NADP}^{+}$and NADH. The cellular content of NADPH is important for macromolecules biosynthesis, as well as reducing power, particularly for mitochondrial antioxidant system. Mitochondria deficient in NNT present higher rates of $\mathrm{H} 2 \mathrm{O} 2$ release and susceptibility to undergo mitochondrial permeability transition. Human deficiency of NNT is associated with cardiac disturbances and glucocorticoid deficiency. Here, we aimed at verifying whether NNT play a role in the development of obesity.

Methods: C57BL6J independent and congenic mice sub-strains, carrying or not a spontaneous NNT mutation, were compared regarding lipid metabolism and adiposity features under low (10\% Kcal) and high (60\% Kcal) fat diets. Independent lines were C57BL/6J from Jackson Lab (B6J, NNT mutant) and C57BL/6JUNI from State University of Campinas (B6Uni, NNT wild type). Congenic lines carrying the mutated NNT allele from the C57BL/6J sub-strain were develop through backcrosses up to the $7^{\text {th }}$ generation $\left(\mathrm{NNT}^{/-}\right.$vs $\left.\mathrm{NNT}^{+/+}\right)$.

Results: Compared with B6UNI mice under low fat diet, B6J (NNT mutant) presented marked increases in adipose tissue depot sizes: epididymal (65\%), subcutaneous (2-fold) and brown adipose tissue (BAT, 25\%). Accordingly, plasma leptin levels were higher in B6J mice (56\%). No differences were observed in food ingestion, fat excretion, dietary fat retention and lipogenesis. The increased adiposity in B6J mutant mice can be explained by lower global in vivo energy expenditure rates: $10 \%$ in the light and $15 \%$ in the dark period. The same results were verified after 16 weeks of high fat diet. In order to exclude other genetic background possible interferences, congenic strains were generated and compared. Under low fat diet, adiposity of $\mathrm{NNT}^{-/}$did not differ from $\mathrm{NNT}^{+/+}$mice. However, under high fat diet, $\mathrm{NNT}^{-/-}$mice presented increased masses of white adipose tissue (WAT, 33\%) and BAT (60\%), as well as of plasma leptin levels (60\%). Energy expenditure rates were markedly diminished in high fat fed $\mathrm{NNT}^{-/}$mice during the light $(16 \%)$ and dark $(18 \%)$ periods. Based on these results we could speculate that NNT play a role in BAT activity and/or WAT adipogenesis through mitochondrial derived reactive oxygen species signaling, under high fat diet condition.

Conclusion: Together these results demonstrated that: 1- C57BL/6J closely related sub-strains present distinct metabolic phenotypes, and, 2- the mitochondrial enzyme NNT plays a critical role in the development of diet induced obesity.

Conflict of interests: None to disclose

Funding: Fapesp and CNPq Brazilian research foundations

\section{T1P123}

Roles of melanocortin receptor signaling pathways in central acyl ghrelin-induced stimulation of ingestive behavior, gastric emptying, and small intestinal transit

\section{Chen, ${ }^{1}{ }^{1}$}

${ }^{1}$ Faculty of Medicine, National Yang-Ming University School of Medicine, Taipei; Division of Gastroenterology and Hepatology, Department of Medicine, Taipei Veterans General Hospital, Taipei, Taiwan

Introduction: The purpose of this study was to investigate the roles of melanocortin receptors in the regulation of central acyl ghrelin-induced stimulation of ingestive behaviour, gastric emptying, and small intestinal transit in conscious rats.

Methods: We examined the effects of á-melanocyte stimulating hormone (á-MSH) and $O$ - $n$-octanoylated ghrelin on food intake, gastric emptying, 
and small intestinal transit in conscious rats that had a chronic implant of intracerebroventricular (ICV) catheters.

Results: ICV injection of $O$ - $n$-octanoylated ghrelin $(0.1 \mathrm{nmol} / \mathrm{rat})$ significantly increased the cumulative food intake up to $8 \mathrm{~h}(P<0.01)$, enhanced non-nutrient semiliquid gastric emptying $(P<0.001)$, and increased the geometric centre and running percentage of small intestinal transit $(P<$ 0.001 ). Pre-treatment with ICV injection of á-MSH (1.0 and $2.0 \mathrm{nmol} / \mathrm{rat}$ ) blocked the acyl ghrelin-induced hyperphagic effect, and a higher dose of á-MSH (2.0 nmol/rat) attenuated the increase in the geometric centre of small intestinal transit $(P<0.01)$, but without altering either the acyl ghrelin-induced gastroprokinetic effect or the increase in the running percentage of small intestinal transit.

Conclusion: These results indicate that the AgRP signaling pathway is involved in central acyl ghrelin-elicited ingestive behaviour and small intestinal transit but without altering central acyl ghrelin-induced acceleration of gastric motility in rats.

None Disclosed

Research relating to this abstract was funded by grants from Taiwan Ministry of Science and Technology (NSC 96-2320-B-010-037- and MOST 105-2314-B-010015-)

\section{T1P124}

Salsalate ameliorates metabolic disturbances by reducing inflammation and oxidative stress and by activating brown adipose tissue in spontaneously hypertensive rats expressing human CRP

Silhavy, J. '; Trnovska, J. ${ }^{2}$ Kuda, O. ${ }^{1}$; Landa, V. ' Zidek, V. ${ }^{1}$; Mlejnek, P. ' Simakova, M. '; Strnad, H. ${ }^{3}$; Oliyarnyk, O. ${ }^{2}$; Skop, V. ${ }^{2}$; Kazdova, L. ${ }^{2}$;

Pravenec, $M$.

${ }^{1}$ Institute of Physiology, Czech Academy of Sciences, Prague, Czech Republic, ${ }^{2}$ Institute For Clinical And Experimental Medicine, Prague, Czech Republic, ${ }^{3}$ Institute For Molecular Genetics, Czech Academy Of Sciences, Prague, Czech Republic

Introduction: Chronic low-grade inflammation plays an important role in the pathogenesis of insulin resistance. In the current study, we tested the effects of salsalate, a non-steroidal anti-inflammatory drug, in an animal model of inflammation and metabolic syndrome, using spontaneously hypertensive rats that transgenically express human C-reactive protein (SHR-CRP).

Methods: We treated 15-month-old male transgenic SHR-CRP rats and nontransgenic SHR with salsalate $(200 \mathrm{mg} / \mathrm{kg} /$ day $)$ mixed as part of a standard diet for 4 weeks. A corresponding untreated control group of male transgenic SHR-CRP and SHR rats were fed a standard diet without salsalate.

Results: In the SHR-CRP transgenic strain, salsalate treatment decreased circulating levels of inflammatory markers TNF-alpha and MCP-1, reduced oxidative stress in the liver and kidney, increased sensitivity of skeletal muscles to insulin action and improved tolerance to glucose. In SHR controls, with no CRP-induced inflammation, salsalate treatment reduced body weight, decreased levels of serum free fatty acids, total and HDL cholesterol and increased palmitate oxidation and incorporation in brown adipose tissue. Salsalate regulated lipid metabolism by affecting expression of genes from PPAR-alpha signaling pathways and inflammation by affecting expression of genes from MAPK signaling and NOD-like receptor signaling pathways.

Conclusion: These findings suggest that salsalate has metabolic effects beyond suppressing inflammation.

Conflict of Interest: None Disclosed

Funding: Research relating to tgis abstract was funded by grant 16-04859S from the Czech Science Foundation.
T1P125

Sedentarism potentiates the hapatic and pancreatic structural changes induced by a fatty diet in rodents.

Bovolini, J. ${ }^{1}$; Andrade, M. ${ }^{2}$; Duarte, J. ${ }^{1}$

${ }^{1}$ Sports Faculty of Porto, ${ }^{2}$ Universidade Federeal De Pernambuco

Introduction: Obesity and overweight are one of the major sources of metabolic disorders as dyslipidaemia, insulin resistance and diabetes. Resulted mainly from positive energy balance which is determined by the rate of energy consumption and energy expenditure through physical activities or exercise. However, metabolic disorders seem to be caused either by high caloric intake and food type consumed independently of obesity and overweight. Considering the sedentary lifestyle ability to induce and potentiate the metabolic disturbances, in this study, we seek to observe the long-term effect of sedentarism and high caloric diary intake, especially from fat, on the pathogenicity potential in essential tissues for balance and metabolic health, such as pancreas and liver.

Methods: 40 Wistar rats (4 weeks of age), randomly divided into four groups: sedentary standard diet ( $\mathrm{SSD}, \mathrm{n}=7)$, active standard diet (ASD, $\mathrm{n}=7)$, sedentary high fat diet (SHFD, $\mathrm{n}=7$ ) and active high fat diet (AHFD, $\mathrm{n}=7$ ); The animals were fed with a standard or a high fat diet (HFD - 70\% of calories from fat; Research Diets, Inc.) for 21 weeks. The active groups had unrestricted free wheel access to voluntary physical activity practice while the sedentary groups remained restricted to their cage space. At the end of protocol, the animals were weighed, euthanized and pancreas and liver samples were collected for histological analysis. The samples were stained with Hematoxylin-Eosin to evaluate hepatic steatosis and Langerhans islets cross-sectional area (CSA) and Sirius Red protocol to evaluate collagen deposition. After stained protocols, the samples were photographed with a light microscope connect to a digital camera (Carl Zeiss, Germany). The photographs were analyzed with NIH ImageJ software (USA) to evaluation of fat tissue deposition and CSA. For collagen quantification was used the software Image Pro Plus 6. Caloric intake and animals body weight were recorded weekly.

Results: surprisingly there were no differences between groups in body weight and caloric intake after 21 protocol weeks. However, HFD induced a hepatic and pancreatic weight reduction in both groups administered with a fat-rich diet $(\mathrm{P}<0.005 \mathrm{vs}$.SSD). Excessive fatty acid dietary availability, even without overweight, also induced significant collagen deposition in both pancreas and liver $(\mathrm{P}<0.005 \mathrm{vs}$.SSD $)$, having the animals from SHFD group presented an expressive liver fat deposition $(\mathrm{P}<0.005 \mathrm{vs}$. SSD) and CSA of Langerhans Islets increase $(\mathrm{P}<0.005 \mathrm{vs} . \mathrm{SSD})$.

Conclusion: the results suggest that the daily consumption of high caloric and fat amounts have an important and deleterious effect on pancreas and liver histological profile, even without relevant clinical manifestation such as overweight or obesity. They also suggest that the sedentary lifestyle seems to exert an amplifying effect on the tissue damage induced by the high consumption of calories and fat, making it a pathogenic combination.

Funding: This study was supported by Capes (Ministry of Education, Brazil) and CIAFEL (UID / DTP / 00617/2013, Portugal).

\section{T1P126}

\section{Short sleep duration in relation to fuel selection in overweight} and obese subjects

Poggiogalle, E. '; Calabretto, M. '; Lubrano, C. ; Gnessi, L. ' ; Mariani, S. ' ; Mancini, C. ' Ciccantelli, B. ' ; Marocco, C. ${ }^{\text {; }}$ Lenzi, A. ${ }^{\text {; }}$ Donini, $L .{ }^{1}$

${ }^{1}$ Sapienza University, Rome, Italy

Introduction: Sleep deprivation represents a novel risk factor potentially associated with obesity and with adverse metabolic effects. The aim of the present study was to investigate the relationship between reduced sleep duration and fuel selection in adults with obesity.

Methods: Participants were recruited at the High Specialization Centre for the Care of Obesity (CASCO), Sapienza University, Rome, Italy. Inclu- 
sion criteria were: age $18-65$ years, body mass index (BMI) $\geq 25 \mathrm{~kg} / \mathrm{m}^{2}$. Use of any medications affecting the central nervous system, any psychiatric disorders and night shift work were considered as exclusion criteria All participants underwent indirect calorimetry (Quark RMR, Cosmed, Italy). Gas exchanges were used to calculate the respiratory quotient (RQ). The equation by Weir was used to calculate the resting energy expenditure (REE). Carbohydrate and fat oxidation was calculated (equations by Frayn et al.), assuming that protein oxidation accounts approximately for $15 \%$ of REE. Sleep duration was objectively assessed through the use of an Actigraph watch. Body composition was evaluated by DXA. 3T magnetic resonance was used to assess visceral (VAT) and subcutaneous adipose tissue (SAT). Participants were divided into two groups according with sleep duration $\geq 6 \mathrm{~h} /$ day or $<6 \mathrm{~h} /$ day.

Results: 118 participants were included (age: $49.6 \pm 12.1$ years, BMI $37.3 \pm 5.9 \mathrm{~kg} / \mathrm{m}^{2}$ ) and short sleep duration was found in $48 \%$ of subjects The RQ was significantly lower in the short sleep duration group than in the group sleeping $\geq 6 \mathrm{~h} /$ day $(0.72$ vs. $0.77, \mathrm{p}<0.05)$. The short sleep duration group oxidized more fat and less carbohydrate than the "normal sleep" group (fat: $109 \pm 56$ vs. $92 \pm 55 \mathrm{mg} / \mathrm{min}, \mathrm{CHO}: 21 \pm 17$ vs. $66 \pm 12 \mathrm{mg} / \mathrm{min}$, all $\mathrm{p}<0.05)$. SAT was lower in subjects sleeping less than $6 \mathrm{~h} /$ day than the group sleeping more than $6 \mathrm{~h} /$ day $(50022 \pm 10404 \mathrm{vs}$. $\left.64303 \pm 95165 \mathrm{~mm}^{3}, \mathrm{p}<0.05\right)$ Higher sleep duration was positively associated with RQ (beta: $3.5^{\star} 10^{-4}$, SE: $1.6^{\star} 10^{-4}, \mathrm{p}=0.03$ ) after adjustment for age, sex and body fat. The average METs/week were not different between groups.

Conclusion: Reduced sleep duration affects substrate metabolism in subjects with excess fat. Implications for the potentially related metabolic disturbances need to be further explored.

\section{References:}

1 Weir JB. New methods for calculating metabolic rate with special reference to protein metabolism. J Physiol 1949; 109: 1-9.

2 Frayn KN. Calculation of substrate oxidation rates in vivo from gaseous exchange. J Appl Physiol 1983; 55: 628-634.

Conflict of Interest: None Disclosed

Research relating to this abstract was funded by the Italian Ministry of Education and Research

\section{T1P128}

\section{Sleeve gastrectomy normalizes defects in enteroendocrine cells in morbidly obese humans}

Wölnerhanssen, B. ; Burdyga, G. ${ }^{2}$; Meyer-Gerspach, A. ${ }^{1}$; Moran, A. ${ }^{2}$; Peterli, R. ${ }^{3}$; Manz, M. ${ }^{4}$; Thumshirn, M. ${ }^{4}$; Daly, K. ${ }^{2}$; Beglinger, C. ; ShiraziBeechey, S. ${ }^{2}$

${ }^{1}$ Department of Research, St. Claraspital Basel, Basel, Switzerland, ${ }^{2}$ Institute Of Integrative Biology, University Of Liverpool, United Kingdom, 3Department Of Surgery, St. Claraspital Basel, Basel, Switzerland, ${ }^{4}$ Department Of Gastroenterology, Clara Hospital Basel, Switzerland

Introduction: Morbidly obese patients exhibit impaired secretion of gastrointestinal peptides that may contribute to the development of obesity and its associated conditions. Bariatric surgery induces an increase in gastrointestinal peptide release. A better understanding of the underlying mechanisms may contribute to the development of non-invasive therapeutic strategies.

Methods: Gastric and duodenal tissues were endoscopically collected from lean controls and morbidly obese subjects before and 3 months af ter laparoscopic sleeve gastrectomy (LSG). Gastrointestinal peptides were measured after a standardized nutrient drink pre- and post- LSG. Tissue morphology was determined by morphometric analysis. Tissue expression levels of chromogranin A, gut hormones, á-defensin, mucin 2 and $\mathrm{Na}+$ /glucose co-transporter 1 (SGLT1) were assessed by combination of immunohistochemistry, western blotting and quantitative PCR. Protein abundance of transcription factors (Hes1, HATH1, NeuroD1, and Ngn3) were determined by western blotting.

Results:In the obese, the total number of enteroendocrine cells (EEC) containing gastrointestinal peptides was significantly reduced $(\mathrm{p}<0.001)$; they were restored post-LSG $(\mathrm{p}<0.001)$. This profile matched the levels of circulating gastrointestinal peptides ( $\mathrm{p}<0.001$, respectively). A significant increase in SGLT1 $(\mathrm{p}<0.05)$ expression was detected in the obese duodenum. Expression levels of transcription factors required for differentiation of absorptive and secretory cell lineages were reduced $(\mathrm{p}<0.05$, respectively).

Conclusion: In obesity, there is deregulation of developmental programming of intestinal epithelial cell lineages. The programing is restored postLSG leading to an increase in secretion of gut hormones and reduced blood glucose levels.

\section{Conflict of Interest: none}

Funding: The trial was funded by the Swiss National Science Foundation and the Biotechnology and Biological Sciences Research Council and Pancosma SA.

\section{T1P129 \\ Slump in microbial gene richness in severe and complicated obesity is partialy restored after bariatric surgery}

Judith, A. '; Prifti, E. '; Dao, M. '; Verger, E. '; Kayser, B. '; Pons, N. ${ }^{2}$; Levenez, F.2. Erhlich, D. ${ }^{2}$; Doré, J. ${ }^{2}$; Zucker, J. ${ }^{1}$; Clément, K. ${ }^{1}$

${ }^{1} 1$ Institute of Cardiometabolism and Nutrition, ICAN, Assistance Publique Hôpitaux de Paris, Pitié-Salpêtrière hospital, Paris, France 2INSERM, UMR S U1166, Nutriomics Team, Paris, France 3Sorbonne Universités, UPMC University Paris 06, UMR_S 1166 I, Nutrio, ${ }^{25 I n r a, ~ U s 1367 ~ M e t a g e n o p o l i s, ~ J o u y-E n-J o s a s, ~}$ France 6Agroparistech, Umr1319 Micalis, Jouy-En-Josas, France

Introduction: Microbial gene richness was associated with adverse metabolic parameters in lean, overweight or moderate obesity $(1,2)$. Bariatric surgery (BS) induces microbiota composition changes, some of which associates with metabolic and body composition improvement $(3,4)$, mostly post-Roux-en-y gastric bypass (RYGB). We aimed to evaluate gene richness in severe obesity and evaluate whether different BS type would differently modulate gut microbiota.

Methods: 61 morbidly obese individuals, candidate to banding (AGB) or RYGB, were prospectively enrolled. 24 patients were followed at 1, 3 and 12 months after surgery. Associations were evaluated between gene richness and metabolic parameters in both BS. 49 overweight and moderately obese individuals (2) were also included to pooled to this BS cohort obtain a wide a spectrum of BMI. Microbiota was analysed using and shotgun metagenomics. Serum metabolomics was performed at baseline using LCMS analyses.

Results: We observed a dramatic increased prevalence of low gene count (LGC), in BS patients, which concerned $75 \%$ of patients. Pooling the two cohorts, we confirm that LGC associated increased BMI, fat mass, insulin-resistance and low-grade inflammation. We also demonstrate that LGC associates with detrimental fat deposition (i.e increased fat-trunk mass $\mathrm{p}=8 \mathrm{e}-0.5$ ), and overt metabolic complications (type- 2 diabetes, HTA and its severity). Gene richness significantly associated with 78 MGS among which, a lot were associated with both adverse body composition and metabolic clinical traits. Finally, 12 serum metabolites were significantly associated with gene richness, MGS and clinical traits. Both BS induced an increase in gene richness that became significant at 1 year, however remaining significantly lower in RYGB compared to AGB, along with weight loss and metabolic improvement. 12 MGS were significantly modified post-BS.

Conclusions: We confirm that decreased gene richness is a signature of obesity and metabolic alterations. In morbid obesity, LGC is much more frequent and to a more severe extend than that of overweight and moderate obesity, in link with more severe metabolic alterations. BS is able to improve these phenotypes but most morbid obese individuals remain LGC. Le Chatelier E, et al. Nature. 2013 Aug 29;500(7464):541-6.

Cotillard A, et al. Nature. 2013 Aug 29;500(7464):585-8.

Kong L-C, et al. . Am J Clin Nutr. 2013 Jul;98(1):16-24.

Aron-Wisnewsky J, Nat Rev Gastroenterol Hepatol. 2012 Oct;9(10):590

Conflict of Interest: None Disclosed 
Funding: PHRC -APHP program called Microbaria "AOM10285/ P100111" registered on clinical trial NCT01454232 and grant agreement HEALTH-F4-2012-305312 (METACARDIS). JAW received a grant from Institut Appert and the Nestle research grand.

\section{T1P130}

\section{Soluble LR11/SorLA represses thermogenesis in adipose tissue and correlates with bmi in humans}

Jiang, M. ${ }^{1}$; Whittle, A. ${ }^{2}$; Ebinuma, H. ${ }^{3}$; Murano, T. ${ }^{\text {; }}$ Nakaseko, C. ${ }^{4}$; Jin, W. ; Schneider, W. ${ }^{6}$; Vidal-Puig, A. ${ }^{2} ;$ Bujo, $H^{1}{ }^{1}$

${ }^{1}$ Department of Clinical-Laboratory and Experimental-Research Medicine, Toho, ${ }^{2}$ University Of Cambridge Metabolic Research Laboratories, ${ }^{3}$ Tsukuba Research Institute, Sekisui Medical Co. Ltd., ${ }^{4}$ Department Of Hematology, Chiba University Hospital, ${ }^{5}$ Department Of Endocrinology, Yanbian University Hospital, ${ }^{6}$ Department Of Medical Biochemistry, Max F. Perutz Laboratories, Medical University Of Vienna

Introduction: Thermogenesis in brown adipose tissue (BAT) is a significant component of energy expenditure. Recent studies using fluorodeoxyglucose (FDG)-Positron Emission Tomography (PET) have shown that decreased thermogenetic activity in BAT is highly associated with the decreased energy expenditure in obese patients with type 2 diabetes. Therefore, defining the mechanism underlying the phenotypic transition between white adipose tissue (WAT) and BAT is of great importance for developing treatment strategies for type 2 diabetes. Here, we show that the soluble form of the low-density lipoprotein receptor relative, LR11/SorLA (sLR11), suppresses thermogenesis in adipose tissue in a cell-autonomous manner.

Methods: LR11-deficient mice were fed high-fat diet for eight to twelve weeks. Primary adipocytes were obtained from human or mouse fat tissues. Serum sLR11 concentrations were determined using a sandwich ELISA system.

Results:Mice lacking LR11 are protected from diet-induced obesity, insulin resistance, dyslipidemia, or hepatic steatosis, associated with an increased browning of WAT. Assessment of energy expenditure using indirect calorimetry confirmed that LR11-deficient mice had a higher metabolic rate than WT mice when fed a high fat diet. Treatment of adipocytes with sLR11 inhibits thermogenesis via the bone morphogenetic protein/ TGF $\beta$ signaling pathway and reduces Smad phosphorylation. In addition, sLR11 levels in humans are shown to positively correlate with body mass index and adiposity.

Conclusion: sLR11 may be applied for the detection and treatment of disturbed metabolic conditions as a novel fine-tuning negative regulator and/or as a biomarker indicative of disturbed energy expenditure to protect obese patients from the progression of type 2 diabetes.

None

Research relating to this abstract was funded by FP7-BetaBAT, BBSRC (BB/ J009865/1), the British Heart Foundation (PG/12/53/29714), MDU MRC, Japan Health and Labour Sciences Research grants for translational research (H22-rinkensui-ippan-001), US-Japan cooperative medical science program, Grants-in-aid fo Scientific Research $(24390231,26461369$, and15K15198), the strategic research foundation at private universities from the Japanese Ministry of Education, Culture, Sports, Science and Technology, Wellcome Trust and the Cambridge Overseas Trust, Ministerio de Educacio'n, through "Programa Nacional de Movilidad de Recursos Humanos del Plan Nacional de I-D.i 2008-2011 (Subprograma de Estancias de Movilidad en el Extranjero 'Jose' Castillejo' para jo'venes Doctores,ref: JC2011-0248), MRC, and the Austrian Science Fund (FWF P-20218 and P-20455).
T1P131

Sphingolipid metabolism in the salivary glands of rats with diet induced insulin resistance and streptozotocin induced diabetes

Garbowska, M. '; Lukaszuk, B. ${ }^{2}$; Miklosz, A. ${ }^{2}$; Wroblewski, I. ${ }^{3}$; Kurek, K. ${ }^{3}$; Chabowski, A. ${ }^{2}$; Zalewska, A. ${ }^{4}$; Zendzian-Piotrowska, $M .{ }^{1}$

${ }^{1}$ Medical University of Bialystok, Department of Hygiene, Epidemiology and Ergonomics, Poland, ${ }^{2}$ Medical University Of Bialystok, Department Of Physiology, Poland, ${ }^{3}$ Medical University Of Bialystok, Department Of Gastroenterology And Internal Medicine, Bialystok, Poland, ${ }^{4}$ Medical University Of Bialystok, Department Of Conservative Dentistry, Bialystok, Poland

Introduction:Diabetes and obesity are considered to be major public health problems affecting hundreds of millions people worldwide. Remarkably, scientific reports regarding salivary glands sphingolipid metabolism in diabetes are virtually non-existent. This is odd given a well-established link between the both in other tissues (e.g. skeletal muscles, liver) and the key role of these glands in the oral health preservation. The aim of this research was to examine sphingolipid metabolism in the salivary glands in (pre)diabetes (evoked by high fat diet feeding or streptozotocin). Methods:Wistar rats were allocated into 3 groups: control, HFD- or STZ-diabetes. The content of major sphingolipid classes in the parotid (PSG) and submandibular (SMSG) salivary glands was assessed via chromatography (TLC, GLC, HPLC). Western Blot analyses were employed to study the levels of key sphingolipids signaling pathway enzymes.

Results:In the HFD group a reduction in ceramide content in the SMSG was found. Yet, no change in ceramide content in the PSG was found, whereas an increase in ceramide amount for SMSG of the STZ rats was observed. This was accompanied by an elevation in SPT1 level and increased sphingomyelin hydrolysis, since we observed a significant drop in the amount of SM.

Conclusion:PSG and SMSG respond differently to (pre)diabetes, with clearer pattern presented by the later gland. An activation of sphingomyelin signaling pathway was observed in the course of STZ-diabetes, i.e. metabolic condition with rapid onset/progression. Whereas, chronic HFD led to an inhibition of sphingomyelin signaling pathway in the salivary glands (manifested in an inhibition of ceramide de novo synthesis and S1P accumulation).

\section{Conflict of Interest: None Disclosed}

Funding: Research relating to this abstract was funded by Medical University of Bialystok grant no 154-18703L (N/ST/MN/15/003/1118), 153-09673L.

\section{T1P132}

\section{Survivin, a key player in cancer progression, increases in obesity and protects adipose tissue stem cells from apoptosis}

Ejarque, M. '; Ceperuelo-Mallafré, V. '; Serena, C. ${ }^{1}$; Pachon, G. ${ }^{2}$; NuñezRoa, C. ${ }^{1}$; Vilarrasa, N. ${ }^{3}$; Tinahones, F. ; Peinado, M. ${ }^{5}$; Vendrell, J. ${ }^{1}$ Fernandez-Veledo, $\mathrm{S}$.

${ }^{1}$ University Hospital Joan XXIII- IISPV, ${ }^{2}$ Brigham \& Women'S Hospital - Harvard Medical School, ${ }^{3}$ University Hospital Bellvitge, ${ }^{4}$ Virgen De La Victoria Clinical University Hospital, ${ }^{5}$ Institute Of Predictive And Personalized Medicine Of Cancer

Introduction: Adiposetissue (AT) has a central role in obesity-related metabolic imbalance through the dysregulated production of cytokines and adipokines. In addition to its known risk for cardiovascular disease and diabetes, obesity is also a major risk for cancer. Human adipocyte-derived stem cells (hASCs), which determine AT expansion, are important players in pathological development of obesity and associated cancer; however, the mechanisms underlying hASC-induced alterations in cancer remain unknown.

Methods: ASCs were isolated from subcutaneous AT of lean and obese subjects. Serum and AT from a cross-sectional study of 111 subjects classified by body-mass index were collected. Apoptosis was measured using 
annexin-propidium iodide staining by flow cytometry. Gene and protein expression were assessed by qPCR and WB.

Results: We investigated the impact of obesity for the expression of survivin, an anti-apoptotic protein upregulated by adipokines and a diagnostic biomarker of tumor onset and recurrence. In the cross-sectional study circulating levels of survivin and gene expression in subcutaneous AT were significantly higher in obese patients and positively correlated with leptin. Within AT, survivin was detected in hASCs, and its expression was significantly increased in obesity and by pro-inflammatory M1 macrophage soluble factors as IL-1b. Analysis of survivin expression in hASCs revealed a complex regulation including epigenetic modifications and protein stability. We uncovered that survivin levels determine the susceptibility of hASCs to apoptotic stimuli. Accordingly, hASCs from an obese setting were protected from apoptosis.

Conclusion: Collectively, these data shed new light on the molecular mechanisms governing AT expansion in obesity through promotion of hASCs that are resistant to apoptosis, and point to survivin as a new molecular player in the communication between hASCs and tumor cells. Thus, inhibition of apoptosis targeting survivin might represent an effective strategy for both obesity and cancer therapy.

Conflict of Interest: The authors declare no potential conflicts of interest.

Funding: This study was supported by grants from the Spanish Ministry of Economy and Competitiveness and the European Regional Development Fund (ERDF) (JV: PI14/00228; SF-V: BFU2015-70454-REDT; SAF2015-65019R). The Spanish Biomedical Research Center in Diabetes and Associated Metabolic Disorders (CIBERdem) (CB07708/0012) an initiative of the Instituto de Salud Carlos III.

\section{T1P133}

\section{Sustainability of changes in energy expenditure variables at 1 year follow-up after initial weight loss with a very-low energy diet}

\section{Nymo S' ${ }^{1}$ Coutinho S. ; Eknes P. ${ }^{1}$; Bomo O. ${ }^{1}$; Truby $H^{2}$; Kulseeng $B^{3}$;} Martins $C^{1}$

${ }^{1}$ Centre for Obesity Research, Department of Cancer Research and Molecular Medicine, Faculty of Medicine, Norwegian University of Science and

Technology, Trondheim, Norway, ${ }^{2}$ Department Of Nutrition, Dietetics \& Food, Monash University, Melbourne, Australia, ${ }^{3}$ Centre For Obesity, Department Of Surgery, St. Olav Hospital-Trondheim University Hospital, Trondheim, Norway

Introduction: Weight loss (WL) activates compensatory mechanisms, which oppose the weight-reduced state. These include a reduction in total energy expenditure (EE), driven mainly by a reduction in resting metabolic rate (RMR), an increase in exercise efficiency (Ef) and potentially also adaptive thermogenesis (AT) and reduced fat oxidation. The aim of this study was to determine if changes in RMR, Ef and fat oxidation, and potential presence of AT, observed with WL were sustained at 1 year (1Y) follow-up.

Method: 36 adults (26 males) (BMI: $36.6 \pm 0.7 \mathrm{~kg} / \mathrm{m}^{2}$ and age: $41.0 \pm 1.6 y e a r s)$ underwent 8 wks VLED, 4 wks refeeding and $1 Y$ follow-up. Body weight and composition (air displacement plephysmography (BodPod)), RMR, respiratory quotient (RQ) and Ef (by graded cycle ergometry at 10, 25 and 50W of power) (all by indirect calorimetry) and AT (both at the level of RMR and exercise induced EE- (EIEE)) were measured at baseline, wk13 (W13) and 1Y. Statistical analysis was performed using liner mixed model, with Bonferroni corrections. Data are presented as mean \pm SEM.

Results: A WL of $20.0 \pm 1.3 \mathrm{~kg}(17.1 \pm 1.1 \%)$ at W13 was associated with a significant reduction in both absolute and adjusted $(/ \mathrm{kg}$ free fat mass (FFM)/day) RMR $(\mathrm{P}<0.001$ and $\mathrm{P}<0.05$, respectively $\rangle$ a significat increase in $\mathrm{Ef}\langle 10 \mathrm{~W}\langle\mathrm{P}<0.001\rangle, 25 \mathrm{~W}\langle\mathrm{P}<0.01\rangle$ and $50 \mathrm{~W}\langle\mathrm{P}<0.001\rangle\rangle$ and $\mathrm{AT}$, at the level of EIEE, at $10 \mathrm{~W}\langle\mathrm{P}<0.001\rangle$. At $1 \mathrm{Y}$ follow-up with sustained WL $\langle 15.2 \pm 1.1 \%\rangle$, RQ was significantly reduced $\langle\mathrm{P}<0.05\rangle$, Ef was still significantly increased $\langle 10 \mathrm{~W}\langle\mathrm{P}<0.001), 25 \mathrm{~W}\langle\mathrm{P}<0.01\rangle$ and $50 \mathrm{~W}$ $\langle\mathrm{P}<0.01\rangle$ and AT, at the level of EIEE, still present at $10 \mathrm{~W}\langle\mathrm{P}<0.001\rangle$.

Conclusion: A $15 \%$ sustained WL at $1 Y$ follow-up was associated with increased Ef and AT (at the level of EIEE at 10W), despite no change in
RMR. These compensatory responses may contribute to the difficulty in maintaining WL in the long-term.

Disclosure: The authors declare no conflicts of interest.

Funding: Liaison Committee between the Central Norway Regional Health Authority (RHA) and the Norwegian University of Science and Technology (NTNU). Allevo, Karo Pharma AS. Sweden, for providing the VLED products (no commercial interest).

\section{T1P134}

\section{Syndecan 4 is highly upregulated in subcutaneous adipose tissue one year after bariatric surgery due to tumor necrosis factor stimulation of adipocytes}

Jürets, A. ${ }^{\text {; }}$ Itariu, B. ' ; Keindl, M. ${ }^{1}$; Leitner, L. ' Prager, G. ${ }^{2}$; Langer, F. ${ }^{2}$; Grablowitz, V. ${ }^{3}$; Zeyda, M. ${ }^{4}$; Stulnig, T.

${ }^{1}$ Christian Doppler Laboratory for Cardio-Metabolic Immunotherapy, Clinical Division of Endocrinology and Metabolism, Department of Medicine III, Medical University of Vienna, Vienna, Austria, '2Department Of General Surgery, Medical University Of Vienna, Vienna, Austria, ${ }^{3}$ Department Of General Surgery, Göttlicher Heiland Hospital, Vienna, Austria, ${ }^{4}$ Department Of Pediatrics And Adolescent Medicine, Clinical Division Of Pediatric Pulmonology, Allergology And Endocrinology, Medical University Of Vienna, Vienna, Austria

Introduction: Syndecan 4 (SDC4), a ubiquitously expressed transmembrane heparin sulphate proteoglycan, is involved in inflammation, tissue remodeling and LDL-uptake. It is upregulated by tumor necrosis factor (TNF), which we recently showed is highly expressed in the subcutaneous adipose tissue one-year after bariatric surgery. We wanted to investigate whether this upregulation affects SDC4 in adipose tissue.

Methods: We investigated gene expression in subcutaneous adipose tissue from 31 morbidly obese, non-diabetic patients $\left(B M I \geq 30 \mathrm{~kg} / \mathrm{m}^{2}\right)$ during and 1 year after bariatric surgery and matched them to a lean control group. Adipose tissue samples were collected during elective abdominal surgery or by needle biopsy. Genes relevant to glucose and lipid metabolism, inflammation, and apoptosis were investigated by RT-PCR. Serum TNF concentration was determined by commercially available ELISA. In the post-obese we looked for independent predictors for SDC4 expression in the investigated baseline characteristics and expressed genes by using an unbiased machine learning approach. Primary human adipocytes were stimulated for $24 \mathrm{~h}$ with TNF before being harvested and SDC4 gene expression was analyzed by RT-qPCR.

Results: SDC4 was upregulated in comparison to the lean control 1.7 times $(\mathrm{p}<0.05)$ in the obese and 20 times $(\mathrm{p}<0.001)$ one year after bariatric surgery. Positive independent predictors for SDC4 expression were serum TNF and TNF expression. Furthermore, stimulating primary adipocytes in vitro by TNF increases SDC4 expression 2.5 times $(\mathrm{p}<0.01)$. Conclusion: SDC4 is highly upregulated in the subcutaneous adipose tissue one year after bariatric surgery due to TNF stimulation. These results indicate an important role of the TNF-SDC4 axis in the catabolism of adipose tissue during surgery-induces weight loss.

\section{Conflict of Interest: None}

Funding: Research relating to this abstract was funded by the Federal Ministry of Economy, Family and Youth and the National Foundation for Research, Technology and Development (to T.M.S.). 
T1P135

\section{Synergic effect of eugenol and cinnamaldehyde on the inhibition of the expression of lipogenic genes in 3T3-L1mature adipocytes}

Martin, A. ${ }^{1}$; Alberto Angel ${ }^{1,2}$, Juana Sánchez', Andreu Palou', ${ }^{1}$ Laboratory of Molecular Biology, Nutrition and Biotechnology (Nutrigenomics and Obesity), University of the Balearic Islands and CIBER Fisiopatología de la Obesidad y Nutrición (CIBEROBN), Palma de Mallorca, ${ }^{2}$ School of Nutrition and Dietetics. Faculty of Health. Universidad Industrial de Santander (UIS), Bucaramanga, Colombia.

Introduction: Eugenol (EU) and Cinnamaldehyde (CNA) are compounds extracted from certain essential oils, especially cloves and cinnamon, that can may have a potential effect on, control of obesity and associated diseases. The aim of this study was to analyse the effect of EU, CNA and the combination of both (EU + CNA) in vitro on the expression of genes involved in lipid metabolism.

Methods: 3T3-L1 mature adipocytes were treated with different concentrations $(0,10,50,100,200,400 \mu \mathrm{M})$ of EU, CNA or EU + CNA.

Results: The treatment with $\mathrm{EU}(400 \mu \mathrm{M})$ results in decreased Fasn and Lipe mRNA expression compared to the control treatment. The treatment with CNA $(400 \mu \mathrm{M})$ decreases the expression of Fasn and increases the expression of Cpt1b compared to the control treatment. With the combination of both (EU + CNA) produced a dose-response inhibition of the expression of the lipogenic genes (Srebf1, Pparg, Fasn) and Lipe and a dose-response induction of the expression of Cpt1b.

Conclusion: The combination of EU and CNA seems to have a synergistic effect on the inhibition of the expression of lipogenic genes in 3T3-L1 mature adipocytes. The combined use of these compounds emerges as a potential strategy in the control of obesity.

\section{T1P138}

\section{Tarumã (Vitex cymosa bertero ex spreng) extract protects against diet induced obesity and with tendency to lower expression of FFAR2/GPR43 on adipose tissue in C57BL/6J mice}

Ballard, C. ${ }^{1}$; Santos, E. ${ }^{2}$; Dubois, M. ${ }^{3}$; Pilon, G. ${ }^{3}$; Cazarin, $C .{ }^{1}$; Marette, A. $^{3}$; Jr., M. ${ }^{1}$

'University of Campinas, ${ }^{2}$ Federal University Of Mato Grosso Do Sul, ${ }^{3}$ Université Laval

Introduction: Tarumã is a Brazilian fruit rich in polyphenols. Beneficial effects of polyphenols against obesity have been reported, but the mechanism is not clear. Free fatty acid receptor 2 (FFAR2/GPR43) is a potential target for regulation of energy balance. The aim of present study was to analyze the consumption of tarumã extract (TAE) on FFAR2/GPR43 expression and the consequences on body weight gain, energy intake, adiposity and lipid homeostasis.

Methods: Eight-week-old C57BL/6J male mice ( $n=12$ /group) were fed either a Standard diet (10\% fat, $L F$ group) or a High Fat High Sucrose diet (45\% fat, HFHS and Tarumã groups). LF and HFHS groups received daily doses $(100 \mathrm{mg} / \mathrm{kg}$ ) of vehicle (water) by gavage, and Tarumã group received $100 \mathrm{mg} / \mathrm{kg}$ of TAE. Body weight gain was assessed twice week and energy intake, three times a week. Adiposity was evaluated byTD-NMR (Bruker ${ }^{\circ}$. After 8 weeks, mice were sacrificed, liver tissue was collected for triglycerides, cholesterol measures and epididymal adipose tissue was collected for FFAR2/GPR43 analysis by western blot. Data was expressed as media \pm SEM. Statistical analysis was performed using ANOVA-one way $(\mathrm{p} \leq 0.05)$. All procedures were approved by the Laval University Animal Ethics Committee.

Results: TAE induced a lower total body weight gain $(21 \%)$, triglycerides $(18 \%)$ and cholesterol (7\%) compared to HFHS vehicle ( $\mathrm{p} \leq 0.05)$. Energy intake and total body fat mass was similar in HFHS and Tarumã groups $(630.0 \pm 12.5 \mathrm{kcal} \times 613.0 \pm 16.0, \mathrm{p}=0.56 ; 13.2 \pm 0.95 \%$ x $11.0 \pm 1.1$, $\mathrm{p}=0,17)$. It was observed a tendency reduction of FFAR2 expression in
Tarumã group. HFHS group obtained higher body weight gain and body fat mass than LF in supporting the establishment of obesity model.

Conclusion: TAE, an extract rich in polyphenols, protected against obesity induced by the HFHS diet, may also suggest a protection for steatosis in the liver. It found a tendency to lower expression of FFAR2/GPR43 on epididymal adipose tissue without change the energy intake and total body fat mass. Further studies are necessary to establish the role of FFAR2/ GPR43 in energy expenditure and its association with polyphenols .

\section{Conflict of Interest: None}

Funding: Research relating to this abstract was funded by National Council for Scientific and Technological Development (CNPq/Brazil)

\section{T1P139}

\section{Testosterone level at android and gynoid types of obesity in} men

\section{Deev D.A.; Vasileva O.V.; Novikova E.G.; Epanchintseva E.A.;} Pinkhasov B.B.; Selyatitskaya V.G.

Research Institute Experimental and Clinical Medicine, Novosibirsk, Russia

Introduction: The purpose of the study was to investigate features of testosterone level inmen with overweight and obesity depending on type of fat distribution in the body.

Methods: The study included 217 men with mean age $37.2 \pm 11.0$ years. Measurements of body weight $(\mathrm{kg})$, body height $(\mathrm{m})$, waist circumference $(\mathrm{WC}, \mathrm{cm})$ and hips circumference $(\mathrm{HC}, \mathrm{cm})$ were taken. The existence of overweight and obesity was determined by calculating the body mass index (BMI). Android (WC/ $\mathrm{HC}>0.95$ ) and gynoid (WC/ $\mathrm{HC}<0.95$ ) types of fat distribution were identified in men with overweight and obesity. Metabolic syndrome (MetS) was diagnosed according to the criteria NCEP-ATP III. Total testosterone level was determined in serum of examined men. Erectile dysfunction was identified by using IIEF-5 international index.

Results: In total sample number of men with normal body weight was $28.1 \%$, with overweight $-42.4 \%$, with obesity $-29.5 \%$. The ratio of android and gynoid types of fat distribution was 0.8:1.0 in men with overweight, and 3.9:1.0 in men with obesity. The MetS was diagnosed in $58.50 \%$ in men with the overweight and android type of fat distribution, and in $20.0 \%$ - with gynoid type of fat distribution; in $84.3 \%$ of men with obesity and android type of fat distribution, and in $30.8 \%$ - with gynoid type of fat distribution. In men with both overweight and obesity who had android type of fat distribution testosterone level was significantly lower (to 25$30 \%$ ) than in men with normal body weight or gynoid type of fat distribution in conjunction with both overweight and obesity. At the same time the testosterone level in men with both overweight and obesity, who had gynoid type of fat distribution, did not differ from that for normal body weight men. Neither in group of men significant correlations were not found between age and testosterone level, while at the same time, mainly in men with android type of fat distribution correlations (direct and inverse) between testosterone level and indicators of the MetS components were found. Significant inverse correlation of testosterone level with the severity of erectile dysfunction was revealed only in men who had android type of fat distribution with overweight and obesity.

Conclusion: Android type of obesity has significant impact on the androgenic status of men, which is manifested in the reduction of testosterone level and in the presence of clinical signs of erectile dysfunction. Gynoid type of obesity doesn't have such strong influence on androgen status in men, which may be due to their significantly lower severity of metabolic disorders and, respectively, the frequency of MetS. 
T1P142

The classical pathway is critical for pathogenic complement activation in patients with abdominal obesity

Sofya, E. ${ }^{1}$;Oxana, D. ${ }^{1}$; Batogab, S. ${ }^{1}$

${ }^{1}$ The National Research Center for Preventive Medicine

Introduction: Recent studies documented the close association between abdominal obesity and the increase of $\mathrm{C} 3$ production in human macrophages. In several reports, $\mathrm{C} 3$ and the degree of $\mathrm{C} 3$ activation (C3a and $\mathrm{C} 3 \mathrm{a}$-desArg) have been linked to cardiovascular disease (CVD). The study aim was to evaluate the $\mathrm{C} 3$-convertase activation of the classical pathway of complement in middle-aged individuals at low cardiovascular risk with abdominal obesity.

Methods: A pilot study, including 45 patients without evidence of atherosclerosis (IMT $<0,9 \mathrm{~mm}$ ) at low CVD risk according to SCORE, was designed. Abdominal obesity was detected according to the IDF criteria (2009). The C3-convertase functional activity (\%) - a key enzyme complex of the classical pathway complement activation, were assessed by using original techniques.

Results: Analysis included 45 participants (mean age: 41(9) years; body mass index: $27(5) \mathrm{kg} / \mathrm{m}^{2}$; and $47 \%$ male). Mean lipid values were as follows: total cholesterol 5.4 (1) mmol/l; LDL-C 3.8 (1) mmol/l; HDL-C 0.98 (0.3) $\mathrm{mmol} / \mathrm{l}$; TG $2.5(1.5-2.1) \mathrm{mmol} / \mathrm{l} .27$ (60\%) participants of the sample had signs of abdominal obesity. There were found significant differences in the hs-CRP of patients with abdominal obesity and without it: 6,5 $\mathrm{mg} / \mathrm{l}(3.3-8.2)$ vs $1,2 \mathrm{mg} / \mathrm{l}(0.8-1.6)$, ( $\mathrm{p}<0,05)$. The hs-CRP levels were associated with WC $(\mathrm{r}=0.5, \mathrm{p}<0.05)$, hypertension $(\mathrm{r}=0.5, \mathrm{p}<0.05)$, TG $(\mathrm{r}=0.4, \mathrm{p}<0.05)$ and mLDL $(\mathrm{r}=0.4, \mathrm{p}<0.05)$, independently of LDL-C. The activity of stabilized C3-convertase was high (mean 18.5 (7.6) $\%)$ in the majority of patients (82\%), independently of BMI, WC, blood pressure, hs-CRP, levels of TG, LDL-C, HDL-C.

Conclusion: Our findings underscore the role of hs-CRP in early atherosclerosis among the asymptomatic middle-age sample with abdominal obesity at low risk of CVD. The observed fact of stabilization of C3 convertase, apparently, can serve as a predictor of the autoimmune nature of abdominal obesity. The association between lipid homeostasis, obesity and innate immune system need to be studied in larger samples.

Conflict of Interest: None Disclosed.

Funding: No Funding.

T1P144

The diagnostic value of serum osteocalcin / HDL-cholesterol level report in assessing the cardiovascular risk in overweight women

Circo, E. '; Ibadula, S. '; Circo, R. ${ }^{1}$; Scrinic, O. ${ }^{1}$

${ }^{1}$ Spitalul Judetean de Urgenta "Sf.Apostol Andrei",Constanta

Aim of the Study: Assessment of serum osteocalcin level in overweight women.

Material and Methods: A retrospective study, conducted over a period of 3 years (2011-2014), on a group of 150 postmenopausal women, the study group was divided into 2 lots within the presence $(n=99)$ or absence $(n=51)$ of metaolic syndrome. Variables assessed: age, waist circumference, body weight, height, body mass index (BMI), blood pressure values (mmHg). Biological: total cholesterol, triglycerides, HDL cholesterol, blood glucose, non HDL - cholesterol, serum osteocalcin.

Results: Women associating MS in comparison with those without MS, showed significantly higher values of BMI, abdominal circumference, triglycerides $(\mathrm{p}<0.001)$. HDL -cholesterol value was significantly higher in subjects without MS $(67.2$ vs $41.6, \mathrm{p}<0.001)$. The group of women with MS had significantly lower values of osteocalcin (12.14 \pm 3.2 vs $17.4 \pm 2.8$, $\mathrm{p}<0.01)$. Serum osteocalcin correlated inversely proportional to adiposity markers represented by BMI $(\mathrm{p}<0.02)$ and glucose $(\mathrm{p}<0.01)$. Serum osteocalcin values were significantly and negatively correlated to the amount of non - HDL - cholesterol ( $\mathrm{p}<0.001)$. Cardio-vascular complications were observed in $86.6 \%$ of cases, of which hypertension (52\%), followed by $22.6 \%$ stable angina of effort.

Conclusions: Establishing a correlation between non-HDL cholesterol and osteocalcin is of real interest being the expression of pro-atherogenic lipid fractions and a parameter of bone metabolism such as ostecalcin. Keywords: overweight, metabolic syndrome, osteocalcin, non-HDL-colesterol, cardiovascular risk.

\section{T1P145}

\section{The effect of bariatric surgery type on levothyroxine dose in} morbidly obese patients with hypothyroidism

Pedro, J. ${ }^{1}$; Cunha, F. ${ }^{1}$; Costa, M. ${ }^{1}$; Magalhães, D. ${ }^{\text {; }}$ Bettencourt-Silva, R. ${ }^{\text {'; }}$ Neves, J. '; Souteiro, P. ; Gonçalves, V. '; Queirós, J. '; Oliveira, S. '; Freitas, P. '; Varela, A. '; Carvalho, D. ${ }^{1}$

${ }^{1}$ Centro Hospitalar São João

Introduction: Bariatric surgery (BS) is an effective treatment for morbid obesity. There are doubts about the effect of different types of BS on the absorption of levothyroxine (Lt4). We compared doses of Lt4 and their variation $(\Delta$ dose $)$, in patients with hypothyroidism who underwent either malabsorptive (MS) or restrictive (RS) surgery and studied predictors of decrease of Lt4 dose.

Methods: Retrospective study of morbidly obese hypothyroid patients submitted to BS between January / 2009 and June / 2015. Excluded: revision surgery or lack of data regarding Lt 4 dose or weight. $\Delta$ dose $=$ Dose Lt4 after lyear - basal Lt4. We compared RS ('sleeve' gastrectomy or adjustable gastric banding) with MS (Roux-en-Y gastric bypass) patients. We built a multivariable logistic regression model to study predictors of Lt4 dose changes.

Results: We studied 90 patients: 53 submitted to MS and 37 to RS. Mean age was $45 \pm 11$ years; $6.7 \%$ were men. Patients submitted to MS lost more weight and had lower basal free T4 (FT4) than those submitted to RS. Lt4 dose decreased in the 1st year in both groups, but there was no significant difference in the Lt 4 dose before BS $(116 \pm 51$ vs $114 \pm 43 ; \mathrm{p}=0.78)$, after one year $(105 \pm 50$ vs $104 \pm 44, \mathrm{p}=0.95)$ or $\Delta$ dose (median (IQR): $0(-25$; $0)$ vs $0(-21 ; 0) ; \mathrm{p}=0.58$ ] between RS and MS. The Lt4 dose remained unchanged in $49(54.4 \%)$ patients, increased in $11(12.2 \%)$ and decreased in 60 (33.3\%), without significant differences between RS and MS. In the multivariate analysis, BS type, age, BMI variation, initial dose of Lt4 or total thyroidectomy were not independent predictors of Lt 4 dose reduction. Conclusions: In more than half of morbidly obese hypothyroid patients submitted to BS the Lt 4 dose remained unchanged 1 year after surgery. There were no differences in dose variation of Lt4 between RS and MS, suggesting that MS may not affect Lt4 absorption. Neither BS type nor BMI variation were independent predictors of LT4 dose changes.

\section{T1P146}

\section{The effect of different durations of a high fat diet on the contractile performance of isolated mouse soleus, EDL and diaphragm muscles}

\section{Hurst, J. ${ }^{1}$; Hill, C. ${ }^{1}$; Jame, ${ }^{1} .{ }^{\prime}$; Cox, V. ${ }^{1}$; Tallis, J. ${ }^{1}$}

${ }^{1}$ School of Health \& Life Sciences, Applied Biological \& Exercise Sciences, Coventry University, Priory Street, Coventry, CV1 5FB, UK

Introduction: Understanding the effect of obesity on skeletal muscle function should be of primary importance given its role in locomotion and metabolism, and the importance of both of these factors on managing weight status. Recent evidence using isolated skeletal muscle has demonstrated a significant muscle specific relationship between obesity and a reduction in contractile performance which is proposed to further contribute to the negative cycle of obesity. Despite this there is a lack of evidence that determines the timeframe in which the decline in muscle performance occurs, and as such, the present study aims to uniquely exam- 
ine the dose response relationship between a high fat diet and changes in skeletal muscle performance using an isolated muscle work-loop model. Methods: 150 female mice (CD-1) were randomly assigned to a control group or a group receiving $2,4,8$, or 12 weeks of a high calorie forage diet $(\mathrm{N}=8$ or greater in each case). At 20 weeks of age the soleus (SOL), extensor digitorum longus (EDL), and diaphragm (DIA) muscles were isolated. The absolute and normalised (relative to muscle mass) isometric tetanic responses, work loop $(\mathrm{WL})$ power and resistance to fatigue were measured for each muscle.

Results: Body mass increased significantly in all treatment groups when compared to the lean controls. When compared to the lean controls SOL of the 8 and 12 week feeding groups produced significantly lower normalised WL power. Absolute WL power Isometric stress, isometric force and fatigue resistance were unaffected. EDL of the 8 and 12-week treatment groups produced significantly lower normalised WL power decreased fatigue resistance and decreased isometric stress but demonstrated no change in isometric force or absolute WL power. DIA from the 12 -week treatment group produced decreased normalised WL power, 8 and 12-week treatment groups produced significantly lower isometric stress whilst isometric force and fatigue resistance remained unaffected. Conclusion: These findings add weight previous literature demonstrating a muscle specific reduction in the contractile performance of isolated skeletal muscle that is likely related to in vivo mechanical role, fibre type and metabolic profile. Moreover, these results are the first to demonstrate that 8 weeks high fat diet feeding is needed to cause a significant change in muscle contractility. Despite a number of studies demonstrating an obesity associated decline in metabolic capacity, insulin sensitivity and the maintenance and regeneration of contractile proteins using short duration feeding regimes ( $<$ than 8 weeks), the present results indicate that longer duration feeding is needed for such mechanisms to cause meaningful changes in muscle performance.

None Disclosed

No Funding

\section{T1P147}

\section{The faecal microbiota in subjects with morbid obesity; the effects of comorbidity, diet and drugs}

\section{Farup, P.'; Winther, R. ${ }^{2}$; Kvehaugen, A. ${ }^{2}$; Aasbrenn, M.}

${ }^{1}$ Norwegian University of Science and Technology, Trondheim, Norway, ${ }^{2}$ Innlandet Hospital Trust, GjøVik, Norway

Introduction: An abnormal faecal microbiota (dysbiosis) is common in subjects with morbid obesity. The microbiota regulates the energy metabolism, and dysbiosis could be a causal factor for obesity.

Aim: To study the prevalence of, and factors associated with faecal dysbiosis in subjects with morbid obesity.

Patients and Methods: Consecutive subjects with BMI $>40$ or $>35 \mathrm{~kg} / \mathrm{m}^{2}$ with complications referred to a hospital unit for bariatric surgery, were evaluated for participation in a clinical study. A medical history including comorbidity and demographics was noted, a clinical examination was performed, and the use of drugs was noted. A validated Food Frequency Questionnaire was used for the calculation of the daily intake of food, nutrients, energy and non-caloric artificial sweeteners (NAS). One unit of NAS was defined as $100 \mathrm{ml}$ beverage with NAS or 2 sweeteners. Faecal dysbiosis was assessed with GA-map ${ }^{\text {nt }}$ Dysbiosis Test (Genetic Analysis, Oslo, Norway) and reported as Dysbiosis Index (DI) scores 1-5. High values indicate more severe dysbiosis and score $\leq 2$ no dysbiosis. The results are given as mean, correlation coefficients ( $r$ and rho), estimates (E) from the ordinal logistic regression, and level of significance (p-values).

Results: Out of 350 consecutive subjects, 159 were included in a clinical study. 104 of the subjects in the study provided a faecal test (men/women: $18(17 \%) / 86(83 \%)$; mean age (SD): 44 years (9)) and were included in the present analyses. Dysbiosis was present in $64 \%$ of the subjects. DIscore: mean (SD): 2.9 (1.3); scores 1-5 were seen in 20 (19\%), 18 (17\%), 35 (34\%), 14 (14\%) and 17 (16\%) subjects respectively. DI was associated with gender (men/women: $3.5 / 2.8, p=0,034)$, intake of coffee $(r=-0.247$, $\mathrm{p}=0.013$ ), diabetes (yes/no: $3.5 / 2.7, \mathrm{p}=0.010$ ), use of metformin (yes/ no: $3.8 / 2.7 ; \mathrm{p}=0.002)$ and intake of NAS (rho $=0.389, \mathrm{p}<0.001)$ in univariable analyses. In mulitvariabel analyses (ordinal logistic regression) adjusted for age, gender, intake of coffee, diabetes and use of metformin, only NAS ( $\mathrm{E}=0.07 ; 95 \% \mathrm{CI} 0.02$ to $0.12 ; \mathrm{p}=0.002)$ and use of metformin $(\mathrm{E}=1.37 ; 95 \% \mathrm{CI} 0.23$ to $2.50 ; \mathrm{p}=0.019)$ were significantly associated with DI.

Conclusion: The prevalence of dysbiosis was high. The significant correlation between faecal dysbiosis and intake of NAS might indicate a causal relationship. The correlation with metformin is well-known. An unfavourable effect of NAS, such as induction or maintenance of obesity, deserves exploration.

Conflicts of interest: None disclosed.

Funding: Innlandet Hospital Trust, Brumunddal, Norway

\section{T1P148 \\ The first experience of liraglutide $\mathbf{3 . 0} \mathbf{~ m g}$ in Russia and its influence on metabolic risk factors in obese patients}

\section{Kseniya, K. ${ }^{1}$; Ekaterina, T. '; Nataliya, M. ' ; Ekaterina, E. '; Oksana, L. ${ }^{1}$ \\ ${ }^{1}$ Endocrinology Research Center, Moscow, Russian Federation}

Introduction: Obesity is the chronic disease is associated with cardiometabolic markers of risk for cardiovascular disease and type 2 diabetes. In 2016 in Russia for the treatment of obese patients was registered liraglutide $3.0 \mathrm{mg}$.

Objective: To study the efficacy of liraglutide $3.0 \mathrm{mg}$ and its impact on metabolic risk factors in patients with obesity.

Methods: 30 patients aged 25-59 years with obesity without type 2 diabetes are examined. The patients were treated liraglutide $3.0 \mathrm{mg}$ for 3 months. Examination included measurement of anthropometric parameters, blood sampling to estimate parameters of lipid and carbohydrate metabolism.

Results: After 3 months of therapy noted a significant reduction in the anthropometric parameters: the median weight loss was $8.3 \mathrm{~kg}$, BMI 4.3 , and waist circumference $-9.0 \mathrm{~cm}$, respectively $(\mathrm{p}<0,005)$. Clinically significant weight loss $(\geq 5 \%)$ reached $100 \%$ of patients, $\geq 10 \%-86,0 \%$ of patients. The therapy of liraglutide $3.0 \mathrm{mg}$ showed a significant reduction in fasting plasma glucose of $9.7 \%$ and up to 120 minutes OGTT by $16.2 \%, 20.8 \%$ insulin, HOMA-IR index by $32.1 \%$, total cholesterol by $9.7 \%$ LDL-cholesterol by $13.2 \%$, triglycerides by $25 \%$ and a significant increase in HDL-cholesterol by $18.2 \%(\mathrm{p}<0.0001)$. All the patients on liraglutide therapy reported significant appetite reduction, increase of satiety and decrease of the food consumption. Liraglutide $3.0 \mathrm{mg}$ therapy was well tolerated by patients, the most frequency adverse event was transient nausea at the beginning of the therapy ( $30 \%$ of patients).

Conclusion: Liraglutide $3.0 \mathrm{mg}$ therapy in obese patients results in a significant body weight reduction and improvement of associated metabolic disorders.

\section{Conflict of Interest: None disclosed}

Funding: No Funding

\section{T1P149}

\section{The importance of postingestive mechanisms in feeding decisions}

\section{Fernandes, A. ; Almeida, J. ${ }^{1}$; Costa, R. $^{1}$; Oliveira-Maia, . $^{1}$}

${ }^{1}$ Champalimaud Research and Clinical Centre, Champalimaud Centre for the Unknown, Lisboa, Portugal

Control of food intake is under regulation of a complex brain circuit involving close relationships between homeostatic and non-homeostatic factors. Non-homeostatic factors are mainly associated to the highly reinforcing properties of food, such as palatability, thought to be the main 
reinforcing property of food. However, recent findings have demonstrated the importance of caloric content in food reinforcement. Here, we proposed to determine the role of postingestive feedback in an instrumental reinforcement-learning task. To isolate postingestive stimulation, sucrose or a non-caloric sweetener (sucralose) was injected in Bl6 mice through an intragastric catheter, contingent upon lever pressing. Mice significantly increased lever pressing to obtain intragastric sucrose, but not sucralose. Consistently, in a simultaneous 2-lever choice task, mice showed a preference for the lever associated to intragastric sucrose over another lever associated to intragastric delivery of sucralose. Furthermore, Trpm5-/mice, where sweet orosensory feedback is abolished, showed increased lever pressing to obtain access to sucrose, but not sucralose, confirming that postingestive mechanisms are sufficient to sustain instrumental behaviours. To explore the importance of dopaminergic neuronal activity for these behaviors, the single-lever instrumental task was repeated in mice with absent NMDA receptors in ventral tegmental area (VTA) dopamine-producing neurons. As expected, both control and $\mathrm{KO}$ mice increased lever pressing to obtain access to sucrose for oral consumption. However, only control, but not KO mice, increased lever pressing to obtain intragastric injections of sucrose, indicating that functional VTA dopaminergic neurons are fundamental to sustain instrumental behaviours through postingestive feedback. Towards clarification of the peripheral mechanisms mediating these postingestive-dependent behavioral effects, we repeated the single-lever instrumental task in $\mathrm{Bl} 6$ mice with lesions of the vagus nerve. In these animal, we observed a significant decrease in lever pressing behavior for intragastric infusion of sucrose, indicating that part of the postingestive feedback depends on integrity of the vagus nerve. Thus, we have demonstrated that mice can learn a reinforcement learning task based exclusively in postingestive reinforcement, and that dopamine-producing neurons in the VTA and vagus nerve integrity are necessary for these postingestive-dependent behavior to be acquired.

Conflit of interests: None Disclosed

Funding: Research relating to this abstract was funded by FCT (SFRH/ BPD/88097/2012 and FCT/HMSP-ICJ/0020/2011) and Bial Foundation (BIAL-BIC-176/10)

\section{T1P150 \\ MASP Activity And The Thrombosis Risk In Patients With Obesity}

Batogab, S. '; Sofya, E. ${ }^{1}$; Oxana, D. ${ }^{1}$

${ }^{1}$ The National Research Center for Preventive Medicine

Introduction: The lectin pathway's mannose binding lectin associated serine proteases (MASP) activates coagulation factors but its role in obesity is unknown. The study aim was to evaluate the MASP activity of the lectin pathway of the complement system activation among middle-aged individuals at low cardiovascular risk with obesity.

Methods: A pilot study was designed that includes 30 patients without evidence of atherosclerosis, with low CVD risk according to SCORE. Abdominal obesity was detected according to the IDF criteria (2009). The MASP activity and the cholesterol of modified LDL level were assessed using original techniques.

Results: Analysis included 30 patients (mean age: 41(9) years; body mass index: 27(6) kg/m2; and 53\% male). Among them 23\% (7) with overweight, $27 \%$ (8) with obesity. $18(63 \%)$ participants of the sample had signs of abdominal obesity ( 9 male and 9 female). Mean lipid values were as follows: total cholesterol: 5.4 (1) mmol/l; LDL-C: 3.7 (1) mmol/l; mLDL-C: 1.04 (0.4) mmol/l; HDL-C: 0.97 (0.2) mmol/l; triglycerides 1.85 $(1.2-2.5) \mathrm{mmol} / \mathrm{l}$. Median of the fasting glucose - $5.4(4.9-5.5) \mathrm{mmol} / \mathrm{l}$. 7 patients with obesity demonstrated the high MASP activity (over 10\%). The MASP activity was associated with mLDL-C (Spearman up to 0.3, $\mathrm{p}<0.05$ ), independently of LDL-C and other variables. Significant differences in the MASP activity among patients with abdominal obesity and without it $(\mathrm{p}<0,05)$ were found. The median of the MASP activity among patients with abdominal obesity was $8.5 \%(5-13)$, while the median of the MASP activity level among patients without abdominal obesity was $4 \%(0-9)$.

Conclusion: The discovered information about high MASP activity among patients with general obesity at low risk of cardiovascular disease can serve as a predictor of the risk of thrombosis. Determination of the functional MASP activity analysis opens a qualitatively new approach to risk assessment of thrombosis.

Conflict of Interest: None Disclosed.

Funding: No Funding.

\section{T1P151}

\section{The relation between PPAR- $Y$ gene expression in visceral and subcutaneous adipose tissues and food group intakes}

\author{
Zarkesh, M. ' ' Yuzbashian, E. ${ }^{2}$; Tabaei, K. ${ }^{3}$; Zadeh-Vakili, A. '; \\ Daneshafrooz, A. '; Asghari, G. '; Mirmiran, P. '; Hedayati, M. '; \\ Mahmoodi, B. ${ }^{2}$
}

${ }^{1}$ Cellular and Molecular Endocrine Research Center, Research Institute for Endocrine Sciences, Shahid Beheshti University of Medical Sciences, Tehran, Iran, ${ }^{2}$ Nutrition And Endocrine Research Center, Research Institute For Endocrine Sciences, Shahid Beheshti University Of Medical Sciences, Tehran, Iran, ${ }^{3}$ School Of Advanced Sciences In Medicine, Tehran Medical Sciences Branch, Islamic Azad University, Tehran, Iran

Objective: The evidence comes from animal studies showed that PPAR- $\gamma$ gene expression in some tissues may be changed by alterations in dietary food intakes. The aim of this study was to evaluate the association of grains, fruits, vegetables, legumes, meats, and dairy consumption with PPAR- $\gamma$ gene expression in adipose tissues among healthy subjects.

Methods: A total of 97 subjects ( 41 non-obese $\left(\mathrm{BMI}<30 \mathrm{~kg} / \mathrm{m}^{2}\right), 18$ obese $\left(\mathrm{BMI} \geq 30\right.$ and $\left.<40 \mathrm{~kg} / \mathrm{m}^{2}\right)$, and 38 morbid obese $\left.\left(\mathrm{BMI} \geq 40 \mathrm{~kg} / \mathrm{m}^{2}\right)\right)$ were eligible. The habitual dietary intakes of participants during the previous year were collected using a valid and reliable food frequency questionnaire. Daily intake of grains, fruits, vegetables, legumes, meats, and the diary was calculated. Visceral and subcutaneous adipose tissues were obtained during open abdominal surgery with minimal impact on dietary intake including appendicitis and hernia. The mRNA levels of PPAR- $\gamma$ in visceral and subcutaneous adipose tissues were assessed using Real-Time PCR.

Results: PPAR- $\gamma$ expression in the visceral adipose tissue of non-obese subjects was negatively correlated with the grain $(r=-0.302, P=0.046)$. Among obese subjects, PPAR- $\gamma$ expression in visceral adipose tissue had the correlation with meat intake $(r=-0.467, P=0.049)$ and in subcutaneous adipose tissue of obese subjects was negatively correlated with grains $(r=-0.533, P=0.026)$. PPAR- $\gamma$ gene expression in visceral adipose tissue was correlated with the grain $(r=-0.343, P=0.043)$, and legumes ( $\mathrm{r}=-0.319, P=0.049$ ), as well as subcutaneous adipose tissue PPAR- $\gamma$ was only correlated with legumes $(r=-0.318, P=0.0049)$ among morbidly obese subjects.

Conclusions: PPAR- $\gamma$ gene expression in visceral adipose tissue has a negative correlation with low-glycemic food group. The results provide some insights into the improvements in dietary habits that can be made for obesity prevention.

Conflict of Interest: None of the authors has any personal or financial conflict of interest.

Funding: No Funding.

Acknowledgment: The authors would like to thank Dr. Mohammad-Reza Ebrahimi for surgical procedures. 
T1P152

\section{The roots of Atractylodes macrocephala Koidzumi prevents high fat diet induced obesity in mice with increasing ampk and PGC-1 $a$ expressions}

\section{Song, M. ${ }^{1}$; Kang, A. ${ }^{2}$; Kang, S. ${ }^{2}$; Jung, $\mathrm{H}^{2}$}

${ }^{1}$ Department of Rehabilitation Medicine of Korean Medicine, College of Korean Medicine, Dongguk University, ${ }^{2}$ Department Of Herbology, College Of Korean Medicine, Dongguk University

Introduction: The root of Atractylodes macrocephala Koidzumi (AMK) has been reported to prevent obesity and improve glucose metabolism; however, the mechanism responsible for these effects has not been elucidated. Interestingly, in a previous study, we found that AMK extract increased mitochondrial biogenesis in mouse skeletal muscle cells and based on these evidence, we hypothesized that AMK extract might prevent obesity by regulating energy metabolism in skeletal muscle.

Methods: Five-week-old C5BL/6 mice were fed a high-fat diet (HFD) containing or not containing AMK (100 or $300 \mathrm{mg} / \mathrm{kg}$ ) or metformin (250 $\mathrm{mg} / \mathrm{kg}$ ) for 16 weeks. Body weight, serum and hepatic lipids and glucose tolerance were examined. Adenosine monophosphate-activated protein kinase $(\mathrm{AMPK})$ and peroxisome proliferator-activatd receptor gamma coactivator-1 alpha (PGC-1á) expressions were investigated in skeletal muscle.

Results: AMK dose-dependently prevented HFD-induced body weight increases despite no significant change in food intake.AMK extract dose-dependently decreased lipid accumulation in livers as determined by oil-red-O staining. In adipose tissues, adipocyte areas were significantly smaller in the AMK extract treatment group than in the HFD group. In addition, hyperglycemia, insulinemia, and glucose tolerance were dose-dependently improved by AMK extract administration. Furthermore, AMK increased the PGC-1á expression and phospholylation of AMPK in skeletal muscle tissues.

Conclusion: The present study demonstrates that AMK can prevent diet-induced obesity, glucose tolerance in mouse models of obesity. The mechanism responsible for the effect of AMK appears to be related to the up-regulation of energy metabolism in skeletal muscle, which at the molecular level may be associated with PGC-1á and AMPK activation.

None Disclosed

Research relating to this abstract was funded by the Korean Health Technology R\&D Project through the Korean Health Industry Development Institute (KHIDI) funded by the Ministry of Health \& Welfare, Republic of Korea (grant number: HI15C0127)

\section{T1P153}

The serum omentin and vaspin level in rat model of type 2 diabetes: Fed with high-fat diet and low dose of streptozotocin.

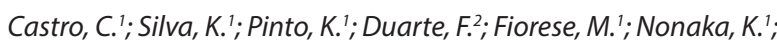
Aníbal, F. ; Duarte, A. ${ }^{1}$; Furino, V. ${ }^{3}$; Fabrizzi, $F^{3}$

${ }^{1}$ Federal University of São Carlos, ${ }^{2}$ Ophthalmic Instrumentation Laboratory Eesc-Usp- Brazil, ${ }^{3}$

Introduction: Vaspin, a visceral adipose tissue-derived serine protease inhibitor and omentin are considered new identified adipokines, mainly produced by adipose tissue and also related to obesity and its comorbidities with type 2 diabetes. Performed investigations indicate in vitro and in animal experimental models that the discussed molecules may favorably influence glucose metabolism and sensitivity of tissues to action of insulin. The analyzes on the concentrations of omentin and vaspin, depends on the degree of sensitivity of the tissue to the action of insulin and in the diabetes it is relatively few and their results are not conclusive. Therefore, in the current research we investigated the behavior of omentin and vaspin in serum and, their relation to inflammation in obesity-related type 2 diabetes in an experimental model for type 2 diabetes associating with a high-fat diet and a low dose of streptozotocin.
Methods: Wistar rats $(\sim 200 \mathrm{~g})$ were randomly divided into 2 groups: a non-diabetic group $(n=6)$ and a diabetic group of rats fed on a high-fat diet $(n=6)$ and a low dose of streptozotocin (Sigma "). All procedures were approved by the Brazilian Ethics Committee (\#008/2013). Body weight and food intake were recorded daily. Blood glucose levels were assessed by the tail in the end of the diabetes induction period (seven weeks), as well as the Insulin Tolerance Test (ITT). Insulin, Omentin, Vaspin, Adiponectin and inflammatory citokines, IL-6, IL-8(CXCL8), TNF- $\alpha$ and C-Reactive Protein (CRP) were analyzed in serum using the enzyme-linked immunosorbent assay (ELISA).

Results:Our results showed that IL- 8 and CRP serum in the diabetic group were significantly higher than in the non-diabetic group. Vaspin and Adiponectin values were lower for the diabetic group than in the non-diabetic group. Omentin, IL- 6 and TNF- $\alpha$ values did not differ between the groups.

Conclusion:Our results provided evidence that both, metabolisms of adipose tissue and adipokine secretion can be affected in the diabetic rats. The omentin showed no difference between the groups, although the vaspin values decreased in the diabetic group.

Conflict of Interest: None Disclosed

Funding: FAPESP (2013/12797-0)

\section{T1P154}

\section{Thermal imaging in the investigation of active brown adipose tissue in obese adults}

\section{Hadi, ${ }^{1}{ }^{1}$; Frascati, A. ${ }^{1}$; Vettor, $R .{ }^{1}$; Rossato, M. ${ }^{1}$}

'Internal Medicine 3, Department of Medicine - DIMED, University of Padova, Via Giustiniani 2, 35100, Padova, Italy.

Introduction: Brown adipose tissue (BAT) is the main site for non-shivering thermogenesis in mammals. It was shown that adult humans possess functionally active BAT depots and that the presence of active BAT influences human metabolism. Hence, this tissue is now regarded as an interesting target for therapies against obesity and associated diseases by increasing energy expenditure. Thermal imaging using infrared thermography (IRT) has emerged as a potential safe, rapid and inexpensive technique for detecting BAT in human. However, little attention has been given on the reliability of this method in obese subjects.

Methods: The present study investigates the capacity of IRT in detecting activated supraclavicular (SCV) BAT in 14 lean and 16 mildly obese young adults after acute cold exposure. Both groups were comparable for age, gender and race. By using IRT we measured the temperature of the skin overlying the SCV/lateral neck region and the sternal area at baseline and after acute cold stimulation. Additionally, energy expenditure was measured by indirect calorimetry and body composition was estimated using bioelectrical impedance analysis (BIA).

Results: Energy expenditure, respiratory quotient (RQ) and SCV skin temperature significantly increased in lean subjects upon cold exposure, while no significant changes were detected in the obese group. Furthermore, cold-induced variations in SCV skin temperature of obese subjects showed a negative correlation with body mass index (BMI).

Conclusion: This study suggests that in lean individuals BAT is a rapidly activated thermogenic tissue possibly involved in the regulation of energy balance and can be indirectly assessed using IRT. Concerning obese subjects, BAT seems less prone to be activated by cold exposure with the degree of adiposity representing a limiting factor for the indirect detection of BAT activation by measuring the skin temperature overlying BAT.

Conflict of interests: The authors declare that they have no conflict of interests 
T1P155

\section{Thermic effect of a protein rich meal in obstructive sleep apnea patients}

\author{
Re, M. ${ }^{1}$; Melo, C. ; Santos, M. ${ }^{1}$; Zimberg, I. ${ }^{1}$; Tufik, S. ; Mello, M. ${ }^{2}$
}

${ }^{1}$ Universidade Federal de São Paulo, ${ }^{2}$ Universidade Federal De São Paulo

Rationale: Higher food intake at evening period is related to negative aspects of sleep quality in healthy subjects. Changes in quantity and nutrients composition of the evening meal could be an important ally for Obstructive sleep apnea treatment.

Objectives: To determine the relationship between habitual food intake, resting energy expenditure and sleep pattern in OSA patients.

Methods:Forty five OSA obese male were included in the study. All participants were submitted to a nocturnal polysomnography, body composition measurements by plethysmography, resting energy expenditure (REE) by indirect calorimetry and completed a 3-day food diary to food intake evaluations.

Measurements and Results: No differences in body composition, REE and food intake was found between moderate and severe OSA groups. A trend for higher energy intake in severe OSA group was observed compared to moderate group $(p=0.08)$. Significant association of AHI with body weight, BMI and REE were found. Higher food intake in evening period was positively correlated with NREM sleep stage 1 , total arousals and AHI and negatively correlated with NREM sleep stage 3 and sleep efficiency. Also, nutrient composition of the evening meal was not correlated with sleep pattern in our sample.

Conclusions: We conclude that in our sample there is no relationship between total energy, macronutrient intake and REE and the severity of OSA, but higher food intake in evening period could be deleterious for sleep quality and OSA severity in moderate and severe sleep apnea patients.

\section{T1P156}

\section{Total antioxidant capacity and apelin gene expression in visceral and subcutaneous adipose tissues among obese and non-obese subjects}

Safarian, M. ${ }^{\text {'; }}$ Yuzbashian, E. ${ }^{2}$; Zarkesh, M. ${ }^{3}$; Asghari, G. ${ }^{2}$; Mahmoodi, B. ${ }^{2}$; Mirmiran, P.'; Hedayati, $M .^{3}$

${ }^{1}$ Biochemistry of Nutrition Research Center, School of Medicine, Mashhad University of Medical Sciences, Mashhad, Iran, ${ }^{2}$ Nutrition And Endocrine Research Center, Research Institute For Endocrine Sciences, Shahid Beheshti University Of Medical Sciences, Tehran, Iran, ${ }^{3}$ Cellular And Molecular Endocrine Research Center, Research Institute For Endocrine Sciences, Shahid Beheshti University Of Medical Sciences, Tehran, Iran

Introduction: Obesity is associated with increased oxidative stress, and the exaggeration of oxidative damage is considered important in obesity complications. Anti-oxidant based treatments have emerged as effective approaches to managing metabolic disturbance induced by the alteration in hormones and mediators. The aim of the study was to demonstrate the relationship between total antioxidant capacity (TAC) and apelin mRNA expression among morbid obese and non-obese subjects.

Methods: Visceral and subcutaneous adipose tissue were collected during open abdominal surgery from 32 morbidly obese (BMI: $\left.45.3 \mathrm{~kg} / \mathrm{m}^{2}\right)$ and 32 age-sex-matched non-obese (BMI: $25.6 \mathrm{~kg} / \mathrm{m}^{2}$ ). Usual dietary intake of participants was measured using a validated semi-quantitative food frequency questionnaire and their dietary TAC was estimated. The gene expressions of apelin in visceral and subcutaneous adipose tissue were assessed by Real-Time PCR.

Results: Dietary TAC was not significantly different between the morbidly obese and not-obese group. Apelin gene expression in visceral adipose tissue was correlated with TAC among morbid obese, but not in nonobese subjects $(\beta=0.527, \mathrm{P}=0.002$ and $\beta=0.338, \mathrm{P}=0.054$, respectively). However, a significant correlation also existed between dietary TAC and apelin gene expression in subcutaneous adipose tissue in both morbidly obese and non-obese subjects $(\beta=0.551, p=0.001$ and $\beta=0.387$,
$\mathrm{P}=0.032$, respectively). After adjustment for energy intake, apelin gene expression in visceral and subcutaneous adipose tissues was correlated with TAC $(\beta=0.460, P=0.010$ and $\beta=0.582, P=0.003$, respectively) among morbidly obese subjects. There was no significant finding for nonobese subjects after adjustment for energy intake.

Conclusions: We demonstrated that dietary antioxidant intakes have the positive correlation with apelin gene expression independent of total energy intake among morbidly obese subjects.

\section{T1P157 \\ Transcriptomic analysis reveals a strong down regulation of immune response pathways in ferret aortic perivascular adipose tissue by cold exposure: Potential implications for cardiovascular health}

Reynés, B. '; Schothorst, E. '; García-Ruiz, E. '; Keijer, J. ${ }^{2}$; Oliver, P. '; Palou, A. ${ }^{\prime}$

'Laboratory of Molecular Biology, Nutrition and Biotechnology, Universitat de les Illes Balears and CIBER de Fisiopatología de la Obesidad y Nutrición (CIBERobn), Palma de Mallorca, Spain, ${ }^{2}$ Human And Animal Physiology, Wageningen University, Wageningen, The Netherlands

Introduction: The role of perivascular adipose tissue (PVAT) in cardiovascular disease (CVD) is under active investigation, given its ability to regulate vascular inflammation, positively and negatively, which, in turn, modulates vascular health. A pro-inflammatory state of PVAT has been related with atherosclerosis development, while an anti-inflammatory state has been shown to be protective. Cold exposure is known to affect immune response and, thus, could affect the pathogenesis of cardiovascular diseases. Here we used a promising animal model (ferrets, Mustela putorius furo) aiming to explore the effect of cold exposure on PVAT transcriptomic profile to gain insight into potential CVD treatment strategies. Methods: We designed a ferret-specific Agilent microarray and performed a global gene expression analysis in aortic PVAT (aPVAT) of cold-exposed ferrets (one week at $4^{\circ} \mathrm{C}$ ) in comparison to controls. Ferrets were selected due to their similarity to humans in terms of immune response and adipose tissue organization and metabolism making them a well-suitable translational animal model.

Results: Our transcriptome analysis using a ferret-specific whole genome microarray revealed a clear and strong down-regulation of immune response-related genes induced by cold exposure in aPVAT. Affected genes, including the top down-regulated, were related to processes such as antigen recognition and presentation, cytokine signalling and immune system maturation and activation. This immunosuppressive effect was depot-specific, as it was not observed in the inguinal subcutaneous depot. Interestingly, the immunosuppressive gene expression pattern observed in aPVAT was also evident in peripheral blood mononuclear cells (PBMC).

Conclusion: These results reveal that cold exposure produces an inhibition of immune response-related pathways in aPVAT, reflected in PBMC, indicative of an anti-inflammatory response, which could contribute to a protective cardiovascular profile. Moreover, PBMC could be used to examine the potential of cold exposure as cardioprotective anti-inflammatory strategy in translational human studies.

None Disclosed

CIBER de Fisiopatología de la Obesidad y Nutrición is an initiative of the ISCIII. This work was supported by the Spanish Government (Ministerio de Economía y Competitividad, INTERBIOBES -AGL2015-67019-P), by the EU FP7 project DIABAT (HEALTH-F2-2011-278373) and by the University of the Balearic Islands (BIOTHERM, FA42/2016). E. García-Ruiz was recipient of a fellowship from the University of the Balearic Islands. 
T1P158

\section{Validation, precision testing \& systems identification of a modern indirect calorimeter}

Corley, B. ${ }^{1}$; Hall, D. ${ }^{1}$; Carroll, D. ${ }^{1}$; Krebs, A. ${ }^{1}$

'University of Otago, Wellington

Introduction: Indirect calorimetry is an essential tool in studies of energy expenditure however the technique is complex. Quality recordings are dependent on operator experience, a robust protocol and a valid, consistent approach to data analysis. Moreover, as a calorimeter measurement is a complex system, the output generated is only relatable to the input signal of interest by understanding the system completely. We outline a method to benchmark operators, test protocols and simultaneously define the input-response relationship of an indirect calorimeter for the purpose of accurate data analysis.

Methods: A nitrogen gas validation technique was used to evaluate validity \& precision of the Promethion Metabolic Cart. A programmable controlled mass-flow of nitrogen was used to simulate physiologic-range oxygen depletion. The mass flow input was synchronously recorded with the output. Ethanol burn tests were performed for comparison. The input-output relationship of the system was estimated from this data and applied to more complex human waveform outputs to determine their input. Results: Oxygen depletion was measured with an accuracy of $98.5 \%$ and a precision of $99 \%$. The ethanol burns resulted in a large overestimation of oxygen depletion despite an accurate respiratory quotient.

Conclusion: Mass flow controlled nitrogen provides a convenient, cheap, $\&$ reproducible means of validating an indirect calorimeter and accurately defining the impulse-response of the system.

Conflict of Interest: None. None of the Investigators have affiliations with Sable Systems Ltd.

Funding: Dr Corley was funded by the Health Research Council of New Zealand.

\section{T1P159}

\section{Various implications of diabetes mellitus and adipose tissue topography in the achievement of osteoporosis and fragility fractures in obese female patients}

Circo, E. ' Ibadula, S. ${ }^{\prime}$; Circo, R. $^{1}$

${ }^{1}$ Spitalul Judetean de Urgenta "Sf.Apostol Andrei",Constanta

Introduction: Osteodensitometry (DXA) performed in women with premature ovarian failure that associate diabetes mellitus (DM) and/or obesity may reveal interesting data and controversial issues.

Aim of the study:To assess the correlation between premature ovarian failure and the bone mineral density (BMD) in obese women with diabetes melliturs and to establish the possible disadvantages between body mass index and body fat distribution.

Methods: Our study included 71 women with obesity and premature menopause with a mean age of $53 \pm 7.4$ years. The patients were divided in 3 groups: Group 1 - 15 women diagnosed with type I DM (insulino-dependent); Group 2 - 23 women with type II DM (non insulino-dependent); Group 3 - 33 women with obesity without DM (either I or II). The patients included in the study were diagnosed with DM at least 5 ago and obesity at least 10 years ago. We calculated BMI (body mass index),WHR (waist-to hip ratio) and measured BMD by DXA.The presence and incidence of fragile fractures was taken into consideration.

Results: The incidence of osteoporosis registered in women with /without DM was: Group 1- n =8 (53.3\%); Group 2- n= 11(47.8\%), Group 3$\mathrm{n}=5(15.1 \%)$. The patients with osteoporosis in our study were $n=24$ $(\mathrm{p}<0.001)$.The values for incidence of osteoporosis in relation to BMI were: Class $1=30 \pm 1.2, \mathrm{n}=34$ cases; Class $2=35 \pm 2.4, \mathrm{n}=27$ cases, Class $3=39 \pm 1.6, \mathrm{n}=10$ cases. The incidence of osteoporosis in relation to fat tissue distribution was considered as generalized $n=15(62,5 \%)$ and abdominal adipose tissue $n=9(37.5 \%)(\mathrm{p}<0.001)$. The incidence of fragile fractures in patients with osteoporosis was $n=10$ being divided in the study groups: Group 1:n $=7(29 \%)$; Group 2: $\mathrm{n}=2(8.3 \%)$; Group $3: \mathrm{n}=1(4.1 \%)$. The topography of fragile fractures in these patients were vertebral fractures: $n=6(25 \%)$, forearm fractures: $n=3(12.5 \%)$, hip fractures $\mathrm{n}=1(4.1 \%)$.

Conclusions: The incidence of osteoporosis and fragile fractures in patients with obesity and without DM is different. DM type 1 is strongly correlated with an increased incidence of low BMI, compared to type II DM and obesity. Abdominal adipose tissue and obesity class 2 and class 3, seem to be "protective factors" against osteoporosis. The incidence of fragile fractures was higher in patients with DM type 1 compared to patients with DM type 2 and those with obesity but without DM.Considering DM and obesity, the protective role of the fat tissue compared to the loss of bony mass seems to be decreased.

\section{T1P160}

\section{What controls eating? Modelling the physiological, behavioral and psychological drivers of energy intake in the context of total energy balance}

\section{Hopkins, M. '; Finlayson, G. '; Duarte, C. ${ }^{\text {; }}$ Gibbons, C. ' Johnstone, A. ${ }^{3}$;} Whybrow, S. ${ }^{3}$; Horgan, G. ${ }^{4}$; Blundell, J. ${ }^{1}$; Stubbs, R. ${ }^{1}$

${ }^{1}$ University of Leeds, ${ }^{2}$ University Of Coimbra, ${ }^{3}$ University Of Aberdeen, ${ }^{4}$ Biomathematics And Statistics Scotland

Background: While food intake is regarded as a psychobiological process, few studies have sought to determine the inter-relationships between the determinants of energy intake from different scientific domains. Therefore, the present study examined the cross-sectional relationships between physiological, behavioral and psychological determinants of energy intake in the context of total energy balance.

Design: Three hundred and fifty-two participants (body mass in$\operatorname{dex}=25.6 \pm 4.4 \mathrm{~kg} / \mathrm{m}^{2}$; 162 males, 190 females) completed either two or seven day assessment periods in which body composition (air displacement plethysmography), resting metabolic rate (indirect calorimetry), total energy expenditure (heart rate flex method), ad libitum energy intake (laboratory test meals and weighed food records), restraint, emotionality and externality (Dutch Eating Behavior Questionnaire) were measured. Results: After controlling for age, sex and the length of measurement period, hierarchical multiple regressionindicated that fat-free mass $(p=0.001)$ and resting metabolic rate $(p=0.032)$, but not but fat mass $(\mathrm{p}=0.122)$, acted as physiological predictors of energy intake. Activity energy expenditure (total energy expenditure - resting metabolic rate) was not a behavioral predictor of energy intake in the whole group $(p=0.213)$, but was a significant predictor when measured over seven days $(p=0.045)$ rather than two days $(\mathrm{p}=0.876)$. Dietary restraint also independently predicted energy intake alongside the physiological and behavioral determinants of energy intake $(\mathrm{p}<0.001)$.

Conclusions: These data indicate that the physiological factors fat-free mass and resting metabolic rate strongly predict energy intake under conditions where food choice is allowed to vary freely. However, behavioral (physical activity) and psychological (dietary restraint) factors are additional (weaker) predictors and should be measured in energy balance studies to better understand potential determinants of energy intake.

Conflict of Interest: None Disclosed.

Funding: Research relating to this abstract was funded by the Food Standards Agency, UK. 
T1P161

Zinc deficiency impairs 3T3-L1 adipogenesis but promotes visceral adipose tissue inflammation and expansion after a 3-month high carbohydrate-diet in mice

Manuel, L. ${ }^{1}$; Kretzer, I. ${ }^{1}$; Saleh, N. ${ }^{1}$; Sierra, J. ${ }^{1}$; Siqueira, C. ; Monteiro, F. $^{1}$

${ }^{1}$ Universidade Federal de Santa Catarina

Introduction: Obese individuals have altered concentrations of several essential minerals, such as zinc ( $\mathrm{Zn})$, which operates in various catalytic activities of several enzymes, besides acting in the adipogenic process. The prevalence of micronutrient deficiencies is higher in obese subjects and commonly associated with low-grade inflammation in hypertrophy of adipocytes, type II diabetes, and insulin resistance. Previous studies have demonstrated that insulin can bind to $\mathrm{Zn}$ improving the solubility of this hormone in the beta cells of the pancreas and may contribute to insulin binding capacity to its receptor. Due to the high prevalence of zinc deficiency in obese patients and the lack of studies on the role of depletion of $\mathrm{Zn}$ in adipogenesis, this study aimed to verify the influence of $\mathrm{Zn}$ deficiency on the in vitro adipogenesis as well as on adipose tissue of mice after a short-term carbohydrate overfeeding.

Methods: In vitro experiments were performed using 3T3-L1 preadipocytes on proliferation assays (viability, morphology, and cell cycle) and cell differentiation assessed by oil-red staining after exposed to Zn-deficient (ZD) or $\mathrm{Zn}$-adequate (ZA) medium. Female Swiss mice were fed either high carbohydrate diet $(\mathrm{HCD})$ with adequate $\mathrm{Zn}$ concentration (HCDZA) or Zn deficient HCD (HCD-ZD) for three months. Fasting plasma leptin and adiponectin were performed and adipose tissues were collected for further histological analysis or adipocyte measurement and determination of the inflammatory adipokine serum amyloid A (SAA). Results: In ZD-treated preadipocytes, a decrease of $51 \%$ of cells in S-phase was observed when cell cycle was evaluated $(\mathrm{p}<0.05 \mathrm{em}=$ "'" $>$ versus ZA-treated cells). During differentiation, cells exposed to ZD exhibited a reduced ability to differentiate demonstrating reduced intracellular lipid accumulation. In vivo experiments demonstrated that $\mathrm{Zn}$ deficiency promoted differences in weight gain from day 11 through day 90 of the experiment $(2.69 \pm 0.71 \%)$ when compared to mice fed with HCD-ZA $(-2.07 \pm 0.9 \%$. $\mathrm{p}<0.05)$. Visceral fat mass from HCD-ZD-treated mice was significantly higher than HCD-ZA mice and showed larger adipose cell size in both histological and contrast phase microscopy analysis. In addition, it was observed a 23-fold increment of SAA in supernatants of isolated adipocytes from mice fed with HCD-ZD, when compared to HCD-ZA-treated mice. Finally, adiponectin was significantly lower in HCD-ZD mice $(5 \pm 0.7$ versus $8 \pm 0.2 \mathrm{ng} / \mathrm{mL}, \mathrm{p}<0.05)$ while leptin increased significantly in this group $(31 \pm 2$ versus $25 \pm 3 \mathrm{ng} / \mathrm{mL}, \mathrm{p}<0.05)$.

Conclusion: Zn deficiency reduces cellular proliferation of 3T3-L1 and the ability of these cells to differentiate. However, a 3-month HCD deficient in $\mathrm{Zn}$ promoted inflammation and expansion of the adipose tissue in mice.

\section{PO2 - Health, Behaviour and Environment}

\section{T2P1}

\section{A novel procedure for integrating three objectively measured dimensions of free-living sedentary time}

\section{Myers, M. '; Gibbons, D. '; Butler, M. ${ }^{2}$; Blundell, P. ; Finlayson, D. ${ }^{1}$ \\ ${ }^{1}$ University of Leeds, ${ }^{2}$ Endava Ltd.}

Introduction: The widely accepted definition of sedentary behaviour (SB) refers to any waking behaviour characterized by an energy expenditure $<1.5$ metabolic equivalents (METs) while in a sitting or reclining posture. At present, there is no single field-based device which accurately measures sleep, activity intensity and posture. The aim of this study was to develop a novel integrative procedure to combine sleep, activity intensity and posture information from two validated activity monitors to quantify free-living sedentary time.

Methods: Sixty-three female participants aged $37.1( \pm 13.6)$ years with a BMI of $29.6( \pm 4.7) \mathrm{kg} / \mathrm{m}^{2}$ were continuously monitored for $5-7$ days to track free-living SB with the SenseWear Armband (for sleep and activity intensity) and the activPAL (for posture). A set of data merging operations controlled via a simple graphical user interface were developed. After accounting for sleep time, differences in sedentary time according to sitting/ reclining $\left(\mathrm{SED}^{\mathrm{AP}}\right)$, activity intensity $<1.5 \mathrm{METs}\left(\mathrm{SED}^{\mathrm{SWA}}\right)$ and the integration of these dimensions $\left(\mathrm{SED}^{\mathrm{INT}}\right)$ were compared.

Results: The three SB measures were positively inter-correlated with the weakest relationship between $S D^{\mathrm{SWA}}$ and $\mathrm{SED}^{\mathrm{AP}}$, followed by SED${ }^{S W A}$ and SED ${ }^{\mathrm{INT}}$, and the strongest relationship was between $\mathrm{SED}^{\mathrm{AP}}$ and $\mathrm{SED}^{\mathrm{INT}}$. There was a significant difference between the three measures of sedentary time. Post-hoc tests revealed all three methods differed significantly from each other. SED ${ }^{\mathrm{SWA}}$ resulted in the most sedentary time (11.74 \pm 1.60 hours/day), followed by $\operatorname{SED}^{\mathrm{AP}}$ (10.16 \pm 1.75 hours/day), and $\operatorname{SED}^{\mathrm{INT}}(9.10 \pm 1.67$ hours/day). Sedentary time accumulated on weekdays compared with weekend days did not differ for any of the measurement Methods: More sedentary time was accumulated in the longest bout category ( $>40$ minutes) when measured with SED ${ }^{\text {SWA }}$.

Conclusion: It is possible to combine information from two validated activity monitors to obtain a measure of free-living SB based on posture and activity intensity during waking hours. The positive correlation suggests that both activity intensity and posture are related aspects of the same phenomenon (sedentary behaviour). However, sedentary time according to posture and sedentary time according to activity intensity are conceptually different. The implications of this methodological distinction for understanding the impact of SB on markers of health and obesity are yet to be determined.

Conflict of Interest: None Disclosed

Funding: Research relating to this abstract was funded by EU projects under grant agreements no 610440 (DAPHNE) and 289800 (SATIN)

\section{T2P2}

A study on understanding about differences of nutrient intake according to metabolic health status in the same BMI group: A cross-sectional study in Korean populations

Byun, A.; Lee, H.; Lee, S.; Shim, K.

Department of Family Medicine, School of Medicine, Ewha Womans University

Background: Metabolic syndrome and obesity has been widely recognized as a major risk factor for cardiovascular disease and various diets are recommended for treatment and prevention. However, there is still a lack of clinical evidence and medical controversy in each diet. Therefore, it will be analyzed the current diet of Koreans and try to find out how they relate to obesity and metabolic syndrome in this study.

Methods:The results of the health questionnaire / screening / nutrition survey were analyzed for adults without chronic baseline disease over 19 years of age by combining data from the Korean National Health and Nutrition Examination Survey (KNHANES, 2013-2014). A total of 6,392 subjects were divided into 2 groups and 8 subgroups based on the body mass index (BMI) and metabolic syndrome (MetS) components: healthy (exhibiting less than 2 MetS components) with the followings: healthy-normal weight (18.5-22.9), healthy-overweight (23-24.9), healthy-obese $(25-29.9)$ and healthy-severe obese $(\geq 30)$; and unhealthy (exhibiting 3 or more MetS components) with the followings: unhealthy-normal weight, unhealthy-overweight, unhealhty-obese and unhealthy-severe obese. In these 8 groups, the amounts of total calories, carbohydrates, protein and fat consumed per day and ratio of each component in total calories were compared. Chi square test, variance analysis and covariance analysis were performed using the SPSS window ver.23 reflecting the composite sample design.

Results: Of the total subjects, 5,038 persons were Metabolic Healthy and 1,354 persons were Metabolic Unhealthy. Eight groups showed statistical- 
ly significant differences in age, sex, smoking status, drinking status, physical activity, household income level, education level, family members waist circumference, systolic/diastolic blood pressure, fasting blood glucose, low density / high density cholesterol and HbA1c. The total calories consumed per day in the eight groups were the highest in the Metabolic Healthy obesity group and the lowest in the Metabolic Unhealthy severe obesity group. After adjusting the confounding variables, the composition of each nutrient was composed of 7: 1: 2 carbohydrate, fat, protein. In the subjects with the same body mass index except for the severe obesity group, the metabolic healthy group showed lower carbohydrate intake and higher fat intake than the metabolic unhealthy group, which was statistically significant $(\mathrm{p}<0.001)$.

Conclusion: In Metabolic Healthy Korean adults, the amount of carbohydrates intake was lower and the amount of fat intake was higher than Unhealthy group. Therefore, the general knowledge that low-fat diets are beneficial to maintaining metabolic health needs to be reconsidered.

Conflict of Interest: Non disclosed

Funding: No Funding

\section{T2P3}

\section{A weight-loss intervention impact on thigh composition, cardiorespiratory fitness, and physical activity: Possible athlete's paradox effect?}

Martins, S. ${ }^{1}$; Rocha, P. ${ }^{2}$; Branco, T. ${ }^{3}$; Palmeira, A. ${ }^{4} ;$ Silva, A. ${ }^{5}$; Minderico, C. ${ }^{6}$; Vieira, P. ${ }^{7}$; Teixeira, P.5 ; Sardinha, L.

${ }^{1}$ Universidade Europeia, Lisbon, Portugal; Instituto de Saúde Ambiental, Faculdade de Medicina, Universidade de Lisboa, Lisbon, Portugal, ${ }^{2}$ Nterdisciplinary Centre For The Study Of Human Performance, Faculty Of Human Kinetics, University Of Lisbon, Lisbon, Portugal, ${ }^{3}$ Instituto Prof. Teresa Branco, Oeiras, Portugal, ${ }^{4}$ Universidade Lusófona De Humanidades E Tecnologias, Lisbon, Portugal; Interdisciplinary Centre For The Study Of Human Performance, Faculty Of Human Kinetics, University Of Lisbon, Lisbon, Portugal, ${ }^{5}$ Interdisciplinary Centre For The Study Of Human Performance, Faculty Of Human Kinetics, University Of Lisbon, Lisbon, Portugal, ${ }^{6}$ Universidade Lusófona De Humanidades E Tecnologias, Lisbon, Portugal, ${ }^{7}$ Universidade Europeia, Lisbon, Portugal

Introduction: Computed tomography (CT) estimates of thigh composition, namely adipose tissue (AT) and skeletal muscle (SM), are associated with health conditions such as diabetes type 2, blood glucose and lipid levels, metabolic syndrome, prothrombotic and atherosclerotic abnormalities, and increased risk of mobility loss (Eastwood et al., 2014; Rocha et al., 2008; Visser et al., 2005). Physical activity (PA) and cardiorespiratory fitness (CRF) are recognized as important health-related protective indicators (WHO, 2016).This study aimed to analyze the impact of a 16-month weight-loss intervention on thigh composition (SM area, SMHU, and AT area), CRF, and PA, along with the associations between changes in the studied variables.

Methods: 48 overweight and obese premenopausal women $(39.2 \pm 5.7 \mathrm{yr}$ $30.0 \pm 3.0 \mathrm{~kg} / \mathrm{m}^{2}$ ), participated voluntarily on a cognitive-behavioral program consisting on a 16-week intervention, followed by randomization to a comparison group, a monthly meetings group, and a monthly meetings plus structured PA group, for a 12-month intervention. Body composition was assessed by CT and DXA. Anthropometry measurements were performed. IPAQ was used for PA estimation, and CRF was tested with gas analysis during a maximal aerobic power treadmill incremental protocol. All measurements were performed at baseline and 16-month.

Results: Changes revealed increases in low density SM, CRF and PA $(P<0.001$ for all). Decreases were observed for low density SM quality, TAT and thigh subcutaneous AT (TSAT) areas, anthropometric variables, and lower limbs FM $(P<0.001$ for all). No differences were found between groups $(\mathrm{P}>0.05)$, even after adjustment for PA. The increase of low density SM area and the decrease of SMHU were inversely associated with the increment in CRF ( $P \leq 0.001$ and $P<0.05$, respectively), and positively related with the reductions of weight $(P \leq 0.001$ and $P<0.05$, respectively), BMI $(P \leq 0.001$ and $P<0.05$, respectively), and other body composition indicators. TAT and TSAT decreases during the 16-month period were positively associated with reductions in weight, BMI, anthropometric variables and lower limbs FM ( $P \leq 0.01$ for all).

Conclusion: A 16-month weight-loss intervention positively influenced low density SM thigh area along with a decrease in low density SM quality and increases in CRF, suggesting the occurrence of the athlete's paradox in overweight and obese premenopausal women (van Loon et al., 2006). Significant improvements were found in each intervention group from baseline to the end of the study concerning body composition, CRF and PA, despite no significant modifications were observed between the groups at 16-month.

Research related to this abstract was funded by the Portuguese Foundation for Science and Technology (Sapiens 358007/99).

T2P4

\section{Ability of nontraditional risk factors for cardiovascular disease to identify high cardiometabolic risk in adolescents}

Sobrinho, C. ' ; Moreira, C. ${ }^{1}$; Abreu, S. ' Lopes, L. ${ }^{1}$; Santos, J. ' ; Mota, J. ${ }^{\text {' }}$; Santos, . $^{1}$

${ }^{1}$ Faculty of Sport, University of Porto, Portugal.

Introduction: Low-grade systemic inflammation has been associated with cardiovascular diseases risk factors. We aimed to evaluate the ability of several inflammatory biomarkers in identifying a high cardiometabolic risk in adolescents.

Methods: A cross-sectional study was conducted with 529 Portuguese adolescents (267 girls) aged $14.3 \pm 1.7$ years. A continuous variable of clustered cardiometabolic risk score [sum of Z-scores of body fat percentage, systolic blood pressure, ratio total cholesterol/HDL, triglycerides, HOMA-IR and cardiorespiratory fitness $\left.{ }^{\star}(-1)\right]$ was computed. Receiver operating characteristic (ROC) curves were used to explore the ability of each biomarker (C3, C4, hs-CRP, Fibrinogen, Leptin, WBC, Albumin, IL-6) to discriminate between low/high cardiometabolic risk. Logistic regression analysis was performed to further study the association of each biomarker and high cardiometabolic risk.

Results: ROC curves analyses showed that C3, C4, hs-CRP, Fibrinogen and Leptin were able to present discriminatory ability in identifying unfavorable cardiometabolic profile in both girls and boys ( $p<0.01$ for all). Logistic regression analyses showed that C3, C4, hs-CRP, fibrinogen and leptin (in both sexes) and with WBC (in boys) were associated with high cardiometabolic risk, independent of age, pubertal stage, socioeconomic status and adherence do a Mediterranean diet ( $p<0.05$ for all).

Conclusion: In conclusion, C3, C4, hs-CRP, fibrinogen and leptin were able to present discriminatory ability in identifying high cardiometabolic risk score. Such biomarkers may have utility in motivating health professionals, public health and adolescents' families toward lifestyle changes, improving prevention efforts early in life.

Conflict of Interest: The authors declare no conflicts of interest.

Funding: Research relating to this abstract was funded byFCT grants: BPD/102381/2014 and BD88984/2012; Brazilian government by CAPES (Coordination of Improvement of Higher Education Personnel) (Proc: 9588-13-2). The Research Centre on Physical Activity Health and Leisure (CIAFEL) is supported by UID/DTP/00617/2013 (FCT) 


\section{T2P5}

Accuracy and inconsistency associated factors of selfreported hypertension among participants of the study of cardiovascular risk in adolescents (ERICA)

Gonçalves, V. ${ }^{1}$; Galvão, T. ${ }^{\text {; }}$ Silva, M. ${ }^{3}$; Lamarca, F. ${ }^{\text {; }}$ Dutra, E. ' Carvalho, K. ${ }^{1}$ ${ }^{1}$ University of Brasilia, Brazil, ${ }^{2}$ State University Of Campinas, Brazil, ${ }^{3} \mathrm{Federal}$ University Of Amazonas, Brazil

Introduction: No studies evaluating the validity of the self-reported diagnosis of high blood pressure (HBP) for the adolescent population were identified. In this context, the objective of this study was to investigate the accuracy of the self-reported diagnosis of HBP in the Brazilian adolescent school population.

Methods: The Study of Cardiovascular Risk in Adolescents (ERICA) was a multicenter, school-based representative national cross-sectional study. Adolescents 12 to 17 years old answered the question, ' 'Have any doctor ever told you that you have or had high blood pressure?' ' Anthropometry, blood pressure, behavioral and sociodemographic aspects were evaluated. HBP was defined by mean systolic or diastolic blood pressure $\geq$ the 95 th percentile of the reference curve. Overweight was classified by BMI for Age greater than Z-score +1 . Sensitivity, specificity, positive predictive value (PPV) and negative predictive value (NPV) with their respective confidence intervals (95\% CI) were calculated for the assessment of accuracy, considering the clinical diagnosis of HBP as standard. Poisson regression was applied to calculate prevalence ration (PR) in order to verify possible inconsistency associated factors of the self-reported diagnosis, defined as a divergent response from the clinical diagnosis.

Results: A total of 73,399 adolescents from 1,247 schools and $122 \mathrm{mu}-$ nicipalities were included in the analysis. The mean age was 14.4 years, with $52.7 \%$ being between 12 and 15 years old, 50.8\% male, and $25.0 \%$ overweight. Most of the adolescents studied in public schools and in the urban zone. The prevalence of HBP by clinical and self-reported diagnosis was, respectively, 9.6\% (95\% CI: 9.0-10.3) and 3.8\% (95\% CI: 3.4-4.2) The sensitivity was $6.2 \%$ (95\% CI: 5.6-6.8), specificity $96.7 \%$ (95\% CI: 96.6-96.8), PPV 15.7\% (95\% CI: 14.3-17.3) and NPV 91.2\% (95\% CI: 91.0-91.4). The following factors were positive associated with the inconsistency of the answers: males (PR 1.35, 95\% CI: 1.21-1.51), older than 15 years (PR 1.11, 95\% CI: 1.01-1.23), students from rural schools (PR 1.85, 95\% CI: 1.60-2.15), overweight (PR 2.61, 95\% CI: 2.40-2.83) and consuming at least one dose of alcoholic beverage in the last month (PR 1.17; 95\% CI: 1.02-1.33). Students from private schools had a lower prevalence than those from public schools (PR 0.77, 95\% CI: 0.67-0.89).

Conclusion: Based on the inaccuracy of self-reported diagnosis of hypertension accompanied by several factors associated with this inconsistency, it is recommended to increase access to the adolescent primary health care in order to guarantee an adequate screening of hypertension through direct measurement of blood pressure.

\section{T2P6}

\section{Active travel to school, sport participation and obesity in adolescents}

\section{Almeida, M. ${ }^{1}$; Sabino, B. $^{2}$}

${ }^{1}$ Faculty of Sport Sciences and Physical Education, University of Coimbra, ${ }^{2}$ Faculty Of Sport, University Of Porto

Introduction: Decreasing levels of physical activity (PA) may be predisposing children to become overweight and obese. Motorized transportation may be partially responsible for the daily physical activity reduction, by replacing active travel to school. The purpose of this study is to analyze the relationship of PA, and specifically Active Transport, with the \% body fat (BF).

Methods: Participants in this study were 1329 adolescents (630 boys and 699 girls), from the $5^{\text {th }}$ and $7^{\text {th }}$ grades (mean age of $11,73 \pm 1,56$ years), from Madeira Island. PA was assessed with the PAQ-C questionnaire (Crocker et al., 1997), and travel method between home and school. They were measured for height, weight, waist circumference, tricipital and calf skinfolds, and \%BF was estimated according to Slaughter et al. (1988), and classified in health categories according to Lohman (1987).

Results: From the overall sample, only $10 \%$ walked daily between home and school, with minimal duration of 20 minutes. About $53,1 \%$ of the participants had Physical education classes as the only organized physical activity and $41 \%$ had high or very high body fat percentage. Results showed significant differences between gender and education level $(\mathrm{p}<0.05)$, with boys presenting, on average, higher scores of PA (boys: $x=2,73 \pm 0,71$ vs girls: $\mathrm{x}=2,23 \pm 0,51$ ), with a decrease on PA levels as age increased. Students that use non-active transport, presented higher \%BF (Non-active transport $=26,7 \%$ vs Active transport $=22,2 \%$; $<<0.05)$. Three predictors of high or very high $\% \mathrm{BF}$ were identified: age $(\mathrm{OR}=1,136 ; 95 \% \mathrm{IC}$ : 1,036-1,246), non-active transport ( $\mathrm{OR}=1,002 ; 95 \% \mathrm{IC}: 1,000-1,005)$ and level of sports participation (OR $=1,212 ; 95 \% \mathrm{IC}: 1,034-1,421)$.

Conclusions: The development of intervention programs targeted to lower levels of adiposity shall include a PA informal component, such as active transport.

Keywords: obesity, eating behaviors, fruit and vegetable environment, youth

None Disclosed

The research relating to this abstract was funded by the Secretary of Education of the Madeira Autonomous Region

T2P7

\section{Analysis of CPT1A gene expression in peripheral blood mononuclear cells as an early marker of metabolic alterations due to the intake of diets with unbalanced macronutrient composition}

\section{Díaz-Rúa, R. ${ }^{1}$; Palou, A. ${ }^{1}$; Oliver, P. $^{1}$}

${ }^{1}$ Laboratorio de Biología Molecular, Nutrición y Biotecnología (Nutrigenómica y Obesidad), Universidad de las Islas Baleares (UIB) y CIBER de Fisiopatología de la Obesidad y Nutrición (CIBEROBN), Palma de Mallorca, España

Introduction: Peripheral blood mononuclear cells (PBMC) constitute a promising tool to identify subjects at risk of developing diet-related diseases. Here we analysed mRNA expression of key energy homeostasis-related genes in a time-course analysis to find out early markers of metabolic alterations due to the sustained intake of high-fat (HF) or high-protein (HP) diets in rats.

Methods: Two unbalanced diets, rich in fat or protein were chronically administered (4 months trial) to adult rats in isocaloric conditions to a control diet. Isocaloric administration was performed to avoid overweight associated with the intake of hyperlipidic diets and to be able to characterise markers of the metabolically obese normal-weight (MONW) syndrome. Key energy homeostatic genes were analysed by real-time RT-PCR in PBMC samples obtained at different time-points of dietary treatment. Different serum parameters related with metabolic syndrome, as well as fat deposition in liver, were also analysed.

Results: Chronic intake of both unbalanced diets (HF and HP) affected PBMC gene expression profile, reflecting physiological deviations from healthy homeostatic equilibrium. The most outstanding results were obtained for Cptla mRNA expression in PBMC: its mRNA levels increased after only one month of exposure to both unbalanced diets and this increased expression was maintained thereafter. Animals with isocaloric administration of a HF diet did not develop obesity but presented obesity-related complications, mimicking the MONW phenotype. In these animals, Cpt1a expression correlated with higher insulin resistance, alteration of serum lipid profile and, particularly, increased fat deposition in liver. Correlation with liver fat deposition was observed even in animals fed a HP diet.

Conclusion: Our results reinforce the use of $\mathrm{PBMC}$ as a tool in nutritional studies and show that Cptla analysis in PBMC could be used as an early biomarker of future complications related to the intake of unbalanced diets. 


\section{Confict of interest: None Disclosed}

Funding: Research relating to this abstract was funded by the Spanish Government (Ministerio de Economía y Competitividad, INTERBIOBES, AGL2015-67019-P) and by the University of the Balearic Islands (IBIFLEX, FA38/2016). The Laboratory of Molecular Biology, Nutrition and Biotechnology is a member of the European Research Network of Excellence NuGO. CIBER de Fisiopatología de la Obesidad y Nutrición is an initiative of the Instituto de Salud Carlos III (ISCIII). RDR was recipient of a fellowship from the Spanish Government.

\section{T2P8}

\section{Are pharmacists ready to contribute to reduce the burden of obesity?}

\section{Pistja, E. ${ }^{1}$; Themeli, A. ${ }^{1}$}

${ }^{1}$ Medical Training Center Santa Maria

Background: Obesity is a growing health concern in Albania. Obesity has been identified as a key risk factor for many chronic diseases including hypertension, dyslipidemia and type 2 diabetes mellitus. This study was conducted to investigate the role of community pharmacists in obesity counseling, and to identify the barriers to counseling in Albania.

Methods: It was used a descriptive cross-sectional study involved 15 community pharmacies that were selected via stratified and systematic random sampling. A pretested self-administered questionnaire collected information on frequency and comfort level with obesity counseling, and the perceived effectiveness of four aspects of obesity management (diet and exercise, prescribed anti obesity medications, diet foods, and nonprescription products and dietary supplements). Information on perceived confidence in achieving positive outcomes as a result of counseling and barriers to counseling was also collected. Descriptive and Spearman' $r$ analysis were conducted using SPSS version 17. Responses with Likert scale rating 1 (low score) to 5 (high score) and binary choices (yes/no) were presented as mean (SD) and $(95 \% \mathrm{CI})$, respectively.

Results: The response rate was $90.6 \%$. The overall mean (SD) responses indicated that pharmacists counseled obese patients sometimes to most of the time, 3.67 (1.19) and were neutral to comfortable with counseling about aspects of obesity management, 3.77 (1.19). Respondents perceived obesity management aspects to be somewhat effective, 3.80 (1.05). Of the four aspects of obesity management, diet and exercise, and diet foods were the highest ranked in terms of frequency of counseling, comfort level and perceived effectiveness. Pharmacists were neutral to confident in achieving positive outcomes as a result of obesity counseling, 3.44 (1.09). Overall mean responses of counseling obese patients by pharmacists were positively correlated with their perceived comfort with counseling and perceived effectiveness of obesity management aspects. The most anticipated barriers to obesity counseling were lack of patient awareness about pharmacists' expertise in counseling and pharmacists' opinions that obese patients lack willpower and are non-adherent to weight reduction interventions.

Conclusions: Strengths, weaknesses and barriers related to obesity counseling by pharmacists in Albania were identified, and suggestions were provided to strengthen that role.

\section{T2P10 \\ Association between maternal and foetal fatty acids profile and birth weight.}

Cinelli, G. '; Fabrizi, M. '; Ravà, L.' ; Signore, F.'; Vernocchi, P.'; Semeraro, M.'; Vallone, C. ${ }^{2}$; Lanciotti, R. ${ }^{3}$; Atti, M. '; Manco, M. ${ }^{1}$

${ }^{1}$ Bambino Gesù Children Hospital, ${ }^{2}$ San Camillo Hospital, ${ }^{3}$ University Of Bologna

Introduction: The aim of the study was to investigate the influence of maternal versus foetal erythrocytes lipid profile at delivery on birth weight. Methods: This was a cohort study of pregnant women recruited at the first trimester. A total of 607 mother-infant pairs (316 males, 52.1\%) were included in the study. Maternal blood and cord blood were collected at delivery and erythrocytes fatty acids were analysed using gas chromatography. Quantile regression analyses were conducted to investigate the association between maternal/foetal erythrocyte lipid profile and birth outcomes.

Results: After optimal adjustment, a significant positive association was observed between birth weight and maternal linoleic acid (LA) (coeff. $=20.02$; 95\%CI 5.43 to $34.62 ; \mathrm{p}=0.01$ ), arachidonic acid (AA) (coeff. $=13.25$; 95\%CI 2.19 to $24.32 ; \mathrm{p}=0.02)$, docosahexaenoic acid (DHA) (coeff. $=30.54$; 95\%CI 10.17 to $50.90 ; \mathrm{p}=0.00$ ), polyunsaturated (PUFA) and omega 6 fatty acids (coeff. $=9.91 ; 95 \%$ CI 3.93 to $15.88 ; \mathrm{p}=0.00$ ) (coeff. $=10.74 ; 95 \% \mathrm{CI}=3.02$ to $18.46 ; \mathrm{p}=0.01$ ). A negative association were found for foetal DHA (coeff.=-25.11; 95\%CI -45.21 to $5.00 ; p=0.01$ ). Finally, we found a major risk of being Small for Gestation Age (SGA) for higher percentage of maternal erucic acid $(\mathrm{RRR}=4.90$; 95\% CI 1.03 to 9.71; $\mathrm{p}=0.05)$; conversely, ecosapentaenoic acid (EPA) results to be protective against the risk of being SGA $(\mathrm{RRR}=0.21$; 95\%CI 0.05 to 0.85 ; $\mathrm{p}=0.03)$.

Conclusion: Our results suggest that maternal PUFA and omega 6 fatty acids are positively related to birth weight, while higher percentage of cord blood DHA is associated with lower birth weight. Moreover, maternal lipid profile may influence the risk of being SGA.

\section{Conflict of Interest: None Disclosed.}

Funding: Research relating to this abstract was funded entirely by the Italian Ministry of Health (GR-2010-2304957)

\section{T2P11 \\ Association between metabolic status and the incidence of psoriasis}

Kwon, H. $^{1}$; Kim, E. ' ; Lee, J. ${ }^{2}$; Yoo, S. ${ }^{1}$

${ }^{1}$ The Catholic University of Korea, ${ }^{2}$ Myongji Hospital

Introduction: Growing evidence suggests that obesity-a major component of metabolic disease-is a risk factor for incident psoriasis. This study was aimed to evaluate the association of obesity and metabolic status with the cumulative incidence of psoriasis

Methods: We used data from a prospective cohort study collected by the National Health Insurance Service in Korea. A total of 418,057 adults aged $>20$ years who were psoriasis-free at baseline were followed-up until the diagnosis of psoriasis or December 2013. Participants were stratified based on the body mass index (BMI) categories and metabolic condition, which was defined as unhealthy in cases with at least one of the following: hypertension, diabetes, and hyperlipidemia.

Results: During the follow-up visit, 11054 (2.6\%) cases were found to have psoriasis. Diabetes, hypertension, hyperlipidemia, and obesity (BMI $\geq 25$ $\mathrm{kg} / \mathrm{m}^{2}$ ) were all found to be risk factors for incident psoriasis. Subjects with the metabolically unhealthy non-obese phenotype (MUNO; hazard ratio [HR], 1.29; 95\% confidence interval [CI], 1.22-1.37) and metabolically unhealthy obese phenotype (MUO; HR, 1.33; 95\% CI, 1. 26-1.41) had a significantly higher risk of psoriasis incidence as compared to those with the metabolically healthy non-obese phenotype, after adjusting for age, sex, smoking, exercise, and income. In the analysis stratified by sex and age groups, the risk of psoriasis development was found to be high among those with the MUNO and MUO phenotypes in both sexes and all age groups.

Conclusion: The metabolic health status was significantly associated with an increased risk of psoriasis in both obese and non-obese individuals. However, further studies are needed to evaluate whether the control of metabolic parameters can lower the incidence of psoriasis. 
T2P12

Association between physical activity with sufficient vitamin D status in brazilian adolescents: The study of cardiovascular risks in adolescents (ERICA)

Silva, A. ' ; Cureau, F. ${ }^{2}$ Dutra, E. ' ; Schaan, B. ${ }^{3}$; Carvalho, K. $^{1}$

${ }^{1}$ Departament of Nutrition, University of Brasília, Federal District, Brazil, ${ }^{2}$ Department Of Internal Medicine, Faculty Of Medicine, Federal University Of Rio Grande Do Sul, Brazil, ${ }^{3}$ Hospital Of Clinics Of Porto Alegre, Brazil

Introduction: Adolescents are at risk for vitamin D deficiency because they have high demand of nutrients and usually present an inactive lifestyle, which interfere on vitamin D synthesis. There is still under-reporting of Vitamin D levels in countries like Brazil with a diversity of cultures and regional climate conditions. The aim of this study was to investigate the association between physical activity with vitamin D status among Brazilian adolescents.

Methods: This is a cross-sectional analysis of a subsample of the Study of Cardiovascular Risk in Adolescents (ERICA), composed of 1,155 students aged between 12 and 17 years living in four State capitals (Rio de Janeiro, Porto Alegre, Brasília and Fortaleza). Sociodemographic features and clinical data were obtained through self-administered questionnaires and standardized anthropometric and blood pressure measurements. Fasting blood samples for serum vitamin D (chemiluminescence of microparticles by immunoassay technique - CMIA) were succeed. Sufficient vitamin D status was defined by a serum level $\geq 30 \mathrm{ng} / \mathrm{ml}$. Prevalence rates were estimated for the total sample and analyzed by vitamin D status. Weighted Poisson regression models were applied to investigate association between sufficient vitamin $\mathrm{D}$ status and being active ( $\geq 300$ minutes per week). Analyses were adjusted for center, sample collection period, type of school, age, skin color, economic status, sexual maturation and nutritional status.

Results: Prevalence of sufficiently active adolescents was about twice as high among boys $(65.9 \%, 95 \%$ CI 61.6- 70.0), as compared to girls (33.3\%, 95\% CI 29.4- 37.4). Sufficient vitamin D status prevalence was $36,4 \%$ (95\%IC: 32.7 - 40.4). After model adjustments, for boys, being active was associated with sufficient vitamin D status ( $P R=1.53,95 \%$ CI $1.15-2.05$ ), not observed among girls.

Conclusion: The association between vitamin D sufficient status and physical activity in boys reinforce the importance of stimulating behavioral changes to promote adolescent health.

\section{T2P13}

\section{Association between sarcopenic obesity and mortality in elderly living in the community}

Rocha, M. ${ }^{1}$; Marucci, M. ${ }^{1}$; Roediger, M. ${ }^{\text {' }}$; Crisostomo, L. ' ; Duarte, Y.' ; Lebrão, M. ${ }^{3}$; Santos, J. ${ }^{4}$

${ }^{1}$ Department of Nutrition, School of Public Health, University of São Paulo/ Brazil, ${ }^{2}$ Department Of Nursing Medical-Surgical School Of Nursing, University Of São Paulo/ Brazil, ${ }^{3}$ Department Of Epidemiology, School Of Public Health University Of São Paulo/ Brazil, ${ }^{4}$ Medical School Of Ribeirão Preto, University Of São Paulo / Brazil

Introduction: Sarcopenic obesity (OS) is associated with metabolic disorders, increased risk of falls, worsening of quality of life and mortality. This study verified the association between OS and death in a cohort of domiciled elderly, according to sex and age groups.

Methods: A longitudinal, epidemiological, and home-based study was carried out using data from the SABE Study (Health, Well-Being and Aging) conducted in the city of São Paulo in 2000 and 2010. In 2000, the population was composed of 2143 elderly people ( $\geq 60$ years), selected by probabilistic sample; In 2010, deaths were verified by the Mortality Information System of the city of São Paulo, from 2000 to 2010. Due to the absence of variables necessary for this study, the population was composed of 1407 elderly people, of which 688 died between 2000 and 2010. The study variables were: a) sarcopenic obesity: considering: obesity [identified by waist circumference (WC) $\geq 80 \mathrm{~cm}$ and $\geq 94 \mathrm{~cm}$ for men and women, respectively] and sarcopenia [by performance in Sit-to-stand test, considering low performance, when the time spent $\geq 75$ th percentile $(\mathrm{P})$ of the population itself; By handgrip strength, being considered reduced strength, when the value was $\leq$ P25, of the population itself; and by muscle mass (MM), estimated by the muscle mass index ( $\mathrm{MMI}=\mathrm{MM}$ (kg) / height $(\mathrm{m})$ ), the MM being calculated by predictive equation, considering MM when the value was $\leq$ P 20, of the own population]; b) sex; c) age groups (60-74 and $\geq 75$ years). The presence of OS was confirmed when all criteria adopted for the diagnosis of obesity and sarcopenia were considered. To verify the association between the variables, a chi-square Rao-Scott test $(\mathrm{p} \leq 0.05)$ was used. The Kaplan-Meier method was used to compare survival curves and the log-rank test to verify if there were differences between the impact of sarcopenic obesity on survival. The magnitude of the association between death and OS was verified using the Poisson regression model $(\mathrm{p}<0.05)$.

Results: Of the 1407 elderly evaluated, 2.4\% $(n=66)$ who died between 2000 and 2010, were identified with OS. The mortality rate was higher: in the elderly with OS [120.2 / 1000 person / year (95\% CI 93-155.3) / 43.6 / 1000 person / year (95\% CI 39.6-48.1) In the $\geq 75$ years group [141.0 / person / year (95\% CI 112.1-177.4) / 63.4 / person / year (CI 95\% 24.8202.7)] and in men (296.0 / 1000 person / year (CI 191.0-466.8) / 109.1 / person / year (95\% CI 82.9-143.9)) ( $\mathrm{p} \leq 0.05)$. After adjustment of the variables, a higher risk for death was observed in the elderly with 60-74 years $(\mathrm{RR}=1.3795 \% \mathrm{CI} 0.73-2.58)$ and in men $(\mathrm{RR}=1.9995 \% \mathrm{CI})$. Conclusion: OS was associated with death risk in men and in the group 60-74 years.

Conflict of interest: None disclosed

Funding: No Funding

T2P14

\section{Association between saturated and trans fatty acids intake and cardiovascular risk factors in Brazilian adolescents - findings from the study of cardiovascular risk in adolescents (ERICA)}

Cabral, C. '; Giannini, D. '; Rodrigues, C. '; Kuschnir, M. ' ; Oliveira, C. Souza, A. $^{2}$

${ }^{1}$ State University of Rio de Janeiro, ${ }^{2}$ Federal University Of Rio De Janeiro

Introduction: Evidence shows that diet rich in saturated and trans fatty acids influences the genesis of cardiovascular risk factors and metabolic syndrome (MS), whose prevalence is increasing among adolescents. The objective of this study was to investigate the association between saturated and trans fatty acids consumption and cardiovascular risks in Brazilian adolescents.

Methods: This study is part of the Study of Cardiovascular Risk in Adolescents (ERICA), which is a national, cross-sectional, school-based study with adolescents aged between 12 and 17 years. Information regarding sociodemographic characteristics, clinical data and environmental factors were collected. Blood samples were taken for total cholesterol and fractions, triglycerides and glucose. The food consumption was evaluated by one 24 hour recall. Descriptive and multivariable logistic regression was performed on the data.

Results: 36.650 students (60.0\% girls) participated in this study. $2.3 \%$ were underweight, $71.5 \%$ well-nourished, $17.6 \%$ overweight and $8.2 \%$ obese. The prevalence of MS was $2.6 \%$ (95\%CI $2.3-2.9)$. The prevalence of inadequacy of fatty acid consumption varied between $72-76 \%$ for saturated and $45-51 \%$ for trans in adolescents with metabolic alterations. Females, students from private schools and adolescents with excess weight had a greater chance of inadequate consumption of the fatty acids analyzed. There was no association between the consumption of fatty acids and SM (total fatty acid: OR [95\% CI]: 0.91 [0.68-1.22]; saturated fatty acid: 0.91 [0.66-1.26], trans fatty acid: 0.99 [0.73-1.33]).

Conclusion: Despite the non-association between fatty acids and SM, lipid consumption was considered high (approximately $50 \%$ higher than recommended) among adolescents who presented metabolic alterations. 
Overweight adolescents had a higher consumption of saturated and trans fatty acids, which are related to cardiovascular risk.

Conflit of interest: None declared

Funding: FINEP, Ministério da Ciência e Tecnologia, Ministério da Saúde, FIRJAN

\section{T2P16 \\ Association of adherence to a healthy dietary pattern and muscular fitness with cardiovascular risk factors in adolescents.}

Sobrinho, C. '; Abreu, S. '; Moreira, C. ' ; Lopes, L. ' ; Mota, J. '; Santos, R. ${ }^{2}$

${ }^{1}$ Research Centre in Physical Activity, Health and Leisure, Faculty of Sport, University of Porto, Portugal, ${ }^{2}$ Early Start Research Institute, Faculty Of Social Sciences, School Of Education. University Of Wollongong, Australia

Introduction: Unhealthy lifestyles are linked to cardiovascular diseases. The aim of this study was to assess the impact of the combined associations of muscular fitness and adherence to the Southern European Atlantic Diet (SEADiet) on the clustering of cardiovascular risk factors in adolescents.

Methods: A total of 467 Portuguese adolescents ( 275 girls and 192 boys, aged 15-18) participated in this cross-sectional study. Muscular fitness was evaluated by Curl Up and Push Up tests, then the sum of the Z-Scores of the two tests was performed to create the muscular fitness score. Dietary intake was obtained using a food frequency questionnaire and adherence to SEADiet was assessed with the SEAD index. A cardiovascular risk score was computed from the following components: age and sex, of triglycerides, systolic blood pressure, total cholesterol/HDL-cholesterol, HOMA-IR and waist circumference.

Results: Our results show that muscular fitness is negatively associated with cardiovascular risk score. Overall, the adolescents classified with low muscular fitness and low adherence to the SEADiet had the poorest cardiovascular profile. Lower levels of cardiovascular risk score were associated with high muscular fitness/low adherence to SEADiet and high muscular fitness/high adherence to SEADiet groups compared to low muscular fitness/low adherence to SEADiet group $(\mathrm{p}<0.05)$. We also found significant differences between low muscular fitness/high adherence to SEADiet group and high muscular fitness/high adherence to SEADiet.

Conclusion: Our findings seem suggest that improving muscular fitness as well an adherence to a healthy diet could be an important strategy to reduce clustered cardiovascular risk in youth.

\section{T2P17}

\section{Association of dairy intake with the clustering of inflammatory biomarkers in portuguese adolescents: LabMed physical activity study}

Abreu, S. '; Gonçalves, C. ${ }^{2}$; Moreira, C. '; Rosário, R. ${ }^{3}$; Lopes, L. ' AgostinisSobrinho, C. ${ }^{1}$; Oliveira-Santos, J. ${ }^{1}$ Mota, J. ${ }^{1}$; Santos, R. ${ }^{4}$

${ }^{1}$ Research Centre in Physical Activity, Health and Leisure, Faculty of Sport, University of Porto, ${ }^{2}$ Ordem Dos Nutricionistas, ${ }^{3} \mathrm{Health}$ Sciences Research Unit, University Of Coimbra, ${ }^{4}$ Research Centre In Physical Activity, Health And Leisure, Faculty Of Sport, University Of Porto; Early Start Research Institute, Faculty Of Social Sciences, School Of Education. University Of Wollongong, Australia

Introduction: Low-grade systematic inflammation has been associated with the development and progression of a number of chronic noncommunicable diseases. Dairy intake has been associated with a lower risk of developing low-grade inflammation state. However, the association of dairy intake with inflammation among adolescents remains unclear. Thus, the aim of this study was to determine the association between dairy intake and biomarkers of inflammation among Portuguese adolescents.

Methods: A cross-sectional study was conducted with 507 Portuguese adolescents aged 12 to 18 years old. A continuous cluster of inflammatory biomarkers was constructed by summing up the sex- and age-adjusted $\mathrm{z}$-scores of C-reactive protein, leptin, adiponectin, interleukin-6 and white blood cell. Diet was evaluated using a food frequency questionnaire, and dairy (milk, yogurt and cheese) consumption was categorized using sex-specific tertiles of each food group amount (g/day). Data were analyzed separately for girls and boys, and a separate multiple linear regression analysis was used to estimate the association between dairy intake and the clustered inflammatory biomarkers score. Total energy intake, dietary adequacy, socioeconomic status, misreporting of energy intake, weight status, cardiorespiratory fitness, pubertal stage, and insulin resistance (HOMA-IR) were used as the main confounders.

Results: Boys in tertile 3 of milk intake had a lower inflammatory biomarkers score than those in lower tertiles (Tertile1: $0.37 \pm 2.5$; Tertile 2: $0.21 \pm 2.84$; Tertile 3: $-0.61 \pm 2.31, \mathrm{p}=0.038$ ). After adjustment, higher intake of milk in boys was inversely related to the cluster of inflammatory biomarkers $(B=-0.988$; $C I$ 95\%: $-1.830,-0.147, p=0.021)$. No significant associations were seen in girls or with yogurt or cheese.

Conclusion: We found that a higher intake of milk was inversely associated with the clustering of inflammatory biomarkers in boys. Prospective and randomized clinical investigations are needed to examine the role of dairy intake in inflammatory condition.

Conflict of Interest: None Disclosed

Funding: This study was supported by FCT grants: BPD/102381/2014 and BD88984/2012; The author César Aparecido Agostinis Sobrinho was given Doctoral scholarship from Brazilian government by CAPES (Coordination of Improvement of Higher Education Personnel) (Proc: 9588-13-2). The Research Centre on Physical Activity Health and Leisure (CIAFEL) is supported by UID/DTP/00617/2013 (FCT). The author Rute Santos has a Discovery Early Career Research Award from the Australian Research Council (DE150101921).

\section{T2P18}

Associations of objectively-measured physical activity, sedentary behavior and cardiorespiratory fitness with metabolic syndrome in Japanese male workers: TMCPF study

Yagi, K. ${ }^{1}$; Chen, T. ${ }^{2}$; Chen, S. ${ }^{3}$; Suwa, M. ${ }^{4}$; Imoto, T. ${ }^{4}$; Kida, A. ${ }^{4}$; Iwase, M. ${ }^{4}$; Yokochi, T. ; Kumagai, $\mathrm{S}^{5}$

${ }^{1}$ Graduate School of Human-Environment Studies, Kyushu University, Fukuoka, Japan, ${ }^{2}$ Faculty Of Arts And Science, Kyushu University, Fukuoka, Japan, ${ }^{3} 1$ Xiangya Nursing School Of Central South University, Changsha, Hunan Province, China, ${ }^{4}$ Toyota Motor Corporation, Aichi, Japan, ${ }^{5}$ Counselling And Health Center, Kyushu University, Fukuoka, Japan

Introduction: The purpose of this cross-sectional study was to investigate the independent associations of objectively- measured physical activity, sedentary behavior (SB), and cardiorespiratory fitness (CRF) with metabolic syndrome (MetS) in Japanese male workers.

Methods: We used the data from the baseline survey of TMCPF (Toyota Motor Corporation Physical Activity and Fitness) study, which was conducted between October 2015 and January 2016. A total of 1,410 male workers aged 35-59 from the Toyota Motor Corporation's male employees participated in this study. SB and moderate-to-vigorous physical activity (MVPA) were objectively-measured by tri-axial accelerometer. We defined the valid data that wear time $>=600 \mathrm{mins} /$ day, $>=4$ valid days. CRF was indirectly estimated by bicycle ergometer. MetS was defined as having $\geq 3$ out of 5 diagnostic criteria according to the Joint Interim Statement 2009 definition. MVPA, SB and CRF levels were coded into tertiles with the lowest tertile serving as the reference group. Multiple logistic regression analysis was used to examine associations of physical activity, SB and CRF with MetS.

Results: A total of 756 participants (mean age: $47.7 \pm 8.0 \mathrm{yr}$ ) with valid data were included in the analyses. Of those 536 (70.9\%) participants were factory workers and $220(29.1 \%)$ participants were office workers. Prevalence of MetS were $11.5 \%$ and $3.8 \%$ respectively. The mean time of MVPA, SB and CRF were $87.8 \mathrm{mins}, 438.6 \mathrm{mins}$ and $41.5 \mathrm{ml} / \mathrm{kg} / \mathrm{min}$, respectively. After adjusting for age, occupation, shift work, drinking, smoking, food intake and medical history of heart disease, lower levels of MVPA and CRF were significantly associated with higher odds of MetS. No significantly association was observed for SB with MetS. After mutual 
adjustment, those in the highest tertiles of MVPA and CRF still showed lower likelihood of having MetS compared to the lowest tertile group with adjusted odds ratios (95\% confidence intervals) of $0.45(0.25-0.84)$ and $0.47(0.28-0.81)$, respectively.

Conclusion: This study showed both MVPA and CRF, but not SB were independently associated with MetS highlighting the importance of increase physical activity and improving CRF for preventing MetS.

Conflict of Interest:Payment received from Toyota Motor Corporation.

Funding: No Funding.

\section{T2P21}

Being fat is being lonely? Psychological aspect of loneliness, body dissatisfaction and relationship to obesity. Preliminary study

Kościcka, K. ';

${ }^{1}$ Uniwersity of Social Science and Humanities, Katowice, Poland

Introdution: Loneliness, body dissatisfaction and obesity are multidimensional phenomenon's. All of them have several different aspects and values, also psychological one.The aim of the study was to compare two group of participants (obese and non-obese) and assess the relationship between loneliness and body dissatisfaction in those two group.

Method: The research sample consisted of 31 obese people $(M=36.35$, $S D=11.34)$ and 40 non-obese $(M=25.20, S D=7.69)$. The following tools were use: The SBS - scale (Dołęga, 2013) and Self-Esteem Scale (Rosenberg, 1965).

Results: Comparing two group of participants, obese sample has lower level of global loneliness comparing to non-obese sample $\left(M_{\text {obse }}=1.97\right.$, $S D=0.64$ vs. Mnon-obese 2.05, $S D=0.62 ; U=437.50 ; p>0.56)$. Relationship between BMI and loneliness was significant (obese vs. non obese $r=0.65 ; p<0.01$ ). The relationship between global loneliness and body dissatisfaction is significant in obese sample ( robese $=0.41 ; p<0.05$ vs. rnon-obese $=-0.15, p>0.05)$.

Conclusions: However, obesity is close connected to medicine area, psychological characteristic connected with obesity are also important issue and may have relationships and influence on different fields.

None Disclosed/Payment received from reaserch.

No Funding/Research relating to this abstract was funded.

\section{T2P22 \\ Bigger bodies - long-term time trends and disparities in obesity in Finland}

Lundqvist, A. ${ }^{\prime}$; Härkänen, $T^{1}$; Jääskeläinen, $T .{ }^{\prime}$; Koskinen, S. ${ }^{1}$

${ }^{1}$ National Institute for Health and Welfare

Introduction: Owing to the detrimental and costly effects of obesity on public health, monitoring obesity trends at a population level and identifying population groups at the greatest risk of weight gain is necessary. Aim of the present study was to examine long-term time trends as well as socioeconomic and sosiodemographic disparities in obesity and severe obesity among Finnish adults.

Methods: Three national health examination surveys with a representative sample of 17668 adult men and women were conducted between 1978 and 2012. Height and weight were measured and obesity was defined as a body mass index (BMI) of $30 \mathrm{~kg} / \mathrm{m}^{2}$ or higher and severe obesity as BMI of $35 \mathrm{~kg} / \mathrm{m}^{2}$ or higher.

Results: During the period 1978-2012, the prevalence of obesity increased from $12.0 \%$ (95\% CI 11.1-12.8) to $23.7 \%$ (95\% 21.5-26.0) among men and from $18.9 \%$ (95\% 17.6-20.1) to $25.8 \%$ (95\% 23.9-27.7) among women. Simultaneously, the prevalence of severe obesity increased from $1.1 \%$ (95\% CI $0.9-1.4)$ to $5.5 \%$ (CI 95\% 4.2-6.7) among men and from $3.9 \%$ (95\% CI 3.3-4.6) to $7.8 \%$ (95\% CI 6.6-9.0) among women. An av- erage BMI increased by $1.7 \mathrm{~kg} / \mathrm{m}^{2}$ (from 25.7 to $27.4 \mathrm{mg} / \mathrm{m}^{2}$ ) in men and by $1.0 \mathrm{~kg} / \mathrm{m}^{2}$ (from 26.1 to $27.1 \mathrm{~kg} / \mathrm{m}^{2}$ ) in women which equals an average increase in weight of $9.40 \mathrm{~kg}$ and $5.94 \mathrm{~kg}$ in men and women, respectively. In general, the strongest upward trend in obesity and severe obesity occurred between first two surveys while during the last decade the increase was smaller. Although there were some differences in time trends by age, education and marital status, increases in both obesity and severe obesity were similar across the different social groups.

Conclusions: The prevalence of obesity and severe obesity has substantially increased during the past three decades in Finland. Nowadays nearly every fourth men and women is obese. The increasing trends are universal across the socioeconomic and sosiodemographic groups suggesting that the obesity epidemic has reached all corners of society. A novel strategy to promote preventive action is urgently needed.

\section{$\mathrm{T} 2 \mathrm{P} 23$}

\section{Binge eating in obesity: Impact of behavior and support satisfaction in continental portugal and São Miguel, Açores}

\author{
Silva, M. ${ }^{1}$; Pimenta, F. ; Leal, I. ${ }^{1}$ \\ ${ }^{1}$ ISPA
}

Introduction: The increasing number of individuals with obesity and binge eating constitutes a serious and global public healthcare problem. As such, it becomes relevant to understand the different singularities experienced by these individuals. This study aimed to investigate if binge eating in adults with obesity is influenced by their eating behavior, social support satisfaction, BMI, perceived health status, lifestyle and socio-demographical variables. It also aimed to compare binge eating between individuals from continental Portugal and São Miguel, Açores - cluster of islands with the largest national obesity incidence.

Methods: This sample consisted of individuals from both continental Portugal $(n=117)$ and São Miguel $(n=70)$. Participants filled in the Binge Eating Scale, the Satisfaction with Social Support Scale and Dutch Eating Behaviour Questionnaire, either online or in paper-pencil format. Both health status and BMI were self-reported. Structural equation modelling was used to build a structural model which had binge eating has the dependent variable.

Results: Results point to an association between binge eating and external eating $(\beta=.559 ; p<.001)$, emotional eating $(\beta=.530 ; p<.001)$ and satisfaction with support from friends $(\beta=-.206 ; p=.016)$. There was no noticeable association between binge eating and BMI $(r=.225 ; p=.609)$, or differences between individuals from continental Portugal and São Miguel regarding binge eating $(t(115.897)=-1.382 ; p=.170)$.

Conclusion: Interventions intending to promote efficient strategies for the management of binge eating should target the development of informal support networks (namely, with friends) that can be combined with health promotion activities (e.g., physical exercise), with the objective of lessening the binge eating severity. The absence of differences between the samples from continental Portugal and the islands in relation to binge eating raises the hypothesis of other variables being able to explain the differences between the prevalence of obese individuals, other than the ones studied (e.g., high sodium diet).

Conflict of Interest: None Disclosed

Funding: This research was funded by the Fundação para a Ciência e Tecnologia FCT (grant SFRH/BPD/77799/2011). William James Center for Research is funded by the Fundação para a Ciência e Tecnologia - FCT (grant UID/PSI/04810/2013). 


\section{T2P24}

\section{Biochemical and technology supported feedback for successful weight loss}

\section{Tejpal, S. ${ }^{1}$, Sanghera, N. ${ }^{1}$; Manoharan, V. ${ }^{1}$; Planas-Iglesias, J. ${ }^{1}$; Klein-} Seetharaman, J.2

${ }^{1}$ Warwick Medical School, University of Warwick, Coventry, UK, ${ }^{2}$ Warwick Medical School, University Of Warwick, Coventry, Uk And Institute For Digital Healthcare, Warwick Manufacturing Group, University Of Warwick, Coventry, Uk

Introduction: The prevalence of obesity has spurred great interest in the use of technology such as apps and activity trackers to assist weight loss efforts. Molecular markers of weight change could provide powerful motivators but current technology does not yet include them. We propose to provide personalized and quantitative feedback on feeding habits and their effect on metabolism using insulin and lactate in urine which could be exploited as biomarkers of weight loss.

Method: Participants over the age of 18 were recruited and asked to omit one main meal of the usual breakfast (B), lunch (L) and dinner (D), and to restrict total daily caloric intake to $1200 \mathrm{kcal}$. An optional snack of $\leq 200 \mathrm{kcal}$ (S) was permitted in addition to the two main meals. A mobile health platform was designed in-house to enable participants maintain diaries of food intake, weight, urine collection and volume and physical activity. Insulin was measured using an immunosandwich assay (Mesoscale Discovery Human Insulin Kit). Lactate was measured using an enzymatic assay in which lactate dehydrogenase was used to interconvert lactate to pyruvate following reduction of nicotinamide adenine dinucleotide.

Result: $\mathrm{BD}(\mathrm{S})$ was the most commonly chosen meal plan. Skipping a meal in diet days resulted in consistent weight loss in comparison to control days. Distinct insulin and lactate profiles in urine were observed and an inverse relation to weight loss was found among the last insulin value of the day and lactate concentrations prior to meal 2 using Pearson Correlation.

Conclusion: The main indicator of successful weight loss is weight change, which is slow or non-existent over short periods of time relevant to behaviour modification. Molecular feedback instead has the potential to be immediate and can thus be used to personalize and optimize dieting behaviour. Future trials will investigate if molecular feedback to individuals produces greater levels of success in longer term weight loss programmes.

Conflict of Interest: The authors declare no competing interest

Funding: The study was funded by Judith Klein-Seetharaman's University of Warwick start-up funds

\section{$\mathrm{T} 2 \mathrm{P} 25$}

Body image quality of life inventory: Preliminary study for the adaptation with a Portuguese sample

\section{Pimenta, F. ${ }^{1}$; Rosas, R. ${ }^{2}$; Campos, J. ${ }^{3}$; Silva, W. ${ }^{3}$; Maroco, J. ${ }^{1}$}

${ }^{1}$ William James Center for Research, ISPA - Instituto Universitário de Ciências Psicológicas, Sociais e da Vida, ${ }^{2}$ Ispa - Instituto Universitário De Ciências Psicológicas, Sociais E Da Vida, ${ }^{3}$ Department Of Food And Nutrition, State University Of São Paulo - Unesp

Introduction: Dissatisfaction with part or totality of the body might have a negative impact in some areas of one's life. Moreover, most instruments usually ignore the influences of body image in life fields. Hence, this study aims to show the preliminary analysis regarding the adaptation of the Body Image Quality of Life Inventory, an instrument that assesses the impact of body image on several individual experiences and areas of life, with a Portuguese sample.

Methods: A total of 360 Portuguese college students filled in all instruments, including the Body Image Quality of Life Inventory (BIQLI), the Body Shape Questionnaire (BSQ) and a socio-demographic questionnaire. Participants had a mean age of 20 years $(\mathrm{SD}=2.18)$ and $77 \%$ were women; weight classes were as follows: $12 \%$ below normal weight, $72 \%$ normal weight and 16\% with excessive weight/obesity. A confirmatory factor analysis (CFA) was conducted. The indices chi-square of degree freedom ( $\chi 2 / \mathrm{df})$, comparative fit index (CFI), Tucker-Lewis Index (TLI) and root mean square error of approximation (RMSEA) were used as goodness of fit. The convergent validity was evaluated by average variance extracted (AVE) and the reliability of BIQLI was evaluated by Cronbach's alpha coefficient $(\alpha)$. The correlation between the BIQLI and the BSQ was calculated to evaluate criterion validity.

Results: BIQLI items' sensitivity was adequate $(|\mathrm{ku}|<7$ and $\mid$ sk $\mid<3)$. CFA entailing all items showed an acceptable fit $(\mathrm{X} 2 / \mathrm{df}=3.330$; CFI $=.934$; $\mathrm{TLI}=.923$; RMSEA = .081 C.I. 90\%].073; .089 [p < 0.001). All factorial weights were above .55, except for items 6 (My experiences at work or at school; $\lambda=.38$ ) and 15 (My activities for physical exercise; $\lambda=0.28$ ). The convergent validity was asserted through AVE (.50). An excellent reliability was established $(\alpha=.96)$. Moreover, criterion validity was given through a moderate and significant relationship with a construct expected to be associated with body image quality of life, namely, body shape concerns $(\mathrm{r}=.39 ; \mathrm{p}<0.001)$.

Conclusion: This preliminary analysis show promising psychometric characteristics of the BIQLI in a Portuguese sample. Nonetheless, it should be noticed that further studies are required (specifically, a separate analysis considering sex, since it is expected that the measurement models show some disparities among men and women). Despite this, the current study presents a valid instrument, which is useful, both in normative and clinical settings of research and intervention.

\section{Conflict of Interest: None}

Funding: Research relating to this abstract was funded by the Fundação para a Ciência e Tecnologia - FCT (grant SFRH/BPD/77799/2011). Also, William James Center for Research is funded by the Fundação para a Ciência e Tecnologia - FCT (grant UID/PSI/04810/2013).

\section{T2P26}

\section{Body mass index and illness perception as determinants of depressive symptomatology among cardiac patients}

\section{Pokrajac-Bulian, A. $^{1}$; Kukic, $M{ }^{1}$}

${ }^{1}$ Faculty of Humanities and Social Sciences, University of Rijeka

Introduction: Overweight and obesity impact the whole cardiovascular system and are linked with many forms ofcardiovascular disease (CVD). Depression is commonly present in patients with CVD. It triples the risk of noncompliance with medical treatment regimens and reduces the chances of successful modifications of other cardiac risk factors. As such, depression is associated with increased cardiovascular morbidity and mortality. The present study aims to exam the determinants of depressive symptomatology in men with CVD.

Methods: The research was conducted on a sample of male cardiac patients. A total of 125 men were enrolled in the study, $47 \%$ of the subjects were overweight and $28 \%$ were obese. The subjects ranged in age between 35 and 89 years $(M=61.97, S D=11.69)$. Depression was assessed using the Hospital Anxiety and Depression Scale (HADS), the patient's perceptions of their illness (identity, timeline, consequences, personal control, treatment control, illness coherence, timeline cyclical and emotional representations) were evaluated using the Revised Illness Perception Questionnaire (IPQ-R), and body mass index (BMI) was calculated as weight divided by height squared.

Results: The regression analysis results indicated that measured variables accounted for a significant proportion of the variance in depression (42\%) in men with CVD. BMI and some of the components of illness perception (namely, identity, treatment control and emotional representations) were significant predictors of depression, even after including age and professional status as control variables in the analysis. The higher level of body mass index, strongly held beliefs about the number of symptoms attributed to the CVD, the more negative beliefs about the effectiveness of the treatment, and negative emotional representations(fear, anxiety, and anger) are found to be potential risk factors for the development of depressive symptoms in men with CVD. 
Conclusion: The results from the present study show that objective as well as subjectivefeatures of an individual may play the significant role in the course of depressive symptomatology among cardiac patients. These findings are of clinical importance, suggesting that working on losing weight and modifying maladaptive illness perceptions may reduce depressive symptomatology in obesemen with CVD, and, consequently, affect health behaviors and CVD recovery.

Conflict of Interest: None Disclosed

Funding: No Funding/Research relating to this abstract was funded by the University of Rijeka, project number 13.04.1.3.07.

\section{T2P27}

Body mass index is associated with static balance and fall-risk in elders independently of age, gender, and moderate-tovigorous physical activity

\section{César, A. ${ }^{1}$; Bohn, L. ${ }^{1}$; Santos, A. ${ }^{1}$; Andaki, A. ${ }^{1}$; Alves, A. ${ }^{\prime}$; Carvalho, J. ${ }^{1}$}

${ }^{1}$ Research Centre in Physical Activity, Health and Leisure. Faculty of Sport. University of Porto. Portugal.

Introduction: Falls occur frequently in growing elderly population and are a leading cause of morbidity and mortality in elders. Researches show that impairment of balance and postural control plays a major role in falling, and lose of balance is associated with intrinsic and extrinsic factors. The biomechanical evidence suggests that increased body mass index (BMI) worsens the postural control. The aim of this study was to observe the association between balance with obesity and physical activity in elders.

Methods: This is a cross-sectional study composed by 170 elders (age mean $72.3 \pm 6.6$ years; $73 \%$ female) independent in daily living activities. Static and dynamic balances were assessed with the Performance-Oriented Assessment of Mobility (POMA) test. Obesity was assessed with body mass index (BMI). Physical activity was objectively measured with accelerometry and data was transformed in minutes per day in moderate-to-vigorous physical activity. Multiple linear regression with "enter" method of variable selection was used. Significant level was set at 0.05 .

Results: Body mass index $(\beta=-0.11,<0.01)$ and age $(\beta=-0.12, p<0.01)$ were associated with static balance, independently of gender and moderate-to-vigorous physical activity.

Conclusions: In elders, obesity seems to negatively impair static balance independently of the ageing process and the level of moderate-to-vigorous physical activity.

\section{Conflict of Interest: None}

Funding: Research related to this abstract was funded by Coordination for the Improvement of Higher Education Personnel (CAPES) No 10143-13-0 and CIAFEL (Faculty of Sport, University of Porto) UID/DTP/00617/2013

\section{T2P28}

\section{Body perception and dissatisfaction in first graders}

\section{Jimenez-Flores, P. ${ }^{1}$; Bacardí-Gascón, M. ${ }^{1}$; Jiménez-Cruz, A. ${ }^{1}$}

${ }^{1}$ Universidad Autónoma de Baja California

Background: Body image refers to a mental self-representation of the size, shape and form of our body; it also involves how we feel about our bodies. The difference between perceived and desired silhouette is defined as body image dissatisfaction (BID). Body image misperception is one of the manifestations of eating disorders. Cases in which alterations of body image exist, symptoms appear at four response levels: psychophysiological, behavioral, cognitive and emotional.

Objective:To evaluate body image perception and body dissatisfaction of a group of children aged 6-7 years by using a validated pictogram adapted for Mexican children.

Material \& methods:Participants were students aged six and seven years old from eight elementary schools in the city of Ensenada, Baja California.
Anthropometric measurements of weight, height and waist circumference were taken. The Children's Body Image Scale (CBIS) was used to assess body dissatisfaction. Body Image Dissatisfaction (BID) was calculated by subtracting the "'ideal figure"' to the "'perceived image"'. Negative results indicate dissatisfaction for wanting to be robust and positive results for wanting to be thinner.

Results: 431 first graders were evaluated, of which $50.5 \%$ were boys. Thirty-nine percent were overweight or obese. Twelve percent of the children perceived their body image correctly, $74 \%$ and $15 \%$ underestimated and overestimated it respectively. A greater percentage of the thin population overestimated their body size; while children with normal, overweight and obesity underestimated it. Seventy-two percent had body dissatisfaction; there was no difference between genders. Mild BID was found in $40.5 \%$ (equally distributed by wanting to be more robust and thinner), moderate in $23.5 \%$ (64\% wanting to be thinner), and severe in $7.5 \%$ (84\% wanting to be thinner) of the children. According to BMI categories, $66 \%$ in thin/normal and 34\% in overweight/obese categories presented BID.

Conclusion: Seventy-four percent of the first graders underestimated their body size. Children present BDI from an early age because they desire to be thinner. Within this age group, there were no significant differences in BID between genders or between different BMI categories.

Acknowledgement: CONACYT

Funding: \#0212727

\section{T2P29}

Body size ideals and dissatisfaction in Ghanaian adolescents: Role of media, diet, physical activity and well-being.

Michels, N. ${ }^{1}$; Amanyah, S. ${ }^{1}$

${ }^{1}$ Ghent University

Aims. To inspire effective health promotion campaigns, we tested the relationship of ideal body size and body size dissatisfaction with lifestyle, well-being and media. In addition, mediation was tested: (a) "can lifestyle and well-being explain overweight as a consequence of unrealistic ideal body size or body size dissatisfaction?" and (b) "can perceived media influence play a role in the effects of actual body size on body size dissatisfaction?".

Methods:This cross-sectional study included 370 Ghanaian adolescents aged 11-18y. Questionnaires included disordered eating (EAT26), diet quality (FFQ), physical activity (IPAQ), well-being (KINDL) and media influence on appearance (SATAQ: pressure, internalization and information). Ideal body size and body size dissatisfaction were assessed using the Stunkard figure rating scale. BMI, skinfolds and waist were measured. Linear regressions were adjusted for gender, age and parental education. Results: Though 64\% preferred the normal-weight ideal, the traditional preference for the overweight ideal was still present. Body size dissatisfaction was higher in adolescents who preferred the overweight or underweight ideal. Body size dissatisfaction was associated with lower well-being $\left(c^{2} \mathrm{p}=0.087\right)$ and more media influence (pressure and internalization; $\left.c^{2} \mathrm{p}=0.058\right)$ but not with physical activity, diet quality or disordered eating. An underweight body size ideal might worsen disordered eating but it was not significantly related to the other predictors of interest. Only a mediation effect by media pressure was found: especially overweight adolescents felt media pressure and this media pressure was associated with more body size dissatisfaction.

Conclusions. To prevent disordered eating and low well-being, obesity interventions should include strategies that reduce body size dissatisfaction and increase body-esteem by not focusing on the thin body size ideal. Changing body size ideals in media might be an appropriate way since media pressure was a mediator in the BMI-dissatisfaction relation. 
T2P32

\section{Changes in legislation as an effective tool of obesity prevention and their application in the practice}

\section{Dana, M. ${ }^{1}$; Jarmila, R. ${ }^{2}$; Alexandra, $K^{3}$ \\ ${ }^{1}$ Charles University, Medical Faculty in Pilsen, ${ }^{2}$ The Regional Hygiene Station In Prague, ${ }^{3}$ The National Institute Of Public Health, Prague}

Czech Republic 28 years ago passed the transformation from socialism to democracy. During that time muscular fitness of schoolchildren was dramatically reduced and the prevalence of obesity and overweight was doubled. The strict socialistic rules completely prohibiting the sale of food in school were broken and schools had commercial profit of that additional sale without any limitation of offered foods and non-alcoholic drinks. The sale in school machine and buffets were conducted on self-regulatory scheme only. The structure of offered food and drinks were monitored by supervisory authorities during 2011, 2014, and 2016 in 1367 schools. The data showed unhealthy foods and drinks high in fat, sugar, and salt as the prevailing kind in supply and demand. In that context Ministry of Schoo Education in cooperation with Ministry of Health managed to enforce legislative limitations (282/2016 Coll.) on foods and drinks which can be offered to children in schools. These criteria were constructed with the help of WHO Regional Office for Europe nutrient profile model, however in softened form. Even though the novel of that law was accepted a big wave of protests started during its application to life.

Conflict of Interest: None disclosed

Funding: The Czech National Action Plan, Health 2020.

\section{T2P33}

\section{Changes in plasma miRNAs expression in healthy overweight and obese subjects: The GLYNDIET study}

\section{Giardina, S. ${ }^{1}$; Alonso, P. ${ }^{2}$; Salas-Huetos, A. ${ }^{2}$; Salas-Salvadó, J. ${ }^{3}$; Bulló, M. ${ }^{4}$}

${ }^{1}$ Human Nutrition Unit URV Reus Spain, ${ }^{2}$ Human Nutrition Unit Urv Reus Spain, lispv Reus Spain, ${ }^{3}$ Human Nutrition Unit Urv Reus Spain,lispv Reus Spain,

Ciberobn-Isciii Madrid, ${ }^{4}$ Human Nutrition Unit Urv Reus Spain ,lispv Reus Spain, Ciberobn-Isciii Madrid

Introduction: MicroRNAs are small non-coding RNA molecules that control gene expression in several biological processes and diseases. Several macro and/or micronutrients can modify the miRNAs expression, however little is known on the effect of long-term energy restricted diets on miRNAs expression. We therefore explored the effect of 3 energy-restricted diets with different glycemic index on plasma miRNAs levels in overweight and obese subjects from the GLYNDIET study.

Methods: GLYNDIET study is a 6-month, parallel, randomized clinical trial conducted on overweight and obese subjects who were randomized to one of 3 different dietary intervention groups: 1) a moderate-carbohydrate and low glycemic index diet (LGI), 2) a moderate-carbohydrate and high glycemic index diet (HGI), and 3) a low-fat and high glycemic index diet (LF). We assessed the genome-wide circulating miRNAs profile in a subsample of 8 patients by TLDS assay. The most relevant changing miRNAs were therefore validated in 103 subjects using TaqMan MicroRNA Assays. ( $\mathrm{n}=36 \mathrm{LGI}, \mathrm{n}=36 \mathrm{HGI}$ and $\mathrm{n}=31 \mathrm{LF}$ ).

Results: A total of 8 miRNAs differently expressed in the representative samples were selected. In the validation study, the between-group analyses showed a significant decreased miR-361 levels in LGI group compared to LF group. While in the intra-group analyses we observed a significant downregulation of all miRNAs screened in subjects after the LGI intervention. We also found miR-139-3p and miR-340 downregulated between the beginning and the of HGI intervention, while in LF group miR-139-3p, miR-432, miR-99p and miR-423-5p were downregulated after the intervention.

Conclusion: Our results suggest that the composition of diets can selectively affect circulating plasma miRNAs levels in healthy overweight and obese subjects.
Conflict of Interest: The authors do not report any conflict of interest

Funding: Supported by the Institut d'Investigació Sanitaria Pere Virgili (PV11059S) and the Fondo de Investigación Sanitaria (PI12/0153), Instituto de Salut Carlos III

\section{T2P34}

\section{Changes in quality of life, dietary, activity and self-monitoring behaviours among severely obese individuals attending a multidiscplinary weight management programme}

\author{
Gaynor, K. ${ }^{1}$; Breen, C. ${ }^{2}$; Dunlevy, C. ${ }^{2}$; O'Malley, E. ${ }^{2}$; Wallace, N. ${ }^{2}$; Yoder, R. ${ }^{2}$;
} O'Shea, D. ${ }^{2}$

${ }^{1}$ Weight Management Service, St. Columcille's Hospital, Loughlinstown, Co. Dublin, Republic of Ireland, ${ }^{2} \mathrm{~S} / \mathrm{S}$

Aims:The Weight Management Service (WMS), St Columcille's Hospital is a specialist service for the management of severe obesity. Patients attend a behavioural programme delivered by a team of clinical psychologists, registered dietitians and chartered physiotherapists. The aim of the programme is development of self-management skills and behaviours that support long-term weight management. This audit examined changes in quality of life, function and self-management behaviours among individuals who attended the service from 2013-2015.

Methods:Data were gathered at baseline and at 8-12 months using self-report questionnaires modified from National Weight Control Registry [1], Weight and Lifestyle Inventory [2], Impact of Weight on Quality of Life (IwQOL)[3] and a 6-minute walk test. Data was analysed using Microsoft Excel 2010 (Microsoft, Washington, USA). Statistical significance was determined using Chi-square (categorical data), paired t-tests (parametric data) and Wilcoxin Signed Rank test (non-parametric data). $\mathrm{P} \leq 0.05$ was considered statistically significant.

Results:Individuals with repeat data were $62 \%$ female, mean age $44.7 \pm 12.1$ yrs, weight $145.7 \pm 28.3 \mathrm{~kg}$ and body mass index $50.9 \pm 8 \mathrm{~kg} /$ $\mathrm{m}^{2}$. Repeat weight was $139.8 \pm 28.8 \mathrm{~kg}$ and body mass index $48.8 \pm 7.3 \mathrm{~kg} /$ $\mathrm{m}^{2}$.

Conclusions: Quality of life, functional outcomes and self-management behaviours that are associated with health, weight loss and longer-term weight maintenance improve significantly among severely obese individuals who attend the WMS at St Columcille's Hospital.

\section{References:}

1 Wing, R.R. and S. Phelan, Long-term weight loss maintenance. American Journal of Clinical Nutrition, 2005. 82(1 Suppl): 222S-225S.

2 Wadden, T.A. and G.D. Foster, Weight and Lifestyle Inventory. Surg Obes Relat Dis, 2006. 2(2):180-99.

3 Kolotkin RL, Crosby RD, Kosloski KD, Williams GR. Development of a brief measure to assess quality of life in obesity. Obesity Research. 2001;9:102-11.

Table 1. to T2P34
\begin{tabular}{|l|l|l|l|l|l|}
\hline & $\mathrm{n}$ & Baseline & $\begin{array}{l}12 \\
\text { months }\end{array}$ & Change & P Value \\
\hline Importance(x/10) & 85 & $8.5 / 10$ & $9 / 10$ & $0.5 / 10$ & 0.121 \\
\hline Confidence(x/10) & 85 & $6.5 / 10$ & $7.5 / 10$ & $1 / 10$ & $<0.05$ \\
\hline $\begin{array}{l}\text { Self-monitor weight at least weekly } \\
\text { (\%) }\end{array}$ & 85 & 27.2 & 53.5 & $25 \%$ & $<0.01$ \\
\hline $\begin{array}{l}\text { Self-monitor food at least weekly } \\
\text { (\%) }\end{array}$ & 66 & 5 & 26.7 & $22 \%$ & $<0.01$ \\
\hline Breakfast(days/week) & 66 & $5.1 \pm 2.6$ & $6.3 \pm 1.7$ & 1.1 & $<0.05$ \\
\hline 1 or more takeaways/week(\%) & 85 & 86.1 & 63.3 & $-23 \%$ & $<0.01$ \\
\hline 1 or more portions of fruit/d(\%) & 78 & 43.2 & 64.2 & $21 \%$ & $<0.05$ \\
\hline 1 or more portions of veg/d(\%) & 101 & 43 & 59.3 & $16 \%$ & $<0.01$ \\
\hline Cooking a meal $>4$ times/week(\%) & 80 & 37.5 & 52.5 & $15 \%$ & 0.06 \\
\hline Physical activity level(mins/week) & 73 & 94.9 & 177.8 & 82.9 & $<0.001$ \\
\hline Gait speed(metres/second) & 129 & $1.1 \pm 0.3$ & $1.2 \pm 0.3$ & $0.1 \pm 0.2$ & $<0.0001$ \\
\hline Sleep(hours) & 130 & $6.5 \pm 1.5$ & $6.6 \pm 1.6$ & 0.1 & 0.34 \\
\hline Lower back pain(x/10) & 78 & $7.4 \pm 2.6$ & $5.6 \pm 3.6$ & $-1.8 \pm 3.8$ & $<0.001$ \\
\hline Knee pain(x/10) & 59 & $6.9 \pm 2.6$ & $5.8 \pm 3.0$ & $-1.1 \pm 3.0$ & $<0.005$ \\
\hline IwQOL score & 45 & 39 & 55 & 16 & $<0.001$ \\
\hline
\end{tabular}




\section{T2P35}

\section{Changes to psychosocial health for obese women during} energy restriction with and without exercise.

\section{Hoek, D. ${ }^{1}$; Miller, C. ; Fraser, S. ${ }^{1}$; Selig, S. ${ }^{1}$; Dixon, J. ${ }^{2}$}

${ }^{1}$ Deakin University Australia, ${ }^{2}$ Baker Idi Heart And Diabetes Institute

Introduction: Obese individuals, and in particular, young women, experience poorer psychosocial health. Exercise and weight loss are both seen to independently, positively, influence quality of life (QOL) and depressive symptoms amongst obese populations. Less is known regarding the possible synergistic or additive effects when exercise and weight loss are combined for treatment.

Methods: Sixty obese women aged $18-50$ years (Mean (SD); BMI $=40.43$ (6.63) $\mathrm{kg} / \mathrm{m}^{2}$; Age $=37$ (9) years) were recruited and allocated to a 12 month program consisting of a very low calorie diet (VLCD) with or without the addition of exercise, EXER and ER respectively. The VLCD was supervised by Accredited Practicing Dietitians and exercise interventions were delivered by Accredited Exercise Physiologists. Dietary and exercise interventions followed a reducing contact protocol over the 12 month program. QOL was assessed using the Short Form 36 Medical Outcomes Survey (SF-36) at baseline, 3, 6 and 12 months intervention.

Results: Mean weight of participants at baseline was EXER: $111.38 \mathrm{~kg}$ (12.48), ER: 114.08 (23.55). Eighteen women withdrew from the study or were lost to follow-up, 31 women completed SF-36 surveys and 29 completed Beck Depression Inventory (BDI) surveys at baseline and 12 months. Mean mental and physical component summary scores (MCS and PCS respectively) at baseline for the SF-36 were EXER MCS: 42.1 (9.9) PCS: 46.8 (6.4) and ER MCS: 43.4 (10.3) PCS: 46.9 (8.2). Mean BDI scores for groups at baseline was EXER: 12.65 (8.08) and ER: 14.08 (11.46), indicative of minimal depressive symptoms. Moderate-severe depressive symptoms were reported by 16 women (EXER $n=7$, ER $n=9)$ at baseline and by four women at 12 months (EXER $n=1$ ER $n=3$ ). There was no group by time interaction in any domain or summary score of the SF-36 or BDI surveys at any time point.

Conclusion: This study shows that the addition of exercise intervention to energy restriction did not improve QOL or depressive symptoms for obese women. QOL results are consistent with those of previous research in similar populations as evidenced by a recent systematic review.

Conflict of Interest: Nestle Health Science provided VLCD product at cost.

Funding: Research relating to this abstract was funded by Deakin University and Baker IDI Heart and Diabetes Institute.

\section{T2P36}

\section{Clinical findings on body mass index in an intellectual disability population and the effect of a healthy lifestyle intervention clinic}

\section{Boylan, P. ${ }^{1} ;$ O'Shea, D. ${ }^{2}$; Mccormack, B. ${ }^{1}$; Woods, C. ${ }^{3}$}

${ }^{1}$ Cheeverstown, ${ }^{2}$ St Vincents University Hospital Dublin $4,{ }^{3}$ Nass General Hospital Kildare

Background: Persons with Intellectual Disability (ID) are supposedly pre-disposed to weight gain and obesity. Data on weight status and weight loss interventions in persons with ID is scarce. Medication, anxiety/stress and metabolic diseases are among factors blamed for weight gain. Persons with ID may not be supported to make good/healthy decisions about calorie intake and food choice.

We sought to ascertain the prevalence of obesity in an ID population and the impact of a 'healthy lifestyle' clinic.

Methods:We analysed data on 149 adults (women $=69$, mean age $=44.6 \pm 14.8$, range $19-95)$ attending the ID service in Cheeverstown.

Prospective data was available on 23 adults (women $=22$, baseline $\mathrm{BMI}=39.1 \pm 7.5 \mathrm{kgs} / \mathrm{m}^{2}$ ) attending a monthly 'healthy lifestyle' clinic, where diet \& lifestyle advice was available to attendees, family members \& staff involved in purchasing \& food preparation.
Bi-annually, visits included food \& exercise diary analysis, weight measurement along with biochemical testing for diabetes \& thyroid dysfunction. Psychotropic/epileptic medications were rationalised under review by Psychiatry/Neurology.

Results: The mean BMI for the total 149 adults with ID is as follows: 69 females with ID $\left(29.67 \mathrm{~kg} / \mathrm{m}^{2}\right.$, range $15.10 \mathrm{~kg} / \mathrm{m}^{2}-53 \mathrm{~kg} / \mathrm{m}^{2}, \pm$ SD $\left.7-75\right)$ is significantly greater than the mean BMI for 80 males with ID ( $26.14 \mathrm{~kg}$ / $\mathrm{m}^{2}$, range $\left.12.10 \mathrm{~kg} / \mathrm{m}^{2}-54.90 \mathrm{~kg} / \mathrm{m}^{2}, \pm \mathrm{SD} 7.32\right)$.

Prospective data on 23 attending Intervention Clinic.

Figure - shows weight loss and change in BMI in those attending the intervention clinic. There were low levels of alcohol consumption and none smoked cigarettes. Age range was $19-69$. The mean weight loss was $6.1 \pm 8.4 \mathrm{kgs}(93.9 \pm 26.6$ vs. $87.9 \pm 23.4 \mathrm{kgs}, \mathbf{p}=\mathbf{0 . 0 0 1})$. GLP-1 was prescribed for 4 people. 12/23 lived at home.

Conclusion:

- $\quad$ Prevalence of obesity In this adult ID cohort is similar compared to the general public.

- Persons with Downs Syndrome had a significantly higher BMI compared to ID only.

- $\quad$ Persons with ID living in the community had significantly higher BMI compared to those in residential care.

- $\quad$ Diet \& Lifestyle interventions and medication rationalisation were successful in producing sustained weight loss.

\section{T2P38}

\section{Comparison of the obesity indices to predict cardiovascular risk factors and metabolic syndrome in Korean and US} populations using representative nationwide sample data

\section{Oh, S. ${ }^{1}$; Kim, J. ${ }^{2}$; Yoon, $Y^{3}{ }^{3}$; Yun, $Y^{4}$}

${ }^{1}$ Department of Family Medicine, Center for Obesity, Metabolism and Nutrition, Dongguk University Ilsan Hospital, Goyang-Si, Gyeonggi-Do, Korea, ${ }^{2}$ Department Of Neurosurgery, Hanil Hospital, Jinju-Si, Gyeongsangnam-Do, Korea, ${ }^{3}$ Department Of Family Medicine, Inje University Ilsan Paik Hospital, Goyang-Si, Gyeonggi-Do, Korea, ${ }^{4}$ Department Of Family Medicine, Seoul National University Hospital, Seoul, Korea

Introduction: Obesity is closely related with cardiovascular disease (CVD) risk factors including diabetes, hypertension, and hyperlipidemia and metabolic syndrome (MetS). The appropriate selection of an obesity indices is particularly important to predict CVD risk factors and MetS. The present study was performed to identify which obesity indices predict better CVD risk factors and to evaluate ethnic differences in the relationship obesity indices with CVD risk factors and MetS.

Methods: A total number of 126,718 (28,377 Korean and 98,314 USA) subjects, aged 19 or more, who participated in the 2008-2011 Korean National Health and Nutrition Examination Survey (NHANES) and in the 1999-2006 US NHANES were analyzed. Six obesity indices including body mass index (BMI), waist circumference (WC) and total body fat mass, total body fat percent, trunk fat mass and trunk fat percent measured by dual-energy $\mathrm{x}$-ray absorptiometry were investigated. Subjects who have two or more of components of Mets except WC was defined as the Mets. Multivariate logistic regression analysis and ROC analysis were conducted to identify the relationships between obesity indices and CVD risk factors and MetS by ethnic groups.

Results: Total body and trunk fat mass in Koreans tended to be lower at the same BMI and WC level when compared to all ethnic groups of US population. Total body and trunk fat percent in Koreans were significantly higher than those in Non-Hispanic Blacks for BMI categories $<25 \mathrm{~kg} / \mathrm{m} 2$ and $\mathrm{WC}<90 \mathrm{~cm}$, but significantly lower for BMI categories $\geq 25 \mathrm{~kg} / \mathrm{m} 2$ and $\mathrm{WC} \geq 90 \mathrm{~cm}$. Koreans had greater odds ratios for diabetes, hypertension, and hypercholesterolemia and MetS according to each unit increase of all obesity indices compared to the other ethnic groups. WC showed the highest prediction rate for diabetes, hypertension, and Mets among the obesity indices, while total body fat mass and total body fat percent had relatively lower prediction rate in all ethnicity groups. 
Conclusions: Koreans showed higher risk for diabetes, hypertension, hypercholesterolemia and MetS despite of the lower total body and trunk fat mass at the same BMI and WC level than other ethnic groups in USA. Our results suggest that the diagnostic criteria for obesity in Korea, which are lower than other ethnic groups, can be an appropriate. Additionally, trunk fat mass and percent were highly predictive for the diagnosis of CVD risk factors and MetS compared with total body fat mass and percent. Thus, trunk fat mass and percent would be more appropriate for using the obesity diagnosis in Korean as well as in US populations.

Conflict of Interest: The authors declared no conflicts of interest.

Funding: No Funding sources

\section{T2P39}

Consumption of sugar-sweetened drinks: Evidence to support government policy of taxation as part of a suite of measures to address obesity

Harrington, J. ${ }^{1}$; Perry, C. ${ }^{2}$; Keane, E. ${ }^{3}$; Perry, I. ${ }^{4}$

${ }^{1}$ University College Cork, ${ }^{2}$ University Of Cambridge, ${ }^{3} \mathrm{HSE},{ }^{4}$ University College Cork

Introduction: The obesity epidemic represents a public health crisis with the potential to reverse recent favourable trends in life expectancy and undermine the financial viability of health systems. Though multifactorial in origin, links between the consumption of sugar-sweetened drinks (SSD) and excessive weight gain in children have been observed. Further excessive consumption is associated with increased prevalence of dental caries. The aim of this study is to provide evidence of the magnitude of the consumption of SSBs in Ireland and to explore the association between SSD consumption and overweight and obesity.

Methods: Cross sectional datafrom 1075 8-11 year old school children. Consumption of SSD was assessed from 3-day food diaries. Height (m) and weight $(\mathrm{kg})$ were measured using standard Methods: BMI was used to define obesity (IOTF definitions). Plausible energy reporters (PER) were classified using Scholfield equation. Results are reported for PER only. Results: Eighteen percent of children with PER were overweight (16\%) or obese $(2 \%)$ compared with $25 \%$ of the total sample. Of those with PER, $82 \%$ were SSD consumers. Mean calories from SSDs increased incrementally between weight categories: SSD contributed $108 \mathrm{kcal}$, and $155 \mathrm{kcal}$ for normal weight and overweight/obese children respectively, equating to $5.8 \%$; and $7.6 \%$ of total calories respectively. Mean intake volumes were significantly higher in children who were overweight or obese compared to normal weight children. Average consumption volume was $383 \mathrm{ml} / \mathrm{d}$ $368 \mathrm{ml} / \mathrm{d}$ and $315 \mathrm{ml} / \mathrm{d}$ for overweight/obese and normal weight children respectively. Adjusting for gender, parental education, physical activity and tv viewing, compared to low volume consumers $(<200 \mathrm{ml} / \mathrm{d})$, high consumers $(>200 \mathrm{ml} / \mathrm{d})$ had an increased odds of being overweigh/obese (OR 1.9; 95\%CI [1.0-3.5]).Further, within quintiles of volume of SSD consumption, there was a clear downward population shift in BMI distribution.

Conclusion: While no single measure will reverse current trends in obesity, given the high level of consumption of SSD and the lack of nutritional value of these products, action needs to be taken to reduce consumption, particularly in high consumer groups e.g. children. There is a compelling case for the introduction of public policy to reduce SSD consumption in the population. The introduction of a tax on SSDs in combination with other public health interventions has the potential to have a measurable effect on the scale of the epidemic of childhood obesity.

\section{T2P41 \\ Cultural pattern of gender and the use of wellness treatments in overweight women}

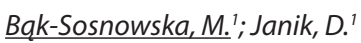

${ }^{1}$ Department of Psychology, Chair of Social Sciences and Humanities, School of Health Sciences in Katowice, Medical University of Silesia in Katowice, Poland

Cultural pattern of gender and the use of wellness treatments in overweight women

Introduction: Gender pattern is a set of psychological characteristics culturally attributed to woman or man. Psychological gender identity forms itself since childhood as a result of socialization and affects human personality and behavior. The goal of the study was to examine the relationships between the use of wellness treatments and the cultural patterns of gender in overweight women.

Methods: The participants were 60 adult overweight women, their average age was $37 \pm 11$, the average BMI was $29,74 \pm 2,82$. The control group consisted of 60 women homogeneous in terms of age and BMI, having never benefited from wellness treatments. Participants completed Sex Role Inventory (SRI) and our own survey.

Results: The most popular form of wellness in the examined group was relaxing massage $(46,7 \%)$, body treatments with peeling and mask $(43,3 \%)$ and spa treatments $(43,3 \%)$. The most important reasons for using wellness treatments were: improving the appearance of body (66.7\%), relaxation $(60 \%)$ and increasing the self-esteem (56.7\%). In the SRI examined group, an indeterminate type of gender was dominant (50\%). Feminine and masculine types were subscribed to the number of $18,3 \%$ participants respectively. The next $13,3 \%$ of participants constituted the individuals of the androgynous type. The results did not differ significantly from the results in the control group $(\mathrm{p}=0.057)$.

Conclusion:There is no relationships between the use of wellness treatments and the cultural pattern of gender in overweight women.

Conflict of Interest: None Disclosed

Funding: No Funding

\section{$\mathrm{T} 2 \mathrm{P} 42$ \\ Determination of the fructose enrichment threshold in diets inducing nonalcoholic fatty liver disease and its characteristics}

Fakhoury-Sayegh, N. '; Sayegh, R. ${ }^{2}$; Track-Smayra, V. ${ }^{\text {; }}$ Obeid, O.4; Saad, S. ${ }^{5}$; Khazzaka, $A .^{6}$

${ }^{1}$ Department of Nutrition, Faculty of Pharmacy, Saint Joseph University, Damascus Road, Beirut, Lebanon, ${ }^{2}$ Department Of Gastroenterology, Faculty Of Medicine, Saint Joseph University, Damascus Road, Beirut, Lebanon, ${ }^{3}$ Department Of Pathology, Faculty Of Medicine, Saint Joseph University, Damascus Road, Beirut, Lebanon, ${ }^{4}$ Department Of Nutrition And Food Sciences, Faculty Of Agricultural And Food Sciences, American University Of Beirut, Beirut, Lebanon, ${ }^{5}$ Department Of Laboratory Sciences, Faculty Of Pharmacy, Saint Joseph University, Damascus Road, Beirut, Lebanon, ${ }^{6}$ Department Of Surgical Research, Faculty Of Medicine, Saint Joseph University, Damascus Road, Beirut, Lebanon

Background/objectives: A fructose enriched diet has been shown to be associated with the induction of parameters of metabolic syndrome and an increase in fatty liver, fibrosis and inflammatory markers. Our objective was to induce, within 16 weeks, the characteristics of nonalcoholic fatty liver disease (NAFLD), using three different diets enriched in varying levels of fructose, and to compare the results to those of a control diet. Another objective was to determine the threshold of fructose enrichment capable of inducing nonalcoholic fatty liver disease and its characteristics. Materials/Methods: Forty (6 weeks old) Wistar male rats, weighing an average of $150 \mathrm{~g}$ were randomly divided into four groups of ten, and each group assigned diets of equal quantity ( $15 \mathrm{~g} / \mathrm{rat})$ but of varying composition in fructose. The first group was fed $15 \mathrm{~g}$ of a standardized diet $(16.9 \%$ of fat, $21.0 \%$ of proteins, $62.1 \%$ of carbohydrates). The second group was fed a high fructose diet ( $30 \%$ fructose), while the third and the fourth 
group were fed a $20 \%$ and $10 \%$ fructose enriched diet respectively. At week 16, the rats were sacrificed and liver and kidneys excised.

Results: At week 16, the second group (30\% fructose enriched diet) had the highest percentage of cells enriched in fat (10\%), an induced macrovacuolar and microvesicular steatosis, a perisinusoidal fibrosis and a significant decrease in adiponectin as compared to week $1(P<0.05)$. The third and the fourth groups showed a mild and absent perisinusoidal fibrosis respectively, and neither showed significant steatosis (5\%). No significant difference was observed in liver, kidney, epidydimal fat and body weight between groups $(P>0.05)$. The control group showed no change in any of the above-mentioned parameters.

Conclusions: Ten percent fructose enrichment could be considered as the threshold for a diet inducing NAFLD.

Keywords: Adiponectin, fructose, Wistar rats, perisinusoidal fibrosis

\section{$\mathrm{T} 2 \mathrm{P} 43$}

Development of physical activity, BMI, and waist circumference (WC) during the first working year in different job sectors.

\section{Dreher, M. ${ }^{1} ;$ Simon, $P^{2}$}

1 Johannes Gutenberg University Mainz, Department of Sports Medicine, Rehabilitation and Disease Prevention, ${ }^{2} J$ ohannes Gutenberg University Mainz, Faculty Of Social Science, Media And Sport Department Of Sporehabilitation And Disease Preventionrts Medicine,

Introduction: The start in work life initiates health-related challenges for apprentices.

In Germany 30.0\% of women and 35.3\% of men between 18 and 29 are overweight ${ }^{1}$. So far, the impact of the first working year on BMI and WC development and related factors like physical activity has not been studied.

Methods: 9 Employees from 5 different job sectors in the rhine/main region took part in a 2 years study. BMI and WC was measured and health related aspects, e.g.

Habitual Physical Activity (HPA) ${ }^{2}$, were assessed via questionnaire in 679 apprentices within the first 3 working months. 616 apprentices took part at a recall in 2015 enabling a follow-up for 412 participants.

Results: Categorized by BMI, 3.9\% were under-, 70.6\% normal-, $21.4 \%$ overweight and $4.1 \%$ obese, while categorized by WC, $86.2 \%$ were normal-, $8.7 \%$ overweight and $4.1 \%$ obese.

Table 1 shows the BMI and the WC in the 5 different job sectors and likewise the total collective was dichotomized according to HPA-Sport Index in 2014 (ACTIVE \& INACTIVE).

There is significant increase of the BMI in laboratory and industrial apprentices, while in healthcare WC rose significantly. A significant difference of the WC development for active and inactive participants was found as well.

Discussion: The results of the study show that depending on the job sector apprentices already have different BMI and WC levels at job start. The development of the WC is influenced by the physical activity before job start.
In the total collective, the BMI merely rose according to the expected increase for respective reference collectives while the increase of the WC might be related to metabolic risk.

\section{References:}

1 Mensink G. et al. (2013). Overweight and obesity in Germany. Bundesgesundheitsblatt, (56), 786-794

2 Baecke, JA. et al. (1982). A short questionnaire for the measurement of habitual physical activity in epidemiological studies. The American Journal of Clinical Nutrition (36), pp 936-942

Conflict of Interest: None Disclosed

Funding: No Funding

\section{T2P44}

\section{Diet quality among Portuguese adolescents: Associations between body composition, physical activity, sleep and socio- economic status}

\section{Silva, A. ${ }^{1}$; Fragoso, I. $^{1}$; Teles, $\mathrm{J}^{1}$ \\ ${ }^{1}$ Faculdade de Motricidade Humana, Universidade de Lisboa}

Introduction: Greater interest into the effects of diet on health and its contribution in some diseases has increased in the last decades ${ }^{1}$. Findings suggest that a healthful diet mirrors other health behavioursand health outcomes $^{2,3}$. The aim of this study was to describe the Diet Quality International-Index (DQI-I) of Portuguese adolescents and its associations with body composition, physical activity, sleep duration and socioeconomic status (SES), considering sex differences.

Methods: A cross-sectional study was conducted. Participants answered a semiquantitative food frequency questionnaire (SQPortFoodFQ) and a Portuguese biosocial questionnaire (RAPIL II). The DQI-I was obtained from SQPortFoodFQ in order to evaluate diet quality. Physical activity levels, sedentary behaviour, amount of sleep and SES were collected from RAPIL II. Anthropometric variables included body fatness and body mass index ${ }^{4,5}$.

Statistics analysis included descriptive expressed by mean and standard error. Student's t test and one-way ANOVA were used to study the association between DQI-I and all variables. Differences among DQI-I components were performed by chi-square test. The significance level was set at $\mu=0.05$.

Results: A total of 669 participants enrolled the study. Male were heavier and taller. They had less body fat and mature later comparing to female.

The mean total DQI-I score was $49.6 \%$ of the 100 highest score. Male score better for adequacy component and female showed better results for moderation. Half of the participants showed great compliance for protein, iron, vitamin C, and cholesterol. Around 50\% of the participants reported no inclusion of vegetable and fruit in their diet. Saturated fat, sodium and empty calorie foods were beyond the recommendations.

There were no difference between the DQI-I and sex, body composition, sedentary behaviours, sleep duration and SES. The strongest variable for diet quality was physical activity.

Table 1. to T2P43 Basic BMI and WC characteristics

* sign. for longitudinal comparisons $\mathrm{P} \leq 0.05$; \# sign. between group comparisons a on mean differences

\begin{tabular}{|c|c|c|c|c|c|c|c|c|c|c|c|}
\hline Sector & Proportion in \% & BMI 14 & BM 15 & 95\% CI Lower & $95 \%$ Cl upper ${ }^{\mathrm{a}}$ & $p$-value & WC 14 & $\begin{array}{l}\text { WC } \\
15\end{array}$ & 95\% Cl Lower & $95 \%$ Cl upper & $p$-value \\
\hline Healthcare & 17.0 & 22.5 & 22.7 & -0.20 & 0.77 & 0.6958 & 76.4 & $79.6^{\#}$ & 1.42 & 5.09 & $<.0001^{*}$ \\
\hline Industrial & 39.3 & $23.6^{\#}$ & 24.0 & 0.13 & 0.74 & $0.0002^{*}$ & 78.5 & 79.2 & -0.48 & 1.82 & 0.6997 \\
\hline Commercial & 7.80 & 21.8 & 22.1 & -0.42 & 1.08 & 0.9324 & 73.3 & 74.8 & -1.33 & 4.36 & 0.4521 \\
\hline $\begin{array}{l}\text { Public sector } \\
\text { (police) }\end{array}$ & 30.1 & 23.1 & 23,3 & -0.12 & 0.64 & 0.4726 & 77.0 & 76.9 & -1.30 & 1.56 & 1.0000 \\
\hline Laboratory & 3.40 & $25.2^{\#}$ & 27.2 & 0.45 & 3.45 & $0.0004^{*}$ & 81.7 & 85.7 & -1.42 & 9.42 & 0.3620 \\
\hline ACTIVE & & 23.7 & 23.9 & -0.00 & 0.43 & $0.0438^{*}$ & 77.9 & $77.6^{\#}$ & -0.41 & 1.02 & 0.6864 \\
\hline INACTIVE & & 23.4 & 23.4 & -0.21 & 0.27 & 0.9876 & 78.4 & $79.4^{\#}$ & 0.10 & 1.72 & $0.0191^{*}$ \\
\hline
\end{tabular}


Conclusion: Active and younger participants (10-13 years old) had better diet quality and the results suggest these variables as predictors for DQI-I

References:

1 Wirt A, Collins CE. Diet quality-what is it and does it matter? Public Health Nutr. 2009;12(12):2473-2492.

2 Papadaki S, Mavrikaki E. Greek adolescents and the Mediterranean diet: factors affecting quality and adherence. Nutrition. 2015;31:345-349.

3 Martínez E, Llull R, Del Mar Bibiloni M, Pons A, Tur J a. Adherence to the Mediterranean dietary pattern among Balearic Islands adolescents. $B r J$ Nutr. 2010;103:1657-1664.

4 Lohman T. The use of skinfold to estimate body fatness on children and youth. J Phys Educ Recreat Danc. 1987;58(9):98-102.

5 World Health Organization. WHO Child Growth Standards: Training Course on Child Growth Assessment: Geneva; 2008.

\section{T2P45 \\ Diet quality predicts visceral adiposity and liver fatness: The multiethnic cohort study}

Maskarinec, G. ' ; Lim, U. '; Jacobs, S. '; Monroe, K. ${ }^{3}$; Ernst, T.4 ; Buchthal, S. ; Shepherd, J. ${ }^{5}$; Wilkens, L. ${ }^{1}$; Marchand, L. '; Boushey, C.

${ }^{1}$ University of Hawaii Cancer Center, ${ }^{2}$ University Of Heidelberg, ${ }^{3}$ University Of Southern California, ${ }^{4}$ University Of Hawaii, ${ }^{5}$ University Of California At San Francisco

Introduction: Non-caloric qualitative aspects of diet, such as healthy dietary patterns, may affect visceral adipose tissue (VAT) and nonalcoholic fatty liver (NAFL), key risk factors for cardiometabolic diseases. This study determined the association of four a priori-defined dietary indexes, i.e., the Healthy Eating Index (HEI-2010), Alternative Healthy Eating Index (AHEI-2010), alternate Mediterranean Diet score (aMED), and Dietary Approaches to Stop Hypertension (DASH), with dual energy X-ray absorptiometry (DXA) and magnetic resonance imaging (MRI)-derived adiposity measures.

Methods: In a prospective design, male and female members of the Multiethnic Cohort (MEC) completed a validated quantitative food frequency questionnaire at cohort entry (1993-95) and at clinic visit (2013-15) when they also underwent whole-body DXA and abdominal MRI scans. Exclusion criteria included smoking, implants, claustrophobia, serious health conditions, substantial weight change, and treatments likely to affect adiposity measures of interest. Linear and logistic regressions adjusted for relevant covariates were performed to estimate associations of dietary indexes with adiposity measures.

Results: In this study population of 1,000 African American, Native Hawaiian, Japanese American, Latino, and white ancestry aged 60-72 years with a body mass index (BMI) of $18.5-40 \mathrm{~kg} / \mathrm{m}^{2}$, higher scores of all four indexes were inversely associated with modest differences in BMI and DXA-derived total adiposity and trunk fat (1-8\%) measured 20 years later. However, for VAT and NAFL, the differences between extreme tertiles were greater by $8-27 \%(\mathrm{p}<0.001)$ across indexes. Participants with the highest scores were $32-63 \%$ less likely to have NAFL or high VAT ( $>150$ $\left.\mathrm{cm}^{2}\right)(\mathrm{p}<0.001)$. For HEI-2010, participants with high scores at cohor entry and at clinic visit experienced the lowest risk of NAFL and high VAT Conclusions. These findings indicate that high diet quality predicts lower VAT and NAFL and emphasize the need to counsel patients about a high quality diet in addition to maintaining a healthy body weight.

Conflict of Interest: None Disclosed

Funding: US National Institutes of Health (P01CA169530, U01CA164973, P30CA071789, \#UL1TR000130)
T2P46

Diet, smoking and physical activity in nondiabetic patients: Is our patient education effective?

Svacina, S. ${ }^{1}$; Pallay, G. ${ }^{2}$

${ }^{1}$ Charles University, 1 st Medical faculy, 3rd Medical Department, ${ }^{2}$ Dr Max Pharmacies, Czech Republic

Every patient should be educated in healthy lifestyle. We have performed personal interview in 27121 patients without diabetes coming consecutively to 366 pharmacies to pick up prescribed drugs in the second half of the year 2016 in Czech Republic. One third of patients was in age over 60, one third 40 to 60 and one third bellow 40. The questionsand and answers contained information about smoking, physical activity and food intake. In Czech Republic patients are educated by physicians and nurses using printed materials and consultations. Small comparison with 10000 diabetic patients was done too.

Results: 1. Obesity degree I and more was present in $26 \%$ patients 2 . Smoking was present in $27 \%$ of patients. 2 . Physical activity more than 30 minutes 3 times weekly or more was present only in $25 \%$ men and in $29 \%$ women. 3. Daily consumption of fresh vegetables was only in $23 \%$ of patients ( $28 \%$ women and $16 \%$ men). Very low intake of fresh vegetables was in smokers ( $12 \%$ ) and very low in patents below 20 years of age $(10 \%)$ and among obese with BMI over 30 (10\%). In patients with hypertension better result were present (23\%). 4. Significantly lower intake of sweet beverages was in smokers, obese patients, older patients and in patients consuming more vegetables. Small comparison of these results shows even worse results in diabetic patients- lower physical acrivity and lowe vegetable intake.

Conclusion: In an actual survey of 21 thousands nondiabetic patients lifestyle is not optimal. The result in obese patients is only partly better in limiting sweet drinks but they are not increasing intake of vegetables and physical activity. The lifestyle is even worse in diabetic patients then in nondiabetic patients. The education needs intensification using perhaps special educators

No conflict of interest

\section{T2P47}

\section{Dietary energy density is positively associated with body} composition in chinese adults: A cross-sectional study

Yin, J. ${ }^{1}$; Chen, Y. ${ }^{1}$; Xue, H. ${ }^{1}$; Cheng, G. $^{1}$

${ }^{1}$ Department of Nutrition, Food Safety and Toxicology, West China School of Public Health, Sichuan University

Introduction: Dietary energy density (ED) might influences body composition. This study was performed to examine whether ED was associated with body composition among Chinese adults.

Methods: A total of 1933 adults (49.2\% men) aged 25-65 years were included from an ongoing prospective study conducted in Southwest China between 2013 and 2014. Dietary data were collected through the validated 24-hour dietary recalls. ED, computed as the amount of energy (kcal) divided by total consumed foods (g), was calculated based on five calculation methods (ED1, foods only; ED2, foods and alcohol; ED3, foods and milk; ED4, foods and energy-containing beverages; ED5, foods and all beverages). Body weight and height were measured to calculate body mass index (BMI), percentage body fat (\%BF), fat mass index (FMI) and fat-free mass index (FFMI). Multiple linear generalized regression models were used to examine the association between ED and body composition parameters.

Results: Among women, ED based on any calculation methods was positively and significantly associated with BMI, FMI, FFMI and \%BF after adjusting for age, education level, smoking, monthly family income per capita and energy expended on moderate-to-vigorous physical activities (MVPAEE) ( $\mathrm{p}<0.01)$. In men, ED1 was positively related to BMI, FMI, FFMI and \%BF ( $<0.05)$; however, ED5, including all beverages in the calculation, was not associated with each of body composition parame- 
ters. Moreover, the changes of body composition contributed by per unit of ED were much higher in women than in men $(\mathrm{p}<0.01)$.

Conclusion: The present study identified the positive association between ED and body composition in both Chinese men and women. Beverages may have profound effects on the association between ED and body composition, especially in men.

Conflict of Interest: The authors reported no potential conflict of interest.

Funding: All phases of this study were supported by research grant from the National Nature Science Foundation of China (No. 81472976), research grant from the National 1000 Young Talents Program.

\section{T2P49}

Divergence in perceptions and attitudes among people with obesity, healthcare professionals, and employers create barriers to effective obesity management: Results of the national action study

Dhurandhar, N. ${ }^{1}$; Kaplan, L. ${ }^{2}$; Golden, A. ${ }^{3}$; Jinnett, $K_{.}{ }^{4}$; Kolotkin, R. $^{5}$; Kyle, T.6. Look, M.7; Nadglowski, J. ; O 'Neil, P. ${ }^{9}$; Parry, T. ${ }^{4}$; Guerrero, G. ${ }^{10}$; LilleøRe, S. ${ }^{11}$; Tomaszewski, K. ${ }^{12}$

${ }^{1}$ Texas Tech University, Lubbock, TX, USA, ${ }^{2}$ Massachusetts General Hospital, Boston, Ma, Usa, ${ }^{3} \mathrm{~Np}$ From Home, Llc, Munds Park, Az, Usa, ${ }^{4}$ Integrated Benefits Institute, San Francisco, Ca, Usa, ${ }^{5}$ Quality Of Life Consulting Pllc, Durham, Nc, Usa, ${ }^{6}$ Conscienchealth, Pittsburgh, Pa, Usa, ${ }^{7}$ San Diego Sports Medicine And Family Health, San Diego, Ca, Usa, ${ }^{8}$ Obesity Action Coalition, Tampa, Fl, Usa, ${ }^{9}$ Medical University Of South Carolina, Charleston, Sc, Usa, ${ }^{10}$ Novo Nordisk Inc, Plainsboro, Nj, Usa, ${ }^{11}$ Novo Nordisk A/S, SøBorg, Denmark, ${ }^{12}$ Kjt Group Inc, Honeoye Falls, Ny, Usa

Background: We investigated barriers, behaviours and perceptions towards obesity management (OM) among people with obesity ( $\mathrm{PwO}$ ), healthcare providers (HCPs) and employers.

Methods: Online surveys using cross-sectional, US-based stratified-sample design. Adult $\mathrm{PwO}\left(\mathrm{N}=3008, \mathrm{BMI}>30 \mathrm{~kg} / \mathrm{m}^{2}\right.$ from self-reported height, weight), 606 HCPs (83\% PCPs, 17\% weight loss [WL] specialists), 153 employers completed surveys.

Results: Despite several "serious" WL attempts, only $23 \%$ of PwO reported $10 \% \mathrm{WL}$ during the past 3 years; $44 \%$ of those maintained WL $>1$ year. Most respondents acknowledged obesity as a disease and felt OM brings health benefits. Among PwO, 82\% considered WL as "completely" their responsibility; $72 \%$ of HCPs felt they had "responsibility to actively contribute" to WL efforts, while only $18 \%$ of employers agreed that employees' WL was "partially" employers' responsibility. While $98 \%$ of $\mathrm{PwO}$ described their weight status as "overweight" (48\%) or "obese" (50\%), only $\sim$ half $(55 \%)$ reported receiving a formal obesity diagnosis. Few (16\%) PwO had a follow-up appointment with their HCP after initial OM conversations. HCPs felt "comfortable" having OM conversations, but efforts were often deprioritized by limited time. Perceived value of employer-sponsored wellness offerings differed between $\mathrm{PwO}$ and employers with $\mathrm{PwO}$ seeing less value. Employers had concerns about OM insurance coverage.

Conclusions: OM perceptions, attitudes, and resulting behaviours varied widely among respondent groups, potentially jeopardising overall OM outcomes. Initial OM conversations between $\mathrm{PwO}$ and HCPs are few and rarely continued at follow-up visits. These results suggest that initiatives fostering patient-HCP dialogue and enhanced employer understanding are needed to support effective OM efforts.
T2P50

\section{Do behavioural weight management programmes exacerbate inequalities?}

\author{
Astbury, N. ${ }^{1}$; Aveyard, P. ${ }^{1}$; Jebb, S. \\ ${ }^{1}$ University of Oxford
}

Introduction: There is a concern that health improvement programmes that require self-regulation, like behavioural weight management, may exacerbate health inequalities and do not appeal to men, but data are scarce. Methods: We calculated the percentage and the odds ratio for the demographic characteristics ofpeople who joined the trial compared with those invited.

Results: We invited 2115 patients (1087 male, 1022 female) with a BMI $>30 \mathrm{~kg} / \mathrm{m}^{2}$ from $10 \mathrm{GP}$ practices in Oxfordshire, England. 278 participants were enrolled, 109 men (10\%) compared with 166 (16\%) women, (OR; 1.74, [95\% CI; 1.34, 2.25] $\chi^{2}$ 95.7, P < 0.001). People with a BMI in their medical record greater than $35 \mathrm{~kg} / \mathrm{m}^{2}$ were more than twice as likely to participate than those with a BMI $<35 \mathrm{~kg} / \mathrm{m}^{2}\left(2.3[1.82,3.05] \chi^{2} 44.6\right.$, $\mathrm{P}<0.001)$ and patients from more deprived areas were more likely to take up the offer than those from less deprived areas $\left(1.387[1.065,1.808] \chi^{2}\right.$ $5.91 \mathrm{P}<0.015)$. There was no difference in the uptake of the offer to participate based on age (above/below 40 years) or the presence of type 2 diabetes or hypertension.

Conclusion: Weight management programmes may appeal more to people who are socioeconomically deprived, women, and are more overweight. They do not appear to exacerbate socioeconomic inequalities in health. These results suggest that a large proportion of the previously reported inequalities occur as a result of the type of intervention offered.

\section{T2P51 \\ Does ethnicity make a difference in the association between obesity and depression?}

Gibson-Smith, D. ${ }^{1}$; Snijder, M. ${ }^{2}$; Bot, M. ${ }^{1}$; Nicolaou, M. ${ }^{2}$; Derks, E. ${ }^{2}$; Stronks, K. ${ }^{2}$; Penninx, B. ${ }^{1}$

${ }^{1}$ VU University Medical Center, Amsterdam, The Netherlands, ${ }^{2}$ Department Of Public Health, Academic Medical Center, Amsterdam, The Netherlands

Introduction: It is generally accepted that obesity and depression are positively associated. This relationship has been shown to vary across American ethnic groups, however no prior research has examined variations among Dutch ethnic minorities. This study investigated the association between obesity and depression among six Dutch ethnic groups (Dutch, South Asian-Surinamese, Afro-Caribbean Surinamese, Ghanaian, Turkish and Moroccan). Additionally, in order to explore potential mechanisms, this study examined the relationship between C-reactive protein (CRP) and depression and potential modification by ethnic groups.

Method: Data on 21,384 men and women (18-70 years) was sourced from the HELIUS (Healthy life in an urban setting) study. The cross-sectional relationship between obesity and depressive symptoms (using the Patient Health Questionnaire-9) was analysed, adjusting for socio-demographics, lifestyle factors and health status. Interaction terms (ethnicity*obesity) were tested and the analysis were subsequently stratified by ethnicity. Logistical regression analysis was also used to examine the relationship between CRP and depressive symptoms.

Results: After adjustment for all covariates, being obese significantly increase the odds of having depressive symptoms (odds ratio $[\mathrm{OR}]=1.24$; $95 \%$ confidence interval $[\mathrm{CI}]=1,11-1.39)$. Interaction terms revealed significant variation between ethnic groups. Obesity was significantly associated with a greater likelihood of depressive symptoms for Dutch $(\mathrm{OR}=$ $1.71 ; 95 \%$ CI $=1.20-2.43)$, African Surinamese $(\mathrm{OR}=1.35$; 95\% CI $=1.04$ $1.74)$ and South Asian Surinamese $(\mathrm{OR}=1.58$; $95 \% \mathrm{CI}=1.21-2.06)$. These differences disappear after inclusion of health status. Increased CRP was also significantly related to depressive symptoms however, this was consistent across all ethnic groups. 
Conclusion: The association between obesity and depressive symptoms varies according to Dutch ethnicity although differences are attenuated by health status. Analysis of CRP data suggests that these ethical differences are not due to CRP levels. Possibly differences in body dissatisfaction or perceived weight based discrimination are more likely mechanisms. Given the worldwide dramatic rise in obesity it is important to address weight reduction strategies and the social stigma and lower self-esteem associated with obesity in order to help reduce the problem of comorbid depressive.

\section{Conflict of Interest: None}

Funding: Research relating to this abstract was funded by EU FP7 MooDFOOD Project 'Multi-country cOllaborative project on the rOle of Diet, FOod-related behaviour, and Obesity in the prevention of Depression', Grant agreement no. 613598

\section{T2P52}

\section{Eating inventory, body adiposity and cardiometabolic health risks in adolescents}

\section{Hainer $\mathrm{V}^{\prime}$; Zamrazilova $\mathrm{H}^{\prime}$; Kunesova $\mathrm{M}^{1}$; Aldhoon-Hainerova $\mathrm{I}^{2}$}

${ }^{1}$ Institute of Endocrinology, Obesity Management Centre, Prague, Czech Republic, ${ }^{2}$ Institute Of Endocrinology, Obesity Management Centre, Prague, Czech Republic; Department Of Paediatrics, 3Rd Faculty Of Medicine, Charles University, Prague, Czech Republic

Introduction: Associations of three factors (dietary restraint, disinhibition and hunger) of the Eating Inventory (EI) with body adiposity and cardiometabolic health risks have been studied in adults (Hainer $\mathrm{V}$ et al 2006, Aldhoon Hainerová I et al. 2013). However, only limited data have been reported about such associations in adolescents (Gallant AR et al. 2010). Thus the aim of our study was to evaluate a relation of the items of EI to anthropometric parameters and essential components of metabolic syndrome (MS) in adolescent girls and boys.

Methods: Anthropometric and biochemical parameters characterizing MS, blood pressure and eating attitudes assessed by EI (Stunkard AJ \& Messick SM,1985) were examined in two cohorts of adolescents aged 13.0-17.9 years: 1) general population: 787 girls with median BMI 21.2 $\mathrm{kg} / \mathrm{m}^{2}$ and 746 boys with median BMI $21.5 \mathrm{~kg} / \mathrm{m}^{2} ; 2$ ) overweight/obese adolescents: 472 girls with BMI $28.8 \mathrm{~kg} / \mathrm{m}^{2}$ and 384 boys with BMI $28.6 \mathrm{~kg}$ / $\mathrm{m}^{2}$. In addition, daily energy and macronutrient intakes were evaluated by computer program using a 3-day dietary intake record. Statistical analysis: Spearman's correlations, Mann-Whitney test.

Results: Girls exhibited significantly higher restraint score than boys in all weight categories $(\mathrm{p}<0.01)$. As expected, in both genders dietary restraint was inversely related to the intake of energy and essential macronutrients while an opposite relationship was found for the hunger score. In general population dietary restraint positively correlated $(\mathrm{p}<0.001)$ with BMI, percent body fat (measured by BIA) and waist $\mathrm{z}$-score in both genders whereas dietary disinhibition was significantly positively related $(p<0.001)$ to measures of adiposity and body fat distribution only in boys. Hunger score did not significantly correlate with anthropometric parameters in both genders. No significant associations of the items EI with essential components defining MS were demonstrated in both girls and boys.

Conclusion: Relationship between the measures of body adiposity and body fat distribution with dietary restraint was revealed in both genders whereas that with dietary disinhibition only in boys. In contrast to adults, in adolescents non-anthropometric components of MS were not associated with the factors of EI.

Conflict of Interest: No Disclosed

Funding: Research related to this abstract was funded by the Czech Ministry of Health [project number 00023761, Institute of Endocrinology, Prague, Czech Republic]
T2P53

Educated women respond to dietary intervention regardless of neighbourhood deprivation - secondary analysis from the ROLO study

\section{O'Brien, E. ${ }^{1}$; Alberdi, G. ${ }^{1}$; Geraghty, A. ${ }^{1}$; Mcauliffe, F.}

${ }^{1}$ UCD Perinatal Research Centre, Obstetrics \& Gynaecology, School of Medicine, University College Dublin, National Maternity Hospital, Dublin, Ireland

Introduction: Low socioeconomic status is associated with excess gestational weight gain (EGWG), reduced diet quality and poorer pregnancy outcomes. We aimed to determine if response to a low glycaemic index (GI) dietary intervention, measured by changes in diet and EGWG, differed across women of high/low educational attainment and neighbourhood deprivation.

Methods: This was secondary data analysis of 521 women recruited to the ROLO study. The intervention consisted of a 2-hour low GI dietary education session. Change in GI was measured using 3-day food diaries preand post-intervention. EGWG was categorised as per the 2009 Institute of Medicine guidelines. Education was self-reported (achieved/did not achieve $3^{\text {rd }}$ level) and neighbourhood deprivation indices (advantaged/ disadvantaged) were assigned. Chi-square (EGWG) and independent sample T-test (change in GI) were utilised for statistical analysis between variables.

Results: The intervention significantly reduced GI and EGWG among women with $3^{\text {rd }}$ level education, residing in both disadvantaged [GI: intervention $-3.30(5.15)$ vs. control $-0.32(4.22), P=0.024$; EGWG: intervention $7(31.8 \%)$ vs. control $23(69.7 \%) ; P=0.006]$ and advantaged areas [GI: intervention -1.13(3.88) vs. control 0.06(3.75), $P=0.020$; EGWG: intervention $41(34.5 \%)$ vs. control 61(55.5\%); $P=0.001]$. Neither GI nor EGWG differed between the intervention and control group among women with less than $3^{\text {rd }}$ level education, regardless of neighbourhood deprivation.

Conclusion: Women with $3^{\text {rd }}$ level education, regardless of the neighbourhood in which they lived, were most receptive to a dietary intervention. A dietary education session was not effective in reducing GI and EGWG among less educated women. New approaches are required in pregnancy interventions to improve pregnancy outcomes for less educated women.

Conflict of Interest: None Disclosed

Funding: Research relating to this abstract was funded by the Health Research Board, Ireland

T2P55

Effects of a health education intervention on sedentary time, moderate-to-vigorous physical activity, and body mass index in individuals with moderate-to-high cardiovascular risk

\section{Bohn, L. ; Sa-Couto, P. ; Castro, A. ${ }^{3}$; Ribeiro, F. ; Oliveira, J. ${ }^{5}$}

${ }^{1}$ Faculty of Sport, University of Porto. Research Centre in Physical Activity, Health and Leisure, Porto, Portugal., ${ }^{2}$ Center For Research And Development In Mathematics And Applications (Cidma), Department Of Mathematics (Dmat), University Of Aveiro, Aveiro, Portugal., ${ }^{3}$ Primary Care Centre Espaço Saúde. Aldoar, Porto, Portugal., ${ }^{4}$ School Of Health Sciences And Institute Of Biomedicine-lbimed, University Of Aveiro, Aveiro, Portugal., ${ }^{5}$ University Of Porto. Research Centre In Physical Activity, Health And Leisure. Porto, Portugal.

Background: Insufficient physical activity, excessive time spent in sedentary activities and obesity are associated with a rising burden of global disease. Effectiveness of programs aiming to promote healthy behaviors in primary care is inconsistent. This study evaluated the effects of a health education and counseling intervention program, in a primary healthcare setting, on sedentary time, moderate-to-vigorous physical activity, and body mass index in individuals with moderate-to-high risk of cardiovascular disease.

Methods: This was a parallel- group study with a non-probabilistic sample with 4 months intervention plus 8 months of follow-up, carried out in a primary healthcare unit. A total of 164 men and women with mod- 
erate-to-high cardiovascular risk assessed according the $2013 \mathrm{ESH} / \mathrm{ESC}$ Guidelines for the Management of Arterial Hypertension, were enrolled. The subjects were allocated to either intervention $(n=87)$ or control group $(n=77)$. The intervention consisted by 3 walk and face-to-face group sessions plus text messages. Primary outcomes were sedentary time, moderate-to-vigorous physical activity (measured by accelerometry) and body mass index.

Results: There was not significant group ${ }^{\star}$ time interaction for sedentary time $[-5.9(7.6) ; \mathrm{p}=0.433]$ and moderate-to-vigorous physical activity [-0.4 (2.5); $\mathrm{p}=0.878]$. For body mass index, a non-significant group ${ }^{\star}$ time interaction was found $[-0.13(0.09) ; \mathrm{p}=0.177]$. Results remain simmilar after adjusting for age, gender and energy intake.

Conclusion: The education and counseling program did not improve daily physical activity (sedentary time and moderate-to-vigorous physical activity), and body mass index in participants with moderate to high cardiovascular risk.

\section{Conflict of Interest: None}

Funding: Research related to this abstract was funded by FCT: PTDC/ DES/122763/2010). The FCT supported the first author (SFRH/BD/78620/2011).

\section{T2P56 \\ Effects of maternal anthropometrics on short- and long-term pregnancy outcomes in South Asian women: A systematic review}

Slack, E. ; Rankin, J. '; Jones, D. ${ }^{2}$; Heslehurst, N. ${ }^{1}$

${ }^{1}$ Newcastle University, ${ }^{2}$ Teesside University

Introduction: There is evidence of a higher risk of obesity related comorbidities (e.g. diabetes) among South Asian populations at a lower body mass index (BMI) than White populations. It is thought that this pattern may also extend to pregnancy; for example there may be a higher risk of some pregnancy outcomes (e.g. gestational diabetes) at a lower pre-pregnancy BMI or weight gain in pregnancy. One reason for the increased risk at a lower BMI is related to differences in body composition, or fat distribution between White and South Asian populations. Therefore, it is important that measures of body composition (i.e. anthropometrics) are investigated as well as BMI. This systematic review investigates associations between maternal anthropometrics (e.g. weight, body fat) and pregnancy outcomes in South Asian women.

Methods: Twelve electronic databases, reference lists and citations of al included studies were searched. Observational studies published in English, reporting maternal anthropometrics and pregnancy outcomes in South Asian women were included. Two researchers performed screening, data extraction and quality assessments. Descriptive synthesis was used to summarise the evidence-base of the independent and combined effects of maternal pre-pregnancy anthropometrics and anthropometric change during pregnancy on pregnancy outcomes.

Results: Nineteen studies were included, reporting 346,319 births. Studies were published between 1981-2015, from the UK $(n=12)$, Australia and Norway ( $\mathrm{n}=2$ each), Spain, USA and Canada ( $\mathrm{n}=1$ each). Exposure variables included pre-pregnancy anthropometrics $(n=18)$, anthropometric change during pregnancy $(n=2)$, or a combination of both $(n=2)$. South Asian women had an increased risk of gestational diabetes mellitus (GDM), stillbirth, and congenital anomalies compared with White women of the same BMI. South Asian women with obesity (BMI $>30 \mathrm{~kg} /$ $\mathrm{m}^{2}$ ) had an increased risk of GDM, stillbirth, macrosomia, hypertensive disorders and caesarean section when compared with South Asian women of recommended BMI (BMI $20-24.9 \mathrm{~kg} / \mathrm{m}^{2}$ ). Anthropometric change was associated with increased risk of GDM in South Asian women, despite a lower total weight gain than White women. The combined effect (pre-pregnancy and change in anthropometrics) on GDM and post-partum weight retention was greater in South Asian women.

Conclusion: The increased risk of adverse pregnancy outcomes at lower anthropometric measures both at the start of and during pregnancy, in
South Asian women compared with White women should be considered in guidelines for weight management during pregnancy.

Conflict of interest: None disclosed

Funding: Research relating to this abstract was funded by a UK Medical Research Council $1+3$ PhD studentship

\section{T2P57 \\ Effects of physical activity-induced weight loss on bone health in obese adolescents}

\section{Elodie, C. ; Julie, M. ${ }^{2}$; Frederic, D. ${ }^{3}$; Martine, D. ${ }^{3}$; Dave, G. ; Geraldine, N. ${ }^{5}$;} David, T. ${ }^{6}$; Daniel, $C .{ }^{6}$

${ }^{1}$ Clermont Auvergne University, ${ }^{2}$ Ssr Ugecam Nutrition Obesité, ClermontFerrand, France, ${ }^{3} \mathrm{Chu}$ Clermont-Ferrand, Clermont-Ferrand, France, ${ }^{4}$ Australian Catholic University, Sydney, Australia, ${ }^{5}$ Australian Catholic University, Melbourne, Australia, ${ }^{6}$ Clermont Auvergne University, France

Introduction: The protective effect of obesity on bone has been lately challenged suggesting a detrimental effect of fat accumulation on bone quality during growth. Recent works have effectively underlined a lower cite-specific bone quality in adolescents with obesity compared with lean ones. This work investigated the effect of a 8-month physical activity intervention on hip structural parameters changes in adolescents with obesity. Methods: Thirty-one obese adolescents (12-15 years old) followed a 8 -month physical activity program and were compared with 23 agedmatched lean ones (without intervention). The intervention consisted in 60-minute moderate intensity exercise sessions combining endurance and resistance exercises, performed 3 times a week. Whole body composition and the geometric contributions to bone strength in the proximal femur (narrow neck and femoral shaft) were quantified by DXA using the Hip Structural Analysis technique before and by the end of the 8-month.

Results: The intervention favoured a significant weight loss and body fat decrease $(p<0.001)$. This weight loss was accompanied by a significant increase of the bending and axial strength at femoral neck and shaft leading obese adolescents to similar raw data compared with the normal weight group. However, bulking ratios were significantly higher at the narrow neck $(6.92 \pm 1.08$ vs $8.25 \pm 2.00 ; p<0.01)$ and the shaft $(2.27 \pm 0.47$ vs $2.73 \pm 0.54 ; p=0.01)$. While a significant increase in shaft bending strength $(\mathrm{p}<0.001)$ was observed in the lean group, a significantly increased bending strength $(\mathrm{p}=0.01)$ at the shaft and a tendency $(\mathrm{p}=0.06)$ of lower bending strength was observed at the neck in the obese group. After adjustment to body weight changes, obese adolescents displayed lower bending and torsional strength as well as lower resistance to axial stresses at the narrow neck $(\mathrm{p}<0.001 ; \mathrm{p}<0.001)$ and the shaft $(\mathrm{p}<0.001$; $\mathrm{p}<0.001)$ sites compared with normal weight. Also, significant higher bulking ratios $(\mathrm{p}=0.027)$ were observed at the narrow neck at 8 months. Finally, after adjustment to fat mass changes, the bulking ratio at the shaft $(\mathrm{p}=0.004)$ was significantly higher in obese adolescents.

Conclusion: Our results suggest that geometric indices of bone strength at weight bearing site are unadapted to excess body weight and that positive adaptations can be observed in response to physical activity-induced weight loss in adolescents with obesity.

Conflict of Interest: None Disclosed

Funding: No Funding 


\section{T2P59}

\section{Evaluation of food and nutritional education actions in the} municipalities of Brazil in 2010.

\section{Ottoni, I. ${ }^{1}$; Bandoni, D.}

${ }^{1}$ UNIFESP - Universidade Federal de São Paulo

Introduction: The Brazilian School Nutrition Program (PNAE) is considered one of the largest and most comprehensive school food programs in the world. One of the guidelines is the inclusion of Food and Nutrition Education as a decisive component in promoting health and preventing overweight. However, there are few references that support these practices. The aim of this study is to evaluate the actions of Food and Nutrition Education in Brazilian municipalities.

Methods: It was used the secondary data obtained from the database of the Efficient Manager of School Feeding Award, formulated from the voluntary enrollment of 786 cities throughout Brazil in the year of 2010. The variables of the study were: Execution of Food Education and Nutrition and execution of School Gardens and Culinary Workshops, stratifying them according to the Great Regions of Brazil, and verifying if there was influence of attendance to the PNAE, financial resources and use of Family Agriculture in the actions execution. Statistical analysis was performed at a significance level of 5\%. The work was approved by the Ethics Committee in Research with the number 02519/2016.

Results: Among the cities enrollees $(n=786), 61.2 \%(n=466)$ had the theme Food and Nutrition Education in their school curriculum, 44.8\% $(\mathrm{n}=348)$ of municipalities use food from Family Agriculture, $71,1 \%$ $(n=322)$ had School Gardens and 32\% $(n=243)$ held Culinary Workshops. There was no statistical difference regarding the performance of Food and Nutrition Education according to the major regions of Brazil The South Region had the highest percentage of cities that carried out Pedagogical gardens (83.1\%) and used Food from Family Agriculture $(61.2 \%)$ with a significant statistical difference $(p<0.05)$. The cities that received the lower financial resources of the National Fund for School Development for kindergarten, elementary and middle school (average: R \$ 1830120,38) carried out more actions of Food and Nutrition Education in the year of study, and the municipalities that attended the largest Number of students in elementary education carried out School Gardens and Workshops more frequently $(\mathrm{p}<0.05)$.

Conclusion: Food and Nutrition Education is present in most of the Brazilian cities enrolled in the award, being an important instrument used to improve health conditions, quality of life e to prevent the weight gain of the population.

Conflict of Interest: None

Funding: No Funding

\section{T2P60 \\ Evaluation of food consumption of women with morbid obesity}

Magno, F.'; Guaraná, H. .' Pedrosa, A. ${ }^{1}$; Ximenes, A. '; Carneiro, J.2.; Rosado, E.

${ }^{1}$ Universidade Federal do Rio de Janeiro, ${ }^{2}$ Hospital Universitário Clementino Fraga Filho

Introduction: Nowadays, excessive intake of food and reduced physical activity promotes serious damage to health. An important epidemiologic brazilian study (POF 2008-2009) indicated high intakes of sugar, saturated fat and sodium and low intakes of fiber in this population. The aim of this study was to evaluate dietary intakes by morbid women.

Methods: Fourty-two morbid obese adult women, aged between 20 and 45 years, BMI between 40 and $60 \mathrm{~kg} / \mathrm{m}^{2}$ and long-term obesity more than 3 years participated in this cross-sectional analytical study. A prospective, three-day non-consecutive dietary intake method was used to evaluate the habitual dietary intake. Calories, macronutrients and micronutrients intake were evaluated. All dietary records were analyzed in the nutritional assessment software Avanutri, version 4.0. All data were analyzed in the statistical analysis program SPSS version 16.0.

Results: Women presented mean caloric intake of $2424.1 \pm 1210.08 \mathrm{Kcal} /$ day, of which $88 \%$ had hypocaloric consumption. Protein intake corresponded to $17.9 \pm 5.9 \%$ of Total Energetic Consumption (TEC) $(0.79 \mathrm{~g} /$ $\mathrm{kg}$ body weight/day). Carbohydrate intake corresponded to $49.17 \pm 1.04 \%$ of TEC, considering that $85.7 \%$ of individuals presented a low intake of carbohydrate. The fat intake corresponded to $32.34 \pm 9.41 \%$ of TEC, of which $69 \%$ presented fat intake above RDA for the Brazilian population. Saturated fatty acid (AGS) and cholesterol intake was $8.27 \pm 4.12 \%$ and $346.07 \pm 275.59 \mathrm{mg} /$ day, respectively. Fiber intake was low $(13.42 \pm 7.28 \mathrm{~g} /$ day). Regarding to micronutrients, the percentage of women with deficient intake was $97.6 \%$ for magnesium, $97.6 \%$ for folic acid, $88.1 \%$ for vitamin D, $85.7 \%$ for iron, $66.7 \%$ for vitamin C, $54.8 \%$ for vitamin A, 52.4\% for zinc, $38.1 \%$ for vitamin B12, 33.3\% for selenium, and $28.6 \%$ for vitamin B1. 52.4\% of women had sodium intakes below $2400 \mathrm{mg} /$ day.

Conclusion: Dietary habits of women with class III obesity follow the profile described in other studies, what contributes to the genesis, development and maintenance of obesity and worsening of nutritional deficiencies in this population, considering the low fiber intake, high fat and cholesterol intake and low micronutrients intake, among them antioxidants. Hypocaloric intake may result from underestimated amounts reported in food registry.

Conflict of Interest: None Disclosed.

Funding: FAPERJ (Fundação de Amparo à Pesquisa do Estado do Rio de Janeiro).

\section{T2P61}

Evaluation of the characteristics of carbohydrates and fatty acids intake in normal weight overweight and obese by gender

\section{Halmy, E. ${ }^{1}$; Kovács, G. ${ }^{2}$; Halmy, L. ${ }^{3}$; Paksy, A. $^{4}$}

${ }^{1}$ Hungarian Society for the Study of Obesity, Budapest, ${ }^{2}$ Platon Health Ltd. Budapest, ${ }^{3}$ Universitiy Hospital, Regensburg, ${ }^{4}$ Hungarian Society For The Study Of Obesity

Introduction: In our earlier nutritional surveys we have found deficiency and excess in comparison to recommendations in the nutrients intake of overweight and obese. We have also found increasing energy intake by BMI but similar ratio of macronutrients intake. The aim of the study was to evaluate the characteristics of main sources of energy intake in normal weight overweight and obese groups.

Material and Method:The dietary questionnaire was made on 462 persons by NutriComp 3.0. After the rejection of under $(<1.1)$ and over $(>2.7)$ reporting cases we examined 287cases(male n: 93, female n: 164). Age: Mean (SD): 50,7 y $(11,8)$, BMI: $37,1 \mathrm{~kg} / \mathrm{m}^{2}(8,9)$.

Results: CH intake in male: Mean (SD) BMI $<25 \mathrm{~kg} / \mathrm{m}^{2}: 308.9 \mathrm{~g}(79.1)$ BMI 25-29.9: 268.8 g (60.1) BMI 30-39.9: 325 g (111.1) BMI $\geq 40369.8 \mathrm{~g}$ (100.3) (ANOVA p < 0.01). CH intake in female: 246.3 (65.1), 259.6 (63.5), 260.9 (73.3), 314.4 (93.6) (ANOVA $\mathrm{p}<0.001$ ). BMI $<25$ vs. BMI $\geq 40$ increasedp $<0.05$ in male, $\mathrm{p}<0.001$ in female. The tendency of $\mathrm{CH}$ intake in en\% decreasing by BMI in male, while the fat intake increasing, the protein did not changed (ANOVA NS). Sugar intake increased inmorbid obese in both gender. Male: $26.3 \mathrm{~g}(9.3), 24.3$ (5.0), 28.7 (11.0), 29.6 (10.4). Female: 21.3 (7.0), 25.3 (10.3), 22.7 (8.7), 26.1 (6.9). Dietary fiber intake did not changed by BMI or $\mathrm{g} / 1000 \mathrm{kcal}$ (ANOVA NS). In femaleBMI $<25$ vs. overweight $(\mathrm{p}<0.05)$, vs. morbid obese $(\mathrm{p}<0.05)$ increased. Fat intake in maleincreased by BMI (ANOVA $\mathrm{p}<0.001$ ). BMI $<25$ vs. obese $(\mathrm{p}<0.05)$, vs. morbid obese: $159.4 \mathrm{~g}(43.5)(\mathrm{p}<0.001)$ increased.In female increasedby BMI (ANOVA $\mathrm{p}<0.01$ ). BMI $<25$ vs. morbid obese $155.4 \mathrm{~g}$ $(27.0)]$ increased $(p<0.01)$. SFA in both gender increased (ANOVA $\mathrm{p}<0.001$ ). MUFA in male increased (ANOVA $\mathrm{p}<0.001$ ), in female NS. In both gender PUFA intake (ANOVA $p<0.001$ ) increased. In morbid obese groupstotal PUFA $(\mathrm{p}<0.001), \mathrm{n}-6$ fatty acid $(\mathrm{p}<0.001)$ increased. PUFA/SFA ratio NS. 
Conclusion: In overweight no significant change in nutrients intake. In obesity both gender showed higher $\mathrm{CH}$ intake vs. $\mathrm{BMI}<25$, in morbid obesity higher sugar intake, but in all groups $\mathrm{CH}$ intake was under 50 en\%. Dietary fiber intake was under the recommendations in all groups. Total fat, SFA and n-6 fatty acid showed higher intake, high n-6/n-3 ratio and low n-3 fatty acid intake characterized in obesity. Our results emphasize to increase the dietary fiber and complex $\mathrm{CH}$ and decrease simple $\mathrm{CH}$. Attention should be taken to the ratio of fatty acids intake in dietotherapy of obesity.

Conflict of Interest: None Disclosed

Funding: No Funding

\section{T2P62}

\section{Exploring consumers' attitudes related to healthy nutrition in} the context of reading labels

\section{Jarosz, M. '; Siuba-StrzelińSka, M. ${ }^{1}$}

${ }^{1}$ National Food and Nutrition Institute

Introduction: Consumers education on reading labels is one of the elements of prevention and treatment of overweight and obesity. The main objective of the study was to obtain information that will help build effective communication to consumers by identifying insights (barriers, be liefs, unmet needs) related to healthy eating and reading labels.

Methods: The study was conducted in May and June 2016 among a randomly selected adults (20-45 years), divided into 6 groups of 4-6 people, in three Polish cities (Warsaw, Torun, Srem). Those were healthy individuals, which are not on any diet, responsible for food shopping in the household. A qualitative consumer research - FGI (Focus Group Interviews) was used.

Results: In most cases consumers do everyday food shopping schematically, choose the same products, following their taste preferences and attachment to the brand. They belived that ingredients of the products are responsible for their quality, but in practice few people thoroughly analyzes them, because they do not have knowledge of what they should pay attention. They are aware that a table of nutritional value is on the package, but the vast majority do not pay attention to it because they see it to be too complicated.

Conclusion: The effective consumers education on reading labels should be simple, based on the idea of smart shopping - you have a choice, make it smart, better. The best way is to give practical and clear message ie. to choose better cheese for you, compare it to another and buy this one with lower salt content.

Funding: Research relating to this abstract was funded by Swiss Contribution within the project "'Prevention of overweight, obesity, and chronic diseases by educating on nutrition and physical activity of the society" under the Swiss-Polish Cooperation Program.

\section{T2P63}

External validation of equations to predict percentage body fat using demographic and anthropometric measurements: Fels longitudinal study 1999-2006

\section{Stevens, J. ${ }^{1}$; Reynolds, K. ${ }^{1}$; Cai, J. ${ }^{1}$; Choh, A. ${ }^{2}$; Czerwinski, S. ${ }^{2}$}

${ }^{1}$ University of North Carolina Chapel Hill, ${ }^{2}$ University Of Texas Health Science Center At Houston, School Of Public Health, Brownsville, Tx

Background: Assessment ofpercent body fat (\%BF) by highly precise methods such as dual-emission X-ray absorptiometry (DXA) is often not feasible. Recently, Stevens et al. published gender-specific equations to predict \%BF using data from NHANES 1999-2006. The purpose of this study is to evaluate the external validity of the equations in adults and youth.

Methods: The Fels Longitudinal Study is an ongoing prospective cohort of predominantly white Americans founded in 1929 in Yellow Springs,
Ohio, USA. Data were collected from 1999 to 2006 from 1070 males and females ages 8 years and older. Total percent body fat was measured using the Hologic QDR 4500A (Hologic Inc., Bedford MA) DXA, and data were corrected using the Schoeller procedure. Triceps and subscapular skinfolds thicknesses; waist, calf, thigh, and arm circumferences; standing height; and weight were measured. These anthropometric variables along with age and race were used in 13 published, gender-specific equations to predict $\%$ BF. Equations included between 2 (height and weight) and 8 anthropometrics. The same equations were applied to children (8-19 years) and adults (20+ years). Equation-predicted \%BF was compared to assessments by DXA.

Results: $\mathrm{R}^{2}$ values for predicted \%BF calculated from the 13 different equations ranged from 0.677 to 0.770 in woman, 0.619 to 0.828 in men, 0.659 to 0.803 in girls and 0.729 to 0.907 in boys. RMSE values ranged from 2.34 to $4.01 \% \mathrm{BF}$ over all groups. As expected, the $\mathrm{R}^{2}$ tended to be lower, and the RMSE higher, in this external validation compared to the published internal validation, but the differences were small. Mean Signed Differences (MSD) indicated that bias by gender and age (youth vs adults) was low $(<1 \% \mathrm{BF})$. Bias across BMI categories was also low at less than $2 \% \mathrm{BF}$.

Conclusions: This was the first study to evaluate the external validity of the Stevens equations for prediction of $\% \mathrm{BF}$. A relatively high percentage of the total variance in the observed $\% \mathrm{BF}$ was explained by the calculated values. Thus, the equations provide a reasonable method for assessment of $\% \mathrm{BF}$ when more precise methods are not feasible.

Acknowledgements:UNC grant 1RO1DK097046; Fels grant NICHD HD012252

\section{T2P64}

\section{Factors associated with the occurrence of obesity in aging}

\section{Araujo, $T^{1}$; Naslavsky, M. ${ }^{2}$; Duarte, ${ }^{1}{ }^{1}$}

${ }^{1}$ School of Public Health, University of São Paulo, ${ }^{2}$ Human Genome Research Center, University Of São Paulo

Introduction: Obesity may be associated with increased disability in Brazilian older adults [1]. However, for Cheng et al. [2] although current results suggest a potential adverse effect of weight change on aging, more research is warranted. In the present study, we aim at identifying factors associated the occurrence of obesity in Brazilian older adults.

Methods: Data were drawn from the SABE survey (for Health, Well-being end Ageing), aging 64 and over, conducted in 2006 (baseline) and 2010 (second interview). Dependent variable: nutritional status change. $\mathrm{Nu}$ tritional status: underweight (Body Mass Index BMI, $<23 \mathrm{~kg} / \mathrm{m}^{2}$ ), normal weight $\left(\mathrm{BMI} \geq 23 \mathrm{~kg} / \mathrm{m}^{2}\right.$ and $\left.\leq 28 \mathrm{~kg} / \mathrm{m}^{2}\right)$, and obese $\left(>28 \mathrm{~kg} / \mathrm{m}^{2}\right)$ independent variables: gender (female, male), age groups (60-69, 70-79 and $\geq 80$ years), smoking (no, yes) and schooling as adjustment (years of study, continuous variable). The chi-square test and multiple logistic regression model $(\mathrm{p}<0.05)$ were used to verify association between variables (STATA / SE 13.0).

Results: The 751 individuals evaluated were divided in two groups (reference group and risk group). The reference group was consisted of the 397 individuals, for those who maintained their normal weight in the two evaluations (2006 and 2010) (31\%), who were underweight (2006) and changed to normal weight $(2010)(7 \%)$ and those who remained underweight in both interviews (14\%). Risk group with 354 individuals, those who remained obese in both evaluations (35\%) and those who had normal weight (2006) and became obese (2010) (12\%). We excluded from the analysis those who were obese, who had normal weight and became underweight (2010) and those who were obese (2006) and changed to normal weight: 28 subjects. The factors associated with obesity in the second interview (risk group) were: age 70-79 years (OR: 1.99, p: <0.001) and $\geq 80$ years (OR: 2.64, p: <0.001); Gender male (OR: $0.42, \mathrm{p}<0.001$ ); and smoking (OR: 0.29, p < 0.001); Adjusted for schooling.

Conclusion: results show that increasing age is a risk factor for obesity in aging, and males and smokers would have lower chances of obesity.

Conflict of Interest: The authors declare that they have no conflict of interests. 
Funding: Research relating to this abstract was funded by CNPq, CAPES e FAPESP.

References:

1 Andrade FCA, Nazan, AINM, Lebrão, ML et al (2013) The Impact of Body Mass Index and Weight Changes on Disability Transitions and Mortality in Brazilian Older Adults. Journal of Aging Research doi:10.1155/2013/905094

2 Cheng FW, Gao X, Jensen GL. Weight Change and All-Cause Mortality in Older Adults: A Meta-Analysis. Journal of Nutrition in Gerontology and Geriatrics 34(4):343-368

\section{T2P65}

\section{Factors associated whith the risk of eating disorders among nutrition students}

\section{Martins, F.'; Vietta, G. '; Pereira, E. '; Silva, A. '; Gama, F. '; Kretzer, M. ${ }^{1}$}

${ }^{1}$ Universidade do Sul de Santa Catarina

Introduction: Eating disorders, bulimia and anorexia nervosa, affect mainly young female adults. Studies of the traceability of risk behaviors are necessary in different, field of professional training, especially nutrition, since this profession refers to a constant concern with physical appearance which may represent a greater risk of developing disorders Food. Considering that the nutritionist is important in interdisciplinary care for these disorders, it is essential to detect these conditions early in these professionals. . This study aimed to identify the prevalence and factors associated with risky eating behaviors in nutrition students from the University of Southern Santa Catarina (UNISUL), Brazil.

Method: A cross-sectional study was carried out with 106 students from all phases of the nutrition course, first to the ninth phase, representing $83.5 \%$ of the students enrolled in the period of June 2016. Data were collected from the Bulimic Investigatory Test of Edinburgh (BITE) And Eating Attitudes Test (EAT-26). Analysis in SPSS 18.0, Chi-square test and p value $<0.05$. Ratio of Prevalence with 95\% Confidence Interval . Approved by the Research Ethics Committee of UNISUL.

Results: students with mean age of 22.3 years, eutrophic (88.6\%) and 5.7\% with overweight / obesity. Regular physical activity was $49.1 \%$, and $33.0 \%$ consumed alcohol. The majority performed below 3 meals per week relative to breakfast $(95.3 \%)$, lunch $(99.1 \%)$, dinner $(87.7 \%)$ and snack $(89.5 \%)$. History of eating disorders in $11.3 \%, 19.3 \%$ with feeling fat / very fat, $22.8 \%$ with irregular menstruation and $24.5 \%$ with depression. The prevalence of risk symptoms for bulimia was $57.5 \%$, of these, $11.5 \%$ with moderate to severe . Behavior suggestive of anorexia in $14.3 \%$. Association $(\mathrm{p}<0.05)$ between and feeling of fat / very fat weight with bulimic symptoms and anorexic behavior. Depression associated with anorexic behavior.

Conclusion: Most students with irregular eating patterns, and more than half presented behavior of bulimic risk. Bulimic and anorexic symptoms are associated with feeling fat / very fat. Anorexic behaviors associated with the presence of depression.

Conflict of Interest: None Disclosed

Funding: No Funding

\section{T2P67 \\ Feasibility and acceptability of a healthy takeaway masterclass aimed at improving cooking practices and menu options in takeaway food outlets}

Hillier-Brown, F.'; Adamson, A. ${ }^{2}$; Goffe, L. ${ }^{2}$; Thomlinson, N. ${ }^{2}$; Adams, J.3.; Penn, L. ${ }^{2}$; Wreiden, W. ${ }^{2}$; Summerbell, C. '; White, M. ${ }^{3}$; Lake, A. ${ }^{4}$; Moore, H. '; Abraham, C. ${ }^{5}$; Araújo-Soares, $V^{2}$

${ }^{1}$ Obesity Related Behaviours Research Group, Durham University, ${ }^{2}$ Institute For Health And Society, Newcastle University, ${ }^{3}$ Centre For Diet And Activity Research (Cedar), University Of Cambridge, ${ }^{4}$ Centre For Public Policy \& Health, Durham University, ${ }^{5}$ Psychology Applied To Heath, University Of Exeter

Introduction: Takeaway and fast-food consumption has been associated with energy dense diets of poor nutritional quality, adverse metabolic health outcomes, obesity and type 2 diabetes. There is growing interest in interventions to improve the healthiness of cooking practices and menu offerings in takeaway food outlets.

Methods: A mixed methods approach was used to evaluate the feasibility and acceptability of a Healthy Takeaway Masterclass; a half-day training session delivered by a local authority public health team to staff of takeaway food outlets consisting of nutrition and cooking skills education; taste tasting and nutrition estimation activities; and action planning and goal (pledge) setting. Owners and managers of 181 takeaway food outlets located in a local authority district in the north east of England were invited to attend one of two training sessions. Self-reported data were requested six weeks post intervention from all businesses. Assessment visits were conducted by a researcher in a sub-sample of businesses pre-training and at six-week follow-up.

Results: Staff from 18 (10\% of invited) takeaway businesses attended the training, and all made at least one 'pledge' to improve the healthiness of their cooking practices or menu options. Staff from 15 of the businesses were contactable at follow-up. All reported achieving at least one of their pledges, with many making additional changes. Changes requiring minimal effort and cost to the business were the most popular (e.g. reducing sugar or salt, or using semi-skimmed instead of whole milk). Using products that were difficult to source without increased costs (e.g. reduced salt and sugar tomato ketchup) or perceived to be unpopular with customers (e.g. wholemeal bread) were unlikely to be tried or sustained. Assessment visits were conducted in seven businesses in which reported changes included reduced salt $(n=5)$ and sugar $(n=2)$ in cooking, improved oil management $(n=4)$ and increased vegetable portions $(n=2)$.

Conclusion: The Healthy Takeaway Masterclass appears to be a feasible and acceptable intervention for improving cooking practices and menu options for food outlets that agreed to take part. However, uptake was low and participants are unlikely to be representative of the whole target population. Food outlets self-reported making a number of 'healthy' changes, but there was minimal objective evidence of change.

Conflict of Interest: None Disclosed

Funding: Intervention was funded by Redcar and Cleveland Borough Council (RCBC). Evaluation was primarily funded by the UK National Institute of Health Research's School for Public Health Research, with a small contribution from RCBC.

\section{T2P68 \\ Fitness mitigates the influence of obesity in health related quality of life in young adults}

Garrido-Miguel, M. '; Álvarez-Bueno, C. '; Cavero-Redondo, I.'; PozueloCarrascosa, D. '; Cruz, L. ' ; Martínez-Hortelano, J. '; Soriano-Cano, A. '; García-Prieto, J.'

${ }^{1}$ Health and Social Research Center. Universidad de Castilla-La Mancha, Cuenca. Spain

Background: The "fat but fit" concept suggests that cardiorespiratory fitness (CRF) attenuates the risk of metabolic and cardiovascular disease independent of adiposity, even among the obese. If this hypothesis could be extended to other constructs such as Health Related Quality of Life (HRQoL) has not been elucidated yet.

Objetive: To examine whether CRF mitigates the largely evidenced negative influence of obesity on HRQoL.

Methods: A cross-sectional study including 770 college students aged 18 to 30 years from Castilla-La Mancha University, Spain. Data of body fact percentage (BF\%), CRF (20-m shuttle run test) and mental HRQoL (SF12 questionnaire) measurements were analyzed. Analysis of covariance tested the differences in mental HRQoL by categories of BF\% mass and CRF, controlling for different sets of confounders. Subsequently, a mediation analysis was performed using the PROCESS macro, developed by Preacher and Hayes.

Results: Mental HRQoL mean scores were significantly higher in students who had good CRF and low values of $\mathrm{BF} \%$. However, the negative relationship between $\mathrm{BF} \%$ and mental HRQoL $(\beta=-0.158 ; \mathrm{P} \leq 0.001)$ was at- 
tenuated losing all its statistical significance when fitness cardiorespiratory (mediator) was included in the regression model $(\beta=-0.054 ; \mathrm{P}=0.432)$. Besides, mediation analyses reported that CRF acts as a full mediator in the relationship between $\mathrm{BF} \%$ and mental HRQoL.

Conclusions: Our data suggest that the "fat but fit" hypothesis could be extended to the HRQoL, because CRF attenuates the negative influence of adiposity on HRQoL.

Conflict of Interest: The authors declare that they have no conflict of interest

Funding: This study was funded mainly by "Fundación para la Investigación Sanitaria en Castilla- La Mancha (FISCAM)” (Ref.- AN/2008/31). Additional Funding was obtained from the "Instituto de Salud Carlos III en Actividades Preventivas y de Promoción de Salud (Ref.-RD06/0018/0038).

\section{T2P69}

\section{Freedom, choice and obesity prevention policies}

\section{Williams, G. ${ }^{1}$;}

${ }^{1}$ Lancaster University

Introduction: It is common to frame state policies to tackle obesity as restrictions on choice and freedom. Opponents speak of the 'nanny state'; both opponents and advocates often speak of 'paternalism,' as if public health interventions may improve welfare - but only at the price of freedom.

Methods: This is a conceptual and normative paper. It analyses the ideas of 'restriction' and 'freedom' to show the difficulties for public health advocates that arise if these ideas are not well understood.

Results: In complex interdependent societies, people's options are always determined by organisations and social contexts. The 'freedom' to eat processed foods is now available to all; the 'freedom' to drive a car to most buildings in most cities is available to anyone who can afford it. At the same time, some options have disappeared for most people, such as opportunities to buy affordable whole foods at local shops or markets, or for younger children walk or cycle to school unsupervised. Measures to restore such options are bound to restrict some freedoms. But they also create new freedoms. For example, policies that restrict marketing to children reduce the pressures that families face from such marketing, and free corporations from competitive pressure to engage in such marketing, while pedestrianisation of streets creates new freedoms for children and adults.

This argument is also applicable, at a deeper level, to the choices that might be 'restricted' by obesity prevention policies. For example, roads depend on statutory restrictions (for example, prohibitions on owning or using these spaces for most purposes). So too do business corporations (for example, private individuals are required to recognise officers and employees as acting on behalf of the company). Public health policies (such as pedestrianisation or regulating corporate activity) change the scope of these restrictions but do not limit freedom per se. In other words, the question is not whether to restrict or increase freedom, but instead, which freedoms are worthwhile and which restrictions are necessary to secure them.

Conclusion: Admittedly, statutory intervention can limit freedom, as we see in authoritarian polities. Where it aims at public goods such as obesity prevention, however, regulation creates new freedoms, in the forms of environments and contexts that enable healthier behaviours. Advocates for policies to combat obesity should beware of defending them as tradeoffs between freedom and welfare. Such policies improve welfare *and* increase freedoms that matter.

Funding: This work was carried out as part of the I.Family study (http://www. ifamilystudy.eu), funded by the European Commission within the Seventh RTD Framework Programme, contract no. 266044.

\section{T2P70 \\ Genetic predisposition to obesity, restrained eating and changes in body weight - a population-based prospective study}

Konttinen, $H_{.}{ }^{1}$; Llewellyn, C. ${ }^{2}$; Silventoinen, K. ${ }^{1}$; Joensuu, A. ${ }^{3}$; Männistö, S. ${ }^{3}$; Salomaa, V. ${ }^{3}$; Jousilahti, P. ${ }^{3}$ Kaprio, J. ${ }^{1}$; Perola, M. ${ }^{3}$; Haukkala, A. ${ }^{1}$

${ }^{1}$ University of Helsinki, Helsinki, Finland, ${ }^{2}$ University College London, London, Uk, ${ }^{3}$ National Institute For Health And Welfare, Helsinki, Finland

Introduction: There is no consensus on whether cognitive control of eating (i.e. restrained eating) is helpful, merely ineffective or actually harmful in weight management. We examined the interplay between known obesity-related genetic variants, restrained eating and changes in body weight and size.

Methods: Participants were Finnish 25- to 74-year-olds who attended the baseline $(\mathrm{n}=5024)$ and follow-up $(\mathrm{n}=3735)$ phases of the DILGOM study. At baseline in 2007, height, weight and waist circumference (WC) were measured in a health examination and participants self-reported their weight at age 20 years. At follow-up in 2014, height, weight and WC were based on measured or self-reported information. We calculated 7-year change in body mass index (BMI) and WC, and annual weight change from age 20 years to baseline. The Three-Factor Eating Questionnaire-R18 was used to assess restrained eating. A weighted polygenic risk score (PRS) was created, using 97 BMI-related loci.

Results: Cross-lagged autoregressive models indicated that baseline restrained eating was unrelated to 7-year change in BMI $(\beta=0.00 ; 95 \% \mathrm{CI}=-$ $0.01,0.02)$, but higher baseline BMI predicted greater 7 -year increases in restrained eating $(\beta=0.08 ; 95 \% \mathrm{CI}=0.05,0.11)$. Similar results were obtained with WC as an outcome. The PRS did not predict 7-year changes in $\mathrm{BMI}$ or WC and no significant restrained eating $\times$ PRS interactions were observed. However, individuals with a higher PRS tended to gain more weight from age 20 years to baseline and this effect was more pronounced in unrestrained than in restrained eaters $(\mathrm{P}=0.038$ for interaction).

Conclusion: Our findings suggest that restrained eating is an indicator of susceptibility to weight gain rather than a factor that causes weight gain in middle-aged adults. There was tentative evidence that restrained eating may be helpful in reducing genetic influences on long-term weight gain over adulthood.

\section{T2P71}

\section{Genetic relationship between the rs9939609 FTO} polymorphism, body mass index and depression Agudo, M. ${ }^{1}$; Gutierrez, B. ${ }^{1}$; Cervilla, J. ${ }^{1}$; Ching-Lopez, A. ${ }^{1}$; Molina, E. ${ }^{2}$;
Mckenney, K. ${ }^{1}$; Ruiz-Perez, I. ${ }^{3}$; Rodriguez-Barranco, M. ${ }^{4}$; Rivera, M. ${ }^{1}$

${ }^{1}$ University of Granada, ${ }^{2}$ University Of Seville, ${ }^{3}$ Andalusian School Of Public Health And Ciberesp, ${ }^{4}$ Andalusian School Of Public Health

Introduction: Obesity and depression are highly prevalent major public health problems that frequently co-occur. Their relationship has been repeatedly examined in numerous studies but the nature of this association is still unclear. Shared aetiological factors have been found between depression and obesity. The role of the FTO gene in body mass index (BMI) and obesity has been confirmed in many studies. Recently, we reported the first study showing that depression modifies the effect of the FTO gene on BMI. The aim of this study is to investigate the genetic influence of the FTO rs9939609 polymorphism, BMI and depression and try to replicate previous findings in a Spanish population-based sample.

Methods: The PISMA-ep is a cross-sectional epidemiological study of mental disorders based on a representative sample of the adult population of Andalusia, Spain. The sample consists of 1,258 individuals, 337 with depression and 921 psychiatrically healthy controls. A DSM-IV diagnosis of major depression was ascertained using the MINI interview. T-tests were used to analyze the association between depression and BMI. Linear regression models for quantitative traits assuming an additive genetic model were applied to test the association between the rs 9939609 polymorphism 
and BMI in the whole sample, in depressive cases and in controls separately. Finally, we performed interaction analysis between the rs 9939609 , depression and BMI. The statistical analyses were carried out with the software PLINK v1.06.

Results: We found that individuals with depression had significantly higher BMI values compared to controls $(\mathrm{p}<0.01)$. Linear regression analysis showed that the rs 9939609 polymorphism was significantly associated with BMI in the whole sample $(\mathrm{p}<0.05)$ and in controls $(\mathrm{p}<0.05)$ No association was found in depressive cases alone $(p>0.05)$. There was no significant interaction between genotype and depression status in relation to BMI ( $\mathrm{p}>0.05)$.

Conclusion: This study provides additional support for an association between BMI and depression in a representative sample of the Spanish population. We found an association between the rs9939609 polymorphism that is in keeping from previous reports. However, our initial results do not support previous findings in which depression moderates the effect of the FTO gene on BMI. Given the relevance of exploring the genetic relationship between obesity and depression and to overcome the issue of insufficient sample size, we are working on the genotyping of the remaining 3,000 samples that constitute the entire PISMA-ep target population.

Conflict of Interest: None Disclosed

Funding: Research relating to this abstract was funded by Consejería de Salud de la Junta de Andalucía and the Marie Curie Research Grants Scheme (FP7-626235)

\section{T2P72}

Habitual physical activity, independent of moderate-vigorous activity, predicts amount of liver fat and metabolic health status

Davies, K. ${ }^{1}$; Sprung, V. ${ }^{1}$; Norman, J. '; Wilding, J. '; Kemp, G. ${ }^{2}$; Cuthbertson, D. ${ }^{1}$

${ }^{1}$ Institute of Ageing and Chronic Disease, University of Liverpool, UK, ${ }^{2}$ Magnetic Resonance And Image Analysis Research Centre (Mariarc), University Of Liverpool, Uk

Introduction: The role of exercise in the prevention and treatment of non-alcoholic fatty liver disease (NAFLD), a consequence of obesity, is well established. Until more recently, previous research has focused on the therapeutic benefit of increased moderate-vigorous activity, as opposed to habitual physical activity. We aimed to investigate the influence of habitual physical activity on metabolic health and in particular, the amount of liver fat.

Methods: Seventy-five healthy individuals ( $35 \pm 9$ years old, $25 \pm 4 \mathrm{~kg} / \mathrm{m}^{2}$ BMI) underwent comprehensive assessments of physical activity patterns using a SenseWear armband, metabolic health using IDF criteria of metabolic syndrome, regional body composition using magnetic resonance imaging and cardio-respiratory fitness using V02 peak.Statistical analysis was performed using i) a Mann-Whitney test, ii) univariate logistic regression models fitted with an outcome of health status, data presented as odds ratio (95\% CI) and iii) univariate linear regression models fitted with an outcome of liver fat, data presented as coefficient (95\% CI).

Results: There was no significant difference in physical activity in terms of sedentary behaviour, number of steps or moderate-vigorous physical activity (MVPA) between metabolically healthy and unhealthy individuals. However, the metabolically unhealthy individuals had significantly lower cardio-respiratory fitness and higher liver fat and visceral fat $(P<0.05)$. For every unit increase in \% liver fat, the odds of being metabolically unhealthy increased by a factor of $1.37(1.14,1.63) ; P<0.001$. Furthermore, for every one hour increased sedentary time, $\%$ liver fat increased by $0.87(\mathrm{P}=0.05)$ while for every daily increase of 1000 steps, $\%$ liver fat decreased by $0.87 ; P=0.016$. Interestingly, there was no significant association between hours of MVPA and liver fat $(P=0.360)$

Conclusion: In these individuals, sedentary behaviour and daily step counts are important determinants of the amount of liver fat and in turn of metabolic health status. These findings reinforce the role of avoiding sedentary behaviour even in the absence of increased MVPA
Conflict of Interest: None disclosed.

Funding: Research relating to this abstract was funded by Diabetes UK and MRC/ Arthritis UK.

\section{T2P74 \\ Healthy ranges of key micronutrients in menu-plans based on dietary recommendations}

\section{Green, $\mathrm{H}_{.}{ }^{1}$; Mainardi, $\mathrm{F}^{2}$; Steullet, $\mathrm{V}^{2}$}

${ }^{1}$ Nestlé Research Center, ${ }^{2}$ Nestlé Institute Of Health Sciences

Introduction: Reference intakes, such as the US Recommended Dietary Allowances (RDAs), provide the average daily amounts of nutrients that will meet the nutrient requirements of virtually everyone in a given healthy population. The aim here was to define the ranges of key micronutrients in two ideal menu-plans, which are based on dietary guidelines. Methods: Two publically-available ideal menu plans (MyPlate and DASH) were used. Each menu plan comprised meals, snacks and beverages for 7 consecutive days. Nutrient compositions were derived using the USDA nutrient database (Release 28). The 13 micronutrients of interest were those used on US food labels, as well as shortfall nutrients and nutrients of concern in the diets of the US population. Micronutrients for which there is no RDA were excluded.The healthy range of each micronutrient was determined from the minimum and maximum value of each nutrient in each menu-plan. This was expressed relative to the RDA for two population groups, women aged 19-30, and women aged 51-65 years. Descriptive statistics and one way ANOVAs were done using Minitab v16. Results: The MyPlate menu-plan provide less calories than DASH $(2043.1 \pm 84.5$ vs $2274.0 \pm 46.0 \mathrm{Kcal} /$ day, $\mathrm{n}=7 \pm$ SEM; $\mathrm{p}<0.05)$. Vitamin E was lower in MyPlate than DASH $(9.92 \pm 1.45$ vs $21.0 \pm 0.9 \mathrm{mg} / \mathrm{day}$, $\mathrm{n}=7 \pm$ SEM $\mathrm{p}<0.001)$, but there were no other differences in the nutrients of interest between the two menu-plans. In the MyPlate menu-plan the minimum values of 8 nutrients were less than $100 \%$ of the RDA for women aged 19-30 years, and 7 for women aged $51-65$ years. In the DASH menu-plan the minimim values of 5 nutrients were less than $100 \%$ of the RDA for both age groups. The maximum values of all nutrients was at least $100 \%$ of the RDA for both menu-plans and both age groups. However, the maximum value of 4 of the nutrients exceeded $200 \%$ of the RDA for at least one menu-plan and one age group.

Conclusion: This analysis shows how challenging it is to devise ideal menu-plans that address the RDAs of multiple nutrients. Even these ideal menu-plans do not meet the RDA for all nutrients on every day of the week. These include shortfall nutrients such as vitamin D, iron, folate and potassium. By contrast, the highest daily values of virtually all nutrients are well in excess of the RDAs.

Conflict of Interest: The authors are all employees of Nestec SA, which is part of Nestlé SA

Funding: No Funding

\section{T2P75 \\ High dietary calcium intake and a lack of dairy consumption are associated with metabolic syndrome in obese males: The Korean national health and nutrition examination survey 2010 to 2012}

\section{Shin, B. '; Kim, H. ' ; Song, S. ${ }^{1}$}

${ }^{1}$ Department of Family Medicine, The Catholic University of Korea College of Medicine, Seoul, Korea

Introduction: Metabolic syndrome (MetS) has been increasing rapidly worldwide and is associated with elevated risks of cardiovascular disease and mortality. The relationship between MetS and dietary calcium intake is controversial. We hypothesized that high dietary calcium intake is associated with MetS in males and that the associations vary according to obesity status. 
Methods: Using data from the Korean National Health and Nutrition Examination Survey V, a cross-sectional survey of Korean civilians was conducted from January 2010 to December 2012 to test these hypotheses. Results: Data from a total of 5946 males were analyzed with no association observed between dietary calcium intake and the presence of MetS. However, males deriving high calcium intake levels from dairy products had a lower prevalence of MetS than did those who did not ingest such products (adjusted odds ratio [OR], 0.75; 95\% confidence interval [CI], $0.58-0.96)$. In obese males, the highest dietary calcium intake was positively associated with the presence of MetS (adjusted OR, 1.61; 95\% CI, 1.12-2.36). However, obese males who consume dairy products had a lower prevalence of MetS than did those who did not consume such products (adjusted OR, 0.51; 95\% CI, 0.29-0.90).

Conclusion: The results suggest that the high dietary calcium intake, in the absence of dairy product consumption, was associated with the increased prevalence of MetS in obese males. Additional studies are required to determine whether dietary calcium intake affects the development of MetS.

Conflict of Interest: None

Funding: No Funding

\section{T2P76}

How adolescent patients enrolled in an outpatient pediatric obesity clinic experience online weight-, food-, and health information

\section{Holmberg, C. ${ }^{1}$; Berg, C. ${ }^{1}$; Dahlgren, J. ${ }^{2}$; Lissner, L. ${ }^{3}$; Chaplin, J. ${ }^{2}$}

${ }^{1}$ University of Gothenburg; Department of Food and Nutrition, and Sport Science, ${ }^{2}$ University Of Gothenburg And Sahlgrenska University Hospital; Department Of Pediatrics And Sahlgrenska Academy, ${ }^{3}$ University Of Gothenburg;

Introduction: This study aimed to explore digital media and information health literacy competencies among pediatric patients undergoing treatment for obesity. How the patients search for and select online information regarding food, body weight, and health, and how they experience this information was also explored.

Methods: Twenty adolescent patients with obesity were interviewed. The participants were between 13-16 years old and enrolled at Swedish university hospital clinic. Participants used a computer with Internet access to demonstrate search procedures and online information sources they used. The interviews were audio recorded and search activities on the computer were recorded via screen capture software. Qualitative content analysis was used to categorize the transcribed interview material.

Results: The participants described that they foremost searched for fun and easy ways to lose weight. Participants expressed that they encountered a wealth of food content in their online social networks which could be inspiring but it could also be tempting and as negative for weight management. Adolescents' described variation in search- and evaluation skills. Some participants assessed the trustworthiness of information by comparing different sources while others selected sources based on convenience and visual appeal. The participants described experiences such as gaining social support with others with obesity that had lost weight, but described being discouraged by unattainably successful fitness models. They also expressed disengagement in weight management due to deceptive commercial information.

Conclusion: Digital media resources proved to be a source for inspiration, information and social support but required critical literacy skills. The conventional division between media- and information literacy is increasingly distorted as more complex social media communication is taking place. Commercial content was often experienced as tailored (e.g. persuasive marketing), and personal information as mediatized (e.g. food marketing transmitted by peers in online social networks). To guide pediatric patients in how to autonomously manage their diet and weight management, it is essential to focus on adolescents' ability to assess online health information and to foster their critical media literacy skills.

\section{Conflict of Interest: None to declar}

Funding: This study was supported by Formas, the Swedish Research Council for Environment, Agricultural Sciences and Spatial Planning, (\#259-2012-38), and EpiLife, Gothenburg's center for epidemiologic studies (Forte 2006-1506). We wish to thank participants and their families, and the clinic staff at the Obesity unit at Queen Silvia Children's Hospital.

\section{T2P77}

\section{How people are able or willing to follow dietary recommendations}

\section{Stritecka, $H^{1}{ }^{1}$; Pastucha, D. ${ }^{2}$}

${ }^{1}$ University of Defence, Faculty of Military health Sciences, ${ }^{2}$ Refit Clinic

Introduction: Nutrition plays an important role in the prevention at many disease. In obesity and overweight, nutrition with physical activity is an essential element of both prevention and treatment. The basic recommendation is to limit and change the composition of fats as well as simple sugars and alcohol. Also important is the source and way of getting information about changing eating habits, their validity and their comprehensibility and grasp by common population.

Methods: In an ongoing survey, we focused on obese patients with CVD. Interviews are conducted in person with randomly addressed clients of GP, obesitologists or cardiologists. At present, 176 interviews were performed (79 M, $97 \mathrm{~F}$, at age 42-69).

Results: For now, you cannot say firm conclusions, but some trends can be observed. Almost 1/3 confirmed that it was sufficiently informed by their doctor about the necessary nutritional changes. $98 \%$ of the educated respondents received only printed form of recommendations without further comment. $80 \%$ of respondents under general recommendations cannot imagine real food or their quantity.

Conclusion: Most of respondents noted that the ideal form of recommendations would be illustrative examples and serving food.The biggest problem is with omega 3 fatty acids and their sources. $67 \%$ of respondents are not able or willing to regularly consume fish and other sources of omega-3 fatty acids do not know. In the conditions of the Czech Republic (CZ) is very difficult to reach fish consumption in the frequency of twice a week. In $\mathrm{CZ}$ is need to take account of the available resources n $3 \mathrm{MK}$ (canola, flax, walnuts).

Supported by:Possibilities of positive lifestyle influence of people with completed oncological treatment and Long term plan of development of organization 


\section{T2P78}

\section{Impact of a low carbohydrate and a high fibre diet in the central obesity of a low socioeconomic female population.}

Ferraz, I. ${ }^{1}$; Gelisk, I. ${ }^{2}$; Ladeia, A. ${ }^{3}$; Lima, M. ${ }^{4}$; Guimarães, A. $^{3}$

${ }^{1} \mathrm{~S} c h o o l$ of Medicine and Health, ${ }^{2}$ Regional College Of Bahia, ${ }^{3}$ Bahiana School Of Medicine, ${ }^{4}$ Bahiana School Of Medicine

Introduction: Central obesity is characterized by the excessive accumulation of fat inside and outside the abdomen and is associated with an increased risk of mortality by type 2 diabetes, hypertension and atherosclerotic vascular disease and especially coronary artery disease. Paradoxically, its incidence is high in low socioeconomic women living in Salvador, a costal eastern Brazilian tropical city.

This combination of poverty and central obesity led to this prospective study with the objective to learn the type of their usual diet and the nutritional changes necessary to overcome this situation.

Methods: Clinical prospective study of females treated at the Interdisciplinary Outpatient Clinic of the Bahiana School of Medicine under an Institutional Multi-professional project foe the study of excessive weight (PEPE). This study has been carried out for 48 months (2012 to 2016) and the patients included had at least one consultation every three months, over 18 years and a waist circumference $>80 \mathrm{~cm}$. Variables included age, race, school years and financial income. The weights were measured with a bioimpedance scale and food consumption was assessed by three twenty-four hour recalls. The principal modifications in the diet included a decrease in carbohydrate and fiber consumption.

Results: 89 obese females were studied, $48.5 \pm 12.4$ years old, $49(54.4 \%)$ of brown skin and income of 1.5 to 2.5 minimal salaries $57(64.04 \%)$. The main modification in the diet included a significant decrease in the ingestion of carbohydrates (231.570.0 97.5g to $215.6 \pm 63.0, \mathrm{p}=0.002)$, and in the ingestion of fibers $(11.83 \pm 6.14 \mathrm{~g}$ to $16.11 \pm 5.96 \mathrm{~g}, \mathrm{p}=0.04)$. These changes in food consumption were associated to a significant decrease in waist circumference $(106 \pm 12.5 \mathrm{~cm}$ to $100.2 \pm 11.3 \mathrm{~cm}, \mathrm{p}=0.03)$ and body fat content $(43.23 \pm 7.7 \% 40.9 \pm 6.4 \%, \mathrm{p}=0.04)$.

Conclusion: A decrease in carbohydrate and an increase in fiber content of the diet resulted in a significant reduction in the degree of central obesity in this low income population. The metabolic impact of these changes is being evaluated.

None Disclosed

Research relating to this abstract was funded by FAPESP

\section{T2P79}

Impact of school environmental programs on anthropometry, nutrition-related knowledge, and fitness in Austrian adolescents

Luger, M. ${ }^{1}$; Luger, E. ${ }^{1}$; Schätzer, M. ${ }^{2}$; Rust, $P^{3}$; Wild, B. ${ }^{4}$; Ladstätter, A. ${ }^{5}$; Rieder, A. ${ }^{6}$; Lechleitner, M. ${ }^{7}$; Hoppichler, F. $^{8}$

${ }^{1}$ Special Institute for Preventive Cardiology And Nutrition - SIPCAN, Salzburg; Division of Social and Preventive Medicine, Centre for Public Health, Medical University of Vienna, ${ }^{2}$ Special Institute For Preventive Cardiology And Nutrition - Sipcan, Salzburg, ${ }^{3}$ Department Of Nutritional Sciences, University Of Vienna, ${ }^{4}$ Department Of Psychology And Medical Sciences, Umit - Private University Of Health Sciences, Medical Informatics And Technology, Hall In Tirol, ${ }^{5}$ Tyrolean Educational Board, Austria, ${ }^{6}$ Division Of Social And Preventive Medicine, Centre For Public Health, Medical University Of Vienna, ${ }^{7}$ Special Institute For Preventive Cardiology And Nutrition - Sipcan, Salzburg; Department Of Internal Medicine, Landeskrankenhaus Hochzirl - Natters, ${ }^{8}$ Special Institute For Preventive Cardiology And Nutrition - Sipcan, Salzburg; Department Of Internal Medicine, Krankenhaus Der Barmherzigen Brüder Salzburg

Introduction: The impact of the school environment on adolescents body weight is poorly understood. Therefore, this study examined the effects of environmental school programs with either physical activity during the breaks, free offer of fruit \& vegetables or a combination of both Methods: 513 adolescents [49\% female; 14 (SD: 1) years] responded to a classroom survey in 16 secondary schools in Austria (Tyrol; 78\% country- side). Body weight $(\mathrm{kg})$, height $(\mathrm{m})$, and waist circumference $(\mathrm{cm})$ were measured and BMI $\left(\mathrm{kg} / \mathrm{m}^{2}\right)$ was calculated. Additionally, nutrition-related knowledge score (based on 20 nutrition-related questions; points), media consumption (h/day), and self-reported fitness using the International Fitness Scale (IFS) were examined.

Results: $22 \%$ of the respondents were overweight and $8 \%$ obese. A combination of both school environmental programs $(n=47)$ compared to one $(\mathrm{n}=393)$ and none $(\mathrm{n}=47)$ showed a significantly lower waist circumference [70.6 (9.1) vs. 72.6 (9.3) vs. $76.6(13.9) \mathrm{cm}$; p < 0.05], BMI [20.5 (4.0) vs. $21.8(3.8)$ vs. $\left.23.1(5.4) \mathrm{kg} / \mathrm{m}^{2} ; \mathrm{p}<0.01\right]$, media consumption [6.3 (3.8) vs. 10.4 (7.0) vs. 11.0 (9.1) h/day; p < 0.001], and a higher nutrition-related knowledge score [7.2 (3.3) vs. 5.4 (5.1) vs. 4.0 (4.8) points; p $<0.01$ ] Self-reported fitness, by using the IFS, was not significantly influenced [both programs: 19.5 (3.3) vs. one program: 19.1 (3.5) vs. no program: 17.7 (4.5) points; $\mathrm{p}=0.084$ ]. No availability of a school environmental program was associated with a 1.11-fold risk of higher BMI (OR 1.11, 95\%-CI 1.01-1.23; $\mathrm{p}<0.05$; adjusted for age, sex, migration background, and socioeconomic status). More adolescents with no program were overweight/obese compared to those with a combination of both school environmental programs (22\% vs. $9 \%$; $\mathrm{p}<0.05)$.

Conclusion: Providing opportunities for physical activity and offering fruit \& vegetables for free at school favourably affects the adolescents' waist circumference, BMI, the percentage of overweight/obesity, media consumption, and the nutrition-related knowledge score. These findings suggest that slight modifications of the school environment might contribute to the overweight/obesity prevention.

Conflict of Interest: None Disclosed

Funding: No Funding

\section{T2P81}

\section{Individual behaviours or sociodemografic factors are more "obesogenic" in school children's parents from Gdańsk}

Walentukiewicz, A. '; Resiak, M. ${ }^{1}$; Kamila, W. ${ }^{1}$; Lysak, A. ${ }^{1}$

${ }^{1}$ University of Physical Education and Sport, Gdansk, Poland

Introduction: Overweight and obesity were recognized in almost $20 \%$ of younger and older schoolchildren in Gdansk. The presented results cover a part of the study on parents of school age children in Gdansk aimed at frequency of occurrence of underweight and overweight concerning sociodemographic and environmental factors.

Methods: The study was carried out in 2016, on 1311 individuals (453 men and 858 women) whose children attended schools in Gdansk. The average age for men was 41 and for women 38 . The study was based on a diagnostic poll method, and overweight was determined by the WHO criteria.

Results: Overweight and obesity was registered in $42.64 \%$ of the subjects (68.11\% of men, $44.64 \%$ of women). Men related overweight and obesity was observed in relation to unhealthy behaviors such as sweetened beverages, fast food, short sleep, and frequent consumption of alcohol. In women adverse factors were associated with sweetened beverages, fast food, short sleep, and physical activity $<150 \mathrm{~min} /$ week. Sociodemographic variables coexisting with overweight and obesity in men are higher age category, lower income, remaining in partnership/marriage and lower education level, and in women higher age category, more children in family (3 and more), lower income, on social welfare, no career, partnership/ marriage and lower education level.

Conclusion: It seems that sociodemographic factors play a higher role among the factors identified in the study especially in women.

Conflict of Interest: None Disclosed

Funding: No Funding 
T2P82

Influence of anthropometric factors on altered postural stability: The relationship between body composition and body oscillation

Maestri, T. ${ }^{1}$; Vignatti, L. ' Nazário, A. ${ }^{2}$; Nazário, N. ${ }^{1}$; Silva, A. ${ }^{1}$

${ }^{1}$ Universidade do Sul de Santa Catarina - UNISUL, ${ }^{2}$ Universidade Federal De Santa Catarina - Ufsc

Introduction: Obesity is a metabolic disease that has reached epidemic proportions(1).This disorder can influence the systems responsible for vital functions. The balance is a result of the harmonious interaction of various systems of the human body: vestibular, visual, somatosensory and musculoskeletal(2), and increased body mass can reduce this interaction, affecting the postural adjustments(3).

Methods: Transversal study, with 85 individuals with aged $\geq 18$ years who attend the University of Southern Santa Catarina (UNISUL). Participants were distributed by Body Mass Index (BMI) in normal weight 18,5 to 24,9 (G1), overweight 25 to 29,9 (G2) and obese $\geq 30$ (G3). The anthropometric data: weight and height were collected using a digital scale (W801 Wiso) and portable stadiometer (Portable Wood Compact - WCS) and the static balance was evaluated by means of the parameter analysis of the pressure center oscillation area (COP) measured by a pressure platform (baropodometry). Were divided according to BMI under the ' 'eyes open' ' and ' 'eyes closed" condition. A bivariate analysis was performed when it oscillated from $0-69.9 \mathrm{~mm}$ was classified as a small oscillation and when oscillated $\geq 70 \mathrm{~mm}$ was classified as a large oscillation and related to BMI. The data was analyzed in the SPSS 18.0, CHI-square test and prevalence rate, with IC $95 \%$, statistic relevance $\mathrm{p} \leq 0.05$. The oscillation averages of each group were performed under the conditions "'eyes open" and ' 'eyes closed"'.

Results: The mean oscillation of the groups was: G1 $=70.68 \mathrm{~mm}$, $\mathrm{G} 2=77.47 \mathrm{~mm}$ and G3 $=72.41 \mathrm{~mm}$ with "'eyes open' ' condition. When subjected to the condition "eyes closed" G1 $=79.82 \mathrm{~mm}, \mathrm{G} 2=84.03 \mathrm{~mm}$ and $\mathrm{G} 3=94.80 \mathrm{~mm}$. The mean age of the groups was: $\mathrm{G} 1=44.16$ years $\mathrm{G} 2=65.74$ years and G3 $=64.20$ years. When analyzing the conditions ' 'open eye' ' and ' closed eye' ' in relation to BMI, there was no statistical significance.

Conclusion: Theincreased body mass in overweight and obese individuals can reduces the ability to make postural adjustments and increases postural instability, mainly in the "eyes closed" condition, due to the average oscillation found between the groups, although there was no statistical significance.

Conflict of Interest: None Disclosed

Funding: No Funding

Reference:

1 YUMUK, Volkan et al. European Guidelines for Obesity Management in Adults. Obesity Facts, [s.I.], v. 8, n. 6, p.402-424, 5 dez. 2015. S. Karger AG. http://dx.doi.org/10.1159/000442721.

2 ALMEIDA, Sionara Tamanini de et al . Análise de fatores extrínsecos e intrínsecos que predispõem a quedas em idosos. Rev. Assoc. Med. Bras., São Paulo, v. 58, n. 4, p. 427-433, Aug. 2012

3 ALONSO, Angélica et al. Anthropometric factors interfering in postural balance. Brazilian Journal of Biomechanics, Year 2012, vol 13, n.25

\section{T2P83}

Influence of school fruit and vegetable scheme on fruit and vegetable consumption among school children during 3 years of evaluation

Wolnicka, K. ${ }^{1}$; Annataraszewska ; Jaczewska-Schuetz, J. ${ }^{1}$; Jarosz, M. ' Niewiarowska, M. ${ }^{2}$

${ }^{1}$ National Food and Nutrition Institute, ${ }^{2}$ Agricultural Market Agency

Objectives: The School Fruit and Vegetable Scheme (SFVS) has been created as one of the priority activities of the European Commission aiming at the development among children of a permanent habit of eating fruit and vegetables. In Poland children receive for free fruit and vegetables $(F \& V)$ portions, eaten at school, 2-3 times a week. The aim of this study was to assess SFVS impact on F\&V consumption among school children during the 3 years of evaluation.

Methods: The study was conducted among pupils of selected I-III classes of primary schools from 2012 to 2015 . The study was conducted in randomly selected schools in the area of 5 selected voivodeships representative for north, south, central, east and west regions of Poland ( 85 schools in first year, 77 - in second, 66 - in third year of study) which participated in the program (intervention group) and did not participate in the program (control group). The first stage of the study was conducted at the beginning of the school year before the introduction of SFVS (stage "0"). The next stages of the study were carried out at the end of F\&V distribution period in class I, II, III. The fruit and vegetables consumption of pupils was evaluated on the basis of 3-day food record method.

Results: At the beginning of the study (stage " 0 ") there was no significant differences in fruit, vegetables and F\&V together intake between control and intervention group. After the first year of SFVS implementation, F\&V consumption was significantly higher in the intervention group than in the control group ( $p=0,001)$. At the same time in the control group fruit consumption decreased, vegetables consumption remained at the same level. In the second year of evaluation study there was increase of consumption $\mathrm{F} \& \mathrm{~V}$ in both groups but the difference between groups was even greater than in the first year in favor of the intervention group $(p=0,0001)$. After the third - the last year of providing children with free servings of $F \& V$, in the intervention group $\mathrm{F} \& \mathrm{~V}$ consumption remained higher than in the control group $(p=0,01)$. Consumption of fruit after the third year of the program was higher by $13.5 \%$ in the intervention group compared to the control group $(\mathrm{p}=0.0001)$.

Conclusions: The evaluation results show that the SFVS has a very strong potential and is an appropriate tool to improve the eating habits of children towards consuming more fruit and vegetables for the future. However, the program has a beneficial effect on increasing consumption in the case of fruit only.

\section{T2P84}

\section{Insights and perceptions of obesity management in older people with obesity: Results of the ACTION (awareness, care, and treatment in obesity management) study}

Look, M. ${ }^{1}$; Golden, A. ${ }^{2}$; Kyle, T. ${ }^{3}$; Dhurandhar, N. ${ }^{4}$; Stevenin, B. ${ }^{5}$; Kothari, M. ${ }^{5}$; Tomaszewski, $\mathrm{K}^{6}$

${ }^{1}$ San Diego Sports Medicine and Family Health, San Diego, CA, USA, ${ }^{2} \mathrm{~Np}$ From Home Llc, Munds Park, Az, Usa, ${ }^{3}$ Conscienchealth, Pittsburgh, Pa, Usa, ${ }^{4}$ Texas Tech University, ${ }^{5}$ Novo Nordisk Inc, Plainsboro, Nj, Usa, ${ }^{6} \mathrm{Kjt}$ Group Inc, Honeoye Falls, Ny, Usa

Background: Continued obesity management challenges remain, especially among older persons who may have greater comorbidities. The ACTION study examined attitudes and behaviours related to obesity management among people with obesity $(\mathrm{PwO})$. This research examines results among people aged $\geq 65$ years.

Methods: This study was a cross-sectional, stratified sampling of $\mathrm{PwO}$ (self-reported BMI $\geq 30 \mathrm{~kg} / \mathrm{m}^{2}$ ) ( $\left.\mathrm{n}=3,008\right)$, who completed online surveys. The instrument assessed attitudes, experiences and behaviours associated with obesity management. Comparisons between older ( $\geq 65$ years) and younger ( $\leq 64$ years) $\mathrm{PwO}$ are presented.

Results: The minority of $\mathrm{PwO}$ were aged $\geq 65$ (31\%). More older $\mathrm{PwO}$ $(66 \%)$ reported feeling that "obesity is a disease," compared with younger $\mathrm{PwO}(64 \%)$. Of the PwO that have discussed weight-management with a healthcare provider (HCP), fewer older PwO (56\%) report that they "seek support" for weight loss compared with younger PwO (65\%). Fewer older $\mathrm{PwO}(50 \%)$ report receiving a "formal diagnosis" of obesity compared with younger $\mathrm{PwO}$ (56\%). Older PwO report greater weight loss "success" in the past year (13\%) compared with younger $\mathrm{PwO}(9 \%)$. Older $\mathrm{PwO}$ report greater comorbidity prevalence and $16 \%$ of older $\mathrm{PwO}$ reported 
that "a specific medical event" influenced their desire to manage weight compared with $12 \%$ of younger $\mathrm{PwO}$.

Conclusion: Older $\mathrm{PwO}$ show important differences in obesity management compared with younger $\mathrm{PwO}$. Considering their greater prevalence of comorbidities, older $\mathrm{PwO}$ may need more appropriate, attentive obesity management from HCPs. Efforts at providing HCPs with a better understanding of how older $\mathrm{PwO}$ perceive and manage their obesity may further improve their outcomes.

\section{T2P85}

\section{Interactions between genetic variants associated with adiposity traits and soft drinks in relation to longitudinal changes in body weight and waist circumference}

Olsen, N. '; Ängquist, L. ${ }^{2}$ Larsen, S. ; Linneberg, A. ${ }^{3}$; Skaaby, T. ${ }^{3}$; Husemoen, L. ${ }^{3}$; Toft, U. ${ }^{3}$; Tjønneland, A. ${ }^{4}$; Halkjær, J. ${ }^{4}$; Hansen, T.5 ; Pedersen, O. ${ }^{5}$; Overvad, K. ${ }^{6}$; Ahluwalia, T. ${ }^{5}$; Sørensen, T. ${ }^{2}$; Heitmann, B. ${ }^{1}$

${ }^{1}$ Research Unit for Dietary Studies at the Parker Institute and Institute of Preventive Medicine, Bispebjerg and Frederiksberg Hospital, Copenhagen, Denmark, ${ }^{2}$ Institute Of Preventive Medicine, Bispebjerg And Frederiksberg Hospital, Copenhagen, Denmar, ${ }^{3}$ Research Centre For Prevention And Health, Copenhagen, Denmark, ${ }^{4}$ Danish Cancer Society Research Center, Copenhagen, Denmark, ${ }^{5}$ Novo Nordisk Foundation Center For Basic Metabolic Research, Section Of Metabolic Genetics, Faculty Of Health And Medical Sciences, ${ }^{6}$ Section Of Epidemiology, Department Of Public Health, Aarhus University, Aarhus, Denmark;

Background: Intake of sugar-sweetened beverages is associated with obesity, and this association may be modified by a genetic predisposition to obesity.

Objective: We examined the interactions between a molecular genetic predisposition to various aspects of obesity and the consumption of soft drinks, which are a major part of sugar-sweetened beverages, in relation to changes in adiposity measures.

Design: A total of 4765 individuals were included in the study. On the basis of 50 obesity-associated single nucleotide polymorphisms that are associated with body mass index (BMI), waist circumference (WC), or the waist-to-hip ratio adjusted for BMI (WHRBMI), the following 4 genetic predisposition scores (GRSs) were constructed: a complete genetic predisposition score including all 50 single nucleotide polymorphisms (GRSComplete), a genetic predisposition score including BMI-associated single nucleotide polymorphisms (GRSBMI), a genetic predisposition score including waist circumference-associated single nucleotide polymorphisms (GRSWC), and a genetic predisposition score including the waist-to-hip ratio adjusted for BMI-associated single nucleotide polymorphisms (GRSWHR). Associations between soft drink intake and the annual change $(\Delta)$ in body weight $(\mathrm{BW})$, WC, or waist circumference adjusted for BMI (WCBMI) and possible interactions with the GRSs were examined with the use of linear regression analyses and meta-analyses.

Results: For each soft drink serving per day, soft drink consumption was significantly associated with a higher $\Delta \mathrm{BW}$ of $0.07 \mathrm{~kg} / \mathrm{y}$ (95\% CI: 0.01 , $0.13 \mathrm{~kg} / \mathrm{y} ; P=0.020)$ but not with the $\triangle \mathrm{WC}$ or $\triangle \mathrm{WCBMI}$. In analyses of the $\triangle \mathrm{BW}$, we showed an interaction only with the GRSWC (per risk allele for each soft drink serving per day: $-0.06 \mathrm{~kg} / \mathrm{y} ; 95 \% \mathrm{CI}:-0.10,-0.02 \mathrm{~kg} / \mathrm{y}$ $P=0.006)$. In analyses of the $\Delta \mathrm{WC}$, we showed interactions only with the GRSBMI and GRSComplete [per risk allele for each soft drink serving per day: $0.05 \mathrm{~cm} / \mathrm{y}(95 \%$ CI: $0.02,0.09 \mathrm{~cm} / \mathrm{y} ; P=0.001)$ and $0.05 \mathrm{~cm} / \mathrm{y}(95 \%$ CI: $0.02,0.07 \mathrm{~cm} / \mathrm{y} ; P=0.001)$, respectively]. Nearly identical results were observed in analyses of the $\triangle \mathrm{WCBMI}$.

Conclusion: A genetic predisposition to a high WC may attenuate the association between soft drink intake and BW gain. A genetic predisposition to high BMI as well as a genetic predisposition to high BMI, WC, and WHRBMI combined may strengthen the association between soft drink intake and WC gain. However, the public health impact may be limited.

\section{T2P86}

\section{Landscape review of obesity research in Scotland}

Jepson, R. '; Estrade, M. ' ; Malden, S. ${ }^{1}$; Archibald, D. ${ }^{1}$ ${ }^{1} \mathrm{SCPHRP}$, University of Edinburgh

Introduction: Obesity rates in Scotland are amongst the highest in Europe. It is therefore important to assess where Scotland's obesity research efforts are being focused in order to counteract this public health issue. This review describes the landscape and characteristics of obesity research that has been undertaken in Scotland in the last decade. Important research gaps are identified, as well as potential collaboration opportunities that could help move Scotland's obesity research agenda forward in a more strategic, coherent way.

Methods: Data on relevant research projects were collected via electronic searches of internet databases and supplemented by email and telephone communication with relevant researchers. Studies were then marked against the inclusion/exclusion criteria. The data collection included studies conducted between January 2006 until October 2016. Research projects and their associated published papers were coded for a range of variables including: study design, geographical area, Funding source, participant characteristics (e.g. weight status, age group, socio-demographics, co-morbidity), ANGELO framework, and type of intervention.

Findings: In total, 180 studies were included in the review which had generated 217 total publications (some studies had associated publications). There are a number of findings from this review that need to be considered within the policy and practice arena. Firstly, there is a relatively small amount of research that has focused on preventing obesity compared to that which has focused on reducing weight in people who are already obese/overweight. The second striking finding is the amount of research that focuses on one particular cause of obesity (diet/physical activity) rather than taking into account multiple causes and pathways.

Conclusion: The observational/descriptive obesity research identified in this review is substantial and shows that surveillance and monitoring of trends/associations relating to obesity has been adequate within the last decade in Scotland. However, it may be time to move on from the observational research to find long-term solutions in the form of sustainable and evidence-based interventions. This project employed a novel methodological approach which will help researchers and policymakers assess, monitor and prioritise obesity research efforts in Scotland.

Conflict of Interest: None

Funding: Research relating to this abstract was funded by NHS (National Health Service) Health Scotland

\section{T2P87 \\ Life events, quality of life, autonomic nervous system, and obesity risk}

Majerèák, I. ${ }^{1}$; Milos, S. ${ }^{2}$; Erika, G. ${ }^{3}$; Antonia, K. ; Michal, K. ; Marta, Z. ${ }^{2}$; Michaela, C. ${ }^{4}$; Jan, P.

${ }^{1} 1$ st Department of Internal Medicine, Faculty of Medicine, Pavol Josef Safarik University in Kosice, the Slovak Republic, ${ }^{2}$ Department Of Psychology Sciences, Faculty Of Social Science And Health Care, Constantine The Philosopher University In Nitra, The Slovak Republic, ${ }^{3}$ Internal Medicine And Cardiology Private Practice Mudr Ivan Majercak, Mudronova 29, Kosice, The Slovak Republic, ${ }^{4}$ Psychagogia, Garbiarska 3948, 03101 Liptovsky Mikulas, The Slovak Republic, ${ }^{5}$ Department Of Psychiatry, Faculty Of Medicine And Dentistry, University Palacky Olomouc, University Hospital, 77520 Olomouc, The Czech Republic

Background: Psychological distress is considered as a component of the obesity risk. The present study aims to determine which psychophysiological, arterial stiffness parameters, anthropometric factors and body composition measures are correlated with life events, depression and quality of life in otherwise healthy adults.

Method: 114 adults were examined using The Social Readjustment Rating Scale, The EuroQol Group 5-Dimension Self-Report Questionnaire, Beck Depression Inventory - Second Edition, Zung Self-Rating Depression 
Scale. Physiological measures included heart rate variability, skin conductance level and skin temperature. Anhropometric and body composition measures chracteristics included weight, height, hip size, waistline, blood pressure, heart rate at rest and after mental activity, muscle mass, fat stock, percentage of the body fat, segmental distribution of muscle and fat mass, fat-free mass and the water content in the body. Finally, data from non-invasive arteriography examination included aortic pulse wave velocity, central aortic blood pressure and augmentationindex.

Results:Life events in last two years correlate with the obesity risk in terms of several anthropometric and body composition measures. Life events in last two years also correlate with worse quality of life and a higher level of depression.

Conclusions: Results suggest some possible mechanisms by which life events may exert adverse effects on anthropometric and body composition measures in otherwisehealthy persons. Primary preventive strategies with the stress management training may prove beneficial.

\section{T2P89 \\ Magnitude of overweight and obesity in older adults of different birth cohorts (1936 to 1940 and 1946 to 1950): Health, wellbeing and aging (SABE) study}

Roediger, M. ${ }^{1}$; Marucci, M. ${ }^{1}$; Dourado, D. ${ }^{1}$; Bueno, D. ${ }^{1}$; Souza, C. '; Duarte, $Y^{2}$; Lebrão, $M .^{3}$

${ }^{1}$ Department of Nutrition, School of Public Health, University of São Paulo/ Brazil, ${ }^{2}$ Department Of Nursing Medical-Surgical School Of Nursing, University Of São Paulo/ Brazil, ${ }^{3}$ Department Of Epidemiology, School Of Public Health, University Of São Paulo/ Brazil

Introduction: The aging process is characterized by several changes, including nutritional status. To understand the trend of these changes it is necessary studies with older adults from different times of birth. This study verifiedthe magnitude of overweight and obesity in older adults of different birth cohorts.

Methods: It were analyzed older adults participants from the Health, Wellbeing and Aging (SABE) Study, carried outin São Paulo-SP/Brazil, in 2000 and 2010.In 2000 (Cohort A ), the population was composed of 2143 older adults ( $\geq 60$ years), selected by probabilistic sample, being selected only 426 of 60 to 64 years born between 1936 and 1940. In 2010 (Cohort C), was composed 715 individuals, selected 329 of 60 to 64 years born between 1946 and 1950. The variable of this study was overweight and obesity, identified by body mass index (BMI), considering BMI $\geq 28$ $\mathrm{kg} / \mathrm{m}$. The prevalence ratio (PR) was calculated to compare the variables of cohorts, by Poisson regression.

Results: A total of 755 individuals, of both sexes, 60 to 64 years born in the periods of 1936 to 1940 and 1946 to 1950 . There was a higher proportion of women $(60 \%)$ and of older adults of cohort C (54\%), with overweight and obesity $(\mathrm{p}=0.0000)$. Women of cohort $\mathrm{C}$ presented higher values of PR for overweight and obesity $(\mathrm{PR}=1.73)$.

Conclusion: These results showed concerning worrying scenarios of condition nutritional for the most recent cohort C(1946 - 1950), situation that damages the health conditions of older adults and can predict the future development of chronic diseases in this population group.

Conflict of Interest: None Disclosed

Funding: Fundação de Amparo à Pesquisa do Estado de São Paulo (FAPESP)

\section{$\mathrm{T} 2 \mathrm{P} 90$}

\section{Maintenance factors of obesity in adults with and without binge eating}

\section{Horta, C. ${ }^{1}$; Pimenta, F. ${ }^{2}$; Maroco, J. ${ }^{2}$; Leal, I. ${ }^{2}$}

${ }^{1} I S P A$ - Instituto Universitario de Ciências Psicológicas, Sociais e da Vida, ${ }^{2}$ Ispa - Instituto Universitario De Ciências Psicológicas, Sociais E Da Vida - William James Center For Research
Introduction: Previous studies have identified a variety of factors (e.g. socio-demographic, lifestyle, health status, psychological, etc.) as associated with obesity. However, most researches developed are quantitative ones, not allowing an in-depth examination of the several factors associated to the excessive weight maintenance. Thus, this study aims at exploring the factors/processes associated to excessive weight maintenance in adults with obesity.

Method: In total, 38 adults with BMI above $30 \mathrm{~kg} / \mathrm{m} 2$ were individually interviewed about the self-perceived obesity maintenance processes/factors. Interviews were audiotaped and transcribed. Participants filled in the Binge Eating Scale and a socio-demographic questionnaire. The qualitative analysis (both thematic and content analysis) was undertaken by two researchers. MAXQDA and SPSS software were used.

Results: Individual processes and factors included lack of perceived control; negative self-concept, low self-esteem and negative body image; lack of an organized meal timetable; negative comments from others; dysfunctional avoidance coping; existence of comorbidities; eating as a way to manage negative emotions (such as stress or depressive mood); lack of support; internal pressure to eat; and pregnancy. Contextual factors were also mentioned (e.g. frequently eating out, unemployment). Thirteen participants presented binge eating behaviour.

Conclusions: Acknowledging the different factors and processes associated to obesity maintenance is important for both intervention and research settings, since they might be a barrier to successful weight loss. These contents should be analysed considering the need for a multi-disciplinary intervention in the context of obesity treatment.

Conflict of Interest: None Disclosed

Funding: This research was funded by the Fundação para a Ciência e Tecnologia FCT (grant SFRH/BPD/77799/2011). William James Center for Research is funded by the Fundação para a Ciência e Tecnologia - FCT (grant UID/PSI/04810/2013).

\section{T2P91 \\ Maternal pre-pregnancy obesity and maternal prenatal depressive symptoms across 41.478 Person-observations}

Kumpulainen, S. '; Lahti, M. '; Tuovinen, S. '; Pesonen, A. '; Heinonen, K. '; Kajantie, E. ${ }^{3}$; Villa, P. ${ }^{4}$; Hämäläinen, E. ; Laivuori, H. ${ }^{6}$; Räikkönen, $K .{ }^{1}$

${ }^{1}$ Institute of Behavioral Sciences, University of Helsinki, Finland, ${ }^{2}$ Institute Of Behavioral Sciences, University Of Helsinki, Finland; Endocrinology Unit, University/Bhf Centre For Cardiovascular Science, Queen'S Medical Research Institute, Edinburgh, Uk, ${ }^{3}$ National Institute For Health And Welfare, Helsinki, Finland; Children'S Hospital, University Of Helsinki And Helsinki University Hospital, Finland; Department Of Obstetrics And Gynaecology, Mrc Oulu, Oulu University Central Hospital And University Of Oul, ${ }^{4}$ Research Programmesunit, Women'S Health, University Of Helsinki, Finland; Department Of Obstetrics And Gynaecology(Pv), University Of Helsinki And Helsinki University Central Hospital, Finland, ${ }^{5}$ Huslab And Department Of Clinical Chemistry, Helsinki University Central Hospital, University Of Helsinki, Finland, ${ }^{6}$ Haartman Institute, Medical Genetics, University Of Helsinki, Finland

Introduction: We examined if pre-pregnancy overweight and obesity are associated with maternal prenatal depressive symptoms, independently of cardiometabolic pregnancy complications.

Methods: Our study sample comprised 3078 pregnant women participating in the Prediction and Prevention of Preeclampsia and Intrauterine Growth Restriction (PREDO) study. Before pregnancy 2070 women were normal weight $\left(\mathrm{BMI}<25 \mathrm{~kg} / \mathrm{m}^{2}\right), 589$ overweight $\left(25-29.99 \mathrm{~kg} / \mathrm{m}^{2}\right)$, and 419 obese $\left(\geq 30 \mathrm{~kg} / \mathrm{m}^{2}\right)$. During pregnancy the women filled in the Center for Epidemiological Studies Depression Scale biweekly from $12-13^{\text {th }}$ gestational week onwards, resulting in 41.478 person-observations of depressive symptoms.

Results: Compared to normal-weight women, overweight and obese women reported consistently higher levels of depressive symptoms throughout pregnancy ( $p<0.001$; normal weight/overweight/obesity $\mathrm{x}$ gestational week interaction on depressive symptoms $>0.05$ ). Multiplicative additive analyses showed that hypertensive pregnancy disorders and/or gestational diabetes did not add to the effects of overweight and obesity on depres- 
sive symptoms. Using normal-weight women without cardiometabolic pregnancy complications as a reference group our analyses showed that the risk of clinically significant depressive symptoms was not higher in normal-weight women with cardiometabolic pregnancy complications (Odds ratio [OR]:1.03, 95\% confidence Interval [95\% CI]:0.85 - 1.27), but was higher in overweight women without (OR:1.28, 95\% CI:1.05-1.55) or with (OR:1.32, 95\% CI:1.01-1.72) cardiometabolic pregnancy complications, and in obese women without (OR:1.51, 95\% CI:1.20-1.90) or with (OR:1.56, 95\% CI:1.20-2.03) cardiometabolic pregnancy complications. Adjustments for age, education, smoking and alcohol use did not alter any of these associations.

Conclusions: Maternal pre-pregnancy overweight and obesity are associated with higher levels of and increased odds for clinically significant depressive symptoms throughout pregnancy. Our findings suggest that pregnancy disorders may not per se trigger higher levels of depressive symptoms, but women with pre-pregnancy overweight and obesity may feel more depressed from the beginning of pregnancy.

Conflict of Interest: None Disclosed.

Funding: The Academy of Finland, Finnish Graduate School of Psychology, the Emil Aaltonen Foundation, EVO, the Finnish Medical Foundation, the Jane and Aatos Erkko Foundation, the Novo Nordisk Foundation, the Päivikki and Sakari Sohlberg Foundation, the Sigrid Juselius Foundation and the Sir Jules Thorn Charitable Trust, University of Helsinki, EraNet Neuron and University of Helsinki Research Funds.

\section{T2P92}

\section{Measurement of the unsatisfaction index and body image distortion in a population of university students}

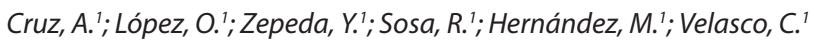
${ }^{1}$ Benemérita Universidad Autónoma de Puebla

Introduction: The concept of body image has been changing and enriched thanks to successive investigations. Body image is a dynamic construct that can be described as an exchange of feelings, perceptions, attitudes and beliefs towards the body between the persons and their social context. The present work tries to explain what is the perception that university students have of their own body, in these times of globalization, consumerism and erroneous ideals of perfection.

Methods: It was requested the participation of 500 students at the Autonomous University of Puebla (BUAP) in the period of August to December 2015, all they completed the BSQ questionnaire and the Montero test for body image.

Results: In the aforementioned population, the majority presented normality: 219 women (43.8\%) and 149 men (29.8\%), mean age was 21 years. Regarding the circumference measure, men presented $36.8 \%$ of low cardiometabolic risk and women $32 \%$ according to this parameter indicative that the majority has the adequate measure of abdominal fat. Eighty three percent of the population presented distortion of their image (difference between perceived and desired body image, referred to altered perception of body dimensions), while $91.6 \%$ presented dissatisfaction (difference between perceived and actual image to negative feelings and emotions towards the body).

Conclusion: Despite the data obtained, the level of concern of young university students is minimal, since $80 \%$ indicated that they do not have an important interest in modifying their body image. In the case of gender distortion and dissatisfaction, there are more women than men with this problem, but it should be mentioned that the difference is not as marked as in the past so the approach of future research needs to be rethought.

\section{T2P94 \\ Modulation of postingestive reward mechanisms by weight loss surgery}

\section{Ribeiro, A. ${ }^{1}$; Fernandes, A. ${ }^{\text {; }}$ Camacho, M. ${ }^{1}$; Oliveira, M. ${ }^{2}$; Duarte, J. ${ }^{2}$;} Costa, R. ${ }^{1}$; Oliveira-Maia, $A .^{{ }^{1}}$

${ }^{1}$ Champalimaud Research and Clinical Centre, Champalimaud Centre for the Unknown, Lisboa Portugal., ${ }^{2}$ Centro Hospitalar De Lisboa Ocidental, Lisboa, Portugal.

Introduction: Flavor-nutrient conditioning (FNC) is the process whereby the preference for a particular flavor is altered by multiple pairings of that flavor with the postingestive consequences of a nutrient. FNC has been extensively demonstrated in rodents but there is significant variability regarding FNC methods and findings in humans. Also, its relevance in the context of obesity and weight-loss surgery has not been explored. We hypothesized that weight-loss surgery reduces postingestive food reward and, to test this hypothesis, developed a novel method for controlled FNC in humans.

Methods: Our FNC protocol was developed and optimized in a control sample of healthy non-obese volunteers $(n=55)$. In a pre-conditioning day, two flavors with similar novelty and pleasantness ratings were selected for each participant. In the following days, subjects drank one of the flavors (conditioned flavor), enriched with maltodextrin (MD, an insipid carbohydrate), and in alternate days the other flavor (controlled flavor), without MD, was offered. Intake and pleasantness measurements were taken daily, including in a post-conditioning day. The optimized protocol was then applied in a cohort of 34 patients, either prior to or after weightloss surgery, specifically, gastric bypass or sleeve gastrectomy.

Results: We did not find, in either sample, significant conditioning-induced differences of flavor pleasantness, for any flavor (conditioned and control). However, regarding intake, in the control sample, FNC induced increased consumption of the flavor paired with MD. In the clinical samples, both the obese group and the gastric bypass patients had a similar significant increase in the intake of the conditioned flavor, that was not observed in the patients receiving sleeve gastrectomy.

Conclusion: Our findings suggest that flavor-nutrient conditioning contributes towards implicit feeding decisions in humans. Furthermore, such mechanisms seem to be altered after weight-loss surgery, particularly by restrictive procedures such as sleeve gastrectomy.

\section{Conflict of Interest: None}

Funding: Research relating to this abstract was funded by a grant from the BIAL Foundation (176/10) to AJO-M and by Fundação para a Ciência e Tecnologia, through a Junior Research and Career Development Award from the Harvard Medical Portugal Program to AJO-M (HMSP/ICJ/0020/2011).

\section{T2P95 \\ Monetary diet cost is positively associated with body composition and obesity among school-aged children in South West China}

Zhang, J. ${ }^{1}$; Xue, H. ${ }^{1}$; Yin, J. ${ }^{\text {; }}$ Li, D. ${ }^{1}$; Chen, Y. ${ }^{1}$; Cheng, G. ${ }^{1}$

${ }^{1}$ West China School of Public Health, Sichuan University, Chengdu, P.R. China

Introduction: Previous studies revealed that diet cost may contribute to childhood obesity. However, pertinent studies remain scarce in China. Our aim was to investigate whether diet cost affects body composition and obesity among school-aged children.

Methods: A cross-sectional study was conducted in 1,605 Chinese Children (48.4\% girls) aged 7-15 years. Dietary data was assessed using 3-d $24 \mathrm{~h}$ dietary recalls. Height, weight, waist circumference and skinfold thickness were measured and body mass index standard deviation score (BMISDS), waist-to-height ratio (WHtR), fat mass index (FMI) and fatfree mass index (FFMI) were derived. Diet costs were calculated by multiplying the amount of consumed food (g/day) by price of each food and summing the products. All food prices were adjusted for preparation and waste using China Food Composition Table. Asdiet cost was observed 
strongly associated with energy intake $(r=0.69)$, diet cost per $1,000 \mathrm{kcal}$ per day was calculated. Multivariate regression models were used to explore the relevance of diet cost on body composition and obesity.

Results: Energy-adjusted diet cost showed a positive association with FMI (ptrend $=0.01)$, BMISDS (ptrend $=0.002)$ and WHtR (ptrend $=0.02)$ after adjusted for potential confounders (age, gender, energy, physical activity, birth weight, family location, parental educational, parental BMI and family income). The association remained significant after further adjusted for diet quality (FMI: ptrend $=0.01$, BMISDS: ptrend $=0.01$, WHtR: ptrend $=0.03$ ). No association was observed between energy-adjusted diet cost with FFMI (ptrend=0.2).

Conclusion: Energy-adjusted diet cost was independently positively associated with FMI, BMISDS and WHtR, in a group of school-aged children in South West China.

Conflict of Interest: The authors have no financial relationships relevant to this article to disclose.

Funding: All phases of this study were supported by research grant from the Sichuan Outstanding Young Scholars Foundation (2014JQ0005)

\section{T2P97}

Neuro-olfactory reprogramming - RNO - a new approach in the treatment of eating behaviors

Pinelli, N. ${ }^{1}$;

${ }^{1}$ indépendant researcher

This clinical research project aims to demonstrate the effectiveness of a multidisciplinary protocol. This protocol combines two disciplines: olfactotherapy and the sciences of education. New morphostatic and staturo-weight measurements (Ospamad) and a new learning method called Neuro-Olfactive Reprogramming (RNO) were created for the purpose of this study. This RNO proposes workshops that are part of a therapeutic education process and could interest the various CSO (specialized obesity centers) in the world.

Objectives: to demonstrate the effectiveness of this protocol and therefore the usefulness of olfactotherapy (aerial diffusion of essential oils), this new RNO-Ospamad approach in the diagnosis and The treatment of overweight and obesity, change the eating behaviors

Medium-term objectives: transfer of this methodology to help some psychological, cognitive-behavioral, memory disorders, neurological rehabilitation and extend it to all sectors of health and well-being in general.

Principle of this research: RNO uses the effects of emotional memory olfactory.

Type of research: biomedical research, randomized controlled study with clinical trials, quantitative evaluation and statistical analysis of data. RNO Workshops

- Neuro-olfactory tests

- Neuro-olfactory education or rehabilitation

- Learning Assessment

Products: non-toxic and organics essential oils for aerial diffusion and inhalation.

Expected Results: Significant reduction in obesity or overweight as demonstrated by a change in the staturo-weight and news morphostatic parameters and identification of useful essential oils.

\section{T2P100 \\ Nutritional knowledge, information sources and health beliefs: Data to improve strategies in obesity prevention}

\section{Melo, A. ; Andrade, G. ${ }^{2}$; Mendes, L. ${ }^{2}$}

${ }^{1}$ Faculdade de Medicina da Universidade de Lisboa, ${ }^{2}$ Escola Superior De Tecnologia Da Saúde De Lisboa

Introduction: Nutrition is considered as the most important modifiable determinant of chronic diseases, such as obesity. Associations between higher levels of nutrition knowledge, healthier food habits and less prev- alence of obesity have been previously established. This study aims to characterize the association between nutritional knowledge, information research about food and nutrition (frequency and information sources), health beliefs and sociodemographic variables.

Methods: Analytical, observational and cross-sectional study. Data were collected through an online questionnaire submitted to a panel of experts and a pre-test. Inclusion criteria to answer the questionnaire were established: individuals with a minimum age of 18 years, residing in continental Portugal or islands, with Internet access, and with non health related training and/or profession. The sample included 818 individuals. Nutritional knowledge was assessed through the brief version of Nutritional Knowledge Questionnaire, validated for the Portuguese population. The perception of health status was assessed through the first question of the Short Form Health Survey 36-Item (SF-36). Perceived susceptibility was about diseases that are most related to unhealthy eating habits, such as obesity. $P \leq 0.05$ was considered statistically significant.

Results: Research frequency was higher in female individuals $(P<0.001)$. Internet was the preferred source of information which main users were women $(P=0.004)$, younger individuals $(P<0.001)$ and individuals with fewer children $(P<0.001)$. Books' utilization was more common in women $(P<0.001)$ and older individuals $(P<0.001)$. Individuals with higher levels of perceived susceptibility to obesity used more frequently nutrition services $(P<0.001)$ and doctor's appointments $(P<0.001)$ to get more information about food and nutrition. Nutritional knowledge was positively associated with Internet $(P<0.001)$ and books' utilization $(P=0.014)$ and negatively associated with the frequency of use of TV programs $(P=0.032)$.

Conclusion: The prominent utilization of the Internet, besides the positive association with nutrition knowledge, makes it an important information source. Furthermore, knowing the relationship between information research, sociodemographic variables and health beliefs is a useful tool to improve nutritional information disclosure in the prevention of obesity.

\section{Conflict of Interest: None Disclosed}

Funding: No Funding

\section{T2P101 \\ Obesity and a sense of coherence, exploring integrative perspectives to expand therapeutic resources}

\section{Paola, Z. ; Abel, O. '; Maximo, R. ${ }^{1}$}

${ }^{1}$ Maximo Ravennna therapeutic Centre

Introduction: Eating habits and sedentary lifestyle are the main determinants of overweight. Despite modifiable behavior, statistics continue to growing in a pandemic way. It is necessary to expand perspectives that enable more therapeutic resources to interrupt this type of behavior. In this sense, emerged in our interdisciplinary team the idea of applying the Sense of Coherence (SOC) as a multidimensional approach variable that we began to implement a year ago. The SOC is an instrument that measures how aligned is the domains of cognition, motivation and behavior in subjects. Achieving alignment of the three domains entails self-knowledge and balance, both of which are health-generating assets. In this research we proposed to evaluate the SOC in its three domains to explore the different moments of a comprehensive treatment for obesity.

Objective: To evaluate if there is any association between SOC and the weight loss of patients in comprehensive therapy.

Method: Observational cross-sectional design. SOC validated surveys were carried out on patients who requested treatment at the Comprehensive Care Center for obesity. The survey was carried out during 9 months, both at the time of admission and also at the end of the therapeutic process. The results of each SOC domain with each variable between the two moments were analyzed and compared.

Results: During the period March-November 2016, 437 surveys were carried out on incoming patients, $76 \%$ women (mean age 43 (SD 14.0), mean BMI 31.7 (SD 6.85), mean SOC: 60 (SD 12.3), and 24\% men (mean age 45 (SD 13.1), mean BMI 37.3 (SD 7.21), and SOC 65 (SD 13.0)). 32\% of 
the participants completed their active decrease in the clinic, of which 78 (42\%) were randomly selected on average of SOC 62 (SD 10.8).

Although there were no statistically significant differences in the mean SOC, a strong association was observed between the BMI drop points in each of the 3 SOC domains. From the manageability domain: the patients with maximum score, ie strong SOC, presented at the same time an average of 10.77 points of decrease of BMI; SOC Moderate 9.61 and mild SOC with 5.55. For the domain of significance: 10.79 for strong SOC; 9.92 for a moderate SOC 8.40 for a mild SOC. For comprehensibility: 10.41 for strong SOC, 9.96 for moderate SOC, and 8.20 for mild SOC.

Conclusion: The greatest strength of weight loss is observed in patients with strong and moderate SOC, so work on coherence as a therapeutic goal could be considered.

Bibliographic: The structure and properties of the sense of coherence scale.Antonovsky,A. 1993 Mar; 36(6):725-33. Sense of Coherence, hardy personality, self-esteem and health.Moreno,b.; Alonso,m.;Alvarez,e.. Revista de Psicología de la Salud, 13, 2,185-207pp.

Conflict of Interest and Funding: It is important to mention for the analysis of implications that the researcher belongs to the institution in which it is investigated, this being the same one that funds the investigation. Private institution that performs activities of private order (individual attention in medical and nutritional clinics and therapeutic groups) and also public order activities (such as collaboration with public canteens, schools, physical activity open to the community in parks)

T2P103

Obesity and inflammatory biomarkers as predictors of metabolic syndrome in Brazilian older adults living in the community dwelling: Health, wellbeing and aging (SABE) study

Roediger, M. ${ }^{1}$; Marucci, M. ${ }^{1}$; Duim, E. ${ }^{2}$; Santos, J. ${ }^{3}$; Lebrão, Y. ${ }^{4}$; Lebrão, M. ${ }^{5}$

${ }^{1}$ Department of Nutrition, School of Public Health, University of São Paulo/ Brazil, ${ }^{2}$ Phd Student In Epidemiology, Public Health School, University Of São Paulo, São Paulo, Brazil, ${ }^{3}$ Medical School Of Ribeirão Preto, University Of São Paulo / Brazil Department Of Nursing Of School Of Nursing, University Of São Paulo, São Paulo, Brazil., ${ }^{4}$ Department Of Nursing Of School Of Nursing, University Of São Paulo, São Paulo, Brazil., ${ }^{5}$ Department Of Epidemiology Of Faculty Of Public Health, University Of São Paulo, São Paulo, Brazil

Introduction: Metabolic syndrome (MetS) is related with chronic diseases that can affect the health conditions of older adults. Researches about metabolic syndrome in this population are limited. This study verified the serum levels of inflammatory biomarkers, metabolic syndrome and factors associated in Brazilian older adults living in the community dwelling. Methods: Cross-sectional study carried out based on a population-based of Health, Wellbeing and Aging (SABE) Study realized in São Paulo, Brazil, including older adults ( $\geq 60$ years-old) in 2010 . The variables of study were: metabolic syndrome (MetS) identified according to the criteria of the National Cholesterol Education Program-Adult Treatment Panel III; socio-demographic information (gender, age, educational level, skin color and income) and health conditions (number of non-communicable diseases, self-assessment of health status, body mass index, physical exercise, sarcopenic obesity, alcohol and tobacco consumption) identified using questionnaire and specific measures; inflammatory biomarkers (uric acid, fibrinogen and C-reactive protein) using sensitive regular immunoassays (ELISA). Multiple logistic regression was used to analyze this association. Results: From the 1.344 older adults participants of SABE Study, 1155 were eligible to be part of this study, being that prevalence of MetS was $57,9 \%$. The adjusted model showed that older adults female (OR, 1.61; p-value 0.001 ), individuals of $60-69$ years (OR, 1.58 ; p-value 0.001 ), obesity (OR, 3.30 ; -value $<0.001$ ), high concentration of C-reactive protein (OR, 1.68 $\mathrm{p}$-value $<0.001)$ and uric acid $(\mathrm{OR}, 2.35$; p-value $<0.001)$ self-assessment of health status as regular or poor (OR 1.47; p-value 0.007) were more likely to have MetS.

Conclusion: Of the variables studied, obesity and elevated inflammatory biomarkers were the more prevalent conditions that predispose the pres- ence of metabolic syndrome in Brazilian older adults living in the community dwelling.

Conflict of Interest: None Disclosed

Funding: Fundação de Amparo à Pesquisa do Estado de São Paulo (FAPESP) / Brazil

\section{T2P104 \\ Obesity and smoking are jointly associated with sickness absence among midlife employees}

\author{
Roos, E. ${ }^{1}$; Lallukka, T. ${ }^{2}$; Lahelma, E. ${ }^{1}$; Rahkonen, $0 .^{1}$ \\ ${ }^{1}$ University of Helsinki, Department of Public Health, ${ }^{2}$ University Of Helsinki, \\ Department Of Public Health; Finnish Institute Of Occupational Health
}

Introduction: Obesity and smoking are some of the strongest preventable risk factors for sickness absence (SA). Unhealthy behaviours may bundle together yet their joint association have rarely been studied. The aim of this study was to examine the joint associations of obesity and smoking with short self-certified and longer, medically certified SA in a multi-occupational midlife employee cohort.

Methods: A Survey among midlife employees of the City of Helsinki, Finland, during 2000-2002 included data on obesity, smoking and covariates $(\mathrm{N}=8960$, response rate $67 \%, 80 \%$ women). These data were prospectively linked with register data on self-certified (1-3 days) and medically certified (4 days or longer) SA spells registered during the five-year follow up after responding to the survey. The linkage was performed only among those with a written consent for the linkage (78\%). Covariates used were age, socio-economic position, working conditions, alcohol consumption, leisure-time physical activity and self-rated health. Men and women were analysed separately. Poisson regression was used.

Results: Among men smoking was associated with self-certified SA (Obese non-smokers RR 1.28, 95\% CI 1.00-1.63, non-obese smokers RR 1.81, 95\% CI 1.54-2.12, obese smokers RR 1.81, 95\% CI 1.34-2.46). Among women, obesity and smoking were jointly associated with self-certified SA (Obese non-smokers RR 1.40, 95\% CI 1.28-1.53, non-obese smokers RR $1.35,95 \%$ CI 1.26-1.45, and obese smokers RR 1.81, 95\% CI 1.59-2.07). The associations attenuated but remained after adjustments for covariates. Among men obesity and smoking were jointly associated with medically certified SA (Obese non-smokers RR 1.72, 95\% CI 1.36-2.18, non-obese smokers RR 1.54, 95\% CI 1.28-1.85, and obese smokers RR 2.68, 95\% CI 2.02-3.55). Among women, the results were similar (Obese non-smokers RR 1.75, 95\% CI 1.60-1.92, non-obese smokers RR 1.58, 95\% CI 1.461.71, and obese smokers RR 2.23, 95\% CI 1.93-2.58). Again, adjustments attenuated the association somewhat but it remained.

Conclusion: Obesity and smoking are jointly associated with SA. Obese smokers have particularly high risk for SA. Support measures for prevention of obesity and smoking cessation are likely to reduce sickness absence.

Conflict of Interest: No conflicts of interest.

Funding: ER is supported by The Finnish Work Environment Fund, TL is supported by the Academy of Finland (Grant \#287488 \& 294096).

\section{T2P105}

Obesity policy and action plan - a whole of government and a whole of society approach, the Irish model?

\section{Eldin, N. '; Devlin, J. ${ }^{\top}$}

${ }^{1}$ Department of Health

Introduction: $60 \%$ of adults and $25 \%$ of children in Ireland are either overweight or obese. The levels have doubled in two decades and this is placing an increasing burden on individuals and society. Causes of overweight and obesity are multiple and complex. No single organisation working on its own can solve this; therefore, there is a need for a 'whole of Government and a Whole of Society' evidence based and sustained effort 
to halt the increase and reverse this trend. Ireland has taken this challenge by developing 'Healthy Weight for Ireland - Obesity Policy and Action Plan, 2016 - 2025'.

Processes \& Methods: 1. Literature review of scale of problem, its contributary factors and what works 2. A: Stakeholder consultation B: Health provider consultation C: Consulting children D: Bilateral meeting with other Government Departments E. Bilateral meetings with major sectors like agri-food, retailers, voluntary etc. F: Consulting international organisations (e.g. WHO, MGI) and academic and research institutes

The Policy and its structure: A Governance structure B Multi-sectoral support C Targets D The 'Ten steps Forward': 1. Embed multi-sectoral actions on obesity prevention of Government departments and public sector organisations 2. Regulate for a healthier environment 3. Secure appropriate support from the commercial sector 4 . Implement a strategic and sustained communication strategy 5. Provide leadership, engagement and coordination 6.Mobilise the health services to better prevent and address overweight and obesity 7. Develop a service model for specialist care for children and adults 8 . Acknowledge the role of physical activity in prevention 9. Allocate resources according to need 10. Develop a multi-annual research, surviellance and evaluation programme

The Action Plan: Contains 60 actions: each action has a lead responsibility, most Lead organisations have partners and each action has a timeframe work

Discussion: This is a Government policy and in spite of the fact that Department of Health takes the lead, all other Government departments have contribute to it. The Policy is funded in three different ways: 1 Through annual budgetary allocation for services .e.g. health, education, social protection, active transport, sport etc. 2 . The Government has set up a Healthy Ireland Fund to enable cross government and cross sectoral programmes to address determinants and inequalities. 3. Specific Funding for the national health services

Closing remarks: we aim to reduce levels by $5 \%$ in ten years in both adults and children and to reduce the gap between rich and poor by $10 \%$. Please have a look at http://.healthyireland.ie/wp-content/uploads/2016/09/A-Healthy-Weight-for-Ireland-Obesity-Policy-and-Action-Plan-2016-2025.pdf

T2P106

\section{Obesity, anxiety and depression in a menopause model}

Boldarine, ${ }^{1}{ }^{1}$; Pedroso, A. ; Neto, N. ' ; Dornelas, A. ${ }^{2}$; Nascimento, C. ${ }^{1}$; Oyama, L. '; Ribeiro, E. ${ }^{1}$

${ }^{1}$ Universidade Federal de São Paulo, ${ }^{2}$ Universidade Federal Do Paraná

Introduction High fat intake plays a key role in obesity, a multifactorial disease in which mood disorders may be part of the co morbidities. Menopausal women frequently present obesity accompanied by anxiety and depression symptoms. To explore the possible connections of these conditions, the present work investigated the metabolic and behavioral effects of ovariectomy and lard intake in rats.

Methods: Fifty-four 3-months-old Wistar rats were either ovariectomized (Ovx) or sham-operated (S) and fed for 3 months with either control diet (SC and OvxC) or high fat diet enriched with lard (SL and OvxL). Food intake and body weight were measured weekly. Anxious behaviors were assessed by the Elevated Plus Maze (EPM) test and depressive behaviors by the Modified Forced Swim (MFS) and Sucrose Positive Contrast (SPC) tests. Serum parameters and fat pads weight were measured. ANOVA was used for statistical analysis, followed by Tukey's test for multiple comparisons.

Results: OvxC had higher body weight gain than SC; OvxL had a higher gain than all other groups. Cumulative food (caloric) intake was higher in SL. The sum of fat pads weight was higher in SL, OvxC and OvxL than in SC, and again OvxL had the highest value. High fat groups showed higher levels of serum glucose while Ovx animals presented higher levels of triacylglycerols, regardless of diet. OvxC presented higher anxiety index in the EPM test, as compared to the other 3 groups. In the MFS test, the Ovx animals had both lower swimming and climbing and higher immobility frequencies. OvxL also had significantly decreased latency to immobility. In the SPC test, high fat animals showed lower intake of the palatable sucrose solution.

Conclusion: Obesity induced by lard intake is associated with higher propensity to develop depression, not influenced by ovarian hormones. Lard intake ameliorates the anxiety caused by ovariectomy, however it further increases obesity. The findings suggest that, although the anxiolytic effect of fat intake may induce its consumption by menopausal women, its obesogenic and depression-inducing consequences must be taken into account.

\section{Conflict of Interest: None Disclosed}

Funding: Research relating to this abstract was funded by CNPq (National Counsel of Technologival and Scientific Development)

\section{T2P107 \\ Obesity, binge eating disorder and food addiction: What do neurobiological studies tell us?}

Balodis, I. ${ }^{1}$; Laliberte, M. ${ }^{2}$; Laliberte, M. ${ }^{2}$

${ }^{1}$ McMaster University, ${ }^{2} \mathrm{St}$. Joseph'S Healthcare Hamilton

Currently, more individuals in the world are overweight than underweight. As obesity becomes one of the most serious public health problems, research is directed at identifying meaningful subgroups based on biobehavioural differences. Understanding similarities and differences between various obese subgroups has implications for identifying at-risk individuals as well as identifying therapeutic targets. Here, we review neurobiological findings in binge eating disorder (BED), a condition associated with particularly severe obesity. Although BED is the most common eating disorder, to date, only a few studies investigate obese individuals with this condition independently from non-BED obese individuals. These early neuroimaging studies in BED mostly examine the neural correlates of palatable food-cue reward. Findings demonstrate heightened neural responses in prefrontal areas to palatable food cues, but reduced responses in these same areas in response to non-food cues in BED relative to nonBED individuals. Early BED studies also show alterations in the neural correlates of inhibitory control and impaired behavioural adaptation.

We also evaluate evidence for the controversial concepts of 'food addiction' and 'eating addiction', and discuss potential unique and overlapping biobehavioural features with BED. We discuss how different forms of eating (i.e. not only bingeing) might affect reward neurocircuitry and contribute to addiction-like tendencies and obesity. Additionally, to study recovery processes, we review studies linking neuroimaging with treatment outcomes in BED. Finally, we will discuss potential biomarkers, review areas of research dearth and suggest important future research directions for the field.

Conflict of Interest: Dr. Iris Balodis and Dr. Michele Laliberte have no conflicts to disclose

Funding: Research relating to this abstract was partially supported from the Peter Boris Centre for Addictions Research at McMaster University and from the Constantine Douketis Award at the Research Institute of St. Joe's Hamilton.

\section{T2P108}

\section{Objective measures of free-living physical activity and sedentary behaviour and their association with control over eating and food preferences in women.}

Oustric, P. ; Finlayson, G. ${ }^{1}$; Myers, A. ' ; Butler, E. ${ }^{2}$; Long, C. ' ; Beaulieu, K. ${ }^{\text {' }}$;

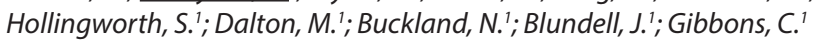

${ }^{1}$ University of Leeds, ${ }^{2}$ Endava Uk Ltd.

Background: The relationship between free-living physical activity (PA) and sedentary behaviour (SB) with control over eating (CE) and food preferences (FP) may throw light on the relationship between PA, eating behaviour and obesity. 
Objective: We examined associations between average time spent in different PA intensities (from sedentary to vigorous) on markers of CE and FP. Differences in CE and FP were also examined across quintiles of moderate-to-vigorous PA.

Methods: Cross-sectional data from 180 women were combined from six studies using identical protocols. Free-living PA and SB was continuously monitored for 5-7 days using armband activity monitors. Markers of CE (positive mood, craving control, craving for savory, craving for sweet) and FP (explicit liking, explicit wanting, and implicit wanting for fat and sweet taste) were assessed using validated tools.

Results: SB was negatively associated with positive mood and positively associated with liking and wanting for high fat relative to low fat foods. The inverse pattern of relationships was observed for moderate and vigorous PA time on FP, but not mood. These findings remained significant after adjusting for potential confounds, but the effects of SB on FP and CE were not independent from MVPA. Analysis of MVPA quintiles revealed a linear trend on FP, with highly active individuals (Q5: $197 \pm 62 \mathrm{~min} /$ day) demonstrating stronger liking and wanting for low fat foods, which was significantly different from less active individuals (Q1-3: $25 \pm 8-83 \pm 9$; $\mathrm{min} /$ day) who showed stronger liking and wanting for high fat foods. Conclusion: Objectively measured free-living PA and SB time had opposing relationships with liking and wanting for high fat foods. Additionally, SB time was related to lower mood. These findings are consistent with the strong link between physical inactivity and obesity and suggest this relationship involves dietary preferences and mood state. Engaging in large amounts of MVPA appears to coincide with a preference for low fat relative to high fat foods, which may act as an additional leverage for appetite control and healthy weight management.

Conflict of Interest: None disclosed

Funding: No Funding

\section{T2P110}

Overweight prevalence and trends in Hungarian adults: The Hungarian diet and nutritional status surveys 2009 and 2014

\section{Erdei, G. ${ }^{1}$; Bakacs, M. '; Nagy, E. '; Kovács, A.}

${ }^{1}$ National Institute of Pharmacy and Nutrition

Introduction: Obesity is an increasing concern worldwide. In order to develop targeted actions and for the monitoring of trends and the impact of interventions, valid and reliable data on obesity prevalence and its risk factors are needed. The Hungarian Diet and Nutritional Status Survey objectively measures the nutritional status, dietary habits and physical activity in Hungarian adults (age 18 y or above) in every 5 years.

Methods: A successive independent samples study is running since 2009 as part of the European Health Interview Survey providing national data that are representative for the non-institutionalized adult population by age and gender. Anthropometric measurements, 3-day dietary record and pedometer are used following a standardized protocol.

Results: In 2014, nearly two-thirds of adults were overweight or obese. $28.2 \%$ of men and $31.5 \%$ of women were obese. Prevalence of morbid obesity were $2.6 \%$ and $3.3 \%$ in men and women, respectively. Abdominal obesity was more prevalent among women than men (55\% vs. $38 \%$ ), and the rate was increasing with age in both genders. In elderly, $55 \%$ of men and almost $80 \%$ of women were abdominally obese. Prevalence data did not change since 2009.

Conclusion: The prevalence of overweight, obesity, and abdominal obesity is high in Hungarian adults, particularly in elderly. However, the rates did not rise in the last 5 years which might be explained by the introduced policy interventions.

Conflict of Interest: None Disclosed

Funding: No Funding

\section{T2P111}

\section{Paternal antenatal vitamin D intake is associated with offspring's height and weight aged five and nine years in the Lifeways cross-generation cohort study, Republic of Ireland}

\section{Mejia-Lancheros, C. ; Mehegan, J. ${ }^{1}$; Murrin, C. ; Kelleher, C.}

${ }^{1}$ UCD School of Public Health, Physiotherapy and Sports Science

Introduction: Maternal vitamin D intake during pre-pregnancy has been found to have an important role in both offspring musculoskeletal and overall health. The antenatal paternal vitamin $\mathrm{D}$ effects on progeny development areless well-known. This analysis investigated the prospective relationship between antenatal paternal Vitamin D intake and offspring height and weight.

Methods: Participants were children and fathers from the Lifeways Cohort Study. Paternal vitamin D intake from baseline food frequency questionnaire and children's height and weight values were available for 213 and 148 father-child dyads when children were aged 5 and 9 years respectively. Height $(\mathrm{cm})$ and weight $(\mathrm{kg})$ of child at both time points were the main outcomes. Father's vitamin D intake $((\mu \mathrm{g})$ reported during the offspring-first prenatal trimester was the main predictor. Paternal (age, energy intake (kcal/day), height, weight, and being the biological father), maternal (age, Vitamin D and energy intake (kcal/day), height, and weight) and children's characteristics (sex; age; vitamin D and energy intake (Kcal/ day) and summer outdoor physical activity aged five) were considered in the analyses. Multivariate linear regression was used to estimate the crude and adjusted associations.

Results: At age $5(\mathrm{~N}=213)$, the children's mean height $(\mathrm{cm})$ and weight (kg) were 112.12(SD5.17) and 20.95(SD 2.94) respectively, whilst the prenatal paternal vitamin D intake( $\mu$ g) was 3.46(SD1.94). At age $9(\mathrm{~N}=148)$, the average height $(\mathrm{cm})$ and weight $(\mathrm{kg})$ for children were 138.61(SD 7.12 ) and $34.09(\mathrm{SD} 6.79)$ respectively, whereas father's vitamin $\mathrm{D}(\mu \mathrm{g})$ intake level was 1.25(SD 0.68). The Pearson correlation coefficients between father's Vitamin D intake and offspring's anthropometric measures at 5 years were: height: $0.295(\mathrm{p}$-value: $<0.001)$ and weight: $0.197(0.004)$, whilst at 9 years were: height: $0.229(0.005)$ and weight: $0.159(0.054)$. After adjustment for paternal, maternal and child characteristics, the paternal vitamin $\mathrm{D}$ intake remained positively and statistically associated with offspring's height (Log-coefficients: 0.019(CI:0.008;0.030) and weight (Log-Coefficient: $0.074(\mathrm{CI}: 0.019 ; 0.130)$ at 5 years old; whilst these associations were attenuated and no longer statistically significant when offspring were 9 years old (height: Log-coefficients $=0.011$ (CI:-0.005;0.028), weight: Log-coefficients $=0.074(\mathrm{CI}:-0.017 ; 0.165)$. The findings remained similar when analyses were repeated with only biological fathers.

Conclusion: Paternal Vitamin D intake was positively and prospectively associated with offspring's height and weight at 5 years old, independently of maternal characteristics, meriting further investigation of familial dietary pathways.

Conflict of interest: The authors declare no conflict of interest.

\section{T2P113}

Physical activity level assessed by a self-report questionnaire and accelerometry in adolescents: A relative validation and the importance of considering non-wear time activities in accelerometry data

\section{Silva, C. ' ;ilva, A. '; Fragoso, I. '; Teles, J.'}

${ }^{1}$ Faculty of Human Kinetics, University of Lisbon

Introduction: Physical activity energy expenditure measurement in large samples, especially in children and adolescents, can be a challenge given the high cost of the most accurate

Methods: Questionnaires have been used in large-scale studies to subjectively evaluate physical activity patterns in free living conditions. Accelerometry had shown promising results recently, providing more objective information on the measurement of these kind of variables. The aim of 
this study was to provide information on the relative validity of the Questionnaire RAPIL II in estimating physical activity level of adolescents in free-living conditions, using an ActiGraph GT3X accelerometer as a comparison method. The importance of considering non-wear time activities in the accelerometer data was also analyzed.

Methods: A total of 127 adolescents (10.53 - 17.66 years) completed the Questionnaire RAPIL II with information on their daily life routines (frequency and duration of several activities), during a typical week, and wore a triaxial ActiGraph GT3X accelerometer during five consecutive days, including two days of weekend. The final variable obtained from both methods was PAL. Any periods when participants did not wear the accelerometers were self-reported in a diary, regarding type and duration of activities performed and correspondent perceived intensity. Validation process was carried using the following procedures: Bland-Altman analysis, intraclass correlation coefficient, linear-weighted kappa coefficient and paired-samples $t$-test.

Results: Bland-Altman method revealed satisfactory agreement between Methods: For the "average individuals", the mean difference between methods when considering non-wear time was between 0.05 and 0.26 METs, approximately. However, as the PAL value increases, the differences variation between methods was higher. Error analysis supported the utilization of RAPIL II on a group basis, instead of individual. RAPIL II tends to overestimate energy expenditure for low PAL levels and underestimate it for higher values. Consideration of non-wear time activities improved accelerometry data.

Conclusion:The Questionnaire RAPIL II showed satisfactory validity on the estimation of PAL on a group basis of a youth sample on free-living conditions, but not at an individual level. Questionnaire RAPIL II can be an low-cost alternative tool in large survey studies. To increase its accuracy, it is recommended its review and an inclusion of intensity level estimation of each activity.

\section{Conflict of Interest: None.}

Funding: Research relating to this abstract was funded by the Science and Technology Foundation (PTDC/DES/113156/2009).

\section{T2P114}

\section{Preconceptual body mass index as predictor for gestational diabetes}

\section{Krzizek, E. '; Herz, C. '; Brix, J. '; Ludvik, B.}

${ }^{1}$ Department of Medicine 1 and Karl Landsteiner Institute for Obesity and Metabolic Diseases, Rudolfstiftung Hospital Vienna, ${ }^{2}$ Department Of Medicine Ii, Medical University Vienna And Karl Landsteiner Institute For Obesity And Metabolic Diseases, Rudolfstiftung Hospital Vienna

Background and aims: Body mass index (BMI) is a risk factor for the development of gestational diabetes (GDM) due to insulin resistance (IR). In this study we aimed to determine a cut-off value of preconceptual BMI as indicator for subsequent IR during pregnancy which might serve as a criterion for earlier screening.

Material and Methods: 505 pregnant women (mean age 31 years [27; 35], mean preconceptual BMI $24.3 \mathrm{~kg} / \mathrm{m}^{2}$ [21.8; 28.5]) were evaluated in this cross sectional study. All patients had a 2 hour $75 \mathrm{~g}$ OGTT during $24^{\text {th }}-28^{\text {th }}$ week of gestation with measurement of glucose and insulin levels for determination of IR by Matsuda-Index and HOMA-IR.

Results: 167 (33.1\%) patients were diagnosed with GDM. 31.1\% ( $\mathrm{n}=52)$ of those showed an elevated IR (Matsuda Index: GDM 3.2 [2.2;3.6] vs. nonGDM 4.5[3.16; 6.67], p < 0.001; HOMA-IR: GDM 2.38[1.58;3.85] vs. nonGDM 1.98[1.29;2.84], p >0.001). After exclusion of potential confounders such as age, week of gestation and number of pregnancies, BMI was determined as independent risk factor for GDM ( $p=0.003)$. Furthermore, patients with $\mathrm{BMI}>26.5 \mathrm{~kg} / \mathrm{m}^{2}$ were significantly more likely to develop IR ( $\mathrm{p}<0.001$, sensitivity $66.7 \%$, specificity $79.3 \%$ ).

Conclusion: Preconceptual BMI is an independent risk factor for GDM. Patients with a $\mathrm{BMI}>26.5 \mathrm{~kg} / \mathrm{m}^{2}$ are more likely to develop IR during pregnancy and might therefore require earlier screening, which needs to be further investigated in a prospective study.

\section{T2P118 \\ Prevalence and incidence of sarcopenic obesity in a cohort of Brazilian older adults}

Crisostomo, L. ${ }^{1}$; Marucci, M. ${ }^{2}$; Roediger, M. ${ }^{1}$; Rocha, M. ${ }^{1}$; Duarte, Y. ${ }^{3}$; Lebrão, M. ${ }^{4}$; Santos, J. ${ }^{5}$

${ }^{1}$ Department of Nutrition, School of Public Health, University of São Paulo/ Brazil, ${ }^{2}$ Department Of Nutrition, School Of Public Health, University Of São Paulo / Brazil, ${ }^{3}$ Department Of Nursing Medical-Surgical School Of Nursing, University Of São Paulo/ Brazil, ${ }^{4}$ Department Of Epidemiology, School Of Public Health, University Of São Paulo/ Brazil, ${ }^{5}$ Medical School Of Ribeirão Preto, University Of São Paulo / Brazil

Introduction: Epidemiological evidence shows that sarcopenic obesity (SO) is associated with accelerated functional decline and high risk of morbidity and mortality, and its impact has become major concern of health professionals. Thus, this study aimed to estimate the prevalence and incidence of SO in the older adults cohort domiciled in São Paulo / Brazil - 2000 and 2010.

Methods: We used data from the SABE Survey (Health, Well-being and Aging), held in São Paulo in 2000 (2.143) and 2010 (795). The study population consisted of older adults ( $\geq 70$ years) of both sexes, who had all the necessary data for this study and who agreed to participate, totaling 871 older adults analyzed in 2000 and 656 older adults in 2010. The variables of study were: 1 .Dependent - sarcopenic obesity, identified according to: obesity, diagnosed by the value of the waist circumference (WC $\geq 94 \mathrm{~cm}$ and WC $\geq 80 \mathrm{~cm}$ for men and women, respectively); sarcopenia, identified by: 1-muscle strength, by handgrip test (kg) (low $\leq$ P25; normal > P25, of the same population), 2-muscle mass (MM), obtained by muscle mass index $\left(\mathrm{MMI}=\mathrm{MM} /\right.$ height $\left.^{2}\right)($ low $\leq \mathrm{P} 20$; normal $>\mathrm{P} 20$, of the same population) and 3-physical performance, identified by the test (time-dependent seconds) to sit down and get up 5 times a chair (S\&L), with their arms crossed on the chest (low $\geq$ P75; normal $<$ P75); 2. Explanatory: sex and age groups (70-79 and $\geq 80$ ). They were classified with SO older adults who presented, simultaneously, WC values adopted and low performance and low MM, or, normal performance but low muscle strength and MM. The prevalence of SO in 2000 and in 2010 was estimated by the number of the cases identified in two moments, where comparisons were made between confidence intervals, to check statistical difference in the older adults (\%) with the second explanatory variables, with a significance level of 5\%. For the calculation of the incidence coefficient, in 2010, it was considered the each individual observation time, determined specifically for each case. For the calculations we used the program: Stata / SE " 10.0 for Windows.

Results: Of the 871 analyzed older adults in $2000,85(7.4 \%)$ were diagnosed with SO [6.5\% women (CI 5.0-8.4) and $4.8 \% \geq 80$ years (CI 3,6-6.4) ] $(\mathrm{p} \leq 5 \%)$, and, in 2010 (656), 73 (9.2\%) were identified with SO [7.2\% women (CI 5.5- 9.4) and $5.3 \% \geq 80$ years (CI $4.0-7,0)](\mathrm{p} \leq 5 \%)$. In 10 years, were identified 43 new cases of SO. Incidence coefficient of SO was 15.29/1000 people/year between 2000 and 2010. Conclusion: The prevalence of SO in 2000 and 2010 was higher in women and the oldest old, and, in both cases, was higher in 2010 compared to 2000. There was no significant difference between the incidence coefficient according to the explanatory variables.

\section{T2P119}

\section{Prevalence and predictors of early gestational weight gain associated with obesity risk in a diverse antenatal population}

Sim, K. ${ }^{1}$; Berkemeier, S. ${ }^{2}$; Cheney, K. ${ }^{3}$; Gordon, A. ${ }^{3}$; Black, K. ${ }^{3}$

${ }^{1}$ The University of Sydney, ${ }^{2}$ Division Of Obstetrics And Gynaecology, Campbelltown Hospital, ${ }^{3}$ Discipline Of Obstetrics, Gynaecology And Neonatology, The University Of Sydney 
Background: Excess gestational weight gain (GWG), particularly in early pregnancy, may be detrimental to both the mother and her offspring and contribute to the intergenerational cycle of obesity. The aim of this study was to investigate the prevalence and predictors of excess GWG in early pregnancy and the women's understanding of the risks to themselves and their offspring stratified by maternal body mass index (BMI).

Methods: This was a secondary analysis $(n=2,131)$ of a cross-sectional study $(n=2,338)$ of pregnant women attending antenatal clinics at their second antenatal visit at four maternity hospitals across Sydney, Australia. An anonymous, self-completed questionnaire was used to investigate knowledge of expected weight gain in pregnancy, understanding of risks associated with excess GWG, anthropometric measures and socio-demographic data.

Results: One third $(34 \% ; n=692)$ of women in the study gained weight in excess of the recommendations. In a multivariate analysis, women who were overweight (OR: 1.69, 95\% CI: 1.33-2.14; $\mathrm{p}<0.005)$ or obese (OR: $1.64,95 \%$ CI: $1.20-2.24 ; \mathrm{p}=0.002)$ at booking visit were more likely to have excess GWG compared to normal weight women. Overweight and obese women were also more likely to estimate a higher weight than what is recommended for their BMI $\left(c^{2}=922.38\right.$; $\left.p<0.005\right)$. Low socio-economic status independently predicted excess GWG (OR: 1.89, 95\% CI $1.49-2.41 ; \mathrm{p}<0.005)$. Half $(51 \% ; \mathrm{n}=1057)$ of the women were unsure about the effect of excess GWG on their baby; $11 \%$ did not believe that excess GWG would affect the weight of the baby and $14 \%$ did not believe that excess GWG would affect longer term outcomes for their baby. Women who gained weight above the Institute of Medicine recommendations were more likely to believe that excessive GWG in pregnancy would not have any adverse future effect on health outcomes of their baby $\left(\chi^{2}=6.95\right.$; $\mathrm{p}<0.05)$ or the weight of the baby $\left(\chi^{2}=16.54 ; \mathrm{p}<0.005\right)$.

Conclusions: The women at particular risk of excess early GWG are those who are overweight and obese and/or residing in lower socio-economic areas. These women need to be targeted for appropriate counselling preconception or in early pregnancy. Given the significant adverse outcomes associated with excess GWG in early pregnancy, preconception or early pregnancy counselling with respect to GWG and intervention research regarding best approach remains a public health priority.

\section{T2P120}

\section{Prevalence of obesity and factors associated in teachers and employees of Brazilian state schools}

\section{Silva, E. '; Roediger, M. ${ }^{2}$; Goulart, R. ${ }^{2}$; Sanches, A. '; Shirassu, M. ';} Waisberg, J.'; Silva, M.

${ }^{1}$ Instituto de Assistência ao Servidor Público Estadual - HSPE/ IAMSPE- SP/Brazil, ${ }^{2}$ Faculdade De Saúde Pública -Fsp, Da Universidade De São Paulo - Usp/ Brazil

Introduction: The obesity is among the factors that contribute for development of chronic diseases, increased absence from work and high public costs generated by the medical licenses of teachers and schools employees. This study verified the prevalence of obesity and associated factors in teachers and employees.

Methods: Retrospective cross-sectional study, using data from teachers and employees of the state schools of the city of São Paulo- Brazil, with adults and the elderly $(n=23,273)$. The study variables were: body obesity; Sociodemographic and clinical conditions (diabetes mellitus, hypertension, high cholesterol, musculoskeletal disorders, emotional disorders, quality of life and sleep); Life habits (physical activity practice) and diet. The measure of the magnitude of effect was assessed by prevalence ratio (PR). Multiple logistic regression was used to verify the association between variables by the Stata / SE 13.0 program.

Results: Obesity was higher in women $(83,5 \%)$, aged 50 years and older $(57 \%)$ and is associated with diabetes mellitus $(\mathrm{PR}=2.93)$,hypertension $(\mathrm{PR}=3.12)$, musculoskeletal diseases $(\mathrm{PR}=1.56)$, sedentary lifestyle $(\mathrm{PR}=1.73)$ and poor feeding quality $(\mathrm{PR}=1.48)$.

Conclusion: Diabetes mellitus, arterial hypertension, musculoskeletal diseases, sedentary lifestyle and poor eating quality are the main causes that influence the magnitude of the prevalence of obesity among teachers and employees of schools.

Conflict of Interest: None Disclosed

Funding: No Funding

\section{T2P121 \\ Prevalence of overweight and obesity among Bulgarian university students from 2008 to 2014}

\section{Birdanova, V. ${ }^{12}$; Penkov, A. B. ${ }^{1}$; Stojnovska, M. R. ${ }^{2}$}

${ }^{1}$ Department of Hygiene, Medical Ecology and Occupational Diseases and Primary Health Department Disaster, Medical University of Pleven, Bulgaria ${ }^{2}$ Department of Hygiene, Medical Ecology and Occupational Diseases and Primary Health Department Disaster, Medical University of Pleven, Bulgaria

Introduction: Obesity is important risk factor for non-communicable diseases as type 2 diabetes, coronary heart disease, musculoskeletal disorders and some cancers. Obesity and overweight has become global health problem for all ages. In Bulgaria children and young people are target groups for prevention of chronic diseases. New studies suggest that increased body weight may affect negatively academic performance in university students.

Objectives: to investigate the prevalence of overweight and obesity among university students over 18 years old from Pleven region (Bulgaria), 2008 to $2014 \mathrm{y}$.

Material/Methods: A cross-sectional study on representative sample of medical university students in Pleven University was carried out during two academic years - 2008/2009 and 2013/2014 year. A total of 1220 subjects $(70.5 \%$ females) of mean age $21.6 \pm 3.04$ were selected. Weight, body mass index (BMI), body fat, fat free mass were measured using Tanita TBF-300 M bioelectrical impedance analysis. Height was measured by standard procedure. Overweight and obesity was defined according WHO criteria.

Results:In university male students, the prevalence of overweight was $29.1 \%$ vs $26.8 \%$ (2008/2014), but obesity increased $6.6 \%$ vs $10 \%$. Inversely, the prevalence of obesity in university women was $4.4 \%-4.6 \%$, but being overweight females magnified $-7.8 \%$ vs $10.7 \%(2008 / 2014)$. It was found significant gender differences in the prevalence of overweight and obesity $(\mathrm{p}=0.001)$. The prevalence of overweight in university men was 2.5 times more than university women. The proportion of obese men was two times higher, compared to obese women. Increased BMI $(>25)$ showed strong significant correlation with body fat for university males $(r=0.76$; $p=0.001)$ and significant moderate correlation for university females $(\mathrm{r}=0.43 ; \mathrm{p}=0.006)$.

Conclusion: The prevalence of overweight and obesity among university students from Pleven region, Bulgaria was increased between 2008 and 2014.

\section{T2P122 \\ Promotion of food security in adolescents using new technologies: Effectiveness analysis of an intervention program}

\section{Prioste, A. '; Fonseca, $\mathrm{H}_{.}^{2}$; Gregório, M. ${ }^{3}$; Canhão, $\mathrm{H}_{.}{ }^{3}$}

${ }^{1}$ Faculdade de Psicologia, Universidade de Lisboa, Portugal; Escola de Psicologia e Ciências da Vida, COPELABS, Universidade Lusófona, Portugal., ${ }^{2}$ Department Of Paediatrics, Hospital De Santa Maria, Faculdade De Medicina, Universidade De Lisboa, Portugal. Rheumatology Research Unit, Instituto De Medicina Molecular, Faculdade De Medicina, Universidade De Lisboa, Lisboa, Portugal., ${ }^{3}$ Faculdade De Ciências Médicas, Universidade Nova De Lisboa

Introduction: Food insecurity concept includes uncertainty or worry about having enough food,inadequate quality of food,lack of consistent access to adequate food and food acquired through socially unacceptable means.Considering that the first United Nation's Millennium Development Goal is to eradicate extreme poverty and hunger,the development 
of strategies to promote of food security is a global need.This study investigated the effectiveness of an intervention program which aimed to promote food security using new technologies.

Methods: We conducted a 6-month intervention with a sample of 149 adolescents $(M=12.56 ; S D=.77)$ and their families.Adolescents were evaluated using anthropometric measurements and self-reported questionnaires to assess nutritional habits and status, and psychological variables. Parents were asked to complete a measure to assess food insecurity in households.All variables were assessed at baseline and at 6-month.Primary endpoints (nutritional habits):dietary patterns,preference towards healthy foods,perceived benefits of healthy eating,fast food intake,attitudes regarding eating.Secondary endpoints:anthropometric measures(BMI,percentage of fat mass, waist circumference) and psychosocial variables(psychological distress,body image).To assess the effectiveness of the program,we compared the two groups(adolescents living in food insecure vs. food secure households) and the changes in variables between baseline and T1 by conducting multiple repeated-measures factorial ANOVAs.

Results: Significant differences were found regarding dietary patterns,preference towards healthy foods,fast food intake,BMI z-score,percentage of fat mass, waist circumference and body dissatisfaction.Regarding nutritional habits, for both groups, the mean score of preference toward healthy foods at baseline was lower than the mean score at T1.Regarding the nutritional status, the results showed that, at baseline and T1,the mean BMI $\mathrm{z}$-score for food insecure group was higher than the food secure group mean score.For both groups, the waist circumference and the percentage of fat mass mean scores at baseline were lower than at T1.

Conclusion: These findings need to be interpreted taking into account the age of the participants which is characterized by the growth spurt and the occurrence of important changes at the level of the body compartments. The lack of significant differences regarding the endpoints might be related to the reduced time of intervention.For a better understanding of the power of this program, these findings should be examined in a long-run follow-up.

\section{Conflict of Interest: None Disclosed.}

Funding: Research relating to this abstract was funded by the Public Health Initiatives Programme (PT06), financed by EEA Grants Financial Mechanism 2009-2014.

\section{T2P123 \\ Provegetarian dietary pattern and the incidence of obesity in the Seguimiento Universidad de Navarra (SUN) cohort}

Sanz-Serrano, J. '; Gea, A. ; Martínez-González, M. ' ; Martín-Calvo, N. '; Ruiz-Canela, M. '; Bes-Rastrollo, M. ${ }^{1}$

${ }^{1}$ University of Navarra

Introduction: Plant-based or also called provegetarian diets have been recommended to both prevent and treat obesity. Obesity is the largest chronic health problem in adults and one of the leading causes of disability and death worldwide. Etiology of obesity is complex and multifactorial. We examined the association of the adherence to a provegetarian diet and the incidence of obesity (BMI>30) in a prospective Mediterranean cohort. Methods: We included 16,181 participants from the SUN cohort (19992016) without obesity, chronic diseases or pregnancy at baseline. Dietary data were assessed at baseline using a semi-quantitative food frequency questionnaire previously validated. Using these data, an overall provegetarian diet index (PVI) was created, where plant foods (vegetables, fruits, grains, nuts, olive oil, legumes and potatoes) received positive scores, while animal foods (animal fats, dairy, eggs, fish/seafood, meat) received reverse scores. The cohort was categorized into quintiles of PVI. We documented 584 incident obesity cases during a median follow-up of 10.3 years. Generalized estimating equations were fitted to assess the association between adherence to the PVI and the incidence of obesity adjusting for potential confounders.

Results: Each quintile of PVI was analyzed compared to the lowest quintile (Q1-lowest provegetarian adherence). PVI was inversely associated with obesity incidence when adjusted for sex and age. The association of obesity with provegetarian quintiles was attenuated when we additionally adjusted for alcohol intake, body mass index (BMI), family history of obesity, snacking between meals, smoking habit, sleeping hours, and physical activity (Q2: Odds Ratio $0.94,95 \%$ CI 0.74-1.19; Q3: Odds Ratio 0.85, 95\% CI 0.67-1.08; Q4: Odds Ratio 0.83, 95\% CI 0.63-1.08; Q5: Odds Ratio $0.57,95 \%$ CI $0.42-0.78$; $p$ for trend $<0.001$ ). Limitations of the study include self-reported diet assessment, with the possibility of measurement error, exposure only at baseline, and the potential for residual or unmeasured confounding given the observational nature of the study design.

Conclusions: Our study suggests that plant-based diets are associated with substantially lower risk of developing obesity. This supports current recommendations to shift to diets rich in plant foods, with lower intake of animal foods.

Conflict of Interest: The authors declare no conflicts of interest

Funding: The SUN Project has received Funding from the Spanish Government-Instituto de Salud Carlos III, and the European Regional Development Fund (FEDER) (RD 06/0045, CIBER-OBN, Grants PI10/02658, PI10/02293, PI13/00615, PI14/01668, PI14/01798, PI14/01764, and G03/140), the Navarra Regional Government $(45 / 2011,122 / 2014)$, and the University of Navarra

\section{T2P124 \\ Psycho- social correlates among obese patients in a tertiary health care centre in India}

Yoosuf, S.';

${ }^{1}$ All India Institute of Medical Sciences

Introduction: Obese individuals may have various psychosocial co-morbidities which are often undiagnosed, and may impact management of obesity. Aim of this study was to find the magnitude of psychiatric co-morbidity among obese patients and to assess their quality of life and the prevailing perceptions regarding obesity.

Methods: This was an a cross sectional study. We included patients with a BMI of $\geq 25.0 \mathrm{~kg} / \mathrm{m}^{2}$, aged 18-65 years. MINI 7.0 questionnaire was applied to screen patients for psychiatric co-morbidity, and psychosocial factors were assessed using a semi-structured questionnaire. WHO QolBref was used to calculate quality of life scores.

Results: A total of 151 patients who were ultimately included in the study, belonged predominantly to middle and upper lower socio-economic classes, and $69.0 \%$ among them were female. About $24.5 \%$ of patients (CI:10.3\%-26.7\%) were found to have a lifetime diagnosis of psychiatric illness, and $89.7 \%$ among these were female. The disorders found included panic disorder, major depressive disorder, suicidality, and generalised anxiety disorder. Female sex was found to be associated with increased incidence of psychiatric comorbidity ( odds ratio: 4.99, 95\% CI: 1.65 15.07). The physical domain of quality of life had significant negative correlation with BMI $(\mathrm{p}<0.001)$. Also, $15.9 \%$ of the obese subjects felt that they were thin, and $21.9 \%$ felt that they were the correct weight. None of the patients who thought of themselves as thin had perceived weight based discrimination or other social issues. Among those who had perceived weight based discrimination, $61.0 \%$ felt that they were obese, while the rest felt that they were either the correct weight or slightly overweight.. Conclusions: Various misperceptions and lack of awareness about obesity may act as a deterrent in reversing the trend of rising obesity. Presence of undiagnosed psychiatric co-morbidities is an important factor that can add to the disease burden in obese patients.

Conflict of interests of authors: none 
T2P125

\section{Psychometric properties of the Repetitive Eating Questionnaire (Rep(eat)-q): A self-report measure for grazing}

Conceição E. '; Simões J. '; de Lourdes $M^{1}$; Ramalho S. '; Pinto-Bastos $A^{1}$; Isabel Brandão' ${ }^{2}$ Vaz AR'; Machado PP'; James E. Mitchell ${ }^{3}$

${ }^{1}$ University of Minho, School of Psychology, Portugal, ${ }^{2}$ Faculty Of Medicine, Hospital São João, University Of Porto, ${ }^{3}$ Neuropsychiatric Research Institute, Fargo, Usa

Grazing, an eating behavior associated with obesity and weight regain, has recently been defined as the repetitive eating of small/modest amounts of food in an unplanned manner and/or not in response to hunger/satiety sensations. Two subtypes were considered: compulsive and non-compulsive. The Repetitive Eating Questionnaire (Rep(eat)-Q) is a self-report measure developed base on this proposed definition. This paper presents the psychometric properties of Rep(eat)-Q.

Participants were 1223 community individuals (70.3\% female; $14-72$ y.o) Assessment included: Rep(eat)-Q, Eating Disorder Examination Questionnaire (EDE-Q), and the Depression Anxiety Stress Scale (EADS) and Impulsive Behavior Scale (Negative Urgency Subscale) (UPPS-NU). For test-retest validity 405 participants filled in Rep(eat)-Q one week after their first assessment.

Rep(eat)-Q scores were significantly higher for women $(\mathrm{t}=3.88, p<.001)$, younger participants $(\mathrm{F}=18,62, p<.001)$, and higher BMI $(\mathrm{F}=7.11, p<.001)$. Rep(eat)-Q showed good internal consistency $(\alpha=93)$, teste-retest reliability $(r=.68)$ and convergent validity with significant correlations with EADS $(r=.34, p<.000)$, EDE-Q $(r=.48$ $p<.000)$ and UPPS scores $(r=.32, p<.000)$. Factor structure revealed two factor supporting a compulsive and a non-compulsive subtype.

Rep(eat) presented good psychometric properties. Research is still needed with clinical samples, in particularly, post-bariatric patients, to test cutoff points associated with risk for poorer outcomes.

T2P127

Quality and quantity of carbohydrate affects the composition of erythrocyte fatty acid membrane in overweight and obese subjects

Giardina, S. ${ }^{1}$; Sala-Vila, A. ${ }^{2}$; Alonso, P. ; Calvo, C. ${ }^{2}$; Sala-Salvadó, J. ${ }^{4}$; Bulló, M. ${ }^{4}$

${ }^{1}$ Human Nutrition Unit URV Reus Spain, ${ }^{2}$ Lipid Clinic Idibaps Barcelona Spain, ${ }^{3}$ Human Nutrition Unit Urv Reus Spain, lispv Reus Spain, ${ }^{4}$ Human Nutrition Unit Urv Reus Spain, lispv Reus Spain, Ciberobn-Isciii Madrid Spain

Background: Cell membrane fatty acid (FA) composition may play a role in obesity and other metabolic diseases. However, the modulatory effect of diet is poorly explored.

Objective: To investigate the effect of moderate-carbohydrate diets with different glycemic index (GI) and a low-fat diet (LF) on red blood cell (RBC) FA membrane composition.

Design: The RBC FA profile was measured in 87 subjects from the GLYNDIET study. Participants were randomly assigned to one of the following energy-restricted diet for 6 months: moderate-carbohydrate/low-GI diet (LGI), moderate-carbohydrate/ high-GI diet (HGI) or LF-diet.

Results: We observed a significant increase in C20:0 and decrease in C20:3n-6 in the LGI and HGI groups compared to LF group. Compared to LF-diet, C22:4n-6 was lower after the HGI while C22:6n-3 was higher after LGI diet. Also, a tendency was found for higher concentrations of long-chain omega-3 polyunsaturated fatty acids (LCn3PUFA) in LG compared to HGI and LF groups. The intra-group analysis showed significantly increased levels of total monounsaturated FA (MUFA) after LG and HGI interventions, as well as a significant increase in C22:5n-6 and a decrease in LCn3PUFA and omega-3-index after the LF diet. The decrease in C20:5n-3 after HGI and LF diets was also significant.

Conclusion: Diets with a moderate amount of carbohydrates and healthy fat, mainly with LGI, modify the RBC fatty acid membrane composition.
Conflict of Interest: The authors do not report any conflict of interest

Funding: Supported by the Institut d'Investigació Sanitaria Pere Virgili (PV11059S) and the Fondo de Investigación Sanitaria (PI12/0153), Instituto de Salut Carlos III

T2P128

Relation among emotional eating, chocolate craving and orthorexic behaviours in underweight, overweight and obese adults: A preliminary study

\section{Brytek-Matera, A. '; Czepczor, K. ${ }^{2}$}

${ }^{1}$ SWPS University of Social Sciences and Humanities, Katowice Faculty of Psychology, ${ }^{2}$ Interdisciplinary Doctoral Studies At The Swps University Of Social Sciences And Humanities, WrocłAw Faculty Of Psychology

Introduction: Research suggest that eating behaviours and eating patterns are related to abnormal weight and Body Mass Index. Furthermore, both men and women experience chocolate craving and have tendency to orthorexic behaviours and emotional eating.

The present study aimed to examine the relationship between emotional eating and attitudes to chocolate and orthorexic behaviours and to assess emotional eating as mediator between orthorexic behaviours and chocolate craving.

Methods: The study comprised of 159 individuals with varied BMI categories: underweight $(N=36)$, overweight $(N=66)$ and obesity $(N=57)$ We used the ORTO-15 test, the Attitudes to Chocolate Questionnaire and the Three-Factor Eating Questionnaire-R18.

Results: In underweight individuals high emotional eating was related to chocolate craving, negative feelings after eating chocolate and dissatisfaction with weight and body image as well as orthorexic behaviours. In individuals with obesity high emotional eating was associated with chocolate craving, chocolate consumption under emotional stress, guilt after eating chocolate and orthorexic behaviours. Furthermore, we found a positive relationship between chocolate craving and orthorexic behaviours.

In underweight individuals orthorexic behaviours emerged as a predictor of emotional eating.

In overweight individuals as well as individuals with obesity orthorexic behaviours and chocolate craving were found to be predictive factors of emotional eating

Emotional eating turned out to be the mediator of the relationship between chocolate craving and orthorexic behaviours in overweight and obese individuals.

Conclusion: The relationship between emotional eating and attitudes to chocolate and orthorexic behaviours is significant in all individuals with abnormal Body Mass Index. In addition, emotional eating is important variable for connection between chocolate craving and orthorexic behaviours in group with overweight and obesity.

Conflict of Interest: None Disclosed

Funding: Research relating to this abstract was funded by a grant from the SWPS University of Social Sciences and Humanities, Katowice Faculty of Psychology

\section{T2P129 \\ Relation between body mass index and periodic eating compulsion}

Pereira, F. ${ }^{1}$; Pereira, A. $^{2}$; Veiga-Branco, M. $^{3}$

${ }^{1}$ Faculdade de Medicina da Universidade de Lisboa, PAIDEA, ${ }^{2}$ Instituto Politécnico De Bragança, ${ }^{3}$ Instituto Politécnico De Bragança, Reci, Paideia

Introduction: Periodic Eating Compulsion (PEC) is most frequently associated to obese and overweight subjects. However, studies indicate that subjects with a normal weight also show PEC episodes.

Methods: A quantitative exploratory study was designed with the purpose to know the ratio between Body Mass Index (BMI) and PEC, involving a sample of 306 people of both genders with different BMI categories $(0.3 \%$ of the sample was underweight, $21.9 \%$ had normal weight, $23.5 \%$ were 
pre-obese, $31.4 \%$ had Grade I obesity, $14.1 \%$ had Grade II obesity and $8.8 \%$ had Grade III obesity). Data was collected by means of the Periodic Eating Compulsion Scale (PCES) at 4 hospitals in the North and Centre regions of the country.

Results: In the overall sample, 219 subjects (71.6\%) were found to have no PEC, 49 subjects (16.0\%) had moderate PEC and a further 38 subject $(12.4 \%)$ had severe PEC. In consonance with the literature in this field, the descriptive study of the study variables showed that all the BMI category groups - with the exception of a low weight patient - showed PEC behaviours.

Conclusion: It was observed that the higher the BMI, the higher the number of subjects with compulsive-type PEC, a fact considered to be pertinent for new studies in this overweight population. The results show the need for nutritional education and renewed vigilance in the population with PEC.

Conflict of Interest: None Disclosed

Funding: No Funding

T2P130

Relationship between body fat distribution obtained by dual-energy X-ray absorptiometry and alcohol consumption: The Fourth and Fifth Korea National Health and Nutrition Examination Survey

Kim, K. $^{1}$; Lee, K. $^{2}$

${ }^{1}$ Gachon University Gil Medical Centre, ${ }^{2}$ Dongincheon Gil Hospital

Background: Alcohol consumption is considered to induce abdominal fat deposition. However, the relationship was usually assessed with the indirect measurement such as body mass index (BMI) and waist circumference (WC).

Objectives: The aim of this study is to show the difference of body fat distribution assessed by dual-energy X-ray absorptiometry (DXA) between normal and problem drinking popuation, and the relationship between the amount of alcohol consumption and body fat distribution, in a large nationwide survey data.

Subjects: This study was based on data obtained from the Fourth and Fifth Korea National Health and Nutrition Examination Survey (KNHANES IVV). DXA measurements were performed on survey subjects over 10 years old from July 2008 through to May 2011. Of these, 18198 individuals, aged 19 years and older for whom DXA data were available, were included. Dietary intake was assessed with 24-hr recall method.

Methods: A pooled analysis combining the datasets from each year together was conducted with a revised pooled weight. For minimizing the influence of sex hormone, the population was divided into three group, men, pre- and post-menopausal women, and the analysis was performed in each group. The comparision between normal and problem drinking population, and the regression analysis were performed with survey analysis methods. Results: In men, problem drinkers had higher BMI and WC, higher whole body fat in $\mathrm{kg}$ (WBF) and trunk fat in $\mathrm{kg}$ (TF), higher trunk fat / leg fat ratio $(\mathrm{TF} / \mathrm{LF})$ and trunk fat / limb fat ratio (TF/LimbF), and lower appendicular skeletal muscle mass / body weight ratio (ASM/Wt), than norma drinkers $(\mathrm{P}<0.05)$. However, therer was no significant difference in percent body fat ( $\mathrm{pWBF}$ ) between both. In premenopausal women, problem drinkers had higher WC, WBF, TF, TF/LF, and TF/LimbF than normal drinkers $(\mathrm{P}<0.05)$, while there were no difference in BMI, pWBF and ASM/Wt between both. In postmenopausal women, there were no significant difference in BMI, WC, WBF, pWBF, TF and AFM/Wt between normal and problem drinkers. However, problem drinking postmenopausal women showed higher TF/LF and TF/LimbF than normal drinkers $(\mathrm{P}<0.05)$. In linear regression models including age and total energy intake, both of TF/LF and TF/LimbF were significantly related with the amount of weekly alcohol consumption in men, pre- and post-menopausal women groups $(\mathrm{P}<0.05)$. The relationship between TF and the amount of weekly alcohol consumption in linear regression models including age and total energy intake was significant in men and premenopausal women $(\mathrm{P}<0.05)$, not in postmenopausal women.
Conclusions: Alcohol consumption is positively related with truncal body fat accumulation in Korean.

Conflict of Interest: None Disclosed

Funding: No Funding

\section{T2P131}

\section{Relationship between obesity and atherogenic dyslipidemia}

Pérez, K. ${ }^{1}$; Parejo, M. '; Cedeño, J. '; Solé, M. ${ }^{1}$; Arnaiz, M. ${ }^{1}$; Fernández, V. ${ }^{1}$; Arrocha, M. ' ; González, N. '; Font, R. '; Sol, G. ' ; Niubó, M. ${ }^{1}$

${ }^{1}$ Centre d'Atenció Primària de Mollerussa

Objective: Determine the correlation between obesity and atherogenic dyslipidemia (AD).

Methods: Cross-sectional study.

The exhibition features a total of 5827 people over 18 years who have a BMI greater than 18.5 , from primary care consultations in a rural community. In all the selected lipid profile has been registered in the last analysis, gender and age.

The diagnosis is established by $\mathrm{AD}$ :

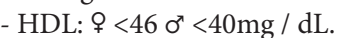

- Triglycerides: $>200 \mathrm{mg} / \mathrm{dL}$.

- One of the following:

- Total Cholesterol / cHDL: $9<4 \sigma^{\pi}<4.5$

- cLDL / cHDL: $9<2.5 \sigma^{\pi}<3$.

- CnoHDL / cHDL: $9<4$ ơ $<4.5$.

- Triglycerides / HDL: $>2$.

Results: The $44.72 \%$ are men. The average age is 53.09 [52.62 to 53.56].

Of the total sample:

The prevalence of AD in obesity is $7.6 \%$, in overweight is $4 \%$, and in normal weight is $1.9 \%(\mathrm{p}<0.000)$.

If divide according to gender:

In Men (2606): The prevalence of AD in obesity is $10.5 \%$, in overweight is $4.2 \%$, and in normal weight is $2.5 \%(\mathrm{p}<0.000)$.

In women (3221): The prevalence of $\mathrm{AD}$ in obesity is $5.7 \%$, in overweight is $3.7 \%$, and in normal weight is $1.5 \%(\mathrm{p}<0.000)$.

If divide into quartiles according to age.

$18-25$ (349): The prevalence of AD in obesity is $4.9 \%$, in overweight is 4.1 , $\%$, and in normal weight is $0 \%(\mathrm{p}=0.005)$.

$26-50$ years old (2491): The prevalence of $\mathrm{AD}$ in obesity is $8.4 \%$, in overweight is $4.4 \%$, and in normal weight is $1.5 \%$ ( $\mathrm{p}<0.000)$.

51-75 years old (2154): The prevalence of $\mathrm{AD}$ in obesity is $8.4 \%$, in overweight is $3.6 \%$, and in normal weight is $3.1 \%(\mathrm{p}<0.000)$.

$>75$ years (833): The prevalence of $\mathrm{AD}$ in obesity is $3.9 \%$, in overweight is 3.9 and in normal weight is $2.8 \%(\mathrm{p}<0.000)$.

Conclusions: There is a clear relationship between obesity and $\mathrm{AD}$, and this relationship its mantaining by gender and age. We also observed higher prevalence of $\mathrm{AD}$ in men, and in 25 to 75 subjects than others. The relationship between obesity and $\mathrm{AD}$ is also maintained in the different age groups, with the exception of patients over 75 years.

\section{T2P132}

\section{Relationship between obesity and chronic obstructive pulmonary disease}

Cedeño, J. '; Parejo, M. ' Perez, K. ' Fernández, V. '; Solé, M. ${ }^{1}$; Arnaiz, M. ${ }^{1}$; González, N. ${ }^{1}$; Arrocha, M. ${ }^{1}$; Niubó, M. ${ }^{1}$; Font, R. ${ }^{1}$

${ }^{1}$ Centre d'Atenció Primària de Mollerussa

Objective: Determine the correlation between obesity and lung disease. Methods: Cross-sectional study.

The exhibition features a total of 7540 people over 18 years who have a $\mathrm{BMI}>18.5$, from primary care in a rural community. In all selected subjects we registered if they have a diagnosis of chronic obstructive pulmonary disease (COPD), gender and age, according to the medical record. 
Results: The $46.31 \%$ are men. The average age is 49.30 [48.87-49.73]. Of the total sample:

The prevalence of COPD in the obese is $5.5 \%, 4.1 \%$ in overweight and $2.8 \%$ in normal weight subjects $(\mathrm{p}<0.000)$.

If we divide the sample by gender:

In men: The prevalence of COPD in the obese is $9.4 \%, 6.4 \%$ in overweight and $3.9 \%$ in normal weight subjects $(\mathrm{p}<0.000)$.

Women: the prevalence of COPD is $3 \%$ in the obese, $1.5 \%$ in overweight and $2 \%$ in normal weight subjects $(\mathrm{p}<0.042)$.

If perform the same calculations dividing the sample by age quartiles, we not found significant differences.

Conclusions: The obesity has a significant association with COPD, independently of gender and age. In men this association is maintained. However in women we observe significant differences between groups of BMI, but there is less overweight than normal weight in COPD patients, which may attribute perhaps to estrogenic component of this group. We don't observe remarkable relationship between these two variables in different age groups, possibly by decreasing the potency of the sample because divided in quartiles of age.

\section{T2P133}

\section{Relationship between obesity and mood disorder: Anxiety and depression}

Parejo, M. '; Cedeño, J. '; Pérez, K. '; Arnaiz, M. '; Fernández, V. '; Solé, M. ${ }^{1}$; Arrocha, M. ${ }^{1}$; González, N. ${ }^{\text {; }}$ Font, R. ${ }^{1}$; Niubó, M. ${ }^{1}$

${ }^{1}$ Centre d'Atenció Primaria de Mollerussa

Introduction: The relationship between obesity and mental disorders is unclear, some studies suggest that the obese have fewer mental health, while others talk about obesity as a protective factor against mental disorders such as anxiety and depression. Furthermore, this relationship seems to be different according to sex and/or ethnic group.

Objective: Determine the correlation between obesity and depression and anxiety.

Methods: Cross-sectional study.

4706 people over 18 years who have a BMI between 18.5 to 25 or greater than 30 inquiries from primary care in a rural community. In all selected registered if they have a diagnosis of depression and anxiety, sex and age, according to computerized medical records

Results: $42.5 \%$ are men. The average age is 47.05 [46.5-47.6].

Of the total sample:

The prevalence of anxiety in obese is $21.3 \%$, and in the normal weight is $19.8 \%(\mathrm{p}=0.227)$.

The prevalence of depression in obese is $20.5 \%$ and $10.3 \%$ in the normal weight $(\mathrm{p}<0.000)$.

By gender:

Men: The prevalence of anxiety in obese is $13.7 \%$, and in the normal weight is $14.3 \%(\mathrm{p}=0.713)$. The prevalence of depression in obese is $10.4 \%$ and $5 \%$ in the normal weight $(\mathrm{p}<0.000)$.

Women: the prevalence of anxiety in obese is $26.4 \%$, and in the normal weight is $24.1 \%(\mathrm{p}=0.195)$. The prevalence of depression in obese is $27.2 \%$ and $14.5 \%$ in the normal weight $(\mathrm{p}<0.000)$.

Age quartiles:

$18-25$ years old (631): The prevalence of anxiety in obese is $11.3 \%$, and in the normal weight is $14.5 \%(\mathrm{p}=0.466)$. The prevalence of depression in obese is $7 \%$ and $1.6 \%$ in the normal weight $(\mathrm{p}=0.03)$.

$26-50$ years old (2282): The prevalence of anxiety in obese is $23.3 \%$, and in the normal weight is $19.2 \%(\mathrm{p}=0.025)$. The prevalence of depression in obese is $13.8 \%$ and $8.6 \%$ in the normal weight $(\mathrm{p}<0.000)$.

$51-75$ years old (1325): The prevalence of anxiety in obese is $21.3 \%$, and in the normal weight is $26 \%(\mathrm{p}=0.044)$. The prevalence of depression in obese is $25 \%$ in normal weight and $17.3 \%$ ( $\mathrm{p}=0.001)$.

$>75$ years (468): The prevalence of anxiety in obese is $18.5 \%$, and in the normal weight is $18.6 \%(\mathrm{p}=0.766)$. The prevalence of depression in obese is $31.5 \%$ and $23.5 \%$ in the normal weight $(\mathrm{p}=0.052)$.
Conclusions: Note that obesity significantly associated with depression, meaning that most obese patients suffer depression. This relationship is maintained regardless of sex and age, with the exception of patients over 75 years.

As for obesity and anxiety, usually show no significant association. But it's observed in the sample divided by age.

\section{T2P134 \\ Representative nutritional knowledge study in Hungary including the factors influencing obesity}

Antal, E. ;

${ }^{1}$ TET Platform Association

Introduction: The prevalence of obesity is growing continuously worldwide, even in Hungary. The overall prevalence rate of overweight and obesity among men is $40 \%$ and $32 \%$, respectively, while overweight and obesity occurred in $32 \%$ of women. The data were collected by a measuring process. (1) Our goal was with this study to collect data among the Hungarian adults regarding their nutritional knowledge, believes, and misbelieves, food safety, labelling,healthy lifestyle and other habits including parameters influencing obesity.

Methods: A representative study with a questionnaire for 1,019 participants in Hungary (CAPI $=569$ and CAWI $=450)$. The sample was representative for Hungarian population in terms of age (15-69), sex, region and settlement type. The study was carried out between June and July in 2016.

Results: Three quarter of the respondents feels informed about healthy diet $(62 \%)$. Barriers to a healthier lifestyle are: money $(66 \%)$, willpower $(39 \%)$, healthier products $(36 \%)$. Half of the respondents are unaware of the recommended daily energy intake (48\%) (and also their own actual intake $(57 \%)$. The majority of the respondents are overweight $(33 \%)$ or obese $(20 \%)$ by BMI according to the self-reported data collection process. However, $62 \%$ thought they have problem with their weight. Interestingly, It should be noted, that $35 \%$ of the population measured their body weight more than 1 year earlier.

Conclusion:Proper nutrition and physical activity are fundamental to achieve or maintain good health. However, the average consumer is often reluctant to give up on good (familiar) taste, alcohol and convenience. Low physical activity level is general. Moreover, many of them are not able (or willing to) spend more money on food. Obesity means a serious medical, public health and economic problem, and it requires higher public awareness and political support.

References: 1,Rurik I, Ungvári T et al: Obese Hungary. Trend and prevalence of overweight and obesity in Hungary, 2015. Orv Hetil. 2016 Jul;157(31):1248-55.

Conflict of Interest: None Disclosed

Funding: No Funding

\section{T2P135}

Restrained eating, guilt and food and weight preoccupation: Analysis of mediation and moderation in a sample of obese, overweight and normal weight adults.

\section{Czepczor, K. ${ }^{1}$; Brytek-Matera, A. ${ }^{2}$}

${ }^{1}$ Interdisciplinary Doctoral Studies at the SWPS University of Social Sciences and Humanities, Wrocław Faculty of Psychology, ${ }^{2}$ Swps University Of Social Sciences And Humanities, Katowice Faculty Of Psychology

Introduction: Subsequent to eating forbidden food (e.g. chocolate), people feel fear, shame and guilt. In order to reduce guilt they introduce restriction on food. Additionally, research shows that food and weight preoccupation may be important element in the relationship between negative emotions after eating chocolate and restrictive eating. 
The aim of the study was to evaluate whether food and weight preoccupation mediated and moderated the effect of guilt after eating chocolate on restrained eating.

Methods: The research sample consisted of 57 participants with obesity, 66 participants with overweight and 236 individuals with normal weight. The mean age was 34.16 years $(S D=12.41)$ in obese adults, 30.47 years $(S D=12.22)$ in overweight adults, and 24.74 years $(S D=7.71)$ in normal weight adults. All participants completed the Three-Factor Eating Questionnaire, the Body Image Avoidance Questionnaire and the Attitudes to Chocolate Questionnaire.

Results: Only among overweight and obese adults food and weight preoccupation completely mediated the relationship between guilt after eating chocolate and restrained eating. In a sample of normal weight adults food and weight preoccupation partially mediated the relationship between guilt and restrained eating.

Moreover, only among participants with normal body weight food and weight preoccupation was a significant moderator in relationship between guilt and restrained eating.

Conclusion: It seems necessary to draw attention to food and weight preoccupation not only among individuals with obesity but also among overweight and normal weight adults.

\section{Conflict of Interest: None Disclosed}

Funding: Research relating to this abstract was funded by the SWPS University of Social Sciences and Humanities, Katowice Faculty of Psychology.

\section{T2P136}

\section{Rethinking obesity and depression}

Brito, M. ${ }^{1}$; Carmo, I. ${ }^{2}$; Bugalho, M. ${ }^{3}$

${ }^{1}$ Hospital de Santa Maria Lisboa - Serviço de Endocrinologia, ${ }^{2}$ Earlier Director In Serviço De Endocrinologia Hospital De Santa Maria, Lisbon, ${ }^{3}$ Hospital De Santa Maria Lisboa-Serviço De Endocrinologia

Introduction - It is well known that depression and obesity are causally related in both senses: depression can contribute to obesity (eating more and more to fill the empty space at orgin of depressioh) and obesity can declench depression (poor bdy-image affects self esteem), It is also known tat ending obesity through bariatric surgery can cause depression and suicide,

Method - In a given population of morbid obese and obese with comorbities, all of them pacients of the author, how are both depression (applying the Hopkins Symptom Distress Checklist SCL-90-R to evaluate it) and obesity correlated to each other and tothe eternal factors sex, BMI, and age? the results are shown in 6 graphics that are exhibited and explained in the poster.

Results -No correlation could be found between BMI and depression, nor between age and depression.

A positive correlation was established between sex and depression: more obese women with depression than men. A qualitative analysis shows that certain items (easy crying, los of sexual interest, diminution of muscular force) are reported by women and not by men, but this seems related to cultural preconceptions.

These results and the known fact that depression and suicide may be a consequence of bariatric surgery reveal that the treatment of obesity has a psychological price that should be evaluated

\section{T2P137}

\section{Risk of type 2 diabetes mellitus in Portuguese adults}

\section{Albuquerque, $C .{ }^{1}$; Aparício, $G{ }^{1}$}

${ }^{1}$ Escola Superior de Saúde/Instituto Politécnico de Viseu

Introduction: The World Health Organization estimates that Diabetes Mellitus (DM) accounts for about 285 million people worldwide and is recognized as one of the most prevalent chronic diseases and as the fourth leading cause of death in the world, which leads us to consider it as a serious and alarming public health problem. In Portugal, $40 \%$ of the population has diabetes or intermediate hyperglycemia. Based on this epidemiological reference, the objective of this study was to evaluate the risk of developing type 2 diabetes mellitus in a group of Portuguese adults in a 10 - year period.

Material and Method: A cross-sectional, observational study was carried out using a sample of 113 Portuguese adults living in the municipality of Viseu. Mostly they were female (62.8\%), and $56.6 \%$ were over 64 years old. The research protocol included a section for the measurement of sociodemographic data and the risk assessment form for Diabetes Mellitus type 2, recommended by the Portuguese National Diabetes Program (Directorate General for Health), whose psychometric properties certify its reliability. Results: In the sample, $37.2 \%$ had a significantly high risk and $23.9 \%$ had a high risk to develop Type 2 DM, especially the female participants (26.8\%), while men, $35.7 \%$ presented moderate risk. The most prevalent metabolic risk factors were: (i) the waist circumference value, which was very high in $58.4 \%$ of the participants (in $70.4 \%$ of women); (ii) the body mass index (BMI), because globally $48.7 \%$ had pre-obesity and $35.4 \%$ obesity; (iii) and hereditary burden, since $31 \%$ reported having parents with type 2 diabetes. As protective factors it was noted that $80.5 \%$ reported never having detected high blood sugar and remain as physically active daily.

Conclusions: The results show that the prevention of diabetes is a real priority, which should go through the person empowerment and its adherence to the changes in healthy behaviors and life styles, such as balanced diet, daily physical activity and maintenance of normal weight. There are many risk factors for develop type 2 diabetes however, the single best predictor is overweight or obesity, so, those changes are considered as the key to decreasing the number of diabetics around the world.

None Disclosed

Funding: No Funding

\section{T2P138}

\section{Self-report dieting predicts changes in body composition}

Sares-Jäske, L. ${ }^{1}$; Heliövaara, M. ${ }^{1}$; Männistö, S. ${ }^{1}$; Knekt, P. ${ }^{1}$

${ }^{1}$ National Institute for Health and Welfare

Dieting has become increasingly common in parallel with the expanding obesity prevalence. However, few epidemiological studies have focused on consequences of dieting. This prospective study explores whether dieting attempts and weight changes predict changes in body composition during an 11-year follow-up.

Methods: The study was based on the representative Finnish Health 2000 Survey carried out in 2000-2001, and on its follow-up examination in 2011-2012. The study sample included 2785 men and women, aged 30-69 at baseline. Information on body mass index (BMI), fat free mass index (FFMI), fat mass index (FMI) and waist circumference (WC) was collected at health examinations.FFMI and FMI were measured with eight-polar bioimpedance devices. Information on dieting attempts, weight loss and weight gain during the previous year was collected using a questionnaire. The statistical analyses were based on a linear regression model including the confounding factors sex, age, education, marital status, leisure-time physical activity, alcohol consumption, smoking, energy intake, a diet quality index, and geographical area.

Results: At baseline a total of $32.8 \%$ of the participants were dieters. Of these, $41.0 \%$ had lost weight whereas the corresponding value in non-dieters was $13.3 \%$. The dieters were fatter than non-dieters. Adjusted for sex and age, the mean BMIs among dieters and non-dieters were $28.9 \mathrm{~kg} / \mathrm{m}^{2}$ and $25.4 \mathrm{~kg} / \mathrm{m}^{2}$, respectively. The corresponding values for WC, FFMI, and FMI were $96.8 \mathrm{~cm}$ vs. $88.2 \mathrm{~cm}, 20.2 \mathrm{~kg} / \mathrm{m}^{2}$ vs. $19.0 \mathrm{~kg} / \mathrm{m}^{2}$, and $8.9 \mathrm{~kg} /$ $\mathrm{m}^{2}$ vs $6.6 \mathrm{~kg} / \mathrm{m}^{2}$, respectively. During the 11 year long follow-up FFMI was reduced $8.6 \%$, while FMI grew $31.6 \%$. The change of FMI differed statistically significantly between dieters and non-dieters, being $2.45 \mathrm{~kg} / \mathrm{m}^{2}$ and $2.24 \mathrm{~kg} / \mathrm{m}^{2}$ respectively $(\mathrm{P}=0.008)$. There was no significant difference in FFMI change $(\mathrm{P}=0.86)$. Simultaneous study of the change in relation to 
dieting and former weight change showed a statistically significant interaction between dieting and weight change for the FMI change $(P=0.05)$ but not for the FFMI change ( $\mathrm{P}=0.71)$. The FMI growth was strongest in those who had previously lost weight being $3.01 \mathrm{~kg} / \mathrm{m}^{2}$ in dieters and 2.61 $\mathrm{kg} / \mathrm{m}^{2}$ in non-dieters, whereas those not dieting and had maintained their weight during the previous year had growth of $2.15 \mathrm{~kg} / \mathrm{m}^{2}$.

Conclusion: During the follow-up FFMI was reduced and FMI grew. The FMI growth was strongest in dieters who had lost weight during the previous year before the baseline examination, suggesting a reverse long-term effect of dieting in the general population.

Conflict of Interest: None Disclosed

Funding: Research was funded by The Finnish Cultural Foundation.

\section{T2P139}

Self-reported sleep duration and daytime napping are associated with risk of overweight/obesity: A cross-sectional study among adults in South West China

\section{Zhang, J. ${ }^{1}$; Xue, H. ${ }^{1}$; Li, D. ${ }^{1}$; Chen, M. ${ }^{\text {; }}$ Cheng, $G .{ }^{1}$}

${ }^{1}$ West China School of Public Health, Sichuan University, Chengdu, P.R. China

Introduction: The increasing prevalence of obesity has become one of the most serious threats to public health in China. Previous studies have shown that sleep duration and daytime napping might be associated with obesity and the associations vary across country and race. We aim to examine whether sleep duration and daytime napping are related to overweight/obesity among adults in South West China.

Methods: A total of 1,452 adults (65\% men) aged 20-70 years were randomly selected. Sleep duration and daytime napping were self-reported using a validated questionnaire. Sleep duration was categorized into 5 groups ( $\leq 5$ hours, $5-6$ hours, $6-8$ hours [reference], $8-9$ hours, $\geq 9$ hours). Height and weight were measured to calculate body mass index. Overweight/obesity was defined according to Working Group on Obesity in China (WGOC) criteria. Logistic regression models were used to estimate the odd ratio (OR) and 95\% confidence interval (95\% CI) of overweight/ obesity, with adjustment for age, gender, occupation, education, marriage, hypertension and diabetes.

Results: Mean sleep duration was 8.0 hours. Approximately $65.4 \%$ of subjects had daytime napping habit. After adjustment for potential confounders, OR $(95 \% \mathrm{CI})$ of overweight/obesity was $0.71(0.57-0.88)$ for nap takers compared with individuals without daytime napping habit. Short sleep duration ( $\leq 5$ hours) was significantly associated with an increased risk of overweight/obesity only among young and middle-aged adults (OR: 1.91, 95\%CI: $1.22-3.00$ ), but not in those aged 60 years and above.

Conclusion: Individuals with daytime napping habit had lower risk of overweight/obesity. Short sleep duration was associated with higher risk of overweight/obesity among young and middle-aged adults in Southwest China.

\section{T2P140}

\section{Serum levels of $25(\mathrm{OH})$ vitamin D in overweight and obese adults}

\section{Nikolova, M. ${ }^{1}$; Penkov, A. ; Boyanov, M. $^{2}$}

${ }^{1}$ Department of Hygiene, Medical Ecology and Nutrition, Medical University Sofia, ${ }^{2}$ Clinic Of Endocrinology, Department Internal Medicine, Alexandrovska University Hospital, Medical University - Sofia

Introduction: Owerweight and obesity are major factor in etiology of insufficiency or deficiency of vitamin $\mathrm{D}$ deficiency in adult populations.

Objective: To investigate the serum levels of $25(\mathrm{OH})$ vitamin D in adults with overweight and obesity in Sofia.

Method / Design: Two hundred sixty four adults with normal BMI (18.5 - $24.9 \mathrm{~kg} / \mathrm{m}^{2}$ ), overweight (BMI: $25.0-29.9 \mathrm{~kg} / \mathrm{m}^{2}$ ) and obesity ( BMI> $30.0 \mathrm{~kg} / \mathrm{m}^{2}$ ) participated - 109 men and 155 women, in the period from
January to April of 2014 and 2015. With normal body weight were 27.3\%; overweight were $24.6 \%$ and obese adults were $48.1 \%$. Food consumption was examined by $24-\mathrm{h}$ recall, food frequency questionnaire (FFQ), and the physical activity level (PAL) by IPAQ. Physical examination (body height and weight, waist circumference, blood pressure) were performed. Body composition was measured on leg to leg body impedance analyzer (Tanita BC 420 MA, Tanita Corp., Tokyo, Japan) and dual-energy X-ray absorptiometry (DXA) on the GELunarProdigyPRO. Further defines the following indexes: W / Ht, FMI, FFMI, ALMI, ALM / BMI. Serum levels of vitamin D were measured as $25(\mathrm{OH}) \mathrm{D}$ Total (Immunotest, Roche Diagnostics, Switzerland).

Results: The mean serum $25(\mathrm{OH})$ vitamin D for the entire sample was $38.15 \pm 22.84 \mathrm{nmol} / 1(39.25 \pm 21.94 \mathrm{nmol} / 1$ in men and $37.37 \pm 23.49$ $\mathrm{nmol} / \mathrm{l}$ in women) and respectively for those with normal BM - $48.8 \pm 25.9$ $\mathrm{nmol} / \mathrm{l}$ (53.45 $\pm 26.45 \mathrm{nmol} / \mathrm{l}$ in men and $47.13 \pm 25.7 \mathrm{nmol} / 1 \mathrm{in} \mathrm{wom}-$ en). The mean serum $25(\mathrm{OH})$ vitamin D levels were $34.16 \pm 20.25 \mathrm{nmol}$ $/ 1$ in group with overweight and obesity $(37.82 \pm 21.09 \mathrm{nmol} / \mathrm{l}$ in owerweight and $32.28 \pm 19.63 \mathrm{nmol} / 1$ in obesity). We were able to find a statistically significant difference in average levels of $25(\mathrm{OH})$ vitamin D in adults with normal BMI and the group with overweight and obesity. The sub-analyses (men versus women) did not produce any additional information.

Keywords: 25(OH )vitamin D, overweight, obesity, fat mass

\section{T2P141}

\section{Sexual satisfaction and binge eating behaviors: Correlation study}

Pereira, F. ; Pereira, A. ${ }^{2}$; Ribeiro, I. ${ }^{2}$; Veiga-Branco, $M .^{3}$

${ }^{1}$ Faculdade de Medicina da Universidade de Lisboa, PAIDEIA, ${ }^{2}$ Instituto Politécnico De Bragança, Instituto Politécnico De Bragança, Reci, Paideia

Introduction: As basic human needs, diet and sexuality depend on the connections of multiple systems (muscular, neuro-endocrine, metabolic and reproductive), as well as aspects related to emotions, feelings and pleasure.

Methods: A transversal, exploratory and quantitative, descriptive and correlational study. The instruments for data collection were the following: a questionnaire to describe the participants, the Index of Sexual Satisfaction (ISS) and the Binge Eating Scale (BES), both of which were translated and adapted to the Portuguese population. The statistical analysis was carried out with the statistics software program SPSS, version 23.

Results: The results of the descriptive analysis show a sample of 306 patients, of which $69.3 \%$ are female and $30.7 \%$ are male, with ages ranging from 18 to 64 years old. the study shows that the Binge Eating Scale (BES) is divided into three levels, with 219 respondents (71.6\% of the sample) who are non-binging, followed by 49 patients ( $16 \%$ of the sample) with moderate binge and finally, in smaller numbers, 38 patients $(12.4 \%$ of the sample) with severe binging. Furthermore, the study shows that even those with a BMI in the healthy weight range $(4.5 \%$ and $1.5 \%$ in this category) show a moderate BES and a severe BES respectively.. The correlational analysis confirmed the existence of a statistically significant $(\rho, 205$; $\alpha, 000)$ positive correlation between the sexual satisfaction of the sample and the level of binge eating. Furthermore, there was a highly significant statistical correlation between the BES, ISS and BMI variables, with a triangulation effect

Conclusion: This study demonstrates that people with normal weight or with an apparently "normal" profile can in fact show binge eating behaviors. In the study related to the level of Sexual Satisfaction (SS) of patients on different scales of the Body Mass Index (IMC), the average rate of ISS increases with the degree of obesity, indicating that these groups are dissatisfied with their sexuality, which increases with the level of BMI.

Conflict of Interest: None Disclosed

Funding: No Funding 
T2P143

\section{Short-term increased sedentary behaviour leads to loss of muscle mass in young individuals}

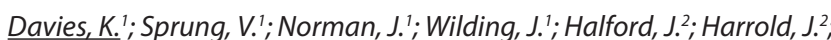
Kemp, G. ${ }^{3}$; Cuthbertson, D.

${ }^{1}$ Institute of Ageing and Chronic Disease, University of Liverpool, UK, ${ }^{2}$ Institute Of Psychology Health And Society, University Of Liverpool, Uk, ${ }^{3}$ Magnetic Resonance And Image Analysis Research Centre (Mariarc), University Of Liverpool, Uk

Introduction: Societal factors (relating to occupation, travel and the domestic environment) reduce our physical activity level relative to our ancestors. Habitually reduced levels of physical activity are an independent risk factor for obesity, poor metabolic health and accelerated musculoskeletal decline. Previous research has focused on increasing habitual physical activity as an alternative to structured exercise, yet little is known about the consequences of decreasing habitual physical activity. We aimed to determine the extent of musculoskeletal decline after 14 days of physical inactivity.

Methods: Twenty eight (age $31 i^{3 / 4}$ yyears, BMI $25 i^{3 / 4} 2 \mathrm{~kg} / \mathrm{m}(\div)$ physically active ( $\mathrm{i}$ Ã10,000 steps per day) individuals underwent comprehensive assessments of i) physical activity using a SenseWear armband ii) regional body composition and muscle volume using MRI and DEXA scans, iii) mitochondrial function using ${ }^{31} \mathrm{P}-\mathrm{MRS}$ and iv) cardio-respiratory fitness using V02 peak analysis. Assessments were completed at baseline and following a 14-day step reduction protocol ( 1,500 steps/day). A dietary journal was completed to ensure no changes to intake throughout the intervention. Statistical analysis was performed using paired t-tests; data are presented as mean $i^{3 / 4}$ SD $[95 \% \mathrm{CI}]$.

Results: The step reduction protocol decreased moderate to vigorous activity (MVPA) from a daily mean of $161_{i}^{3} / 486 \mathrm{~min}$ to $36 ; 3 / 429 \mathrm{~min}$, a mean reduction of $125 \mathrm{~min}[94,155] P<0.005$. In parallel daily mean sedentary time increased by $129 \mathrm{~min}[35,224] P=0.009$. Following the period of inactivity, cardio-respiratory fitness significantly decreased $(2.9 \mathrm{ml} / \mathrm{min}$ $1 / \mathrm{kg}[1.3,4.6] ; P=0.001)$ and with it a significant loss in skeletal muscle mass with a reduction in total (whole body) lean mass $(0.36 \mathrm{~kg}[0.04$, $0.70] ; P=0.031)$ and leg lean mass $(0.21 \mathrm{~kg}[0.06,0.37] ; P=0.009)$. Skeletal muscle mitochondrial function, measured by $\mathrm{PCr}$ recovery rate, did not change significantly $P=0.106$.

Conclusion: In a cohort of habitually active, healthy young individuals that met the recommended MVPA guidelines, only 14 days of increased sedentary behaviour induces small but significant reductions in muscle mass that are accompanied by a decrease in cardio-respiratory fitness such decrements that can lead to chronic metabolic disorders and premature mortality. The results emphasise the importance of minimising physical inactivity.

Conflict of Interest: None disclosed.

Funding: Research relating to this abstract was funded by Diabetes UK and MRC/ Arthritis UK.

\section{T2P144}

\section{Social capital and children's experiences of physical activity:} Influence of family and peers

\section{Everley, S.}

${ }^{1}$ University of Chichester

Introduction: Nearly one third of children in the UK aged 2 to 15 are overweight and children are becoming obese at an earlier age (Gov. 2016). To support children in establishing and maintaining a physically active lifestyle, it is necessary to understand what they value in physical activity (PA) and who influences them in this.

Methods: 112 children aged 5-10 yrs, from three primary schools drew themselves being physically active and were interviewed about their pictures. Interviews centred on:

social interactions fields (locations) in which PA occurred

cultural significance of PA as affecting social relations and sustainability Results: Findings indicate cultural and social capital significantly affect engagement in PA. Outcomes demonstrate:

Key influencers of PA shift from family to peers between school years 1 and 2 (children aged 5/6yrs and 6/7yrs).

Children accumulate social capital through culturally acceptable forms of PA

Social capital features significantly in potential to participate in PA

Social capital affects the hierarchical organisation of PA in free play at school

Conclusion: There is a clear relationship between social capital and children's participation in PA. Establishing patterns PA requires stability of environment and social networks. Schools provide a natural conduit through which to implement policy targeting PA health behaviours, linking to families.

Caution needs to be exercised where children find engaging in social networks problematic: the 'dark side' of social capital may actually lead to exclusion from PA opportunities. Further investigations need to be made into social capital and its function in health behaviours of children.

\section{T2P145}

\section{Soft drink consumption is associated with increased risk of metabolic syndrome only in women}

Lee, J. ${ }^{1}$; Kang, Y. $^{1}$; Kim, J. ${ }^{1}$

${ }^{1}$ Kyung Hee University

Introduction: Prospective study on the association between soft drink consumption and risk of incident metabolic syndrome (MetS) has not been examined in Asians.

Methods: We explored the gender-specific association between soft drink consumption and risk of MetS in Korean adults during 10 years of follow-up. A total of 5,797 subjects who were free of MetS at baseline were studied. Soft drink consumption was assessed using a semi-quantitative food frequency questionnaire. Time-dependent Cox proportional hazard model was used to examine hazard ratios (HRs) for risk of MetS and its components in relation to soft drink consumption.

Results: In women, the multivariable-adjusted HR for developing MetS was 1.8-fold higher in frequent consumers of soft drinks ( $\geq 4$ servings/ week) compared with rare consumers $(95 \% \mathrm{CI}=1.23-2.64)$. The adjusted HRs for elevated BP increased by 2-fold (95\% CI $=1.24-3.14)$, and for hypertriglyceridemia by 1.9 -fold ( $95 \% \mathrm{CI}=1.19-2.88$ ) in frequent consumers of soft drinks compared with rare consumers. However, in men, there was no association between soft drink and risk of incident MetS or its components.

Conclusion: Frequent soft drink consumption was associated with increased risk of developing MetS and its components only in middle-aged and older Korean women suggesting gender difference on the risk of MetS related to diet.

Conflict of Interest: None Disclosed/Payment received from the authors

Funding: Research relating to this abstract was funded by the Basic Science Research Program of the National Research Foundation of Korea (NRF), funded by the Ministry of Education, Science, and Technology (NRF2016R1D1A1B03931307).

\section{T2P146}

\section{Stealth interventions to prevent and treat obesity: Focusing on process motivators to achieve greater magnitude and more sustained behavior change}

\section{Robinson, T. N}

${ }^{1}$ Stanford University, USA

Motivation is considered a key factor in behavior change. Stealth interventions focus on rewards inherent in the process of change itself, or process 
motivators. This intervention design paradigm contrasts with the traditional approach of attempting to motivate behavior change by focusing on health-related outcomes. Stealth intervention principles have been applied to successfully prevent and control weight gain in both population-based and overweight populations of children and young adults in interventions to reduce screen time, increase physical activity, and improve diet. These examples show that interventions can directly target process motivation with design characteristics known to increase intrinsic motivation for participation. Other stealth interventions focus on activities that are already highly motivating for participants, such as dance classes and team sports, while promoting healthful behaviors as side effects. Finally, stealth interventions may piggyback onto motivating social and ideological movements, such as environmental sustainability and humane treatment of animals, to achieve more sustained behavior changes. Many existing social and ideological movements have behavioral goals that overlap with those of obesity prevention and control, and may represent effective alternative approaches to addressing obesity in individuals, groups, and society. Advantages of the stealth intervention approach are its basis in theory and empirical research, it can be applied across all intervention levels, it is solution-oriented, and it suggests new potential alliances and synergies in the battle against obesity.

\section{Conflict of Interest: None}

Funding: Research relating to this abstract was funded by the U.S. National Institutes of Health; U.S. Department of Energy; Precourt Energy Efficiency Center, Stanford University; Stanford Child Health Research Institute;

\section{T2P147}

\section{Substitution models of water for other beverages, and the incidence of obesity and weight gain in the SUN cohort}

Fresán, U. ${ }^{1}$; Gea, A. '; Bes-Rastrollo, M. '; Ruiz-Canela, M. '; MartínezGonzalez, M. ${ }^{1}$

${ }^{1}$ University of Navarra

Introduction: Obesity is a major epidemic for developed countries nowadays. Its main cause is energy imbalance, of which contributing factors include a sedentary lifestyle, epigenetic factors and excessive caloric intake through food and beverages. A high consumption of caloric beverages, such as alcoholic or sweetened drinks, may particularly contribute to weight gain, and lower satiety has been associated with the intake of liquid instead of solid calories. Our objective was to evaluate the association between the substitution of a serving per day of water for another beverage (or group of them) and the incidence of obesity and weight change in a Mediterranean cohort.

Methods: We followed 15,765 adults without obesity at baseline. The intake of 17 beverage items was assessed at baseline through a validated food-frequency questionnaire. The outcomes were average change in body weight in a four-year period and new-onset obesity and their association with the substitution of one serving per day of water for one of the other beverages.

Results: During the follow-up, 873 incident cases of obesity were identified. In substitution models, the consumption of water instead of beer or sugar-sweetened soda beverages was associated with a lower obesity incidence (the Odds Ratio (OR) 0.80 (95\% confidence interval (CI) 0.68 to 0.94 ) and OR 0.85 (95\% CI 0.75 to 0.97 ); respectively) and, in the case of beer, it was also associated with a higher average weight loss (weight change difference $=-328 \mathrm{~g}$; $(95 \% \mathrm{CI}-566$ to -89$))$.

Conclusion: This study found that replacing one sugar-sweetened soda beverage or beer with one serving of water per day at baseline was related to a lower incidence of obesity and to a higher weight loss over a four-year period time in the case of beer.

Conflict of Interest: The authors declare no conflicts of interest.

Funding: The Seguimiento Universidad de Navarra (SUN) Project has received Funding from the Spanish Ministry of Health (Grants PI14/01668, PI14/01764,
PI14/01798), the Navarra Regional Government (122/2014), and the University of Navarra.

T2P148

Sustainable diets in fighting obesity and creating healthier food consumption patterns among schoolchildren in Poland

\section{Halicka, E. ; Rejman, K. '; Kaczorowska, J.'}

${ }^{1}$ Warsaw University of Life Sciences

Introduction: According to FAO 2010 consensus sustainable diets are those diets with low environmental impacts which contribute to food and nutrition security and to healthy life for present and future generations. This definition reflects the recognition that the health of human beings cannot be isolated from the health of ecosystems. In Poland the number of children with excess body weight is still growing and actions aimed at improving their dietary patterns and food choices as well as increasing physical activity need to be reinforced.

Methods: In 2015 a survey was developed and conducted among a sample of 1035 parents living in urban areas with children aged 6-8 years. The study included questions on demographic characteristics, children's food consumption habits and level of parental involvement in shaping children's food-related behaviors. Special attention was paid to the respondents' knowledge and awareness of factors influencing decisions regarding food (incl. household waste management), nutrition and health.

Results: Research results showed that only $28 \%$ of the interviewed parents (average age was 39 years) had heard the term "sustainable diet" and only $35 \%$ of them indicated correctly that such diets have less impact on the environment. In general the studied group of parents felt responsible for talking to their children about the link between nutrition and health, the need to limit food waste and protecting natural resources. Statistical analysis of primary data on dietary patterns and food choice determinants associated with sustainable behaviors allowed to distinguish two clusters: one focusing on fulfilling the child's individual "ego" needs $(n=449)$, the other - on the collective (ecological) "allo" needs $(n=586)$. These groups differed statistically in: number of children, income level and type of school that the children attended.

Conclusion: The collected data indicates that among the parents of schoolchildren in Poland there is a low level of awareness in the topic of sustainable diets however half of the studied respondents showed a pro-collective approach and therefore would feel empowered if they gained knowledge on these issues. Sustainability of diets should be incorporated in public health policies to strengthen the message and effectiveness of preventing obesity and unhealthy choices among schoolchildren.

Conflict of Interest: None Disclosed

\section{T2P149}

\section{Tableware design as a tool for reducing obesity problems}

\section{Cinovics, N. ${ }^{1}$;}

${ }^{1}$ Art Academy of Latvia

Introduction: Nowadays more than 2.1 billion people ( $30 \%$ of the world's population) have obesity or overweight. (Ng et al., 2014) There are many ways how to reduce overweight - exercises, stress reduction, diets and other, however it has been little studied how tableware design can contribute to weight loss by changing eating habits.

Methods: Qualitative case study methodology was used. Data were collected using Internet search and evaluated according to inclusion/exclusion criteria. Cases (examples of tableware design aimed at reducing overweight) were systematically divided into four groups - cutlery, dish, tableware accessories and other design products. After collection of case studies (random selection) in-depth analysis of each case was performed. Results: Different design methods for changing eating habits are applied in the analyzed cases - form, graphic, weight, technologies and light. Graphic as a method is applied mostly to depict optimal portion sizes. 
Weight as a method is used mostly in cutlery to prolong the process of eating. However, mostly the designed tableware to reduce overweight is focusing on vision, because all the mentioned elements above can be related to vision when eating, which is also emphasized by B. Wansink: "We tend to eat with our eyes and not our stomach, because our stomach is a crude measure of how much we've eaten.... (Jessica Gross, 2016)

Conclusion: By using all human senses (including synesthesia) in combination with design methods, it is possible to create a tableware design aiming to reduce overweight. Innovative tendencies can be observed in tableware design development nowadays, however, there still is a need to create more diverse approaches to help people to reduce obesity. Based on the analysis of literature and case studies the author has developed a hypothesis about a new three dimensional form of a dish as a design method for changing eating habits.

\section{References:}

1 Ng, M., Fleming, T., Robinson, M., Thomson, B., Graetz, N., Margono, C., .. Gakidou, E. (2014). Global, regional, and national prevalence of overweight and obesity in children and adults during 1980-2013: a systematic analysis for the Global Burden of Disease Study 2013. Lancet, 6736, 1-16. http://doi. org/10.1016/S0140-6736(14)60460-8

2 Jessica Gross. (2016). Want to eat well? Forget about willpower. Retrieved from http://ideas.ted.com/want-to-eat-well-forget-about-willpower/?utm campaign=social\&utm_medium=referral\&utm_source=t.co\&utm_content=ideas-blog\&utm_term=social-science

Conflict of Interest: None Disclosed

Funding: No Funding

T2P150

\section{Technology-based interventions in the treatment of overweight and obesity: A systematic review}

\section{Pouwels, S. ${ }^{1}$; Raaijmakers, L. ${ }^{2}$; Berghuis, K. ${ }^{2}$; Nienhuijs, S. ${ }^{2}$}

${ }^{1}$ Franciscus Vlietland, Schiedam, The Netherlands, ${ }^{2}$ Department Of Surgery, Catharina Hospital, Eindhoven, The Netherlands

Introduction: The prevalence of obesity increases worldwide. The use of technology-based interventions can be beneficial in weight loss interventions.

Objectives: This review aims to provide insight in the effectiveness of technology-based interventions on weight loss and quality of life for patients suffering overweight or obesity compared to standard care.

Methods: Data was searched from the earliest date of each database up to February 2015. Cochrane Collaboration's tool for assessing risk of bias was used for rating the methodological quality

Results: Twenty-six trials met inclusion criteria. Twelve studies showed significant effects on weight loss compared to controls. Most interventions used a web-based approach (42\%). Interventions were screened for five technical key components: self-monitoring, counsellor feedback and communication, group support, use of a structured program and use of an individually tailored program. No significant results for quality of life were found. Outcomes on program adherence were reported in six studies. No significant results were found between weight loss and program adherence. However, interventions with a technological component did show higher adherence rates compared to control groups.

Conclusion: Evidence is lacking about the optimal use of technology in weight loss interventions. However, when the optimal combination of technological components is found, technology-based interventions can be a valid tool for weight loss. Furthermore, more outcomes on quality of life and information about the effect of technology-based intervention after bariatric surgery are needed.
T2P151

\section{The 5AsT-MD pilot: Improving education and training in obesity management in family medicine residents.}

Campbell-Scherer, D. '; Wicklum, S. ${ }^{2}$; Luig, T. ${ }^{3}$; Li, S. ${ }^{3}$; Lafrance, R. ${ }^{4}$; Connors, A. ${ }^{5}$; Heatherington, M. ${ }^{1}$; Klein, D. ${ }^{1}$; Moniz, K. ${ }^{1}$; Sharma, A. ${ }^{3}$

${ }^{1}$ Department of Family Medicine, University of Alberta, Edmonton, AB, Canada, ${ }^{2}$ Department Of Family Medicine, Cumming School Of Medicine, University Of Calgary, Calgary, Ab, Canada, ${ }^{3}$ Department Of Medicine, Division Of Endocrinology, University Of Alberta, Edmonton, Ab, Canada, ${ }^{4}$ Department Of Psychiatry And Pediatrics, University Of Alberta, And Primary Health Care, Alberta Health Services, Edmonton, Ab, Canada, ${ }^{5}$ Primary Health Care, Alberta Health Services, Edmonton, Ab, Canada

Introduction: The prevention and management of obesity requires a lifecycle approach that is ideally suited for family physicians. While effective tools exist to support weight counselling, research shows that medical residents and practicing physicians still feel ill-prepared and lack knowledge and confidence to effectively address weight concerns with patients. There is a pressing need to address this gap and create evidence-based obesity management training programs for family medicine residents so they can better support patients. We developed a comprehensive educational program for residents based on the 5 As of Obesity Management ${ }^{\text {mit }}$ (Ask, Assess, Advise, Agree, Assist- 5As), a framework and suite of resources to improve residents' knowledge and confidence in weight counselling. Methods: This educational intervention combines lectures with experiential learning methods, including content expert presentations, bariatric suit experience, practice with standardized and in-clinic patients, and narrative reflections. The participants were 42 first year family medicine residents enrolled in an educational institution in Alberta, Canada. We assessed changes in residents' attitudes, beliefs and level of self-confidence. Narrative reflections were analyzed thematically.

Results: Findings of the 5AsT-MD pilot program show increases of residents' understanding of the complexity and chronicity of obesity, and an uptake of the 5 As framework and tools by residents to improve confidence and success in their weight management practice. Experiential learning elements of the program proved crucial in increasing residents' ability to empathically engage with patients and to critically reflect on the implications for their practice.

Conclusion: Results underscore the need for training programs for family medicine. Furthermore, findings reflect the utility of the $5 \mathrm{As}$ to improve residents' confidence and competency in obesity management counselling across the age continuum.

Conflict of Interest: DL Campbell-Scherer has an unrestricted educational grant from a Novo Nordisk; AM Sharma is a member of an Advisory Board with Novo Nordisk: Advisory Board for anti-obesity drug; and was a member of the Data Safety Monitoring Board for an anti-obesity trial (Takeda). S Wicklum, T Luig, S Li, $\mathrm{R}$ LaFrance, A Connors, M Heatherington D Klein and K Moniz have nothing to disclose.

Funding: The 5AsT study is funded by Alberta Innovates-Health Solutions, and an unrestricted educational grant from a commercial organization (Novo Nordisk: Advisory Board for anti-obesity drug). The researchers are independent of the study funders. The funders had no access to study data, no involvement in the design or execution of the study and no involvement in analysis

\section{T2P154}

The cross-sectional association between eating frequency and body composition among adults in South China

\section{Chen, Y. ${ }^{1}$; Xue, H. ' Yin, J. ' Zhang, J. ${ }^{\text {; }}$ Cheng, G. ${ }^{2}$}

${ }^{1}$ Department of Nutrition, Food Safety and Toxicology, West China School of Public Health, Sichuan University, Chengdu 610041, Sichuan, China, ${ }^{2}$ Department Of Nutrition, Food Safety And Toxicology, West China School Of Public Health, Sichuan University, No.16, Section 3, Renmin Nan Road, Chengdu, Sichuan, China 610041

Introduction: Epidemiology evidence is discrepant on the association between eating frequency (EF) and body composition. The aim of the 
present study was to explore whether EF was associated with body composition in Chinese adults.

Methods: A total of 1017 adults (53.8\% men) aged 25-65 years were included from an on-going prospective cohort study carried out in Southwest China since 2013. Dietary data were collected by validated 24-h dietary recalls. Misreporters of total EI who had extreme values were excluded from the study to minimize the potential recall bias. EF was calculated with two different methods based on the energy intake (EI) and the eating time interval: all eating occasions providing at least $50 \mathrm{kcal}$ (or 210 $\mathrm{kJ}$ ) with a minimum time interval between occasion at least $15 \mathrm{~min}$ or 30 min. Each EF was divided into meal frequency (MF) and snack frequency (SF). Body height and weight were measured to calculate body mass index (BMI), percentage of body fat (\%BF), fat mass index (FMI) and fat-free mass index (FFMI). Multiple linear regression models were used to examine the association between EF and body composition parameters.

Results: Overall, participants ate more frequently had higher daily intake (energy, protein, fat and carbohydrate) and \%BF $(\mathrm{p}<0.01)$ and lower FFMI $(\mathrm{p}<0.05)$ compared to participants with less EF. No difference was observed in the prevalence of overweight/obesity among different categories of eating frequency. Both EF and SF had positive association with \%BF ( $<<0.01)$ and FMI $(\mathrm{p}<0.01)$, negative association with FFMI $(\mathrm{p}<0.01)$ and null relationship with BMI after further adjustment for age, occupation, personal monthly income, smoking status, alcohol, physical activity, energy intake, protein intake, fat intake and carbohydrate intake. However, null associations were found between MF and body composition parameters.

Conclusion: Our results indicate that higher EF and SF were associated with higher body fatness and lower fat-free body mass in Chinese adults.

Conflict of Interest: The authors have no financial relationships relevant to this article to disclose.

Funding: All phases of this study were supported by research grant from the National Nature Science Foundation of China (No.81472976)

\section{T2P155}

\section{The dangerous link between obesity and depression}

Rivera, M. ${ }^{1}$; Rovira, P. ${ }^{2}$; Gutierrez, B. ${ }^{1}$; Ching-Lopez, A. '; Molina, E. ; MartinLaguna, M. ${ }^{\text {; }}$ Ibanez-Casas, I. ' 'Mckenney, K. ${ }^{1}$; Ruiz-Perez, I. ${ }^{4}$; RodriguezBarranco, M. ${ }^{5}$; Cervilla, J. ${ }^{1}$

${ }^{1}$ University of Granada, ${ }^{2}$ Universitat Autònoma De Barcelona, ${ }^{3}$ University Of Seville, ${ }^{4}$ Andalusian School Of Public Health And Ciberesp, ${ }^{5}$ Andalusian School Of Public Health

Introduction: Obesity and depression are leading causes of disease burden and disability, as well as major public health concerns worldwide. Both conditions are highly prevalent and major risk factors for chronic physical diseases such as type 2 diabetes, cardiovascular disease and hypertension. The reason why obesity and depression cluster together is not totally understood and several mechanisms have been proposed. There are many factors driving this observation, such as individual lifestyle choices, socioeconomic factors, psychosocial stress, medication, as well as biological and genetic factors. There are also a limited number of cross-national studies on comorbid mental and medical conditions.

Methods: This work will highlight the evidence of shared genetics effects that may contribute to the link between obesity and depression. We will also examine the prevalence of obesity in people with depression compare with psychiatrically healthy controls from the PISMA-ep study. The PISMA-ep is a cross-sectional study of a representative sample of the Andalusian population (Spain). The total sample includes 1,005 participants with diagnosis of a psychiatric disorder and 3,502 psychiatrically healthy controls. The MINI interview was used to establish the diagnostic of depression. Height and weight data from each individual was used to calculate body mass index (BMI), as a measure of obesity, using the formula: weight $(\mathrm{kg}) /$ height $(\mathrm{m})^{2}$. T-tests were used to analyze the association between depression and BMI. First, the analyses were carried out in the whole sample, and then separately in depression cases and controls. The statistical analyses were performed using SPSS 15.0 software.

Results: Initial results from a subsample of 337 individuals with depression and 921 controls showed a statistically significant association between depression and BMI. The individuals with depression had significantly higher BMI values compared to controls (cases: $\mathrm{BMI}=27.54$ $(\mathrm{SD}=5.75)$; controls: $\mathrm{BMI}=26.75(\mathrm{SD}=4.76)$; $\mathrm{t}=-2.34, \mathrm{P}=0.009)$.

Conclusion: The association between obesity and depression has repeatedly been reported in many studies. Although the nature and direction of this association remains unclear, both disorders may share some genetic variance. Our results show an association between BMI and depression confirming the results from previous studies. This study highlights the importance of addressing obesity in people with depression, as both disorders are associated with substantial personal and societal economic costs worldwide.

\section{Conflict of Interest: None Disclosed.}

Funding: Research relating to this abstract was funded by the Marie Curie Research Grants Scheme, grant FP7-626235 and by a NARSAD Young Investigator Grant from the Brain \& Behavior Research Foundation.

\section{T2P156}

\section{The eating behavior patterns among comorbid obese people}

\section{Sofya, E. ' Julia, D. '; Oxana, D. ${ }^{1}$}

${ }^{1}$ The National Research Center for Preventive Medicine

Introduction: The study aim was to evaluate a potential association between eating disorders and metabolic profile among people with obesity and cardiovascular disease.

Methods: A pilot study, including 38 patients with cardiovascular disease (CAD and hypertension), was designed. Abdominal and general obesity was detected according to the WHO criteria. The Dutch Eating Behavior Questionnaire (DEBQ) was used to assessment of restrained, emotional, and external eating behavior.

Results: Analysis included 38 participants (mean age: 57 (14) years; body mass index: $28(5,6) \mathrm{kg} / \mathrm{m} 2$; and $45 \%$ male). Mean lipid values were as follows: total cholesterol: 5.5 (1) mmol/l; LDL-C: 4 (1) mmol/l; HDL-C: 0.98 (0.3) $\mathrm{mmol} / \mathrm{l}$; triglycerides $2.5(1.5-2.6) \mathrm{mmol} / \mathrm{l}$. 16 (42\%) participants of the sample had signs of general obesity, 31 (82\%) - had signs of abdominal obesity. We have not identified eating disorders only in 7 patients. Restrained eating had been revealed in $23(61 \%)$, emotional eating - in $12(32 \%)$ patients, and external eating - in 20 (52\%) patients. The combination of the two types of eating disorders was detected in 8 patients. Eating disorders were associated with BMI, WC, triglyceride level $(r=0.5$, $\mathrm{p}<0.05)$, low physical activity $(\mathrm{r}=0.6, \mathrm{p}<0.05)$, low fruit and vegetables $(\mathrm{r}=0.5, \mathrm{p}<0.05)$ and high consumption of processed meat $(\mathrm{r}=0.6$, $\mathrm{p}<0.05)$.

Conclusion: Our findings underscore the role of behavioral risk factors in obesity and cardiovascular disease progression.

Conflict of Interest: None Disclosed.

Funding: No Funding.

\section{T2P157}

\section{The effect of timing and composition of gestational weight gain in obese pregnant women on infant birth weight: $A$ prospective cohort study.}

\section{Redfern, K. ${ }^{1}$; Rees, G. '; Pinkney, J. \\ ${ }^{1}$ Plymouth University}

Introduction: Numerous maternal factors, such as body mass index (BMI), gestational weight gain (GWG), diet, physical activity (PA) and the development of gestational diabetes mellitus (GDM), have been shown to impact infant birth weight - a key determinant of infant health ${ }^{1}$. In the $\mathrm{UK}$, antenatal care tends to be based on pre-pregnancy BMI and wom- 
en are not weighed routinely during pregnancy nor are there guidelines for GWG. However, it is widely acknowledged that maternal obesity and GWG in excess of the American Institute of Medicine guidelines are associated with increased risk of fetal macrosomia and recent studies have suggested a role of the timing and composition of GWG beyond that of $\mathrm{BMI}^{2,3}$. The purpose of this study is to examine the effects of timing and composition of GWG on neonatal anthropometric outcomes in a prospective cohort study amongst women with a BMI $\geq 30$ in Plymouth, UK. Methods: Data are presented for 32 women from an ongoing study. Women were recruited at 12 weeks gestation. Maternal height, weight, body composition using skinfolds, were collected at baseline and repeated at 28 and 36 weeks gestation. Following delivery, infant weight and gestational age were obtained, and neonatal anthropometric measurements were recorded within 72 hours of delivery.

Results: Total GWG varied considerably among the participants (mean $8.57 \mathrm{~kg}$, standard deviation $6.16 \mathrm{~kg}$, range $-3.00 \mathrm{~kg}-22.20 \mathrm{~kg}$ ). Multiple regression analysis identified several significant predictors of birth weight centile $\left(\mathrm{F}(14,17)=3.846, \mathrm{p}=0.005\right.$, adj. $\left.\mathrm{R}^{2}=0.562\right)$. These were maternal pre-pregnancy BMI, total GWG, rate of GWG (total, trimester 2 and trimester 3) and infant length (all p < 0.05). Maternal sum of skinfolds was associated with birth weight centile in trimester $1(\mathrm{p}=0.001)$ but not in trimesters 2 or 3 ( $\mathrm{p}=0.099$ and $\mathrm{p}=0.291$, respectively).

Conclusions: The timing and composition of GWG in women with obesity is highly variable. The current study shows the sum of triceps, biceps and subscapular skinfolds in the first trimester to be an interesting potential predictor of infant birth size that will be further investigated in a larger sample. Diet and activity patterns contributing to weight gain will also be explored.

\section{References:}

1 Scott-Pillai R et al. (2013) The impact of body mass index on maternal and neonatal outcomes: a retrospective study in a UK obstetric population, 2004-2011. BJOG.120(8):932-9.

2 Davenport MH et al. (2013) Timing of excessive pregnancy-related weight gain and offspring adiposity at birth. Obstet Gynecol.122(2 Pt 1):255-61.

3 Kent E et al. (2013) Correlation between birth weight and maternal body composition. Obstet Gynecol.121(1):46-50.

Conflict of interest: None disclosed

Funding: No Funding

\section{T2P159}

\section{The impact of exposure to cartoons promoting healthy eating} on children's food preferences and choices

Vaz, A. ${ }^{1}$; Gonçalves, S. '; Gonçalves, S. ' Conceição, E. ' ; Conceição, E. ${ }^{\text {; }}$ Silve, C. ; Silve, C. ; Ferreira, R. ; Ferreira, R. ; Machado, P. '; Machado, P.2 ; Boyland, E. ; Boyland, $E^{3}$

${ }^{1}$ Universidade do Minho, ${ }^{2}$ Universida Do Minho, ${ }^{3}$ University Of Liverpool

Few studies have analyzed the effects of the use of cartoons in promoting healthy eating behavior in children. The present study aimed to explore whether or not a cartoon with healthy eating messages would have a positive effect on children's (1) food preferences and attitudes; and (2) food choices. Participants ( $\mathrm{n}=143$, aged 4 to 8 years) were randomized to one of two groups: control $(n=73)$ who were exposed to cartoons without any reference to food or nutritional messages, and experimental $(n=70)$ who were exposed to cartoons with healthy eating messages. Duration of viewing was 20 minutes for each group, after which each child was given the opportunity to eat ad libitum for 10 minutes from a small selection of snack foods ( 2 healthy and 2 unhealthy items). Measures of hunger, cartoon recognition and liking; attitudes to healthy eating; and food preferences were also taken. Children exposed to the cartoon containing healthy eating messages chose significantly more healthy food items than the children in the control group. These results are promising and could inform the development of health promotion campaigns for children.

Keywords: cartoons, food preferences, food attitudes, food choices, children
T2P160

\section{The impact of obesity on cancer risk}

\section{Gribsholt, S. ${ }^{1}$; Veres, K. $^{2}$; Farkas, D. ${ }^{2}$; Ording, $A .{ }^{2}$; Richelsen, B. $^{3}$. SøRensen, $H^{2}$}

${ }^{1}$ Department of Endocrinology and Internal Medicine, Department of Clinical Epidemiology, Aarhus University Hospital, ${ }^{2}$ Department Of Clinical Epidemiology, Aarhus University Hospital, ${ }^{3}$ Department Of Endocrinology And Internal Medicine, Aarhus University Hospital

Introduction: Previous studies have reported a 16-33\% increased cancer risk associated with obesity. However, recent long-term studies assessing the overall cancer risk among persons with obesity are sparse. Thus, we aimed to evaluate the association between obesity and the risk of various forms of cancer.

Methods: In this nationwide Danish cohort study, we used medical databases to identify all patients with a diagnosis (inpatient, outpatient, or emergency room) of obesity $(\mathrm{N}=192,375)$, recorded from 1978 through 2013, and followed them up until a first-time cancer diagnosis, death, emigration, or 30 November 2013, yielding up to 35 years of follow up. We computed standardized incidence ratios (SIRs) for cancer as the observed to expected number of cancers based on national cancer incidence rates. Results: The overall SIR of cancer was 1.05 (95\% confidence interval (CI); 1.03-1.07), excluding the first year of follow up. The SIR was 1.09 (95\% CI; 1.05-1.12) among men and 1.03 (95\% CI; 1.00-1.05) among women. The SIR of cancer was similar among patients with and without a diagnosis code on diabetes; diabetes: 1.10 (95\% CI; 1.04-1.17), no diabetes: 1.04 (95\% CI; 1.02-1.07). The first year after obesity diagnosis the SIR of cancer was 1.80 (95\% CI; 1.72-1.87).

Conclusion: Obesity is associated with increased cancer risk, though the long follow up time resulted in lower estimates than previously reported.

Conflicts of interest: None disclosed

Funding: The Program for Clinical Research Infrastructure (PROCRIN) established by the Novo Nordisk Foundation and the Lundbeck Foundation

\section{T2P161}

\section{The influence of socioeconomic status on gestational weight gain: A systematic review}

\section{O'Brien, E. ; Alberdi, G. ' McAuliffe, F.}

${ }^{1}$ UCD Perinatal Research Centre, Obstetrics \& Gynaecology, School of Medicine, University College Dublin, National Maternity Hospital, Dublin, Ireland

Gestational weight gain is associated with pregnancy outcome and recommendations for gestational weight gain were published by the Institute of Medicine in 2009. Despite a large number of publications that have examined the association between socioeconomic status and gestational weight gain, the findings in the literature are inconsistent. This, therefore, is a systematic review of current evidence relating to the association between socioeconomic status and gestational weight gain.

Methods: Six electronic databases were searched, with the final search run on 1st July 2016. The PRISMA Statement guidelines were followed and a modified version of the RTI Item Bank was used to assess risk of bias in individual studies. The primary outcome was inadequate, adequate or excessive gestational weight gain, as per the Institute of Medicine 2009 guidelines.

Results: The review identified 16 studies for inclusion. Maternal educational attainment was the most commonly identified socioeconomic status measures relating to gestational weight gain, with a positive skew in the number of studies that indicated that those who are less well educated are most at risk of gaining weight outside of the recommendations. Other measures of socioeconomic status were not significantly associated with gestational weight gain.

Conclusions: Low educational attainment is likely to be associated with women gaining outside the Institute of Medicine recommendations for gestational weight gain. Healthcare providers should provide additional support to pregnant women who are most at risk of gaining outside the 
recommendations, thus reducing the gap in health inequalities between those of high and low socioeconomic status.

Conflict of Interest: None Disclosed

Funding: Research relating to this abstract was funded by the Health Research Board, Ireland

\section{T2P162}

The influence of the arrangement of different food images on participants' attention: An experimental eye-tracking study

\section{Hummel, G. ${ }^{\text {; }}$ Zerweck, I. ${ }^{2}$; Salazar-Winter, S. ; Ehret, J. '; Stroebele-} Benschop, N. ${ }^{1}$

${ }^{1}$ University of Hohenheim, Institute of Clinical Nutrition, ${ }^{2}$ University Of Stuttgart, Institute Of Biology

Introduction: Food images are powerful stimuli that are believed to capture attention and have the potential to induce cravings. Neuroimaging research revealed greater brain responses to high (HED) vs. low energy dense (LED) food pictures, and previous eye-tracking studies also describe differences in reaction time between HED and LED food images. No study so far has looked at the potential impact of the arrangement of different kinds of food images on participants' attention.

Methods: A total of 50 students ( 24 men and 26 women) completed a free viewing-task wearing mobile eye-tracking glasses. Food images were $30 \mathrm{HED}$ and 30 LED foods presented in pairs in a random order. LED food images were subdivided into cut up and unprepared food images (eg. sliced pepper vs. entire cauliflower). HED food images were subdivided into sweet and savory foods (eg. muffin vs. sausage). Dwell time and eye movements to food images were collected.

Results: No differences in dwell time between HED and LED food images were found. However, significantly greater visual attention were found for cut up LED food images compared to unprepared LED food images. No differences in dwell time between sweet and savory HED were found.

Conclusion: Cut up LED food images appear to capture visual attention longer compared to unprepared LED food images, as well as sweet and savory HED food images. Advertising healthy foods with ready to eat food images might be promoting its intake. More research in this area is necessary in order to understand how the presentation of food stimuli can influence eating behavior.

Conflict of Interest: None disclosed

Funding: No Funding

\section{T2P163}

\section{The level of cardiorespiratory endurance and the excess and} insufficient body weight in schoolgirls

Łysak, A. ${ }^{1}$; Dobosz, J. ${ }^{2}$; Walentukiewicz, A. ${ }^{1}$; Wilczynska, D. ${ }^{1}$; Skonieczny, P. ${ }^{1}$; Zajt, J.'

${ }^{1}$ Gdansk University of Physical Education and Sport, ${ }^{2} J$ Jóf PiłSudski University Of Physical Education In Warsaw

Introduction: The issue of the relation between developmental disharmonies in the level of body fat and the level of cardiorespiratory endurance as related to their influence on health hazards - is still important and thus an interesting matter for study for numerous authors.

Methods: The research material is part of an extensive study undertaken in schools in the Pomorskie voivodship. The present analysis covers girls between 7 and 19 years of age who were divided according to their leve of biological maturity as evidenced by menarche. 1224 girls were studied (627 non-menstruating, 597 menstruating). The schools were picked at random from a list of all educational institutions. An analysis of the excess and insufficient body weight was performed with the use of IOTF criterium and the level of cardiorespiratory endurance measured with the Cooper test. The results of the 12-minute running test were compared with the norms pertinent to the age and gender of the studied. The assessment of the level of biological maturity was conducted in an interview. The statistical analysis was performed with the use of Statistica software.

Results: There are no statistically relevant differences in the extent of the excess and insufficient body weight in menstruating and non-menstruating girls $(p=0,0610)$. Similar differences were not found in the results of the Cooper test $(\mathrm{p}=0,4447)$. Statistically relevant relation was found between the level of body fat and the results of the Cooper test. The low level of cardiorespiratory endurance was connected to the excess body weight regardless of the level of biological maturity of girls.

Conclusion: The results should lead to the search for practical and effective solutions to modify health behaviour of schoolchildren and youth.

Conflict of Interest: None Disclosed

Payment received from National Science Centre and Ministry of Science and Higher Education The study used data carried out under the project N N404 078036 funded by National Science Centre and Ministry of Science and Higher Education

\section{T2P164 \\ The metabolic consequences of short-term increased sedentary behaviour}

Davies, K. ${ }^{1}$; Sprung, V. ${ }^{1}$; Norman, J. ${ }^{1}$; Wilding, J. ${ }^{1}$; Halford, J. ${ }^{2}$; Harrold, J. ${ }^{2}$; Kemp, G. ${ }^{3}$; Cuthbertson, D.

${ }^{1}$ Institute of Ageing and Chronic Disease, University of Liverpool, UK, ${ }^{2}$ Institute Of Psychology Health And Society, University Of Liverpool, Uk, ${ }^{3}$ Magnetic Resonance And Image Analysis Research Centre (Mariarc), University Of Liverpool, Uk

Introduction: In the western world, we now adopt lifestyles low in levels of habitual physical activity; a risk factor for obesity and its manifestation of health problems. Sedentary behaviour is now considered a major public health threat. We assessed the metabolic effects of short-term changes in physical activity by implementing a step reduction model ( $>80 \%$ daily reduction).

Methods: Twenty eight (age $31 \pm 9$ years, BMI $25 \pm 2 \mathrm{~kg} / \mathrm{m}^{2}$ ) habitually active participants were assessed before and after 14 days of step reduction. Habitual physical activity (SenseWear armband), body composition (magnetic resonance imaging and dual-energy X-ray absorptiometry), biochemical profile (fasting plasma samples) and cardio-respiratory fitness (maximal oxygen consumption test) were assessed. A dietary journal was completed to ensure no changes to intake throughout the intervention. Statistical analysis was performed using paired t-tests; data are presented as mean $[95 \% \mathrm{CI}]$.

Results: The intervention successfully decreased daily step count by $80 \pm 7 \%$, a mean of 10,118 daily steps $[9,167,11,069] P<0.005$. Daily sedentary time increased by a mean of $129 \mathrm{~min}[35,224] P=0.009$. This reduction in physical activity was accompanied by decreased cardio-respiratory fitness $\left(2.9 \mathrm{ml} / \mathrm{min}^{-1} / \mathrm{kg}[1.3,4.6] ; P=0.001\right)$; waist circumference increased by $(0.7 \mathrm{~cm}[0.3,1.1] ; P=0.001)$; central fat by $(0.7 \%[0.2$, $1.2] ; P=0.011)$ and android fat by $0.9 \%[0.2,1.5] P=0.010$, and there was a trend for an increase in gynoid fat $(0.5 \%[0.1,1.0] P=0.067)$. Plasma triglycerides also increased significantly by $0.2 \mathrm{mmol} / 1[0.1,0.3] P=0.03$. Liver fat did not significantly change $P=0.197$.

Conclusion: As little as 2 weeks of increased sedentary behaviour results in reduced cardio-respiratory fitness, accumulation of central fat and appearance of associated components of metabolic syndrome including increased waist circumference and triglycerides. Further, we observed a predilection for increased android fat storage, a well-established risk factor that precedes obesity-related health problems.

Conflict of Interest: None disclosed.

Funding: Research relating to this abstract was funded by Diabetes UK and MRC/ Arthritis UK. 
T2P165

The phenomenon of 'food addiction': Relationship between 'food addiction', eating behavior, health status and score of binge eating in a morbidly obese German sample

\section{Hauck, C. ${ }^{1}$; Weiß, A. ${ }^{1}$; Ellrott, $P .{ }^{1}$}

${ }^{1}$ Institute for Nutrition and Psychology at the Georg-August University Göttingen, Goettingen, Germany

Introduction: While the number of obesity is growing, part of the current research is focusing on, food addiction'('FA') as one potential contributing factor (1). The construct of 'FA' was developed based on the DSM-5 criteria for substance-related and addictive disorders (2). This study addresses possible relationships between 'FA', rigid control of eating behavior, mental health and binges in morbidly obese patients.

Methods: A total of 64 persons answered questions on binge episodes (DSM-5) and filled in the German versions of the questionnaires Yale Food Addiction Scale (YFAS) 2.0, enlarged Three-Factor Eating Questionnaire (TFEQ+), and Short Form 36 Health Survey (SF-36).

Results: The prevalence of 'FA' was $29.7 \%$ with an average amount of three symptoms. The score of rigid control of eating behavior (TFEQ+) was able to predict the symptoms of 'FA'. The amount of 'FA'-symptoms could predict the score of mental health (SF-36) and the amount of binge-symptoms (DSM-5).

Conclusion: The latter suggests an overlap between 'FA'- and classic symptoms of existing eating related disorders. Research is needed concerning positioning ' $F A$ ' as a distinct or part of an established disorder $(3,4)$.

\section{References:}

1 Gearhardt AN, Corbin WR, Brownell KD (2016) Development of the Yale Food Addiction Scale Version 2.0. Psychol Addict Behav 30(1): 113-121

2 Association AP (2013) Diagnostic and statistical manual of mental disorders. DSM-5, 5. ed. American Psychiatric Publishing, Washington DC u.a.

3 Hauck C, Weiß A, Ellrott T (2016) Zusammenhang zwischen,Food Addiction', gezügeltem Essverhalten, mentalem Gesundheitsstatus und Heißhungersymptomen bei morbider Adipositas. Adipositas 4/2016

4 Hauck, C, Weiß, A, Schulte, E, Meule A, Ellrott, T, "Prevalence of 'food addiction' as measured with the Yale Food Addiction Scale 2.0 in a representative German sample and its association with sex, age and weight categories."(submitted)

Conflict of Interest: None Disclosed

Funding: No Funding.

\section{T2P166}

The prevalence of obesity and metabolic syndrome among adults living in different altitudes

Lin, W. ${ }^{1}$; Yang, K. ${ }^{2}$; Lin, B. ${ }^{3}$; Genden, K. ${ }^{4}$ Yang, W. ${ }^{5}$; Wu, P. ; Fu, C. ${ }^{5}$; Hung, H. ${ }^{7}$; Li, C. ${ }^{8}$; Lin, C.

${ }^{1}$ School of Medicine, China Medical University and Department of Family Medicine, China Medical University Hospital, Taichung, Taiwan, ${ }^{2}$ Department Of Family Medicine, National Taiwan University Hospital, Bei-Hu Branch, Taipei, Taiwan, ${ }^{3}$ Syosset High School, New York, United States, ${ }^{4}$ Executive Secretary Of Tibetan Primary Health Care Center, Choglamsar, Leh Ladakh, India, ${ }^{5}$ Department Of Pediatrics, National Taiwan University Hospital, Hsin-Chu Branch, Hsinchu, Taiwan, ${ }^{6}$ Department Of Family Medicine, National Taiwan University Hospital, Taipei, Taiwan, ${ }^{7}$ Department Of Community And Family Medicine, National Taiwan University Hospital, Hsin-Chu Branch, Hsinchu, Taiwan, ${ }^{8} \mathrm{School}$ Of Medicine, China Medical University And Department Of Medical Research, China Medical University Hospital, Taichung, Taiwan

Introduction: To examine the prevalence of obesity and metabolic syndrome (MetS) among adults living in different altitudes.

Method: A total of 149 Tibetan immigrants aged 20 years and over were recruited in 2016 in Ladakh, India where the altitude is about 3000 3500 meters. To compare the effects of altitude on the prevalence of obesity and MetS, we chose another similar race who lives in a low altitude area Age and gender were matched (1:4) with another community cohort in Taiwan where the altitude is low (near sea level). Anthropometric indices and biochemical factors were measured. Obesity classified by body mass index (BMI) was defined using the World Health Organization Asia-Pacific criteria. MetS was defined using the American Heart Association and the National Heart, Lung and Blood Institute definitions.

Results: Subjects living in high altitudes have significantly higher prevalence of obesity and MetS than subjects living in low altitudes. The prevalence of overweight, general obesity, central obesity, and metabolic syndrome was $19.6 \%, 33.4 \%, 36.7 \%$, and $30.2 \%$ among subjects living in low altitude and $13.4 \%, 57.0 \%, 61.1 \%$, and $26.2 \%$ among subjects living in high altitude, respectively. The prevalence of general and central obesity was higher in people living in high altitudes. On the other hand, the prevalence of MetS was lower in people living in high altitude.

Conclusion: People living in highaltitude areas have a higher prevalence of general and central obesity but not MetS than people living in low altitude. Altitude may play an important role in the development of obesity.

Conflict of Interest: None disclosed.

Funding: No Funding.

\section{T2P167}

The prospective relationship between self-evaluation and loss of control of eating over 6 months in a sample of participants of a weight management programme

\section{Duarte C. ; Stalker, C. ${ }^{2}$; Catarino, F. ${ }^{2}$; Basran J. ${ }^{2}$; Gilbert, P. ${ }^{2}$; Horgan, G. ${ }^{3}$;} Morris, L. ${ }^{4}$; Stubbs, R. J. ${ }^{5}$

${ }^{1}$ Cognitive and Behavioural Centre for Research and Intervention, University of Coimbra, Rua do Colégio Novo, Apartado 6153, 3001-802 Coimbra, Portugal, ${ }^{2}$ College Of Life And Natural Sciences, University Of Derby, Derby, De22, 1 Gb, ${ }^{3}$ Biomathematics And Statistics Scotland, Rowett Institute Of Nutrition And Health, Foresterhill House, Aberdeen Ab25 2Zd, ${ }^{4}$ Slimming World, Clover Nook Road, Somercotes, Alfreton, Derbyshire De55 4Rf, ${ }^{5}$ Appetite Control And Energy Balance Research Group, School Of Psychology, University Of Leeds Ls2 9Jt

Introduction: Cross sectional data suggest that negative self-evaluation is associated with loss of control of eating (binge eating symptomology) in some participants of a commercial weight management programme. The current study investigated the prospective effect of negative self-evaluation on binge eating symptomatology, mediated by weight-related negative affect, over a six-month period.

Methods: 214 on-going participants of a commercial weight management programme completed an online survey including standardised psychometric measures of eating and weight-related self-criticism, shame, negative affect and binge eating symptoms at baseline, 3 and 6 months. The mediator effect of negative affect on the prospective association between negative self-evaluation and binge eating symptomatology was tested through a path analysis with maximum likelihood method and through bootstrapping (5000 samples) with CI95\%. The model controlled for weight-related negative affect at baseline and binge eating symptomatology at baseline and 3 months.

Results: Baseline measures of eating and weight-related self-criticism and shame were strongly associated. There was a significant prospective indirect effect of baseline hated-self form of self-criticism on binge eating symptomatology at 6 months, mediated by negative affect measured at 3 months $(\mathrm{b}=0.02$ CI95\% [.004, .059], $p=0.015)$. The model presented a very good model fit: $\mathrm{X}^{2}=17.76, \mathrm{p}=0.087$; CFI $=0.995$; TLI $=0.988$; RM$\mathrm{SEA}=0.054$ CI90\% [0.000, 0.098], $\mathrm{p}=.399$.

Conclusion: Results support the hypothesis that intense, negative aspects of self-evaluation significantly predict binge eating symptomatology and that this effect is mediated by negative weight-related emotions. These findings suggest that approaches to eating and weight that improve self-evaluation may help reduce binge eating symptomology in susceptible individuals participating in weight management programmes.

Conflict of Interest: R James Stubbs is a consultant to Slimming World through University of Leeds Consulting (Trading as Consulting Leeds).

Funding: Cristiana Duarte was funded by Fundação para a Ciência e a Tecnologia - SFRH/BD/76858/2011. 
This study was funded by a grant in aid of research from Slimming World UK, to the University of Derby.

\section{T2P168 \\ The relationship between obesity and depression in Korean adults}

\section{Hong, S. ${ }^{1} ; \underline{\text { Hur, } \text { Y. }^{2}}$}

${ }^{1}$ Department of Internal Medicine, Seoul Paik Hospital, Inje University College of Medicine, ${ }^{2}$ Department Of Family Medicine, Seoul Paik Hospital, Inje University College Of Medicine

Introduction: Previous literatures about relationship between obesity and depression have discrepant outcomes.

Some studies reported that obese people tend to have depression, and other studies did not. However, there are not many studies conducted in Asians. Additionally, there are few studies conducted with large-scaled, National-wide, Korean population-based sample. The aim of this study was to investigate the relationship between obesity and depression in Korean adults using data from a population-based sample of Korean National Health and Nutritional Examination Survey (KNHANES) of 2014.

Methods: This study analysed a total of 4,026 (men 1,692, women 2,334), aged 19-69 years, participated in KNHANES of 2014. Current depression was defined to have as score of $\geq 10$ on the score of the nine-item Patient Health Questionnaire. Height and weight were measured and BMI was calculated. The socio-demographic factors and disease morbidity were surveyed through face-to face interview. The participants asked to complete a questionnaire about and health-related behaviors. We considered age, educational level, household income, habitation, marital status, comorbidities, smoking, alcohol drinking habits, and stress perception as the covariates. Chi-square test and multivariate logistic regression analyses were performed to examine therelationship between obesity and depression.

Results: Depression was diagnosed in $5.7 \%$ (men: $3.9 \%$, women: $7.0 \%$ ) of study subjects. According to body weight status, there was a significant difference in depression prevalence (underweight: $16.2 \%$, normal-weight: $5.5 \%$, overweight $4.3 \%$, obesity $6.9 \%$ ). Compared to normal-weight group underweight group had a higher adjusted odds ratio (OR) for depression $(\mathrm{OR}=3.27,95 \%$ confidence interval 1.22 to $8.75,2.00,1.12$ to 3.57$)$ in men and women respectively. Overweight $(0.60,0.32$ to 1.13$)$ and obese men (BMI $\geq 30$ ) had lower ORs for depression $(0.62,0.17$ to 2.27 ), but these were not significant. Compared to normal-weight women, obese women had higher adjusted ORs $(1.75,0.79$ to 3.88$)$ for depression, while overweight women had lower ORs for depression $(0.90,0.56$ to 1.45$)$, but these were insignificant.

Conclusion: This study shows the difference of depression risks depending on body weight status. Underweight was related to high risk of depression in both men and women. And also we cannot ignore obesity condition as the risk of depression.

Conflict of Interest: The authors declare no conflict of interest.

Funding: No Funding

\section{T2P170}

The role of illness perception in predicting long-term glycemic control in overweight and obese patients with type 2 diabetes mellitus

\section{Pokrajac-Bulian, A. '; Majanović, S.'; Štimac, D. ${ }^{2}$}

${ }^{1}$ Faculty of Humanities and Social Sciences, University of Rijeka, Croatia, ${ }^{2} \mathrm{School}$ Of Medicine, University Of Rijeka, Clinical Hospital Center Rijeka, Croatia

Background and aims: The global epidemic of obesity largely explains the dramatic increase in the prevalence of T2DM over the past years (1). The patients' self-care behaviours, including maintenance of glycemic control, may be driven by their illness perceptions of their T2DM (2). Patient with a strong belief in their ability to control their T2DM may be more likely to engage in lifestyle management than a patient who believes their T2DM to be outside their own personal control (3).

Objectives: The aim of the research was to examine gender differences in beliefs about the disease, anxiety and depression in overweight and obese patients with T2DM. We wanted to assess if any of the patients' psychological characteristics, their health beliefs, age or BMI could be used to predict long-term glycemic control.

Materials and Methods: A total of 88 patients ( 42 women and 46 men) were enrolled in the study, $47 \%$ of the subjects were overweight and $53 \%$ were obese. Depression and anxiety were assessed using the Hospital Anxiety and Depression Scale (HADS), the Revised Illness Perception Questionnaire (IPQ-R) assessed the six components of the illness representation in Leventhal's Self-Regulatory Model.

Results: Women experience greater emotional reaction to diabetes (worry, depression, fear, anger) and are generally more anxious than men. The regression analysis results indicated that in women, beliefs that their treatment does not help in the regulation of the disease and that the disease has a number of negative consequences for their lives (financial, family, social) can be used to predict poor long-term glycemic control. In addition, older age, higher BMI and the female patient's belief that her own behaviour can affect her disease and makes her able to control it, can also predict poor long-term glycemic control. An interesting result is that among the variables that we studied not one has proved useful in predicting longterm glycemic control in man.

Conclusion: Results of this study suggest that the cognitive components of female patients' representations of illness, especially consequences, personal and treatment control have greater associations with long-term glycemic control and may be the best targets for interventions. It is necessary to take account of gender specificities in the management of T2DM and further research is needed to identify predictors of long-term glycemic control in men.

References:

1 Eckel RH et al. Diabetes Care 2011; 34(6):1424-1430; (2) Harvey JN, Lawson VL. Diabet Med 2009; 26:5-13; (3) Mc Sharry J, Moss-Morris R, Kendrick T. Diabet Med 2011; 28:1300-1310

Acknowledgment: This research is a part of the project 'Biopsychosocial aspects of obesity' (number: 13.04.1.3.07) supported by grant from University of Rijeka, Croatia.

\section{T2P171}

The school fruit and vegetable scheme as an effective strategy leading to positive changes in some eating behaviours of children.

Taraszewska, A. '; Wolnicka, K. '; Jaczewska-Schuetz, J. '; Jarosz, M. ${ }^{1}$; Niewiarowska, M. ${ }^{2}$

${ }^{1}$ National Food and Nutrition Institute, ${ }^{2}$ Agricultural Market Agency

Objectives: The School Fruit and Vegetable Scheme (SFVS) has been created as one of the priority activities of the European Commission aiming at the improvement of nutrition habits of children. In Poland children receive for free fruit and vegetables (F\&V) portions, eaten at school, 2-3 times a week

Methods: The study was conducted in primary schools in Poland, among 2531 pupils (starting at the age of 7-years old) on the basis of anonymous questionnaires and food diaries in two types of schools: participating in the SFVS (intervention group) and not-participating (control group), before launching the distribution of free F\&V (October 2012) and at the end of the two consecutive school years of F\&V distribution at school (June 2013, 2014).

Results: The awareness of the recommended daily amount of F\&V in everyday diet increased significantly more among children in the intervention group. The second year of the program contributed significantly to increase children's awareness about the link between the consumption of $\mathrm{F} \& \mathrm{~V}$ with health. In the intervention group on open-ended question "'What should you do in order to live a healthy life?" there was a significant increase in response: "eat vegetables" and "eat fruit", ' 'be more 
physically active," and "'do not eat sweets". The children when asked what they like to eat or drink - more frequently in the intervention group showed fruit. The SFVS proved to have an influence on lowering the barriers in F\&V consumption. There were significantly less responses ' I do not eat fruits because colleagues do not eat" ", 'I prefer to eat something sweet instead of fruit" 'and 'I do not eat vegetables because colleagues do not eat" in the intervention group.

Conclusions: The School Fruit and Vegetable Scheme as an effective strategy leads to positive changes in knowledge and some eating behaviours of children. The fact that F\&V were readily accessible at school as well as their consumption by schoolmates made eating F\&V more attractive for those children who had not consumed them for various reasons.

\section{$\mathrm{T} 2 \mathrm{P} 172$}

The television as an affordable and effective way to promote healthy diet and physical activity to prevent or treat obesity

Alamo, P. ${ }^{1}$; Castañeda, A. ${ }^{1}$; García, J. ${ }^{1}$; Arazola, D. ${ }^{1}$; Cubiles, M. ${ }^{2}$; Enguix, A. ${ }^{2}$; Pichardo, J.2

${ }^{1}$ La Báscula, ${ }^{2}$ University Of Sevilla, Statistic Department

In the last decades obesity has more than doubled and is, with overweight, the second leading cause of preventable death. Despite multiple strategies against obesity, no country to date has reduced the number of obese people. The way we currently treat obesity is not effective and many patients have already given up hope. To achieve World Health Organization's target to reverse this tendency we need dramatic and different actions to engage the civil society in creating demand for healthy style of life.

The objective of this study is to demonstrate that social media, such as the television can be used to convince the society that a healthy nutrition and physical activity are affordable, effective and necesary to prevent and to treat the obesity.

Methodology: 61 individuals ( 34 women and 27 men) with obesity (mean BMI 45,51) were recruited to follow, during 22 weeks, an intensive life style intervention in order to lose weight in a healthy manner. They were not isolated or moved from their usual environment. This programme included endocrinological and nutritional

assessment, promotion of physical activity and psychological support BMI were measured every week. Time to leave obesity between men and women was analysed with a survival analysis.

Results: BMI decreased in all the cases. Analysing Time to leave obesity during approximately the 30 th week , $25 \%$ of men were no longer obese and during the 39 th week $25 \%$ of women leave the obesity too.

Conclusion: We demonstrate to the audience that improving the quality of the diet and increasing the physical activity is a realistic way to lose weight. This evidence can encourage the people to act in their own self-interest changing their style of life in order to prevent or to reduce their overweight.

\section{T2P174}

\section{Trends in body mass index among adults aged 25-69 years in Germany, 1990-2011}

\section{Schienkiewitz, D. '; Lange, D. '; Mensink, D. ${ }^{1}$}

${ }^{1}$ Robert Koch-Institut

Introduction: In Germany, the prevalence of obesity has increased in the last decades [1]. It may be interesting to know to what extend this trend is a result of changes in the whole range of BMI or particular within the upper levels of the distribution.

Methods: We used measurements of body weight and height from 25-69 year old adults of three German National Health Interview and Examination Surveys conducted in 1990-1992 ( $\mathrm{n}=7466), 1997-1999(\mathrm{n}=5825)$ and 2008-2011 $(\mathrm{n}=5375)$ and examined trends in mean BMI $\left(\mathrm{kg} / \mathrm{m}^{2}\right)$ and BMI-categories defined according to WHO criteria. Means and prev- alence estimates were standardized to the German population Dec $31^{\text {st }}$ 2010 using SAS 9.4 survey procedures for complex sample designs.

Results: In the general population, mean BMI increased significantly from $26.7 \mathrm{~kg} / \mathrm{m}^{2}$ (95\%-CI: $\left.26.6-26.9\right)$ in $1990-1992$ to $27.0 \mathrm{~kg} / \mathrm{m}^{2}(26.8-$ 27.2) in 1997-1999, but was unchanged between 1997-1999 and 2008-11 (27.0, 26.8-27.2). Among men, mean BMI was higher in 2008-11 (27.5, $27.3-27.7)$ than in $1990-1992(27.0,26.8-27.1)$, especially among the age group 25-34 years (1990-1992: 25.5, 25.2-25.8, 2008-11: 26.1, 25.6-26.7) and 55-69 years (1990-1992: 27.7, 27.5-28.0; 2008-11: 28.5, 28.1-28.9), while there was no overall change among women. Over the decades, the BMI distribution among men has become wider and the increase is more pronounced in the upper percentiles. From 1990-1992 to 2008-2011 the prevalence of overweight decreased slightly but not significantly, whereas the prevalence of normal weight remained stable among men and women. During this period the prevalence of obesity increased from $18.9 \%$ (17.2$20.8)$ to $24.5 \%(22.1-27.0)$ among men and from $21.6 \%(19.8-23.4)$ to $23.0 \%$ (20.9-25.3) among women. Among those with obesity, the prevalence of obesity class I decreased significantly among men, but not among women, whereas among men the prevalence of obesity class II increased significantly from $11.7 \%(9.0-15.1)$ to 16.8 (13.3-20.9). Among women the prevalence of obesity class III increased from $8.6 \%(6.7-10.9)$ to $12.6 \%$ (9.8-16.0).

Conclusion: Between 1990 and 2011 mean BMI in adults has increased among men, especially among the younger and older age groups. This has resulted in an increased prevalence of obesity, particularly in the most severe level of obesity. To ensure that obesity prevention is targeted appropriately, it is important to monitor changes across the full BMI distribution as well as different classes of BMI.

\section{Reference:}

1 Mensink GB et al. Overweight and obesity in Germany: results of the German Health Interview and Examination Survey for Adults (DEGS1). Bundgesbl. 2013 May;56(5-6):786-94.

None Disclosed

The German National Health Interview and Examination Surveys were funded by the German Federal Ministry of Health.

\section{T2P175 \\ Use of progression criteria to support decision making in community based interventions}

Bryant, M. ; Dharni, N. '; Dickerson, J.2; Willan, K. ${ }^{2}$; Mceachan, R. ${ }^{2}$; Howell, $M .^{3}$

${ }^{1}$ University of Leeds, ${ }^{2}$ Bradford Institute Of Health Research, ${ }^{3}$ Bradford Trident

Introduction: Monitoring implementation and delivery of complex community based interventions is a challenge. Information collected usually includes process type measures like recruitment or attendance but there is no clear evaluation criteria for commissioners to aid decisions on whether to continue or decommission interventions. We developed a methodology informed by recent MRC guidance to identify 'progression' criteria and applied this to three early years parenting interventions (two group based courses, and one to one midwifery support) delivered as part of the Better Start Bradford (BSB) programme (www.betterstartbradford.org.uk).

Methods: We ran a workshop with 30 stakeholders including intervention delivery teams, commissioners, BSB facilitators and academics to determine the most important criteria their cut-offs for programmes to be 'Green' (meeting criteria targets), 'Amber' (falling short of targets) and 'Red' (targets not being met, instigating actions to resolve issues and decommissioning discussions). Participants individually ranked the importance of seven criteria modified from the MRC guidance for progression criteria (recruitment, reach, implementation, fidelity, attendance, satisfaction and data) for each intervention using an anonymous electronic voting system. They were then asked to decide the point at which interventions progress from 'Amber' to 'Red' using the same anonymous system for all seven criteria. Targets for each criteria were specific to each intervention (eg recruiting 10 parents per programme) but we applied proportions to 
permit generalisability across interventions (100\% of target recruitment). We considered meeting $100 \%$ of a target as reaching 'Green' status.

Results: The top three ranked progression criteria were recruitment, reach and implementation for the group based interventions and satisfaction, reach and fidelity for the one-to-one intervention. Median cut-offs to determine when an intervention moves from Amber to Red ranged from $65 \%$ for recruitment $(45-70 \%)$ to $87.5 \%(60-90 \%)$ for fidelity (i.e. $87 \%$ of intervention delivered according to manual). The median cut-off for satisfaction was $80 \%(60-90 \%)$ Responses were similar across participants, although median cut-offs were consistently higher from those whose roles involved facilitation of implementation and commissioning.

Conclusion: This methodology for monitoring the progression of interventions has resulted in a clear pathway which supports commissioners and intervention teams in decision making at local levels. In addition, we have developed a clear strategy for review, that includes timelines, and roles and responsibilities for decision-making.

Conflict of Interest: None disclosed

Funding: Big Lottery Funded as part of the A Better Start programme

\section{T2P176}

Waist to height ratio: Simpler and better screening anthropometric measurement for cardiometabolic risks than BMI in Turkish adults

\section{Kumas, M. ${ }^{1}$; ArITICl, G. $^{2}$}

${ }^{1}$ Acıbadem University, Health Sciences Faculty, Department of Nutrition and Dietetics, Istanbul, Turkey, ${ }^{2} \mathrm{AcIBadem}$ University

Introduction:Obesity is strongly associated with chronic metabolic diseases.There has been a well documented increase in the prevalence of obesity, assesed by body mass index(BMI), leads to an increased level of cardiometabolic risks. However, there are some evidences that central obesity, which is assessed by waist circumference and waist to height ratio is more assosciated with cardiometabolic risk factors. Waist to height ratio(WtHR) is anthropometric index used in the assessment of central obesity and it has strong correlation with central adiposity.Therefore the aim of this study was, to compare and investigate WtHR with BMI to see which anthropometric index is more reliable to predict cardiometabolic risks in Turkish adults.

Methods:The study population comprises 100 non-smoker Turkish volunteers aged between 20 years and 65 years. We excluded the volunteers who smoke regularly and use fish oil as supplements, due to their effects on high density lipoprotein cholestrol levels.Participants completed a specific questionare to acquire some background information, eating and lifestyle behaviours such as alcohol use, physical activity, smoking, supplement use, family history.Some biochemical parameters such as; fasting blood glucose, total cholestrol, LDL cholesterol, HDL cholestrol triglycerides were analyzed in all patients.Overall; anthropometric, biochemical and clinical parameters were measured.

Results:Sample size was 100.This sample includes 64 female and 36 male The mean WHtR in all adults was $0.52 \pm 0.25$, females $0.50 \pm 0.27$ had a significant lower WHtR than males $0.54 \pm 0.21(\mathrm{p}<0.05)$. WHtR had the highest correlation with metabolic parameters. WHtR had the highest correlation with triglycerides, fasting blood glucose, total cholestrol, systolic blood pressure in all adults, males and females in comparison to $\mathrm{BMI}$, a classical anthropometric index. In adults the correlation of WHtR with the traditional anthropometric parameters was highest for WC $(\mathrm{r}=0.923)$, followed by BMI $(\mathrm{r}=0.879)$.

Conclusion: In the light of high prevalence of obesity, it is necessary to find new anthropometric measurements to help identifying people that are at the greatest risk under the cardiometabolic diseases. Our data suggests that WHtR is better than other anthropometric indices in identifying obesity and associated with cardiometabolic diseases. These data provides that the use of WtHR shows the reliability of predicting cardiometabolic risk factors.In conclusion, WHtR is a simpler and more accurate anthropometric index identifying obesity associated with cardiometabolic risk among Turkish adults.We found a stronger correlation between WHtR with metabolic parameteres than classical anthropometric indices such as WC and BMI.

\section{T2P177 \\ Weight change and depression in South Korean adults: The Korea National Health and Nutrition Examination Survey 2011-2012}

\section{Kim, Y.'; Lee, K. ${ }^{2} ;$ Yoo, S. ${ }^{3}$}

${ }^{1}$ Korea University College of Medicine, Department of Family Medicine, ${ }^{2}$ Korea University College Of Medicine, Department Of Pediatrics, ${ }^{3}$ The Catholic University College Of Medicine, Department Of Endocrinology And Metabolism

Introduction: Obesity is one of the public health problems in South Korea. It has been known that psychological diseases such as depression was associated with obesity. Some studies found that subjects with obesity had higher rates of depression and weight change were found in the subjects with depression. Therefore, we evaluated the relationship between weight change and depression in the representative sample of Korean whole population.

Methods: We analyzed 17813 participants (7550 men and 10263 women) from the Korea National Health and Nutrition Examination Survey data 2011-2012. Subjects with depression was defined as those who received a diagnosis of depression by a doctor. Weight change was checked during 1 year and divided into three groups; no change, decreased, and increased. Multivariate logistic regression analyses were performed to examine the relationship between weight change and depression.

Results: Compared with no change group, the prevalence of depression was higher in the weight decreased group and weight increased group. Increased weight was associated with increased prevalence of depression in total subjects (Odds ratio (OR); 95\% Confidence interval $(\mathrm{CI})=$ $1.566 ; 1.218-2.013)$, and in female subjects (OR;95\% $\mathrm{CI}=1.603 ; 1.21$ to 2.122) compared with no weight change group. In obese group (body mass index $\geq 25 \mathrm{~kg} / \mathrm{m}^{2}$ ), weight change was not associated with the prevalence of depression, but increased weight was associated with the depression in non-obese group (OR;95\% CI = 1.670; 1.195-2.334).

Conclusion: Weight gain during 1 year was associated with the prevalence of depression, especially in women and non-obese group. Further prospective studies are needed to reveal causal relationship between weight change and depression.

Conflict of Interest: None Disclosed.

Funding: No Funding

\section{T2P178 \\ Weight management in a patient with Turner's syndrome and learning difficulty}

Kushe, M. ' ; Haridass, S. ' ;oseph, V. ${ }^{1}$; Khan, N. '; Rajeswaran, C. ${ }^{1}$

${ }^{1}$ The Midyorks NHS Hospitals Trust

Introduction: Prevalence of obesity is significantly higher in adults with intellectual disabilities than in the general population. The increase in the rate of obesity in these group of people has been linked to various factors like lack of understanding on balanced diet and limited access to healthy food.

Case report: A 26 year old lady with Turner's syndrome was referred to our weight management service. She had Type 2 diabetes, asthma, hypertension, learning difficulty and deafness. She was reviewed along with her partner who was her main carer. On her first assessment, she weighed $113.8 \mathrm{~kg}$ with BMI of $51.26 \mathrm{~kg} / \mathrm{m} 2$. She was provided a tailored weight management programme. Dietitian provided a simple and personalised dietetic intervention, which she and her partner could understand and follow. Physiotherapist helped in improving her activity at the same time. Insulin and oral hypoglycaemic agents were titrated during each visit by 
the diabetes specialist nurse. Her HbAlc dropped from 106 to $90 \mathrm{mmol} /$ mol in 6 months along with a weight loss of $24 \mathrm{~kg}$. There was a drop in BMI to $40 \mathrm{~kg} / \mathrm{m} 2$. Interestingly, there was no reduction in total daily dose of insulin.

Conclusion: Managing obesity is very challenging in people with Turner's syndrome. Although there are few studies on weight management in patients with learning difficulty, there is no guidance on how to effectively manage obesity in this group of people. Our experience shows that weight management in Turner's syndrome can be effectively achieved through a specialist multidisciplinary approach tailored and simplified to the person's need.

Conflict of Interest: None Disclosed

Funding: No Funding

\section{T2P179}

\section{Wellness treatments and self-esteem in overweight women}

\section{Bak-Sosnowska, M. ${ }^{\text {; }}$ Janik, D. ${ }^{\text {' }}$}

${ }^{1}$ Department of Psychology, Chair of Social Sciences and Humanities, School of Health Sciences in Katowice, Medical University of Silesia in Katowice, Poland

Introduction: Wellness is a conscious action on the body applying a variety of resources and environmental conditions to protect the health, maintain or improve the performance and optimize physiological processes of recreation. The goal of the study was to examine the relationship between the use of wellness treatments and the level of self-esteem and body esteem in overweight women.

Methods: The participants were 60 adult overweight women, their average age was $37 \pm 11$, the average BMI was $29,74 \pm 2,82$. The control group consisted of 60 women homogeneous in terms of their age and BMI, having never benefited from wellness treatments. The participants completed: Self-Esteem Scale (SES), Body-Esteem Scale (BES), Figure Rating Scale (FRS) and our own survey.

Results: In SES, $51,7 \%$ of the participants examined had low results, $41,6 \%$ of them scored average and $6,7 \%$ high. There were no differences between the examined and the control group $(p=0,14)$. In BED subscale, the examined group achieved the following results in terms of Sexual Attractiveness: $43,4 \%$ scored low, $43,4 \%$ average, and $13,2 \%$ high; as regards Weight Concern: $56,6 \%$ came out with low results, while the next $43,3 \%$ with average; in terms of Physical Condition: 51,7\% achieved low scores, $38,3 \%$ of them - average, $10 \%$ - high. Differences between groups occur only in the case of Physical Condition $(p=0.0107)$. Woman enjoying wellness treatments had significantly better physical condition. In FRS, the examined group perceived the present figure as a figure considerably stouter than ideal $(\mathrm{p}<0.0001)$. There were no differences between the groups in terms of the current figure, the ideal figure and the degree of divergence between the actual and the ideal figure.

Conclusion: Overweight women using wellness treatments differ from overweight women not using wellness in terms of physical condition. They do not differ in the overall self-assessment and in the other parameters of the body-evaluation.

Conflict of Interest: None Disclosed

Funding: No Funding

\section{T2P180}

\section{What are the developmental complex factors that predict} obesity in a 20-year-old adolescents in Japan?

\section{Kimura, A. $^{1}$.}

${ }^{1}$ Graduate School of Health Sciences Gunma PAZ College

Introduction: Although the incidence of obesity has been reported less frequently in young people 20-years-old(20s) in Japan, there is an in crease in the incidence of obesity in young people 20s born after 1995. It is thought that weight from birthday weight to 20 years old plays an important role in predicting obesity in a 20-year-old adolescent. We are aiming to evaluate the influence of developmental complex factors predicting obesity in a 20 -years-old adolescent.

Methods: The developmental factor influence predicting obesity in 20 s adolescents was analyzed by a retrospective cohort study. Predictors were birth weight, body weight(BW) of 3-year-old, BW of 12-year-old, BW of 15-year-old. Presence of exercise habits in high school days, presence of physical inactivity for 20 s, presence of vegetables and fruits intake in 20 s 's meal, presence of breakfast intake habits for 20s, and presence of blood glucose spike(GS) in 20s. We analyzed using a logistic regression model with the presence of obesity as a dependent variable by IBM-SPSS ver20. Results: 67 subjects (mean age $21 \pm 0.4$ years old, 51\% women) were included in the analysis. The frequency of obesity was $13.4 \%(1.079$ to 78.219). After adjustment of the individual factors of the effect on obesity, A best predict model is composed the BW of a 12-year-old, and gender, constant(error). The only significant predictor was the BW of a 12 -yearold 1.222 (1.038 to 1.438$)$. gender $0.05(0.003$ to 0.831 ), constant 0.0098 . Differences in effect by gender were shown. Interestingly,there did not show the relationship to prediction of obesity. These factors were the vegetable and fruit intake at 20-year-old, birth weight, BW of 3-year-old and 15-year-old, exercise habits in high school days, physical inactivity for $20 \mathrm{~s}$, breakfast intake habits for $20 \mathrm{~s}$, GS in $20 \mathrm{~s}$. Odds are the birth weight $0.998(0.992$ to 1.004$)$, the body weight of 3-year-old 1.62(0.364 to 7.201), the body weight of 12-year-old $1.109(0.882$ to 1.395$)$, the body weight of 15-year-old $1.369(0.92$ to 2.037$)$, the presence or absence of exercise habits in high school days 0 , the presence or absence of body inactivity for 20 -year-old 0.001 , the presence or absence of vegetables and fruits intake in 20-year-old 's meal 5.622(0.067 to 471.68), the presence of breakfast intake habits for 20-year-old 0 , and the presence or absence of blood glucose spike in 20-year-old 0.185(0.003 to 12.814).The influence of economic depression in Japan around 2008 also may be affecting the widespread adoption of cheap glucose foods imported ingredients from traditional Japanese cuisine.

Conclusion: Our findings probably shows body weight of the 12-yearold associated around 2008 as a developmental complex factor predicting obesity at a 20 -year-old adolescents.

\section{T2P181 \\ Which one is better as an intermittent fasting model, overnight or daylight fasting? A comparison study according to the metabolic parameters in humans}

\section{Aksungar, F. ${ }^{1}$; Kolay, M. ${ }^{2}$; Pak, M. ${ }^{2}$; Pinarbasi, A. ${ }^{2}$; Serteser, M. ' Unsal, I. ${ }^{1}$}

${ }^{1}$ Acibadem University, School of Medicine, Department of Biochemistry, Acibadem Labmed Clinical Laboratories, ${ }^{2}$ Acibadem University, School Of Medicine, Department Of Biochemistry

Introduction: Scientists are well aware of the advantages of reducing calories in both animals and humans in having healthier life span. Recently the question is "Are there any alternatives to daily calorie restriction (CR)?". Intermitten fasting (IF) is shown to be an alternative feeding model in terms of improving metabolic health. IF model involves alternating cycles of fasting and eating which may result in reduced calories in total in a given time. Our group compared intermittent fasting and daily calory restriction (CR) models in several studies and we clearly found positive effects of IF. In the present study we have compared the changes in metabolic parameters between overnight fasting and daylight fasting which are two different IF models for humans.

Methods: Twenty-nine subjects ( 17 female, 12 male) were included in the study. Subjects fasted overnight and 4days later the same subjects fasted during the daylight hours. Fasting duration was 15 hours in both model and there was no eating and drinking. Blood and urine sampling were performed at the end of the fasting period and 1 hour after the meal. Glucose, insulin, glucagon, ketone body, thyroid stimulating Hormone (TSH), triglycerides and alkaline phosphatase (ALP) levels were measured in 4 samplings (fasting and fed state) in each subject in each model. 
Results: Keton body production in daylight fasting was higher than overnight fasting $(0.17 \pm 0.13$ vs $0.09 \pm 0.05 \mathrm{mmol} / \mathrm{L} \mathrm{p}<0.001)$ while insulin levels were lower in daylight fasting $(\mathrm{p}<0.5)$. TSH levels were statistically very high in fed state, in daylight fasting $(\mathrm{p}<0,001)$. Finally ALP, glucagon and glucose levels were not statistically different in two models. Conclusion: This comparison study is a part of an ongoing IF study in which subjects are fasting for 15 hours daily for 30 consecutive days. Our preliminary results, representing the first days of the study, suggest that daylight fasting results in more ketone body production which can be a positive effect on body metabolism. Physiologically produced high ketone levels are shown to have beneficial effects specially on neurons. The differences between the two models may be attributed to sleep/active status of the body which reflects the parasympathetic/sympathetic nervous system dominance, respectively.

\section{T2P183}

\section{Young people's experiences and attitudes on the use and} retention of lifestyle apps in six countries. A qualitative study.

\section{Nikolaou, C. ${ }^{1}$; Lean, M. $^{2}$}

${ }^{1}$ Universite Catholique de Louvain, ${ }^{2}$ University Of Glasgow

Introduction: Lifestyle mobile apps are increasingly popular, but most lack any scientific input in design or evaluation. The highest rate of unwanted weight-gain is in teenagers and young adults, who favour webbased and mobile technologies, but the use of lifestyle or weight-control apps by this age group is underexplored.

Methods: A recruitment questionnaire was devised, and a link targeting young people aged13-24 years old in Belgium, UK, Finland, Greece, Singapore, and New Zealand was posted on Facebook for an 86-hour period. Those completing the questionnaire were then randomised to attend online focus groups, stratified by country, age, and gender. The online discussions were asynchronous and hosted in the password-protected Webspace of Proboards. A topic guide was developed to guide the online discussions. Data were analysed qualitatively using thematic analysis. Results: Over 84 hours, 834 adolescents aged $13-18$ years and 1,447 young adults aged 19-24 years answered the recruitment questionnaire. Of those randomised to focus groups, $89.0 \%$ of adolescents and $91.0 \%$ of young adults responded and contributed. Eight focus groups of 371 and 658 individuals were conducted; four with adolescents: females $=65.0 \%$, mean age $=15.2$ (SD1.5) years, and four with young adults, females $=61.0 \%$, mean age $=19.3(1.4)$ years. The adolescents reported seldom using lifestyle mobile apps, except for those incorporated into their smart-phones, but they are interested in using apps on weight gain prevention, identifying as main themes for app use: cost, personalization, and appropriate content. The young adults commonly reported using lifestyle apps downloaded from app stores, favouring those free of cost, and have greater interest than adolescents in environmental issues around diet and health. The main themes they identified for app use were: data security, cost, and personalization. Adolescents and young adults from Singapore were most interested in mobile apps and reported spending more time in social media but other countries followed up closely.

Conclusion: Young people willingly respond to surveys on social media and are interested in using mobile apps for weight-gain prevention, provided they are personalised with age-relevant content, free, and secure.

Conflict of Interest: None Disclosed

Funding: Research relating to this abstract was funded by a Marie Curie fellowship to Dr Charoula Nikolaou.
PO3 - Childhood Obesity

T3P1

\section{A change in the serum leptin level is associated with the improvement of the binge eating symptoms of women with overweight or obesity undergoing weight loss intervention}

\section{Nozaki, T. ${ }^{1}$; Sawamoto, R. '; Nishihara, T. ; Hata, T. ; Furukawa, T.' ;} Komaki, G. ${ }^{2}$; Sudo, N.

${ }^{1}$ Department of Psychosomatic Medicine, Graduate School of Medical Sciences, Kyushu University, ${ }^{2}$ School Of Health Sciences Fukuoka, International University Of Health And Welfare

Introduction: Patients with obesity seeking weight loss treatment often have a binge eating problem; however, the biological mechanisms related to the development of the binge eating are not clear. This study was done to determine if hormones are related to the binge eating of women with overweight or obesity who participated in a weight loss intervention (WLI) based on cognitive behavior therapy.

Methods: Ninety women with overweight or obesity (age 46.8 \pm 11 ; BMI $31.5 \pm 4.7 \mathrm{~kg} / \mathrm{m}^{2}$ ) completed seven months of WLI. Body fat percentage (BFP) was measured using dual energy $\mathrm{x}$-ray absorptiometry and serum levels of the biomarkers ghrelin, leptin, adiponectin, IL-6, IGF-1, and insulin were measured before and after the treatment. Binge eating status was assessed by the Binge Eating Scale (BES).

Results: The baseline BES score, adjusted for age and BFP, was significantly associated with the leptin, Il-6, and insulin levels. $\triangle \mathrm{BFP}$ was significantly associated with the $\triangle \mathrm{BES}$ score independently of the baseline BES score. The leptin, insulin, and IL-6 levels were significantly decreased and the ghrelin and adiponectin levels significantly increased after treatment. However, only $\triangle$ leptin was associated with the $\triangle B E S$ score independently of the baseline BES score and $\triangle \mathrm{BFP}$.

Conclusion: The present study shows that a change in the serum leptin level is related to the improvement of binge eating symptoms, regardless of the degree of body fat loss among women with overweight or obesity during WLI.

Conflict of Interest: None Disclosed

Funding: This study was supported by a Research Grant (23-2) for Nervous and Mental Disorders from the Ministry of Health, Labour and Welfare of Japan and a Grant-in-Aid for Scientific Research (25460902) from the Japan Society for the Promotion of Science, and a Budget for clinical research base development project (GAQQ250001) from the Ministry of Health, Labour and Welfare of Japan.

T3P2

A community-based lifestyle intervention to prevent type 2 diabetes in British ethnic minority adults at high risk: Beforeand-after study

\section{Rodrigues, A. ${ }^{1}$; White, M. ${ }^{2}$; Sniehotta, F.' ${ }^{1}$ Penn, L. ${ }^{\prime}$}

${ }^{1}$ Institute of Health \& Society, Newcastle University, UK, ${ }^{2}$ Centre For Diet And Activity Research (Cedar), Mrc Epidemiology Unit, University Of Cambridge, Uk

Introduction: Evidence from randomised-controlled trials suggested that behavioural interventions with goals of weight loss, increased physical activity (PA) and healthy diet (increased fibre and reduced fat consumption), can reduce risk of developing type 2 diabetes (T2D) in adults at high-risk. This study aimed to assess recruitment and retention, and likely effectiveness at 12 month follow-up of a behavioural intervention, culturally adapted for British ethnic minority adults at high risk of T2D.

Methods: Adults over 18 years of age of non-white ethnicity with a Finnish Diabetes Risk Score (FINDRISC) $\geq 11$ were eligible. An 8-week supported programme to promote increased PA, healthy eating, and weight management was delivered separately to women and men by fitness trainers as free weekly one-hour group PA sessions, each followed by behavioural counselling, with support to 12 months in leisure and commu- 
nity settings. We assessed recruitment and retention rates, and change in behavioural and health-related outcomes at 6 and 12 months.

Results: From 885 registers of interest, 379 women and 107 men were recruited to the programme. Additional efforts were made to encourage the recruitment of men, including organising screening events in places where men were likely to attend, such as at the Mosque and through a local taxi firm. Baseline mean (SD) age was 38.7 (9.8)years, FINDRISC score 14.0 (2.9), weight $76.5(15.6) \mathrm{kg}$, BMI $30.0(5.6) \mathrm{kgm}^{-2}$, waist circumference $100.4(13.9) \mathrm{cm}$ and PA levels (self-report) daily total $51.6(8.0)$ MET-hours/day equivalent. Follow-up at 12 months was completed by 374 (77\%; 330 women and 44 men) with mean change (95\% CI) from baseline in participants' weight $-2.7(-3.5$ to -1.9$) \mathrm{kg}$, waist circumference $-4.9(-5.9$ to -3.9$) \mathrm{cm}$, and PA level $1.8(-0.3$ to 3.8)MET-hours per day equivalent. The changes in weight and waist circumference were significant for women only (weight: $-3.0[-3.8 ;-2.2]$; waist: $-5.3[-6.4 ;-4.2]$ ).

Conclusions: Recruitment and retention of UK ethnic minority women to a culturally adapted behavioural intervention for prevention of T2D was feasible and the intervention is likely to be effective in reducing weight at 12 months. However, recruitment and retention of men was less successful and requires further development.

\section{T3P3}

\section{A comparison in baseline characteristics of individuals joining a weight loss programme comprised of a VLCD with behaviour-change sessions delivered via either an eHealth or a face to face platform}

Johnston, K. '; Philippou, J. '; Dyson, L. '; Cox, J.'; Hewlett, B. ${ }^{1}$

${ }^{1}$ LighterLife UK Ltd

LighterLife is a commercial provider of very low calorie diet (VLCD) weight loss programmes which are delivered in conjunction with behavioural-change therapy underpinned by group support via one of two means: eHealth platform (LL2U) or face to face (F2F). We have shown previously in age and BMI matched individuals that there is no difference in initial weight loss between these two delivery methods ${ }^{1}$. However, an assessment of demographic characteristics at baseline of those who remain engaged over the longer-term may prove useful in facilitating an individual's choice of programme such that their likelihood of longer term weight loss success is increased. As such, the objective of this study was to evaluate and compare the baseline characteristics of individuals embarking on either a F2F or LL2U weight loss programme.

Baseline participant data for overweight and obese first-time clients during 2015/16 were obtained from the LighterLife database. Individuals with complete registration records, including their age, gender, starting BMI, use of medication for type 2 diabetes (T2DM) and whose records indicated that they both purchased foodpacks and engaged with groupbased behavioural change activities, were selected for inclusion in this study. Descriptive statistics include means and standard deviations (SD) for continuous data and comparisons between groups were performed using unpaired t-tests (OpenStat, version 2013 http://www.statpages.org/ miller/openstat).

Data for $5122(12.8 \%$ males $)$ individuals were included and there were significantly more females than males (4465 vs. $657 ; \mathrm{p}<0.001)$. Whilst $81 \%$ of all participants registered with the F2F programme, a significantly higher number of females (20\%) engaged with LL2U than did males (13\%).

Results show that the average age and BMI respectively for all participants was $43.78 \pm 12.09$ years and $34.62 \pm 6.53 \mathrm{~kg} / \mathrm{m}^{2}$ (females) and $42.12 \pm 11.76$ years and $36.48 \pm 6.51 \mathrm{~kg} / \mathrm{m}^{2}$ (males). There were no significant differences between groups (LL2U vs. F2F) in age (females: $43.31 \pm 10.61$ vs. $43.90 \pm 12.44$; males: $42.51 \pm 10.23$ vs. $42.06 \pm 11.97$ ) or starting BMI (females: $34.58 \pm 6.80$ vs. $34.63 \pm 6.46$; males: $35.82 \pm 5.59$ vs. $36.58 \pm 6.64) .0 .76 \%$ of females and $1.67 \%$ of males indicated that they were taking medication for T2DM with the majority of these (98\%) engaging with the F2F programme.
These data show that there are demographic differences between groups of first-time clients who engage with either LL2U or F2F for initial weight loss. Further analysis looking at differences in longer term-engagement and weight loss success between these methods of delivery is currently underway.

\section{References:}

1 Johnston KL et al. Obesity Facts, Volume 8, Supplement 1, T4:PO.015 (2015)

Conflict of Interest: All authors are employed by LighterLife

\section{T3P4}

\section{A low glycaemic diet in pregnancy induces DNA methylation variation in blood of newborns at risk of macrosomia: Results from the ROLO study}

\section{Geraghty, A. '; Fransquet, P. ; Alberdi, G. '; O'Brien, E. '; Saffery, R. ${ }^{2}$; McAuliffe, $F^{3}$}

1UCD Perinatal Research Centre, Obstetrics \& Gynaecology, School of Medicine, University College Dublin, National Maternity Hospital, Dublin, Ireland, ${ }^{2}$ Cancer And Disease Epigenetics, Murdoch Children'S Research Institute, Melbourne, Victoria, Australia, ${ }^{3}$ Ucd Perinatal Research Centre, Obstetrics And Gynaecology, School Of Medicine, University College Dublin, National Maternity Hospital, Dublin 2, Ireland

Background: The in-utero environment is key to healthy fetal development. The epigenetic profile of the developing fetus is sensitive to environmental influence, with mounting research suggesting a role of variable DNA methylation in fetal programming of risk for a range of common non-communicable diseases. Research shows maternal diet may influence individual DNA methylation patterns in offspring but research in humans is limited.

Methods: Sixty sex-matched infants from a cohort of the ROLO (Randomised cOntrol trial of LOw glycaemic index to prevent recurrence of fetal macrosomia) study were analysed. Using genome-scale profiling, we investigated DNA methylation in neonatal cord blood using the Illumina MethylationEPIC BeadChip. Principal components analysis was undertaken and epigenome-wide associations were examined using linear regression of methylation values at over $850,000 \mathrm{CpG}$ sites. Associations with maternal and fetal factors were also explored.

Results: Principal component and regression analysis revealed widespread variation in the DNA profile of newborns of mothers exposed to the dietary intervention, accounting for $13 \%$ of total variation within the methylation dataset. No association was found with maternal early-pregnancy BMI or infant sex. Pathway analysis identified common influences of the dietary intervention on multiple aspects of cellular function. Locus-specific replication of a subset of these sites in a larger sample failed to replicate the original genome-wide findings in terms of the direction of effect however some potential sex-specific effects were noted.

Conclusions: Using a small discovery sample, we identified widespread differential methylation in progeny of mothers exposed to a low glycaemic dietary intervention. Novel genes were identified that may be associated with the intervention. These findings provide preliminary evidence for a role of dietary interventions in modifying the potentially detrimental epigenetic variation associated with transmission of poor metabolic health from mothers to progeny.

\section{Conflict of Interest: None}

Funding: Research relating to this abstract was funded by the Health Research Board Ireland, the Health Research Centre for Health and Diet Research, The National Maternity Hospital Medical Fund and the European Union's Seventh Framework Programme (FP7/2007-2013), project EarlyNutrition under grant agreement no. 289346 


\section{T3P5}

\section{A new swallowable intragastric balloon for weight loss not needing endoscopy: Early experience in combination with a VLCKD}

lenca, R. ${ }^{1}$; Ernesti, I. ${ }^{1}$; Casella, G. ${ }^{2}$; Francomano, D. ${ }^{3}$; Cipriano, M. ${ }^{2}$; Genco, $A .^{2}$

${ }^{1}$ department of experimental medicine-medical physiopathology, food science and endocrinology section, Sapienza University of Rome, Italy, ${ }^{2}$ Department Of Surgical Sciences, Surgical Endoscopy Unit, Sapienza University Of Rome, Italy, ${ }^{3}$ Department Of Xperimental Medicine- Medical Physiopathology, Food Science And Endocrinlogy Section, Sapienza University Of Rome

Background: Intragastric balloons (IGBs) have been used as weight loss devices for decades ${ }^{1}$. Their mechanism of action is likely multifactorial, but they appear to make it easier to adhere to a low calorie diet by inducing satiety ${ }^{2}$. However, all IGBs have historically required endoscopy and sedation resulting in a low adoption rate and high cost.

Aims: To evaluate the efficacy and safety of a new swallowable IGB (Elipse $^{\mathrm{Tx}}$ Balloon, Allurion Technologies, MA, USA) not needing endoscopy or anesthesia in combination with a very low calorie ketogenic diet (VLCKD) in the final month of therapy.

Methods: This was a prospective, non-randomized study in 50 obese individuals (BMI 30-45 kg/m²) who refused other IGBs treatment and were offered the Elipse Balloon. The balloon is swallowed under fluoroscopy and then filled with $550 \mathrm{~mL}$ of liquid. The balloon remains in the stomach for 16 weeks after which it spontaneously opens, empties, and is excreted. Follow-up was performed every 2 weeks. In the last 4 weeks of treatment, a ketogenic diet ( $700 \mathrm{kcal} /$ day $)$ was introduced to enhance weight loss At balloon excretion, patients were transitioned to a Mediterranean diet. Results: $42(29 \mathrm{M} / 13 \mathrm{~F})$ patients were enrolled. Mean age was $46.4 \pm 10.6$ years, mean initial weight was $109.7 \pm 21.9 \mathrm{~kg}$, and mean initial BMI was $38.6 \pm 6.7 \mathrm{~kg} / \mathrm{m}^{2}$. After 16 weeks, the mean weight loss was $15.2 \pm 2,6 \mathrm{~kg}$, mean percent excess weight loss was about $31 \%$, and mean BMI reduction was $4.9 \pm 1.1 \mathrm{~kg} / \mathrm{m}^{2}$. At 4, 8 and 12 weeks a mean weight loss of 5,4 kg, $8,9 \mathrm{~kg}$ and $11,5 \mathrm{~kg}$ was observed, respectively. In the VLCKD period, we observed a mean weight loss of $3,7 \pm 0,9 \mathrm{~kg}$, similar to that observed between weeks 4 and 8 . There were no serious adverse events. All other adverse events including nausea, vomiting, and abdominal pain were either self-limiting or resolved with medication. Significant reductions were also observed in obesity-related co-morbidities.

Conclusion: The Elipse Balloon appears to be a safe and effective weight loss method. Furthermore, the introduction of a VLCKD improves weight loss. The procedureless nature of the Elipse Balloon may make it amenable to a larger population of obese patients not responding to diet treatment and a variety of clinicians (e.g. nutritionists, dietitians, and internists) who currently do not have access to endoscopic or surgical weight loss devices.

References:

1 Genco A. et al. BioEnterics Intragastric Balloon: The Italian Experience with 2,515 Patients. Obesity surgery. 2005 Sep;15(8):1161-4

$2 \mathrm{Kim} \mathrm{SH}$. et al. Current status of intragastric balloon for obesity treatment. World J Gastroenterol. 2016 Jun 28;22(24):5495-504

Conflict of Interest: The authors have no conflict of interest

\section{T3P6}

\section{A randomised controlled trial assessing the impact of intermittent energy restriction (IER) on weight loss and insulin sensitivity in healthy men and women with central obesity. "The Met-IER study"}

Pinto, A. '; Bordoli, C. '; Buckner, L. ; Kaplan, P. ${ }^{1}$; Arenal, I. ' Jeffcock, E. '; Kim, C. ; Johnston, K. ${ }^{2}$; Hall, W. ${ }^{1}$

${ }^{1}$ Faculty of Life Sciences and Medicine, Kings College London, ${ }^{2}$ Lighterlife Uk Ltd

The effectiveness of intermittent energy restriction (IER) diets for cardio-metabolic health compared with continuous energy restriction (CER) diets is unclear. Using a parallel-design RCT in 45 healthy men and women (aged 35-75 y) with central obesity, we compared the shortterm effects of IER and CER on cardio-metabolic risk factors. IER was 2 consecutive days of very low energy diet (VLED; LighterLife UK Ltd) plus a 5 -d healthy eating pattern for $4 \mathrm{wk}$. CER comprised a $-500 \mathrm{kcal} / \mathrm{day}$ healthy eating pattern. The primary outcome was insulin sensitivity using revised-QUICKI (R-QUICKI). Secondary outcomes included HOMA-IR, fasting glucose and insulin. Treatment effects were assessed at endpoint (at least 2 days after the last VLED day for IER) by ANCOVA (adjusted for baseline, sex and compliance). Baseline weights were (mean $\pm \mathrm{SD}, \mathrm{n}=43$ ) $102 \pm 17 \mathrm{~kg}$ in men and $83 \pm 17 \mathrm{~kg}$ in women. Percentage weight loss did not significantly differ between groups: IER, $-3.1 \%$ (95\%CI -3.9, -2.3); CER, $-2.7 \%$ (95\%CI $-3.5,-1.9)$. Insulin sensitivity significantly increased in the whole cohort (R-QUICKI: mean baseline $0.39 \pm 0.04$; mean change in R-QUICKI 0.02, 95\% CI 0.01, 0.04, $P<0.0001$ ), but there were no significant treatment differences at endpoint (IER group: $\mathrm{n}=21$, mean 0.41 , 95\% CI 0.38, 0.43; CER: $\mathrm{n}=22$, mean $0.41,95 \%$ CI 0.38, 0.44). There were no treatment differences for fasting HOMA-IR and plasma insulin. Plasma glucose was $7 \%$ higher (95\% CI $1,13, P=0.025)$, and fasting NEFA was non-significantly $13 \%$ lower (95\% CI -25, 2, $P=0.080)$, following IER compared with CER. Statistically significant acute differences within the IER group (post- $2 \mathrm{~d}$ IER versus CER diet endpoints) included decreased insulin $(P=0.011)$, and HOMA-IR $(P=0.008)$, with no acute differences effects on R-QUICKI. Selecting either a standard CER diet or " $5: 2$ " IER diet, in combination with adherence to healthy eating guidelines, results in equivalent improvements in insulin sensitivity. Acute benefits of a 2-d VLED for insulin resistance are transient but may provide cumulative benefit over a longer time.

Conflicts of Interest: KLJ is employed by LighterLife UK Ltd

Funding: Research relating to this abstract was funded by LighterLife UK Ltd

\section{T3P7}

\section{A systematic review update of psychosocial pretreatment predictors of weight control}

\section{Carraça, E. ${ }^{\text {; }}$ Santos, I. ' ; Mata, J. ${ }^{2}$; Teixeira, . $^{1}$}

${ }^{1}$ CIPER, Faculty of Human Kinetics, University of Lisbon, ${ }^{2}$ Faculty Of Social Sciences (Health Psychology), University Of Mannheim, Germany

Objective: Systematically identifying pretreatment characteristics that predict which participants will successfully lose weight is important to improve intervention efficacy and clinical practice. This study provides a research update on pretreatment predictors as follow-up on a 2005 review. Method: Results of 36 recent original studies from peer-reviewed journals were merged with results from the 2005 review. The 2005 categories were largely maintained to allow comparability. A meta-analysis of data was performed where feasible given the data. Results: Fewer previous weight loss attempts were the most consistent pretreatment predictor of weight loss. Other frequently assessed variables, including body image and exercise self-efficacy, were often but not consistently correlated with subsequent weight loss. Importantly, several variables were consistently identified as non-predictors of weight loss. To date, many psychosocial factors remain too little studied to allow reliable conclusions regarding their predictive value. However, frequently studied predictors and non-predictors show consistent effects on weight loss, also in comparison to the 2005 review. Conclusion: Given the importance of previous weight loss attempts for successful weight management, interventions should evaluate related metabolic or psychosocial factors to better understand how previous dieting affects future weight management success. Several potentially negative factors can now be reliably discarded as predictors of weight loss, suggesting that individuals can successfully lose weight despite scoring high on these factors. In the face of a comparably thin empirical basis for many pretreatment predictors, further research is essential to move the field forward. Implications of the current state of research and necessary steps to improve intervention efficacy and clinical practice are discussed. Keywords: Correlates, Moderators, Obesity, Weight Management, Success 
T3P8

\section{Adding compassion-focused modules to a commercial weight management program: Effect on shame, self-criticism, self- reassurance and well-being.}

\section{Stalker, C'; Catarino, F²; Duarte, C33 Basran J'; Gilbert, P'; Horgan, G';} Morris L'; Stubbs R. J. ${ }^{1}$

${ }^{1}$ College of Life and Natural Sciences, University of Derby, Derby, DE22 1GB., ${ }^{2}$ College Of Life And Natural Sciences, University Of Derby, Derby, De22 1Gb., ${ }^{3}$ Cognitive And Behavioural Centre For Research And Intervention, University Of Coimbra, Rua Do Colégio Novo, Apartado 6153, 3001-802 Coimbra, Portugal

Introduction: We have previously shown in cross sectional data how shame and self-criticism were associated with disinhibition, perceived hunger and historical weight loss. Compassion-focused therapy (CFT) is a talking therapy that aims to reduce shame and self-criticism. This intervention examined whether adding compassion-focused online video exercises into a commercial weight-management programme (WMP) (the intervention) helped reduce shame, self-criticism and negative affect compared to the regular programme.

Methods: This was a two-arm, non-randomised parallel design (i) control arm: multicomponent WMP (ii) intervention arm: The same multicomponent WMP with the addition of 2 days training for weight management group leaders (Consultants) and 8 short online video exercises available to participants throughout the trial. 18 Consultants were allocated to intervention vs control based on matched performance criteria. 964 ongoing participants, who had on average lost 10.8\% (Control) and 9.8\% (Intervention) consented to join the trial arm to which their Consultant was allocated. The Weight-Focused External Shame, Body Image Shame, Weight-focused Self-Criticising and Self Reassuring, DASS and Warwick Edinburgh Wellbeing scales were administered to participants at 0, 3, 6 and 12 months. Intervention and control were compared by mixed model ANOVA of differences from baseline, with adjustment for baseline values of all outcome variables. PCA was used to avoid problems of multicollinearity among these baseline covariates. Only the first component was found to differ between groups. The baseline value of the variable being modelled was included as a separate covariate. Consultant ID was included as a random effect.

Results: The intervention significantly reduced external shame, inadequate self, hated-self and negative affect between 3-12 months ( $p<0.04$ 0.001 ; Cohen's $d=0.18-0.23$ ). Body image shame decreased over 3 and 6 ( $\mathrm{p}<0.002 ; \mathrm{d}=0.27$ ) but not 12 months; positive affect and self-reassurance increased over $3-12$ months $(p<0.004 ; d=0.21-0.25)$. The intervention had a transient 3-month effect on DASS and well-being outcomes (all $\mathrm{p}<0.036 ; 0.19-0.32$ ). The intervention reduced drop out by $10 \%$ over 12 months.

Conclusion: A light touch, web-based CFT intervention improved emotional/psychological well-being over 3 months and reduced drop out in participants of a commercial WMP and may benefit those who struggle with self-evaluation and psychological adjustment.

Conflict of Interest: RJS is a consultant to Slimming World through University of Leeds Consulting.

Funding: CD was funded by Fundação para a Ciência e a Tecnologia - SFRH/ $\mathrm{BD} / 76858 / 2011$. This study was funded by a grant i from Slimming World UK.

\section{Adding compassion-focused modules to a commercial weight management programme: Effect on control of eating and weight outcomes.}

\section{Stalker C. ; Catarino, F'; Duarte, C2 ; Basran J. ${ }^{3}$; Gilbert, P'; Horgan, G4;} Stubbs R. J. ${ }^{5}$

${ }^{1}$ College of Life and Natural Sciences, University of Derby, Derby, DE22, 1GB., ${ }^{2}$ Cognitive And Behavioural Centre For Research And Intervention, University Of Coimbra, Rua Do Colégio Novo, Apartado 6153, 3001-802 Coimbra, Portugal, ${ }^{3}$ Ollege Of Life And Natural Sciences, University Of Derby, Derby, De22, 1 Gb., ${ }^{4}$ Biomathematics And Statistics Scotland, Rowett Institute Of Nutrition And Health, Foresterhill House, Aberdeen Ab25 2Zd, ${ }^{5}$ Appetite Control And Energy Balance Research Group, School Of Psychology, Faculty Of Medicine And Health, University Of Leeds, Ls2 9Jt

Compassion-focused therapy (CFT) is a talking therapy that aims to reduce shame and self-criticism and help people be compassionate to themselves. This intervention examined whether adding compassion-focused online video exercises into a commercial weight-management programme (WMP) (the intervention) helped people control their eating behaviours and therefore body weight compared to the regular programme.

Methods: This was a two-arm, non-randomised parallel design (i) control arm: multicomponent WMP (ii) intervention arm: The same multicomponent WMP with the addition of 2 days training for weight management group leaders (Consultants) and 8 short online video exercises available to participants throughout the trial. 18 Consultants were allocated to intervention vs control based on matched performance criteria. 964 ongoing participants, who had on average lost 10.8\% (Control) and 9.8\% (Intervention) consented to join the trial arm to which their Consultant was allocated. Restraint, Disinhibition, Perceived Hunger Flexible Control, Rigid Control (TFEQ), and binge eating (Gormally Binge Eating Scale), Restrictive Eating and Eating Guilt (Revised Rigid Restraint-Eating Scale) were measured at $0,3,6$ and 12 months. Weight was recorded during weekly. Intervention and control were compared by mixed model ANOVA of differences from baseline, with adjustment for baseline values of all outcome variables. PCA was used to avoid problems of multicollinearity among these baseline covariates. Only the first component was found to differ between groups. The baseline value of the variable being modelled was included as a separate covariate. Consultant ID was included as a random effect.

Results: There were slight, significant $(\mathrm{p}<0.01)$ weight losses from month $0-12$ in both arms $(1.1 \mathrm{~kg}$ and $1.6 \mathrm{~kg})$, but no intervention effect on weight loss at 3, 6 or 12 months. There was no intervention effect on restraint, disinhibition, susceptibility to hunger or restrictive eating at any time point. There was an effect on eating guilt $(\mathrm{P}<0.001$; Cohen's $\mathrm{d}=0.23-0.26)$ and binge eating ( $\mathrm{P}<0.022 ; \mathrm{d}=0.27-0.35)$ (from 3-12 months).

Conclusion: Inclusion of light-touch online digital exercises via a webpage in a WMP had no effect on control of eating or weight outcomes but improved binge eating symptomology and eating guilt. Such approaches may provide specific tools to meet the differing needs of programme participants.

Conflict of Interest: RJS is a consultant to Slimming World through University of Leeds Consulting.

Funding: This study was funded by a grant from Slimming World UK.

\section{T3P10}

\section{Age as a determinant of weight loss after bariatric surgery}

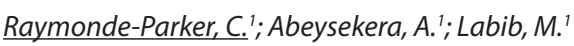

${ }^{1}$ Russells Hall Hospital, The Dudley Group NHS Foundation Trust

Introduction: There have been conflicting data regarding the effect of age on bariatric surgery outcomes. Some studies have shown that older patients lose less weight following bariatric surgery and also are at greater risk of developing complications. However, some recent studies 
have shown that older patients achieve weight loss comparable to that of younger patients.

Methods: We audited the outcome at one and three years in 30 patients aged $<45$ years and 30 patients $\geq 45$ years (matched for gender and type of surgical procedure) who underwent either gastric bypass or sleeve gastrectomy operations, in order to assess the difference in \% excess body weight (\%EBW) loss between the two groups. Patients who did not attend for either their 1 year or 3 year follow up appointments were excluded from selection.

Results: The pre-surgery mean BMI was $50.0 \pm 9.1 \mathrm{~kg} / \mathrm{m}^{2}$ in those aged $<45$ years and $52.1 \pm 11.9 \mathrm{~kg} / \mathrm{m}^{2}$ for those aged $\geq 45$ years $(\mathrm{p}=0.45)$. At one year, the mean \%EBW loss in the younger group (70.2 $\pm 15.7 \%)$ was significantly greater than the \%EBW in the older group $(58.5 \pm 17.6 \%)$ $[\mathrm{p}<0.05]$. However, at three years post-surgery, the mean \%EBW loss in the younger group $(67.0 \pm 20.3 \%)$ was not significantly different from $\%$ EBW loss in the older group $(60.5 \pm 24.1 \%)$ [p $=0.26]$.

Conclusion: Our audit showed that younger patients lost more weight compared to older patients at one year after bariatric surgery but, at three years, weight loss was not significantly different between younger and older patients. This suggests that behavioural modifications somewhat converge between younger and older patients and that, in the longer term, age does not seem to influence bariatric surgery outcomes.

\section{T3P12}

\section{Anemia and hemoglobin levels in post-menopausal women after bariatric surgery}

Santos, F. ' Capitão, R. ; Roque, C. '; Duarte, J. '; Ferrinho, C. ' Lacerda, J.2.; Fonseca, R. '; Limbert, C. '; Azinheira, J. '; Tapadinhas, A. '; Vasconcelos, C. ${ }^{1}$ Hospital Egas Moniz - Serviço de Endocrinologia, ${ }^{2}$ Hospital Egas Moniz

Introduction: Bariatric surgery (BS) is an effective strategy to achieve long-term weight reduction in obese patients, however, particularly in case of malabsorptive procedures, nutritional deficiencies (such as iron deficiency) and anemia can develop during the follow-up. Female gender has also long been associated with lower hemoglobin $(\mathrm{Hb})$ levels which is mainly attributed to menstrual blood losses. Our work aims to compare $\mathrm{Hb}$ and ferritin levels and the need for iron supplementation between men and post-menopausal women submitted to BS.

Methods: A retrospective study was conducted on male and postmenopausal female obese patients (BMI $>35 \mathrm{~kg} / \mathrm{m}^{2}$ ) submitted to BS. We included patients which had measurements of $\mathrm{Hb}$, ferritin, folic acid and vitamin B12 at four different periods postoperatively: 1 to 6 months; 6,01 to 12 months; 12,01 months to 5 years or more than 5,01 years after surgery. We collected prescription data from the electronic system. The data was analyzed using SPSS software.

Results: A total of 123 patients (44 male and 79 female) with ages at the time of surgery between 21 and 70 years old (mean age of 43 years in male and 56 years in female patients $-\mathrm{p}<0,05)$ were included. Most patients $(78,9 \%)$ were submitted to a restrictive procedure. The diagnosis of anemia was made at some point during follow-up in $29,1 \%$ of women and in $15,9 \%$ of men $(p=0,102)$. There was need for iron supplementation in $9,1 \%$ of men and $19 \%$ of women $(p=0,145)$ and $5,1 \%$ also had intravenous supplementation. Taking into account only patients not on any iron supplementation, women had lower mean $\mathrm{Hb}$ than men in all periods more than 6 months after surgery $(\mathrm{p}<0,05)$ and their ferritin was also lower in this period although not reaching statistical significance. Linear regression (stepwise method) found female sex (but not age) to be the only factor capable of predicting lower $\mathrm{Hb}$ concentration in all periods more than 6 months after surgery. Malabsorptive procedures were also associated in a linear regression model with lower $\mathrm{Hb}$ concentration more than 5 years after surgery.

Conclusion: Postmenopausal women submitted to BS were found to have a higher frequency of anemia throughout follow-up compared to men. Surprisingly, sex was the only variable capable of integrating a model to predict $\mathrm{Hb}$ concentration postoperatively, more than 6 months after surgery. Iron status, represented by ferritin levels, and vitamin B12 and folic acid concentrations however, were not statistically different between sexes and, as such, other factors should be sought to further justify this difference although lack of statistical power could have had some interference with these results:

Conflict of Interest: None Disclosed.

Funding: No Funding.

\section{T3P13}

\section{Applicability, reliability and diagnostic performance of FibroScan for liver evaluation in severe obese patients candidate to bariatric surgery}

Barsamian, C. ${ }^{1}$; Czernichow, S. ${ }^{2}$; Sasso, M. ${ }^{3}$; Miette, V. ${ }^{3}$; Bedossa, $P^{4}$; Parlier, D. ${ }^{5}$; Poghosyan, T.5 ${ }^{5}$ Bouillot, J. ${ }^{5}$; Emile, J. ${ }^{5}$; Carette, C. ${ }^{1}$

${ }^{1}$ Service de nutrition, Hôpital Européen Georges Pompidou, APHP, Paris, France, ${ }^{2}$ Service De Nutrition, Hôpital Européen Georges Pompidou, Aphp, Paris, France; Université Paris Descartes, Paris, France, ${ }^{3}$ Echosens, Paris, France, ${ }^{4}$ Hôpital Beaujon, Aphp, Clichy, France, ${ }^{5} \mathrm{Hôp}$ ital Ambroise Paré, Aphp, Boulogne-Billancourt, France

Introduction: The XL probe of the FibroScan was recently developed to realize liver stiffness measurements (LSM) in overweight patients. This study aims to assess the Fibroscan applicability, reliability and diagnostic performances in severe obese patients candidates to bariatric surgery.

Methods: 127 severe obese patients candidate to bariatric surgery, with no known chronic liver disease and having a FibroScan examination before the surgery were recruited. Per-operative liver biopsy (LB) samples were collected during surgery. Histological assessment was performed by two expert pathologists in a double-blind manner with consensus. Fibrosis was assessed according to the Kleiner score and NASH according to the FLIP algorithm. Fibroscan applicability (number of patients with Fibroscan feasible with at least 10 valid measurements) and reliability according to Boursier's criteria (IQR/median $\mathrm{LSM} \leq 30 \%$ in patients with a $\mathrm{LSM}>7.1$ $\mathrm{kPa}$ ) was evaluated. Diagnostic performance was assessed using ROC curves analysis and the area under the ROC curve (AUROC). A p-value $<0.05$ was considered as statistically significant.

Results: Patients were for $78 \%$ female gender, age $=41$ [17] years and $\mathrm{BMI}=43.0[8.2] \mathrm{kg} / \mathrm{m}^{2} .43 \%$ of patients had at least minimal fibrosis, $19 \%$ had at least severe fibrosis and $2 \%$ had cirrhosis. $69 \%$ of patients had steatosis, 29\% had NASH and 29\% had no liver lesions. 126 patients had a Fibroscan examination feasible giving to the examination a $99 \%$ applicability in severe obese patients in our center. 10 patients $(8 \%)$ were measured using the M probe, 116 (92\%) using the XL probe. Liver stiffness measurement (LSM) was reliable in $92 \%$ of the patients. Patients with unreliable FibroScan examination had a significantly higher BMI than patients with reliable examination (48.0 [7.7] versus $42.9(8.1), \mathrm{p}=0.03)$.

AUROC for LSM for the detection of $F \geq 2$ and $F \geq 3$ and $F=4$ was 0.82 [0.73;0.91], 0.90 [0.83-0.96] and $0.96[0.89 ; 1]$, respectively. Cutoffs maximizing Youden index were respectively 8.0 , 8.7 and $15.6 \mathrm{kPa}$. Sensitivity $(\mathrm{Se})$, specificity $(\mathrm{Sp})$, positive and negative predictive values (PPV and NPV) were: $\mathrm{Se}=0.75 / \mathrm{Sp}=0.79 \mathrm{PPV}=0.46 / \mathrm{NPV}=0.93$ for $\mathrm{F} \geq 2$, $\mathrm{Se}=0.92 / \mathrm{Sp}=0.80 \mathrm{PPV}=0.35 / \mathrm{NPV}=0.99$ for $\mathrm{F} \geq 3$ and $\mathrm{Se}=1 / \mathrm{Sp}=0.93$ $\mathrm{PPV}=0.18 / \mathrm{NPV}=1$ for $\mathrm{F}=4$.

Conclusion:Severe obese patients candidate to bariatric surgery with no history of liver disease have a large prevalence of liver lesions. FibroScan applicability and reliability in this type of patients is high. Its performance for the diagnosis of significant fibrosis and steatosis are good. FibroScan appears as a reliable and noninvasive tool for screening chronic liver disease severity in severe obese patients.

Conflict of Interest: MS and VM are Echosens employee.

Funding: Research relating to this abstract was funded by Echosens. 
T3P15

Association between high blood pressure and anthropometric measures in healthcare professionals in Rio de Janeiro, Brazil

Tavares, C. ${ }^{1}$ Q Queiroz, F. ; Ansel, J. ' ${ }^{1}$ Costa, L. ${ }^{2}$; Souza, S. ${ }^{2}$

${ }^{1}$ Fundação Técnico-Educacional Souza Marques, ${ }^{2}$ Instituto Estadual De Cardiologia Aloysio De Castro - lecac

Introduction: Cardiovascular diseases (CVD) are the main cause of morbimortality in the world ${ }^{1}$. Health promotion campaigns enable the screening of risk factors for CVD, of which central obesity (CO) and high blood pressure (BP) stand out. Although body mass index is used to assess obesity, studies show that it is inaccurate to estimate $\mathrm{CO}^{2}$ and it is difficult to measure in itinerant campaigns. Waist circumference (WC), waist-hip ratio (WHR) and neck circumference (NC) also estimate $\mathrm{CO}^{1}$, although $\mathrm{NC}$ is underused in population-based studies. These campaigns are scarce for healthcare professionals.

Objectives: To evaluate systolic and diastolic blood pressure (SBP,DBP), NC, WC, hip circumference (HC) and WHR in healthcare professionals from a public health unit specialized in cardiology in Rio de Janeiro, Brazil.

Methods: Cross-sectional descriptive study submitted and approved by the Research Ethics Committee where it was performed. BP levels elevated: $\geq 140 \times 90 \mathrm{mmHg}$. WC was high and very high respectively in: women: $>80 \mathrm{~cm}$ and $>88 \mathrm{~cm}$; men: $>94 \mathrm{~cm}$ and $>102 \mathrm{~cm}$. WHR was elevated in women: $\geq 0.85 \mathrm{~cm}$; men: $\geq 0.90 \mathrm{~cm}^{3}$. Statistical analyzes were performed by SPSS v.21, considering statistically significant $\mathrm{p}<0.05$.

Results: 203 subjects, with age:43.2(12.3), SBP:117.5(16), DBP:77.8(10.7), $75 \%$ women, $53.2 \%$ nursing staff. Of these, 159 were not under hypotensive medication: 125 women, with SBP, DBP, NC, WC, HC and WHR 112.5(12.6); 75.5(9.8); 33.5(2.3); 85.3(10.2); 105.3(8); 0.81(0.6); 34 men $119.6(13.5) ; 78.2(9.8) ; 39.3(2.4) ; 94.8(10) ; 105.1(6.7) ; 0.90(0.06)$. Stratifying sample according to $\mathrm{WC}, \mathrm{SBP}$ and $\mathrm{DBP}$ were: adequate $\mathrm{WC}$ 111.2(12.6), 72.9(8.7); High/very high WC: 116(13.2), 78.2(10); according to WHR, SBP and DBP were: 112.8(12.6)vs.117.6(14), 74.9(9.8) vs.79.3(9.3). Statistical significance was observed between groups. Stratifying according to optimal or elevated BP, NC in women and men was: 33.3(2.3)vs.34.5(2) and 39.1(2.7)vs.39.5(2), with $\mathrm{p}<0.05$ only in women. Conclusion: Although a low frequency of high BP was observed, SBP and DBP means were higher when WC and WHR scores were higher. The same was observed in NC, suggesting its relevance for epidemiological purposes. The findings show the importance of monitoring these anthropometric indexes for prevention of hypertension and CVD, including healthcare professionals.

\section{References:}

1 WHO.Global atlas on cardiovascular disease prevention and control, 2011.

2 Joris, PJ et al. Diet-induced weight loss improves not only cardiometabolic risk markers but also markers of vascular function: a randomized controlled trial in abdominally obese men, 2016.

3 WHO. Waist circumference and waist-hip ratio: report of a WHO expert consultation, 2008.

Conflict of Interest: No conflict of interest is reported.

Funding: No external Fundings.
T3P16

\section{Association between methylation levels of PPARGC1A gene from leukocytes with insulin sensitivity indexes in normoglycaemic Chilean women}

Santos, J. ${ }^{1}$; Krause, B. ${ }^{2}$; Cataldo, R. ${ }^{1}$; Milagro, F. ${ }^{3}$; Gallegos, R. ${ }^{1}$; PrietoHontoria, P.4; Riezu-Boj, J. ${ }^{3}$; Bravo, C. ; Mennickent, P. '; Galgani, J. '; Martínez, J. ${ }^{3}$

${ }^{1}$ Department of Nutrition, Diabetes and Metabolism, School of Medicine, Pontificia Universidad Católica de Chile, ${ }^{2}$ Centro De Investigaciones Médicas. School Of Medicine, Pontificia Universidad Católica De Chile, ${ }^{3}$ Centro De Investigación En Nutrición, Universidad De Navarra Pamplona (Spain) And Ciberobn. Madrid, Spain., ${ }^{4}$ Universidad Sek. Santiago, Chile.

Introduction: PPARGC1A is a gene epigenetically regulated by DNA methylation in pancreatic islets and insulin-sensitive tissues. Additionally, PPARGC1A promoter methylation in blood at 5-7 years may predict adiposity from 9 to 14 years (Clarke-Harris et al. Diabetes 2014; 63: 2528-37). The aim of this study was to evaluate the association between methylation levels of PPARGC1A in leukocytes with indexes of insulin sensitivity derived from oral and endovenous glucose loads in normoglycaemic women.

Subjects and Methods: A standard 2-hour Oral Glucose Tolerance Test (OGTT; 75 g of glucose) and a short Minimal-Model Intravenous Glucose Tolerance Test (IVGTT; $0.3 \mathrm{~g} / \mathrm{kg}$ body weight; 13 blood samples during 50 minutes) were performed in 66 Chilean normoglycaemic women (age: $27 \pm 6.2$ years, BMI $24.1 \pm 3.5 \mathrm{~kg} / \mathrm{m} 2$ ). The following indexes were calculated: HOMA-S (from fasting glucose and insulin), Matsuda-ISICOMP (from OGTT) and the Calculated-Sensitivity Index CSi (from IVGTT). Bisulphite-treated DNA from leukocytes was interrogated for methylation in $6 \mathrm{CpG}$ sites of the promoter region of PPARGC1A gene by pyrosequencing (PyroMark Q96; positions -816, -783, -652, -617, -521 y -515). We also evaluated methylation from $17 \mathrm{CpG}$ sites across PPARGC1A using the Illumina Infinium Human Methylation 450 BeadChip ( 5 of them within the promoter region; positions -922, -841, -816, -384, and -136). Non-parametric correlation tests were used to analyse associations between pairs of variables.

Results: The only CpG position measured both by pyrosequencing and the Illumina array (position -816; cg11270806) showed a rho $=0.52$ $(\mathrm{p}=0.006)$. Methylation levels of all $\mathrm{CpG}$ sites measured by pyrosequencing were positively correlated $(\mathrm{p}<0.05)$, suggesting an integrated methylation of the promoter region. No significant associations were found between methylation of the promoter with insulin sensitivity indexes. Methylation levels of the intronic CpG site cg09427718 was significantly correlated with the CSi (rho =-0.29; $\mathrm{p}=0.047$ ), showing a similar trend for the association with Matsuda-ISICOMP index ( $r h o=-0.26 ; p=0.054$ ). Additionally, methylation level in the intronic site cg06772578 showed significant association with HOMA-S ( rho $=0.36 ; \mathrm{p}=0.008)$. None of these relationships remained significant after correction for multiple comparisons.

Conclusion: Methylation levels in the promoter region of PPARGC1A from leukocytes do not appear to be associated with insulin sensitivity indexes in normoglycaemic Chilean women. Further studies will focus on the relevance of near-significant signals of intronic CpG sites in PPARG$\mathrm{C} 1 \mathrm{~A}$ with systemic insulin sensitivity indexes .

Conflict of Interest: None

Funding: Chilean FONDECYT 1150416, Spanish. CIBERobn. Madrid

\section{T3P17}

Association of sarcopenia with metabolic syndrome in Korean cancer survivors

Kim, H. ${ }^{1}$; Moon, J. ${ }^{1}$ Kong, M. ${ }^{1}$; Huh, J. ${ }^{2}$

${ }^{1}$ Department of Family Medicine, Jeju National University \& Hospital, Jeju, Korea, ${ }^{2}$ Department Of Urology, Jeju National University \& Hospital, Jeju, Korea 
Introduction: Subjects diagnosed and treated for cancer may experience weight loss, especially in the skeletal muscles. The aim of this study was to investigate the prevalence of sarcopenia and its association with metabolic syndrome in Korean cancer survivors.

Methods: We used the Korean National Health and Nutrition Examination Survey data from 2008 to 2011 and enrolled a total of 312 cancer survivors older than 40 years. Appendicular skeletal muscle mass (ASM) was measured using dual-energy X-ray absorptiometry and was adjusted by weight (ASM/Wt). Sarcopenia was defined as 1 standard deviation (SD) below the mean for young healthy adults.

Results: According to the definition, our cut-off value of sarcopenia was $30.52 \%$ in men and $23.80 \%$ in women. For men and women, values of $33.6 \%$ and $32.7 \%$ were used to define sarcopenia according to ASM/Wt, respectively. The prevalence of metabolic syndrome was $34.8 \%$ in men and $38.2 \%$ in women. After adjusting for age, smoking status, alcohol consumption, exercise, education level and income, both men and women with sarcopenia in Korean cancer survivors had increased risk of metabolic syndrome (OR 3.71, 95\% CI: $1.28-10.69$ and OR 5.53, 95\% CI: $2.36-13.00$, respectively).

Conclusion: In conclusion, sarcopenia adjusted by weight was closely associated with metabolic syndrome in Korean cancer survivors.

\section{T3P19}

\section{Association of weight status, hyperhomocysteinemia and risk of chronic kidney disease in a Taiwanese adult population}

\section{Lai, S. ${ }^{1}$; Tsai, Y. ${ }^{1}$; Chang, S. ${ }^{2}$}

${ }^{1}$ Department of Family Medicine, Chang Gung Memorial Hospital, Keelung, Taiwan, ${ }^{2}$ Department Of Family Medicine, Chang Gung Memorial Hospital, Taoyuan, Taiwan

Introduction: Obesity is associated with increased risk of cardiovascular disease and chronic kidney disease (CKD). Hyperhomocysteinemia refers to increased oxidative stress, and decreased antioxidant defense function have been found to be associated with the risk of chronic kidney disease (CKD). However, little has been studied about the relationship among obesity, homocysteine and CKD. We investigate the association between body mass index (BMI), homocysteine level and impaired kidney function in a Taiwanese adult population.

Methods: This is a retrospective cross-sectional study of 24826 subjects $\geq 18$ year undergoing a health check-up during Jan 2012-Dec 2014. Subjects were divided into normalweight $\left(\mathrm{BMI}<24 \mathrm{~kg} / \mathrm{m}^{2}\right)$ and overweight/ obesity (BMI $>24 \mathrm{~kg} / \mathrm{m}^{2}$ ) groups according to the criteria of the Ministry of Health and Welfare in Taiwan. CKD was defined as estimated glomerular filtration(eGFR) rate less than $60 \mathrm{ml} / \mathrm{min}$ per $1.73 \mathrm{~m}^{2}$ and/or the presence of albuminuria. The upper quartile of serum homocysteine leve (>11.81 umol/L) was defined as hyperhomocysteinemia. Multivariate linear regression model was developed to analyze the relationship between BMI, serum homocysteine and eGFR. A multivariate logistic regression model was used to assess the relationship among weight categories, hyperhomocysteinemia, and CKD. ROC curve and Youden index were developed to define the discrimination power of homocysteine level for CKD in both normalweight and overweight/obese groups.

Results: Overweight/obese subjects with high homocysteine levels had the higher odds ratio for CKD compared with normalweight subjects ( 1.843 vs $1.384, \mathrm{P}<0.001$ vs $\mathrm{P}=0.023$ ). In females, overweight/obesity with hyperhomocysteinemia has Odds Ratio(OR) of $3.401(\mathrm{P}<0.001,95 \%$ Confidence Interval (CI): 2.06-5.61) for CKD; while in male, the OR was 1.655 ( $\mathrm{P}<0.001,95 \% \mathrm{CI}: 1.38-1.99)$. The prevalence of CKD in the quartile groups of hymocysteine were $2.5 \%, 2.7 \%, 3.4 \%$ and $5.2 \%(P<0.001)$. For every 1 unit increase of BMI $\left(\mathrm{kg} / \mathrm{m}^{2}\right)$, the eGFR declined by $0.496 \mathrm{~mL} /$ $\mathrm{min} / 1.73 \mathrm{~m}^{2}$. The area under curve (AUC) of the ROC of serum homocysteine level for CKD in normalweight and overweight/obesity groups were $0.556(\mathrm{P}=0.002,95 \% \mathrm{CI}: 0.52-0.59)$ and $0.603(\mathrm{P}<0.001,95 \% \mathrm{CI}$ : $0.58-0.63)$. The Youden index of homocysteine cut-off value was 10.70 and $11.08 \mathrm{umol} / \mathrm{L}$ in normalweight and overweight/obesity subjects.
Conclusions: Overweight/obesity with higher homocysteine levels is associated with increased risk for CKD especially in female. Further studies exploring on whether the effect of weight loss or homocysteine-lowering therapy such as folic acid, vitamin B6, B12 supplement may prevent or slow the progression of the residual renal function is warranted.

Conflict of Interest: None

Funding: None

\section{T3P20}

\section{Band off to bypass: A ten year experience at a public teaching hospital}

\section{Carroll, D. ${ }^{1}$; Kwok, D. ${ }^{2}$; Patel, D. ${ }^{2}$; Bowden, D. ${ }^{2}$; Hopkins, D. ${ }^{2}$}

${ }^{1}$ University of Queensland, ${ }^{2}$ Department Of Surgery, Royal Brisbane And Women'S Hospital

Introduction: In Australia, there is limited public-funded access to bariatric revisionary procedures. These procedures are necessary, particularly where previous procedures, commonly the laparoscopic adjustable gastric band (LAGB), may have significant associated morbidity. Our institution has been providing relatively limited public access to revisionary bariatric procedures in patients with prior LAGB for ten years. However, there continues to be considerable demand, and improving access of this population to such procedures is essential. We therefore decided to investigate the safety and efficacy of revisionary band to bypass procedures in our ten year experience at a public teaching hospital, where fellows and trainees assist or perform the procedure under the guidance of an experienced bariatric surgeon.

Methods: A retrospective case series of 92 consecutive revisionary band to bypass procedures from 2007 to November 2016 was performed using a prospectively maintained bariatric surgical database. All patients had prior LAGB's, either at our institution or elsewhere.

Results: 80 patients met the inclusion criteria. 69 (86.3\%) were women, with a mean age of 48 years. Median follow-up was 1.2 years (IQR $0.56-$ 2.1). Seven $(8.8 \%)$ patients were found to have post-operative leak, with one mortality (1.3\%). 18 (22.8\%) patients were re-admitted for issues related to their bypass surgery. Mean excess weight loss at the end of follow-up was $47.9 \%$ (SD 40.9\%). 27 (33.8\%) cases were performed primarily by a bariatric fellow. There was no significant difference in length of stay, excess weight loss or leak rate among these cases.

Conclusion: This study demonstrates that revisionary band to bypass procedures may be performed safely and with good efficacy in a tertiary teaching hospital, and we hope that these results may be extended to other public hospitals within Australia to improve access to similar complex revisionary surgery.

Conflict of Interest: None Disclosed

Funding: None

\section{T3P21}

\section{Bariatric surgery - an analysis of the prescription costs}

Paredes, S. ${ }^{1}$; Ribeiro, L. ${ }^{2}$; Obesity, M. ${ }^{1}$; Alves, M. ${ }^{1}$

${ }^{1}$ Hospital de Braga, ${ }^{2}$ Faculty Of Medicine, University Of Porto

Introduction: Bariatric surgery (BS) has grown in popularity in recent years, with demonstrated weight loss and improvement and resolution of comorbidities. Despite these data, BS is costly and doubts about its affordability have been raised. Overall, uncertainties remain about whether BS is cost effective, if the cost reductions expected are sustained over time and when a return of the investment occurs.

Methods: Retrospectively, databases of 385 patients who underwent BS between $01 / 2011$ and 08/2015 were analyzed. For the purpose of evaluating health benefits, namely the improvement or resolution of comorbidities, patients' treatments were compared in the preoperative period and 
at 12 months after BS. This study focused on cardiovascular comorbidities such as type 2 diabetes mellitus, hypertension, dyslipidemia and sleep apnea, therefore only patients treated for at least one of these comorbidities were included. Prescription drug data were obtained accordingly to the information available in medical charts. The number of drugs used per patient were compared pre and postoperatively as well as the use of continuous positive airway pressure (CPAP). The cost of a daily supply of each drug was obtained from the national public registry and averaged, taking into account the percentage financed by National Health Service (NHS). Results: Two-hundred and one patients were analysed, with a mean age of 47 years-old and a mean body mass index (BMI) of $44.2 \mathrm{Kg} / \mathrm{m}^{2}$. The majority were women $(79.6 \%)$ and sleeve gastrectomy was the predominant surgery $(83.1 \%)$; the remaining were submitted to gastric bypass. Twelve months after surgery, mean BMI was $30.96 \mathrm{Kg} / \mathrm{m}^{2}$. The mean prescribed drugs per patient per day was $3.05 \pm 2.2$ before and $1.59 \pm 2.0$ after surgery. Globally, $38.2 \%$ of individuals discontinued the use of drugs. Antihypertensive, antidiabetic and antidyslipidemic drugs decreased 46.3\%, 49.5\% and $43 \%$, respectively, after surgery. Mean insulin dose per day $(\mathrm{n}=16)$ was $99.31 \pm 77.2$ and $25.5 \pm 36$ units, respectively before and after surgery. The number of patients using CPAP for sleep apnea decreased from 41 to 21 . Globally, preoperatively, costs were on average $1.02 \pm 1.4 €$ per patient per day, whereas postoperatively costs were on average $0.43 \pm 0.8 €$ per patient per day $(\mathrm{p}<0.001)$. Patients exhibited an improvement in their comorbidities which, remaining stable, could allow a reimbursement of surgery in 27.34 years.

Conclusion: Bariatric surgery can decrease medication requirements, resulting in significant cost savings to NHS. This surgery is pricey, but the decrease of expenditures in medications may allow a reimbursement of the investment, and this is more evident in patients with more associated comorbidities.

Conflict of Interest: None

No Funding was received for this work

\section{T3P22 \\ Bariatric surgery and lipid profile - effects of the type of surgery}

Paredes, S. ${ }^{1}$; Ribeiro, L. ${ }^{2}$; Obesity, M. ${ }^{1}$; Alves, M. ${ }^{1}$

${ }^{1}$ Hospital de Braga, ${ }^{2}$ Faculty Of Medicine, University Of Porto

Introduction: Bariatric surgery improves lipid profile. Different surgical procedures seem to induce different variations in lipid profile (1). It was objective of this study to analyse the lipid profile before and one year after two different types of bariatric surgery and to study the predictors of its variation.

Methods: A retrospective study which included 385 patients undergoing bariatric surgery between 01/2011 and 08/2015 was conducted. Pre- and postoperative data were collected and analysed using SPSSv22 $(\mathrm{p}<0.05)$. Analytical parameters were compared using the T-test for independent and matched samples; multiple linear regression was used to evaluate the predictors of lipid profile variation.

Results: Preoperatively, the mean age was $42.14 \pm 10.6$ years-old and the mean body mass index (BMI) was $43.6 \pm 6.2 \mathrm{~kg} / \mathrm{m}^{2}$. The majority were women $(86.2 \%)$ and sleeve gastrectomy (SG) was the predominant surgery $(88.1 \%)$ : the remaining were submitted to gastric bypass (GB). Twelve months after surgery, mean BMI was $29.9 \pm 4.9 \mathrm{~kg} / \mathrm{m}^{2}$. There were no statistically significant differences in the preoperative values of total cholesterol (TC), high density lipoprotein cholesterol (HDL-c) and low density lipoprotein cholesterol (LDL-c) among patients undergoing SG or GB. Both surgeries caused significant improvement in all components of the lipid profile, although the reduction of TC and LDL-c was larger in patients submitted to GB. The increase in HDL-c was similar in both surgical types. The type of surgery was found to be a predictor of TC and LDL-c variation. Gender and glycated hemoglobin were predictors of HDL-c variation. HOMA-IR was shown to be a predictor of TC variation, whereas excess weight loss (EWL) was found to be a predictor of LDL-c variation.

Conclusion: GB causes a significantly greater reduction in TC and LDL-c when compared to SG. Knowledge of the effect of the different types of surgeries on lipid profile may be useful in clinical practice: patients with high LDL-c may benefit from a hybrid procedure such as GB, whereas patients with atherogenic dyslipidemia could be suggested a SG, since it is a less aggressive procedure, with similar results regarding triglycerides and HDL-c.

Conflict of Interest: None disclosed

Funding: No Funding was received for this work

References:

1 Benaiges $D$ et al., Impact of restrictive (sleeve gastrectomy) vs hybrid bariatric surgery (Roux-en-Y gastric bypass) on lipid profile. Obes Surg. 2012;22:1268-75

\section{T3P23}

\section{Bariatric surgery induced weight loss leads to improvements in cardiorespiratory fitness and physical function: A longitudinal study}

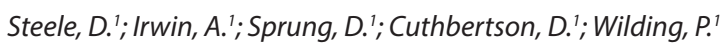 \\ ${ }^{1}$ University of Liverpool
}

Introduction: Obesity is associated with impairments in everyday physical functioning $(\mathrm{PF})$, leading to disability, and in cardio-respiratory fitness (CRF), an independent predictor of mortality. The impact of significant weight loss induced by bariatric surgery on these outcomes has not been well studied. Thus, the aim of this study is to assess the impact of surgery-induced weight loss on PF and CRF and, furthermore, to examine the relationship between these important health outcomes.

Methods: 16 patients (13 female, age $49 \pm 8$ years, BMI $48.0 \pm 5.1 \mathrm{~kg} / \mathrm{m}^{2}$ ) undergoing Roux-en-Y gastric bypass or sleeve gastrectomy were recruited and assessed prior to and 4 months following surgery. Body weight and body composition (bioimpedance analysis) were measured. Habitual physical activity (PA) (>3 METs; SenseWear armband), 6-minute walk distance (6MWD), sit-to-stand test (STS), grip strength (dynamometer) and CRF (maximal oxygen consumption) were all assessed. Statistical analysis was performed using paired $t$-tests and simple correlation; data is presented as mean (95\% CI).

Results: Patients weight reduced by $31.2 \mathrm{~kg}$ (28.1-34.4); 24.3\% (22.426.3). There were improvements observed in 6MWD [79.9m (62.2-97.5; baseline $393.9 \mathrm{~m} ; P<0.001)]$, STS [2.6 (1.9-3.3; BL 13; $P=0.001)]$ and CRF $\left[3.28 \mathrm{ml} / \mathrm{min} / \mathrm{kg}^{-1}(2.29-4.27 ; \mathrm{BL} 17.4 ; P<0.001)\right]$ following surgery. Duration of habitual PA [6.7 minutes per day (-0.53 to 13.9; BL 35.1; $P=0.1)$ ] did not increase. Positive correlations between the $\%$ weight loss coming from fat and improvements in CRF $(\mathrm{r}=0.6 ; P=0.03)$, grip strength $(\mathrm{r}=0.67 ; P=0.006)$ and $6 \mathrm{MWD}(\mathrm{r}=0.72 ; P=0.003)$ were identified. There was no correlation between change in PA and measures of PF or CRF.

Conclusion: Significant weight reduction with bariatric surgery leads to improvements in measures of fitness and physical function. Significantly, these changes were not accompanied by increased levels of PA. Improved understanding of the relationship of rapid weight loss with physical activity, physical functioning and fitness could aid the development of guidelines and inform future lifestyle interventions for these patients. Further work is required to identify the benefits and optimum deign of these interventions and should recognize the possible importance of maintaining lean body mass. 


\section{T3P24}

Body composition and biochemical variables of overweight/ obese breast cancer survivors submitted to 36 week of combined training

Trindade, A. '; Paulo, T. ${ }^{\prime}$; Aro, B. ${ }^{1}$; Viezel, J. ${ }^{1}$; Seidinger, S. ' ; Junior, I.

${ }^{1}$ Universidade Estadual Paulista

Introduction: Breast cancer survivors under hormonal treatment present several changes in body composition which can affect metabolic profile, mainly in those with excesso of body fat and intervention with exercise can be improve the unhealthy effects of treatment.

Objective: Compare long term effects of combined training on body fat and biochemical variables of overweight/obese breast cancer survivors under treatment with aromatase inhibitor and women breast cancer-free. Methods: The sample was women (aged 50+ years), BMI over $25 \mathrm{~kg}$ / $\mathrm{m}^{2}$, distributed in four groups: breast cancer exercise (BCE $\mathrm{n}=18$ ); cancer-free exercise (CFE $n=24)$; breast cancer control $(B C C n=9)$ - both breast cancer groups were under treatment with aromatase inhibitor; and cancer-free control (CFC $\mathrm{n}=13$ ). The BCE and CFE performed combined training three times/week for 36 weeks. Training was composed by 40 minutes of machine-based strength exercise followed by 30 minutes of treadmill exercise.BCC and CFC groups performed stretching two times/ week in on-consecutive days. The percentage of body fat (BF\%) was estimate by Dual-Energy X-ray Absorptiometryn(DEXA).The biochemical variables were estimate by $12 \mathrm{~h}$ fasting blood sample (total cholesterol, HDL, LDL, tryacylglicerol and glycemia). Comparisons between groups were made by repeated measure of ANOVA ajusted per age in SPSS program ( version 22.0). Significance was set at 5\%.)

Results: The $\mathrm{BCE}$ presented a significant decreasing in $\mathrm{BF} \%(\mathrm{p}=0.001)$ and HDL $(p=0.005)$; CFE showed lower BF\% $(p=0,001)$, HDL $(\mathrm{p}=0,001)$ and glycemia $(\mathrm{p}=0,006$; the CFC presented statistically increase in LDL ( $\mathrm{p}=0.048)$.

Conclusion: Our results indicate the potential efficacy of combined training during 36-wk to decrease body fat and improve metabolic profile in breast cancer survivors under treatment with aromatase inhibitor and cancer free women. We also advise that clinicians could recommend combined training as non-pharmacological treatment for improved unhealthy consequences of breast cancer treatment.

Conflict of Interest: None Disclosed

Funding: Research relating to this abstract was funded by CAPES and CNPq.

\section{T3P25 \\ Bone turn-over after Roux-en-Y gastric bypass in obese individuals with type 2 diabetes compared to non-operated matched type 2 diabetes controls.}

\section{Madsen, L. '; Espersen, R. '; Ørnstrup, M. ${ }^{3}$; Langdahl, B. ; Richelsen, B. ${ }^{3}$} ${ }^{1}$ Department of Endocrinology and Internal Medicine, Aarhus University Hospital, Denmark; Department of Clinical Medicine, Aarhus University, Denmark; Danish Diabetes Academy, Odense University Hospital,

Denmark, ${ }^{2}$ Department Of Endocrinology And Internal Medicine, Denmark; Department Of Clinical Medicine, Arhus University, Denmark, ${ }^{3}$ Department Of Endocrinology And Internal Medicine, Aarhus University Hospital, Denmark

Introduction: Roux-en- $\mathrm{Y}$ gastric bypass surgery (RYGB) results not only in restricted food uptake and weight loss but also in malabsorption of e.g. calcium and vitamin $\mathrm{D}$, which are important nutrients for bone metabolism. We hypothesized that bone turn-over after RYGB could be higher than in controls. Moreover, we aimed to determine whether such an influence on bone turn-over could be explained by malabsorption or by post-operative diabetes status, since individuals with type 2 diabetes (T2D) might have lower bone turn-over than individuals without T2D.

Methods: I this clinical trial, 99 RYGB operated obese individuals with T2D were matched with 49 non-operated T2D controls in accordance to age, gender, and current body mass index (BMI). Bone turn-over was evaluated by measuringprocollagen type 1 amino terminal propeptide
(P1NP), osteocalcin, C-terminal cross-linked telopeptide (CTX), bone specific alkaline phosphatase (BAP), 25-OH vitamin D, calcium, magnesium, phosphate, parathyroid hormone (PTH), and hemoglobonAlc (HbAlc). Moreover, anthropometrics, use of calcium and vitamin D supplementation (CalD), and smoking were recorded.

Results:Median time since RYGB was 6.07 years (IQR: $5.41 ; 7.00)$. The RYGB group displayed significantly higher levels of PTH (7.3 pmol/1 (95\% CI: 6.6; 8.0) vs. $5.4 \mathrm{pmol} / \mathrm{l}(95 \% \mathrm{CI}: 4.8 ; 8.6)$,) and $25-\mathrm{OH}$ vitamin D (80 $\mathrm{nmol} / \mathrm{l}(95 \%$ CI:74; 86) vs. $56 \mathrm{pmol} / \mathrm{l}$ (95\% CI:51; 55), than controls, the latter probably as a result of calD supplementation in the RYGB group $(87 \%$ vs. $33 \%$, p-value $=0.00)$. Furthermore, median $\mathrm{HbAlc}$ was significantly lower in the RYGB group than in controls $(47 \mathrm{mmol} / \mathrm{mol}(95 \% \mathrm{CI}$ : $46 ; 61)$ vs. $59 \mathrm{mmol} / \mathrm{mol}(95 \%$ CI: $55 ; 62)$. Finally, the median value of CTX after RYGB was $75 \%$ higher ( $\mathrm{p}$-value $=0.00$ ), P1NP after RYGB was $41 \%$ higher ( $p$-value $=0.000$ ), and osteocalcin after RYGB was $72 \%$ higher ( $\mathrm{p}$-value $=0.00)$ than in the control group, while BAP after RYGB was non-significantly elevated $(7 \%$, p-value $=0.29)$. When adjusting for $\mathrm{PTH}$, $25-\mathrm{OH}$ vitamin $\mathrm{D}$, smoking status, CalD intake, and $\mathrm{HbAlc}$ in a multiple regression model, CTX ( $36 \%$, p-value $=0.01)$ and osteocalcin $(31 \%$, p-value $=0.00)$ stayed significantly higher than in the control group, whereas the difference in P1NP $(19 \%$, p-value $=0.09)$ rendered insignificant.

Conclusion: After six years, RYGB operated T2D individuals have a significantly higher bone turn-over than non-operated T2D controls, in spite of adjustment for calcium and vitamin D supplementation and diabetes status. Thus, RYGB individuals may be at increased risk of developing osteoporosis.

\section{Conflict of Interest: None disclosed.}

Funding: Research relating to this abstract was funded by Aarhus University, Danish diabetes Academy founded by the Novo Nordisk Foundation, AP Møller Fonden, and Central Denmark Region.

\section{T3P26}

\section{Branched-chain amino acid catabolism rather than amino acids plasma concentrations predict diet-induced changes in insulin resistance}

Haufe, S. ${ }^{1}$; Engeli, S. ; Kaminski, J. ${ }^{2}$; Witt, H. ${ }^{3}$; Rein, D. ${ }^{4}$; Kamlage, B. ${ }^{4}$; Mähler, A. ; Schulz-Menger, J.6; Boschmann, M. ${ }^{5}$; Jordan, J. ${ }^{7}$

${ }^{1}$ Hannover Medical School, ${ }^{2}$ University Of Potsdam, ${ }^{3}$ Metanomics Gmbh, ${ }^{4}$ Metanomics Health Gmbh, ${ }^{5}$ Charité University Medical School, ${ }^{6} \mathrm{Helios}$ Klinikum Berlin-Buch, And University Medicine Berlin, ${ }^{7}$ Hannover Medical School, And German Aerospace Center

Introduction: 3-hydroxyisobutyrate (3-HIB), a catabolic intermediate of the BCAA valine stimulating muscle fatty acid uptake, has been implicated in the pathogenesis of insulin resistance. We hypothesized that circulating 3-HIB herald's insulin resistance and that metabolic improvement with weight loss relate to BCAAs and 3-HIB changes.

Methods: We analyzed plasma and urine in 109 overweight to obese individuals before and after six months on hypocaloric diets reduced in either carbohydrates or fat. We calculated the homeostasis model assessment index (HOMA-IR) and whole body insulin sensitivity from oral glucose tolerance tests and measured intramyocellular fat by magnetic resonance spectroscopy.

Results: BCAAs and 3-HIB plasma concentrations were inversely related to insulin sensitivity but not to intramyocellular fat content at baseline. With $7.4 \pm 4.5 \%$ weight loss, mean BCAA and 3-HIB plasma concentrations did not change, irrespective of dietary macronutrient content. Individual changes in 3-HIB with 6-month diet but not in BCAAs correlated with changes in whole body insulin sensitivity and HOMA-IR independently of BMI changes.

Conclusion: 3 -HIB relates to insulin sensitivity but is not associated with intramyocellular fat content in overweight to obese individuals. Moreover, changes in 3-HIB rather than BCAAs predict metabolic improvements with weight loss.

Conflict of Interest: None Disclosed 
Funding: This study was part of a joint project between metanomics $\mathrm{GmbH}$ (Berlin, Germany) and Charité - University Medical School, which was supported by the Federal Ministry of Education and Research (BMBF- 0313868) the German Obesity Network of Competence (01 Gl0830).

\section{T3P27}

\section{Can we develop a successful risk score for gestational diabetes?}

\section{Huvinen, E. ${ }^{1}$; Koivusalo, P. ${ }^{1}$; Eriksson, P. ${ }^{2}$}

${ }^{1}$ Department of Obstetrics and Gynecology, University of Helsinki and Helsinki University Hospital, Finland, ${ }^{2}$ Department Of Chronic Disease Prevention, National Institute For Health And Welfare, Helsinki, Finland, Folkhälsan Research Center, Helsinki, Finland, Unit Of General Practice And Primary Health Care, University Of Helsinki And Helsinki University Hospita

Introduction: The global epidemic of type 2 diabetes and gestational diabetes (GDM) requires methods for early detection of individuals at risk Risk scores have been successfully developed for type 2 diabetes ${ }^{1}$, but the GDM scores are not performing equally well ${ }^{2}$. The aim of this study was to investigate the risk profiles of GDM women, taking simultaneously into account the marked heterogeneity of $\mathrm{GDM}^{3}$.

Methods: This is a secondary analysis of the RADIEL (Finnish Gestational Diabetes Prevention) study including 510 women at high GDM risk $\left(\mathrm{BMI}>30 \mathrm{~kg} / \mathrm{m}^{2}\right.$ and/or previous GDM) recruited either in pre-pregnancy or in first trimester. We divided the participants according to BMI, parity and GDM history. Age, weight, family history of diabetes and first trimester markers of inflammation, lipid and glucose metabolism served as potential predictors for GDM.

Results: The cumulative GDM incidence was 37.4\% (95\% CI: 33.2 to 41.8 ) in the first trimester and $49.4 \%$ (95\% CI: 45.0 to 53.8 ) in the second trimester. Among the non-obese $\left(\mathrm{BMI}<30 \mathrm{~kg} / \mathrm{m}^{2}\right)$ women with a history of previous GDM, the cumulative incidence in the second trimester was $59 \%$ and among obese women with previous GDM 74\%. Obese primiparous women showed an increased GDM risk with higher fasting glucose values increasing GDM risk OR 3.76 (95\% CI: 1.48 to 9.53) but in the other subgroups there were no risk predictors.

Conclusions: Our "risk model", simply based on degree of adiposity and history of previous GDM, performed as well as more complicated/sophisticated models in identifying a high GDM-risk group already in the first trimester. Due to the heterogeneity of GDM, it might be impossible to achieve a universal risk score; instead, focus should be on universal OGTT screening.

\section{References:}

1 Abbasi A, Peelen LM, Corpeleijn E, et al. Prediction models for risk of developing type 2 diabetes: Systematic literature search and independent external validation study. BMJ. 2012; 345:e5900

2 Lamain-de Ruiter M, Kwee A, Naaktgeboren CA, et al. External validation of prognostic models to predict risk of gestational diabetes mellitus in one dutch cohort: Prospective multicentre cohort study. BMJ. 2016; 354:4338.

3 Huvinen E, Grotenfelt NE, Eriksson JG, et al. Heterogeneity of maternal characteristics and impact on gestational diabetes (GDM) risk-implications for universal GDM screening?. Ann Med. 2016; 48:52-58.

4 Koivusalo SB, Rono K, Klemetti MM, et al. Gestational diabetes mellitus can be prevented by lifestyle intervention: The finnish gestational diabetes prevention study (RADIEL): A randomized controlled trial. Diabetes Care. 2016; 39:24-30.

No conflicts of interest.

Funding: Juho Vainio Foundation(this abstract) and numerous Finnish health foundations (RADIEL study).

\section{T3P28 \\ Cardiac structure and function before and after bariatric surgery: A new perspective}

\section{Pouwels, S. ${ }^{1}$; Lascaris, B. ${ }^{2}$; Houthuizen, P. ${ }^{3}$; Dekker, L. ${ }^{3}$; Nienhuijs, S. ${ }^{4}$;} Bouwman, A. ${ }^{2}$; Buise, M. $^{2}$

${ }^{1}$ Franciscus Vlietland, Schiedam, The Netherlands, ${ }^{2}$ Department Of Anaesthesiology, Intensive Care And Pain Medicine, Catharina Hospital, Eindhoven, The Netherlands, ${ }^{3}$ Department Of Cardiology, Catharina Hospital, Eindhoven, The Netherlands, ${ }^{4}$ Department Of Surgery, Catharina Hospital, Eindhoven, The Netherlands

Obesity, defined as a body Mass Index of $\geq 30 \mathrm{~kg} / \mathrm{m}^{2}$, is the most common chronic metabolic disease worldwide and its prevalence has been strongly increasing. Obesity is associated with various diseases such as cardiovascular disease, type 2 diabetes, hypertension, obstructive sleep apnoea syndrome, rheumatoid arthritis and neoplasms. Obesity has deleterious effects on cardiac function. The purpose of this review is to evaluate the cardiac function and structure changes and hemodynamic responses to obesity before and after excessive weight loss. Secondly, to elucidate the possible clinical implications of cardiac remodelling after bariatric surgery for perioperative and anesthesiological care.

\section{T3P29 \\ Cardiometabolic and serum antioxidant profile of metabolically healthy and unhealthy severe obese adolescents}

Stenzel, A. '; Matos, A. ${ }^{2}$; Jesus, P. '; Pereira, S. '; Saboya, C. ${ }^{3}$; Ramalho, A. ${ }^{\text {' }}$

${ }^{1}$ Researcher of the Center for Research on Micronutrients (NPqM)/ Federal University of Rio de Janeiro, ${ }^{2}$ Adjunct Professor At Fluminense Federal University And Researcher Of The Center For Research On Micronutrients (Npqm)/ Federal University Of Rio De Janeiro, ${ }^{3}$ Brazilian Society Of Bariatric And Metabolic Surgery

Introduction: The study aimed to compare the cardiometabolic changes of severe obese adolescents classified as metabolically healthy (MHO) or unhealthy (MUO) and assess the distribution of frequency and severity of other metabolic relevance variables not comprised in the classification criteria and the serum inadequacy of micronutrients with antioxidant function.

Methods: This is an observational study, comprising adolescents with severe obesity submitted to anthropometric, biochemical and clinical evaluations. Body weight, height and waist circumference (WC) were measured and body mass index (BMI) calculated. Lipid profile, C-reactive protein (CRP) and glucose were assessed. Insulin resistance was quantified by the Homeostasis Model Assessment - Insulin Resistance (HOMA-IR). Serum concentrations of vitamins $\mathrm{A}$ (retinol and $\beta$-carotene), $\mathrm{E}$ and $\mathrm{C}$ and of the minerals zinc and selenium were quantified, and systemic arterial hypertension (SAH) and non-alcoholic fatty liver disease (NAFLD) were diagnosed. For the classification of MHO and MUO, the criteria used were those proposed by the IDF and the NCEP ATPIII. Statistical tests were used and a 5\% significance level was adopted.

Results: The sample consisted of 60 adolescents with severe obesity, $64.4 \%$ female. A total of $36.7 \%$ and $46.7 \%$ of MHOs were identified according to the IDF and the NCEP ATPIII, respectively. Considering the variables comprising the classification criteria, significant differences were found for HDL-c and HAS between MHOs and MUOs, regardless of the criterion used. Other metabolic relevance variables, such as CRP, total cholesterol, triglycerides and insulin resistance were also elevated in both groups, although with lower prevalence and severity in MHOs. A lower frequency of NAFLD was found in MHOs, with a significant difference $(\mathrm{p}=0.000)$. High percentages of inadequacy of micronutrients were observed in the overall sample, and a significant difference between the groups was only observed for selenium $(\mathrm{p}=0.029)$. A significant negative correlation was found between $\beta$-carotene and glucose $(r=-0.282 ; p=0.02)$, retinol and glucose $(\mathrm{r}=-0.309 ; \mathrm{p}=0.01)$ and retinol and WC $(\mathrm{r}=-0.220 ; \mathrm{p}=0.03)$. 
Conclusion: The adolescents classified as MHOs presented important metabolic changes also observed in MUOs, albeit with lower prevalence and severity, demonstrating that, despite their greater protection to these changes, MHOs should also be constantly monitored in order to avoid progression or, even, to reverse such changes aiming to effectively reduce cardiometabolic risk.

Conflict of interest: None.

Funding: FAPERJ/CNPq.

\section{T3P30}

\section{Cardiovascular risk improvement and event prevention after} gastric bypass are higher in diabetic and elderly patients

Pereira, P. '; Morais, T. '; Pereira, S. ${ }^{\prime}$; Almeida, R. ${ }^{2}$; Guimarães, M. ${ }^{2}$; Monteiro, $M$.

${ }^{1}$ Clinical and Experimental Endocrinology, Unit for Multidisciplinary Research in Biomedicine UMIB, Instituto de Ciências Biomédicas Abel Salazar (ICBAS), University of Porto, Portugal, '2Department Of General Surgery, Centro

Hospitalar De Entre O Douro E Vouga

Introduction: Obesity and associated co-morbidities are well recognized risks for cardiovascular $(\mathrm{CV})$ disease and mortality. Bariatric surgery has shown to decrease several independent $\mathrm{CV}$ risk factors, as well as to prevent the occurrence of $\mathrm{CV}$ events when compared to non-operated controls. The aim of the current study was to assess the occurrence of CV events in a large cohort of patients submitted to Roux-en-Y gastric bypass (RYGB) and to compared it with the predicted events rate as estimated by pre-operative CV risk calculation.

Methods: Framingham CV Risk Score was calculated in patients $(\mathrm{n}=261)$ before and 2 years after RYGB surgery, while occurrence of CV events was monitored up to 7 years after the procedure.

Results: Before surgery, estimated CV risk adjusted for the follow-up time predicted the occurrence of $6.1 \pm 0.6$ cardiovascular disease (CVD) events, $3.9 \pm 0.4$ coronary heart disease $(\mathrm{CHD})$ events, $1.5 \pm 0.2$ myocardial infarctions $(\mathrm{MI}), 0.8 \pm 0.1$ strokes, $0.3 \pm 0.1$ deaths from coronary heart disease (DCHD) and $0.34 \pm 0.1$ deaths from cardiovascular disease (DCVD). Two years after surgery there was a significant reduction in the estimated CV risk, at 10 years, that was significantly higher in diabetic and patients over 50 years old. After a mean follow-up time of $5.4 \pm 0.01$ years, none of the CV events predicted pre-surgery occurred.

Conclusion: RYGB decreased significantly CV risk and prevented the occurence of estimated CV events. Diabetic and older patients seem to be the patients subgroups that benefit the most from the CV risk reduction derived from weight loss attained trough the obesity surgery procedure.

\section{T3P31}

\section{Cardiovascular risk improvement and event prevention after gastric bypass are higher in diabetic and elderly patients}

Pereira, P. ; Morais, T. ${ }^{1}$; Pereira, S. ${ }^{1}$; Almeida, R. ${ }^{2}$; Guimarães, M. ${ }^{2}$; Nora, M. ${ }^{2}$; Monteiro, $M$.

${ }^{1}$ Clinical and Experimental Endocrinology, Unit for Multidisciplinary Research in Biomedicine UMIB, Instituto de Ciências Biomédicas Abel Salazar (ICBAS), University of Porto, Portugal, ${ }^{2}$ Department Of General Surgery, Centro Hospitalar De Entre O Douro E Vouga

Introduction: Obesity and associated co-morbidities are well recognized risks for cardiovascular $(\mathrm{CV})$ disease and mortality. Bariatric surgery has shown to decrease several independent $\mathrm{CV}$ risk factors, as well as to prevent the occurrence of $\mathrm{CV}$ events when compared to non-operated controls. The aim of the current study was to assess the occurrence of $\mathrm{CV}$ events in a large cohort of patients submitted to Roux-en-Y gastric bypass (RYGB) and to compared it with the predicted events rate as estimated by pre-operative CV risk calculation.
Methods: Framingham CV Risk Score was calculated in patients $(\mathrm{n}=261)$ before and 2 years after RYGB surgery, while occurrence of CV events was monitored up to 7 years after the procedure.

Results: Before surgery, estimated CV risk adjusted for the follow-up time predicted the occurrence of $6.1 \pm 0.6$ cardiovascular disease (CVD) events, $3.9 \pm 0.4$ coronary heart disease $(\mathrm{CHD})$ events, $1.5 \pm 0.2$ myocardial infarctions $(\mathrm{MI}), 0.8 \pm 0.1$ strokes, $0.3 \pm 0.1$ deaths from coronary heart disease (DCHD) and $0.34 \pm 0.1$ deaths from cardiovascular disease (DCVD). Two years after surgery there was a significant reduction in the estimated CV risk, at 10 years, that was significantly higher in diabetic and patients over 50 years old. After a mean follow-up time of $5.4 \pm 0.01$ years, none of the CV events predicted pre-surgery occurred.

Conclusion: RYGB decreased significantly CV risk and prevented the occurence of estimated CV events. Diabetic and older patients seem to be the patients subgroups that benefit the most from the $\mathrm{CV}$ risk reduction derived from weight loss attained trough the obesity surgery procedure.

\section{T3P32 \\ Cardiovascular, metabolic and mental comorbidities in the long-term after bariatric surgery and relation to weight loss}

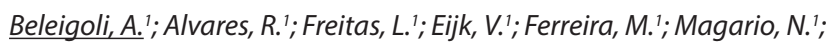
Diniz, M.'; Diniz, M. ${ }^{1}$

${ }^{1}$ Federal University of Minas Gerais, Brazil

Objectives: To assess comorbidities prevalence in the long-term after gastric bypass (GB), and the clinical and demographic factors associated with it.

Methods: Retrospective cohort with $>=5$ years of follow-up. Arterial hypertension $(\mathrm{AH})$ was defined as blood pressure $>140 / 90 \mathrm{mmHg}$ or use of antihypertensive medications; diabetes mellitus (DM2) as fasting glyce$\mathrm{mia}>=126 \mathrm{mg} / \mathrm{dL}$, or post-dextrosol $>=200 \mathrm{mg} / \mathrm{dL}$, or use of oral antidiabetic drugs or insulin; depression was defined by clinical history or use of antidepressants. Medians and proportions of pre and post-operative characteristics were compared by Wilcoxon and McNemar tests, respectively. Results: 115 patients (95; 86\% women), aged 40.2 (SD 13.2) years were followed-up by 8.7 (SD 2.9) years. Mean pre and post-operative BMI were 51.9 (SD 8.3) and $36.1(6.7) \mathrm{kg} / \mathrm{m}^{2}(\mathrm{p}<0.001)$ with mean percentage excess BMI loss (\%EBL) of $58 \%$ (SD 22.1). Comparing the pre and post-operative periods the prevalence of $\mathrm{AH}(78.4 \%$ versus $54.1 \%, \mathrm{p}<0.001)$, DM2 $(36.9 \%$ versus $9 \%, \mathrm{p}<0.001)$ were significantly reduced, but that of depression was not $(59.8 \%$ versus $53.6 \%, \mathrm{p}=0.25)$. \%EBL was associated with improvement of $\mathrm{AH}(\mathrm{p}=0.004)$, and depression $(\mathrm{p}=0.02)$, but not with DM2 ( $=0.53)$.

Conclusion: GB promotes significant \%EBL and comorbidities control in the long-term. The strong relation between $\mathrm{AH}$ reduction and weight loss after GB points out to the need of improving measures to maintain weight loss in the long-term. Conversely, DM2 prevalence reduction which was not related to \%EBL, suggests that weight loss is not the only mechanism involved in metabolic control after bariatric surgery. Neuro-hormonal alterations in the gastrointestinal-adipose tissue-brain axis induced by bariatric surgery might play a key role. The maintenance of depression strengthens the need of close psychological follow-up in the long-term. Acknowledgement: This work was funded by Fapemig and CNPq. The authors have no conflicts of interest.

\section{ТЗР33}

\section{Central obesity measured by controlled attenuation parameter but not body mass index is more important factor to predict significant steatosis}

Lee, J. ${ }^{1} ; K_{i m}, K^{2}$

${ }^{1}$ department of internal medicine, seonam university college of medicine, myongji hospital, ${ }^{2}$ Department Of Internal Medicine, Yonsei University College Of Medicine 
Background: The central obesity significantly contribute to occurrence of hepatic steatosis, but the relationship between controlled attenuation parameter (CAP) value, which is noninvasive method of measuring hepatic steatosis, and visceral fat area (VFA) by computed tomography (CT) which represents central obesity, is not investigated yet.

Methods:A total of 304 consecutive subjects who underwent general health examination including abdominal ultrasonography, transient elastography and abdominal fat CT at one tertiary center in Korea were enrolled prospectively. In this study, significant steatosis was diagnosed by ultrasonographic finding and $\mathrm{CAP}>250 \mathrm{~dB} / \mathrm{m}$.

Results: Multivariate linear regression analysis revealed that VFA was significantly related with CAP, whereas body mass index (BMI) was related with CAP only in univariate analysis. In the multiple logistic regression analysis, VFA (odd ratio [OR], 1.010; 95\% confidence interval $[\mathrm{CI}], 1.001-1.019 ; \mathrm{P}=0.028)$ and TG (OR, 1.006; 95\% CI, 1.001-1.011; $\mathrm{P}=0.022$ ) were selected as independent risk factor for significant hepatic steatosis. When population was stratified according to VFA $i \ddot{U} 100 \mathrm{~cm}^{2}$, $100<\mathrm{VFA}_{i} \mathrm{U} 200 \mathrm{~cm}^{2}$, VFA $>200 \mathrm{~cm}^{2}$, patients with a higher VFA were at a greater risk of significant hepatic steatosis with OR of $4.838(\mathrm{P}<0.001$; 95\% CI, 2.912-8.039) for $100<\mathrm{VFA}_{i} \mathrm{U} 200 \mathrm{~cm}^{2}$; OR of $7.474(\mathrm{P}<0.001$; $95 \% \mathrm{CI}, 2.462-22.693$ ) for VFA $>200 \mathrm{~cm}^{2}$, as compared to those with VFA ¡Ü $100 \mathrm{~cm}^{2}$.

Conclusions: Our data demonstrated that VFA was significantly related with significant hepatic steatosis assessed by CAP, suggesting that surveillance of hepatic steatosis need to be performed according the parameter which represents central obesity, not just BMI.

\section{T3P34}

Changes in blood phospholipid profile of patients submitted to Roux-en- $Y$ gastric bypass are associated with improved triacylglycerol levels

\section{Lima, T. '; Fiamoncini, J.2; Junior, J. ' ; Junior, J. ; Taglieri, C. ; Souza, H.} Daniel, H. ${ }^{2}$; Lima, T. $^{3}$

${ }^{1}$ Clinica IMEC, ${ }^{2}$ Technische Universität München, ${ }^{3}$ Medical School, University Of Sao Paulo

Obesity severely affects human health and Roux-en-Y gastric bypass (RYGB) is an effective method to achieve sustained weight loss and diabetes remission. In addition, bariatric surgery patients may provide important data regarding metabolic adaptations due to remarkable changes in food intake, with a view to clinical biomarker development. Therefore, we employed a metabolomics approach in 20 obese patients who underwent RYGB. Fasting plasma samples were collected before, 15 and 90 days after surgery in order to assess clinical chemistry markers. Total blood samples (dry blood spots) were collected and subjected to phospholipid profiling using LC-MS/MS. As expected, RYGB resulted in significant weight loss with normalization of biochemical parameters. By analyzing 109 species of phospholipid in patients' blood, we found a decrease in the concentration of phosphatidylcholines (C34:2, C36:1, C36:2, C36:3, C38:3), lyso-phosphatidylcholines (C18:0, C18:2) and ether-phosphatidylcholines (C36:1, $\mathrm{C} 36: 2$, C38:1, C38:2) early after surgery. On the other hand, sphingomyelin (C16:0, C18:0, C18:1, C24:1) levels increased. These effects remained 90 days after surgery, indicating that patients' phospholipid metabolism alterations after the surgery were not only due to food restriction. The decrease in triacylglycerol levels correlated positively with the decrease of PC36:3 ( $\mathrm{r}=0.65, \mathrm{p}<0.0001)$, PC38:0 ( $\mathrm{r}=0.59, \mathrm{p}<0.0001), \mathrm{PC} 40: 1$ $(\mathrm{r}=0.53, \mathrm{p}<0.0001)$ and PC40:4 $(\mathrm{r}=0.43, \mathrm{p}<0.0016)$. The decrease of IMC correlated positively with PC20:3 $(r=0.45, \mathrm{p}<0.008)$ and PCae 36:3 $(\mathrm{r}=0.62, \mathrm{p}<0.001)$. The increase in Oleoyl-sphingomyelin levels correlated negatively with the decrease of gamma-glutamyltransferase $(r=-0.51$, $\mathrm{p}<0.02$ ). Our data indicate that there is an increase in long chain fatty acid catabolism in patients after RYGB surgery, as palmitic (C16:0), stearic (C18:0), oleic (C18:1), linoleic (C18:2) and arachidonic (C20:4) acids concentration decreased in phosphatidylcholines. Catabolism of C20 fatty acids, saturated or polyunsaturated, correlated with triacylglycerol levels.
T3P35

Changes in body composition induced by a very low-calorieketogenic diet in the treatment of obese patients: Evaluation by DXA scan, multifrequency bioelectrical impedance and air displacement plethys

Gomez-Arbelaez, D. '; Crujeiras, A. 2; Bellido, D. ${ }^{3}$; Castro, A. ${ }^{2}$; OrdoñezMayan, L. ${ }^{2}$; Carreira, J. ${ }^{4}$; Galban, C. ${ }^{4}$; Martinez-Olmos, M. ${ }^{2}$; Sajoux, I. ; Casanueva, $F^{2}$

${ }^{1}$ Division of Endocrinology, Department of Medicine, Complejo Hospitalario Universitario de Santiago (CHUS), Santiago de Compostela, Spain, ${ }^{2}$ Division Of Endocrinology, Department Of Medicine, Complejo Hospitalario Universitario De Santiago (Chus), And Ciber Fisiopatología De La Obesidad Y Nutrición, Santiago De Compostela, Spain, ${ }^{3}$ Division Of Endocrinology, Complejo Hospitalario Universitario De Ferrol And Coruña University, Ferrol, Spain., ${ }^{4}$ Intensive Care Division, Complejo Hospitalario Universitario De Santiago (Chus), Santiago De Compostela, Spain., ${ }^{5}$ Medical Department Pronokal, Pronokal Group, Barcelona, Spain

Introduction: A common concern when using low-calorie diets as a treatment for obesity is the reduction in fat-free mass, mostly muscular mass that occurs together with the fat mass loss, and the best methodologies to evaluate body composition changes. This study was aimed to evaluate the very low-calorie-ketogenic (VLCK) diet-induced changes in body composition of obese patients and to compare three different methodologies employed to evaluate that changes.

Methods: Twenty obese patients followed a VLCK diet for 4 months. Body composition was performed by dual-energy X-ray absorptiometry (DXA), multifrequency bioelectrical impedance (MF-BIA) and air displacement plethysmography (ADP) techniques. Muscular strength was also assessed. Measurements were performed at four points matched with the ketotic phases (basal, maximum ketosis, ketosis declining and out of ketosis).

Results: After 4-months the VLCK diet induced a $-20.2 \pm 4.5 \mathrm{~kg}$ weight loss, at expenses of reductions in fat mass (FM) of $-16.5 \pm 5.1 \mathrm{~kg}$ (DXA), $-18.2 \pm 5.8 \mathrm{~kg}$ (MF-BIA), and $-17.7 \pm 9.9 \mathrm{~kg}$ (ADP). A significant decrease was also observed in the visceral FM. The mild but significant reduction in fat-free mass occurred at maximum ketosis mainly due to changes in the total body water and was recovered thereafter. No changes in muscle strength were observed. A strong correlation was evidenced between the three methods of body composition.

Conclusion: The VLCK diet-induced weight loss was mainly at the expense of FM and visceral mass, preserving muscle mass and strength. From the three employed body composition techniques, the multifrequency bioelectrical impedance seems more convenient in the clinical setting.

Conflict of interest: DB and FFC received advisory board fees and or research grants from Pronokal Protein Supplies Spain. IS is Medical Director of Pronokal Spain SL

Funding: This work was supported by the PronoKal Group " and grants from the Fondo de Investigacion Sanitaria, PE13/00024 and PI14/01012 research projects and CIBERobn (CB06/003) from the Instituto de Salud Carlos III (ISCIII)-Subdireccion General de Evaluacion y Fomento de la Investigación; Fondo Europeo de Desarrollo Regional (FEDER)

\section{T3P36}

\section{Characterization of metabolically healthy obesity and relation with vitamin $D$ in a bariatric surgery population}

\section{Coelho, C. ${ }^{1}$; Fernandes, V. ${ }^{1}$; Monteiro, A. ${ }^{\text {; }}$ Paredes, S. ${ }^{1}$; Obesity, M. ${ }^{2}$;} Souto, S. '; Correia, F. ${ }^{3}$

${ }^{1}$ Endocrinology Department, Braga Hospital, Portugal, ${ }^{2}$ Braga Hospital, Portugal, ${ }^{3}$ Faculty Of Nutrition And Food Science, University Of Porto, Portugal

Introduction: Obesity not associated with metabolic syndrome is defined has metabolically healthy obesity. The prevalence is variable. Certain clinical and analytic features are recognised in obese patients with and without metabolic syndrome. However, it remains unknown the pathophysiology 
involved. The aim of this study was to determine the prevalence, characterize and compare metabolically healthy obese with metabolically unhealthy obese in an obese population undergoing bariatric surgery with particular interest in vitamin D.

Methods: This is a retrospective study that involved 217 patients followed at Braga Hospital, outpatient consultation of Endocrinology/General Surgery. Metabolically healthy obesity was defined has absence of metabolic syndrome and body mass index equal or superior to $30 \mathrm{~kg} / \mathrm{m}^{2}$. Metabolic syndrome was classified according to the National Cholesterol Education Program, Adult Treatment Panel (NCEP ATP III) updated version

Results: Metabolically healthy obesity prevalence was 47,3\% ( $\mathrm{n}=103)$. The patients with metabolically healthy obesity were younger $(\mathrm{p}<0,001)$ with less vitamin $\mathrm{D}$ deficiency $(\mathrm{p}=0,023)$ and magnesium $(\mathrm{p}=0,043)$. Furthermore, they presented less hepatic steatosis $(p=0,017)$ and less insulin resistance $(\mathrm{p}<0,001)$. There were no differences in body mass index $(\mathrm{p}=0,445)$.

Conclusion: Metabolically healthy obesity prevalence is elevated. Vitamin $\mathrm{D}$ deficiency was predominant. However, we found differences in between metabolically healthy obesity and metabolically unhealthy obesity concerning vitamin $\mathrm{D}$. These findings suggest that vitamin $\mathrm{D}$ could be relevant in metabolically healthy obesity.

Conflict of Interest: None disclosed

Funding: No Funding

\section{T3P37 \\ Circulating concentrations of ANGPTL8/betatrophin are altered in dyslipidemia}

Gomez-Ambrosi, J. '; Pascual-Corrales, E. '; Catalán, V. '; Rodríguez, A. '; Ramírez, B. '; Romero, S. ${ }^{3}$ V Vila, N. ${ }^{3}$; Ibáñez, P. ${ }^{3}$; Margall, M. ${ }^{3}$; Silva, C. ${ }^{3}$; Gil, M. ${ }^{4}$; Salvador, J. ${ }^{5}$; Frühbeck, G. ${ }^{6}$

${ }^{1}$ Metabolic Research Laboratory, Clínica Universidad de Navarra, IdiSNA, CIBEROBN, Pamplona, Spain, ${ }^{2}$ Department Of Endocrinology \& Nutrition, Clínica Universidad De Navarra, Pamplona, Spain, ${ }^{3}$ Department Of Endocrinology \& Nutrition, Clínica Universidad De Navarra, Idisna, Ciberobn, Pamplona, Spain, ${ }^{4}$ Department Of Biochemistry, Clínica Universidad De Navarra, Idisna, Ciberobn, Pamplona, Spain, ${ }^{5}$ Department Of Endocrinology \& Nutrition, Clínica Universidad De Navarra, Ciberobn, Pamplona, Spain, ${ }^{6}$ Metabolic Research Laboratory, Department Of Endocrinology \& Nutrition, Clínica Universidad De Navarra, Idisna, Ciberobn, Pamplona, Spain

Introduction: ANGPTL8/betatrophin is a secreted protein initially related to $\beta$-cell replication. Recent data in humans and mice models suggest that ANGPTL8/betatrophin is involved in lipid metabolism. Our aim was to study the circulating levels of ANGPTL8/betatrophin in patients with dyslipidemia defined as having high or low levels of HDL-cholesterol (HDL-C) or triglycerides (TG), respectively.

Methods: Serum levels of ANGPTL8/betatrophin were measured by using a commercial ELISA kit in 177 individuals. We studied two different case-control dyslipidemic cohorts including individuals with high $(n=43)$ or low $(n=46)$ circulating concentrations of HDL-C or with low $(n=48)$ or high $(n=40)$ levels of TG.

Results: Circulating concentrations of ANGPTL8/betatrophin were significantly lower $(\mathrm{P}<0.001)$ in subjects with dyslipidemia in both men (control $27.8 \pm 15.2$ vs dyslipidemia $17.0 \pm 11.2 \mathrm{ng} / \mathrm{mL}$ ) and women (control $50.0 \pm 22.2$ vs dyslipidemia $27.0 \pm 16.5 \mathrm{ng} / \mathrm{mL}$ ). The magnitude of the differences was higher in dyslipidemic patients classified according to HDL-C than in those classified according to TG levels. ANGPTL8/betatrophin levels were lower in subjects with T2D $(\mathrm{P}<0.001)$ but the impact of T2D vanished $(P=0.257)$ when the effect of dyslipidemia was included in the analysis. A highly significant correlation was found between ANGPTL8/betatrophin and HDL-C levels $(r=0.56 \mathrm{P}<0.001)$. The multiple linear regression model that best predicted HDL-C concentrations included ANGPTL8/betatrophin $(\mathrm{P}<0.001)$, AST/ALT ratio $(\mathrm{P}=0.002)$, TG $(\mathrm{P}=0.011)$ and QUICKI $(\mathrm{P}=0.035)$ as predictive variables explaining $46 \%$ of the total variability of HDL-C levels $(\mathrm{P}<0.001)$.
Conclusion: We conclude that serum ANGPTL8/betatrophin concentrations are altered in patients with dyslipidemia. ANGPTL8/betatrophin arises as a potential actor in dyslipidemia with a strong association with HDL-C and also as a promising therapeutic tool for the treatment of dyslipidemia.

Conflict of Interest: The authors declare that they have no conflict of interest.

Funding: Supported by FIS-FEDER, Instituto de Salud Carlos III (ISCIII) (PI12/00515, PI13/00460 and PI14/00950) and CIBEROBN, Spain.

\section{T3P38 \\ Circulating vaspin levels are not influenced by gluteal cryolipolysis}

\section{Lee, K. ${ }^{1}$; Hwang, I. ${ }^{2}$; Ko, K. ${ }^{2}$; Suh, H. ${ }^{2}$; Kim, K. ${ }^{2}$}

${ }^{1}$ Bariatric clinic, Family Medicine, Dongincheon Gil Hospital, Gachon University, ${ }^{2}$ Family Medicine, Gil Medical Center, Gachon University

Whereas cryolipolysis was prevalent as a notable device to remove local fat by cooling, vaspin was recently considered as a kind of adipocytokines released from visceral adipose tissues.

The purpose of this study is to investigate whether vaspin, metabolic and inflammation markers are influenced by cryolipolysis.

As a case-controlled, prospective, clinical trial, a total of 28 healthy premenopausal women who would like to remove unwanted fat on thigh were recruited.

Inclusion and exclusion criteria were described as below.

Inclusion criteria premenopausal healthy women

subjects with body mass index $\geq 18$ subjects with visible fat on both thigh subjects to understand and agree to this study protocol

The radio frequency method was applied on right thigh as sham procedure for 30 minutes through $3000 \mathrm{~Hz}$ - amplitude modulated frequency at once. Zeltiq System (Zeltiq Aesthetics Inc., Pleasanton, CA) was developed. This device consists of a control console, with a treatment applicator attached by a cable.

We measured weight, body fat (bioelectric impedance: Biospace, Korea), vaspin, the cardio-metabolic markers such as insulin, fasting plasma glucose, total cholesterol, HDL cholesterol, LDL cholesterol, triglycerides, ESR and hs CRP levels in completed 15 subjects at initial, $4^{\text {th }}$, and $12^{\text {th }}$ week visit after 8 hour fasting.

The Wilcoxon signed-rank test was applied to evaluate the difference of anthropometrical, percent of body fat, metabolic variables and inflammation markers at initial, 4th week and 12th week visit. SPSS packages for window version 15 was performed, and a probability value of less than 0.05 was considered as statistically significant at both sides.

No significant statistical difference of vaspin, metabolic variables such as total, HDL, LDL cholesterol, triglyceride, and fasting blood glucose was elucidated in the comparison of mean values at initial, 4 th week and 12 th week.

In conclusion vaspin, metabolic risk variables and inflammation markers in premenopausal healthy women for 12 weeks are not influenced by gluteal fat removal using by cryolipolysis.

Table 1.

\begin{tabular}{|l|l|l|l|}
\hline & Initial & $4^{\text {th }}$ Week & $12^{\text {th }}$ Week \\
\hline Vaspin $(\mathrm{pg} / \mathrm{mL})$ & $0.45 \pm 0.15$ & $0.71 \pm 0.20$ & $0.45 \pm 0.09$ \\
\hline Insulin $(\mathrm{ng} / \mathrm{L})$ & $7.03 \pm 0.93$ & $8.66 \pm 0.89$ & $10.34 \pm 1.97$ \\
\hline Free Fatty Acid (mmolL) & $613.60 \pm 67.43$ & $637.07 \pm 114.42$ & $531.80 \pm 100.93$ \\
\hline BMI(kg/m $\left.{ }^{2}\right)$ & $23.21 \pm 0.79$ & $23.45 \pm 0.83$ & $23.29 \pm 0.90$ \\
\hline Body Fat (\%) & $32.13 \pm 1.21$ & $31.97 \pm 1.34$ & $32.83 \pm 1.56$ \\
\hline $\begin{array}{l}\text { Fasting Blood Glucose } \\
\text { (mg/dL) }\end{array}$ & $88.53 \pm 1.35$ & $87.33 \pm 2.03$ & $88.13 \pm 3.97$ \\
\hline Total Cholesterol $(\mathrm{mg} / \mathrm{dL})$ & $173.47 \pm 2.03$ & $173.33 \pm 6.55$ & $175.73 \pm 5.46$ \\
\hline Triglyceride(mg/dL) & $69.73 \pm 6.50$ & $74.33 \pm 7.42$ & $82.80 \pm 9.63$ \\
\hline HDL Cholesterol $(\mathrm{mg} / \mathrm{dL})$ & $65.33 \pm 3.03$ & $64.33 \pm 2.87$ & $63.27 \pm 3.26$ \\
\hline
\end{tabular}


T3P40

Clinical usefulness of immunoreactive insulin measurement in the course of OGTT

\section{Assyov, $Y^{1}{ }^{1}$; Kamenov, $Z{ }^{1}$}

${ }^{1}$ Department of Internal Diseases, Medical University - Sofia

Introduction: Obesity and insulin resistance are major risk factors for type 2 diabetes, metabolic syndrome and cardiovascular morbidity and mortality. The aim of this study is to determine whether immunoreactive insulin (IRI) measurements in the course of an oral glucose tolerance test (OGTT) bears clinical usefulness in the evaluation of high-risk individuals.

Methods: This cross-sectional study included 813 obese patients , mean age $43.7 \pm 14.9$ years with mean BMI $37.9 \pm 6.2$ who were admitted in a tertiary endocrine department for the evaluation of the metabolic risk. All subjects underwent an oral glucose tolerance test with measurement of IRI (0, 60, $120 \mathrm{~min})$. HOMA-IR and QUICKI indices were calculated. Biochemical measurements included lipid profiles, liver enzymes, uric acid concentrations and serum creatinine. Weight, height, waist circumference and arterial blood pressure were measured.

Results: Correlation analyses determined QUICKI index to be strongest associated with the evaluated biochemical parameters, with IRI measured on the $120 \mathrm{~min}$ being the best of the three IRI measurements. ROC analyses determined that the measurement of IRI on the $120 \mathrm{~min}$ had the best specificity, sensitivity and PPV for distinguishing subjects with arterial hypertension ( $\mathrm{AUC}=0.57$, CI $0.53-0.62 \mathrm{p}<0.001$ ), metabolic syndrome $(\mathrm{AUC}=0.64, \mathrm{CI} 0.60-0.68, \mathrm{p}<0.001)$ and dyslipidemia $(\mathrm{AUC}=0.63, \mathrm{CI}$ $0.58-0.68, \mathrm{p}<0.001)$. Multiple regression analyses determined that of all the aforementioned parameters, only IRI, measured on the $120 \mathrm{~min}$ and HOMA-IR added further information for distinguishing high-risk individuals in a grouping regression model, after adjustment for potential confounders

Conclusion: Measurement of immunoreactive insulin in the course of an OGTT as an adjunct to traditional biochemical parameters is useful for the determination of the individual metabolic risk. Immunoreactive insulin, measured on the $120^{\text {th }}$ minute of the test appears to have the best discriminative value for distinguishing high-risk individuals.

\section{Conflict of Interest: None}

Funding: The current work was funded by project number № 4651/22.07.2015, contract № 3-C/2015 by Medical University - Sofia.

\section{T3P41}

\section{Comparative analysis of respiratory muscle strength before and after bariatric surgery using five predictive equations}

Pouwels, S. ${ }^{1}$; Buise, M. ${ }^{2}$; Smeenk, F. ${ }^{3}$; Teijink, J. ${ }^{4}$; Nienhuijs, S. ${ }^{4}$

${ }^{1}$ Franciscus Vlietland, Schiedam, The Netherlands, ${ }^{2}$ Department Of Anesthesiology, Catharina Hospital, Eindhoven, The Netherlands, ${ }^{3}$ Department Of Respiratory Medicine, Catharina Hospital, Eindhoven, The Netherlands, ${ }^{4}$ Department Of Surgery, Catharina Hospital, Eindhoven, The Netherlands

Introduction: Obesity is the most common chronic metabolic disease worldwide, with detrimental effects on respiratory function. Less is known about the recommended reference values for respiratory muscle strength in the morbidly obese population.

Objectives: This study aimed to evaluate respiratory muscle strength in the morbidly obese population, before and after bariatric surgery, and to compare these estimates with the predictive values using different mathematical equations available

Methods: A multidisciplinary team screened patients referred to a bariatric centre preoperatively. Their Maximum Inspiratory Pressure (MIP) was measured at screening and 3, 6 and 9 months postoperative. Predictive values were calculated using five different mathematical equations. Visual inspection of Bland-Altman plots was performed to determine the agreement between the equations studied.
Results:In total 122 patients were included in this study, among them were 104 females and 18 men, with a mean age was $43.02 \pm 11.11$ years and mean BMI was $43.10 \pm 5.25 \mathrm{~kg} / \mathrm{m}^{2}$. There were no significant differences between the predicted MIP (according to Neder, Harik-Khan, Enright, Costa and Wilson equations) and the actual obtained MIP preoperatively $(\mathrm{p}>0.05)$. Also there were no significant between the predictive values and the postoperative MIP values. $(\mathrm{P}>0.05)$ Bland Altman analysis showed that the Enright equation was best suitable for predicting the MIP. Conclusion

Of the five mathematical equations studied, that of Enright and collegues was found best suitable for predicting the MIP in the obese population studied.

\section{T3P42 \\ Comparative study of the nutritional status of vitamin A in pregnant women and in women who became pregnant or did not after Roux-en-Y gastric bypass}

\section{Cruz, S. ${ }^{1}$; Matos, A. ${ }^{1}$; Machado, S. ${ }^{2}$; Cruz, S. ${ }^{2}$; Pereira, S. ' ; Saboya, C. ${ }^{3}$; Ramalho, $A{ }^{1}$}

${ }^{1}$ Researcher of the Center for Research on Micronutrients (NPqM)/ FEDERAL UNIVERSITY OF RIO DE JANEIRO, ${ }^{2}$ Federal University Of Rio De Janeiro, ${ }^{3}$ Brazilian Society Of Bariatric And Metabolic Surgery

Introduction: The changes in digestive physiology after Roux-en-Y Gastric Bypass (GRYR), as well as gestation, maximizes the risk of vitamin A deficiency (DVA), both can result in harm to maternal and child health. The study aimed to compare the nutritional status of vitamin A among women who became pregnant or did not after RYGB and in pregnant who did not undergo surgery, and to assess the impact of VAD on the mother and child health

Methods: This is a cross-sectional study of the analytical type. The woman was divided into: Group 1 (G1) 80 pregnant women, Group 2 (G2) 40 pregnant women who had previously undergone RYGB, both in their third gestational trimester, and Group 3 (G3) 77 non-pregnant women who had previously undergone RYGB. Serum concentrations of retinol and $\beta$ - carotene, night blindness (NB), gestational and neonatal intercurrences (urinary tract infection, iron deficiency anemia, hypertensive syndrome of pregnancy, presence of dumping syndrome, birth weight, gestational age at birth $(\mathrm{GAB})$ and weight/GAB correlation) was investigated. The significance level adopted was $\mathrm{p}<0.05$.

Results: RYGB, per se, had a greater impact on the inadequacy of retinol, $\beta$-carotene, and on the increased percentage of $\mathrm{NB}$ when compared to non-surgical pregnant women. When surgery was associated with pregnancy, more than $75 \%$ of inadequacy of retinol and $\beta$ - carotene were noted, as well as a higher percentage of individuals with NB. G2 also showed increased odds for developing gestational and neonatal intercurrences, when compared to G1.

Conclusion: RYGB provides greater negative impact on the nutritional status of vitamin A compared to pregnancy, and surgery associated with pregnancy can provide even greater risks and, consequently, increased risks for the mother and child health.

None disclosed

Research relating to this abstract was funded by FAPERJ and CNPq

FAPERJ and CNPq

References:

1 WORLD HEALTH ORGANIZATION (WHO). Vitamin and Mineral Nutrition Information System (VMNIS), WHO. Available at: <http://www.who.int/vmnis/ en/index.html>. Accessed in: Dec 2011.

2 Saunders C, Leal MD, Neves PA, Padilha PC, Silva LB, Schilithz AO. Determinants of gestational night blindness in pregnant women from Rio de Janeiro, Brazil. Public Health Nutr 2015; 9:1-10. 


\section{T3P44}

\section{Comparison of an electronic versus traditional food diary for assessing dietary intake - a validation study}

Fuller, N. '; Fong, M. '; Gerofi, J. '; Ferkh, F.'; Leung, C. '; Leung, L. ' Zhang, S. '; Skilton, M. '; Caterson, I. ${ }^{1}$

${ }^{1}$ The Boden Institute, The University of Sydney

Introduction: Paper-based estimated food diaries are often used in research to collect dietary data, despite this method being burdensome for both participants and researchers. Such food diaries are often time consuming, labour intensive, and rely on participant literacy and therefore may lead to greater rates of under-reporting.

Methods: This study assessed the validity of the 'Boden Food Plate', a novel web-based electronic application, compared to a paper-based three-day estimated food diary. Participants were also asked to rate their satisfaction with the new electronic diary. Sixty seven participants completed both the electronic and paper-based diaries at two different time-points.

Results: Baseline BMI of participants (mean \pm standard deviation (SD)) was $30.4 \pm 2.9 \mathrm{~kg} / \mathrm{m} 2$, body weight was $87.6 \pm 13.4 \mathrm{~kg}$, and age was $42.3 \pm 7.7$ years. Fifty four percent $(n=41)$ of the cohort were female. Bland Altman plots for total energy, and percentage of total energy intake from fat, carbohydrate, and protein, indicated that the two methods of dietary data collection were in agreement. Participants rated the electronic food diary as easier to use and more fun than the traditional paper-based estimated food diary.

Conclusion: These results show that the Boden Food Plate would be as valid and reliable as current data collection methods and is therefore a practical, and easier, means of collecting data on dietary energy and macronutrient for future studies.

Conflict of Interest: None

Funding: None

\section{T3P46 \\ Comparison of long term evolution of nutritional parameters after gastric bypass and restrictive bariatric surgery}

\section{Ledoux, S. '; Sami, O. '; Calabrese, D. ${ }^{2}$; Msika, S. '; Coupaye, M.}

${ }^{1}$ Explorations Fonctionnelles, Centre de prise en charge de l'Obésité, Hôpital Louis Mourier (AP-HP), 92700 Colombes and Faculté Paris Diderot, France, ${ }^{2}$ Service De Chirurgie, Centre De Prise En Charge De L'Obésité, Hôpital Louis Mourier (Ap-Hp), 92700 Colombes And Faculté Paris Diderot, France

Background: Long term prospective data on nutritional complications after bariatric surgery are scarce and whether gastric bypass (GBP) induces more severe deficiencies than pure restrictive surgery (RS) in the long term has not been assessed. Our aim was to compare long term evolution of nutritional parameters after GBP or RS in patients attending a specialized centre.

Methods: We selected from our prospective database started in 2004 subjects who underwent bariatric surgery 5 years ago or more and performed nutritional assessments in our centre. We identified 328 subjects (226 after GBP and 102 after RS including 85 gastric bandings and 17 sleeve gastrectomies). Multivitamins were systematically prescribed after GBP but not after RS and additional supplements (including vitamin B12, 25OH-vitamin $\mathrm{D}$, calcium, and iron) were introduced if deficiencies were recorded during follow-up.

Results: Initial BMI was similar $\left(47.1 \pm 7.9\right.$ vs $\left.45.8 \pm 8.0 \mathrm{~kg} / \mathrm{m}^{2}\right)$ in GBP and RS groups but patients in RS group were younger ( $36 \pm 11$ vs $42 \pm 10$ years, $\mathrm{p}<0.001)$. Mean follow-up was $7 \pm 2$ years in each group but caloric intake was lower $(1388 \pm 468$ vs. $1519 \pm 609 \mathrm{kcal} / 24 \mathrm{~h}, \mathrm{p}=0.034)$ and weight loss was greater $(30.6 \pm 9.8$ vs. $20.4 \pm 13.4 \%, \mathrm{p}<0.001)$ after GBP. The number of nutritional deficiencies was not significantly different in average between groups $(5.0 \pm 2.4$ vs $4.7 \pm 2.4$, after GBP and RS respectively). However, despite more patients taking multivitamins in GBP group ( 84 vs $11 \%$ in RS group, $\mathrm{p}<0.001$ ), they also need more often additional complements ( $70 \mathrm{vs} 16 \%, \mathrm{p}<0.001)$ to maintain the same nutritional status. Furthermore, nutritional deficiencies were increased in subjects who did not take multivitamins after GBP $(6.1 \pm 2.3$ vs $4.8 \pm 2.4$, $p=0.004)$. Vitamin $D$, calcium and iron were the most often deficient parameters after both procedures, but some parameters were specifically altered, including vitamins B1 and C after RS and zinc and vitamin B12, after GBP, with increased homocysteine, notably in those without supplementation. Severe malnutrition or severe anaemia were rare $(<5 \%)$ but almost all were observed after GBP.

Conclusion: Long term nutritional status is preserved both after RS and GBP in patients attended a specialized centre. However, nutritional follow-up is necessary even in long term, to adapt nutritional supplementation, notably after GBP, in order to avoid specific nutritional deficiencies.

Conflict of interest: None disclosed

Funding: No Funding

\section{T3P48 \\ Comparison of nutritional status and neonatal outcomes in pregnant women according to type of bariatric surgery}

\section{Coupaye, M. ' 'Legardeur, H. ${ }^{2}$; Sami, O. '; Calabrese, D. '; Msika, S. ;} Mandelbrot, L. ' ${ }^{\text {; Ledoux, } S \text {. }}$

${ }^{1}$ Centre de prise en charge de l'Obésité, Hôpital Louis Mourier (AP-HP), Colombes and Université Paris Diderot, France, ${ }^{2}$ Service De GynécologieObstétrique, Hôpital Louis Mourier (Ap-Hp), Colombes And Université Paris Diderot, France

Introduction: Bariatric surgery is known to influence pregnancy outcomes but whether it alters nutritional status during pregnancy is poorly known. Our aim was to compare nutritional parameters during pregnancy and their potential link with neonatal outcomes after Roux-Y-gastric bypass (GBP) and restrictive surgery (RS, i.e. sleeve gastrectomy (SG) and gastric banding $(\mathrm{GB})$ )

Methods: All women with singleton pregnancies who had at least one nutritional evaluation during pregnancy in our institution between 2006 and 2016 were included. We evaluated nutritional deficiencies according to standard and specific norms for pregnancy (Abbassi-Ghanavati 2009). Results: 97 women had 106 pregnancies during the study period: 60 had a GBP and 37 a restrictive surgery (29 SG and 8 GB). Mean weight before pregnancy and weight gain during pregnancy were not significantly different $(82.8 \pm 14.0$ vs. $89.9 \pm 24.8 \mathrm{~kg}$, and $8.2 \pm 6.5$ vs. $7.9 \pm 8.0 \mathrm{~kg}$, respectively after GBP and RS). Mean interval between surgery and pregnancy was also similar $(27.9 \pm 22.2$ vs. $20.5 \pm 16.8$ months $)$ but weight loss after GBP was higher $(41.7 \pm 12.5$ vs. $30.2 \pm 13.7 \mathrm{~kg})$. The number of nutritional deficiencies was not different between groups ( $8.0 \pm 2.6$ vs. $7.1 \pm 2.9$ with the standard norms, $3.5 \pm 2.0$ vs. $2.9 \pm 1.9$ with the specific norms, after GBP and RS respectively). However, women took more multivitamins and additional supplements after GBP than after RS (93\% vs. $77 \%, \mathrm{p}=0.046$, and $1.9 \pm 1.4$ vs. $0.9 \pm 1.3$ other supplements, $\mathrm{p}=0.0015$ ). Moreover, whereas albumin and hemoglobin were not significantly different, GBP specifically altered some nutritional parameters, including serum ferritin, calcium, PTH and vitamin E. The interval between surgery and pregnancy was inversely correlated with ferritin $(R=-0.316$, $\mathrm{p}=0.008)$. Gestational age at delivery was lower after GBP $(38.4 \pm 2.6$ vs. $39.5 \pm 1.9$ weeks, $\mathrm{p}=0.04)$ and birth weight also tended to be lower $(3025 \pm 639$ vs. $3269 \pm 529 \mathrm{~g}, \mathrm{p}=0.08)$. Thus, $Z$ score was not significantly different between groups $(-0.41 \pm 1.1$ vs. $-0.26 \pm 1.1)$. Z score was positively correlated with maternal weight in the second trimester $(R=0.265$, $\mathrm{p}=0.04)$, and weight gain during pregnancy $(\mathrm{R}=0.401, \mathrm{p}=0.003)$ but not with nutritional parameters. Interestingly, fasting insulinemia was significantly lower after GBP (5.7 \pm 5.4 vs. $8.2 \pm 5.6 \mathrm{mUI} / \mathrm{l}, \mathrm{p}=0.03)$ and correlated with $\mathrm{Z}$ score $(\mathrm{R}=0.391, \mathrm{p}=0.03)$.

Conclusion: In women attending a specialized centre, nutritional deficiencies do not seem to significantly alter neonatal outcomes but larger studies are needed to confirm these results. However, after GBP, women need to take more supplements to maintain the same nutritional status 
and some parameters are more altered than after RS. Careful nutritional monitoring is necessary after both procedures.

\section{T3P50 \\ Conservative multidisciplinary intervention in the treatment of obesity. Preliminary data after 1 year}

Doctor, E. '; Psychologist, E. ${ }^{1}$

${ }^{1}$ NIMI Ringerike AS (Norwegian Institute of Sports Medicine)

Introduction: Obesity is a chronic disease that requires long-term strategies for its management. Studies have reported health benefits with weight loss of only $5 \%$ of the body weight. (1)

Methods: In a multidisciplinary inpatient treatment program for severely obese, 189 patients (of 276 initially) have been followed for 1 year. The treatment consists in gradual life-style intervention that targets exercise, nutrition and psychological coping strategies for behavior change management. The program lasts 5 years and it has a standardized length $(9+1+1+1$ weeks $)$ the first year and $2 \times 1$-week visits annually during the next 4 years. This is preliminary data, after 1 of 5 years of treatment. Results: Initial average total body weight was 123,2. After the first 9 weeks, the average total body weight registered $9,1 \%$ weight reduction, compared to initial weight. $64,6 \%$ have succeed weight loss $\geq 8 \%$ and $34,9 \%$ have succeed a weight loss $\geq 10 \%$.

At the end of the first year, the average total body weight registered 10,2\% reduction compared to the initial weight. $59,8 \%$ had $\geq 8 \%$ weight loss, $42,9 \%$ had $\geq 10 \%$ and $8,5 \%$ had $\geq 20 \%$ weight loss, meanwhile $79,9 \%$ had $\geq 5$ weight loss.

Conclusion: The most weight-loss was registered after 6 months $(13,2 \%$ reduction compared to initial weight). At the end of the first year the average total-body weight increases compared to the most weight loss, but it remains lower than the initial average total body weight. A large part of the patients have succeeded weight loss $\geq 5 \%$ after 1 year.

Reference:

1 Knowler WC, Barrett-Connor E, Fowler SE, et al. Reduction in the incidence of type 2 diabetes with lifestyle intervention or metformin. N Engl J Med $2002 ; 346: 393$

The data used is collected from NIMI Ringerike which treats obesity conservative. No Funding

\section{T3P51}

\section{Detecting leakage in bariatric patients: Value of clinical, paraclinical, and radiological parameters}

Duprée, A. ${ }^{1}$; Wolter, S. ${ }^{1}$; Busch, P. ${ }^{1}$; Miro, J. ${ }^{1}$; Izbicki, J. ${ }^{1}$; Aberle, J. ${ }^{1}$; Mann, O. ${ }^{1}$

${ }^{1}$ University Medical Center Hamburg-Eppendorf

Backround: Major complications in bariatric surgery are rare but in case of occurrence severe. Due to the elevated BMI diagnosis of major complications seems to be more challenging than in norm weight patients. We aimed to show the relevance of clinical and paraclinical parameters, as well as radiologic findings in diagnosis of major complications after bariatric procedure.

Method: All patients operated from 2010-2012 were retrospective studied after bariatric surgery with special view on clinical parameters (tachycardia, blood pressure,temperature), paraclinical parameters (CRP, Leukocytes), and realized CT scans for diagnosis of leakage which required an operative intervention.

Results: In 588 bariatric procedures performed, a major complication rate of 2,9\% occurred, 2,2\% leaks in sleeve gastrectomy and 0,7\% in RYGB.

Univariate analysis showed a significant higher risk in development of leakage if CRP (OR 2,47; p < 0,01)or leucocytes $(\mathrm{OR} 1,26 ; \mathrm{p}<0,005)$ double to first postoperative value. Multivariate Analysis showed a significant higher risk of leakage development for doubling of postoperative CRP
(OR 4,836; p < 0,001), while leucocytes, as well as clinical parameters showed no significance.

CT scans showed $50 \%$ false-negative findings. Sensitivity for detecting leakage remained only $25 \%$.

Conclusion: In the detection of major complications after bariatric surgery the CT scan captures a subordinated role. Clinical aspects and experience as well as blood parameters, especially CRP doubling in the postoperative course are of overriding importance in diagnosis of post bariatric complications, especially if there are no seminal findings in CT scan. Significant trends towards E/e' decrease were demonstrated only in Lisinopril-treated patients ( $\Delta \mathrm{E} / \mathrm{e}^{\prime} 1.2$ Lisinopril and 0.8 Perindopril , $\left.\mathrm{p}<0.05\right)$. Conclusion: LV diastolic function in obese $\mathrm{AH}$ patients demonstrated impairment of the I type. LV diastolic function was improved in response with ACE inhibitor treatment. Both Lisinopril and Perindopril treatment improved in LV diastolic function parameters, whereas only Lisinopril treatment was more significantly associated with trends in E/e' improvement in 3 months' short-term follow-up.

\section{T3P54}

\section{Dietary phylloquinone consumption and the risk of incident} cataracts in an elderly Mediterranean population

Barcia, M. ${ }^{1}$; Bulló, M. ${ }^{2}$; García-Gavilán, J. ${ }^{1}$; Canela, M. ${ }^{3}$; Corella, D. ${ }^{4}$; Estruch, R. ${ }^{5}$; Fitó, M. ${ }^{6}$; Layana, A. ${ }^{7}$; Arós, F. ${ }^{8}$; Fiol, M. ${ }^{\text {; }}$ Lepetra, J. ${ }^{10}$; SerraMajem, L. ${ }^{11}$; Pintó, X.12; García-Arellano, A. ${ }^{3}$; Vinyoles, E. ; Sorli, J. ${ }^{13}$; SalasSalvadó, J. ${ }^{14}$

${ }^{1}$ Human Nutrition Unit, Biochemistry and Biotechnology Department, Faculty of Medicine and Health Sciences, University Hospital of Sant Joan de Reus, IISPV, Universitat Rovira i Virgili, Reus, Spain., ${ }^{2}$ Human Nutrition Unit, Biochemistry And Biotechnology Department, Faculty Of Medicine And Health Sciences, University Hospital Of Sant Joan De Reus, lispv, Universitat Rovira I Virgili, Reus, Spain; And Ciberobn Physiopathology Of Obesity And Nutrition, In, ${ }^{3}$ Ciberobn Physiopathology Of Obesity And Nutrition, Instituto De Salud Carlos lii, Madrid, Spain; And University Of Navarra, Medical School, Department Of Preventive Medicine And Public Health, Pamplona, Spain., ${ }^{4}$ Ciberobn Physiopathology Of Obesity And Nutrition, Instituto De Salud Carlos lii, Madrid, Spain; And Department Of Preventive Medicine, University Of Valencia, Valencia, Spain., ${ }^{5}$ Ciberobn Physiopathology Of Obesity And Nutrition, Instituto De Salud Carlos lii, Madrid, Spain; And Department Of Internal Medicine, August Pi I Sunyer Institute Of Biomedical Research (Idibaps), Hospital Clinic, University Of Barcelona, Barcelona, Spain, ${ }^{6} \mathrm{Ciberobn}$ Physiopathology Of Obesity And Nutrition, Instituto De Salud Carlos lii, Madrid, Spain; And Cardiovascular Risk And Nutrition (Regicor Study Group), Hospital Del Mar Medical Research Institute, Barcelona Biomedical Research Park, Barcelona, Spain, ${ }^{7}$ Ciberobn Physiopathology Of Obesity And Nutrition, Instituto De Salud Carlos lii, Madrid, Spain And Department Of Ophthalmology, University Of Navarra, Pamplona, Spain., ${ }^{8}$ Ciberobn Physiopathology Of Obesity And Nutrition, Instituto De Salud Carlos lii, Madrid, Spain, And Department Of Cardiology, Hospital Txangorritxu, Vitoria, Spain., ${ }^{9} \mathrm{Ciberobn}$ Physiopathology Of Obesity And Nutrition, Instituto De Salud Carlos lii, Madrid, Spain, And Department Of Cardiology, Hospital Universitario Son Dureta, Palma De Mallorca, Spain., ${ }^{10}$ Ciberobn Physiopathology Of Obesity And Nutrition, Instituto De Salud Carlos lii, Madrid, Spain; And San Pablo Health Center, Sevilla, Spain., ${ }^{11}$ Ciberobn Physiopathology Of Obesity And Nutrition, Instituto De Salud Carlos lii, Madrid, Spain; And Research Institut Of Biomedical And Health Sciences, University Of Las Palmas De Gran Canaria, Las Palmas, Spain., ${ }^{12}$ Ciberobn Physiopathology Of Obesity And Nutrition, Instituto De Salud Carlos lii, Madrid, Spain; And Lipids And Vascular Risk Unit, Internal Medicine, Hospital Universitario De Bellvitge, Barcelona, Spain, ${ }^{13}$ Ciberobn Physiopathology Of Obesity And Nutrition, Instituto De Salud Carlos lii, Madrid, Spain; Anddepartment Of Preventive Medicine, University Of Valencia, Valencia, Spain., ${ }^{14} \mathrm{Ciberobn}$ Physiopathologhuman Nutrition Unit, Biochemistry And Biotechnology Department, Faculty Of Medicine And Health Sciences, University Hospital Of Sant Joan De Reus, lispv, Universitat Rovira I Virgili, Reus, Spain; And Ciberobn Physiopathology Of Ob

Background: Cataract, the most frequent cause of blindness in developed countries, is strongly associated with aging. The exactly mechanisms underlying cataract formation are still unclear but growing evidence suggest a potential role of inflammatory and oxidative processes. Therefore, anti- 
oxidant and anti-inflammatory factors of the diet, as phylloquinone, could play a protective role.

Aim: The aim of this study is to examine the association between dietary phylloquinone intake and the risk of incident cataracts among an elderly Mediterranean population.

Methods: A prospective analysis in 5860 participants from the PREvención con DIeta MEDiterránea (PREDIMED) Study was conducted. Dietary phylloquinone intake was evaluated using a validated food frequency questionnaire. The time to the cataracts event was calculated as the time between recruitment and the date of the appearance of the event, the time to the last visit of the follow-up, date of death, or the end of the study. Hazard ratios (HRs) and 95\% confidence intervals (CIs) for cataracts incidence were estimated with the use of multivariable Cox proportional hazards models.

Results: After a median of 5.6 years of follow-up we documented a total of 768 new cataracts. Subjects in the highest tertile of dietary phylloquinone intake had a significant lower risk of cataract than those in the lowest tertile (HR: 0.72; 95\% CI: 0.60, 0.88); P for trend $=0.002$ ) after adjusting by potential confounders.

Conclusion: High intake of dietary phylloquinone is associated with a reduced risk of cataracts in an elderly Mediterranean population.

\section{T3P55}

\section{Dietary protein content modulates the amino-acid and IGF1 responses to sucrose overfeeding in humans.}

Jegatheesan 1, P. '; Surowska, A. '; Campos, V. '; Cros, J. '; Stefanoni, N. '; Rey, V.' ; Schneiter, P.'; Bandt, J. '; Tappy, L.'

${ }^{1}$ Department of Physiology, University of Lausanne, Lausanne, Switzerland, ${ }^{2}$ Ea4466, Faculty Of Pharmacy, Paris Descartes University, Sorbonne Paris Cité, 75006 Paris, France

Introduction: High sucrose diets have been shown to decrease muscle anabolism through insulin resistance, and changes in blood amino acid concentrations. Whether this is also the case in humans remain unknown. In this study, we compared the short-term effects of high vs low protein intake during a short-term hypercaloric high-sucrose diet on fasting blood amino acids and IGF1 concentrations.

Methods: We performed a randomized, crossover clinical study on 12 healthy young male and female volunteers. Each participant was studied after a 3-day run-in diet containing 100\% daily energy requirement ( $45 \%$ starch, $10 \%$ sucrose, $33 \%$ lipid, $12 \%$ protein), and after 7 -day on hypercaloric diet containing $150 \%$ daily energy requirement with $45 \%$ starch, $50 \%$ sucrose, $10 \%$ lactose, and either $37.5 \%$ lipid and $7.5 \%$ protein (HSLP) or $15 \%$ lipid and $30 \%$ protein (HSHP). Fasting plasma insulin, glucagon and IGF1 concentrations were assessed before and after each intervention by RIA, and plasma amino acids concentrations by HPLC.

Results: High-protein, high sucrose overfeeding significantly increased plasma glucagon area under the curve $(26.0 \pm 2.5$ vs $21.3 \pm 1.4 \mathrm{ng} /$ $\left.\mathrm{ml}^{\star} 300 \mathrm{~min}\right)$ and IGF- 1 area under the curve $(31.4 \pm 1.9 \mathrm{vs} 25.9 \pm 1.7 \mu \mathrm{g} /$ $\left.\mathrm{dl}^{*} 300 \mathrm{~min}\right)$ compared with low-protein, high sucrose overfeeding. This was associated with changes in amino acids profiles, particularly a lower plasma alanine $(386 \pm 11$ vs $464 \pm 33 \mu \mathrm{M})$ and glycine $(170 \pm 11$ vs $229 \pm 25 \mu \mathrm{M}$ ) levels and a higher plasma branched amino acids (valine $(273 \pm 17$ vs $193 \pm 11 \mu \mathrm{M})$, leucine $(140 \pm 7$ vs $116 \pm 5 \mu \mathrm{M}))$, tyrosine $(53 \pm 2$ vs $43 \pm 2 \mu \mathrm{M})$, lysine $(177 \pm 7$ vs $122 \pm 5 \mu \mathrm{M})$, and phenylalanine $(53 \pm 2 \mathrm{vs} 42 \pm 1 \mu \mathrm{M})$ concentrations after high protein than low protein overfeeding. Plasma insulin did not differ between the two groups.

Conclusion: Sucrose overfeeding differentially alters blood IGF1 and amino-acid concentrations according to dietary protein content. This may be associated with decreased muscle protein synthesis when dietary protein intake is low.

\section{Conflict of Interest: None Disclosed.}

Funding: Research relating to this abstract was funded by a grant from the Swiss National Science Foundation (320023-138428), and by Institute Benjamin De lessert.

\section{T3P56 \\ Disordered eating, weight and shape concerns among bariatric patients and controls}

Lev-Ari, L. ' ; Zohar, A. '; Bachner-Melman, R. '; Globus, I. ${ }^{2}$; BursteinKlomek, A. ${ }^{3}$; Latzer, $Y^{4}{ }^{4}$

${ }^{1}$ Ruppin Academic Center, ${ }^{2}$ University Of Haifa, ${ }^{3}$ Interdisciplinary Center (Idc), ${ }^{4}$ University Of Haifa; Rambam Health Care Campus

The obesity epidemic is gaining momentum across the globe, as the weight loss battle intensifies. Obesity is a risk factor for many medical and psychological ailments and studies on weight loss interventions consistently indicate that the chances of losing weight and keeping it off are slim. For the morbidly obese, bariatric surgery might be the last resort for weight loss. The Centers for Disease Control and Prevention (CDC) reports almost 180,000 bariatric operations conducted in 2013 in the United States alone. The literature shows that surgery is insufficient to keep weight off in the long run, without a major change in diet, physical activity and lifestyle. Failure to adhere to a strict post-surgery diet is one of the highest risk factors for weight regain following surgery. Notably, only a few studies have compared disordered eating behaviors of bariatric surgery candidates and individuals with overweight and obesity who do not undergo surgery. This study used the Eating Disorders Examination Questionnaire (EDE-Q) to compare the eating behaviors and weight and shape concerns of 87 (22.8\%) bariatric surgery candidates (bariatric), 91 (23.9\%) overweight community volunteers (over-weight) and 203 (53.3\%) normal weight community volunteers (normal weight). No gender differences were observed between the groups. Bariatrics had the least education (mean years $=12.05, \mathrm{SD}=1.35$ ), followed by the normal weight group $($ mean $=15.21, \mathrm{SD}=3.54)$ and the over-weight group had the highest level of education ( mean $=16.15, \mathrm{SD}=4.04 ; \mathrm{F}_{(2,344)}=37.05, \mathrm{p}<.000$ ), which was, therefore, included as a covariate in all analyses. Significant differences were observed between the three groups on all EDE-Q measures, with the normal weight group scoring lowest on all measures. The bariatrics scored highest in eating concerns, weight concerns and shape concerns. The over-weight group showed the highest level of restraint, overate most frequently, exercised most excessively and used the most laxatives. There were significant correlations between BMI and all EDE-Q measures in the whole sample, but these correlations were not significant within the bariatric group alone. A three-way logistic regression, in which the EDE-Q subscales were entered as predictors, differentiated the bariatric group from both other groups, but did not differentiate between the over-weight and normal-weight groups. Thus, the members of the bariatric group are distinctly different in their eating behavior and weight and shape concerns from community volunteers, regardless of their BMI. Better understanding of the unique qualities of bariatric surgery candidates, is needed in order to maximize their chances of profiting from this potentially life-changing intervention.

\section{T3P57 \\ Does a previous daily soft drink intake impair the effects of bariatric surgery?}

Haenni, A. ${ }^{1}$; Johansson, H. ${ }^{1}$; Nelson, D. ${ }^{2}$

${ }^{1}$ Dept of Surgery, Falu lasarett and Uppsala University, Sweden, ${ }^{2}$ Dept Of Surgery, Falu Lasarett

Introduction: A high intake of soft drinks has been associated with weight gain, higher risk for type 2 diabetes and cardiovascular morbidity. Aim: To investigate if a history of high soft drink intake impairs the effects of bariatric surgery.

Methods: The eating and drinking habits were assessed by a questionnaire during the investigation for bariatric surgery. The glucometabolic effects of gastric bypass in 25 subjects with a high, i.e., daily, intake of soft drinks were in this retrospective study compared to those of 29 age-matched patients without any such consumption. All patients were treated with gastric bypass, GBP, and given the same kind of dietary advice and multivita- 
min and mineral supplementation after surgery. All subjects were asked to quit all soft drink intake before surgery.

Results: Before surgery there were no significant difference between the two groups regarding BMI, fasting blood glucose and HbAlc levels, or prevalence of diabetes. A history of psychiatric disease was more frequent among the soft drink consumers, $72 \%$ compared to $38 \%$ in the non-consumers, $\mathrm{p}<0,05$. No difference was noted regarding alcohol or drug abuse. During the one year follow-up BMI fell by $31 \%$ among the previous soft drink consumers and by $28 \%$ among the non-consumers with no statistical difference between the two groups. The sagittal abdominal diameter decreased by $28 \%$ in both groups. The fasting glucose concentration and HbAlc fell by $13 \%$ and $15 \%$ among the previous soft drink consumers and by $11 \%$ and $15 \%$ among non-consumers, respectively (ns). The alterations in blood pressure, liver enzymes, lipid concentrations, were similar in the two groups. Psychiatric disease or medication, mostly continued from the period before surgery, remained more frequent, although not reaching statistical significance, after surgery among the previous soft drink consumers compared to non-consumers, $52 \%$ vs $31 \%$, respectively .

Conclusion: The weight lowering and glucometabolic effects after gastric bypass surgery are similar for previous daily consumers of soft drinks when compared to non-consumers. A history of psychiatric disease seems to be more common among soft drink consumers.

Conflict of Interest: None Disclosed

Funding: No Funding

\section{T3P58}

\section{Does gender and ethnic differences in fasting blood leptin persist after adjusting for differences in body composition?}

Hunma, S. ; Ramuth, H. ; Miles-Chan, J. ${ }^{2}$; Schutz, Y. ${ }^{2}$; Montani, J. ${ }^{2}$; Joonas, N. ${ }^{1}$; Dulloo, A. ${ }^{2}$

${ }^{1}$ Obesity Unit, Victoria Hospital, Ministry of Health and Quality of Life, Mauritius, ${ }^{2}$ Department Of Medicine, Division Of Physiology, University Of Fribourg, Fribourg, Switzerland

Introduction:There are conflicting findings as to the predictive power of leptin for weight gain, type 2 diabetes or cardiovascular diseases, and about gender differences in leptin beyond that explained by various indices of adiposity. These discrepancies have been attributed to the use of body mass index (BMI) and waist circumference (WC) as surrogates of total or central adiposity rather than body fat per se, lack of dietary control prior to blood sampling, and/or to statistical procedures employed for adjusting plasma/serum leptin. In the light of these arguments, we have re-investigated the earlier findings from the island of Mauritius that adults of Indian (South Asian) descent have higher levels of leptin than Creoles (African/Malagasy descent) beyond that explained by adiposity, albeit on the basis that these ethnic differences in leptin remained after adjustments for BMI and/or WC (Lilja et al. Int J Obes 2010;34:878-85).

Methods: In a healthy adult Mauritian cohort (age 20-42 years; total $\mathrm{n}=160 ; 80$ men and 80 women; BMI range: $15-41 \mathrm{~kg} / \mathrm{m}^{2}$ ) belonging to the two main ethnic groups - Indians and Creoles - we evaluated overnight fasted serum leptin, BMI, WC, total body fat by the deuterium oxide dilution technique and expressed as a percentage of body weight (total body fat\%), central adiposity (TrunkFat\%) by abdominal bioimpedance analysis using the ViScan device, and applied regression analysis for statistical adjustment of logarithmically-expressed serum leptin for the various adiposity and body composition measures.

Results: The higher serum leptin values observed in women than in men $(\mathrm{p}<0.001)$ over the range of BMI or WC studied are totally or almost completely abolished when BMI and WC were replaced by total body fat\% and TrunkFat\%, respectively, independently of whether those of extreme BMI were included or excluded in the analysis. Furthermore, the leptinBMI and leptin-WC linear relationships are found to be more elevated in Indians than in Creoles in men, but these differences are abolished when BMI and WC are replaced by total body fat $\%$ or TrunkFat $\%$. In women, no significant between-ethnic difference is observed in serum leptin nor in total body fat $\%$ and TrunkFat $\%$.

Conclusion: In healthy young adults, in which leptin-adiposity relationships were analysed as linear regressions of logarithmically-expressed leptin versus total or central body fat $\%$, the differences in body fat $\%$ explained all the gender or ethnic differences in serum leptin.

Authors declare no conflict of interest

Research was supported by International Atomic Energy Agency, Mauritian Ministry of Health \& University of Fribourg - Switzerland.

\section{T3P59}

\section{Does nonlinear resistance training reduce metabolic syndrome in people living with HIV? A randomized clinical trial}

\section{Zanetti, H. ' Cruz, L. ' Lourenço, C. ${ }^{1}$; Neves, F. ${ }^{2}$; Silva-Vergara, M. ${ }^{2}$;} Mendes, . $^{3}$

${ }^{1}$ Postgraduate Program in Physical Education - Federal University of Triângulo Mineiro - Uberaba/MG, Brazil, ${ }^{2}$ Postgraduate Program In Infectious Disease And Tropical Me Dicine - Federal University Of Triângulo Mineiro - Uberaba/Mg, Brazil, ${ }^{3}$ Sports Science Department - Federal University Of Triângulo Mineiro Uberaba/Mg, Brazil

Introduction: to evaluate the effectiveness of a 12-week nonlinear resistance training (NLRT) program on metabolic syndrome (MetS) components in people living with human immunodeficiency virus (PLHIV) and diagnosed with MetS.

Methods: Subjects were randomly assigned to either a NLRT $(n=10)$ or control (CON; $\mathrm{n}=11$ ) group. The NLRT group exercised three times per week during the 12 week supervised training program. The CON group was asked to maintain their daily habits. At baseline (pre) and after the intervention (post), both groups completed anthropometric, blood pressure, and biochemical assessments. A $2 \times 2$ repeated measures ANOVA (significance level of 5\%) was performed to calculate the effects of intervention duration (time), group, and the interaction between the two.

Results: There was a significant time* group interaction for lean body mass $(\mathrm{LBM})(+5.5 \%, \mathrm{p}<.0001)$, body fat mass $(\mathrm{BFM})(-10.1 \%, \mathrm{p}<.0001)$, body fat percentage $(\mathrm{BF} \%)(-11.4 \%, \mathrm{p}<.0001)$, waist circumference (WC) $(-1 \%, \mathrm{p}=.001)$, high-density lipoprotein $(\mathrm{HDL})(+31 \%, \mathrm{p}<.0001)$ levels, fasting glucose $(\mathrm{FG})(-13.7 \%, \mathrm{p}=.012)$, and glycated hemoglobin $(\mathrm{HBA} 1 \mathrm{C})(-9 \%, \mathrm{p}<.0001)$. After the intervention, the NLRT group included fewer participants with abnormal levels of triglycerides (TG), HDL, systolic blood pressure (SBP), diastolic blood pressure (DBP), and FG while the CON group had more participants with abnormal levels of TG, SBP, and DBP.

Conclusion: Twelve weeks of NLRT was effective in ameliorating some components of MetS in PLHIV

Conflict of Interest: None

Funding: This work was supported by FAPEMIG - Fundação de Amparo a Pesquisa do Estado de Minas Gerais, Brazil.

\section{T3P60}

Double-blind, randomised, placebo-controlled three-way crossover clinical investigation to evaluate the benefit of IQPVV-102 in reducing postprandial glucose level in overweight Caucasian subjects

\section{Bothe, G. ' Teng, E. $^{2}$; Chong, P. ${ }^{3}$; Khoo, J. ${ }^{2}$; Uebelhack, R. ${ }^{\text {' }}$}

${ }^{1}$ Analyze \& Realize GmbH, ${ }^{2}$ Inqpharm Europe Ltd, ${ }^{3}$ Zaluvida Corporate Ltd

Introduction: IQP-VV-102, which contains a proprietary grape extract (grape marc) and L-arabinose, has been proven to promote weight loss in overweight and obese individuals. In this study we evaluated the effect of IQP-VV-102 on reducing postprandial glucose (PPG) AUC levels. 
Methods: 30 overweight, but otherwise healthy Caucasians (BMI 25$29.9 \mathrm{~kg} / \mathrm{m}^{2}$ ), both male and female, aged $18-60$ years were randomly allocated to verum and placebo in a single-dose crossover design, with 3-day washout periods. Two doses of IQP-VV-102 were investigated - 1290mg (V1) and 2580mg (V2) - together with placebo control (P). Blood sampling for PPG, insulin and triglycerides were performed after shortly taking each of the respective investigational products followed by the test meal - marked as 0 mins - and subsequently at 15, 30, 45, 60, 90, 120 and 180 mins.

Results: There were statistically significant differences for in total PPG AUC levels at 120mins, both between the V2 group and the $\mathrm{P}$ group $(\mathrm{p}=0.0028)$, and between the $\mathrm{V} 1$ and $\mathrm{P}$ groups $(\mathrm{p}=0.037) . \mathrm{IQP}-\mathrm{VV}-102$ also reduced postprandial insulin AUC levels significantly $(\mathrm{p}<0.05$

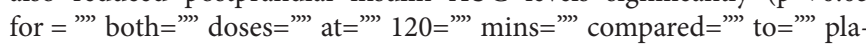
cebo="'"--0="' 05--="'>

There were no clinically relevant changes in safety laboratory parameters and no adverse effects. $100 \%$ of the subjects rated the tolerability of the products with good or very good.

Conclusion: IQP-VV-102 favourably affects PPG levels and insulin response. In addition to promoting weight loss, the results here suggest that IQP-VV-102 may have important clinical value in reducing PPG level without augmenting insulin responses.

Conflict of interest: Emily Teng and JooLian Khoo are currently employed by InQpharm Europe Ltd. Pee-Win Chong was employed by InQpharm Europe Ltd at the time of the study.

Funding: Research was funded by InQpharm Europe Ltd.

\section{T3P61}

\section{Early weight-loss and weight regain after gastric bypass predict weight outcome at 10 years.}

Despland, C. ${ }^{1}$; Rueger, S. ${ }^{2}$; Favre, L. ${ }^{\text {; }}$ Suter, M. ${ }^{3}$; Laferrere, B. ${ }^{4}$; Pralong, F. ; Kutalik, Z. ${ }^{2}$; Vionnet, $N{ }^{1}$

${ }^{1}$ Service of Endocrinology, Diabetology, and Metabolism, Lausanne University Hospital, 1011 Lausanne, Switzerland " ${ }^{2}$ Institute Of Social And Preventive Medicine, Lausanne University Hospital, Lausanne, Switzerland; Swiss Institute Of Bioinformatics, Lausanne, Switzerland, ${ }^{3}$ Department Of Surgery, Lausanne University Hospital, Lausanne, Switzerland / Department Of Surgery, Hôpita Riviera-Chablais, Aigle-Monthey, Switzerland, ${ }^{4}$ New York Obesity Nutrition Research Center, Department Of Medicine, Columbia University College Of Physicians And Surgeons, New York, Ny

Introduction: Roux-en-Y gastric bypass surgery (RYBP) is an effective therapeutic intervention resulting in $\sim 30 \%$ total weight loss and resolution and/or improvement of obesity-associated co-morbidities. However weight loss response varies significantly between patients, ranging from 10 to $50 \%$ total weight loss at 3 years. Few predictors of long-term weight loss have been identified due to the lack of long-term ( $>3$ years) follow-up studies. The goal of this study was to model weight trajectories to predict long-term weight loss, in a large cohort of patients followed up to 10 years after RYGB. We hypothesized that early weight loss to nadir, and weight regain trajectories at 3 years, would predict long-term weight loss after RYGB.

Methods: A cohort of 1608 patients who underwent RYGB by the same surgical team in two different hospitals was followed up from 1999 till 2014. Weight and BMI were measured before surgery, at 6 and 12 months after surgery and then yearly. Of the 1608 patients included, 561 had data available 10 years after surgery. Weight outcomes (body weight or BMI at 10 years after operation) were modelled, using normal linear regression, as a function of key variables such as nadir weight at 1 year and weight change between nadir and 3 years post surgery.

Results: Mean age, BMI and gender distribution at pre-surgery were: $41 \pm 11.1$ years old, $45,5 \pm 5.9 \mathrm{~kg} / \mathrm{m}^{2}$ and $\mathrm{M} / \mathrm{F} 25.4 \% / 74.6 \%$ respectively Mean and range of nadir weight loss at 1 year were $(34.4 \% \pm 7,12-57)$, and weight loss at 3 years $(33.9 \% \pm 8.2,6.75-58.3)$. Weight at 10 years varied between $8 \%$ weight gain and $57.2 \%$ weight loss.
Of various weight outcome measures, such as BMI change, total weight change, excess weight loss or \% excess weight loss, the outcome that can be best modelled in the normal linear model framework was BMI change. Thus we studied the role of early weight loss and regain trajectories to predict BMI change 10 years after operation. We could demonstrate that $70 \%$ of the 10 -year-BMI-value variability can be captured (adjusted $\mathrm{r}^{2}=0.7$ ) by the combination of maximum weight loss (nadir weight post-surgery) up to 1 year post-surgery and by the early weight regain from nadir to year 3 (the 1-3 year period after surgery). As nadir weight may not always occur up to one year post surgery, we also tested the relation between BMI at nadir and BMI at 1 year and showed that BMI at 1 year after surgery was an excellent predictor of the BMI at nadir $\left(r^{2}>0.8\right)$.

Conclusion: Identifying determinants of the $0-1$ year weight loss and the 1-3 year weight regain are sufficient to capture the long term (10 year) BMI evolution. Weight loss until one year can be used as a reasonable substitute for weight loss at nadir in prospective studies.

Conflict of Interest: None Disclosed

Funding: No Funding

\section{T3P62}

\section{Eating behavior and body weight loss in women long after gastric bypass surgery.}

Novelli, I. '; Fonseca, L. ' Gomes, D. ${ }^{2}$; Dutra, E. ' Carvalho, K. ${ }^{1}$

${ }^{1}$ University of Brasilia, Brazil, ${ }^{2}$ University Of Para, Brazil

Introduction: Successful weight loss and maintenance after Roux-en-Y gastric bypass (RYGB) may be related to eating behavior. The objective of this study was to assess the main eating behavior domains in women long after RYGB surgery and possible associations with excess weight loss (EBWL).

Methods: This cross-sectional study investigated women at least two years after RYGB in a university hospital. The Three-Factor Eating Questionnaire (TFEQ-R21) was applied to evaluate the following eating behaviours domains: cognitive restraint, emotional eating, and uncontrolled eating. The score of each eating behavior domain ranges from 0 to 100 . Higher scores indicate a greater incidence of eating behaviors associated with the respective domain. The patients were grouped according to surgery outcome: successful group ( $S G, n=72$ ), defined as present at least $50 \%$ of the preoperative EBWL; and unsuccessful group (UG, $\mathrm{n}=23$ ) when EBWL\% did not reach $50 \%$. Linear regression analysis was used to investigate whether any of the eating behavior domains was associated with \%EBWL, adjusted for age and education level in Model 1, and for postoperative time in Model 2. The 95\%CI was calculated, and the significance level was set at $5 \%(\mathrm{p}<0.05)$.

Results: The mean age was $47.3 \pm 9.8$ years, and $60 \%$ of the sample referred only elementary school level. The mean postoperative time was $59 \pm 21$ months $(56.2 \pm 19.4$ in the SG; and $69.9 \pm 23.6$ months in the UG; $\mathrm{p}=0.016)$. In both groups the cognitive restraint domain reached the highest score, followed by emotional and uncontrolled eating. The emotional eating domain was negatively associated with EBWL $(B=-0.286$; $\mathrm{p}=0.033$ ), regardless of age, education level, and postoperative time.

Conclusion: The cognitive restraint domain was the main type of eating behaviour observed in women long after RYGB surgery. Patients with higher emotional eating levels are at higher risk of having insufficient weight loss after RYGB. These results reinforce the understanding that behavioral factors affect weight loss and maintenance, and should be considered by the multidisciplinary teams that monitor bariatric patients over a long time.

Conflict of Interest: The authors have no business associations that might pose a conflict of interest in relation to this article.

Funding: This study was sponsored by CNPq Brazilian Agency under process number 476459/2011-7; 
T3P63

Effect of a 6-week very low calorie diet on anthropometric characteristics and blood clinical chemistry parameters in subjects with obesity

\section{Cloetens, L. ' ; Sedin, Å. ; Landin-Olsson, M. ${ }^{2}$}

${ }^{1}$ Biomedical Nutrition, Pure and Applied Biochemistry, Lund University, Lund, Sweden, ${ }^{2}$ Department Of Endocrinology, Lund University Hospital, Lund, Sweden

Introduction: Obesity is worldwide increasing in an alarming way and nutritional research is needed to prevent obesity-related disorders.

Methods: A very low calorie diet (VLCD) (650 kcal/day) was followed by obese subjects for 6 weeks. Anthropometric characteristics including body composition using a BIA-device and fasting blood clinical chemistry parameters were measured before and after VLCD. This study is part of an ongoing study investigating weight-maintenance after weight-loss in which subjects are randomised into different types of diets. Statistical significance was set at $\mathrm{p}$-value $<0.05$.

Results: 35 obese subjects (BMI $34.5 \pm 2.9 \mathrm{~kg} / \mathrm{m}^{2}, 52 \pm 10 \mathrm{y}, 74 \%$ female) were included in the study. All subjects completed the study without adverse effects. Two subjects had to be excluded because they did not reach the aim (-5\% of weight) and 1 subject because of diagnosed diabetes. After VLCD significant decreases (all p < 0.001) compared to baseline were observed in weight $(-10.7 \pm 2.2 \mathrm{~kg})$, BMI $\left(-3.6 \pm 0.5 \mathrm{~kg} / \mathrm{m}^{2}\right)$, wais circumference $(-9.2 \pm 3.6 \mathrm{~cm})$, sagittal abdominal diameter $(-3.0 \pm 1.3 \mathrm{~cm})$ and body fat $(-3.2 \pm 1.5 \%)$, fat-free mass $(-3.4 \pm 1.8 \mathrm{~kg})$ and total body water $(-2.5 \pm 1.4 \mathrm{~kg})$. Insulin $(-6.5 \pm 6.1 \mathrm{mIE} / \mathrm{L}, \mathrm{p}<0.001), \mathrm{Hb} 1 \mathrm{Ac}$ $(-2.3 \pm 2.0 \mathrm{mmol} / \mathrm{mol}, \mathrm{p}<0.001)$, total cholesterol $(-1.0 \pm 0.8 \mathrm{mmol} / \mathrm{L}$ $\mathrm{p}<0.001)$, triglycerides $(-0.4 \pm 0.6 \mathrm{mmol} / \mathrm{L}, \mathrm{p}<0.001)$, HDL-cholesterol $(0.2 \pm 0.3 \mathrm{mmol} / \mathrm{L}, \mathrm{p}=0.001)$ and LDL-cholesterol $(-0.9 \pm 0.7 \mathrm{mmol} / \mathrm{L}$, $\mathrm{p}<0.001)$ were significantly different from baseline. Framingham risk score was reduced by $1.2 \pm 2.3 \%(\mathrm{p}=0.006)$. Blood pressure, glucose ApoB/ApoA1, C-reactive protein and liver enzymes remained unchanged. Conclusion: A 6-week VLCD is an attractive and efficient weight-loss method for healthy obese subjects. VLCD also contributes to the prevention of cardiovascular disease and diabetes.

Acknowledgement: We would like to thank Cambridge Viktprogram for sponsoring the study.

\section{T3P64}

\section{Effect of regular magnesium supplementation on} dyslipidemia in obese insulin resistant patients

\section{Wulfsohn, . $^{1}$; Ravenna, M. ${ }^{1}$}

${ }^{1}$ Therapeutic Center Máximo Ravenna

Introduction: Obese insulin resistant patients usually present associated dyslipidemia (DLP) as part of a metabolic syndrome.

Magnesium supplementation is frequent in use in very low-calorie diets (VLCD) and regulates the metabolism of lipids and carbohydrates.

Methods: Retrospective review of medical records. Sixty patients were selected (30 female) with BMI > 30, mean age 46 years, with glycemia 100 - $125 \mathrm{mg} / \mathrm{dl}$, insulinemia > $15 \mathrm{mcU} / \mathrm{mL}$ and HOMA > 3, without taking antidiabetic drugs, and blood cholesterol, LDL, HDL and triglycerides were measured. They underwent a VLCD treatment plan alternating with LCD, with multivitamin and potassium. Half of patients received regular supplements of magnesium citrate. All attended cognitive behavioral therapeutic groups oriented to lose weight with physical activity and monthly medical- nutritional monitoring which was reassessed at 6 months.

Results: Both groups had significant decreases in weight and insulin resistance index at 6 months. Both decreased blood lipids.

Patients who regularly took magnesium showed higher statistically decreases than those who did not: Total Cholesterol $-27,12$ vs $-6,15$; LDL $-29,47$ vs $-5,94$; HDL 4,40 vs 1,36 ; triglycerides $-40,43$ vs $-13,03$.

Conclusions: Regular magnesium supplementation improves dyslipidemia in obese insulin resistant patients.
Conflict of Interest: Research relating to this abstract was funded by Temis Lostaló Laboratory (Argentina).

T3P66

Effectiveness of a digitally delivered behavioural intervention for weight loss maintenance in obese adults: The randomised controlled NULevel trial

Sniehotta, F.' ; Evans, E. '; Sainsbury, K. '; Adamson, A. '; Batterham, A. ${ }^{2}$; Brown, H. ${ }^{1}$; Mccoll, E. '; Olivier, P. ${ }^{1}$; Rothman, A. ${ }^{3}$; Vieira, R. ${ }^{1}$; Howel, D. ${ }^{1}$; Vale, L. ${ }^{1}$; White, M. ${ }^{4}$; Wright, P. ; Araújo-Soares, V.

${ }^{1}$ Newcastle University, ${ }^{2}$ Teesside University, ${ }^{3}$ University Of Minnesota, ${ }^{4}$ Cambridge University, ${ }^{5}$ Newcastlle University

Introduction: Scalable weight loss maintenance interventions for obese adults are lacking but vital for the health and economic benefits of weight loss to be fully realised. We examined the effectiveness of a technology-mediated behavioural intervention to support weight loss maintenance in obese adults after clinically-significant weight loss ( $\geq 5 \%)$, compared to standard lifestyle advice.

Methods: In open label superiority randomised controlled trial, we recruited 288 adults after weight loss of $\geq 5 \%$ within the previous 12 months, from a pre-weight loss BMI of $\geq 30 \mathrm{~kg} / \mathrm{m}^{2}$. We randomly allocated participants 1:1 to receive either standard lifestyle advice via newsletter (control condition) or a technology-assisted behavioural weight loss maintenance programme (intervention condition). The intervention included a single face-to-face meeting, self-monitoring and feedback on weight, diet and physical activity via links embedded in SMS messages. All participants received wirelessly-connected weighing scales and participants in the intervention arm were instructured to weigh themselves daily. After 12 months we measured the primary outcome, change in weight $(\mathrm{kg})$ from baseline, and also self-weighing, anthropometric variables, physical activity (via accelerometry) and psychological process variables. The study was powered to detect a between-group weight difference of $\pm 2.5 \mathrm{~kg}$ at 12 months.

Results: 253 participants (88\%) were retained at 12 months. Mean weight gain from baseline to 12 months was $1.6 \mathrm{~kg}$ in the intervention group $(\mathrm{n}=124)$ and $1.4 \mathrm{~kg}$ in the control group $(\mathrm{n}=129)$. There were no significant intervention effects on weight at 12 months (difference in adjusted mean weight change from baseline: -0.23 ; $95 \%$ CI -2.4 to $1.6, p=0.8$ ). Intervention participants weighed themselves more frequently than control participants ( 4 vs 2 times per week) but did not differ on anthropometric characteristics or physical activity. Intervention participants reported greater satisfaction with weight outcomes, greater dietary self-efficacy and greater weight loss maintenance confidence.

Conclusion: There was no difference in the weight loss maintenance of participants who received the NULevel behavioural intervention compared to participants who received standard lifestyle advice via newsletter. Differences in behavioural and psychological variables between the trial arms suggest that some of the hypothesised intervention mechanisms could be substantiated.

Conflict of Interest: None Disclosed

Funding: This research was funded by the UK National Prevention Research Initiative (NPRI) Phase 4 (grant grant MR/J000477/1) through the UK Medical Research Council. This trial was registered before recruitment commenced, on 20th March 2014 (see http://www.isrctn.com/ISRCTN14657176).

\section{T3P68}

\section{Effects of bariatric surgery on inspiratory muscle strength}

Pouwels, S. ${ }^{\text {; }}$ Aarts, M. ${ }^{2}$; Said, M. ${ }^{2}$; Teijink, J. ${ }^{2}$; Nienhuijs, S. ${ }^{2}$

${ }^{1}$ Franciscus Vlietland, Schiedam, The Netherlands, ${ }^{2}$ Department Of Surgery, Catharina Hospital, Eindhoven, The Netherlands

Introduction: The respiratory function is affected by obesity due to an increased deposition of fat on the chest wall. 
Objectives: The objective of this study was to investigate the strength of the inspiratory respiratory muscles of obese individuals and the possible influence of bariatric surgery.

Methods: Of the patients referred to our bariatric centre between the $3^{\text {rd }}$ of October 2011 and the $3^{\text {rd }}$ of May 2012 the Maximum Inspiratory Pressure (MIP) was measured at screening and 3, 6 and 9 months postoperative.

Results: The mean age of the 124 included patients was $42.9 \pm 11.0$ years and mean BMI was $43.1 \pm 5.2 \mathrm{~kg} / \mathrm{m}^{2}$. The mean predicted MIP preoperatively was $127 \pm 31 \mathrm{in} \mathrm{cm} \mathrm{H} 2 \mathrm{O}$ and the mean measured MIP was $102 \pm 24$ in $\mathrm{cm} \mathrm{H} 2 \mathrm{O}$. Three patients $(2.4 \%)$ received training. Three months after surgery the MIP was $76 \pm 26 \mathrm{~cm} \mathrm{H} 2 \mathrm{O}$, after 6 months $82 \pm 28 \mathrm{~cm} \mathrm{H} 2 \mathrm{O}$ and after 9 months $86 \pm 28 \mathrm{~cm} \mathrm{H} 2 \mathrm{O}$. All postoperative measurements were significant lower than preoperatively $(\mathrm{P}<0.05)$. No significant difference was found between patients who had a sleeve gastrectomy compared to a gastric bypass $(\mathrm{P}=0.06, \mathrm{P}=0.165$ and $\mathrm{P}=0.124$ after 3,6 and 9 months respectively). The only influencing factor for the preoperative MIP was age $(\mathrm{p}=0.014)$.

Conclusion: The preoperative MIP values were significantly lower than the predicted MIP values and a significant decrease in inspiratory pressures was found at 3, 6 and 9 months after bariatric surgery.

\section{T3P69}

\section{Effects of dietary protein content on metabolic responses to sucrose overfeeding in healthy humans}

Surowska, A. ${ }^{1}$; Jegatheesan, P. ${ }^{1}$; Campos, V. ${ }^{1}$; Cros, J. ${ }^{1}$; Zwygart, K. ${ }^{2}$; Kreis, R. ${ }^{2}$; Boesch, C. ${ }^{2}$; Tappy, L. ${ }^{1}$

${ }^{1}$ Department of Physiology, Faculty of Biology and Medicine, University of Lausanne, Lausanne, Switzerland, ${ }^{2}$ Department Of Clinical Research, University Of Bern And Institute Of Diagnostic Interventional And Pediatric Radiology, University Hospital, Bern, Switzerland

Introduction: Excess energy intake is a contributor to the worldwide epidemic of metabolic disease. The impact of dietary macronutrient composition at identical high energy intake remains, however, not known. In this study, we compared the short-term effects of a high-sucrose, high protein (HSHP) or low protein (HSLP) diet on ectopic lipids and cardiovascular risk factors.

Methods: We performed a randomized, crossover clinical study on 12 healthy young male and female volunteers. Each participant was studied after a 3-day run-in diet containing $100 \%$ daily energy requirement (starch $45 \%$, sucrose $10 \%$, lipid $33 \%$, protein $12 \%$ ), and after 7 -day on hypercaloric diet containing $150 \%$ daily energy requirement with starch $45 \%$, sucrose $50 \%$, lactose $10 \%$, and either lipid 37,5 and protein $7,5 \%$ (HSLP) or lipid 15\% and protein 30\% (HSHP). Intrahepatic (IHCL) and intramuscular (IMCL) lipid content were measured by magnetic resonance spectroscopy. Energy metabolism (indirect calorimetry) and plasma metabolites were monitored in fasting conditions and over 6 hours postprandially.

Results: HSLP increased IHCL more than HSHP (to $642 \pm 105 \%$ vs $224 \pm 39 \%$ of basal values, $\mathrm{p}<0.001$ ); it also increased IMCL more (to $124.0 \pm 3.0 \%$ vs $111.4 \pm 2.6 \%, \mathrm{p}=0.004)$. Postprandial energy expenditure was higher after HSHP than HSLP $(1.6 \pm 0.1 \mathrm{kcal} / \mathrm{min}$ vs $1.4 \pm 0.1$ $\mathrm{kcal} / \mathrm{min}, \mathrm{p}<0.001$ ), but HSLP and HSHP increased to the same extent fasting and postprandial carbohydrate oxidation and plasma glucose, insulin and triglyceride concentrations. They also decreased fasting and postprandial fat oxidation to the same extent.

Conclusion: Higher deposition of ectopic lipids in the liver and muscle was observed after HSLP diet than HSHP. Postprandial energy expenditure was also increased after HSHP compared to HSLP. These results suggest that not only overfeeding as such, but also the type and amount of further macronutrients play an important role in the extent of ectopic lipid storage.

Conflict of Interest: None Disclosed.

Funding: Research relating to this abstract was funded by a grant from the Swiss National Science Foundation (320023-138428), and by Institute Benjamin Delessert.

\section{T3P70}

Effects of electrical muscle stimulation on waist circumference in adults: A randomized controlled trial

\section{Lee, S. ${ }^{1}$;Choi, E. ${ }^{2}$; Kim, Y. ${ }^{3}$}

${ }^{1}$ Pusan National Universiy Yangsan Hospital, ²Daedong Hospital, ${ }^{3}$ Pusan National Universiy Hospital

Electrical muscle stimulation (EMS) excites motor nerve electrically to constrict the skeletal muscle fiber. We expect that EMS makes metabolic benefits similar to that of an exercise. Therefore, we conducted a double-blind, randomized, sham-controlled trial to compare the efficacy and safety of a 12-week program using an EMS device with an identical device providing transcutaneous electrical nerve stimulation (TENS) as control in adults with abdominal obesity.

Methods: In a randomized trial, a total of 60 participants assigned to one of two groups: the EMS group $(n=30)$ or the TENS group $(n=30)$ for 12 weeks, constituting the intent-to-treat population. They all were between 18-65 years of age and had an WC $>90 \mathrm{~cm}$ for men and $>80 \mathrm{~cm}$ for women as abdominal obesity defined based on the Asia-Pacific criteria of the International Diabetes Federation. The primary outcome was waist circumference and the secondary were satisfaction score, free fatty acid (FFA), visceral and subcutaneous adipose tissue. The subjects were asked not to change their ordinary diet and exercise for the study period.

Results: When comparing changes in WC during study period, a clearer decrease in EMS group was observed than TENS group after 12 weeks $(5.2 \pm 2.8 \mathrm{~cm}$ vs. $2.9 \pm 3.3 \mathrm{~cm}, \mathrm{P}=0.005)$. In EMS group, WC slightly declined more sharply in the latter half then in the first half of the study. According to the degree of WC loss, $70.0 \%$ of EMS group lost $4 \mathrm{~cm}$ more off of their WC while $46.7 \%$ of TENS group lost lesser than $2 \mathrm{~cm}$ of their WC $(\mathrm{P}=0.008)$. After 12 weeks, the self-rated satisfaction score in EMS group was higher than in TENS group $(3.7 \pm 0.7$ vs. $3.3 \pm 0.7, \mathrm{P}<0.05)$. Also, fasting FFA levels were significantly higher in EMS than TENS group at week $12(\mathrm{P}=0.006)$. All participants completed the protocol without adverse symptoms.

Conclusions: This 12-week program of EMS modestly reduced the waist in abdominal obese adults without side effects. Replication of this study will be needed in order to confirm the results of this first-stage study and additional studies are required to assess the long term effects of EMS in abdominal obese people.

Conflict of Interest: None Disclosed

Funding: None

T3P71

Effects of gastroplasty Roux-en-Y on body weight and clinical metabolic comorbidities at the bariatric surgery service of an University Hospital

\section{Silva, C. ${ }^{1}$; Rosado, E. ${ }^{2}$; Carneiro, J. ${ }^{1}$; Rosa, F. ${ }^{\text {; }}$ Souza, A. ' Magno, F. ${ }^{2}$}

${ }^{1}$ Hospital Universitário Clementino Fraga Filho, ${ }^{2}$ Universidade Federal Do Rio De Janeiro

Introduction: Conventional obesity treatments show little effect in the long term, leading to increase in the search for bariatric surgery as an alternative for the control of comorbidities. The aim of this study was to To evaluate the performance of type 2 diabetes mellitus, high blood pressure and dyslipidemia in patients submitted to Roux-en-Y gastric bypass in late follow-up.

Methods: Retrospective analysis of 59 patients from the bariatric surgery program of an University Hospital at Rio de Janeiro was performed. Anthropometric (height and body weight) and laboratorial (LDL-c, HDL-c, VLDL-c, triglycerides (TG) and glucose) data at pre-operative and post-operative periods were accessed from medical records.

Results: $83 \%$ of patients were female, with a mean age of $43 \pm 11$ years old. $76 \%$ and $36 \%$ of the patients in the present study had hypertension and hypertension, respectively. The mean Post-operative time was $7 \pm 3$ years. There was weight and body mass index (BMI) reduction in postopera- 
tive period $\left(133 \pm 24 \mathrm{~kg}\right.$ vs $91 \pm 22 \mathrm{~kg}$ and $49 \pm 8 \mathrm{k} \mathrm{g} / \mathrm{m}^{2}$ vs $33 \pm 6 \mathrm{~kg} / \mathrm{m}^{2}$, respectively). We also observed reduction in glucose (101.00 \pm 26.99 vs $89,11 \pm 15.19)$, total cholesterol $(179.00 \pm 37.95$ vs $167.48 \pm 28.50)$, LDL-c $(104.30 \pm 33.12$ vs $91.46 \pm 24.58)$, VLDL-c $(25.40 \pm 11.12$ vs $15.68 \pm 7.40)$ TG ( $143.35 \pm 86,35$ vs $82.45 \pm 37.39)$, and higher HDL levels ( $43.53 \pm 8.23$ vs $57.90 \pm 15.60)$. Remission for diabetes and dyslipidemia was registered in $81 \%$ and $94 \%$ patients, respectively. From patient who still present hypertension in the postoperative moment, $40 \%$ were using one or more types of hypertension drugs.

Conclusion: RYGB has shown be an effective procedure in the long term, leading to weight loss and remission for diabetes and dyslipidemia.

Conflict of Interest: None Disclosed

Funding: No Funding

\section{T3P72}

Effects of hesperidin supplementation on weight, glycemic control, lipid profile and inflammatory factors in patients with type 2 diabetes: A randomized, controlled clinical trial

Eghtesadi, S. ; Mohammadi, M. ${ }^{2}$; Vafa, M. ${ }^{3}$; Khademhaghighian, H.4. Heidari, I. ${ }^{5}$; Salehi, M. ${ }^{6}$ Amiri, F. ${ }^{7}$; Alipour, R. ${ }^{8}$; Eghtesadi, M. ${ }^{9}$

${ }^{1}$ Iran University of Medical Sciences, School of Public Health,Tehran, Iran, ${ }^{2}$ Iran University Of Medical Sciences, School Of Public Health, Tehran Iran, ${ }^{3}$ Iran University Of Medical Sciences, School Of Public Health, Tehran, Iran, ${ }^{4}$ Ahwaz University Of Medical Sciences, School Of Paramedicine,Ahwaz, Iran., 5 Iran University Of Medical Sciences, School Of Medicine,Tehran, Iran, ${ }^{6}$ Iran University Of Medical Sciences, School Of Public Health, Tehran, Iran., ${ }^{7}$ Iran University Of Medical Sciences, School Of Public Health,Tehran, Iran, ${ }^{8}$ Iran University Of Medical Sciences,School Of Public Health, Tehran, Iran, ${ }^{9}$ Azad Universty, Tehran Medical Branch, School Of Medicine,Tehran, Iran

Intrduction: Diabetes mellitus is a common chronic disease and a major public health problem globally. This study was carried out to investigate the effects of hesperidin supplementation on body weight, markers of glucose and lipid, insulin levels, insulin resistance and inflammation.

Methods: Forty-five patients with type 2 diabetes participated in this randomized double-blind controlled clinical trial. Subjects consumed 500 $\mathrm{mg} /$ day hesperidin supplement in the intervention group $(\mathrm{n}=23)$ and 500 $\mathrm{mg} /$ day placebo in the control group $(\mathrm{n}=22)$, for 8 weeks. $10 \mathrm{cc}$ blood samples were drawn and body weight and BMI were measured at the baseline and at the end of the study. Three days dietary recalls and records were obtained at the beginning and at the fourh week of trial.The levels of fasting blood glucose (FBG), insulin, hemoglobin Alc (HbAlc), total cholesterol (TC), triglyceride (TG), high density lipoprotein cholesterol (HDL-C), low density lipoprotein cholesterol (LDL-C), insulin resistance, inflammatory factors: IL6 and hs- CRP were measured and compared within and between treatment groups. Data were processed through SPSS software by using independent $t$ and paired $t$ test.

Results: Mean weight and BMI of patients did not change significantly in both groups at the beginning and the end of the 8th week of trial.Hesperidin supplementation led to significant decrease in FBG $(\mathrm{P}=0.041)$ and $\mathrm{HbAlc}(\mathrm{P}=0.028)$ levels, compared with control group. A significant increase in serum insulin, decrease in TC and FBG $(\mathrm{P}=0.018, \mathrm{P}=0.049$ and $\mathrm{P}=0.014$, respectively) were observed in the hesperidin group after the intervention, whereas no significant changes in the placebo group were shown at baseline and at the end of the study. Inflammatory factors, high-sensitivity C-reactive protein (hs-CRP) and interleukin-6 (IL-6) were not significantly changed in the hesperidin group compared to the control group.

Conclusions:hesperidin supplementation lowered the plasma level of total cholestrol and improved glycemic control and insulin resistance in patients with type 2 diabetes but no changes in weight and BMI were observed.

\section{Coflict of Interest: None}

Funding: Research relating to this abstract was funded by Iran University of Medical Sciences, Tehran, Iran
T3P73

Effects of juçara (Euterpe edulis Mart.) supplementation in the inflammatory state of obesity: A pilot study

\section{Jamar, G. ${ }^{1}$; Santamarina, A. '; Mennitti, L. ' ; Argentato, P. '; Dourado, V. ${ }^{2}$;} Rosso, V. ${ }^{3}$; Pisani, L. ${ }^{3}$

${ }^{1}$ Post Graduate Program of Interdisciplinary Health Sciences, Federal University of São Paulo (Unifesp), Santos, São Paulo, Brazil, ²Department Of Human Movement Sciences, Federal University Of São Paulo (Unifesp), Santos, São Paulo, Brazil, ${ }^{3}$ Department Of Biosciences, Federal University Of São Paulo (Unifesp), Santos, São Paulo, Brazil.

Introduction: Juçara (Euterpe edulis Mart. - a species native to the Atlantic Forest/Brazil) is a promising source of antioxidants in addition to its anti-inflammatory properties. Anthocyanins, which are pigments of the flavonoids family with beneficial in preventing diet-induced obesity and possibly reduce disease risk and promoting health, are the main phytochemicals present in this fruit. The juçara pulp has an important nutritional value, with proteins, sugars, fiber and a lipid fraction with high content of polyunsaturated fatty acids, oleic acid and a lower content of saturated lipids. However, no studies have been found that relate the potential of juçara to physiological and metabolic aspects in humans.

Objective: To analyze the effects of juçara supplementation in body composition, metabolic parameters, and inflammatory process in obesity. Methods: 36 subjects with overweight and obesity (BMI 30 to $39.9 \mathrm{~kg} /$ $\mathrm{m}^{2}$ ) of both sexes aged between 31 and 59 years were included in this double-blind randomized study. They were assessed for resting energy expenditure by indirect calorimetry (QUARK); anthropometry (weight, height, BMI, NC, WC and WHtR); metabolic parameters (TAG, TC, HDL-col, LDL-col, and glucose); and inflammatory profile (PAI-1 and adiponectin). Subsequently, they were randomized 2 groups to use or not (placebo) supplementation with $50 \mathrm{~g}$ freeze-dried juçara pulp for 6 weeks.

Results: After supplementation, juçara group showed a significant increase of adiponectin $(0.95 \mu \mathrm{g} / \mathrm{ml}, \mathrm{p}=0.01)$ and fat-free mass $(1.7 \mathrm{Kg}$, $\mathrm{p}<0.01)$, and reduction of body fat $(-1.3 \mathrm{Kg}, \mathrm{p}=0.03)$. Both groups presented significant alteration in the lipid profile (TC and HDL-col reduction and LDL-col increase). Other measures were not changed significantly. There was no difference between juçara and placebo groups among the measures.

Conclusion: The juçara supplementation improved body composition and low-grade inflammation in obese and could contribute to reducing chronic disease development.

Conflict of interest: None disclosed.

Funding: CAPES-REUNI

\section{T3P75}

Effects of vildagliptin and metformin during post-prandial lipemia on microvascular function, low-grade inflammation, blood viscosity and oxidative stress on recently diagnosed T2DM obese women

Schiappacassa, A. ${ }^{1}$; Aguiar, L. ${ }^{2}$; Maranhão, P. ${ }^{1}$; Souza, M. '; Panazzolo, D. ${ }^{1}$ Bouskela, E. ${ }^{1}$

${ }^{1}$ Universidade Estadual do Rio de Janeiro, ${ }^{2}$ Universidade Estadual Do Rio De Janeiro

Introdution:Type 2 diabetes mellitus (T2DM) and obesity are related to cardiovascular (CV) risk, and microvascular dysfunction is present at its early stages. Postprandial lipemia (PPL) is a CV risk. Incretins increase insulin secretion and this effect is reduced in T2DM while the metabolic changes during PPL are more accentuated. Metformin is the first choice drug to treat T2DM, while vildagliptin is one of the most prescribed ones. Since both drugs have CV protective effects,our purpose was to compare during PPL the acute effects on microvascular reactivity, oxidative stress, inflammatory markers, blood viscosity and GI peptides of vildagliptin or metformin in obese T2DM subjects. 
Methods: 38 women with T2DM recently diagnosed and medically-naïve, aged between 19 and 50 years, $\mathrm{BMI} \geq 30 \mathrm{~kg} / \mathrm{m}^{2}$; were subjected to microvascular measurements and sample collections at fasting and after the ingestion of a high-fat diet at 30, 60, 120 and $180 \mathrm{~min}$ and randomized 1:1 to ingest a pill of metformin $(850 \mathrm{mg}$ ) or vildagliptin $(50 \mathrm{mg})$ before the meal. We evaluated endothelial function through videocapillaroscopy and Laser-Doppler flowmetry, blood viscosity, inflammatory and oxidative stress markers, GI peptides and metabolic profile in all time points.

Results: At the baseline, no differences in any variable were noticed between groups. Compared to metformin, vildagliptin promoted a reduction in DPP4 activity $(\mathrm{p}=0.004)$ and an increment in glucagon-like peptide $1(p=0.04)$. There was no superiority of one drug over the other on postprandial metabolic profile, inflammatory markers, oxidative stress and blood viscosity. Metformin promoted a sharp rise in functional capillary density during PPL compared to vildagliptin while vildagliptin increased vasomotion.

Conclusions: At microcirculation, vildagliptin increased vasomotion while metformin induced higher capillary recruitment during PPL suggesting different mechanisms of action. Additionally, both drugs promoted better responsiveness of the microvascular reactivity during PPL. Study partially funded by Novartis S.A.

\section{T3P76}

\section{Effects of week-on, week-off energy restriction compared to continuous energy restriction}

\section{Headland, M. ${ }^{1}$; Clifton, P. ; Keogh, J. ${ }^{1}$}

${ }^{1}$ University of South Australia

Introduction: Intermittent energy restriction (IER) has become a popular alternative to continuous dieting for weight loss but there is little longterm data on its efficacy. The aim was to investigate the effect of week-on, week-off energy restriction (IER), compared to continuous energy restriction (CER) on weight loss and weight loss maintenance after 12 and 24 months.

Methods: Participants were overweight or obese (BMI $34.04 \pm 5.3 \mathrm{~kg} / \mathrm{m}^{2}$, $51.8 \pm 11.3$ years) ( $\mathrm{n}=107,22$ men and 85 women), who. Weight, body composition, and a fasting blood sample for lipids, glucose and FGF-21, were taken at 0,8 weeks CER and 16 weeks IER, and at 12 and 24 months. Results: Attrition was $56 \%$ at 12 and $60 \%$ at 24 months. Weight loss was $-6.2 \pm 2.9 \mathrm{~kg}$ CER, $-6.4 \pm 2.3 \mathrm{~kg}$ IER; $(\mathrm{p}=0.8)$ at 8 weeks and $-6.3 \pm 5.2 \mathrm{~kg}$ CER, $\mathrm{n}=27,-4.9 \pm 3.4 \mathrm{~kg}$ IER, $\mathrm{n}=20$; $(\mathrm{p}=0.3)$ at 12 months. There was no difference between the groups for either fat mass or lean muscle mass after 8 weeks ER or 12 months ER. At 12 months FGF-21 $(\mathrm{P}<0.02)$ and triglycerides concentrations decreased $\left(-0.2 \pm 0.5 \mathrm{mmol}^{-1} \mathrm{P}<0.01\right)$ while the decrease in fasting glucose approached significance $(-0.2 \pm 0.5 \mathrm{mmol}$ $\left.\mathrm{l}^{-1} \mathrm{P}=0.06\right)$ with no difference between groups. Total cholesterol, HDI and LDL levels did not change. Weight change between baseline and the $24 \mathrm{mnths}$ between the groups approached significance $(-4.2 \pm 3.5 \mathrm{~kg}$ CER, $\mathrm{n}=24,-1.6 \pm 3.2 \mathrm{~kg}$ IER, $\mathrm{n}=19 ;(\mathrm{p}=0.06))$. Weight increased between 12 and 24 months but was not significantly different $(1.9 \pm 2.5 \mathrm{~kg}$ CER, $3.1 \pm 3.7 \mathrm{~kg}$ IER $(\mathrm{p}=0.2)$ ).

Conclusion: Weight loss experienced in the first 12 months was the same in each group. However by 24 months the difference in overall weight loss between the groups is approaching statistical significance, and regain experienced between 12 and 24 months is numerically larger in the IER. The large attrition whilst being similar in both groups limits generalisation of the

Results: This study is ongoing.

Conflict of Interest: None Disclosed

Funding: PMC is supported by a NHMRC Principal Research Fellowship. This research was jointly funded through this fellowships and the University of South Australia. MH is funded by a University of South Australia Postgraduate Award.

\section{T3P78}

\section{Energy balance during a two year follow up after Roux en Y gastric bypass = an unexplained gap between energy expenditure and food intake}

\section{Segrestin, B. ${ }^{1}$; Maitrepierre, C. ${ }^{1}$; Laville, M. ${ }^{1}$; Robert, M. ${ }^{2}$; Disse, E. ${ }^{3}$}

${ }^{1}$ Research center on human nutrition (CRNH Rhône Alpes), ${ }^{2}$ Fédération Hospitalo Universitaire Do It, ${ }^{3}$ Claude Bernard University Lyon 1

Understanding the energy balance after Roux en Y gastric bypass(RYGBP) is important to avoid weight regain. Some data has been published on food intake(FI) after RYGBP (1) but rarely simultaneously with facts on Energy expenditure(EE).The aim of our study was to analyse FI regarding EE during a two year follow up after surgery.

Methods: We conducted a 2year longitudinal study of 44 patients after RYGBP. We performed before surgery and at the end of each period after surgery an assessment of resting EE (REE) by indirect calorimetry and body composition by bioelectrical impedance analysis. Total EE (TEE) was estimated to be $1.53 \mathrm{x}$ REE (estTEE). Food intake(FI) was estimated by a trained dietician with a $24 \mathrm{~h}$ hour dietary recall . The 2 year follow up will be described in 4 periods: $1^{\text {st }}$ period $=0-3$ months, $2^{\text {nd }}$ period $=3-6$ months, $3 \mathrm{rd}$ period $=6-12$ months, 4 th period $=12-24$ months. The formulas used are:

Available energy $=$ FI + energy from stores-energy lost in feces $(\sim 7 \%$ of FI) Energy from stores $=$ Fat mass loss in $\mathrm{kg}^{\star} 9300 \mathrm{kcal}+$ Fat free mass loss in $\mathrm{kg}{ }^{\star} 1100 \mathrm{kcal}$,

Energy gap $=$ estTEE-available energy

T-test was performed using SPPSS 19 software.

Results: The patients were mainly female (84\%),aged $43.2 \pm 7.9$ years old, with a BMI of $44.5 \pm 3.7 \mathrm{~kg} / \mathrm{m}^{2}$. Excess BMI loss(\%) from the 1 st to the 4 th period was $39 \pm 13 \%, 57 \pm 16 \%, 67 \pm 23 \%, 68 \pm 27 \%$.

REE decreased after the first period $(\mathrm{p}<0.01)$, and then stabilized.We found an energy gap which increased from $323 \pm 100 \mathrm{kcal}$ in the 1st period to $1018 \pm 49 \mathrm{kcal}$ in the 4 th period.

Conclusion: We found an important energy gap between EE and the available energy(FI and energy used from stores) during the follow up. This cannot be fully explained by misreport of FI (2) or production of short chain fatty acids by gut microbiota (3). Further research is needed to understand the energy balance after RYGBP.

No Conflict of interest Disclosed.

No Funding was obtained .

References:

1 Kruseman M et al JADA. 2010 Apr;110(4):527-34. Dietary, weight, and psychological changes among patients with obesity, 8 years after gastric bypass.

2 Lopes TS et al EJCN. 2016 Nov;70(11):1259-1264. Misreport of energy intake assessed with food records and $24-\mathrm{h}$ recalls compared with TEE estimated with DLW.

3 Blaut, PNS 2015 Aug;74(3):227-34.Gut microbiota and energy balance: role in obesity.

Table 1. Table summarizing REE, estTEE, Energy from stores, FI and the energy gap during the follow up.

\begin{tabular}{|l|l|l|l|l|}
\hline & 1 st period & $2^{\text {nd }}$ period & $3^{\text {rd }}$ period & $4^{\text {th }}$ period \\
\hline REE(kcal/d) & $1809 \pm 39$ & $1644 \pm 30$ & $1620 \pm 32$ & $1604 \pm 36$ \\
\hline estTEE(kcal/d) & $2768 \pm 59$ & $2516 \pm 46$ & $2478 \pm 49$ & $2454 \pm 55$ \\
\hline $\begin{array}{l}\text { Energy from } \\
\text { stores(kcal/d) }\end{array}$ & $1769 \pm 111$ & $788 \pm 81$ & $355 \pm 59$ & $15 \pm 24$ \\
\hline $\begin{array}{l}\text { Food Intake(k- } \\
\text { cal/d) }\end{array}$ & $681 \pm 18$ & $1112 \pm 34$ & $1337 \pm 42$ & $1523 \pm 48$ \\
\hline $\begin{array}{l}\text { Energy gap } \\
\text { (kcal/d) }\end{array}$ & $323 \pm 100$ & $687 \pm 84$ & $878 \pm 77$ & $1018 \pm 49$ \\
\hline
\end{tabular}




\section{T3P82}

Evaluation of different scores to predict nonalcoholic fatty liver disease in overweight or obese patients with type 2 diabetes

Angelidi, A. ' ; Angelidi, M. ${ }^{1}$; Papazafiropoulou, A. ${ }^{1}$; Anagnostopoulou, K. ${ }^{1}$; Vagena, E. ' Velissaris, V. '; Markakis, V. ${ }^{1}$; Kamaratos, A. ' ; Melidonis, \&. ${ }^{1}$

'Diabetes Center, 'Tzaneio" General Hospital of Piraeus, Greece

Introduction: Non-alcoholic fatty liver disease (NAFLD) is the commonest cause of abnormal liver function tests and liver disease in the western countries. The objective of this study was to evaluate whether some of the most common, non-invasive and easily assessed diagnostic models of NAFLD can be used to screen for the presence of NAFLD in overweight or obese patients with type 2 diabetes (T2D).

Methods: Study population included 110 (58 men) overweight or obese patients $\left(\mathrm{BMI} \geq 25 \mathrm{Kg} / \mathrm{m}^{2}\right)$ aged $60.1 \pm 9.5$ years, with T2D. Anthropometric, clinical, and laboratory data were evaluated. NAFLD was diagnosed using ultrasound. NAFLD liver fat score, HAIR (Hypertension, ALT, Insulin Resistance), BARD, APRI (AST to Platelet Ratio Index), FIB-4 and LAP (Lipid Accumulation Product) scores were also estimated. Discrimination capability was assessed based on the area under the receiver operating characteristic curve (AUC), sensitivity (Se), specificity (Sp), positive (PPV) and negative (NPV) predictive values were calculated.

Results: NAFLD, using ultrasound, was present in 77 patients (70\%) Analysis showed that NAFLD liver fat score for a cutoff of $\geq-1.44$ had Se: $93 \%$, Sp: 72\%, AUC: 0.95, PPV: $89 \%$ and NPV: $82 \%$, HAIR score for a cutoff of $\geq 0.50$ had Se: $62 \%$,Sp: $44 \%$, AUC: 0.58 , PPV: $72 \%$ and NPV: $33 \%$, BARD score for a cutoff of $\geq 2.50$ had Se: $51 \%$, Sp: $64 \%$, AUC: 0.59 , PPV: $77 \%$, NPV: $36 \%$, APRI for a cutoff of $\geq 0.23$ had Se: $49 \%$, Sp: $64 \%$, AUC: 0.55, PPV: $76 \%$ and NPV: $35 \%$, FIB- 4 score for a cutoff of $\geq 1.00$ had Se: 53\%, Sp: 54\%, AUC: 0.52 , PPV: $73 \%$ and NPV: $33 \%$ and LAP score for a cutoff of $\geq 30.93$ had Se: $94 \%$, Sp: $82 \%$, AUC: 0.89 , PPV: $92 \%$ and NPV: $85 \%$.

Conclusion: NAFLD liver fat and LAP scores showed good sensitivity and specificity for the diagnosis of NAFLD. Both are two simple, accurate enough and non-invasive tools potentially useful to predict NAFLD in overweight or obese patients with T2D in everyday clinical practice.

Conflict of Interest: None Disclosed

Funding: Hellenic Society of Lipidology, Atherosclerosis and Vascular Disease

\section{T3P84}

\section{Evaluation of nutritional interventions in obesity}

\section{Nuijten, . $^{1}$;}

${ }^{1} \mathrm{a} 2 \mathrm{~m}$

Background:Obesity is now universally acknowledged as a major public health problem, both in children and adults. This raises vital public health questions concerning what interventions are necessary to control the obesity epidemic. The judgment of the clinical benefit of medicinal products by reimbursement authorities is based on traditional clinical trial outcomes, but coverage decisions are also based on effectiveness, cost-effectiveness and budgetary impact. A similar development in a value message is increasingly important for nutritionals, especially if the comparative treatments are traditional medical treatments like drugs or surgery in obesity management.

Objective: The objective is to present specific methodological issues for the evaluation of nutritional interventions in obesity.

Methods: Currently medical nutrition interventions in obesity tend to be excluded from reimbursement processes even though they also can have a positive impact on patients' clinical outcomes. The role of nutritional intervention in obesity to optimize the use of scarce resources is therefore of interest for healthcare decision makers. A favourable impact of nutrition in obesity will improve healthcare expenditures and quality of life. In health economics, an analysis of the costs and effects of a healthcare technology by means of a cost-effectiveness analysis has become an established tool for reimbursement decisions for innovative medicines. Therefore the evaluation of nutritionals may require similar evaluation techniques than for medical treatments in obesity. The next step is to consider the most appropriate reimbursement procedures for nutrition in obesity. Conclusion: Up to recently, no specific valuation approach has been published for the evaluation nutritional interventions in obesity in spite of clear clinical and economic benefits for payers, which may be similar to traditional medical treatments like drugs or surgery in obesity management. To correspond to the increasing value of nutritionals in obesity, there is a need to generate adapted methodologies for the evaluation of nutrition in obesity and policy changes for inclusion in the reimbursement package.

\section{T3P85}

\section{Ex vivo studies using human peripheral blood mononuclear cells to test metabolic effects of $n-3$ long-chain polyunsaturated fatty acids}

Cifre, M. ${ }^{1}$; Díaz-Rúa, R. ${ }^{1}$; Varela-Calviño, R. ${ }^{2}$; Reynés, B. ${ }^{1}$; Pericás-Beltrán, J. ${ }^{3}$; Palou, A. ${ }^{1}$; Oliver, $P{ }^{1}$

${ }^{1}$ Laboratory of Molecular Biology, Nutrition and Biotechnology, Universitat de les Illes Balears and CIBER de Fisiopatología de la Obesidad y Nutrición (CIBERobn), Palma de Mallorca, Spain, ${ }^{2}$ Department Of Biochemistry And Molecular Biology, University Of Santiago De Compostela, Santiago De Compostela, Spain, ${ }^{3}$ Research Group On Evidence, Lifestyles \& Health, Universitat Illes Balears, Palma De Mallorca, Spain

Introduction: There is great interest to study effectiveness of food bioactive compounds ameliorating obesity-related metabolic alterations. Health protective effects of food compounds can be easily assessed using animal models; however, translation to humans is not always simple. Culture of peripheral blood mononuclear cells (PBMC) is emerging as an interesting possibility to test food compounds. We tested the usefulness of an ex vivo human PBMC system as a tool to analyse the effects of n-3 long-chain PUFA (LCPUFA), not only on immune response, but also as a surrogate system to reflect known effects of these bioactives on lipid metabolism. Moreover, we aimed to determine if the effects diverge between normoweight (NW) and overweight/obese (OW-OB) subjects.

Methods: We set up an in vitro system of human PBMC from NW and OW-OB individuals to study direct effects of physiological doses $(10 \mu \mathrm{M})$ of docosahexaenoic (DHA), eicosapentaenoic (EPA), or their combination. After treatments, mRNA expression of selected genes involved in immune response and lipid metabolism was analysed by real-time RTPCR in PBMC.

Results: PBMC reflected increased beta-oxidation capacity (Cpt1a expression) characteristic of liver and adipose tissue, but only after DHA treatment, with independence of BMI. However, an impaired n-3 LCPUFA response was evident in OW-OB for the studied lipogenic genes: both LCPUFA diminished Fasn and Srebp $1 c$ mRNA expression in NW, but no effect was observed for DHA in PBMC from OW-OB. This insensitivity was also evident for inflammatory genes: DHA, EPA or the combination decreased the expression of these genes in NW; nevertheless, no effect was observed in OW-OB after DHA treatment, and EPA effect was impaired. Considering the different genes analysed, Slc27a2, Il6, and Tnfo PBMC analysis resulted of special interest to determine obesity-related insensitivity to LCPUFA treatment.

Conclusion: An in vitro system of human PBMC reflects n-3 LCPUFA effects on lipid metabolism/inflammation and appears as a proper tool for food bioactive screening to promote functional food development. Moreover, we have observed general insensitivity to n-3 LCPUFA related to higher BMI, so that this system also offers the possibility to set up proper dietary strategies aimed for obese population to fully benefit from the positive effects of these compounds.

Conflict of Interest: None disclosed. 
Funding: Research relating to this abstract was funded by the Spanish Government (INTERBIOBES -AGL2015-67019-P- and EPIMILK -AGL2012-33692-) and by the University of the Balearic Islands (IBIFLEX, FA38/2016). CIBER de Fisiopatología de la Obesidad y Nutrición is an initiative of the ISCIII. MC is a recipient of a fellowship from the Spanish Government.

\section{T3P86}

\section{Experiences in affective-sexual obesity contexts}

Pereira, F. ; Pereira, A. ${ }^{2}$; Veiga-Branco, M. ${ }^{3}$

${ }^{1}$ Faculdade de Medicina da Universidade de Lisboa, PAIDEIA, ${ }^{2}$ Instituto Politécnico De Bragança, ${ }^{3}$ Instituto Politécnico De Bragança, Reci, Paideia

Introduction: The adverse effects of obesity are well documented; however, a growing topic is that relationship with sexuality. Studies show that obesity is associated with a decrease in sexual desire and involvement, avoidance of sexual encounters difficulties in sexual performance, reduced number of sexual partners, and the females to report poor quality of sexual life.

Methods: With the objective to find relationships between the level of Body Mass Index (BMI) and sexual experiences, we proceeded to a quan titative descriptive methodology through the BMI calculation to a sample of 306 users of Obesity Therapy (30.7\% gender male and $69.3 \%$ female) aged 18 and 65 years.

Results: The results are Grade I obesity in most (96 to 31.4\%) and pre-obesity in $23.5 \%$ (72) of respondents, but obesity and pre-obesity, particularly Obesity Grade I (63- 65.6\% of individuals) is more prevalent in marita status ' 'Married' '. Respondents with higher BMI have lower qualifications. Regarding the number of sexual partners, it appears that the higher the BMI, the lower the number of sexual partners that the sample states: 63 of respondents Obesity Grade I self reports a sexual partner, and the normal weight respondents, this number varies between 1 and 20, while the class III obesity varies from 1 to 4 partners. Individuals with normal weight and pre-obesity present frequency of sexual relations / week between 2/3 times / week, while individuals with Obesity Grade II and Grade III have this frequency $1 / 2$ times/week.

Conclusion: BMI assumes influence of variable outlines the quality of affective and sexual experiences. It is considered urgent and relevant to invest in Sexual Education and Nutrition at the level of these populations. Keywords: Obesity, sexual relations, BMI, sexual partner, sexuality, sexual desire, sexual experience, affective-sexual, relationship, sexual performance, Nutrition

Conflict of Interest: None Disclosed

Funding: No Funding

\section{T3P89}

\section{Fasting plasma glucose levels in impaired fasting glucose participants and coronary artery calcification}

\section{Kang, S. ${ }^{1}$; Song, S. ${ }^{1}$}

${ }^{1}$ the Catholic University of Korea

Introduction: Prediabetes is associated with an increased risk of cardiovascular disease (CVD). While the association of impaired glucose tolerance with CVD has been shown in many studies, the relationship between impaired fasting glucose (IFG) and CVD remains in unclear. The purpose of this study was to compare the coronary artery calcium scores (CACS) of participants with normal fasting glucose versus those with IFG, according to fasting plasma glucose (FPG) levels, and to assess whether differences in CACS were independent of important confounders.

Methods: This was a cross-sectional study that was conducted through the Health Promotion Center of the University Hospital (Gyeonggi-do, Republic of Korea), during the period 2010-2014. CACS were assessed by multidetector computed tomography in 1,012 asymptomatic individuals. Anthropometric parameters and metabolic profiles were also acquired.
Study subjects were divided into four glucose groups, excluding participants with a history of CVD or diabetes mellitus.

Results: Out of 1,012 participants, $346(34.2 \%)$ had a CACS $>0$. The incidence of CAC differed according to FPG level $\left(\chi^{2}=44.22, p<0.001\right)$, and the mean CACS increased with FPG. After adjustment for other factors, those subjects with FPG $\geq 110 \mathrm{mg} / \mathrm{dl}$ had a significantly higher risk of coronary artery calcification than did subjects with normal fasting glucose. $(110 \leq \mathrm{FPG}[\mathrm{mg} / \mathrm{dl}]<120, \mathrm{OR}=2.507, p=0.002 ; 120 \leq \mathrm{FPG}[\mathrm{mg} /$ dl] $<126$, OR $=3.568, p=0.001$ )

Conclusion: FPG values in the IFG patients are positively correlated with CAC, and IFG (especially FPG $\geq 110 \mathrm{mg} / \mathrm{dl}$ ) could be an independent risk factor for CAC. These associations were not significantly altered after adjustment for other possible confounders.

Conflict of Interest: None disclosed.

Funding: No Funding.

\section{T3P90}

\section{Fat and fat-free mass contribution to weight loss during an intensive diet and exercise intervention}

\section{Branco, T. ${ }^{1}$; Martins, S. ${ }^{2}$, Vieira, $P_{.}^{2}$; Raimundo, $A .{ }^{3}$}

${ }^{1}$ Instituto Prof. Teresa Branco, Oeiras, Portugal, ${ }^{2}$ Universidade Europeia, Lisbon, Portugal; Instituto De Saúde Ambiental, Faculdade De Medicina, Universidade De Lisboa, Lisbon, Portugal, ${ }^{3}$ Universidade De Évora

Introduction: Due to overweight and obesity epidemic proportions and its comorbidities in most industrialized countries, weight loss is a serious health and economic concern (WHO, 2016). Different weight loss (WL) approaches can be used but an important objective is to maximize the loss of fat mass (FM) while minimizing the loss of metabolically active fat free mass (FFM). Research suggest that adding vigorous exercise to a WL program may help spare FFM in severely obese patients during massive weight reduction (Johannsen et al., 2012). The aim of the present study was to assess FM and FFM contribution to massive WL in a unique group of severely obese individuals undergoing a 16-wk competitive program of diet restriction and vigorous exercise.

Methods: 12 severely obese adults $\left(28.9 \pm 6.2 \mathrm{yr} ; 44.4 \pm 7.7 \mathrm{Kg} / \mathrm{m}^{2}\right), 6$ women, participated voluntarily in the16-week intensive diet and exercise intervention as part of the Portuguese version "The Biggest Loser" televised WL competition. Participants were housed together at an isolated ranch outside Lisbon. The exercise component of the competition consisted of $2-3 \mathrm{~h} / \mathrm{d}(7 \mathrm{~d} / \mathrm{wk})$ of directly supervised vigorous circuit training and/or aerobic training. Participants were encouraged to exercise up to an additional $3 \mathrm{~h} / \mathrm{d}$. Dietary intake was monitored consisting of a calorie-restricted diet greater than $70 \%$ of their baseline energy requirements as calculated by the following: $21.6 \mathrm{kcal} / \mathrm{kg} . \mathrm{d}$ x FFM (kilograms) $+370 \mathrm{kcal} / \mathrm{d}$ (Cunningham, 1991). At wk 16 (4 months), all the participants were assessed. Body composition was estimated by dual-energy X-ray absorptiometry and anthropometric measurements were performed.

Results: At 4-month, participants lost, on average, $25.1 \pm 9.0 \mathrm{Kg}$ of initial body weight (range: $-39.3 \mathrm{Kg}-(-12.6 \mathrm{Kg})$ loss), with a BMI mean reduction of $8.5 \pm 3.2 \mathrm{~kg} / \mathrm{m}^{2}$ representing $19.6 \pm 7.3 \%$ decrease $(P<0.001$ for all). FM decreased in the whole body $(-21.0 \pm 1.3 \mathrm{Kg}$ and $-38.2 \pm 16.3 \%)$, representing $-13.9 \pm 6.8 \mathrm{Kg}(-21.8 \pm 9.7 \%)$ in the trunk, $-11.9 \pm 3.9 \mathrm{Kg}$ $(-37.0 \pm 15.1 \%)$ in lower limbs (LL), and $-1.1 \pm 1.3 \mathrm{Kg}(-21.9 \pm 34.7 \%)$ in upper limbs (UL) $(P<0.001$ for all). FFM was reduced in the whole body $(-4.1 \pm 4.3 \mathrm{Kg}$ and $-5.8 \pm 5.4 \% ; P<0.01)$ and in LL $(-2.4 \pm 1.7 \mathrm{Kg}$ and $8.3 \pm 5.3 \% ; P \leq 0.001)$, while no changes were observed in the trunk and $\mathrm{UL}(P>0.05$ for all). The relative contribution of FM and FFM to WL was, respectively, $85.7 \%$ and $14.3 \%$ in the whole body, $85.6 \%$ and $14.4 \%$ in the trunk, $76 \%$ and $24 \%$ in LL, and $90.7 \%$ and $9.3 \%$ in UL.

Conclusion: Participants in this very controlled intervention experienced a massive WL with absolute mass reductions in the whole body, trunk and LL, and preservation in the UL. FM decrease was the major contributor to total body and regional WL, while FFM presented a minor contribution to weight reduction particularly in UL. 
T3P91

Flaxseed consumption could improve adiponectin level and reduce central obesity in overweight or obese women. $A$ randomized control clinical trial

Faghih, S. $^{1}$; Ahmadnia, $\mathrm{H}^{1}$

${ }^{1}$ Department of Community Nutrition, School of Nutrition and Food Sciences, Shiraz University of Medical Sciences, Shiraz, Iran.

1. Department of Community Nutrition, School of Nutrition and Food Sciences, Shiraz University of Medical Sciences, Shiraz, Iran.

Background: Overweight and obesity are strongly associated with the development of several chronic diseases in human including diabetes, cardiovascular disease and high blood pressure. Flax seed is known as an effective compound for treatment of obesity due to high content of alpha-linolenic acid, fiber and lignans. We aim to evaluate the effects of Flaxseed consumption on serum adiponectin, leptin, and weight loss in overweight or obese women.

Methods: A randomized controlled clinical trial was conducted on $60 \mathrm{pa}$ tients with overweight or obesity. Participants were randomly allocated to two groups; a) weight loss diet plus $25 \mathrm{~g} /$ day brown milled Flaxseed and b) weight loss diet plus $25 \mathrm{~g} /$ day raw powder rice, for 12 weeks. Anthropometric indices and serum leptin and adiponectin were measured at baseline and at the end of the intervention. To assess dietary intakes three 24-hour records were taken at baseline, middle and end of the study. Results: After 12 weeks of intervention, weight, body mass index (BMI) and waist to hip ratio (WHR) decreased significantly in flaxseed group but not the controls. Reduction of waist circumference (WC) $(\mathrm{P}=0.001)$ and WHR $(\mathrm{P}=0.003)$ were significantly more in flaxseed group compared to control group. There was a significant reduction in serum leptin and an increase in serum adiponectin of flaxseed group after intervention $(\mathrm{P}<0.001$ for both). Increase of adiponectin level was significantly more in flaxseed group compared to the controls $(\mathrm{P}=0.002)$.

Conclusion: Flaxseed consumption could reduce WC and WHR in a low energy diet. Also it may improve adiponectin level as an important adipocytokine in regulation of body composition and weight. So flaxseed could be used as a supplement to attenuate central obesity.

Keywords: obesity, flaxseed, adiponectin. leptin

Conflict of interest: There is no conflict of interest

Funding: Research related to this abstract was funded by Shiraz University of Medical Sciences

\section{T3P92}

Follow-up study after laparoscopic adjustable gastric banding: Modifications in inflammatory state and iron metabolism

Catarino, C. ${ }^{1}$; Ferreira, C. ${ }^{2}$; Pereira, P. ${ }^{3}$; Catarino, A. ; Monteiro, L. ${ }^{4}$; Coimbra, S. ; Silva, A.

${ }^{1}$ UCIBIOREQUIMTE, Department of Biological Sciences, Laboratory of Biochemistry, Faculty of Pharmacy, University of Porto, Porto, Portugal, ${ }^{2}$ Laboratory Of Biochemistry, Faculty Of Pharmacy, University Of Porto, Porto, Portugal, ${ }^{3}$ Ucibiorequimte, Department Of Biological Sciences, Laboratory Of Biochemistry, Faculty Of Pharmacy, University Of Porto, Porto, Portugal; Health Science Research Centre, University Of Beira Interior, Covilhã, Portugal, ${ }^{4}$ Hospital Of Prelada-Dr. Domingos Braga Da Cruz, Porto, Portugal, ${ }^{5}$ Ucibiorequimte, Department Of Biological Sciences, Laboratory Of Biochemistry, Faculty Of Pharmacy, University Of Porto, Porto; Cespu, Institute Of Research And Advanced Training In Health Sciences And Technologies (linfacts), Gandra-Prd, Portugal

Introduction: Obesity is characterized by a low-grade inflammation that has been associated with atherosclerosis and insulin resistance. The aim of this study was to evaluate the changes in inflammatory process, iron metabolism and lipid profile of obese individuals after weight loss following laparoscopic adjustable gastric banding.

Methods: Twenty obese individuals were evaluated at baseline (T0) and about a year after surgery (T1). Leukocyte count was evaluated; C-reactive protein (CRP), interleukin (IL)-6, hepcidin, iron status, lipid profile, glucose and insulin were also measured; the homeostasis model assessment of insulin resistance (HOMA-IR) was calculated.

Results: At T1 a significant decrease in body mass index (BMI), hip circumference, waist circumference and waist-to-height ratio was observed; there was also a significant decrease in CRP, IL-6, leukocyte count, hepcidin and ferritin; significant improvement in lipid profile and significant reductions in glucose and insulin, as well as improvement in HOMA-IR were also observed.

Before bariatric surgery, at T0, we found significant correlations between IL-6 and serum iron levels; hepcidin was also correlated with ferritin and transferrin. After bariatric surgery these correlations were not observed; even though, we found a significant positive correlation between variation $(\Delta)$ of IMC and $\triangle$ HOMA-IR.

Conclusion: In summary, body weight reduction in obese individuals, achieved after about a year of gastric band surgery, was associated with reduction in the inflammatory response, favouring iron bioavailability, contributing to reduce cardiovascular risk. Moreover, the lower lipid risk profile and the higher insulin sensitivity observed might contribute to prevent/improve type 2 diabetes mellitus.

Conflit of Interest: None Disclosed.

Funding: FCT/MEC through national funds and co-financed by FEDER, under the Partnership Agreement PT2020, from UCBIO (UID/MULTI/04378/2013 POCI/01/0145/FEDER/007728)

\section{T3P93}

Gastroesophageal acid reflux disease and bariatric surgery: Sleeve gastrectomy versus Roux-en-Y gastric by-pass

\section{Zúñiga, Á. ${ }^{7}$; Guererro, M. ${ }^{2}$; Perez, M. ${ }^{3}$; Aparici, A. ${ }^{3}$; Acarino, A. ${ }^{3}$;} Velasquez, M. ${ }^{1}$; Vilallonga, R. $^{4}$; Fort, J. ${ }^{4}$; Mesa, J. ${ }^{2}$; Ciudin, $A .{ }^{2}$

${ }^{1}$ Endocrinology and Nutrition Department, Vall d'Hebrón University Hospital, Barcelona, Spain., 'Endocrinology And Nutrition Department, Vall D’Hebrón University Hospital, Barcelona, Spain, ${ }^{3}$ Gastroenterology Department, Vall D’Hebron University Hospital, Barcelona, Spain., ${ }^{4}$ Endocrine And Metabolic Surgery Department, Vall D’Hebron University Hospital, Barcelona, Spain

Background: Bariatric surgery (BS) is the most effective treatment for obesity. Laparoscopic sleeve gastrectomy (LSG) and Roux-en-Y gastric bypass (RYGB) are the most commonly used techniques today. Gastroesophageal acid reflux disease (GERD) is common after BS but little is known about the GERD before the surgery. Additionally, there are conflicting data whether or not GERD occurs and / or worsens after BS, especially LSG. The Impedance $\mathrm{pH}$-metry monitoring is a sensitive test for the diagnosis of GERD.

On these bases, the aim of our study was to evaluate the changes in Impedance $\mathrm{pH}$-metry monitoring before and 3 months after LSG and RYGB. Methods: we have designed an observational and prospective study, as a "proof of concept". We have performed impedance $\mathrm{pH}$-metry monitoring in 6 patients before and 3 months after BS. The main exclusion criterion was the absence of any previous symptoms or diagnose of GERD. In all the patients any protons pump inhibitors therapy was discontinuated at least 1 month before performing the impedance $\mathrm{pH}$ metry.

Results: We have evaluated 6 patients: 3 patients underwent LSG and 3 patients underwent RYGB. The mean age was $48.7 \pm 8.1$ years, $66,6 \%$ were women and presented a baseline BMI of $43.9 \pm 4.5 \mathrm{~kg} / \mathrm{m}^{2}$. The patients in the LSG group presented a higher \%EWL at 3 month follow-up then the RYGB ( $46.15 \pm 6.8 \%$ and $31.35 \pm 15.1 \%$ respectively). No differences were seen between the two groups in terms of baseline BMI $(43.78 \pm 8.6 \mathrm{~kg} /$ $\mathrm{m}^{2}$ versus $44.2 \pm 7,3 \mathrm{~kg} / \mathrm{m}^{2}$, respectively). All cases presented hypotonia of the lower esophageal sphincter before and after BS, being more marked after LSG. In 4 of 6 cases GERD was diagnosed by pH-metry before BS (2 patients in the LSG group and 2 patients in the RYGB group). In 2 of 3 cases, GER deteriorated after LSG, whereas after the RYGB there were no changes in the ph-metry in the 3 cases. One patient that underwent LSG required reintervention due to severe GERD. 
Conclusions: In our study, GERD was detected in most of the patients before the surgery. Our results showed that LSG worsened the presence of GERD after 3 months after the BS, whereas the RYGB does not seem to have any influence.

\section{T3P94}

Gender and age, but not obesity, affects serum dexamethasone and/or cortisol levels post overnight 1 mg dexamethasone suppression test

Dotan, I. ${ }^{\text {; }}$ Osher, E. ${ }^{2}$; Greenman, Y. ${ }^{2}$; Tordjman, K. ${ }^{2}$; Limor, R. ${ }^{2}$; Gilad, S. ${ }^{2}$; Moshe, Y.'; Novack, V. ${ }^{3}$; Toledano, R. ${ }^{3}$; Stern, N. ${ }^{2}$

${ }^{1}$ Tel Aviv-Sourasky Medical Center and Sackler Faculty of Medicine, Israel and McGill University Health Center, Montreal, Canada, ${ }^{2}$ Tel Aviv-Sourasky Medical Center And Sackler Faculty Of Medicine, Israel, ${ }^{3}$ Soroka University Medical Center And The Faculty Of Health Sciences, Ben-Gurion University Of The Negev, Beer Sheba, Israe

Background. Cushing's syndrome (CS) is often considered, though rarely encountered, in the work-up of obesity. The overnight $1 \mathrm{mg}$ dexamethasone (Dex) suppression test (ODST) is widely used in this setting. Confusingly, obese subjects were shown to be either Dex hypersensitive (better suppressors) or require higher Dex dose to achieve proper suppression. The latter is due to a higher volume of distribution that consequently lowers the achieved serum Dex, with inadequate Hypothalamus-Pituitary-Adrenal axis suppression. Here we re-assess this debatable issue.

Aim. To evaluate the outcome of ODST in obese versus non-obese subjects, not suspected as having CS.

Methods: Blood samples for baseline cortisol (at 8:00 AM) were obtained from 59 volunteers whose BMI ranged from 17.9 to $47.5 \mathrm{~kg} / \mathrm{m}^{2}$. The morning (8:00-9:00 AM) following ODST (at 11:00 PM) blood samples for serum cortisol and Dex levels were collected and determined by ECLIA (Cobas 411, cortisol) and HPLC (cortisol, Dex).

Results: All but one of the participants properly suppressed serum cor tisol level to $<1.8 \mu \mathrm{g} / \mathrm{dL}$; All but 5 suppressed to $<1.4 \mu \mathrm{g} / \mathrm{dL}$. Men with $\mathrm{BMI} \leq 30$ had a significantly higher serum Dex level than women with a similar BMI $(0.67 \pm 0.38$ vs. $0.36 \pm 0.19 \mathrm{ug} / \mathrm{dL}, \mathrm{p}=0.01)$. The difference in Dex levels did not translate, however, into gender-related differences in attained cortisol. There was no correlation between Dex and post-Dex cortisol or serum Dex in the 5 individuals who failed to show suppres sion to $\leq 1.4 \mathrm{ug} / \mathrm{dL}$. Post Dex serum cortisol correlated positively with age ( $\mathrm{r}=0.45$ by RIA, $\mathrm{r}=0.27$ by HPLC, adjusted for BMI; $\mathrm{p}<0.05$ for both tests), but not with BMI. Subdivided by body mass, subjects with $\mathrm{BMI} \geq 25$ vs. those with $\mathrm{BMI}<25$, had substantially higher HPLC- (but not ECLIA) determined cortisol levels $(1.16 \pm 0.57$ vs. $0.86 \pm 0.33 \mu \mathrm{g} / \mathrm{dL}, \mathrm{p}=0.044)$. Conclusions. Age, but not elevated BMI, is linked to lesser suppression of cortisol by Dex. Therefore, ODST is equally reliable in normal weight, overweight and obese subjects, with no need for Dex dose adjustment. Because of some disparity between HPLC and RIA-determined cortiso levels in the low concentration range seen with suppression, borderline and unexpected results should be clarified by the measurement of cortisol and Dex by HPLC

Conflict of Interest: None Disclosed

Funding: No Funding

\section{T3P95}

\section{Gender as a determinant of weight loss after bariatric surgery}

Raymonde-Parker, C. ${ }^{1}$; Abeysekera, A. ${ }^{\text {; }}$ Labib, M. ${ }^{1}$

\section{${ }^{1}$ Russells Hall Hospital, The Dudley Group NHS Foundation Trust}

Introduction: Women's reduced health-related quality of life, particularly in self-esteem, sexual life, and greater rates of depression may play an important role in their decision to seek bariatric surgery. On the other hand, men seem to be less concerned about their body image compared to women and they don't tend to seek bariatric surgery until they face more serious health problems. Despite this, there is little research regarding whether gender is a predictor of weight loss following bariatric surgery with some studies reporting better results for men but others reporting no gender differences.

Methods: We audited the outcome at one and three years in 20 male and 20 female patients (matched for age and type of surgical procedure) who underwent either gastric bypass or sleeve gastrectomy operations, in order to assess the difference in \% excess body weight (\%EBW) loss between the two groups. Patients who did not attend for either their 1 year or 3 year follow up appointments were excluded from selection.

Results: The pre-surgery mean BMI was $48.6 \pm 6.6 \mathrm{~kg} / \mathrm{m}^{2}$ in men and $50.7 \pm 10.2 \mathrm{~kg} / \mathrm{m}^{2}$ in women $(\mathrm{p}=0.45)$. At one year, the mean $\% \mathrm{EBW}$ loss was $61.1 \pm 17.1 \%$ for men and $65.8 \pm 17.8 \%$ for women $(p=0.39)$. The mean \%EBW loss at three years was $61.7 \pm 23.1 \%$ for men and $64.7 \pm 24.9 \%$ for women $(\mathrm{p}=0.70)$.

Conclusion: Our audit showed that despite gender differences in attitudes and reasons for seeking bariatric surgery, there was no difference in weight loss following bariatric surgery between men and women. The existing literature regarding gender influence on weight loss following bariatric surgery is not conclusive perhaps due to the heterogeneity of these studies and that gender difference in weight loss was not assessed as a primary outcome. Our audit showed that irrespective of the reasons for bariatric surgery, weight loss men and women was not different at one year and three years after bariatric surgery.

\section{T3P97}

Glasgow prognostic score is associated with severity of obesity but not with complication rate in bariatric patients

Wolter, S. ; Duprée, A. ' ; Miro, J. ${ }^{1}$; Schroeder, C. ; Busch, P. ; Aberle, J. ${ }^{1}$; Vashist, $Y^{\prime}{ }^{\prime}$;Mann, $O$.

${ }^{1}$ University Medical Center Hamburg-Eppendorf

Introduction: Adipose tissue contributes to a state of chronic inflammation. Pro-inflammatory adipokines are elevated in obese patients and are associated with increased insulin resistance. The acute phase proteins albumin and C-reactive protein (CRP) represent broadly available indicators of systemic inflammation. Hypoalbuminemia and CRP are associated with poor prognosis in patients undergoing surgery. The Glasgow Prognostic Score (GPS) combines albumin and CRP into a risk stratification score for prognosis of clinical outcome. Bariatric surgery is the most effective option to provide long-term weight loss and to improve comorbid conditions like T2DM in morbidly obese patients. The surgical treatment of obese patients in general is associated with higher complication rates compared to patients with normal weight. The aim of this study was to define risk groups for patients undergoing bariatric surgery by using the Glasgow Prognostic Score.

Methods: We conducted a retrospective analysis of bariatric primary procedures, either laparoscopic Roux-Y Gastric Bypass (RYGB) or Sleeve Gastrectomy (SG). We excluded patients undergoing revisional surgery after primary bariatric procedures due to its higher complication rate. After the final analysis, the Clavien-Dindo Classification was applied to differentiate the severity of complications. For evaluation of the GPS preoperative blood test results not older than 6 months were used. Patients with both an elevated CRP ( $>10 \mathrm{mg} / \mathrm{L})$ and hypoalbuminemia $(<35 \mathrm{~g} / \mathrm{L})$ were allocated a score of 2 (GPS2). Accordingly, patients with neither of these abnormalities were allocated a score of 0 (GPS0). Remaining patients in whom only one of the biochemical parameter was abnormal were assigned a score of 1 (GPS1).

Results: Of 761 evaluated patients, 95 patients (12,5\%) experienced one or more postoperative complications. Major complication rate (Clavien Dindo $\geq 3)$ was $6,4 \%(n=49)$. Leakage rate was $0,92 \%$ and mortality rate was $0,39 \%$. There was no association between GPS and complication rate ( $p$ 0.547 ) and no association between GPS and the severity of complications (p 0.888). An elevated GPS was more common in super obese patients $(\mathrm{p}<0.0001)$ and in patients with hypertension $(\mathrm{p} 0.029)$. A tendency to- 
ward higher a GPS in patients with type II diabetes was seen, but did not reach statistical significance (p 0.051).

Conclusions: An elevated Glascow Prognostic Score (GPS) is more common in super obese bariatric patients and seems to be associated with metabolic syndrome. Nevertheless, GPS fails to predict postoperative complications in patients undergoing bariatric surgery.

No conflicts of interest regarding the topic of this abstract.

No Funding received.

\section{T3P98}

\section{Hepatic fibrosis is associated with lower BMI-reduction and muscle mass after gastric bypass}

Luger, M. ${ }^{1}$; Kruschitz, R. ${ }^{2}$; Luger, E. ${ }^{3}$; Schindler, K. ; Trauner, M. ${ }^{5}$; Krebs, M. ; Rieder, A. ; Hoppichler, F.'; Ludvik, B. ${ }^{8}$

${ }^{1}$ Division of Endocrinology and Metabolism, Department of Internal Medicine III, Medical University of Vienna; Special Institute for Preventive Cardiology And Nutrition - SIPCAN, Salzburg; Division of Social and Preventive Medicine, Centre for Public Health, ${ }^{2}$ Division Of Endocrinology And Metabolism, Department Of Internal Medicine lii, Medical University Of Vienna; Department Of Internal Medicine, Elisabethinen-Krankenhaus Klagenfurt, ${ }^{3}$ Division Of Social And Preventive Medicine, Centre For Public Health, Medical University Of Vienna; Special Institute For Preventive Cardiology And Nutrition - Sipcan, Salzburg, ${ }^{4}$ Division Of Endocrinology And Metabolism, Department Of Internal Medicine lii, Medical University Of Vienna, ${ }^{5}$ Division Of Gastroenterology And Hepatology, Department Of Internal Medicine lii, Medical University Of Vienna, ${ }^{6}$ Division Of Social And Preventive Medicine, Centre For Public Health, Medical University Of Vienna, ${ }^{7}$ Special Institute For Preventive Cardiology And Nutrition - Sipcan, Salzburg; Department Of Internal Medicine, Krankenhaus Der Barmherzigen Brüder Salzburg, ${ }^{8}$ Department Of Medicine 1 And Karl Landsteiner Institute For Obesity And Metabolic Diseases, Rudolfstiftung Hospital Vienna

Introduction: Recent studies revealed that low muscle mass was associated with significant hepatic fibrosis [1-2]. Therefore, we assessed the association between $\mathrm{BMI}$ reduction and muscle mass loss with hepatic fibrosis in bariatric patients.

Methods: Baseline liver biopsy in the context of a randomized controlled trial [3] was performed in patients with omega-loop gastric bypass. Fatfree mass was assessed by Dual Energy X-ray Absorptiometry at baseline, 6 and 12 months and appendicular skeletal muscle mass (ASMM) was calculated. Low muscle mass was defined as ASMM/weight below 2 standard deviation (SD) than the sex-specific mean of a young population (cut-offs: $<0.283$ for men and $<0.235$ for women [4]).

Results: 46 patients [78\% females, 42 (13) years, BMI $44(4) \mathrm{kg} / \mathrm{m}^{2}$ ] were included in the analysis and $30 \%$ demonstrated significant fibrosis $[\mathrm{F} \geq 2$; $9 \%$ advanced and $4 \%$ cirrhosis]. At 6 and 12 months after surgery, they lost 27 (6) \% and 35 (8) \% of their initial BMI, with no differences at baseline. At 6 and 12 months postoperatively, fibrotic patients showed a significantly lower BMI reduction [mean difference between $\mathrm{F} \geq 2$ and $\mathrm{F} \leq 1:-3.5$ (1.6) \% and $-8.1(1.7) \% ; \mathrm{p}<0.001]$ and lower muscle mass index $[-0.012$ (0.009) and $-0.037(0.011) ; \mathrm{p}=0.016]$, with no differences at baseline. The prevalence of low muscle mass (ASMM/weight) was $46 \%$ at baseline, $5 \%$ at 6 , and $4 \%$ at 12 months postoperatively. By using logistic regression, fibrotic patients showed a 4 -fold higher risk of remaining a lower muscle mass over time [OR 3.9; 95\% CI 1.01-15.50; $\mathrm{p}=0.050$ ].

Conclusion: Patients with hepatic fibrosis lost significantly less BMI but more muscle mass in relation to weight compared to those without. Consequently, we suggest that fibrotic patients undergoing bariatric surgery require special postoperative attention.

\section{References:}

1 Lee YH et al. J Hepatology. 2015

2 Lee YH et al. Hepatology. 2016

3 Luger M, et al. Trials. 2015

4 Poggiogalle E et al. JNHA. 2016

Conflict of Interest: None Disclosed

Funding: This work was partly supported by the Austrian Biobanking and BioMolecular resources Research Infrastructure (BBMRI.at) funded by the Austrian
Federal Ministry of Science, Research and Economy (BMWFW GZ 10.470/0016II/3/2013) and by the non-profit organization "Special Institute for Preventive Cardiology And Nutrition - SIPCAN save your life" (Salzburg) for personnel costs; Trial registration number: Clinicaltrials.gov (NCT02092376)

\section{T3P101 \\ Impact of a multidisciplinary weight management service on musculoskeletal pain in obese individuals.}

Maclellan, G. '; Dunlevy, C. ${ }^{2}$; O'Malley, E. '; Blake, C. '; Breen, C. ${ }^{2}$; Gaynor, K. ${ }^{2}$; Wallace, N. ${ }^{2}$; Yoder, R. ${ }^{2}$; Casey, D. ${ }^{2}$; Mehegan, J.'; O'Shea, D. ${ }^{2}$; Fullen, B. ${ }^{1}$

${ }^{1}$ University College Dublin, School of Public Health, Physiotherapy \& Sports Science, Belfield, Dublin 4, Republic of Ireland, ${ }^{2}$ Weight Management Service, St. Columcille'S Hospital, Loughlinstown, Co. Dublin, Republic Of Ireland

Introduction:Musculoskeletal (MSK) pain is prevalent in obesity, especially in weight-bearing joints. Multidisciplinary weight management services (WMS) effectively manage weight; however, pain is not often investigated as a primary outcome measure post-WMS participation.

Methods:This retrospective-design study established changes in anthropometric and pain outcomes in patients who attended a national WMS. Data were entered into the Statistical Packages for the Social Sciences (V.20). Descriptive and inferential statistics were used to analyze pre-post WMS changes in anthropometric and pain outcomes, and to evaluate variables predictive of clinically significant change (CSC) in pain scores. Ethical approval from the participating hospital was granted.

Results:In total, 806 patients attended the WMS [male, 34.6\% $(n=279)$; female, $65.4 \%(n=527)$; mean age, 44.7 years]. Baseline mean weight was $145.6 \mathrm{~kg}$; mean BMI was $50.7 \mathrm{~kg} / \mathrm{m}^{2}$. The majority of patients reported MSK pain $(90.9 \% ; n=628)$. Only $59.1 \%(n=476)$ attended their reassessments. Overall, $72.2 \%(n=343)$ lost weight; with significant reductions in weight and BMI $(p<0.05)$. Low back pain (LBP) prevalence and pain scores reduced significantly $(p<0.05)$; significant differences were reported between weight loss categories $(p<0.05)$. Measures of CSC were reported for pain outcomes: LBP, 32\% $(n=77)$; and knee pain, $23.2 \%$ $(n=46)$. Significant associations were found between changes in pain, anthropometric, self-report, and functional outcomes $(p<0.05)$. Models were then developed to illustrate variables predictive of CSC in LBP and knee pain scores $(p<0.05)$.

Conclusion:Musculoskeletal pain prevalence and severity is high in this obese cohort. There were significant reductions in weight, LBP and knee prevalence, and LBP scores post-WMS with increased improvements in pain prevalence for those who lost weight. These services are effective for weight management and subsequent MSK pain symptom reduction. The inclusion of pain management strategies within the WMS may provide a more holistic approach to obesity management.

Conflict of Interest: No conflicts of interest have been identified.

Funding: No Funding was received for the research relating to this abstract.

\section{T3P102 \\ Impact of bariatric surgery in reducing cardiovascular risk - application of Framingham Risk Score and SCORE risk}

Oliveira, S. ${ }^{1}$; Souteiro, P.'; Neves, J. ${ }^{1}$; Pedro, J. ${ }^{1} ;$ Magalhães, D. ${ }^{1}$;

Gonçalves, V. ${ }^{1}$; Bettencourt-Silva, R. ${ }^{1}$; Costa, M. ${ }^{1}$; Santos, A. ${ }^{2}$; Varela, A. ${ }^{1}$;

Queirós, J. ${ }^{1}$; Freitas, P. ${ }^{1}$; Carvalho, D. ${ }^{1}$; Group), M. ${ }^{1}$

${ }^{1}$ Centro Hospitalar São João, ${ }^{2}$ Faculty Of Medicine, University Of Porto

Background: Obesity is associated with an increased risk of cardiovascular (CV) morbidity and mortality. Framingham risk score (FRS) and SCORE risk (SR) are 2 models that estimate, respectively, the risk of developing $\mathrm{CV}$ disease or fatal $\mathrm{CV}$ event over a period of 10 years, regardless of weight, making them ideal for the purpose of the present study: to evaluate the impact of bariatric surgery (BS) in reducing the CV risk of obese patients. 
Methods: We performed a retrospective longitudinal study of 1448 obese patients undergoing BS between January/2010 and June/2015 at our centre. Patients with no record of the FRS (American Heart Association 2008) or SR (European Society of Cardiology 2007) criteria were excluded.

Results: A total of 558 patients were included in SR analysis, 492 (88.2\%) women, mean age $42.6 \pm 10.3$ years. Of these patients, $338(60.6 \%)$ were submitted to roux-en-Y gastric bypass (RYGB), 123 (22.0\%) to adjustable gastric banding (AGB) and $97(17.4 \%)$ to sleeve gastrectomy (SG). The 4 $(0.7 \%)$ patients who were at high risk $(5-6, \geq 10 \%)$ before BS transited into the low-intermediate risk category $(0-4,<10 \%)$ during the first postoperative year, which was maintained in the second. Conversely, $1(0.2 \%)$ patient went from low-intermediate risk to high risk in the same period. Of the patients submitted to AGB only $1(0.8 \%)$ presented high risk after 12 months of BS, while in RYGB and SG all (100\%) were in the low-intermediate risk category after the first year.

For the FRS, the longitudinal analysis included 76 patients over 4 years, 74 $(97.4 \%)$ women, with a mean age of $43.6 \pm 10.7$ years. Of these patients, 38 (50.0\%) were submitted to RYGB, $34(44.7 \%)$ to AGB and $4(5.3 \%)$ to SG Preoperative FRS was $9.5 \pm 1.1 \mathrm{pts}$, with a statistically significant reduction to $7.0 \pm 0.8$ pts after 12 months of BS, starting to increase gradually from 24 months $(7.8 \pm 0.9$ pts $)$ to 48 months $(10.9 \pm 1.0 \mathrm{pts})$. No statistically significant differences were found between the 3 types of surgery in the subgroup analysis.

Conclusion: BS has a favorable effect in reducing the CV risk of obese patients, analyzed through FRS and SR. The greatest impact occurs in the first year, and there seems to be no significant difference between the 3 types of surgery, which should be confirmed in future studies.

\section{T3P103 \\ Impact of bariatric surgery on albuminuria reduction and its predictive factors}

Oliveira, S. ${ }^{1}$; Neves, J. '; Souteiro, P. ${ }^{1}$; Pedro, J. ${ }^{1}$; Magalhães, D. ${ }^{1}$;

Gonçalves, V. ${ }^{\text {; }}$ Bettencourt-Silva, R. ${ }^{\text {; }}$ Costa, M. ${ }^{\prime}$; Santos, A. ${ }^{2}$; Varela, A. ${ }^{\text {' }}$; Queirós, J. '; Freitas, P. ${ }^{1}$; Carvalho, D. ${ }^{1}$; Group), M. ${ }^{1}$

${ }^{1}$ Centro Hospitalar São João, ${ }^{2}$ Faculty Of Medicine, University Of Porto

Background: Albuminuria is an independent predictive factor of cardiovascular and renal risk, with an important prognostic value and has recently been recognized as the main risk factor for increased morbidity and mortality in obese patients. The aim of the present study was to evaluate the impact of bariatric surgery (BS) on albuminuria reduction and its predictive factors.

Methods: We performed a retrospective study of 1448 obese patients undergoing BS between January/2010 and June/2015 at our centre. Patients with no record of urinary albumin excretion were excluded. For longitudinal and subgroups analysis, we excluded those whith a lack of albuminuria consistent registration during 2 years of follow-up.

Results: The longitudinal analysis included 75 patients, 69 (92.0\%) women, mean age $43.0 \pm 10.9$ years and BMI $42.9 \pm 5.2 \mathrm{~kg} / \mathrm{m}^{2}$. Of all patients 54 (72\%) were submitted to roux-en-Y gastric bypass (RYGB), 19 (25.3\%) to sleeve gastrectomy (SG) and only $2(2.7 \%)$ to adjustable gastric banding (AGB). Preoperative albuminuria was $22.8 \pm 55.4 \mathrm{mg} / \mathrm{g}$, observing a statistically significant reduction of $67.2 \%$ after 12 months of BS $(7.5 \pm 8.4$ $\mathrm{mg} / \mathrm{g})$, with a tendency for stabilization after 24 months $(7.1 \pm 8.4 \mathrm{mg} / \mathrm{g})$. In the subpopulation of diabetic patients $(41 ; 54.6 \%)$, the reduction profile was similar, whereas in the hypertensive group $(33 ; 44.0 \%)$ we observed a higher initial reduction, of $71.3 \%$. The subgroup of patients with a BMI> $45 \mathrm{~kg} / \mathrm{m}^{2}(19 ; 25.3 \%)$ was the one that found the greatest benefit, with a reduction of $81.1 \%$ in the first year and $22.3 \%$ in the second. Contrary to RYGB, where an initial reduction of $72 \%$ was observed but with a slight increase from 12 to 24 months (14.9\%), in the SG, we observed a lower initial reduction $(38.7 \%)$, but increased in the second year $(44 \%)$, which is an interesting distinctive data of these 2 types of BS. In the multivariate analysis, only the BMI proved to be an independent predictive factor for albuminuria reduction in these patients $(\mathrm{p}<0.001)$.
Conclusion: BS results in a significant and sustained reduction of albuminuria in obese patients, with BMI being an independent predictive factor. The greatest impact occurs in the first 12 months and in the subgroup of patients with a BMI $>45 \mathrm{~kg} / \mathrm{m}^{2}$. Thus, BS should be considered early in the treatment of obese patients with renal impairment, and may prevent progression to manifest disease.

\section{T3P104 \\ Impact of bariatric surgery on nonalcoholic fatty liver disease assessed through validated fibrosis scores}

\section{Gonzalez, D. ${ }^{1}$; Viñuelas, I. ${ }^{1}$, Dominguez, O. ${ }^{1}$; Martin, A. ${ }^{1}$; Blanco, A. ${ }^{1}$}

${ }^{1}$ University Hospital La Paz

Introduction: Nonalcoholic fatty liver disease (NAFLD) is common among morbid obese with estimated prevalence of $90 \%$. Weight loss play a main role in the control of this disease. We aimed to determine the evolution of NAFLD by means of validated fibrosis scores 12 months after surgery and measure the impact of bariatric surgery (BS).

Methods: It is a retrospective observational study which enrolled obese who underwent BS at a University Hospital in Madrid, Spain during 2014 and 2015. Demographics, anthropometric, clinical and analytical features were collected at baseline and compared at 12 months after BS. NAFLD liver fibrosis and FIB-4 scores were calculated.

Results: We included 50 patients (37 women, $74 \%$ ) whose mean age was $43.84 \pm 11.98$ years. At baseline, mean weight was $124.98 \pm 19.88 \mathrm{~kg}$, body mass index (BMI) $45.74 \pm 5.93 \mathrm{~kg} / \mathrm{m}^{2}$, basal glycaemia $104 \pm 25 \mathrm{mg} / \mathrm{ld}$., glycated hemoglobin (HbA1c) $5.93 \pm 0.79 \%$, alanine aminotransferase (ALT) 27 (12 - 125) IU, aspartate aminotransferase (AST) $22(11$ - 126) IU, gamma glutamyl transpeptidase (GGT) 35 (7 - 216) IU, alkaline phosphatase (AP) $72(17$ - 159) IU. 24 (48\%) patients were considered as having altered transaminases. Type 2 diabetes (T2D), hypertension (HT) and dyslipidemia was present in 20 (40\%), 30 (60\%) and $16(32 \%)$, respectively. NAFLD liver fibrosis and FIB-4 scores were $1.859(-1.755-4.631)$ and $0.72(0.22-3.05)$, respectively.

In the postoperative period, mean weight was $84.17 \pm 15.93 \mathrm{~kg}$, BMI $30.76 \pm 4.52 \mathrm{~kg} / \mathrm{m}^{2}$, basal glycaemia $85 \pm 12 \mathrm{mg} / \mathrm{ld}$., HbAlc $5.28 \pm 0.31 \%$, ALT 20 (9 - 133) IU, AST 20 (11 - 80), GGT 18 (6 - 237), AP 75 (8 - 183). $17(34 \%)$ were considered as having altered transaminases. T2D, HT and dyslipidemia was present in $3(6 \%), 14(28 \%)$ and $2(4 \%)$, respectively. NAFLD liver fibrosis and FIB-4 scores were $0.412(-2.643-4.661)$ and 0.78 $(0.38-1.79)$, respectively.

Mean weight loss was $40.75 \pm 14.59 \mathrm{~kg}$, mean percentage of excess weight loss after surgery was $73.23 \pm 20.79 \%$, and mean percentage of excess BMI loss was $72.92 \pm 19.36 \%$.

Weight, BMI, basal glycaemia, HbA1c, ALT, GGT, total cholesterol, HDL cholesterol and triglycerides significantly improved after BS. NAFLD fibrosis score significantly decrease after surgery, but FIB- 4 did not. Those two scores were strongly correlated between them.

Conclusions: BS is an effective intervention to control the progression of NAFLD evaluated through clinical parameters, analytical values and fibrosis scores, of which NAFLD score is better than FIB-4 score due to the fact that it consider the weight to calculate it.

Conflict of Interest: None.

Funding: No Funding.

\section{T3P105 \\ Impact of central obesity on blood pressure levels of healthcare professionals}

Queiroz, F. '; Tavares, C. '; Ansel, J. '; Costa, L. ${ }^{2}$; Souza, S. ${ }^{2}$

${ }^{1}$ Fundação Técnico-Educacional Souza Marques - Escola de Medicina, ${ }^{2}$ Instituto Estadual De Cardiologia Aloysio De Castro - lecac 
Introduction: Central obesity (CO) screening is an important strategy for primary prevention of systemic arterial hypertension (SAH), since it is one of the main risk factors (RF) for its development ${ }^{1}$. The maintenance of body mass and blood pressure (BP) at adequate levels is fundamental to prevent cardiovascular diseases $(\mathrm{CVD})^{2}$. Health promotion campaigns with RF screening are frequent for general population, but scarce for healthcare professionals.

Objectives: To evaluate CO and BP in healthcare professionals of a public health unit specialized in cardiology in Rio de Janeiro, Brazil.

Methods: Cross-sectional descriptive study submitted and approved by the Research Ethics Committee where it was carried out. The invitation to participate was made during a visit to the sectors. Variables evaluated gender, age, professional category, waist circumference (WC), hip circumference (HC), waist-hip ratio (WHR), systolic and diastolic BP (SBP, DBP). BP was optimal up to $120 \times 80 \mathrm{mmHg}$ and elevated from $121 \times 81 \mathrm{mmHg}^{1}$. WC was high and very high in: women: $>80 \mathrm{~cm},>88 \mathrm{~cm}$; men: $>94 \mathrm{~cm}$, $>102 \mathrm{~cm}$. WHR was elevated in: women: $\geq 0.85 \mathrm{~cm}$; men: $\geq 0.90 \mathrm{~cm}^{3}$. Statistical analysis were performed using SPSS v.21, considering statistically significant $\mathrm{p}<0.05$.

Results: 194 subjects were evaluated, with mean age of $43 \pm 12.3$ years, $76 \%$ women, $88 \%$ adults, $12 \%$ elderly, $53.6 \%$ nursing staff, PAS $118 \pm 16.3$, PAD: $78 \pm 10.8$, of which 35 had diagnosis of SAH, in pharmacotherapy (gHAS) and 159 without (gnHAS). Stratifying the sample in gHAS and gnHAS, it was observed: age: $53 \pm 9.8 v s .41 \pm 11.6$; adequate WC: $8.5 \%$ vs.39.7\%; high WC: $28.5 \%$ vs.27\%; very high WC: $63 \% v s .33 .3 \%$; adequate WHR: $31.4 \% v s .72 .3 \%$; high WHR: $68.6 \% v s .27 .7 \%$; optimal BP: $28.6 \% v s .78 \%$; high BP: $71.4 \% v s .22 \%$, SBP: $133 \pm 19.8 v s .114 \pm 13.1$; DBP: $85 \pm 12 v s .76 \pm 9.8$; in women: WC: $96.2 \pm 9.9 v s .85 .3 \pm 10.2$, HC: $110.8 \pm 10.9 v s .105 .3 \pm 7.8$, WHR: $0.87 \pm 0.06 v s .0 .81 \pm 0.06$; in men: WC: $105.8 \pm 14.9 v s .94 .8 \pm 9.9$, HC: $108.5 \pm 10.5 v s .105 .1 \pm 6.7$, WHR: $0.96 \pm 0.08 v s .0 .90 \pm 0.06$. $\mathrm{P}<0.05$ was observed in all differences between means, except in men HC.

Conclusion: The findings highlight the importance of early screening of $\mathrm{CO}$ for $\mathrm{SAH}$ prevention, indicating that after onset of disease its control may be inefficient, leading to increased CVD mortality, including healthcare professionals, and emphasize the need for individual and collective strategies to reverse this scenario.

\section{References:}

1 Malachias M et al.7th Brazilian Arterial Hypertension Guidelines. Arq Bras Cardiol, 2016.

2 WHO.Global atlas on cardiovascular disease prevention and control, 2011.

3 WHO.Waist circumference and waist-hip ratio: report of a WHO expert consultation, 2008.

Conflict of Interest: The authors declare that there is no conflict of interest in the development of the research.

Funding: This research was developed with own resources.

\section{T3P106}

Impact of high-BMI (body mass index) on non-communicable diseases in the European Union

Chakraborty, S. ${ }^{1}$; Balanda, K. ${ }^{2}$; Perry, I. ${ }^{1}$; Kabir, Z. ${ }^{1}$

${ }^{1}$ University College Cork, ${ }^{2}$ Institute Of Public Health, Dublin

Introduction: The World Health Organisation (WHO) states that atleast 2.8 million people die annually globally as a result of being overweight or obese.Approximately 35.8 million(2.3\%) of the global DALYs are from overweight and obesity.It is thus important to quantify the NCD-burden attributable to high-BMI to aid in the policy making.

Aim: To quantify NCD deaths and burden attributable to high-BMI between 1990 and 2015 across the 28 nations of the European Union(including UK).

Methods: Publicly available data from US Institute of Health Metrics and Evaluation was abstracted to estimate deaths,Disability-Adjusted-Life-Years (DALYs) and Years-lived-with-disability (YLDs) from
NCDs attributable to high-BMI employing the comparative risk assessment (CRA) framework of the Global Burden of Disease methodology.

Results: NCDs related DALYs attributable to high-BMI accounted for approximately 11.35 million (95\%Uncertainity interval $\mathrm{UI}=7.2 \mathrm{mil}$ lion-16.2million) and 10.2 million $(95 \% \mathrm{UI}=6.6 \mathrm{million}-14.6 \mathrm{million})$ in 1990 and 2015 respectively.

Deaths from NCDs attributable to high-BMI showed declining trends from $487 \times 10^{3}(11.1 \%)$ in 1990 to $455 \times 10^{3}$ (9.6\%) in 2015 .

There has been a rise in the NCD related YLDs from 20.2 million $(4.3 \%)$ to 30.3 million $(5.3 \%)$.

The contributions of high-BMI to NCD related deaths and burden metrics was to these major NCDs- diabetes, ischemic heart disease,stroke and uterine cancer.

Conclusions: NCD burden attributable to high-BMI in the European Union is still quite substantial $\left(\sim 455 \times 10^{3}\right.$ deaths and $\sim 10.2$ million DALYs).Some of the observed patterns particularly increase in YLDs are due to population ageing.More cost-effective and population based policies and interventions targeting the inequalities of gender and health is the need of the hour.

\section{Conflict of Interest-None}

Funding-Partial Funding by Centre for Health and Diet Research

\section{T3P107 \\ Impact of regular orange juice consumption with or in- between meals on glucose regulation in healthy subjects}

\section{Hägele, F. ${ }^{1}$; Büsing, F.'; Nas, A. '; Sprügel, L. '; Kaelble, A. '; Aschoff, J. ';} Carle, R.'; Bosy-Westphal, A. ${ }^{1}$

${ }^{1}$ Institute of Nutritional Medicine, University of Hohenheim, Stuttgart, Germany, ${ }^{2}$ Institute Of Food Science And Biotechnology, University Of Hohenheim, Stuttgart, Germany

Background: Consumption of sugar sweetened beverages (SSB) is an established risk factor for obesity and type 2 diabetes although the underlying mechanisms are less clear. Timing of SSB consumption may play a role since a regular intake of SSB between meals has been shown to increase the development of obesity. On the other hand a higher daylong glycemia was observed with 6 compared to 3 high carbohydrate meals. The aim was to investigate the impact of orange juice consumption with or in-between meals on the regulation of glucose metabolism.

Methods: Twenty-six healthy adults (13 women and 13 men; $25.2 \pm 3.1 \mathrm{y}$; BMI $23.5 \pm 4.0 \mathrm{~kg} / \mathrm{m}^{2}$ ) participated in an ongoing randomized $2 \times 2$-wk cross-over intervention and consumed orange juice (20\%Ereq) i) together with 3 meals (WM) or ii) in-between 3 meals (BM). Daylong glycemia (iAUC) was assessed by continuous glucose monitoring for 6 days during each intervention phase. In a subgroup $(n=12)$, insulin secretion was assessed using C-peptide excretion and basal and postprandial insulin sensitivity (IS) were measured using HOMA- and Matsuda-Index.

Results: Glucose variability was higher with WM compared to BM (MAGE: $2.08 \pm 0.3$ vs. $2.52 \pm 0.5 ; \mathrm{p}<0.01$ ) whereas daylong glycemia and insulin secretion did not differ between interventions. Postprandial IS decreased after the BM-intervention (Matsuda: $-2.02 \pm 2.4 ; \mathrm{p}<0.05$ ) but remained unchanged after WM. Basal IS did not change during both interventions.

Conclusion: Orange juice consumption with meals lead to increased glucose variability which is a proposed cardiovascular risk factor. On the other hand orange juice consumption in-between meals lead to impaired postprandial insulin sensitivity that may be due to a higher voluntary energy intake with in-between meal snacking.

Conflict of interest: The authors declare no conflict of interest

Funding: Research relating to this abstract was funded by Verband der deutschen Fruchtsaft-Industrie e.V. 
T3P108

\section{Impact of regular orange juice or cola consumption on uric acid levels in healthy adults}

\section{Büsing, F. ${ }^{1}$; Hägele, F. '; Nas, A. '; Kaelble, A. '; Sprügel, L. ' ; Aschoff, J. ${ }^{2}$;} Carle, R. ${ }^{2}$; Bosy-Westphal, $A{ }^{1}$

${ }^{1}$ Institute of Nutritional Medicine, University of Hohenheim, Stuttgart, Germany, ${ }^{2}$ Institute Of Food Science And Biotechnology, University Of Hohenheim, Stuttgart, Germany

Introduction: Epidemiological studies have found an association between fructose-rich beverage consumption and increased serum uric acid levels no matter whether the fructose comes from sugar-sweetened soda or orange juice. In contrast, hyperuricemia in rats was lowered by orange juice consumption. Orange juice may therefore be less detrimental in terms of gout risk when compared with sugar-sweetened beverages.

Methods: In an ongoing randomized cross-over $2 \times 2$-wk intervention, 19 healthy individuals ( 10 men, 9 women, $24.7 \pm 3.2$ years, BMI $23.1 \pm 3.3$ $\mathrm{kg} / \mathrm{m}^{2}$ ) either consumed orange juice (20\% of energy requirement, 57.4 $\mathrm{g}$ glucose, $55.1 \mathrm{~g}$ fructose) or an equal amount of sugar (57.9 g glucose, $55.6 \mathrm{~g}$ fructose) with caffeine-free cola between meals. Body weight and serum uric acid level were measured at the beginning and end of each 2-wk intervention.

Results: Body weight remained unchanged within both intervention periods. Levels of uric acid were normal at baseline $(4.5 \pm 1.2 \mathrm{mg} / \mathrm{dL}$ before orange juice and $4.6 \pm 1.1 \mathrm{mg} / \mathrm{dL}$ before cola consumption, $\mathrm{p}=0.573)$ and did not change with 2 -wk cola consumption $(+0.1 \pm 0.7 \mathrm{mg} / \mathrm{dL}, \mathrm{p}>0.05)$ whereas they decreased with the orange juice intervention $(-0.4 \pm 0.5 \mathrm{mg} /$ $\mathrm{dL}, \mathrm{p}<0.001)$. The decrease in uric acid levels was more pronounced at higher baseline levels $(\mathrm{r}=-0.62, \mathrm{p}<0.01)$.

Conclusion: These results do not support an increased risk of gout with orange juice consumption. Quite the contrary, regular consumption of larger amounts of orange juice lowered serum uric acid levels. This may either be due to a flavonoid-dependent inhibition of xanthine oxidase or because of a uric acid lowering effect of vitamin $\mathrm{C}$.

Conflict of Interest: The authors declare no conflict of interest

Funding: Research relating to this abstract was funded by Verband der deutschen Fruchtsaft-Industrie e.V.

\section{T3Р109 \\ Impact of weight loss and ketosis on subjective and objective appetite markers}

Lyngstad, A. ' 'Nymo, S. '; Coutinho, S. '; Kulseng, B. ${ }^{2}$;ruby, H. ${ }^{3}$; Martins, C. ${ }^{1}$ ${ }^{1}$ Department of Cancer Research and Molecular Medicine, Faculty of Medicine, Norwegian University of Science and Technology (NTNU), Trondheim, Norway, ${ }^{2}$ Department Of Cancer Research And Molecular Medicine, Faculty Of Medicine, Norwegian University Of Science And Technology (Ntnu), Trondheim, Norway, Centre For Obesity And Innovation (Obece), Clinic Of Surgery, St. Olav University Hospital, Trondheim, No, ${ }^{3}$ Department Of Nutrition, Dietetics \& Food, Monash University, Melbourne, Australia.

Introduction: Diet-induced weight loss (WL) is usually followed by increased appetite, which may lead to over eating and potentially weight regain. To date, the impact of ketogenic diets on appetite responses is inconsistent. The objective of this study was to assess the effect of WL and ketosis on subjective and objective appetite markers.

Methods: Forty-nine adults (34 males) with obesity (BMI: $36.5 \pm 0.6 \mathrm{~kg}$ / $\mathrm{m}^{2}$, age: $40.3 \pm 1.5$ years) completed 8 weeks of a ketogenic very-low energy diet (VLED), followed by 4 weeks of refeeding and weight stabilization. Fasting/postprandial subjective feelings of hunger, fullness, desire to eat (DTE) and prospective food consumption (PFC) were assessed and plasma concentrations of active ghrelin (AG), total peptide YY (PYY), active glucagon-like peptide 1 (GLP-1) and insulin were measured every 30 minutes for 2.5 hours. Measurements were taken at baseline, week 9 (W9), in ketosis, and week 13 (W13), no ketosis. Statistical analysis was performed using linear mixed model, with Bonferroni adjustments. Data are presented as mean \pm SEM.

Results: A $16 \pm 0.34 \%$ WL was seen at W9 and maintained at W13. Feelings of hunger and DTE in fasting did not change at W9, but were significantly higher at W13 compared with both W9 and baseline $(\mathrm{p}<0.01$ for hunger and $\mathrm{p}<0.01$ and $\mathrm{p}<0.05$ for DTE, respectively). PFC feelings in fasting did not change at W9 or W13 compared to baseline, but increased from W9 to W13 ( $\mathrm{p}<0.05)$.A significant increase in postprandial fullness $(\mathrm{p}<0.05)$ and a decrease in PFC $(\mathrm{p}<0.01)$ were observed at W9, but no change at W13 compared with baseline or W9. Fasting/postprandial AG plasma concentrations did not change at W9, but increased significantly from W9 to W13 ( $\mathrm{p}<0.01$ for both) and were higher at W13 compared with baseline ( $\mathrm{p}<0.001$ for both). A significant decrease in fasting PYY and GLP-1 were seen at W9 ( $\mathrm{p}<0.05$ for both), but no difference between W9 and W13 or baseline and W13. Fasting/postprandial insulin plasma concentrations were significantly reduced at W9 and W13 compared to baseline ( $\mathrm{p}<0.001$ for all).

Conclusion: A ketogenic VLED induces WL effectively in adults without increasing subjective or objective measures of appetite. However, once participants are out of ketosis, an increase in appetite should be expected, which may drive up food intake and may eventually lead to weight regain.

Acknowledgments: Thanks to all participants for their time and commitment, study nurses Sissel Salater and Hege Bjøru at the ObeCe, St. Olav University Hospital, Ingrid Hals for support with lab work (NTNU) andAllevo (Karo Pharma AS. Sweden) for providing the VLED products.

\section{T3P110 \\ Implementation and evaluation of the'5As of obesity management' in primary care: Results from the 5 As team (5AsT) study}

\section{Campbell-Scherer, D. '; Asselin, J. ${ }^{2}$; Osunlana, A. ${ }^{3}$; Ogunleye, A. ${ }^{3}$;} Fielding, S. ${ }^{4}$; Anderson, R. ${ }^{4}$; Cave, A. ${ }^{3}$; Johnson, J. ${ }^{3}$; Sharma, A. ${ }^{3}$

${ }^{1}$ Department of Family Medicine, University of Alberta, Edmonton, AB, Canada, ${ }^{2}$ University Of Lethbridge, ${ }^{3}$ University Of Alberta, ${ }^{4}$ Edmonton Southside Primary Care Network

Introduction: Despite advances in obesity management, primary care providers are poorly prepared to address obesity. Evidence is lacking on effective strategies to change behavior of providers, and on organization of care for persons with obesity. The objective of this study was to evaluate a theoretically informed intervention designed to improve obesity management in primary care.

Methods: Allocation concealed, blinded (outcome measure, analysts), randomised controlled trial, with convergent mixed-methods evaluation. Participants included registered nurses/nurse practitioners, mental health workers, and dieticians from a Primary Care Network in Alberta, Canada. The intervention was based upon self-assessed needs and involved structured material and team-based, facilitated learning collaboratives supported by practice facilitation. The main outcome measure was the number of visits for obesity management by RN/NPs. Qualitativedata from semi-structured interviews, field notes, and logbooks were used to identify contextual factors affecting provider ability to improve management. Results: The intervention group did not show a significant increase in weight management visits at baseline (rate ratio $1.30,95 \%$ confidence interval 0.83 to $2.03, \mathrm{p}=0.248$ ) or nine-month post-intervention (rate ratio $1.38,95 \%$ confidence interval 0.87 to $2.19, \mathrm{p}=0.166$ ). Mixed-methods analysis revealed important factors such as increased confidence and high functioning teams which influenced uptake of the approach and increased weight management encounters.

Conclusion: Multi-level changes in practice were observed, with strong innovations-values fit of the '5As of obesity management' and impacts on team function and shifts in provider behavior. Although not statistically significant, weight management encounters increased in the intervention group, and were maintained 9 months post intervention. Qualitative findings have implications for the effective implementation of obesity management strategies in primary care. 
Conflict of Interest: DL Campbell-Scherer, J Asselin, AM Osunlana, AA Ogunleye, S. Fielding, R Anderson, J Johnson have nothing to disclose. AM Sharma is member of an Advisory Board with a commercial organization (Novo Nordisk: Ad visory Board for anti-obesity drug); AM Sharma was a member of the Data Safety Monitoring Board for an anti-obesity trial (Takeda). A Cave has nothing to disclose.

Funding: The 5AsT study is funded by Alberta Innovates-Health Solutions, with significant in kind support from the Edmonton Southside Primary Care Network The researchers are independent of the study funder. The funder had no access to study data, no involvement in the design or execution of the study and no involvement in analysis.

\section{T3P111}

Importance of oral health awareness in patients with diabetes mellitus type 2, a systematic literature review

\section{Jankovic-Pejicic, A. ${ }^{1}$; Popovic-Pejicic, P. ${ }^{2}$}

${ }^{1}$ School of dentistry, Medical University Vienna, ${ }^{2}$ Medical Faculty University Of Banjaluka Bosnia And Herzegowina

Diabetes and periodontitis are chronic diseases widely spread with increasing prevalence in each population. Periondontitis is the major cause of tooth loss in adults and has been considered as serious complication of diabetes. Studies have suggested a bidirectional relationship between periodontitis and diabetes. Diabetes promote the occurence, the progression and the severity of periodontitis. Conversely, periodontitis was shown to be a risk factor for poor glycemic control in patients with diabetes due to bacteria and their byproducts in the inflammed periodontal tissue constituting a chronic source of systemic chalenge to the host, increases the risk of diabetes-associated complications and possibly even of ist onset.

Method: In the current literature a large variation in the methodologic approaches and reporting results of data denied a meta-analysis, whereupon a systematical review was performed.

Results: It is still not sufficienty communicated within the medical com munity, because the periodontal disease is stil considered as the concern of only dentists. Physicians and dentists need to be aware of the relationship between periodontitis and diabetes and take adaquate steps to minimaze negative outcomes in patient with diabetes mellitus Tooth loss due to periodontitis seriously decreases chewing abillity which is related to quality of life and general health, possibly reflecting the impact on chewing food choice and enjoyment of meals and diet and also indicated the importance of oral health to general well-beeing.

Conclusion: Periodontal therapy which decreases the intraoral bacterial bioburden and reduces periodontal inflammation can have a significant impact on systemic inflammatory status and improves glycemic control in many patients with diabetes and periodontitis.Recognition of the bilateral relationship between oral and systemic helath will chalenge physicians and dentists to work together closely in the future when managing patients with diabetes and periodontal disease

\section{T3P112}

Improved and more effective algorithms to screen for nutrient deficiencies after bariatric surgery

Pouwels, S. '; Bazuin, I. ${ }^{2}$; Houterman, S. ${ }^{3}$; Nienhuijs, S. ; Smulders, F. ${ }^{4}$; Boer, A. ${ }^{2}$

${ }^{1}$ Franciscus Vlietland, Schiedam, The Netherlands, ${ }^{2}$ Department Of Clinical Chemistry, Catharina Hospital, Eindhoven, The Netherlands, ${ }^{3}$ Department Of Education And Research, Catharina Hospital, Eindhoven, The Netherlands, ${ }^{4}$ Department Of Surgery, Catharina Hospital, Eindhoven, The Netherlands

Objective: Most bariatric guidelines recommend frequent lab monitoring of patients to detect nutrient and vitamin deficiencies as early as possible. The aim of this study was to optimize the cost effectiveness of the nutrient panel, by developing an algorithm, which detects nutrient deficiencies at lower costs.

Methods: In this retrospective study, 2055 patients who had undergone Laparoscopic Roux-Y Gastric Bypass (LRYGB) and Laparoscopic Sleeve
Gastrectomy (LSG) surgery at Catharina Hospital Eindhoven between January 2009 and December 2013. Perioperative biochemical measurements ( 7 days before and 127 days after surgery) and measurements $>549$ days before surgery were excluded. For analysis, the most recent pre- and postoperative measurements were selected for each biochemical parameter separately. Firstly step the amount of moderate and severe deficiencies were calculated. Secondly, we investigated whether each variable (vitamins A, B1, B6, B12, D, folate, ferritin, zinc and magnesium) could predict the presence of deficiency.

Results: In total, 561 (LRYGB) and 831 (LSG) patients had at least preand postoperative values of vitamin $A, B 1, B 6, B 12, D$, folate, ferritin, zinc or magnesium. The algorithm reduces vitamin $\mathrm{D}, \mathrm{B} 12, \mathrm{~B} 6, \mathrm{~B} 1$ and ferritin examinations by $15 \%, 11 \%, 28 \%, 28 \%$ and $38 \%$, respectively, without missing clinically relevant deficiencies. The corresponding potential cost savings was $14 \%$.

Conclusion: This study identified substantial cost savings in laboratory test for both LRYGB and LSG procedures. The potential cost reduction of $14 \%$ might even be increased to $42 \%$ when less frequent moderate deficiencies are not screened anymore, while $>99.0$ of moderate deficiencies will be detected.

\section{T3P114 \\ Indice of visceral adiposity but not BMI is associated to type 2 diabetes in obese patients}

Fonseca, R. ${ }^{1}$; Duarte, J. ${ }^{1}$; Roque, C. ${ }^{1}$; Ferrinhos, C. ${ }^{1}$; Ferreira, R. ${ }^{\prime}$; Saraiva, C. ${ }^{1}$; Gouveia, C. '; Santos, Z.' ; Ribeiro, O. '; Nascimento, C. '; Azinheira, J. '; Vasconcelos, C. ${ }^{1}$

${ }^{1}$ Hospital Egas Moniz (CHLO)

Background and aims: Bariatric surgery (BS) significantly reduces body weight and may have an impact on cardiovascular (CV) events including $\mathrm{CV}$ death, hospitalization due to heart failure and all-cause mortality. Whether improvement in $\mathrm{CV}$ risk is related to treatment effects on total body and visceral adiposity (VA) is unknown. Therefore, we explored the effect of 3 types of BS on anthropometry and validated surrogate indices of VA and total fat mass in 806 patients from our obesity clinic

Materials and Methods: Retrospective single center study of all the patient's with waist circumference measured in at least two observations from 2006-2015. We calculated the annual mean of the indexes of VA (waist circumference (WC), index of central obesity (ICO; ratio WC/ height) and total adiposity (BMI)) and sitting blood pressure measured. Biochemistry in fasting state was recovered from hospital laboratory and the annual mean of each parameter was calculated. Ongoing therapies and eventual bariatric surgery were collected. Data was analyzed in SPSS software using descriptive methods, $\mathrm{T}$ test for continuous variables, chisquare for categorical. Results are presented by mean $+/$ - standard deviation.

Results: A total of 806 patients were found with a mean age of $47.8+/-11.3$ years at first visit, with a predominance of female gender (80.5\%). Many of these patients had received some bariatric surgery including sleeve gastrectomy $(\mathrm{n}=136)$, gastric bypass $(\mathrm{n}=178)$, gastric banding $(\mathrm{n}=292)$ with 38 reoperations in the last group. Comorbidities were common and $74.8 \%$ had some form of dyslipidemia, $75.6 \%$ hypertension, $42.6 \%$ type 2 diabetes, $17.6 \%$ sleep apnea, $6.1 \%$ previous cardiovascular events. The $\mathrm{WC} /$ height ratio was associated with the presence of type 2 . No association was found to hypertension, dyslipidemia or the presence of previous cardiovascular events. We found no differences in statin use, antidiabetic, anti-hypertensive or anti-platelet therapies between the two groups.

Conclusion: These findings suggest that index of central obesity is associated with type 2 diabetes and high CV risk. Further analyses are needed to determine the potential contribution of these indexes to select the patients for more intensive programs of weight reduction and diabetes prevention. 
Table 1. to T3P114

\begin{tabular}{|l|l|l|l|l|}
\hline & T2D & N & Mean & SD \\
\hline \multirow{2}{*}{ Waist circumference (WC) } & Y & 489 & 113,0 & 15,5 \\
\cline { 2 - 5 } & $\mathrm{N}$ & 450 & 113,4 & 16,0 \\
\hline \multirow{2}{*}{ WC / Height ratio } & $\mathrm{Y}$ & 489 & 0,7 & 0,1 \\
\cline { 2 - 5 } & $\mathrm{N}$ & 450 & 0,7 & 0,1 \\
\hline \multirow{2}{*}{ Weight $(\mathrm{kg})$} & $\mathrm{Y}$ & 485 & 100,8 & 18,2 \\
\cline { 2 - 5 } & $\mathrm{N}$ & 445 & 103,0 & 21,6 \\
\hline \multirow{3}{*}{ BMI } & $\mathrm{Y}$ & 485 & 38,8 & 7,0 \\
\cline { 2 - 5 } & $\mathrm{N}$ & 445 & 38,5 & 7,0 \\
\hline \multirow{2}{*}{ Fat Mass \% } & $\mathrm{Y}$ & 213 & 32,4 & 12,2 \\
\cline { 2 - 5 } & $\mathrm{N}$ & 219 & 33,8 & 9,9 \\
\hline
\end{tabular}

T3P115

Influence of Bifidobacterium lactis isolated or combined with oligofructose on anthropometric and laboratory variables of obese women

Crovesy, L. '; Ostrowski, M. '; Mota-Soares, M. '; Rosado, E.

${ }^{1}$ Federal University of Rio de Janeiro

Introduction: Obesity is an important public health problem which is increasing in the world. Currently, the gut microbiota composition has been appointed as one of causal factors of the disease. For this reason, gut microbiota modulation of obese subjects seem to be an alternative therapeutic adjuvant of obesity. Probiotics and symbiotics may be cited as modeling agents of gut microbiota. The aim was evaluate if Bifidobacterium lactis (B. lactis) isolated or combined with oligofructose can to promote weight loss and to improve glycaemia and lipid profile of obese women.

Methods: Clinical trial, double-blind, randomized, parallel and controlled was conducted with 32 obese class I and II women (Body mass index (BMI) between $30-39.99 \mathrm{~kg} / \mathrm{m}^{2}$ ). The women received a hypocaloric dietetic plan and capsules containing $10^{\circ} \mathrm{CFU}$ of $B$. lactis and sachet containing $5 \mathrm{~g}$ of maltodextrin (probiotic group - G1), or capsule with $10^{\circ} \mathrm{CFU}$ of $B$. lactis and sachet containing $5 \mathrm{~g}$ of oligofructose (symbiotic group - G2), or capsule and sachet placebo (control group - G3) for 60 days. Body weight, waist circumference, dietary and laboratory (blood glucose and lipid profile) variables were evaluated in baseline and after intervention.

Results: Women presented BMI of $33.51 \pm 2.44$ (G1), $36.34 \pm 10.08$ (G3), and $33.78 \pm 3.07$ (G2), high waist circumference and LDL-cholesterol, low HDL-cholesterol and insulin resistance. In baseline, BMI was lower and triglycerides were higher in G1, compared G2, and fiber intake was lower in G2. After intervention, triglycerides and protein intake were lower in differ between G1 compared with G3. All groups decreased energy intake, but only G2 and G3 showed reduction in BMI. In comparison of baseline and after intervention by group, G2 decreased body weight and body fat mass.

Conclusion: It seems that $B$. lactis and oligofructose for 60 days had better effects on weight loss and body fat mass than probiotic, however $B$. lactis isolated was able to reduce triglyceride.

\section{T3P116 \\ Influence of body mass index on blood pressure in hypertensive patients}

Kyriazis, I. '; Sgouros, K. '; Stefani, D. '; Pouliou, E. '; Georgakopoulos, P.'; Tsamis, I. '; Petropoulou, S. '; Lelekis, M. ${ }^{1}$ ${ }^{1}$ INTERNAL MEDICINE Dpt. ,KAT GENERAL HOSPITAL , ATHENS, GREECE

Introduction: Although the increased body mass is an established risk factor for developing a cardiovascular disease, there is an ongoing investigation into whether or not there is a threshold above which the correlation between obesity and cardiovascular risk is less evident. This correlation is described by J-Curve graph and also extends at lower rates of somatometric measurements.

Objective: Evaluation of sub-clinical effect of fat deposits on risk factors, target-organ damage, including cardiac parameters, and hypertension.

Methods: 187 patients from hypertension outpatients clinic were enrolled in this observational study. Medical history was taken, complete physical examination and routine biochemical tests were performed for all patients. Clinical and 24-hour blood pressure (BP) measurement as well as echocardiography was performed.

Results: Patients enrolled were mainly women (55\%) aged $51.21 \pm 11$ years. $38 \%$ of patients were obese and $31 \%$ overweight. The body mass index (BMI) was found to be profoundly associated with the office blood pressure levels of 24-hour measurement, lipid profile, and glucose level, but not with the central blood pressure levels, the pulse wave velocity (PWV) and renal function. More precisely, overweight, obese and underweight patients had increased mean systolic and diastolic blood pressure values of 24-h measurements compared with normal -weight patients $(123 / 75 \mathrm{mmHg}, 125 / 76 \mathrm{mmHg}, 128 / 72 \mathrm{mmHg}$ vs $117 / 70 \mathrm{mmHg}$ ( $\rho$ $<0.001$ ), higher average blood pressure values in the morning and evening $(\mathrm{p}<0.001)$, higher levels of diastolic blood pressure measured in the office $(p<0,001)$, with no significant differences in systolic blood pressure levels measured in the office, central blood pressure levels and PWV ( $p=$ NS). As expected, the glucose values and the lipid profile were found to be highly associated with BMI, since the higher the BMI, the higher glucose, LDL and triglycerides levels were $(\mathrm{p}<0.001)$.

Conclusions: The overweight, obese and underweight individuals have increased cardiovascular risk factors, and it seems that the J-curve is present.

\section{T3P117 \\ Influence of obesity in the diagnosis and treatment of gestational diabetes}

Tavares, P.' ; Machado, C. '; Ferreira, E. '; Monteiro, S. '; Rocha, G. '; Oliveira, $M$.

${ }^{1}$ Centro Hospitalar Vila Nova de Gaia/Espinho, Endocrinology department, ${ }^{2}$ Centro Hospitalar Vila Nova De Gaia/Espinho

Introduction: Obesity is a modifiable risk factor for the development of gestational diabetes (GD). Exposure to altered glycemia in utero induces changes in fetal metabolism with increased risk of diabetes and obesity for that individual and the pregnant woman.

This study sought to evaluate the prevalence of obesity in women with GD and differences in diagnosis and treatment of GD according to Body Mass Index (BMI).

Methods: Retrospective observational study that included pregnant women with GD followed in our hospital between 2011 and 2015.

Results: We included 462 women with a mean age of 33 years $( \pm 5.4)$. In preconception $179(38.7 \%)$ were in normal range, 147 (31.8\%) were overweight and $136(29.4 \%)$ were obese. 56 had a history of GD and most of them (71.8\%) were overweight/obese. In 350 women the diagnosis of GD was made in the $1^{\text {st }}$ or $2^{\text {nd }}$ trimester. The majority $(52,2 \%)$ of obese women were diagnosed in the $1^{\text {st }}$ trimester while most $(63,9 \%)$ of the normal weight women were diagnosed in the $2^{\text {nd }}$ trimester $(p<0,05)$. Obese women were diagnosed mainly due to high fasting plasma glucose while normal weight women were mainly diagnosed by oral glucose tolerance test $(\mathrm{p}<0,05)$.

With increasing BMI, insulin was required in higher proportion of patients $(38.8 \%$ of normal weight, $56.2 \%$ of overweight and $62.2 \%$ of obese patients) $(\mathrm{p}<0.001)$. Also, obese patients required higher insulin dose $(\mathrm{p}<0.05)$. During pregnancy mean weight gain was $10.2 \mathrm{~kg}( \pm 5.9)$. The average increase was $12 \mathrm{~kg}( \pm 5.3)$ for normal weight, $10.2 \mathrm{~kg}( \pm 5.8)$ for overweight and $7.7 \mathrm{~kg}( \pm 5.8)$ for obese women $(\mathrm{p}>0.05)$. There was no correlation between weight gain and insulin dose.

There were no differences in the three groups of BMI in relation to the birth weight, maternal-fetal complications and the reclassification test. 
Conclusion: The majority of pregnant women with GD have a preconception $\mathrm{BMI}>25 \mathrm{~kg} / \mathrm{m} 2$. There were differences in the diagnosis and treatment of GD according to the BMI. There were no differences in the three groups of BMI in relation to the weight of the newborn or maternal-fetal complications.

During pregnancy, the mean weight increase was in accordance with the recommendations.

Conflict of Interest: None Disclosed

Funding: No Funding

\section{T3P118}

\section{Insights and perceptions of obesity management in people} with obesity: Results of the national ACTION study

Look, M. ${ }^{1}$; Golden, A. ${ }^{2}$; Dhurandhar, N. ${ }^{3}$; Jinnett, K. ${ }^{4}$; Kaplan, L. ${ }^{5}$; Kolotkin, R. ${ }^{6}$; Kyle, T. ${ }^{7}$; Nadglowski, J. ${ }^{8}$; O 'Neil, P. ${ }^{9}$; Parry, T. ${ }^{4}$; Guerrero, G. ${ }^{10}$; LilleøRe, S. ${ }^{11}$; Tomaszewski, K. ${ }^{12}$

${ }^{1}$ San Diego Sports Medicine and Family Health, San Diego, CA, USA, ${ }^{2} \mathrm{~Np}$ From Home Llc, Munds Park, Az, Usa, ${ }^{3}$ Texas Tech University, Lubbock, Tx, Usa, ${ }^{4}$ Integrated Benefits Institute, San Francisco, Ca, Usa, ${ }^{5}$ Massachusetts General Hospital, Boston, Ma, Usa, ${ }^{6}$ Quality Of Life Consulting Pllc, Durham, Nc, Usa, ${ }^{7}$ Conscienhealth, Pittsburgh, Pa, Usa, ${ }^{8}$ Obesity Action Coalition, Tampa, Fl, Usa, ${ }^{9}$ Medical University Of South Carolina, Charleston, Sc, Usa, ${ }^{10}$ Novo Nordisk Inc, Plainsboro, Nj, Usa, ${ }^{11}$ Novo Nordisk A/S, SøBorg, Denmark, ${ }^{12}$ Kjt Group Inc, Honeoye Falls, Ny, Usa

Background: The ACTION (Awareness, Care, and Treatment In Obesity maNagement) study examined Wagner's Chronic Care Model's Self-Management Support element by uncovering and identifying attitudes and behaviours related to obesity management among people with obesity (PwO).

Methods: A cross-sectional, US-based, stratified sampling of people with obesity ( $\mathrm{PwO}$ ). Inclusion criterion: $\mathrm{BMI} \geq 30 \mathrm{~kg} / \mathrm{m}^{2}$ based on self-reported height and weight. Adult $\mathrm{PwO}(\mathrm{N}=3,008 ; 51 \%$ female $)$ completed online surveys. The instrument assessed attitudes, experiences and behaviours associated with medical and employer-based obesity management.

Results: $65 \%$ of PwO reported feeling that obesity is a "disease". Despite disease recognition, $82 \%$ perceive that they have "complete responsibility" for their weight loss (WL). Of the 73\% that have discussed "losing weight" or "being overweight" with an HCP, 36\% report that they do not "seek support" from their HCP for WL. PwO believe that $10 \% \mathrm{WL}$ is a valuable goal. Nonetheless, the average WL goal set between themselves and their HCP is reported as $20 \%$. Among $\mathrm{PwO}, 49 \%$ say that they know what to do but cannot "make themselves" do so; $38 \%$ are not sure that they could achieve WL even "if they set their mind to it".

Conclusions: Obesity treatment outcomes may be hindered by suboptimal self-management strategies among $\mathrm{PwO}$ or their perceived inability to manage obesity. This study highlights the need for $\mathrm{PwO}$ to gain increased understanding of obesity as a chronic, biological, and often progressive disease that requires a combination of self-management and multidisciplinary support. Initiatives to give HCPs a better understanding of the perceptions and beliefs of PwOs may foster more productive interactions and improve treatment outcomes.

\section{T3P119 \\ Intragastric balloon placement for the management of obesity: Improving patient selection}

Adame, R. ${ }^{1}$; Alvizo, A. ${ }^{2}$; Gomez, E. ${ }^{2}$; Garza, F. ${ }^{2}$; Serna, U. ${ }^{2}$; Martinez, M. ${ }^{2}$

${ }^{1}$ Hospital San José Tec de Monterrey / SSNL, ${ }^{2}$ Hospital San José Tec De Monterrey

Introduction: Intragastric balloon placement has been an available procedure in Mexico during the past decade or more. As a BariatricClinic, we encourage patients with lower BMIs to apply to Nutrition and Patient Support Groups before considering a procedure in them. The intragastric balloon has been an attractive procedure to this population because its reversibility, itsambulatory basis, its feasibility in lower BMI patients and unfortunately in our country, because of the lower price. Its final price as a package of procedure plus the visits during 6 months equals the price of a gastric plication or non-adjustable banding, unfortunately, this procedures are still very common in our area.

Objective: Determine the best group of patients suitable for endoscopic balloon placement according to their BMI based in a series of 152 cases.

Materials and methods: A total of 152 patients with BMIs from 27 to71 were included in this study. OrberaTM Intragastric balloon was filled with 550 to $700 \mathrm{cc}$ plus $3 \mathrm{cc}$ of Methylene Blue and placed endoscopically with sedation on an ambulatorysetting; in all except 1 patient (BMI 71-no sedation, just oral local anesthetic). All patients were treated 12 hours before with Omeprazole, Buthylhyoscine and Meclizine, and for 2 to 5 days after as well dependingon the symptoms. All of them were removed endoscopically 6-12 monthsafter, this range varied because some patients refused to have the ball removed. All of them were followed at least monthly by Registered Dietitian and monitored twice during this period by a surgeon in our team to detect abnormal symptoms.

Results: BMI below 30 (n18-11.84\%), were the group with worst tolerance and lower weight loss (9.78 kg avg), 2 premature extractions in this group, 5 readmissions to the clinic to treat symptoms via IV fluids \& meds. Between BMI of 30-35 (n83-54.60\%) the avg weight loss was $14.7 \mathrm{~kg}$. No premature extractions, 1 readmission.

Between BMI of 35-50 (n 42-27.63\%), theavg weight loss was of $17.3 \mathrm{~kg}$. No premature extractions, 2 readmissions.

BMI of 50 and more had avg weight loss of $27.1 \mathrm{~kg}$. 1 rupture at 4 months in patient with severe gastritis, blueurine.

No other complications other than the previously discussed, no mortality. Conclusion: Patients with BMI between 30-35 are the group with best response, we dont reccomend it below BMI 30. Above $35 \mathrm{BMI}$, weight loss is not enough but helps in future bariatric procedures by reducing the liver size and making inoperable patients for other reasons. The intragastric ballon is usefull as a bridge for further restrictive surgery.

\section{T3P122}

\section{Is there a role for glucagon in weight loss mediated metabolic improvements?}

Silvestre, M. ${ }^{1}$; Goode, J. ${ }^{2}$; Vlaskovsky, P. ${ }^{1}$; Mcmahon, C. ${ }^{\text {; }}$ Tay, A. ${ }^{\text {; }}$ Poppitt, S. ${ }^{1}$

'University of Auckland, 2University Of Tasmania

Introduction: It has become clear that an "insulinocentric" view of glucose homeostasis is incomplete and that glucagon may also have an important role in the maintenance of normal plasma glucose levels. However, weight loss studies have not yet specifically addressed the role of glucagon as a mediator of improved glucose regulation and decreased risk of T2D in response to weight change. The aim of our study was to perform a meta-analysis of the literature on the role of glucagon in improving glucose homeostasis following weight loss in individuals with, or at high risk of developing T2D.

Methods: A comprehensive electronic search of weight loss intervention and observational studies was conducted in Medline, Scopus, Web of Science, PubMed and Embase, published up to the 31st of July 2015. Data collection and validity assessments were carried out by 4 investigators, independently. A meta-analysis using random effect model was performed. Meta-regression models were used to address heterogeneity between studies. Primary outcomes included: a) BMI/body weight change, b) fasting glucagon concentration. Secondary outcomes included: a) fasting glucose, b) fasting insulin concentration and c) methods of weight reduction, including traditional weight loss and bariatric surgery.

Results: Fasting glucagon concentration decreases in parallel with weight loss and glucose improvements and these changes are independent of the weight loss method - bariatric surgery or traditional diet - but dependent on the duration of the calorie restriction. 
Conclusion: Glucagon may be involved in weight-loss related improvements but a causal effect is yet to be established. Bariatric surgery does not cause weight-independent improvements in metabolism.

Conflict of Interest: None Disclosed

Funding: MPS and SDP were funded by the Health and Research Council of New Zealand; grant number 14/191

\section{T3P123}

Juçara pulp (Euterpe edulis Mart.) Supplementation improves $\omega 3$ / $\omega 6$ ratio in serum and increases fat-free mass in obese subjects without dietary intervention

Santamarina, A. '; Jamar, G. '; Mennitti, L. ${ }^{1}$; Argentato, P. ${ }^{2}$; Rosso, V. ${ }^{3}$; Dourado, V. ${ }^{4}$; Moreira, R. ${ }^{5}$; Oyama, L. ${ }^{6}$; Pisani, L. ${ }^{3}$

${ }^{1}$ Post Graduate Program of Interdisciplinary Health Science of Universidade Federal de São Paulo (UNIFESP), ${ }^{2}$ Post Graduate Program In Food, Nutrition And Health Of Universidade Federal De São Paulo (Unifesp), ${ }^{3}$ Department Of Bioscience Of Universidade Federal De São Paulo (Unifesp), ${ }^{4}$ Department Of Human Moviment Science Of Universidade Federal De São Paulo (Unifesp), ${ }^{5}$ Department Of General Physiology - Institute Of Biosciences In Universidade De São Paulo, ${ }^{6}$ Department Of Nutrition Of Universidade Federal De São Paulo (Unifesp)

Introduction: The control and treatment of obesity using bioactive food compounds has gained scientific and clinical attention. Brazil has pronounced biodiversity of native species with potential health benefits. An example of this is the fruit of juçara palm tree (Euterpe edulis Mart.) which has nutritional composition rich in dietary fiber $(28.3 \mathrm{~g} / 100 \mathrm{~g})$, monounsaturated fatty acids (MUFA) (38.57\%) and polyunsaturated fatty acids (PUFA) (20.09\%). It is also remarked for having high levels of flavonoids, such as anthocyanins $(239.16 \mathrm{mg} / 100 \mathrm{~g})$. Therefore, it could contribute to prevention and treatment of obesity and comorbidities as metabolic syndrome.

Methods: Were included in the study 27 subjects of both genders with obesity (BMI between 30.0 and $39.9 \mathrm{~kg} / \mathrm{m}^{2}$ ) and insufficiently active lifestyle aged 31 to 59 years, randomly divided into two supplementation groups of $05 \mathrm{~g}$ juçara freeze-dried pulp (approximately 170mg of anthocyanin) or $05 \mathrm{~g}$ of placebo (maltodextrin) during 6 weeks. Before and after supplementation the volunteers were interviewed for characterization of the sample group, and submitted to anthropometric and body composition (BIA) evaluation. Blood was also collected to obtain serum for analysis of the fatty acid profile by gas chromatography.

Results: Juçara supplementation was able to increase fat-free body mass and reduced body fat percentage after 6 weeks. The freeze-dried juçara pulp was also effective in increasing the omega- 3 / omega- 6 fatty acids ratio in the serum of the volunteers. Regarding the placebo group, juçara pulp also elevated the levels of omega-3 polyunsaturated fatty acids, especially long-chain fatty acids that include eicosapentaenoic (EPA) and docosahexoic (DHA) fatty acids.

Conclusion: Our results suggest that juçara pulp is a potential tool in the fight against obesity and its related diseases.

None Disclosed

Supported by Fundação de Amparo à Pesquisa do Estado de São Paulo (FAPESP 2015/13875-0).

\section{T3P124}

Leptin/adiponectin ratios using either total or high-molecular weight adiponectin as biomarkers of systemic insulin sensitivity in normoglycaemic women

Bravo, C. '; Cataldo, R. '; Galgani, J.' ; Santos, J. ${ }^{1}$

${ }^{1}$ Department of Nutrition, Diabetes and Metabolism. School of Medicine. Pontificia Universidad Católica de Chile, ${ }^{2}$ Department Of Nutrition. Diabetes And Metabolism, School Of Medicine. Pontificia Universidad Católica De Chile
Introduction: Leptin and adiponectin are adipokines closely related to variation of Body Mass Index (BMI).The Leptin/Adiponectin Ratio (LAR) has shown negative associations with insulin sensitivity indexes. On the other hand, High-Molecular Weight Adiponectin (HMWA) was proposed as the most biologically active form of this insulin-sensitizing adipokine. There are no studies assessing the relative merits of Leptin/HMWA ratio over LAR as a biomarker of systemic insulin sensitivity. In this study, we assess the association between leptin, total adiponectin, HMWA, LAR and Leptin/HMWA ratio with indexes of insulin sensitivity in normoglycaemic women.

Methods: A standard 2-hour Oral Glucose Tolerance Test (OGTT; 75 g of glucose) and a short Minimal-Model Intravenous Glucose Tolerance Test (IVGTT; $0.3 \mathrm{~g} / \mathrm{kg}$ body weight; 13 blood samples during 50 minutes) were performed in 58 Chilean normoglycaemic women (age: $27 \pm 6.3$ years, BMI $23.6 \pm 3.2 \mathrm{~kg} / \mathrm{m}^{2}$ ). The following indexes were calculated: HOMA-S (from fasting glucose and insulin), Matsuda-ISICOMP (from OGTT) and the Calculated-Sensitivity Index CSi (from IVGTT). Plasma leptin, total adiponectin and HMWA were measured by immunoassays. Correlation and regression analyses were used to assess associations between pairs of study variables. Receiver Operating Characteristic (ROC) curve analysis was used to compare the discriminatory capacity of LAR and Leptin/ HMWA ratio to distinguish insulin-resistant versus insulin-sensitive subjects.

Results: Plasma leptin levels were positively associated with BMI and negatively with insulin sensitivity indexes, whereas the opposite was found both for adiponectin and HMWA. LAR was negatively associated with HOMA-S $(r=-0.49 ; \mathrm{p}<0.0001)$, Matsuda-ISICOMP $(r=-0.54$; $\mathrm{p}<0.0001)$ and CSi $(\mathrm{r}=-0.38 ; \mathrm{p}=0.007)$. Multiple linear regression and residual analysis showed that both associations of LAR and Leptin/ HMWA ratio with insulin sensitivity indexes remained significant after adjustment by BMI. In comparison to LAR, Leptin/HMWA ratio did not increase neither linear fit ( $\mathrm{r} 2$ ) nor the magnitude of association with insulin sensitivity indexes (slope of multiple linear regression). The discriminatory capacity of both ratios to classify insulin-resistant versus insulin-sensitive subjects was similar in the ROC curve analysis for HOMA-S $(\mathrm{p}=0.84)$, Matsuda-ISICOMP $(\mathrm{p}=0.43)$ or CSi $(\mathrm{p}=0.50)$.

Conclusion: LAR and Leptin/HMWA ratio showed consistent negative associations with different systemic insulin sensitivity indexes derived from basal as well as from oral or intravenous glucose loads. The use of HMWA to generate Leptin/HMWA ratio did not show any advantage over LAR as a biomarker of systemic insulin sensitivity in normoglycaemic women.

Conflict of Interest: None

Funding: FONDECYT 1150416

\section{T3P125 \\ Liraglutide $3.0 \mathrm{mg}$ treatment for obese patients, a real life experience}

Shehadeh, N. ${ }^{1}$; Zaid, W. ${ }^{2}$; Zuckerman, N. ${ }^{1}$; Marji, M. ${ }^{2}$; Sagit, Z. ${ }^{1}$

${ }^{1}$ Institute of Diabetes, Endocrinology and Metabolism, Rambam Health Care Campus, Haifa, Israel; Bruce Rappaport Faculty of Medicine- Technion, Haifa, Israel, ${ }^{2}$ Rambam Health Care Campus, Haifa, Israel

Obesity, the $21^{\text {st }}$ century's epidemic, is a chronic condition associated with various medical complications and co-morbidities. According to recent studies comorbidities can be minimized by loosing $5-10 \%$ of initial body weight. However, weight loss of this magnitude isn't easy to accomplish and maintain solely by lifestyle modifications, and often require further pharmaceutical therapy for achieving better outcomes. The GLP-1 receptor analog Liraglutide (Saxenda), was recently approved for use in weight reduction in obese patients.

Methods In our obesity clinic at Rambam Medical Care Campus, We have prescribed Liraglutide $3.0 \mathrm{mg} /$ day combined with lifestyle and dietary counselling for 153 obese patients, some of them already suffering from diabetes complication or co-morbidities. Out of them, 66 patients have 
already completed 3 months of follow up, and their data was analyzed. The recommended starting dose of Liragtlutide was $0.6 \mathrm{mg}$ SC once a day, gradually increased to a maximal dose of $3.0 \mathrm{mg} /$ day. We followed patients for adherence to treatment, weight change, and adverse effects by recurrent clinic visits and telephone calls follow-up.

Results At baseline, mean age ( \pm SD) was $44.98 \pm 11.74$ years, $74 \%$ (49) of the patients were females. Mean pre-treatment weight was $98.83 \pm 17.04$ $\mathrm{kg}$ and mean BMI $34.8 \pm 4.74 \mathrm{~kg} / \mathrm{m}^{2}$. At week 12 , the mean weight loss was $7.94 \pm 6.35 \mathrm{~kg}$, (95\% CI 6.37-9.5); p < 0.0001. Weight loss was more significant in male compared with fe males, $-10.31 \pm 8.68 \mathrm{~kg}(-8.67 \%)$ and $-7.11 \pm 5.18 \mathrm{~kg}(-7.64 \%)$ respectively $(\mathrm{p}<0.01)$. A total of 46 patients $(69 \%)$ reduced at least 5\% of their initial body weight, and 17 patients (25\%) reduced at least $10 \%$ of their initial body weight during the first 3 months of follow up. 31 patients (46.9\%) developed adverse side effects mainly gastrointestinal (mainly mild nausea), and 9 of them (13.6\%) stopped treatment before 3 months. No severe adverse effects were reported.

Conclusions In our obesity clinic, a combination treatment of Liraglutide $3.0 \mathrm{mg} /$ day with lifestyle and dietary changes for a 3 months period was associated with a significant weight loss, and minimal adverse effects.

Conflict of Interest: Nehama Zuckerman: ADVISORY PANEL: NovoNordisk. Naim Shehadeh: ADVISORY PANEL: NovoNordisk, Sanofi, BI.

Funding: none.

\section{T3P126}

\section{Long-term effects of Roux-en-Y gastric bypass surgery on quality of life in severely obese Brazilian patients}

Beleigoli, A. '; Alvares, R. ; Magario, N. ' Eijk, V. '; Freitas, L. '; Ferreira, M. '; Diniz, M. ${ }^{1}$

${ }^{1}$ Federal University of Minas Gerais, Brazil

Introduction: Quality of life (QoL) improves substantially in the first years after gastric bypass surgery (GBP). However, physical and psycho-social issues that ensue after surgery might impact on QoL in the long-term. Objectives: We aimed to evaluate QoL and associated factors in the long-term after GBP in severely obese patients in the Hospital das Clínicas of the Federal University of Minas Gerais (UFMG), Brazil.

Methods: Retrospective cohort study of 115 patients operated for $\geq$ 5 years. The SF-36 questionnaire, which provides physical (PCS) and mental (MCS) component scores of QoL, as well as consultation of patient records, clinical exam and laboratorial tests were applied to all patients who signed an informed consent form. Pre and post-operative characteristics were compared by Wilcoxon or McNemar tests. Ordinal logistic regression models were performed to investigate the association between PCS and MCS and demographic, clinical and laboratorial characteristics. The study was approved by the Ethics Comitte of UFMG. Results: Female sex was predominant $(95 ; 82.6 \%)$. Mean age and pre-operative BMI were 40.2 (13.2) years and 51.9 (8.3) kg/m². Over 8.7 (2.9) years of follow-up, mean percentage of excessive BMI loss (\%EBL) was $58 \%$ (22.1). The prevalence of hypertension and diabetes decreased $(\mathrm{p}<0.001)$ after surgery, but not of depression $(\mathrm{p}=0.25)$. Median PCS and MCS were 48.54 (9.83) and 48.71 (15.85), respectively. Higher PCS were associated with \%EBL (OR1.03; 95\%CI1.01-1.06) and improvement of metabolic comorbidities (OR7.88; 95\%CI1.08-57.3). None of the characteristics investigated was associated with MCS.

Conclusion: Physical well-being in the long-term after GBP depends on maintenance of weight loss and good control of metabolic comorbidities. Acknowledgement: This work was supported by Fapemig and CNPq. The authors have no conflicts of interest.

\section{T3P128 \\ Metabolic activity in the visceral and subcutaneous adipose tissues by FDG-PET/CT in obese patients}

\section{Monteiro, A. $^{1}$; Ferreira, G. $^{2}$; Duarte, $H_{.}{ }^{2}$}

${ }^{1}$ Endocrinology department of Braga Hospital, ${ }^{2}$ Nuclear Medicine Department Of Ipo Porto

Introduction: The increased visceral adipose tissue (VAT) and subcutaneous adipose tissue (SAT) seems to have a different impact on adverse metabolic risk profile. The emerging role of the 18FDG-PET/CT in the study of the metabolic activity and inflammation in adipose tissue indicates that it might be a reliable tool to complement the risk stratification in obesity. The aims of this study were the investigation possible differences in in VAT and SAT FDG uptake between patients with and without obesity.

Methods: Retrospective study of adult patients who underwent whole body 18FDG-PET/CT scanning for clinical purposes (diagnosis or staging of cancer) in July and August of 2016. Statistical analysis: SPSS ${ }^{\mathrm{m} m}$ software v.20. Statistical significance: $\mathrm{p}<0.05$.

Results: We assessed 18FDG-PET/CT scans from 156 patients (58.3\% of males) with mean age of $61.0 \pm 14.1$ years. Half of the patients had a BMI $\geq 25.0 \mathrm{~kg} / \mathrm{m}^{2}$ and $15.4 \%(\mathrm{n}=24)$ were obese. In both groups, the mean FDG uptake were higher in VAT than in SAT. There were no differences in FDG uptake in VAT between the groups. Obese patients had lower density of adipose tissue, both SAT and VAT. Abdominal circumference and density of VAT had a positive predictive value in the mean FDG uptake in VAT. While in SAT, the mean FDG uptake can be positively predicted by density in SAT and VAT and by mean uptake in muscle. Moreover, higher BMI and maximum uptake in myocardium are independent and negatively associated with the mean FDG uptake.

Conclusion: Through a non-invasive 18FDG PET/CT scanning, this study demonstrated a significant higher metabolic activity in VAT, comparatively to SAT, in both obese and non-obese patients. According to our results, abdominal circumference was an important determinant in FDG uptake in VAT. We also demonstrated that obese patients had differences in adipose tissue quality. Our findings reinforce the importance of the adipose tissue quality and distribution for metabolic risk stratification.

\section{T3P129}

Metabolic effects of replacing sugar-sweetened by artificially sweetened beverages in overweight subjects with or without hepatic steatosis: A randomized control clinical trial

\section{Campos, V. ${ }^{1}$; Despland, C. ' ${ }^{1}$ Kreis, R. $^{2}$; Schneiter, P. ${ }^{1}$; Boesch, C. ${ }^{3}$; Tappy, L. ${ }^{1}$}

${ }^{1}$ University of Lausanne, Switzerland, ${ }^{2}$ Inselspital Bern, Switzerland, ${ }^{3}$ Inselspital Bern

We conducted a 12-week trial involving 26 overweight male and female adults (BMI $>25$ ) consuming 2 or more servings/day sugar-sweetened beverages (SSB). Participants were randomized to either a 12-week intervention with replacing SSB with artificially sweetened beverages (ASB arm, 7M, 6F) or a control arm without intervention (CTRL arm, 6M, 7F). At the end of run-in (week 4) and again at the end of intervention (week 16) they took part to a 8.5 hour metabolic investigation during which their plasma glucose, insulin, glucagon, lactate, triglyceride (TG), non-esterified fatty acids (NEFA), and uric acid concentrations were measured over a $30 \mathrm{~min}$ in fasting conditions $(-30-0 \mathrm{~min})$ and then every 2 hours over $480 \mathrm{~min}$. with ingestion of a standard breakfast at time $0 \mathrm{~min}$ and a standard lunch at time $240 \mathrm{~min}$. Breakfast and lunch were consumed together with a $3 \mathrm{dl}$ SSB at week 4 and with either an ASB (ASB arm) or a SSB (CTRL arm) at week 16. After analyzing the whole group, a secondary analysis was performed on 14 subjects with hepatic steatosis based on their intrahepatic fat concentration measured by magnetic resonance spectroscopy (7 randomized to ASB, 7 to CTRL) and 12 subjects without hepatic steatosis (6 randomized to ASB and 6 to CTRL). 
Consumption of SSB was similar in both groups at inclusion. Ingestion of meals increased plasma glucose, insulin, lactate and TG concentrations and decreased NEFA concentrations, but with no significant difference of integrated postprandial responses between week 4 and week 16 in both ASB and CTRL. In subjects without hepatic steatosis, peak plasma insulin after breakfast was slightly, but significantly lower at week 16. In subjects with hepatic steatosis, peak plasma insulin and lactate after breakfast and peak insulin after lunch were significantly decreased in the ASB arm. ASB however did not significantly lower post-prandial plasma glucose and TG concentrations in either group.

These results indicate that, in overweight, high SSB consumers, replacing SSBs with ASB during 12 week slightly reduced postprandial insulin and lactate concentrations, but did not significantly alter post-prandial TG concentration in spite of the lower energy and fructose content of the meals. These effects were globally the same in subjects without and with hepatic steatosis.

This work was supported by the Swiss National Science Foundation and the Raymond Berger Foundation.

\section{T3P130 \\ Microarray analysis of liver gene expression in severely obese patients after eight weeks of treatment with long chain $n-3$ PUFA}

Itariu, B. '; Bilban, M. ${ }^{2}$; Amor, M. ${ }^{1}$; Prager, G. ${ }^{3}$; Langer, F. ${ }^{3}$; Stulnig, T. ${ }^{1}$

${ }^{1}$ Department of Internal Medicine III, Clinical Division of Endocrinology and Metabolism and Christian Doppler-Laboratory for Cardio-Metabolic Immunotherapy, Medical University of Vienna, ${ }^{2}$ Department Of Laboratory Medicine, Medical University Of Vienna, ${ }^{3}$ Department Of General Surgery, Div. Bariatric Surgery, Medical University Of Vienna

Introduction: Long chain n-3 polyunsaturated fatty acids ( $n-3$ PUFA) have favourable metabolic effects due to their triglyceride lowering and anti-inflammatory properties. Metabolic dysfunction in obesity leads to an increased cardiovascular risk, which predisposes to premature death Our aim was to evaluate the effect of 8 weeks of treatment with n-3 PUFA gene expression in liver tissue of severely obese patients, specifically on genes related to cardio-metabolic disorders.

Methods: Severely obese patients $(6 \mathrm{~m} / 23 \mathrm{f})$ were randomly treated with either $3.6 \mathrm{~g} / \mathrm{d} n-3$ PUFA $(\mathrm{n}=14)$ or the same amount of butterfat as control $(n=15)$ for 8 weeks. After treatment patients underwent elective bariatric surgery, during which we obtained liver biopsy specimens. For whole genome gene expression microarray analysis (Affimetrix Gene Chip Prime View Human Gene Expression Array") was performed. We analysed data by gene set enrichment analysis.

Results: From a total of 2956 analysed gene sets, 120 gene sets were dysregulated with a false detection rate $<25 \%$ and $p<0,05$. For further evaluation, we selected gene sets relevant to liver metabolism and inflammation. We found that $n-3$ PUFA influenced pathways specific for glucagon receptor signalling, immune regulation, inflammatory response, IGF-1, angiogenesis and insulin action.

Conclusion: This was to our knowledge the first microarray analysis to investigate the effects of n-3 PUFA treatment on human liver gene expression. We conclude that n-3 PUFA have pleiotropic effects on metabolic and inflammatory pathways.

Conflict of Interest: None

Funding: This work was supported by the Austrian National Bank (P12735) and the by the Federal Ministry of Economy, Family and Youth and the National Foundation for Research, Technology and Development (all to T.M.S.).
T3P131

"Milk, dairy products and fruit" dietary pattern is associated with reduced asthma prevalence and severity in obesityasthma phenotype

Barros, R. ${ }^{1}$; Moreira, A. ${ }^{2}$; Padrão, P. ; Teixeira, V. ${ }^{4}$; Lopes, C. ${ }^{5}$; Severo, M. ${ }^{5}$; Delgado, L. ${ }^{2}$; Moreira, P. ${ }^{4}$

${ }^{1}$ Faculty of Nutrition and Food Sciences, University of Porto, Porto, Portugal, ${ }^{2}$ Laboratory Of Immunology, Basic And Clinical Immunology Unit, Faculty Of Medicine, University Of Porto, And Immunoallergology Department, Centro Hospitalar São João, Porto, Portugal, ${ }^{3}$ Faculty Of Nutrition And Food Sciences And Ispup-Epiunit, University Of Porto, Porto, Portugal, ${ }^{4}$ Faculty Of Nutrition And Food Sciences And Research Centre On Physical Activity And Health, University Of Porto, Portugal, ${ }^{5}$ Ispup - Epiunit And Department Of Clinical Epidemiology, Predictive Medicine And Public Health, Faculty Of Medicine, University Of Porto, Porto, Portugal

Background: Dietary patterns have been suggested as modifiable factors with impact in both obesity and asthma conditions. Moreover, obesity has also been associated with a more persistent and severe asthma phenotype. We aimed to explore the association between dietary patterns and asthma prevalence, incidence and control in overweight and obese adult population.

Methods: A representative sample of 32644 adults, 53\% female, from the 4th Portuguese National Health Survey was analysed. The following asthma definitions were used: ever asthma (ever medical doctor asthma diagnosis), current asthma (asthma within the last 12 months), current persistent asthma (required asthma medication within the last 12 months), current severe asthma (attending an emergency department because of asthma within the last 12 months), and incident asthma (asthma diagnosis within the last 12 months). Body mass index (BMI) was calculated based on self-reported weight and height according the WHO classification. Dietary patterns (DP) were identified by Latent Trait models based on self-reported dietary intake. Unconditional logistic regression was performed to estimate the association between DP and asthma, in a total of 17901 participants being overweight or obese $\left(\mathrm{BMI} \geq 25.0 \mathrm{~kg} / \mathrm{m}^{2}\right)$. Age, gender, BMI, education, family income, proxy reporting information and smoking were analysed as confounders.

Results: Prevalence of ever asthma was 5.3\%, current asthma 3.5\%, current persistent asthma 3.0\%, current severe asthma $1.4 \%$, and incident asthma $0.2 \%$. Prevalence of obesity was $16 \%$, overweight $37.6 \%$, normal weight $44.6 \%$ and underweight $0.2 \%$. Considering DP analysis, five factors were identified: F1 ("milk, dairy products and fruit" DP), F2 ("vegetable soup, pulses and bread"), F3 ("high fat, sugar and salt"), F4 ("fish, fruit and vegetables"), F5 ("sugary and fatty foods"). After adjustment, "Milk, dairy products and fruit" DP was inversely associated with asthma prevalence $(\mathrm{OR}=0.87,95 \% \mathrm{CI}=0.78,0.98)$ and current severe asthma $(\mathrm{OR}=0.79$, $95 \% \mathrm{CI}=0.62,0.99)$, in participants with overweight or obesity. No other significant associations were observed between dietary patterns and asthma prevalence, incidence or control in this sample.

Conclusion: Our results suggest a protective association between "milk, dairy products and fruit" dietary pattern and the prevalence and severity of obesity-asthma phenotype, providing rational for future intervention studies and dietary patterns recommendations.

Conflict of interest: None

Funding: No Funding

*2017 EASO Award Application 
T3P132

\section{Mindful eating and weight loss, results from a randomized trial}

\section{Dunn, C. ${ }^{1}$; Thomas, C. ${ }^{2}$; Whetstone, L. $^{3}$; Aggarwal, S. ${ }^{1}$}

${ }^{1} \mathrm{NC}$ State University, ${ }^{2} \mathrm{Nc}$ Division Of Public Health, ${ }^{3}$ University Of California Sacramento

Introduction: Overweight and obesity increase the risk of chronic disease including hypertension, type 2 diabetes, and cardiovascular disease. Public health organizations support the small changes approach, ${ }^{2}$ which emphasizes the combination of diet and physical activity and suggests reductions in energy intake and increases in energy expenditure to prevent weight gain and or promote weight reduction. In recent years, mindful eating, increasing one's awareness and focus on eating, has been introduced as a possible strategy for weight management. The purpose of the present study was to examine the effectiveness of Eat Smart, Move More, Weigh Less (ESMMWL) in increasing mindful eating as measured by the Mindful Eating Questionnaire (MEQ). The secondary objective was to investigate the relationship between mindful eating and weight loss.

Methods: Participants were part of a randomized controlled trial evaluating the effectiveness of ESMMWL, an online, 15-week weight management program $(n=80)$. The primary outcome was mindful eating as measured by the MEQ, a 28-item questionnaire that assesses five domains of mindful eating. Independent samples t-tests and $\mathrm{X}^{2}$ tests were used to compare baseline measures for intervention and wait-list control groups. Analyses of covariance with baseline measure as covariates were conducted to compare change in mindfulness scores between the two groups. Mean mindfulness scores were analyzed using repeated measures ANOVA with Greenhouse-Geisser correction for violations of sphericity. The relationship between weight loss and mindfulness was analyzed with Pearson correlations.

Results: ESMMWL participants lost more weight than participants in the waitlist control group. For all subscales and the summary score, participants who completed ESMMWL had a significantly larger increase in their mindfulness scores than those in the waitlist control group.

Conclusion: Results suggest that there is a beneficial association between mindful eating and weight loss. The current study contributes to the mindfulness literature as there are very few studies that employed rigorous methodology to examine the effectiveness of an intervention on mindful eating.

Authors have no conflicts of interest.

Funding for the ESMMWL program from NC State Health Plan. There was no Funding for this research.

\section{Rerferences:}

1 Dixon JB. The effect of obesity on health outcomes. Molecular and Cellular Endocrinology. 2010; 316: 104-108.

2 Hill JO. Can a small-changes approach help address the obesity epidemic? A report of the Joint Task Force of the American Society for Nutrition, Institute of Food Technologists, and Internal Food Information Council. Am J Clin Nutr. 2009; 89: 477-484.

\section{T3P133}

\section{Modelling the epidemiological impact of a brief physician intervention for obesity using a microsimulation model}

Retat, L. '; Pimpin, L. '; Webber, L. '; Jebb, S. ${ }^{2}$; Aveyard, P. ${ }^{2}$

${ }^{1}$ UK Health Forum, 2 University Of Oxford

Introduction: In a parallel, two-arm randomised UK trial, a brief physician-delivered referral of patients with $\mathrm{BMI} \geq 30 \mathrm{~kg} / \mathrm{m}^{2}$ to a weight management group for 12 weeks was effective in reducing population mean weight, compared to physician advice to lose weight ${ }^{1}$. However, the longterm epidemiological impacts of these interventions are not known.

Methods: Using a microsimulation ${ }^{2}$, two scenarios representing the brief intervention and its control were modelled for 20 years (2015-2035), and compared to a baseline assuming natural progression in UK BMI popu- lation trends. An $80 \%$ probability of visiting the physician was assumed in both scenarios, and a referral uptake of $40 \%$ was assumed in the intervention scenario. In the first year, the population BMI change observed in each trial arm was applied in the relevant scenario. Over the next 5 years, $100 \%$ linear regain in weight was assumed if the individual lost weight in both scenarios. Individuals then followed national BMI trends. The model population was representative of the trial population's age sex and BMI. Individuals' annual BMI was associated with a risk of disease. The incidence for 12 chronic conditions between each scenarios and the natural progression scenario was compared. Cost-effectiveness analyses using microsimulation are in preparation.

Results: By 2035, the intervention scenario resulted in more incident cases avoided than the control scenario, when compared to the natural progression scenario: for every 100,000 individuals, 386 cases of disease were avoided, including cases of pre-diabetes, diabetes and hypertension (332, $124 \& 67$ cases per 100,000 respectively). However, the intervention scenario also resulted in additional cumulative incidence for some diseases, notably CHD (88 cases/100,000) and stroke (31 cases/100,000). In contrast, the control arm consistently resulted in a negative difference in total cumulative incidence of disease, when compared to the baseline scenario. This ranged from an additional 76 cases of disease/100,000 simulated individuals in 2020, to 1,137 cases/ 100,000 in 2035 .

Conclusion: Microsimulation showed that an effective brief obesity intervention of referral to a weight loss programme resulted in more cases of disease avoided up to 20 years after the intervention has taken place. Further health economic analyses should help inform on the cost-effectiveness of such an intervention.

The authors would like to acknowledge all members of the trial team.

\section{References:}

1 Aveyard $\mathrm{P}$ et al. Screening and brief intervention for obesity in primary care: a parallel, two-arm, randomised trial. The Lancet 2016; 388(10059): 2492-500.

2 McPherson K et al. Tackling obesities: future choices: Modelling future trends in obesity and the impact on health: Citeseer; 2007.

\section{T3P134}

\section{Multidimensional improvements induced by an intensive obesity inpatients rehabilitation program}

Budui, S. '; Giordano, F. '; Berteotti, M. ' ; Franceschini, L. ' Gilli, F. '; Lovisi, M. '; Salvetti, S. '; Sartori, E. ' Raschella, G. '; Schena, F. '; Taddei, M. '; Petroni, M. ${ }^{1}$; Busetto, L. ${ }^{3}$

${ }^{1}$ Solatrix clinique, ${ }^{2}$ Cerism Sport Mountain And Health Research Center, University Of Verona, ${ }^{3}$ Center For The Study And The Integrated Management Of Obesity, University Of Padova, Italy

Introduction: the aim of this study is to present preliminary data on the effectiveness of an intensive inpatient rehabilitation program specifically developed for patients with severe obesity.

Methods: residential rehabilitative treatment is managed by a multidisciplinary team during a 3-week period and it is characterized by the integration of nutritional and physical/functional rehabilitation with psychological/educational intervention. At admission and at discharge all patients are evaluated by anthropometric assessment (weight, body mass index (BMI), waist and neck circumferences), laboratory tests (glycaemia, $\mathrm{HbA1c}$, lipid profile), sychological tests (SF-36 and BES) and physical performance assessment (VO2 peak by incremental cycle ergometer test, six minutes walking test (6MWT), chair stand test).

Results: 136 subjects (61\% females, median age 52.7 years) with obesity (mean BMI $43.2 \mathrm{~kg} / \mathrm{m}^{2}$ ) and comorbidities (diabetes mellitus 31\%, hypertension $58 \%$, hepatosteatosis $76 \%$, sleep apnea $68 \%$, cardiopathy $31 \%$, artropathy $76 \%$, eating disorders $6 \%$, psychiatric diseases $27 \%$ ) were admitted from $13 / 4 / 15$ to $30 / 1 / 16$. Considering metabolic parameters, we observed glycemic control in $68 \%$ of patients with uncontrolled diabetes at admission (HbAlc 53.4 vs $50 \mathrm{mmol} / \mathrm{mol}$ ), total cholesterol reduction of $16 \%$, LDL-cholesterol reduction of $19 \%$, reduction in triglycerides of $9 \%$. Blood pressure control was achieved in all patients with uncontrolled 
hypertension at admission. When analyzing anthropometric changes, we observed a $3.9 \%$ weight and BMI reduction, and a reduction of $3.8 \%$ and $3.3 \%$ in waist and neck circumference. For physical performance, we observed a $4.7 \%$ improvement in VO2 peak, with longer distances in 6MWT and higher number of standings. Finally, psychometric tests showed improvements in quality of life perception and binge eating disorder.

Conclusions: our preliminary data prove that a 3-weeks rehabilitation program determined a clinically significant multi-dimensional improvement in patients with severe obesity. Although these results are encouraging, long-term follow-up data are needed in order to confirm the efficacy of our rehabilitation setting.

Conflict of Interest: Disclosed conflict of interest

Funding: No Funding/payment relating to this abstract

\section{T3P136}

\section{Muscle mass after gastric bypass: Characterization of weight loss and determinants of change}

Magalhaes, D. '; Silva, L. '; Oliveira, S. '; Neves, J. '; Souteiro, P. '; Pedro, J.' ; Guerreiro, V. ${ }^{1}$; Bettencourt-Silva, R. ${ }^{1}$; Costa, M. ${ }^{1}$; Correia, F. ${ }^{2}$; Varela, A. ${ }^{1}$; Queirós, J.' ${ }^{1}$ Freitas, P. ${ }^{1}$; Carvalho, D. ${ }^{1}$; Amtco, G. ${ }^{3}$

${ }^{1}$ Department of Endocrinology, Diabetes and Metabolism of Centro Hospitalar de São João, Porto, Portugal, ${ }^{2}$ Faculty Of Nutrition And Food Science, University Of Porto, Portugal, ${ }^{3}$ Multidisciplinary Group For Surgical Management Of Obesity At Centro Hospitalar De São João, Porto, Portugal

The constituents of weight loss following bariatric surgery are poorly known. Skeletal muscle mass (MM) plays a major role in glucose disposa and glycaemic regulation. Its significant loss can cause functional impairment and a poorer quality of life.

Aims: To evaluate the constituents of weight loss after gastric bypass and to analyse the determinants of MM changes following this kind of bariatric surgery.

Methods: We investigated a cohort of 119 obese patients who underwent gastric bypass. Using bioelectrical impedance analysis, the subjects body composition was assessed before, and 1, 6 and 12-month post-surgery. We analysed their metabolic [total, HDL and LDL cholesterol, triglycerides, fasting plasma glucose (FPG) and A1c] and inflammatory [white blood cells count (WBC) and C-reactive protein (CRP)] status before and 12 months after gastric bypass.

Results: At the end of the first month after surgery, the median weight loss was 10.5 (IQR 6.45) kg, with fat mass (FM) and MM loss representing $2.6 \%$ and $31.1 \%$, respectively. At 6-month, the median weight loss was 29.2 (IQR 11.5 ) $\mathrm{kg}, 34.4 \%$ of FM and $14.6 \%$ of MM. At $12-$ month, the median weight loss was 39.0 (IQR 13.2) kg, made up of $42.9 \% \mathrm{FM}$ and $12 \% \mathrm{MM}$. There was a significant improve of metabolic and inflammatory parameters at 12 -month post-surgery $(p<0.001)$. At 12 -month the MM variation $(\triangle \mathrm{MM} 12 \mathrm{~m})$ showed a positive correlation with the variation in the same time of CRP $(\triangle \mathrm{CRP} 12 \mathrm{~m})$, weight $(\Delta$ weight $12 \mathrm{~m})$ and total body water $(\Delta \mathrm{TBW} 12 \mathrm{~m})$. In a multiple regression model, only $\Delta \mathrm{TBW} 12 \mathrm{~m}$ demonstrated to be independently associated with $\Delta \mathrm{MM} 12 \mathrm{~m}$, exhibiting a strong positive correlation with our dependent variable.

Conclusion: The contribution of MM to weight loss decreased at 6 and 12 months of follow-up, with an increasing contribution of FM to the total weight loss. The $\Delta \mathrm{MM} 12 \mathrm{~m}$ has a strong positive correlation with $\triangle \mathrm{BTW} 12 \mathrm{~m}$. We found very few predictors of the $\triangle \mathrm{MM} 12 \mathrm{~m}$ after gastric bypass, so monitoring its evolution is of crucial importance given the physical and metabolic consequences that can result from its significant decrease.
T3P137

Musculoskeletal pain profile of obese individuals attending a multidisciplinary weight management service.

Maclellan, G. '; Dunlevy, C. ${ }^{2}$; O 'Malley, E. ${ }^{2}$; Blake, C. ${ }^{1}$; Breen, C. ${ }^{2}$; Gaynor, K. ${ }^{2}$; Wallace, N. ${ }^{2}$; Yoder, R. ${ }^{2}$; Casey, D. ${ }^{2}$; Mehegan, J. ${ }^{1}$; O'Shea, D. ${ }^{2}$; Fullen, B. ${ }^{1}$

${ }^{1}$ University College Dublin, School of Public Health, Physiotherapy \& Sports Science, Belfield, Dublin 4, Republic of Ireland, ${ }^{2}$ Weight Management Service, St. Columcille'S Hospital, Loughlinstown, Co. Dublin, Republic Of Ireland

Introduction: Obesity is associated with numerous chronic diseases, including musculoskeletal (MSK) pain, which impacts on quality of life (QoL). There is, however, limited research providing a comprehensive MSK pain profile of an obese cohort.

Methods: This retrospective study was undertaken using data from the patient database at the national weight management service (WMS) in the Republic of Ireland. Following ethical approval from the participating hospital, all anonymized patient data were entered into the Statistical Package for the Social Sciences (V.20) to develop a pain profile, investigate relationships between measures of pain, sleep and function, and explore variables predictive of having low back pain (LBP) and knee pain.

Results: Overall, 915 individuals attended the WMS from January 2011 to September 2015 [male, $34.8 \%(\mathrm{n}=318)$; female, $65.2 \%(\mathrm{n}=597)$; mean age of 44.6]. Mean BMI was $50.7 \mathrm{~kg} / \mathrm{m}^{2} ; 91.9 \%(\mathrm{n}=835)$ were Class III obese (BMI $\geq 40$ ). Approximately $92 \%$ reported MSK pain: LBP, $68.7 \%$ $(\mathrm{n}=539)$ [mean NRS 7.4]; knee pain, 58\% $(\mathrm{n}=447)$ [mean NRS 6.8]. Class III obese patients and patients with multi-site pain had lower QoL, physical activity levels, reduced sleep, and poorer functional outcomes $(\mathrm{p}<0.05)$ than lower obesity classifications and patients without pain. $\mathrm{Re}$ lationships were found between demographic, pain, self-report, and functional measures $(\mathrm{p}<0.05)$. Patients who were older, slept fewer hours, and had poorer functional outcomes were more likely to have LBP or knee pain $(\mathrm{p}<0.05)$

Conclusion: Multi-site MSK pain is prevalent and severe in obese patients and is negatively associated with nearly all self-report (sleep and QoL) and functional outcome measures. Due to the high MSK pain prevalence, pain management strategies must be considered when treating obesity.

Conflict of Interest: No conflicts of interest were identified.

Funding: No Funding was received for the research relating to this abstract.

\section{T3P138 \\ Nadir of weight change and weight regain after three to ten years of three types of bariatric surgery}

Roque, C. ${ }^{1}$; Ferrinho, C. '; Capitão, R. '; Santos, F. '; Bello, C. '; Oliveira, M. '; Duarte, J. '; Vasconcelos, C. '; Cardoso, J. '; Tapadinhas, A. ' ; Tomás, C. ${ }^{1}$

${ }^{1} 1$ - Obesity Multidisciplinary Team, Hospital de Egas Moniz, C.H.L.O. Lisboa

Objective: Obesity is a chronic disease that is associated with many health problems. Bariatric surgery (BS) induces weight loss and reduces complications. The authors analyze the clinical impact of 3 types of BS in long term and the amount of weight regain.

Methods: Retrospective observational, single center study. BS procedures included laparoscopic adjustable gastric banding (LAGB), sleeve gastrectomy (SG) and Roux-en-Y gastric bypass (RYGB). Adult patients that underwent a single BS procedure and that had a minimal follow up of 3 years were included. Clinical and biochemical data were collected from the hospital medical records and laboratory software. We assessed percent weight change from baseline, its nadir and the prevalence of comorbidities. Data was analyzed in SPSS software using descriptive methods, T test for continuous variables, $\mathrm{Chi}^{2}$ for categorical and results presented by median-SE. Results: A total of 398 patients ( $85 \%$ women) with a median age of $46.2 \pm 0.5$ years were included. At the time of BS the median BMI and weight were $45.7 \mathrm{~kg} / \mathrm{m}^{2}$ and $118.7 \pm 0.9 \mathrm{~kg}$ respectively. Comorbidities were common: $82 \%$ had dyslipidemia, $75.6 \%$ hypertension, $49 \%$ type 2 diabetes, 36\% sleep apnea, 29\% liver steatosis and $6.5 \%$ previous cardiovascular events They completed longitudinal observations for $5.8 \pm 0.1$ years 
and the minimum weight (median decrease of $30.3 \%$ weight) was reached in $2.9 \pm 0.1$ years. Summary of main results.

Conclusions: A substantial weight loss was observed after BS. The nadir of lost weight was greater with RYGB. SG patients achieved their nadir the soonest. The maximal weight lost for LAGB occurs later, possibly due to long time band adjustments. However, is possible that this difference is greater due to the exclusion of the patients with LAGB that needed a subsequent $\mathrm{BS}$.

\section{T3P139}

\section{New diabetes genetic risk assessment tool for the prevention of type 2 diabetes in Mexico}

Zulueta, M. ${ }^{\text {; }}$ Orozco, L. ${ }^{2}$; Juárez-Torres, E. ${ }^{3}$; Ramirez-Campos, E. ; MarinMedina, M. ${ }^{4}$; Mendizabal, L. ; Simón, L. ; Berumen, J. ${ }^{6}$

${ }^{1}$ Patia Biopharma, Mexico, ${ }^{2}$ Instituto Nacional De Medicina Genómica, México, ${ }^{3}$ Hospital General De México/Facultad De Medicina, Universidad Nacional Autónoma De México, Mexico, ${ }^{4}$ Huella Génica, México, ${ }^{5}$ Patia Biopharma, Spain, $\left.{ }^{6}\right)$ Unidad De Medicina Genomica, Hospital General De México/Facultad De Medicina, Universidad Nacional Autónoma De México, México

Background: Mexico is experiencing the most rapid increase ever recorded in childhood and adult Type 2 Diabetes (T2D) prevalence. Shifts in dietary and physical activity patterns in combination with strong genetic predisposition may be contributing to this. Genome-wide association studies (GWAS) have identified many single nucleotide polymorphisms (SNPs) associated with T2D. Our objective was to create a useful public health and research tool. We developed a T2D genetic risk test for mexican and latinamerican mestizo populations.

Methods: We selected SNPs from the largest meta-analyses of GWAS available, prioritizing those with the highest predictive power (effect size $x$ allele frequency) in latinamerican mestizo population, and located in genes involved in a variety of cellular processes in T2D development (SLC16A11, HNF1A, TCF7L2, CDKN2A/B, CDKAL1, SLC30A8, IGF2BP2, FTO, PPARG, HHEX/IDE, ADCY5, JAZF1, WFS1, INS-IGF2, KCNQ1, KCNJ11). A genetic risk score was calculated based on the combined odds ratio (COR) of 16 SNPs and the frequency distribution of cases and controls through ascendant COR intervals. We ran a theoretical model in which 0,1 or 2 risk alleles (RA) of each SNP were assigned randomly according to reported ORs, RA frequency and Hardy-Weinberg law. T2D risk (\%) was calculated with a linear regression. We then tested the model in a mexican sample cohort of 500 cases and 500 controls. The efficiency of the models was evaluated with ROC curves. The contribution of each SNP and other clinical factors was measured with a multivariate logistic regression (MLR). Genotyping was performed in a time and cost effective platform.
Results:Results of the theoretical and sample cohort models were very similar. AUC, sensitivity and specificity were $0.680,71 \%$ and $55.1 \%$, respectively. Adding BMI to the model slightly increased the AUC to 0.692 . Addition of parents history of diabetes substantially increased prediction (AUC up to 0.79 , sensitivity $68 \%$, specificity $79 \%$ ).

Conclusions:Utilization of genetic markers in combination with clinical characteristics may improve accuracy of diabetes risk prediction and reinforce the adoption of healthier lyfe style habits.

\section{T3P140}

\section{No changes of serum Fetuin-a levels six months after bariatric surgery}

Verras, C. '; Simos, Y. ${ }^{2}$; Angelidi, A. ${ }^{1}$; Ayiomamitis, G. ${ }^{3}$; Fousteris, E. ${ }^{\prime}$; Konitsiotis, S. ${ }^{2}$; Evangelou, A. ${ }^{2}$; Kiortsis, D. ${ }^{2}$; Melidonis, A. ${ }^{1}$

${ }^{1}$ Diabetes Center, Tzanio General Hospital, Piraeus, Greece, ${ }^{2}$ Laboratory Of Physiology, Medical School Of University Of loannina, loannina, Greece, ${ }^{3} 2 \mathrm{Nd}$ Surgical Department, Tzanio General Hospital, Piraeus, Greece

Introduction: The liver secreted glycoprotein Fetuin-A has been recently associated with insulin resistance, atherosclerosis and obesity. However, there are limited data regarding the effects of bariatric surgery in morbidly obese patients on serum Fetuin-A. The objective was to examine any possible correlation between Fetuin-A with these biomarkers in morbidly obese patients prior to and six months after bariatric surgery.

Methods: We recruited 20 morbidly obese patients (mean BMI: $43.0 \pm 3.0$ $\mathrm{kgr} / \mathrm{m}^{2}$ ) who were admitted to the surgery clinic of our hospital to undergo programmed bariatric surgery (laparoscopic sleeve gastrectomy). $40 \%$ of our sample was male and $20 \%$ had type 2 diabetes. Fasting blood samples collected before (baseline) and 6 moths after surgery (follow up) were analyzed for glucose, $\mathrm{HbAlc}$, lipidemic profile, hs-CRP, fibrinogen, insulin and Fetuin-A. HOMA-IR index and lipid accumulation product (LAP) to all participants were performed. Bioelectric impedance was performed in all patients for the assessment for fat mass and fat free mass at baseline and at 6 month follow up.

Results: The mean body weight loss during the study period was $31.5 \pm 11.81 \mathrm{kgs}$, of which $25.54 \pm 21.48 \mathrm{Kgs}$ were fat mass. Mean $( \pm \mathrm{SD})$ serum Fetuin-A levels at baseline were $0.27( \pm 0.05) \mathrm{g} / \mathrm{l}$ which did not show difference compared to the $0.26( \pm 0.07) \mathrm{g} / \mathrm{l}$ of the follow up re-examination $(\mathrm{p}=0.808)$. Baseline Fetuin-A levels correlated with total cholesterol levels $(\mathrm{r}=0.582, \mathrm{p}<0.05)$ and LDL-cholesterol $(\mathrm{r}=0.540, \mathrm{p}<0.05)$ prior to bariatric surgery. No correlation to the rest of the study parameters at baseline. Follow up Fetuin-A levels showed no correlation to any study parameters. The changes of Fetuin-A levels (follow up - baseline) correlated with baseline triglycerides levels $(\mathrm{r}=0.592, \mathrm{p}<0.01)$.

Conclusion: To our knowledge this is the first study examining medium term effects of bariatric surgery on the serum fetuin-A levels. Our study

Table 1. to T3P138

\begin{tabular}{|c|c|c|c|c|c|c|c|c|}
\hline LAGB $(n=248)$ & RYGB $(n=67)$ & $S G(n=83)$ & \begin{tabular}{|l|} 
Total \\
$(\mathrm{n}=398)$
\end{tabular} & & & & & \\
\hline & Median & SE & Median & SE & Median & SE & Median & \\
\hline Age at BS (Years) $p<0.001$ & 45,0 & 0,7 & 40,3 & 1,1 & 53,4 & 1,1 & 46,2 & 0,5 \\
\hline Weight pre BS (Kg) $p=0.019$ & 120,2 & 1,2 & 120,0 & 2,3 & 115,0 & 1,8 & 118,7 & 0,9 \\
\hline BMI pre BS $p=0.021$ & 46,5 & 0,4 & 45,2 & 0,8 & 44,1 & 0,7 & 45,7 & 0,3 \\
\hline $\begin{array}{l}\text { Time To Nadir (Years) } \\
p<0.001\end{array}$ & 3,7 & 0,1 & 2,0 & 0,2 & 1,8 & 0,1 & 2,9 & 0,1 \\
\hline Nadir Weight posT BS (Kg) & 84,0 & 1,1 & 75,6 & 1,6 & 78,0 & 1,7 & 81,5 & 0,8 \\
\hline \% Weight nadir $p<0.001$ & 28,9 & 0,8 & 37,5 & 1,1 & 30,8 & 0,9 & 30,3 & 0,6 \\
\hline $\begin{array}{l}\text { Time To Last Visit (Years) } \\
p<0.001\end{array}$ & 7,0 & 0,2 & 4,0 & 0,2 & 4,7 & 0,1 & 5,8 & 0,1 \\
\hline $\begin{array}{l}\text { Weight Last Visit }(\mathrm{Kg}) \\
\mathrm{p}<0.001\end{array}$ & 89,0 & 1,2 & 79,0 & 1,9 & 82,0 & 1,8 & 85,0 & 0,9 \\
\hline$\%$ Weight lost Last Visit ns & 27,6 & 0,8 & 30,0 & 1,6 & 30,0 & 1,6 & 28,5 & 0,7 \\
\hline BMI Last Visit $p<0.001$ & 34,1 & 0,4 & 29,4 & 0,6 & 30,9 & 0,7 & 32,7 & 0,3 \\
\hline
\end{tabular}


demonstrated that Fetuin-A levels remained unchanged besides the significant weight loss achieved within the first 6 months after bariatric surgery, possibly indicating that this time period after the surgery is too short to reveal significant changes. Further research on this field with larger populations need to be done.

\section{T3P141}

\section{NOR = neuro-olfactive reprogramming}

\section{Pinelli, N. ${ }^{1}$;}

${ }^{1} 2010$

This clinical research project aims to demonstrate the effectiveness of a multidisciplinary protocol. This protocol combines three disciplines: $\mathrm{Ol}$ factory sensation, psychology and education. New morphostatic and staturo-weight measurements and a new learning method called Neuro-Olfactive Reprogramming (NOR) were created for the purpose of this study. This NOR proposes workshops that are part of a therapeutic education process and could interest the various SOC (specialized obesity centers) in the world.

Objectives: to demonstrate the effectiveness of this protocol and therefore the usefulness of Olfactory sensation this new approach in the diagnosis and The treatment of overweight and obesity, change the eating behaviors Medium-term objectives: transfer of this methodology to help some psychological, cognitive-behavioral, memory disorders, neurological rehabilitation and extend it to all sectors of health and well-being in general.

Principle of this research: NOR uses the effects of emotional memory olfactory.

Type of research: biomedical research, randomized controlled study with clinical trials, quantitative evaluation and statistical analysis of data.

NOR Workshops:

- Neuro-olfactory tests

- Neuro-olfactory education

Results: reduction of obesity or an overweight, improvement the staturo-weight and the morphostatic parameters.

\section{T3P143}

\section{Assessment of nutrient intake to obese people before and after a nutritional intervention}

Posea, M. ${ }^{1}$; Dragomir, A. ${ }^{2}$; Radulian, G. $^{3}$

${ }^{1}$ AIS Clinics and Hospital, Bucharest, Romania, 2"Carol Davila" University Of

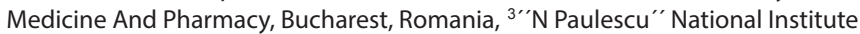
Of Diabetes, Nutrition And Metabolic Diseases, Bucharest, Romania

Introduction: In the last decades, obesity became a major problem of public health and there is a huge need of knowing eating habits and of trying to change them in order to achieve a healthy lifestyle for a long period of time. In Romania, a cross sectional study from 2014 regarding obesity and health related lifestyle factors in the general population (the ORO Study) showed that the prevalence of obesity was about $21.3 \%$ and the most frequent unhealthy eating habits were irregular meals and eating while watching TV, both in normal weight and obese subjects

Methods:We evaluated the intake's energy, vitamins and minerals in a sample of 126 overweight and obese subjects before and after a nutritional intervention. We also performed anthropometric's measurements, paraclinic's and laboratory's tests.

Results: All the antropometric and paraclinic's data improved in the end Regarding laboratory's tests, we found that the prevalence of normal plasma cholesterol is higher in the end, from $43.64 \%$ to $63.64 \%(\chi 2=4.42$, $\mathrm{p}=0.035$ ). At the final of the programme, the same vitamins from food are in excess as before, but vitamin $\mathrm{C}$ intake increased significantly and the vitamin B1 decreased significantly. Of the 8 minerals studied, four have seen significant decreases of the intake, following nutritional intervention: calcium $(\mathrm{p}=0.02)$, magnesium $(\mathrm{p}=0.027)$, iron $(\mathrm{p}=0.02)$ and copper $(\mathrm{p}=0.027)$. In the end, the level of the calcium and magnesium are below daily recommended intake (DRI) in the subjects' diet, with $68.81 \%$ of DRI, respectively $71.86 \%$ of DRI. Iron is the only mineral which met the DRI(105.7\% of DRI) and the intake of copper, zinc, manganese, phosphorus and selenium is above.

Conclusion: The nutritional intervention led to the improvement of all anthropometrics' and paraclinic 's data. Assessing the effect of nutritional intervention on dietary intake of vitamins and minerals in overweight and obese showed an unbalance of micronutrient consumption. The research results support the need for balanced nutritional recommendations as to achieve a healthy lifestyle that can be implemented in the long term.

Conflict of Interest: None

Funding: No Funding relating to this abstract

\section{T3P144 \\ Nutrition education materials from cardiac short stay units are lacking information to guide and motivate weight loss}

\section{Ferreira, L. ${ }^{1}$; Miller, K. ${ }^{2}$; Botelho, R. $^{1}$; Dutra, E. ${ }^{1}$}

${ }^{1}$ Department of Nutrition, Faculty of Health, University of Brasília, Brasília, Federal District, Brazil, ${ }^{2}$ Faculty Of Health Sciences, Simon Fraser University, Burnaby, British Columbia, Canada

Introduction: Overweight and obesityare conditions that increase the risk and damage caused by many chronic diseases. Among them, stable Coronary Artery Disease (CAD) is a condition that reduces quality of life, life expectancy and productivity (WHO, 2011a). The modifiable risk factors that increase the risk of CAD and the complications related to it are also related to dietary habits. Changes in lifestyle are necessary to manage CAD and prevent secondary events (WHO, 2011b). That is why health professionals rely on printed educational materials to reach as many patients as possible, and those materials need to be tailored to the audience and comply to recent evidence to be effective in reducing risk of secondary events in CAD.

Methods: This was a Quality Improvement study. Four guidelines for secondary prevention of CAD (ECS 2010, AHA Nutrition Committee 2006, AHA/ACCF 2011, AHA/ACC 2006) were summarized into 25 topics. 3 Hospitals in British Columbia (Canada) provided all their printed materials used for nutrition education following CAD diagnose. Materials were scored according to the presence of guideline topics and using a Plain Language checklist validated by the Centers for Disease Control and Prevention (CDC, 2014).

Results: The topics extracted from the guidelines referred to CAD secondary risk factors. The guidelines were consistent in recommending that prevention of weight gain should be focused, but that individuals lost at least $5 \%$ of body weight if BMI was higher than $25 \mathrm{~kg} / \mathrm{m}^{2}$ at baseline. They also called for participation in programs focusing on behavior change, and that patients ate more home cooked meals. At last, the guidelines recommended the balance between calorie expenditure and intake. The materials scored low with regards to compliancy to dietary guidelines and Plain Language checklist scores. They only mentioned 1 topic about weight loss out of the 5 present in the guidelines. The materials were scored as unsuited for patients regarding plain language and actionability.

Conclusion: The educational materials might not be effective to instruct patients regarding weight loss. It is recommended that dietitians redevelop them, using the most recent evidence in this field and the language level fit for their audience.

\section{T3P145}

\section{Nutritional deficiencies after sleeve gastrectomy}

Paredes, S. $^{1}$; Alves, M. ${ }^{1}$; Obesity, M. ${ }^{1}$; Ribeiro, L. $^{2}$

${ }^{1}$ Hospital de Braga, ${ }^{2}$ Faculty Of Medicine, University Of Porto

Introduction: Malabsorptive procedures are associated with several nutritional deficiencies (1) since the omission of part of the intestine pre- 
vents the absorption of nutrients, whereas restrictive procedures have, in theory, a lesser potential to cause these deficiencies. Nevertheless, recent studies have also demonstrated nutritional deficits in restrictive procedures such as adjustable gastric banding. In respect to sleeve gastrectomy (SG), it is known that nutritional deficits are rarely evaluated (2). The aim of our study was to investigate nutritional deficiencies before and 1 year after SG.

Methods: Retrospectively, databases of 339 patients who underwent SG between $01 / 2011$ and 08/2015 were analyzed. The majority were women (86.4\%), with a mean age $41.8 \pm 10.6$ years-old and a mean body mass index (BMI) of $43.8 \pm 6.3 \mathrm{Kg} / \mathrm{m}^{2}$. One year after surgery, mean BMI was $29.9 \pm 5 \mathrm{Kg} / \mathrm{m}^{2}$. Electronic medical records were accessed to extract data on anthropometry, biochemistry and clinical status.

Results: Preoperatively, vitamin D deficiency was the most common nutritional deficit (34.8\%), although the majority of the individuals presented with insufficient vitamin D levels (63.8\%). There was a raising prevalence of nutritional abnormalities 1 year after surgery when compared to the preoperative period. One year after surgery, there was a significant increase in the prevalence of anemia (5.7\% vs $16.1 \%)$, ferritin deficiency $(10.3 \%$ vs $22.6 \%)$, folic acid deficit (7.3\% vs $17.6 \%)$ and vitamin B12 deficiency (4.6\% vs $18.9 \%$ ). There were no significant differences before and after surgery with respect to albumin. Elevated homocysteine levels, which can be related to folic acid deficiency, increased 1 year after surgery (6.2\% vs $21.3 \%)$. Even after supplementation, the prevalence of vitamin D deficiency has decreased, but not significantly.

Conclusion: Nutritional deficits are very common in candidates for bariatric surgery and vitamin D seems to be the most common deficiency. Although it is a restrictive procedure, after SG, micronutrient deficiencies can persist and even aggravate. Consequently, monitoring, screening and, when necessary, supplementation is essential in this population.

Conflict of Interest: None disclosed

Funding: No Funding was received for this work

References:

1 van der Beek ES., et al., Nutritional deficiencies in gastric bypass patients; incidence, time of occurrence and implications for post-operative surveillance. Obes Surg. 2015; 25:818-23

2 Pech, N., et al., Complications and nutrient deficiencies two years after sleeve gastrectomy. BMC Surg. 2012;5:12-13

\section{T3P146}

\section{Nutritional deficiencies in the first year after sleeve gastrectomy}

Gomes, V. ${ }^{1}$; Ferreira, F. '; Gomes, A. ' ; Barbosa, D. ${ }^{1}$; Bugalho, M. ${ }^{1}$

${ }^{1}$ Endocrinology,Diabetes and Metabolism Department,Hospital de Santa Maria, Lisbon, Portugal

Introduction: Sleeve gastrectomy (SG) has become popular as a single-stage procedure for the treatment of morbid obesity and its co-morbidities. SG is a restrictive procedure and the risk of developing nutritional deficiencies after surgery is considered low and, therefore, often not tested. However, this low risk status can probably be questioned, since resection of the gastric fundus can impact the absorption of a number of micronutrients, such as iron and vitamin B12. The aim of this study was to analyze the prevalence of nutritional deficiencies during the first year after SG.

Methods: Retrospective study of 97 obese patients, who underwent SG at a tertiary medical center, between January 2014 and November 2015. Patients were tested for anemia, albumin and micronutrient deficiencies (iron, zinc, folic acid, vitamin B12, vitamin D, calcium, phosphorus and magnesium) preoperatively and at 3, 6 and 12 months after surgery. Weight and BMI (body mass index) were also evaluated. Patients were instructed to take multivitamin/mineral tablets according to our hospital protocol. We determined the prevalence of de novo nutritional deficiencies in each evaluation after SG. Statistical analysis was carried out with SPSS software, version 20.
Results: $90,7 \%$ of the patients were female and the mean age was $46,8 \pm 10,3$ years (range: 20-67). Mean initial weight and BMI were $119,1 \pm 20,1 \mathrm{~kg}$ and $46,1 \pm 6,6 \mathrm{~kg} / \mathrm{m}^{2}$, respectively. At 12 months, there was a significant reduction in weight to $85,7 \pm 17,8 \mathrm{~kg}(\mathrm{p}<0,0001)$ and in BMI to $35,1 \pm 5,7 \mathrm{~kg} / \mathrm{m}^{2}$ ( $\left.\mathrm{p}<0,0001\right)$. At 3 months, $6 \%$ of the patients were anemic. Iron, zinc, folic acid, vitamin B12 and vitamin D deficiencies were found in $28 \%, 20 \%, 6 \%, 2 \%$ and $28 \%$, respectively. At 6 months, $9 \%$ of patients presented anemia. Deficiencies of iron, zinc, folic acid, vitamin B12 and vitamin D were 14\%,29\%,27\%, 6\% and 28\%, respectively. At 12 months, anemia was found in $16 \%$ of the patients. Deficiencies of iron, zinc, folic acid, vitamin B12, and vitamin D were 15\%, 33\%, 24\%, 3\% and $35 \%$, respectively. Calcium deficiency was detected, only at 12 months, in $4 \%$. There was no deficiency of magnesium, phosphorus and albumin during the 12 months follow-up.

Conclusion: Iron, zinc, folic acid and vitamin D were the most prevalent micronutrient deficiencies. These results strengthen the importance of routine screening to ensure an adequate supplementation and to prevent and/or correct postoperative deficiencies.

Conflict of Interest: None

Funding: No Funding

\section{T3P147}

\section{Obesity and diabetes type 2 in Republic of Macedonia - recent activities for improved management}

\section{Spasovski, M. ${ }^{1}$; Ristovska, G. ${ }^{2}$; Spiroski, I. ${ }^{3}$; Pangovski, I. ${ }^{4}$}

${ }^{1}$ Institute for Social Medicine, Faculty of Medicine University Ss. Cyril and Methodius, Skopje, ${ }^{2}$ Institute For Public Health,Republic Of Macedonia, ${ }^{3}$ Institute For Public Health, Republic Of Macedonia, ${ }^{4}$ Institute For Social Medicine,Faculty Od Medicine University Ss. Cyril And Methodius, Skopje,

Introduction: National data show that mortality from diabetes has increased by more than 1.6 times since 1991 in the Republic of Macedonia and the country has one of the highest rates of diabetes mortality in the European Region of WHO. Aim of the study was to evaluate prevalence of obesity in adult population as risk factor for diabetes type 2 .

Methods: Food consumption survey was performed in 2015 by the Institute of Public Health, supported by the Ministry of Health and the World Health Organization office in the country. Data was collected for food consumption and dietary habits, anthropometric characteristics of nutritional status and physical activity, on representative sample of population aged 18 and above. The survey included 226 men and 278 women, with average age of 46 years.

Results:Out of all participants, $36.4 \%$ of men and $31.2 \%$ of women were overweight, and $19.6 \%$ of men and $17.2 \%$ of women were obese. The prevalence of overweight and obesity in Macedonia presented by the survey is in line with the WHO estimates which we used previously since there hasn't been any survey on obesity for adult population in the country before.

Conclusions: Preventive measures for reduction of obesity and diabetes mortality have already been undertaken. Those were introduced by Health Insurance Fund and performed by primary care physicians. Thegoals of each primary care doctor are defined on the basis of the age and gender structure of the patients. The preventive goals in the performance package include preventivecheck-ups for diabetes mellitus and obesity, education and follow up for patients with risk. There is a need of evaluation of the effectiveness of the measures, as well as introduction of regular national risk factor survey for adults. 


\section{T3P148}

Obesity and diabetes type 2 in Republic of Macedonia - recent activities for improved management

\section{Spasovski, M. ${ }^{1}$; Ristovska, G. ${ }^{2}$; Spiroski, I. ${ }^{3}$; Pangovski, I. ${ }^{4}$}

${ }^{1}$ Institute for Social Medicine, Faculty of Medicine University Ss. Cyril and Methodius, Skopje ${ }_{\mu}{ }^{2}$ Institute Of Public Health, Republic Of Macedonia, ${ }^{3}$ Institute For Public Health, Republic Of Macedonia, ${ }^{4}$ Institute For Social Medicine,Faculty Od Medicine University Ss. Cyril And Methodius, Skopje,

National data show that mortality from diabetes has increased by more than 1.6 times since 1991 in the Republic of Macedonia and the country has one of the highest rates of diabetes mortality in the European Region of WHO. Aim of the study was to evaluate prevalence of obesity in adult population as risk factor for diabetes type 2 .

Methods: Food consumption survey was performed in 2015 by the Institute of Public Health, supported by the Ministry of Health and the World Health Organization office in the country. Data was collected for food consumption and dietary habits, anthropometric characteristics of nutritional status and physical activity, on representative sample of population aged 18 and above. The survey included 226 men and 278 women, with average age of 46 years.

Results:Out of all participants, $36.4 \%$ of men and $31.2 \%$ of women were overweight, and $19.6 \%$ of men and $17.2 \%$ of women were obese. The prevalence of overweight and obesity in Macedonia presented by the survey is in line with the WHO estimates which we used previously since there hasn't been any survey on obesity for adult population in the country before.

Conclusions: Preventive measures for reduction of obesity and diabetes mortality have already been undertaken. Those were introduced by Health Insurance Fund and performed by primary care physicians. Thegoals of each primary care doctor are defined on the basis of the age and gender structure of the patients. The preventive goals in the performance package include preventivecheck-ups for diabetes mellitus and obesity, education and follow up for patients with risk. There is a need of evaluation of the effectiveness of the measures, as well as introduction of regular national risk factor survey for adults.

\section{T3P149}

\section{Outcome of hospitalized intensive clinical treatment of obesity and its evaluation after one year}

\section{Braga, S. ${ }^{1}$; Leal, V. ${ }^{2}$; Reis, D. ${ }^{2}$; Sholl, J. ${ }^{2}$; Freitas, L. ${ }^{2}$ \\ ${ }^{1}$ Clinica da Obesidade, ${ }^{2}$ Clínica Da Obesidade}

Introduction: Obesity is a chronic disease associated with the development of several comorbidities. Their treatment is difficult and with high chances of failure and poor adherence. The objective of this study is to describe the treatment outcome based on a low and very low calorie diet associated with physical activity in obese individuals.

Methods: This is a retrospective cohort that accompanies obese adults hospitalized at an obesity treatment clinic in Camaçari, Bahia, Brazil, between May 2012 and October 2015, and its evolution for a period of one year after discharge from hospital. Treatment was divided into two phases. The first phase was intensive, hospitalized and the patients were submitted to a diet of between 500 and $1000 \mathrm{Kcal} /$ day, practice of physical activities and multidisciplinary follow-up. The second phase is called maintenance, in which the patient should attend the clinic monthly in order to maintain the result obtained in the initial phase. A treatment success was considered a weight loss greater than or equal to $50 \%$ of the excess weight and adherence to treatment was defined as a frequency of at least $50 \%$ in maintenance

Results: Eighty six obese women aged 20-59 years, 60 (73\%) women, with a mean weight of $121( \pm 23) \mathrm{kg}$ and a BMI of $43.6( \pm 6) \mathrm{kg} / \mathrm{m}^{2}$ were hospitalized for intensive treatment. The length of hospital stay lasted approximately $179( \pm 70)$ days. At the end of the initial phase of treatment, 21 (24\%) reach overweight and 80 (93\%) of the sample was successful in the treatment. When evaluating the maintenance phase, adherence to treat- ment was $44.2 \%$ at the end of the first semester after discharge from hospital and $36.1 \%$ at the end of one year. Successful treatment with weight loss $\geq 50 \%$ of excess weight was maintained in $90.3 \%$ of the 31 adherent patients at the end of one year.

Conclusion: The intensive treatment of obesity based on physical activity and diet of low and very low calorie, in addition to multidisciplinary follow up was effective and obtained a high success among the patients with adherence to the follow up one year after the medical discharge of the intensive phase. This fact alerts us to the need to seek ways to increase adherence to the treatment of obesity.

Conflict of Interest: There is no conflict interest

Funding: There was No Funding

\section{T3P151}

\section{Patients with high visceral adiposity index (VAI) show a larger improvement of insulin resistance after bariatric surgery}

Brix, J. ${ }^{1}$; Krzizek, E. ${ }^{1}$; Kopp, H. $^{1}$; Ludvik, B. ${ }^{1}$

${ }^{1}$ Rudolfstiftung Hospital; Department of Medicine I

Introduction: Although BMI and waist circumference (WC) have many limitations, they are still the most used criteria in the determination of obesity. In contrast to WC or BMI, the Visceral Adiposity Index (VAI) can determine visceral adiposity dysfunction (VAD). In our study we investigated whether VAI is related to the metabolic outcome of bariatric surgery.

Methods: We investigated 131 patients (mean age: $40 \pm 12$ years; mean BMI: $45.1 \pm 6.7 \mathrm{~kg} / \mathrm{m}^{2}$ ) with morbid obesity (MO) before and 2 years after bariatric surgery. Anthropometric data, cardiovascular risk factors and glucose tolerance test were performed. VAI was calculated as Waist Circumference $/(39.58+(1.89 * \mathrm{BMI})) *($ Triglycerides / 0.81) * $(1.52 /$ HDL-cholesterol) in women and Waist Circumference /(39.68+(1.89 * BMI $)$ * (Triglycerides / 1.03) * (1.31 / HDL cholesterol) in men and age stratified pre-defined cut off points ( $<30$ years: $2.52 ;>30<42$ years: $2.23 ;>42<52$ years: $1.92 ;>52<66$ years: 1.93 ; $>66$ years: 2.00$)$ were used to determine VAD. A non-obese healthy person has a VAI of 1 .

Results: After bariatric surgery, VAI dropped significantly $(2.5[1.6,3.9]$ vs $1.3(0.8,1.9] ; \mathrm{p}<0.001)$. Compared to $59.7 \%$ before bariatric surgery, only $20.6 \%$ of the patients still had VAD postoperatively. Patients with VAD before bariatric surgery had a significantly larger decrease in HOMA-IR $(4.0[2.8,6.0]$ vs $3.3[1.3,5.8] ; \mathrm{p}=0.035),(\mathrm{HbA} 1 \mathrm{c}(0.4[0.2,0.9]$ vs $0.3[0.1,0.8] \% ; \mathrm{p}=0.011)$ and a trend towards improvement in fasting glucose $(12.0[3,24]$ vs $9.5[0.3,17.5] \mathrm{mg} / \mathrm{dl} ; \mathrm{p}=0.059)$. There were no differences regarding weight $(\mathrm{p}=0.632)$ or BMI $(\mathrm{p}=0.872)$ compared to patients without VAD before surgery.

Conclusion: We were able to demonstrate that in patients with VAD the amelioration of insulin resistance after bariatric surgery is more profound compared to patients without VAD, independent of weight loss. Therefore, patients with preoperatively elevated VAD might benefit more from bariatric surgery.

Conflict of Interest: None Disclosed

Funding: No Funding

\section{T3P152}

\section{Patients' follow-up adherence after bariatric surgery}

Souteiro, P. ${ }^{1}$; Pedro, J. ' ; Magalhães, D. ${ }^{1}$; Neves, J. ; Oliveira, S. '; Belo, S. '; Guerreiro, V. ${ }^{1}$; Bettencourt-Silva, ${ }^{1}{ }^{1}$; Costa, M. ${ }^{1}$; Freitas, $P^{1}{ }^{1}$; Varela, A. ${ }^{1}$; Queirós, J. ; Carvalho, D. ${ }^{\text {; }}$ Group, A. ${ }^{2}$

${ }^{1}$ Department of Endocrinology, Diabetes and Metabolism, Centro Hospitala São João, Porto, Portugal, ${ }^{2}$ Multidisciplinary Group For Surgical Management Of Obesity, Centro Hospitalar São João, Porto, Portugal

Introduction: Surgical treatment of obesity implies regular hospital follow-up in the first years after surgery. This should be conducted by a spe- 
cialized team in order to perform the required therapeutic adjustments to the patients' new clinical situation.

Objective: To evaluate the adherence to hospital follow-up of patients undergoing bariatric surgery and to study the factors that could explain their dropout.

Methods: Cross-sectional study of 1449 patients followed by the Multidisciplinary Group for Surgical Management of Obesity. Baseline anthropometric and analytical parameters were evaluated over 4 years after surgery. Patients' absence to two consecutive clinical and analytical evaluations was considered as a dropout.

Results: In the second year of follow-up 48 (3.3\%) patients that underwent bariatric surgery abandoned hospital visits. The dropouts total number at the 3rd and 4th post-operative years was of 128 (14.6\%) and $119(22.4 \%)$ patients, respectively. Only 8 patients returned to the follow-up visits after being considered as dropouts. Male patients presented higher abandon rates at the 2nd $(\mathrm{OR}=1.96$, CI $1.01-3.85, \mathrm{p}<0.05)$ and 3rd follow-up years $(\mathrm{OR}=2.04$, CI 1.27- 3.23, $\mathrm{p}<0.001)$ after the surgical procedure. Patients with hypothyroidism before surgery had a higher adherence to visits at the $3 \mathrm{rd}(\mathrm{OR}=3.70, \mathrm{CI} 1.14-12.5, \mathrm{p}<0.05)$ and 4rd years analysis $(\mathrm{OR}=4.76$, CI $1.15-20.0, \mathrm{p}<0.05)$. No statistically significant differences were found regarding the type of surgery, patients' age, the presence of hypertension, dyslipidaemia and diabetes, or the weight loss percentage. Conclusions: Adequate postoperative follow-up is essential for the success of surgical treatment of obesity. Identifying the profile of patients who abandon the follow-up can help multidisciplinary teams to promote post-surgery treatment adherence.

\section{T3P153 \\ Pistachio intake modulates urinary gut microbiota-related metabolites in pre-diabetic subjects}

Hernández-Alonso, P.' ; Cañueto, D. '; Giardina, S. '; Salas-Salvadó, J.' ; Cañellas, N. ${ }^{2}$; Correig, X. ${ }^{2}$; Bulló, $M .{ }^{1}$

${ }^{1}$ Human Nutrition Unit-URV-IISPV, Reus, Spain; and CIBEROBN-ISCIII, Madrid, Spain, ${ }^{2}$ Metabolomics Platform-Urv-lispv, Tarragona, Spain; And Ciberdem-Isciii, Madrid, Spain, ${ }^{3}$ Human Nutrition Unit-Urv-lispv, Reus, Spain

Introduction: Nut consumption could modulate different metabolic pathways involved in several metabolic diseases. We therefore investigated whether chronic consumption of pistachio modifies the urine metabolome in pre-diabetic subjects.

Methods: We designed a randomized crossover clinical trial in 39 pre-diabetic subjects. Subjects consumed a pistachio-supplemented diet (PD, $50 \%$ carbohydrates, $33 \%$ fat, $17 \%$ protein including $57 \mathrm{~g} / \mathrm{d}$ of pistachios daily) and a control diet (CD, 55\% carbohydrates, $30 \%$ fat, $15 \%$ protein) for 4 months each, separated by a 2 -week wash-out. Nuclear magnetic resonance (NRM) was performed to determine changes in 24-h urine metabolites.

Results: Significant changes in urine metabolites according to the different intervention periods were found in uni- and multivariate analysis. Score plot of the first two components of the multilevel partial least squares discriminant analysis (ML-PLS-DA) showed a clear separation of the two intervention periods. Three metabolites related with gut microbiota metabolism (i.e. hippurate, p-cresol sulfate and dimethylamine) were found decreased in PD compared to the $\mathrm{CD}(P<0.05)$. Moreover, cis-aconitate (intermediate of the tricarboxylic acid; TCA) was also found decreased following PD compared to $\mathrm{CD}$. Intra-group analysis showed that creatinine levels were significantly increased in PD $(P=0.023)$, whereas trimethylamine $\mathrm{N}$-oxide (TMAO) was found significantly reduced following $\mathrm{PD}(P=0.034)$.

Conclusion: Our results suggest that chronic pistachio consumption may modulate some urinary metabolites related to gut microbiota metabolism and the TCA cycle; all associated with metabolic alterations related to insulin resistance and type 2 diabetes.

Jordi Salas is a non-paid member of the Scientific Committee of the Nut and Dried Fruit Foundation.
This study was in part funded by the American Pistachio Growers (USA) and Paramount Farms. Pablo Hernández-Alonso was the recipient of a predoctoral fellowship from the Generalitat de Catalunya's Department of Universities [FI-DGR 2014]

\section{T3P154}

\section{Profile of Greeks who seek dietitian's advice}

\section{loannis, N. ${ }^{1}$;}

${ }^{1}$ Hellenic Open University

The purpose of this study was to investigate the profile of Greeks that seek dietitian's advice. The findings of this study could be later compared with similar studies in other countries and examine whether there are similarities worldwide between the people who ask for help about their body weight.

The study was conducted among ' clients"' who seek dietitian's advice in a private dietitian's office between during the years 2015-2016. The individuals that were included in the study were people who visited at least for 4 weeks their dietitian. The dietitian had to fill in a questionnaire while discussing with their client. The questionnaire included questions about anthropometric characteristics, personal goals, previous weight loss attempts, eating habits, physical activity and others.

The results showed that the percentage of men who visit dietitians in Greece is very low compared to the percentage of the women. Men seek for help to lose weight only after they achieve a high BMI, while women most often aim to lose weight for cosmetic reasons, even though their BMI might be a potential risk factor for their health. In addition, men usually make less weight loss attempts compared to women. Moreover, there is a group of 3-5 foods that are the foods that most people choose not to eat. Regarding physical activity, men walk less than women, but both genders are willing to start exercising to improve their weight.

The profile of the Greeks who seek for dietitian's advice, seems to be different between men and women. Dietitian's advice can play a key role in the lifestyle modification and improvement of the overall health of Greeks. Greek Ministry of Health should invest on Public health care by increasing dietitians in Public Hospitals because obesity is a serious problem in Greece and citizens can't afford a visit to a private dietitian.

\section{T3P155}

\section{Post bariatric surgery hypoglycaemia management: A case series}

\section{Haridass, S. ${ }^{1}$; Kushe, M. ${ }^{1}$; Joseph, V. ${ }^{1}$; Rajeswaran, $C^{1}$ \\ ${ }^{1}$ The Midyorks NHS Hospitals Trust}

Introduction: Bariatric surgery is an effective intervention for morbid obesity, resulting in substantial reduction in obesity associated co-morbidity. Number of bariatric surgeries performed worldwide is gradually increasing. Consequently, we are encountering more medical complications post bariatric surgery including hypoglycaemia. Hypoglycaemia occurring post bariatric surgery can be disabling leading to weight gain and poor quality of life.

Discussion: Here we discuss a group of patients who have had disabling hypoglycaemic episodes post bariatric surgery. We followed a systematic protocol to investigate and treat hypoglycaemia. First line investigations involved ruling out the common causes of hypoglycaemia including overtreatment of diabetes, nutritional issues, adrenal insufficiency, malignancies, liver and renal diseases. We treated dumping syndrome with low glycaemic index food, small and frequent meals. In the event of poor response to these measures, medical management including alpha-glucosidase inhibitors, somatostatin analogues and diazoxide were prescribed. Some patients may not respond to any of the above treatment measures and may need second-line investigations including mixed meal test, analysing hormone levels like GLP-1, GIP, PeptideYY and FGF-19 to appropriately target any abnormality. Continuous naso-jejunal feeding has also been used to diagnose and manage a small group of patients. Conclusion: 
Disabling hypoglycaemic episodes post bariatric surgery is an exigent medical issue, requiring a clear protocol for investigation and management. Endocrinologists need to be aware of this systematic approach to diagnose, manage and also be aware of newer medications including Exendin 9-39.

Conflict of Interest: None

Funding: None

\section{T3P156}

\section{Postprandial GLP-1 secretion is inversely proportional to BMI}

Blomster, H. ' ; Elofsson, H. ' Gunnarsson, R. ' ; Ritzén, H. ' Schwanbeck, M. '; Lundequist, A. ${ }^{1}$; Öberg, D. ${ }^{1}$; Grufman, $L .{ }^{1}$

${ }^{1}$ Mercodia $\mathrm{AB}$

Introduction: The incretin Glucagon-like peptide 1 (GLP-1) has received a lot of attention due to its ability to stimulate the release of insulin and suppress glucagon secretion. Intriguingly, GLP-1 contributes to reduction in visceral and subcutaneous fat deposits, which is especially evident in individuals treated with GLP-1 receptor antagonists. As a consequence of GLP-1's effect on body fat, the analog Liraglutide has been approved by FDA to facilitate body weight loss in non-diabetic obese individuals. A number of studies investigating the association of type 2 diabetes and the ability to secrete GLP-1 have arrived at conflicting conclusions; some studies suggest that there is an inverse correlation between BMI and postprandial GLP-1 levels whereas others show no such association. The differences may be due to differences in population, the type of meal used or the assays used to quantify GLP-1 concentrations.

In the absence of DPP4-inhibitors, GLP-1 is rapidly converted from the active $7-36 \mathrm{NH}_{2}$ to the metabolite $9-36 \mathrm{NH}_{2}$. Since the total levels of GLP-1 in fasted individuals in the circulation is less than $15 \mathrm{pmol} / \mathrm{L}$, accurately capturing differences in the the pre- and postprandial levels of GLP-1 requires very sensitive and robust assays. Thus, to study the association between body weight and GLP-1 secretion, we developed an ELISA employing chemiluminescence to increase sensitivity.

Methods: A sandwich ELISA was constructed, which subsequently was validated according to CLSI and FDA/EMA guidelines. The total GLP-1 plasma levels in a cohort of 120 fasting individuals were determined.

Twenty healthy individuals (BMI 19-35, age 26-56) donated plasma prior to and after consuming a standardized mixed meal. The total GLP-1 levels in preprandial and postprandial ( 2 \& 4 hours) plasma were determined. The relationship between absolute levels of GLP-1 pre- and postprandially, as well as the increase in GLP-1 postprandially, was investigated.

Results: A very robust, accurate and sensitive ELISA was developed with no cross-reactivity to similar peptides, a sensitivity of $1 \mathrm{pmol} / \mathrm{L}$ (LLOQ), and a precision of $<11 \%$. Using the validated ELISA, we determined a reference range of $0.9-18.5 \mathrm{pmol} / \mathrm{L}$ for total GLP-1 in healthy fasting individuals. Subsequently, we generated results indicating that obese individuals secrete less GLP-1 upon consuming a mixed-meal.

Conclusion: The sandwich ELISA provides reliable data, which combined with the reference range will be of great help for research into mechanisms involving GLP-1. Preliminary data suggest that obesity might be associated with impaired GLP-1 release. With GLP-1 being an important incretin, it is of interest to investigate whether the impairment can be used to predict the onset of type 2 diabetes.

\section{T3P157}

Predictive model of weight loss after RYGB with 60 months of follow-up: A useful tool to manage the post-operative outcomes.

\section{Seyssel, K. ${ }^{1}$; Suter, M. ${ }^{2}$; Pattou, F. ${ }^{3}$; Verkindt, H. ${ }^{3}$; Disse, E. ; Robert, M. ${ }^{5}$;} Tappy, L. ${ }^{1} ;$ Giusti, V. ${ }^{6}$

${ }^{1}$ Department of Physiology, Faculty of Biology and Medicine, University of Lausanne, Lausanne, Switzerland, ${ }^{2}$ Department Of Surgery, Lausanne University Hospital, Lausanne, Switzerland / Department Of Surgery, Hôpital RivieraChablais, Aigle-Monthey, Switzerland, '3Endocrine Surgery, Inserm 1190, European Genomic Institute For Diabetes, Lille University Hospital, Lille, France, ${ }^{4}$ Claude Bernard Lyon 1 University, Lyon, France; Department Of Endocrinology Diabetology And Nutrition, Lyon Sud University Hospital, Hospices Civils De Lyon, Lyon, France / Fédération Hospitalo-Universitaire Do-It, Centre Intégré Et Spécialisé De L'Obésit, ${ }^{5}$ Fédération Hospitalo-Universitaire Do-It, Centre Intégré Et Spécialisé De L'Obésité De Lyon, Université Lyon 1, Crnh-Ra, Hospices Civils De Lyon, Lyon, France / Department Of Digestive Surgery, Center Of Bariatric Surgery, Edouard Herriot Hospital, Hospic, ${ }^{6}$ Metabolic Center, Fribourg Hospital $\mathrm{Hfr}$, Fribourg, Switzerland

Introduction: Roux-en-Y gastric bypass (RYGB) is one of the most effective bariatric procedure to achieve weight loss in morbidly obese patients Different factors are known to impact weight loss outcomes after surgery, such as age, gender, preoperative weight but also patient's motivation. Weight loss prediction could be helpful to select good candidates for bariatric surgery, maintain motivation during follow-up and limit the failures. Therefore, it appears interesting to develop a realistic predictive tool of weight loss for each patient based on their preoperative parameters.

Methods: A Swiss cohort of 444 patients, who underwent a RYGB surgery was used in order to develop a predictive model of weight loss. We used multiple linear regression models to predict weight loss at each time points of follow-up $(3,6,12,18,24,36,48,60$ months) considering simple anthropometric parameters as age, height, gender and preoperative weight. Equations were as follows: Weight loss $(\mathrm{kg})=$ Constant $+\mathrm{A}^{*}$ (Preoperative weight $(\mathrm{kg}))+\mathrm{B}^{\star}($ Age $($ years $))+\mathrm{C}^{\star}(\operatorname{Sex}(\mathrm{M}$ or $\mathrm{F}))+\mathrm{D}^{\star}($ Height $(\mathrm{m}))$. In order to validate our model, we applied our equations on two different cohorts of patients having undergone RYGB (Lille France ABOS cohort and Lyon France cohort) to generate predicted weight loss (kg), and we compared predicted weight to real weight using spearman's correlation. Results: The results of the models are presented in the table 1 below. Correlation between predicted weight and real weight was highly significant in both cohorts used for validation of the model. We found a $\mathrm{R}=0.81$ at 12 months and $\mathrm{R}=0.68$ at 60 months for the Lille's cohort $(\mathrm{p}<0.001$ for all). In the same manner, we found for the Lyon's cohort a $\mathrm{R}=0.93$ at 3 months, $\mathrm{R}=0.87$ at 6 months, $\mathrm{R}=0.81$ at 12 months, $\mathrm{R}=0.78$ at 18 months, $\mathrm{R}=0.71$ at 24 months, $\mathrm{R}=0.66$ at 36 months, $\mathrm{R}=0.62$ at 48 months and $\mathrm{R}=0.71$ at 60 months ( $\mathrm{p}<0.001$ for all).

Conclusion: Our statistical model to predict weight loss outcomes after gastric bypass seems accurate. It could be an interesting tool to select good candidates for bariatric surgery and to improve the outcomes during the follow-up.

Conflict of Interest:none

Funding:no

\section{T3P158}

Pregnancy after bariatric surgery: Time to improve awareness!

Francesco, F. '; Alesandro, S. ; Alice, B. '; Monica, M. '; Paola, V. '; Carlo, S. ';

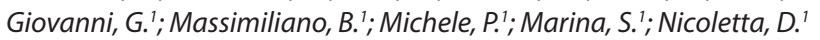

${ }^{1}$ San Raffaele Scientific Institute, Milan, Italy

Background As surgical techniques are evolving the indications for bariatric surgery are widening, with an increasing number of women of childbearing age turning to this approach for metabolic care. The impact of both obesity and surgical-induced weight loss on pregnancies starting closely after bariatric surgery are well documented in the literature. Aim 
Table 1. to T3P157

\begin{tabular}{|c|c|c|c|c|c|c|c|c|c|c|c|c|c|c|c|c|}
\hline & \multicolumn{2}{|l|}{$3 \mathrm{mo}$} & \multicolumn{2}{|l|}{$6 \mathrm{mo}$} & \multicolumn{2}{|l|}{$12 \mathrm{mo}$} & \multicolumn{2}{|l|}{$18 \mathrm{mo}$} & \multicolumn{2}{|l|}{$24 \mathrm{mo}$} & \multicolumn{2}{|l|}{$36 \mathrm{mo}$} & \multicolumn{2}{|l|}{$48 \mathrm{mo}$} & \multicolumn{2}{|l|}{$60 \mathrm{mo}$} \\
\hline & Value & $P$ & Value & $P$ & Value & $P$ & Value & $P$ & Value & $P$ & Value & $P$ & Value & $P$ & Value & $P$ \\
\hline $\begin{array}{l}\text { A: } \\
\text { Preoperative } \\
\text { weight }(\mathrm{kg})\end{array}$ & 0.17 & $<0.001$ & 0.26 & $<0.001$ & 0.4 & $<0.001$ & 0.47 & $<0.001$ & 0.5 & $<0.001$ & 0.5 & $<0.001$ & 0.48 & $<0.001$ & 0.46 & $<0.001$ \\
\hline $\begin{array}{l}\text { C: } \\
\text { Sex }(F)\end{array}$ & -1.4 & 0.001 & -0.82 & 0.1 & 1.1 & 0.1 & 1.4 & 0.07 & 2.2 & 0.01 & 2.8 & $<0.001$ & 3.1 & $<0.001$ & 3.3 & $<0.001$ \\
\hline $\begin{array}{l}\text { D: } \\
\text { Height (m) }\end{array}$ & -0.99 & 0.8 & -3.2 & 0.6 & -3.4 & 0.7 & -11.5 & 0.2 & -9.7 & 0.3 & -9.4 & 0.3 & -9.5 & 0.3 & -5.9 & 0.6 \\
\hline $\mathrm{R} 2$ & 0.44 & & 0.46 & & 0.45 & & 0.48 & & 0.48 & & 0.47 & & 0.42 & & 0.37 & \\
\hline
\end{tabular}

of our study was to document the awareness of women about pregnancy after bariatric surgery.

Methods We identified 79 women of childbearing age (18 to 45 years) who underwent bariatric surgery (13 BIB, 27 LAGB and 27 Sleeve gastrectomy) at the San Raffaele Scientific Institute from 01/02/2010 to 31/07/2016. Sixty-seven of them (84.8\%) accepted to complete a closed-answer questionnaire about awareness of pregnancy after bariatric surgery. Median time after bariatric surgery was 26.5 months (IQR 7.7 to 60.7 ). We also e-mailed 37 accredited for bariatric surgery in Italy to enquire about the information on pregnancy after bariatric surgery provided to their patients of childbearing age. Response was received from 11 centers, $29.7 \%$ of the total (6,176 patients treated over the years 2013-2015, of whom 78\% were women of childbearing age).

Results Although women were well aware (73\% of the responders) that obesity affects fertility and the probability of conception, the majority of them ( $\geq 85 \%)$ was unaware of the potential impact that obesity itself and bariatric surgery have on a pregnancy. Seventy-three percent of the women reported to have received no information on the recommended waiting time to start a pregnancy after bariatric surgery and over $85 \%$ of them were unaware of the need for nutritional counselling or for specific nutritional supplements during pregnancy after bariatric surgery. The response rate did not vary according to women' interest in a future pregnancy nor to the type of bariatric surgery.

Conclusions The majority of the bariatric surgery centers reported to provide some counselling on pregnancy after bariatric surgery to women of childbearing age, although only one center had prepared a dedicated leaflet on this topic to give to patientsOur study document that in Italy the impact of both obesity and surgical-induced weight loss on pregnancies after bariatric surgery are poorly acknowledged by both women of childbearing age and health care teams. There is a need to increase awareness of women and their partners on pregnancy after bariatric surgery and to include this topic in training programs for health professionals caring for women of childbearing age undergoing bariatric surgery.

No Funding was received for this work

\section{T3P159}

\section{Prevalence of obesity comorbidities in $\mathbf{3 4 6 8}$ consecutive obese and superobese patients: The role of the degree of obesity}

Capoccia, D. ${ }^{\prime}$; Coccia, F. ${ }^{2}$; Testa, M. ${ }^{2}$; Guarisco, G. ${ }^{2}$; Rendina, R. ${ }^{2}$; Leonetti, F. ${ }^{2}$ ${ }^{1}$ Sapienza University, Rome, Italy, ${ }^{2}$ Sapienza University

Background: correlation between degree of obesity, including severe obesity, and prevalence of associated comorbidities is unclear.

Aim: to analyze the prevalence of type 2 diabetes (DM2), hypertension (HY) and dyslipidemia (DYS) in different classes of BMI.
Methods: 3468 consecutive patients $(27 \% \mathrm{M})$ since 2000 , age $44.1 \pm 12 \mathrm{yrs,}$ body weight range $89-223.4 \mathrm{Kg}$ and BMI range $30-99 \mathrm{Kg} / \mathrm{m}^{2}$, were studied and divided in 5 classes of BMI (I 30-35, II 35-40, III 40-50, IV 50-60,V $>60$ ). For each class, prevalence of DM2, HY and DYS was analized and transaminases were detected.

Results: in the entire cohort we found: prevalence of DM2 21,1\%, HY34.1\%, DYS 50.1\%; AST and ALT $23.6 \pm 10.4$ and $32.9 \pm 19.7 \mathrm{U} / \mathrm{L}$. In I class(671 patients) we found: prevalence of DM2 $28.1 \%, \mathrm{HY} 29.7 \%$ and DYS37.5\%; AST and ALT $23.5 \pm 15 \mathrm{and} 31.5 \pm 29 \mathrm{U} / \mathrm{L}$; in diabetic patients HbA1c $8.2 \pm 2.2 \%$.In II class (937 patients) we found: prevalence of DM2 20.5\%,HY31,2\% and DYS 49,4\%;AST and ALT $22.3 \pm 11.6$ and $31 \pm 23 \mathrm{U} / \mathrm{L}$; in diabetic patients HbAlc $7.9 \pm 1.9 \%$.In III class (1350 patients)we found: prevalence of DM2 26\%,HY35.9\%andDYS49.6\%;AST and ALT $24.5 \pm 13.4$ and $34.7 \pm 25.3 \mathrm{U} / \mathrm{L}$; in diabetic patients HbA1c $7.5 \pm 1.9 \%$. In IV class (414 patients) we found: prevalence of DM2 28.5\%,HY40.4\%and DYS 51.1\%; AST and ALT $23.1 \pm 14.5$ and $33.6 \pm 22.6 \mathrm{U} / \mathrm{L}$; in diabetic patients $\mathrm{HbAlc} 7.6 \pm 1.7 \%$.In $\mathrm{V}$ class (96 patients) we found: prevalence of DM2 32.3\%, HY42.7\% and DYS41.7\%; AST and ALT $22.6 \pm 11.1$ and $30.5 \pm 16.9 \mathrm{U} / \mathrm{L}$; in diabetic patients HbA1c $7.9 \pm 1.9 \%$. In I class prevalence of HY and DYS was significantly lower compared to other classes $(\mathrm{p}<0.01)$, instead of DM2 which prevalence was higher compared to II class $(p<0.01)$ and not significantly different from other classes. In diabetic patients of I class,HbAlc was significantly higher compared to III class and not significantly different from other classes. ALT levels were significantly higher in III and IV classes $(\mathrm{p}<0.01)$ as expected whereas AST levels did not show significant differences.

Conclusions: prevalence of HY and DYS increased with degree of obesity instead of DM2 that didn't show a lower prevalence in I class compared to others. Moreover, in diabetic patients HbAlc did not increase with increasing BMI.

\section{T3P160}

\section{Promoting satiation and satiety within a weight management} programme improves appetite control and weight loss.

Buckland, N. ${ }^{1}$; Camidge, D. ${ }^{1}$; Croden, . $^{1}$; Hetherington, M. ${ }^{1}$; Blundell, J. ${ }^{1}$; Finlayson, $\mathrm{G}^{1}$

'University of Leeds

Introduction: Weight management programmes (WMPs) that promote satiation and satiety by encouraging behaviour changes towards unrestricted intake of low energy density (LED) food choices might be more effective than self-led traditional calorie reduction WMPs. The SWIPSS trial examined the effect of a commercial weight loss programme promoting unrestricted intake of LED foods [Slimming World, UK (SW)] on body weight and weight loss experience compared to a self-led calorie-reduction standard care WMP [Live Well, NHS standard care (SC)]. The tri- 
al also examined the effects of meals varying in energy density (consistent with SW approach) on appetite and energy intake.

Methods: Ninety-six women who were overweight or obese (age: $41.8 \pm 1.4 \mathrm{y}$; BMI: $33.3 \pm 0.4 \mathrm{~kg} / \mathrm{m}^{2}$ ) were recruited from $\mathrm{SW}$ and $\mathrm{SC}$ weight management arms of a 14-week clinical trial. Weight loss experience was assessed with weekly questionnaires. In weeks 3 and 12 as part of laboratory intensive probe days, participants' diurnal appetite ratings and ad libitum meal intake were assessed in response to iso-caloric LED and high energy dense breakfast and lunch meals.

Results: LED meals increased participants' subjective sensations of fullness and reduced sensations of hunger throughout the day and, reduced total day energy intake compared to high energy dense meals (all $p s<.001)$. The SW group lost more weight and were more likely to achieve clinical weight loss $(>5 \%)$ compared to the SC group. The SW group experienced greater control over eating, found the WMP easier to adhere to, experienced more enjoyment losing weight, were more satisfied with the WMP and were more motivated to continue the programme compared to the SC group.

Conclusion: A commercial WMP supporting behaviour changes towards LED food choices is an effective strategy for weight loss with health benefits and improved experience above self-led calorie reduction programmes [CT\#NCT02012426].

Conflict of Interest: None

Funded by Slimming World, UK

\section{T3P162}

\section{Psychometric predictors of drop out from a commercial weight management programme during a 12-month online compassion focused web-based intervention.}

\section{Stubbs, R. ${ }^{1}$; Stalker, $C^{2}$; Catarino, $F^{2}$; Duarte, $C^{3}$; Basran J $^{2}$; Gilbert, $P^{4}$;} Morris $L^{5} ;$ Horgan, $G^{6}$

${ }^{1}$ Appetite Control and Energy Balance Research Group, School of Psychology, Faculty of Medicine and Health, University of Leeds LS2 9JT, ${ }^{2}$ College Of Life And Natural Sciences, University Of Derby, Derby, De22, 1Gb., ${ }^{3}$ Cognitive And Behavioural Centre For Research And Intervention, University Of Coimbra, Rua Do Colégio Novo, Apartado 6153, 3001-802 Coimbra, Portugal., ${ }^{4}$ College Of Life And Natural Sciences, University Of Derby, Derby, De22, 1Gb, ${ }^{5}$ Slimming World, Clover Nook Road, Somercotes, Alfreton, Derbyshire De55 4Rf.,

${ }^{6}$ Biomathematics And Statistics Scotland, Rowett Institute Of Nutrition And Health, Foresterhill House, Aberdeen Ab25 2Zd.

Introduction: While attrition rates in weight management programmes are generally high, it is not clear to what extent aspects of self-evaluation, emotion regulation, depression, anxiety and stress affect adherence to longer term behaviour change programmes.

Methods: This study used data from a two-arm, non-randomised parallel design using online compassion focused web-based exercises in a commercial weight management programme to model predictors of drop out over a 12 month period in 964 participants. Predictors of drop out were modelled by logistic regression using drop out as the dependent variable and baseline measures of age, group, weight, psychological adjustment, self-evaluation, eating behaviour characteristics and compassion variables as independent variables. An odds ratio $<1.0$ indicates a lower, and $>1.0 \mathrm{a}$ higher, risk of drop out within 12 months.

Results: Table 1 shows predictors of drop out at 12 months with associated odds ratios, confidence intervals and $\mathrm{p}$ values.

Conclusion: Negative aspects of psychological adjustment and self-evaluation, indices of loss of control of eating predicted drop out; indices of wellbeing and control of eating predicted continued programme adherence by 12 months. These data suggest new intervention targets to enhance continued engagement with multicomponent weight management programmes.

Conflict of Interest: RJS is a consultant to Slimming World through University of Leeds Consulting.
Funding: CD was funded by Fundação para a Ciência e a Tecnologia - SFRH/ $\mathrm{BD} / 76858 / 2011$.This study was funded by a grant from Slimming World UK.

Table 1.

\begin{tabular}{|l|l|l|l|l|}
\hline & $\begin{array}{l}\text { Odds } \\
\text { ratio }\end{array}$ & L.CI & U.CI & p. value \\
\hline Age & 0.960 & 0.950 & 0.971 & $<0.001$ \\
\hline Group (control) & 1.58 & 1.061 & 2.252 & 0.011 \\
\hline Weight & 1.886 & 1.252 & 2.841 & 0.002 \\
\hline Psychological adjustment & & & & \\
\hline Depression & 2.431 & 1.447 & 4.085 & 0.001 \\
\hline Anxiety & 2.497 & 1.56 & 3.997 & $<0.001$ \\
\hline Stress & 1.724 & 1.094 & 2.716 & 0.017 \\
\hline Wellbeing & 0.529 & 0.348 & 0.803 & 0.002 \\
\hline Self- Evaluation & & & & \\
\hline External shame & 1.573 & 1.029 & 2.403 & 0.033 \\
\hline Body Image Shame & 1.913 & 1.27 & 2.883 & 0.002 \\
\hline Inadequate self & 1.362 & 0.902 & 2.056 & 0.133 \\
\hline Reassured self & 0.87 & 0.577 & 1.31 & 0.495 \\
\hline Hated Self & 1.473 & 0.846 & 2.566 & 0.162 \\
\hline Weight focused negative affect & 2.281 & 1.518 & 3.426 & $<0.001$ \\
\hline Weight focused positive affect & 0.534 & 0.327 & 0.873 & 0.011 \\
\hline Eating Behaviour & & & & \\
\hline Binge eating symptoms & 1.983 & 1.306 & 3.01 & 0.001 \\
\hline Flexible Control & 0.566 & 0.371 & 0.864 & 0.007 \\
\hline Rigid control & 1.354 & 0.871 & 2.106 & 0.169 \\
\hline Disinhibition & 1.626 & 1.08 & 2.448 & 0.017 \\
\hline Susceptibility to hunger & 1.472 & 0.979 & 2.214 & 0.058 \\
\hline Dietary restraint & 0.65 & 0.442 & 0.956 & 0.025 \\
\hline Restrictive eating & 0.879 & 0.584 & 1.324 & 0.529 \\
\hline Eating guilt & 1.879 & 1.261 & 2.801 & 0.002 \\
\hline Compassion Items & & & & \\
\hline Self-compassion & 0.713 & 0.465 & 1.095 & 0.115 \\
\hline Compassion to others & 0.853 & 0.563 & 1.293 & 0.445 \\
\hline Compassion from others & & & & \\
\hline
\end{tabular}

T3P163

Quality of life assessment determined by the SF-36 brazil instrument among type 2 diabetes patients on insulin treatment

Junqueira, N. ${ }^{1}$; Rosa, É. ${ }^{1}$; Amato, A. ${ }^{1}$

${ }^{1}$ University of Brasilia

Introduction: Type 2 diabetes (T2D) has a negative impact on quality of life, which is attributed to several aspects, including possible need to deal with subcutaneous injections of insulin or puncture of the digital pulp for capillary glucose measurement. Insulin therapy is an important therapeutic strategy for DM2 in several clinical settings. However, insulin therapy is also associated with an increased risk of hypoglycemia and weight gain, in addition to the discomfort associated with the route of administration. The objective of this study was to compare quality of life measures between patients on insulin treatment and patients treated with oral antihyperglycemic agents only.

Methods: Thirty-eight T2D patients (26 on insulin treatment) were selected from the endocrinology clinic of the University Hospital of Brasília. Demographic information (age, gender, marital status, occupation and family residence) and clinical characteristics (presence of chronic pain, time since diagnosis of T2D and duration of treatment with insulin) were obtained. The patients answered the Brazil SF-36 quality of life evaluation questionnaire, which includes information on functional capacity, physical aspects, pain, general health, vitality, social aspects, emotional aspects and mental health. The results from patients on insulin treatment were 
compared to those form patients on antihyperglycemic agents only using using T-test of independent samples, with a $\mathrm{p}$ value of less than 0.05 indicating statistical significance.

Results: Patients on insulin therapy had a higher average time since diagnosis of T2D when compared to those who treated with oral antihyperglycemic agents only $(16 \pm 8 ; 9 \pm 6$ years, respectively, $\mathrm{p}=0.028)$. There was no significant difference in sociodemographic characteristics between groups. Among the quality-of-life domains assessed by the SF-36 instrument, it was observed that patients on insulin therapy had lower scores of functional capacity $(\mathrm{p}<0.001)$, physical limitations $(\mathrm{p}<0.001)$ and vitality $(p=0.037)$ when compared to patients on antihyperglycemic agents only. There was no significant difference between the other five domains between groups.

Conclusion: Patients on insulin therapy showed a higher average time since T2D diagnosis and lower quality of life assessed by the SF-36, especially with respect to physical, functional and social aspects. Although it is not possible to define causality between insulin therapy and lower quality of life, these findings indicate the need to explore this association in order to investigate potential aspects of T2D management that can be the focus of strategies to improve the quality of life of patients.

\section{T3P164}

\section{Reduction in diabetes mellitus pharmacological treatment burden after bariatric surgery}

Roque, C. ${ }^{1}$; Fonseca, R. ${ }^{\prime}$; Capitão, R. ${ }^{1}$; Santos, F. ${ }^{1}$; Duarte, J. ' ; Oliveira, M. ${ }^{1}$; Vasconcelos, C.

${ }^{1}$ Hospital de Egas Moniz C.H.L.O. E.P.E. Lisboa

Introduction: Obesity and type 2 diabetes mellitus (T2DM) are chronic conditions associated with increased morbidity and mortality that require multiple treatment strategies. In the obese population, T2DM management frequently involves multiple pharmacological agents. Bariatric surgery (BS) has shown to improve significantly metabolic control in these patients. Furthermore, subsequent T2DM control might vary with different bariatric surgical procedures. We aim to identify the reduction in T2DM pharmacological treatment burden after BS and if there are differences with selected surgical techniques.

Methods: Retrospective study involving T2DM patients treated with sleeve gastrectomy or roux-en-y gastric bypass from 2006 to 2015 . Those with a minimum of 12 months of post-operative follow up were included. Laboratory test results, pharmacological history and electronic prescription data before and after surgery, were evaluated. Descriptive statistic methods were applied.

Results: A total of 110 patients with T2DM performed BS during the cited period. The mean age at the time of surgery was 51 (30-65) years old, $82 \%$ were females. Sleeve was the technique in 72 and bypass in 38 patients. The mean operative follow up time was $48(12-144)$ months. At the time of surgery, the mean A1c was 7.1 (5.5-13) \% and the mean fasting glycaemia was 148 (67-418) mg/dl. Before surgery, all patients were treated pharmacologically, the mean of drugs taken was 1.78 (1-4) per patient. These drugs were insulin $(\mathrm{n}=20)$ and/or oral and/or injectable agents: 52 patients were treated with 1 drug, 30 with 2,18 with 3 and 5 with 4 drugs. After surgery, the final mean fasting glycaemia was $103(69-232) \mathrm{mg} / \mathrm{dl}$ and the mean A1c was 6.1 (4.4-10.5) \%. The number of drugs was reduced after surgery to a mean of $0.58(0-3)$ per patient; 72 patients were taking no drugs at all, 18 were taking 1 agent, 5 were taking 2 and 11 were taking 3 agents. The 72 patients off drugs had final A1c $<6.5 \%$ and fasting glycaemia $<120 \mathrm{mg} / \mathrm{dl}$. The global reduction of pharmacological agents was of $81.7 \%$. Only 14 patients did not reduce any agent, but 5 of them had lower final A1c and fasting glycaemia. When comparing procedures, there was a significant reduction of A1c and fasting glycaemia with both, but it was more marked with bypass $(\mathrm{p}=0.01)$. After bypass, there was also greater reduction of hypoglycaemic agents use $(\mathrm{p}=0.03)$ and greater chance of T2DM remission of $84.2 \%$ compared to $55.6 \%$ in the sleeve group.

Conclusions: Our study demonstrates a significate reduction in T2DM pharmacological treatment burden. There was a total reduction of $81.7 \%$ in drug use and $65.5 \%$ patients were free of pharmacological treatment. We observed better results in the gastric bypass group when compared with the sleeve group.

\section{T3P165 \\ Relationship between obesity indices and male sex hormone according to age groups}

Huh, J.' ; Lee, Y. ${ }^{2}$; Kong, M. ${ }^{2}$; Kim, H. ${ }^{2}$

${ }^{1}$ Department of Urology, Jeju National University \& Hospital, Jeju, Korea, ${ }^{2}$ Department Of Family Medicine, Jeju National University \& Hospital, Jeju, Korea

Introduction: The decrease in testosterone level during the aging process is associated with decrease in muscle mass and increase in visceral fat and body fat percent. The present study investigates the relationship between various obesity indices and level of testosterone according to different age groups.

Methods: A total of 156 individuals who underwent specific health exams in a local university hospital from January 2013 to July 2015 were included. Obesity indices were represented as BMI, waist circumference (WC), body fat percent (BFP), visceral fat area (VFA), subcutaneous fat area (SFA), and visceral to subcutaneous ratio (VSR). Subjects were categorized into four age groups: $35-44$ years, $45-54$ years, 54-64 years, and over 65.

Results: In almost all age groups obesity indices showed an inverse relation to testosterone level while VSR showed no significant association with either total or free testosterone levels. All obesity indices (BMI, WC, BFP, VFA, and SFA), except for VSR, manifested a statistically significant inverse correlation with total testosterone level, especially among subjects in the age group of 35-44 years and those greater than 65 years. However, free testosterone level failed to show a significant correlation with any of the obesity indices. No significant difference was noted in the correlation coefficient value among the different age groups.

Conclusion: BMI and WC significantly increased in all age groups as total testosterone level decreased. Body fat percent, visceral fat area and subcutaneous fat area showed a significant inverse correlation with total testosterone in the relatively young aged subjects and in the old aged groups.

\section{T3P167 \\ Response to GLP-1 receptor agonist treatment pre-bariatric surgery does not predict post-surgery weight loss in patients with type 2 diabetes: A retrospective study}

\section{Tang, T. ${ }^{1}$; Abbott, S. ${ }^{2}$; Singhal, R. ${ }^{2}$; Wilson, V. ${ }^{3}$; Bellary, S. ${ }^{4}$; Tahrani, A. ${ }^{5}$}

${ }^{1}$ Life and Health Sciences, Aston University, Birmingham, UK, ${ }^{2}$ Department Of Bariatric And Upper Gi Surgery, Heart Of England Nhs Foundation Trust, Birmingham, Uk, ${ }^{3}$ Department Of Diabetes And Endocrinology, Heart Of England Nhs Foundation Trust, Birmingham, Uk, ${ }^{4}$ School Of Life And Health Sciences, Aston University, Birmingham, Uk, ${ }^{5}$ Institute Of Metabolism And Systems Research, University Of Birmingham, Birmingham, Uk

Aims: Glucagon-like peptide 1 (GLP-1) is implicated in the weight loss achieved by bariatric surgery. We hypothesised that the weight loss response to GLP-1 receptor agonist (GLP-1 RA) before surgery predicts the post-BS weight loss.

Methods: A retrospective study of patients with Type 2 diabetes who received GLP-1 RA before undergoing bariatric surgery at a single centre in the UK. Data was collected from the bariatric surgery database and the patients' health records. Only patients with 12 months' postoperative follow-up were included. All patients attended the tier three weight management services before surgery

Results: Forty-five patients (60\% women, age $50.7 \pm 11.4$ years) were included; 26 had laparoscopic adjustable gastric banding (LAGB), and 19 had either laparoscopic sleeve gastrectomy or gastric bypass (LSG/RYGB). The pre-operative median body mass index was $48.3 \mathrm{~kg} / \mathrm{m}^{2}(\mathrm{IQR}=44.5-54)$ and $44 \mathrm{~kg} / \mathrm{m}^{2}(42.9-48.2)$ in the LAGB and LSG/RYGB groups respective- 
ly. The median weight loss at 12 months post-bariatric surgery was $7.9 \%$ (2.9\%-9.9\%) vs 24.8\% (19.5\%-25.9\%) for LAGB and LSG/RYGB respectively. GLP-1 RA treatment resulted in 5.7\% (1.3\% - 7.8\%) and $4.8 \%(2.1 \%$ $-7.8 \%$ ) weight loss in the LAGB and LSG/RYGB groups respectively. There was no correlation between the weight loss following GLP-1 RA treatment and the weight loss 12 months post-LAGB $(r=0.024, \mathrm{p}=0.909)$ and postLSG/RYGB ( $\mathrm{r}=-0.313, \mathrm{p}=0.193)$.

Conclusions: In patients with type 2 diabetes, weight change induced by pre-operative GLP-1 RA treatment did not predict post-bariatric surgery weight loss. Lack of weight loss following GLP-1 RA treatment should not be considered as a reason for not referring patients to bariatric surgery.

Conflict of Interest: None disclosed.

Funding: Research relating to this abstract was funded by the Department of Diabetes and Endocrinology, Heart of England NHS Foundation Trust, Birmingham, UK; and the School of Life and Health Sciences, Aston University, Birmingham, UK

\section{T3P168}

\section{RYGB related decrease in bone mineral density}

Polovina, S. ${ }^{1}$; Milin, J. ${ }^{2}$; Dumanovic, M. ${ }^{1}$; Micic, D. ${ }^{3}$; Pejkovic, D. ${ }^{4}$; Micic, D. ${ }^{4}$ ${ }^{1}$ Clinic for Endocrinology, Diabetes and Diseases of Metabolism, Clinical Center of Serbia, ${ }^{2}$ Medical Faculty University Of Belgrade, ${ }^{3}$ Emergency Center, Clinical Center Of Serbia, ${ }^{4} \mathrm{~S} / \mathrm{S}$

Introduction: Resent studies have shown increased fracture risk after bariatric surgery. That late complication is common after malabsorbtive bariatric procedures

Aim: The aim of our study was to investigate possible correlation between calcium and PTH levels and bone mineral density two years after R-en-Y gastric bypass (RYGB).

Material and Methods: In study were included 39 obese patients, $41.25 \pm 9.6$ years of age, BMI $42.82 \pm 5.5 \mathrm{~kg} / \mathrm{m}^{2}$. In all patients were measured PTH and serum calcium before and in between 12 and 24 months after RYGB. DXA BMD on lumbar spine and femoral neck was measured on Hologic densitometer. In statistic analyses were performed Student T test, Wilcoxon signed rank test and Spearman's rho.

Results: There was significant increase $(\mathrm{p}=0.001)$ of PTH level after the baritric surgery $($ med $=46.5(34.6-60.1)$ vs med $=55.6(40.7-79.5)$. Sig nificant decrease $(\mathrm{p}<0.001)$ was observed for lumbal spine after RYGB $(\mathrm{med}=0.40(0-1.10)$ vs $\mathrm{med}=0.20(-0.50-0.70)$. Significant increase $(\mathrm{p}<0.001)$ was observed for bone mineral density on femoral neck af ter surgery $(\mathrm{med}=0.75(-0.10-1.26)$ vs med $=0.55(-0.48-1.07)$. There was no statistical significant change in serum calcium level. No correlation ( $\mathrm{p}>0.05$ ) was observed between change of lumbar and hip BMD and changes of PTH levels.

Conclusion: Our investigation have shown that PTH level increased and bone mineral density on lumbar spine decline after two years from RYGB, but there was no correlation between change in PTH level and change in bone mineral density. This result requires further investigations regarding possible influences of other factors that could affect bone mineral density after bariatric surgery.

\section{T3P169}

\section{Salivary amylase and food intake in obese adolescents submitted to high intensity intermittent exercise}

Brunholi, C. '; Camparoto, M. ${ }^{2}$; Andrade, M. ${ }^{2}$; Monteiro, P. '; Codogno, J. '; Dias, J. '; Junior, I.

${ }^{1}$ Universidade Estadual Paulista, ${ }^{2}$ Universidade Do Oeste Paulista - Unoeste

Introduction: Obesity is a chronic disease, which the main causes are insufficient physical activity and inadequate food intake. Food intake may be influenced by the perception of food taste in the oral cavity that is facilitated by the action of saliva and its components, especially salivary amylase. Therefore, investigations about the possible effects of exercise in the salivary amylase production of obesse individuals may be importance in the treatment of obesity.

Methods: Non-randomized intervention study with 21 obese adolescents aged between 11 to $17 \mathrm{y}$ of both genders was performed. Were carried anthropometry, Dual Energy X-ray Absorptiometry (DEXA), sexual maturation by Tanner Stages and analysis of food intake through three-day dietary records. Salivary flow and concentration of amylase were collected, in non-consecutive 3-days, using a standard procedure. The participants performed 20 weeks intervention program consisting by 1) nutritional orientation twice a week and 2) two sets of six times of $40 \times 20 \mathrm{sec}$ of high intensity intermittent exercise with five min of active resting between sets, three times/week. Training intensity was estimated using the maximal stress test. For the statistics, descriptive analysis was performed, and for comparisons pre e post intervention, paired t-test was used to compare the variables of body composition, macronutrient consumption, salivary flow rate and salivary amylase concentration. The SPSS software (version 22.0) was used and significance was set at $5 \%$.

Results: The percentage body fat decrease after intervention $(p<0.05)$, as well as in the carbohydrate and lipid intake $(p<0.05)$, but the salivary flow $(\mathrm{p}=0.384)$ and salivary amylase concentration $(\mathrm{p}=0.380)$ did not change after 20 weeks of intervention.

Conclusion: Our results indicate that 20 weeks intervention with high intensity intermittent exercise and nutritional orientation was effective to decrease body fat and carbohydrate and lipid intake, but did not change salivary flow and the amylase in obese adolescents, after 20 week of intervention.

Conflict of Interest: None Disclosed

Funding: Research relating to this abstract was funded by CAPES and CNPq.

\section{T3P170 \\ "Schoolmistress, can i have a glass of water please?" Describing fluid intake of children at school}

\section{Morin, C. ; Bottin, J.' ; Guelinckx, I. ${ }^{1}$ \\ ${ }^{1}$ Danone Research}

Introduction: Since the majority of children (4-9 years) drink less than the adequate intake (AI) of total water intake from fluids set by European Food Safety Authority ${ }^{1}$, identifying opportunities to increase fluid intake seem pertinent. As children spend a large time at school and their intake, especially at this age, is funnelled by the environment, this analysis aimed to describe total fluid intake (TFI; sum of drinking water and all other beverages) according to location of intake, with a focus on intake at school.

Methods: 1044 children (53\% males) participating in the harmonized Liq. $\mathrm{In}^{7}$ cross sectional surveys in 3 European countries, Mexico, and China completed a 7-day fluid specific record. The record captured fluid types, consumed volumes, and moments and location of all intakes. The primary care giver was asked to complete the fluid records. To increase accuracy of the reported volume consumed, a photographic booklet with standard fluid containers was provided. Fluid intake on any location other than school or home is not reported here.

Results: Mean TFI (SD) ranged from $1.02(0.35) \mathrm{L} / \mathrm{d}$ in France to 1.68 $(0.63) \mathrm{L} / \mathrm{d}$ in Spain. In all countries 69 to $87 \%$ of TFI was consumed at home and only 6 to $18 \%$ at school, except for China where $22 \%$ of TFI was consumed at school and only $59 \%$ at home. In China $40 \%$ of children and in Spain and Poland up to 54\% drank at school on a daily basis, yet in France and Mexico almost half of the children did not drank at school at all. Among school drinkers in France, Spain and China water contributed the most to TFI at school ( $42 \%$ to $78 \%$ ), followed by sugared-sweetened beverages (SSB) or milk. In Poland the largest contributor to TFI at school were SSB $(24 \%)$ followed by an equal contribution $(21 \%)$ of water and hot beverages. In Mexico the largest contributor were also SSB (44\%) followed by water (38\%) and milk (14\%). In all countries the contribution of milk to TFI was lower at school than at home, whereas that of water higher. The 
contribution of SSB to TFI was increased at school compared to that at home in Spain (19\%; 11\%), Poland (24\%; 14\%) and Mexico (44\%; 36\%). Conclusion: In most countries children do not drink on a daily basis at school. Among school drinkers, the average water intake was only 1 small glass of 110 to $240 \mathrm{ml}$. Therefore identifying barriers to drinking water at school, encouraging the promotion of water intake at school by teachers and making water available at school seem a relevant way forward towards an adequate water intake.

${ }^{1}$ Iglesia I. et al 2015. Total fluid intake of children and adolescents: cross-sectional surveys in 13 countries worldwide. EJN 54 Suppl 2: 57-67

CM, JB and IG are full-time employees of Danone Research, funder of this research.

\section{T3P171}

Self-reassurance and self-compassion mediate changes in binge eating symptomology over 3 months during a 12-month compassion-focused intervention in a commercial weight management programme

Duarte, C. ${ }^{1}$; Stalker, C. ${ }^{2}$; Catarino, F. ${ }^{2}$; Basran J. ${ }^{2}$; Gilbert, P.2 ; Horgan, G. ${ }^{3}$; Morris, L. ${ }^{4}$; Stubbs, R. J. ${ }^{5}$

${ }^{1}$ Cognitive and Behavioural Centre for Research and Intervention, University of Coimbra, Rua do Colégio Novo, Apartado 6153, 3001-802 Coimbra, Portugal, ${ }^{2}$ College Of Life And Natural Sciences, University Of Derby, Derby, De22, 1 Gb, ${ }^{3}$ Biomathematics And Statistics Scotland, Rowett Institute Of Nutrition And Health, Foresterhill House, Aberdeen Ab25 2Zd, ${ }^{4}$ Slimming World, Clover Nook Road, Somercotes, Alfreton, Derbyshire De55 4Rf, ${ }^{5}$ Appetite Control And Energy Balance Research Group, School Of Psychology, University Of Leeds Ls2 9Jt

Introduction: Compassion-focused therapy (CFT) is a talking therapy that aims to reduce shame and self-criticism and help people to be compassionate to themselves. This intervention examined whether adding compassion-focused online video exercises into a commercial weight-management programme (WMP) (the intervention) helped people control their eating behaviours compared to the regular programme.This study aimed to investigate self-reassurance related to aspects of eating, body weight and shape and self-compassion, as mechanisms that mediated the change in binge eating 3 months after the start of the-intervention.

Methods: This was a two-arm, non-randomised parallel design (i) control arm: multicomponent WMP (ii) intervention arm: The same multicomponent WMP with the addition of 2 days training for weight management group leaders (Consultants) and 8 short online video exercises available to participants throughout the trial. 18 Consultants were allocated to intervention or control based on matched performance criteria. 964 ongoing participants, who had on average lost $10.8 \%$ (Control) and 9.8\% (Intervention) consented to join the trial arm to which their Consultant was allocated. The mediation effect of self-reassurance and self-compassion on changes in binge eating was examined using PROCESS (model 4; Hayes, $2013)$ in complete cases $(n=556)$ in two separate mediation analysis. The indirect effect was tested through bootstrapping (with 5000 samples) with $95 \%$ CI. Baseline values of the outcome and mediator were included as covariates.

Results: At 3 months changes in binge eating were mediated by increases in self-reassurance $\left(\mathrm{R}^{2}=0.63, \mathrm{~F}(3,552)=319.75, \mathrm{p}<0.001\right) ; \mathrm{b}=0.35$, $\mathrm{SE}=0.14$, CI95\% $[0.13,0.70]$ and self-compassion $\left(\mathrm{R}^{2}=0.64, \mathrm{~F}(3,530)=\right.$ $312.15, \mathrm{p}<.001) ; \mathrm{b}=0.28, \mathrm{SE}=0.11, \mathrm{CI} 95 \%[0.11,0.54]$

Conclusion: Inclusion of "light-touch" online digital CFT exercises in a weight management programme improved participants self-reassuring and self-compassion scores, which in turn appear to have had some effect in reducing binge eating symptomology.

Conflict of Interest: R James Stubbs is a consultant to Slimming World through University of Leeds Consulting (Trading as Consulting Leeds).

Funding: Cristiana Duarte was funded by Fundação para a Ciência e a Tecnologia - SFRH/BD/76858/2011.

This study was funded by a grant in aid of research from Slimming World UK, to the University of Derby.
T3P172

\section{Serum IGF-II and IGF2R in diabetes and obesity: Relation to weight, fasting glucose and weight loss intervention in an overweight, pre-diabetic NZ population.}

\section{Lee, K. ${ }^{\text {; }}$ Silvestre, M. ${ }^{2}$; Raben, A. ${ }^{3}$; Fogelholm, M. ${ }^{4}$; Poppitt, S. ${ }^{2}$}

${ }^{1}$ University of Auckland, ${ }^{2}$ University Of Auckland (Preview Consortium),

${ }^{3}$ University Of Copenhagen (Preview Consortium), ${ }^{4}$ University Of Helsinki (Preview Consortium)

New Zealand has a high rate of diabetes with the prevalence of diabetes and especially diabetic complications being highest in our Maori and $\mathrm{Pa}$ cific populations [1]. Obesity is a major risk factor for diabetes [2]. IGF-II and IGF2R have been previously linked to weight and diabetes although data has so far been conflicting. They have also been found to predict whether an individual is likely to be able to lose weight after dietary or surgical intervention [3-5]. Pre-diabetic overweight individuals; a demographic which would benefit the most from dietary intervention to prevent the development of T2D have not yet been studied. We have therefore measured levels of total IGF-II and IGF2R in samples collected in New Zealand as part of a large multi-national diet trial (PREVIEW). IGF-II was found to be higher in females but did not correlate with weight, BMI nor FPG in a cohort of 223 samples collected during participant screening phase. IGF2R was measured in 150 of these individuals and IGF2R was slightly higher in Maori but not correlated to FPG and only weakly correlated to BMI. IGF-II and IGF2R were also measured in 130 participants before and after a short-term weight-loss intervention (participants lost an average of $11 \%$ ). Males lost $1.9 \%$ more weight than females but neither serum marker was useful as a predictor of weight loss nor changed with weight loss. These serum markers are unlikely to be useful in targeting weight loss intervention in individuals with risk factors for T2D. The PREVIEW study is continuing long-term weight loss interventions and therefore correlations with long-term weight loss and initial serum markers can be assessed in the future. References: 1. International Diabetes Federation, Diabetes Atlas. 6th ed. 2013. 2. MoH et al, Nutrition and the Burden of Disease: New Zealand 1997-2011. 2003, MoH: Wellington. 4. Heald et al. The American Journal of Medicine, 2006. 119(2): p. 167. e9-167. 5. Narayanan, et al. Exp Clin Endocrinol Diabetes, 2013. 121(06): p. 361-367.

\section{T3P173 \\ Short-term changes in cardiovascular hemodynamics in response to bariatric surgery and weight loss using the Nexfin ${ }^{\circledR}$ non-invasive continuous monitoring device: A pilot study}

Pouwels, S. ${ }^{\text {; }}$ Lascaris, B. ${ }^{2}$; Nienhuijs, S. ${ }^{3}$; Bouwman, A. ${ }^{2}$; Buise, M. ${ }^{2}$

${ }^{1}$ Franciscus Vlietland, Schiedam, the Netherlands, ${ }^{2}$ Department Of Anaesthesiology, Intensive Care And Pain Medicine, Catharina Hospital, Eindhoven, The Netherlands, ${ }^{3}$ Department Of Surgery, Catharina Hospital, Eindhoven, The Netherlands

Background: Compared to healthy individuals, obese patients have significantly higher systolic and diastolic blood pressure; mean arterial pressure, heart rate and cardiac output. Aim of this study was to evaluate cardiovascular hemodynamic changes before and 3 months after bariatric surgery.

Methods: Patients scheduled for bariatric surgery between the 29th of September 2016 and the $24^{\text {th }}$ of March 2016 were included and compared with 24 healthy individuals. Hemodynamic measurements were performed preoperatively and 3 months after surgery, using the Nexfin non-invasive continuous hemodynamic monitoring device (Edwards Lifesciences / BMEYE B.V., Amsterdam, the Netherlands).

Results: Eighty subjects were included in this study, respectively 56 obese patients scheduled for bariatric surgery and 24 healthy individuals. Baseline hemodynamic measurements showed significant differences in cardiac output $(6.5 \pm 1.6$ versus $5.7 \pm 1.6 \mathrm{l} / \mathrm{min}, \mathrm{p}=0.046)$, mean arterial pressure $(107 \pm 19$ versus $89 \pm 11 \mathrm{mmHg}, \mathrm{p}=0.001)$, systolic $(134 \pm 24$ versus 
$116 \pm 18 \mathrm{mmHg}, \mathrm{p}=0.001)$ and diastolic blood pressure ( $89 \pm 17$ versus $74 \pm 10 \mathrm{mmHg}, \mathrm{p}=0.001)$ and heart rate $(87 \pm 12$ versus $76 \pm 14 \mathrm{bpm}$ $\mathrm{p}=0.02$ ) between obese and healthy subjects. Three months after surgery, significant changes occurred in mean arterial pressure $(89 \pm 17 \mathrm{mmHg}$, $\mathrm{p}=0.001)$, systolic $(117 \pm 24 \mathrm{mmHg}, \mathrm{p}=0.001)$ and diastolic blood pressure $(71 \pm 15 \mathrm{mmHg}, \mathrm{p}=0.001)$, stroke volume $(82.2 \pm 22.4 \mathrm{ml}, \mathrm{p}=0.03)$ and heart rate $(79 \pm 17 \mathrm{bpm}, \mathrm{p}=0.02)$

Conclusion: Three months after bariatric surgery significant improvements occur in hemodynamic variables except cardiac output and cardiac index, in the patient group.

\section{T3P174}

\section{Short-term effects of vildagliptin and metformin on endothelial function, low-grade inflammation and oxidative stress on recently diagnosed medically-naïve T2DM obese women}

Schiappacassa, A. ${ }^{1}$; Aguiar, L. ${ }^{1}$; Maranhão, P. ${ }^{1}$; Souza, M. ${ }^{1}$; Panazzolo, D. ${ }^{1}$; Bouskela, E. ${ }^{1}$

${ }^{1}$ Universidade Estadual do Rio de Janeiro

Introduction: Obesity is the main risk factor for type 2 diabetes mellitus (T2DM) and excessive visceral fat triggers a low-grade inflammatory process, mediated by the activation and release of cytokines and higher flow of free fat acids that contributes to insulin resistance, increased oxidative stress, and impaired endothelial function. Metformin and vildagliptin have known vasculo-protective actions, but the value of these drugs on medically-naïve T2DM during short-term use warrants study. Our purpose was to observe this effect on endothelial function, oxidative stress, inflammatory markers and blood viscosity.

Methods:38 obese medically-naïve T2DM women, aged between 19 and 50 years, BMI $\geq 30 \mathrm{~kg} / \mathrm{m}^{2}$; were subjected to measurements of endothelial function (venous occlusion plethysmography), blood viscosity, inflammatory and oxidative stress markersat fasting state at baseline and randomized $1: 1$ to ingest metformin ( $850 \mathrm{mg}$ twice/day) or vildagliptin (50 mg twice/day) during 30 days; and then, re-evaluated.

Results:Groups were considered as similar at baseline, as well as after 30 days of treatment, except for glucagon-like peptide 1 (GLP1), which increased after vildagliptin use compared to metformin $(p=0.03)$. Intragroup analysis showed that vildagliptin promoted a reduction in insulin $(\mathrm{p}=0.008)$, C-peptide $(\mathrm{p}=0.05)$ and oxidized LDL $(\mathrm{p}=0.02)$, and an increment of adiponectin $(\mathrm{p}=0.04)$ and GLP1 $(\mathrm{p}=0.009)$; while metformin reduced weight $(\mathrm{p}=0.03)$, glycaemia $(\mathrm{p}=0.0005)$, total cholesterol $(p=0.02)$, HDL-c $(p=0.03)$, LDL-c $(p=0.01)$ and DPP4 activity $(\mathrm{p}=0.03)$ and increasedTNF- $\alpha(\mathrm{p}=0.008)$. No significant differences in blood viscosity were noted, but vildagliptinuse resulted in improvement of endothelial reactivity compared to metformin.

Conclusion: Although some positive changes were noted with the use of metformin, vildagliptin administered during only 30 days in medically-naïve T2DM obese women resulted in improvement of endothelial function, oxidative stress and metabolic profile. Study partially funded by Novartis S.A.

\section{T3P175 \\ Single-incision sleeve gastrectomy versus laparoscopic sleeve gastrectomy: A case-control study}

\section{Chahine, E. '; Hayek, M. ' D'Alessandro, A. ${ }^{\text {' }}$; Dirani, M. ${ }^{\text {; }}$ ' Chouillard, E. ${ }^{1}$ POISSY-SAINT-GERMAIN-EN-LAYE MEDICAL CENTER}

Introduction: Single incision laparoscopic approach to the abdominal cavity is, nowadays, gaining popularity. Theoretical advantages include reduced abdominal wall complications, less postoperative pain, reduced genesis of adhesions, shorter hospital stay, faster return to activity and productivity, and preserved cosmesis. Obese patients may theoretically benefit most from single incision techniques due to a would-be lower ab- dominal wall-related morbidity. We undertook a retrospective case-control study evaluating the Single Incision Sleeve Gastrectomy (SSG) as compared to the multiple incision laparoscopic Sleeve Gastrectomy (LSG).

Methods and Procedures: Between January 2008 and September 2016, our group performed sleeve gastrectomy in 1350 patients with morbid obesity. Of these, 400 patients who had SSG (Group A) were retrospectively matched for age, sex, and body mass index (BMI), respectively, to 400 patients who had LSG (Group B).

The two groups were compared regarding operative parameters, post-operative pain management, fistula occurrence, and mid-term outcome at 2 years' follow-up

Results: The conversion rate (i.e., any additional port insertion or laparotomy) was $5.6 \%$ in Group A and $0.9 \%$ in Group B ( $\mathrm{p}<0.05)$. No major operative incident was deplored. Mortality was $0 \%$ in both groups. Average operative duration was 102 minutes in Group A (50-288 minutes) and 69 minutes in Group B $(42-181)(\mathrm{p}<0.05)$. Oral liquid diet was resumed on post-operative day (POD) 1 (1-4) in Group A and on POD 2 (1-4) in Group B $(p<0.05)$. The postoperative morbidity rate was $4.6 \%$ in Group $A$ and $7.4 \%$ in Group B ( $>0.05)$. Cumulative postoperative Morphine doses were significantly lower in Group A patients $(\mathrm{p}<0.05)$. Two leaks were observed in Group A patients (0.5\%) and 4 leaks in Group B patients $(1 \%)(\mathrm{p}>0.05)$. The median duration of hospital stay was 2 days $(0-7)$ in Group A and 2.4 days $(2-12)$ in Group B ( $p>0.05)$.

The quality of life (QoL) score and the cosmetic perception at both 1 and 12 months, respectively favored significantly Group A patients $(\mathrm{p}<0.05)$. Mean follow-up was 16 months in Group A (11-56) and 19 months in Group B (12-66). Weight loss patterns were comparable in both groups $(\mathrm{p}>0.05)$. Two patients in each group had an incisional hernia.

Conclusions: SSG has longer operative duration as compared to LSG. However, SSG seems to be associated to lower post-operative pain and shorter hospital stay. Leak rate, short-term weight loss and 2-year incisional hernia rate are comparable.

Finally, as compared to LSG, SSG is associated to better cosmetic benefits and QoL scores at both 1 and 12 months postoperatively, respectively.

Conflict of Interest: None Disclosed/Payment received

Funding: No Funding/Research relating to this abstract was funded

\section{T3P176}

\section{Six days of sleep restriction do not potentiate the metabolic effects of mixed sugar-fat overfeeding}

\section{Cros, J. ${ }^{1}$; Rosset, R. '; Cornette, F. ${ }^{2}$; Haba-Rubio, J. ${ }^{2}$; Heinzer, R. ${ }^{2}$,}

Pouymayou, B. ${ }^{3}$; Buhler, T. ${ }^{3}$; Kreis, R. ${ }^{3}$; Boesch, C. ${ }^{3}$; Schneiter, P. ; Tappy, L. ' ; Lecoultre, $V .^{1}$

${ }^{1}$ Department of Physiology, Faculty of Biology and Medicine, University of Lausanne, Lausanne, Switzerland, ${ }^{2}$ Center For Investigation And Research In Sleep, University Hospital, Faculty Of Biology And Medicine, Lausanne, Switzerland, ${ }^{3}$ Department Of Clinical Research And Department Of Radiology, University Of Bern, Bern, Switzerland

Introduction: Consumption of energy-dense foods has long been suspected to play a role in the development of abdominal obesity, insulin resistance, non-alcoholic fatty liver disease and the metabolic syndrome. It has also been proposed that sleep restriction (SR) and sleep disorders may be involved as well. The aim of this study was to assess in healthy volunteers whether a hypercaloric, high fat-high sugar diet and SR had synergic effects on intrahepatocellular lipids (IHCL) accumulation and insulin-resistance.

Methods: Ten sedentary volunteers ( 5 men and 5 women; mean \pm SD; age: $24 \pm 4$ y, weight: $63.3 \pm 8.2 \mathrm{~kg}$, BMI: $\left.21.6 \pm 1.6 \mathrm{~kg} / \mathrm{m}^{2}\right)$ were studied before and after a 6-day overfeeding period (OF: $130 \%$ daily energy needs, with $15 \%$ excess sugar and $15 \%$ excess fat), with either normal sleep [control, C; 8 h time in bed/day (TIB)] or SR (4h TIB). Before (Pre) and after OF (Post), IHCL content was measured by ${ }^{1} \mathrm{H}$-magnetic resonance spectroscopy. Insulin resistance, hepatic glucose production (HGP; 6,6 d2-glucose), as well as adipose tissue lipolysis (ATL; d5-glycerol) were assessed 
in fasting conditions and in response to a 4-hour, 75-g oral ${ }^{13} \mathrm{C}$-labelled glucose tolerance test (OGTT). Exogenous glucose oxidation (EGO) was quantified from expired ${ }^{13} \mathrm{CO} 2$.

Results: IHCL were significantly $(\mathrm{p}<0.01)$ increased by $\mathrm{OF}$ in $\mathrm{C}$ (from $13.2 \pm 9.5$ to $22.2 \pm 23.0 \mathrm{mmol} / \mathrm{L}$ ) and SR (from $11.2 \pm 10.4$ to $21.6 \pm 22.8$ $\mathrm{mmol} / \mathrm{L}$ ). As compared to Pre, fasting HGP (C, Pre: $2.06 \pm 0.23$, Post $2.14 \pm 0.38$; SR, Pre: $2.07 \pm 0.13$, Post: $2.12 \pm 0.15 \mathrm{mg} / \mathrm{kg} / \mathrm{min})$ and ATL (C, Pre: $0.33 \pm 0.32$, Post: $0.16 \pm 0.06$; SR, Pre: $0.22 \pm 0.08$, Post: $0.32 \pm 0.28$ $\mathrm{mg} / \mathrm{kg} / \mathrm{min}$ ) remained unchanged after OF (all $\mathrm{p}=\mathrm{NS}$ ). During the OGTT, HGP suppression was significantly blunted after OF in both conditions (C, Pre: $-75 \pm 7$, Post: $-72 \pm 12$; SR, Pre: $-73 \pm 7$, Post: $-69 \pm 8 \%$; $\mathrm{p}<0.05$ ), whereas EGO was significantly increased (C, Pre: $24.3 \pm 2.0$, Post: $26.4 \pm 2.8$; SR, Pre: $24.9 \pm 3.1$, Post: $25.2 \pm 2.4 \mathrm{~g} / 4 \mathrm{~h} ; \mathrm{p}<0.05)$. Af ter OF, ATL suppression in response to OGTT remained unaltered (C, Pre: $-45 \pm 24$, Post: $-36 \pm 19$; SR, Pre: $-49 \pm 11$, Post: $-52 \pm 21 \%$; $\mathrm{p}=\mathrm{NS}$ ) Changes in IHCL, glucose metabolism and ATL after OF were not significantly different in SR as compared to $\mathrm{C}$ (all $\mathrm{p}=\mathrm{NS}$ ).

Conclusion: Six days of a high fat-high sugar OF significantly increased IHCL and altered glucose kinetics in response to an OGTT, suggesting selective alterations of hepatic metabolism. However, the effects of OF were not potentiated by SR.

Conflict of Interest: None Disclosed

Funding: Research relating to this abstract was funded by a grant from the Swiss National Science Foundation to Prof. Luc Tappy.

\section{T3P177}

\section{Six months supervised physical training after Roux-en-Y- gastric bypass increases physical activity levels and health related quality of life}

\section{Stolberg, C. ${ }^{1}$; Mundbjerg, L. ${ }^{2}$; Bladbjerg, E. ${ }^{3}$; Juhl, C. ${ }^{4}$; Gram, B. ${ }^{5}$}

${ }^{1}$ Department of Medicine/Endocrinology, Hospital of Southwest Jutland and . Institute of Regional Health Research, University of Southern Denmark/Hospital of Southwest Jutland, ${ }^{2}$ : Department Of Medicine/Endocrinology, Hospital Of Southwest Jutland And Open, Odense Patient Data Explorative Network Odense University Hospital, Odense, Denmark, ${ }^{3}$ Unit For Thrombosis Research, Department Of Public Health, University Of Southern Denmark/Department Of Clinical Biochemistry, Hospital Of Southwest Jutland, ${ }^{4}$ Department Of Medicine/Endocrinology, Hospital Of Southwest Jutland, ${ }^{5}$ nstitute Of Regional Health Research, University Of Southern Denmark/Hospital Of Southwest Jutland

Obesity is associated with impaired health-related quality of life (HRQoL) and physical inactivity.While Roux-en-Y-Gastric bypass (RYGB) is known to improve HRQoL, no clear evidence exists whether RYGB per se increases the overall physical activity (PA). In addition, supervised physical training intervention following RYGB may further improve HRQoL and increase overall PA among RYGB patients.

Sixty patients with morbid obesity, qualified for RYGB, were 6 months post-surgery randomized 1:1 to 26 weeks exercise intervention (INT) or a control group (CON). The intervention consisted of aerobic capacity training and resistance training $(2 \times 40 \mathrm{~min} /$ week $)$, supervised by physiotherapists. Overall PA was measured for seven consecutive days by Actigraph GTX3 accelerometer (ACC), and HRQoL was measured using the SF-36, with norm based scores (NBS) standardized to a population normal distribution $($ mean $=50, S D=10)$. All assessments were performed pre-surgery, 6 (start of intervention), 12 (end of intervention), and 24 months post-surgery.

Results: Pre-surgery (mean \pm SD): HRQoL: NBS from all eight domains of the SF36 were ranging from $44.6 \pm 7.3$ to $50.0 \pm 9.5$.

PA: Sedentary time: $11.5 \pm 1.5 \mathrm{~h} /$ day; low and light PA (LLPA): $188 \pm 51$ $\mathrm{min} /$ day; moderate-vigorous PA (MVPA): $5 \pm 9 \mathrm{~min} /$ day.

Post-surgery (6 months) (mean \pm SD): RYGB caused improvements in all eight domains of SF36, ranging from $\triangle$ NBS: $4.4 \pm 10.3$ to $\triangle$ NBS: $12.3 \pm 7.7$ $(\mathrm{p}<0.002)$, all NBS post-surgery were above 51 . No significant changes in PA were observed.
Post-intervention (12 and 24 months) (mean \pm SE): The intervention caused additional improvement in the SF36 domain "general health": $\triangle$ NBS: $3.8 \pm 1.7(\mathrm{p}=0.025)$ in INT compared to CON 24 months after RYGB. No further improvements in HRQoL.

LLPA increased in INT compared to CON 12 months after RYGB: $\triangle L L P A$ : $35 \pm 16 \mathrm{~min} /$ day $(\mathrm{p}=0.026)$, but no significant changes in sedentary time or MVPA were observed. No significant differences in PA between groups were observed at the 24 months examination.

This study shows that candidates for RYGB were physically inactive before surgery and no improvements in PA levels measured by accelerometry were induced by surgery alone. Supervised physical training after RYGB improved general health at the 24 months examination and markedly improved low and light PA immediately after the end of intervention. However, the effect on PA did not persist at the 24 months examination. A potential explanation for this could be the length of the intervention period, and further research is needed to understand how an increase in PA can be maintained.

Funding: Dept of Endocrinology and Research Centre, Hospital of Southwest Jutland; SDU; Region of Southern Denmark; Karola Jørgensen fond; Edith og Vagn Hedegaard Jensens fond.

\section{T3P178}

\section{Skeletal effects of Roux-en-Y gastric bypass in obese type 2 diabetes patients measured by DXA and HR-pQCT}

Espersen, R. ${ }^{1}$; Madsen, L. ${ }^{2}$; Ørnstrup, M. ${ }^{3}$; Langdahl, B. ${ }^{3}$; Richelsen, B. ${ }^{3}$ ${ }^{1}$ Department of Endocrinology and Internal Medicine, Aarhus University Hospital, Denmark; Department of Clinical Medicine, Aarhus University, Denmark, ${ }^{2}$ Department Of Endocrinology And Internal Medicine, Aarhus University Hospital, Denmark; Department Of Clinical Medicine, Aarhus University, Denmark; Danish Diabetes Academy, Odense University Hospital, Denmark, ${ }^{3}$ Department Of Endocrinology And Internal Medicine, Aarhus University Hospital, Denmark

Introduction: Roux-en-Y gastric bypass (RYGB) is a highly effective treatment of severe obesity leading to sustained weight loss and improvements in comorbidities. RYGB induces a degree of malabsorption that may affect vitamin $\mathrm{D}$ and calcium uptake, which ultimately may have an effect on bone health. Thus, our aim was to investigate whether RYGB operated type 2 diabetes (T2DM) patients have impaired bone health compared to a non-operated matched control group.

Methods: 95 T2DM patients who underwent RYGB operation in the period 2006-2011 (median time since operation was 6.07 (IQR: 3.41; 7.00) years) and 49 non-operated T2DM controls matched on age, sex, and postsurgical BMI were included.

The patients were examined with Dual-energy X-ray Absorptiometry (DXA) of the lumbar spine, total hip and forearm, and High Resolution Peripheral Quantity Computed Tomography (HR-pQCT) of distal tibia and distal radius

Results: Mean T-scores for the RYGB operated were $-0.3(-0.6 ; 0.0)$ at the lumbar spine, $-0.4(-0.6 ;-0.1)$ in the total hip, and $-1.8(-2.1 ;-1.5)$ in the forearm. Mean T-scores for non-operated were $0.1(-0.4 ; 0.5), 0.4(0.0$; $0.7)$, and $-0.7(-1.0 ;-0.4)$.

OR for having osteoporosis or osteopenia in the operated group compared to the non-operated group was $1.9(0.9 ; 4.0)$.

HR-pQCT showed in the distal tibia that trabecular thickness was $12.8 \%$ $(7.3 ; 17.9)$ lower, and cortical thickness was $10.1 \%(1.9 ; 17.6)$ lower among the operated. In the distal radius, trabecular number was $13.5 \%(7.0 ; 19.5)$ lower, and trabecular thickness was $12.7 \%(7.4 ; 17.6)$ lower among the operated.

Conclusion: DXA showed significantly lower T-scores in the total hip and forearm among the RYGB operated. HR-pQCT showed that in the weight-bearing tibia both cortical and trabecular bone was impaired among the operated compared to the non-operated. In radius, only the trabecular bone was impaired.

Thus, a RYGB-induced reduction in bone health was observed which in the long run may increase the risk for osteoporosis and fractures. 
T3P179

\section{Skeletal effects of Roux-en-Y gastric bypass in obese type 2 diabetes patients measured by DXA and HR-pQCT.}

Espersen, R. ${ }^{1}$; Madsen, L. ${ }^{2}$; Ørnstrup, M. ${ }^{3}$; Langdahl, B. ${ }^{3}$; Richelsen, $B .^{3}$ ${ }^{1}$ Department of Endocrinology and Internal Medicine, Aarhus University Hospital, Denmark; Department of Clinical Medicine, Aarhus University, Denmark, ${ }^{2}$ Department Of Endocrinology And Internal Medicine, Aarhus University Hospital, Denmark; Department Of Clinical Medicine, Aarhus University, Denmark; Danish Diabetes Academy, Odense University Hospital, Denmark, ${ }^{3}$ Department Of Endocrinology And Internal Medicine, Aarhus University Hospital, Denmark

Introduction: Roux-en-Y gastric bypass (RYGB) is a highly effective treatment of severe obesity leading to sustained weight loss and improvements in comorbidities. RYGB induces a degree of malabsorption that may affect vitamin $\mathrm{D}$ and calcium uptake, which ultimately may have an effect on bone health. Thus, our aim was to investigate whether RYGB operated type 2 diabetes (T2DM) patients have impaired bone health compared to a non-operated matched controls.

Methods: Ninety-five T2DM patients who underwent RYGB operation in the period 2006-2011 and 49 non-operated T2DM controls matched on age, sex, and BMI at clinical investigation were included. The patients were examined with Dual-energy X-ray Absorptiometry (DXA) of the lumbar spine, total hip and forearm, and High Resolution Peripheral Quantity Computed Tomography (HR-pQCT) of distal tibia and distal radius.

Results: Median time since operation was 6.07 (IQR: $3.41 ; 7.00$ ) years. Mean T-scores for the RYGB operated were -0.3 (95\% CI: $-0.6 ; 0.0)$ at the lumbar spine and -0.4 (95\% CI: $-0.6 ;-0.1)$ in the total hip. Mean T-scores for non-operated were 0.1 (95\% CI: $-0.4 ; 0.5)$ and 0.4 (95\% CI: $0.0 ; 0.7)$ T-scores in the forearm were -1.8 (95\% CI: $-2.1 ;-1.5)$ in the RYGB operated group and -0.7 (95\% CI: $-1.0 ;-0.4)$ in the non-operated group. Odds ratio for having osteoporosis or osteopenia in the operated group compared to the non-operated group was 1.9 (95\% CI: $0.9 ; 4.0)$.

HR-pQCT showed in the distal tibia that the trabecular thickness was $12.8 \%$ (95\% CI: 7.3; 17.9) lower, and cortical thickness was $10.1 \%$ (95\% CI: $1.9 ; 17.6)$ lower among operated compared to non-operated. In the distal radius, trabecular number was $13.5 \%$ (95\% CI: 7.0; 19.5) lower, and trabecular thickness was $12.7 \%$ (95\% CI: $7.4 ; 17.6)$ lower among the operated compared to non-operated.

Conclusion: DXA showed significantly lower T-scores in the total hip and forearm among the RYGB operated. HR-pQCT showed that in the weight-bearing tibia both cortical and trabecular bone was impaired among the operated compared to the non-operated. In radius, only the trabecular bone was impaired. Thus, a RYGB-induced reduction in bone health was observed which in the long run may increase the risk for osteoporosis and fractures.

Conflicts of Interest: None

Funding: This project was funded by Aarhus University, Osteoporoseforeningen and Fonden til Lægevidenskabens Fremme.

\section{T3P180}

\section{STEP-ing up smoking cessation: Weight-related concern of participants who want to quit smoking}

Leblanc, A. ${ }^{1}$; Reid, R. ${ }^{1}$; Mark, A. ${ }^{2}$; Tulloch, H. ${ }^{1}$; Aitken, D. ${ }^{1}$; Mullen, K. ${ }^{1}$; Pipe, $A{ }^{\prime}$

${ }^{1}$ University of Ottawa Heart Institute, ${ }^{2}$ Institute For Clinical Evaluative Sciences

Introduction: Smoking is a fundamental risk factor for many chronic, non-communicable diseases and acknowledged as a leading cause of preventable death worldwide. Obesity is also acknowledged as a significant risk factor for non-communicable diseases and weight management is of ten cited as a primary reason for starting, or not trying to quit smoking. The use of nicotine replacement therapy (NRT) is designed to provide patients with a dose of nicotine sufficient to approach levels obtained during smoking in order to alleviate symptoms of withdrawal, reduce cravings, and ease smokers in the transition to being smoke free. The Self-Directed Titrated Nicotine Patch Versus Standard Treatment for Smoking Cessation in Smokers Motivated to Quit (STEP) study evaluates the efficacy of titrating the dose of NRT based on smoking history compared to standard treatment employing fixed-dose NRT in conjunction with behavioural counseling.

Methods: STEP was a 52-week, parallel, two-group, randomized control study. Smokers who were ready to quit (i.e., within the next 30 days) were randomized to the usual care group (10 weeks of declining, standard-dose, transdermal nicotine patch); or, STEP group (10 weeks of titrated transdermal nicotine patch). All participants receive six counseling sessions and completed follow-up surveys at 26-, and 52-weeks. The primary outcome of the STEP study was biochemically confirmed smoking cessation at 52-week follow-up. The aim of this preliminary analysis was to provide demographic and health information of all participants.

Results: Complete data was available for 302 participants, usual care group: $\mathrm{n}=151,39.1 \%$ female, mean age: $47.1 \pm 11.1$; STEP group: $\mathrm{n}=152,34.2 \%$ female, mean age: $48.9 \pm 11.6$. Average body mass index (BMI) for both the usual care group $(28.06 \pm 5.8)$ and the STEP group $(28.8 \pm 5.6)$ put participants at an increased risk for obesity. At baseline, no differences were found in BMI, dyslipidemia, hypertension, or presence of cardiovascular disease between groups. At 52-week follow-up, difference in BMI between groups approached significance $(\mathrm{p}=0.058)$. At baseline, both groups (usual care: 7.3\%; STEP: 6.6\%) cited "weight gain" as a reason for smoking relapse in the past. Mild-to-severe weight gain/ increased appetite due to cessation was cited by both groups at the end of the program (usual care: $25.2 \%$; STEP: $19.8 \%$ ), and at 52-week follow-up (usual care: $22.5 \%$; STEP: $14.5 \%$ ) but cited less at follow-up in the STEP group $(\mathrm{p}=0.045)$.

Conclusions: Future work will determine if the STEP program was effective in aiding participants in transitioning to cessation at 52-week follow-up. We also aim to determine if other factors (e.g., baseline and follow-up weight status) were influential in smoking cessation.

\section{T3P181}

\section{Supervised physical training following gastric bypass surgery improves long-term weight loss}

\section{Mundbjerg, L. ${ }^{\text {; }}$ Stolberg, C. ${ }^{2}$; Bladbjerg, E. ${ }^{3}$; Gram, B. ${ }^{4}$; Juhl, C. ${ }^{1}$}

${ }^{1}$ Department of Medicine/Endocrinology, Hospital of Southwest Jutland, ${ }^{2}$ Open, Odense Patient Data Explorative Network, Odense University Hospital, Odense, Denmark, ${ }^{3}$ Unit For Thrombosis Research, Department Of Public Health, University Of Southern Denmark/Department Of Clinical Biochemistry, Hospital Of Southwest Jutland, ${ }^{4}$ Institute Of Regional Health Research, University Of Southern Denmark/Hospital Of Southwest Jutland

Introduction: Roux-en-Y gastric bypass (RYGB) surgery results in a profound weight loss and improves glucose and cholesterol levels in obese subjects, however, over time some weight regain occurs in a large proportion of patients.

The aim of this study was to investigate the effect of six months of supervised exercise intervention post-surgery on weight loss, weight loss maintenance, glucose and cholesterol levels and cardiorespiratory fitness (VO2max).

Materials and methods: We conducted a prospective randomized controlled trial and included 60 subjects with morbid obesity qualified for RYGB. Six months post-surgery, subjects were randomized to either six months of supervised exercise intervention (INT) $(n=32)$ or a control group $(\mathrm{CON})(\mathrm{n}=28)$. The exercise was supervised by physiotherapists 2 x $40 \mathrm{~min} /$ week.

Preoperative, 6 (start of intervention), 12 (end of intervention), and 24 months post-surgery, body weight and aerobic capacity (VO2max, L/min) were measured and glucose, HbAlc, cholesterols, and triglycerides were analyzed.

Results: Twenty-seven subjects (84.4\%) in INT and 25 subjects $(89.3 \%)$ in CON completed the 12 months examination, while $22(68.8 \%)$ and 20 
(71.4\%) subjects completed the 24 months examination in INT and CON, respectively.

Results are presented as mean \pm SD. RYGB caused a significant weight loss from $126.6 \pm 20.9 \mathrm{~kg}$ pre-surgery to $99.2 \pm 18.5 \mathrm{~kg}$ six months post-surgery. Total cholesterol, LDL cholesterol, HbAlc and glucose decreased significantly (all $\mathrm{p}<0.002)$. There was no change in VO2max $(\mathrm{p}=0.677)$. After the intervention at the 12 months examination, body weight was $90.5 \pm 18.0 \mathrm{~kg}$ in INT and $90.9 \pm 18.6 \mathrm{~kg}$ in CON. Supervised exercise intervention resulted in significantly lower weight regain in INT at 24 months follow up, where participants had a body weight of $89.1 \pm 19.7 \mathrm{~kg}$ while CON had a body weight of $92.9 \pm 21.3 \mathrm{~kg}(\mathrm{p}<0.002)$.

At the 12 months examination, there was a significant increase in HDL cholesterol in INT $(1.14 \pm 0.21$ to $1.37 \pm 0.29 \mathrm{mmol} / \mathrm{L})$ compared to CON $(1.13 \pm 0.24$ to $1.25 \pm 0.24 \mathrm{mmol} / \mathrm{L}),(\mathrm{p}<0.035)$. No improvements were found in LDL cholesterol, total cholesterol, HbAlc or glucose at 12 or 24 months examination in INT or CON.

At 12 months, VO2max increased significantly in INT compared to CON $(\mathrm{p}<0.002)$, however, this difference could not be maintained at 24 months of follow up ( $\mathrm{p}=0.762)$.

Conclusion: Supervised physical training following RYGB improves long-term weight loss. Aerobic capacity and HDL cholesterol increase af ter completion of the exercise intervention, however, the improvements cannot be maintained one year after the intervention.

Funding: Funded by Dept. of Endocrinology and IRS, Hospital of Southwest Jutland; SDU; Karola Jørgensens Fond; Edith og Vagn H. Jensens Fond; Fam. Hede Nielsens Fond.

\section{T3P182}

\section{Systematic review and meta-analysis of outcomes after revisional bariatric surgery following a failed adjustable gastric band}

Sharples, A. '; Charalampakis, V. '; Daskalakis, M. '; Singhal, R. ${ }^{1}$; Tahrani, A. ${ }^{1}$ ${ }^{1}$ Heart of England NHS Foundation Trust

Introduction: Laparoscopic adjustable gastric band (LAGB) related complications have been reported in significant numbers of patients often leading to band removal. Increasingly revisional bariatric surgery (RBS) is offered, most commonly either band to roux-en-y gastric bypass (B-RYGB) or band to sleeve gastrectomy (B-SG). We conduct a systematic review and meta-analysis of studies to evaluate the efficacy of RBS following failed LAGB.

Methods: Medline, Embase, The Cochrane Library and NHS Evidence were searched for English language studies assessing patients who had undergone LAGB and who subsequently underwent RBS (either B-RYGB or B-SG). Studies were included if they presented postoperative data on weight change, obesity-related comorbidities, or quality of life and included more than 10 patients.

Results: Thirty-six studies met our criteria for inclusion. In total, there were 2617 patients. B-RYGB was performed in $60.5 \%$. There was only one death within 30 days reported $(0.0004 \%)$. The overall pooled morbidity rate was $13.2 \%$ (8.9\% early and $8.1 \%$ late complications). There was no difference between B-RYGB and B-SG in overall morbidity, leak rate or return to theatre. Percentage excess weight loss (\%EWL) for all patients combined at 6, 12 and 24 months was $44.5 \%, 55.7 \%$ and $59.7 \%$ respectively. There was no statistical difference in \%EWL between B-RYGB and B-SG at any time point. The rates of remission of diabetes, hypertension and obstructive sleep apnoea were $46.5 \%, 35.9 \%$ and $80.8 \%$ respectively. Only two studies looked at quality of life and both demonstrated an improvement following revisional surgery.

Conclusion: The existing evidence, although limited suggests that RBS is associated with generally good outcomes similar to those experienced after primary surgery. Further, high quality, research is required to assess long-term weight loss, comorbidity and quality of life outcomes.
T3P183

Tackling obesity in areas of high social deprivation: Randomised controlled trial of a task-based weight management group programme

Mcrobbie, H. ${ }^{1}$; Hajek, P. ${ }^{1}$; Peerbux, S. ${ }^{1}$; Kahan, B. ${ }^{2}$ Trépel, D. ${ }^{3}$; Parrott, S. ${ }^{3}$; Griffiths, C. ${ }^{4}$; Snuggs, S. ${ }^{\prime}$; Smith, K. ${ }^{1}$; Eldridge, S. ${ }^{2}$

${ }^{1}$ Health and Lifestyle Research Unit, Wolfson Institute of Preventive Medicine, Queen Mary University of London, ${ }^{2}$ Pragmatic Clinical Trials Unit, Queen Mary University Of London, ${ }^{3}$ Department Of Health Sciences, The University Of York, ${ }^{4}$ Centre For Primary Care And Public Health, Blizard Institute, Queen Mary University Of London

Introduction: Obesity is a rising threat to health that is now also contributing to health inequalities. Weight management programmes are needed that are effective and reach underprivileged groups. We examined whether a task-based weight management group programme structured to cater for underprivileged clients (Weight Action Programme; WAP) has better long-term effects than a 'best practice' intervention provided in primary care by practice nurses (PN).

Methods: 330 adults from areas of high social deprivation with a body mass index $(\mathrm{BMI}) \geq 30 \mathrm{~kg} / \mathrm{m}^{2}$ or $\mathrm{BMI} \geq 28 \mathrm{~kg} / \mathrm{m}^{2}$ with co-morbidities were randomised (2:1) to the WAP or PN arms. WAP comprised eight weekly group sessions providing verifiable tasks concerning diet, physical activity and self-monitoring, followed by ten monthly sessions. PN provided advice on diet and physical activity based on NHS 'Change4Life' materials in four sessions over eight weeks. The analysis of weight change at 12 months used a mixed-effects linear regression model adjusted for baseline variables.

Results: Less than half of the participants were in paid employment. 88\% of participants in each study arm provided at least one recorded outcome and were included in the primary analysis. Weight loss at 12 months was greater in the WAP arm $(-4.2 \mathrm{~kg}$ vs. $-2.3 \mathrm{~kg}$; difference $=-1.9 \mathrm{~kg}, 95 \% \mathrm{CI}$ : -3.7 to $-0 \cdot 1 ; \mathrm{p}=0.04)$. Participants in the WAP arm were also more likely to lose at least $5 \%$ of their baseline body weight ( $41 \%$ vs. $27 \%$, OR $=14.61$ 95\% CI: $2 \cdot 32$ to $91 \cdot 96, \mathrm{p}=0 \cdot 004)$.

Conclusion: A task-based programme was more effective than a 'best practice' intervention within primary care. WAP can provide a public-domain template for an economical programme that can reach clients from diverse ethnic and socio-economic backgrounds.

Conflict of interest: None Disclosed

Funding: This project was funded by the National Institute for Health Research Heath Technology Assessment (project number 09/127/34). The views and opinions therein are those of the authors and do not necessarily reflect those of the Health Technology Assessment, NIHR, NHS or the Department of Health.

\section{T3P184 \\ “Teacher, can i have a glass of water please?" Describing fluid intake of adolescents at school}

Morin, C. '; Bottin, J.'; Guelinckx, l.'

${ }^{1}$ Danone Research

Introduction: In many countries, the majority of adolescents drink less than the adequate intake (AI) for total water intake from fluids set by European Food Safety Authority ${ }^{1}$. Therefore identifying opportunities to increase fluid intake seem pertinent. Since adolescents spend a large time at school, the aim of this analysis was to describe total fluid intake (TFI; sum of drinking water and all other beverages) according to location of consumption, with a focus on intake at school.

Methods: 1275 adolescents (10-17 yrs; 52\% males) participating in the harmonized Liq. $\mathrm{In}^{7}$ cross sectional surveys in 4 European countries, Mexico, and China completed a 7-day fluid specific record. The record captured fluid types, consumed volumes, and moments and location of all intakes. For children below 12 years, the primary care giver of the child was asked to complete the fluid records. To increase accuracy of the reported volume consumed, a photographic booklet with standard fluid containers 
was provided. Fluid intake on any other location than school and home is not reported here.

Results: Mean TFI (SD) ranged from 1.26 (0.46) L/d in France to 1.75 (0.61) L/d in Spain. In all countries 69 to $90 \%$ of TFI was consumed at home and only 5 to $20 \%$ at school, except for China where $27 \%$ of TFI was consumed at school and only $56 \%$ at home. In France only $50 \%$ of adolescents reported drinking at school of which still $54 \%$ drank only on 1 or 2 days. The proportion of school drinkers was higher in Mexico (56\%), UK (62\%), Spain (77\%), and Poland (87\%) than in France. Yet the highest proportions were observed in China: $95 \%$ of all adolescents reported to drink at school, and $54 \%$ of them on a daily basis. Among school drinkers the most consumed fluids were water and sugar-sweetened beverages (SSB) ranging from 0.11 to $0.29 \mathrm{~L} / \mathrm{d}(23 \%$ to $87 \%$ of TFI at school) and from 0.03 to $0.26 \mathrm{~L} / \mathrm{d}$ (10 to $49 \%$ of TFI at school) respectively. Compared to at home, the contribution of milk to TFI was lower at school in all countries. Water contribution to TFI at school was higher than at home in France (87\%; 45\%), Spain (56\%, 51\%), whereas SSB contribution at school was higher than at home in Poland (41\%; 13\%) and Mexico (49\%; 41\%).

Conclusion: In most countries adolescents do not drink on a daily basis at school. Among school drinkers, the average water intake was only one 1 glass of 250 to $300 \mathrm{ml}$. Therefore identifying barriers to drinking water at school, and encouraging the promotion of water intake at school by teachers seems a relevant way forward towards an adequate intake.

\section{References:}

1 Iglesia I. et al 2015. Total fluid intake of children and adolescents: cross-sectional surveys in 13 countries worldwide. EJN 54 Suppl 2: 57-67

\section{T3P185}

\section{The comparative effects of intermittent versus continuous energy restriction on postprandial glucose and lipid metabolism following $\mathbf{5 \%}$ weight-loss}

\section{Antoni, R. ${ }^{1}$; Johnston, $\mathrm{K}^{2}{ }^{2}$; Collins, A. ${ }^{1}$; Robertson, M. ${ }^{1}$}

${ }^{1}$ Faculty of Health and Medical Sciences, University of Surrey, ${ }^{2}$ Lighterlife Uk Ltd

Introduction: The intermittent energy restriction (IER) approach to weight-loss involves short periods of substantial (>70\%) energy restriction (ER) interspersed with normal eating ${ }^{(1)}$. Previous work has demonstrated the capacity of substantial ER (70-100\%) over 24 hours to elicit acute, weight-loss independent alterations to postprandial glucose and lipid metabolism in a dose response manner ${ }^{(2)}$. The present study aimed to build on our earlier work by examining the effects of IER following 5\% weight-loss on postprandial metabolism, and comparing this to similar weight-loss achieved through a more modest, continuous energy restriction (CER).

Methods: 26 (13 male) overweight/obese participants (46 $\pm 3 y$, BMI: $\left.30.1 \pm 1.0 \mathrm{~kg} / \mathrm{m}^{2}\right)$ were randomised to either an IER $(2638 \mathrm{~kJ}$ delivered using a formula based very low calorie diet (VLCD) (LighterLife UK Ltd) for 2 consecutive days/week, $\mathrm{n}=14$ ) or CER (2510kJ below requirements) diet group. 6-hour postprandial responses to a test meal as well as changes in anthropometry (fat mass, fat free mass, waist circumference) were assessed at baseline and following attainment of a $5 \%$ weight-loss. Between-group changes were assessed using analysis of covariance (ANCOVA) with post-treatment values as the dependent variable, and baseline values of each parameter as the covariate. The Mann Whitney U test was used as the non-parametric alternative to ANCOVA. Within-group changes were assessed using paired t-tests (or non-parametric Wilcoxon signed ranks test).

Results: Time to attain a $5 \%$ weight loss was similar between IER and CER diet groups (median 59 [interquartile range: 41-70] days and 73 [interquartile range: 48,128 ] days respectively, $\mathrm{p}=0.246$ ), as were changes in body composition ( $\mathrm{p} \geq 0.430)$. Postprandial data: Both interventions comparatively $(\mathrm{p}=0.903)$ reduced postprandial insulinaemia (neither diet significantly altered glycaemia), however the relative reduction in postprandial triacylglycerol responses was greater following IER $(p=0.042)$. Conclusion: Our data suggests that a modest $5 \%$ weight-loss through IER exerts more favourable effects on postprandial lipaemia, an important car- diovascular risk factor, when compared to similar weight-loss achieved through CER. These data are novel and now require replication in larger study cohorts, as well as mechanistic evaluation.

\section{References:}

1 Antoni R et al. (2014) Res Endocrinol 2014 Article ID 459119

2 Antoni R et al. (2016) Br J Nutr 115(6), 951-959

Conflicts of interest: KLJ is employed by LighterLife UK Ltd.

Funding: Research relating to this abstract was funded by LighterLife UK Ltd

\section{T3P186}

The effect of a physical activity consultation on the BMI of overweight adolescents. Results from a pediatric outpatient obesity clinic.

\section{Silva, A. ${ }^{1}$; Fonseca, H. $^{2}$}

${ }^{1}$ Lisbon Academic Medical Centre, ${ }^{2}$ Department Of Pediatrics, Hospital De Santa Maria, Lisbon

Introduction: Adolescent overweight is a major public health concern. It is associated with several short and long-run adverse health conditions. Physical Activity (PA) is recognized as having many obesity-related health benefits. However, the majority of the overweight adolescents still present low levels of PA. PA consultation can be a cost-effective way of enhancing $\mathrm{PA}$ and improve weight status of these adolescents. The main aim of this study was to analyze whether adolescents who are attending a PA consultation in a tertiary health care setting, showed more therapeutic success at six months, based on body mass index (BMI) $\mathrm{z}$-score and waist circumference (WC), compared to those following the standard approach (Pediatrician and Dietitian).

Methods: Clinical records from 396 patients, aged 10-17 (Mean age $=12.8$; $\mathrm{SD}=2.8$ ), were analyzed: PA consultation (PAc) group, $n=198$; Standard consultation (STc) group, $n=198$. Baseline differences between groups were analyzed using Chi-square and independent sample t-tests. Changes within groups and between groups were analyzed using paired sample t-tests and independent sample t-tests, respectively.PAc consisted in a patient-centered, one-to-one session, combining motivational interview techniques and cognitive behavior approaches to strengthen individual's motivation to achieve a positive PA behavior change.

Results: No statistically significant differences between the two groups were found at baseline. Patients in both groups decreased their BMI $\mathrm{z}$-score. The PAc has shown a higher decrease in the BMI z-score (PAc $-0.12, p<.0001$; STc $-0.05, p<.0001)$. The WC of the PAc group has increased $2.2 \mathrm{~cm}$ less than the STc's $(p<.005)$. The difference in waistto-height ratio between the PAc and STc groups was of $0.015(p<.005)$, showing a trend to decrease in the PAc group $(-0.005, p=172)$.

Conclusion: Although the success of adolescent obesity management cannot be based only on BMI and WC, a PA consultation on the top of the traditional Pediatric and Dietetic interventions may further improve the BMI z-score outcome at six months. Waist-to-Height ratio may be more suitable than waist circumference per se to track changes in abdominal adiposity among adolescents who are still growing. 
T3P187

The effect of green tea extract supplementation on weight changes, serum malondialdehyde and blood iron indices in pulmonary TB patients: A randomized controlled trial

Eghtesadi, S. ${ }^{1}$; Honarvar, M. ${ }^{2}$; Gill, P.3. Jazayeri, S. ${ }^{4}$; Vakili, M.5. Shamsardekani, M. ${ }^{6}$; Abbasi, A. ${ }^{7}$

${ }^{1}$ Iran University of Medical Sciences,School of Public Health Tehran, Iran; Azad University, Science and Research Branch,School of Medical Sciences,Tehran, Iran, ${ }^{2}$ Iran University Of Medical Sciences, School Of Public Health, Tehran, Iran: Golestan University Of Medical Sciences, Health Bureau,Gorgan,Iran, ${ }^{3}$ Departments Of Physiopharmacology\&Nanobiomedicine, Research Center For Immunogenetics,Faculty Of Medicine, Mazandaran University Of Medical Sciences ,Sari, Iran., ${ }^{4}$ Research Center For Prevention Of Cardiovascular Disease;Department Of Nutrition, School Of Public Health, Iran University Of Medical Sciences, Tehran, Iran., ${ }^{5}$ Health Management And Social Development Research Center,Department Of Health ,Golestan, Iran., ${ }^{6}$ Department Of Pharmacognosy, Faculty Of Pharmacy And Medicinal Plants Research Center, Tehran University Of Medical Sciences, Tehran , Iran., ${ }^{7}$ Department Of Infection, Faculty Of Medicine, Golestan University Of Medical Sciences, Gorgan, Iran.

Intoduction: High prevalence of TB in Golestan Province in north of Iran motivated us to use supplementation with green tea extract to promote healing.The aim of this study was to investigate the effect of green tea consumption on weight changes, iron status and improving process of pulmonary tuberculosis treatment

Methods: This double-blinded randomized clinical trial study, was conducted on patients with TB, who were assigned randomly to the green tea group (41 patients) receiving $500 \mathrm{mg}$ catechin of green tea extract and the control group (39 subjects) receiving placebo for two months since the beginning of concomitant anti-TB treatment. Height and weight were measured at first and two and six months thereafter. Demographic , food frequency questionnaires and 24-hour dietary recalls of three non-consecutive days were completed. After obtaining $10 \mathrm{ml}$ of venous blood, $\mathrm{He}$ moglobin (Hb), Transferrin, Ferritin, Total iron binding capacity (TIBC), Iron and Serum malondialdehyde (MDA) were measured at the beginning and the end of the study. Data were processed using independent and paired t-test, McNemar, Wilcoxon, Kaplan-Meier, Log-rank test, Cox regression model and nutrition 4 sottware.

Results:At the beginning of the survey, two groups were similar according to age, gender and clinical status $(\mathrm{p}>0.05)$. Average daily energy intake of patients was $1518 \pm 434 \mathrm{kcal}$, distribution of which was as follow: carbohydrates (58\%), protein (17\%) and fat (22\%).Vitamin D and Zinc intake of patients were less and iron intake was higher than the DRI. Weight changes in both groups of placebo and green tea had tendency of increase with a significant difference at two and six month follow ups $(\mathrm{p}<0.0001)$. However, there were no significant changes due to intervention compared to placebo. ANCOVA test showed mean difference level (Pvalue) in both groups for $\mathrm{Hb}$, iron, TIBC, transferrin and ferritin as of: $0.004,0.56,0.65$, 0.38 and 0.16 , respectively which means that increase of hemoglobin in the green tea group was significant compared to the placebo group. There was just a $9.2 \mathrm{nmol} / \mathrm{ml}$ difference between the two groups for MDA at the beginning of study, which was not statistically significant $(\mathrm{p}=0.078)$ whereas, it was increased to $24.8 \mathrm{nmol} / \mathrm{ml}$ after the intervention, indicating a significant difference $(\mathrm{p}<0.001)$. The decline value was estimated $-45.45 \pm 14.69 \mathrm{nmol} / \mathrm{ml}$ for catechin group and $-19.91 \pm 18.38 \mathrm{nmol} / \mathrm{ml}$ for placebo group.

Conclusion:Green tea as an adjuvant to TB treatment can reduce MDA concentration and improve hematopoiesis and hemoglobin level,but no significant effect on weight gain compared to placebo group.

Conflict of Interest: None

Funding: Research was funded by Golestan and Iran Universities of Medical Sciences.

\section{T3P188}

The effects of sprint interval exercise training on hepatic and peripheral insulin sensitivity (IS), as well as intrahepatic triglyceride (IHTG), in men with nonalcoholic fatty liver disease (NAFLD).

Sargeant, J. ${ }^{\text {; }}$ Bawden, S. ${ }^{2}$; Simpson, E. ${ }^{2}$; Gowland, P. ${ }^{2}$; Dorling, J. ${ }^{\text {; }}$; Nimmo, M. ${ }^{3}$; Macdonald, I. ${ }^{2}$; Aithal, G. ${ }^{2}$; King, J. ${ }^{1}$

${ }^{1}$ Loughborough University, UK, ${ }^{2}$ University Of Nottingham, Uk, ${ }^{3}$ University Of Birmingham, Uk

Introduction: Sprint interval training (SIT) is a novel form of exercise (high intensity, short duration) which improves IS in healthy, overweight adults despite a total exercise volume much lower than current guidelines. This study investigated the effects of SIT on hepatic and peripheral IS, and IHTG, in men with NAFLD.

Methods: 9 overweight/obese men with NAFLD (mean \pm SD; BMI: $32 \pm 3$ $\mathrm{kg} \cdot \mathrm{m}^{-2}$; IHTG: $\left.15.6 \pm 8.4 \%\right)$ were assessed at baseline, after a control phase ( $\geq 3 \mathrm{wks}$ ), and then following 6 wks of SIT (4-6 maximal 30-second cycling sprints, 3 times per wk). Dual-step hyperinsulinaemic, euglycaemic clamps (insulin infusions: 20 and $50 \mathrm{mU} \cdot \mathrm{m}^{-2} \cdot \mathrm{min}^{-1}$ ) with simultaneous infusion of $\left[6,6-{ }^{2} \mathrm{H} 2\right]-2$-glucose tracer were used to assess suppression of endogenous glucose production (\%EGPsupp) and whole-body glucose uptake $(M)$ as measures of hepatic and peripheral IS. IHTG was measured using $3 \mathrm{~T}{ }^{1} \mathrm{H}$-magnetic resonance spectroscopy. Participants also completed a peak oxygen uptake ( $\mathrm{VO} 2$ peak) test. Data were analysed by repeated measures ANOVA and post-hoc tests as appropriate. Outcome data are presented as relative change after SIT (mean \pm SEM).

Results: There were no significant changes from baseline to post-control (all $\mathrm{p}>0.05)$. SIT improved VO2 peak $(13.5 \pm 2.4 \%, \mathrm{p}<0.001)$ and reduced IHTG $(-12.4 \pm 9.8 \%, \mathrm{p}=0.03)$ despite no change in body weight $(\mathrm{p}=0.17) . M$ increased over the 3 visits $(\mathrm{p}=0.03, \mathrm{n}=8)$, but the mean change after SIT was not significant $(17 \pm 9 \%, \mathrm{p}=0.11)$ due to large variability in response $(-23$ to $+64 \%)$. There were no changes after SIT in basal EGP or \%EGPsupp ( $\mathrm{p} \geq 0.49 ; \mathrm{n}=7)$.

Conclusion: 6 wks of SIT improves VO2 peak and reduces IHTG without significant changes in body weight. Hepatic IS remains unchanged with SIT whilst the peripheral IS response is highly variable between individuals.

Conflict of Interest: IM is on the Scientific Advisory Boards for Nestlé, Ikea and Mars Inc.

Funding: This study was supported by the NIHR Leicester-Loughborough Diet, Lifestyle and Physical Activity Biomedical Research Unit based at University Hospitals Leicester and Loughborough University, UK and the NIHR Nottingham Digestive Diseases Biomedical Research Unit based at Nottingham University Hospitals and the University of Nottingham, UK.

\section{T3P189}

\section{The fat score, a fibrosis score of adipose tissue: Predicting} weight loss outcome after gastric bypass

Lassen, P. '; Charlotte, F. ${ }^{2}$; Liu, Y. '; Bedossa, P. ${ }^{3}$; Naour, G. ${ }^{2}$; Tordjman, J. ' ; Poitou, C. '; Bouillot, J. ${ }^{4}$; Genser, L. '; Zucker, J. ' ; Sokolovska, N. ' ; AronWisnewsky, J. ${ }^{1}$; Clement, $K{ }^{1}$

${ }^{1}$ Institute of Cardiometabolism and Nutrition, ICAN, F-75013, Paris, France, INSERM UMRS 1166 Team 6 Nutriomics, ${ }^{2}$ Ap-Hp, Pitie Salpetriere Hospital, Department Of Pathology, Uimap, Upmc Universite Paris 06, Paris, F-75013 France, ${ }^{3} \mathrm{Ap}-\mathrm{Hp}$, Pitie-Salpetriere Hospital, Department Of Pathology, Uimap, Upmc Universite Paris 06, Paris, F-75013 France; ${ }^{4} \mathrm{Ap}-\mathrm{Hp}$, Department Of General, Digestive And Metabolic Surgery, Ambroise Pare University Hospital,Versailles Saint-Quentin University, F-92100, Boulogne, France

Context: Bariatric surgery (BS) induces major and sustainable weight loss in many patients, but a significant proportion of patients display poor weight loss response. Factors predicting poor responders (PR) must be identified to improve patient care. Quantification of subcutaneous adipose tissue (scAT) fibrosis is negatively associated with post-BS weight 
loss outcome, but whether scAT fibrosis could be a predictive factor applicable in clinical routine remains to be demonstrated.

Objective: To create a semi-quantitative score evaluating scAT fibrosis and test its predictive value on weight loss response after gastric bypass surgery.

Material and Methods: We created a semi-quantitative score (FAT score) integrating peri-lobular (PLF) and peri-cellular fibrosis (PCF). Using this FAT score, we characterized 183 peroperative scAT biopsies (stained with picrosirius red) of severely obese patients ( 85 from a training cohort, 98 from a confirmation cohort) who underwent Roux-en-Y gastric bypass (RYGB). PR to RYGB was defined as $<28 \%$ of total weight-loss at one year (lowest tertile). We tested the link between the FAT score and PR in univariate and multivariate models.

Results: FAT scoring was directly associated with increasing scAT fibrosis measured by a standard quantification method ( $\mathrm{p}$ for trend $<0.001$ ). FAT score inter-observer agreement was good $(\nmid \hat{\mathrm{E}}=0.76)$. FAT score ¡Y 2 was significantly associated with PR. The association remained significant after adjustment for age, diabetes status, hypertension, percent fat-mass and IL-6 (adjusted OR = 3.6, IC 95\% [1.8, 7.2]; $\mathrm{p}=0.003$ ).

Conclusion: The FAT score is a new simple semi-quantitative evaluation of human scAT fibrosis that may be used to help identifying potential patients with reduced body weight loss after gastric bypass.

\section{T3P190}

\section{“The healthy lifestyle pyramid - an innovative relief board used in obesity prevention among pupils with visual impairment"}

\section{Wrzesińska, M. ${ }^{1}$; Barreda, G. ; Pijpker, R. ; Czarnecka, K. ${ }^{4}$}

${ }^{1}$ Department of Psychosocial Rehabilitation, Medical University of Lodz, Poland, ${ }^{2}$ Department Of Health Promotion And Development, University Of Bergen, Norway, ${ }^{3}$ Department Of Social Science, Health And Society, Wageningen University And Research, Wageningen, The Netherlands, ${ }^{4}$ Department Of Molecular Bases Of Medicine I Chair Of Internal Medicine, Medical University Of Lodz, Poland

Introduction: Young people with disabilities are at greater risk of developing obesity because of a low level of physical activity and improper health behavior connected with disability or secondary condition. It has been estimated that between $18.4 \%$ and $63 \%$ of the children and adolescents with visual impairment (VI) develop overweight or obesity before adulthood. Interventions targeting children and adolescents to improve health literacy are recommended to promote healthy diets and to prevent obesity. Methods: The Design Thinking and Universal Design methods were used to create the Healthy Lifestyle Pyramid for students with VI. This is an innovative educational relief board in $\mathrm{A} 2$ format that presents the basic recommendations regarding nutrition and healthy lifestyle. The board contains pictures especially selected for the poor-sighted and text descriptions in a big black font including Braille transcription.

A pilot study of an intervention was conducted in two Polish Special Schools for pupils with VI. 37 poor-sighted students without other disabilities aged 14-18 years old (15.70 \pm 0.98$)$ took part in the study. Participants were assigned to use the Pyramid in the study group $(n=16)$ and the traditional paper leaflet with the common recommendations in the control group $(\mathrm{n}=21)$. The intervention, describing the same healthy guidelines necessary in obesity prevention, was performed during one school class. The control test containing 9 questions, adapted to visual perception, was prepared to verify of the acquired knowledge. A pretest (T1), before the intervention, posttest (T2), directly after the intervention, and secondary posttest (T3), three months after were performed in both groups.

Results: A higher percentage of correct answers regarding the healthy guidelines between the pretest (T1) and posttest (T2) was noted in the study group ( $47.9 \%$ vs $\left.81.5 \% ; \mathrm{ch}^{2}=34.14 ; \mathrm{p}=0.000\right)$ and in the control group $\left(52.9 \%\right.$ vs $79.4 \%$; $\left.\mathrm{chi}^{2}=29.53 ; \mathrm{p}=0.0000\right)$. However, the presence of correct answers increased in the study group by 33.6 percentage point and in the control group by 26.4 percentage point. In regards to the sec- ondary posttest (T3), reduction in the number of correct answers was observed in both groups, however a smaller decrease was noted in the study group (19.6 percentage point $v s 24.5$ percentage point).

Conclusion: Usefulness of the Healthy Lifestyle Pyramid in improving nutritional knowledge of students with VI was observed. Additional interventions with the Pyramid are necessary to prove the impact on developing dietary behaviors in large groups of schoolchildren with and without disability.

\section{Conflict of interest: None disclosed}

Fundings: Research relating to this abstract was funded by MSHE in Poland no 502-03/5-127-03/502-54-177

\section{T3P191 \\ The impact of a complication-centric approach on the evaluation of obese patients.}

\section{Balsa A.M. ${ }^{1}$ : Alves M. ${ }^{1}$ :Azevedo T. ${ }^{1}$ Dantas R. ${ }^{1}$ 'Guimarães J. ${ }^{1}$}

${ }^{1}$ Centro Hospitalar Baixo Vouga

Introduction: The WHO definition and classification of obesity is based on anthropometric parameters but it lacks clinical prespective. Giving its limitations, the AACE and ACE proposed in 2014 an Advanced framework for a new diagnosis of obesity as a chronic disease, basing therapeutic options on the presence and severity of obesity-related complications.

Goal: To evaluate the impact of an approach based on obesity-related complications in the evaluation of obese patients and to assess its correlation with BMI

Methods: Retrospective study of patients followed-up in Obesity outpatient in CHBV during 2016, collecting data from the clinical files and classifying the patients according to $\mathrm{WHO}$ and these AACE/ACE guidelines. Results: we included 359 patients, ( 80 male, 279 female) with a mean age of $43.88 \pm 13.92 \mathrm{y}$ and a mean BMI of $41.3 \pm 5.4 \mathrm{Kg} / \mathrm{m}^{2}$. Considering the WHO classification: 38 (10.6\%) had class I obesity, 122 (34\%) class II and 199 (55.4\%) class III. A total of 239 (66.6\%) would be eligible to bariatric surgery. According to AACE/ACE 61 (17\%) had no identified obesity-related complications and therefore were stage $0,169(47.1 \%)$ had one or more mild-to-moderate complications - stage 1, and 129 (35.9\%) had at least one severe complication - stage 2 , and were eligible to more aggressive treatment. Mean BMI for each stage was $40.4,40.7$ and $42.52 \mathrm{Kg} / \mathrm{m}^{2}$, respectively. Spearman correlation coefficient $(0.156)$ didn't show a linear dependence between BMI and stage. The main disparities between classifications were found for the $9(23.7 \%)$ of the WHO class I patients that were stage 2 on AACE/AE and $80(40.2 \%)$ of the class III patients that had no obesity-related complications.

Discussion: A patient-oriented approach for obesity should focus mainly on preventing and improving obesity-related complications rather than solely reducing body weight. The clinical evaluation identified fewer patients eligible to surgery, but potentially the ones benefitting the most. As weight and BMI didn't correlate directly with the presence and severity of obesity-related complications, clinical assessment should be fundamental to set therapeutic goals and strategies.

\section{T3P192}

\section{The impact of an mhealth-supported lifestyle intervention on dietary and physical activity outcomes among overweight and obese pregnant women: A randomised controlled trial.}

\section{Ainscough, K. ${ }^{1}$; Kennelly, M. '; Lindsay, K. '; O'Sullivan, E. '; Gibney, E. ';} Mcauliffe, $F$.

${ }^{1}$ UCD Perinatal Research Centre, Obstetrics \& Gynaecology, School of Medicine, University College Dublin, National Maternity Hospital, Dublin, Ireland, ${ }^{2} U$ cd Institute Of Food And Health, School Of Agriculture And Food Science, University College Dublin, Ireland

Background: Improving lifestyle behaviours among overweight and obese women could improve pregnancy outcomes. However, an efficient 
and effective intervention, suitable for translation into clinical care, is required. Our aim was to investigate the impact of a 'healthy lifestyle package, including smartphone-app support, among overweight and obese pregnant women, on glycaemic index (GI), glycaemic load (GL), diet quality as measured by the Alternate Healthy Eating Index modified for Pregnancy (AHEI-P), behavioural stage-of-change for physical activity (PA), and PA levels.

Design: A randomised controlled trial of a mobile health technology (mHealth)-supported behavioural lifestyle intervention among overweight and obese pregnant women. Women $(n=565)$ were randomised in early pregnancy $(15.7 \pm 2.3$ weeks $)$ to an intervention $(n=278)$ or control group $(\mathrm{n}=287)$. The intervention was grounded in behaviour-change theory and consisted of nutrition and exercise advice, supported by a smartphone app, and follow-up email and in-person contact from the research team. The control group received routine antenatal care, with no specific dietary and exercise advice. At 16 and 28 weeks' gestation, dietary data using 3-day food diaries, stage-of-change score for PA (1-5; where a higher score is indicative of increased readiness to engage in exercise), and PA data were collected. Data collection methods were previously validated in pregnancy. Data were log transformed where not normally distributed. Data presented are for those who responded at 16 and 28 weeks, analysed by general linear models and $\chi 2$.

Results: There were no differences between groups at baseline. The effect of the intervention on diet and PA parameters, adjusting for baseline data is shown in Table 1.

Conclusion: A randomised controlled trial of a behavioural lifestyle intervention, supported by a smartphone app, succeeded in improving maternal dietary intakes, diet quality, and PA behaviours. These findings are significant for obstetric patients that may benefit from easily-accessible lifestyle advice as part of routine care to improve diet and exercise. This highlights the potential for mHealth to aid interventions aiming to help women overcome barriers to lifestyle changes.

Table 1. Comparison of dietary and physical activity outcomes at 28 weeks' gestation

\begin{tabular}{|l|l|l|l|}
\hline & Intervention & Control & $P^{*}$ \\
\hline & $(\mathrm{n}=147)$ & $(\mathrm{n}=143)$ & \\
\hline $\mathrm{GI}$ & $56.5 \pm 4.5$ & $57.9 \pm 4.8$ & 0.001 \\
\hline $\mathrm{GL}$ & $117.4 \pm 33.2$ & $131.7 \pm 36.1$ & $<0.001$ \\
\hline AHEI-P Score & $48.3 \pm 10.6$ & $46.6 \pm 9.7$ & 0.021 \\
\hline MET-minutes per wk & $631.5 \pm 426.6$ & $467.5 \pm 322.3$ & 0.002 \\
\hline $\begin{array}{l}\text { Maintained/increa- } \\
\text { sed stage-of-change } \\
\text { for PA score** }\end{array}$ & $130(90.3)$ & $101(72.7)$ & 0.001 \\
\hline
\end{tabular}

${ }^{*}$ Data are mean \pm SD. P values calculated by general linear models.

${ }^{*}$ Data are $\mathrm{n}(\%)$. P values calculated by $\chi^{2}$.

\section{T3P194}

\section{The impact of overweight and obesity on the motor and secretory activity of the upper gastrointestinal tract}

\section{Sergey, T. $^{1}$; Vladimir, S. ${ }^{1}$; Nadejda, L. $^{1}$}

${ }^{1}$ North-western State Medical University named after I.I.Mechnikov

Introduction: comorbidity of obesity and gastroesophageal reflux disease (GERD) is well known. The main theories explaining this relationship are mechanical theory - increased intraabdominal, intragastric pressure, increased the amount of acid gastroesophageal reflux (GER); endocrine theory - production of proinflammatory cytokines by adipose tissue. In our work we have attempted to identify the link between obesity and non-acidic reflux variants.

Methods: Patients with symptomatic GERD performed physical examination, fibrogastroduodenoscopy, daily $\mathrm{pH}$-impedancemetry of upper gastrointestinal tract, impedancemetry of body; testing by SF-36, Spiel- berger and_Beck questionnaires. The study involved 50 patients 51,86 \pm 14 years old, 24 men and 26 women; non-erosive reflux disease (NERD) - 23, reflux esophagitis (RE) -27.

Results: In our study, we detected no association of overweight and obesity with GERD symptoms, form and personality profile of patients. There was not significant differences in BMI between groups NERD and RE, but the patients with abdominal obesity were more common in group of RE: waist circumference (WC) / hips circumference (HC) ER vs. NERD $-0.89 \pm 0.6$ vs. $0.82 \pm 0.7(\mathrm{p}<0.05)$. WC of patients correlated with the amount of alkalin GER (0.53); weakly acidic bolus time min and \% (0.52); bolus alkaline time in minutes and \% (0.56); overall reflux time in minutes and \% (0.48 and 0.52$)$. WC/HC correlated with the number of proximal GER (0.46), slightly acidic bolus time in minutes and \% (0.44 and $0.43)$; overall reflux time in minutes and \% (0.51 and 0.52$)$. BMI had no correlation with any of the $\mathrm{pH}$-metrics dates. The degree of obesity was correlated with the amount of alkaline reflux (0.7); bolus alkaline time in minutes and \% (0.66 and 0.63$)$. Body fat \% correlated with the amount of alkaline reflux (0.52) negatively correlated with the total time of the esophagus acidity $(-0.45)$; the number of acid reflux duration more than 5 minutes $(-0.46)$; the duration of the longest reflux $(-0.47)$; index DeMeester $(-0,52)$.

Conclusion: The presence of abdominal obesity is associated with features of the GERD pathogenesis, which can potentially lead to the resistance to standard acid-suppressive therapy. The increasing of WC and WC/HC lead to significant increasing of GER bolus in the esophagus due to the presence of weakly acidic and alkaline bolus, but not of acidic. This fact indicates the motor discoordination not only at the level of the lower esophageal sphincter but pyloric sphincter and biliary system in patients with abdominal obesity. This fact may indicate that patients with comorbidity of abdominal obesity and GERD may require alternative therapeutic approach due to non-acid impacts.

No Conflict of Interest

\section{T3P195 \\ The influence of nutrition and physical activity on glycemic control in patients with diabetes in Bosnia and Hercegowina- Republic of Srpska}

\section{Popović-Pejičlć, S. ${ }^{1}$; Jankovic-Pejicic, $A{ }^{2}$ \\ ${ }^{1}$ Medical faculty,University of Banjaluka Bosnia\&Hercegowina, ${ }^{2} \mathrm{School}$ Of Dentistry, Medical University Of Vienna}

Materials and Methods: The study included 1088 people, aged (>18), chosen randomly from registries of patients with Diabetes Type 1 (DM1) and Type 2 (DM2) in the primary health clinics. Specially created index of healthy nutrition was used (Healthy diet score), to estimate the participant's type of food intake. Their socioeconomic status was estimated on the basis of the parameters of social standing.

Results: Participants with DM1 comprised 4.6\%, those with DM2 95.4\% of the study group, $50.1 \%$ of whom were obese. There were $55.1 \%$ women and $45.9 \%$ men and patients older than 55 with DM2 comprised $86.3 \%$ $61.8 \%$ of study participants lived in single-income households. $62.3 \%$ of participants total monthly income was under $600 \mathrm{KM}$ (Euro 300).Poor glycoregulation $(\mathrm{HbAl} \mathrm{c} \geq 6.5 \%$ ) was seen in $61.8 \%$ participants and it was worse with the duration of illness with statistical significance $\left(\chi^{2}=62.070\right.$, $\mathrm{df}=2, \mathrm{p}=0.000)$. There were $51.8 \%$ without education beyond partial or high school education, and they had a statistically significant worse glycoregulation $\left(\chi^{2}=15.650, \mathrm{df}=16, \mathrm{p}=0.016\right)$. Macrovascular complications, including the most common cardiovascular diseases $(82.1 \%)$, occurred at the same rates regardless of the quality of glycoregulation in the study group, while microvascular complications were more common in patients with DM2 with poor glycoregulation with statistical significance $\left(\chi^{2}=5.220, \mathrm{df}=1, \mathrm{p}=0.022\right)$.In their daily food intake, items with saturated fats and sugars dominated the diet.Rhythm of 5-6 meals per day is present in $18 \%$ study subjects. Participants with DM1 have a better rhythm of food consumption, as they consume breakfast more often, with 
statistically significant difference $\left(\chi^{2}=6.667, \mathrm{df}=2, \mathrm{p}=0.036\right)$, as well as dinner $\left(\chi^{2}=9,969, \mathrm{df}=2, \mathrm{p}=0,007\right)$ in comparison with participants with DM2. The ideal Healthy Diet Score was noted in only $7.5 \%$ of participants. Daily physical activity was noted in $35.6 \%$ of subjects, $15 \%$ were smokers, and $63.5 \%$ reported alcohol consumption.

Conclusion: Morbidity of diabetes in Republic of Srpska with respect to type, sex and age structure are on par with the trends in the world. Patients according to diet, frequency of alcohol consumption, smoking and physical activity, as well as socioeconomic status, occupation and education correspond to the status of underdeveloped or developing countries, which among other factors explains the poor quality of glycemic control and the incidence of chronic complications.

\section{T3P196}

\section{The long-term metabolic outcomes of weight loss in patients with type 2 diabetes: A longitudinal analysis following laparoscopic adjustable gastric banding combined with medical care}

\section{Mistry, P. ${ }^{1}$; Currie, V. ${ }^{1}$; Super, P. ${ }^{1}$; Roux, C. ${ }^{2}$; Singhal, R. ${ }^{1}$; Tahrani, A. ${ }^{3}$}

${ }^{1}$ Upper GI Unit and Minimally Invasive Unit, Heart of England NHS Foundation Trust, Birmingham, UK, '2Department Of Pathology, University College Dublin, Dublin, Ireland, ${ }^{3}$ Institute Of Metabolism And Systems Research, University Of Birmingham, Birmingham, Uk, Department Of Diabetes And Endocrinology, Heart Of England Nhs Foundation Trust, Birmingham, Uk, Centre Of Endocrinology Diabetes And Metabolism, Birmingham Health Par

Introduction: The long-term outcomes of weight loss maintenance induced by laparoscopic adjustable gastric banding (LAGB) in patients with type 2 diabetes mellitus (T2DM) (beyond 3 years) are scarcely reported. In addition, there is limited evidence assessing the impact of long-term weight loss induced by LAGB on lipids and blood pressure (BP) in patients with T2DM. The aim of this study was to determine the long-term impact of weight loss on patients with Type 2 diabetes mellitus following LAGB combined with medical care.

Methods: A longitudinal analysis of adults with Type 2 diabetes who had LAGB between 2003 and 2008 and were followed-up till 2013. Data was obtained from electronic patients' records, the local bariatric database and from the patients' primary care physicians.

Results: Two-hundred patients (age $47 \pm 9.7$ years, weight $150.3 \pm 29.8$ $\mathrm{kg}$, body mass index (BMI) $52.8 \pm 9.2 \mathrm{Kg} / \mathrm{m}^{2}$, glycosylated haemoglobin (HbAlc) $7.9 \pm 1.9 \%$, women $\mathrm{n}=123(61.5 \%)$, insulin treatment $\mathrm{n}=71$ (35.5\%)) were included. The mean follow-up was $62.0 \pm 13.0$ months (range 18 to 84 months). By the end of follow-up there were significant reductions in body weight $(-24.4 \% \pm 12.3 \%(38 \pm 22.7 \mathrm{~kg}))$, HbAlc $(1.4 \pm 2.0 \%)$, systolic blood pressure (BP) $(-11.7 \pm 23.5 \mathrm{mmHg})$, and lipids profiles. The proportion of patients requiring insulin reduced from $36.2 \%$ to $12.3 \%(\mathrm{p}<0.001)$. Greater reductions in $\mathrm{HbAlc}$ were predicted by higher baseline $\mathrm{HbAlc}$, younger age, and not receiving insulin pre-operatively. Diabetes duration, pre-intervention BMI and \% weight change were not associated with changes in HbAlc, systolic BP, total cholesterol or triglycerides following LAGB.

Conclusions: LAGB when combined with multidisciplinary medical care significantly improved metabolic outcomes in patients with Type 2 diabetes independent of diabetes duration, baseline BMI and weight loss (except in regards to diastolic BP). Diabetes duration and baseline BMI should not be considered barriers to improve metabolic outcomes by long term weight loss induced by LAGB in combination with medical care.

Conflict of Interest: None Disclosed

Funding: AAT is funded by the National Institute for Health Research (UK). The views presented are neither those of NIHR or The Department of Health

\section{T3P197}

The power of football: A socio-psychological approach to promoting behaviour change in overweight and sedentary men in the European fans in training project

Silva, M. ${ }^{\prime}$; Teixeira, P. ; Gray, C. ${ }^{2}$; Hunt, K. ${ }^{2}$; Bunn, C. ${ }^{2}$; Roberts, G. ${ }^{3}$; Wyke, S. ${ }^{2}$; Consortium, $\mathrm{O}^{4}$

${ }^{1}$ Faculty of Human Kinetics, University of Lisbon, ${ }^{2}$ University Of Glasgow, ${ }^{3}$ Norwegian School Of Sport Sciences, ${ }^{4}$ (Fp7 Grant 602170)

Introduction: Achieving sustained health behaviour change remains a challenge, and certain at-risk population groups hard to engage. Advancing the science of behaviour change requires a good understanding of how interventions are informed by theory and how they can better test it. The development of the European Fans in Training (EuroFIT) program was based on sociological and motivational theory to engage male football supporters in four European countries in initiating and maintaining improvements in physical activity, sedentary, and dietary behaviours.

Methods: EuroFIT tests the utility of a culturally- and gender-sensitized lifestyle program in about 1200 middle-aged (30-65y) men in Portugal, Norway, the Netherlands, and the UK. Men participate in a 12 -week behaviour change program, taking place in their football clubs. The strong affiliation and loyalty to clubs are leveraged to increase relatedness and personal interest in the program. Advances in technology, providing real-time, self-relevant feedback (SitFit), as well as connection to other participants (MatchFit) are used to sustain engagement and self-regulation.

Results: The EuroFIT intervention is currently being evaluated in a randomised controlled trial in the 15 participating clubs, led by club coaches. Alongside other perspectives (e.g., from sociology and gender studies), Self-Determination Theory (SDT) and Achievement Goal Theory are integral components of the core intervention and measurement protocol. Specifically, the program is designed to help men develop autonomous goals, gradually build competencies through optimally challenging physical activity and dietary changes, and strengthen relatedness through meaningful connections to group members and the club.

Conclusion: In this presentation, we will describe the elements of EuroFIT that highlight theorethical constructs and how they were linked to the intervention strategies developed, while discussing both form and function. Results will allow to test the efficacy of the intervention as partially mediated by sdt constructs such as need satisfaction and autonomous motivation, resulting in sustained healthy lifestyles and improved health and well-being.

\section{Conflict of Interest: None Disclosed}

Funding: This project has received Funding from the European Union's Seventh Framework Programme for research, technological development and demonstration: grant agreement no 602170 .

\section{T3P198}

The relation of urine phthalate metabolite is specific for type 2 diabetes and is not mediated by $\mathrm{BMI}$ and related metabolic diseases.

Svacina, S. ${ }^{1}$; Matejkova, D. ${ }^{2}$; Mullerova, D. ${ }^{3}$; Vrbik, K. ${ }^{4}$; Pavlouškova, J. ${ }^{4}$; Piecha, R. ${ }^{1}$; Maly, M. ${ }^{4}$

${ }^{1}$ Charles University, 1 st Medical faculy, 3rd Medical Department, ${ }^{2}$ Hospital Mariánské Láznì, Czech Republic, ${ }^{3}$ Faculty Hospital Pilsen, Czech Republic, ${ }^{4}$ State Health Insitute, Czech Republic

Human exposure to organic pollutants may be associated with metabolic outcomes. We have investigated urine samplesof patients $(n=170)$ from metabolic outpatient department . Using standard metabolic syndrome criteria and used therapy we have classified patients as dyslipidaemic $(n=87)$, hypertensive $(n=96)$ and type 2 diabetes $(n=56)$. bmi was also measured. The 24 hours samples were sampled in phthalate free bottles. Bisphenol A and 15 metabolites of phthalates were evaluated in relation to creatinine excretion. All were analysed with enzymatic cleavage of glucuronide using ultra-high-performance liquid chromatography-electro- 
spray ionization tandem mass spectrometry in one laboratory with External Quality control.

Results: 1. Using multiple linear regression with 3 variables - diabetes, hypertension and dyslipidemia - only diabetes showed significant results $\mathrm{p}<0.05$ in four phthalate metabolites.

2. In linear regression with two variables ( diabetes, bmi) the influence of bmi was slightly negative but not significant in all 4 metabolites .

3. There was not any relation of bisphenol A level to diabetes, hypertension, dyslipidemia and bmi.

4. There was no relation of phtthalate levels to age and gender.

Conclusion: Urine levels of four phthalate metabolites are significantly related to type 2 diabetes. This influence is not mediated by bmi. We have found no relation to bmi, dyslipidemia, hypertension, gender and age. Phthalate levels can be in causal relation to beta cell dysfunction (significant relation to only four specific metabolites) rather then to specific diabetes behavior (e.g. diet, skin care), where the relation would be more complex to all phthalate metabolites.

Supported by a research grant of Czech ministry of Health nt 14182-3.

No conflict of interest

\section{T3P200}

\section{The role of genetic factors in predicting the results of obesity} treatment with sibutramine

Marina, G. '; Ekaterina, T. ${ }^{2}$; Anna, V. ${ }^{3}$ Andrey, A. ${ }^{4}$; Anastsiya, $P^{4}{ }^{4}$; Kseniya, K. ${ }^{2}$; Oksana, L. ${ }^{2}$

${ }^{1}$ Endocrinology Research Centre, Moscow, Russia, ${ }^{2}$ Endocrinology Research Centre, Moscow, Russian Federation, ${ }^{3}$ Voronezh State Medical Univercity, Voronezh, Russia, ${ }^{4}$ Laboratory Of Genetic Ingeneering Systems Lagis, Moscow, Russia

Introduction: Sibutramine is serotonin-norepinephrine reuptake inhibitor. The aim of the study was to evaluate the influence of sert and GNB3 genes polymorphism on the results of obesity treatment with sibutramine. Methods: 66 obese patients (57 females and 9 males), mean age $39.29 \pm 12.64$ years, who took $10 \mathrm{mg}$ of Reduxin (sibutramine + microcrystalline cellulose) were included in the study. Clinical and biochemical parameters were performed at baseline and after 3 month of treatment. To diagnose eating disorders and depression we used validated questionnaire ' The types of eating disorders" (debq) and Beck Depression Scale. Also sert and GBN3 genes polymorphisms were assessed. After 3 months of therapy the patients were divided into two groups: 1$)<5 \%$ of body weight reduction - non-responders 2) clinically significant weight loss $\geq$ $5 \%$-responders.

Results: In the 2nd group we observed a significant in body weight loss $11.59 \mathrm{~kg}$ (95\% ci 8.95-14.23) (22.33\%, p <0.05), decrease of diastolic blood pressure $2.81 \mathrm{~mm} \mathrm{Hg}$ (95\% ci $0.77-4.86)$ (3.21\%, p < 0.05). Adverse events were reported in six cases - insomnia in 3 cases, nausea in 2 cases, dry mouth in 1 case; none of them was the reason for therapy withdrawal. In the 2 nd group the presence of sert s-allele was significantly associated with higher rates of external type of eating behavior. In the 1st group s-allele carriership (ss and sl genotypes was associated with less weight loss $-2.8 \mathrm{~kg}$ (versus 1-allele $-3.28 \mathrm{~kg}$ ) and higher fasting blood glucose at baseline $5.38 \pm 0.63 \mathrm{mmol} / \mathrm{l}$ (versus l-allele of $5.04 \pm 0.91 \mathrm{mmol} / \mathrm{l}$ ).

cc genotype of GBN3 in the second group was associated with higher systolic blood pressure (sbp) at baseline $(129.27 \pm 9.16 \mathrm{mmHg})$ and higher sbp $(127.36 \pm 8.16 \mathrm{mmHg})$ and diastolic blood pressure $(78.36 \pm 4.3)$ after 3 months of treatment compared with ct genotype $(117.27 \pm 12.5$; $115.45 \pm 10.6 ; 72.91 \pm 6.0 \mathrm{~mm} \mathrm{Hg}$, respectively) $(\mathrm{p}<0.05)$. In the carriers of c-allele (cc and ct genotype) we revealed more severe depression symptoms in comparison with t-allele carriers (average score on the Beck Depression Scale was $11.68 \pm 4.3$ versus $6.4 \pm 4.0$, respectively), as well as higher rates of triglycerides, both before and after treatment $(1.5 \pm 0.51$; $1.28 \pm 0.38 \mathrm{mmol} / \mathrm{l}$ and $0.87 \pm 0.3 ; 0.93 \pm 0.25 \mathrm{mmol} / \mathrm{l}$, respectively)

Conclusion: 1) Weight loss during Reduxin treatment was lower in the carriers of s-allele of sert gene than in the carriers of the 11 genotype; 2 )
GBN3 gene cc genotype was associated with higher blood pressure at baseline and during Reduxin treatment, as well as with more severe depression symptoms.

Conflict of interest: None disclosed

Funding: Research relating to this abstract was funded by Promomed

\section{T3P201}

\section{The role of melatonin and sleep disturbances in the pathogenesis of metabolic disorders in polycystic ovary syndrome}

\section{Absatarova, $Y^{1}$; Andreeva, . $^{1}$ \\ ${ }^{1}$ Endocrinology Research Centre}

Introduction: Polycystic ovary syndrome (pcos) is the pathology with different metabolic disorders including obesity and insulin resistance.Disruption of circadian rhythms exacerbates the tissue sensitivity to insulin and leads to anovulation. Melatonin regulates the 'sleep-wake'" cycle and reproductive function. It is one of the main components of the antioxidant system. Oxidative stress is ubiquitous in patients with pcos. Disrupted balance of the antioxidant system may worsen the normal growth and maturation of follicles. Women with pcos have high content of lipid peroxidation products, causing the damage of the oocytes.

Objective. To study the role of melatonin in the pathogenesis of metabolic disorders in women with pcos.

Methods: 2 groups of patients with pcos aged from 17 to 35 years:30 patients with a bmi $>25 \mathrm{~kg} / \mathrm{m}^{2}$ and 30 patients with $\mathrm{bmi}<25 \mathrm{~kg} / \mathrm{m}^{2}$. The survey through questionnaires scoring subjective sleep characteristics; examination of glucose and insulin blood levels, melatonin level in blood sampling at 08.00 and in follicular fluid.pcos was diagnosed on the basis of eshre/asr (2007) criteria. The control group: 60 healthy women without menstrual disorders.Sampling a follicular fluid during laparoscopic ovarian drilling for the treatment of infertility in women with pcos. Control group: women who underwent a diagnostic laparoscopy for the tubal factor of infertility.

Result. Patients with pcos have more frequent sleep disorders compared to the control group. The presence of overweight increased the probability of sleep abnormalities by $12.2 \%$. Patients with pcos had significantly higher levels of melatonin in the blood $(\mathrm{p}<0.05)$ compared with the control group. Melatonin level in a follicular fluid was lower in women with pcos compared with the control group. There was a significant inverse correlation of the degree of sleep disorders and insulin level $(\mathrm{p}<0.05)$.

Conclusion: Sleep disorders in patients with pcos and obesity and reduced melatonin concentration in follicular fluid can worsen metabolic disorders and lead to anovulation in women with pcos.

None Disclosed.

No Funding.

\section{T3P202}

\section{The role of sleeve gastrectomy in the evolution of nonalcoholic fatty liver disease and metabolic parameters}

Sava, E. ' Sirbu, A. ' ; Martin, S. ; Leca, B. ' Arbanas, T. ${ }^{2}$; Copaescu, C. ${ }^{3}$; Fica, $S{ }^{1}$

${ }^{1}$ Endocrinology Department, Elias University Hospital, Carol Davila University of Medicine and Pharmacy, Bucharest, Romania, ${ }^{2}$ Clinic Of Gastroenterology, Hepatology And Digestive Endoscopy, Elias Emergency University Hospital, Bucharest, Romania, ${ }^{3}$ Ponderas Hospital, Bucharest, Romania.

Introduction: Nonalcoholic fatty liver disease (nafld), a disease highly prevalent among the morbidly obese population, is a condition defined by the excessive accumulation of fat that is not related to alcohol consumption. Bariatric surgery has potential benefits in improving factors such as weight loss, insulin resistance, lipid profile, and inflammation that contribute to the pathogenesis of nafld. The study aims to evaluate meta- 
bolic status, changes in liver function and steatosis evolution 5 years after laparoscopic sleeve gastrectomy (lsg).

Methods: 76 patients with mean age of $44 \pm 10.56$ years were included in the study. Prior hepatic disease, autoimmune hepatitis and alcohol consumption were excluded. Physical examination and biochemical analyses including liver function tests, hepatic markers, lipid profile and homeostasis model assessment of insulin resistance (homa-IR) score were also performed. The diagnosis of nafld was achieved based on transabdominal ultrasonograpghic imaging and the degree of liver steatosis was graded into four groups based on liver echogenity: 0(absent), 1(mild), 2(moderate), 3(severe). The patients were reevaluated 5 years post lsg.

Results: The average bmi decreased from $44.4 \pm 7.8$ to $34.15 \pm 5.5 \mathrm{~kg} / \mathrm{m}^{2}$ at follow-up ( $\mathrm{p}<0.001)$. After 5 years we observed an improvement of metabolic parameters: decrease in homa index $(5.77 \pm 5$ vs $3 \pm 4.5, p<0.001)$, glucose $(110 \pm 44 \mathrm{mg} / \mathrm{dl}$ vs $97.8 \pm 21.5 \mathrm{mg} / \mathrm{dl}, \mathrm{p}=0.009)$ and triglyceride level $(159.8 \pm 120 \mathrm{mg} / \mathrm{dl}$ vs $103 \pm 61 \mathrm{mg} / \mathrm{dl}, \mathrm{p}<0.001)$. At baseline $80.3 \%$ were diagnosed with moderate and severe hepatic steatosis, after 5 years the percent decreased at $14.55 \%$, with severe steatosis remained present only in those who had ewl (excess weight loss) $<50 \%$. The mean diameters of the right and left liver lobes were $177 \pm 21 \mathrm{~mm}$ and $86 \pm 20 \mathrm{~mm}$ at baseline vs $160 \pm 19 \mathrm{~mm}$ and $74 \pm 16 \mathrm{~mm}$ after 5 years $(\mathrm{p}<0.001)$. A positive correlation between right hepatic lobe reduction, bmi and ew was observed $(\mathrm{r}=0.422, \mathrm{p}<0.002 ; \mathrm{r}=0.313, \mathrm{p}=0.028)$. Improvements in liver enzymes during weight loss were also observed: aspartate aminotransferase (ast) $(24.8 \pm 14$ vs $20.62 \pm 8.58$ ui/l, $\mathrm{p}=0.03)$, alanine aminotransferase (alt) $(34.78 \pm 30$ vs $21 \pm 12 \mathrm{UI} / \mathrm{l}, \mathrm{p}<0.001)$, gamma-glutamyl transferase (ggt) $(45 \pm 39$ vs $31 \pm 30 \mathrm{ui} / \mathrm{l}, \mathrm{p}<0.001)$ and alkaline phosphatase ( $97 \pm 50$ vs $66.9 \pm 23 \mathrm{ui} / \mathrm{l}, \mathrm{p}<0.001)$. A correlation between homa index variation, alt and ggt reduction was established $(\mathrm{r}=0.442, \mathrm{p}<0.001$ and $\mathrm{r}=0.467, \mathrm{p}=0.003$ ).

Conclusion: Our data supports that weight loss after lsg ameliorated nafld, metabolic status and liver function, results that remained stationary 5 years post lsg, and therefore it proves to be an effective method to prevent progress into nonalcoholic steatohepatitis with or without hepatic fibrosis/cirrhosis.

\section{Conflict of Interest: None}

No Funding

\section{T3P203}

\section{The use of meal replacements for weight loss: A systematic review and meta-analysis}

Astbury, N. ${ }^{1}$; Piernas-Sanchez, C. ; Hartmann-Boyce, J. '; Lapworth, S. '; Roberts, N. '; Jebb, S. ${ }^{1}$

${ }^{1}$ University of Oxford

Introduction: Meal replacements $(\mathrm{mr})$ are portion controlled or pre-packaged foods intended to aid weight loss. Although $\mathrm{mr}$ are widely available and have been shown to be effective in small short-term research studies, they are not recommended in most current guidelines for the management of obesity. A systematic review of six studies in 2003 observed significantly greater weight loss at three months but was inconclusive in the longer term.

Methods: We searched (to August 2016) for randomised controlled trials in adults ( $\geq 18$ years) with bmi $\geq 25 \mathrm{~kg} / \mathrm{m}^{2}$, comparing the use of one or more $\mathrm{mr}$ daily with comparator interventions, with weight loss reported at 1 year or longer. Standard Cochrane review methods were used. Our primary outcome was weight change at 12 months (mean; 95\% ci), using baseline observation carried forward to account for missing data.

Results: We identified and extracted data from 18 eligible studies and 17 studies, randomising 3596 participants to $\mathrm{mr}$ and 3244 to a comparator were included in the meta-analysis. Overall $\mathrm{mr}$ groups lost more weight than comparator groups $\left(\mathrm{n}=17,-2.48 \mathrm{~kg} ;-4.53,-0.42 ; \mathrm{I}^{2}=100 \%\right)$. In studies not including a behavioural programme (bp), participants randomised to use mr lost more weight than usual dietary advice ( $\mathrm{n}=7$ : $-2.96 \mathrm{~kg} ;-3.97,-1.95 ; \mathrm{I}^{2}=66 \%$ ). Participants randomised to $\mathrm{mr}$ as part of a bp lost more weight more than bp alone $(\mathrm{n}=6$ : $-1.42 \mathrm{~kg} ;-1.97,-0.86$; $\left.\mathrm{I}^{2}=0 \%\right)$, usual dietary advice $\left(\mathrm{n}=2:-3.98 \mathrm{~kg} ;-7.06,0.91 ; \mathrm{I}^{2}=45 \%\right)$, or a minimal control ( $\left.\mathrm{n}=2:-4.4 \mathrm{~kg} ;-10.87,2.07 ; \mathrm{I}^{2}=100 \%\right)$.

Conclusion: Recommending $\mathrm{mr}$ increases weight loss at $1 \mathrm{y}$, suggesting that their use should be reconsidered within clinical guidelines for weight management.

\section{T3P204 \\ The visceral adiposity index (VAI): A useful selection criteria for bariatric surgery}

Brix, J. ${ }^{1}$; Krzizek, E. ${ }^{1}$; Kopp, H. $^{1}{ }^{\text {; Ludvik, B. }}{ }^{1}$

${ }^{1}$ Rudolfstiftung Hospital; Department of Medicine I

Introduction: Due to worldwide increasing numbers of patients with morbid obesity (mo), the selection of the patients who should be preferred to undergo bariatric surgery becomes more important. Waist circumference seems to be a good parameter but it cannot distinguish between subcutaneous and visceral fat.

Methods: We included 722 patients with mo (76.9\% women, mean bmi $44.9 \pm 6.6 \mathrm{~kg} / \mathrm{m}^{2}$, mean age $40 \pm 12$ years). Anthropometric data, cardiovascular risk factors and a glucose tolerance test were assessed. vai was calculated as Waist Circumference $/(39.58+(1.89 *$ bmi $)) *($ Triglycerides $/ 0.81) *(1.52 /$ hdl-cholesterol $)$ in women and Waist Circumference / $(39.68+(1.89 * \mathrm{bmi})) *($ Triglycerides $/ 1.03) *(1.31 /$ hdl cholesterol $)$ in men and age stratified pre-defined cut off points ( $<30$ years: $2.52 ;>30<42$ years: $2.23 ;>42<52$ years: $1.92 ;>52<66$ years: 1.93 ; $>66$ years: 2.00$)$ were used to determine were used to determine visceral adiposity dysfunction (vad). A non-obese healthy person has a vai of 1 .

Results: $59.7 \%(n=431)$ patients exhibited vad. Of those patients, median vai was $3.4(2.7,4.8)$. Even though men have a higher bmi $(p=0.008)$, there is no significant gender difference regarding vai $(p=0.232)$. We could find a strong association of higher vai and type 2 diabetes and Impaired Glucose Tolerance (Chi Square 43.768; $\mathrm{p}<0.001)$. Patients with vad have a higher homa-IR $(8.2 \pm 2.6$ vs $5.9 \pm 2.1 ; \mathrm{p}<0.001)$ and a higher HbAlc $(6.5 \pm 1.4$ vs $5.9 \pm 0.7 \%$; p $<0.001)$

Conclusion: vai might be a supportive tool for the selection of patients, who are at a higher risk to exhibit a metabolically unfavourable profile and might benefit more from bariatric surgery.

Conflict of Interest: None Disclosed

Funding: No Funding

\section{T3P205 \\ Time to glycemic control - an observational study of 3 different operations}

Pouwels, S. ; Celik, A. ; Karaca, F.'; Çağıltay, E. ; Ugale, S. ; Etikan, I.5. Büyükbozkırlı, D. ${ }^{2} ;$ KılıÇ, Y. ${ }^{2}$

${ }^{1}$ Franciscus Vlietland, Schiedam, The Netherlands, ${ }^{2}$ Metabolic Surgery Clinic, Sisli, Istanbul, Turkey, ${ }^{3}$ Dept. Of Endocrinology, Gata HaydarpaşA Training \& Research Hospital, Istanbul, Turkey, ${ }^{4}$ Kirloskar Hospital, Bariatric \& Metabolic Surgery Clinic, Hyderabad, India, ${ }^{5}$ Near East University Faculty Of Medicine Department Of Biostatistics

Background: Medical treatment fails to provide adequate control for many obese patients with type 2 diabetes mellitus (T2DM). A comparative observational study of bariatric procedures was performed to investigate the time at which patients achieve glycemic control within the first 30 postoperative days following sleeve gastrectomy (sg), mini-gastric bypass (mgb), and diverted sleeve gastrectomy with ileal transposition (dsit).

Methods: Included patients had a body mass index (bmi) $\geq 30 \mathrm{~kg} / \mathrm{m}^{2}$, T2DM for $\geq 3$ years, $\mathrm{HbA} 1 \mathrm{C}>7 \%$ for $\geq 3$ months, and no significant weight change ( $>3 \%$ ) within the prior 3 months. Surgical procedures performed were $\operatorname{sg}(n=49)$, mgb $(n=93)$, and dsit $(n=109)$. The primary endpoint was the day within the first postoperative month on which mean fasting 
capillary glucose levels reached $<126 \mathrm{mg} / \mathrm{dL}$. Multivariate logistic regression analysis was used to identify predictors of glycemic control.

Results: The cohort included 251 patients with a mean bmi of $36.04 \pm 5.76$ $\mathrm{kg} / \mathrm{m}^{2}$; age, $52.84 \pm 8.52$ years; T2DM duration, $13.09 \pm 7.54$ years; $\mathrm{HbA1C}, 8.82 \pm 1.58 \%$. On the morning of surgery, mean fasting plasma glucose was $177.63 \pm 51.3 \mathrm{mg} / \mathrm{dL}$; on day $30,131.35 \pm 28.7 \mathrm{mg} / \mathrm{dL}$ $(\mathrm{p}<0.05)$. Mean fasting plasma glucose of $<126 \mathrm{mg} / \mathrm{dL}$ was reached in the dsit group $(124.36 \pm 20.21 \mathrm{mg} / \mathrm{dL})$ on day 29 , and in the mgb group $(123.61 \pm 22.51 \mathrm{mg} / \mathrm{dL})$, on day 30 . The sg group did not achieve the target mean capillary glucose level within postoperative 30 days.

Conclusion: During the first postoperative month, glycemic control $(<126 \mathrm{mg} / \mathrm{dL})$ was achieved following dsit and mgb, but not sg. Preoperative bmi and postprandial C-peptide levels were independent predictors of early glycemic control following dsit.

\section{T3P206}

\section{Transforming growth factor- $\beta$ in cardiovascular prognosis in patients with obesity and diabetes mellitus type $\mathbf{2}$}

\section{Teona, S. ${ }^{1}$; Irina, B. ${ }^{1}$; Ekaterina, ${ }^{1}{ }^{1}$; Marina, S. ${ }^{1}$; Aleksandr, I. ${ }^{1}$; Larisa, N. ${ }^{1}$ ${ }^{1}$ Endocrinology Research Centre}

Introduction: Obesity and diabetes mellitus type $2(\mathrm{dm} 2)$ are commonly associated with cardiovascular diseases, and particularly with coronary heart disease (chd). But some data indicate protective role of adipose tissue in patients with 1st degree of obesity for cardiovascular prognosis. Obesity and high blood glucose level activate fibrogenic pathways in many cell types, including induction of Transforming Growth Factor- $\beta$ (tgf- $\beta$ ) synthesis. Exploring the contribution of metabolic dysregulation in cardiac remodeling and chd development in obese patients remains a great challenge.

Objective: The aim of the study was to determine the role of tgf- $\beta$ level in cardiovascular prognosis among patients with obesity and $\operatorname{dm} 2$.

Methods: tgf- $\beta$ was detected in peripheral blood samples of 63 patients, aged 48-65 years. Patients were divided in 3 groups: all participants were obese (bmi 30.0-39.9 kg/m²). First group included 21 patients with chd (confirmed by coronary angiography) and $\mathrm{dm} 2,2^{\text {nd }}$ group -22 patients with $\operatorname{dm} 2$ and excluded chd after Tredmil-Test, and the $3^{\text {rd }}$ group -20 obese patients with normal glucose metabolism and excluded chd.

Results: A comparison of tgf- $\beta$ level in studied groups revealed significant differences $(p=0.046)$. The most significant difference was between $1^{\text {st }}$ and $3^{\text {rd }}$ groups $(174620.6[12178.35 ; 26282.04]$ vs. 27909,04 [23564,76;33879,09]; $\mathrm{p}=0,024)$

Spearman correlation analysis showed negative correlation between tgf- $\beta$ with atherogenic fraction of cholesterol $-1 \mathrm{dl}$ in the $2^{\text {nd }}$ group $(r=-0.426$, $\mathrm{p}<0.05)$. Moreover, tgf- $\beta$ had a negative correlation with the degree of stenosis of the internal carotid artery in patients of the $2^{\text {nd }}$ group $(r=-0.426$, $\mathrm{p}<0.05)$. In contrast, patients with verified chd ( $1^{\text {st }}$ group) had a negative correlation with the duration of obesity $(\mathrm{r}=-0.395, \mathrm{p}<0.05)$ and pathological remodeling processes of the heart muscle (left ventricular posterior wall thickness $(\mathrm{r}=-0.386, \mathrm{p}<0.05)$ and interventricular septum $(\mathrm{r}=-0.335, \mathrm{p}<0.05))$, as well as with the number of hemodynamically significant stenosis in the coronary arteries that determine the severity of chd $(\mathrm{r}=-0.455, \mathrm{p}<0.05)$.

Conclusion: Although the strong association between obesity, $\mathrm{dm} 2$ and cardiovascular diseases are well-documented, mechanisms of fibrotic cardiac remodeling, coronary atherosclerosis and their impact on cardiac function remain poorly understood. Considering the increasing worldwide prevalence of obesity, there is an urgent need to understand the cellular and molecular basis of cardiac remodeling and dysfunction in obese subjects.

Conflict of Interest: None Disclosed

\section{T3P210}

\section{Understanding features associated with weight loss in an} online weight management community

\author{
Beleigoli, A. ${ }^{1}$; Pappa, G. ${ }^{\text {; }}$ Cunha, T. ${ }^{1}$; Bicalho, P. '; Silva, A. '; Jr, W. ${ }^{1}$; \\ Ribeiro, $A{ }^{1}$ \\ ${ }^{1}$ Federal University of Minas Gerais, Brazil
}

Background: Online social communities have been increasingly used as tools for weight management. Whether and how users' characteristics, activities and engagement in these communities relate to weight change is not totally understood. Objectives: We investigated the behavior (online activity patterns, interaction with other users and discussed topics) of active users in $r$ /loseit, a weight management community of the online social network Reddit. Additionally, our objective was to understand the association between online users' behavior and real-world variables with weight change in the community.

Methods: Public available data of unidentifiable active users (i.e., users with at least one post or comment) who had information about initial bmi, a minimum 30-day weight change, gender and age were collected in r/loseit from August 2010 to November 2014. Measures of online activity in the community and interaction among these users, as well as a semantic analysis of their posts and comments by a Latent Dirichlet Allocation (lda) method were performed. We investigated the association between a minimum 30-day reported weight change and online user activity patterns, discussed topics in posts and comments and real-world variables by linear regression models.

Results: Complete data were available for 754 unidentifiable users. Women were predominant $(456 ; 60.5 \%)$. Mean age was 26 (5.0) years. Most of the users $(701 ; 92.6 \%)$ lost weight over the follow-up. Overall, 514 users $(68 \%)$ moved to a lower bmi category while participating in the loseit community. Among the users who reported being initially overweight $(n=129)$ or obese $(n=598), 70(54.8 \%)$ and $444(74.1 \%)$ reported being at a lower bmi category at the end of the follow-up, respectively. Median (iqr) percentage of days online was 3.8 (1.5-11.1). The 10 most discussed topics on posts and comments were related to healthy food, clothing, calories count, workout, looks, habits, support and unhealthy food. Female gender, bmi at baseline, high levels of online activity, and discussing topics related to social support were independently associated with weight loss. Conclusions: Our findings suggest that among active users of a weight management community, self-declaration of higher bmi levels, which may represent greater dissatisfaction with weight excess, high online activity and engagement in discussions that might provide social support, are associated with greater weight loss. These findings have the potential to aid health professionals to assist patients in online interventions, by focusing efforts in increasing engagement and/or starting discussions on topics of higher impact on weight change. Acknowledgement: amb, wmj and alr are funded by capes. The authors have no conflicts of interest.

\section{T3P211 \\ Use of ultrasonography as a diagnostic method of the different abdomen fat layers and its prognostic implication with obesity-related metabolic syndrome}

Cuatrecasas, G. ${ }^{1}$; Cabo, F. ; Patrascioiu, I. ' Aranda, G. ; Coves, M. '; Aguilar, G. ${ }^{1}$; Calbo, M. ${ }^{3}$; March, S. ${ }^{3}$; Bretxa, C. ${ }^{3}$; Cuatrecasas, G. ${ }^{1}$

${ }^{1}$ Dpt. Endocrinology, Clinica Sagrada Familia, Barcelona, ${ }^{2}$ Ecografía. Cpen S.L. Dpt. Endocrinology, Clinica Sagrada Familia, Barcelona, ${ }^{3}$ Dietitian. Cpen S.L. Dpt. Endocrinology, Clinica Sagrada Familia, Barcelona

Introduction: Ultrasonography is a diagnostic method increasingly used in endocrinology outwards clinics. There are preliminary data of their usefulness in the measurement of the different layers of abdominal fat. Our aim was to observe associations between different fat thickness with clinical and analytical parameters related to obesity comorbidities.

Methods: $\mathrm{n}=107$, mean age 54 y.o, 27 males (M), 80 females (F) (60\%> 50 y.o), who had a conventional abdominal ultrasonography (us) (12 mhz 
linear and 3-6 mhz convex probes), thickness of 6 different consecutive layers of abdominal fat at the L4 level were assessed (bifurcation point of the abdominal aorta in 2 iliac arteries): total subcutaneous (sc), superficial sc, deep sc, pre-peritoneal, omental (peri-aortic), Hepatic steatosis area $\left(\mathrm{cm}^{2}\right)$ and hepatic us noise $(\mathrm{dB})$ (visceral fat) and finally pre-renal ( $\mathrm{R}$ and L) (retroperitoneal fat). We reviewed clinical history to obtain: bmi, Waist circumference (wc), Glucose, Total cholesterol, ldl, hdl, Triglycerides, $\mathrm{dm} 2$ diagnosis, Hypothyroidism and Metabolic Syndrome according to atpiii criteria.

Results: mean bmi $30 \mathrm{Kg} / \mathrm{m}^{2}$ (sd 4), mean wc $102 \mathrm{~cm}$ (sd 11) in $\mathrm{F}$ and 32 $\mathrm{Kg} / \mathrm{m}^{2}$ (sd 5), wc $111 \mathrm{~cm}$ (sd 7) in M. $19.8 \%$ had a bmi<25 Kg/m², 29.2\% were overweight, $51 \%$ had Obesity ( $81 \%$ Grade I, $10 \%$ gii, $9 \%$ giii). We found a different sex distribution pattern at the sc layer level (mean range $56.8 \mathrm{~mm} \mathrm{~F}$ and $41.4 \mathrm{~mm} \mathrm{M}$ ), retroperitoneal fat layer $(44.7 \mathrm{~mm}$ $\mathrm{F}$ and $78,8 \mathrm{~mm} \mathrm{M})$ and omental fat layer $(45.5 \mathrm{~mm} \mathrm{~F}$ and $76.9 \mathrm{~mm} \mathrm{M})$ $(\mathrm{p}<0.001$ for omental fat). There were only differences $(\mathrm{p}<0.001)$ for omental fat measures according to different bmi groups $(r=0.493)$. There was also a significant correlation only for omental fat with wc $(\mathrm{p}<0.001$, $\mathrm{r}=0.55)$, Glucose $(\mathrm{p}<0.001, \mathrm{r}=0.364)$ and hdl-cholesterol $(\mathrm{p}=0.044$, $\mathrm{r}=0.228)$. Preperitoneal $(\mathrm{p}<0.006)$, retroperitoneal $(\mathrm{p}<0.003)$ and specially omental $(\mathrm{p}<0.001$, ci $14-31 \mathrm{~mm})$ fat thickness correlate with the presence of Hepatic steatosis. Menopause also predisposes to greater omental fat $(\mathrm{p}<0.001$, ci $6-25 \mathrm{~mm})$ as well as Metabolic Syndrome (ms) $(\mathrm{p}<0.001$, ic $9-25 \mathrm{~mm})$. We obtained a roc curve with a $39 \mathrm{~mm}$ cut-off point for omental fat thickness, predictive for $\mathrm{ms}$ (auc $0.713 ; \mathrm{p}<0.006$ ) with $71 \%$ sensitivity and $55 \%$ specificity.

Conclusion: us is an easy and valid method for the measurement and stratification of different abdominal fat layers. There are gender differences in both sc and internal fat (intra- and retroperitoneal) already reported by tc/dexa. us highlights the clinical importance of strictly omental (periaortic) fat layer, thicker in patients with ms with good correlations with bmi, wc, glucose and hdl-cholesterol. We propose a cut-off point of $39 \mathrm{~mm}$ to consider the thickness of the omental layer as pathological and predictive of metabolic comorbidities.

\section{T3P212}

\section{Validation of the Nexfin ${ }^{\circledR}$ non-invasive continuous blood pressure monitoring validated against Riva-Rocci/Korotkoff in a bariatric patient population}

Pouwels, S. ${ }^{1}$; Lascaris, B. ${ }^{2}$; Nienhuijs, S. ${ }^{3}$; Bouwman, A. ${ }^{2}$; Buise, M. ${ }^{2}$

${ }^{1}$ Franciscus Vlietland, Schiedam, The Netherlands, ${ }^{2}$ Department Of Anaesthesiology, Intensive Care And Pain Medicine, Catharina Hospital, Eindhoven, The Netherlands, ${ }^{3}$ Department Of Surgery, Catharina Hospital, Eindhoven, The Netherlands

Study Objective: The present study aimed to validate the Nexfin monitor and to assess the accuracy compared to classical sphyganomanometry (Riva-Rocci/Korotkoff (rrk)) blood pressure (bp) measurements in patients with obesity scheduled for bariatric surgery.

Design: Validation study

Setting: Outpatient clinic for bariatric surgery

Patients: 33 patients scheduled for bariatric surgery

Measurements: The validation process was done according to the protocols developed by the European Society of Hypertension from 2010 The Nexfin monitor (Edwards Lifesciences / bmeye B.V., Amsterdam, the Netherlands) calculates beat-to-beat blood pressure from finger pulse wave analysis. Measurements of systolic and diastolic bp were obtained using classical sphygmomanometry and the Nexfin alternatingly.

Main Results: In total 99 pairs of bp measurements were used. The device failed pass phase 1 as 65 systolic readings fell within $5 \mathrm{mmHg}$ (73 required). And 61, 76 and 90 diastolic readings fell within 5, 10 and 15 $\mathrm{mmHg}$ respectively. Finally, it failed to pass phase 2 as 23 patients for systolic and 25 for diastolic had at least $2 / 3$ of their comparisons falling within $5 \mathrm{mmHg}$ (24 required) but 10 subjects for systolic and 8 for diastolic had all three comparisons more than $5 \mathrm{mmHg}$ different from the rrk readings (zero allowed). Mean differences were $7.8 \pm 6.9 \mathrm{mmHg}$ for sbp and $8.0 \pm 7.2 \mathrm{mmHg}$ for $\mathrm{dbp}$.

Conclusion: Using the revised protocol, the Nexfin device was not able to pass validation. However using the original protocol, the Nexfin ${ }^{\circledR}$ device passed phase 1 and 2.1 of the validation process and failed to pass phase 2.2 .

\section{T3P213 \\ Vitamin B12, iron and folate deficits after bariatric surgery: Results from a 10-year prospective, real-world study}

Lunger, L. ${ }^{1}$; Melmer, A. ' ; Fritz, J. ' Tschoner, A. '; Engl, J. ' ; Wykypiel, H. ${ }^{1}$;

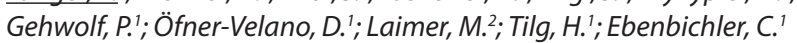

${ }^{1}$ Medical University of Innsbruck, ${ }^{2}$ University Clinic Bern

Bariatric surgery leads to pronounced and sustained weight loss. However, nutrient uptake of vitamin B12, iron and folate is impeded, ultimately leading to anemia.

Methods: Vitamin B12 (vb12) and iron metabolism was determined before and 10-years after bariatric surgery and correlated to weight loss and the incidence of anemia. Postoperatively, patients followed non-standardized supplementation attempts at either the study centre or the primary practitioner. In total, 636 patients were eligible for this study. After exclusion of 496 patients ( $n=463$ declined to participate, $n=33$ had insufficient sample material at baseline), 140 patients were included in the analysis. Of those, 68 patients received adjustable gastric banding (agb), 50 patients received gastric bypass (non-agb) and 22 agb patients were re-operated to non-agb.

Results: vb12 decreased significantly $(-46.3 \pm 182 \mathrm{pmol} / \mathrm{l} ; \mathrm{p}=0.003)$, depending on weight loss $(\mathrm{p}=0.032)$ but neither depending on the type of surgical intervention $(\mathrm{p}=0.432)$ nor baseline levels of vb12 $(\mathrm{p}=0.152)$. In total, 8 patients developed vb12 deficiency. Folate did not change significantly. Ferritin decreased significantly $(-35.8 \pm 119.5 \mu \mathrm{g} / \mathrm{l} ; \mathrm{p}=0.02)$ and correlated with baseline ferritin $(\mathrm{p}=0,001), 52$ patients developed ferritin deficiency. Hemoglobin decreased significantly $(-5.57 \pm 13.26 \mathrm{~g} / \mathrm{l}$; $\mathrm{p}=0.001)$ and correlated to baseline hemoglobin $(\mathrm{p}=0,001), 15$ patients developed anemia.

Conclusion: vb12 and ferritin deficiencies were frequent 10 years after bariatric surgery following inconsistent supplementation. High preoperative concentrations of nutrients cannot prevent the development of nutrient deficiencies. Standardized supplementation at specialist centres is crucial in postoperative care of bariatric patients.

Conflict of Interest: None Disclosed.

\section{T3P214 \\ Vitamin D after three types of bariatric surgery with long term follow up}

Capitão, R. ${ }^{1}$; Santos, F. ${ }^{2}$; Barreiros, C. ${ }^{2}$; Ferrinho, C. ${ }^{2}$; Saraiva, C. ${ }^{2}$; Tomás, C. ${ }^{2}$; Ferreira, R. ${ }^{2}$; Azinheira, J. ${ }^{2}$; Duarte, J. ${ }^{2}$; Vasconcelos, $C^{2}{ }^{2}$

${ }^{1} \mathrm{CHLO}$ - Hospital Egas Moniz, ${ }^{2}$ Hospital Egas Moniz, Chlo, E.P.E.

Introduction: Hypovitaminosis D (hvd) is a frequent and may be present in up to $77 \%$ of population, depending on the cutoff values used and the studied population. One of the risk factors for hvd is obesity. Nevertheless, surgical techniques for treating obesity can lead in some way to malabsorption processes that can decrease even more the levels of Vitamin D.

Objective: To determine the prevalence of hvd in obese patients followed in a consult for surgical treatment of obesity (sto) and to assess whether it is related to the amount of weight lost (wl), time after surgery and type of sto.

Methods: This is a one center longitudinal observational study. The authors selected all patients followed for sto from 2012-2016 with serum levels of 25-hydroxivitamin D [25(oh)D] determined after sto at hospital laboratory. The cutoffs adopted for $25(\mathrm{oh}) \mathrm{D}$ levels were $<25 \mathrm{mmol} / \mathrm{dL}$ for deficiency, and $25-75 \mathrm{mmol} / \mathrm{dL}$ for insufficiency. The follow-up time, $\% \mathrm{wl}$ 
and $25(\mathrm{oh}) \mathrm{D}$ supplementation were recorded. We analyzed data in spss software using descriptive methods, Pearson correlation, anova and T test for continuous variables. The results presented by mean \pm std.

Results: The authors analyzed 171 patients, $86 \%$ female, with an age of $49.6 \pm 10.6$ years and 245 measurements of $25(\mathrm{oh}) \mathrm{D}$. The follow-up from the sto to the $25(\mathrm{oh}) \mathrm{D}$ measurement was $4,8 \pm 3,9$ years. Vitamin D supplementation was given to $26,3 \%$ of patients before measurement of 25(oh)D. The initial and final weights were $121.2 \pm 20 \mathrm{~kg}$ and $89.1 \pm 20.9 \mathrm{~kg}$ respectively. The mean value of $25(\mathrm{oh}) \mathrm{D}$ was $56.3 \pm 25.4 \mathrm{mmol} / \mathrm{dL}, 7.3 \%$ were deficient and $74.3 \%$ were insufficient for $25(\mathrm{oh}) \mathrm{D}$ levels, despite prior Vitamin D supplementation in $33,3 \%$ and $19,5 \%$ respectively. The sto were bypass (38.6\%), sleeve (36.3\%) and adjustable gastric band (25.1\%). No statistically significant correlations were found between $25(\mathrm{oh}) \mathrm{D}$ levels and the follow-up time or \%wl. Patients with vitamin D deficiency had a greater wl (28\% vs $25 \%$ ) and longer follow-up after surgery (6.2 vs 4.1 years) however these differences were not statistically significant. We found no differences between the 25(oh)D levels and the type sto.

Conclusion: 25(oh)D deficiency and insufficiency were common and were present even in those on Vitamin D supplementation. The type of surgery, amount of time after surgery, and \%wl, did not contribute to the deficiency of this vitamin. This fact probably means that, the obesity itself, or the $25(\mathrm{oh}) \mathrm{D}$ values prior to sto, are probably more significant for the hvd than the possible sto's iatrogenic effect along the time. On the other hand, the resolution of obesity may contribute to ameliorate its effect as a cause of hvd and counterbalance the lower ingestion or malabsorption induced by sto.

Conflict of Interest: None disclosed

Funding: No Funding

\section{T3P215 \\ Vitamin D levels after a gastric bypass: Should we be concerned?}

\section{Jesus, A. ;}

${ }^{1}$ Faculty of Nutrition and Food Sciences of the University of Porto

Introduction: Obesity is a public health problem that increases year af ter year with the consequent growth in the number of bariatric surgeries. Among the various techniques, Roux-en-Y gastric bypass (rygb) is the most common worldwide. In addition to promoting long-term weight loss and resolution of comorbidities, it can lead to implications such as nutritional deficits. The relationship between obesity and low levels of vitamin $\mathrm{D}$ has been observed but its causality is not well defined. The literature shows contradictory findings with regard to the definition of the deficiency level of vitamin D and about the optimal dose of supplementation before and after a gastric bypass.

Therefore, the aim of the present work is to review the implications of serum vitamin $\mathrm{D}$ levels in subjects undergoing rygb and supplementation recommendations based on the current knowledge.

Methods: Computerised literature research was conducted in PubMed Scopus, and Web of Science. Investigations were limited to humans but were not restricted by language or date. Reference lists of all relevant systematic reviews, guidelines and primary studies were researched.

Results: The results of vitamin D deficiency in the literature are heterogeneous, depending on the definition of deficiency, the type of test used, demographic differences, characteristics of the sample, among other factors. However, many authors find vitamin D deficiency in obese individuals between $41 \%$ and $97 \%$. After the rygb the prevalence of vitamin D deficiency is in the order of $50 \%$ to $80 \%$. Studies that have evaluated vitamin D treatment using standard doses of ingestion ranging from 200 iu to $400 \mathrm{iu}$ and 800 iu show that these doses are ineffective but which positively affect serum vitamin D levels.

Conclusion: Since vitamin D has shown an important role, not only in bone function but also in immune and cell function, additional research is needed with more randomised controlled trials to develop evidence-based guidelines to prevent and treat vitamin D deficiency in obese subjects, before and after a gastric bypass, through safe sun exposure, diet and a proper supplementation.

Conflict of Interest: The author declares no conflicts of interest.

Funding: No Funding/Research relating to this abstract was funded by any institution

\section{T3P217}

\section{Weight loss prior to bariatric surgery does not predict greater long-term post-operative weight loss: A longitudinal analysis}

\section{Abott, S. ; Fong, T. ${ }^{2}$; Bell, L. ' ; Singhal, R. ${ }^{1}$; Tahrani, A. ${ }^{3}$}

${ }^{1}$ Birmingham Heartlands Hospital, uk, ${ }^{2}$ Aston University, Uk, ${ }^{3}$ University Of Birmingham, Uk

Introduction: The weight loss achieved following bariatric surgery varies considerably amongst individuals. Patients who adhere to lifestyle changes before surgery might be more likely to achieve greater weight loss after surgery as they may be more able to adhere to the required dietary changes post-operatively. We aimed to examine whether pre-operative weight change can predict long-term post-operative weight change following bariatric surgery.

Methods: A retrospective cohort study was undertaken of all patients who underwent a primary laparoscopic adjustable gastric banding (lagb) or laparoscopic roux-en-y gastric bypass (lrygb) in a single tertiary bariatric centre in the uk between $1^{\text {st }}$ January 2012 and $31^{\text {st }}$ December 2013. Data was collected from the electronic patient records. Weight and body mass index (bmi) were collected at the first surgical appointment, the day of surgery, and 24 months post-operatively. Pre-operative weight change was calculated from the first surgical appointment to the day of surgery; a median time period of 102 and 119 days for lagb and lrygb groups respectively

Results: 266 patients met the inclusion criteria of which 160 underwent lagb (mean \pm sd age $47 \pm 11$ years, baseline weight $146.1 \pm 30.6 \mathrm{~kg}$, baseline bmi $52.2 \pm 9.5 \mathrm{~kg} / \mathrm{m}^{2}, 72 \%$ women) and 106 underwent lrygb (age $46 \pm 11$ years, baseline weight $139.5 \pm 24.2 \mathrm{~kg}$, baseline bmi $50.0 \pm 7.4 \mathrm{~kg} /$ $\mathrm{m}^{2}, 78 \%$ women). lagb and rygb resulted in $22.5 \%$ and $32.5 \%$ weight loss respectively by 24 months. Pre-operative weight loss target correlated with 24-month post-operative weight change in the lagb $(r=0.271, p=0.004)$ but not the lrygb $(\mathrm{r}=-0.214, \mathrm{p}=0.352)$ patient group. After adjusting for gender, age and baseline weight $\left(\mathrm{R}^{2}=0.373\right)$, greater pre-operative weight loss was associated with less 24 months post-operative weight loss in patients who underwent lrygb $(B=-0.925, \mathrm{p}=0.003)$ while pre-operative weight loss did not predict post-surgical weight loss in the lagb group ( $\mathrm{B}=$ $0.294, \mathrm{p}=0.108$ )

Conclusions: Greater weight loss before bariatric surgery does not predict greater weight loss following surgery; in fact greater weight loss before lrygb predicted less weight loss post-operatively. Patients should not be denied bariatric surgery on the basis of not achieving satisfactory pre-operative weight loss.

\section{T3P218 \\ Weight loss, reflux and reoperations: Our first 100WEIGHT loss, reoperations and reflux - 10 years of lap. sleeve gastrectomy. our first 100 patients. patients treated with lap. sleeve gastrectomy}

Felsenreich, D. ${ }^{1}$; Langer, F. ${ }^{1}$; Panhofer, P. ${ }^{1}$; Kefurt, R. ${ }^{1}$; Kristo, I. ${ }^{1}$; Sperker, C. ${ }^{2}$; Schermann, M. ${ }^{2}$; Beckerhinn, P. ; Krebs, M. ${ }^{3}$; Prager, G. ${ }^{1}$

${ }^{1}$ University Clinic for Surgery, Vienna Medical University, Austria, ${ }^{2}$ Vienna Rudolfsstiftung Hospital, Department For Surgery, Austria, ${ }^{3}$ Klosterneuburg Hospital, Department For Surgery, Austria, ${ }^{4}$ University Clinic For Internal Medicine Vienna Medical University, Austria

Background: Laparoscopic Sleeve gastrectomy (lsg) is currently the most frequently performed procedure for obesity and its comorbidities worldwide. As a restrictive surgical method it involves the resection of a major 
part of the stomach, thus creating a narrow sleeve. Aspects of interest in this context are de-novo reflux and its possible effects, such as esophagitis and Barrett's esophagus, as well as adequate weight loss in a long-term follow-up.

Method: This cross-sectional study of the first 100 lsg patients was conducted in a multi-center setting (participating bariatric centers were Vienna Medical University, Klosterneuburg Hospital, Vienna Rudolfsstiftung Hospital). The mean follow-up was between 10 and 14 years.

Data on weight loss success, complications and reoperations was collected from all participating patients. Non-converted patients were also asked to complete questionnaires about their quality of life (baros, sf36, giqol, bql). Patients also received gastroscopies (including biopsies), manometries and $24 \mathrm{~h}$ pH-metries.

Results: These first 100 patients, treated in one of the three bariatric centers mentioned above, had their Sleeve gastrectomy between 2003 and 2006. A third of them was converted to a Roux-en-Y gastric bypass within the follow-up period. Today, half of the patients who were not converted suffer from active gastritis and ulcers; Barrett's metaplasia at the gastroesophageal junction was found in $15 \%$. The $24-\mathrm{h}$ pH-metry and manometry's results were pathological for $50 \%$ of the non-converted patients. Primary Sleeve patients as well as those who were converted in the follow-up period managed an Excess Weight Loss (\% ewl) of 50\% at 10 years or more. Data on patients' quality of life will be presented at the congress as well. Conclusion: The results of this longterm study reveal that 10 years after lsg a number of patients has had to deal with conversions and/or postoperative reflux and weight regain. This suggests that a careful selection of patients is necessary when considering lsg.

\section{T3P219 \\ Weight regain after vertical gastric plication: Sleeve gastrectomy or Roux-en-Y gastric bypass as a salvage procedure? Analysis of the first 100 consecutives.}

\section{Chahine, E. ${ }^{1}$; Hayek, M. ${ }^{1}$; Dirani, M. ${ }^{1}$; D'Alessandro, A. ${ }^{1}$; Chouillard, E. ${ }^{1}$}

${ }^{1}$ POISSY-saint-germain-EN-LAYE medical center

Introduction: Bariatric surgery is the best available long-term treatment in patients with morbid obesity. Vertical Gastric Plication (gp) has been recently performed as a weight loss procedure in France. Despite its relative, short-term safety and efficacy, long-term results of gp are still controversial. The goal of this study was to assess the indications and outcome of revision for weight regain in patients with gp.

Methods: Patients were prospectively included in a database, with regular assessment of both results, and complications, respectively. Weight regain or insufficient weight loss were initially treated conservatively in all patients after a through clinical, biological, endoscopic and radiologic assessment. If conservative treatment was unsuccessful or in case of anatomical anomaly, surgical revision was indicated.

Results: Between February 2010 and September 2015, 100 patients had gp. Of these, 20 were lost to follow-up. The rate of patients with excess weight loss (ewl) > 50\% was only 50\%. The remaining 40 patients had either inadequate weight loss (22 patients) or weight regain (18 patients) and eventually required revisional surgery. Roux en Y Gastric Bypass (rygb) was performed in 24 patients (60\%). Sleeve Gatrectomy (sg) was performed in 16 patients $(40 \%)$. Median interval from gp to revision was 29 months (range, 18-41). Mean operative time was $168 \mathrm{~min}$ (range, 100228). Median length of stay was 3 days (range, 2-5). Major complications occurred in 2 patients (5\%) including one fistula and one intra-abdominal abscess, both after sg.

Conclusions: Vertical Gastric Plication is associated to high rates of weight regain or inadequate weight loss. As compared to sg, rygb seems to be a safer revisional procedure after gp.

None Disclosed/Payment received

No Funding/Research relating to this abstract was funded

\section{T3P221}

\section{What do resident physicians know about nutrition?}

Coelho, C. ${ }^{1}$; Souto, S. ${ }^{1}$

${ }^{1}$ Hospital de Braga

Introduction: Nutrition is one of the pillars of disease prevention. However, the curriculum of medical schools does not contemplate this field in a satisfactory manner. The aim of this survey was to better understand clinical nutrition knowledge among resident physicians in a university hospital.

Methods: A questionnaire was sent to the institutional email address of 201 resident physicians working in Braga Hospital during 2015 and shared on a digital platform. The questionnaire was based on a non-validated questionnaire used in a population of Canadian physicians(1). It included 12 multiple-choice questions and general queries about nutrition training in university.

Results: Completed questionnaires were received from $24,8 \%$ physicians $(n=50)$. The average of correct answers was $58 \%$. There were no differences in between gender or medical specialities. Training in nutrition during university was below 10 hours in the majority of the physicians, $82 \%$. There were two questions with $100 \%$ correct answers: a common nutrient deficiency in alcoholics (correct answer - vitamin B1/thiamin) and the nutrient strongly associated with the prevention of neural tube defects (correct answer - folate). These two questions were also the highest scoring in the Canadian physicians survey. On the other hand, the question with the least correct answers, 14\%, was: which substance raises the blood hdl-cholesterol level (correct answer - alcohol).

Conclusion: There is a substantial lack of training in nutrition in Portuguese physicians.

Reference:

1 Temple nj Journal of the American College of Nutrition Survey of Nutrition Knowledge of Canadian Physicians. 1999;(February 1999):37-41

Conflict of Interest: None disclosed

Funding: No Funding

\section{T3P224 \\ Will there be a difference between weight loss among individuals with grade III obesity and superobesity undergoing the same intensive hospital treatment?}

\section{Braga, S. ; Leal, V. ${ }^{1}$; Reis, D. '; Sholl, J. ' ${ }^{1}$ Freitas, L. ${ }^{1}$}

${ }^{1}$ Clínica da Obesidade

Introduction: Obesity is a chronic disease, with a multifactorial etiology, which causes important damages to the health of the individual. The severity of obesity is characterized by the Body Mass Index (bmi), the higher the bmi, the greater the risk of morbidity and mortality

Methods: This is a retrospective cohort that compared the weight evolution of two groups with different bmi. Group 1 was composed of obese individuals with grade 3 (bmi $40-49.9 \mathrm{~kg} / \mathrm{m}^{2}$ ) and group 2 with super obese individuals ( $\mathrm{bmi} \geq 50 \mathrm{~kg} / \mathrm{m}^{2}$ ). All patients were hospitalized at an obesity treatment clinic in Camaçari, Bahia, Brazil, between the years of 2012 to 2016 and their weight evolution six months after discharge was recorded. During the treatment, patients were submitted to the very low calorie diet (500 to $1000 \mathrm{Kcal} /$ day), daily practice of physical activity and multidisciplinary follow-up. The success of the treatment was considered when there was weight loss over $50 \%$ of the excess weight

Results: 82 medical records were analyzed, of which 52 (62.6\%) were women and the mean age was $39.9( \pm 11)$ years. At admission, $67(82 \%)$ were grade 3 obese (mean bmi of $43.4( \pm 3) \mathrm{kg} / \mathrm{m}^{2}$ ) and $15(18 \%)$ were diagnosed as having superobesity (mean bmi of $54.6( \pm 5) \mathrm{kg} / \mathrm{M}^{2}$ ), mean length of hospital stay was $121( \pm 17)$ days and $158( \pm 20)$ days, respectively. At the end of treatment, there was a reduction of $64.3( \pm 9) \%$ of the excess body weight between the individuals in group 1 and $68.7( \pm 10) \%$ among the individuals in group 2, resulting in a final bmi of $31.5( \pm 3) \mathrm{kg}$ $/ \mathrm{m}^{2}$ and $34.3( \pm 3) \mathrm{kg} / \mathrm{m}^{2}$ respectively. When weight loss is evaluated 
in grams per day, group 1 reduced $198( \pm 7)$ grams per day and group 2 reduced $271( \pm 20)$ grams per day, a statistically significant difference $(\mathrm{p}<0.05)$. Only $3(4.5 \%)$ individuals evolved with weight loss less than $50 \%$ of excess weight and did not reach treatment success, all of them were allocated in group 1. After six months of hospital discharge, $78 \%$ of the patients belonging to group 1 returned, bmi varied to $32.9( \pm 3) \mathrm{kg} / \mathrm{m}^{2}$ and excess weight loss was set at $56.7( \pm 18) \%$. The success rate of the sample was $71 \%$. In group $2,87 \%$ of the individuals returned, the mean bmi was $37( \pm 5) \mathrm{kg} / \mathrm{m}^{2}$ and the excess weight reduction was $58.4( \pm 17) \%$, of this group $69 \%$ maintained treatment success.

Conclusion: The intensive hospitalized treatment of obesity was effective and presented a high success rate. Patients with superobesity presented greater weight loss than patients with bmi between 40 and $49.9 \mathrm{~kg} / \mathrm{m}^{2}$. After six months of hospital discharge, both groups had a similar evolution, however, a longer follow-up period is necessary for these results to be confirmed.

There is no conflict interest

There was No Funding

\section{T3P225}

Yogurt consumption, body weight and metabolic profile in youth with a familial predisposition to obesity

Panahi, S. ${ }^{1}$; Gallant, A. '; Tremblay, A. '; Pérusse, L. '; Després, J. '; Bouchard, C. ${ }^{2}$; Drapeau, V. $^{1}$

${ }^{1}$ Université Laval, ${ }^{2}$ Pennington Biomedical Research Center

Introduction: Yogurt consumption has been associated with better body composition and metabolic health and a healthier lifestyle (diet quality and higher levels of physical activity), but no study has considered the role of genetic susceptibility to obesity in this relationship. The objective of this study was to compare physiological and lifestyle markers of health between yogurt consumers and non-consumers as a function of their genetic predisposition to obesity (family history of obesity, fho).

Methods: A total of 198 youth (mean age: $20 \pm 4$ years) from the Québec Family Study were first classified based on their yogurt consumption [yogurt consumers $(\mathrm{yc}+) \mathrm{n}=61$ or non-consumers ( $\mathrm{yc}-) \mathrm{n}=137$ ] and then as having positive (fho+; $\mathrm{n}=112$ ) or negative (fho-; $\mathrm{n}=86$ ) fho (defined as the presence or absence of at least one obese parent, respectively, based on a bmi $\geq 30 \mathrm{~kg} / \mathrm{m}^{2}$ ). Body weight and percent body fat (pbfat), metabolic profiles, diet quality, dietary intakes and lifestyle profiles were compared between groups. A two-way anova was conducted to assess the impact of $\mathrm{yc}$, fho and their interaction on the outcome measures between the two independent variables (yc and fho). All analyses were performed on data adjusted for age and sex, Nutrient-Rich Foods Index (a marker of diet quality), physical activity participation and pbfat, except those on body composition, which were not adjusted for pbfat.

Results: A main effect of fho, but not yc, was observed for variation in body weight and composition, but there was no interaction between yc and fho for these measures. However, there was a significant interaction between yc and fho for fasting insulin $(P=0.02)$ and insulin area under the curve (auc) $(P=0.02)$ after adjustment for studied covariates. Specifically, fho+ and yc+ youth had lower fasting plasma insulin and insulin auc compared to fho+ and yc-youth while these outcomes were similar to fho- individuals, regardless of yc.

Conclusion: Yogurt consumption in youth is associated with lower insulin levels, particularly in those with a familial predisposition to obesity, and this association appears to be independent of body composition and lifestyle factors considered in the present study.

Funding: Research relating to this abstract was funded by the Yogurt in Nutrition Initiative Grant.
PO4 - Management and Intervention

\section{T4P1}

\section{A possible effect of bedroom temperature on BMI in school children and students}

\section{Bednaříková, R. '; Wimer, G. ${ }^{2}$; Ditrich, T.}

${ }^{1}$ Dept. of Biology, Faculty of Education, University of South Bohemia, Czech Republic, ${ }^{2}$ Dept. Of Secondary, Adult, \& Physical Education, Armstrong State University, Ga, Usa

Introduction:Since 2009, when a mechanism of activation of brown adipose tissue (bat) by cold exposure in adult humans was shown, many authors speculated about significance of cold exposures in fighting against obesity. Recently wide surveys tried to find a connection between mean annual temperature and prevalence of obesity with inconsistent Results. However people in industrialized countries rarely experience the outdoor ambient temperature due to dominantly indoor lifestyle. In this research we looked for relationship between bmi and mean bedroom temperature (mbt).

Methods:We conducted the research in within different age classes: 1) children (3-10 years; $\mathrm{N}=77) ; 2$ ) teenagers (11-18 yrs; $\mathrm{N}=64) ; 3$ ) adults (19- 30 yrs; $\mathrm{N}=121)$. All subadults were from the Czech Republic, adults were students from University of South Bohemia (Czech Republic) and Armstrong State University (ga, usa). All participants were weighted and measured and they (or parents of the children) self-reported a bedroom temperature for at least 7 consecutive days. The ambient temperature has a direct effect only if a light insulation (clothing and bed cover) is used during night. The bmi of participants were thus weighted. The total insulation weight was set as a sum of clothing weight and a bed cover weight. Weighted bmi was correlated with mbt with respect to the age class (see above) and sex.

Results: Whereas the correlation between mbt and bmi was weak and nonsignificant in both male and female children and teenage males, the correlation was highly significant in teenage females $\left(r=0.41 ; p<10^{-4}\right)$. The correlation between mbt and bmi was similarly significant in both us and Czech adult males $(r=0.21 ; p=0.008$, resp. $r=0.23 ; p=0.008)$. Conversely in adult females the correlation between mbt and bmi was positive but weak and nonsignificant $(\mathrm{r}=0.1 ; \mathrm{p}=0.09)$ in Czech adult females and negative and highly significant in us adult females $\left(\mathrm{r}=-0.32 ; \mathrm{p}<10^{-6}\right)$.

Conclusion:We show that the room temperature may have an effect on bmi in humans. A strong positive correlation between the bedroom temperature and bmi in teenage females shows a possible important factor in reducing weight in adolescent females as decrease in bedroom temperature by $2{ }^{\circ} \mathrm{C}$ could result in bmi decreased by 1.3 . Similar result in adult males also suggests the same possible effect of bedroom temperature. Interestingly, opposite results showed up in Czech and us females. We speculate that this difference might be contributed to either cultural differences or as a temperature discomfort in hot environment (typical in summer Georgia) in overweight people, resulting in actively decreasing room temperature via ac.

Funding:The research was funded by the Grant Agency of University of South Bohemia 118/2016/S. 
T4P2

Active versus inactive parents and their influence on children involved in a school intervention program to promote physical activity.

\section{Lagoa, M. ${ }^{1}$; Silva, G. ${ }^{2}$; Duncan, M. ${ }^{3}$; Mota, J. ${ }^{4}$; Aires, L. ${ }^{1}$}

${ }^{1}$ Research Centre in Physical Activity, Health and Leisure (ciafel) Faculty of Sports - University of Porto. University Institute of Maia (ismai), Portugal, ${ }^{2}$ Research Centre In Physical Activity, Health And Leisure (Ciafel) Faculty Of Sports - University Of Porto. Centre Of Research On Sports, Health And Development (Cidesd) University Institute Of Maia (Ismai), Portugal, ${ }^{3}$ School Of Life Sciences, Coventry University, Coventry, Uk, ${ }^{4}$ Research Centre In Physical Activity, Health And Leisure (Ciafel) Faculty Of Sports - University Of Porto.

Introduction: Insufficient Physical activity (pa) and high sedentary time are considered critical contributors to childhood overweight/obesity. Parental support is an increasingly relevant factor in helping children to adopt a healthy lifestyle but data regarding its influence is lacking. This study analysed the effect of parental pa levels on children's moderate vigorous pa (mvpa) after a school-based intervention.

Methods:Portuguese children $(\mathrm{n}=101$, mean age $7.76 \pm 1.21$ years); $31.7 \%$ were overweight and $37.6 \%$ obese took part of a school-based intervention, named acorda Project i.e. obese children and adolescents involved in pa and diet counselling. The 8 month program comprised 5 sessions per week of exercise with moderate to vigorous intensities. Children's evaluation consisted of daily mvpa, assessed over 7 days using accelerometers. Body Mass Index (bmi) was also determined from height and body mass. Parental pa was assessed by International Physical Activity Questionnaire allowing to determine the proportion of those whom met the acsm/aha pa guidelines for pa. To analyse how variables changed over time, univariate analysis of covariance, controlling for gender, with Bonferroni adjustments were calculated for each parental pa groups.

Results: Children's mvpa was significantly higher after intervention programme $\left(F=9.66, p<0.002, \eta^{2}=0.05\right)$. Furthermore, children's mvpa was significantly higher, when parents were considered sufficiently active (pa father group: fmvpa $=5.01, \mathrm{p}<0.028, \eta^{2}=0.06$; pa mother group: fm$\mathrm{vpa}=6.043, \mathrm{p}<0.016, \eta^{2}=0.06$ ). For inactive/insufficiently active parent groups the differences in children's mvpa were not significant (pa father group: fmvpa $=3.91, \mathrm{p}>0.051, \eta^{2}=0.05$; pa mother group: fmvpa $=2.71$, $\left.\mathrm{p}>0.103, \eta^{2}=0.04\right)$.

Conclusion: Our results showed that during a school-based intervention program activeparents might play a positive role on their children pa than inactive or insufficiently active ones. A combination of healthy behaviours intervention program in schools including parents could help to improve children's mvpa levels.

Conflict of Interest: None Disclosed

Funding: fct- (sfrh/bd/101410/2014 and uid/dtp/00617/2013)

\section{T4P3}

\section{Adiposity changes throughout adolescence and high- sensitivity C-reactive protein levels in young adulthood}

\section{Cabral, M. ${ }^{1}$; Araújo, J. ; Ramos, E. ${ }^{1}$}

${ }^{1}$ EPIUnit - Institute of Public Health - University of Porto (ispup)

Introduction: Growing evidence suggests an important role of adiposity in the development of a systemic inflammatory state that contributes to increased cardiovascular risk. However, it remains unclear whether different variations in adiposity pose an additional risk.

Objective: To investigate, in a longitudinal perspective, how changes in adiposity from 13 to 21 years impact on high-sensitivity C-reactive protein (hs-crp) levels measured at age 21.

Methods: We analysed data from a population-based cohort (epiteen) ( $\mathrm{n}=1623$ ). Body mass index (bmi) was measured at 13, 17 and 21y. Three bmi trajectories ('normal', 'high, declining' and 'high, increasing') were previously identified using mixture growth models. hs-crp was evaluated at 21 years, and high levels were defined as hs-crp above the 75th percen- tile. Logistic regression was fitted to estimate the association between bmi trajectories from 13 to 21 and hs-crp, adjusted for sex, parental education and bmi at $21 y$.

Results: Median $\left(25^{\text {th }}-75^{\text {th }}\right.$ percentiles) hs-crp levels at $21 \mathrm{y}$ significantly increased with increasing values of bmi across all ages evaluated. The 'high, increasing' trajectory presented the highest hs-crp concentrations at 21 years [median $\left(25^{\text {th }}-75^{\text {th }}\right.$ percentiles $\left.): 1.8(0.8-3.9) \mathrm{mg} / \mathrm{L}\right]$, whereas the 'high, declining' and 'normal' trajectories presented similar levels [0.7 (0.4-1.4) and $0.7(0.3-1.8) \mathrm{mg} / \mathrm{L}$, respectively]. After adjustment for potential confounders and considering as reference the 'normal' bmi trajectory, the 'high, increasing' trajectory was associated with higher odds of having high levels of hs-crp at $21 \mathrm{y}$ (or: 2.2, 95\%ci 1.5-3.1), while the 'high, declining' trajectory(or: $0.8,95 \%$ ci $0.5-1.2$ ) presented a profile similar to the 'normal' trajectory.

Conclusion: In addition to the effect of adiposity at 21 years old,increasing adiposity throughout adolescence was associated with a pro-inflammatory profile at early adulthood, whereas for the "high, declining" trajectory a profile similar to the "normal" trajectory was observed.

Conflict of Interest: None Disclosed.

Funding: This study was supported through feder from the Operational Programme Factors of Competitiveness - compete and through national Funding from the Portuguese Foundation for Science and Technology - fct (Portuguese Ministry of Education and Science) within the project ptdc/dtp-epi/6506/2014, and by the Epidemiology Research Unit - Institute of Public Health, University of Porto (uid/ $\mathrm{dtp} / 047507 / 2013)$. The individual doctoral grant to $\mathrm{mc}$ (pd/bd/105824/2014) by the Portuguese Foundation for Science and Technology - fct is gratefully acknowledged.

\section{T4P4 \\ Adiposity in adolescents and its association with cardiovascular risk factors}

\section{Vargas, F. ' Gonçalves, V. ' Dutra, E. ' Pizato, N. $^{\text {; }}$ ' Carvalho, $\mathrm{K}^{\prime}$ \\ ${ }^{1}$ University of Brasilia, Brazil}

Introduction: The Body Mass Index (bmi) is the most common parameter for diagnosing adolescent obesity, but it does not directly measure body fat (bf). The present study aimed to compare the profile of markers of cardiovascular risk factors according to bmi and bf in adolescents.

Methods: Cross-sectional study with a adolescent sample $(\mathrm{n}=56 ; 71 \%$ girls) from public and private schools located in the Federal District, Brazil. Blood pressure measurements and biochemical tests were performed. The nutritional status was evaluated by bmi $\left(\mathrm{kg} / \mathrm{m}^{2}\right)$ and bf $\%$ measured by multifrequency bioimpedance (InBody 720). T-test or Mann-Whitney were used to compare normal weight and overweight adolescents, according to bmi or bf $\%$ and Pearson or Spearman correlation of clinical and biochemical variables with bmi, bf\%. Statistical significance was set as $\mathrm{p}$ $<0.05$.

Results: The participants classified as overweight according to bmi had significantly greater levels of total cholesterol, triglycerides, systolic and diastolic blood pressure than eutrophic adolescents. When nutritional status was classified according to body fat, those with excess bf had significantly greater triglycerides levels. Systolic, diastolic blood pressure, triglycerides and insulin levels were positively associated with both bmi $(\mathrm{r}=0.57 ; \mathrm{p}<0.001 ; \mathrm{r}=0.35, \mathrm{p}=0.005 ; \mathrm{r}=0.42, \mathrm{p}=0.001$, $\mathrm{r}=0.38, \mathrm{p}=0.003, \quad$ respectively $), \quad$ and $\mathrm{bf} \% \quad(\mathrm{r}=0.48, \mathrm{p}<0.001$; $\mathrm{r}=0.34, \mathrm{p}=0.01 ; \mathrm{r}=0.39, \mathrm{p}=0.002, \mathrm{r}=0.39, \mathrm{p}=0.003$, repectively). hdl-cholesterol was negatively associated only with bmi $(r=-0.29$, $\mathrm{p}=0,03$ ), while total cholesterol was significantly associated only with bf\% $(r=0.27, p=0.049)$.

Conclusion: Nutritional status, evaluated by either bmi or bia, was associated to the main cardiovascular risk factors, supporting the fact that the excess weight and/or body fatness increase the risk of metabolic disturbances even in youth. 
T4P7

An innovative family and home-based intervention for the prevention and management of pediatric obesity: The ProxOB program

Magalie, M. '; Amélie, R. ' Margaux, D. '; Chloé, D. ${ }^{1}$; Alicia, F. ' Elisabeth, D. ${ }^{1}$; Christelle, J. ${ }^{1}$; Pauline, B. ${ }^{2}$; Marie, B. $^{2}$; Julie, M. ${ }^{3}$; David, T. ${ }^{4}$; Yves, . $^{5}$

${ }^{1}$ Service de Nutrition Clinique, cso caloris, chu Clermont-Ferrand, France, ${ }^{2}$ Service De Santé Publique, Chu Clermont-Ferrand, France, ${ }^{3}$ Ssr Nutrition Obésité Ugcam, Clermont-Ferrand, France, ${ }^{4}$ Ame2P, Université Clermont Auvergne, France, ${ }^{5}$ Service De Nutrition Clinique, Cso Caloris, Chu ClermontFerrand, France \& Unité De Nutrition Humaine, Umr1019, Crnh, ClermontFerrand, France

Introduction: The prevention and treatment of children obesity have to rely on the implication of their parents and other family members. While family-based interventions are effectively considered as a gold standard, they remain time and personnel consuming and most of the time face organizational issues. The development of home-based family interventions melting with the family organization and daily routine might improve their adherence and commitment and should favor a better follow-up. The aim of this project was to propose simple, realistic and durable therapeutic behavioral modifications using personalized interventions built up on the family needs, demands and possibilities.

Methods: 20 families (36 parents and 41 children) entered the ProxOB program that proposed a 6- month home-based therapeutic education composed of 16 sessions covering adapted physical activity, eating behaviors and parental education, led by appropriate health care professionals Anthropometric measurements, quality of life (sf36 questionnaire) and behavioral assessment were done before, at the end and 6 months post intervention.

Results: $71 \%$ of the parents and $29 \%$ of the children were diagnosed as obese while $21 \%$ of both children and adults were overweight at the beginning of the program. By the end of the intervention, a significant improvement of the parents corpulence (bmi $34.4 \mathrm{vs} 35.5 \mathrm{~kg} / \mathrm{m}^{2}, \mathrm{p}=0.0187$ ) as well as of their quality of life ( 4 of 8 parameters, $\mathrm{p}<0.05$ ) was found. There were tendency to an improvement of children $\mathrm{z}$-score, sleep length, decreased tv watching, snacking. The accession rate was 95\% (participation of the whole family in all sessions) and $85 \%$ of families were satisfied that the sessions take place at home.

Conclusion: This pilot home- and family-based ProxOB program offers an innovative and effective approach to prevent and treat overweight and obesity in both children and their parents. These results led us to design a larger program involving more families (44 new families) and using more objective and extensive evaluation Methods.

\section{T4P8}

\section{Association between gene polymorphisms and obesity and physical fitness in Korean children}

\section{Lee, S. ${ }^{1}$; Kim, H. ${ }^{2}$}

${ }^{1}$ Pusan National University Yangsan Hospital, ${ }^{2}$ Department Of Physical Education, Kyungnam University College Of Education

Introduction: Obesity is affected by genetic factors and environmental influences. This research was undertaken to identify single nucleotide polymorphisms (snps) related to obesity and physical fitness and then to conduct comparative analyses of interactions between physical fitness and obesity-associated genotypes.

Methods: To analyze the relationships between physical fitness and the major snps previously reported to be related to obesity, 68 snps in 32 genes were genotyped in 71 Korean children. Tests were conducted to evaluate five elements of physical fitness (speed, aerobic endurance, muscular endurance, muscular strength and flexibility).

Results: The results obtained showed significant $(\mathrm{P}<0.02)$ differences in physical fitness scores for genotypes of cnr1, lep, hhex, gc, lrp5, npy2R, ppy, ucp2, cdkn2B, and adipoq. Ten physical fitness-related genotypes were newly identified during the present study.
Conclusion: The study suggests classification of genotypes by physical fitness level could be used as an index for predicting the risk of obesity and for selecting individuals for an effective intervention program. Furthermore, the study shows even children participating in the same physical fitness improvement program can exhibit different genotype dependencies.

Conflict of Interest: None Disclosed

Funding: None

\section{T4P9}

\section{Association between hair cortisol concentration and adiposity measures among children and parents from the "Healthy Start" study}

\section{Larsen, S. ${ }^{1}$; Fahrenkrug, J. ${ }^{2}$; Olsen, N. ${ }^{1}$; Heitmann, $B .{ }^{1}$}

${ }^{1}$ Research Unit for Dietary Studies at The Parker Institute, The Capital Region, Copenhagen, Denmark, ²Department Of Clinical Biochemistry, Faculty Of Health Sciences, Bispebjerg Hospital, Dk-2400, Copenhagen Nv, Denmark

Background: Previous studies have suggested a direct association between hair cortisol concentration (hcc) and Body Mass Index (bmi), as well as other adiposity measures. However, these studies have mostly been conducted among adult populations.

Objective: To examine the association between hcc and different measures of adiposity among a selected group of children predisposed to obesity and their parents.

Methods: We conducted a cross-sectional study based on 363 children and their parents (301 mothers and 231 fathers) participating in the "Healthy Start" study. Linear regression analysis was used to investigate associations between hcc and adiposity measures while taking into account possible confounding factors. Analyses were performed examining the association between hcc and bmi, fat mass and fat free mass index Z-scores, as well as waist circumference and waist-hip ratio among the children. Likewise, the association between hcc and bmi among the parents was explored. Finally, we examined the association between parental hcc and children's adiposity measures.

Results: hcc was directly associated with a higher bmi among the fathers $\left(0.49 \mathrm{~kg} / \mathrm{m}^{2}\right.$ [95\% ci: $\left.0.09,0.90, \mathrm{P}=0.02\right]$ per $\left.100 \mathrm{pg} / \mathrm{mg}\right)$ and the mothers $(0.93 \mathrm{~kg} / \mathrm{m} 2$ [95\% ci: $0.24,1.61, \mathrm{P}=0.01]$ per $100 \mathrm{pg} / \mathrm{mg})$. We found no clear evidence of an association between hcc and adiposity measures among children. However, a high maternal hcc was associated with a high fat mass index and low fat free mass index z-score in the offspring $(0.14 \mathrm{sd}$ [95\% ci: $0.02,0.26, \mathrm{P}=0.02]$ and -0.17 sd $[95 \% \mathrm{ci}:-0.30,-0.05, \mathrm{P}=0.01]$ per $100 \mathrm{pg} / \mathrm{mg}$, respectively).

Conclusion: Our study found no evidence of an association between hcc and measures of adiposity among children predisposed to obesity. However, hcc may be directly associated with bmi among men and women, and maternal hcc may be related to a higher fat mass and a lower fat free mass among their children. 
T4P10

\section{Association of parental obesity with cardiometabolic risk factors in their children: The CASPIAN-V study}

Ejtahed, H. ${ }^{1}$; Heshmat, R. ${ }^{2}$; Qorbani, M. ${ }^{3}$; Hasani-Ranjbar, S. ; Motlagh, M. ${ }^{4}$; Ziaodini, $H^{5}{ }^{5}$; Larijani, B. ${ }^{6}$; Kelishadi, R. $^{7}$

${ }^{1}$ Obesity and Eating Habits Research Center, Endocrinology and Metabolism Molecular -Cellular Sciences Institute, Tehran University of Medical Sciences, Tehran, Iran., ${ }^{2}$ Chronic Diseases Research Center, Endocrinology And Metabolism Population Sciences Institute, Tehran University Of Medical Sciences, Tehran, Iran., ${ }^{3}$ Department Of Community Medicine, Alborz University Of Medical Sciences, Karaj, Iran., ${ }^{4}$ Department Of Pediatrics, Ahvaz Jundishapur University Of Medical Sciences, Ahvaz, Iran., ${ }^{5}$ Institute Of Education And Training, Tehran, Iran., ${ }^{6}$ Endocrinology And Metabolism Research Center, Endocrinology And Metabolism Clinical Sciences Institute, Tehran University Of Medical Sciences, Tehran, Iran., ${ }^{7}$ Pediatrics Department, Child Growth And Development Research Center, Research Institute For Primordial Prevention Of Non-Communicable Disease, Isfahan University Of Medical Sciences, Isfahan, Iran.

Introduction: Considering the importance of the family environment on childhood obesity and cardiometabolic disorders, the aim of this study is to investigate the association of parental obesity with cardiometabolic risk factors in their children.

Methods: This study was performed on 14002 students (aged 7-18 years) and one of their parents recruited through multistage, stratified cluster sampling from urban and rural areas of 30 provinces of Iran, as part of a national surveillance program. Fasting blood sample was obtained from a subsample of 3843 randomly selected students. Demographic, anthropometric and clinical parameters were collected.

Results: The prevalence of obesity in children, fathers and mothers was $11.4,10.6$ and $24.2 \%$, respectively. The most commonly observed metabolic abnormality was low hdl-C (29.5\%). The prevalence of metabolic syndrome and dyslipidemia in children was 5 and $55.7 \%$, respectively. Significant correlations were observed between the body mass index (bmi) and waist circumference of parents and weight, height, bmi, waist circumference, systolic blood pressure and diastolic blood pressure of their children $(P<0.05)$. In the multivariate model, the risk of excess weight (or: 1.35 , 95\%ci: $1.23-1.49$ ), obesity (or: $1.47,95 \%$ ci: $1.30-1.66$ ), abdominal obesity (or: $1.21,95 \%$ ci: $1.11-1.33$ ) and elevated bp (or: $1.24,95 \%$ ci: 1.09-1.40) were higher in those students whose parents had excess weight compared with other students. Parental obesity did not have significant association with metabolic syndrome and dyslipidemia in children.

Conclusions: Parental history of obesity could be used as a practical approach for the early identification of children at risk of cardiometabolic complications.

None Disclosed

No Funding

\section{T4P11}

\section{Associations between physical activity, sedentary behavior, and indicators of metabolic strain in children and adolescents with obesity}

\section{Sørensen, C. '; Nielsen, T. '; Schnurr, T. ${ }^{2}$; Bech, B. ${ }^{2}$; Holm, J. ${ }^{1}$}

${ }^{1} T$ he Children's Obesity Clinic, Department of Pediatrics, Copenhagen University Hospital Holbæk, Denmark, ${ }^{2}$ The Novo Nordisk Foundation Center For Basic Metabolic Research, Section Of Metabolic Genetics, University Of Copenhagen, Denmark

Introduction: An increased sedentary lifestyle is associated with the degree of obesity in children and adolescents ${ }^{1-5}$. A combination of obesity and a physically inactive lifestyle increases the risk of co-morbidities ${ }^{1-5}$. The aim of this study was to examine the associations between physical activity/inactivity and selected biomarkers.

Methods: This cross-sectional study was based on baseline-data from The Danish Childhood Obesity Biobank. The study included 93 children with overweight or obesity (bmi $>90$ percentile) between $5-17$ years enrolled in childhood obesity treatment. Physical activity and sedentary behavior (sb) were measured objectively with an ActiGraphGT3X+ accelerometer ( $\geq 4$ days wear time incl. one day in the weekend and $\geq 10$ hours daily). Furthermore, blood pressure, fasting plasma total cholesterol, low-density lipoprotein, high-density lipoprotein (hdl), triglycerides, glucose and homa-ir, and percentage of body fat measured by dual-energy X-ray, as well as degree of obesity were measured. Linear regression analyses were applied to determine the associations between the sedentary behavior ( $\mathrm{sb}$ ) and moderate to vigorous physical activity (mvpa) and the above indicators of metabolic strain. All analyses were adjusted for age, sex, and puberty status as potential confounders.

Results: On average in the study population, sb was $523,1 \mathrm{~min} /$ day $(8.72$ hours) and the mvpa was $62.8 \mathrm{~min} /$ day (1.1 hours). The study identified a positive correlation between mvpa and hdl $(\beta: 0.003,95 \mathrm{CI}[0.001 ; 0.005]$, $p=0.0046)$. In addition, the study identified negative associations between mvpa and the percentage of body fat $(\beta:-0.032,95 \mathrm{CI}[-0.062$;$0.001], p=0.044)$. No associations were found between sb and mvpa and blood pressure, glucose, homa ir, total cholesterol, low-density lipoprotein, or triglycerides.

Conclusion: In a group of Danish children and adolescents with overweight and obesity the level of physical activity (sb and mvpa) was associated with fasting plasma hdl concentrations, and the percentage of body fat.

\section{References:}

1 Cliff, D. P. et al. Pediatr.Obes. 21(2)382-385. 2 (2013)

2 Velásquez-Rodríguez, C.M. et al. BioMed Pediatr. 14:258 doi: 10.1186/14712431-14-258 (2014).

3 Vale, S. et al. J.Pediatr. 167(1) 167;98-102 (2015).

4 Meyer, A. A. et al. J.Am. Coll. Cardiol.48(9), 1865-1870 (2006).

5 Rizzo, N. S. et al. Am. J. Clin. Nutr., 87, 586-592 (2008).

Interest of conflicts: No conflict of interest.

Funding: The study was supported by the Innovation Fund Denmark, www.innovationsfonden.dk (grant 0603-00484B and grant 0603-00457B), the Novo Nordisk Foundation, www.novonordiskfonden.dk (grant nnf15OC0016544), and the Region Zealand Health and Medical Research Foundation, www.regionsjaelland.dk.

\section{T4P12}

\section{Birth weight, parents' body mass index and education level and nutritional status in a population between 6 and 18 years old}

\section{Sousa, B. ${ }^{1}$; Pinto, C. ${ }^{2}$; Oliveira, B. $^{2}$; Almeida, $M .^{2}$}

${ }^{1} \mathrm{School}$ of Sciences and Health Technologies, Universidade Lusófona de Humanidades e Tecnologias, ${ }^{2}$ Faculty Of Nutrition And Food Sciences Of The University Of Porto

Introduction: Higher birth weight and parents' Body Mass Index (bmi) is associated with greater nutritional status of children/adolescents. Lower parents' education is associated with highest prevalence of obesity occurred in the children/adolescents. The aim of this study is to relate birth weight, parents' bmi and education level to nutritional status of a sample of children and adolescents between 6 and 18 years old $(n=6699)$, resident in the Autonomous Region of Madeira.

Methods: Birth weight, duration of gestation, mother and father bmi and education level were collected. Weight and height of children and adolescents were measured and bmi was calculated to determine their nutritional status, according to the International Obesity Task Force (iotf) criteria. Results: Nutritional status was higher in boys between 10 and 18 years old, and in child/adolescent between 6 and 14 years old, whose parents had higher education. The higher birth weight and parents' bmi were also associated with a higher nutritional status.

Conclusion: In this population, the higher parental education was associated with higher nutritional status of children/adolescents between 6 and 14 years old.

Conflict of Interest: None

Funding: No Funding 
T4P13

BMI-for-age at birth is associated with BMI-for-age in female children from 24 to 48 months old

\section{Amoroso, A. ${ }^{\text {; }}$;}

${ }^{1}$ Montepio Geral Associação Mutualista

Introduction: According to the who most recent statistics there were 42 million children from 0 to 5 years old overweight or obese in 2013 and these numbers could achieve 70 million by the year 2025. Most commonly these children will become overweight/obese adults in an increased risk to suffer from cardiovascular diseases, diabetes and cancer, among others. In the last three decades the Developmental Origins of Health and Disease (dohaD) paradigm has gathered evidences that early intervention is the best policy when it comes to promote health, and obesity is not an exception. In this study, the relation between bmi-for-age was measured at birth and compared with bmi-for age in children from 24 to 48 months old.

Methods: This study used body mass index-for-age (bmi-for-age), according to the who child growth standards. Data from length and weight at birth and height and weight at present data were extracted from 172 children who participated in the study Pro.Mo Saúde - Babies (2016) The association between bmi-for-age at birth and bmi-for-age at 24 to 48 months old was tested using anova and a Scheffe Post Hoc test and ChiSquare.

Results: anova showed a statistically significant positive relation between bmi-for-age at birth and bmi-for-age in 24 to 48 months old baby girls ( $\mathrm{p}=0.010)$, but not for baby boys. Chi-Square also showed a statistically significant relation for baby girls $(\mathrm{p}=0.034)$, but not for boys.

Conclusion: The results are consentaneous with dohaD paradigm that states that health interventions should be considered as early as possible. It is needed to address obesity policies to gestational period and perhaps even previously. The sex differences obtained in this study need to be further investigated.

None Disclosed

Research relating to this abstract was funded by Montepio Geral - Associação $\mathrm{Mu}$ tualista

\section{T4P14}

\section{Body composition in obese children}

Irina, L. ${ }^{1}$; Galina, O. ${ }^{1}$; Valeria, N. ${ }^{1}$; Anastasia, $K{ }^{1}$

${ }^{1}$ Federal Almazov North-West Medical Research Centre

Objectives: To investigate the fat and body cell mass ( $\mathrm{bcm})$ in children with excess body weight and obesity

Methods: We examined150teenagersaged 10 to 17 years suffering from excess body weight (body mass index from 25,49 $\pm 2,18$ ) and obesity (body mass indexfrom 30,47 $\pm 4,61$ ). The control group included 30 teenagerswith normal body mass index $(19,45 \pm 2,18)$. Bio-impedance analyzer «Diamant-aist» («Diamond», Russia) assessed body composition.

Results: In the control group, body fat percentage was $26 \%$ (in girls) and $14 \%$ (in boys). In the group of children with an excess weight of $33 \%$ and $27 \%$ respectively. In the group of obese children, the figure was $40 \%$ (in girls) and $37 \%$ (of boys), $\mathrm{p}<0,001$.

In the control group, body cell mass was 55\% (in boys), $47 \%$ (in girls). In the group of children with excess body weight - $43 \%$ in girls, $47 \%$ in boys In the group of children with obesity bcm was $41 \%$ (in boys), $38.5 \%$ in girls $(\mathrm{p}<0.001)$

Conclusion: In children with excess body weight and obesity grows as fat as $\mathrm{bcm}$. However, the increase fat and $\mathrm{bcm}$ is not proportional. Increase of fat is more than $200 \%$, and body cell mass no more than $34 \%$. This fact can effect on the metabolic processes in the body.
T4P15

\section{Body fat percentage, nutritional and physical activity knowledge in adolescents}

Ferro-Lebres, V. ${ }^{1}$; Ribeiro, J.2 ; Silva, G. ${ }^{2}$; Moreira, P. ${ }^{3}$

${ }^{1}$ Diagnostic and Therapeutic Technologies Department, School of Health Sciences - Polytechnic Institute of Braganza, Portugal, ${ }^{2}$ Research Center In Physical Activity, Health And Leisure, Faculty Of Sports, University Of Porto, Portugal, ${ }^{3}$ Faculty Of Nutrition And Food Sciences, University Of Porto, Portugal

Introduction: Knowledge enhancement has been referred as a mean to improve nutrition and physical activity, and reduce obesity. The objective of this work was to identify the difference of nutrition and physical activity knowledge according body fat and physical activity levels in adolescents. Methods: A cross sectional study with 734 adolescents was designed. Body fat was measured using electric bio-impedance, physical activity data were objectively measured by accelerometer. Adolescents were divided in 2 physical activity groups: high and low physical activity; 2 body fat groups: normal fat and overfat and 4 groups resulted from the combination of body fat and physical activity. Nutrition and physical activity knowledge were assessed by questionnaires.

Results: Body fat percentage revealed that $30.8 \%$ of adolescents were overfat or obese, and daily moderate to vigorous physical activity was in average 47.9 ( $s d=27.49)$ minutes. High physical activity adolescents presented higher physical activity knowledge $(\mathrm{p}=0.044)$ and the overfat/ low physical activity group scored the worse on experts' nutritional recommendations knowledge.

Conclusion: Knowledge is not the single factor in the obesity equation, but it seems essential to reduce overfat and obesity. Interventions that address several nutrition and physical activity determinants should be designed.

\section{Conflict of Interest: None Disclosed}

Funding: This work was supported by fct, grant number fcomp-01-0124-feder-028619 (fct: ptdc/dtp-des/1328/2012) and the Research Center supported by: uid/dtp/00617/2013

T4P16

\section{Body mass index profile in adolescents and its relation with weight concerns}

Pereira, A. '; Veiga-Branco, M. ${ }^{2}$; Pereira, F. $^{3}$

${ }^{1}$ Instituto Politécnico de Bragança, ${ }^{2}$ Instituto Politécnico De Bragança, Paideia, ${ }^{3}$ Universidade De Lisboa De Faculdade De Medicina

Introduction: The percentage of obese adolescents has increased during the past years, which as lead the World Health Organization to consider obesity as the main epidemic of the xxi century. However, adolescents are not immune to body image and weight concerns. This sensibility regarding weight might be an asset, allowing a healthy eating behaviour.

Objectives: Analyse the adolescent's body mass index (bmi); Evaluate the relation between the bmi and the weight concerns.

Methods: Restrictive, quantitative and transversal study. By gathering a simple random sample, and considering a sampling error of $3.5 \%$, a sample of 600 adolescents (44\% of the male sex and $56 \%$ of the female sex) with ages among 12-18 years old and attending the eb 2/3 Schools, High Schools and Professional Schools from Bragança, was studied. The bmi was determined through the calculation of the ration between the measure of the body mass in kilograms and the height in meters, squared (mass $(\mathrm{kg}) /$ height $\left.(\mathrm{m})^{2}\right)$, and classified with the criteria established by the Centers for Disease Control and Prevention. For the collection of data regarding weight concerns, it was used a self-administered questionnaire adapted from Luciana Apetito et al (2010). For the statistics analysis, it was used the spss programme version 14.0 (2005) for Windows, Microsoft. Results: $7,2 \%$ of boys and $5,7 \%$ of girls, in the age group $12-14$ years old, and $15,9 \%$ and $10,1 \%$, respectively, in the age group of $15-18$ years old, had overweight/obesity. At a $5 \%$ significance level, it was demonstrated that the bmi was statistically dependent of the sex (et: 5.171; vp: 0,160) and 
the age group (et: 1,900; vp: 0,593). Also, $61,2 \%$ of boys and $83,6 \%$ of girls stated that they worry about their weight. The concerns regards weight are statistically associated to the bmi observed (et:17,225; vp: 0,001)

Conclusion: The results showed that $23,1 \%$ of boys and $15,8 \%$ of girls have a bmi above the recommended amounts and, also, that it is statistically dependent of weight concerns. From this point of view, the interventions among young people must be multifaceted, never forgetting the elements that constitute the human being (psychological and biological). Keywords: Adolescents, Body mass index, weight concerns

Conflict of interests: none

Financing: none

T4P17

\section{Body weight stabilization (plateau) as a treatment goal in adolescents with obesity}

Starodubova, A. ${ }^{1}$; Pavlovskaya, E. ${ }^{2}$; Strokova, T. $^{2}$; Kosyura, S. ${ }^{3}$

${ }^{1}$ Institute of Nutrition, Pirogov Russian National Medical Research University, ${ }^{2}$ Institute Of Nutrition, ${ }^{3}$ Pirogov Russian National Medical Research University

Aim. To examine the role of body weight stabilization as an obesity treatment goal in adolescents.

Patients and Methods: The prospective clinical intervention study included 120 adolescents with obesity (2 standard deviations above the who Growth Reference median). The median of initial age - 13 years old girls $-62 \%$. Examination included anthropometric and body composition measurements (bioelectrical impedance analysis), clinical and laboratory methods (plasma levels of lipids, fasting glucose levels and insulin resistance). Weight management program included diet and physical exercises during 2 -weeks stay in a specialized inpatient department, regular visits to outpatient department; nutrition, physical activity, lifestyle and behavioral counseling for adolescents and parents. Follow-up period was 12 months Weight stabilization was diagnosed when dynamic of body weight from initial was less than 0,05 body mass index standard deviation score (bmi sds) ( $1^{\text {st }}$ group, $\mathrm{n}=18$ ), decrease/increase of weight were diagnosed when there were a decrease/increase of final weight (12 months visit) more than 0,05 sds bmi $\left(2^{\text {nd }}(n=56)\right.$ and $3^{\text {rd }}$ groups $\left.(n=46)\right)$.

Results: There was a decrease of rates of dyslipidemia (from 69 to 45\%), impaired glucose tolerance (from 25 to $12,5 \%$ ), non-alcoholic fatty liver disease (from 64 to $48 \%$ ), hypertension (from 43 to $30 \%$ ), and metabolic syndrome (idf, 2007) (from 25 to 14\%) during 12 months weight management program in total group. Weight management program was well tolerated by adolescents in all groups. During 1-year of treatment $62 \%$ of adolescents did not increase their weight ( $1^{\text {st }}$ and $2^{\text {nd }}$ groups).

There were no differences between groups in age, gender, bmi, degree of obesity. Fat mass $(\mathrm{kg})$ increased in the $2^{\text {nd }}$ and decreased in the $3^{\text {rd }}$ grp during one year of treatment $(\mathrm{p}<0,01)$, without any significant dynamic in the $1^{\text {st }}$ grp. But there were a decrease of the proportion of fat mass (\%) from initial in the 1 st and $2^{\text {nd groups }}$, and an increase in the $3^{\text {rd }}$ group $(\mathrm{p}<0,01)$. Skeletal muscle mass was increased in all three groups $(\mathrm{p}<0,01)$, but in the $3^{\text {rd }}$ group it increased more than in the $1^{\text {st }}$ and the $2^{\text {nd }}$ groups $(\mathrm{p}<0,01)$, without any difference between $1^{\text {st }}$ and $2^{\text {nd }}$ groups $(p>0,05)$. Plasma levels of total cholesterol and high-density lipoproteins increased in all groups $(\mathrm{p}<0,05)$. Plasma levels of triglycerides and glucose increased only in the $3^{\text {rd }}$ group. $(p<0,05)$.

Conclusion: Body weight stabilization as a result of 12 months weight management program as well as decrease of body weight was accompanied by the decrease of the proportion of fat mass, and stable levels of triglycerides and glucose in adolescents with obesity. We suggest that body weight stabilization may be considered as an appropriate treatment goal in adolescents with obesity.

\section{T4P19 \\ Breastfeeding and nutritional status in a population between 6 and 18 years old}

Sousa, B. ${ }^{1}$; Pinto, C. ${ }^{2}$; Oliveira, B. ${ }^{2}$; Almeida, M. ${ }^{2}$

${ }^{1}$ School of Sciences and Health Technologies, Universidade Lusófona de Humanidades e Tecnologias, ${ }^{2}$ Faculty Of Nutrition And Food Sciences Of The University Of Porto

Introduction: The increasing prevalence of overweight and obesity is a worldwide serious public health problem. Some studies have reported a protective effect of breastfeeding in prevention of future overweight or obesity, however others are inconclusive and influenced by confusing factors. The aim of this study is to evaluate the effect of breastfeeding on the nutritional status of a sample of children and adolescents between 6 and 18 years old $(n=6699)$, resident in the Autonomous Region of Madeira. Methods: The data about the breastfeeding practice and duration, and clinical and parents data were collected retrospectively. Weight and height of children and adolescents were measured and Body Mass Index was calculated to determine their nutritional status, according to the International Obesity Task Force (iotf) criteria.

Results: In this population, $84.4 \%$ were breastfed and the average of duration of breastfeeding was $5.9( \pm 7.6)$ months. The exclusive breastfeeding was held in $63.0 \%$ of children/adolescents and the average duration was $2.3( \pm 2.0)$ months. We found a prevalence of overweight and obesity of $37 \%$ (6-10 years-old), $38.8 \%$ (10-14 years-old) and 30.3\% (14-18 yearsold). It was found that breastfeeding was not significantly related to the nutritional status of the child/adolescent.

Conclusion: Therefore awareness campaigns for the parents about the nutritional health of their children should be strengthened.

Conflict of Interest: None

Funding: No Funding

\section{T4P20 \\ Cafeteria diet intake in nursing rats alters mir-222 levels in breast milk}

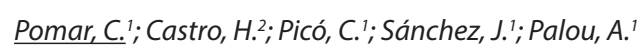

${ }^{1}$ Laboratorio de Biología Molecular, Nutrición y Biotecnología (Nutrigenómica y Obesidad), Universidad de las Islas Baleares (uib) y ciber Fisiopatología de la Obesidad y Nutrición (ciberobn), Palma de Mallorca, España., ${ }^{2}$ Facultad De Nutrición Y Salud Pública, Universidad Autónoma De Nuevo León, Nuevo León, México

Introduction: miRNAs are small endogenous non-coding rnas that regulate metabolic processes including adipocyte differentiation, glucose/insulin homeostasis, among others, and hence a deregulation of their pathways may be related to the pathogenesis of obesity. The objective of the present study was to investigate in nursing rats the impact of a cafeteria diet intake (cafeteria dams) in mir-222 levels in breast milk. mir-222 was selected for its potential relation with obesity. To discern the effects of the adiposity per se, breast milk from obese dams fed with cafeteria diet until one month before gestation (post-cafeteria dams) was also analysed.

Methods: Milk samples were collected from control, cafeteria and post-cafeteria dams at three time points of lactation (days 5, 10, and 15) and levels of mir-222 were determined. After weaning, pups from the different groups of dams were killed under feeding or after $12 \mathrm{~h}$ fasting conditions. The expression of key genes related to energy metabolism in white adipose tissue (wat) and liver were analysed.

Results: The levels of mir-222 were higher in breast milk of cafeteria dams, but not in post-cafeteria dams, compared to controls. After weaning, the expression levels of Prkaal (a possible target of miR-222) in the offspring of control dams were increased upon fasting conditions, both in liver and wat. Unlike controls, this increase was not observed in the offspring of cafeteria dams, which also presented an altered response to fed/fasting conditions in the expression of metabolic genes regulated by the ampk: 
Srebf1, Fasn, Scd1 y Gck in liver, and Pparg, Srebf1 y Scd1 in wat. These alterations were not observed in the offspring of post-cafeteria dams. Conclusion: The intake of a cafeteria diet in nursing rats leads to increased mir-222 levels in milk. The greatest supply of mir-222 to offspring through the milk could be potentially related to the altered mRNA expression of Prkaal and to the impairment of the metabolic response to fed/fasting conditions in key genes involved in energy homeostasis. However, future research is needed to establish a real cause-effect relationship.

Conflict of Interest: None Disclosed

Funding: Research relating to this abstract was funded by agl2015-67019-P

\section{T4P25}

\section{Children's perception about their mothers' body image and their association with BMI}

\section{Solorio, A.; Bacardí-Gascón, M.; Jiménez-Cruz, A.}

Universidad Autónoma de Baja California, Children's perception about their mothers' body image and their association with bmi

Background \& aims: A correct perception of the body image is a factor that can induce to have a behavior on health favoring a lifestyle for a healthier one. The aim of this study was to evaluate children's perception about their mothers' body image and to identify the factors associated with the perception of the weight of their mothers using a set of images. Objectives: To assess the perception of the maternal corporal image of elementary school children and their ability to correctly identify the weight of their mothers through a set of images and determine the association between the weight status, school level and family income and sex.

Material \& methods: A pictogram with a scale of women's bodies was applied to 431 children aged 9 to 12 from eight public schools in the city of Tijuana, Baja California, and anthropometric and sociodemographic data were collected from their mothers through a questionnaire. The associations between maternal bmi categories, schooling, age, place of origin and monthly income of the mother and the children's perception of the body image of the mothers with the Chi-square test with the spss program were analyzed.

Results: Fifty percent of the children perceived correctly the weight of their mothers, while $38 \%$ underestimated and $12 \%$ overestimated. There was an association between the perception of the children and the mother's bmi $(P=0.0001)$ and the mothers' age $(P=0.017)$.

Conclusion: The higher bmi and age of the mothers shows a higher percentage of children who underestimated the body image of their mothers.

\section{Acknowledgement: conacyt}

References:

1 De La O A, Jordan kc, Ortiz K, Moyer-Mileur lj, Stoddard G, Friedrichs M, Cox $\mathrm{R}$, Carlson ec, Heap E, Mihalopoulos nl. "Do parents accurately perceive their child's weight status?," J. Pediatric Heatlh Care, vol. 23, no. 4, pp. 216-221.

2 Guevara-Cruz, M., Serralde-Zuniga, A. E., Vazquez-Vela, M. E. F., Blancas, L., \& Islas-Ortega, L. (2012). Association between maternal perceptions and actual nutritional status for children in a study group in Mexico. Nutrición Hospitalaria, 27, 209-212.

3 Jiménez-Cruz A, Bacardí-Gascón M. “The fattening burden of type 2 diabetes to Mexicans: projections from early growth to adulthood". Diabetes Care 2004.

\section{T4P26}

Children's psychosocial stress and gut health in a pathway to inflammation and obesity: Gut barrier, fecal short-chain fatty acids and gut bacteria

Michels, N. '; Wiele, T.' ; Henauw, S. ${ }^{1}$

${ }^{1}$ Ghent University

Objectives: We and others have previously shown that already during childhood, stress is related to unhealthier diet, higher adiposity and inflammation. A common underlying pathway might be changed gut bac- teria, but mechanisms underpinning the stress-gut axis are poorly understood. This might inspire probiotic interventions. We investigated the relationship of stress measures with bacterial produced short-chain fatty acids (scfa), gut bacteria and gut barrier; and its independence of diet, adiposity and inflammation.

Methods: In 113 Belgian children (8-16y), bmi, a fecal sample, blood sample, hair sample and questionnaire data were collected. Biological measures of stress included hair cortisol (most proximal $3 \mathrm{~cm}$ ) and 5-minute heart rate variability (high frequency hrv). Self-report measures of stress included emotional problems and negative events. Fecal calprotectin was determined as a marker of intestinal inflammation and an indirect indicator of gut barrier integrity. Fecal scfa (butyrate, propionate, acetate, valerate, isobutyrate, isovalerate) and bacteria were measured by gas chromatography and Miseq Illumina. Serum c-reactive protein (crp) and tnfalpha were used as inflammatory parameters. Linear regression analyses for stress versus gut were adjusted for sex, age, socio-economic status, bmi, crp, tnfalpha, fiber intake and protein intake.

Results: Stress as measured by emotional problems was significantly associated with higher butyrate $(B=0.263)$, valerate $(\beta=0.230)$, isovalerate $(B=0.231)$ and isobutyrate $(B=0.233)$. Heart rate variability reflecting higher parasympathetic activity was related to lower valerate levels $(\beta=-0.217)$ and higher Firmicutes/Bacteroidetes ratio $(B=0.178)$. Hair cortisol was not associated with the fecal data. Fecal calprotectin was not related to the other fecal or stress measures. These significances were present with or without adjusting. Interestingly, Firmicutes/Bacteroides was positively associated with all scfa. Concerning the relation of fecal measures with bmi or inflammation, only calprotectin was related to higher tnfalpha.

Conclusions: In healthy children, chronic stress (as measured by hrv and emotional problems but not by hair cortisol) is manifested in terms of higher scfa concentrations rather than in intestinal inflammation measured by calprotectin or in Firmicutes/Bacteroidetes ratio. This was independent of diet, bmi or inflammation. The rather counterintuitive associations with the health-beneficial butyrate point to the need for further detailed sequencing and contrasting of this healthy population with children with clinical depression to hopefully detect more clear-cut differences (data will be available by the congress date). In addition, we are following-up this cohort for longitudinal data.

\section{T4P27}

\section{Children's psychosocial stress and metabolic health}

\section{Michels, N. ${ }^{1}$; Henauw, S. ${ }^{1}$}

${ }^{1}$ Ghent University

Aims: Psychosocial stress has been detected as an extra lifestyle risk factor for metabolic health due to lifestyle and hormonal changes. We have already shown more comfort food consumption and adiposity in stressed children but it is not yet known whether the metabolic effects can already start during childhood.

Method: In +-350 children, stress was measured by reports (events, emotions, behavioral problems), salivary cortisol (total-day and morning output aucg) and heart rate variability (hrv). Metabolic syndrome (MetS) risk was measured by measured by (1) waist circumference (wc); (2) diastolic (dbp) and systolic (sbp) blood pressure; (3) high-density lipoprotein (hdl) and triglycerides (trg); (4) glucose homeostatic model assessment (homa). Total metabolic risk was calculated using the sum of sex- and age-specific $z$-scores of the components $z W C+(z S B P+z D B P) / 2+(z T R G-$ zHDL)/2+zHOMA.C-reactive protein as inflammatory parameter; leptin as appetite regulator and adiponectin as insulin-sensitizing/anti-inflammatory parameter were available.

Results: Blood pressure, glucose homeostasis and triglycerides were the relevant MetS components related to stress: events positive with homa, internalizing problems positive with homa and triglycerides, aucg positive with homa and lf/hf positive with blood pressure but negative with triglycerides. When considering the total MetS, only internalizing problems were directly positively related (beta $=0.236$ ). Cortisol was the dominant 
biomarker as an enhancer (i.e. moderator) of the effects of stress reports on MetS. Physical activity and diet were not mediators nor moderators in the stress-MetS association. Behavioral/emotional problems, cortisol and hrv were also related to higher inflammation and leptin levels, sometimes in a sex-specific way. Adiponectin was only negatively related to total-day cortisol and subcategories of the stress reports: anger and conduct problems.

Conclusions: This study shows that stress can have unfavourable metabolic effects during childhood. Implications are the incorporation of stress management and emotion regulation in prevention or intervention and also the need to monitor physiological health in children with high stress levels. Longitudinal follow-up is ongoing.

\section{T4P28}

\section{Children's understanding of physical activity and health}

\section{Everley, S.}

${ }^{1}$ University of Chichester

Introduction: Only $21 \%$ of boys and $16 \%$ of girls meet minimum recom mended guidelines for physical activity (pa) in the uk (Scholes and Mindell, 2013) and the uk Government has targeted the use of 'sport' as a potential solution to the problem (hmgov.Cabinet Office, 2015). Justification for interventions however, lacks consideration of children's perspectives. Understanding the nature of children's engagement in physical activity may help establish interventions that ensure healthy active lifestyles.

Aims: Understand young children's perceptions of physical activity and health Identify key factors that influence engagement in physical activity and other health behaviours

Methods: 28 primary school children aged 5 and 6 yrs (12 girls and 16 boys) drew themselves being physically active and were interviewed individually about their drawings. They also modelled a representation of 'health' individually with the researcher whilst constructing a narrative around the process and their understanding of the concept.

Constructing narratives in association with arts based data decodes meaning and facilitates interpretation. Using multiple approaches enables topics to be revisited and developed in depth and complexity.

Results: pa largely took place within family environments

Activities were associated with unstructured play/lifestyle engagement (not sport)

- Children were active in social situations $(n=26)$

- Relationships with parents and extended family was central to engagement.

- Children valued activity as part of home culture

- 'Health' was associated with diet rather than pa

Conclusion: In order to harness existing interest in pa and encourage continued active healthy lifestyles there is a need to:

- Educate families and children with respect to the value of physical activity in health

- Develop the availability of culturally accessible physical activity for children in schools

- Utilise existing social groups to encourage physical activity so that as children move away from family to peer influence they are able to maintain active lifestyles

\section{T4P29}

\section{Children's birth weight and parental obesity as risk factors of} children obesity

lubina, O. ${ }^{1}$; Napituhina, I. ${ }^{1}$; Agadžanjana, K. ${ }^{1}$; Dzivite-Krišāne, I. '; Sprudzāne, I. '; Vètra, A. '; Gailìte, J. ${ }^{1}$

${ }^{1}$ Children's Clinical University Hospital

Introduction: According to Centre of Disease Prevention and Control data in Latvia, overweight and obesity of 2012 / 2013th school year, seven- year-old children was $24.1 \%$ for boys and $20.9 \%$ for girls. Children obesity risk factors are birth weight and parental obesity.

Methods: Data about anthropometric parameters (weight, height, bodymass index (bmi)), birth weight, parental obesity was collected and analysed by a multidisciplinary team in Children's Clinical University Hospital (Riga, Latvia)between August 2014 and August 2016

Results: 218 children participated in the research. Mean age is 12.2 (sd \pm 2.7 y.o.), mean weight is 79.3 ( $\mathrm{sd} \pm 22.9 \mathrm{~kg}$ ), mean height is 1.61 (sd $\pm 0.13 \mathrm{~m}$ ), mean bmi is 29.9 ( $\mathrm{sd} \pm 5.5 \mathrm{~kg} / \mathrm{m}^{2}$ ), mean waist circumference 97.9 ( $\mathrm{sd} \pm 14.1 \mathrm{~cm})$, mean birth weight is 3634 ( $\mathrm{sd} \pm 586 \mathrm{~g})$. In $21 \%$ of the cases, birth weight was above $4 \mathrm{~kg}$. Both parents were overweight in $36 \%$, mothers were overweight in $29 \%$, fathers $-27 \%$ of the observations.

Conclusion: Children's birth weight is not significant risk factor in childhood obesity. Parental weight plays more significant role in childhood obesity development, so parental education in obesity treatment should be one of the main topics during weight control programme.

Conflict of Interest: None Disclosed

Funding: No Funding

\section{T4P30}

\section{Cholecalciferol supplementation does not improve arterial stiffness in obese adolescents: A double-blind randomized trial vidado.}

Christopher, M. ; Antonia, P. ; Catherine, D. ${ }^{3}$; Catherine, T. ${ }^{3}$; Cecile, R. ${ }^{4}$; Cecile, M. ${ }^{4}$; Marie-Josephe, A. ${ }^{3}$; Agnes, . $^{1}$

${ }^{1}$ LaPEC, University of Avignon, France, ${ }^{2}$ Vascular Medicine And Laboratory, Nimes University Hospital, France, ${ }^{3}$ Nort, Amu, Inra, Inserm, Marseille, France, ${ }^{4}$ Ssr Institut Saint Pierre, Palavas-Les-Flots, France

Introduction: As obesity in children was associated with lower concentration of vitamin D and arterial stiffness, an early marker of cardiovascular risk, we aimed to examine the effects of cholecalciferol (vd) supplementation on body composition, arterial stiffness and inflammatory status in obese adolescents.

Methods: Twenty-six obese adolescents (oa) (bmi-Z score $=4.0 \pm 06$ ) 12-17 y-old, and sixteen puberty-matched normal-weight counterparts (bmi-Z score $=0.2 \pm 0.8$ ) as controls were enrolled. Obese adolescents were randomized into two arms, (1) supplemented with $4000 \mathrm{iu} / \mathrm{d}$ of vd $(n=13)$ and $(2)$ received a placebo $(n=13)$. All oa followed a weight-loss program consisting of a reduction of $500 \mathrm{kcal}$ per day combined with 180 $\mathrm{min} /$ week of supervised moderate-to-vigorous intensity exercise. Serum 25(oh)D, body composition, inflammatory markers (il-6, crp) and common carotid artery (cca) compliance were measured at inclusion and after 3-month supplementation. Delta changes of the parameters (expressed in $\%)$ were compared between the both arms.

Results: At inclusion, 70\% oa had lower 25(oh)D concentration $(<50$ nmol L ${ }^{-1}$ ) compared to normal-weight adolescents (30\%). oa displayed lower cca compliance and higher il- 6 and crp values than controls. After three months, 25(oh)D increased but was not significantly different between the both arms, supplemented or placebo ( $59 \%$ vs $36 \% \mathrm{p}=0.08$ ). Similarly, the delta changes of cca compliance (7 vs 17\%), abdominal fat mass $(-14$ vs $-14 \%)$ and inflammatory markers $(-42 \%$ vs $-33 \%$ for il-6, $-37 \%$ vs $-61 \%$ for crp) were not significantly different between the two arms. However, the delta change of $25(\mathrm{oh}) \mathrm{D}$ was significantly correlated with the delta change of abdominal fat mass $(r=-0,49, p=0,02)$.

Conclusion: Vitamin D supplementation during weight loss did not improve the increase cca compliance. This result is consistent with a recent meta-analysis (Rodriguez et al, 2016). Vitamin D supplementation during weight loss did not enhance the decrease of inflammatory markers. Interestingly, the greater abdominal fat mass loss was obtained in oa who had the greater 25(oh)D increase.

Reference:

1 Rodríguez et al. Effect of vitamin D supplementation on measures of arterial stiffness: a systematic review and meta-analysis of randomized controlled trials. Clin Endocrinol. 2016; 84(5):645-57. 


\section{Conflict of Interest: None}

Funding: Research relating to this abstract was funded by Nimes University Hospital (France).

\section{T4P31}

\section{Comorbidities in childhood obesity}

Mihaela, T. ${ }^{1}$; Georgiana, R. ${ }^{1}$; Mihaela, M. ${ }^{1}$; Elena, . $^{1}$

${ }^{1}$ University of Medicine and Pharmacy Grigore T. Popa lasi

Introduction: Obesity in children has a strong impact on all system and organs, being associated with metabolic and cardiovascular complications, which significantly increase mortality and morbidity rate. The aim of our study was to evaluate the presence of comorbidities in obese children.

Methods: The study group consist in 78 overweight and obese children, hospitalized in a Pediatric Clinic from Saint Mary Hospital Iasi, 42 girls and 37 boys, aged $2-18$ years, with a mean age of $11.9 \pm 3.5 \mathrm{DS}$ in girls and $9.5 \pm 3.3 \mathrm{SD}$ in boys. Based on the weight and height measurement, body mass index (bmi) was calculated and converted to standard deviation scores (sds) using the cdc reference data. Overweight was defined as a bmi within the $85^{\text {th }}$ to $95^{\text {th }}$ percentile. Obesity was defined as a bmi over the $90^{\text {th }}$ percentile. Hypertriglyceridemia was defined as $\operatorname{tg}>95^{\text {th }}$ percentile for age and sex. Cardiovascular risk assessment was defined by serum cholestero value in normal, borderline, medium and high according to sinupe 2000. Results: From the study group, $58 \%$ of children were obese, $67 \%$ of boys and $50 \%$ of girls. Severe obesity was found in $15 \%$ of the patients. Visceral obesity was found in $9 \%$ of the children. Obesity was associated with hyperglycemia in $25.6 \%$ children. Elevation of triglycerides was found in $28.2 \%$ of the patients and decreased hdl-cholesterol was present in 2 patients. Metabolic syndrome was defined in 3 patients. Medium and high cardiovascular risk was found in $14.1 \%$, respectively $4 \%$ of children. Regression analysis of cholesterol showed a significant correlation with the values of $\operatorname{sbp}(\mathrm{r}=0.72, \mathrm{p}=0.0027,95 \% \mathrm{ci})$. Thus, high levels of cholesterol risk for hypertension $\mathrm{hr}=2.06$ (95\% ci: $1.81-3.48)$.

Conclusion: We found a high risk of metabolic and cardiovascular complications in children. Childhood obesity is a complex disease needing thorough examination in order to early identify comorbidities and individualize the therapeutically approach.

\section{T4P32 \\ Comparing methods of assessing parental perceptions of their obese child's weight status}

Cutler, L. '; Parkinson, K. '; Arnott, B. '; Jones, A. '; Araújo-Soares, V. '; Mann, K. ${ }^{1}$; Tovee, M. ${ }^{2}$; Pearce, M. ${ }^{1}$; Ells, L. ${ }^{3}$; Harris, J. ${ }^{4}$; Adamson, A. ${ }^{1}$

${ }^{1}$ Newcastle University, ${ }^{2}$ University Of Lincoln, ${ }^{3}$ Teesside University, ${ }^{4}$ University Of St Andrews

Introduction: Accurate parental perceptions of their child's weight status is considered an important factor in childhood obesity prevention as underestimation is suggested to be a barrier for behaviour change ${ }^{1}$. Evidence indicates that parents of obese children do not typically describe them as such when assessed using a categorical questionnaire item ${ }^{2}$. Research indicates that parents are more sensitive to visual cues such as skinfolds than body mass index $(\mathrm{bmi})^{3}$ when judging their child's body weight. Therefore, this study explored different methods of assessing parents' perceptions of their obese child's weight status.

Methods: Perceptions of childhood obesity were assessed in parents of 4-5 and 10-11 year old children using two methods a categorical question (How would you describe your child's weight at the moment? underweight; healthy weight; overweight; very overweight); and an age- and sex-specific visual body image scale (bis) of known bmi (Looking at the pictures below which body shape looks most like your child? A-G). The responses to these questions were compared to their child's objectively measured weight status. Child weight status was determined using uk90 criteria $^{4}$ (very overweight perception refers to obese).

Results: From 2,933 families recruited from 15 regions across England, 89 parents had an obese child (53\% male, 65\% 10-11 years) with complete data for this analysis. Of these, using the categorical question, $29 \%$ reported their child as a healthy weight, $64 \%$ as overweight and only $7 \%$ as very overweight. In contrast, using the bis, $16 \%$ selected a healthy weight body image, $25 \%$ an overweight body image, and $60 \%$ a very overweight body image.

Conclusions: Different methods of assessing parental perceptions of their child's weight status yield different results; parents were more accurate at matching their obese child to a very overweight body image compared to reporting a weight status category. This raises important questions about whether these methods are capturing parental recognition of their child's weight status and careful consideration of the methods available should be taken in future research when assessing and interpreting parental perceptions.

\section{Conflict of Interest: None disclosed}

Funding: National Prevention Research Initiative; Gateshead Council; Newcastle City Council; North Tyneside Council; Redcar \& Cleveland Borough Council; Sunderland City Council. L.C is funded by an nihr doctoral fellowship award.

\section{References:}

1 Manios et al (2015) Public Health Nutr

2 Rietmeijer-Mentink et al (2013) Mater Child Nutr.

3 Parkinson et al (2011) Int J Obes.

4 Cole, et al (1995) Arch Dis Child.

\section{T4P34 \\ Comparison of changes in body mass index metrics and bioelectrical impedance analysis with dual X-ray absorptiometry in obesity treatment of children and adolescents}

\section{LøKling, H. ${ }^{1}$; Roelants, M. ${ }^{2}$; Benestad, B. ${ }^{3}$; Hjelmesæth, J.3.; Morken, M. ${ }^{4}$; Júlíusson, $P^{5}$}

${ }^{1}$ Department of Clinical Medicine, University of Bergen, Norway, ${ }^{2}$ Department Of Public Health And Primary Care, Ku Leuven, University Of Leuven, Belgium, ${ }^{3}$ Morbid Obesity Centre, Vestfold Hospital Trust, University Of Oslo, Norway, ${ }^{4}$ Department Of Clinical Medicine, University Of Bergen, Norway And Department Of Internal Medicine, Haukeland University Hospital, Bergen, Norway, ${ }^{5}$ Department Of Paediatrics, Haukeland University Hospital, Bergen, Norway And Department Of Clinical Science, University Of Bergen, Norway

Introduction: The change in bmi standard deviation score (sds) is often used to evaluate treatment effects in children and adolescents with obesity, despite known limitations. The percentage above the bmi cut-off for overweight or obesity has been suggested as a better alternative. In the present study we assessed the association between the changes in several measures of adiposity, using the change in percentage body fat (\%bf), measured by dxa, as the reference method.

Methods: Data were extracted from the medical records at the Obesity Outpatient Clinic at Haukeland University Hospital, Bergen, Norway. Eligible patients had dxa measurements at baseline and follow-up. Height, weight and bioelectrical impedance analysis (bia) were measured within 3 months of the baseline and follow-up dxa measurements. The follow-up period ranged from $12-54$ months. bmi, bmi sds and percentage above the International Obesity Task Force's overweight limit (\%iotf25) were calculated. Monotonicity of the association between the change in \%bf based on dxa and the change in \%bf (bia) and bmi metrics was assessed using Spearman's rank correlation.

Results: Fifty-nine obese children and adolescents ( 31 boys) with a mean age of 11.6 (range 5.7-17.7) years at baseline were eligible for analysis. Mean (sd) bmi sds and \%iotf25 was 2.98 (0.42) and 148.7 (14.8), respectively. The change in \%bf measured by dxa was moderately correlated with the change in absolute bmi $(r=0.51, \mathrm{p}<0.01)$ and \%iotf $25(\mathrm{r}=0.45$, $\mathrm{p}<0.01)$, and weakly correlated with the change in bmi sds $(r=0.26$, 
$p=0.045)$. The changes in $\% b f$ assessed by dxa and bia were also moderately correlated $(\mathrm{r}=0.51, \mathrm{p}<0.01)$.

Conclusion: The data suggest that \%iotf 25 might be preferred over bmi sds to monitor children and adolescents with (severe) obesity. Although absolute bmi was even better correlated with \%bf measured by dxa, the bmi changes naturally with age and would therefore not be suitable to track children over longer periods of time.

Conflict of Interest: None Disclosed

Funding: No Funding

\section{T4P35}

\section{Concomitant gastrointestinal tract pathology in overweight and obese children}

\section{Gurova, M. ${ }^{1}$; Guseva, A. ${ }^{2}$}

${ }^{1}$ Belgorod National Research University, ${ }^{2}$ Kursk Regional Children Hospital

Introduction: Complex of metabolic changes in case of obesity accompanied with hyperinsulinemia and development of insulin resistance leads to the pathological shifts in homeostatic parameters with the following affections of all organs and systems. Meanwhile the spectrum of gastro-intestinal tract (git) pathology in overweight children is not well studied.

Methods: For this purpose we have examined 60 obese children - the $1^{\text {st }}$ group (average age $12.8 \pm 0.84$ years old, body mass index (bmi) $27.5 \pm 1.24$ ) and 65 overweight children - the $2^{\text {nd }}$ group (mean age $13.02 \pm 1.16$ years old, bmi $23.6 \pm 1.09$ ). Criteria of inclusion were primary overweight and obese patients. Insulin resistance (ir) was estimated by calculation homa-ir index. Ultrasonographic and upper git endoscopic investigations were performed.

Results: Abdominal pain with predominant localization in the epigastrium and right hypochondrium was revealed as a leading complaint in patients of both groups - $88.3 \%$ vs $78.3(\mathrm{p}=0.15)$. Such dyspeptic complaint as heartburn was more frequently detected in obese patients $-75 \%$ vs $51.7 \%(\mathrm{p}=0.008)$. Presence of other dyspeptic complaints including meteorism, bloating, and constipation was comparable in both groups. Beside that $48 / 80 \%$ children of the $1^{\text {st }}$ group and $53 / 81.6 \%$ of the $2^{\text {nd }}$ group had some asthenic complaints such as weakness, irritability, headaches and emotional instability.

Chronic gastritis was confirmed by endoscopy in all the investigated children of both groups with non-significant prevalence of $H$. pylori incidences in obese children $(48 / 80 \%$ vs $43 / 66.15 \%, p=0.328)$.

The spectrum and frequency of gastrointestinal associated pathology were comparable in obese and overweight children with the exception of higher rate of steatohepatosis and gastro-esophageal reflux disease in obese children: biliary sludge $-38 / 63.3 \%$ in the $1^{\text {st }}$ group vs $31 / 47.7 \%$ in the $2^{\text {nd }}$ group $(\mathrm{p}=0.072)$, dysfunction of sphincter Oddy $-44 / 73.3 \%$ in the $1^{\text {st }}$ group vs $31 / 47.6 \%$ in the $2^{\text {nd }}$ group $(\mathrm{p}=0.056)$, steatosis of pancreas $51 / 85 \%$ vs $42 / 70 \%(\mathrm{p}=0.062)$, steatohepatosis $-40 / 66.6 \%$ vs $11 / 16.9 \%$ $(\mathrm{p}=0.0026)$ and gastro-esophageal reflux disease $-14 / 23.3 \%$ vs $3 / 5 \%$ $(p=0.018)$. Were found the strong positive correlation between insulin resistance and steatohepatosis $(r=0.82, \mathrm{p}=0.0013)$.

Conclusion: The character of complaints and spectrum of gastrointestinal tract associated pathology in obese and overweight children were identical with the exception of higher rate of steatohepatosis and gastro-esophageal reflux disease in obese children. The strong positive correlation between insulin resistance index and liver affection were revealed.

Conflict of Interest: None Disclosed

Funding: No Funding
T4P36

Cut off values of neck circumference to predict overweight and obesity according to pubertal stage in Brazilians adolescents

Santos, I. ${ }^{1}$; Passos, M. ${ }^{2}$; Sampaio, I. ${ }^{2}$; Fisberg, M. ${ }^{2}$; Ganen, A. ${ }^{\prime}$

${ }^{1}$ Centro Universitário São Camilo, ${ }^{2}$ Universidade Federal De São Paulo

Introduction: The development degree in adolescents should not be determined just by the chronological age, once it is influenced by other environmental and intrinsic factors. The associating between pubertal staging with anthropometric data, such as neck circumference (nc) is considered essential for screening adolescents at risk of morbidities, especially cardiovascular. These measures have proved to be a useful tool for identifying overweight and obesity, as well as its association with chronic noncommunicable diseases.

Methods: A total of 557 adolescents aged 10 to 15 years were recruited from public schools, who were classified according to pubertal stage, by Tanner criteria. The interest variables, nc and the independent variables, such asgender, age, weight were evaluated. The predictive power and cutoff points of nc for overweight/obesity prevention were identified using roc curves.

Results: According to the nutritional status, the adolescents were 58\% eutrophic, $25.2 \%$ overweight and $13.9 \%$ obese, there was no difference between genders. It was observed a strong correlation between $\mathrm{nc}$ and weight and bmi, in both genders. The nc cutoff points showed high sensitivity and specificity in girls and boys prepubescent, however, in post pubertal boys, it was verified a high sensitivity $(1,00)$ and lower especifity $(0,40)$.

Conclusion: The creation of nc cutoff points according to the pubertal stage, specific for the Brazilian adolescent population, proved to be an excellent, non-invasive and low cost tool for identifying overweight/obesity, and it is extremely relevant, due to changes in body composition resulting from puberty events.

Conflict of Interest: None Disclosed

Funding: No Funding

\section{T4P37}

\section{Cytokine status of children with morbid obesity}

Valeria, N. ${ }^{1}$; Ekaterina, A. ' Irina, L. ${ }^{1}$; Marina, K. ${ }^{2}$; Ludmila, V. ${ }^{2}$; Viktoria, K. ${ }^{3}$; Natalia, $K^{4}$

${ }^{1}$ Federal Medical Research Center Almazov, ${ }^{2}$ Saint Petersburg Pediatric State Medical University, ${ }^{3}$ Children'S Regional Hospital, ${ }^{4}$ The Federal State Budgetary Institute «The Nikiforov Russian Center Of Emergency And Radiation Medicine»

Objectives: To estimate the cytokine levels in the blood of children with morbid obesity.

Methods: We examined 10 children aged 12 to 18 years suffering from morbid obesity (body mass index $>35$ ) and 20 children with obesity with body mass index from 24-to 34). Examination methods included clinical examination by pediatrician, endocrinologist, study of alt, ast, total protein, amylase, lipid profile, blood glucose, serum insulin, homa-index definition. Levels of il-10, il -6, il-8, il-1ß, ifn- $\gamma$, tnf- $\alpha$ in blood serum was detected by the means of elisa.

Results: Cytokine status of morbid obese children demonstrated significant differences in il $-6(36,69 \pm 4,8 \mathrm{pg} / \mathrm{ml}$ and $17,52 \pm 2,4 \mathrm{pg} / \mathrm{ml}$, $\mathrm{p}<0,05)$. Levels of il-10, il-8, il- $1 \beta$, ifn- $\gamma$, tnf- $\alpha$ in serum in both groups were identical. There were a significant positive correlations between the body mass index and the level of il $-6(\mathrm{r}=0,39, \mathrm{p}<0,05)$, between homa index and the level of il $-6(\mathrm{r}=0,42, \mathrm{p}<0,05)$ and between serum triglycerides level and the level of il $-6(\mathrm{r}=0,42, \mathrm{p}<0,05)$.

Conclusion: Cytokine status of children with morbid obesity was characterized by increased levels of pro-inflammatory cytokine il -6 compared with obese patients with body mass index from 24-to 34. Cytokine il -6 level connected with methabolic changes in obese patients. 
T4P38

\section{Determining adherence of children and adolescents in Santa Maria de Feira (Portugal) and Kırıkkale (Turkey) to Mediterranean diet}

ÇakıR, B. ${ }^{1}$; KıLıNç, F. ${ }^{1}$; Hiçyılmaz, M. ${ }^{2}$; Santos, A. ${ }^{3}$

${ }^{1}$ Kırıkkale University Faculty of Health Sciences, Turkey, ${ }^{2} \mathrm{~K}$ RIIKkale National Education Directorate, Turkey, ${ }^{3}$ Municipality Of Santa Maria De Feira, Portugal

Introduction: This study was carried out in Portugal (Santa Maria de Feira District), both in the Mediterranean country, and in Turkey (Kırıkkale District) in order to determine the adherence of dietary habits of children and adolescents to the Mediterranean diet (md), which is considered as a healthy diet all over the world.

Methods: Within the scope of llp Program Comenius Regio Partnership project, entitled as Health4Life, 536 children aged 6-15 years (243 children, 6-10 years and 293 children,11-15 years) in Kirıkkale and 321 children and adolescents aged 7-18 years in Santa Maria de Feira were evaluated. The project has been carried out in the coordination of Kirıkkale National Education Directorate in Turkey, in cooperation with Kirıkkale University Health Sciences Faculty and in the partnership of Santa Maria de Feira Municipality in Portugal. The Mediterranean Diet Quality Index (kidmed) was used to determine adherence to the md. The index consists of 16 questions, 4 negative ( -1 point), 12 ( +1 point) positive and $<3$ points="' shows="' low="' $4-7=$ ="' medium ="' and="'> $>8$ points "opti mal" diet quality.

Results: It was found out that $0.9 \%$ of Portuguese children had low, 33\% of them had medium and $66 \%$ of them had optimal diet quality. However, it was found out that $5.8 \%$ of the children aged $6-10$ years had low $42.82 \%$ of them had medium and $51.4 \%$ of them had optimal diet quality, whereas $13 \%$ of the $11-15$ year olds had low, $58.4 \%$ of them had medium and $28.7 \%$ of them had the optimal diet quality in Turkish children. In 6-10 years old Turkish children, in terms of adherence to md, the differences between genders were found to be statistically significant $(\mathrm{p}<0.05)$ whereas the differences between ages were found to be statistically insignificant $(p>0,05)$. The differences which children the ages of 11-15 had about adherence to the md were found to be statistically insignificant according to gender and age ( $\mathrm{p}>0,05)$. In Portuguese children, skips breakfast situation were found to be at $7.5 \%$, and $9 \%$ consumed commercially baked goods or pastries for breakfast.In Turkish children-6-10 years and 11-15 years skipping breakfast situation respectively were found to be at $11.9 \%$ and $17.4 \%$, and $42 \%$ of all ages consumed commercially baked goods or pastries for breakfast.It was found that consumption of vegetable and fruit were respectively $43.0 \%, 41.1 \%$ in Portuguese children, those were $49.4 \%, 73.7 \%$ in Turkish children-6-10 years and $29.4 \%, 68.3 \%$ in Turkish children-11-15 years. While consumption of nuts, sweets and candy for Portuguese children 19.9\% and 11.2\%, in Turkish children-6-10 years were $72.0 \%$ and $62.6 \%$ and $11-15$ years were $60.1 \%$ and $75.1 \%$.

Conclusion: This study shows that Portuguese children are better adherence to Mediterranean diet than Turkish children.

\section{T4P39}

\section{Dietary and meal pattern and association to body size in adolescents}

Viljakainen, J. ${ }^{1}$; Figueiredo, R. ${ }^{2}$; Simola-Ström, S. ${ }^{2}$; Roos, E. '; Weiderpass, E. ${ }^{3}$; Rounge, $T^{4}$

${ }^{1}$ Folkhälsan Research Center, Finland; University of Helsinki, Finland, ${ }^{2}$ Folkhälsan Research Center, Finland, ${ }^{3}$ Folkhälsan Research Center, Finland; Cancer Registry Of Norway; University Of Troms $\varnothing$ - The Artic University Of Norway, Karolinska Institutet, Sweden, ${ }^{4}$ Folkhälsan Research Center, Finland; Cancer Registry Of Norway

Introduction: Overweight and obesity in adolescents is a major health problem and about $23.8 \%$ of boys and $22.6 \%$ of girls were overweight or obese globally in 2013. The prevalence of overweight and obesity has increased in Finnish adolescents during the last decades and data from one of the largest prospective cohort study, the Finnish Health in Teens Study
(Fin-hit, www.finhit.fi $\mathrm{N} \approx 11523$ ), show that about $16 \%$ of 9-12 -year-old Finnish adolescents are overweight and about 3\% are obese. Childhood overweight and obesity increase risk of cardiovascular disease, type 2 diabetes and mortality. Different dietary and meal patterns, such as high intake of fats and sugar, snacking and skipping breakfast have been associated with overweight and obesity in adolescents. The aim of our study is to investigate the association between dietary and meal patterns and body size in Finnish adolescents.

Methods: The adolescents, aged 9-12 year old at baseline, answered a web questionnaire including questions on lifestyle related health behavior on diet. Diet questions consisted of 7 different alternatives on dietary intake and meal questions consist of 6 different alternatives on meal consumption. In addition, adolescents had their height, weight and waist circumference measured at school. bmi was calculated and the adolescents were classified as underweight, normal weight, overweight or obese according to age and gender specific Cole's cut-offs. Unsupervised two-step clustering identifying the number of different dietary and meal clusters.

Results: Preliminary results shows that adolescents can be divided into three different dietary clusters: healthy, less healthy and unhealthy. Associations between dietary and meal clusters and bmi groups will be assessed by Chi-square test and Loglinear models. Adjusted variables are sex and age. Conclusion: We will present dietary and meal pattern clusters that potentially may be associated with bmi in Finnish adolescents and discuss how the results may guide recommendations in nutrition and weight development to prevent childhood overweight and obesity.

\section{Conflict of Interest: None}

Funding: Research relating to this abstract was funded by Samfundet Folkhälsan, the Academy of Finland, Medicinska Understödsföreningen Liv och Hälsa rf, the Swedish Cultural Foundation, Signe and Ane Gyllenberg Foundation and the Yrjö Jahnsson Foundation.

\section{T4P40 \\ Dietary and physical activity characteristics of overweight Greek children}

Papadopoulou, S. ${ }^{1}$; Methenitis, S. ${ }^{2}$; Kokkinopoulou, A. ' Pagkalos, I. ${ }^{3}$; Hassapidou, M. ${ }^{1}$

${ }^{1}$ Department of Nutrition and Dietetics, Alexander Technological Educational Institute of Thessaloniki, Thessaloniki, Greece, ${ }^{2}$ Department Of Physical Education And Sport Science, National And Kapodistrian University Of Athens, Athens, Greece, ${ }^{3}$ Department Of Electrical And Computer Engineering, Aristotle University Of Thessaloniki, Thessaloniki, Greece

Introduction: Pediatric obesity constitutes a rising epidemic. Dietary and sedentary behaviours leading to obesity are established during childhood. For the prevention of childhood obesity,it is essential to identify the modifiable risk factors. The purpose of the study was to explore the role of dietary intakes and physical activity variables in normal weight and overweight high school students in Thessaloniki, Greece.

Methods: The participants were 1,003 students from Junior High and High Schools (14.62 \pm 1.5 years) were divided according to their age-adjusted bmi into normal weight and overweight groups. Participants were chosen using stratified sampling. Dietary intake, food choices and physical activity variables were evaluated using specific questionnaires. Statistical analyses (descriptive statistics, t-test and Pearson's correlation coefficient) were performed with spss Statistics v.20 ( $p<0.05)$.

Results: Significant positive correlations were found between bmi and sports energy drinks consumption ( $\mathrm{r}$ : 0.223-0.991, $\mathrm{p}=0.000$ ). However, significant but negative correlations were found between bmi and daily energy expenditure per $\mathrm{kg}$ of body weight $(\mathrm{r}:-0.253--0.548, \mathrm{p}=0.000)$. Conclusion: Overweight children had lower energy expenditure per kg of body weight, while having higher total amount of daily intake of protein fat and carbohydrates when comparing to the normal weight children. Furthermore overweight children consumed more sports energy drinks.

Conflict of interest: None disclosed

Funding: No Funding 
T4P41

\section{Dietary patterns in toddlers with excess weight. The 2016 Pitnuts study.}

Weker, H. ${ }^{1}$; Baranska, M. '; Riahi, A. ' 'Strucinska, M. '; Wiech, M. '; Bzikowska, A. ' Rowicka, G. '; Dylag, H. ${ }^{1}$; Klemarczyk, W. ${ }^{1}$; Socha, P. ${ }^{2}$

${ }^{1}$ Institute of Mother and Child, ${ }^{2}$ The Children'S Memorial Health Institute

Introduction: Proper nutrition ensures the optimal physical and mental child's development, prevents childhood obesity and reduces the risk of noncommunicable diseases in adulthood. We aimed to assess the dietary patterns in children aged 1-3 years with excess weight to compare them with models of safe nutrition.

Methods: A national cross-sectional study was conducted in Poland from May to July 2016. Parent-child pairs were selected by a systematic, nationwide, random sampling technique. Analysis of dietary patterns was undertaken on a subsample of 173 toddlers with excess weight (bmi $\mathrm{z}$-score $>1 \mathrm{sd}$ ). Diets of children were evaluated on a basis of 3-day dietary record which provided data to compute children's daily food rations by means of a nutritional software Dieta 5.0. Information on feeding practices were collected in a questionnaire survey. To determine dietary patterns of toddlers with excess weight we performed cluster analysis ( $\mathrm{k}$-means method) selecting variables describing mean daily amounts of foods consumed by children. We examined differences in feeding practices in identified clusters of children $\left(\mathrm{Chi}^{2}\right.$ test and Kruskal-Wallis Anova rank test, p-value set at 0.05).

Results: We identified three clusters of excess weight toddlers aged 1 to 3 years with different dietary patterns. First cluster (Baby Food Diet) included 58 children in the second year of life (median age 20 months) whose diet was based on junior formula and baby food. Remaining two clusters consisted of children in the third year of life (median age approx. 26 months). In the second cluster we found 33 children on Milk and Cereals Diet characterised by large consumption of cow's milk, milk products and cereals. The third cluster comprised of 82 children on Sandwich and Sugar Diet whose dietary pattern was predominated by consumption of bread, cured meats, fats, as well as products being sources of sugars (sweets, juices and sweet beverages, fruits). In daily food rations of these children we observed decreased amounts of milk, partially replaced with sweet dairy desserts and yoghurts. Our results also confirm incorrect feeding practices in all studied toddlers with excess weight, including snacking between meals, eating before bedtime and drinking sweet beverages during night. Conclusions: Dietary patterns of toddlers with excess weight do not meet the recommendations of safe nutrition model. More educational efforts are required to increase the parents' awareness and the adherence to current guidelines.

Conflict of Interest: None Disclosed

Funding: The study conducted under a project of Nutricia Foundation No. $161 / 2016$.

\section{T4P42}

\section{Eating foods surplus to requirements: Where, with whom and why are adolescents eating non-core foods?}

\section{Toumpakari, Z. ${ }^{1}$; Tilling, K. ${ }^{2}$; Haase, A. ' Johnson, L. ${ }^{1}$}

${ }^{1}$ School for Policy Studies, University of Bristol, ${ }^{2}$ School For Social And Community Medicine, University Of Bristol

Introduction: Non-core food refers to all foods surplus to nutritional requirements e.g. soft drinks, chips, confectionery, while a diet high in non-core foods during adolescence is associated with increased adiposity and Body Mass Index (bmi). Understanding the role of the physical and social environment relative to individual factors in adolescents' consumption of non-core foods is crucial for developing effective interventions that reduce consumption and subsequently reduce adolescents' obesity levels. Methods: Mixed methods were used first drawing on quantitative multilevel data from the National Diet and Nutrition Survey 2008-11/12. Three-day food diaries provided information on the environmental con- text of each eating occasion (eo) including 'where' (home, school, eateries, etc.) and 'with whom' (parents, friends, etc.) adolescents $(n=884)$ consumed non-core foods. Linear multilevel models investigated associations between eating context and non-core food energy intake (kcal) per eo, adjusting for variables at the eo and adolescent level. Qualitative methods were then employed to understand why adolescents consume non-core foods in certain physical and social environments, as well as factors they attend to in each of these eating contexts. Eighteen face-to-face interviews were conducted with 14-16 year old adolescents from the wider Bristol area. Thematic analysis was applied to interview data, organised using nvivo.

Results: From both methods, 'Eateries', i.e. restaurants, fast food outlets, cafes, etc. emerged as an important environment in which adolescents' consumed more non-core food. In adjusted models, non-core food intake more than doubled in eos in 'Eateries' compared with 'Home' (2.51 95\% ci $2.14,2.95)$. Adolescents preferred eating at 'Eateries' because of perceived exciting food options compared to home and increased socialising. Their food choices were mostly influenced by norms of (un)healthy eating and social aspects, whereas in the case of friends a more functional way of eating became important.

Conclusion: Eateries represent a high-risk environment where adolescents eat more non-core food because they enjoy the food and socialising. Interventions could target reformulation and reduction of portion sizes in eateries to reduce consumption of non-core foods and therefore reduce adolescents' odds for becoming obese.

Conflict of Interest: None Disclosed

Funding: No Funding

\section{T4P43}

Effect of a 10-month multidisciplinary weight loss program on quality of life and health perception in adolescents with obesity

\author{
Julie, M. ${ }^{1}$; Maud, M. ${ }^{2}$; Daniel, C. ${ }^{3}$; Yves, B. ${ }^{4}$; Martine, D. ${ }^{4}$; David, . $^{5}$
}

${ }^{1} \mathrm{SSR}$ ugecam Nutrition Obesité, Clermont-Ferrand, France, ${ }^{2}$ Clermont Auvergne University, France, ${ }^{3}$ Clermont-Auvergne University, France, ${ }^{4} \mathrm{Chu}$ ClermontFerrand, Clermont-Ferrand, France, ${ }^{5} \mathrm{Clermont}$ Auvergne University, France

Introduction: Obese adolescents usually report lower Quality of Life compared with their lean counterparts. While there are today many evidences regarding the beneficial effects of multidisciplinary interventions inducing weight loss on body composition, metabolic profile or physical fitness, the impact of such interventions on obese adolescents' quality of life remains less explored. The aim of this work was to assess the effect of a 10-month multidisciplinary intervention on the quality of life and health perception in adolescents with obesity.

Methods: Thirty-six 12-15 years old obese adolescents (iotf cut-off points; Tanner stage 3-4) completed a 10-month multidisciplinary intervention combining nutritional counselling, physical activity (Three times a week, moderate intensity exercises) and health-related therapeutic education. At baseline (T0), after 5 months (T1) and by the end (T2) of the program, anthropometric parameters and body composition ( $\mathrm{dxa}$ ) were assessed and the adolescents' quality of life (sf-36) and Health Perception (Health Perception Scale) were evaluated.

Results: Body weight, Body Mass Index and Fat Mass percentage were significantly reduced at T1 and T2 $(\mathrm{p}<0.001)$ while Fat-Free Mass remain stable. The Physical Functioning, Role Physical and General Health items of the sf-36 increased significantly with the intervention. While the Mental dimension of the sf-36 questionnaire remained unchanged, the Physical Dimension improved significantly $(\mathrm{p}<0.01)$. The Physical Fitness $(p<0.001)$, Health-Balanced Diet $(p<0.01)$ and General Health $(\mathrm{p}<0.05)$ items of the Health perception questionnaire improved significantly while the Ideal Weight one decreased $(\mathrm{p}<0.01)$. The General Health Perception Score was significantly improved at both T1 and T2 $(\mathrm{p}<0.05)$. There was no relationship between the Quality of Life and 
Health Perception modifications and the observed weight, bmi or FatMass improvements.

Conclusion: A 10-month multidisciplinary program favours improves physical quality of life and health perception in adolescents with obesity, independently of the induced weight-loss and body composition improvements.

\section{Conflict of Interest: None Disclosed}

Funding: Research relating to this abstract was funded by Auvergne-Rhône-Alpes County and the French National Academy of Medicine.

\section{T4P44}

\section{Effectiveness of the MOVI-KIDS physical activity intervention improving children's academic achievement}

\section{Álvarez-Bueno, C. ' ; Cavero-Redondo, I. '; Garrido-Miguel, M. '; Notario-} Pacheco, B. '; Martínez-Andrés, M. '; Sánchez-López, M. ${ }^{2}$

${ }^{1}$ Health and Social Research Center. Universidad de Castilla-La Mancha, Cuenca. Spain, ${ }^{2}$ Health And Social Research Center. Universidad De Castilla-La Mancha, Ciudad Real. Spain

Introduction: The aim of this study was to analyse the effectiveness of a physical activity (pa) intervention on academic achievement of children's aged from 4 to 7 years old, by weight status.

Methods: This randomized cluster trial involved 4-to-7 years old children from 21 schools in Castilla-La Mancha region (Spain).

The pa program was aimed at increasing weekly pa time through three weekly $60 \mathrm{~min}$ sessions,at school facilities, including: sport and playground games, dance and activities on motor skills development.

Variables that were measured:

Weight and height, as mean of two measurements.

Height, using a wall stadiometer (Seca ${ }^{\circledR} 222$ ), with the child barefoot, upright and the sagittal midline touching the backboard.

$b m i$, as weight $(\mathrm{kg}) /$ height $(\mathrm{m})^{2}$, and categorized as normal weight and as overweight/obese, according to iotf criteria.

Academic achievement measured by the Battery of General and Differential Aptitudes scales for children (badyG),tincluding: logical reasoning; verbal, numerical and spatial factors, and general intelligence.

ancova models were used to analyse the pre-post intervention changes in academic achievement dimensions, by children's weight status. Two models were estimated: a) model 1: unadjusted, and b) model 2: adjusted by age, gender and socioeconomic level.

Results:Data are reported as mean \pm sd. es: Effect size

Conclusions: pa interventions are effective for significantly improving academic achievement in both, normal weight and overweight/obese children aged from 4 to 7 years old overall, greater effect has been observed in overweight/obese children's general intelligence.

Conflict of interest: None disclosed.
Funding:This study was funded by the Ministry of Economy and Competitiveness-Carlos iii Health Institute (fis pi12/02400)

\section{T4P45}

\section{Effects of between generations changes in nutrition type on adipokine levels in female Sprague Dawley rat}

\section{Grgić, A. ${ }^{1}$; Kačarević, Ľ. ${ }^{\text {; }}$ Selthofer, R. $^{1}$; Čekić, N. ${ }^{1}$; Radić, $R .{ }^{1}$ \\ ${ }^{1}$ Faculty of Medicine Osijek, University of Osijek}

Introduction: Previous studies established that between generations changes in feeding protocol can have significant impact on physiology of adipose tissue. The aim of the study was to determinate effects of mothers nutrition and nutrition of the offspring on circulation adipokine levels in all groups of rats.

Methods: Ten female rats were randomly divided in two groups, first group fed with food containing high content of saturated fatty acids (hfd) and the second with standard laboratory chow (cd). After coupling and lactation period their offspring were further randomly divided into two subgroups fed hfd or cd forming four study groups: a) cd-cd, b) cd-hfd, c) hfd-cd and d) hfd-hfd. The dams and offspring at the age of 37 and 18 weeks, respectively, were subjected to biochemical analysis of the blood adiponectin, il- 6 and tnf- $\alpha$ levels. Additionally body weight was recorded and bmi calculated.

Results: There was no significantdifference in body weight and bmi between groups. Higher il-6 concentration was measured in hfd group of dams $(P=0.009)$, as well as lower adiponectin concentration $(P=0.009)$. Significantly higher concentrations of il- 6 and tnf- $\alpha$ were observed in hfdhfd and hfd-cd groups ( $P=0.004$ and $P=0.004$, respectively), as well as low adiponectin level $(P=0.004)$.

Conclusion: High fat diet consuption is not necessary correlated with high body weight and bmi. Nevertheless, maternal hfd consumption predisposes offspring to increased risk of disturbances in adipokine levels resulting in metabolic abnormalities.

\section{T4P46 \\ Effects of early infant feeding interventions on parental feeding practices: A systematic review}

\section{Matvienko-Sikar, K. ' ; Toomey, E. '; Delaney, L. ; Harrington, J. '; Byrne, M. '; Kearney, $P$. \\ ${ }^{1}$ University College Cork, ${ }^{2}$ National University Of Ireland Galway}

Introduction: Interventions targeting childhood obesity increasingly focus on early infant feeding practices, but a comprehensive examination of intervention effects is lacking. Systematically evaluating behavioural and dietary outcomes is crucial to interrogate mechanisms of change in obesity-focused infant feeding interventions. The aim of this study is to

Table 1. to T4P44

\begin{tabular}{|c|c|c|c|c|c|c|c|c|c|c|c|c|c|c|}
\hline & \multicolumn{6}{|l|}{ Normal weight } & & \multicolumn{6}{|c|}{ Overweight/Obesity } & \\
\hline & & & Model 1 & & Model 2 & & & & & Model 1 & & Model 2 & & \\
\hline $\begin{array}{l}\text { BADIG } \\
\text { Variables }\end{array}$ & $\begin{array}{l}\text { MOVIKIDS } \\
\text { Intervention } \\
\text { Group } \\
n=62\end{array}$ & $\begin{array}{l}\text { Control } \\
\text { Group } \\
n=133\end{array}$ & $\begin{array}{l}\Delta \text { Mean } \\
\text { Differen- } \\
\text { ce }\end{array}$ & $p$ & $\begin{array}{l}\Delta \text { Mean } \\
\text { Diffe- } \\
\text { rence }\end{array}$ & $\mathrm{p}$ & ES & $\begin{array}{l}\text { MOVIKIDS } \\
\text { Intervention } \\
\text { Group } \\
n=24\end{array}$ & $\begin{array}{l}\text { Control } \\
\text { Group } \\
n=39\end{array}$ & $\begin{array}{l}\Delta \text { Mean } \\
\text { Difference }\end{array}$ & $p$ & $\begin{array}{l}\Delta \text { Mean } \\
\text { Difference }\end{array}$ & $\mathrm{p}$ & ES \\
\hline $\begin{array}{l}\text { Logical } \\
\text { reasoning }\end{array}$ & $10.74 \pm 8.19$ & $9.10 \pm 7.12$ & 1.72 & 0.058 & 2.25 & 0.155 & 0.22 & $12.26 \pm 8.92$ & $7.25 \pm 7.23$ & 5.28 & 0.006 & 5.88 & 0.021 & 0.63 \\
\hline $\begin{array}{l}\text { Verbal } \\
\text { Factor }\end{array}$ & $5.74 \pm 4.30$ & $0.95 \pm 6.29$ & 4.79 & $<0.001$ & 4.39 & $<0.001$ & 0.83 & $5.29 \pm 4.96$ & $0.56 \pm 6.62$ & 4.73 & 0.004 & 5.19 & 0.004 & 0.78 \\
\hline $\begin{array}{l}\text { Numerical } \\
\text { Factor }\end{array}$ & $12.11 \pm 9.16$ & $8.10 \pm 7.08$ & 4.01 & 0.001 & 3.60 & 0.004 & 0.51 & $12.17 \pm 8.47$ & $4.46 \pm 9.00$ & 7.70 & 0.001 & 7.32 & 0.006 & 0.87 \\
\hline $\begin{array}{l}\text { Spatial } \\
\text { Factor }\end{array}$ & $6.29 \pm 7.56$ & $3.00 \pm 7.18$ & 3.29 & 0.004 & 3.81 & 0.001 & 0.45 & $6.58 \pm 6.84$ & $1.87 \pm 6.43$ & 4.71 & 0.008 & 4.24 & 0.018 & 0.71 \\
\hline $\begin{array}{l}\text { General } \\
\text { Intelligence }\end{array}$ & $24.14 \pm 15.91$ & $12.05 \pm 13.98$ & 12.09 & $<0.001$ & 11.81 & $<0.001$ & 0.83 & $24.04 \pm 14.77$ & $6.90 \pm 13.54$ & 17.14 & $<0.001$ & 16.76 & $<0.001$ & 1.22 \\
\hline
\end{tabular}


conduct a systematic review of the effects of infant feeding interventions delivered in a healthcare context for children under 2 years, on feeding behaviours, dietary intake, and weight outcomes.

Methods: The search strategy consisted of grey literature and the following databases between March and August 2016: cinahl, the Cochrane Library, embase, Medline, PubMed, PsycINFO, and Maternity and Infant Care. Eligibility criteria included: full-term infants $\leq 2$ years; interventions promoting healthy feeding practices to prevent overweight and obesity, delivered in a healthcare context; inclusion of an active or norma care comparator; examination of at least one feeding outcome. Quality assessment was conducted using the Cochrane Effective Practice and Organization of Care risk of bias criteria.

Results: Fifteen papers, representing 9 trials, met inclusion criteria. Infant feeding interventions demonstrate inconsistent effects on feeding practices, dietary intake, and weight outcomes. There is tentative evidence of improvements in parental responses to food refusal, encouragement, and food provision between meals. Interventions incorporating a responsive feeding focus demonstrate greater improvements in outcomes. Methodological issues, including outcome measurement variability, were observed

Conclusion: This is the first systematic review of infant feeding intervention effects on feeding behaviours, dietary intake, and weight outcomes. Inconsistent effects and methodological limitations are apparent across trials. Robust measurement and evaluation of behavioural feeding outcomes is essential to identify mechanisms of change for reducing childhood obesity in infant feeding interventions.

Conflict of Interest: No conflict of interest

Funding: Research relating to this abstract was funded by a Health Research Board Interdisciplinary Capacity Enhancement Award

\section{T4P47}

Effects of maternal exposure to different types of fatty acids during pregnancy and lactation on the adiposity and inflammatory responses in the $21-d-o l d ~ p u p s$

\section{Mennitti, L. ; Oyama, L. ${ }^{2}$; Santamarina, A. ${ }^{1}$; Nascimento, C. ${ }^{2}$; Pisani, L. ${ }^{3}$}

${ }^{1}$ Programa de Pós-graduação Interdisciplinar em Ciências da Saúde, Universidade Federal de São Paulo (unifesp), Santos, sp, Brazil, ${ }^{2}$ Departamento De Fisiologia, Disciplina De Fisiologia Da Nutrição, Universidade Federal De São Paulo (Unifesp), São Paulo, Sp, Brazil, ' ${ }^{3}$ epartamento De Biociências, Instituto De Saúde E Sociedade, Universidade Federal De São Paulo (Unifesp), Santos, Sp, Brazil

Introduction: Fatty acids (fa) composition of maternal diet during pregnancy and/or lactation seems to influence in the foetal programming, epigenetic pattern and healthy phenotype of the offspring. Therefore, we investigated the effects of maternal exposure to normolipidic diets based in different types of fa during pregnancy and lactation on the adiposity and pro-inflammatory responses of 21 -d-old pups.

Methods: On the first day of pregnancy, Wistar female rats were divided into four groups: control (C), saturated fa-SFAs (S), Trans fa-TFAs (T) or n-3 polyunsaturated fa-pufa (pufa). Diets were maintained during pregnancy and lactation. After delivery, male offspring were maintained in the same group as their mother (C21, S21, T21 and pufa21). Pups were weighed and measured weekly. At 21st, pups were euthanized. Retroperitoneal adipose tissue (ret) and liver were collected. Carcass protein content was evaluated. Protein levels were determined by Western Blotting. $\mathrm{p} \leq 0.05$ was considered significant.

Results: Body weight, body weight gain and ret relative weight were higher in the S21 and T21 groups than in the C21 group. In contrast, we observed a decrease of the same parameters in the pufa21 group, without changes in the length and protein carcass content. Moreover, T21 group presented an increase in the liver protein expression of il10R $\alpha$ and phospho-ikka+ $\beta$ compared to $\mathrm{C} 21$ group. In the pufa 21 group there were reductions in phospho-ikka+ $\beta$ and phospho-nfkB p50 liver protein levels relative to the $\mathrm{T} 21$ group.
Conclusion: While maternal intake of sfas and tfas during pregnancy and lactation seems to have a negative impact in pup's adiposity and pro-inflammatory responses, the early exposure to $n-3$ pufas may be considered beneficial for the health status of the 21-d-old offspring.

Conflict of Interest: The authors declare no conflicts of interest.

Funding: $\mathrm{PhD}$ scholarship (process number 2014/10683-0) by Fundação de Amparo à Pesquisa do Estado de São Paulo (fapesp).

\section{T4P48}

Effects on physical activity patterns among preschool children predisposed to overweight and obesity in the Danish Healthy Start intervention

Händel, M. ' ; Larsen, S. ; Rohde, J. '; Stougaard, M. '; Olsen, N. '; Heitmann, $B$.

${ }^{1}$ Research Unit for Dietary Studies, the Parker Institute, Bispebjerg-Frederiksberg Hospital

Introduction: There is limited evidence to support the effectiveness of primary interventions aiming to prevent excess weight gain among young children. Evaluating behavioral changes, such as physical activity (pa), may add to future development of efficient interventions. The objective was to evaluate the effect of the 15 months intervention on pa outcomes in the Healthy Start intervention that focused on changing diet, pa, sleep and stress management among normal weight but obesity-prone preschool children.

Methods: From a baseline study population of 635 normal weight children from the greater Copenhagen area, who had either a birth weight $\geq$ $4,000 \mathrm{~g}$, mothers with a pre-pregnancy body mass index of $\geq 28 \mathrm{~kg} / \mathrm{m}^{2}$, or mothers with an educational level $\leq 10$ years of schooling, parents of 307 children had given information on pa at both the baseline and follow-up examinations. pa was obtained from a 7 days recording in the Children's Physical Activity Questionnaire. Time used for sport activities were combined with outdoor playing time to achieve a proxy of total pa level.

Results: Linear regression analyses revealed that the intervention group spent more time being physically active per week in per protocol (pp), as well as in the intention to treat analyses (pp analyses: intervention: 400 min/week; 95\% ci: 328, 472; control: $325 \mathrm{~min} /$ week; 95\% ci: 281, 369; $\mathrm{p}=0.03$ ), although no significant intervention effects were seen for each of the subcategories making up total pa, e.g. sports participation, outdoor activities, screen time, or commuting frequency.

Conclusion: Our results suggest that the overall time spent being physically active was increased among normal weight obesity-prone children, as a result of the Healthy Start intervention.

Conflicts of interest: None disclosed

Funding: The Healthy Start study was funded by the Tryg Foundation (grant no.: 7984-07, 7106-09, and 7-10-0330), the Danish Medical Research Council (grant no.: 271-07-0281), and the Health Insurance Foundation (grant no.: 2008B101).

Acknowledgements: The authors would like to thanks all the participating children and parents from the Healthy Start project. Moreover, we would like to acknowledge Fanney Thorsteinsdottir, Naghmeh Rahmanfard, Lauren Lissner, Gabriella Eiben, Kenneth Christiansen, Lone Viggers, Ellis Tauber-Lassen, Helle Grønbæk, Åshild Skogerbø, Hanne-Lise Falgreen Eriksen and the Healthy Start project group.

\section{T4P49 \\ Efficacy of a home-based weight management intervention for 2-6 year old children and their parents: Results of a randomized controlled pilot trial}

\section{Martin, C. ${ }^{1}$; Apolzan, J. ${ }^{1}$; Hawkins, K. ${ }^{1}$; Hall, L. ${ }^{1}$; Davis, A. ${ }^{1}$; Chatham, J. ${ }^{2}$}

${ }^{1}$ Pennington Biomedical Research Center, ${ }^{2}$ Georgia State University

Introduction: Approximately $32 \%$ of children in the United States are overweight or obese (Ogden, 2014), and $20 \%$ of children in Britain are overweight by age 5 years (Stuart \& Panico, 2016). Obesity in youth is 
associated with cardiometabolic risk factors that are present by adolescence. Intensive family-based weight management interventions delivered in clinics are efficacious, but not widely available. Alternative strategies to deliver efficacious interventions are needed. This pilot study tested the efficacy of a home-based weight management intervention for children and their parents.

Methods: 16 parent/child dyads $(\mathrm{N}=32)$ were recruited. Children were 2-6 years old, had body mass index (bmi) percentile $>75^{\text {th }}$. Primary caregivers with $\mathrm{bmi}>25 \mathrm{~kg} / \mathrm{m}^{2}$ were also eligible to receive weight loss treatment delivered in the home. Dyads were randomized to 19 weeks of either: 1) a health education control group (C), or 2) a weight management intervention to modify diet and activity via parent training and positive child-parent interactions (weight management or wm group). Analysis of covariance evaluated if change from week 0 to 19 in children's bmiz-score (and parental weight change and secondary endpoints) differed by group (baseline values were covariates).

Results: Children's mean+sd weight and bmiz-score at baseline were $23.2+8.6 \mathrm{~kg}$ and $1.5+1.0$. Compared to $\mathrm{C}$, the wm group experienced reduced bmiz from baseline to week 9 and 19 (Week 19: $0.50+0.13$ vs. $-0.14+0.13$, respectively, $. \mathrm{p}<.01)$. The caregiver's baseline weight and bmi were $89.2+23.3 \mathrm{~kg}$ and $33.5+6.6$. An outlier in the control group was observed $(-23.9 \mathrm{~kg})$. Caregiver weight change $(\mathrm{kg})$ did not differ between groups with the outlier, but did differ significantly at week 19 without the outlier (Control, $2.0+1.6$ vs. wm $-3.5+1.5 \mathrm{~kg}, \mathrm{p}<.05$ ).

Conclusion: A weight management intervention to modify diet and activity via parent training and positive child-parent interactions delivered in the home significantly attenuated children's body mass gain and had positive effects on the body weight of caregivers. This intervention approach warrants further study.

Conflicts of Interest: None

Funding: Regents of Georgia State University and National Institutes of Health grants U54 gm104940 and P30 dk072476

\section{T4P50}

\section{Endothelial function and arterial stiffness in obese adolescents - A relation to baroreflex function}

Czippelová, B. ${ }^{1}$; Turianiková, Z. ' Krohová, J. ' Lazarová, Z. ' ; Javorka, M. ${ }^{1}$ ${ }^{1}$ Comenius University in Bratislava, Jessenius Faculty of Medicine in Martin (jfm $\mathrm{cu})$, Department of Physiology jfm cu and Biomedical Center Martin jfm cu, Martin, slovakia

Introduction:The atherosclerotic process starts early in the childhood and is accompanied by structural and functional changes of the vessel wall with a potential to impair the baroreflex function. Obesity is regarded as a risk factor contributing to the development of atherosclerosis. The aim of our study was to assess the early atherosclerotic changes and their relation to baroreflex function in obese adolescents.

Methods: Two non-invasive methods designated for the quantification of early atherosclerotic changes were employed: rh-pat method (Reactive hyperemia peripheral arterial tonometry - rhi index) - a method for examination of endothelial function and a method for the arterial stiffness measurement - cavi index (Cardio-ankle vascular index). Baroreflex sensitivity (brs) was calculated from the continuous recordings of rr intervals from ecg and finger arterial blood pressure as a causal gain from systolic blood pressure to rr interval oscillations using bivariate autoregressive model. Study groups involved 22 obese (10f, age: $15.28 \pm 2.8 \mathrm{y}$, bmi: $30.78 \pm 2.3 \mathrm{~kg} . \mathrm{m}^{-2}$ ) and 22 non-obese (10f, age: $15.98 \pm 2.46 \mathrm{y}$. , bmi: $20.68 \pm 2.1 \mathrm{~kg} \cdot \mathrm{m}^{-2}$ ) matched adolescents.

Results: We found no significant difference in rhi $(\mathrm{p}=0.473)$ suggesting similar endothelial function in both groups. A significant difference in cavi ( $\mathrm{p}=0.001$ ) was found with cavi lower in obese group (caviob $=4.49 \pm 0.84$ vs. cavicont $=5.17 \pm 0.39$ ). We found no difference in brs $(\mathrm{p}=0.573)$ between groups.

Conclusion: In contrast to our expectations of increased arterial stiffness associated with obesity previously observed in adults, we found lower val- ues of cavi in obese adolescents, indicating a lower stiffness in this group. This could lead to increased brs in obese subjects but no difference in brs between groups was found. The mechanisms responsible for these findings require future study.

Conflict of Interest: None Disclosed

Funding: Research supported by grants apvv-0235-12, vega $1 / 0087 / 14$ and itms project "BioMed Martin" no.26220220187

\section{T4P51 \\ Evaluation of the impact of using TV/computer on the physical activity in obese children}

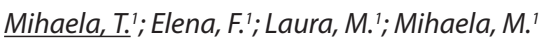 \\ ${ }^{1}$ University of Medicine and Pharmacy Grigore T. Popa lasi
}

Introduction: Early intervention in obese children will improve medium and long-term results in preventing complications and comorbidities of obesity. Physical activity is an important element in the strategic plan for the prevention and treatment of obesity along with changing eating habits. The aim of our study was to assess the physical activity and the impact of computer and tv use on obese children and teen's lifestyle.

Methods: We conducted a prospective study on 38 obese and overweight children, aged 10 to 18 years, hospitalized in ' Saint Mary" Children's Hospital Iasi. Children and their caregivers completed a questionnaire assessing physical activity and the impact of television and computer use on their lifestyles and an informed consent. They were excluded from the study adolescents with comorbidities that limit exercise (hypertension, cardiomyopathy, heart failure) or patients who refused to complete the questionnaire.

Results: The majority of the adolescents preferred to spend their free time watching television or using the computer. Spending time with family, socializing with friends in the expense of exposure to tv/computer is found only in $20.1 \%$ of cases, while playing a sport is preferred by $10.5 \%$ of them. Involving parents in managing the child's leisure time is observed only in $10.5 \%$ of cases. A lifestyle moderate impairment due to the use of $\mathrm{tv} / \mathrm{com}$ puter was found in $42.1 \%$ of adolescents and great impairment of daily activities has been recognized by $7.9 \%$ of respondents. Time spent watching tv was 1-3 hours/day for $78.94 \%$ of adolescents and between 3-5 hours/ day for $21.05 \%$ of them. In front of the computer, $89.5 \%$ of respondents said they spend more than 1 hour. Among the reasons for preferring tv/ computer in their free time $-36.84 \%$ of teens say they relax so and just $21.05 \%$ of them use the computer to obtain information. Spending long time in front of the tv or computer by the adolescents in the study group was manifested by: fatigue $(57.9 \%)$ and dependence $(21.05 \%)$, isolation and boredom ranked equally with $10.5 \%$ each. Frequently perceived positive effects of using tv/computer in adolescents were: relaxation $(47.36 \%)$, information (31.57\%) and communications (10.52\%).

Conclusions: We observe a preference of obese children on spending time on tv or computer in expense of doing physical activity. The correlation of the analyzed parameters showed a major impact of the tv or computer on the sedentary lifestyle of obese adolescents.

\section{T4P52 \\ Excess weight and its relation with raising blood pressure in children}

\section{Pinto, R. ${ }^{1}$; Mello, L. ${ }^{2}$ J Junior, $R^{3}$}

${ }^{1}$ Faculdade de Medicina de Ribeirão Preto usp, ${ }^{2}$ Faculdade De Medicina De Ribeirão Preto-Usp, ${ }^{3}$ Faculdade De Medicina De Ribeirão Preto - Usp

Objetive:To correlate the presence of childhood hypertension with the Body Mass Index (bmi) and the practice of physical activity in schoolchildren.

Methods:Cross-sectional study that assessed 505 schoolchildren through a structured questionnaire and anthropometric data. 
Results:A total of $30.9 \%$ of the students were overweight. When performing linear regression analysis considering the genders, there was positive correlation between the bmi and systolic blood pressure (sbp) variables for both the male $\left(\mathrm{R}^{2}=0.3494, y=60.1099+1.8576 \mathrm{x} ; \mathrm{p}<0.0001\right)$ and female students $\left(\mathrm{R}^{2}=0.3391, y=62.5432+1.7759 \mathrm{x} ; \mathrm{p}<0.001\right)$. The same was observed when the bmi and diastolic blood pressure ( $\mathrm{dbp}$ ) variables were analyzed for the male $\left(\mathrm{R}^{2}=0.2295, y=34.5611+1.1014 \mathrm{x} ; \mathrm{p}<0.001\right)$ and female groups $\left(\mathrm{R}^{2}=0.2179 ; y=36.0179+1.0359 \mathrm{x} ; \mathrm{p}<0.001\right)$. In the population studied less than $35 \%$ reported practicing some form of regular physical activity, 59\% of the students spent 3 hours or more in front of the tv and $74 \%$ used video games daily. Of the 505 students interviewed $71 \%$ did not practice any activity with energy expenditure in their free time.

Conclusions: The association between high blood pressure and excess weight worries health professionals. Knowing that prevention is a longterm process that requires the involvement of multidisciplinary professionals, planning with an implementation strategy is necessary to reduce these indicators in order to minimize future risks of hypertension

\section{T4P53}

\section{Exploring barriers and facilitators of fruit and vegetable consumption in pre-school children: A meta-synthesis using the theoretical domains framework (TDF)}

\section{O'Malley, ${ }^{1}{ }^{1}$; Summerbell, C. ${ }^{1}$; Moore, $H^{1}$; ; Heslehurst, N. $^{2}$ \\ ${ }^{1}$ Durham University, ${ }^{2}$ Newcastle University}

Introduction: Fruit and vegetable $(\mathrm{F} \& \mathrm{~V})$ consumption plays a significan role in chronic disease prevention ${ }^{1}$. Eating behaviours are established at a young age, particularly in the preschool years ${ }^{2}$. Responsibility of food provision predominantly lies with primary caregivers. Identifying barriers and facilitators to F\&V provision is key to designing effective and sustainable interventions for healthy diets and obesity prevention in this age group ${ }^{3}$. This mixed methods systematic review aimed to identify determinants of parental and caregiver $F \& V$ provision behaviours.

Methods: Eight databases were searched. Reference lists and citations of included studies were hand searched (prospero crd42015024627). Data were analysed using the tdf, an integrative framework of 12 domains representing the theoretical constructs of 33 behaviour change theories ${ }^{4}$. The domains were used as an a priori analytical framework to explore barriers and facilitators of primary caregiver $\mathrm{F} \& \mathrm{~V}$ provision to preschool children. Results: Twenty nine studies were included ( $n=15$ qualitative, $n=10$ survey and $n=4$ mixed methods). Twenty seven studies included paren$\mathrm{tal} /$ carer data, one professional caregiver (teacher) and one both. The total number of codes assigned to the dataset was 807 and the domains most frequently identified included 'social influences', 'nature of behaviours', and 'knowledge'. Family members had both a positive and negative impact on provision with children's preference and neophobic tendencies identified as important barriers (social influences). Encouragement, bribery and disguising of $F \& V$ were methods regularly used to facilitate consumption whilst authoritative parenting practices had an overall negative impact (nature of behaviours). Primary caregivers were aware of the health benefits of $F \& V$, but often lacked or had incorrect knowledge relating to specific nutrients (knowledge). Other barriers identified included; cost, time, availability and accessibility.

Conclusion: Results suggest that those interventions which could potentially help increase F\&V provision must involve the wider family. Intervention components which may prove successful should include; positive role modelling of healthful behaviours, nutrition specific education, practical and resource skills and crucially they must address parenting techniques to facilitate consumption. Evidence in relation to professional caregivers is sparse and warrants further investigation.

\section{References:}

1 Wang, P.Y et al (2016) Journ of Diab Invest, 7(1): 56-69

2 Betoko, M.A et al (2013) Europ Journ of Clin Nutrition, 67, 631-637

3 Lakshman, R et al (2013) Syst Rev, 2

4 Michie, S et al (2005) bmj, Quality and Safety, 14: 26-33
Conflict of Interest: None disclosed

Funding: School for Public Health Research (fuse) PhD

\section{T4P54}

\section{Exploring the cardiorespiratory health of children referred for obesity treatment}

\section{O'Malley, D. ${ }^{1}$; Connolly, $K^{2}{ }^{2}$; Guidon, . $^{2}$}

${ }^{1}$ Temple Street Childrens University Hospital, ${ }^{2}$ School Of Physiotherapy, Royal College Of Surgeons In Ireland, Dublin, Ireland.

Introduction: Risk factors for cardiovascular disease have been identified by five years of age in children who are overweight (Flynn et al 2006). The benefit of high levels of childhood cardiorespiratory fitness on cardiovascular health in adulthood is well known (Ruiz et al. 2015) and improving activity levels and fitness in children leads to improved cardiovascular and metabolic profiles (Woo et al., 2004).This study explored the cardiorespiratory fitness, risk of hypertension and the relationship between obesity and fitness in children attending an obesity clinic.

Methods: A retrospective observational study was conducted involving children and adolescents with a bmi $\geq 98$ thpercentile referred for obesity treatment at Temple Street Children's University Hospital. Height and weight were measured and bmi (Kg/M2), bmi centiles and bmi sds were calculated (uk90). Cardiorespiratory fitness was measured with a modified Balke Treadmill test to $90 \%$ age-predicted heart-rate max and vo2peak measures were estimated using established equations (acsm, 2000). Throughout the exercise test, heart rate, and rate of perceived exhaustion (borg) were recorded. Blood pressure (bp) readings were recorded pre and post exercise test in the sitting position. Age- and gender- adjusted bp percentiles were calculated using pre-test bp measurements and the risk of hypertension was defined according to established guidelines.

Results: The mean age of the children $(\mathrm{n}=316)$ was 11.72 years $(\mathrm{sd}+/$ 3.03) and mean bmi sds and bmi Percentile were 3.15 (sd+/- 0.6) and 99.7 $(\mathrm{sd}+/-0.7)$. Sixty-one percent of children referred $(\mathrm{N}=195)$ completed an exercise test with $30 \%(\mathrm{~N}=59)$ managing to reach their $90 \% \mathrm{hr}$ max. Mean estimated vo2peak was $24.69 \mathrm{ml} / \mathrm{kg} / \mathrm{min}(+/-5.4)$ for girls and $26.6 \mathrm{ml} / \mathrm{kg} / \mathrm{min}(+/-12.4)$ for boys. In those that undertook the exercise test, fifteen percent $(\mathrm{N}=29)$ were at risk of pre-hypertension while $40 \%$ $(\mathrm{N}=78)$ were at risk of hypertension.

Conclusion: A substantial number of children referred for weight management present with cardiorespiratory and cardiovascular impairments including impaired aerobic fitness and hypertension. Further work is required to examine factors that predict aerobic fitness and to explore whether treatment can improve cardiovascular and cardiorespiratory health outcomes.

Conflict of Interest: The authors have no conflict of interest.

Funding: This study was funded by a grant from the rcsi Student Summer Research programme 2015.

\section{T4P55 \\ Fast food intake and dietary behaviours in South Korea childhood and adolescent: KNHANES 10-14 database}

Lee, H. $^{1}$; Ahn, A. ${ }^{2}$; Choi, J. ${ }^{2}$; Lim, H. ${ }^{3}$

${ }^{1}$ Research Institute of Medical Nutrition, Kyung Hee University, Seoul 02447, Republic of Korea, ${ }^{2}$ Department Of Family Medicine, Research Institute Of Medical Science, Konkuk University School Of Medicine, Republic Of Korea, ${ }^{3}$ Republic Of Korea, Department Of Medical Nutrition, Graduate School Of EastWest Medical Science, Kyung Hee University, Yong-In 17104, Republic Of Korea

Introduction: Westernized and irregular nutritional Intakes are important problem among children and adolescents in South Korea. Especially, consumption of fast food (ff) is well known to associate with negative impacts on nutrition and health. Hence, this study examined ff consumption status and dietary behaviors among Korean children and adolescentsusing national representative data. 
Methods: From the database of the 5th and 6th Korea National Health and Nutrition Examination Survey (knhanes, 2010-2014), total of 5744 subjects aged 6-18 years who participated were included. Subjects were classified into four groups: Non-consumer $(n=2761)$, Western-ff $(n=450)$ as consumed hamburger, pizza, sandwiches, french fries and so on, from $\mathrm{ff}$ restaurants, Korean-ff $(\mathrm{n}=2051)$ as consumed street, delivery, and instant food such as kimbob (seaweed rice rolls), tteokbokki (spicy rice cake), ramen (noodles) and so on, and Both-consumer $(n=481)$. Intakes of the ff, nutrients and food group were collected using 24-hour recall. Dietary behaviour referred to the rate of skipping of breakfast, rate of having a meal with family, and frequency of eating out and nutrition labelling awareness were investigated. All statistical analyses were performed using the sas considering the complex sampling design, strata, clusters, and weights. Results: Energy intake from the ff were both-consumer (968 kcal/day), Western-ff (563 kcal/day), Korean-ff (435 kcal/day). Intake of the energy (2389.1 kcal/day) and fat $(61.5 \mathrm{~g} /$ day $)$ were the highest of the Both-consumer group.Protein intake were the highest in the Western-ff group (82.6 $\mathrm{g} /$ day) and Both-consumer group $(80.2 \mathrm{~g} /$ day), while, sodium intake was the highest in the Korean-ff (3797.7 mg/day). Compared with the \%Dietary reference intake for Korean, intake of vitamin C was significantly lower in Korean-ff (94.4\%) and both-ff (83.6\%) than Non-consumer (111.8\%) and Western-ff (108.3\%). Rate of the skipping of breakfast was the highest in the both-consumer group $(28.1 \%)(\mathrm{p}=0.0023)$, which was the higher in girl than boy $(\mathrm{p}=0.0107)$. Rate of having a meal with family was the lowest in the western-ff group $(70.2 \%)(p=0.0016)$. Above $30 \%$ of the Western-ff, Korean-ff, and Both-consumer were eating out per day, compared to in the Non-consumer group $(28.4 \%)(\mathrm{p}<0.0001)$, which were higher in boy and adolescents group. In adolescents, regardless $\mathrm{ff}$ and sex, nutrition labelling awareness did not significant.

Conclusion: Intakes of the ff, nutrients and food group and dietary behaviours were difference by ff consumption, which also difference in the sex and adolescents groups.

\section{T4P58}

\section{Friends make children less sedentary and neighbourhoods make them more active}

\section{Barnett, ${ }^{2}{ }^{1}$; Henderson, M. ${ }^{2}$; Contreras, G. $^{3}$}

${ }^{1}$ INRS-Institut Armand-Frappier/cr chu Sainte-Justine, ${ }^{2}$ Université De Montréal/ $\mathrm{Cr}$ Chu Sainte-Justine, ${ }^{3}$ nrs-Institut Armand-Frappier

Introduction: Sedentary behavior ( $\mathrm{sb}$ ) and physical inactivity are distinct constructs for which separate research and intervention paradigms may be warranted. To this end, we compared individual- and neighbourhood-level risk factors of each among youth at risk of obesity.

Methods: Data are from quality, a cohort study of the natural history of obesity in Quebec, Canada. Baseline data were obtained in 2005-2008 when children were aged $8-10 y(n=512$ families). Activity level was measured using accelerometers at age 8-10 years and again at age 10-12 years. At each time point, children were categorized as inactive if they did less than 60 minutes/day of moderate to vigorous physical activity and as excessively sedentary if they recorded less than 100 counts/minute for at least $50 \%$ of the day. Children were required to have worn the device for at least 4 days and for at least 10 hours/day. Child-level factors included sex, sleep duration, and weekly frequency seeing friends; neighbourhood-level factors included density of fast food outlets, convenience stores, and parks; school proximity, street connectivity, land use mix, disorder, social and material deprivation, and parental perceived safety. Separate logistic regression models were estimated for each of inactivity and excessive sb. We tested models using the identical set of baseline risk factors at both time points. Analyses were restricted to 413 children with complete data at age $8-10 y$, and to 283 children with complete data at age $10-12 y$. Models controlled for child's obesity status, father and mother's obesity status, and parental education.

Results: At both time points, girls were $75 \%$ to $85 \%$ more likely to be inactive than boys, but were equally likely to be excessively sedentary. Also at both time points, each additional weekly outing with friends reduced the likelihood of being sedentary by $20 \%$, but did not reduce the likelihood of being inactive. Only area-level disorder was associated with being excessively sedentary, and only in 10-12y olds; in contrast, several factors increased the likelihood of being inactive, including area deprivation at age $8-10 y$ (or: $1.7 ; 1.0-3.0$ ) and perceived lack of safety at age $10-12 \mathrm{y}$ (or: 2.8: 1.1-6.3). Moreover, the likelihood of being inactive decreased by $24 \%$ for each quintile increase in land use mix.

Conclusion: Our findings suggest that physical inactivity and sedentary behavior are driven by largely distinct paradigms. It seems likely that each may be favourably impacted through increases in light physical activity. Although interventions need to target all spheres of influence, enhancing the built environment may be more effective to reduce physical inactivity, while leveraging social and peer groups may be more effective to reduce sedentary behaviors.

\section{T4P60 \\ Healthier eating behaviors among Portuguese school-aged children: Association with overweight/obesity}

\section{Sabino, B. ${ }^{1}$; Almeida, M. $^{2}$}

${ }^{1}$ Faculdade de Desporto - Universidade do Porto, ${ }^{2}$ Faculdade De Ciências Do Desporto E Educação Física - Universidade De Coimbra

Introduction: Eating behaviors, amongst other factors, play a major role in becoming overweight and obesity. Therefore, it is important to understand behaviors, such as vegetable and fruit intake and other healthy behaviors, as well as attitudes and related environment. The objective of this study is to examine the association between nutrition related behaviors and body mass index (bmi) in young people.

Methods: This study was a cross-sectional study involving 2048 youths (1116 boys and 932 girls), aged 9 to 16 years. Socio-demographic characteristics, attitude towards fruits and vegetables, fruit and vegetable environment, and healthy behaviors were assessed by questionnaire. Weight status (bmi) was measured.

Results: The prevalence of overweight/obesity was $32.9 \%$, higher in girls than boys. Young people who reported a more favorable attitude and environment to fruits and vegetable intake adopted healthier eating behaviors $(\mathrm{p}<0.05)$. bmi and healthy behaviors were inversely correlated $(\mathrm{p}<0.05)$. Higher levels of healthy eating behaviors were associated with an increasing friendly environment for fruits and vegetables intake (or:1.16; [1.111.20]), attitude towards fruits (or:1.20; $[1.15-1.25]$ ), and attitude towards vegetables (or:1.26;[1.21-1.31]).

Conclusions: Positive attitudes and an enabling home and school environment to fruit and vegetables consumption were related to healthy eating behaviors in adolescents. So, obesity-based intervention and prevention should consider incorporating strategies related with psychosocial measures to change dietary behaviors by promoting friendly environments for fruits and vegetables consumption.

Keywords: obesity, eating behaviors, fruit and vegetable environment, youth

Conflict of Interest: None Disclosed

Funding: The research relating to this abstract was funded by fct - Portuguese Foundation for Science and Technology through doctoral grant conceded to the first author (sfrh/bd/89150/2012).

\section{T4P61 \\ Healthy lifestyles have your say - hear the children \& young people voices}

Eldin, N. ${ }^{1}$; Martin, S. ${ }^{2}$; Horgan, D. ${ }^{2}$; Scanlon, M. ${ }^{2}$; O 'Donnell, A. ${ }^{3}$

${ }^{1}$ Department of Health, ${ }^{2}$ National University Of Ireland, Cork, ${ }^{3}$ Department Of Children And Youth Affairs

Introduction: In preparation for a National Obesity Policy and Action Plan (2016), it was felt that the children and young people should inform 
the policy. This paper describes the views of children and young people on factors that help and hinder them in having a healthy lifestyle

Methods: Two consultations were held - one with 34 young people aged 13 to 17 years, selected from 11 of the 31 Comhairle na nÓg (The Irish Youth Parliament), and one with 48 primary school children between the ages of eight and 12 years. Every effort was made to have a representative sample of the Irish child and youth populations. All children and young people had freely consented to participate and their parents assented to the process and participation. Methods used were innovative, age-appropriate and strengths based. They included the use of warm-up exercises, lifelines, body mapping, floor mats, consensus workshops and voting Results: Children and young people who were involved in the consultation appear to be well informed as to the general factors which contribute to healthy and unhealthy lifestyles. Healthy lifestyles are often associated with having supportive families and friendship groups, a good balance between school work and leisure, and positive personal attributes (self-esteem, confidence and motivation). Participants multi-faceted understanding of health was not limited to physical aspects but included mental and emotional health. There were some differences of emphasis related to age, with the older age group engaging more with issues relating to mental health and peer relationships, while younger children focused more on balanced diet and exercise. Another key finding of our research is that young people see health as being influenced not only by personal choices, but by broader contextual issues, including family and friends, schools, the physical environment, community settings and wider societal issues. As a result they informed and influenced a number of actions in the National Obesity Policy and Action Plan (2016) e.g. a 'whole of school' approach to healthy lifestyle programme, integrated health \& wellbeing model for early childhood services, reducing the obesogenic environment and community based family centred programmes (Actions 1.1, 1.2, 2.1, $5.5,6.3$ and 9.2)

Conclusion: Children and young people's constructions of health and healthy lifestyles are multi-faceted and can colour national policies.

\section{Conflict of interest: None}

Funding: Research relating to this abstract was funded by the Department of Health, Ireland.

\section{T4P62}

\section{High BMI in late adolescence predicts future severe liver disease and hepatocellular carcinoma: A national, population- based cohort study in $\mathbf{1 . 2}$ million men}

\author{
Hagström, H. ${ }^{1}$; Tynelius, P. ${ }^{2}$, Rasmussen, $F^{3}$ \\ ${ }^{1}$ Centre for Digestive Diseases, Division of Hepatology, Karolinska University \\ Hospital, Stockholm, Sweden and Department of Medicine, Huddinge, \\ Karolinska Institutet, Stockholm, Sweden, ${ }^{2}$ Centre For Epidemiology And \\ Community Medicine, Stockholm County Council, Health Care Services, \\ ${ }^{3}$ Department Of Public Health Services, Karolinska Institutet, Stockholm, \\ Sweden
}

Introduction: A high body mass index (bmi) is associated with an increased long-term risk for severe liver disease. It is unclear if this risk differs across bmi categories, and if the association is partially attributed to development of type 2 diabetes mellitus (T2DM).

Methods: We used register-data from more than 1.2 million Swedish men enlisted for conscription 1969-1996 with complete baseline data on bmi and covariates. Data regarding new events of severe liver disease and T2DM during follow-up were obtained by record-linkage of population-based registers. We used Cox regression to estimate adjusted hazard ratios (hr) for future inpatient care or mortality in severe liver disease and incidence of hepatocellular carcinoma (hcc) across bmi categories, using bmi of $18.5-22.5 \mathrm{~kg} / \mathrm{m}^{2}$ as reference.

Results: During a follow-up of more than 34 million person-years, 5281 cases of severe liver disease including 251 cases of hcc were identified. An association with severe liver disease was found for overweight (hr 1.49, 95\%ci 1.35-1.64) and for obese men (hr 2.17, 95\%ci 1.82-2.59). Develop- ment of T2DM further increased the risk for severe liver disease across all bmi categories, for instance men with obesity and T2DM had a higher risk of severe liver disease (hr 3.28,95\%ci 2.27-4.74) than men with obesity free of T2DM (hr 1.72, 95\%ci 1.72-2.54).

Conclusions: A high bmi in late adolescent men was associated with an increased risk of future severe liver disease, including hcc. Development of T2DM during follow-up was associated with a further increased risk of severe liver disease, independent of baseline bmi.

Conflict of Interest: None

Funding: No specific Funding for this study

\section{T4P63 \\ High-fat meal, systemic inflammation and glucose homeostasis in obese children and adolescents}

Morandi, A. '; Fornari, E. '; Opri, F.'; Olivieri, F.'; Piona, C. '; Bonadonna, R. ${ }^{2}$; Maffeis, $C .{ }^{\prime}$

${ }^{1}$ Pediatric Diabetes and Metabolic Disorders Unit, University Hospital of Verona, Verona, Italy, ${ }^{2}$ Division Of Endocrinology, Department Of Clinical And Experimental Medicine, University Hospital Of Parma, Parma, Italy

Introduction: We aimed to assess, in obese youths, relationships between Il-6, fat-meal induced endotoxemia and glucose homeostasis.

Methods: Twenty obese children/adolescents (9-17 years old, 11 boys) underwent a standard ogtt and, 7-14 days later, a 5-hours fat meal test (fat $69 \%$ of energy, sfa $31.5 \%$, mufa $35 \%$,pufa $33.5 \%$ ), with serial measures of il-6 and two markers of lps exposure and translocation, lps-binding protein (lbp) and soluble cd14 (sCD14).

Results: il-6 correlated with both basal (homa-ir) and post-prandial (Matzuda-index) insulin sensitivity $(\mathrm{r}=0.61[0.24-0.82], \mathrm{p}=0.005$, $\mathrm{r}=-0.53[0.12-0.78], \mathrm{p}=0.03$, respectively). il- 6 did not change after meal whereas lbp and sCD14 decreased significantly, indicating lps translocation. Neither basal sCD14 and lbp, nor their incremental concentrations correlated with il-6 or glucose homeostasis.

Conclusion: In our sample il- 6 was associated with insulin sensitivity but not with lps exposure, suggesting that meals with balanced content of sfa/ mufa/pufa might not be associated with lps-induced inflammation and metabolic impairment.

\section{T4P65}

If you build it will they come? An analysis of the recruitment of families to a community-based, multi-disciplinary childhood weight-management programme

\author{
Kelleher, M. '; O'Malley, D. ${ }^{2}$; Harrington, D. '; Shiely, D. '; Perry, P.
}

${ }^{1}$ University College Cork, ${ }^{2}$ The Children'S University Hospital, Dublin

Introduction: International recommendations agree that initiatives to treat and manage childhood obesity should be family-focused and combine healthy eating, physical activity and behavioural components. However, to be effective, these interventions must engage with families and achieve good recruitment, uptake and retention rates. The aim of this study was to analyse the recruitment process for W82GO-community; a government-funded, community-based, multidisciplinary pilot weight management programme for families of children with obesity and to present participation and retention rates.

Methods: For the current study, W82GO-community was piloted in two community sites from April 2015 for 12 months. The programme was offered by the government free of charge and was delivered by a multi-disciplinary team of community health professionals. Children aged 4-7 years were screened in school via school public health nurses $(n=2064)$. Eligible children (obese $\geq 98$ th percentile) were identified and their families subsequently offered an initial assessment and a place on the pilot programme. 
Results: The screening process identified 121 children who were eligible for initial assessment. Of this 121, school public health nurses reported having referred $94(77.6 \%)$ families. Less than half $(n=42)$ of families accepted the invitation to the initial assessment and of this, $19(20.2 \%)$ subsequently started the programme. Just eight families completed the programme.

Conclusion: Detailed descriptions of recruitment and participation rates are limited. Most studies report the number of final participants instead of including the number screened, contacted and recruited. Although almost $80 \%$ of eligible families were referred to the programme, participation and retention rates were low. This highlights the need for sufficient time and resources to be dedicated to the recruitment stage. Special at tention should be given to developing strategies to boost recruitment and minimise attrition, and further research is required to explore the factors influencing these low levels of engagement.

\section{No competing interests exist}

This research was funded by the Health Research Board spheRE/2013/1. The Health Research Board (hrb) supports excellent research that improves people's health, patient care and health service delivery. We aim to ensure that new knowledge is created and then used in policy and practice. In doing so, we support health system innovation and create new enterprise opportunities

\section{T4P66}

\section{Impact of a web-based intervention to improve nutritional literacy in families of pre-school children: The Nutriscience program}

\section{Gregório, M. ${ }^{1}$; Almeida, C. ${ }^{1}$; Barros, R. ${ }^{1}$; Padrão, P. $^{1}$; Moutinho, N. ${ }^{2}$;}

\section{Azevedo, J.3}

${ }^{1}$ Faculty of Nutrition and Food Sciences of the University of Porto, ${ }^{2}$ Faculty Of Economics Of The University Of Porto, ${ }^{3}$ Faculty Of Arts Of The University Of Porto Portugal

Introduction: Tackling childhood obesity is a major public health challenge. Traditional nutrition education strategies for childhood obesity prevention have shown little effect. Therefore, we aimed to investigate the impact of innovative web-based intervention programs in nutritional literacy of families of pre-school children.

Methods: The Nutriscience project is a prospective follow-up evaluation program, for families and educators of pre-school children. The program consists in a web-based intervention, using an on-line interactive platform, focused on fruit and vegetables, sugar, and salt topics. A gamification approach was developed, using a convenience sample from a national schools' network. The strategy includes a mix between participants' interactions in a social network, raising awareness through educational materials, serious games apps, and nutritional challenges. A parental self-reported questionnaire assessing sociodemographic characteristics and nutritional literacy (20 questions on 4 different dimensions: nutrients, food portions, national food wheel guide groups and food labelling) was administered at the baseline and at the end of the intervention. A nutritional literacy score was obtained for each family through the proportion of correct answers. A General Linear Model for Repeated Measures was used to analyse the improvement of the nutritional literacy score over the intervention.

Results: Intervention program included 550 families from 24 institutions, during 3 months. A total of 1265 and 327 recipes, photographs, videos and comments published by families' and educators were uploaded, respectively. At the end of the intervention 83 families have completed both evaluation assessments. The final mean (sd) score was significantly higher than the baseline one: 77.1 (16.2) \% vs 70.7 (16.2) \%; $p<0.001$. There was a mean (sd) score improvement for all dimensions of nutritional literacy, however significantly differences were just observed for the nutrients dimension [84.3 (15.7) \% vs $87.6(15.4) ; p=0.035$ ], and for the dimension of the national food wheel guide [50.9 (33.8) \% vs. $63.9(35.2) \% ; p=0.002)]$. The nutritional literacy score's improvement was not significantly different according the families' income or educational level.
Conclusion: Our results suggest that an intervention program, based on a digital and entertaining interactive platform, could be a useful and easily disseminated educational tool to improve nutritional literacy in families of pre-school children and prevent childhood obesity, independent of socioeconomic and educational level.

Conflict of Interest: None

Funding: eea Grants Program

\section{T4P67}

Impact of high-intensity circuit-training in physical capacity and cardiometabolic risk markers in overweigh adolescents

Moreira-Gonçalves, D. ${ }^{1}$; Schmidt, C. ${ }^{2}$; Ferreira, R. ${ }^{3}$; Neuparth, M. ${ }^{2}$; Canotilho, C. ${ }^{2}$; Serrão, F. ${ }^{2}$; Sobral, M. ${ }^{2}$; Oliveira, J. ${ }^{2}$

${ }^{1}$ Research Center of Physical Activity, Health and Leisure (ciafel), Faculty of Sport, University of Porto; Department of Surgery and Physiology, Faculty of Medicine, University of Porto, ${ }^{2}$ Research Center Of Physical Activity, Health And Leisure (Ciafel), ${ }^{3}$ University Of Aveiro

Introduction: Despite the well-known benefits of exercise training for improving cardiometabolic health in overweight adolescents, it remains to be determined what is the best mode to provide maximal benefits. The purpose of this work was to evaluate how a high-intensity circuit-training (hict) program affects key physiological health markers in overweigh adolescents.

Material and Methods: 18 students (age $16 \pm 0.9$ yrs and bmi $28.2 \pm 0.26$ ) were submitted to hict program (3 days/week, 1hour/session, for 9 weeks in their school facilities. Before and after the hict program, anthropometrics and body composition [body weight (bw), waist circumference (wc), and fat mass (fm) and free fat mass (ffm)], physical fitness (Fitnessgram), blood pressure and fasting biochemical analysis [Glucose (Gluc), low density lipoprotein-cholesterol (ldl-C), triglycerides (tg), alanine aminotransferase (alt) and aspartate transaminase (ast)] were assessed.

Results: hict induced significant reductions in bw $(77.15 \pm 13.01$ vs. $75.96 \pm 12.35 \mathrm{Kg})$, wc $(93.10 \pm 10.43$ vs. $90.31 \pm 11.66 \mathrm{~cm})$, fm $(27.80 \pm 6.61$ vs. $24.64 \pm 6.29 \mathrm{Kg})$ and an increase in $\mathrm{ffm}(49.37 \pm 9.33$ vs. $51.33 \pm 9.29 \mathrm{Kg})(\mathrm{P}<0.001)$, and in systolic $(128 \pm 12.7$ vs. $117 \pm 7.2$ $\mathrm{mmHg})$ and diastolic $(66 \pm 7.8$ vs. $62 \pm 4.4 \mathrm{mmHg})$ blood pressure $(\mathrm{P}<0.001)$. Regarding physical fitness, the hict resulted in a significant improvement in the curl-up $(38.1 \pm 25.6$ vs. $59.5 \pm 28.9$ rep $)$, push-up $(9.7 \pm 6.9$ vs. $16.5 \pm 6.4$ rep $)$, horizontal jump (131.3 \pm 25.6 vs. $141.3 \pm 28.9$ $\mathrm{cm})$ and $20 \mathrm{~m}$ shuttle run test $(29.1 \pm 11.9$ vs. $38.9 \pm 6.4$ laps $)(\mathrm{P}<0.001)$. Finally, Gluc $(85.9 \pm 12.4$ vs. $76.8 \pm 12.3 \mathrm{mg} / \mathrm{dL})$, ldl-C $(151.7 \pm 25.8$ vs $93.9 \pm 27.8 \mathrm{mg} / \mathrm{dL}), \operatorname{tg}(66.5 \pm 21.4$ vs. $62.3 \pm 23.5 \mathrm{mg} / \mathrm{dL})$, alt $(18.1 \pm 5.5$ vs. $12.0 \pm 4.1 \mathrm{U} / \mathrm{L})$ and ast $(18.0 \pm 6.5$ vs. $13.9 \pm 3.1 \mathrm{U} / \mathrm{L})$ were all significantly reduced $(\mathrm{P}<0.001)$ after the hict program.

Conclusion: Our results support the notion that a short-term high-intensity circuit-training program is an effective strategy to positively modulate physiological health markers in overweigh adolescents.

Conflict of interest: None Disclosed

Funding: European Regional Development Fund through the Operational Competitiveness Programme and fct (uid/dtp/00617/2013)

\section{T4P68 \\ Implementation of a community-based, multi-disciplinary, family-focused childhood weight management programme: Barriers and facilitators for success}

Kelleher, M. ${ }^{1}$; Harrington, D. ${ }^{1}$; Shiely, D. ${ }^{1}$; Perry, P. ${ }^{1}$; Mchugh, D. ${ }^{1}$

${ }^{1}$ University College Cork

Introduction: Research suggests that family-focused weight management programmes that incorporate physical activity, diet and behavioural components can be effective in treating paediatric obesity. However, little is known about the translation of these interventions into real-world set- 
tings or the factors affecting implementation. This qualitative study explores the barriers and facilitators experienced by those implementing a government-funded, multi-component childhood weight management programme in the community setting.

Methods: Semi-structured interviews were conducted with a sample of 29 national and local level stakeholders responsible for implementing the programme including professionals from dietetics, psychology, public health nursing, physiotherapy and health promotion. Framework analysis was used to identify barriers and facilitators which were then mapped onto six levels of factors influencing implementation as outlined by Grol and Wensing: the innovation, the individual professional, the patient, the social context, the organisational context, and the external environment. Results: For all participants, barriers arose due to the multi-disciplinary nature of the programme, including the lack of role clarity, lack of understanding of other disciplines as well as the added complexities of working in different locations. Participants' recognition of the need for a weight management programme and their own personal interest in the area were the main drivers behind implementation while the presence of supportive colleagues were further enabling factors.

Conclusion: This study highlights the complexities associated with implementing multi-disciplinary childhood weight management programmes in the community setting from a wide range of stakeholder perspectives. Our results suggest the assignment of clear roles and responsibilities, the provision of sufficient practical training and resources as well as organisational support play pivotal roles in overcoming barriers to change. This evidence should be used to develop an implementation plan to support the translation of efficacious interventions into real world settings.

\section{No competing interests exist}

This research was funded by the Health Research Board spheRE/2013/1. The Health Research Board (hrb) supports excellent research that improves people's health, patient care and health service delivery. We aim to ensure that new knowledge is created and then used in policy and practice. In doing so, we support health system innovation and create new enterprise opportunities

\section{T4P69}

\section{Indicating obesity status of the children and adolescents in Santa Maria de Feira (Portugal) and Kırıkkale (Turkey)}

\section{Kılınç, F. ${ }^{1}$; Çakır, B. ; Hiçyılmaz, M. ${ }^{2}$; Santos, $A .^{3}$}

${ }^{1}$ Kırıkkale University, Faculty of Health Sciences,Turkey, ${ }^{2}$ KıRıKkale Provincial Directorate Of National Education, Turkey, ${ }^{3}$ Municipality Of Santa Maria De Feira, Portugal

Introduction: Obesity is a globally important public health problem Childhood obesity is increasing just like the adult obesity all over the world. The World Health Organization (who) states that more than 20\% of the children and adolescents in Europe are overweight while one third of them are obese. This study has been carried out in order to indicate the obesity status of the school-age children in Portugal (Santa Maria de Feira) and Turkey (Kırıkkale) and to encourage those who has been specified as overweight and obese for healthy nutrition and physical activities.

Methods: In this study, 617 children (male:329; female:320), between the ages of 6-15 in Kirlkkale and 649 children and adolescents between the ages of 10-17 in Santa Maria de Feira has been evaluated within the Health4Life Programme Comenius Regio Partnership project. The project has been carried out under the coordinatorship of Kirikkale Provincial Directorate of National Education in coordination with Kirıkkale University, Faculty of Health Sciences in Turkey and with the partnership of Municipality of Santa Maria de Feira in Portugal. The body mass index of the children has been calculated by measuring their weights and heights and overweight and obesity status have been indicated by using the who child growth reference data for 5-19 years.

Results: It has been figured out in Turkish children that out of 327 (male:146, female:181) between the ages of $6-10,15.3 \%$ is obese, $18.4 \%$ is overweight, $8.6 \%$ underweight and out of 290 children between the ages of $11-15$, (male:163, female:127), $11 \%$ is obese, $21 \%$ is overweight and $10 \%$ is underweight. Overweight and obesity in males between the ages of 6-10 (overweight:\%19.1 and obese:\%17.1) are higher than the females (overweight:\%17.7 and obese:\%13.8). It has been indicated that overweight and obesity in males between the ages of 11-15 (overweight:\%22.1 and obese:\%11.0) are higher than the females (overweight: $18.1 \%$ and obese: $9.5 \%)$. It has been seen that out of 617 Turkish children between the ages of $6-15,12.96 \%$ are obese, $19.28 \%$ are overweight and $9.2 \%$ are underweight.It has been found out that out of 649 children, in Santa Maria da Feria $10.6 \%$ is obese, $16.6 \%$ is overweight, $5.4 \%$ is underweight. Besides, it has been similarly in Kirlkkale, found out that overweight and obesity in males (overweight:18\% and obese:15\%) is higher than females (overweight: $14 \%$ and obese: $7 \%$ ).

Conclusion: In this study, it has been found out that overweight and obesity in children and adolescents in Kirıkkale and Santa Maria de Feira is common and it is higher for males. It is required to increase the awareness of the children and their parents about healthy nutrition through education and media campaigns and need more effective programmes to prevent the obesity.

\section{T4P70 \\ Influence of obesity on physical working capacity at teenagers.}

\section{Valeria, N. ${ }^{1}$; Irina, L. ' Olga, P. ; Oksana, G. ${ }^{\prime}$; Irina, B. ${ }^{2}$; Pavel, V. ${ }^{3}$}

${ }^{1}$ North-Western Federal Medical Research Center Almazov, ${ }^{2}$ Children's Regional Hospital, Saint-Petersburg, ${ }^{3}$ North-Western State Medical University Named After I.I. Mechnikov

Objectives: Definition of influence of obesity on physical working capacity at teenagers.

Methods: We examined 30 teenagers aged 14 to 17 years (mean age $15,9 \pm 0,2$ years) suffering from obesity (body mass index from 24-to 42 ) and 30 teenagers with normal body mass index. Methods included clinical examination by pediatrician, endocrinologist, study of lipid profile, blood glucose, serum insulin, homa-index definition. The maximal treadmill test using the Bruce protocol was performed.

Results: Group of obese children demonstrated more frequent dyspnea during exercise $(80 \%$ è $40 \%, \mathrm{p}<0,01)$ and more rare high $(23 \%$ è $67 \%, \mathrm{p}<$ $0,01)$ and medium $(47 \%$ è $17 \%, \mathrm{p}<0,05)$ stress tolerance, higher levels of systolic blood pressure $(140,3 \pm 3,4$ è $125,8 \pm 2,5 ; \mathrm{p}<0,01)$ and heart rate $(168,8 \pm 1,2$ è $161,8 \pm 2,6 ; \mathrm{p}<0,05)$ after exercise. There was a significant negative correlation between the body mass index and stress tolerance $(r$ $=-0,85, \mathrm{p}<0,01)$, significant negative correlation between the insulin resistance and stress tolerance $(r=-0,86, p<0,01)$ and gender differences (girls was worse tolerance test) $(\mathrm{r}=-0,92, \mathrm{p}<0,01)$.

Conclusion: Increased body mass index, insulin resistance and female contribute to poorer tolerability of physicalical activity at teenagers should be considered when designing individual exercise programmes.

\section{T4P71}

Influence of parental nutritional status on nutritional status of their offspring at 0-36 months. Results from EPACI Portugal 2012

\section{Nazareth, M. ${ }^{1}$; Pinto, E. ${ }^{1}$; Lopes, C. ${ }^{2}$; Rêgo, C. ${ }^{3}$}

${ }^{1}$ Centre for Biotechnology and Fine Chemistry - Associated Laboratory, Faculty of Biotechnology, Universidade Católica Portuguesa, ${ }^{2}$ Ispup-Epiunit, University Of Porto, ${ }^{3}$ Children And Adolescent Centre, Cuf Hospital, Center For Health Technology And Services Research (Cintesis), Faculty Of Medicine, University Of Porto, Porto, Portugal

Introduction: It is known that maternal obesity is a strong predictor of overweight and obesity in children. Furthermore, literature clearly demonstrates that there is an association between parents and children obesity prevalence supported by the share of the genetic and obesogenic environment. However, there is a significant lack of knowledge, regarding the onset age of the clinical expression of obesity in children. 
Methods: This study was based in epaci Portugal 2012 (Estudo do Padrão Alimentar e de Crescimento na Infância) (Childhood Feeding and Growth Patterns Study), a national representative study, with a mixed design (cross sectional between 12-36 months and retrospective cohort since birth), involving 2230 children, which took place between May 2012 and June 2013. A study protocol were applied which includes, weight (g) and length $(\mathrm{cm})$ at birth, 4, 6, 9, 12, 18, 24 and 36 months, gathered from individual child health bulletin and at evaluation. Body Mass Index (bmi) $\mathrm{Z}$-score was calculated and classified according to who reference values. Self-reported parents' anthropometric data were collected and respective bmi were calculated and classified according who cut-offs. Mother weight gain during pregnancy was also collected.Binary Logistic regression models were fitted to quantify the association between parental and children nutritional status.

Results: $60 \%$ of the fathers and $37.6 \%$ of the mothers were overweight (ow) from those $14.9 \%$ and $12.2 \%$ are obese (ob) respectively. Prevalence of "risk of ow" (risk-ow) (zsBMI>1) in children was $32.6 \%$, with no significant differences between 2 nd and 3 rd year of life, increasing from $14.6 \%$ to $24.7 \%$ and $25.1 \%$ since birth to 12 and 24 months, respectively. $53.1 \%$ and $46.6 \%$ of mothers with pre-pregnancy ow and ob, increased weight above recommendation during pregnancy. Pre-pregnancy and current mother bmi are significantly associated with children risk-ow at all ages from birth until evaluation moment. Children of obese mothers were more prone to be on risk-ow at birth $[1.72(1.24 ; 2.40)], 6$ months $[1.53$ $(1.07 ; 2.18)]$ and 12 months $[1.79(1.32 ; 2.42)]$. Gestational weight gain above recommendations increased the risk-ow at 12 months $[1.30(1.00$ 1.67)]. Father's nutritional status was not significantly associated with infants' nutritional status.

Conclusion: A high prevalence of overweight/obesity was observed in Portuguese toddlers, since early age. Pre-pregnancy bmi and gestational weight gain in mothers were associated with bmi of their offspring. Urgent and earlier effective interventions are needed in order to stop the transgenerational transmission of obesity.

None Conflict of Interest

Funding: Milupa Danone Early Life Nutrition funded Epaci Portugal 2012, the study where this paper is grounded

\section{T4P72 \\ Intake of fruits, vegetables and vegetable soup in Azorean adolescents according to waist-to-height ratio}

Gonçalves-Figueiredo, A. '; Santos, R. ${ }^{2}$; Moreira, C. ${ }^{3}$; Lopes, L. ${ }^{3}$; AgostinisSobrinho, C. ${ }^{3}$; Oliveira-Santos, J. ; Gonçalves, C. ; Rosário, R. ${ }^{5}$; Mota, J. ${ }^{3}$; Abreu, $S^{3}$

${ }^{1}$ ISEIT - Piaget Institute, ${ }^{2}$ Research Centre In Physical Activity, Health And Leisure, Faculty Of Sport, University Of Porto; Early Start Research Institute, Faculty Of Social Sciences, School Of Education. University Of Wollongong, Australia ${ }^{3}$ Research Centre In Physical Activity, Health And Leisure, Faculty Of Sport, University Of Porto, ${ }^{4}$ Ordem Dos Nutricionistas, ${ }^{5} \mathrm{Health}$ Sciences Research Unit, University Of Coimbra

Introduction: Several studies have suggested that the consumption of fruits, vegetables and vegetable soup is reduced during adolescence. The consumption of fruits and vegetables have been negatively associated with obesity. This study will analyze the intake of fruits, vegetables and vegetable soup amongst a group of Azorean adolescents with and without abdominal obesity (ao), by gender.

Methods: A cross-sectional study was conducted with 1460 adolescents, aged 15-18 from the Azorean Archipelago, Portugal in 2008. Abdominal obesity (ao) was defined by a waist-to-height ratio at or above $0 \cdot 500$. Diet was evaluated using a food frequency questionnaire. Data were analyzed separately for girls and boys and T-test was used to examine differences between independent groups.

Results: The prevalence of adolescents with ao was $35.3 \%$ (girls: $44.3 \%$ and boys: $22.4 \%$ ). Girls had a higher intake of vegetables and vegetable soup compared to boys (vegetables: $142.7 \pm 188.9 \mathrm{~g} /$ day vs. $116.7 \pm 153.3$ g/day; vegetable soup: $162.9 \pm 186.0 \mathrm{~g} /$ day vs. $121.7 \pm 159.7 \mathrm{~g} / \mathrm{day}$, respectively, $\mathrm{p}<0.05$ for all). A lower intake of vegetable soup was verified only in girls with ao compared to girls without ao $(148.3 \pm 149.3 \mathrm{~g} / \mathrm{day}$ vs. $174.4 \pm 210.1 \mathrm{~g} /$ day, $\mathrm{p}=0.033$, respectively). No significant differences were seen between adolescents with and without ao regarding fruits and vegetables intake in both sexes $(\mathrm{p}>0.05)$.

Conclusion: We found that girls with ao had a lower intake of vegetable soup than those without ao. The consumption of vegetables is part of a healthy diet that have been associated the prevention of chronic non-communicable diseases (ncd), including ao. In adolescence, the consumption of vegetables and vegetable soup is even more important, considering that micro needs are increased. Further studies are needed to examine the association between ao and consumption of fruits and vegetables.

Conflict of Interest: None Disclosed

Funding: This study was supported by fct grants: bpd/102381/2014 and bd88984/2012; The author César Aparecido Agostinis Sobrinho was given Doctoral scholarship from Brazilian government by capes (Coordination of Improvement of Higher Education Personnel) (Proc: 9588-13-2). The Research Centre on Physical Activity Health and Leisure (ciafel) is supported by uid/dtp/00617/2013 (fct). The author Rute Santos has a Discovery Early Career Research Award from the Australian Research Council (de150101921).

\section{T4P74 \\ Lifestyle treatment of children and adolescents with obesity in Bergen, Norway: Impact of follow-up frequency and self- monitoring during the first year}

\section{Kommedal, K.'; Roelants, M. 2; SkjåkøDegård, H. ; Danielsen, $Y^{4}$;} Morken, $M .^{5}$; Júlíusson, $P^{6}{ }^{6}$

${ }^{1}$ Department of Clinical Medicine, Faculty of Medicine and Dentistry, University of Bergen, Norway, ${ }^{2}$ Department Of Public Health And Primary Care, Ku Leuven University Of Leuven, Belgium, ${ }^{3}$ Department Of Clinical Science, Faculty Of Medicine And Dentistry, University Of Bergen, Norway, ${ }^{4}$ Department Of Clinical Psychology, Faculty Of Psychology, University Of Bergen, Norway, ${ }^{5}$ Department Of Clinical Medicine, Faculty Of Medicine And Dentistry, University Of Bergen, Norway; Department Of Internal Medicine, Haukeland University Hospital, Norway, ${ }^{6}$ Department Of Clinical Science, Faculty Of Medicine And Dentistry, University Of Bergen, Norway; Department Of Pediatrics, Haukeland University Hospital, Bergen, Norway

Introduction: Overweight and obesity in children and adolescents is a major public health problem. A good and simple tool to measure the degree of adiposity is needed for the follow-up of patients and to assess treatment effect. bmi standard deviation scores (sds) are often used in children and adolescents with obesity, despite known limitations. The percentage above the iotf 25 cutoff (\%iotf25) has been proposed as a better bmi metric. We aimed to compare changes in bmi sds and \%iotf25 during the first year of treatment at the Obesity outpatient clinic, Haukeland University Hospital, and to examine the effect of follow-up frequency and self-monitoring as potential determinants of treatment success.

Methods: Patients were enrolled in multidisciplinary, family-based, tailored lifestyle treatment. Height and weight at baseline and one-year follow-up, and number of clinic visits, intermediate visits to their local health care nurse and weightings at home during the one-year follow-up were registered. Paired t-tests were used to compare changes in both bmi metrics according to age group and sex, and simple linear regression were used to analyze the impact of contact and weighing frequency.

Results: In total, 181 children and adolescents ( 83 boys) with a mean age of 11.6 years (range 2.8-18.5) completed one year of follow-up (54 dropped out). Baseline mean (sd) bmi sds and \%iotf25 were 3.06 (0.42) and 150.9 (14.5), respectively. After one year, bmi sds decreased on average with $0.06(\mathrm{p}=0.012)$ and \%iotf25 with $2.6(\mathrm{p}=0.001)$. However, in the case of bmi sds, there was only a significant change in children younger than 12 years of age $(-0.14 ; p<0.001)$, and not in those above 12 years $(0.02, p=0.6)$. Changes in \%iotf 25 were comparable and significant in both age groups $(-2.9, \mathrm{p}=0.003$ and $-2.4, \mathrm{p}=0.047)$. For both parameters, the reduction was significant in boys $(-0.16$ SDS, $\mathrm{p}<0.0001$ and $-4.2 \%$, 
$\mathrm{p}<0.001$ ), but not girls (0.02SDS, $\mathrm{p}=0.4$ and $-1.3 \%, \mathrm{p}=0.2)$. Further, simple linear regression analyses showed a larger reduction in bmi sds with the frequency of visits to the local health care nurse (coded none, some, monthly: $\mathrm{b}=-0.085, \mathrm{p}<0.01)$ and the frequency of weightings at home (none, some, weekly; $b=-0.058, p=0.028$ ). For the \%iotf 25 , the frequency of visits to the local health care nurse was the only significant predictor of change $(b=-2.31, \mathrm{p}=0.04)$.

Conclusion: Both change and determinants depended on the bmi metric used as outcome variable. Though moderately effective, outpatient lifestyle treatment can be improved giving intermediate controls by a local health care nurse.

Conflict of interest None disclosed

No Funding

\section{T4P76}

Long-term changes in skinfolds thickness in children and adolescents aged 3-18 from Krakow (Poland), within the last 30 years (from 1983 to 2010)

\section{Kryst, \&. ${ }^{\text {; }}$ BilińSka, I. ${ }^{2}$; Kowal, M. ' ; Woronkowicz, A. '; Sobiecki, J. ${ }^{1}$}

${ }^{1}$ University of Physical Education, Department of Anthropology, Krakow, ${ }^{2}$ Mieszko I College Of Education And Administration, Faculty Of Public Health, Poznan

Introduction: In Poland, even in the late twentieth century, the problem of obesity was not significant. However, recent studies have shown an increase in the prevalence of overweight and obesity. Socio-economic changes, in last decades, approached Poland to Western Europe. A lifestyle of Poles (physical activity and diet) have changed radically. The aim of the study was to investigate changes in adiposity in children and adolescents over the last decades.

Methods: Two cross-sectional studies were made in 1983 and 2010. The analysis included 10324 children and adolescents aged 3-18 living in Krakow (Poland). Data on selected skinfolds (triceps, subscapular, abdominal, suprailiac, calf) were collected and compared between the series of studies.

Results: The total body fat (sum of 5 skinfolds) was higher in contemporary boys. In girls, there were not noticed such changes. In both sexes, the triceps and supscapular skinfolds thickness decreased. In the case of abdominal skinfold - there were no significant changes in boys, while contemporary girls had a smaller thickness of this skinfold. In boys, there were noticed reduction in suprailiac skinfold thickness. In the case of girls, the reverse trend was reported - contemporary girls were characterized by thicker suprailiac skinfold. Children studied in 2010 were characterized by thicker calf skinfold.

Conclusion: Changes in adiposity are worrying, especially in boys. The priority should therefore be effective prevention and intervention programs. They can prevent further deepening of the problem among Polish children, by the time it will be as severe as in Western Europe.

\section{Conflict of Interest: None Disclosed}

Funding: Research relating to this abstract was funded by Polish Council for Science and Technology (nn404 177 035).

\section{T4P77}

\section{Maternal consumption of saturated, trans or $\mathbf{n - 3}$ polyunsaturated fatty acids during pregnancy and lactation differently affects the lipid profile and glucose tolerance of 21-d-old pups}

Mennitti, L. '; Oyama, L. '; Santamarina, A. ' ; Nascimento, C. ${ }^{2}$; Pisani, L. $^{3}$

${ }^{1}$ Programa de Pós-graduação Interdisciplinar em Ciências da Saúde, Universidade Federal de São Paulo (unifesp), Santos, sp, Brazil, ${ }^{2}$ Departamento De Fisiologia, Disciplina De Fisiologia Da NutriçãO, Universidade Federal De SãO Paulo (Unifesp), SãO Paulo, Sp, Brazil, ${ }^{3}$ Departamento De BiociêNcias, Instituto De SaúDe E Sociedade, Universidade Federal De SãO Paulo (Unifesp), Santos, Sp, Brazil

Introduction: Maternal nutrition, particularly the fatty acids (fa) diet composition during pregnancy and lactation could modify the foetal programming and offspring phenotype related to obesity and insulin resistance. Thus, we evaluated the changes triggered by maternal consumption of different types of fa during pregnancy and lactation in the lipid profile and glucose tolerance of 21-d-old pups.

Methods: Pregnant Wistar rats were allocated into four groups: control (C), saturated fa-SFAs (S), Trans fa-TFAs (T) or n-3 polyunsaturated fa-pufa (pufa). Diets were normolipidic and were maintained during pregnancy and lactation. After delivery, male offspring composed the groups: C21, S21, T21 and pufa21. At 21st, pups were euthanized. Serum concentration of triacylglycerol (tag), total cholesterol and insulin were determined by specific kits. Hepatic tag deposition, Homeostasis Model Assessment of Basal Insulin Resistance (homa-ir) and oral glucose tolerance test (ogtt) was performed. Protein expressions were analysed by Western Blotting.

Results: In the pufa21 group, there were a decrease in the pup's serum concentrations of tag and total cholesterol, hepatic tag content and glycaemia at baseline, 90 and 120 minutes; accompanied by an increase in the liver protein expression of catalase. Although T21 group has presented reductions in hepatic tag content and serum glucose concentration at baseline, the glycaemia was not maintained during and at the end of the ogtt. Insulin serum concentration, homa-ir and liver proteins levels of total Akt, phospho-Akt and irâ were unchanged among groups.

Conclusion: Early exposed to tfas during pregnancy and lactation seems to impair offspring glucose response, while maternal consumption of $n-3$ pufa-based diets improve the glucose tolerance, lipid serum profile, tag hepatic accumulation and pro-oxidant status in the 21-d-old pups.

Conflict of Interest: There is no conflict of interest.

Funding: fapesp/Brazil granted PhD scholarship (2014/10683-0).

\section{T4P78}

Maternal obesogenic diet during pregnancy and suckling: Study of proteins in the inflammatory pathway, serotonin metabolism and notch signaling in hippocampus of offspring mice

Mendes-Da-Silva, C. ${ }^{1}$; Dias, C. ${ }^{1}$; Curi, H. ${ }^{1}$; Camargo, M. ${ }^{1}$; Vieira, T. ${ }^{1}$; Gomez, P. ' ; Lambertucci, R. ' ; Lemes, S. ${ }^{2}$; Payolla, T. ${ }^{2}$; Torsoni, M. ${ }^{2}$

${ }^{1}$ Laboratory of Neuroscience and Nutrition, Department of Biosciences Federal University of Sao Paulo (unifesp)/Campus Baixada Santista. Santos/ sp/Brazil, ${ }^{2}$ Laboratory Of Metabolism Disorders - Faculty Of Applied Sciences State University Of Campinas (Unicamp). Limeira/Sp/Brazil.

Introduction: The maternal high-fat diet (hfd) during pregnancy and suckling can promote neurological damage to offspring, affecting the memory and learning hippocampus-dependent, the serotonin neurotransmitter metabolism and the Notch signaling pathway. These possible effects may be associated with pro-inflammatory mechanisms promoted by hfd.

Methods: Female Swiss mice were fed a control diet (cd - 14.7\% fat) or hfd ( $45.0 \%$ fat) throughout pre-mating until suckling. After weaning the offspring received standard chow until 30 postnatal days of age (p30). 
Were measured: body weight, adipose tissue, liver, brain (only offspring), glucose, total cholesterol, ldl or 'bad cholesterol', hdl or 'good cholesterol' and triglycerides of dams and pups. In addition, in the offspring's hippocampus were performed proteins for inflammatory pathways (jnk, pJNK and tnf- $\alpha$ ), serotonergic system (enzyme tryptophan hydroxylase 2 (tph2), serotonin transporter (sert) and the 5-ht1A receptor) and for Notch signaling pathway (Notch1, Hes5 and Mash1) by western blotting and rt-pcr.

Results: The findings show that dams and offspring hfd exhibit increased body weight gain and adipose tissue. Moreover, hfd offspring exhibited decrease of hdl when compared cd. In addition, hfd offspring showed increased protein and gene expressions of $\mathrm{pJNK}$ and tnf- $\alpha$ respectively, and decrease in expression of tph2 e 5-ht1A. There wasn't change in Notch signaling pathway.

Conclusion: Our findings demonstrate that maternal hfd (obesogenic diet) promote pro-inflammatory molecular effects and also affect the serotonin metabolism (tph2 and 5-ht1A changed) on hippocampus of the offspring $\mathrm{p} 30$. The hippocampus and brain serotonin play role important in molecular and cellular processes related to neurogenesis.

The authors declare that they have no conflict of interest.

Research relating to this abstract was funded by Fundação de Amparo à Pesquisa do Estado de São Paulo (fapesp - processes 2014/26146-4 and 2015/08441-1)

\section{T4P79}

\section{Medium- and long-chain acylcarnitine profiles in plasma are altered in the offspring of cafeteria fed dams during lactation in response to fed/fasting conditions}

Pomar, C. '; Kuda, O. ${ }^{2}$; Sánchez, J. '; Rombaldova, M. ${ }^{2}$; Picó, C. '; Kopecky, J.2.; Palou, $A$.

${ }^{1}$ Laboratorio de Biología Molecular, Nutrición y Biotecnología (Nutrigenómica y Obesidad), Universidad de las Islas Baleares (uib) y ciber Fisiopatología de la Obesidad y Nutrición (ciberobn), Palma de Mallorca, España., ${ }^{2}$ Department Of Adipose Tissue Biology, Institute Of Physiology Academy Of Sciences Of The Czech Republic, V.V.I., Prague, Czech Republic

Background/Aim: Deregulation of fatty acid oxidation (fao) is recognized as an important feature in the pathophysiology of obesity and insulin resistance, and species of acylcarnitines (ac) in plasma have been shown to be increased in these conditions. We have previously described that the consumption of a cafeteria diet throughout lactation in rats produces permanent consequences in the offspring metabolic health associated with greater fat accumulation, without higher body weight. Here we aimed to analyse whether changes in plasma ac profiles at early stages could serve to identify potential early biomarkers of these alterations.

Material/Methods: Rat dams were fed with a control or a cafeteria diet throughout lactation. After weaning, the offspring (males and females) of each group were killed half of them under ad libitum feeding conditions and the other half after $12 \mathrm{~h}$ fasting. We studied in the offspring the plasma ac profiles and the expression levels of genes related to fatty acid oxidation in liver.

Results: Under feeding conditions, the offspring of cafeteria diet fed dams presented higher circulating levels of most of medium- and long-chain ac species compared to controls. This could be tentatively related to the known greater maternal intake of fat and its higher presence in milk under these dietary conditions. However, while in the control group most of medium- and long-chain ac increased in response to fasting, this response was generally impaired in the offspring of cafeteria diet fed dams. In addition, the offspring of cafeteria diet fed dams also presented a higher hepatic expression of Ppara, Fgf 21 and Cptla genes, both under ad libitum and fasting conditions, compared to controls.

Conclusion: Maternal intake of a cafeteria diet during lactation in rats impairs the offspring medium- and long-chain ac profiles in plasma and the response to fed/fasting conditions, which could be indicative of the deregulation of fao. Thus, plasma levels of ac species are suggested as early biomarkers of metabolic dysfunction, particularly when measured under the fed/fasting stress

Conflict of Interest: None Disclosed

Funding: Research relating to this abstract was funded by agl2015-67019-P and supported in part by the Czech Science Foundation under grant number 14-3684G.

\section{T4P80}

\section{Metabolic outcomes in overweight adults according to birth weight and overweight onset}

\section{Garruti, G. ' Natuzzi, A. '; Rotondo, A. ' ; Mandoi, A. '; Mallardi, G. '; Giorgino, $F$. \\ ${ }^{1}$ Center for the Study of Obesity, Section of Internal Medicine, Endocrinology and Metabolic Diseases, D.E. T.O., University of Bari "Aldo Moro"}

Background: Low-birth weight is a risk factor for type 2 diabetes (T2D) and high-birth weight is a risk factor for obesity (plos one 2012, 7(10):e47776). Few studies analyzed the prevalence of diabetes, insulin-resistance (InsR), and metabolic syndrome (MetS) in adults who were already overweight during adolescence according to birth weight.

Methods: A cohort of 336 overweight subjects ( $75.3 \%$ women, mean age: $44.9 \pm 14.9$ years, mean bmi: $\left.38.0 \pm 7.4 \mathrm{~kg} / \mathrm{m}^{2}\right)$ was stratified according to birth weight, overweight onset (childhood/adolescence, adulthood), bmi, waist, fasting glycaemia, total cholesterol, hdl, ldl, triglycerides, homa-ir, $\mathrm{HbA1c}$, arterial blood pressure levels, fasting insulin levels, MetS occurrence. Compliance to a 6 -months life style program was assessed by nutritional interview and \% excess weight loss (\%ewl).

Results: Low-birth weight ( $<2500 \mathrm{~g})$ was found in $19.9 \%$ of cases, normal-birth weight (2500 g - $3999 \mathrm{~g})$ in $30 \%$ of cases, high-birth weight (> $3.999 \mathrm{~g}$ ) in $17.2 \%$ of cases. In $32.9 \%$ of cases information about birth weight was not available. Overweight appeared in childhood/adolescence in $74 \%$ of cases and in adulthood in $14 \%$ of cases. $76 \%$ of low-birth weight subjects became overweight during childhood/adolescence. In subjects with childhood/adolescence overweight, $18 \%$ of cases had T2D, $62.93 \%$ a positive family history for T2D and $22 \%$ InsR. In patients with adulthood onset of overweight, $10 \%$ had $\mathrm{T} 2 \mathrm{D}, 42 \%$ a positive family history for T2D and 42\% InsR. Subjects with childhood/adolescence overweight showed basal triglyceride levels and \%ewl significantly higher than those with adulthood overweight.

Conclusions: In our cohort, the early overweight occurrence was associated with a higher prevalence of T2D and a positive family history for T2D, but also a better compliance to a 6-months life style program as compared with adulthood onset of overweight.

\section{T4P81 \\ Metabolic signatures in an adolescent with Silver Russell syndrome and outcomes after bariatric surgery}

Mosbah, $H_{\text {. }}$; Dubern, B. '; Mosbah, H. ${ }^{1}$; Tordjman, J.'; Genser, L. '; Bedossa, P.'; Bouillot, J. ${ }^{4}$; Andreelli, F.'; Faucher, P.'; Oppert, J.' ; Clément, K.'; Netchine, I.'; Poitou-Bernert, C.'

${ }^{1}$ Hôpital Pitié Salpêtrière, ${ }^{2}$ Hôpital Trousseau, ${ }^{3} \mathrm{Hôpital} \mathrm{Beaujon,}{ }^{4} \mathrm{Hôpital}$ Ambroise Paré

Introduction: Silver-Russell syndrome (srs) is a rare imprinting genetic disorder with hypomethylation of the $11 \mathrm{p} 15$ paternal igf2/H19 region. This syndrome is characterized by pre and postnatal growth retardation, dysmorphic features, feeding difficulties. It could be at risk of early-onset obesity, type 2 diabetes (T2D) and cardiovascular complications in adulthood. No detailed phenotype is available regarding nutritional and metabolic status of young adults with srs. Bariatric surgery has never been described in this context.

Methods: Here we report the case of an obese srs adolescent referred for bariatric surgery. Medical history and phenotype lead to the diagnosis of srs with hypomethylation of the igf $2 / \mathrm{H} 19$ paternal region. We described her metabolic parameters and body composition. We report the evolution 
of these parameters three years after a sleeve gastrectomy (sg). We performed a histologic analysis of her subcutaneous adipose and liver tissue. Results: This srs patient, aged 17, was obese (Body Mass Index (bmi) $37.6 \mathrm{~kg} / \mathrm{m}^{2}$ ). She suffered from T2D with high insulin-resistance and renal disease, dyslipidemia and steato-hepatitis. Body composition showed an increased total fat mass (52.2\%) mainly troncular. Subcutaneous adipose tissue analysis revealed marked inflammation (macrophages cd68+: 17\%) and fibrosis. Liver biopsy showed mild steatosis, lobular inflammation and fibrosis. Decision was made to perform a sg. The postoperative course was uneventful. After three years, the patient had a remarkable improvement of her condition with a $33 \%$ total weight loss (bmi: $25 \mathrm{~kg} / \mathrm{m}^{2}$ ), remission of $\mathrm{T} 2 \mathrm{D}$, correction of dyslipidemia and microalbuminuria, and normalization of liver blood tests. Inflammatory markers (leptin, adiponectin, il-6, crp) improved significantly.

Conclusion: Despite an intra-uterine growth retardation, srs adult patients are at higher risk of early-onset obesity and severe metabolic complications. Results of bariatric surgery were comparable with those reported in common obesity in terms of metabolic improvement, mobility and self-esteem, without adverse effects.

\section{T4P82 \\ Moderate versus high intensity interval exercise trainings on energy intake and appetite feelings in adolescents with obesity.}

Maud, M. ' Julie, M. ${ }^{2}$; Valérie, J. ${ }^{3}$; Lore, M.4 Elodie, C. ${ }^{4}$; Charlotte, C. ${ }^{5}$; Yves, B. ${ }^{3}$; Martine, D. ${ }^{3}$; David, T. $^{4}$

${ }^{1}$ Clermont-Auvergne University, France, ${ }^{2}$ Ssr Ugecam Nutrition Obesité, Clermont-Ferrand, France, ${ }^{3} \mathrm{Chu}$ Clermont-Ferrand, Clermont-Ferrand, France, ${ }^{4}$ Clermont Auvergne University, France, ${ }^{5} \mathrm{Cmi}$ Romagnat, France

Introduction: Obese adolescents have been recently found to decrease their daily energy intake in response to physical activity programs with a greater impact of intensive trainings. However, previously published studies only considered energy intake as a secondary outcome and used self-reported food diaries. This pilot study was designed to compare the effects of a moderate intensity versus High Intensity Interval Training on daily ad libitum energy intake and appetite feelings in adolescents with obesity.

Methods: Thirty 12-15 years old obese adolescents (iotf cut-off points; Tanner stage 3-4) were randomly assigned to: i) a control group (no intervention, con, $\mathrm{n}=10$ ); ii) a moderate intensity training group (mod, $\mathrm{n}=10$ ); iii) High Intensity Interval Training group (hiit, $\mathrm{n}=10$ ). The intervention lasted 12 weeks and consisted in 3 sessions per week of 60 minutes each. At baseline and by the end of the 12 weeks, anthropometric parameters and body composition (dxa) were evaluated, daily ad libitum energy intake assessed and appetite feelings estimated using visual analogue scales.

Results: Body weight was significantly reduced in response to both mod $(\mathrm{p}<0.05)$ and hiit $(\mathrm{p}<0.001)$ without any change in the control group. Both hiit and mod $(\mathrm{p}<0.05)$ groups significantly reduced their percentage of Fat Mass $(p<0.001)$. A higher Fat Mass decrease was found in the hiit group compared with the mod one $(\mathrm{p}<0.01)$. Ad libitum daily energy intake did not change significantly in all groups. There is no relationship between the individual degree of ei changes and the variations of body weight, fat mass percentage and Fat-Free Mass, whatever the group. While the percentage of energy derived from proteins and carbohydrates did not vary significantly between groups and time, the energy derived from fat intake was significantly lower by the end of the intervention in the hiit group $(p<0.001)$. Hunger was significantly higher and satiety lower after the intervention in the hiit group with no change in the two other groups. Conclusion: None of the moderate intensity and High Intensity Interval Trainings significantly affect daily ad libitum energy intake in obese adolescents despite reduced satiety and increased hunger feelings after hiit.

Conflict of Interest: None Disclosed
Funding: Research relating to this abstract was funded by Auvergne-Rhône-Alpes County and the French National Academy of Medicine.

\section{T4P84}

\section{No difference in musculoskeletal consultations at the GP between overweight and normal weight children}

\section{Leeuwen, J. '; Middelkoop, M. '; Paulis, W. '; Bueving, H. '; Bindels, P.';} Koes, $B{ }^{1}$

${ }^{1}$ Erasmus Medical Centre

Introduction: Childhood overweight and obesity are associated with self-reported musculoskeletal complaints, injuries, and fractures as early as childhood. In the current study we investigated the (potential) association between the frequency and type of musculoskeletal consultations at the general practice (gp) and weight status of children during a two year follow-up.

Methods:Data from a prospective longitudinal cohort study including $\mathrm{n}=733$ children aged $2-18$ years presenting in general practices in the Netherlands were used. Height and weight were measured at baseline, 6 months, 1 and 2 year follow-up. At those same moments children and parents filled out questionnaires about complaints, health related topics (time spent on physical activity, food intake, etc.) and general demographics. Electronic medical files were available for 617 children and were used to collect information on the frequency and type of consultations of the children at the gp during the two-year follow-up period. Poisson regression and logistic regression were used to calculate the associations between weight status and the frequency and type of gp consultations.

Results: Of the 617 included children, 111 (18\%) were overweight or obese and 506 (82\%) was non-overweight. Overweight children were significantly older (mean age 9.8 years sd (3.6) versus $7.8(4.0), \mathrm{p}=0.004$ ). Overweight children consulted the gp for any type of complaint significantly more often during the 2-year follow up than non-overweight children (mean 7.3 (5.7) vs 6.7 (5.4), or 1.09, 95\%ci 1.01-1.18). Though, no significant difference was seen in the number of musculoskeletal consultations during two year follow-up between overweight and non-overweight children (or 1.20, 95\%ci 0.86 - 1.68). Additionally, no significant difference between overweight and non-overweight children was seen for the number of consultations for any of the further specified musculoskeletal disorder.

Conclusion: No association was found between childhood weight status and the frequency and type of musculoskeletal consultations in gp during a two year follow-up. It therefore seems that musculoskeletal complaints caused by excessive weight do not present in general practice at such a young age.

\section{Conflict of Interest: None disclosed}

Funding: No Funding

\section{T4P85}

\section{Nutritional status among Polish school age children}

\author{
Jarosz, M. '; Charzewska, J.'; Wolnicka, K. ' ; Jaczewska-Schuetz, J.'; \\ Taraszewska, A. '; Siuba-Strzelińska, M.' \\ ${ }^{1}$ National Food and Nutrition Institute
}

Introduction: Obesity in developmental age has both immediate and long-term harmful consequences for health. Therefore, it is important to know the extent of the problem in each country. The aim of the study was to assess the nutritional status of school age children in Poland.

Methods: The study was conducted from April to June 2013, in 2 primary schools and 2 secondary schools in each of the 16 provinces in Poland. A total of 64 schools have been included in the study throughout the country including 3266 children aged 11 to 16 years (1 619 girls and 1647 boys). Measurements of the height and weight were made, on the basis of which the bmi (Body Mass Index) was calculated and the nutritional status of 
school age children using the classification of Cole et al. 2000 and 2007 was assessed.

Results: The overweight and the obesity were observed in $22.3 \%$ of examined children, a normal body weight - in $69.1 \%$, however the deficiency of the body weight appeared at the $8.6 \%$ of children. The excess body weight has more often appeared at boys (25.5\%) than at girls (19.2\%), in contrast with the underweight which was more often related to girls (11\%) than to boys $(6.2 \%)$.

Conclusion: Overweight and obesity is a serious problem among school age children in Poland. There is a need to implement educational programs in schools, which would raise the awareness of children, parents and teachers about healthy nutrition and physical activity.

Funding: Study relating to this abstract was funded by Swiss Contribution within the project' 'Prevention of overweight, obesity, and chronic diseases by education on nutrition and physical activity of the society" under the Swiss-Polish Cooperation Program

\section{T4P86 \\ Obesity and genetic susceptibility - a study in Portuguese women}

Brito, M. ${ }^{1}$; Veiga, L. ${ }^{1}$; Nunes, J. ${ }^{2}$

${ }^{1}$ Escola Superior de Tecnologia da Saúde de Lisboa, Portugal, ${ }^{2}$ Centro Hospitalar De Lisboa Central, Epe

Introduction: Obesity arises from a complex interaction between genetic variance, environment, and lifestyle changes, and has become a major public health problem as a result of its increasing prevalence in most developed countries. Several genes have been implicated as risk factors for obesity, namely paraoxonase-1 (pon-1), adiponectin (AdipoQ), leptin (lep), leptin receptor (lepr), ghrelin (ghrl), melanocortin-4 receptor (mc4R), angiotensin I converting enzyme (ace), apolipoprotein A-V (ApoA5), fat mass and obesity-associated protein (fto), interleucin 6 (il 6), peroxisome proliferator-activated receptor gamma (ppar $\gamma$ ), transcription factor 7-like 2 (tcf7L2) and tumour necrosis factor alpha (tnfa), among others.

We aimed to investigate whether several polymorphisms in the mentioned genes were risk factors for obesity in a Portuguese female population and to assess the association between those polymorphisms with obesity-related traits.

Methods: One hundred healthy normal-weight women and 112 obese women were included in the study. Inclusion criteria for obese subjects were body mass index $>30 \mathrm{~kg} / \mathrm{m}^{2}$. All polymorphisms mentioned above were assessed by rt-pcr, with TaqMan probes. Statistical analysis was performed by spps version 21 .

Results: Significant differences in genotypic frequencies between control and obese women were observed for several polymorphisms, with risk for obesity observed for Pon1 Q192R (risk allele R), Pon1 M55L (risk genotype ml), AdipoQ G276T (risk allele T), AdipoQ G11377C (risk allele G and risk genotype gc), tnfa G308A (risk allele A), Leptin G2548A (risk allele $\mathrm{G}$ and risk genotype gg and ga) and Leptin Receptor K109 R (risk allele R). In a multivariate logistic analysis significant association with obesity was observed for Pon1 Q192R, AdipoQ G11377C and fto. Significant association was observed between polymorphisms and obesity related traits, namely AdipoQ G276T associated with percent of fat mass (increase in $\mathrm{tt}$ ), AdipoQ G45T associated with bmi (increase in gg), $\mathrm{fta} \mathrm{A} / \mathrm{T}$ associated with weight and percent of fat mass (increased in aa), ApoA5 T1131C associated with bmi and waist perimeter (increased in gg), ace $\mathrm{I} / \mathrm{D}$ - associated with percent of fat mass (increased in dd) and Ghrelin R51Q associated with waist perimeter (increased in rq).

Conclusion: Presented data is useful for the clinical management of obese women. Nevertheless, additional data are required, namely inclusion of males and youth subjects, to fully characterize the involvement of these loci in the development of obesity within the Portuguese population.

\section{T4P87}

\section{Obesity and hypertension among intellectually disabled} children

\author{
Gao, Y. ${ }^{1}$; Zhang, Y. ${ }^{1}$; Wang, J. ${ }^{2}$; Kwok, H. ${ }^{1}$; Huang, W. ${ }^{1}$ \\ ${ }^{1}$ Hong Kong Baptist University, ${ }^{2}$ Chinese University Of Hong Kong
}

Introduction: Both childhood obesity and hypertension have attracted high attention from researchers due to their important impacts on health and wellness in both childhood and later adulthood. Increasing trends have been observed in most countries in the world in recent decades, including China. Although epidemics of the two diseases have been well documented in general child population, situations among children with intellectual disability (id) remain unclear. This study aimed at investigating the prevalence rates of obesity and hypertension among children with mild and moderate id in Hong Kong. Their relationship with sociodemographic and lifestyle variables was also examined.

Methods: It was a cross-sectional study, conducted among students with id in Hong Kong in 2015. Each participant was measured height, weight, and blood press at school following standard procedures. Parents reported socio-demographic characteristics, children's physical activity (pa), sedentary behaviours (sb), eating habits, and sleeping duration on a self-administered questionnaire. Univariate and multivariate regression were performed to estimate the relationship between potential risk factors and obesity and hypertension.

Results: We recruited 558 students (age: 6-21 years, $12.2 \pm 3.9$ years; boys: $68.8 \%$ ) from 12 special schools, of which 524 (93.9\%) had bmi data and $476(85.3 \%)$ had hypertension data. Overall, $31.3 \%$ of the participants were overweight or obese, and $9.8 \%$ and $24.7 \%$ of them were pre-hypertensive and hypertensive respectively. All studied lifestyle variables played a role in obesity and hypertension except pa for obesity. In addition, obese children were also at elevated risk for hypertension.

Conclusion: Children with id are more likely to be obese and hypertensive than their general counterparts. Actions should be taken immediately to protect them.

\section{T4P88}

\section{Obtaining comprehensive estimates of the costs of childhood obesity in Europe}

Balanda, P. ;

${ }^{1}$ Institute of Public Health in Ireland

Background: Comprehensive estimates of the impacts and costs help raise awareness of childhood obesity and to properly place it in policy debates. Work Package 4 of the eu Joint Action on Childhood Obesity (janpa wp4) (www.janpa.eu) aims to develop the economic rationale for action on childhood obesity by:

Estimating the lifetime impacts and costs of childhood obesity in seven eu countries

Assessing the effect of $1 \%$ and $5 \%$ reductions of mean bmi on these impacts and costs

Exploring the feasibility of extending the methodology to other eu countries

Aim: The aim of this presentation is to compare the challenges of estimating the impacts and costs of childhood obesity to those for adult obesity, and to describe how the challenges are being handled in the janpa wp 4 project.

Methods: wp4 is a simulation modeling exercise based on a substantial modifications of the ukhf's simulation modeling software). The modelling requires extensive research and data inputs and adopts a societal economic perspective. This includes reviews of childhood and adulthood impacts, recent costing studies and the development of study protocols for a modeling exercise.

Results: Costing studies can focus on either the experience of a whole population or the lifetime experiences of those who are overweight and obese. Comprehensive costing studies usually involve complex simulation 
modeling exercises and the janpa wp4 modelling uses a closed cohort of children. It adopts a societal economic perspective that includes (direct) healthcare costs and other costs such as loses in adult productivity (due to premature deaths and work absenteeism) and lifetime incme losses. Conclusions: The inadequacy of existing research and data and the need to undertake this exercise isn sevenb countries across Europe poses considerable methodological challenges. Addressing these inadequacies will also have benefits for the management of other chronic conditions and build capacity at the country-level.

\section{T4P89 \\ Outpatient treatment of childhood obesity: Results from a multi-disciplinary group intervention pilot study}

Ramalho, S. '; Silva, D. ${ }^{2}$; Silva, S. ${ }^{3}$; Marinho, A. ${ }^{4}$; Viana, V. ${ }^{5}$; Vasconcelos, C. ${ }^{3}$; Conceição, E. '; Guerra, A. ${ }^{2}$

${ }^{1}$ School of Psychology, University of Minho, ${ }^{2}$ Faculdade De Ciências Da Nutrição E Alimentação Da Universidade Do Porto; Hospital Pediátrico Integrado, Centro Hospitalar São João;Faculdade De Medicina Da Universidade Do Porto, ${ }^{3}$ Hospital Pediátrico Integrado, Centro Hospitalar São João, ${ }^{4}$ Faculdade De Ciências Da Nutrição E Alimentação Da Universidade Do Porto, ${ }^{5}$ Faculdade De Ciências Da Nutrição E Alimentação Da Universidade Do Porto; Hospital Pediátrico Integrado, Centro Hospitalar São João

Introduction: Childhood obesity rates are increasing in health care centers worldwide. Due to the health consequences of childhood obesity, long-term clinical support is needed and the development of alternative interventions for childhood obesity gains attention. The aim of this study is to describe the implementation and the preliminary results of a pilot intervention project for childhood obesity in outpatient treatment.

Methods: This is a 6-month psychoeducational multidisciplinary group intervention conducted in São João Hospital Center, in the north of Portugal. Its objective is twofold: reduce children weight and educate them and their families about healthy eating and lifestyle. Participants included 7 patients in outpatient treatment for childhood obesity, aged 10 to 12 years, with a body mass index (bmi) above the 85th percentile and their parents. It includes monthly group sessions (2 hours) with nutritional and psychological counseling, as well as cognitive behavioral monthly tasks. Nutritionists, psychologists, exercise physiologists and pediatricians conducted the intervention. Psychological and anthropometric measures were collected at the baseline and 6 months after the beginning of the intervention. Results: A total of 6 children ( 3 boys and 3 girls) completed the 6-month multidisciplinary group intervention, mean age 11.17 years $(\mathrm{sd}=0.75)$, and at least one of their parents enrolled in the intervention, mean parent bmi $28.05 \mathrm{~kg} / \mathrm{m}^{2}$ (sd = 3.41). Average number of years in nutrition consultation was 3.40 years $(s d=2.79)$. Statically significant decreases were found between baseline and end of the intervention assessments in the children bmi $z$-scores $(Z=-2.20, p=0.027)$, body fat percentage $(Z=$ $-2.20, p=0.028)$ and waist circumference $(Z=-2.20, p=0.028)$. Overal children quality of life also improved between the two assessment moments $(Z=-2.02, p=0.043)$.

Conclusion: The preliminary results seem to indicate a positive health effect of this type of intervention. Further research is needed to investigate the efficacy and cost-effectiveness of this type of intervention to childhood obesity in an outpatient treatment setting.

Conflict of Interest: None Disclosed

Funding: This research was partially supported by Foundation for Science and Technology through a doctoral scholarship (sfrh/bd/104182/2014) to Sofia Ramalho

\section{T4P90}

\section{Parental feeding practices and their determinants}

Gomes, M. ${ }^{1}$; Pimenta, F. ${ }^{2}$; Marôco, J. ${ }^{2}$; Leal, I. ${ }^{2}$

${ }^{1}$ ISPA - Instituto Universitário, ${ }^{2} I s p a$ - Instituto Universitário; William James Center For Research
Introduction: The prevalence of child obesity has been increasing in recent years and, since parents have a crucial influence over their child's eating habits, the aim of the present study is to ascertain if the parental feeding practices are determined by the parents own eating behavior, perceived self-efficacy, socio-demographic and health-related variables.

Methods: This convenience sample consists of 252 participants, both mothers and fathers of children between the age of 2 and 18 years old, with a mean bmi $25 \mathrm{~kg} / \mathrm{m}^{2}$. The participants filled in the Parental Feeding Style Questionnaire (Pfsq), the Generalized Expectations of Control Scale (Beegc-20) and the Dutch Eating Behavior Questionnaire (Debq). Structural equation modeling was used to build a structural model having parental feeding practices (Pfsq) as the dependent variable.

Results: The results indicated that self-efficacy predicts the encouragement $(\beta=.290 ; p=.012)$, emotional eating $(\beta=-.319 ; p<.001)$ and instrumental eating $(\beta=-.295 ; p<.001)$. The emotional eating (Debq) associates with emotional eating $(\beta=.171 ; p=.004)$. Also, the external eating (Debq) correlates itself with control $(\beta=-.155 ; p=.031)$, instrumental eating $(\beta=.273 ; p<.001)$ and emotional eating $(\beta=.137 ; p=.034)$.

Conclusion: These results indicate that parents with greater capacity of general self-efficacy tend to practice healthier parental feeding practices, being equally verified that the type of eating behavior of the parents predicts the parental feeding practices with their children. This shows their influence on the feeding environment of the children and their importance as providers of healthy/unhealthy feeding practices. Given that negative parental feeding is associated with similar patterns of adult (parent) own eating, primary screening in health care context might be useful to detect cases were children with overweight or unhealthy eating might translate their parents own eating habits; these cases/parents might benefit from support to manage their behavior in order to impact more positively their children's feeding practices.

Conflict of Interest: None Disclosed

Funding: Research relating to this abstract was funded by the Fundação para a Ciência e Tecnologia - Fct (grant Sfrh/Bpd/77799/2011). William James Center for Research is funded by the Fundação para a Ciência e Tecnologia - Fct (grant Uid/ Psi/04810/2013).

\section{T4P91}

\section{Parents' knowledge about infant feeding: Relationship with the child's nutritional status}

\section{Pinto, L. ${ }^{1}$; Aparício, G. '; Duarte, J.}

${ }^{1}$ Escola Superior de Saúde/Instituto Politécnico de Viseu

Introduction: Obesity is the most prevalent paediatric nutritional disease worldwide. This pathology results from the interaction of several factors, including unhealthy nutrition what is naturally linked to the role of the family in the development of eating behaviour. It is recognized that children should be taught early to develop healthy eating habits, having the family a key role in this process. Objectives: To relate parents 'knowledge about infant feeding to the child's nutritional status.

Methods: A cross-sectional, descriptive-correlational study was carried out on a non-probabilistic sample of 114 parents of pre-school (56.1\%) and school children (43.9\%), mean age 5.53 years $(\mathrm{Sd}=1.93)$ who attended the child health consultations at a Health Center in the Northern region of Portugal. Mothers had a mean age of 34.40 years $(S d=5.77)$ and fathers 36.50 years $(S d=9.155)$. Data were collected to sociodemographic characterization and the questionnaire on ' 'Parent Knowledge on Infant Feeding (Qai)"', (Aparício, Cunha, Duarte \& Pereira, 2012) was applied. Children's anthropometric evaluation and the Nchcs cut-offs (Cdc, 2000) were used to classify the nutritional status.

Results: Of the parents, $74.6 \%$ living in urban areas, had a mean of 1.98 children $(\mathrm{Sd}=0.922), 43 \%$ had secondary education, $20.2 \%$ was graduate and $69.3 \%$ were unskilled workers. Overall, the level of knowledge about infant feeding was classified as sufficient in $43.9 \%$ of the parents, $30.7 \%$ good and $25.4 \%$ insufficient, standing out the mothers positively, but not statistically significant in relation to fathers. Overall, $57.0 \%$ of the children 
were eutrophic and $29.9 \%$ overweight (including $13.2 \%$ obesity). Parents of normal weight children had higher mean values in Qai, while parents of overweight children had lower mean values, with significant differences in normal weight and obesity percentiles $(p=0.020)$. Parents with better knowledge tend to have children with normal weight and the lowest knowledge was related to child's overweight. The multivariate regression model explains about $9 \%$ of the observed variance in the "dietary rules" factor and 23\% in "food and health" factor.

Conclusion: These results show the relationship between parents' knowledge of infant diet and the child's weight. This fact justifies the investment in prevention that should be initiated at an early stage of child development. Promoting family empowerment, with guidelines for its autonomy in decisions making about healthy eating, encouraging even small changes without criticizing, may be one of the strategies for the prevention of childhood obesity.

Conflict of Interest: None Disclosed

Funding: No Funding

\section{T4P92}

\section{Physical activity and physical fitness in pediatric obesity: What are the very first clinical steps? recommendations from the european childhood obesity group (ECOG).}

David, $T^{1}$; Susanne, R. ${ }^{2}$; Marie-Laure, $F^{3}{ }^{3}$; Nathalie, F.4 ${ }^{4}$ Daniel, $W^{5}{ }^{5}$; Grace, ${ }^{6}{ }^{6}$

${ }^{1}$ Clermont Auvergne University, ${ }^{23}$. Department Of Sport Science And Kinesiology, Paris Lodron-University, Salzburg, Austria, ${ }^{3} 4$. Pediatric Endocrinology Department, Bicêtre Paris Sud University Hospital, Paris, ${ }^{4} 5$. Obesity Prevention And Care Program Contrepoids, Service Of Therapeutic Education For Chronic Diseases, Department Of Community Medicine, Primary Care And Emergency, University Hospitals Of Geneva And University Of Geneva, Geneva, Switzerland, 56 . Department Of Pediatrics, Paracelsus Medical University, Salzburg, Austria; Obesity Research Unit, Paracelsus Medical University, Salzburg, Austria, ${ }^{6} 7$. Physiotherapy Department, Temple Street Children'S University Hospital, Temple Street, Dublin, Ireland

Introduction: Although paediatricians are perceived by most parents as valued advisors concerning their children's weight management, they face a number of barriers which can limit their weight-related counselling and the referral to and delivery of lifestyle-based treatment. Such barriers can explain why paediatricians and practitioners often express a lack of effective counselling skills, especially regarding physical activity. There is a pressing need to increase their core treatment skills, self-confidence and efficacy related to physical activity and fitness assessment. The aim of this work is to provide practitioners with the basic and essential first steps that should be considered when it comes to physical activity and fitness in youth with obesity.

Methods: Three working group sessions have been conducted during the last European Childhood Obesity Group meeting in Thessaloniki (Greece) in October 2016. These working groups have been directed by specialists in the fields of physical activity and paediatric obesity. Attendance was composed of practitioners including paediatricians, nurses, coaches, physiotherapists, General practitioners and dieticians mainly. They have been asked to define physical activity and physical fitness, to describe how they would assess both in their practice and to explicit their potential barriers and limitations in this field.

Results: The discussions revealed that for practitioners, physical activity mainly represents structured exercise sessions. Practitioners reported questioning the child about engagement in exercise sessions but rarely about active transportation or daily activities. Similarly, very few explored whether their patients were at the very least reaching the age-related recommended Pa levels and sedentary behaviours. The attendees expressed a clear need for a clear definition of fitness. Themed discussions with Ecog participants revealed the necessity to clearly inform and debate the differences and similarities between $\mathrm{Pa}$ and $\mathrm{Pf}$ and between $\mathrm{Pa}$ recommendations and adapted physical activity interventions.
Conclusion: Practitioners assessing a child for obesity should introduce the theme of physical activity and fitness at initial assessment. This seems necessary for practitioners to adequately consider the role and importance of $\mathrm{Pa}$ and $\mathrm{Pf}$ when it comes to assessing obesity in youth; and to encourage a greater understanding of when and to whom an onward referral may be necessary.

Conflict of Interest: None Disclosed

Funding: No Funding

\section{T4P93 \\ Physical activity intervention program (movi-kids study) on preventing obesity in preschoolers}

Cavero-Redondo, I.' ; Alvarez-Bueno, C. '; Garrido-Miguel, M. '; Martinez-

Vizcaíno, V. '; Diez-Fernandez, A. '; Fernandez-Infante, A. ${ }^{2}$

${ }^{1}$ Health and social research center, Universidad de Castilla-La Mancha, Cuenca, Spain, ${ }^{2}$ Health And Social Research Center, Universidad De Castilla-La Mancha, Ciudad Real, Spain

Introduction: This study aims to assess the effectiveness of a physical activity intervention (Movi-Kids) in schoolchildren for preventing obesity, improving fitness, and reducing cardiovascular risk during the adiposity rebound period in children from 4-to-7 years of Castilla-La Mancha (Spain).

Methods: This was a cross-over randomised cluster trial that involved 21 schools located in the provinces of Cuenca and Ciudad Real, Castilla-La Mancha region (Spain).

Adiposity parameters included: Body Mass Index (Bmi) (calculated with weight and height), waist circumference and percentage fat mass were measured twice by standard procedures.

Physical fitness parameters included: cardiorespiratory fitness, as measured by $20-\mathrm{m}$ shuttle run test, lower limbs muscular strength, as measured by standing long jump test, and analyzed divided by weight, and velocity/agility, by $4 \times 10 \mathrm{~m}$ shuttle run test.

Maximum-likelihood random-effects regression models were used to estimate cluster effects. Effect estimates described differences between mean change in Movi-Kids intervention group versus control group, adjusted by age, gender, socioeconomic level, weight status, and school's cluster factor. Results: Table 1. Data are reported as mean $\pm \mathrm{Sd}$.

Conclusion: Our data suggest that Movi-Kids physical activity intervention was effective on reducing waist circumference and improving physical fitness (but significantly only velocity/agility and lower limbs strength) at early ages.

\section{Conflict of intertest: None Disclosed}

Funding: This study was funded by the Ministry of Economy and Competitiveness-Carlos Iii Health Institute (Fis Pi12/02400).

\section{T4P94}

\section{Platelet to lymphocyte ratio and HOMA-IR in pediatric obesity and overweight}

\section{Santos, C. ${ }^{1}$; Picoito, J. ${ }^{1}$; Teixeira, A. ${ }^{1}$; Rodrigues, C. ${ }^{1}$; Ferreira, S. ${ }^{1}$}

${ }^{1}$ Pediatrics Department, Centro Hospitalar Cova da Beira, Covilhã, Portugal

Introduction: There is a complex interplay between obesity, inflammation and insulin resistance. We aimed to evaluate the relationship between inflammatory markers, as platelet-to-lymphocyte ratio (Plr) and neutrophil-to-lymphocyte ratio (Nlr), and insulin resistance, measured by homeostasis model assessment of insulin resistance (Homa-Ir), in a sample of overweight and obese children and adolescents.

Methods: In this cross-sectional retrospective study, a total of 92 pediatric patients were enrolled (77 obese, 15 overweight) from our general pediatric outpatient clinic. All subjects had a complete blood cell count, glucose, insulin and lipid panel measurements performed in fasting blood samples. 
Results: Homa-Ir was positively associated with Plr $(\mathrm{P}<0,001)$ and Nlr $(\mathrm{p}<0,05)$. After adjustment for age, sex and Bmi, the association between Homa-Ir and Plr remained statistically significant.

Conclusion: The positive and independent association between Plr and Homa-Ir may stem from a pro-inflammatory status associated with obesity, and from the complex interplay between platelets, insulin signaling and inflammation. As Plr is an easily available inexpensive marker of inflammation, it is a potential biomarker of insulin resistance and severity of obesity and it could be useful in the follow-up of these patients in daily clinical practice.

Conflict of Interests: None disclosed.

Funding: No Funding.

\section{T4P95}

\section{Portuguese adolescents incidence of overweight and obesity and relation with access to audiovisual and playful means}

Oliveira, A. ' ; Vázquez, J.2 ; Albuquerque, C. ${ }^{1}$; Oliveira, M. ${ }^{3}$; Cunha, M. '; Andrade, A. ${ }^{7}$; Bica, ${ }^{1}{ }^{1}$

${ }^{1} \mathrm{Cl} \&$ Dets - Instituto Politécnico de Viseu - Portugal, ${ }^{2}$ Upsa - Salamanca -Spain, ${ }^{3}$ Aces Dão-Lafões - Portugal

Background: Currently, more than 300 million people are considered obese and 1.2 billion, or $34 \%$ of the world population is considered to have overweight. Particularizing this epidemiological framework in adolescence, with the data demonstrate that in the Who European Region $27 \%$ of teenagers, aged 13 and 33\% aged 11 are overweight. In Portugal $31 \%$ of adolescent males and $18 \%$ of female adolescents are overweight and obesity affects $24 \%$ to $17 \%$ for each gender respectively (Who, 2013). Objectives: Analyze the existence of causality determinants associated with access to audiovisual and playful means (frequency hours watching television and frequency of hours to entertain with computer games) as determining factors in the body mass index of adolescents.

Methods: This study was observational and transversal, quantitative, non-experimental, descriptive and correlational, was conducted with 1467 adolescents with a mean age of $14.01( \pm 1,43 \mathrm{Dp})$, mostly sex female $(52.3 \%)$, living in rural areas $(63.32 \%)$ in various districts of mainland Portugal, attending between the 5 th and 12 th grade public education, it is carried out anthropometric assessment and stratification index obesity based on the framework of the National Center for Health and Statitics (Nchs) of Cdc (2000).
Results: It was possible to see that $7.2 \%$ of adolescents are obese, $14.5 \%$ pre-obesity, $74.3 \%$ normal weight and $4 \%$ underweight. In boys: $10.0 \%$ are obese and $15.1 \%$ show pre-obesity, while girls have $4.6 \%-13.8 \%$ and obesity - pre-obesity. The inferential analysis confirms significant effect of some of the determinants studied in body mass index (Bmi) showing the teenagers, who are described below.

Study of the relationship between the determinants associated with access to audiovisual habits and playful means and body mass index in adolescents:

The highest number of hours of television viewing is associated with a higher body mass index $\left(\chi^{2}=28.120, \mathrm{p}=, 021\right)$; in particular, are obese (res. $=2.1$ ) and pre-obese (res. $=2.5$ ) more hours viewing Tv.

They are also teenagers that more hours are entertained with computer games that have a higher body mass index $\left(\chi^{2}=17.438, \mathrm{p}=, 043\right)$.

Conclusion: The resulting inferences of this study invite us to reflect on the importance of creating and monitoring of action programs in the field of early prevention or contingency for an effective reduction of adverse health behaviors by adolescents. Clearly this task, being urgent, must be coordinated between the whole school community, with the direct involvement of parents and guardians in order to develop intervention strategies addressed the real needs of adolescents.

Keywords: Bmi, Obesity, Adolescents, Audiovisual Habits.

\section{T4P96}

\section{Positive trend towards a healthier nutrition in school-aged children from sofia. Results from the EPHE study}

\section{Handjieva-Darlenska, ${ }^{1}{ }^{1}$; Kuzeva, A. ${ }^{2}$; Handjiev, S. $^{2}$}

${ }^{1}$ Medical University, Sofia, Bulgaria, ${ }^{2}$ Bulgarian Association For The Study Of Obesity And Related Diseases

Introduction: Childhood obesity in Bulgaria has dramatically increased during the last decades. According to WHO Bulgaria is on the fifth place of childhood obesity among 53 member countries in Europe. The reasons of these striking numbers are clear: a sedentary lifestyle and a poor nutrition. Thereafter, the aim of our study was to evaluate the nutritional and physical activity behavior of school-aged children in Sofia.

Methods: A total number of 205 children (46,8\% - boys and 52,7\% - girls, with a mean age of 7.97) from three schools in Sofia and their parents have participated. A self-administrated parental questionnaire was distributed to parents. The questionnaire focus was on fruit and vegetable consumption, soft drinks and fruit juices consumption, water intake,

Table 1. to T4P93 ${ }^{*}$ Lower values indicates better Results; Icc=intercluster correlation.

\begin{tabular}{|c|c|c|c|c|c|c|c|}
\hline & \multicolumn{2}{|l|}{ Baseline } & \multicolumn{2}{|c|}{ After intervention } & \multicolumn{3}{|l|}{ Effect estimate } \\
\hline Adiposity & $\begin{array}{l}\text { Movikids } \\
\text { Intervention } \\
\text { Group } \\
n=619\end{array}$ & $\begin{array}{l}\text { Control Group } \\
n=815\end{array}$ & $\begin{array}{l}\text { Movikids } \\
\text { Intervention } \\
\text { Group } \\
\mathrm{n}=619\end{array}$ & $\begin{array}{l}\text { Control Group } \\
n=815\end{array}$ & $\begin{array}{l}\text { Estimate } \\
(95 \% \mathrm{Ci})\end{array}$ & $P$ value & ICC \\
\hline Percentage Fat mass & $20.05 \pm 7.33$ & $20.04 \pm 7.97$ & $20.08 \pm 5.85$ & $19.73 \pm 5.85$ & \begin{tabular}{|l|}
$-1.42(-2.99$ to \\
$0.15)$ \\
\end{tabular} & 0.076 & 0.10 \\
\hline Waist circumference & $55.88 \pm 6.63$ & $55.38 \pm 6.87$ & $56.57 \pm 7.05$ & $56.49 \pm 7.40$ & $\begin{array}{l}2.27(0.69 \text { to } \\
3.85)\end{array}$ & 0.005 & 0.06 \\
\hline Bmi & $15.93 \pm 2.63$ & $15.76 \pm 2.72$ & $16.08 \pm 2.48$ & $15.94 \pm 2.48$ & $\begin{array}{l}-0.22(-0.73 \text { to } \\
0.29)\end{array}$ & 0.407 & 0.08 \\
\hline$\%$ Overweight/obesity & 21.00 & 19.02 & 20.84 & 19.63 & - & - & - \\
\hline \multicolumn{8}{|l|}{ Physical fitness } \\
\hline Cardiorespiratory fitness & $49.65 \pm 6.51$ & $48.75 \pm 7.67$ & $50.20 \pm 6.15$ & $50.18 \pm 6.81$ & $\begin{array}{l}2.64(-1.05 \text { to } \\
6.33) \\
\end{array}$ & 0.161 & 0.05 \\
\hline Velocity/Agility* & $17.07 \pm 5.24$ & $17.26 \pm 6.17$ & $15.96 \pm 6.28$ & $16.48 \pm 7.51$ & \begin{tabular}{|l|}
$-2.24(-3.31$ to \\
$-1.18)$ \\
\end{tabular} & $<0.001$ & 0.14 \\
\hline Flexibility & $27.62 \pm 6.49$ & $27.96 \pm 7.16$ & $26.70 \pm 7.43$ & $26.65 \pm 7.78$ & $\begin{array}{l}1.91(-1.47 \text { to } \\
5.30)\end{array}$ & 0.268 & 0.01 \\
\hline Strength & $4.53 \pm 2.00$ & $4.48 \pm 2.27$ & $4.51 \pm 1.79$ & $4.46 \pm 2.02$ & $\begin{array}{l}0.80(0.33 \text { to } \\
1.27)\end{array}$ & 0.001 & 0.02 \\
\hline
\end{tabular}


sedentary behavior, and their family-environmental determinants, sleep habits of the child, socio-demographic characteristics of the family, and milk consumption.

Results: Children from good income families had significantly higher fruit, fruit juices and soft drinks consumption compared to the children from not good income families $(\mathrm{p}<0.001, \mathrm{p}<0.05, \mathrm{p}<0.05$, respectively). The children from not good income families spent significantly more total screen time compared to the children from high-income families, 25.7 hours vs. 18 hours, respectively, $\mathrm{p}<0.05$. strong="'> Conclusion: Our study has demonstrated a positive trend towards a healthier nutrition based on a high fruit consumption. However, the sedentary lifestyle is very popular in school-aged children in Sofia which could increase the risk for obesity and its metabolic complications.

Conflict of interest: None Disclosed

Funding: Research relating to this abstract was funded by Ephe project

\section{T4P97}

\section{Prediction of metabolic health in 19-year old Swedish students}

Mårild, S. ' ; Sjöberg, A. '; Wikland, K. '; Chaplin, J. '; Lissner, L. '; Chaplin, J.'; Dahlgren, J.'

${ }^{1}$ Göteborg University, Sweden

Introduction: To assess the overall metabolic state, or health, in young adults a continuous metabolic score is a useful option. The aim of the study was to find variables predicting the metabolic score in 19 year old subjects using data of each subject's growth pattern from birth to puberty, heredity, parental features and a lifestyle index in adult age.

Methods: The study population was recruited from a population of 18-19 year-old students participating since birth in a longitudinal growth study. 1685 subjects living in Göteborg, Sweden with complete information on weight, length at birth and growth data at ten years of age were eligible and contacted by a survey team. Those accepting to participate had the following variables measured: body weight, height, waist circumference, blood pressure, blood glucose, Hdl- and Ldl- cholesterol, triglycerides and questionnaire-derived information on heredity, parental features and lifestyle. The metabolic score was calculated by adding the z-scores for waist circumference, blood pressure, glucose and (triglycerides + Hdl-cholesterol)/2. The analysis of the association between the score and possible predictors was done separately by sex. First univariate associations were analyzed, then the most significant predictors were included in a stepwise forward multiple linear regression model.

Results: A total of 517 (female 256) 18-19 year-old subjects were included in the study. The socioeconomic position was higher and body mass index (Bmi) at ten years of age was lower in the study group compared to remaining subjects in the growth study. The median (min;max) metabolic score was $-0.060(-7.3 ; 12.7)$ for men and $-0.232(-11.1 ; 12.3)$ for women and median body mass index was 21.7 (men) and $21.0 \mathrm{~kg} / \mathrm{m}^{2}$. The incidence of cardiovascular disease was $22 \%$ for fathers and $13 \%$ for mothers. Some 15 predictors of a total of 75 tentative predictors had significant $(\mathrm{p}<0.01)$ associations to the metabolic score in univariate analysis. The models in the multivariable logistic regression analysis had a low explanatory power (R-square 0.11 for men, 0.13 for women). The Bmi standard deviation score at 10 years of age was found to be a significant predictor in both sexes. Other significant predictors were birth weight and use of contraceptives at 18 years of age in females and age at adiposity rebound and maternal Bmi in males.

Conclusion: The Bmi standard deviation score at 10 years of age appears to predict metabolic state in both sexes in young adult age, which is of interest for preventive interventions.

\section{T4P98 \\ Predictive power of birth weight and early life body mass index on adiposity measures of adolescents}

\section{Oliveira-Santos, J. ' ; Santos, R. ${ }^{2}$; Moreira, C. ${ }^{1}$; Abreu, S. ' Lopes, L. ${ }^{\text {; }}$;}

Agostinis-Sobrinho, C. ' Mota, J. ${ }^{1}$

${ }^{1}$ Research Centre in Physical Activity, Health and Leisure (Ciafel) - Faculty of Sport, University of Porto, ${ }^{2}$ Early Start Research Institute, Faculty Of Social Sciences. University Of Wollongong, Australia

Introduction: The tracking of excessive adiposity from early childhood into adolescence and adulthood has been studied ${ }^{1,2}$. However, it remains unclear how early it may be the ideal period to start preventing overweight and obesity later in life. We aimed to assess the ability of birth weight (Bw), body mass index (Bmi) at birth, and Bmi from month 6 until 6 years of age in predicting an unfavourable status of several adiposity measures in adolescents.

Methods: A cross-sectional study with 539 adolescents (292 girls, 247boys) with a mean age of $13.94 \pm 1.62$ years took place in five schools from the north and centre of Portugal.

Anthropometric data on birth and growth were collected from individual health booklets of each subject. Actual weight, height and waist circumference (Wc) were measured, and Bmi and waist to height ratio (WhtR) were subsequently calculated. Body fat percentage (Bf\%) was estimated by bioimpedance (Tanita Bc532 InnerScan). Adolescents were classified as overweight/obese/overfat according to national and international reference values.

Receiver operating characteristic curves were used to explore the ability of Bw, Bmi at birth, and Bmi at months 6, 12, 18, 24, and at 3, 4, 5 and 6 years of age to predict unfavourable status for each adiposity measure in adolescence.

Results: Bw and Bmi at birth presented no predictive power for unfavourable categories for any measure of adiposity in adolescents of both sexes. In girls, except Bmi at month 6 who failed to predict WhtR, Bmi from 6 months to 6 years of age presented an increasing predictive power for all the adiposity measures in adolescence.

In boys, Bmi at 6 months presented no predictive power for any measure of adiposity; from 12 months of age onwards, Bmi presented predictive power for unfavourable Bmi at adolescence; from 24 months ahead for Wc, and from 3 years onwards for Bf\% and WhtR.

Conclusion: Our data suggest that, with regard to prediction and consequent prevention of an unfavourable category in various measures of obesity during adolescence, the growth period from 6 months onwards may be more valuable than the period relative to birth.

\section{References:}

1 Evensen, E., et al. (2016). Tracking of overweight and obesity from early childhood to adolescence in a population-based cohort - the Tromsø Study, Fit Futures. Bmc Pediatrics, 16 (1): 64.

2 Freitas, D., et al. (2012). Tracking of fatness during childhood, adolescence and young adulthood: a 7-year follow-up study in Madeira Island, Portugal. Ann Hum Biol, 39 (1): 59-67.

\section{Conflict of Interest: None.}

Funding: Research relating to this abstract was funded by the Portuguese Foundation for Science and Technology (Fct) grants: Sfrh/Bd/88984/2012 and Uid/ Dtp/00617/2013, and by Ciafel (Faculty of Sport, University of Porto).

\section{T4P99}

\section{Presence of obesity in children with sickle cell anemia}

Cople-Rodrigues, C. ${ }^{1}$; Mataratzis, P. ${ }^{1}$; Lemos, D. ${ }^{2}$; Calixto-Lima, L. ${ }^{3}$; Citelli, M. ${ }^{4}$; Oliveira, C. ${ }^{1}$

${ }^{1}$ State of Rio de Janeiro University, ${ }^{2}$ Federal Of Rio De Janeiro University, ${ }^{3}$ Cancer Institute, ${ }^{4}$ State Of Rio De Janeiro University

Introduction: Sickle cell anemia (Sca) is a chronic hematologic disease that presents with intense hemolysis and vaso-occlusive events. Historically children with Sca have malnutrition and short stature. This study aims to describe the nutritional status of children with Sca and to study 
the association of nutritional status with laboratory markers of hemolysis and body iron status.

Methods: We evaluated 89 children aged 24 to 71 months with a diagnosis of Sca and 48 children without the disease (control). Anthropometric evaluation (Bmi/A -body mass index/age; H/A- height/age), body fat $\%$, as well as laboratory analysis of hemoglobin, \%reticulocytes, lactate dehydrogenase, transferrin, iron and ferritin.

Results: Children with Sca showed a predominance of adequate Bmi/A $(81.1 \%)$, followed by the risk of overweight $(14.1 \%)$ and the same percentage of underweight and overweight/obesity $(2.4 \%)$. In the contro group, $48.9 \%$ were normal weight, followed by the risk of obesity $(21.3 \%)$ and overweight / obesity (27.7\%). There was difference between groups: Bmi/A, body fat\%, hemoglobin, transferrin (lower in Sca children); \% Reticulocytes, Ldh, Leukocytes, Iron and Ferritin (higher in Sca children). All markers of hemolysis and body iron status were associated with Bmi/A - \%reticulocytes (95\% confidence interval [Ci] -0.108, -0.030; p < 0.001), Ldh (95\% Ci -0.003, 0.000; p < 0.019), hemoglobin (95\%Ci 0.153, $0.367 ; \mathrm{p}<0.000)$, transferrin (95\% Ci 0.003, 0.007; p < 0.000), iron $(95 \%$ $\mathrm{Ci}-0.006,-0.001 ; \mathrm{p}<0.012)$ and ferritin $(95 \% \mathrm{Ci}-0.001,0.000 ; \mathrm{p}<0.006)$ and the short stature was associated with $\mathrm{Hb}(95 \% \mathrm{Ci} 0.057,0.248$; $\mathrm{p}<0.002)$, transferrin $(95 \% \mathrm{Ci} 0.000,0.004 ; \mathrm{p}<0.014)$ and iron $(95 \% \mathrm{Ci}$ $-0.006,-0.001 ; \mathrm{p}<0.010)$.

Conclusion: There was a change in the nutritional profile in the children evaluated, with the appearance of obesity. It is also observed that there is an association between hemolysis markers and body iron status with nutritional status. Any emphasis on improving the clinical management of sickle cell disease should be prioritized so that nutritional status is preserved.

Conflict of Interest: There is no conflict of interest

Funding: This study was funded by the Blood and Hemodynamic Coordination of the Brazilian Ministry of Health.

\section{T4P101}

\section{Prevalence of overweight and obesity among 7-year-old bulgarian schoolchildren in 2016}

Duleva V'; Chikova-Iscener E'; Petrova $S^{1}$; Dimitrov $P^{1}$; Bojilova $D^{1}$

${ }^{1}$ National Center of Public Health and Analyses

Introduction: As many other European countries, Bulgaria has also faced significant increase in the prevalence of overweight and obesity among children. In the period 1998-2011 the prevalence of overweight and obesity among children aged 7-18 years has more than doubled. Data from different nationally representative cross-sectional surveys for 7-year-old children, recorded a trend for significant increase of overweight and obesity in 3 survey periods $(1998,2008,2011)$, while study in 2013 showed a stabilization of the rates in boys and decline of those among girls (Petrova S et al., 2015). The aim of the present study is to assess the current prevalence of obesity and overweight among 7-year-old schoolchildren.

Methods: From March to May 2016 a cross-sectional study was carried out in Bulgaria on nationally representative final effective sample of 3378 schoolchildren aged 7-years under the protocol developed by Who as part of the Who European Childhood Obesity Surveillance Initiative (Cosi). Height and weight were measured with standardized anthropometric equipment. Overweight and obesity were assessed by Bmi-for-age using the Who Growth Reference 2007 definitions.

Results: In 2016 the prevalence of overweight (including obesity) among boys was $31.8 \%$ and $30.1 \%$ among girls. Obesity rates were $16 \%$ and $12 \%$ (boys and girls respectively).

Conclusion: The prevalence of overweight and obesity among 7-year-old schoolchildren in 2016 compares closely with the one measured in 2013 (Duleva V et al., 2014), reconfirming the observed trend for plateauing. Nevertheless, those rates remain high and represent important public health problem that needs further targeted action in the related health and nutrition policies.
Conflict of Interest: None Disclosed

Funding: No Funding

\section{T4P102}

\section{Prevalence of overweight and obesity among school-aged children in northeast Romania}

Elena, F. ${ }^{1}$; Mihaela, M. ${ }^{1}$; Mihaela, T. $^{1}$

${ }^{1}$ University of Medicine and Pharmacy Grigore T. Popa lasi

Introduction: The prevalence of obesity and overweight has dramatically increased in Europe and also in Romania in the last 10 years. Evaluation of the nutritional status in school aged children is a base for further preventive and interventional programs in children obesity.In this study, we assess the prevalence of overweight and obesity among school-aged children from Northeast Romania.

Methods: The study cohort consists of 692 children from schools in two district of Northeast Romania, 378 boys (54\%) and 318 girls (46\%), aged 6.08 to 16.4 years, mean age 9.8 years $\pm 2.08 \mathrm{Sd}$. Based on the weight and height measurement, body mass index $(\mathrm{Bmi})$ was calculated. Obesity and overweight was defined according to Cdc recommendations. Wc percentile were calculated using database from Fernandez et al., 2005 and abdominal obesity was defined like Wc above $90^{\text {th }}$ percentile. Continuous variables with normal distribution were expressed as mean \pm standard deviation. The categorical variables were expressed as absolute frequency and relative frequency. Data were analyzed using Spss V.21 software. Statistical significance was set to $\mathrm{p}<0.5$.

Results: The prevalence of overweight was $14 \%$ of the study group, without significant difference between male and female (14.8\% vs $12.8 \%$ ). The prevalence of obesity found in this study was $11 \%$. Male encountered a significant higher percentage of obesity then female, $16 \%$ vs $5 \%(p<0.01)$. Bmi values were significantly higher $(\mathrm{F}=0.57, \mathrm{p}=0.0123,95 \% \mathrm{Ci})$ in obese boys (Bmi mean 23.903 $\pm 2.62 \mathrm{Sd}$ ) compared to obese girls (Bmi mean $22.54 \pm 1.98 \mathrm{Sd}$ ). The prevalence of abdominal obesity was $13.4 \%$. We calculated the Hazard Ratio (Hr) for overweight according to children's age adjusted by sex. The risk for overweight in boys older than 12 years was $\mathrm{Hr}=2.81$ (95\% Ci: 1.93-3.65) compared with those aged less than 12 years. In girls, calculated risk in the age group over 12 was $\mathrm{Hr}=1.76$ (95\%Ci: 1.12-4.57).

Conclusion: The prevalence of overweight and obesity is high in schoolaged children. These results are part of a nutritional screening study conducted in schools in Northeast Romania to identify children and adolescents with overweight and obesity, in order to include them in an optimal management program.

\section{T4P103 \\ Prevalence of risk behaviors for eating disorders and associated factors in high school adolescents in Brazil}

\section{Kretzer, M. '; Vietta, G. '; Kretzer, I. ${ }^{2}$; Sousa, C. ${ }^{2}$; Pereira, E. ${ }^{\prime}$}

${ }^{1}$ Universidade do Sul de Santa Catarina, ${ }^{2}$ Universidade Do Vale Do Itajai

Introduction: Eating disorders affect mainly female adolescents, causing significant losses, both biological and psychosocial. In adolescence it takes time for assimilation and acceptance of the body image due to the transformations proper to the age The need for acceptance by the social group makes the adolescent vulnerable to eating disorders. Studies in school children are needed to alert the need for investments in nutritional education programs in childhood and adolescence. Objective to evaluate the prevalence and factors associated with risky eating behaviors high school adolescents in Brazil.

Method: A cross-sectional study with 3,322 female adolescents of Brazil, high school students, distributed from the first to the third year, in 2015, from a public domain database of the National School Health Survey (Pense) of the Brazilian Institute of Geography and Statistics (Ibge). Behavior of bulimic risk evaluated according to induction of vomiting, use of 
laxatives or remedies to lose weight without medical follow-up. Analysis in Spss 18.0, Chi-square test and $\mathrm{p}<0.05$.

Results: mean age of 16.05 years, with $35.4 \%$ in the age group of 11 to 15 years. Eutrophic nutritional status (74.4\%), 16.3\% overweight and 7.0\% obesity. Schooling of the mother below incomplete secondary school (39.1\%). Frequency of feeding up to 5 days per week at breakfast (57.0\%), consumption of fried salty (68.6\%), soft drinks (69.1\%), processed foods (54.9\%), fast food 91.0\%). Consumption of vegetables (50.1\%) and fresh fruit (61.9\%). School offers snack to $67.6 \%$. Habit to feed in front of the Tv $(51.4 \%)$ and $54.9 \%$ remain 4 or more hours daily in front of the Tv. Physical activity practice at school (32.3\%) and out of school (48.6\%). Already consumed alcohol (70.1\%), tobacco (25.4\%), illicit drug (12.8\%). History of bullying (48.8\%). It considers an important body image (86.1\%), satisfaction with the body (58.3\%), and feeling fat / very fat in $27.1 \%$. Prevalence of bulimic symptoms in $2.9 \%$. Risk behavior for associated eating disorder $(\mathrm{p}<0.05)$ with daily consumption of breakfast less than 4 times a week, low fruit consumption, alcohol use, smoking and illicit drugs, history of bullying, body image dissatisfaction And feeling fat / very fat.

Conclusion: High school studentsfrom Brazil present inadequate eating habits, with a high prevalence of processed and ultraprocessed foods. Psychosocial factors related to alcohol consumption, smoking and drugs are associated with risk behaviors for eating disorders.

Keywords: Eating Disorders,risk behaviors, female adolescents

Conflict of Interest: None Disclosed

Funding: No Funding

\section{T4P104}

\section{Promising intervention strategies for prevention of obesity and other risk factors for non-communicable diseases in early childhood}

\section{Martin, A. ${ }^{1}$; Hughes, A. ${ }^{2}$; Reilly, J. ${ }^{2}$}

${ }^{1} 1$. Usher Institute for Population Health Sciences and Informatics, University of Edinburgh, Scotland; 2. Physical Activity for Health Group, School of

Psychological Sciences and Health, University of Stathclyde, Scotland, ${ }^{2}$ Physical Activity For Health Group, School Of Psychological Sciences And Health, University Of Stathclyde, Scotland

Introduction: Rates of child obesity are increasing in countries that are experiencing rapid economic growth and demographic transition. The evidence base for interventions to prevent obesity and other risk factors for non-communicable diseases (Ncds) is inadequate for global recommendations and national policy. Hence, a research initiative to generate evidence that will inform national policy and decision-making to prevent obesity in children and reduce risk of Ncds in adult life is needed. The aim of this research was to support the development of a research initiative protocol. The objective was to identify intervention strategies associated with beneficial effects on obesity and Ncds.

Methods: In May 2016, the Cochrane Library and Pubmed were systematically searched for systematic reviews of interventions to prevent obesity and Ncds in healthy children. No language limits were set. Using a narrative synthesis approach, effectiveness of interventions that included a dietary and/or physical activity component were synthesised by intervention function and level of socio-ecological influence. The quality of included systematic reviews was assessed independently using the Risk of Bias in Systematic Reviews (Robis) tool.

Results: $23 / 82$ screened reviews were included. Eleven reviews (8 primary studies) and 19 reviews (36 primary studies) targeted obesity outcomes (Bmi z-score, prevalence, body fat) in children aged 0-23 months and $2-5$ years, respectively. No study reported on Ncd risk factors and only 2 were from low-middle income countries. All but one quality appraised review were of high risk of bias. In children aged 0-23 months, beneficial interventions typically included education and modelling strategies targeting both diet and physical activity, and involved the child, parents and clinical care staff. In children aged 2-5 years, beneficial interventions typically also targeted multiple behaviours and employed incentives in addition to other intervention functions like education, training, modelling, and persuasion. Successful interventions involved the child, parents and child care staff. Environmental restructuring to facilitate behaviour change was only effective in 1 of 4 studies. There was no evidence that studies including strategies to increase the means/reduce the barriers of behaviour change resulted in obesity prevention.

Conclusion: Successful interventions to prevent childhood obesity exist in high-income countries and target multiple behaviours, employ multiple intervention functions and involve all three major agents of child development: the child, parents and clinic/child care.

Conflict of Interest: The authors declare no conflict of interest.

Funding: This research was funded by the World Health Organisation: Hqmca161093/ 2016-652723-0

\section{T4P105}

Questionnaire on lifestyle for 8-10 year-old children: Assessment of sensitivity to change

Monique, R. '; Gaelle, B. ${ }^{2}$; Valerie, D. ${ }^{3}$; Alain, D. ${ }^{4}$

${ }^{1}$ Lille2 University Ea2694, ${ }^{2}$ Flvs Association, ${ }^{3}$ Lille2 Universitu Ea2694, ${ }^{4}$ Lille2 University Ea 2694

Introduction: Vivons en Forme (Vif) is a community based program aimed at helping municipalities that adhere to it to implement actions in line with the recommendations of the French Nutrition Program. Most of these actions are directed towards school-aged children. To evaluate these actions we constructed a questionnaire designed to assess the lifestyle of children and asked both to children and parents. The aim of this work is to study the sensitivity to change

Methods: The study was carried out in seven Ce2 / Cm1 / Cm2 classes in two different cities. The questionnaire was asked at the beginning and end of the school year in schools conducting actions about breakfast skipping (Br), morning snack (Sn), nibbling in front of the tv (Tvn). The children $(n=153)$ completed the questionnaire in the classroom; Parents were given the questionnaire and 122 answered. Comparisons were made by paired chi-square test..

\section{Results:}

Conclusion For children, the questionnaire shows changes in agreement with the actions carried out. The lack of sensitivity observed in the parents can be explained by the discrepancy already reported in a previous study showing that the parents underestimate unhealthy behaviours. These results suggest that in children aged $8-10$ years the questionnaire measures declared changes. The results suggests that it is not useful to send a questionnaire to parents.

Funding: Research relating to this abstract was funded by Nestle France Fundation

Table 1 to T4P105

\begin{tabular}{|l|l|l|l|l|l|l|}
\hline & \multicolumn{2}{|l|}{ Children } & \multicolumn{3}{l|}{ Parents } \\
\hline & Beginning & End & $\mathrm{p}$ & Beginning & End & $\mathrm{p}$ \\
\hline $\operatorname{Br}(\%)$ & 93 & 98 & .001 & 98 & 98 & Ns \\
\hline Sn (\%) & 63 & 57 & .002 & 70 & 64 & Ns \\
\hline Tvn (\%) & 59 & 41 & .001 & 27 & 21 & Ns \\
\hline
\end{tabular}




\section{T4P106}

Register of primary health care visits (avohilmo) as data source for monitoring the prevalence of overweight among Finnish children

Mäki, P. '; Lehtinen-Jacks, S. ${ }^{2}$; Vuorela, N. ; Levälahti, E. '; Koskela, T.'; Saari, A. ${ }^{4}$; Mölläri, K. ${ }^{1}$; Mahkonen, R. $^{1}$; Salo, J.5; Laatikainen, . $^{6}$

${ }^{1}$ National Institute for Health and Welfare, ${ }^{2}$ University Of Tampere, National Institute For Health And Welfare, ${ }^{3}$ Helsinki University Hospital, ${ }^{4}$ University Of Eastern Finland, Kuopio University Hospital, ${ }^{5}$ National Institute For Health And Welfare, Oulu University Hospital, ${ }^{6}$ National Institute For Health And Welfare, University Of Eastern Finland,

Introduction: Almost all children in Finland regularly participate in health check-ups administered by child welfare clinics and school healthcare services. The growth of children is systematically monitored, and data on height and weight recorded in the local electronic patient records. Consequently possibilities for monitoring children's obesity have existed for long, but nationwide data on the prevalence of overweight and obesity among children in Finland have been lacking.Since 2011, real-time data have been collected from primary health care services of the public sector to a register of Primary Health Care Visits (Avohilmo), including information on height and weight measured in the child welfare and school healthcare services. The data collection covers all health centres and municipalities in Finland. Our aim was to evaluate the feasibility of utilising the Avohilmo register data for nationwide monitoring of the prevalence of overweight and obesity among children between 2 and 16 years of age. Methods: Data for this study were collected from the Avohilmo register All information of visits to the child welfare clinics and school health services between 1 June 2014 and 31 May 2015 were included. Only those municipalities where the coverage of child growth data was $65 \%$ or more were included in the analyses of prevalence of overweight and obesity. The age of a child was defined as 2-6 years + one month and 7-16 years + three months. The final data included 96,341 children. Overweight and obesity were defined using the international Bmi cut-offs for overweight and obesity (Iotf).

Results: The coverage and quality of height and weight data differed substantially between municipalities. About one fifth of the municipalities had the coverage of $65 \%$ or more, and approximately half of the municipalities had the coverage of less than $10 \%$.In those municipalities where the coverage was $65 \%$ or more, $19 \%$ of boys and girls were classified as overweight, and $5 \%$ of boys and $4 \%$ of girls as obese.

Conclusions: The prevalence figures observed using the Avohilmo data are in line with previous studies using other methods of data collection. The Avohilmo register has the potential to serve in the nationwide monitoring of the prevalence of overweight and obesity among Finnish children. However, the validity of the local record-keeping and the coverage of the Avohilmo register data has to be improved.

T4P107

Relationship between meeting 24-hour movement guidelines and measures of adiposity in children

\section{Katzmarzyk, $P^{1}$; Staiano, $A .^{1}$}

${ }^{1}$ Pennington Biomedical Research Center

Introduction: The Canadian 24-h Movement Guidelines provide recommendations for moderate-to-vigorous physical activity (Mvpa), sedentary behaviour (television viewing) and sleep for children and youth. The purpose of this study was to evaluate the relationship between adherence to these guidelines and adiposity in a biracial sample.

Methods: The sample included 357 white and African American children aged 5-18 years. Physical activity, television viewing and sleep duration were measured using questionnaires, and meeting the 24-h guidelines was defined as: $\geq 60 \mathrm{~min} /$ day of Mvpa on at least 5 days per week, $\leq 2 \mathrm{~h} /$ day of Tv time, and sleeping 9-11 h/night (ages 5-13 y) or 8-10 h/night (ages 14-18 y). Height and weight were measured using standard techniques and Bmi \%iles were computed using Cdc reference data. Obesity was defined as $\mathrm{Bmi} \geq 95^{\text {th}} \%$ ile. Total fat mass $(\mathrm{kg})$ was measured by Dxa, and abdominal subcutaneous (Sat; $\mathrm{kg}$ ) and visceral fat (Vat; $\mathrm{kg}$ ) were measured using Mri. General linear models and logistic regression were used to evaluate the associations among meeting the 24-hour guidelines and adiposity. Statistical significance was set at $\mathrm{p}<0.05$.

Results: Overall, $35 \%, 31 \%$ and $52 \%$ of the sample met the guidelines for Mvpa, Tv time, and sleep, respectively. A total of $27 \%$ of the sample met none of the guidelines, whereas $36 \%, 28 \%$ and $8 \%$ of the sample met 1,2 or all 3 of the guidelines, respectively. Meeting the Mvpa guideline was associated with significantly lower fat mass, Sat mass; meeting the Tv guideline was associated with significantly lower Bmi, fat mass, Sat mass; meeting the sleep guideline was associated with significantly lower Bmi, fat mass, Sat mass and Vat mass. The odds of obesity (Or; $95 \% \mathrm{Ci})$ were successively lower across a higher number of components achieved: 0 (1.00; reference), 1 (0.79; 0.46-1.35), $2(0.70 ; 0.38-1.29)$, and $3(0.12 ; 0.03-0.56)$.

Conclusion: A small proportion of this sample met all 3 of the 24 -h movement guidelines. Meeting more components of the guidelines was associated with lower amounts of adiposity and lower odds of obesity.

Conflict of Interest: None disclosed.

Funding: Research relating to this abstract was funded by Nih-Niddk-1RC1DK086881.

\section{T4P108 \\ Renal function markers in obese boys - influence of 6-month soccer and traditional physical activity programs}

\section{Lousa, I. ; Nascimento, H. ${ }^{2}$; Rocha, S. ${ }^{2}$; Reis, F. ${ }^{3}$; Nunes, S. ${ }^{3}$; Rêgo, C. ${ }^{4}$; Santos-Silva, A. ${ }^{2}$; Seabra, A. ${ }^{\text {'; }}$ Ribeiro, S. ${ }^{2}$; Belo, L. ${ }^{2}$}

${ }^{1}$ Department of Biological Sciences, Laboratory of Biochemistry, Faculty of Pharmacy, University of Porto, Porto, Portugal, ${ }^{2}$ Ucibiorequimte, Department Of Biological Sciences, Laboratory Of Biochemistry, Faculty Of Pharmacy, University Of Porto, Porto, Portugal, ${ }^{3}$ Laboratory Of Pharmacology \& Experimental Therapeutics, Institute For Biomedical Imaging And Life Sciences (Ibili),Faculty Of Medicine And Cnc.lbili Consortium University Of Coimbra, Coimbra, Portugal, ${ }^{4}$ Children And Adolescent Centre, Cuf Hospital, Center For Health Technology And Services Research (Cintesis), Faculty Of Medicine, University Of Porto, Porto, Portugal, ${ }^{5}$ Research Centre In Physical Activity, Health And Leisure (Ciafel), Faculty Of Sport, University Of Porto, Portugal

Introduction: Obesity associates with increased risk of renal injury, and renal function seems to improve after weight loss in obese patients. However, few data exists regarding renal changes at pediatric ages. We evaluated the effects of soccer and traditional physical activity programs on changes in body composition and renal function markers in obese boys. Methods: 39 obese boys (mean age of 10.7 years) were divided in 3 groups: soccer (Sg, $n=13)$, traditional physical activity (Ag, $n=13)$ and sedentary control $(\mathrm{Cg}, n=13)$. Soccer and traditional activity programs involved 6-month extra-school Pa 3x/week for 60-90 min at an average intensity of $70-80 \%$ of maximal heart rate.Anthropometry measurements (weight, height and waist circumference) were collected and body composition was measured with dual-energy X-ray absorptiometry. Plasma concentrations of creatinine and cystatin $\mathrm{C}$ were also measured using an automatized standardized enzymatic in vitro assay and a commercial enzyme-linked immunoassay, respectively.

Results: At baseline $(n=39)$, age and lean mass were positively and significantly correlated with creatinine concentration. In a lesser significant manner, lean mass also positively correlated with cystatin C levels. After 6 months, both intervention groups presented a significantly decrease in body mass index (Bmi) z-score, body fat percentage (Bfp) and waist circumference, while the Cg presented a significant increase in Bfp. Lean mass increased in the three groups. A slight but significant increment in creatinine levels was observed in Sg after intervention (mean 0.51 vs 0.59 $\mathrm{mg} / \mathrm{dL}, P<0.001$ ) that was not correlated with changes in lean mass or Bfp. No significant changes were observed for cystatin $\mathrm{C}$ in any group. Conclusions: Age and lean mass were major predictors of creatinine levels in obese boys. Physical activity improved body composition, and soccer increased slightly creatinine levels but that increase was not directly 
related with changes in lean mass. Cystatin C may work as a more specific marker of renal function in this population, as it seems to be more independent of alterations in body composition. The study of longer physical activity interventions on renal function involving wider age-range obese pediatric patients is worthy.

Funding: Research relating to this abstract was funded by the Union European Football Association(Uefa), the Portuguese Football Federation (Fpf) and Nort Portugal Regional Coordination and Development Commission (Ccdr-N) / Norte2020 / Portugal 2020 (Norte-01-0145-Feder-000024).

\section{T4P109 \\ Risk evaluation of type 2 diabetes mellitus in a sample of Portuguese teens}

\section{Aparício, G. ${ }^{1}$.}

${ }^{1}$ Escola Superior de Saúde/Instituto Politécnico de Viseu

Introduction: World Diabetes Day is celebrated on November 14 and was established by the International Diabetes Federation (Idf) and the World Health Organization (Who) in response to the growing prevalence of diabetes, which in Portugal affects $40 \%$ of the population. One of the major modifiable risk factors for the development of type 2 diabetes Mellitus (type $2 \mathrm{Dm}$ ) is obesity. The American Diabetes Association (Ada) recommends assessing the risk of type 2 diabetes in children over 10 years of age, especially facing to risk factors such as obesity and family history of type 2 diabetes. Objectives: To assess the nutritional status and other risk factors for The development of type 2 diabetes mellitus in a group of Portuguese teens.

Methods: A cross-sectional and observational study carried out during World Diabetes Day celebrations in 2016, in a sample of 90 children / young people (mean age 14.52 years; $\mathrm{Sd}=2.10$ ), who participated in the activities promoted by the City Council of the municipality of Viseu, in partnership with other institutions. The research protocol included a section for the measurement of sociodemographic data and an adaptation to the paediatric population of the risk assessment form for type 2 Diabetes Mellitus, recommended by the Portuguese National Diabetes Program (Directorate General for Health) were used. The assessment of teens anthropometric data and the classification of the nutritional status were performed using the Nchs cut-off points (Cdc, 2000).

Results: Overall, $74.4 \%$ had normal weight $15.6 \%$ pre-obesity and $6.7 \%$ obesity ( $7.9 \%$ of boys). Characterizing the lifestyle, $61.1 \%$ reported eating vegetables daily, $80 \%$ periodically ate candies ( $16.6 \%$ daily), $86.7 \%$ fastfood (3.3\% daily) and $35.6 \%$ fruit and / or vegetable (3.3\% never consumed), however $78.9 \%$ had daily physical activity of at least 1 hour. Of the teens, $94.4 \%$ had already heard speaking in diabetes, and as other risk factors, $6.6 \%$ had as well detected a point value of hyperglycaemia, $51.1 \%$ reported having a family member with type 2 diabetes, specifically the parents (10.9\%) and the grandparents in $89.1 \%$ of the participants.

Conclusion: Portugal is one of the European countries with the highest prevalence of children's overweight. Thus, the risk assessment for type 2 diabetes should be started early in life, in order to intervene in modifiable factors, such as overweight as the single best predictor. Developing healthy lifestyles, such as balanced diet, daily physical activity and appropriate weight maintenance, are healthy lifestyles that should start in childhood.

Conflict of Interest: None Disclosed

Funding: No Funding

\section{T4P110 \\ Risk evaluation of type 2 diabetes mellitus in a sample of Portuguese teens}

\section{Aparício, G. ${ }^{1}$; Albuquerque, $C^{2}$}

${ }^{1}$ Escola Superior de Saúde/Instituto Politécnico de Viseu, ${ }^{2} \mathrm{Ci}$ Dets - Instituto Politécnico De Viseu - Portugal
Introduction: World Diabetes Day is celebrated on November 14 and was established by the International Diabetes Federation (Idf) and the World Health Organization (Who) in response to the growing prevalence of diabetes, which in Portugal affects $40 \%$ of the population. One of the major modifiable risk factors for the development of type 2 diabetes Mellitus (type $2 \mathrm{Dm}$ ) is obesity. The American Diabetes Association (Ada) recommends assessing the risk of type 2 diabetes in children over 10 years of age, especially facing to risk factors such as obesity and family history of type 2 diabetes. Objectives: To assess the nutritional status and other risk factors for The development of type 2 diabetes mellitus in a group of Portuguese teens.

Methods: A cross-sectional and observational study carried out during World Diabetes Day celebrations in 2016, in a sample of 90 children / young people (mean age 14.52 years; $\mathrm{Sd}=2.10$ ), who participated in the activities promoted by the City Council of the municipality of Viseu, in partnership with other institutions. The research protocol included a section for the measurement of sociodemographic data and an adaptation to the paediatric population of the risk assessment form for type 2 Diabetes Mellitus, recommended by the Portuguese National Diabetes Program (Directorate General for Health) were used. The assessment of teens' anthropometric data and the classification of the nutritional status were performed using the Nchs cut-off points (Cdc, 2000).

Results: Overall, $74.4 \%$ had normal weight $15.6 \%$ pre-obesity and $6.7 \%$ obesity (7.9\% of boys). Characterizing the lifestyle, $61.1 \%$ reported eating vegetables daily, $80 \%$ periodically ate candies (16.6\% daily), $86.7 \%$ fastfood (3.3\% daily) and $35.6 \%$ fruit and / or vegetable $(3.3 \%$ never consumed), however $78.9 \%$ had daily physical activity of at least 1 hour. Of the teens, $94.4 \%$ had already heard speaking in diabetes, and as other risk factors, $6.6 \%$ had as well detected a point value of hyperglycaemia, $51.1 \%$ reported having a family member with type 2 diabetes, specifically the parents $(10.9 \%)$ and the grandparents in $89.1 \%$ of the participants.

Conclusion: Portugal is one of the European countries with the highest prevalence of children's overweight. Thus, the risk assessment for type 2 diabetes should be started early in life, in order to intervene in modifiable factors, such as overweight as the single best predictor. Developing healthy lifestyles, such as balanced diet, daily physical activity and appropriate weight maintenance, are healthy lifestyles that should start in childhood.

Conflict of Interest: None Disclosed

Funding: No Funding

\section{T4P111}

\section{Sources of variability in childhood obesity and related behaviours}

Katzmarzyk, P. '; Broyles, S. '; Chaput, J. ${ }^{2}$; Fogelholm, M. ${ }^{3}$; Hu, G. ${ }^{\text {; }}$ Kuriyan, R. ${ }^{4}$; Kurpad, A. ; Lambert, E. ; Maher, C. ; Maia, J.'; Matsudo, V. ${ }^{8}$; Olds, T.6; Onywera, V. ${ }^{9}$; Sarmiento, O. ${ }^{10}$; Tremblay, M. ${ }^{2}$; Tudor-Locke, C. ${ }^{11}$; Zhao, $P^{12}$

${ }^{1}$ Pennington Biomedical Research Center, ${ }^{2}$ Children'S Hospital Of Eastern Ontario, ${ }^{3}$ University Of Helsinki, ${ }^{4}$ St. John'S Research Institute, ${ }^{5}$ University Of Cape Town, ${ }^{6}$ University Of South Australia, ${ }^{7}$ University Of Porto, ${ }^{8}$ Centro De Estudos Do LaboratOrio De Aptidao Fisica De Sao Caetano Do Sul, ${ }^{9}$ Kenyatta University, ${ }^{10}$ Universidad De Los Andes, ${ }^{11}$ University Of Massachusetts, ${ }^{12}$ Tianjin Women'S And Children'S Health Center

Introduction: The purpose of this study was to estimate the percentage of variance in obesity-related variables explained at the individual, school and study-site levels in a sample of children from countries varying widely in level of development.

Methods: The sample included 6411 children 9-11 y of age from 12 countries (Australia, Brazil, Canada, China, Colombia, Finland, India, Kenya, Portugal, South Africa, United Kingdom, and United States). The sampling design involved recruitment of children (level 1) nested within schools (level 2), which were nested within study sites (level 3). Height, weight and waist circumference were obtained using standard techniques and Bmi was calculated; moderate-to-vigorous physical activity (Mvpa) 
was measured by accelerometry; and healthy and unhealthy dietary scores were obtained from principal components analysis of food frequency questionnaire data. Sources of variability were analysed using multi-level statistical models.

Results: The variance in all traits was largely explained by individual-level factors: Bmi (91.5\%), waist circumference (92.8\%), Mvpa (73.3\%), healthy diet score $(88.4 \%)$, and unhealthy diet score $(66.1 \%)$. However, the variance explained at the school level ranged from $3.6 \%$ for waist circumference to $17.1 \%$ for Mvpa, and variance explained at the site level ranged from $3.6 \%$ for Bmi and waist circumference to $25.1 \%$ for unhealthy diet score. In general, more variance was explained at the school and site levels for lifestyle behaviours than for the anthropometric variables.

Conclusion: Given that $7 \%$ to $34 \%$ of the variance in obesity-related variables was explained at the school and site levels, interventions that incorporate policy and environmental changes may enhance obesity-prevention efforts that have traditionally focused on individual-level changes. Conclusion: This study highlights the shortage of dosage guidelines in $\mathrm{Ow} / \mathrm{Ob}$ children. We found as suspected a large inter-individual variability in dosage regimens even in drugs with narrow therapeutic intervals or drugs which has loading doses important for clinical effects. The clinicians are left with "best practice", as evidence based dosage regimens are missing for several drugs prescribed during childhood.

\section{T4P113}

\section{Sympathetic nervous system activity is decreased in obese adolescents}

Javorka, M. ${ }^{1}$; Czippelová, B. '; Turianiková, Z. ; Krohová, J. '; Lazarová, Z. ' ; Pozorčlaková, K. ${ }^{2}$; Bánovčln, P. $^{2}$

${ }^{1}$ Department of Physiology and Biomedical Center Martin, Comenius University in Bratislava, Jessenius Faculty of Medicine, Martin, Slovakia, ${ }^{2}$ Clinic Of Children And Adolescents, Comenius University In Bratislava, Jessenius Faculty Of Medicine, Martin, Slovakia

Introduction: Dysregulation of the autonomic nervous system can be an important factor contributing to the etiology and pathogenesis of obesity and its cardiovascular complications. A changed activity of the sympathetic nervous system can significantly influence not only the function of fat tissue but also the cardiovascular system function. It is assumed that a decreased sympathetic activity can contribute to the pathogenesis of obesity. On the other hand, an increased sympathetic activity was proposed as an important risk factor involved in the development of obesity related cardiovascular complications, including hypertension. Sympathetic activity can be studied non-invasively using several methods quantifying hemodynamics related measures or the magnitude of spontaneous blood pressure oscillations. The aim of our study was to describe the sympathetic activity in obese adolescents in comparison with control group.

Methods: We investigated 22 obese (10f, age: $15.28 \pm 2.8 \mathrm{y}, \mathrm{Bmi}$ $30.78 \pm 2.3 \mathrm{~kg} . \mathrm{m}^{-2}$ ) and 22 non-obese (10f, age: $15.98 \pm 2.46$ y., Bmi: $20.68 \pm 2.1 \mathrm{~kg} . \mathrm{m}^{-2}$ ) gender and age matched adolescents. At supine rest, we recorded continuously and non-invasively on the beat-to-beat basis (300 beats long recordings) heart rate (from Ecg), systolic blood pressure (Sbp) (volume clamp method) and impedance cardiography measures. Following sympathetic nervous system activity related indices were calculated from the recordings: magnitude of low frequency $(0.04-0.15 \mathrm{~Hz})$ oscillations in Sbp (Lf Sbp), preejection period (Pep), velocity index and Heather index from Icg, and peripheral vascular resistance.

Results: While mean heart rate tended to be lower in obese group $(p=0.078)$, no significant difference between groups in mean Sbp was found $(p=0.197)$. From the sympathetic nervous system activity related indices, Lf Sbp $(p=0.009)$, velocity index $(p<0.001)$, Heather index $(\mathrm{p}=0.009)$ and peripheral vascular resistance $(\mathrm{p}=0.001)$ were all significantly lower in obese adolescents compared to controls. No significant difference in Pep was found $(\mathrm{p}=0.919)$.

Conclusion: In contrast to obese adults, where previous studies found a sympatho-vagal balance shifted towards a sympathetic dominance, we found a decreased sympathetic activity related indices in obese adoles- cents. This phenomenon could be involved in the pathogenesis of obesity in the early phase of its development.

Conflict of interest: None Disclosed.

Funding: Research supported by grants Apvv-0235-12, Vega 1/0087/14 and Itms project “BioMed Martin” no.26220220187.

\section{T4P115}

\section{The association between parental separation during childhood and obesity in adulthood: A Danish twin study}

\section{Petersen 1, J. ${ }^{1}$; Heitmann, B. ${ }^{2}$; Kyvik, K. ${ }^{3}$; Vamosi, M. ${ }^{4}$}

${ }^{1} 1$ Research Unit for Dietary Studies at the Parker Institute, Bispebjerg and Frederiksberg Hospital, Copenhagen, Denmark, ${ }^{2}$ Research Unit For Dietary Studies At The Parker Institute, Bispebjerg And Frederiksberg Hospital, Copenhagen, Denmark, ${ }^{3}$ Department Of Clinical Research, University Of Southern Denmark, Odense, Denmark 3Odense Patient Data Explorative Network (Open),, ${ }^{4}$ Aarhus University, Denmark

Objective: The purpose of this study was to examine if parental separation during childhood was associated with obesity in adulthood.

Methods: A co-twin case-control study of 146 adult same-sexed twin pairs with discordant body mass index (Bmi) (i.e. one of the twins should have a Bmi between 20-25 kg/m², and the co-twin has a Bmi $\geq 30 \mathrm{~kg} / \mathrm{m}^{2}$ ), were selected from Danish Twin Registry (Dtr). In total of 236 out of $289(81.7 \%)$ eligible twin individuals participated in an interview and a physical examination. Childhood parental separation prior to age 17 was self-reported. Logistic and linear regression was applied for the statistical analysis using Stata 13.0.

Results: The twin who was separated from the father at least 1 year prior to age 17 had a $22 \%$ higher risk [ $\mathrm{Or}=1.22,95 \% \mathrm{Ci}(0.46-3.34), \mathrm{p}=0.65$ ] of developing obesity in adulthood, as compared to the co-twin who did not experience separation from the father. Separation from the mother showed reverse results [Or $=0.90,95 \% \mathrm{Ci}(0.32-2.46), \mathrm{p}=0.82]$. None of these results were significant.

Conclusions: Generally, while controlling for genetic and shared environmental factors, separation from parents in childhood was unrelated to obesity in adulthood. Further studies with bigger sample sizes are needed to examine this in greater detail.

Acknowledgement: None

\section{T4P116}

\section{The association of parental obesity with physical activity and} sedentary behaviors of their children: The CASPIAN-V study

\section{Ejtahed, H. ${ }^{1}$; Angorani, P. ${ }^{1}$; Qorbani, M. ${ }^{2}$; Hasani-Ranjbar, S. '; Heshmat, R. ${ }^{3}$;} Motlagh, M. ${ }^{4}$; Ziaodini, $H_{.}{ }^{5}$; Larijani, B. ${ }^{6}$; Kelishadi, . $^{7}$

${ }^{1}$ Obesity and Eating Habits Research Center, Endocrinology and Metabolism Molecular -Cellular Sciences Institute, Tehran University of Medical Sciences, Tehran, Iran., ${ }^{2}$ Department Of Community Medicine, Alborz University Of Medical Sciences, Karaj, Iran., ${ }^{3}$ Chronic Diseases Research Center, Endocrinology And Metabolism Population Sciences Institute, Tehran University Of Medical Sciences, Tehran, Iran., ${ }^{4}$ Department Of Pediatrics, Ahvaz Jundishapur University Of Medical Sciences, Ahvaz, Iran., ${ }^{5}$ Institute Of Education And Training, Tehran, Iran., ${ }^{6}$ Endocrinology And Metabolism Research Center, Endocrinology And Metabolism Clinical Sciences Institute, Tehran University Of Medical Sciences, Tehran, Iran., ${ }^{7}$ Pediatrics Department, Child Growth And Development Research Center, Research Institute For Primordial Prevention Of Non-Communicable Disease, Isfahan University Of Medical Sciences, Isfahan, Iran.

Introduction: Low physical activity and sedentary behaviors, two important determinants of childhood obesity, might be influenced by parents' life style and weight status. This study aims to determine the association of parental weight status on children's physical activity $(\mathrm{Pa})$ and screen time (St).

Methods: This study is conducted on 14400 Iranian schools students, aged 7-18 years, and one of their parents participated in the large national school-based surveillance program (Caspian-V). The children's screen- 
based and physical activities were evaluated based on the World Health Organization-Global School Student Health Survey (WHO-GSHS) questionnaire. . Parents' height, weight and waist circumference (Wc) were measured using standardize methods Excess weight, general obesity and abdominal obesity were defined according to the body mass index (Bmi) $\geq 25, \mathrm{Bmi} \geq 30 \mathrm{~kg} / \mathrm{m}^{2}, \mathrm{Wc} \geq 95 \mathrm{~cm}$, respectively.

Results: Mean (standard deviation) age of students was 12.3 (3.2) years and the prevalence of low $\mathrm{Pa}$ and high St was $58.2 \%$ and $17.7 \%$, respectively. In multivariate model, the parental general obesity and abdominal obesity increased the odds of children low $\mathrm{Pa}$, by $21 \%$ and $13 \%$, respectively. Parental excess weight, general obesity and abdominal obesity increased the odds of combination of low Pa/high St in children by $33 \%, 26 \%$ and $20 \%$, respectively.

Conclusions: This study showed that parental excess weight was associated with increased screen-based activities and low $\mathrm{Pa}$ in children. Focus on parents' weight status, as an important factor influenced by their life style, can be helpful for preventing of sedentary behaviors in their children.

Conflict of Interest: None Disclosed

Funding: No Funding

T4P117

\section{The effect of a multidisciplinary intervention program on} blood pressure and physical fitness in overweight and obese children

\section{Leeuwen, J. ${ }^{1}$; Visser, M. ${ }^{1}$; Koes, B. ${ }^{1}$; Middelkoop, M. ${ }^{1}$}

${ }^{1}$ Erasmus Medical Center

Introduction: Already in children, elevated Bmi is strongly associated with elevated blood pressure, which can lead to diseases later in life including type 2 diabetes mellitus, left ventricular hypertrophy and dyslipidemia. It has been shown that cardiorespiratory fitness is a stronger predictor for all-cause mortality than Bmi. Therefore, this study will investigate the effect of a multidisciplinary intervention on blood pressure (Bp) and physical fitness in overweight and obese children.

Methods: A prospective cohort study was conducted among 154 children in the age of 6-12 years old. All children subscribed to Kids4Fit, a 12 week multidisciplinary intervention for overweight and obese children in deprived areas in Rotterdam, the Netherlands. The Kids4Fit-intervention was led by a dietitian, physiotherapist and child psychologist. Blood pressure was measured and a shuttle-run test (Srt) was performed, both at the start of the waiting list-period (average of 3 months - baseline), at start of the intervention, at the end of the intervention and after 52 weeks. The effect of the intervention on Bp and Srt-scores were analyzed using mixed model analyses adjusting for confounders and the change of the outcome measure during the waiting list period. A general linear model $(\mathrm{Glm})$ with repeated measurements was used to compare the effect on Bp levels between a 'hypertension group', a 'prehypertension group' and a 'normal group'. The effect on change in physical fitness was compared between the 'low-fit', 'moderately-fit' and 'high-fit' group.

Results: The study population consisted of 66 (42.9\%) boys, the mean age at baseline was 8.5 (1.9) years, and 21 (14.7\%) children had parents who were both born in the Netherlands. The mixed model analyses showed no significant effect of the intervention on both systolic and diastolic Bp. Though, a significant effect of the intervention was found on physical fitness $(\beta-0.08,95 \% \mathrm{Ci}-0.11 ;-0.05)$ Mean shuttle-run test (Srt) score increased from $6.5(2.8)$ at baseline, to $7.8(2.8)$ at the start of the intervention and 8.1 (3.1) at the end of the intervention. One year after the intervention, the Srt-score went back to 7.5 (2.6). The Glm showed a significant difference in time between children with a hypertensive Bp status at baseline compared to those with a normal Bp (Md 24.52, Se 6.82).

Conclusion: A multidisciplinary intervention in children positively affects Bp levels and physical fitness. However, effects do not seem to hold in the long-term.

Conflict of Interest: None disclosed.
Funding: This study is funded by Fonds Achterstandwijken Rotterdam, the Netherlands.

T4P118

The effect of impaired glucose metabolism in children and adolescents during weight reduction treatment

Kloppenborg, J. '; Gamborg, M. ${ }^{2}$; Fonvig, C. ${ }^{3}$; Nielsen, T. ${ }^{4}$; Pedersen, O.5. Johannesen, J. ${ }^{6}$; Hansen, T. ${ }^{5}$; Holm, J.'

${ }^{1}$ The Children's Obesity Clinic, Department of Pediatrics, Copenhagen University Hospital Holbæk, Denmark, ${ }^{2}$ Institute Of Preventive Medicine, Copenhagen University Hospital, Denmark, ${ }^{3}$ The Children'S Obesity Clinic, Department Of Pediatrics, Copenhagen University Hospital Holbæk And Department Of Pediatrics, Odense University Hospital, Denmark, ${ }^{4}$ The Children'S Obesity Clinic, Department Of Pediatrics, Copenhagen University Hospital Holbæk, ${ }^{5}$ The Novo Nordisk Foundation Center For Basic Metabolic Research, Section Of Metabolic Genetics, University Of Copenhagen And University Of Copenhagen, Faculty Of Health And Medical Sciences, Denmark ${ }^{6}$ Department Of Pediatrics, Copenhagen University Hospital Herlev And University Of Copenhagen, Faculty Of Health And Medical Sciences, Denmark, ${ }^{7}$ The Children'S Obesity Clinic, Department Of Pediatrics, Copenhagen University Hospital Holbæk, Denmark And The Novo Nordisk Foundation Center For Basic Metabolic Research, Section Of Metabolic Genetics, University Of Copenhagen And University Of Copenhag

Introduction: Whether specific metabolic variables, including insulin resistance, hyperinsulinemia, and impaired glucose tolerance (Igt) - also described as prediabetes, have an impact on weight changes is controversial, due to conflicting results in both adults and children. This study aims to investigate whether children and adolescents exhibiting an impaired glucose metabolism are more obese at treatment entry and less likely to reduce their degree of obesity during childhood obesity treatment.

Methods: The present study is an observational study, including children and adolescents from the Children's Obesity Clinic, Holbæk, Denmark. Anthropometrics, pubertal development, socioeconomic status (Ses), and fasting concentrations of plasma glucose, serum insulin, serum C-peptide, and whole blood glycosylated hemoglobin (HbAlc) were collected at treatment entry and at follow-up. Proxies of insulin sensitivity (Homa2-Is) and $\beta$-cell function (Homa2-B) were calculated with the Homeostasis Model Assessment 2 program.

Results: 569 (333 boys) patients, median 11.5 years of age (range 6-22) and median body mass index (Bmi) z-score 2.94 (range 1.34-5.54) were included. The mean Bmi $\mathrm{z}$-score reduction was $0.31( \pm 0.46)$ after 13 months (range 6-18) of treatment. At treatment entry, patients with impaired estimates of glucose metabolism were more obese than normoglycemic patients. A low concentration of C-peptide in girls at baseline was associated with a poorer weight loss during treatment $(\mathrm{p}=0.02)$. Changes in insulin concentrations were positively associated with changes in Bmi $\mathrm{z}$-score in both sexes $(\mathrm{p}<0.0001 ; \mathrm{p}=0.0005)$. During treatment, values of glucose, HbA1c, Homa2-Is, and Homa2-B did not change or impact the treatment outcome, regardless of age, sex, Ses, or degree of obesity at treatment entry.

Conclusion: The capability to reduce weight during multidisciplinary chronic care treatment in children and adolescents with overweight/obesity is not influenced by an impaired glucose metabolism.

\section{References:}

1 Weiss R et al. Lancet. 2003 Sep 20;362(9388):951-7.

2 Chiavaroli V et al. Endocr Res. 2013;38(1):48-57.

3 Reinehr T. Nat Rev Endocrinol. 2013 Oct;9(10):607-14.

4 Holm J-C et al. Int J Pediatr Obes ljpo Off J Int Assoc Study Obes. 2011 Aug;6(3-4):188-96.

Conflict of Interest: None All authors declare that there are no conflicts of interest.

Funding: This study is part of the research activities in The Danish Childhood Obesity Biobank, funded by The Region Zealand Health Scientifics Research Foundation, The Danish Innovation Foundation, The Novo Nordisk Foundation, and an unrestricted educational grant from Novo Nordisk. 
T4P119

The influence of plasmatic fatty acids in inflammatory and cardiovascular biomarkers in obese adolescents submitted to a long-term interdisciplinary treatment.

Ganen, A. ${ }^{1}$; Masquio, D. ${ }^{1}$; Estadella, D. ${ }^{2}$; Oyama, L. ${ }^{2}$; Souza, G. ${ }^{2}$; Santamarina, A. ${ }^{2}$; Moreira, R. ${ }^{3}$; Sanches, P. ${ }^{2}$; Tock, L. ${ }^{2}$; Mello, M. ${ }^{2}$; Tufik, S. ${ }^{2}$; Dâmaso, A. ${ }^{2}$; Nascimento, C. ${ }^{2}$

${ }^{1}$ Centro Universitário São Camilo, ${ }^{2}$ Universidade Federal De São Paulo, 3Universidade De São Paulo

Background: Obesity presents a strong association with the higher cardiovascular risk factors, however, the role of serum fatty acids composition in carotid intima media thickness (cIMT) is not fully investigated in obese adolescents undergoing long-term weight loss therapy.

Objectives: Assess the influence of fatty acids composition in cIMT and inflammatory cardiovascular biomarkers.

Methods: 53 post-pubertal obese adolescents were enrolled. The blood samples were collected to glycemia, insulinemia, lipid profile and adipokines. Serum fatty acid composition was performed by technical of chromatography in fizzy phase. cIMT, visceral and subcutaneous fat was determined by ultrasound.

Results: The intervention promoted significant reduction in body fat, visceral and subcutaneous fat, Homa-Ir and lipid profile. Leptin, leptin/adiponectin ratio, Pai-1, Crp and Icam decreased; and adiponectin increased significantly after one year of treatment, corroborating with a reduction in cIMT. Additionally, it was noted a positive correlation between cIMT with visceral fat, Homa-Ir, Tg, Pai-1 and C20:0, and conversely negative correlation with C20:4n6. Interesting, in the multiple regression analysis, C20:4n 6 was confirmed to be an independent negative predictor of cIMT. Moreover, Pai-1 was positively predicted by visceral fat and negatively by C20:3n6; while, Crp was positively associated with body fat and negatively with C18:1n9. Saturated fat acid was associated with decrease in adiponectin, and conversely polyunsaturated fatty acids increased it.

Conclusions: the plasmatic fatty acid composition showed to be a strong predictor of cIMT and cardiometabolic risks. However, the long-term weight loss may protect obese adolescents avoid the development of precocious cardiovascular diseases.

\section{Conflit of interest: None Disclosed}

Funding: Research relating to this abstract was funded by Capes, Afip, Fapesp (2011/50356-0; 2011/50414-0) Fapesp (Cepid/Sleep \#9814303-3 S.T) Cnpq, Cenesp.

\section{T4P120}

\section{The relationship between body mass index and sit \& play in preschoolers}

\section{Silva-Santos, S. ${ }^{1}$; Santos, A. '; Vale, S. ${ }^{2}$; Mota, J. ${ }^{1}$}

${ }^{1}$ Research Centre in Physical Activity, Health and Leisure. Faculty of Sport. University of Porto. Portugal., ${ }^{2}$ Research In High School Of Education. Polytechnic Institute Of Porto. Portugal

Introduction: Excess childhood adiposity can exert negative health effects across the life-course. The obesity is positively associated with physical activity $(\mathrm{Pa})$ and inversely associated with sedentary behaviour ( $\mathrm{Sb}$ ). Although children behave sedentarily while sitting and playing there is effectively movement in this behaviour. We considered the transition sedentary behaviour ( $\mathrm{Sb})>148$ c.p.m and light physical activity (Lpa) $\leq$ 800c.p.m as sit \& play (Sp), that is, when preschoolers are sitting but there is some movement playing. No studies to date have examined howBMI is related to $\mathrm{Sp}$. The aim of this study was to examine association between body mass index (Bmi) and Sp in preschool children.

Methods: The sample comprised 315 children aged $3-6$ yrs (51.8\% girls) mean age $4.59 \pm 0.79$ yrs, recruited in schools located in the metropolitan area of Porto, Portugal. Total physical activity (Tpa) and Sp were assessed through the accelerometer. The cut point used to $\mathrm{Sp}$ was $>148 \mathrm{cpm}$ until $\leq 800 \mathrm{cpm}$ (Espana-Romero et al., 2013).The Bmi was assessed as mass (kg)/stature squared $\left(\mathrm{m}^{2}\right)$. The Spss 21.0 program was used to statistical analyses.

Results: We have found that mean values for Bmi were $17.1 \pm 2.1 \mathrm{~kg} / \mathrm{m}^{2}$ for girls, and $16.8 \pm 1.7 \mathrm{~kg} / \mathrm{m}^{2}$ for boys, respectively. Multiple linear regression analysis showed significant relationship between $\mathrm{Bmi}$ and $\mathrm{Sp}$ for girls $(B=0.34 ; \mathrm{p}=0.01)$ but not for boys $(\mathrm{B}=0.08 ; \mathrm{p}=0.38)$. Both models were adjusted to age and Tpa.

Conclusion: This study has shown an association between Bmi and Sp, that is, Bmi may be influenced by Sp for girls and not for boys. These could be related by the fact that the girls have more Bmi and spend more time in Sp than boys.

\section{Conflict of Interest: None}

Funding: Research relating to this abstract was funded by Ciafel (Faculty of Sport, University of Porto) and by the following Portuguese Foundation for Science and Technology (Fct) grants: Uid/Dtp/00617/2013. Sfrh/Bd/86538/2012.

\section{T4P121}

The use of a text-messaging program during weight loss treatment: Baseline results regarding fruits and vegetables intake, screen time and physical activity in obese children and adolescents.

\section{Silva, C. ${ }^{1}$; Saint-Maurice, P. ${ }^{2}$; Gonçalves, S. ' ; Antunes, $H_{.}^{3}$; Conceição, E. ${ }^{\text {; }}$} Machado, P. ${ }^{1}$

${ }^{1}$ School of Psychology of University of Minho, ${ }^{2}$ lowa State University, Usa, ${ }^{3}$ Pediatric Gastroenterology, Hepatology And Nutrition Unit, Hospital De Braga, Braga, Portugal; Life And Health Sciences Research Institute (Icvs); School Of Health Sciences, University Of Minho, Braga, Portugal

Introduction: Unhealthy diets and sedentary lifestyle have been associated with the risk of being overweight. The current study is a part of a randomized clinical trial developed to analyze the effectiveness of an additional monitoring tool ( $\mathrm{Sms}$ ) to promote healthy behaviors during weight loss treatment for overweight youth. Baseline results regarding fruits and vegetables intake, screen time and physical activity were reported. Behavioral differences between boys and girls and children and adolescents were analyzed. Finally, caregivers' behaviors were also explored.

Methods: One-hundred and seven overweight participants aged from 8 to 17 years old participated in the study $(\mathrm{M}=11.83, \mathrm{Sd}=2.3)$, as well as their corresponding caregivers $(N=107)$. The recruitment took place in the Hospital during the first medical appointment in the pediatric nutrition care service. Overall, $41.1 \%$ were girls and $58.9 \%$ boys.

Results: In this sample, overall results showed that the majority of overweight and obese patients often do not include vegetables in their diet. Additionally, $74.2 \%$ of the total patients reported low levels of physical activity (less that 60-minutes of physical activity), showing that they spend more time in front of the screen (two-to-four hours a day). Gender and age was not associated to fruits and vegetables consumption or physical activity level. However, age group emerged as a significant predictor for meeting the screen time guidelines. Specifically, children were more likely to spend less than two hours in front of the screen than adolescents. Finally, the majority of the parents/caregivers were considered sedentary and did not achieve the $\mathrm{Pa}$ recommendations.

Conclusions: Overweight and obese participants showed low consumption of vegetables, more hours in screen time and low levels of physical activity before weight loss treatment. These findings can be important and could bring some important clinical implications for the rest of the treatment. Also, it will be interesting to evaluate the effectiveness of a text-messaging program in promoting these behaviors in this clinical setting. We will soon be able to examine these behavioral trends in more detail once the longitudinal data is analyzed.

Conflict of Interest: No financial interests exist.

Acknowledgments: This research was supported by a Fundação para a Ciência e a Tecnologia/Foundation for Science and Technology $\mathrm{PhD}$ grant to the first author (Sfrh/Bd/80468/2011), by a European Union Compete program grant to Eva Con- 
ceição (If/01219/2014), and co-financed by Feder under the Pt2020 Partnership Agreement (Uid/Psi/01662/2013)

T4P122

The utilization of billing codes of obesity as a first recognition of the problem: A pediatric university practice review

Yafi, M. ${ }^{1}$; Rubio, N. ${ }^{1}$; Rivera-Davila, M. ${ }^{1}$; Sanders, M. ${ }^{1}$; Yetman, R. $^{1}$

${ }^{1}$ UT Health The University of Texas Health Science Center at Houston

Introduction: Pediatric obesity is a global challenge. In the Usa, it is estimated that more than one third of children are overweight or obese. Childhood obesity increases the risk of adult-onset obesity and cardiovascular disease; therefore, the early management of obesity during its pediatric and adolescent phase can prevent serious consequences of obesity-related health problems in adulthood, since adult obesity is often more severe if it starts as childhood overweight problem.

The first step in dealing with obesity is to recognize it by the health care provider; this allows a candid discussion with the family and child to try lifestyle modification approach. Many primary care providers in the Usa have been reluctant to use the diagnoses of obesity for billing, since insurance companies have historically denied reimbursement for it as a medical diagnosis. This could also undermine recognizing obesity as a medical problem and less prioritizing its documentation and management approach.

Method: We reviewed all Icd9/10 billing codes used for overweight, obesity (childhood, simple, central, morbid) and abnormal weight gain by the health care providers in the Department of Pediatrics at The University of Texas Health Science Center at Houston in one fiscal year of September 2015 through the end of August 2016, and compared it to the total pediatrics visits in both general and all specialty pediatric-billed visits during the same duration.

Results: There were 104643 pediatric bills; 63.6\% were general pediatrics while $36.4 \%$ were specialty pediatrics. Only 3197 bills (3\%) were related to overweight, obesity and abnormal weight gain diagnoses as a single or part of multiple diagnoses: 1425 bills ( $45 \%$ ) were from general pediatrics, while 1772 (55\%) were from specialty.

Conclusion: The billing submitted for pediatric overweight and obesity diagnoses in our institution does not reflect the rate of obesity in Texas (33\%) nor in the Usa. This shows that pediatricians are still reluctant to use these codes during general and specialty pediatric visits. While this may not necessarily mean that pediatricians are not addressing the obesity problem,it clearly shows the need to documemnt obesity as a medical problem.

Proper education of physicians and insurance companies to recognize and utilize the billing codes for pediatric obesity is the first step towards effective monitoring and intervention. A better understanding by providers and insurers of the nuances affecting billing for overweight and obese children (diagnosis definition, miss or delay, or apathy) should improve diagnosis capture and documentation, and improve outcomes for children more prone to illnesses related to obesity.

Conflict of Interest: None

Funding: None

Refrences:

1 www.cdc.gov
T4P123

Thermography-based assessment of brown fat activity in children: Effects of age, gender, and responsiveness to a meal

Sánchez-Infantes, D. '; Cereijo, R. ${ }^{2}$; Llorens, P. ; Moreno, J. ; Nebreda, V. ${ }^{4}$; García, M. ${ }^{4}$; Giralt, M. ${ }^{2}$; Villarroya, $F^{2}$

${ }^{1}$ Department of Endocrinology and Nutrition, Institut d'Investigació Germans Trias I Pujol, Barcelona, Spain, ${ }^{2}$ Department Of Biochemistry And Molecular Biomedicine, University Of Barcelona And Ciberobn, Spain, ${ }^{3}$ Department Of Biochemistry And Molecular Biomedicine, University Of Barcelona, ${ }^{4}$ Institute For Medical And Molecular Genetics (Ingemm). La Paz University Hospital, Madrid, Spain.

Introduction: Brown adipose tissue (Bat) activity is protective against obesity and associated metabolic alterations. positron emission tomography (Pet)-scan is the standard method for measuring Bat activity in humans; however, costs and ethics considerations preclude an extensive use, especially in pediatric populations. High resolution infrared thermography at the supraclavicular area has been proposed as a potential non-invasive method to assess Bat activity. Here we determined thermography-based Bat activity in relation to age, gender and responsiveness to cold and to a single meal.

Methods: Study population were male and female healthy, non-obese, children from two age ranges: (3-5 year-old, $\mathrm{N}=7$; and 5-7 year-old, $\mathrm{N}=6$ ), adolescents ( 16 year-old $\mathrm{N}=7$ ) and adults ( $50-55$ year-old, $\mathrm{N}=7$ ). Thermal imaging was performed essentially as reported (Symonds et al. J Pediatr. 2012,161:892). A 2.3 Megapixel Infrared Camera (Flir Systems) was used. Individuals were at $21^{\circ} \mathrm{C}$ ambient temperature, in a sitting position and without clothing in the upper chest area. Thermal infrared pictures were obtained before and 5 min after placement of the hand into a bowl of water at $18^{\circ} \mathrm{C}$. The procedure was performed before and $20 \mathrm{~min}$ after ingestion of a single meal averaging $300 \mathrm{Kcal}$ (59\% carbohydrate, $6 \%$ protein, $35 \%$ fat). The peak temperature and the surface area with a significant rise in temperature were determined at the supraclavicular fossa area as indices of activated Bat.

Results: The highest rates of thermography-estimated Bat activity were found in children. Significant, but lower, Bat activity appeared in adolescents, and a minor activity was found in adults. In children, Bat activity was induced not only by cold but also after a single meal. The basal skin temperature at the supraclavicular Bat area correlated negatively with the Bmi in children.

Conclusion: Thermography-based estimations of Bat activity evidence a strong age-dependent decline. Bat activation was responsive not only to cold but also to a single meal, at least in children. Thermography-based methodologies appear useful for studies of Bat activity in pediatric populations in response to obesogenic nutritional and environmental factors.

\section{T4P124}

Time(s) and challenges of families with young children: A qualitative study with implications for obesity prevention programs

Rosário, R. ${ }^{1}$; Oliveira, C. ${ }^{1}$; Silva, M. ${ }^{1}$; Lopes, O. ${ }^{2}$; Tinoco, R. ${ }^{3}$; Lopes, C. ${ }^{3}$; Rainho, M. ${ }^{4}$

${ }^{1}$ University of Minho, ${ }^{2}$ Sports Medical Centre, ${ }^{3}$ Gualtar Family Unit Centre, ${ }^{4}$ University Of Tras-Os-Montes E Alto Douro

Introduction: In recent years there is an increase of childhood overweight and obesity all over the world, along with deep changes in family structure and organization. Whilst there is a body of evidence that involvement of family is determinant of well succeed intervention programs aiming at preventing obesity(1), the evidence is scarce about in depth understanding of families with young children's challenges in nowadays. This study aims to explore, describe and understand the tasks associated to families with young children attending a childcare setting.

Methods: We developed an exploratory, qualitative study. Participants included 2 childcare centres staff, 2 primary care centre staff, and 2 parents with a 3 years old child. We developed two focus group sessions in 
June 2016, which lasted between 60 and 120min, following standardized criteria(2). Questions were designed to include a range of issues in the discussion, such as "What are the challenges of families with young children?". To elicit practices about obesity prevention, questions like: "How childcare centres/families are important for healthy lifestyles in children?". The study was approved by ethics commission of Portugal's north health administration(90/2016). The interviews were transcribed verbatim and content analysis was developed to identify categories, guided by Bardin(3). Results: Four categories emerged from the study to illustrate families with young children tasks. Time to be a child, time to be family, time for childcare centres and time for health, are the different and complex times for these families. Time is considered a central element in the organization of the family's system "I have difficulty to manage everything and give my child a "no"... it is easier to say "yes"(mother). Family is in constant interaction with other systems, including education and health. "Childcare centres began as an answer to families, and we must develop a dialogical work with parents...Child's eating is the most difficult issue" (childcare centre staff).

Conclusion: This study allowed the understanding of the challenges and tasks of families with young children. Childcare centres are important for families, however parents have other "times" to attend. This research contributes to further studies that wish to involve families in programs such as prevention of obesity.

\section{References:}

1 Natale R, et al. Role Modelling as an Early Childhood Obesity Prevention Strategy: Effect of Parents and Teachers on Preschool Children's Healthy Lifestyle Habits. 2014. J Dev Behav Pediatr 35(6):378-87.

2 Krueger, et al. (2009). Focus groups: a practical guide for applied research (4 ed.). Usa: Sage. 4.Bardin, L. (2015). Análise de conteúdo. Lisboa: Edições 70.

\section{T4P125}

\section{Validation of a questionnaire for assessing psychosocial factors of junk food consumption in elementary school children}

\section{Hernández-Alcántara, G. ${ }^{1}$; Bacardí-Gascón, ${ }^{1}{ }^{1}$; Jiménez-Cruz, A. ${ }^{1}$} Castillo-Ruiz, O. ${ }^{2}$

${ }^{1}$ Universidad Autónoma de Baja California, ${ }^{2}$ Universidad Autónoma De Tamaulipas

Background \& aims: Psychosocial factors have a direct impact on children's eating habits, and they can encourage junk food consumption. This, combined with low physical activity, has been associated with increased childhood obesity.

Objectives: To assess construct validity of an instrument used to identify and measure the psychosocial factors that influence the consumption of junk food among elementary school children.

Material \& methods: The questionnaire consisted in 23 items and was applied to a sample of 1329 second and fourth graders from public primary schools in Tijuana (northwestern Mexico). To assess the hypothesized dimensions of the questionnaire, factorial exploratory analysis was performed. The internal consistency (Cronbach's alpha) of questionnaire items and psychosocial factors were assessed. To test the factor structure obtained in the exploratory analysis, confirmatory factor analysis was conducted in a second sample of 1541 second and fourth graders in schools from Reynosa (north of Mexico). The analysis was conducted using Spss and Amos.

Results: From the exploratory analysis, six factors (accessibility at home, habit, accessibility outside the home, active modeling, perception and passive modeling) explained $55.86 \%$ of the variance. Cronbach's alpha were $0.73,0.74,0.61,0.76,0.51$ and 0.56 for each scale. The confirmatory analysis confirmed and showed and acceptable fit of the 6-factor mode $(\chi 2=446.497 ; \mathrm{gl}=172 ; \mathrm{p}<0.001 ; \chi 2 / \mathrm{gl}=2.60 ; \mathrm{Gfi}=0.973 ;$ Agfi $=0.964$ Rmsea $=0.032$; and Cfi $=0.956$ ). Test-retest reliability was acceptable (intraclass correlation coefficient $=0.61$ )
Conclusion: The questionnaire provides a valid tool for assessing psychosocial factors which are potential determinants of junk food consumption among elementary school children.

Acknowledgement

Conacyt Funding \#0212727

\section{T4P126}

Waist circumference percentile in children from municipalities of developed and developing countries

Andaki, A. ${ }^{1}$; Mendes, E. '; Tinoco, A. ${ }^{2}$; Santos, A. ${ }^{3}$; Sousa, B. ${ }^{4}$; Vale, S. ${ }^{5}$; Mota, J. ${ }^{6}$

${ }^{1}$ Federal University of Triângulo Mineiro, ${ }^{2}$ Federal University Of Viçosa, ${ }^{3}$ Research Centre In Physical Activity Health And Leisure, Faculty Of Sports University Of Porto, Porto, Portugal., ${ }^{4}$ Escola De Ciências E Tecnologias Da Saúde, Lusófona University, Health Service Of The Autonomous Region Of Madeira, Portugal, ${ }^{5}$ Department Of Sport Science. High School Of Education, Polytechnic Institute Of Oporto, Portugal, ${ }^{6}$ Research Centre In Physical Activity Health And Leisure, Faculty Of Sports University Of Porto, Porto, Portugal

Introduction: The obesity epidemic affects developed and developing countries. Anthropometric measures have widely been used to quantify and classify the obesity status among children according sex, age and race-ethnicity. Accordingly, waist circumference (Wc) was considered one the most common anthropometric index used for defining abdominal obesity and it has been used to predict cardiovascular risk factors (Cvrf). The aim of this study was to design reference percentile curves of Wc in 6-10-year-old children from municipalities of developed (Portugal) and developing (Brazil) countries and to compare these results with other international references.

Methods: This cross-sectional study enrolled a 6-10-year-old probability sampling from Portugal and Brazil. Portuguese data were collected in the metropolitan area of Porto located in the North of Portugal, and Autonomous Region of Madeira. In Brazil data were collected in both cities, Uberaba and Viçosa, located in the South-Eastern region. Wc measurements were taken at midpoint between the iliac crest and the lower rib. Univariate analysis of covariance adjusted by height was carried out to test the main effects of age, sex, countries and interactions. Wc percentile curves were performed with the Lms Chart Maker.

Results: The sample comprised 6,475 children. Portuguese children presented a curvilinear increase in Wc curves and Brazilian Wc curves have shown a linear increase with age in both boys and girls. Overweight and obesity prevalence was $15.9 \%, 4.7 \%$ in Portuguese children and $12.9 \%, 4.5 \%$ in Brazilian children, respectively. There was a main effect $(\mathrm{p}<0.0001)$ for age and countries, and an interaction effect for sex ${ }^{\star}$ age $(\mathrm{p}=0.001)$ and countries ${ }^{\star}$ age $(\mathrm{p}<0.0001)$. Portuguese boys and girls have shown higher values for the 50th, 75th and 95th percentiles than Brazilian boys and girls.

Conclusion: Wc reference percentile curves were drawn for 6-10-yearold Portuguese and Brazilian children. Portuguese children have higher Wc values than Brazilian children and other children from developed countries.

\section{Conflict of Interest: None}

Funding: This work was supported by Fapemig - Fundação de Amparo a Pesquisa do Estado de Minas Gerais, Brazil.

\section{T4P127 \\ Weight gain, feeding and eating in the first year of life of babies of smoking and non-smoking mothers}

Lev-Ari, L. '; Bachner-Melman, R. ${ }^{\prime}$; Zohar, A. '; Mankuta, D. ${ }^{2}$

${ }^{1}$ Ruppin Academic Center, ${ }^{2}$ Haddassa Medical Center

Babies born to mothers who smoke during their pregnancy (Sdp) tend to be born underweight, but by middle childhood are at risk for overweight 
and obesity. We studied maternal feeding practices, maternal disordered eating, and child temperament during the first year of life to seek early potential mediators of this weight gain process. The weight and height of babies born to $88 \mathrm{Sdp}$ mothers and 107 babies born to non-smoking mothers were measured and recorded one to two days after birth. One year later, the babies' height and weight were reported by the mothers, who also completed measures of their own disordered eating, their child feeding practices and the infant's temperament and behavior. Variables predicting the babies' Bmi at 12 months of age were examined for Sdp mothers and nonsmoking mothers using regression analysis, controlling for maternal education. As expected, Sdp babies had significantly lower Bmis at birth, but this difference had disappeared at one year of age. Length of breastfeeding correlated negatively with babies' Bmi at 12 months for all babies, however, Sdp mothers were less likely to breastfeed their babies. Babies' Bmi at one year was predicted by different variables for nonsmoking and Sdp mothers. For nonsmoking mothers, babies Bmi at birth $(\beta=.30, \mathrm{p}<.05)$ predicted Bmi at one year, but maternal eating attitudes or child feeding practices did not. The Bmi at age one of Sdp babies was predicted by maternal disordered eating $(\beta=.69, \mathrm{p}<.001)$ and maternal pressure for her child to eat $(\beta=.29, \mathrm{p}<.001)$, but not by the babies' Bmi at birth. The babies' temperament scores were similar in the two groups, with a slight advantage for the Sdp babies; however, in the non-smoking group there were significant correlations between temperamental difficulties and the baby's Bmi at 12 months; the Sdp babies' temperament scores were not significantly correlated with Bmi suggesting that feeding to sooth was used more by the non-smoking mothers. These results support the hypothesis that in contrast to nonsmoking mothers, Sdp mothers want their children to gain weight, pressure them at an early age to eat, and do not perceive an association between Bmi and the baby's distress. This interim picture after the first year of life may provide insight, beyond the "thrifty gene hypothesis", into the process by which the children of smoking mothers transition from being, on the average, underweight newborns to being overweight children and adults.

\section{T4P128 \\ When maternal misperception of their child's weight status begins?}

\section{Roznowski, J.' ${ }^{1}$ i Leks, B. '; Roznowska, A. ${ }^{2}$; Kopecky, M. ${ }^{3}$}

${ }^{1}$ NZOZ Twoj Lekarz, ${ }^{2}$ Student Of Medical University Gdansk, ${ }^{3}$ Palacky University Olomouc

Introduction: While the number of overweight and obese children is skyrocketing worldwide, raising awareness of weight status among mothers plays a key role in improving physical development of their children.

Methods: Data were obtained from 163 child-mother pairs (children aged 9 m.o., 2 y.o., 4 y.o., 6 y.o.) from routine check-ups, during which dietary habits questionnaire and anthropometric measurements of mother and child were collected. Additionally, mothers were asked if in their opinion their child should weight less, was about the right weight or should weight more. Mothers' Bmi and Cole index in all children were calculated. Chisquare tests were performed to analyse differences in demographic data and variables.

Results: At the baby's 9-month check-up, due to frequent healthcare routine visits, the majority of mothers (62\%) accurately estimated their children's weight. Unfortunately, from the age of 2 years mother's perception of their child's weight status becomes incorrect and this tendency continues until the age of four and further up till the age of six years old Only $16.7 \%, 25 \%$ and $4.3 \%$ of mothers could correctly assessed weight of their children, respectively. $\left(\chi^{2}=18.9, p=0.008\right)$. Breastfeeding for at least 6 months has significantly improved the awareness of accurate children's weight status $\left(45.6 \%\right.$ vs. $\left.24.4 \% ; \chi^{2}=7.9, p=0.004\right)$. In the contrast to some recent literature, in our study neither mother's Bmi nor their educational level have significant impact on the parental perception of child's weight. Conclusion: Increasing parents' awareness of their children's weight is the first step in preventing children's obesity. Continual feedback program should be provided since the beginning of children's life. It is extremely important that developmental paths established in early years are of the most concern since they determine child's further growth.

Conflict of Interest: None Disclosed

Funding: No Funding

\section{T4P130 \\ Who Grows Most In Ireland? An Analysis Of Weight Status Changes In Early Childhood}

\author{
Jabakhanji, S. '; Boland, F.'; Ward, M. 2; Biesma, R. ${ }^{1}$ \\ ${ }^{1}$ Royal College of Surgeons in Ireland, ${ }^{2}$ Trinity College Dublin
}

Introduction: Ireland is expected to reach the highest European prevalence of overweight (Ow) and obesity by 2030 [1] and 1 in 4 children are already Ow or obese [2]. However, few studies have addressed early life risk factors and interventions [3]. The aim of this study was to investigate changes in weight status and identify factors associated with weight transitions in young children in Ireland.

Methods: Data on 11,134 children were collected in the nationally representative Growing Up in Ireland (Gui) cohort study. Height and weight were measured at 9 months, 3 years and 5 years of age. Multinomial logistic regression was used to identify risk factors associated with weight status, and of temporal change between normal weight $(\mathrm{Nw})$ and $\mathrm{Ow}$.

Results: In this cohort, $40 \%$ of all children maintained a $\mathrm{Nw}$ from 9 months to 5 years and $15.9 \%$ were persistently Ow or obese (World Health Organization child growth standard). Over time, prevalence rates of Ow, including obesity, changed from $39 \%$ in infants to $43 \%$ at 3 years and $30 \%$ at 5 years. Compared to children with $\mathrm{Nw}$ at all ages, children with high birthweight or early rapid weight gain had a significantly higher risk of Ow at any age and the risk of persistent $\mathrm{Ow}$ was highest. Additionally, non-Irish white background protected from infant Ow, and having an Asian or Ow mother increased the risk of Ow after 9 months. Furthermore, the risk of becoming Ow after 9 months and remaining Ow was higher for girls, children watching more Tv, children living in rural regions and children with Ow fathers, Ow mothers or mothers who smoked during pregnancy. Returning to Nw after 3 years was associated with late introduction to solid food.

Conclusion: In this cohort of young children in Ireland, risk factors of obesity are related to different growth patterns. Policy-makers should identify channels through which prevention programmes can reach highrisk families before susceptible children reach a critical age. Moreover, effective interventions targeting parental weight and maternal smoking may be needed.

\section{Conflict of Interest: None Disclosed}

Funding: Research relating to this abstract was funded by the Government of Ireland Postgraduate Scholarship.

Acknowledgement: We want to thank the Gui study team, in particular the Economic \& Social Research Institute and Trinity College Dublin, and all families and children involved.

\section{References:}

1 Deborah Condon. Ireland to top obesity tables by 2030. irishhealth.com 2014 12/5/2014 [cited 2015 21/9/2015]; Available from: http://www.irishhealth.com/article.html?id $=23606$

2 Growing Up in Ireland NIsci, Key findings: infant cohort (at 3 years). 2011.

3 Waters, E., et al., Interventions for preventing obesity in children. Cochrane Database Syst Rev, 2011(12): p. Cd001871. 
T4P131

Why doesn't obesity treatment (diet and physical activity)

work?

Morinder, G. ; Janson, A. ${ }^{2}$

${ }^{1}$ National Childhood Obesity Centre, Karolinska University Hospital and Department of Clinical Science, Intervention and Technology, Karolinska Institutet, ${ }^{2}$ National Childhood Obesity Centre, Karolinska University Hospital And Department Of Women'S And Children'S Health, Karolinska Institutet

Obesity in children and adolescents is a major public health problem. As treatment is based on behavioral change, neuropsychiatric disorders may complicate implementation and outcome. The aim of this study was to describe the prevalence of neuropsychiatric disorders, emotional and behavior problems, and migrant background in obese children and adolescents admitted to a specialized pediatric obesity clinic in Sweden.

Methods: Cross-sectional analysis based on chart review of all children and adolescents undergoing treatment at the National Childhood Obesity Centre, Stockholm, Sweden in December 2016. The children $(n=348,160$ girls, 1-19 yrs, mean 13,8 yrs) were all classified as obese according to Iotf at referral.

Results: Of 348 subjects 130 (37\%) were diagnosed with or undergoing neuropsychiatric investigation regarding Adhd, Add, Dcd, autism or Asperger's syndrome. 189 (54\%) subjects had ongoing or had previously had contact with social services or centers for children's mental health. 182 (52\%) subjects had two parents of non-Nordic background.

Conclusion: Multidisciplinary teams working with obese children and adolescents need to consider the impact of neuropsychiatric disorders, emotional and behavior problems and migrant background on treatment. Focusing only on diet and physical activity is inadequate for successful weight loss and improved health in a majority of these children and adolescents. Intensive teamwork including collaboration with schools, social services, and centers for children's mental health is necessary. Further, understanding and awareness of cultural similarities and differences regarding views and perceptions of obesity, physical activity and diet are important for therapeutic alliance and successful treatment.

Conflict of Interest: None Disclosed

Funding: No Funding 
A, A. T1P 93

AARTS, M. T3P 68

AARTS, S. T1P 25

AASBRENN, M. T1P 147

ABBASI, A. T3P 187

ABBOTT, S. T3P 167

ABDOUNI, L. T1P 43

ABEL, O. T2P 101

ABERLE, J. T3P 97, T3P 51

ABEYSEKERA, A. T3P 10, T3P 95

ABRAHAM, C. T2P 67

ABREU, S. T2P 4, OS1:OC 21, T2P 17, T4P 72, T4P 98, T2P 16

ABREU-VIEIRA, G. T1P 81

ABSATAROVA, Y. T3P 201

ACARINO, A. T3P 93

ACEVEDO, L. RS14:4, RS14:5

ADAME, R. T3P 119

ADAMS, J. T2P 67

ADAMSON, A. T2P 67, OS6:OC 37, T4P 32, T3P 66

AGADŽANJANA, K. T4P 29

AGGARWAL, S. T3P 132

AGNES, V. T4P 30

AGOSTINIS-SOBRINHO, C. T2P 17, T4P 72, T4P 98

AGUDO, M. T2P 71

AGUIAR, L. T3P 75, T3P 174

AGUILAR, G. T3P 211

AHERN, A. OS7:OC 63

AHLUWALIA, T. T1P 113, T2P 85

AHN, A. T4P 55

AILANEN, L. T1P 46

AINSCOUGH, K. T3P 192

AIRES, L. T4P 2

AITHAL, G. T3P 188

AITKEN, D. T3P 180

AKOLI KOUDOU, Y. OS4:OC 10

AKSUNGAR, F. T2P 181

ALAIN, D. T4P 105

ALAJEELI, F. T1P 94

ALAMO, P. T2P 172

ALBERDI, G. T3P 4, T2P 53, T2P 161

ALBUQUERQUE, C. T2P 137, T4P 95, T1P 106, T4P 110

ALBUQUERQUE, K. T1P 42, T1P 75

ALDHOON-HAINEROVA, I. T2P 52

ALDISS, P. T1P 26

ALEKSANDR, I. T3P 206

ALEXANDRA, K. T2P 32

ALHONIEMI, E. T1P 109

ALICIA, F. T4P 7

ALIPOUR, R. T3P 72

ALMEIDA, C. T4P 66

ALMEIDA, $\mathrm{H}$. OS3:OC 5

ALMEIDA, J. T1P 149

ALMEIDA, M. T4P 19, T4P 12

ALMEIDA, M. T2P 6, T4P 60

ALMEIDA, R. T3P 30, T3P 31

ALONSO, P. T2P 127, T2P 33

ALONSO-ALONSO, M. T1P 111
ALT, F. T1P 11

ALVARES, R. T3P 126, T3P 32

ÁlVAREZ-BUENO, C. T4P 44, T2P 68

ALVAREZ-BUENO, C. T4P 93

ALVES, A. T2P 27

ALVES, M. T1P 20

ALVES, M. T3P 145, T3P 21, T3P 22

ALVES, M. T3P 191

ALVIZO, A. T3P 119

ALZOUBI, K. T1P 104

AMANYAH, S. T2P 29

AMATO, A. T3P 163

AMATO, V. T1P 52

AMÉLIE, R. T4P 7

AMIL, M. OS3:OC 2

AMIR, Z. RS14:3

AMIRI, F. T3P 72

AMOR, M. T3P 130

AMOROSO, A. T4P 13

ANAGNOSTOPOULOU, K. T3P 82

ANASTASIA, K. T4P 14

ANASTSIYA, P. T3P 200

ANDAKI, A. T4P 126, T2P 27

ANDERSEN, A. OS10:OC 44

ANDERSEN, E. RS17:2

ANDERSON, R. T3P 110

ANDERSSON-ASSARSSON, J. OS11:OC 58

ANDRADE, A. T4P 95, T1P 106

ANDRADE, G. T2P 100

ANDRADE, I. T1P 39, T1P 98

ANDRADE, M. T1P 125

ANDRADE, M. T3P 169

ANDRADE, P. T1P 88

ANDRADE, S. T1P 10

ANDREA, M. T1P 60

ANDREELLI, F. T4P 81

ANDREEVA, E. T3P 201

ANDREY, A. T3P 200

ANGELIDI, A. T3P 140, T3P 82

ANGELIDI, M. T3P 82

ANGORANI, P. T4P 116

ÄNGQUIST, L. T1P 113, T2P 85, OS10:OC 46, OS10:OC 44

ANIBAL, F. T1P 41, T1P 153

ANNA, V. T3P 200

ANNEMIEKE, H. OS2:OC 51

ANSEL, J. T3P 105, T3P 15

ANSELMINO, M. OS11:OC 60

ANTAL, E. T2P 134

ANTONI, R. T3P 185

ANTONIA, K. T2P 87

ANTONIA, P. T4P 30

ANTONIO, D. OS11:OC 56

ANTUNES, H. T4P 121

APARICI, A. T3P 93

APARÍCIO, G. T4P 91, T2P 137, T4P 109, T4P 110

APOLZAN, J. T4P 49

APPEL, E. T1P 113

ARANDA, G. T3P 211

ARAÚJO, A. PL7:2
ARAÚJO, J. T4P 3, OS10:OC 48

ARAÚJO,T. T1P 65

ARAÚJO, T. T2P 64

ARAÚJO-SOARES, V. T2P 67, OS6:OC 37

T4P 32, T3P 66

ARAZOLA, D. T2P 172

ARBANAS, T. T3P 202

ARCHIBALD, D. T2P 86

ARENAL, I. T3P 6, OS2:OC 53

ARGENTATO, P. T3P 73, T3P 123

ARITICI, G T2P 176

ARNAIZ, M. T2P 131, T2P 132, T2P 133

ARNOTT, B. OS6:OC 37, T4P 32

ARO, B. T3P 24

ARONNE, L. RS 3:3

ARON-WISNEWSKY, J. T3P 189, OS11:OC 55

ARÓS, F. T3P 54

ARROCHA, M. T2P 131, T2P 132, T2P 133

ARSENIJEVIC, D, T1P 115

ARTS, I. T1P 108, RS1:1, RS1:4

ARVIDSSON, L. OS1:OC 23

ASCHOFF, J. T3P 107, T3P 108

ASGHARI, G. T1P 78, T1P 151, T1P 23, T1P 14, T1P 156, T1P 57

ASSELIN, J. T3P 110

ASSYOV, Y. T3P 40

ASTBURY, N. T3P 203, T2P 50

ASTIARRAGA, B. OS11:OC 60

ASTRUP, A. T1P 108, RS1:4

ATANASIU, V. T1P 5

ATTI, M. T2P 10

AUCOTT, L. AS4:1

AVEYARD, P. T2P 50, T3P 133, OS7:OC 63

ÁVILA, A. T1P 69, T1P 19

AYALA-LOPEZ, N. AS5:6

AYIOMAMITIS, G. T3P 140

AZEVEDO, J. T4P 66

AZEVEDO, T. T3P 191

AZINHEIRA, J. T3P 214, T3P 114, T3P 12

BAAK, M. T1P 63, T1P 40, RS3:1

BACARDÍ-GASCÓN, M. T2P 28, T4P 125, T4P25

BACHNER-MELMAN, R. T3P 56, T4P 127

BACKHOLER, K. OS5:OC 35

BACQUER, O. T1P 37

BAGNATO, V. T1P 12

BÄHR, I. T1P 36

BAKACS, M. T2P 110

BAKER, J. OS10:OC 46, OS10:OC 44,

OS8:OC 29, R12:1

BAKKER, L. OS9:OC 15

BAK-SOSNOWSKA, M. T2P 179, T2P 41

BAKULA, M. T1P 34

BALANDA, K. T3P 106, T4P88

BALARATNAM, J. RS 14:3

BALIBREA, J. T1P 50

BALLARD, C. T1P 138, T1P 18

BALODIS, I. T2P 107

BALSA, A. M. T3P 191

BAMBRA, C. OS5:OC 34 
BANDONI, D. T2P 59

BANDT, J. T3P 55

BÁNOVČIN, P. T4P 113

BANWELL, C. OS5:OC 36

BARANSKA, M. T4P 41

BARBOSA, D. T3P 146

BARBOT-TRYSTRAM, L. OS9:OC 13

BARCIA, M. T3P 54

BARDINI, R. OS9:OC 18

BARDOS, $\mathrm{H}$. OS5:OC 31

BARNETT, T. T4P 58

BARQUISSAU, V. RS2:3

BARRADA, J. AS2:1

BARREDA, G. T3P 190

BARREIROS, C. T3P 214

BARRON, A. T1P 89

BARROS, A. T1P 56

BARROS, R. T4P 66, T3P 131

BARSAMIAN, C. T3P 13

BASRAN, J. T3P 162, T3P 8, T3P 9, T2P 167, T3P 171

BASTIEN, V. T1P 31

BATINI, F. T1P 88

BATISTA, L. T1P 42,T1P 75

BATOGAB, S. T1P 142, T1P 150

BATTERHAM, A. T3P 66

BATTERHAM, R. OS11:OC 59

BAUR, L. AS4:1, RS16:1

BAWDEN, S. T3P 188

BAZUIN, I. T3P 112

BEAULIEU, K. OS4:OC 8, T2P 108

BECERRIL, S. T1P 58, T1P 83

BECH, B. T4P 11

BECKERHINN, P. T3P 218

BECK-SICKINGER, A. T1P 82

BEDNAŘÍKOVÁ, R. T4P 1

BEDOSSA, P. T3P 189, T3P 13, T4P 81

BEEK, C. OS7:OC 62, OS2:OC 51

BEGEY, A.L. OS3:OC 6

BEGLINGER, C. T1P 48, T1P 128, OS2:OC 50, T1P 54

BELEIGOLI, A. T3P 126, T3P 32, T3P 210

BELL, J. T1P 59, OS7:OC 65, RS1:3

BELL, L. T3P 217

BELLARY, S. T3P 167

BELLICHA, A. OS11:OC 57

BELLIDO, D. T3P 35

BELLIGOLI, A. T1P 118, OS9:OC 18

BELLO, C. T. T3P 138

BELLUZZI, E. T1P 61

BELO, L. T4P 108

BELO, S. T3P 152

BEL-SERRAT, S. RS6:4

BELTOWSKI, J. T1P 1

BENESTAD, B. T4P 34

BERG, C. T2P 76

BERG, S. T1P 25

BERGHUIS, K. T2P 150

BERKEMEIER, S. T2P 119

BERNHARDT, I. T1P 104

BERTEOTTI, M. T3P 134

BERUMEN, J. T3P 139

BES-RASTROLLO, M. T2P 147, T2P 123

BETRY, C. T1P 9

BETTENCOURT-SILVA, R. T1P 145, T3P 103,

T3P 102, T3P 136, Т3P 152

BETTINI, S. T1P 118
BEUZELIN, D. RS2:3

BICA, I. T4P 95, T1P 106

BICALHO, P. T3P 210

BIERI, O. T1P 48, OS2:OC 50

BIESMA, R. T4P 130

BIJELIĆ, N. T1P 34

BIJNEN, M. RS 2:4

BILBAN, M. T3P 130

BILIŃSKA, I. T4P 76

BINDELS, P. T4P 84

BIRDANOVA, V. T2P 121

BISSINGER, R. T1P 104

BISSOLATI, M. T3P 158

BJERREGAARD, L. OS8:OC 29, OS10:OC 46

BLAAK, E. T1P 40, T1P 108, OS7:OC 62,

$\mathrm{RS} 2: 4, \mathrm{RS} 1: 4$

BLACK, K. T2P 119

BLACK, R. RS11:3

BLADBJERG, E. T3P 177, T3P 181

BLAKE, C. T3P 137, T3P 101

BLANCO, A. T3P 104

BLOMSTER, H. T3P 156

BLÜHER, M. T1P 110, T1P 82, PL1:3

BLUNDELL, J. OS1:OC 19, OS4:OC 8, OS4:OC9, T2P 108, T1P 160, T3P 160 BOELSEN-ROBINSON, T. OS5:OC 35

BOER, A. T3P 112

BOESCH, C. T3P 129, T3P 69, Т3Р 176

BOHN, L. T2P 55, T2P 27

BOIRIE, Y. T4P 82, T1P 37, T1P 115

BOJILOVA, D. T4P 101

BOLAND, F. T4P 130

BOLDARINE, V.T. T2P 106, T1P 45

BOLTON, J. T1P 95

BOMO, O. T1P 133

BONADONNA, R. T4P 63

BONGARTZ, U. T1P 11

BONNEL, S. T1P 95

BOPARAI, A. RS 14:3

BORDIGATO, E. T1P 118

BORDOLI, C. T3P 6, OS2:OC 53

BORGA, M. T1P 59, OS7:OC 65, RS1:3

BORGES, D. T1P 35

BORGHAMMER, P. T1P 32

BORGO, C. OS9:OC 18

BORGWARDT, S. T1P 48, OS2:OC 50, T1P 54

BOSCHMANN, M. T3P 26

BOSY-WESTPHAL, A. T3P 107, T3P 108,

T1P 68, AS6:3

BOT, M. T2P 51, OS1:OC 22

BOTELHO, R. T3P 144

BOTHE, G. T3P 60

BOTTIN, J. T3P 184, T3P 170

BOUILLOT, J. T3P 189, T3P 13, T4P 81, OS11:OC 57, OS11:OC 55

BOULE, N. T1P 47

BOURDEAUDHUIJ, I. OS1:OC 23, OS5:OC 31

BOUSHEY, C. T2P 45

BOUSKELA, E. T3P 75, T3P 174

BOUWMAN, A. T3P 173, T3P 212, T3P 28

BOVOLINI, J. T1P 125

BOWDEN, D. T3P 20

BOYANOV, M. T2P 140

BOYLAN, P. T2P 36

BOYLAND, E. OS7:OC 63, T2P 159

BRAGA, S. T3P 149, T3P 224
BRANCO, T. T2P 3, T3P 90

BRANDÃO, I. T2P 125

BRAVO, C. T3P 124, T3P 16

BREDA, J. PL1:1

BREEN, C. T3P 137, T3P 101, T2P 34

BREINING, P. T1P 32

BRETXA, C. T3P 211

BRISSMAN, M. OS6:OC 39

BRITO, M. T2P 136

BRITO, M. T4P 86, T1P 4

BRIX, J. T3P 151, T3P 204, T2P 114

BROT-LAROCHE, E. OS9:OC 13

BROUWER, I. AS2:1

BROWN, H. T3P 66

BROYLES, S. T4P 111

BRUG, J. OS5:OC 31

BRUNHOLI, C. T3P 169

BRYANT, M. OS6:OC 38, T2P 175

BRYTEK-MATERA, A. T2P 135, T2P 128

BUCHTHAL, S. T2P 45

BUCKLAND, N. OS4:OC9, T2P 108, T3P 160

BUCKNER, L. T3P 6, OS2:OC 53

BUDGE, H. T1P 26

BUDUI, S. T3P 134

BUENO, D. T2P 89

BUEVING, $H$. T4P 84

BUGALHO, M. T3P 146, T2P 136

BUHLER, T. T3P 176

BUISE, M. T3P 173, T3P 212, T3P 28, T3P 41

BUJO, H. T1P 130

BULLÓ, M. T2P 127, T2P 33, T3P 54

BUNN, C. T3P 197, RS17:2

BURCHIELLI, S. T1P 87

BURDYGA, G. T1P 128

BURGOS, R. T1P 50

BURINI, A. T3P 158

BURNYSHEVA, I. T4P 70

BURSTEIN-KLOMEK, A. T3P 56

BUSCH, P. T3P 97, T3P 51

BUSE, J. RS14:5

BUSETTO, L. T1P 118, T3P 134, OS9:OC 18

BÜSING, F. T3P 107, T3P 108

BUTLER, E. T2P 108, T2P 1

BÜYÜKBOZKIRLI, D. T3P 205

BYRNE, M. T4P 46

BYUN, A. T2P 2

BZIKOWSKA, A. T4P 41

CABO, F. T3P 211

CABRAL, C. T2P 14

CABRAL, M. T4P 3, OS10:OC 48

CAĞILTAY, E. T3P 205

CAI, J. T2P 63

CAIAZZO, R. OS7:OC 66

CAIRNS, J. OS5:OC 34

ÇAKIR, B. T4P 38, T4P 69

CALABRESE, D. T3P 46, T3P 48

CALABRETTO, $M$. T1P 126

CALBO, M. T3P 211

CALDARA, A. OS4:OC 11

CALEYACHETTY, R. OS2:OC 54

CALIXTO-LIMA, L. T4P 99

CALONNE, J. T1P 77, T1P 115

CALVO, C. T2P 127

CAMACHO, M. T2P 94

CAMARGO, M. T4P 78

CAMASTRA, S. OS11:OC 60 
CAMIDGE, D. OS4:OC9, T3P 160

CAMPAROTO, M. T3P 169

CAMPBELL-SCHERER, D. T3P 110, T2P 151

CAMPOS, B. T1P 99

CAMPOS, J. T2P 25

CAMPOS, R. T1P 12

CAMPOS, V. T3P 129, T3P 69, T3P 55

CAMPS, S. T1P 22

CANFORA, E. OS7:OC 62

CANHÃO, H. OS5:OC 33, T2P 122

CANNON, B. T1P 81

CANOTILHO, C. T4P 67

CAPITÃO, R. T3P 214, T3P 12, T3P 164, T3P 138

CAPOCCIA, D. T3P 159

CAPRIO, C. T1P 3

CARAYOL, J. T1P 95

CARDENAS, G. T1P 50

CARDENOUX, C. T4P 82

CARDOSO, J. T3P 138

CARETTE, C. OS8:OC 28, T3P 13

CARLE, R. T3P 107, T3P 108

CARLSSON, L. OS11:OC 58

CARMO, I. T2P 136, T1P 30

CARMO, M. T1P 75

CARNEIRO, J. T1P 72, T2P 60, T3P 71, T1P 71

CARO, R. T1P 61

CARRAÇA, E. T3P 7

CARREIRA, J. T3P 35

CARREIRA, M. C. OS3:OC 2

CARREIRA, M. T1P 10

CARROLL, D. T3P 20

CARROLL, D. T1P 158

CARVALHO, D. T1P 145, T3P 103, T3P 102, T3P 136, T3P 152

CARVALHO, E. AS5:3

CARVALHO, J. T2P 27

CARVALHO, K. OS8:OC 27, T2P 5, T4P 4, T2P 12, T3P 62

CARVALHO, S. AS3:3

CASANUEVA, $\mathrm{F}$ T1P 10

CASANUEVA, F. T3P 35

CASANUEVA, F.F. OS3:OC 2

CASELLA, G. T3P 5

CASEY, D. T3P 137, T3P 101

CASTAÑEDA, A. T2P 172

CASTAÑO, J.P. OS3:OC 4

CASTILLO-RUIZ, O. T4P 125

CASTRO, A. T2P 55

CASTRO, A. T3P 35

CASTRO, C. T1P 41, T1P 153

CASTRO, H. T4P 20

CATALÁN, V. T1P 58, T1P 83, T1P 67, T3P 37

CATALDO, R. T1P 27, T3P 124, T3P 16

CATALINA, P. T1P 60

CATARINO, A. T3P 92

CATARINO, C. T3P 92

CATARINO, F. T3P 162, T3P 9, T3P 8, T2P 167, T3P 171

CATERSON, I. T3P 44

CATHERINE, D. T4P 30

CATHERINE, T. T4P 30

CAUSSY, C. T1P 9

CAVADAS, C PL7:4

CAVE, A. T3P 110

CAVERO-REDONDO, I. T4P 44, T4P 93, T2P 68
CAZARIN, C. T1P 138,T1P 18

CECILE, M. T4P 30

CECILE, R. T4P 30

CEDEÑO, J. T2P 133, T2P 132, T2P 131

CEKIĆ, N. T4P 45

CELIK, A. T3P 205

CEPERUELO-MALLAFRÉ, V. T1P 132

CEREIJO, R. T1P 85, T4P 123

CERVILLA, J. T2P 155, T2P 71

CÉSAR, A. T2P 27

CESARO, L. OS9:OC 18

CHABOWSKI, A. T1P 131

CHAHINE, E. T3P 175, T3P 219

CHAKRABORTY, S. T3P 106

CHAN, L. OS6:OC 40

CHANDRAN, M. T1P 93

CHANG, E. T1P 44

CHANG, S. T3P 19

CHAPLAIS, E. T4P 82

CHAPLIN, J. T2P 76, T4P 97

CHAPLIN, J. T4P 97

CHAPUT, J. T4P 111

CHARALAMPAKIS, V. T3P 182

CHARLES, M. A. OS4:OC 10

CHARLOTTE, F. T3P 189

CHARREIRE, $\mathrm{H}$. OS5:OC 31

CHARSLEY, J. OS1:OC 20

CHARZEWSKA, J.T4P 85

CHATHAM, J. T4P 49

CHATTERJEE, P. T1P 89

CHEN, C. T1P 123

CHEN, M. T2P 139

CHEN, S. T2P 18

CHEN, T. T2P 18

CHEN, Y. T2P 95, T2P 47, T2P 154

CHENEY, K. T2P 119

CHENG, G. T2P 47, T2P 139, T2P 95, T2P 154

CHIKOVA-ISCENER, E. T4P 101

CHING-LOPEZ, A. T2P 155, T2P 71

CHLOÉ, D. T4P 7

$\mathrm{CHOH}, \mathrm{A} . \quad \mathrm{T} 2 \mathrm{P} 63$

CHOI, E.. T3P 70

CHOI, J. T4P 55

CHONG, P. T1P 11, T3P 60

CHOUILLARD, E. T3P 175, T3P 219

CHRISTELLE, J. T4P 7

CHRISTOPHER, M. T4P 30

CHUNG, A. OS5:OC 35

CIANGURA, C. OS11:OC 57

CICCANTELLI, B. T1P 126

CIFRE, M. T3P 85

CINELLI, G. T2P 10

CINELLI, L. T1P 42

CINOVICS, N. T2P 149

CIOCIARO, D. OS11:OC 60

CIOFFI, I. OS4:OC 11

CIPRIANO, M. T3P 5

CIRCO, E. T1P 159, T1P 144

CIRCO, R. T1P 159, T1P 144

CITELLI, M. T4P 99

CIUDIN, A. T1P 50, T3P 93

CLEMENT, K. T3P 189

CLÉMENT, K. T4P 81, OS11:OC 57

OS9:OC 13, OS11:OC 55, T1P 129

CLIFTON, P. M. T3P 76

CLOETENS, L. T3P 63

COCCIA, F. T3P 159
CODOGNO, J. T3P 169

COELHO, C. T3P 221, T3P 36

COELHO, J. T1P 51

COIMBRA, S. T3P 92

COLEMAN, D. OS6:OC 38

COLLINS, A. L. T3P 185

COLLINS, S. OS1:OC 20

COLONELU, E. T3P 50

COMANESHTER, D. S. OS11:OC 55

COMPERNOLLE, S. OS5:OC 31

CONCEIÇÃO, E. T4P 121, T2P 125, T4P 89, T2P 159

CONCEIÇÃO, G. T1P 90, T1P 92, AS5:5, OS3:OC 1

CONNOLLY, K. T4P 54

CONNORS, A. T2P 151

CONSORTIUM, O. T3P 197

CONSORTIUM, T. D. RS1:4, T1P 108

CONSTÂNCIA, M. RS19:1

CONTALDO, F. T1P 3, T1P 52, T1P 116 OS4:OC 11

CONTRERAS, G. T4P 58

COPAESCU, C. T3P 202

COPLE-RODRIGUES, C. T4P 99

COPPACK, S. RS 14:3

CORDEIRO, A. T1P 99

CORELLA, D. T3P 54

CORLEY, B. T1P 158

CORNETTE, F. T3P 176

CORREIA, F. T3P 136, T3P 36

COSTA, L. T1P 41

COSTA, L. T3P 15, T3P 105

COSTA, M. T1P 145, T3P 103, T3P 102, T3P 136, T3P 152

COSTA, P. T1P 112

COSTA, R. T1P 149, T2P 94

COUPAYE, M. T1P 9, T3P 46, T3P 48

COUTINHO, S. T3P 109, RS6:3, T1P 133, OS4:OC 7

COVES, M. J. T3P 211

COX, J.S. T3P 3

COX, V.M. T1P 38, T1P 146

CRISOSTOMO, L. T2P 118, T2P 13

CRISTEA, B. T1P 5

CRODEN, F. OS4:OC9, T3P 160

CROS, J. T3P 69, T3P 176, T3P 55

CROVESY, L. T3P 115

CROYDEN, D. OS6:OC 40

CRUJEIRAS, A. B. OS3:OC 2, T1P 10, T3P 35

CRUZ, A. T2P 92

CRUZ, C. OS3:OC 5

CRUZ, L. T2P 68

CRUZ, L. T3P 59

CRUZ, M. T1P 88

CRUZ, S. T3P 42

CRUZ, S. T3P 42

CUATRECASAS, G. T3P 211

CUATRECASAS, G. T3P 211, T1P 111

CUBILES, M. D. T2P 172

CUNHA, F. T1P 145

CUNHA, M. T4P 95, T1P 106

CUNHA, T. T3P 210

CUREAU, F. T2P 12

CURI, H.T. T4P 78

CURRIE, V. T3P 196

CUTHBERTSON, D. J. T2P 72, T2P 164, T2P 143, T3P 23 
CUTLER, L. OS6:OC 37, T4P 32

CZARNECKA, K. T3P 190

CZEPCZOR, K. T2P 135, T2P 128

CZERNICHOW, S. OS8:OC 28, OS7:OC 66, T3P 13

CZERWINSKI, S. T2P 63

CZIPPELOVÁ, B. T4P 50, T4P 113

D'ALESSANDRO, A. T3P 175, T3P 219

DAHEL-MEKHANCHA, C. T1P 2

DAHLGREN, J. T2P 76, T4P 97

DALTON, M. T2P 108

DALY, K. T1P 128

DÂMASO, A. T4P 119, T1P 12

DAMMEN, L. OS2:OC 51

DANA, M. T2P 32

DANESHAFROOZ, A. T1P 78,T1P 151, T1P 23, T1P 14

DANIEL, C. T2P 57, T4P 43

DANIEL, H. T3P 34

DANIEL, W. T4P 92

DANIELS, L. A. OS6:OC 40

DANIELSEN, Y. S. T4P 74

DANTAS, R. T3P 191

DAO, M. T1P 129

D'AURIZIO, R. OS9:OC 14

DAVE, G. T2P 57

DAVID, T. T4P 7, T4P 92, T2P 57, T4P 43

DAVIES, K.A. T2P 72, T2P 164, T2P 143

DAVIS, A. T4P 49

DEARY, I. J. OS10:OC 45

DEEV, D. T1P 8,T1P 139

DÉJEAN, S. RS2:3

DEJONG, C. H. OS7:OC 62

DEKKER, J. T3P 221

DEKKER, L. T3P 28

DELANEY, L. T4P 46

DELGADO, L. T3P 131

DER, G. OS10:OC 45

DERKS, E. T2P 51, OS1:OC 22

DESOYE, G. RS19:2

DESPLAND, C. T3P 129, T3P 61

DEVLIN, J. T2P 105

DHARNI, N. T2P 175

DHURANDHAR, N. T2P 84, T3P 118, T2P 49

DIAS, C.T. T4P 78

DIAS, J. S. T3P 169

DÍAZ-LAGARES, A. OS3:OC 2

DÍAZ-RÚA, R. T2P 7, T3P 85

DICKER, D. OS11:OC 55, RS12:2

DICKERSON, J. T2P 175

DIEZ-FERNANDEZ, A. T4P 93

DIMITROV, P. T4P 101

DINH, H. OS5:OC 36

DINIZ, M. T3P 126, T3P 32

DINIZ, M. T3P 32

DIRANI, M. T3P 175, T3P 219

DISSE, E. T3P 157, T3P 78

DISSE, E. T1P 9

DITARANTO, F. T1P 87

DITRICH,T. T4P 1

DIXON, J. T2P 35

DOBERSTEIN, $\mathrm{H}$. T1P 36

DOBOSZ, J. T2P 163

DOMINGOS, A. PL4:1

DOMINGUEZ, O. M. T3P 104

DONELLA-DEANA, A. OS9:OC 18
DONG, L. OS9:OC 13

DONGHYUN, K. T1P 13

DONINI, L. T1P 126

DORÉ, J. T1P 129, PL5:1

DORLING, J. T3P 188

DORNELLAS, A. T2P 106, T1P 45

DOTAN, I. T3P 94

DOUGKAS, A. T1P 29

DOURADO, D. T2P 89, OS8:OC 30

DOURADO, V. T3P 123, T3P 73

DOZIO, N. T3P 158

DRAGOMIR, A. T3P 143

DRAI, J. OS9:OC 13

DREHER, M. T2P 43

DREISCHER, P. T1P 104

DUARTE, A. T1P 41,T1P 153

DUARTE, C. T1P 160, AS3:3

DUARTE, C. T3P 162, T3P 9, T3P 8, T3P 171, T2P 166

DUARTE, F. T1P 12,T1P 153

DUARTE, H. T3P 128

DUARTE, J. T3P 214, T2P 94, T3P 114, T3P 12, T3P 138, T3P 164

DUARTE, J. T4P 91

DUARTE, J. T1P 125, RS17:1

DUARTE, Y. T2P 118, T2P 89, T2P 13 , OS8:OC 30, T2P 64

DUBERN, B. T4P 81

DUBOIS, M. J. T1P 138, T1P 18

DUCLOS, M. T4P 82

DUHITA, M. OS7:OC 61

DUIM, E. T2P 103

DULEVA, V. T4P 101

DULLOO, A. G. OS7:OC 61,T1P 53, T1P 77, T3P 58, T1P 115, AS6:2

DUMANOVIC, M. S. T3P 168

DUNCAN, M. T4P 2

DUNLEVY, C. T3P 137, T3P 101, T2P 34

DUNN, C. T3P 132

DUPRÉE, A. T3P 97, T3P 51

DURRER, D. T1P 77

DUTRA, E. S. OS8:OC 27, T2P 5, T4P 4, T2P 12, T3P 144, T3P 62

DYLAG, H. T4P 41

DYSON, L. T3P 3

DZIVITTE-KRIŠĀNE, I. T4P 29

EBENBICHLER, C. T3P 213

EBERT, T. T1P 110

EBINUMA, $\mathrm{H}$. T1P 130

EBLING, F. T1P 26

EDWARDS, K. T1P 97

EGHTESADI, M. T3P 72

EGHTESADI, S. T3P 187, T3P 72

EHRET, J. T2P 162

EIBEN, G. OS1:OC 23

EIJK, V. G. T3P 126, T3P 32

EJARQUE, M. T1P 132

EJTAHED, H. T4P 10, T4P 116

EKATERINA E. T1P 148

EKATERINA, A. T4P 37

EKATERINA, T. T1P 148, T3P 200, T3P 206

EKNES, P. T1P 133

ELDIN, N. T2P 105, T4P 61

ELDRIDGE, S. T3P 183

ELENA, F. T4P 31, T4P 51, T4P 102

ELIAS, C. OS11:OC 56
ELIE, C. OS11:OC 56

ELISABETH D. T4P 7

ELLS, L. OS6:OC 37, T4P 32

ELODIE, C. T2P 57

ELOFSSON, H. T3P 156

ELY, P. B. T1P 35

EMILE, J. T3P 13

EMMANUEL, J. RS 14:3

ENCANTADO, J. AS3:3, AS3:4

ENGELI, S. T3P 26

ENGL, J. T3P 213

ENGUIX, A. T2P 172

EPANCHINTSEVA, E. A. T1P 139

ERDEI, G. T2P 110

ERHLICH, D. T1P 129

ERIKA, G. T2P 87

ERIKSSON, P. T3P 27

ERMES, M. AS3:3, AS3:4

ERNESTI, I T3P 5

ERNST, T. T2P 45

ESHGHI, S. T1P 47

ESPERSEN, R. T3P 25, T3P 178, T3P 179

ESTADELLA, D. T4P 119

ESTE, C. D. OS5:OC 36

ESTRADE, M. T2P 86

ESTRUCH, R. T3P 54

ETIKAN, Í. T3P 205

EVANGELOU, A. T3P 140

EVANS, E. T3P 66, AS3:1, AS3:2, AS3:3

EVARISTO, S. OS1:OC 21

EVELO, C.T. RS1:4

EVERLEY, S. T2P 144, T4P 28

EVISON, K. OS6:OC 42

EZQUERRO, S. T1P 58,T1P 83

FABRIS, R. T1P 118, OS9:OC 18

FABRIZI, M. T2P 10

FABRIZZI, F. T1P 41, T1P 153

FAGUNDES, M. J. T1P 30

FAHRENKRUG, J. T4P 9

FAKHOURY-SAYEGH, N. T2P 42

FALCÃO-PIRES, I. T1P 90, T1P 92, AS5:5, OS3:OC 1,T1P 51

FARES, E. T1P 77

FARIA, C. T1P 37

FARIAS, T. T1P 88

FARKAS, D. T2P 160

FARUP, P. T1P 147

FASSHAUER, M. T1P 110

FAUCHER, P. T4P 81

FAVARETTO, F. OS9:OC 18

FAVERO, M. T1P 61

FAVRE, L. T3P 61

FELSENREICH, D. T3P 218

FERKH, F. T3P 44

FERNANDES, A. T1P 149, T2P 94

FERNANDES, V. T3P 36

FERNANDEZ, M. T1P 42, T1P 75

FERNÁNDEZ, V. T2P 133, T2P 132, T2P 131

FERNANDEZ-INFANTE, A. T4P 93

FERNANDEZ-REAL, J. OS3:OC 2

FERNÁNDEZ-VEJA, A. T1P 114

FERNANDEZ-VELEDO, S. T1P 132

FERRANNINI, E. OS11:OC 60

FERRAZ, I. T2P 78

FERREIRA, C. T3P 92

FERREIRA, E. T3P 117 
FERREIRA, F. T3P 146

FERREIRA, G. T3P 128

FERREIRA, L. T3P 144

FERREIRA, M. T3P 126, T3P 32

FERREIRA, R. T4P 67, T2P 160

FERREIRA, R. T3P 214, T3P 114

FERREIRA, S. T4P 94

FERRINHOS, C. T3P 214, T3P 12, T3P 138 , T3P 114

FERRO-LEBRES, V. T4P 15

FESTUCCIA, W. T1P 117

FIAMONCINI, J. T3P 34

FICA, S. T3P 202

FICHEUR, G. OS7:OC 66

FIDILIO, E. T1P 50

FIELDING, S. T3P 110

FIGUEIRA, M. L. T1P 30

FIGUEIREDO, R. A. T4P 39

FINK, G.D. AS5:6

FINLAYSON, G. OS1:OC 19, OS4:OC 8, OS4:OC9, T2P 108, T1P 160, T3P 160, T2P 1

FIOL, M. T3P 54

FISBERG, M. T4P 36

FISCHER, A. T1P 81

FITÓ, M. T3P 54

FOGELHOLM, M. T3P 172, T4P 111

FOLETTO, M. T1P 118, OS9:OC 18

FOLGUEIRA, C. T1P 10

FONG, M. T3P 44

FONG, T. T3P 217

FONSECA, A. T1P 72,T1P 71

FONSECA, $\mathrm{H}$. OS5:OC 33, T2P 122, T3P 186

FONSECA, L. T3P 62

FONSECA, R. T3P 114, T3P 12, T3P 164

FONT, R. T2P 133, T2P 132, T2P 131

FONTANELLA, C. T1P 61

FONTOURA, D. T1P 51, T1P 90, T1P 92, AS5:5

FONVIG, C. T4P 118

FORHAN, A. OS4:OC 10

FORNARI, E. T4P 63

FORT, J. T1P 50, T3P 93

FOUSTERIS, E. T3P 140

FRAGOSO, I. T1P 21, T2P 113, T2P 44

FRANCESCHINI, L. T3P 134

FRANCO, O. OS10:OC 43

FRANCOMANO, D. T3P 5

FRANSQUET, P. T3P 4

FRASCATI, A. T1P 154

FRASER, S. T2P 35

FREDERIC, D. T2P 57

FREITAS, L. T3P 126, T3P 32

FREITAS, L. T3P 149, T3P 224

FREITAS, M. T1P 117

FREITAS, N. T1P 39, T1P 98

FREITAS, P. T1P 145, T3P 103, T3P 102, T3P 136, T3P 152

FRESÁN, U. T2P 147

FRIBORG, O. RS 3:4

FRIGERIO, F. T3P 158

FRITZ, J. T3P 213

FRÜHBECK, G. T1P 58, T1P 83, T1P 67, T1P 114, T3P 37

FULLEN, B. T3P 137, T3P 101

FULLER, N. R. T3P 44

FURINO, V. T1P 41, T1P 153

FURUKAWA, T. T3P 1
GAAL, L. RS14:1

GADE, H. RS3:4

GAELLE, B. T4P 105

GAENS, K. RS 2:4

GAGGINI, M. OS11:OC 60

GAILITTE, J. T4P 29

GALBAN, C. T3P 35

GALE, C. OS10:OC 45

GALGANI, J. T1P 27, OS9:OC 17, T3P 124, T3P 16

GALINA, O. T4P 14

GÁLL, Z. T1P 24

GALLAND, B. OS10:OC 47

GALLEGOS, R. T3P 16

GALVÃO, T. OS8:OC 27, T2P 5

GAMA, F. T2P 65

GAMAN, L. T1P 5

GAMBORG, M. T4P 118

GANEN, A. P. T4P 119, T4P 36

GAO, Y. T4P 87

GARBOWSKA, M. T1P 131

GARCIA, M. T4P 123

GARCÍA-ARELLANO, A. T3P 54

GARCÍA-GAVILÁN, J. F. T3P 54

GARCÍA-PRIETO, J. C. T2P 68

GARCÍA-RUIZ, E. T1P 157, AS5:2

GARRIDO-MIGUEL, M. T4P 44, T4P 93, T2P 68

GARRUTI, G. T4P 80

GARZA, F. T3P 119

GASPAROTTO, A. T1P 35

GASTALDELLI, A. OS11:OC 60

GAVALDÀ-NAVARRO, A. T1P 85

GAVRYSHEVA, O. T4P 70

GAYNOR, K. T3P 137, T3P 101, T2P 34

GAZZETTA, P. G. T3P 158

GEA, A. T2P 147, T2P 123

GEBHARDT, C. T1P 110

GEHWOLF, P. T3P 213

GELISK, I. T2P 78

GENCO, A. T3P 5

GENSER, L. T3P 189, T4P 81, OS9:OC 13, OS11:OC 55

GEORGAKOPOULOS, P. T3P 116

GEORGIANA, R. T4P 31

GERAGHTY, A. T3P 4, T2P 53

GERALDINE, N. T2P 57

GEROFI, J. T3P 44

GHASHGHAEINIA, M. T1P 104

GIANNINI, D. T2P 14

GIARDINA, S. T2P 127, T2P 33

GIBBONS, C. T2P 108, T1P 160, T2P 1

GIBNEY, E. T3P 192

GIBSON-SMITH, D. T2P 51, OS1:OC 22, AS2:3

GIL, M. J. T3P 37

GILAD, S. T3P 94

GILBERT, P. T2P 167, T3P 162, T3P 9, T3P 8, T3P 171

GILDER, K. RS14:4, RS14:5

GILHAM, B. OS5:OC 35

GILL, P. T3P 187

GILL, T. AS4:1

GILLI, F. T3P 134

GIL-ORTEGA, M. AS5:4

GIORDANO, F. T3P 134
GIORGINO, F. T4P 80

GIRALT, M. T1P 85, T4P 123

GIRAUDET, C. T1P 37

GIRIBES, M. T1P 50

GIUSTARINI, D. T1P 104

GIUSTI, V. T3P 157

GLOBUS, I. T3P 56

GLONTI, K. OS5:OC 31

GNESSI, L. T1P 126

GODLBERG, M. OS8:OC 28

GOEDECKE, J. OS3:OC 3, T1P 74

GOFFE, L. T2P 67

GÖGENUR, I. OS10:OC 46

$\mathrm{GOH}, \mathrm{H}$. T1P 22

GOKHALE, K. OS2:OC 54

GOLDE, R. OS2:OC 51

GOLDEN, A. T2P 84, T3P 118, T2P 49

GOLDSTEIN, N. T1P 82

GOMES, A. T3P 146

GOMES, D. T3P 62

GOMES, M. T4P 90

GOMES, V. T3P 146

GOMEZ, E. T3P 119

GOMEZ, P. T4P 78

GÓMEZ-AMBROSI, J. T1P 58, T1P 83, T1P 67, T3P 37

GOMEZ-ARBELAEZ, D. T3P 35

GONÇALVES, C. T2P 17, T4P 72

GONÇALVES, I. OS3:OC 5

GONÇALVES, N. T1P 51, T1P 92, OS3:OC 1, AS5:5,

GONÇALVES, S. T2P 159, T4P 121

GONÇALVES, V. S. OS8:OC 27, T2P 5, T4P 4 GONÇALVES, V. T1P 145, T3P 103, T3P 102

GONÇALVES-FIGUEIREDO, A. T4P 72

GONZALEZ, D. T3P 104

GONZÁLEZ, N. G. T2P 133, T2P 131, T2P 132

GONZÁLEZ-IZQUIERDO, A. OS3:OC 2

GOODE, J. T3P 122

GOOSSENS, G. T1P 105, T1P 40, OS7:OC 62, RS2:4, RS1:4

GORDON, A. T2P 119

GORITZ, V. T1P 36

GOULART, R. T2P 120

GOUVEIA, A. OS3:OC 5

GOUVEIA, C. T3P 114

GOVINDHARAJULU, P. T1P 22

GOWLAND, P. T3P 188

GRABLOWITZ, V. T1P 134

GRAÇA, P. RS15:2

GRACE, O. T4P 92

GRAM, B. T3P 181, T3P 177

GRANZOTTO, M. T1P 61

GRARUP, N. T1P 113

GRAY, A. OS10:OC 47

GRAY, C. T3P 197, RS17:2

GREEN, H. T2P 74

GREEN, M. OS5:OC 32

GREENMAN, Y. T3P 94

GREEVENBROEK, M. RS1:4

GREGÓRIO, M. J. T4P 66, OS5:OC 33, T2P 122

GRGIĆ, A. T1P 34, T4P 45

GRIBSHOLT, S. T2P 160

GRIFFITHS, C. OS5:OC 32

GRIFFITHS, C. T3P 183

GRILO, C. OS7:OC 64

GROEN, H. OS2:OC 51 
GRUFMAN, L. T3P 156

GUARANA, $\mathrm{H} . \quad$ T1P 72, T2P 60, T1P 71

GUARISCO, G. T3P 159

GUELINCKX, I. T3P 184, T3P 170

GUERERRO, M. T3P 93, T1P 50

GUERRA, A. T4P 89

GUERREIRO, V. T3P 136, T3P 152

GUERRERO, G. T3P 118, T2P 49

GUIDON, M. T4P 54

GUILLET, C. T1P 37,T1P 115

GUIMARÃES, A. T2P 78

GUIMARÃES, J. T3P 191

GUIMARÃES, M. T1P 20, T3P 30, T3P 31

GUNNARSSON, R. T3P 156

GUROVA, M. T4P 35

GUSEVA, A. T4P 35

GUTIERREZ, B. T2P 155, T2P 71

GUZMÁN-RUIZ, R. OS3:OC 4, T1P 114

GUZZARDI, M. A. OS9:OC 14, T1P 87

GWENNAELLE, B. T1P 31

HAASE, A. M. T4P 42

HABA-RUBIO, J. T3P 176

HADI, H. T1P 154, T1P 61

HAENNI, A. T3P 57

HÄGELE, F. T3P 107, T3P 108, T1P 68

HAGSTRÖM, H. T4P 62

HAIM, Y. T1P 82

HAINER, V. T2P 52

HAJEK, P. T3P 183

HALFORD, J. OS7:OC 63, T2P 164, T2P 143

HALICKA, E. T2P 148

HALKJ/ER, J. T2P 85

HALL, D. T1P 158

HALL, L. T4P 49

HALL, W. T3P 6, OS2:OC 53

HALMY, E. T2P 61

HALMY, L. T2P 61

HALSETH, A. RS14:4, RS14:5

HÄMÄLÄINEN, E. T2P 91

HAMILTON-SHIELD, J. OS6:OC 38

HÄNDEL, M. N. T4P 48

HANDJIEV, S. T4P 96

HANDJIEVA-DARLENSKA, T. T4P 96, T1P 66

HANKEMEIER, T. T1P 108, RS1:4

HANNA, M. OS10:OC 47

HANSEN, D. RS 2:4

HANSEN, T. T1P 113, T2P 85, T4P 118

HARIDASS, S. A. T3P 155, T2P 178

HARJUMAA, M. AS3:3, AS3:4

HÄRKÄNEN, T. T2P 22, OS8:OC 26

HARRINGTON, J. T4P 68, OS6:OC 41, T4P 65, T2P 39, T4P 46

HARRIS, J. OS6:OC 37, T4P 32

HARROLD, J. T2P 163, T2P 143

HARTMANN-BOYCE, J. T3P 203

HASANI-RANJBAR, S. T4P 10, T4P 116

HASSAPIDOU, M. T4P 40

HATA, T. T3P 1

HATCH, B. OS10:OC 47

HAUCK, C. T2P 165

HAUFE, S. T3P 26

HAUKKALA, A. T2P 70

HAWKINS, K. T4P 49

HAYEK, M. T3P 175, T3P 219

HEADLAND, M. T3P 76

HEATH, A. OS10:OC 47
HEATHERINGTON, M. T2P 151

HEDAYATI, M. T1P 78, T1P 151,T1P 23, T1P 14, T1P 156, T1P 57

HEIDARI, I. T3P 72

HEINEN, M. RS6:4

HEINONEN, K. T2P 91

HEINONEN, S. OS9:OC 15

HEINZER, R. T3P 176

HEITMANN, B. L. T2P 85, AS3:1, T4P 115,

T4P 48, T4P 9, AS3:3, AS3:4

HELIÖVAARA, M. T2P 138

HENAUW, S. T4P 26, T4P 27

HENDERSON, M. T4P 58

HERMES, G. OS7:OC 62

HERNÁNDEZ, M. L. T2P 92

HERNÁNDEZ-ALCÁNTARA， G. T4P 125

HERZ, C. T2P 114

HERZIG, K. T1P 109

HESHMAT, R. T4P 10, T4P 116

HESLEHURST, N. T4P 53, T2P 56

HETHERINGTON, M. OS4:OC9, OS1:OC 24, T3P 160

HEUDE, B. OS4:OC 10

HEWLETT, B. T3P 3

HIBBERD, A. T1P 109

HIÇYILMAZ, M. T4P 38, T4P 69

HIGUCHI, M. T1P 89

HILL, A. OS1:OC 20, AS4:1

HILL, C. T1P 38, T1P 146

HILBERT, A. OS1:OC22

HILLIER-BROWN, F. T2P 67, OS5:OC 34

HIRANO, Y. T1P 119, T1P 28

HJELMESAETH, J. RS3:4, T4P 34

HOBBS, M. OS5:OC 32

HOEFIG, C. T1P 81

HOEK, D. T2P 35

HOLECKI, M. T1P 70

HOLLINGWORTH, S. T2P 108

HOLMBERG, C. T2P 76

HOLST, J. OS7:OC 62

HONARVAR, M. T3P 187

HONG, S. T2P 168

HOPKINS, D. T3P 20

HOPKINS, M. OS4:OC 8, T1P 160

HOPPICHLER, F. T3P 98, T2P 79

HORÁCIO, J. G. T1P 112, T1P 30

HORGAN, D. T4P 61

HORGAN, G. AS3:4, T1P 160, T2P 167

T3P 9, T3P 162, T3P 171

HORGAN, G. T3P 8

HORTA, C. T2P 90

HOSSEINZADEH, Z. T1P 104

HOUSTEK, J. T1P 55

HOUTERMAN, S. T3P 112

HOUTHUIZEN, P. T3P 28

HOWEL, D. T3P 66

HOWELL, M. T2P 175

HU, G. T4P 111

HUANG, K. OS9:OC 16

HUANG, W. T4P 87

HUGHES, A. T4P 104

HUH, J. S. T3P 165, T3P 17

HUMMEL, G. T2P 162

HUNMA, S. T3P 58

HUNSBERGER, M. OS1:OC 23

HUNT, K. T3P 197, RS17:2

HUOTARI, V. AS3:3, AS3:4
HUR, Y. T2P 168

HURST, J. T1P 146

HUSEMOEN, L. T2P 85

HUVINEN, E. T3P 27

HWANG, I. C. T3P 38

IACOBELLIS, C. T1P 61

IACOVONI, J. RS2:3

IACOVONI, J. RS2:3, T1P 95

IBADULA, S. T1P 159, T1P 144

IBÁÑEZ, P. T3P 37

IBANEZ-CASAS, I. T2P 155

IENCA, R. T3P 5

IFTIKHAR, $M$. RS 14:3

IMOTO, T. T2P 18

IOANNIS, N. T3P 154

IOZZO, P. OS9:OC 14, T1P 87

IRELAND, R. RS17:3

IRINA, B. T3P 206

IRINA, L. T4P 37, T4P 14

IRVINE, L. OS7:OC 63

IRWIN, A. T3P 23

ISACCO, L. T1P 115

ITARIU, B. T3P 130, T1P 134

IWASE, M. T2P 18

IZBICKI, J. T3P 51

IZQUIERDO, A. T1P 10

J. R., W. T3P 210

J.R., R. OS3:OC 1,T1P 138, T1P 18

JÄÄSKELÄINEN, T. T2P 22, OS8:OC 26, OS8:OC 25

JABAKHANJI, S. B. T4P 130

JACCARD, A. OS7:OC 63

JACOBS, S. T2P 45

JACOBSEN, P. B. RS3:3

JACQUET, J. T1P 53

JACQUET, P. T1P 53

JACZEWSKA-SCHUETZ, J. T2P 83, T2P 171, T4P 85

JADDOE, V. OS10:OC 43

JALEEL, A. T1P 93

JAMAR, G. T3P 73, T3P 123

JAME, R. T1P 146

JAMES, R. T1P 38, T1P 102

JAN, P. T2P 87

JANAKOVA, Z. OS4:OC 12

JANIK, D. T2P 179, T2P 41

JANKOVIC-PEJICIC, A. T3P 195, T3P 111

JANSON, A. T4P 131, OS6:OC 39

JARMILA, R. T2P 32

JAROSZ, M. T2P 83, T2P 171, T2P 62, T4P 85

JAVORKA, M. T4P 50, T4P 113

JAYO, N. T1P 49

JAZAYERI, S. T3P 187

JEAN-FRANÇOIS, T. T1P 31

JEBB, S. T3P 203, T2P 50, T3P 133 ,

OS7:OC 63, RS11:2

JEFFCOCK, E. T3P 6, OS2:OC 53

JEGATHEESAN, P. T3P 69, T3P 55

JENSEN, B. OS10:OC 46

JEPSON, R. T2P 86

JEREBINE, A. OS5:OC 35

JÉRÔME, G. T1P 31

JESUS, A. T3P 215

JESUS, P. T3P 29

Jl, B. T1P 89 
JIANG, M. T1P 130

JILANI, H. OS1:OC 23

JIMÉNEZ-CRUZ, A. T2P 28, T4P25, T4P 125

JIMENEZ-FLORES, P. L. T2P 28, T4P25, T4P125

JIN, W. T1P 130

JINNETT, K. T3P 118, T2P 49

JOCKEN, J. W. T1P 40, T1P 108, RS 2:4, RS1:4, T1P105, OS7:OC 62

JOENSUU, A. T2P 70

JOHANNESEN, J. T4P 118

JOHANSSON, $\mathrm{H}$. T3P 57

JOHANSSON, L. OS6:OC 39

JOHNSON, J. T3P 110

JOHNSON, L. T4P 42

JOHNSTON, K. OS2:OC 53, T3P 3, T3P 6, T3P 185

JOHNSTONE, A. T1P 160

JOKINEN, R. OS9:OC 15, T1P 6

JONES, A. OS6:OC 37, T4P 32

JONES, D. T2P 56

JONG, J. T1P 81

JOONAS, N. T3P 58

JORDAN, H. OS5:OC 32

JORDAN, J. T3P 26, RS18:1

JORGENSEN, M. T1P 113

JOSEPH, V. T3P 155, T2P 178

JOUSILAHTI, P. T2P 70

JUANA, S. T1P 60

JUÁREZ-TORRES, E. T3P 139

JUHL, C. T3P 181, T3P 177

JULIA, D. T2P 156

JULIE, M. T4P 7, T2P 57, T4P 43

JÚLÍUSSON, P. T4P 34, T4P 74

JUNG, $H$. T1P 152

JUNG, J. T1P 96

JUNG, S. T1P 44

JUNIOR, A. T1P 12

JUNIOR, I. T3P 169, T3P 24

JUNIOR, J. T3P 34

JUNIOR, J. T3P 34

JUNIOR, R. T4P 52

JUNQUEIRA, N. T3P 163

JÜRETS, A. T1P 134

KABIR, Z. T3P 106

KAČAREVIĆ, Z. T1P 34, T4P 45

KACZOROWSKA, J. T2P 148

KAELBLE, A. T3P 107, T3P 108

KAHAN, B. T3P 183

KAHLHÖFER, J. T1P 68

KAJANTIE, E. T2P 91

KALENDOVA, V. T1P 33

KALFAS, S. T1P 97

KALLEN, C. RS1:4

KAMARATOS, A. T3P 82

KAMENOV, Z. T3P 40

KAMINSKI, J. T3P 26

KAMLAGE, B. T3P 26

KANG, A. T1P 152

KANG, J. T1P 15

KANG, S. T3P 89

KANG, S. T1P 152

KANG, Y. T2P 145

KAPLAN, L. M T3P 118, T2P 49

KAPLAN, P. T3P 6, OS2:OC 53

KAPRIO, J. OS9:OC 15, T2P 70
KARACA, F. T3P 205

KAROUNE, R. T1P 2

KARPE, F. RS2:2

KASIM, A. OS5:OC 34

KATZMARZYK, P. T4P 111, T4P 107

KAYE, S. T1P 6

KAYSER, B. T1P 129

KAZDOVA, L. T1P 55, T1P 124

KEANE, E. T2P 39

KEARNEY, P. T4P 46

KEFURT, R. T3P 218

KEIJER, J. T1P 157, AS5:2

KEINDL, M. T1P 134

KELISHADI, R. T4P 10, T4P 116

KELLEHER, C. T2P 111, RS6:4

KELLEHER, M. T4P 68, OS6:OC 41, T4P 65

KELLER, J. T1P 68

KEMENY, A. OS9:OC 14, T1P 87

KEMP, G. T2P 72, T2P 164, T2P 143

KENNELLY, M. T3P 192

KEOGH, J. T3P 76

KESWELL, D. OS3:OC 3

KHADEMHAGHIGHIAN, $\mathrm{H}$. T3P 72

KHALAJ, A. T1P 78, T1P 23, T1P 14

KHAN, N. T2P 178

KHAZZAKA, A. T2P 42

KHOO, J. T1P 11, T3P 60

KIDA, A. T2P 18

KIELSTEIN, H. T1P 36, T1P 96

KILINÇ, F. T4P 38, T4P 69

KILINÇ, Y. T3P 205

$\mathrm{KIM}, \mathrm{C} . \quad$ T3P 6, OS2:OC 53

KIM, E. T2P 11

$\mathrm{KIM}, \mathrm{H} . \quad$ T3P 165, T3P 17

$\mathrm{KIM}, \mathrm{H} . \quad$ T2P 75

$\mathrm{KIM}, \mathrm{H} . \quad$ T4P 8

KIM, H. T1P 91

KIM, J. T2P 38

KIM, J. T2P 145

KIM, K. T2P 130

KIM, K. T3P 33

KIM, K. T3P 38

KIM, Y. T2P 177

KIM, Y. T3P 70

KIM, Y. T1P 44

KIMURA, A. T2P 180

KIMURA, K. T1P 7

KING, J. A T3P 188

KINNUNEN, T. AS3:3, AS3:4

KIORTSIS, D. T3P 140

KIRK, A. OS11:OC 59

KITAHARA, A. T1P 119, T1P 28

KITAMOTO, T. T1P 119, T1P 28

KLEIN, D. T2P 151

KLEIN-SEETHARAMAN, J. T2P 24

KLEMARCZYK, W. T4P 41

KLEPPE, M. RS6:3

KLIMCAKOVA, E. T1P 73

KLOPPENBORG, J.T. T4P 118

KNEKT, P. T2P 138, OS8:OC 25

KNÜPPEL, A. AS2:4

KO, K. T3P 38

KÖBERLE, M. T1P 104

KOBOLD, A. OS2:OC 51

KOC, M. T1P 73

KOES, B. T4P 84, T4P 117

KOIVUSALO, S. T3P 27
KOKKINOPOULOU, A. T4P 40

KOLAY, M. T2P 181

KOLCSÁR, M. T1P 24

KOLK, B. T1P 40, T1P 108, RS1:4

KOLOTKIN, R. T3P 118, T2P 49

KOMAKI, G. T3P 1

KOMMEDAL, K. G. T4P 74

KONG, M. H. T3P 165, T3P 17

KONIECZNA, J. T1P 80

KONITSIOTIS, S. T3P 140

KONTTINEN, H. T2P 70

KOPECKY, J. T4P 79, T1P 33

KOPECKY, M. T4P 128, T4P 129

KOPP, H.P. T3P 151, T3P 204

KORALKOVA, P. T1P 104

KOS, K. T1P 79

KOŚCICKA, K. T2P 21

KOSKELA, T. T4P 106

KOSKINEN, S. T2P 22

KOSUTZKA, Z. OS4:OC 12

KOSYURA, S. T4P 17

KOTHARI, M. T2P 84

KOVÁCS, A. T2P 110

KOVÁCS, G. T2P 61

KOVACS, P. T1P 110

KOWAL, G. T4P 76

KRACMEROVA, J. T1P 73

KRAUSE, B. T3P 16

KREBS, A. T1P 158

KREBS, M. T3P 218, T3P 98

KREIS, R. T3P 129, T3P 69, T3P 176

KREMERS, S. RS7:1

KRETZER, I. T1P 161

KRETZER, I. T4P 103

KRETZER, M. T2P 65, T4P 103

KRISHNA, M. T1P 93

KRISTO, I. T3P 218

KROHOVÁ, J. T4P 50, T4P 113

KRUSCHITZ, R. T3P 98

KRYST, L. T4P 76

KRZIZEK, E. T3P 151, T2P 114, T3P 204

KSENIYA, K. T1P 148, T3P 200

KUCHARIKOVA, P. T1P 33

KUCHENBECKER, W. OS2:OC 51

KUDA, O. T4P 79, T1P 124

KUKIC, M. T2P 26

KULSENG, B. T3P 109, OS4:OC 7, RS6:3, T1P 133

KUMAGAI, S. T2P 18

KUMAS, M. N. T2P 176

KUMPULAINEN, S. T2P 91

KUN, I. T1P 24

KUNESOVA, M. T2P 52

KUREK, K. T1P 131

KURIYAN, R. T4P 111

KURPAD, A. T4P 111

KURZEME, A. OS5:OC 35

KUSCHNIR, M. C. T2P 14

KUSHE, M. T3P 155, T2P 178

KUTALIK, Z. T3P 61

KUZEVA, A. T4P 96

KUZMINOVA, I.O. T1P 8

KVEHAUGEN, A. T1P 147

KWOK, D. T3P 20

KWOK, H. T4P 87

KWON, H. T2P 11

KYLE, T. . T2P 84, T3P 118, T2P 49, OS1:OC22 
KYRIAZIS, I. T3P 116

KYVIK, K. T4P 115

LAATIKAINEN, T. T4P 106

LABIB, M. T3P 10, T3P 95

LACERDA, J. T3P 12

LADEIA, A. T2P 78

LADSTÄTTER, A. T2P 79

LAFERRERE, B. T3P 61

LAFRANCE, R. T2P 151

LAGOA, M. T4P 2

LAHELMA, E. T2P 104

LÄHTEENMÄKI, L. AS3:1, AS3:2

LAHTI, M. T2P 91

LAI, S. T3P 19

LAIMER, M. T3P 213

LAIVUORI, H. T2P 91

LAKE, A. T2P 67

LAKERVELD, J. OS5:OC 31, RS4:3

LALIBERTE, M. T2P 107

LALIBERTE, M. T2P 107

LALLUKKA, T. T2P 104

LAMARCA, F. T2P 5

LAMBERT, E. T4P 111

LAMBERTUCCI, R. T4P 78

LANCIOTTI, R. T2P 10

LAND, J. OS2:OC 51

LANDA, V. T1P 55, T1P 124

LANDECHO, M. F. T1P 67

LANDIN-OLSSON, M. T3P 63

LANG, F. T1P 104

LANGDAHL, B. T3P 25, T3P 178, T3P 179

LANGE, D. T2P 174

LANGER, F. T3P 218, T3P 130, T1P 134

LANGIN, D. T1P 95, RS2:3, T1P 73

LAPWORTH, S. T3P 203

LARIJANI, B. T4P 10, T4P 116

LARISA, N. T3P 206

LARSEN, S. C. T4P 9, T2P 85, AS3:4, T4P 48

LASCARIS, B. T3P 173, T3P 212, T3P 28

LASSEN, P. T3P 189

LATZER, Y. T3P 56

LAUGERETTE, F. OS9:OC 13

LAURA, M. T4P 51

LAURENT, Y. T1P 31

LAUSTSEN, C. T1P 32

LAUZON-GUILLAIN, B. OS4:OC 10

LAVILLE, M. T1P 9, T3P 78

LAWRENCE, J. OS10:OC 47

LAYANA, A. T3P 54

LAZAROVA, Z. T4P 50, T4P 113

LEAL, I. T2P 23, T2P 90, T4P 90

LEAL, V. T3P 149, T3P 224

LEAN, M. T2P 182

LEBLANC, A. T3P 180

LEBRÃO, M. T2P 118, T2P 89, T2P 13,

T2P 103, OS8:OC 30

LEBRÃO, Y. T2P 103

LECA, B. T3P 202

LECHLEITNER, M. T2P 79

LECOULTRE, V. T3P 176

LEDOUX, S. T1P 9, T3P 46, T3P 48

LEE, B. RS7:3

LEE, H. T2P 2

LEE, H. T4P 55

LEE, J. T2P 11, T3P 33

LEE, J. T2P 145
LEE, K. T2P 130, T3P 38

LEE, K. T2P 177

LEE, K. T3P 172

LEE, S. T2P 2

LEE, S. T4P 8, T3P 70

LEE, S. T1P 44

LEE, Y. T3P 165

LEEUWEN, J. T4P 84, T4P 117

LEGARDEUR, $\mathrm{H}$. T3P 48

LÉGER, B. RS2:3

LEHTINEN-JACKS, S. T4P 106

LEINHARD, O. T1P 59, OS7:OC 65, RS1:3

LEITE, S. T1P 51

LEITE-MOREIRA, A. OS3:OC 1, AS5:5, T1P 90, T1P 92, T1P 51

LEITNER, L. T1P 134

LEKS, B. T4P 128, T4P 129

LEKŠAN, I. T1P 34

LELEKIS, M. T3P 116

LEMES, S. T4P 78

LEMOGNE, C. OS8:OC 28

LEMOS, D. T4P 99

LEMOS, L. OS2:OC 52

LENAERTS, K. OS7:OC 62

LENZI, A. T1P 126

LEONETTI, F. T3P 159

LEONOVA, I. T4P 70

LEOW, M. T1P 22

LEPETRA, J. T3P 54

LEPPÄNEN, J. AS3:4

LETURQUE, A. OS9:OC 13

LEUNG, C. T3P 44

LEUNG, L. T3P 44

LEVÄLAHTI, E. T4P 106

LEV-ARI, L. T3P 56, T4P 127

LEVENEZ, F. T1P 129

LEWIS, J. T1P 26

LI, D. T2P 139, T2P 95

LI, S. T2P 151

LILLEØRE, S. K. T3P 118, T2P 49

LIM, D. T1P 91

LIM, H. T4P 55

LIM, U. T2P 45

LIMA, M. L. T2P 78

LIMA, T. T3P 34

LIMA, T. T3P 34

LIMBERT, C. T3P 12

LIMOR, R. T3P 94

LINDSAY, K. L. T3P 192

LINGE, J. T1P 59, OS7:OC 65, RS1:3

LINNEBERG, A. T2P 85

LIPWORTH, S. RS 14:3

LISSNER, L. T2P 76, OS1:OC 23, T4P 97

LIU, Y. T3P 189, OS11:OC 55

LLEWELLYN, C. T2P 70

LLORENS, P. T4P 123

LOBSTEIN, T. RS4:1

LØKLING, H. L. T4P 34

LONG, C. OS1:OC 19, OS4:OC 8, T2P 108

LOOK, M. T2P 84, T3P 118, T2P 49

LOPES, C. T4P 124

LOPES, C. T4P 71, T3P 131, OS1:OC 24

LOPES, D. T1P 69

LOPES, L. T2P 4, T2P 16, OS1:OC 21, T2P 17 ,

T4P 72, T4P 98

LOPES, O. T4P 124, PL7:2

LÓPEZ, O. T2P 92
LOPEZ-ALCALÁ, J. T1P 114

LÓPEZ-MIRANDA, J. OS3:OC 4, T1P 114

LORDA, P. T1P 111

LOURDES, M. T2P 125

LOURENÇO, A. T1P 51, T1P 90, T1P 92, AS5:5

LOURENÇO, C. T3P 59

LOUSA, I. T4P 108

LOVISI, M. T3P 134

LU, C. OS9:OC 16

LUBRANO, C. T1P 126

LUDMILA, V. T4P 37

LUDVIK, B. T3P 98, T3P 151, T2P 114,

T3P 204, PL1:2

LUGER, E. T3P 98, T2P 79

LUGER, M. T3P 98, T2P 79

LUIG, T. T2P 151

LUKASZUK, B. T1P 131

LUNDEQUIST, A. T3P 156

LUNDQVIST, A. T2P 22, OS8:OC 26, OS8:OC 25

LUNGER, L. T3P 213

LUQUE, R. OS3:OC 4

LUTGENS, E. T1P 25

LUTOV, Y.V. T1P 8

LYNGSTAD, A. T1P 62, T3P 109

LYSAK, A. T2P 81

LYSAK, A. T2P 81, T2P 163

LYYTINEN, J. OS8:OC 25

MACCHI, V. T1P 61

MACDONALD, I. T3P 188

MACEDO, G. T1P 69, T1P 19

MACEDO, J. T1P 69, T1P 19

MACHADO, C. T3P 117

MACHADO, P. T2P 125, T2P 159, T4P 121

MACHADO, S. T3P 42

MACHIDA, K. T1P 7

MACKENBACH, J. OS5:OC 31

MACLELLAN, G. T3P 137, T3P 101

MADSEN, L. R. T3P 25, T3P 178, T3P 179

MAESTRI, T. T2P 82

MAFFEIS, C. T4P 63

MAGALHÃES, D. T3P 136, T1P 145, T3P 103, T3P 102, T3P 152

MAGALHÃES, J. OS3:OC 5

MAGALIE, M. T4P 7

MAGAREY, A. OS6:OC 40

MAGARIO, N. T3P 126, T3P 32

MAGEROWSKI, G. T1P 111

MAGNO, F. C. T1P 72, T2P 60, T3P 71, T1P 71

MAHER, C. T4P 111

MAHKONEN, R. T4P 106

MÄHLER, A. T3P 26

MAHMOODI, B. T1P 151, T1P 23, T1P 14, T1P 156, T1P 57

MAIA, J. T4P 111

MAINARDI, F. T2P 74

MAITREPIERRE, C. T1P 9, T3P 78

MAJANOVIĆ, S. T2P 170

MAJERĖÁK, I. T2P 87

MÄKELÄ, K. T1P 109

MÄKI, P. T4P 106

MALAGON, M. M. OS3:OC 4, T1P 114

MALDEN, S. T2P 86

MALLARDI, G. T4P 80

MALY, M. T3P 198 
MANCINI, C. T1P 126

MANCO, M. T2P 10

MANDELBROT, L. T3P 48

MANDOI, A. T4P 80

MANKUTA, D. T4P 127

MANN, K. OS6:OC 37, T4P 32

MANN, O. T3P 97, T3P 51

MÄNNISTÖ, S. T2P 138, T2P 70, OS8:OC 26, OS8:OC 25

MANOHARAN, V. T2P 24

MANUEL, L. T1P 161

MANZ, M. T1P 128

MAORET, J. RS2:3

MARANHÃO, P. T3P 75, T3P 174

MARCH, S. T3P 211

MARCHAND, L.T2P 45

MARCHI, M. T3P 158

MARCO, N. OS11:OC 56

MARCUS, C. OS6:OC 39

MARETTE, A. T1P 138, T1P 18

MARGALL, M. T3P 37

MARGAUX, D. T4P 7

MARI, A. OS11:OC 60

MARIANI, S. T1P 126

MARIE, B. T4P 7

MARIE-JOSEPHE, A. T4P 30

MARIE-LAURE, F. T4P 92

MARILD, S. T4P 97

MARIMAN, E. T1P 40, T1P 63, T1P 105

MARINA, G. T3P 200

MARINA, K. T4P 37

MARINA, S. T3P 206

MARINHO, A. T4P 89

MARIN-MEDINA, M. T3P 139

MARJI, M. T3P 125

MARK, A. T3P 180

MARKAKIS, V. T3P 82

MAROCCO, C. T1P 126

MAROCO, J. T2P 90, T2P 25

MARQUES, M. T1P 95

MARQUES, M.M. AS3:1, AS3:3, AS3:4

MARRA, M. T1P 3, T1P 52, T1P 116, OS4:OC 11

MARRÓN, E. T1P 111

MARTA, Z. T2P 87

MARTIN, A. T3P 104

MARTIN, A. T1P 135

MARTIN, A. T4P 104

MARTIN, C. K. T4P 49

MARTIN, S. T3P 202

MARTIN, S. T4P 61

MARTIN, V. T1P 37

MARTÍN-CALVO, N. T2P 123

MARTINE, D. T2P 57, T4P 43

MARTÍNEZ, J. T3P 16

MARTINEZ, M. L. T3P 119

MARTÍNEZ-ANDRÉS, M. T4P 44

MARTÍNEZ-GONZALEZ, M. T2P 147, T2P 123

MARTÍNEZ-HORTELANO, J. T2P 68

MARTINEZ-OLMOS, M. T3P 35

MARTINEZ-VIZCAÍNO, V. T4P 93

MARTIN-LAGUNA, M. T2P 155

MARTINS, A, T1P 56

MARTINS, C. OS2:OC 52

MARTINS, C. T1P 133, OS4:OC 7

MARTINS, C. T3P 109, T1P 62, RS6:3
MARTINS, F. T2P 65

MARTINS, F. RS2:3

MARTINS, S. T3P 90, T2P 3

MARUCCI, M. T2P 118, T2P 89, T2P 13

T2P 103, OS8:OC 30

MASKARINEC, G. T2P 45

MASQUIO, D. T4P 119, T1P 12

MASUDA, Y. T1P 119

MASURIER, J. T4P 82

MATA, J. T3P 7

MATARATZIS, P. T4P 99

MATEJKOVA, D. T3P 198

MATOS, A. T3P 42, T3P 29

MATOS, J. T1P 90, T1P 92, AS5:5

MATOS, M. AS3:3, AS3:4

MATSUDO, V. T4P 111

MATSUMOTO, J. T1P 119,T1P 28

MATSUMOTO, K. T1P 119

MATTA, J. OS8:OC 28

MATTEVI, V. T1P 35

MATTHEIS, L. T1P 96

MATVIENKO-SIKAR, K. T4P 46

MAUD, M. T4P 43 N EXISTE ESTE NOME NO

TRABALHO

MAXIMO, R., T2P 101

MCAULIFFE, F. T2P 53, T2P 161, T3P 4, T3P 192

MCCOLL, E. T3P 66

MCCORMACK, B. T2P 36

MCCORMACK, L. OS6:OC 42

MCCULLOCH, L. T1P 79

MCEACHAN, R. T2P 175

MCHUGH, D. T4P 68, OS6:OC 41

MCKENNA, J. OS5:OC 32

MCKENNEY, K. T2P 155, T2P 71

MCMAHON, C. T3P 122

MCROBBIE, H. T3P 183, OS6:OC 42

MCSHEA, K. T1P 102

MEHEGAN, J. T3P 137, T3P 101, T2P 111, RS6:4

MEINHARDT, N. T1P 35

MEJIA-LANCHEROS, C. T2P 111

MEKHANCHA, D. E. T1P 2

MELIDONIS, A. T3P 82, T3P 140

MELLO, L. T4P 52

MELLO, M. T4P 119, T1P 155

MELMER, A. T3P 213

MELO, A. T2P 100

MELO, C. T1P 155

MEMBRIVES, A. OS3:OC 4

MENDES, E. T3P 59, T4P 126

MENDES, L. T2P 100

MENDES-DA-SILVA, C. T4P 78

MENDEZ-GIMENEZ, L. T1P 58

MENDIZABAL, L. T3P 139

MENNICKENT, P. T3P 16

MENNITTI, L. T3P 123, T4P 47, T3P 73 ,

T4P 77

MENSINK, G. T2P 174

MESA, J. T1P 50, T3P 93

METHENITIS, S. T4P 40

METZ, L. T4P 82

MEYER-GERSPACH, A. T1P 48, T1P 128, OS2:OC 50, T1P 54

MICHAELA, C. T2P 87

MICHAELSEN, K. OS10:OC 44

MICHAL, K. T2P 87
MICHALSKI, M. C. OS9:OC 13

MICHELS, N. T4P 26, T2P 29, T4P 27

MICIC, D. T3P 168

MIDDELKOOP, M T4P 84, T4P 117

MIETTE, V. T3P 13

MIGUET, M. T4P 82

MIHAELA, M. T4P 102, T4P 51, T4P 31

MIHAELA, T. T4P 102, T4P 51, T4P 31

MIKKELSEN, M. L. AS3:4

MIKLOSZ, A. T1P 131

MILAGRO, F. T3P 16

MILAN, G. OS9:OC 18

MILANI, A. T1P 35

MILES-CHAN, J. L OS7:OC 61, T1P 77 T3P 58, T1P 115

MILIN, J. T3P 168

MILLER, C. T2P 35

MILLER, J. OS6:OC 40

MILLER, K. T3P 144

MILOS, S. T2P 87

MINDERICO, C. S. T2P 3

MIN-KYU, C. T1P 13

MIR, L. T1P 95

MIRANDA-SILVA, D. T1P 90, T1P 51

MIREILLE, C. T1P 31

MIRMIRAN, P. T1P 78, T1P 151, T1P 23, T1P 14, T1P 156, T1P 57

MIRO, J. T3P 97, T3P 51

MIRONOVA, D. T1P 66

MISTRY, P. T3P 196

MITCHELL, J. E. T2P 125

MITTAG, J. T1P 81

MIZGIER, M. OS9:OC 17

MLEJNEK, P. T1P 55, T1P 124

MOELLER, N. T1P 79

MOHAMMADI, M. T3P 72

MOHAMMED, N. OS2:OC 54

MOHOVA, I.G. T1P 8

MOJZIKOVA, R. T1P 104

MOL, B. OS2:OC 51

MOLINA, E. T2P 155, T2P 71

MÖLLÄRI, K. T4P 106

MOLNAR, D. OS1:OC 23

MONCADA, R. T1P 58, T1P 83, T1P 67

MONIQUE, R. T4P 105

MONIZ, K. T2P 151

MONNARD, C. T1P 77

MONROE, K. T2P 45

MONTANI, J. OS7:OC 61, T1P 77, T3P 58 T1P 115

MONTASTIER, E. T1P 95

MONTEIRO, A. T3P 128, T3P 36

MONTEIRO, F. T1P 161

MONTEIRO, L. T3P 92

MONTEIRO, M. OS11:OC 59, T3P 30, T3P 31

MONTEIRO, M. T1P 56, T1P 20

MONTEIRO, P. T3P 169

MONTEIRO, R. RS13:1

MONTEIRO, S. T3P 117

MOON, J. T3P 17

MOOR, M. T1P 48, OS2:OC 50

MOORE, H. T2P 67, OS5:OC 34, T4P 53

MOORES, C. J. OS6:OC 40

MORAIS, T. T3P 30, T3P 31

MORAN, A. T1P 128

MORANDI, A. T4P 63

MOREIRA, A. PL7:2 
MOREIRA, A. T1P 20

MOREIRA, A. T3P 131

MOREIRA, B. P. T1P 56

MOREIRA, C. T2P 4, OS1:OC 21, T2P 17, T4P 72, T4P 98, T2P 16

MOREIRA, P. PL7:2

MOREIRA, P. T3P 131, T4P 15

MOREIRA, R.G. T3P 123, T4P 119

MOREIRA-GONÇALVES, D. T1P 51, T4P 67

MORENO, J. T4P 123

MORENO-NAVARRETE, J. M. OS3:OC 2

MORGEN, C. OS10:OC 44

MORIN, C. T3P 184, T3P 170

MORINDER, G. T4P 131, OS6:OC 39

MORKEN, M. T4P 34, T4P 74

MORÓN, S. T1P 85

MORRIS, L. T2P 167, T3P 171

MORRIS, L. T3P 162, T3P 8

MORTENSEN, E. OS8:OC 29

MOSBAH, H. T4P 81, T4P 81

MOSHE, Y. T3P 94

MOTA, J. T2P 4, T4P 2, OS1:OC 21, T2P 17 T4P 72, T4P 98, T4P 120, OS2:OC 52,

T4P 126, T2P 16, RS16:2

MOTA-SOARES, M. T3P 115

MOTLAGH, M. T4P 10, T4P 116

MOURA, C. OS3:OC 1

MOUTINHO, N. T4P 66

MRACEK, T. T1P 55

MSIKA, S. T3P 46, T3P 48

MULLEN, K. T3P 180

MÜLLER, M. AS6:1

MULLEROVA, D. T3P 198

MUNDBJERG, L. T3P 177, T3P 181

MURANO, T. T1P 130

MURATORI, F. T1P 87

MURRIN, C. T2P 111, RS6:4

MUSCUREL, C. T1P 5

MUTSAERTS, M. OS2:OC 51

MYERS, A. T2P 1, T2P 108

MYETTE-CÔTÉ, E. T1P 47

MYÖHÄNEN, M. T1P 6

NABERS, D. OS2:OC 50

NACCARATO, M. T1P 52

NADEJDA, L. T3P 194

NADGLOWSKI, J. T3P 118, T2P 49, OS1:OC22

NAGY, E. T2P 110

NAIK, R. T1P 89

NAKAJIMA, V. T1P 19

NAKASEKO, C. T1P 130

NAOUR, G. T3P 189

NAPITUHINA, I. T4P 29

NAS, A. T3P 107, T3P 108, T1P 68

NASCIMENTO, C. M. T1P 45, T1P 98,

T2P 106, T4P 47, T4P 77, T4P 119, T1P 39

NASCIMENTO, C. T3P 114

NASCIMENTO, D. T1P 90

NASCIMENTO, H. T4P 108

NASCIMENTO, C.M.O. T1P 39

NASLAVSKY, M. T2P 64

NASSAU, F. RS17:2

NATALIA, K. T4P 37

NATALIYA, M. T1P 148

NATHALIE, F. T4P 92

NATUZZI, A. T4P 80

NAVARRO-RUIZ, M. C. OS3:OC 4
NAZARETH, M. T4P 71

NAZÁRIO, A. C. T2P 82

NAZÁRIO, N. O. T2P 82

NEBREDA, V. T4P 123

NEDERGAARD, J. T1P 81

NELSON, D. T3P 57

NESSLANY, P. OS7:OC 66

NETCHINE, I. T4P 81

NETO, N. T2P 106

NEUPARTH, M. T4P 67

NEVES, F. F. T3P 59

NEVES, J. S. T1P 145, T3P 103, T3P 102,

T3P 136, T3P 152

NEZZAL, L. T1P 2

NGUYEN-TU, M.S. OS3:OC 6

NICOLAOU, M. T2P 51, OS1:OC 22

NICOLAS, V. T1P 31

NIELSEN, T. T4P 118, T4P 11

NIENHUIJS, S. T3P 173, T3P 212, T3P 28,

T3P 41, T3P 68, T2P 150, T3P 112

NIEWIAROWSKA, M. T2P 83, T2P 171

NIJPELS, G. OS5:OC 31

NIKOLAOU, C. T2P 183, AS4:1

NIKOLOVA, M. T2P 140

NIMMO, M. T3P 188

NIO-KOBAYASHI, J. T1P 7

NIRANTHARAKUMAR, K. OS2:OC 54

NISHIHARA, T. T3P 1

NIUBÓ, M. C. T2P 133, T2P 132, T2P 131

NIXON,P. OS6:OC 38

NOGUEIRAS, R. T1P 114

NOLAN, C. F. RS15:4

NONAKA, K. T1P 153

NORA, M. T1P 20, T3P 31

NORMAN, J. T2P 72, T2P 164, T2P 143

NOTARIO-PACHECO, B. T4P 44

NOVACK, V. T3P 94

NOVELLI, I. T3P 62

NOVIKOVA, E. G. T1P 139

NOVIKOVA, V. T4P 70

NOZAKI, T. T3P 1

NUIJTEN, M. T3P 84

NUNES, J. T4P 86, T1P 4

NUNES, M. T1P 117

NUNES, S. T4P 108

NUÑEZ-ROA, C. T1P 132

NUTTER, D. AS3:4

NYMO, S. T1P 133, T3P 109, OS4:OC 7, RS6:3

O'BRIEN, E. T2P 53, T2P 161, T3P 4

O'DONNELL, A. T4P 61

O'MALLEY, C. T4P 53

O'MALLEY, D. T4P 54, T4P 65

O'MALLEY, E. T3P 137, T3P 101, T2P 34

O'NEIL, P. T3P 118, T2P 49

O'SHEA, D. T3P 137, T3P 101, T2P 34, T2P 36

O'SULLIVAN, E. T3P 192

OBEID, O T1P 43

OBEID, O. T2P 42

OBERG, D. T3P 156

OERS, A. OS2:OC 51

OFNER-VELANO, D. T3P 213

OGUNLEYE, A. A. T3P 110

$\mathrm{OH}, \mathrm{S}$. T2P 38

OKAMATSU-OGURA, Y. T1P 7

OKSANA, L. T1P 148, T3P 200

OLABI, A. T1P 43
OLDS, T. T4P 111

OLIVEIRA, A. OS1:OC 24

OLIVEIRA, A. T1P 4

OLIVEIRA, A. T4P 95, T1P 106

OLIVEIRA, B. T4P 19, T4P 12

OLIVEIRA, C. T4P 99, T2P 14

OLIVEIRA, C. T4P 124

OLIVEIRA, H. T1P 121

OLIVEIRA, J. T2P 55, T1P 51, T4P 67

OLIVEIRA, M. T3P 117

OLIVEIRA, M. T2P 94

OLIVEIRA, M. T3P 164, T3P 138

OLIVEIRA, M. T4P 95, T1P 106

OLIVEIRA, P. T1P 56, T1P 20

OLIVEIRA, S. T3P 103, T3P 102, T3P 136, T1P 145, T3P 152

OLIVEIRA-MAIA, A. T1P 149, T2P 94

OLIVEIRA-SANTOS, J. T2P 17, T4P 72, T4P 98

OLIVER, J. AS5:2

OLIVER, P. T2P 7, T3P 85, T1P 157

OLIVIER, P. T3P 66

OLIVIERI, F. T4P 63

OLIYARNYK, O. T1P 124

OLSEN, N. T2P 85, T4P 9, T4P 48

ONG, K. OS4:OC 10

ONUFRIO, M. T1P 52, OS4:OC 11

ONYWERA, V. T4P 111

OOSTERHUIS, G. OS2:OC 51

OPPERT, J. OS5:OC 31, OS11:OC 57, OS9:OC 13, OS11:OC 55, T4P 81

OPRI, F. T4P 63

ORDING, A. T2P 160

ORDOÑEZ-MAYAN, L. T3P 35

ØRNSTRUP, M. T3P 25, T3P 178, T3P 179

OROZCO, L. T3P 139

ORTIZ, A. T1P 50

OSHER, E. T3P 94

OSLER, M. OS10:OC 46

OSTMAN, E. T1P 29

OSTROWSKI, M. T3P 115

OSUNLANA, A. M. T3P 110

OTTONI, I. T2P 59

OUSTRIC, P. T2P 108

OVELLEIRO, D. OS3:OC 4

OVERVAD, K. T2P 85

OXANA, D. T1P 142, T2P 156, T1P 150

OYAMA, L. M. T2P 106, T3P 123, T4P 119, T1P 98, T4P 47, T4P 77, T1P 45 T1P 39

PAANS, N. AS2:2

PACHON, G. T1P 132

PADRÃO, P. T4P 66, T3P 131, PL7:2

PAGANELLI, M. T3P 158

PAGANO, M. C. T1P 116

PAGKALOS, I. T4P 40

PAINTER, R. OS2:OC 51

PAIXÃO, R. T1P 88

PAK, M. T2P 181

PAKSY, A. T2P 61

PALA, V. OS1:OC 23

PÁLL, J. A. T1P 24

PALLAY, G. T2P 46

PALMEIRA, A. L. AS3:3 AS3:4

PALOU, A. T1P 60, T4P 20, T4P 79, T1P 135,

T2P 7, T1P 80, T3P 85, T1P 157, AS5:2

PALUMBO, M. OS11:OC 60

PANAHI, S. T3P 225 
PANAZZOLO, D. T3P 174, T3P 175

PANETTA, D. T1P 87

PANGOVSKI, I. T3P 147, T3P 148

PANHOFER, P. T3P 218

PAOLA, Z. T2P 101

PAPADOPOULOU, S. T4P 40

PAPAZAFIROPOULOU, A. T3P 82

PAPPA, G. T3P 210

PARDO, F. OS8:OC 27

PAREDES, S. T3P 36, T3P 145, T3P 21, T3P 22

PAREJO, M. T2P 133, T2P 132, T2P 131

PARIZOTTO, N. ,T1P 12

PARKINSON, K. OS6:OC 37, T4P 32

PARLIER, D. T3P 13

PARROTT, S. T3P 183

PARRY, T. T3P 118, T2P 49

PASANISI, F. T1P 3, T1P 52, T1P 116, OS4:OC 11

PASCUAL-CORRALES, E. T3P 37

PASSOS, M. T4P 36

PASTEL, E. T1P 79

PASTUCHA, D. T2P 77

PATEL, B. T3P 20

PATRAC, V. T1P 37

PATRASCIOIU, I. T3P 211

PATTOU, F. OS7:OC 66, T3P 157

PAULI, D. OS1:OC22

PAULINE, B. T4P 7

PAULIS, W. T4P 84

PAULO, T. T3P 24

PAVLISOVA, J. T1P 33

PAVLOUŠKOVA, J. T3P 198

PAVLOVSKAYA, E. T4P 17

PAYOLLA, T. T4P 78

PEARCE, M. OS6:OC 37, T4P 32

PECHT, T. OS11:OC 55

PEDERSEN, O. T1P 113, T2P 85, T4P 118

PEDERSEN, S. T1P 32,

PEDERSON, S. AS3:1

PEDRAZA-ARÉVALO, S. OS3:OC 4

PEDRO, J. T1P 145, T3P 103, T3P 102,

T3P 136, T3P 152

PEDROSA, A. T2P 60

PEDROSO, A. T1P 42, T1P 75, T2P 106, T1P 45

PEERBUX, S. T3P 183

PEETERS, A. OS5:OC 35, RS11:1

PEINADO, M. T1P 132

PEJKOVIC, D. S. T3P 168

PELLEGRINI, M. OS9:OC 14

PELTONEN, M. OS11:OC 58

PENKOV, A. T2P 140

PENN, L. T2P 67, T3P 2

PENNINX, B. T2P 51, OS1:OC 22, AS2:1

PEREIRA, A. T4P 16, T3P 86, T2P 129, T2P 141

PEREIRA, B. PL7:2

PEREIRA, E. T2P 65, T4P 103

PEREIRA, F. T3P 86, T2P 129, T2P 141, T4P 16

PEREIRA, P. T3P 30, T3P 31

PEREIRA, P. T3P 92

PEREIRA, S. T3P 30, T3P 31

PEREIRA, S. T1P 99, T3P 42, T3P 29

PEREZ, K. T2P 133, T2P 131

PEREZ, K. T2P 132

PEREZ, M. T3P 93

PERICÁS-BELTRÁN, J. T3P 85
PEROLA, M. T2P 70

PERRY P. T4P 68, OS6:OC 41, T4P 65

PERRY, C. T2P 39

PERRY, I. T3P 106, T2P 39

PESONEN, A. T2P 91

PETERLI, R. T1P 48, T1P 128, OS2:OC 50

PETERSEN1, J. T4P 115

PETRAN, M. T1P 5

PETRONI, M. T3P 134

PETROPOULOU, S. T3P 116

PETROVA, S. T4P 101

PETROVIC, N. T1P 81

PHILIPPOU, J. T3P 3

PICHARDO, J. T2P 172

PICO, C. T1P 80

PICÓ, C. T4P 20, T4P 79

PICOITO, J. T4P 94

PIECHA, R. T3P 198

PIERNAS-SANCHEZ, C. T3P 203

PIETILÄINEN, KIRSI H. OS9:OC 15, T1P 6

PIETROBELLI, A. OS1:OC22

PIGEYRE, M. OS7:OC 66

PIJPKER, R. M. T3P 190

PILON, G. T1P 138, T1P 18

PIMENTA, F. T2P 23, T2P 90, T2P 25, T4P 90

PIMPIN, L. T3P 133, OS7:OC 63

PINARBASI, A. T2P 181

PINELLI, N. T2P 97, T3P 141

PINKHASOV, B. T1P 8,T1P 139

PINKNEY, J. T2P 157

PINTO, A. T3P 6, OS2:OC 53

PINTO, C. T4P 19, T4P 12

PINTO, E. T4P 71

PINTO, K. T1P 153

PINTO, L. T4P 91

PINTO, R. T4P 52

PINTÓ, $X$. T3P 54

PINTO-BASTOS, A. T2P 125

PINTO-BASTOS, A. T2P 125

PIONA, C. T4P 63

PIPE, A. T3P 180

PIRINEN, E. T1P 6

PISANI, L. T4P 47, T4P 77, T3P 73, T3P 123

PISTJA, E. T2P 8

PIZATO, N. T4P 4

PLANAS-IGLESIAS, J. T2P 24

PLOEG, H. RS17:2

POGGIOGALLE, E. T1P 126

POGHOSYAN, T. T3P 13

POITOU-BERNERT, C. T3P 189, T4P 81,

OS9:OC 13, OS11:OC 57, OS11:OC 55

POKRAJAC-BULIAN, A. T2P 170, T2P 26

POLOVINA, S. T3P 168

POMAR, C. T4P 20, T4P 79

PONS, N. T1P 129

POOBALAN, A AS4:1

POPOVIC-PEJICIC, S. T3P 111, T3P 195

POPPITT, S. T3P 122, T3P 172

PORTILLO, M. T1P 60

PORZIONATO, A. T1P 61

POSEA, M. T3P 143

POULIOU, E. T3P 116

POUWELS, S. T3P 173, T3P 212, T3P 28,

T3P 41, Т3Р 68, Т2Р 150, Т3Р 205, Т3Р 112

POUYMAYOU, B. T3P 176

POZORCLAKOVÁ, K. T4P 113

POZUELO-CARRASCOSA, D. T2P 68
PRÀ, C. T1P 118

PRAGER, G. T3P 218, T3P 130, T1P 134

PRALONG, F. T3P 61

PRAVENEC, M. T1P 55, T1P 124

PRENDERGAST, L. T1P 97

PREVEDELLO, L. T1P 118, OS9:OC 18

PRIETO-HONTORIA, P. L. T3P 16

PRIFTI, E. T1P 129

PRIOSTE, A. N. OS5:OC 33, T2P 122

PROIETTO, J. T1P 97

PROTOPOPOVA, O. T4P 70

PUHL, R.M. OS1:OC22

PUTAALA, H. T1P 109

QORBANI, M. T4P 10, T4P 116

QUARESMA, R. PL7:6

QUEIRÓS, J. T1P 145, T3P 103, T3P 102, T3P 136, T3P 152

QUEIRÓS, L. T1P 69

QUEIROZ, F. T3P 105, T3P 15

R, VINK T1P 105

RAAIJMAKERS, L. T2P 150

RABANAL, Y. T1P 114

RABEN, A. T3P 172

RADIC, R. T4P 45

RADULIAN, G. T3P 143

RAHKONEN, O. T2P 104

RÄIKKÖNEN, K. T2P 91

RAIMUNDO, A. T3P 90

RAINHO, M. T4P 124

RAJESWARAN, C. T3P 155, T2P 178

RAJU, S. T1P 16

RAMALHO, A. T1P 99, T3P 42, T3P 29

RAMALHO, S. T2P 125, T4P 89

RAMÍREZ, B. T1P 67, T3P 37

RAMIREZ-CAMPOS, E. T3P 139

RAMOS, E. T4P 3, OS10:OC 48

RAMOS, M. T1P 35

RAMUTH, H. T3P 58

RANKIN, J. T2P 56

RAPOSO, H. T1P 121

RAPPOU, E. T1P 6

RASCHELLA, G. T3P 134

RASMUSSEN, F. T4P 62

RATEL, S. T1P 37

RAVÀ, L. T2P 10

RAVENNA, M. T3P 64

RAVUSSIN, E. OS9:OC 17

RAYMONDE-PARKER, C. T3P 10, T3P 95

RAZA, G. T1P 109

RAZOLLI, D. S. T1P 65

RE, M. T1P 155

RECH, D. T1P 35

REDFERN, K. T2P 157

REDOLAR-RIPOLL, D. T1P 111

REES, G. T2P 157

RÊGO, C. T4P 71, T4P 108

REHFELD, J. T1P 54

REID, R. T3P 180

REILLY, J. T4P 104

REIN, D. T3P 26

REIS, D. T3P 149, T3P 224

REIS, F. T4P 108

REJMAN, K. T2P 148

RENDINA, R. T3P 159

RENEHAN, A. OS10:OC 46 
RESIAK, M. T2P 81

RETAT, L. T3P 133, OS7:OC 63

REY, V. T3P 55

REYNÉS, B. T3P 85, T1P 157, AS5:2

REYNOLDS, K. T2P 63

RIAHI, A. T4P 41

RIBEIRO, A. T2P 94

RIBEIRO, A. L. T3P 210

RIBEIRO, E. B. T2P 106, T1P 98, T1P 45, T1P 39

RIBEIRO, F. T2P 55

RIBEIRO, I. T2P 141

RIBEIRO, J. T4P 15

RIBEIRO, L. T3P 145, T3P 21, T3P 22

RIBEIRO, O. T1P 112, T1P 30, T3P 114

RIBEIRO, S. T4P 108

RICHELSEN, BJøRN T2P 160, T1P 32, T3P 25 ,

T3P 178, T3P 179

RIEDER, A. T3P 98, T2P 79

RIEZU-BOJ, J. I. T3P 16

RIIS-VESTERGAARD, M. T1P 32

RINNANKOSKI-TUIKKA, R. T1P 6

RIO-MORENO, M. OS3:OC 4

RISING, R. T1P 68

RISSANEN, A. OS9:OC 15, T1P 6

RISTOVSKA, G. T3P 147, T3P 148

RITTIG, N. T1P 79

RITZÉN, H. T3P 156

RIVERA, M. T2P 155, T2P 71

RIVERA-DAVILA, M. T4P 122

RIZZO, F. OS9:OC 14

ROBERT, M. T3P 157, T3P 78

ROBERTS, G. T3P 197, RS17:2

ROBERTS, N. T3P 203

ROBERTSON, M. T3P 185

ROBINSON, T. T2P 146

ROCHA, G. T3P 117

ROCHA, M. T2P 118, T2P 13

ROCHA, P. T2P 3

ROCHA, S. T4P 108

ROCHA-RODRIGUES, S. OS3:OC 5

RODA, C. OS11:OC 57

RODRIGUES, A. OS3:OC 5

RODRIGUES, A. M. T3P 2

RODRIGUES, C. S. T2P 14

RODRIGUES, C. T4P 94

RODRIGUES, P. OS3:OC 1

RODRÍGUEZ, A. T1P 58, T1P 83, T1P 67,

T3P 37, T1P 114

RODRIGUEZ-BARRANCO, M. T2P 155, T2P 71

ROEDIGER, M. T2P 120, T2P 118, T2P 89,

T2P 13, T2P 103, OS8:OC 30

ROEKENES, J. T1P 62

ROELANTS, M. T4P 34, T4P 74

ROHDE, J. T4P 48

ROMBALDOVA, M. T4P 79

ROMERO, S. T3P 37

ROMON, M. OS7:OC 66

ROMU, T. T1P 59, OS7:OC 65, RS1:3

ROOS, E. T2P 104

ROOS, E. T4P 39

ROQUE, C. T3P 114, T3P 12, T3P 164 T3P 138

ROSA, E. . OS4:OC 11

ROSA, E. C. T3P 163

ROSA, F. T3P 71
ROSADO, E. L. T3P 115, T1P 72, T2P 60,

T3P 71, T1P 71

ROSÁRIO, R. T2P 17, T4P 72, T4P 124, PL7:2

ROSAS, R. T2P 25

ROSEBOOM, T. J. OS2:OC 51

ROSENVINGE, J. RS3:4

ROSSATO, M. T1P 154, T1P 61

ROSSET, R. T3P 176

ROSSI, R. T1P 104

ROSSIGNON, F. T1P 37

ROSSMEISL, M. T1P 33

ROSSMEISLOVA, L. T1P 73

ROSSO, V. T3P 73, T3P 123

ROSSUM, E. RS18:3

ROTHMAN, A. J. T3P 66

ROTONDO, A. T4P 80

ROUMANS, N. T1P 40,T1P 63, T1P 105

ROUNGE, T. T1P 16, T4P 39

ROUSSEL, B. T1P 73

ROUSSET, M. OS9:OC 13

ROUX, C. T3P 196, T1P 54

ROVANI, J.C. T1P 121

ROVIRA, P. T2P 155

ROWICKA, G. T4P 41

ROZNOWSKI, J. T4P 128, T4P 129

RUBIO, N. T4P 122

RUDICH, A. OS11:OC 55, T1P 82

RUEGER, S. T3P 61

RUIZ-CANELA, M. T2P 147, T2P 123, T3P54

RUIZ-PEREZ, I. T2P 155, T2P 71

RUOHONEN, S. T1P 46

RUSSO, P. OS1:OC 23

RUST, P. T2P 79

RUTTER, H. OS5:OC 31, RS7:2

SÁ, R. T1P 88

SAAD, S. T2P 42

SÄÄKSJÄRVI, K. OS8:OC 25

SAARI, A. T4P 106

SAARINEN, L. T1P 6

SABINO, B. T2P 6, T4P 60

SABOYA, C. T1P 99, T3P 42, T3P 29

SA-COUTO, P. T2P 55

SADANANTHAN, S. A. T1P 22

SAFARIAN, M. T1P 23, T1P 14,T1P 156, T1P 57

SAFFERY, R. T3P 4

SAGGESE, P. OS9:OC 14

SAGIT, Z. T3P 125

SAIBENE, A. T3P 158

SAID, M. T3P 68

SAINSBURY, A. T1P 62

SAINSBURY, K. T3P 66, AS3:1, AS3:2

SAINT-MAURICE, P. T4P 121

SAJOUX, I. T3P 35

SALAS, $X . \quad$ OS1:OC22, RS8:1

SALAS-HUETOS, A. T2P 33

SALAS-SALVADÓ, J. T2P 33, T3P 54, T2P 127

SALATER, S. RS6:3

SALA-VILA, A. T2P 127

SALAZAR, M. OS3:OC 5

SALAZAR-WINTER, S. T2P 162

SALEH, N. T1P 161

SALEHI, M. T3P 72

SALEM, J. OS9:OC 13

SALIZZATO, V. OS9:OC 18

SALLES, J. T1P 37
SALO, J. T4P 106

SALOMAA, V. T2P 70

SALOMÄKI, $\mathrm{H} . \quad$ T1P 46

SALVADOR, J. T1P 83, T1P 67, T3P 37

SALVADORI, P. A. T1P 87

SALVETTI, S. T3P 134

SAMI, O. T3P 46, T3P 48

SAMMARCO, R. T1P 3, T1P 52, T1P 116, OS4:OC 11

SAMPAIO, I. T4P 36

SAMPAIO-PINTO, V. T1P 90

SANCHES, A. T2P 120

SANCHES, P. T4P 119

SÁNCHEZ, J. T1P 135

SÁNCHEZ, J. T4P 20, T4P 79, T1P 80

SÁNCHEZ-CEINOS, J. OS3:OC 4

SÁNCHEZ-INFANTES, D. T4P 123

SÁNCHEZ-LÓPEZ, M. T4P 44

SANDBU, R. RS3:4

SANDERS, M. T4P 122

SANDHOLT, C. T1P 113

SANGHERA, N. T2P 24

SANGUINETTI, E. OS9:OC 14, T1P 87

SANNA, M. T1P 118, OS9:OC 18

SANTALIESTRA-PASÍAS, A. OS1:OC 23

SANTAMARINA, A. T3P 123, T4P 119, T4P 47, T4P 77, T3P 73

SANTARPIA, L. T1P 116

SANTORO, N. RS16:3

SANTOS, A. PL7:5

SANTOS, A. T3P 103, T3P 102,

SANTOS, A. T4P 120, T4P 126, T2P 27,

SANTOS, A. T4P 38, T4P 69

SANTOS, C. T4P 94

SANTOS, E. T1P 138, T1P 18

SANTOS, F. T3P 12, T3P 164, T3P 138, T3P 214

SANTOS, I. T4P 36

SANTOS, I. T3P 7, AS3:4

SANTOS, J. T2P 118, T2P 13, T2P 103

SANTOS, J. T1P 27, T3P 124, T3P 16

SANTOS, J. T2P 4

SANTOS, M. T1P 155

SANTOS, M. OS3:OC 1

SANTOS, R. T2P 4, OS1:OC 21, T2P 17, T4P 72, T4P 98, T2P 16

SANTOS, Z. T3P 114

SANTOS-SILVA, A. T4P 108

SANZ, E. T1P 10

SANZ-SERRANO, J. T2P 123

SARAIVA, C. T3P 214, T3P 114

SARASIN, G. T1P 61

SARDINHA, L. T2P 3, RS9:1

SARES-JÄSKE, L. T2P 138

SARGEANT, J. T3P 188

SARIS, W. T1P 108, RS1:4

SARMIENTO, O. T4P 111

SARTORI, E. T3P 134

SASSI, M. T1P 35

SASSO, M. T3P 13

SAUNDERS, J. OS5:OC 32

SAVA, E. T3P 202

SAVONTAUS, E. T1P 46

SAWAMOTO, R. T3P 1

SAYEGH, R. T2P 42

SBRACCIA, P. RS3:2

SCANLON, M. T4P 61 
SCAVINI, M. T3P 158

SCHAAN, B. T2P 12

SCHAEFFERKOETTER, J. T1P 22

SCHALKWIJK, C. G. RS 2:4

SCHÄTZER, M. T2P 79

SCHENA, F. T3P 134

SCHERMANN, M. T3P 218

SCHIAPPACASSA, A. T3P 75, T3P 174

SCHIENKIEWITZ, D. T2P 174

SCHINDLER, K. T3P 98, PL1:2

SCHLEINITZ, D. T1P 110

SCHMIDT, C. T1P 51, T4P 67

SCHNEIDER, W. J. T1P 130

SCHNEITER, P. T3P 129, T3P 176, T3P 55

SCHNURR, T. T4P 11

SCHOLZ, M. T1P 110

SCHON, M. OS4:OC 12

SCHOTHORST, E. M. T1P 157, AS5:2

SCHROEDER, C. T3P 97

SCHULZ-MENGER, J. T3P 26

SCHUTZ, Y. OS7:OC 61, T1P 77, T3P 58

SCHWANBECK, M. T3P 156

SCOTT, E. RS6:1

SCOTT, S. AS3:3, AS3:4

SCRINIC, O. T1P 144

SEABRA, A. T4P 108

SEDIN, A. T3P 63

SEEBACHER, F. T1P 102

SEGRESTIN, B. T3P 78

SEIDINGER, S. C. T3P 24

SELIG, S. T2P 35

SELTHOFER, R. T4P 45

SELYATITSKAYA, V. G. T1P 8, T1P 139

SEMERARO, M. T2P 10

SENA, C. T1P 90

SENDHIL, V. T1P 22

SENE-FIORESE, M. T1P 153, T1P 12,T1P 41

SENN, A. T1P 48

SERAPHIM, P. T1P 117

SERENA, C. T1P 132

SERGEY,T. T3P 194

SERNA, U. G. T3P 119

SERRA, R. T1P 118

SERRA-MAJEM, L. T3P 54

SERRÃO, F. T4P 67

SERTESER, M. T2P 181

SEVERO, M. T3P 131

SEYSSEL, K. T3P 157

SGOUROS, K. T3P 116

SHAMI, A. T1P 25

SHAMSARDEKANI, M. T3P 187

SHAN, K. RS14:4, RS14:5

SHARMA, A. T3P 110, T2P 151, PL3:1, RS8:1

SHARMA, V. OS6:OC 38

SHARPLES, A. T3P 182

SHARPLES, P. OS6:OC 38

SHEHADEH, N. T3P 125

SHEPHERD, J. T2P 45

SHIELY, D. T4P 68, OS6:OC 41, T4P 65

SHIN, B. T2P 75

SHIN, W. T1P 7

SHIN, Y. T1P 44

SHIM, K. T2P 2

SHIRASSU, M. T2P 120

SHIRAZI-BEECHEY, S. T1P 128

SHOLL, J. T3P 149, T3P 224

SHULKINA, S. T1P 76
SHULMAN, A. OS11:OC 58

SIERRA, J. T1P 161

SIGAUDO-ROUSSEL, D. OS3:OC 6

SIGNORE, F. T2P 10

SIKLOVA, M. T1P 73

SILHAVY, J. T1P 55, T1P 124

SILVA, A. T1P 51, OS3:OC 1

SILVA, A. T2P 113

SILVA, A. T2P 12

SILVA, A. T2P 3

SILVA, A. T2P 82

SILVA, A. T3P 186

SILVA, A. T3P 210

SILVA, A. T3P 92

SILVA, A. T2P 65

SILVA, A. T2P 44

SILVA, C. T1P 21, T2P 113

SILVA, C. T3P 71

SILVA, C. T4P 121

SILVA, C. T1P 83, T1P 67, T3P 37

SILVA, D. T1P 112

SILVA, D. T4P 89

SILVA, E. OS2:OC 52

SILVA, E. T2P 120

SILVA, F. T1P 41

SILVA, G. T4P 2, T4P 15

SILVA, K. T1P 153

SILVA, L. T3P 136

SILVA, M. OS8:OC 27, T2P 5

SILVA, M. T2P 120

SILVA, M. T2P 23

SILVA, M. T4P 124

SILVA, M. S. RS17:2

SILVA, M. T3P 197

SILVA, N. T1P 88

SILVA, S. T4P 89

SILVA, T. OS2:OC 52

SILVA, W. T2P 25

SILVA-SANTOS, S. T4P 120

SILVA-VERGARA, M. T3P 59

SILVE, C. T2P 159

SILVENTOINEN, K. T2P 70

SILVESTRE, M. T3P 122, T3P 172

SILVESTRI, E. T1P 3

SIM, K. T2P 119

SIMAKOVA, M. T1P 55, T1P 124

SIMÕES, J. T2P 125

SIMOLA-STRÖM, S. T4P 39

SIMON, C. T1P 9

SIMÓN, L. T3P 139

SIMON, P. T2P 43

SIMOS, Y. T3P 140

SIMPSON, E. T3P 188

SINGHAL, R. T3P 196, T3P 182, T3P 217, T3P 167

SIQUEIRA, C. T1P 161

SIRBU, A. T3P 202

SIUBA-STRZELIŃSKA, M. T2P 62, T4P 85

SJÖBERG, A. T4P 97

SKAABY, T. T2P 85

SKILTON, M. T3P 44

SKJÅKØDEGÅRD, H. T4P 74

SKONIECZNY, P. T2P 163

SKOP, V. T1P 55, T1P 124

SLACK, E. T2P 56

SMEENK, F. T3P 41

SMIDT, H. OS7:OC 62
SMIRNOVA, E. T1P 76

SMITH, K. T3P 183

SMITH, S. RS14:4

SMULDERS, F. T3P 112

SNAJDER, D. T1P 34

SNIEHOTTA, F. T3P 2, T3P 66, AS3:1, AS3:2, AS3:3, AS3:4

SNIJDER, M. T2P 51, OS1:OC 22

SNUGGS, S. T3P 183

SOBIECKI, J. T4P 76

SOBRAL, M. T4P 67

SOBRINHO, C. T2P 4, T2P 16

SOCCI, C. T3P 158

SOCHA, P. T4P 41

SOFYA, E. T1P 142, T2P 156, T1P 150

SOKOLOVSKA, N. T3P 189, OS11:OC 55

SOL, G. T2P 131

SOLÉ, M. T2P 133, T2P 132, T2P 131

SOLORIO, A. T4P 25

SONG, M. T1P 152

SONG, S. T3P 89, T2P 75

SØRENSEN, C. T4P 11

SØRENSEN, $\mathrm{H}$. T2P 160

SøRENSEN, T. T1P 113, T2P 85, OS8:OC 29, OS10:OC 46, OS10:OC 44

SORIANO-CANO, A. T2P 68

SORLI, J. T3P 54

SOSA, R. T2P 92

SOUSA, B. T4P 19, T4P 12, T4P 126

SOUSA, C. T4P 103

SOUSA, I. . T1P98

SOUSA, I. F. T1P 39

SOUSA, M. T1P 56, T1P 20

SOUTEIRO, P. T1P 145, T3P 103, T3P 102, T3P 136, T3P 152

SOUTO, S. T3P 221, T3P 36

SOUZA, A. T2P 14

SOUZA, A. T3P 71

SOUZA, C. T2P 89

SOUZA, G. T4P 119

SOUZA, H. T3P 34

SOUZA, M. T3P 75, T3P 174

SOUZA, S. T3P 105, T3P 15

SPASOVSKI, M. T3P 147, T3P 148

SPERANZA, E. T1P 116

SPERKER, C. T3P 218

SPIELMANN, J. T1P 96

SPIROSKI, I. T3P 147, T3P 148

SPRUDZĀNE, I. T4P 29

SPRÜGEL, L. T3P 107, T3P 108

SPRUNG, V. T2P 72, T2P 164, T2P 143, T3P 23

SRAMKOVA, $\mathrm{V} . \quad$ T1P 73

STAIANO, A. T4P 107

STALKER, C. AS3:4, T3P 162, T3P 8, T2P 167, T3P 171, T3P 9

STARODUBOVA, A. T4P 17

STEELE, T. T3P 23

STEFANI, D. T3P 116

STEFANONI, N. T3P 55

STEHOUWER, C. RS 2:4, RS1:4

STEINBECK, K. AS4:1

STENZEL, A. T3P 29

STERN, N. T3P 94

STEULLET, V. T2P 74

STEVENIN, B. T2P 84

STEVENS, J. T2P 63

STICH, V. RS2:3, T1P 73 
ŠTIMAC, D. T2P 170

STOIAN, I. T1P 5

STOLBERG, C. T3P 177, T3 P181

STOUGAARD, M. T4P 48

STOYAN, I. T1P 31

STRAZDINS, L. OS5:OC 36

STRIEN, T. AS2:1

STRITECKA, H. T2P 77

STRNAD, H. T1P 55, T1P 124

STROEBELE-BENSCHOP, N. T2P 162

STROICA, L. T1P 5

STROKOVA, T. T4P 17

STRONKS, K. T2P 51, OS1:OC 22

STRUCINSKA, M. T4P 41

STUBBS, R. J. T1P 160, T2P 167, T3P 9,

T3P 8, T3P 171, T3P 162, RS9:2, AS3:1,

AS3:3, AS3:4, AS6:4

STULNIG, T. M. T3P 130, T1P 134

STUMVOLL, M. T1P 110

SUDO, N. T3P 1

SUH, H. T3P 38

SUHARA, T. T1P 89

SUHRCKE, M. OS7:OC 63

SUMITHRAN, P. T1P 97

SUMMERBELL, C. T2P 67, OS5:OC 34, T4P 53

SUN, L. T1P 22

SUPER, P. T3P 196

SURENDRAN, A. T1P 93

SUROWSKA, A. T3P 69, T3P 55

SUSANNE, R. T4P 92

SUTER, M. T3P 157, T3P 61

SUWA, M. T2P 18

SVACINA, S. T2P 46, T3P 198

SVENDSEN, M. T1P 62

SVENDSTRUP, M. T1P 113

SVENSSON, P. OS11:OC 58

SYMONDS, M. T1P 26, AS5:1

SZOSTACZUK, N. T1P 80

TABAEI, K. T1P 78, T1P 151

TÁBI, T. T1P 24

TADDEI, M. T3P 134

TAGLIERI, C. T3P 34

TAHRANI, A. T3P 196, T3P 182, T3P 217, T3P 167

TALLIS, J. T1P 38, T1P 102, T1P 146

TANG, T. T3P 167

TANGUY, M. OS11:OC 57

TAPADINHAS, A. T3P 12, T3P 138

TAPPY, L. T3P 129, T3P 69, T3P 176, T3P 55, T3P 157

TARASZEWSKA, A. T2P 83, T2P 171, T4P 85

TASHIMA, A. T1P 45

TAVARES, C. T3P 105, T3P 15

TAVARES, P. T3P 117

TAY, A. T3P 122

TAYLOR, B. OS10:OC 47

TAYLOR, R. OS10:OC 47

TEIJINK, J. T3P 41, T3P 68

TEIXEIRA, A. T4P 94

TEIXEIRA, G. T1P 117

TEIXEIRA, M. T1P 117

TEIXEIRA, P. RS17:2, T3P 7, T2P 3, AS3:1, AS3:2, AS3:3, AS3:4, T3P197

TEIXEIRA, V. T3P 131

TEJPAL, S. T2P 24

TELES, J. T1P 21, T2P 113, T2P 44
TELLESCHI, M. T1P 87

TENG, E. T1P 11,T3P 60

TEONA, S. T3P 206

TERRES, M. T1P 35

TESTA, M. T3P 159

THEMELI, A. T2P 8

THENET, S. OS9:OC 13

THIVEL, D. T4P 82

THOMAS, C. T3P 132

THOMAS, D. OS1:OC22

THOMAS, G. OS2:OC 54

THOMEE, S. AS4:1

THOMLINSON, N. T2P 67

THUESEN, B. T1P 113

THUMANN, B. OS1:OC 23

THUMSHIRN, M. T1P 128

TIIHONEN, K. T1P 109

TILG, H. T3P 213

TILLING, K. T4P 42

TINAHONES, F. T1P 132

TINOCO, A. T4P 126

TINOCO, R. T4P 124

TJONNELAND, A. T2P 85

TOCK, L. T4P 119, T1P 12

TOFT, U. T2P 85

TOKUYAMA, H. T1P 119, T1P 28

TOLEDANO, R. T3P 94

TOMÁS, C. T3P 214, T3P 138

TOMASZEWSKI, K. T2P 84, T3P 118, T2P 49

TÖNJES, A. T1P 110

TOOM, M. T1P 25

TOOMEY, E. T4P 46

TOOTLA, M. OS3:OC 3

TORCIVIA, A. OS11:OC 57, OS9:OC 13

TORDJMAN, J. T3P 189, T4P 81

TORDJMAN, K. T3P 94

TOREKOV, S. T1P 62

TORGERSEN, H. C. L. OS4:OC 7

TORNATITIS, M. OS1:OC 23

TORSONI, M. T4P 78

TOTMAN, J. T1P 22

TOULANY, M. T1P 104

TOULIS, K. OS2:OC 54

TOUMPAKARI, Z. T4P 42

TOVEE, M. OS6:OC 37, T4P 32

TOWNSEND, D. T1P 22

TRACK-SMAYRA, V. T2P 42

TRAUNER, M. T3P 98

TRAVEZ-GARCIA, A. T1P 114

TREMBLAY, A. T3P 225

TREMBLAY, M. T4P 111

TRÉPEL, D. T3P 183

TRINDADE, A. T3P 24

TRIPODI, M. T1P 87

TRNOVSKA, J. T1P 124

TRUBY, H. T1P 133, T3P 109, OS4:OC 7

TSAI, Y. T3P 19

TSAMIS, I. T3P 116

TSCHONER, A. T3P 213

TSUBOTA, A. T1P 7

TUDOR-LOCKE, C. T4P 111

TUFIK, S. T1P 155, T4P 119

TULLOCH, H. T3P 180

TUOVINEN, S. T2P 91

TURIANIKOVÁ, Z. T4P 50, T4P 113

TURNER, D. OS7:OC 63

TYNELIUS, P. T4P 62
UEBELHACK, R. T1P 11, T3P 60

UETA, T. T1P 69

UGALE, S. T3P 205

UKROPCOVA, B. OS4:OC 12

UKROPEC, J. OS4:OC 12

UNSAL, I. T2P 181

VACCARO, A. T1P 116

VAFA, M. T3P 72

VAGENA, E. T3P 82

VÄHÄTALO, L. T1P 46

VAILLANT, J. OS9:OC 13

VAKILI, M. T3P 187

VALE, L. T3P 66

VALE, M. T1P 88

VALE, S. T4P 120, T4P 126

VALENTÍ, V. T1P 58, T1P 83, T1P 67

VALERIA, N. T4P 37, T4P 14

VALERIE, D. T4P 105

VALKOVIC, P. OS4:OC 12

VALLONE, C. T2P 10

VALSESIA, A. T1P 108, T1P 95, RS1:4

VAMOSI, M. T4P 115

VANDENDORPE, S. OS7:OC 66

VARELA, A. T1P 145, T3P 103, T3P 102, T3P 136, T3P 152

VARELA-CALVIÑO, R. T3P 85

VARGAS, F. T4P 4

VASCONCELOS, C. T3P 214, T3P 114, T3P 12 , T3P 164, T3P 138

VASCONCELOS, C. T4P 89

VASHIST, Y. T3P 97

VASILEVA, O.V. T1P 139

VAZ, A. T2P 125, T2P 159

VÁZQUEZ, J. T4P 95, T1P 106

VÁZQUEZ-MARTÍNEZ, R. OS3:OC 4, T1P 114

VEDANI P. T3P 158

VEIDEBAUM, T. OS1:OC 23

VEIGA, L. T4P 86, T1P 4

VEIGA-BRANCO, M. T4P 16, T3P 86, T2P 129, T2P 141

VELAGAPUDI, V. T1P 6

VELASCO, C. T2P 92

VELÁSQUEZ, M. T1P 50

VELASQUEZ, M. T3P 93

VELISSARIS, V. T3P 82

VELLOSO, L. T1P 65

VENDRELL, J. T1P 132

VENEMA, K. OS7:OC 62

VERAS, A. T1P 117

VERBOVEN, K. RS 2:4

VERCESI, A. T1P 121

VERDIJK, L. B. RS12:3

VERES, K. T2P 160

VERGER, E. T1P 129

VERKINDT, H. T3P 157, OS7:OC 66

VERMA, S. T1P 22

VERNOCCHI, P. T2P 10

VERRAS, C. T3P 140

VESTERGAARD, $\mathrm{H} . \quad$ T1P 113

VËTRA, A. T4P 29

VETTOR, R. T1P 118, OS9:OC 18, T1P 154, T1P 61

VIANA, V. T4P 89

VICTOR, H. T1P 74

VIDAL-PUIG, A. T1P 130 
VIDGEN, H. OS6:OC 40

VIEIRA, P. T2P 3, T3P 90

VIEIRA, R. T3P 66

VIEIRA, T. T4P 78

VIEJO-SOBERA, R. T1P 111

VIETTA, G. T2P 65, T4P 103

VIEZEL, J. T3P 24

VIGNATTI, L. T2P 82

VIGUERIE, N. T1P 95, RS2:3, T1P 73

VIKTORIA, K. T4P 37

VILA, N. T3P 37

VILALLONGA, R. T3P 93

VILARRASA, N. T1P 132, RS5:2

VILCA, C. T1P 42, T1P 75

VILELA, S. OS1:OC 24

VILJAKAINEN, J. T4P 39

VILLA, P. T2P 91

VILLARROYA, F. T1P 85, T4P 123, RS2:1

VILLA-VIALANEIX, N. T1P 95

VINCENZO, O. T1P 3

VINDIGNI, V. OS9:OC 18

VINK, R. T1P 40, T1P 63

VINKER, S. OS11:OC 55

VIÑUELAS, I. C. T3P 104

VINYOLES, E. T3P 54

VIONNET, N. T3P 61

VIRTANEN, K. T1P 6

VISSER, M. T4P 117, AS2:1

VITORINO, R. T1P 90, T1P 92, AS5:5

VLADIMIR, S. T3P 194

VLASKOVSKY, P. T3P 122

VOGEL, N. OS2:OC 51

VOGELZANGS, N. T1P 108, RS1:4

VOORTMAN, T. OS10:043

VORONTZOV, P. T4P 70

VRBIK, K. T3P 198

VUORELA, N. T4P 106

WAISBERG, J. T2P 120

WALENTUKIEWICZ, A. T2P 81, T2P 163

WALLACE, N. T3P 137, T3P 101, T2P 34

WANG, J. T4P 87

WARD, M. T4P 130

WATTS, S. W. AS5:6

WEBBER, L. T3P 133, OS7:OC 63

WEIDERPASS, E. T4P 39, T1P 16

WEISZ, A. OS9:OC 14

WEKER, H. T4P 41

WEKKER, V. OS2:OC 51

WEST, J. T1P 59, OS7:OC 65, RS1:3

WESTERSTÅHL, M. OS6:OC 39

WETZELS, S. RS 2:4

WHARTON, S. RS3:3

WHETSTONE, L. T3P 132

WHITE, M. T2P 67, T3P 2, T3P 66

WHITTLE, A. T1P 130

WHYBROW, S. T1P 160

WICKLUM, S. T2P 151

WIECH, M. T4P 41

WIEDER, T. T1P 104

WIELE, T. T4P 26

WIKLAND, K. T4P 97

WILCZYNSKA, D. T2P 163

WILD, B. T2P 79

WILDING, J. T2P 72, T2P 164, T2P 143

WILDING, P. T3P 23

WILKENS, L. T2P 45
WILLAN, K. T2P 175

WILLIAMS, G. T2P 69

WILSON, V. T3P 167

WIMER, G. T4P 1

WIND, P. OS9:OC 13

WINKENS, L. AS2:1

WINTHER, R. T1P 147

WITT, H. T3P 26

WOHLAND, T. T1P 110

WÖLNERHANSSEN, B. T1P 48, T1P 128,

OS2:OC 50, T1P 54

WOLNICKA, K. T2P 83, T2P 171, T4P 85

WOLTER, S. T3P 97, T3P 51

WOODS, C. T2P 36

WORONKOWICZ, A. T4P 76

WOUTERS, K. RS 2:4

WRAW, C. OS10:OC 45

WREIDEN, W. T2P 67

WRIGHT, P. T3P 66

WROBLEWSKI, I. T1P 131

WRZESIŃSKA, M. T3P 190

WU, P. T2P 166

WULFSOHN, R. T3P 64

WYKE, S. T3P 197, RS17:2

WYKYPIEL, H. T3P 213

XIMENES, A. T2P 60

$X U, Q . \quad T 1 P 105$

XUE, H. M. T2P 47, T2P 154, T2P 139, T2P 95

YAFI, M. T4P 122

YAGI, K. T2P 18

YAMAGA, M. T1P 119, T1P 28

YANG, K. T2P 166, OS9:OC 16

YANG, W. T2P 166

YARDIM, N. RS8:3

YETMAN, R. T4P 122

YIN, J. T2P 47, T2P 95, T2P 154

YODER, R. T3P 137, T3P 101, T2P 34

YOKOCHI,T. T2P 18

YOKOTE, K. T1P 28, T1P 119

YOO, S. T2P 11, T2P 177

YOON, Y. T2P 38

YOOSUF, S. T2P 124

YUN, Y. T2P 38

YUZBASHIAN, E. T1P 78, T1P 151, T1P 23,

T1P 14, T1P 156, T1P 57

YVES, B. T4P 7, T4P 43

ZADEH-VAKILI, A. T1P 78, T1P 151

ZAID, W. T3P 125

ZAJT, J. T2P 163

ZALEWSKA, A. T1P 131

ZAMRAZILOVA, $\mathrm{H} . \quad$ T2P 52

ZANCHI, D. T1P 54

ZANETTI, H. T3P 59

ZARKESH, M. T1P 151,T1P 14,T1P 156,

T1P 57, T1P 23

ZEEVI, D. RS1:2

ZEGA, A. T1P 87

ZENDZIAN-PIOTROWSKA, M. T1P 131

ZEPEDA, Y. T2P 92

ZERWECK, I. T2P 162

ZEYDA, M. T1P 134

ZHANG, J. T2P 139, T2P 95, T2P 154

ZHANG, S. T3P 44

ZHANG, Y. T4P 87

ZHAO, P. T4P 111
ZIAODINI, H. T4P 10, T4P 116

ZIDEK, V. T1P 55, T1P 124

ZIMBERG, I. T1P 155

ZINS, M. OS8:OC 28

ZOETENDAL, E. OS7:OC 62

ZOHAR, A. T3P 56, T4P 127

ZUCKER, J. T3P 189, OS11:OC 55, T1P 129

ZUCKERMAN, N. T3P 125

ZULFIQAR, T. OS5:OC 36

ZULUETA, M. T3P 139

ZÚÑIGA, A. T3P 93

ZWINDERMAN, A. OS2:OC 51

ZWYGART, K. T3P 69 